
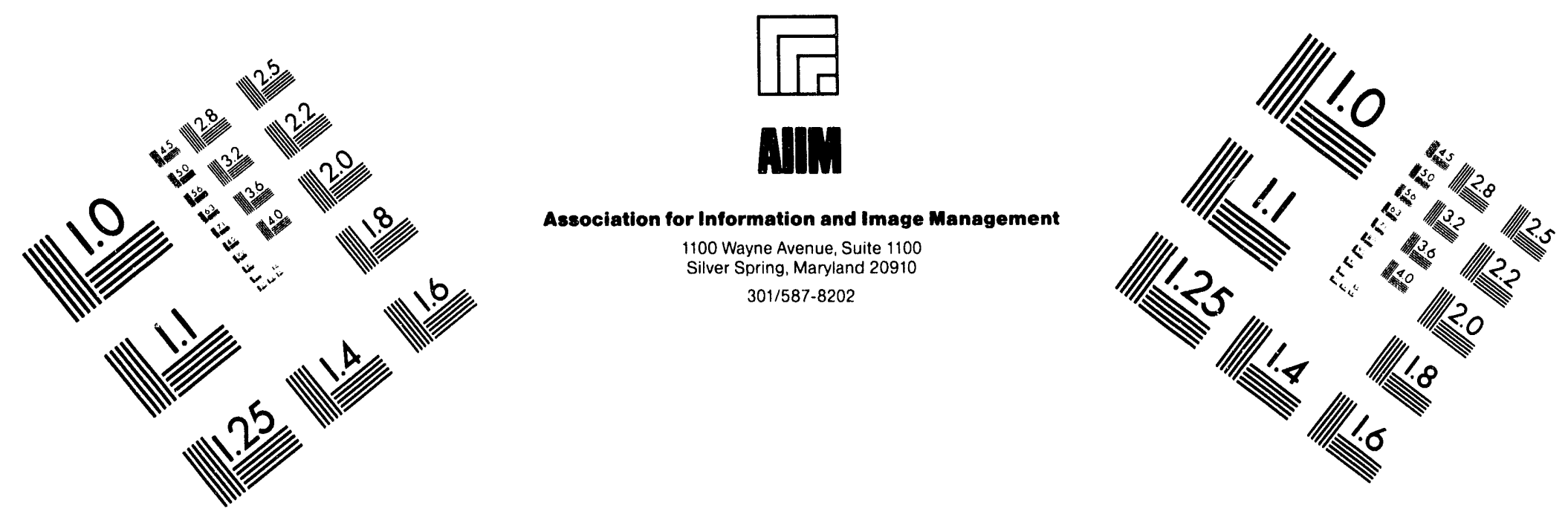

\title{
Centimeter
}

$\begin{array}{llllllllllllllll}1 & 2 & 3 & 4 & 5 & 6 & 7 & 8 & 9 & 10 & 11 & 12 & 13 & 14 & 15 & \mathrm{~mm}\end{array}$

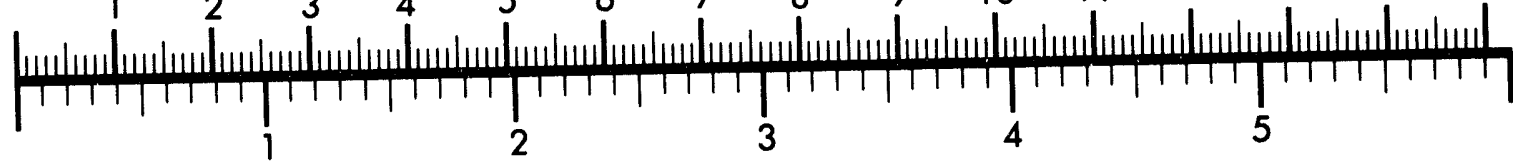
Inches
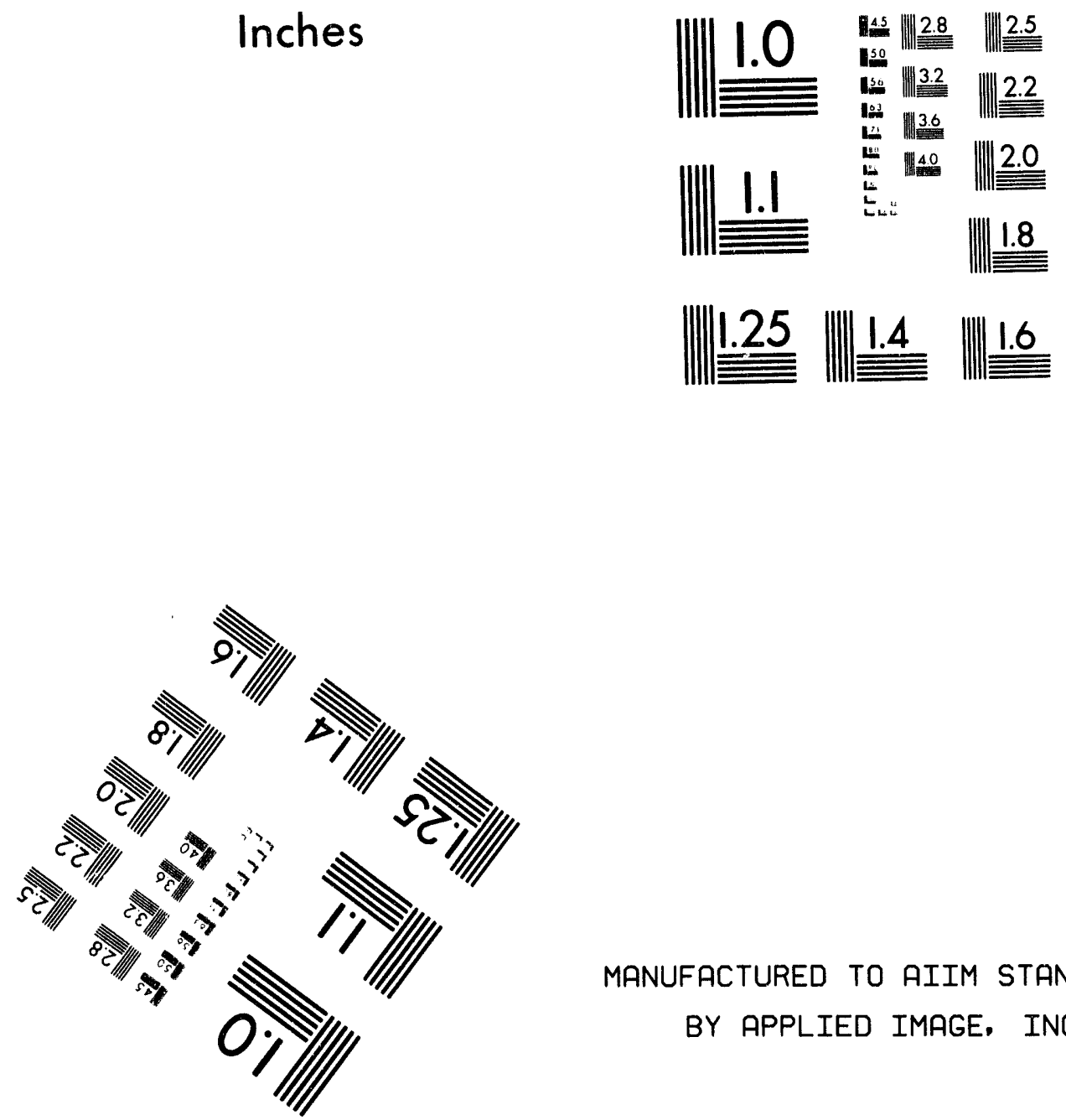

MANUFACTURED TO AIIM STANDARDS

BY APPLIED IMAGE, INC.

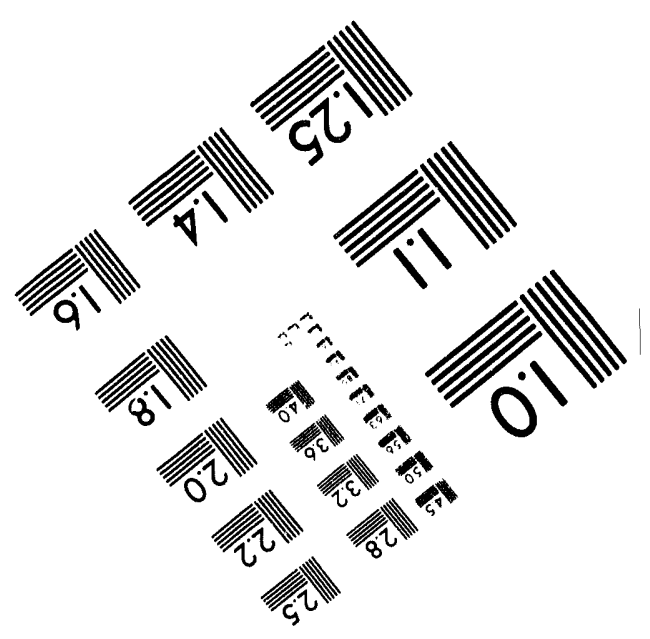



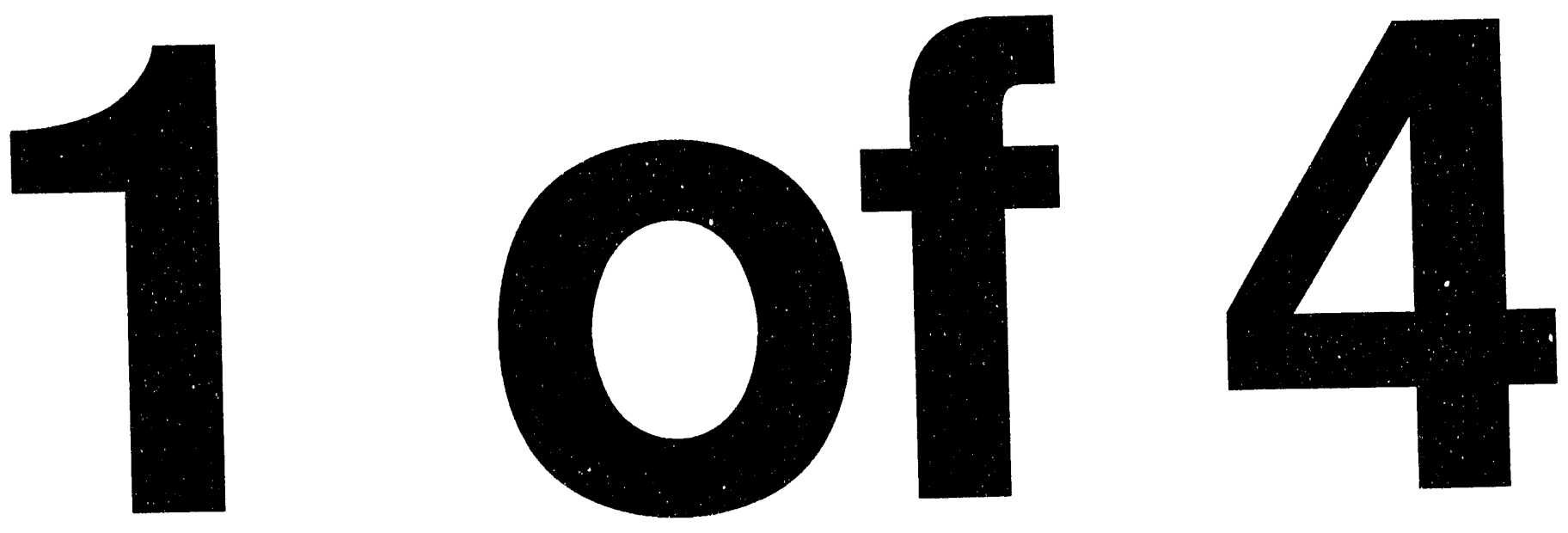
DOE/EIA-0531(92)

Distribution Category UC-950

\section{Electric Trade in the United States 1992}

\section{September 1994}

\section{Energy Information Administration}

Office of Coal, Nuclear, Electric and Alternate Fuels

U.S. Department of Energy

Washington, DC 20585 


\section{Contacts}

Questions regarding the contents of this report may be directed to:

Survey Management Division

Energy Information Administration, EI-52

U.S. Department of Energy

1000 Independence Avenue, S.W.

Washington, DC 20585

FAX (202/254-5765)

Questions of a general nature should be directed to:
Howard Walton (202/254-5500),

Director of the Survey Management Division;

Neal Moerschel (202/254-5640),

Chief of the Electric Data Systems Branch;

Larry Prete (202/254-5671),

Team Leader of the Annual Systems Team.

Principal data analyst and author for the Electric Trade in United States report was John W. Makens of the Survey Management Division. 


\section{Contents}

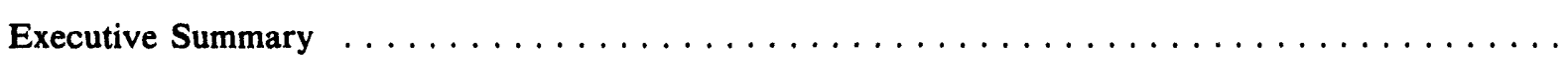

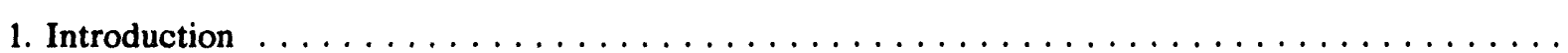

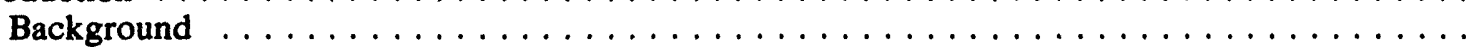

The Electric Utility Industry Structure $\ldots \ldots \ldots \ldots \ldots \ldots \ldots \ldots \ldots \ldots$

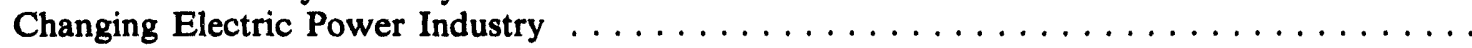

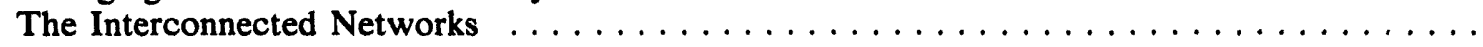

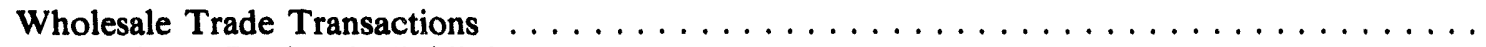

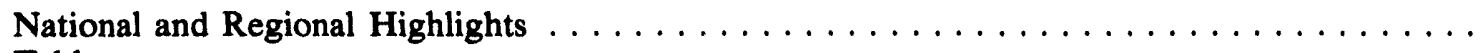

Tables

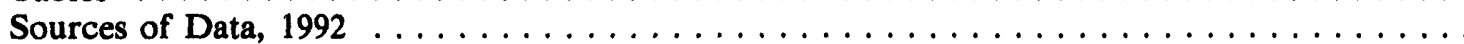

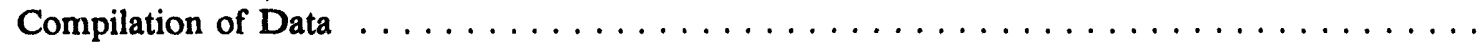

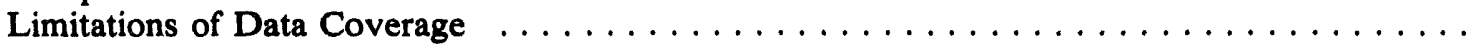

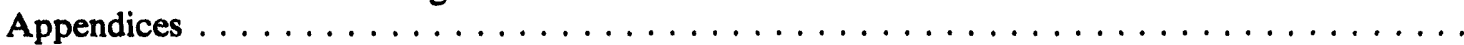

Glossary . . . . . . . . . . . . . . . . . . . . . . . . . . . .

vii

Power Transactions by Investor-Owned Utilities $\ldots \ldots \ldots \ldots \ldots \ldots \ldots \ldots$

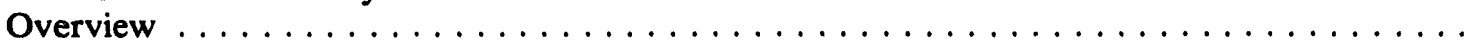

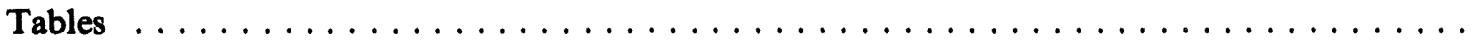

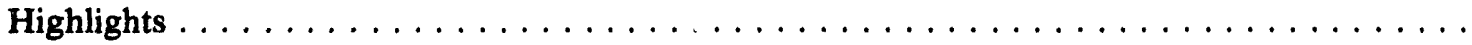

Summary

3. Bulk Power Transactions by Federal and Other Publicly Owned Utilities

Overview

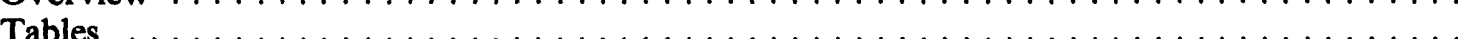

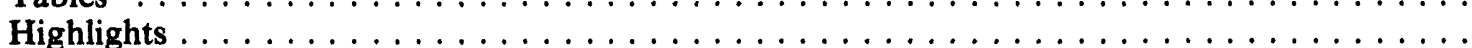

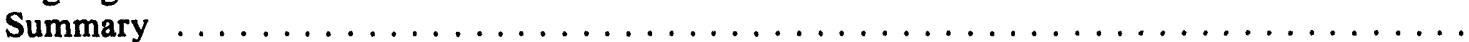

4. Bulk Power Transactions by Cooperative Borrowers $\ldots \ldots \ldots \ldots \ldots \ldots \ldots \ldots \ldots \ldots \ldots$

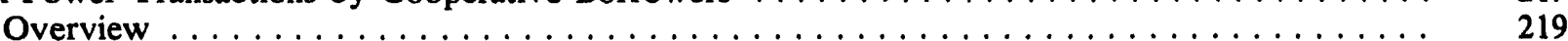

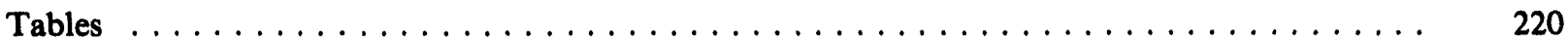

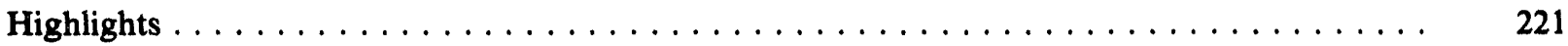

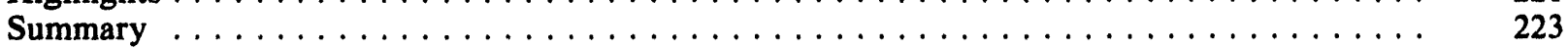

5. Selected Information on the Physical Transmission System $\ldots \ldots \ldots \ldots \ldots \ldots \ldots \ldots \ldots \ldots$

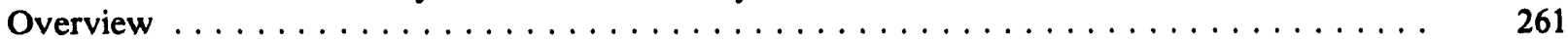

Tables $\ldots \ldots \ldots \ldots \ldots \ldots \ldots \ldots \ldots \ldots \ldots$

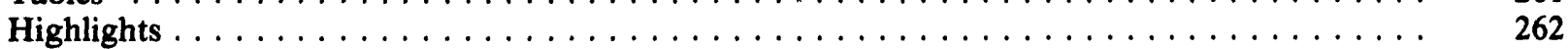

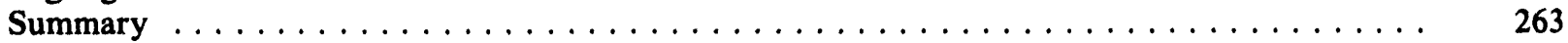

Appendices

A. Federal Energy Regulatory Commission Regulation of the Electric Utility Industry . . . . . 301

B. State, NERC Region, and Data Sources for Electric Utilities . . . . . . . . . . . . . . 307

C. Fundamentals of Electric Power Transmission and Integrating Nonutility Generators . . . . 339

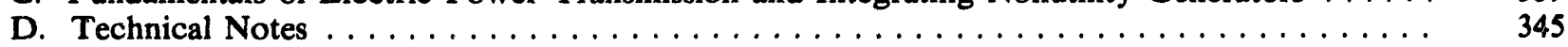

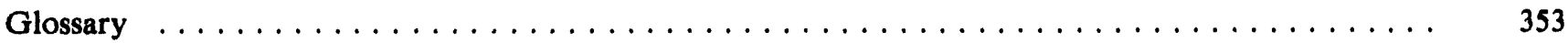


1. Number of Electric Utilities by Class of Ownership Within NERC Region, $1992 \ldots \ldots$. . .

Page

2. Energy Account Balance by Class of Ownership, $1992 \ldots \ldots \ldots \ldots \ldots \ldots \ldots \ldots$

3. Components of Source and Disposition of Electricity by NERC Region, $1992 \ldots \ldots \ldots$.

4. Electric Trade in the United States by NERC Region, 1986 Through $1992 \ldots \ldots \ldots \ldots \ldots$

5. Intra- and Interregional Purchases and Sales for Resale by Selected Ownership Classes,

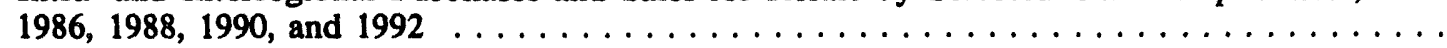

6. Firm and Nonfirm Purchases and Sales for Resale by Investor-Owned Utilities, by NERC

Region, 1986, 1988, 1990, and $1992 \ldots \ldots \ldots \ldots \ldots \ldots \ldots \ldots \ldots \ldots \ldots$

7. Wheeling Trade in the United States by NERC Region, 1986 Through $1992 \ldots \ldots \ldots \ldots$

8. Electricity Purchases by Investor-Owned Utilities, by NERC Region, $1992 \ldots \ldots \ldots \ldots$

9. Electricity Sales for Resale by Investor-Owned Utilities, by NERC Region, $1992 \ldots \ldots$. . .

10. Investor-Owned Utility Exchanges of Electricity with All Ownership Classes, by NERC

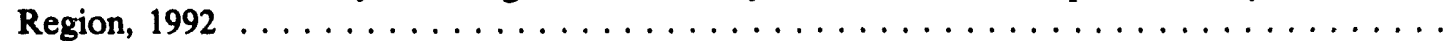

11. Noninvestor-Owned Utility Exchanges of Electricity, by NERC Region, $1992 \ldots \ldots \ldots \ldots$

12. Electricity Wheeling for All Ownership Classes by Investor-Owned Utilities, by NERC

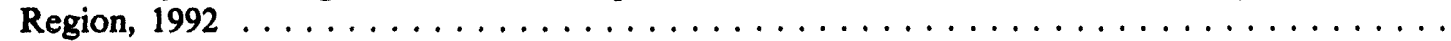

13. Electricity Wheeling by All Ownership Classes to Investor-Owned Utilities, by NERC

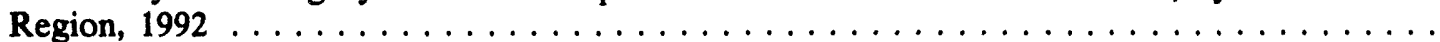

14. Purchases by Cooperative Borrowers, by NERC Region, $1992 \ldots \ldots \ldots \ldots \ldots \ldots \ldots$

15. Receipts by Investor-Owned Utilities Within and Between NERC Regions, $1992 \ldots \ldots \ldots$

16. Deliveries by Investor-Owned Utilities Within and Between NERC Regions, $1992 \ldots \ldots$

17. Electricity Transactions by Investor-Owned Utilities in Alaska and Hawaii, $1992 \ldots \ldots$. . .

18. U.S. Electricity Trade with Canada and Mexico by NERC Region, $1992 \ldots \ldots \ldots \ldots$. . . .

19. Electricity Purchases by Investor-Owned Utilities, by State, $1992 \ldots \ldots \ldots \ldots \ldots \ldots$

20. Electricity Sales for Resale by Investor-Owned Utilities, by State, $1992 \ldots \ldots \ldots \ldots \ldots$. . .

21. Electricity Exchanges by Investor-Owned Utilities, by State, $1992 \ldots \ldots \ldots \ldots \ldots \ldots \ldots$

22. Electricity Purchases by Investor-Owned Utilities Not Reported to the Federal Energy Reg-

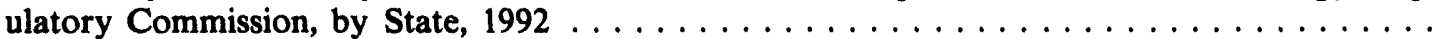

23. Electricity Wheeling by Investor-Owned Utilities, by State, $1992 \ldots \ldots \ldots \ldots \ldots \ldots \ldots \ldots$

24. Electricity Wheeling to Investor-Owned Utilities, by State, $1992 \ldots \ldots \ldots \ldots \ldots \ldots \ldots$

25. Electricity Purchases by Federal Utilities, Fiscal Year Ending September 30, $1992 \ldots \ldots$

26. Electricity Sales for Resale by Federal Utilities, Fiscal Year Ending September 30, 1992 . . .

27. Electricity Purchases by State and Other Government Utilities, Fiscal Year $1992 \ldots \ldots$. . .

28. Electricity Sales for Resale by State and Other Government Utilities, Fiscal Year 1992 . . . .

29. Electricity Purchases by Municipal Utilities, Fiscal Year $1992 \ldots \ldots \ldots \ldots \ldots \ldots \ldots \ldots$

30. Electricity Sales for Resale by Municipal Utilities, Fiscal Year $1992 \ldots \ldots \ldots \ldots \ldots \ldots \ldots$

31. Electricity Purchases by Publicly Owned Utilities Not Reported on Form EIA-412, by

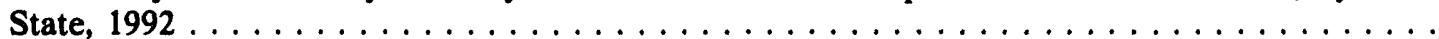

32. Electricity Exchanges and Wheeling by Publicly Owned Utilities, $1992 \ldots \ldots \ldots \ldots \ldots$

33. Electricity Purchases by the Power Supply Segment of Cooperative Borrowers, by State,

1992 . . . . . . . . . . . . . . . . . . . . . . . . . . . . . . . . . . . . . . . .

34. Electricity Purchases by the Distribution Segment of Cooperative Borrowers, by State, 1992

35. Electricity Purchases by Electrical Cooperatives Not Reported to the Rural Electrification

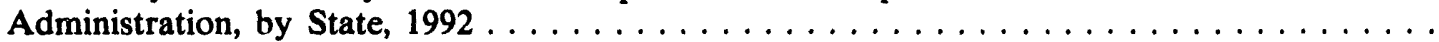

36. Electricity Exchanges and Wheeling by Cooperative Borrowers, by State, $1992 \ldots \ldots \ldots$.

37. Transmission Line Statistics of Investor-Owned Utilities, by NERC Region, $1992 \ldots \ldots$. .

38. Transmission Line Statistics of Investor-Owned Utilities, $1992 \ldots \ldots \ldots \ldots \ldots \ldots \ldots$

39. Transmission Line Statistics of Public Utilities, by NERC Region, Fiscal Year $1992 \ldots \ldots$. .

40. Transmission Line Statistics of Public Utilities, Fiscal Year $1992 \ldots \ldots \ldots \ldots \ldots \ldots \ldots$

41. Transmission Lines Added by Investor-Owned Utilities, by NERC Region, $1992 \ldots \ldots \ldots$

42. Transmission Lines Added by Investor-Owned Utilities, $1992 \ldots \ldots \ldots \ldots \ldots \ldots \ldots$

43. Watthour Meter and Line Transformer Statistics of Investor-Owned Utilities, by NERC Re-

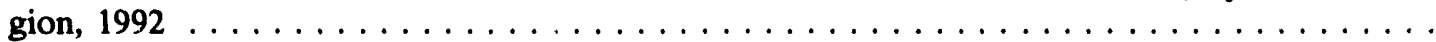

44. Watthour Meter and Line Transformer Statistics of Investor-Owned Utilities, $1992 \ldots \ldots$

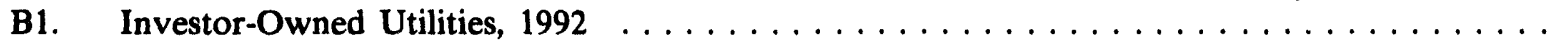

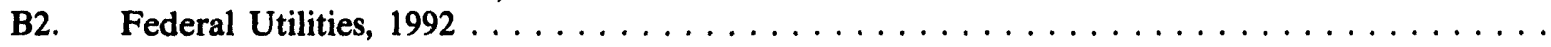

B3. State and Other Government Utilities, $1992 \ldots \ldots \ldots \ldots \ldots \ldots \ldots \ldots$

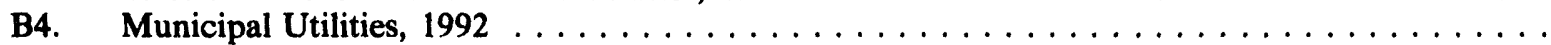




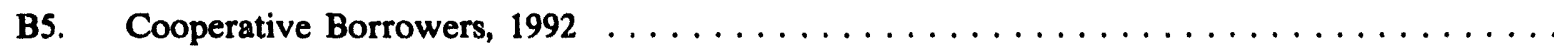

B6. Electric Utilities Not Reported Elsewhere in Surveys Reporting Detailed Transactions, 1992

B7. Electric Power Marketers Authorized by FERC, as of December 31, $1993 \ldots \ldots$ 


\section{Illustrations}

Page

1. Electric Utility Density by County in the United States $\ldots \ldots \ldots \ldots$

2. North American Electric Reliability Council Region Map for the United States . . . . . . . .

3. Electricity Purchases and Costs by Investor-Owned Utilities by NERC Region, 1992 . . . . 9

4. Electricity Sales for Resale and Revenue by Investor-Owned Utilities by NERC Region,

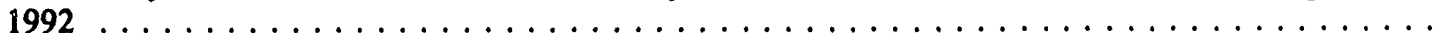

5. U.S. Electricity Trade with Canada and Mexico by NERC Region, $1992 \ldots \ldots \ldots$

6. Electricity Purchases and Costs by the Top Ten Investor-Owned Utilities, $1992 \ldots \ldots$

7. Electricity Sales for Resale and Revenue by the Top Ten Invi stor-Owned Utilities, 1992 . . .

8. Electricity Purchases and Costs by Federal Utilities, Fiscal Year $1992 \ldots \ldots \ldots$

9. Electricity Sales for Resale and Revenue by Federal Utilities, Fiscal Year 1992 . . . . . . . .

10. Electricity Purchases and Costs by the Top Ten State and Other Government Utilities, Fis-

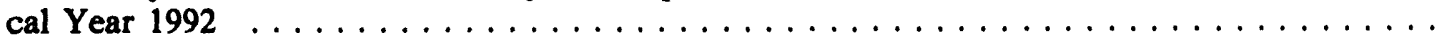

11. Electricity Sales for Resale and Revenue by the Top Ten State and Other Government Utilities, Fiscal Year $1992 \ldots \ldots \ldots \ldots \ldots \ldots \ldots \ldots \ldots \ldots \ldots \ldots$

12. Electricity Purchases and Costs by the Top Ten Municipal Utilities, Fiscal Year $1992 \ldots \ldots$

13. Electricity Sales for Resale and revenue by the Top Ten Municipal Utilities, Fiscal Year

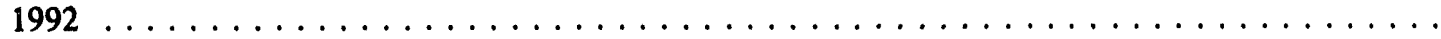

14. Electricity Purchases by Cooperative Borrowers by State, $1992 \ldots \ldots \ldots$. . . . . . . . . . . .

15. Electricity Purchase Costs by Cooperative Borrowers by State, $1992 \ldots \ldots$. . . . . . . . . . .

16. Electricity Purchases and Costs by the Top Ten Cooperative Borrowers, $1992 \ldots \ldots$

17. Electricity Purchases by Cooperative Borrowers, $1992 \ldots \ldots \ldots \ldots \ldots$

18. Selected High-Voltage Transmission Lines in the Contiguous United States . . . . . . . . 


\section{Executive Summary}

Electric Trade in the United States 1992 (ELECTRA) is the fourth in a series of reports on wholesale power transactions prepared by the Electric Data Systems Branch, Survey Management Division, Office of Coal, Nuclear, Electric and Alternate Fuels, Energy Information Administration (EIA). The electric trade data are published biennially. The first report presented 1986 data. This report provides information on the industry during 1992.

Since EIA began publishing wholesale electric trade data, electricity purchases have increased by 77 percent between 1986 and 1992. This is illustrated in a regional comparison of purchases and sales for resale by investor-owned electric utilities (Table ES-1) for 1986, 1988,1990 , and 1992. Transactions by investor-owned electric utilities represent over 55 percent of the transactions in the bulk power market.

Some of the underlying reasons that affect growth in U.S. wholesale electric trade include the following:

- Overa'1 U.S. consumption of electric energy continues tc increase. Many electric utilities are increasing eiectricity purchases to satisfy customer demand in lieu of constructing new power plants.

- Electric utility electricity purchases from nonutility power producers have grown by an average of 27 percent per year since 1986. More electric generating facilities are being established whose primary line-of-business is generating electricity under Federal statutes covering nonutility generators.

- Competition in the wholesale electricity market continued to expand in 1992. The Energy Policy Act of 1992, signed into law in November 1992, is expected to increase competition in the future. The Act established a new class of nonutility generators, called "Exempt Wholesale Generators," and amended portions of the Public Utility Holding Company Act of 1935.

- Ove all electric system reliability is maintained as utilities cooperate in wholesale electric trade during contingency and emergency situations. Backup power purchases by investor-owned utilities, defined as transactions associated with a demand charge only, increased from $\$ 64$ million in 1986 to $\$ 181$ million in 1992.
- Wholesale transmission services (wheeling) will grow as the result of implementing the Energy Policy Act of 1992.

Electric trade is an important part of the electric power industry as approximately half of all electricity generated is purchased (or exchanged) in the wholesale market before being sold to ultimate consumers. Results of the 1992 date include:

- Purchases from all sources by major investorowned utilities totaled 342.5 billion kilowatthours of firm power for $\$ 16.4$ billion and 299.7 billion kilowatthours of nonfirm energy for $\$ 9.5$ billion. The combined amount of firm and nonfirm energy in 1992 represent an 18-percent increase over the amount purchased in 1990, and an increase in costs of 19.5 percent. Firm purchases, in billion kilowatthours, increased by 5.5 percent with the largest increases being posted by investor-owned utilities in the Northeast Power Coordination Council ( 74.9 billion kilowatthours or a 9.6 billion kilowatthour change). The Western Systems Coordinating Council (with 83.0 billion kilowatthours) had the largest regional total.

- Municipal electric utilities delivered to investorowned utilities 9.3 billion kilowatthours of firm power for a cost of $\$ 479.6$ million and 5.9 billion kilowatthours of nonfirm energy for a cost of $\$ 202.9$ million. This represents a total decrease of 5.4 percent in purchases and a 4.0 percent increase in cost. Investor-owned electric utilities reported paying 16.9 percent more than in 1990 and received 11.2 percent less in nonfirm energy for purchases from municipal utilities.

- Purchases by cooperatives have changed on a fairly consistent basis since 1986. Every 2 years equaled about a 10-million kilowatthour increase. In 1992, cooperative utilities reported purchasing 281 billion kilowatthours at a cost of $\$ 11.6$ billion. This represents an increase of 2.8 percent in power purchased and a 4.2-percent increase in associated costs from 1990 levels. Of the 281 billion kilowatthours purchased by cooperatives, over 175 billion kilowatthours (62 percent) costing $\$ 7.9$ billion, came from other cooperative utilities. This was over three times as much power as was purchased by cooperative utilities from Federal utilities. 
- In 1992, the U.S. electricity purchases from Canada returned to pre-1990 levels (35.2 billion kilowatthours in 1992), while trade with Mexico remained nearly constant $(2.0$ billion kilowatthours in 1992). In 1992, the Province of British Col.mbia led in exports to the United States (11.8 billion kilowatthours), while the Province of Quebec increased its exports to the northeastern part of the United States to 8.9 billion kilowatthours. The Province of Manitoba came in a close third by selling 8.5 billion kilowatthours to the upper midwestern United States. Exports of electricity to Canada were 7.9 billion kilowatthours. Utilities in the western United States were first in sales by selling over 3 billion kilowatthours for consumption in the Province of British Columbia.
- Investor-owned electric utilities reported adding 2,565 miles of new transmission lines of a size of 138 kilovolts $(\mathrm{kV})$ or greater to their electrical systems in 1992. The cost for land right-of-way, new structures, conductors, and line materials for these additions and upgrades was nearly $\$ 375$ million. Florida Power and Light, whose electrical system was severely damaged by Hurricane Andrew, led the other reporting utilities in adding new (or in this case replacing) higher transmission voltage support for its electrical system. Public Service Company of Coloradn had the largest increase, but this represented the addition of existing lines acquired through the purchase of the assets of a bankrupt cooperative.

Table ES1. Comparison of Electric Trade Data by North American Electric Rellablity Councll Reglon for Years 1986, 1988, 1990, and 1992

\begin{tabular}{|c|c|c|c|c|c|c|}
\hline \multirow{2}{*}{ NERC Region } & \multirow{2}{*}{ Coographic Region } & \multicolumn{4}{|c|}{ Elllion Kllowatthours } & \multirow{2}{*}{$\begin{array}{l}\text { Porcent } \\
\text { Change } \\
1986-1802\end{array}$} \\
\hline & & 1992 & 1990 & 1888 & 986 & \\
\hline & \multicolumn{6}{|c|}{ Purcheses by Inveator-Owned Utilities } \\
\hline ECAR .................. & $\begin{array}{l}\text { Indlana; Ohio; West Virginia; and portions of } \\
\text { Kentucky, Maryland, Michigan, } \\
\text { Pennsytvania, and Virginia }\end{array}$ & 95.7 & 79.2 & 34.7 & 36.6 & 161.5 \\
\hline MAIN ..................... & $\begin{array}{l}\text { Illinois; and portions of lowa, Michigan, } \\
\text { Missouri, and Wisconsin }\end{array}$ & 28.6 & 21.8 & 8.1 & 6.8 & 320.6 \\
\hline MAPP(US) .......... & $\begin{array}{l}\text { Minnesota; North Dakota; and portions of } \\
\text { lowa, Montana, Nebraska, South Dakota, } \\
\text { Wisconsin, and Wyoming }\end{array}$ & 26.4 & 20.0 & 15.6 & 15.5 & 70.3 \\
\hline NPCC(US) & $\begin{array}{l}\text { Connecticut; Maine; Massachusetts; Now } \\
\text { Hampshire; New York; Rhode Island; and } \\
\text { Vermont }\end{array}$ & 141.9 & 104.1 & 102.3 & 103.3 & 37.4 \\
\hline SERC & $\begin{array}{l}\text { Alabama; Florida; Georgia; North Carolina; } \\
\text { South Carolina; Tennessee; and portions } \\
\text { of Kentucky, Mississippi, and Virginia }\end{array}$ & 86.8 & 88.5 & 52.6 & 38.1 & 127.8 \\
\hline SPP ....................... & $\begin{array}{l}\text { Arkansas; Louisiana; Kansas; Oklahoma; and } \\
\text { portions of Mississippl, Missouri, New } \\
\text { Mexico, and Texas }\end{array}$ & 49.1 & 37.7 & 40.7 & 27.2 & 80.5 \\
\hline WSCC(US) ......... & $\begin{array}{l}\text { Arizona; California; Colorado; Idaho; Nevada; } \\
\text { Oregon; Utah; Washington; and portions } \\
\text { of Montana, Nebraska, New Mexico. } \\
\text { South Dakota, and Wyoming }\end{array}$ & 132.0 & 141.3 & 102.6 & 97.4 & 35.5 \\
\hline \multirow[t]{2}{*}{$\begin{array}{l}\text { Contgurous } \\
\text { U.8. Total ... }\end{array}$} & & 668.6 & 563.3 & 415.8 & 371.6 & 77.2 \\
\hline & \multicolumn{6}{|c|}{ Sales for Reale by Investor-Owned Utillties } \\
\hline 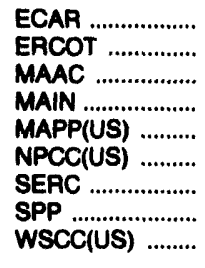 & Same As Above & $\begin{array}{r}132.0 \\
8.0 \\
45.8 \\
26.5 \\
18.6 \\
95.5 \\
89.3 \\
45.3 \\
53.3\end{array}$ & $\begin{array}{r}117.6 \\
9.7 \\
19.1 \\
29.2 \\
18.0 \\
70.5 \\
93.5 \\
35.5 \\
51.0\end{array}$ & \begin{tabular}{r|}
99.5 \\
10.7 \\
14.7 \\
12.2 \\
9.4 \\
66.6 \\
76.3 \\
36.2 \\
40.2
\end{tabular} & $\begin{array}{r}79.1 \\
10.4 \\
14.4 \\
14.8 \\
6.5 \\
78.9 \\
70.4 \\
36.1 \\
36.1\end{array}$ & $\begin{array}{r}66.8 \\
-23.1 \\
218.8 \\
79.1 \\
186.2 \\
21.0 \\
26.9 \\
25.5 \\
47.4\end{array}$ \\
\hline $\begin{array}{l}\text { Contipuous } \\
\text { U.S. Total ... }\end{array}$ & & 514.4 & 444.2 & 368.0 & 346.6 & 48.4 \\
\hline
\end{tabular}


The primary sources of utility data for this publication are shown in Table ES2.

Table ES2. Electriclty Data, by Source

\begin{tabular}{|c|c|}
\hline Form & Data Utilized \\
\hline 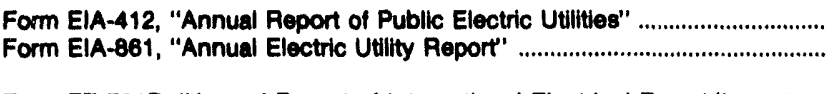 & $\begin{array}{l}\text { Detalled transactions for sales for resale and purchases. } \\
\text { Aggregated transactions for sales for resale, purchases, exchanges, } \\
\text { and wheoling. }\end{array}$ \\
\hline $\begin{array}{l}\text { Form FE-781R, "Annual Report of International Electrical Export//mport } \\
\text { Data" }\end{array}$ & $\begin{array}{l}\text { Detailed transactions for exports from and imports to the United } \\
\text { States }\end{array}$ \\
\hline 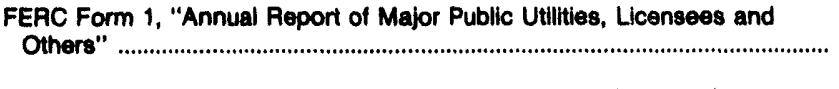 & $\begin{array}{l}\text { Detailed transactions for sales for resale, purchases, exchanges, and } \\
\text { wheeling }\end{array}$ \\
\hline $\begin{array}{l}\text { FERC Form 1-F, "Annual Report of Nonmajor Public Utilities and } \\
\text { Licensees" }\end{array}$ & $\begin{array}{l}\text { Detalled transactions for sales for resale, purchases, exchanges, and } \\
\text { wheeling. }\end{array}$ \\
\hline 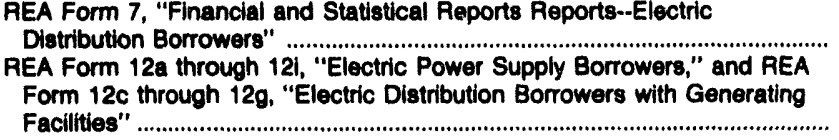 & Detalled transactions for purchases. \\
\hline
\end{tabular}




\section{Introduction}

\section{Background}

Wholesale trade in electricity plays an important role for the U.S. electric utility industry. Wholesale, or bulk power, transactions allow electric utilities to reduce power costs, increase power supply options, and improve reliability. In 1992, the wholesale trade market totaled 1.8 trillion kilowatthours, about 66 percent of total sales to ultimate consumers.

This publication, Electric Trade in the United States 1992 (ELECTRA), is the fourth in a series of reports on wholesale power transactions prepared by the Electric Data Systems Branch, Survey Management Division, Office of Coal, Nuclear, Electric and Alternate Fuels, Energy Information Administration (EIA). The electric trade data are published biennially. The first report presented 1986 data, and this report provides information on the electric power industry during 1992.

The electric trade data collected and presented in this report furnish important information on the wholesale structure found within the U.S. e'ectric power industry. The patterns of interutility trade in the report support analyses of wholesale power transactions and provide input for a broader understanding of bulk power market issues that define the emerging national electric energy policies. The report includes information on the quantity of power purchased, sold, exchanged, and wheeled: the geographical locations of transactions and ownership classes involved; and the revenues and costs. Inforniation on the physical transmission system are being included for the first time in this publication. Transmission data covering investor-owned electric utilities were shifted from the Financial Statistics of Selected Investor-Owned Electric Utilities to the ELECTRA publication. Some of the prominent features of this year's report include information and data not published before on transmission lines for publicly owned utilities and transmission lines added during 1992 by investor-owned electric utilities.

\section{The Electric Utility Industry Structure}

The utilities covered in this publication include all U.S. electric utilities in the 50 States and the District of Columbia. Electric utilities fall into three basic ownership classifications: investor-owned (privately owned), publicly owned, and cooperative. Publicly owned utilities may be Federally owned, State-owned, or municipally owned. In 1992, there were 3,232 electric utilities in the United States. Of these, 2,027 were publicly owned (including 10 Federal utilities), 943 were rural electric cooperatives, and 262 were investor-owned utiitities (Table 1). Some of these electric utilities have service territories extending beyond a single county or parish. Others just serve a municipality or part of a county. Most counties in the United States have more than a single utility and some parts of the country have more than 10 electric utilities operating in a county (Figure 1). Many of the Nation's electric utilities are exclusively distribution utilities, that is, they purchase wholesale power from other utilities and distribute, on their own distribution lines, the electricity to ultimate consumers. For example, fewer than $1,000^{1}$ of the 3,232 utilities in the United States are engaged in power generation. In order to move electricity from utilities with generating capability to the distribution utilities, an extensive system of high voltage transmission lines is operated by the Nation's largest utilities. This transmission network permits the electric trade between utilities, because without it electricity could not be moved from power plants to the thousands of distribution systems and millions of consumers of electric power.

\section{Investor-Owned Utilities}

There are several basic organizational forms among investor-owned companies. The most prevalent is the single corporation. Another common form is the holding company in which a parent company is established to own one or more operating utility companies which are integrated with one another. The Public Utility Holding Company Act of 1935 requires that the utilities

\footnotetext{
'Energy Information Administration Form EIA-860, "Annual Electric Generator Report."
} 
owned by a holding company operate as a single integrated utility system.

Most of the investor-owned utilities sell power at retail rates to several different classes of customers and at wholesale rates to investor-owned, Federal, State, and local government utilities, public utility districts, and rural electric cooperatives. A wholesale customer can be a distribution utility with little or no generating capacity of its own or another integrated utility that purchases power to supplement its own generation or for economic reasons.

\section{Publicly Owned Utillitles}

Publicly owned electric utilities represent 63 percent of the total number of U.S. electric utilities but accounted for only 16 percent of sales to ultimate consumers in 1992. Publicly owned utilities include Federal utilities, State and other government owned utilities (irrigation districts, public power districts, and State authorities and other organizations), and municipal utilities.
Federal power plants generate less than 9 percent of the electricity in the United States, primarily from hydroelectric facilities operated by the U. S. Army Corps of Engineers and the Bureau of Reclamation of the U.S. Department of Interior. Electricity is also generated by the Bureau of Indian Affairs and the International Boundary and Water Commission. Energy generated by these producers is marketed (along with any of their own generation) by the five Federal power marketing administrations: Bonneville (in the northwestern United States), Southeastern, Southwestern, Western Area, and Alıska. The power marketing administrations are part of the U.S. Department of Energy and operate in all States except those in the Northeast, upper Midwest, and Hawaii. The Tennessee Valley Authority generates electricity from coal and nuclear power as well as hydropower. It is the largest Federal power producer and markets electricity in both the wholesale and retail markets. In addition, the U.S. Department of Defense sells a small quantity of power, generated by Federal nonutilities, to the power grid.

Approximately 20 percent of the electricity generated by Federal utilities is sold to retail customers which are usually large industrial customers or Federal instal-

Figure 1. Electric Utility Density by County in the United States

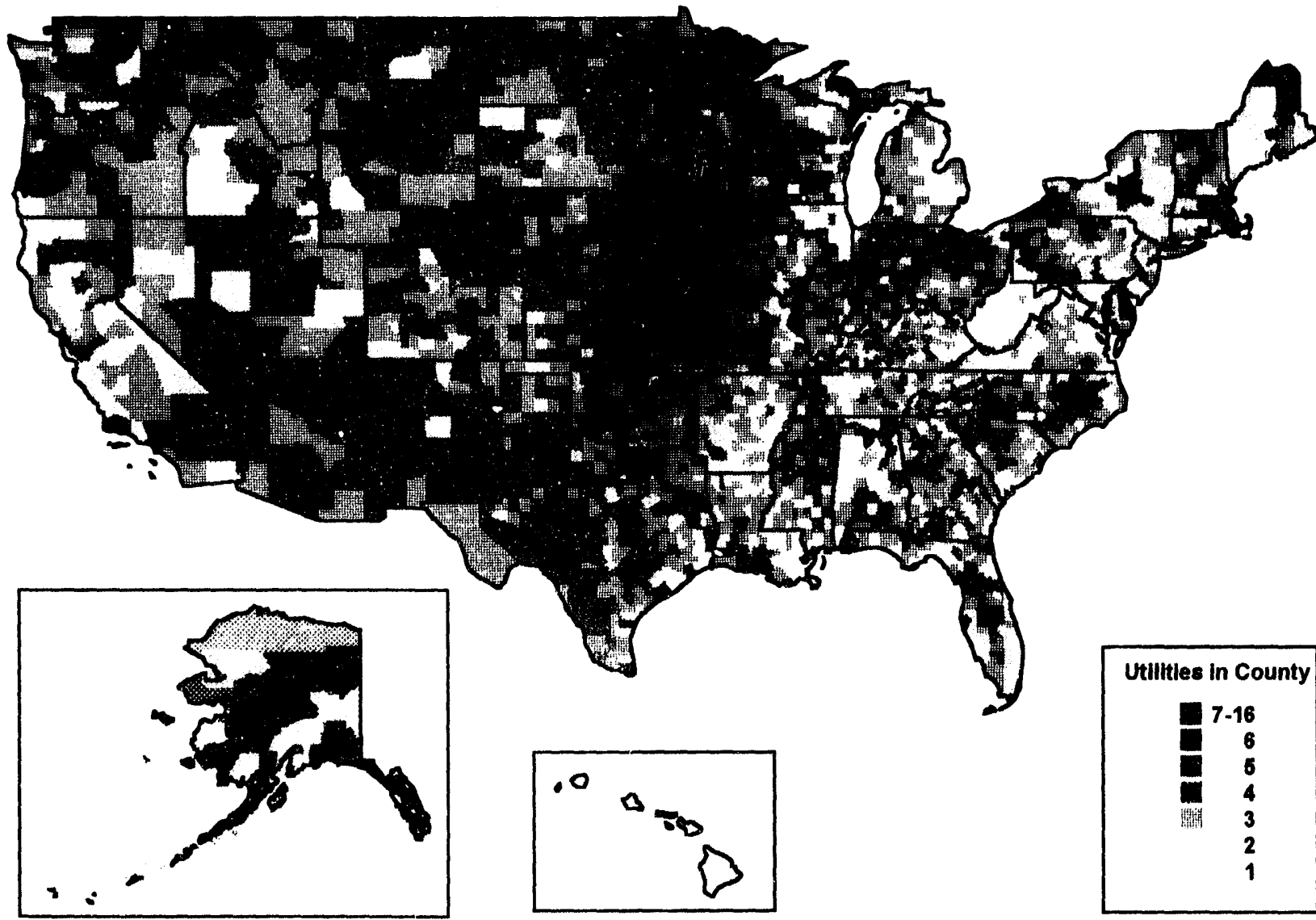

Note: Countles are considered to be the "first-order subdivisions" of each State, regardless of their local designation (county, parish, borough, etc.).

Source: Energy Information Administration, research by the Office of Coal, Nuclear, Electric and Alternate Fuels. 
lations. Most of the remaining energy is sold to publicly owned utilities and rural cooperatives for resale. These wholesale customers have a legal preference right to Federal electricity. Only the surplus remaining after meeting the energy requirements of preference customers is sold to the investor-owned utilities.

Municipal utilities account for almost 58 percent of the number of electric utilities in the United States, but produce less than 5 percent of total net generation. Municipal utilities tend to be concentrated in cities where the loads are small. They exist in every State except Hawaii, but are concentrated, predominately in the Midwest and Southeast. In general, municipal utilities tend to have lower costs than investor-owned utilities because they generally have access to bonds supported by tax based funds and do not pay certain taxes or dividends. They also have hizh density service areas.

The other publicly owned utilities include State authorities, public power districts, irrigation districts, and other State organizations. State authorities are utilities that function in a manner similar to Federal utilities. They generate or purchase electricity from other utilities and market large quantities wholesale to groups of utilities within their States at lower prices than the individual utilities would otherwise pay. The public power districts tend to be concentrated in Nebraska, Washington, Oregon, Arizona, and California. Appendix Tables B2 through B4 contain listings of the public power municipals, State authorities, and Federal utilities used in this publication.

\section{Rural Electric Cooperatives}

Most rural electric cooperative utilities are formed and owned by groups of residents in rural areas to supply power to those areas. Some cooperatives may be owned by a number of other cooperatives. (There are really three types of cooperatives: (1) distribution only; (2) distribution with power supply; and (3) generation and transmission.) Cooperatives currently operate in 46 States, and represent 29 percent of the total utilities in the country. Most distribution cooperatives resemble municipal utilities in that they often do not generate electricity, but purchase it from other utilities. The other type (generating and transmission cooperatives) are usually referred to as power supply cooperatives. These cooperatives are usually owned by the distribution cooperatives to whom they supply wholesale nower. Distribution cooperatives resemble Federal utilities by supplying electricity to other utility customers from their generating capability. Cooperatives accounted for 7.5 percent of sales to ultimate consumers in 1992.

\section{Changing Electric Power Industry}

The electric power industry is undergoing fundamental changes reflected by its evolving structure. For many years, the industry was dominated by locally franchised, vertically integrated utilities, regulated by State regulatory agencies. This picture has been changing rapidly in recent years with the passage of new legislation introducing a new type of business entity, changing regulations, and an increasing reliance by utilities on the wholesale purchase of electricity.

\section{Nonutility Generation in Electric Trade}

The growing role of nonutility generators (NUG) in electricity supply reflects the acceptance of alternative power supplies in the wholesale electric power market. The near monopoly of electric generation by regulated electric utilities has ended, as many new participants generate electricity and sell power to electric utilities, thus changing long-established institutional relationships. The outlook is for greater participation by nonutility generators in wholesale power supply. The changing roles and relationships between utility and nonutility participants in the wholesale power market are uncertain and have raised several unresolved technical, economic, and regulatory issues.

Differences remain between traditional electric utilities and nonutility power generators for many reasons, including the passage of new legislation and the application of regulations designed specifically for nonutility generators. For example, a traditional definition of a utility might simply include any business entity whose principal business is generating and selling electricity. This definition would exclude industrial generators and cogenerators, the principal nonutility generators organizations producing power but which are primarily engaged in business activities other than power sales.

A more common definition of an electric utility includes three elements: a franchised service territory; the obligation to serve all customers within this exclusive area; and the regulations that constitute this compact. This definition, however, ignores utilities without retail customers such as generation and transmission companies. All generators of electricity without a service area and with a contractual obligation to serve would be considered nonutilities under this definition. Even with this definition, there are various classes of nonutility generators, some of which reflect regulatory distinctions and others, which have evolved over time and may be less precise and overlapping: independent power producers (IPP), nonutility generators (NUG), ${ }^{2}$

\footnotetext{
${ }^{2}$ No specific legislative or regulatory definition exists for the term "nonutility." Therefore, it cannot be assumed that if an entity does not qualify as a utility that it is then a nonutility.
} 
small power producers (SPP), qualifying facilities (QF), and industrial power producers.

Current legislation and regulations may not always draw clear distinctions. For example, a producer of electricity that would appear to be a nonutility generator might be considered a utility if it is interconnected to transmission facilities in certain ways. Moreover, various laws and regulations may not always draw clear distinctions and may define utility broadly to include anyone selling electricity. The clearest exception is for firms, which are not electric utilities, that sell power from qualifying facilities (QF) as defined under the Public Utility Regulatory Policies Act (PURPA). The Energy Policy Act of 1992 defined a new class of nonutility generator, called exempt wholesale generators (EWG), to encourage the development of conventicnal generating facilities that will not meet the QF requirements. These EWGs are not considered electric utilities and are exempted from Public Utility Holding Company Act requirements. Despite the variety of definitions, nonutility power producers are generally considered to be the nontraditional producers of electric energy (i.e., producers that are neither traditional Federal, State, investor-owned, cooperative, nor municipal utilities).

The Federal Government and other users of electric generation data are interested in this nontraditional generation group because, as nonutility power producers increase their level of production of electric energy, the group becomes a more important segment of the U. S. electric power industry. Nonutility power producers sold over 166 billion kilowatthours in 1992 (Table 2). As one of the fastest growing segments of the electric power industry, nonutility power producers may be responsible for a significant portion of new power plant construction. This group is, therefore, of great interest to analysts of the electric power industry. Analysis of the electric utility industry is complicated by the changing structure of the industry--which now includes a significant number and wide range of nonutility participants--and by the changing mix of transactions that characterize electric trade.

Table 1. Number of Electric Utilities by Class of Ownershlp Within NERC Region, 1992

\begin{tabular}{|c|c|c|c|c|c|c|}
\hline NERC Reglon & $\begin{array}{l}\text { Investor- } \\
\text { Owned }\end{array}$ & Federal' & $\begin{array}{l}\text { State and Other } \\
\text { Government }\end{array}$ & Municipals & Cooperatives & U.S. Total \\
\hline \multicolumn{7}{|l|}{ Number of Utilities } \\
\hline ASCC & 24 & 1 & 5 & 32 & 22 & 84 \\
\hline ECAR & 46 & 1 & 4 & 222 & 110 & 383 \\
\hline 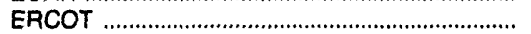 & 6 & 1 & 3 & 63 & 62 & 135 \\
\hline MAAC & 19 & 0 & 0 & 50 & 19 & 88 \\
\hline MAIN & 19 & 0 & 1 & 142 & 46 & 208 \\
\hline MAPP & 16 & 0 & 36 & 461 & 187 & 700 \\
\hline NPCC & 61 & 0 & 5 & 121 & 12 & 199 \\
\hline SERC & 21 & 2 & 5 & 316 & 189 & 533 \\
\hline SPP & 16 & 1 & 8 & 290 & 156 & 471 \\
\hline WSCC & 31 & 4 & 81 & 172 & 140 & 428 \\
\hline 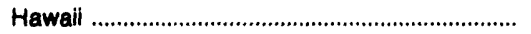 & 3 & 0 & 0 & 0 & 0 & 3 \\
\hline 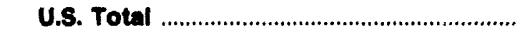 & 262 & 10 & 148 & 1,869 & 943 & 3,232 \\
\hline \multicolumn{7}{|l|}{ Percent } \\
\hline ASCC & 9 & 10 & 3 & 2 & 2 & 3 \\
\hline ECAR & 18 & 10 & 3 & 12 & 12 & 12 \\
\hline ERCOT & 2 & 10 & 2 & 3 & 7 & 4 \\
\hline MAAC & 7 & 0 & 0 & 3 & 2 & 3 \\
\hline MAIN & 7 & 0 & 1 & 8 & 5 & 6 \\
\hline MAPP & 6 & 0 & 24 & 25 & 20 & 22 \\
\hline NPCC & 23 & 0 & 3 & 6 & 1 & 6 \\
\hline SERC & 8 & 20 & 3 & 17 & 20 & 16 \\
\hline SPP & 6 & 10 & 5 & 16 & 17 & 15 \\
\hline 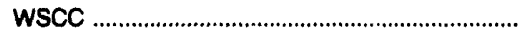 & 12 & 40 & 55 & $\theta$ & 15 & 13 \\
\hline 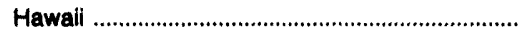 & 1 & 0 & 0 & 0 & 0 & * \\
\hline
\end{tabular}

1 The Federal Government both produces electricity and sells it at wholesale. Federal producers include the U.S. Army Corps of Engineers, Bureau of Indian Affairs, Bureau of Reclamation, and the international Boundary and Water Commission. The power marketing administrations of the U.S. Department of Energy are also federally owned utilities; they include the Alaska, Bonneville, Southeastern, Southwestern, and Western Area Power Administrations. The Tennessee Valley Authority both generates and markets electricity.

The value of the number is less than 0.5 percent.

Notes: A Aggregate 1992 bulk power data supplied by investor-owned electric utilities were matched to the original filings made to the FERC. The FERC has again required investor-owned electric utilities to refile 1992 bulk power data. These revised data are shown in Tables 5-24 and represent the reclassifination of data among purchased power, exchanges, and sales for resale.accounts. The difference between the data series is the result of the reclassifications and adjustments made to the data. -NERC is the North American Electric Reliability Council. See glossary for a list of regions and Figure 2 for a map of the regions. Percentages may not total 100 because of independent rounding.

Source: Energy information Administration, Form ElA-861, "Annual Electric Utilty Report." 
Floure 2. North American Electric Rellability Councll Reglon Map for the United States

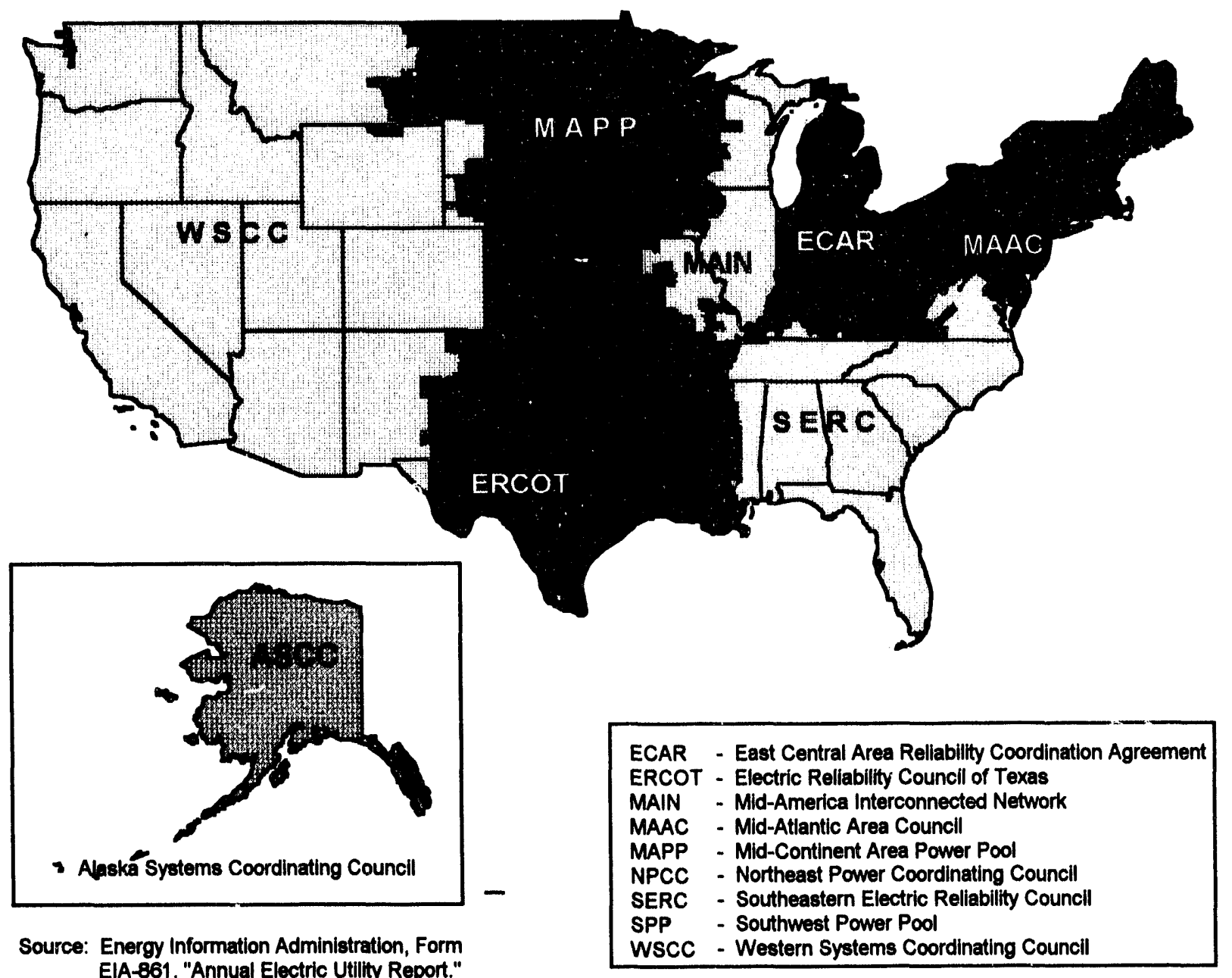

\section{Transmiselon Services and Energy Broker/Marketers}

The Energy Policy Act of 1992 (EPACT) amended the Federal Power Act (FPA) to allow any electric utility to apply to the Federal Energy Regulatory Commission (FERC) for an order requiring another utility to provide transmission services (wheeling). Prior to EPACT, FERC could not mandate an electric utility to provide wheeling services for wholesale electric trade. This change in the law will permit operators of electric generating equipment to sell wholesale power (sales for resale) to noncontiguous utilities. The amendment to the FPA will also affect electricity marketers, a relatively new group under FERC's jurisdiction. Marketers are business entities engaged in buying and selling electricity, but with no generating or transmission facilities. Marketers take ownership of the electricity and are involved in interstate trade. A number of energy marketers that may be involved in interstate electricity trade have filed with FERC and have had their rates authorized (Appendix Table B7). When ownership of the electricity is not taken, then those transactions are not regulated by FERC (the parties handling the transactions are called brokers).

\section{The Interconnected Networks}

The U.S. bulk power system has evolved into three major networks (power grids), which also include smaller groupings or power pools. The major networks consist of extra-high voltage connections between individual utilities designed to permit the transfer of electrical energy from one part of the network to another. These transfers are restricted, on occasion, because of a lack of contractual arrangements or because of inadequate transmission capability (See Appendix C).

The three networks are (1) the Eastern Interconnected System, consisting of the eastern two-thirds of the United States; (2) the Western Interconnected System 
Table 2. Energy Account Balance by Class of Ownershlp, 1992 (Million Kilowatthours)

\begin{tabular}{|c|c|c|c|c|c|c|}
\hline Item & $\begin{array}{l}\text { Investor- } \\
\text { Owned }\end{array}$ & Federal' & $\begin{array}{c}\text { State and Other } \\
\text { Government }\end{array}$ & Municipals & Cooperatives & U.S. Total" \\
\hline 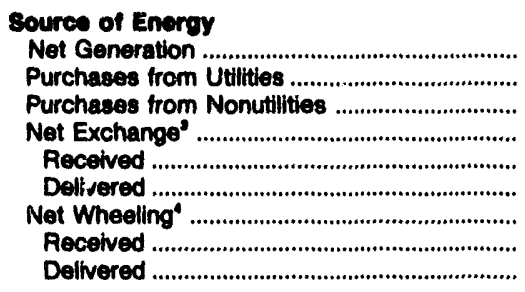 & $\begin{array}{r}2,115,878 \\
480,388 \\
164,461 \\
6,980 \\
123,257 \\
116,278 \\
2,943 \\
239,985 \\
237,042\end{array}$ & $\begin{array}{r}239,629 \\
18,802 \\
306 \\
-10,886 \\
38,689 \\
49,575 \\
2,443 \\
51,825 \\
49,381\end{array}$ & $\begin{array}{r}168,243 \\
73,789 \\
225 \\
1,068 \\
8,165 \\
7,097 \\
143 \\
5,861 \\
5,719\end{array}$ & $\begin{array}{r}127,289 \\
252,275 \\
904 \\
-515 \\
11,073 \\
11,588 \\
62 \\
15,559 \\
15,497\end{array}$ & $\begin{array}{r}154,054 \\
311,069 \\
387 \\
-152 \\
22,474 \\
22,625 \\
165 \\
5,308 \\
5,142\end{array}$ & $\begin{array}{r}2,805,092 \\
1,146,323 \\
166,283 \\
-3,504 \\
203,658 \\
207,162 \\
5,756 \\
318,538 \\
312,782\end{array}$ \\
\hline 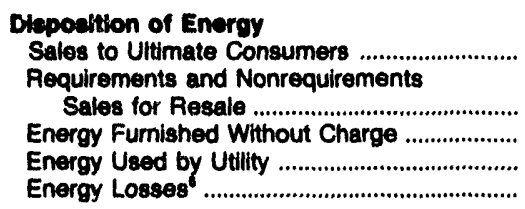 & $\begin{array}{r}2,112,230 \\
\\
505,220 \\
1,598 \\
5,788 \\
155,786\end{array}$ & $\begin{array}{r}48,768 \\
\\
186,130 \\
378 \\
1,289 \\
13,730\end{array}$ & $\begin{array}{r}91,968 \\
\\
138,738 \\
1,105 \\
4,340 \\
7,319\end{array}$ & $\begin{array}{r}303,419 \\
\\
54,085 \\
1,274 \\
3,562 \\
17,674\end{array}$ & $\begin{array}{r}206,939 \\
235,776 \\
54 \\
670 \\
22,084\end{array}$ & $\begin{array}{r}2,763,324 \\
1,119,948 \\
4,409 \\
15,651 \\
216,592\end{array}$ \\
\hline $\begin{array}{l}\text { Federal authorities use different accountir } \\
\text { generation or purchases. } \\
\text { Source and disposition of energy do not b } \\
\text { tions by some utilities of losses from transmiss } \\
\text { Reported as power exchange in account } \\
\text { Reported as transmission services in acco } \\
\text { System of Accounts. } \\
\text { ihese values are not measured; however } \\
\text { disposition of enargy are equivalent. } \\
\text { Notes: -Aggregate } 1992 \text { bulk power dat } \\
\text { FEAC has again required investor-owned elect } \\
\text { reclassification of data among purchased powe } \\
\text { clsselfications and adjustments made to the d } \\
\text { data. Total annual net generation data are pu } \\
\text { source and disposition are the aggregation o } \\
\text { net exchange and net wheeling (except for im } \\
\text { and exports are included in purchases, sales f } \\
\text { specifics of contracts, simultaneous energy tra } \\
\text { the classification and quantity of each transact } \\
\text { ing. }\end{array}$ & $\begin{array}{l}\text { as } 26 \text { milli } \\
\text { y others. } \\
\text { Purchased P } \\
\text { 56, "Transmi } \\
\text { represent Ic } \\
\text { plied by inve } \\
\text { ilities to refil } \\
\text { changes, and } \\
\text { Annual net } \\
\text { od by the Er } \\
\text { reported fo } \\
\text { are include } \\
\text { lale, net excr } \\
\text { ions, the uni } \\
\text { mong utilities }\end{array}$ & $\begin{array}{l}\text { In was include } \\
\text { Transactions,' } \\
\text { For Others," } \\
\text { and unaccour } \\
\text { owned electric } \\
2 \text { bulk power } \\
8 \text { for resale ac } \\
\text { ation data sh } \\
\text { information A } \\
h \text { utlity; these } \\
\text { let generation. } \\
8 \text {, and/or whe } \\
\text { ed receipt ano } \\
\text { not exist. -T }\end{array}$ & $\begin{array}{l}\text { led in source but } \\
\text { " of the FERC U } \\
\text { and in account } 56 \\
\text { Inted for energy. } \\
\text { c utilities were m } \\
\text { data. These rev } \\
\text { ccounts. The diffe } \\
\text { lown here should } \\
\text { administration fro } \\
\text { data are not ad } \\
\text { Sales for resale } \\
\text { eeling. Due to th } \\
\text { d delivery of ene } \\
\text { otals may not eq }\end{array}$ & $\begin{array}{l}\text { isposition. T } \\
\text { n System of } \\
\text { ransmission } \\
\text { oe values ar } \\
\text { do the ori } \\
\text { data are sho } \\
\text { e between th } \\
\text { used only in } \\
\text { Form ElA-7 } \\
\text { due to doul } \\
\text { included in } \\
\text { mplexity of } e \\
\text { hadvertent fle } \\
\text { um of comoo }\end{array}$ & $\begin{array}{l}\text { filings made t } \\
\text { Tables 5-24 } \\
\text { ta series are t } \\
\text { parison with o } \\
\text { Monthly Powe } \\
\text { ounting. Purch } \\
\text { to ultimate co } \\
\text { c power trans } \\
\text { ind losses, un }\end{array}$ & $\begin{array}{l}\text { Uniform } \\
\text { Ft source and } \\
\text { FERC. The } \\
\text { represent the } \\
\text { sult of the re- } \\
\text { Form EIA-861 } \\
\text { ant Report." } \\
\text { from utilities, } \\
\text { ners. Imports } \\
\text { is that involve } \\
\text { ity in reporting }\end{array}$ \\
\hline
\end{tabular}

consisting primarily of the Southwest and areas west of the Rocky Mountains; and (3) the Texas Interconnected System. The latter is not interconnected with the other two networks (except by certain direct current lines). The two other networks have limited interconnections to each other. Both the Western and Texas Interconnect are linked with different parts of Mexico. The Eastern and Western Interconnects are completely integrated with most of Canada or have links to the Quebec Province power grid. Virtually all U.S. utilities are interconnected with at least one other utility by these three major grids. The exceptions are in Alaska and Hawaii. The interconnected utilities within each power grid coordinate operations and planning, and buy and sell power among themselves for the benefit of the customers.

Overall reliability planning and coordination of the interconnected power systems are the responsibility of the North American Electric Reliability Council (NERC). The NERC was voluntarily formed in 1968 by the electric utility industry as a result of the 1965 power failure in the Northeast. The regional councils cover the 48 contiguous States, part of Alaska, ${ }^{3}$ and portions of Canada and Mexico (Figure 2) and are responsible for the overall coordination of bulk power policies that affect the reliability and adequacy of service in their areas. These councils also regularly exchange operating and planning information among their member utilities. The boundaries of the NERC regions follow the service areas of the electric utilities in the region. The service area of many electric utilities do not follow State boundaries. Hence, electric trade data are not available by State, but are presented by NERC regions.

\section{Wholesale Trade Transactions}

Wholesale electricity trade has become a major contributor to systems operations in the U.S. electric utility industry. In this publication, wholesale, or bulk power,

${ }^{3}$ The Alaska System Coordinating Council was an associate member of NERC in 1988 and now they are a member. 
transactions are categorized and defined in accordance with FERC reporting requirements. Wholesale power transactions include purchases, sales for resale, exchanges, and wheeling (i.e., transmission services). Purchase transactions involve buying power from electric utilities and nonutility producers of electricity. Sales for resale transactions refer to power sold by one electric utility to one or more utilities for distribution to ultimate consumers. Exchayges involve trading electric energy when supply and demand conditions are mutually advantageous and reversible for the participating parties. Exchanges are often based on timedependent excess capacity or diversity in load requirements. With exchange transactions, monetary repayment or replacement of energy may extend over several years.

Most bulk power transfer! involve neigliooring utilities whose systems are direcily interconnected. At other times, however, bulk power transfers involve a third utility situated between two unconnected utilities that want to enter into a bulk power transaction. In that transaction, the third (intervening) utility allows its transmission lines to be used for bulk power transfers between the iwo other utilities. This intervening utility is providing a form of transmission service known as wheeling. Wheeling is generally defined as the movement of electricity from one system to another over the transmission facilities of an intervening system. (Technically, there is two-party wheeling: (1) seller and wheeler and (2) purchaser.) Wheeling is conducted under terms and conditions that ensure safety and reliability and that prevent the wheeling utility from being unfairly burdened with added costs.

Table 3 provides an overview of wholesale and retail transactions in the electric utility system by NERC region. The table includes data on net generation, purchases, energy losses, sales to ultimate consumers, and electric trade transactions. Electricity is purchased by all classes of electric utilities. Purchases from nonutilities accounted for 6 percent of sales to consumers in 1992. These purchases are made generally by utilities in whose service territories the nonutilities are located. Losses represent energy lost in transmission, distribution, and power unaccounted for, which amounted to almost 8 percent of sales to consumers in 1992. Electricity inflows--purchases, exchanges-in, and wheeling-in--represent more than 60 percent of sales to consumers. Table 4 presents a historical view of electric trade flows (including net generation) and how components of electric trade changed for years 1986 through 1992.

Chapters 1 through 4 of this publication focus on purchases, sales for resale, exchanges, and wheeling of electricity (columns 4 and 5 of Table 3). Data for energy flows represent the summations of flows of energy between utilities in accordance with the provisions of their service agreements. These flows represent accounting transactions rather than metered flows. Detailed coverage of wholesale trade activities by NERC region for each class of ownership is presented in Chapters 2 through 4 . The electricity transactions reported in the remaining chapters do not include transmission and distribution losses. Chapter 5 presents selected data on the physical transmission systems.

\section{Categorles of Wholesale Trade Transactions}

There are two main types of bulk power transactions: coordination transactions and requirement transactions. The duration of the sale and the type of capacity sold are key factors in determining the type and value of the transaction. The length of the sale can be 1 hour, several months, a year, or longer. Another type of transaction is an exchange in which the energy usually is returned in kind at a date agreed upon by both utilities.

Firm sales usually involve the sale of capacity, meaning that either the associated energy will be taken by the purchaser or the purchaser will pay for the capacity during those periods when the energy is not taken. This payment is called a demand charge. The delivery is scheduled as part of the normal operating conditions of the affected electric systems. Firm capacity may be made available from the selling utility's entire pool of generating facilities or from a specific generating unit The capacity offered in these transactions is usually for a specified period of time and negotiated in advance of the trade. The associated energy purchased through a capacity sale usually has a separate cost from the demand charge.

One of the most common types of firm power transactions involves the transfer of full or partial requirements power. Requirement contracts involve electric utilities that have either insufficient or no generating capability to satisfy their customer load. These utilities negotiate long-term, firm power contracts in which the terms and conditions obligate the selling electric utility to provide the buying electric utility a level of service equivalent to the seller's requirement to support its retail customers. These contracts may involve more than one electric utility.

Nonfirm sales, sometimes called energy, economy, or interruptible sales, rarely have a demand or capacity charge included in the price of the transaction. These transactions are typically for short periods and subject to curtailment or cessation of delivery by the supplier in accordance with prior agreements or under specified conditions. Electric utilities engage in these transactions in order to gain operational savings, such as avoiding the use of more expensive fuels and selling electricity generated by the spillage of excess reservoir water through a water-driven turbine-generator.

In addition to domestic wholesale transactions, U.S. utilities have engaged in international trade agreements with utilities from Canada and Mexico that cover a 
Table 3. Components of Source and Diepositlon of Electriclty by NERC Reglon, 1992

\begin{tabular}{|c|c|c|c|c|c|c|c|}
\hline NEAC Aeglon & $\begin{array}{c}\text { Not } \\
\text { Generation }\end{array}$ & $\begin{array}{l}\text { Energy } \\
\text { Furnlahed } \\
\text { and Used } \\
\text { by Utllty }\end{array}$ & $\begin{array}{l}\text { Nonutllity } \\
\text { Purchases }\end{array}$ & $\begin{array}{l}\text { Purchases, } \\
\text { Exchange in, } \\
\text { Wheeling in' }\end{array}$ & $\begin{array}{l}\text { Sales for } \\
\text { Resale, } \\
\text { Exchange Out, } \\
\text { Wheelling Out' }\end{array}$ & $\begin{array}{l}\text { Total Region } \\
\text { Energy Loses }\end{array}$ & $\begin{array}{l}\text { Sales to } \\
\text { Ulitimato } \\
\text { Conaumer }\end{array}$ \\
\hline 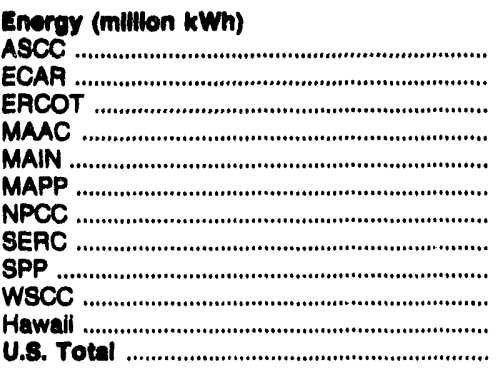 & $\begin{array}{r}4,735 \\
483,530 \\
180,442 \\
183,330 \\
200,288 \\
120,053 \\
202,078 \\
637,803 \\
242,514 \\
522,863 \\
6,565 \\
2,805,092\end{array}$ & $\begin{array}{r}48 \\
1,130 \\
289 \\
2,132 \\
678 \\
603 \\
841 \\
2,519 \\
1,616 \\
10,183 \\
10 \\
20,060\end{array}$ & $\begin{array}{r}1 \\
10,420 \\
23,668 \\
16,433 \\
347 \\
576 \\
38,116 \\
15,304 \\
5,457 \\
55,637 \\
2,324 \\
168,283\end{array}$ & $\begin{array}{r}3,019 \\
179,798 \\
106,383 \\
76,243 \\
55,464 \\
124,758 \\
191,454 \\
363,385 \\
144,878 \\
423,132 \\
4 \\
1,668,518\end{array}$ & $\begin{array}{r}3,020 \\
212,728 \\
102,886 \\
59,416 \\
40,708 \\
116,200 \\
178,603 \\
357,147 \\
137,540 \\
431,563 \\
3 \\
1,639,893\end{array}$ & $\begin{array}{r}349 \\
30,301 \\
14,019 \\
13,660 \\
14,154 \\
11,301 \\
17,709 \\
47,688 \\
18,372 \\
48,508 \\
529 \\
216,592\end{array}$ & $\begin{array}{r}4,338 \\
429,591 \\
203,206 \\
210,798 \\
200,571 \\
117,283 \\
233,395 \\
609,139 \\
235,320 \\
511,352 \\
8,332 \\
2,763,324\end{array}$ \\
\hline $\begin{array}{l}\text { Porcent } \\
\text { ASCC } \\
\text { ECAR } \\
\text { ERCOT } \\
\text { MAAC } \\
\text { MAIN } \\
\text { MAPP } \\
\text { NPCC } \\
\text { SERC } \\
\text { SPP } \\
\text { WSCC } \\
\text { HaWall }\end{array}$ & $\begin{array}{r}17 \\
7 \\
7 \\
7 \\
4 \\
7 \\
23 \\
9 \\
10\end{array}$ & $\begin{array}{r} \\
6 \\
1 \\
11 \\
3 \\
3 \\
4 \\
13 \\
8 \\
51\end{array}$ & $\begin{array}{r}6 \\
14 \\
10 \\
-\quad r \\
\\
\\
22 \\
9 \\
3 \\
33 \\
1\end{array}$ & $\begin{array}{r}11 \\
6 \\
5 \\
3 \\
7 \\
11 \\
22 \\
8 \\
25\end{array}$ & $\begin{array}{r}13 \\
6 \\
4 \\
2 \\
7 \\
11 \\
22 \\
8 \\
28\end{array}$ & $\begin{array}{r}-r \\
14 \\
6 \\
6 \\
7 \\
5 \\
8 \\
22 \\
8 \\
22\end{array}$ & $\begin{array}{r}16 \\
7 \\
8 \\
7 \\
4 \\
8 \\
22 \\
9 \\
19 \\
\quad\end{array}$ \\
\hline
\end{tabular}

1 Inciudes all trade into and within the region and purchases, exchange in, and wheeling in for utllities operating within the region.

2 Includes all trade out of and within the region and sales for resale, exchange out, and wheeling out for utllities operating within the region.

- The value of the number is less than 0.5 percent.

Notes: -Aggregate 1992 bulk power data supplied by investor-owned electric utilities were matched to the original filings made to the FERC. The FERC has again required investor-owned electric utilities to refile 1992 bulk power data. These revised data are shown in Tables 5-24 and represent the reclassification of data among purchased power, exchanges, and sales for resale accounts. The difference between the data series are the result of the reclassifications and adjustments made to the data. •Annual net generation data shown here should be used only in comparison with other Form ElA-861 data. Total annual net generation data are published by the Energy Information Administration from its Form ElA-759, "Monthly Power Plant Report." -NERC is the North American Electric Reliability Council. See glossary for a list of regions and Figure 2 for a map of the regions. $\bullet$ Totals may not equal sum of components because of independent rounding. Percentages may not total 100 because of independent rounding.

Source: Energy Information Administration, Form ElA-861, "Annual Electric Utility Report."

number of transaction options. These options include purchasing nonfirm energy from relatively inexpensive renewable resources, such as hydroelectric facilities in Canada and geothermal facilities in Mexico; acquiring additional generating capability to support the requirements for supply at U.S. electric utilities; the holding of reservoir water to be turned into electricity; and sharing the benefits of coordinated planning between the systems.

\section{Regional Recelpts and Dellveries of Wholesale Power}

A large amount of the electricity generated in the United States is traded under wholesale purchases and sales for resale contracts. The vast majority of wholesale transactions for investor-owned, Federal, and cooperative utilities involve utilities within the same NERC regional boundaries. Many of the differences between intra- and interregional wholesale power transactions result from the historical development of multiple transmission links among clusters of neighboring utilities. In addition, the development of shared interconnection and coordination agreements among these groups of utilities help delineate the regional operating boundaries of electric utilities.

Investor-owned electric utilities have led the other ownership classes in both intra- and interregional purchases and sales for resale. Interregional sources provided 9 percent of investor-owned purchases in 1992. Cooperatives made interregional purchases of 5 percent while Federal utilities purchased almost 9 percent (Table 5).

As shown in Table 6, both firm purchases and sales for resale represented at least 52 percent of investorowned utilities transactions (miscellaneous purchases and sales for resale are included in the total). Utilities in the WSCC region purchased the most firm electricity, while the utilities in the ECAR sold the most in 1992. Utilities in NPCC purchased the most nonfirm energy, and ECAR utilities sold the most nonfirm power. Nonfirm purchases nearly doubled from 1986 to 1992 while firm purchases increased by over 75 percent during that same time period.

Total electrical energy transported under transmission service agreements (wheeling) has held to about the same level during the period of 1990 to 1992 (Table 
7). However, the utilities in the WSCC region, which as a group have reported the highest total, during this same period have also reported declines in all years. Utilities located in SERC, have reported increases over these same years and this region has held the number two position for the same years.

\section{National and Regional Highlights}

The FERC Form 1, "Annual Report of Major Electric Utilities, Licensees and Others," is used to collect annual data on electricity trade by quantity, cost, and revenue from investor-owned utilities. These data were used to construct the tables in Chapters 1 and 2 and show the quantity and value of firm and nonfirm electricity trade reported.

Purchases from all sources by major investor-owned utilities totaled 342.5 billion kilowatthours of nirm power for $\$ 16.4$ billion and 299.7 billion kilowatthours of nonxirm energy for $\$ 9.5$ billion in 1992 (Table 8).
The combined costs of firm and nonfirm energy represent a 20-percent increase over the cost of those purchases reported in 1990. Firm purchases, in billion kilowatthours, increased by 5.5 percent with the two largest increases posted in MAPP $(7.5$ billion kilowatthours change or 120 percent) and NPCC ( 9.6 billion kilowatthours change or 15 percent). In addition to the energy purchased, demand-charge-only payments totaling $\$ 181$ million also were made to other utilities in 1952. Investor-owned utilities purchased 137.1 billion kilowatthours of firm power from other investor-owned utilities at a cost of $\$ 5.9$ billion, and nonfirm purchiases totaled 98.7 billion kilowatthours for $\$ 2.7$ billion (Table 8). Investor-owned utilities bought from Federal utilities 2.2 billion kilowatthours of firm power for \$53.6 million and 12.2 billion kilowatthours of nonfirm power for a cost to the investor-owned utilities of $\$ 277.6$ million. Cooperative electric utilities received \$607.6 million from the sales of 16.6 billion kilowatthours of firm power, to investorowned utilities and received $\$ 494.9$ million for 19.2 billion kilowatthours of nonfirm energy. Municipal electric utilities delivered 9.3 billion kilowatthours of firm power to investor-owned utilities for $\$ 479.6$ million and also delivered 5.9 billion kilowatthours of

Figure 3. Electricity Purchases and Costs by Investor-Owned Utiittles by NERC Region, 1992

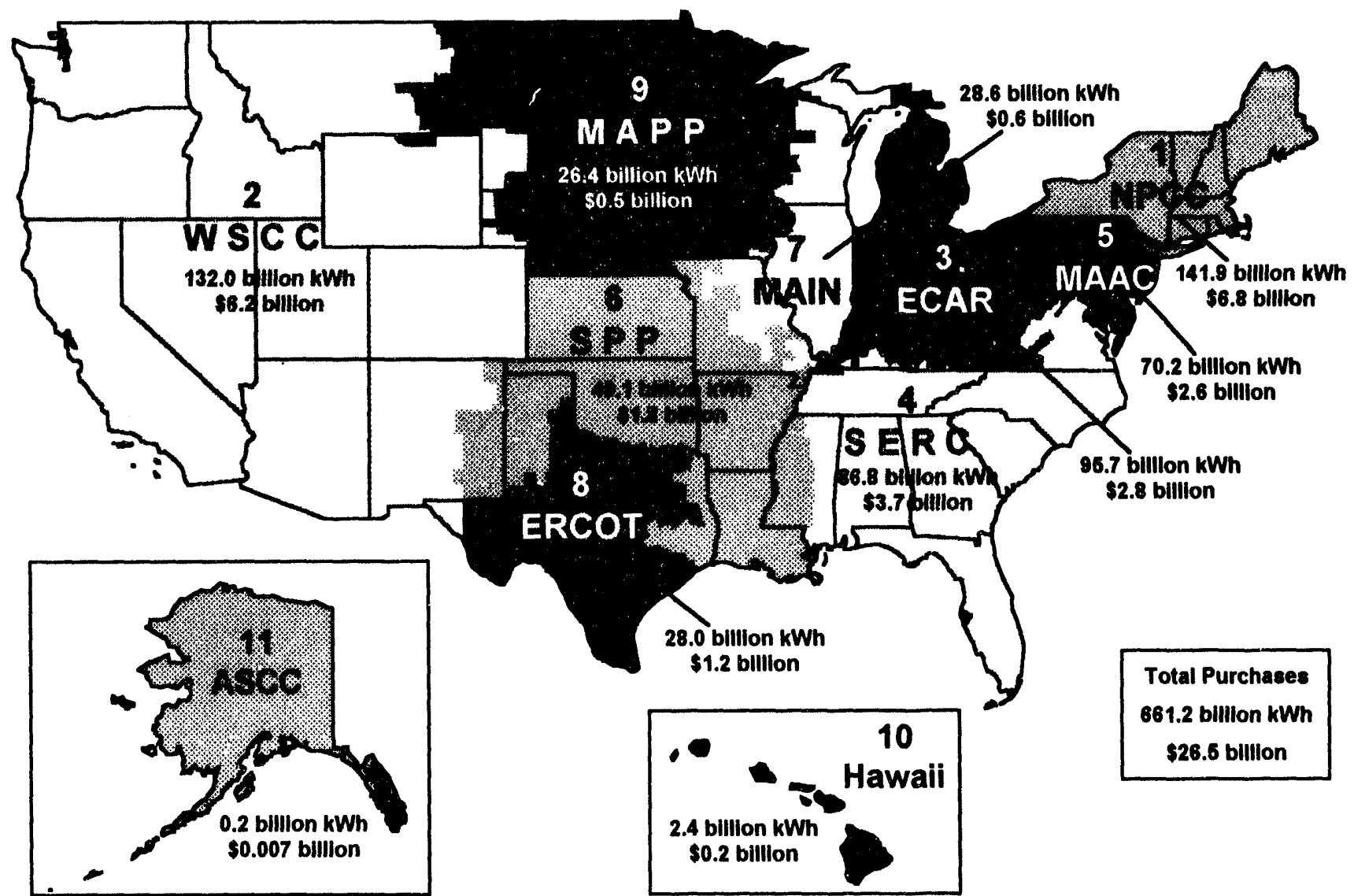

Notes: Number appearing above NERC region acronym (Hawaii is not a NERC region) indicates rank by kilowatthours (kWh) purchased. Hawaii includes Hawail Electric Light Company purchases (utility does not file a FERC Form 1).

Source: Federal Energy Regulatory Commission, FERC Form 1, "Annual Report of Major Electric Utilities, Licensees and Others." 
nonfirm energy for a cost of \$202.9 million to investorowned utilities (Figure 3). Sales for resale data are shown in Table 9 and in Figure 4.

Investor-owned utilities exchanged more than 112 billion kilowatthours of electricity with other utilities for both receipts and deliveries (Table 10). More exchange activity occurred in WSCC than in any other NERC region with 45.9 billion kilowatthours in receipts and 48.1 billion kilowatthours in deliveries. The MAPP region was second. Trade in the WSCC region was dominated by investor-owned trade with Federal utilities in 1992 as in 1990. Total exchange transactions with Federal utilities in WSCC remained relatively stable for the years 1990 and 1992 . Exchange receipts from Federal utilities increased by 8 percent and deliveries increased by less than 3 percent. By contrast, in ECAR total receipts from all sources declined by over 96 percent and deliveries declined by 97 percent. Total exchanges by all investor-owned utilities declined substantially from 1990 with receipts decreasing by almost 40 percent and deliveries decreasing by 43.5 percent (reflecting in part the adjustments to the account from the implementation of FERC Accounting Resolution, AR-14, See Appendix A).

The exchanges reported with noninvestor-owned utilities in 1992 (Table 11), were mostly with Federal utilities. Cooperative utilities were a distant second, which is in contrast to 1990 when municipal utilities exchanged more power than cooperatives. In 1992, there was a substantial decrease from 1990 levels in receipts and deliveries for municipal utilities in WSCC. This resulted in an overall decrease in all NERC regions of 58 percent for receipts and 60 percent for deliveries. In 1990, municipal utilities were second in exchanges to Federal utilities, but in 1992 they were third behind Federal and cooperative utilities. Only in the WSCC, SERC and ERCOT regions were any appreciable exchange activity experienced among noninvestorowned utilities simila: to 1990 levels. Federel trade was predominant in WSCC and SERC, but cooperative utilities dominated in ERCOT.

Figure 4. Electricity Sales for Resale and Revenue by Investor-Owned Utilitles by NERC Reglon, 1992

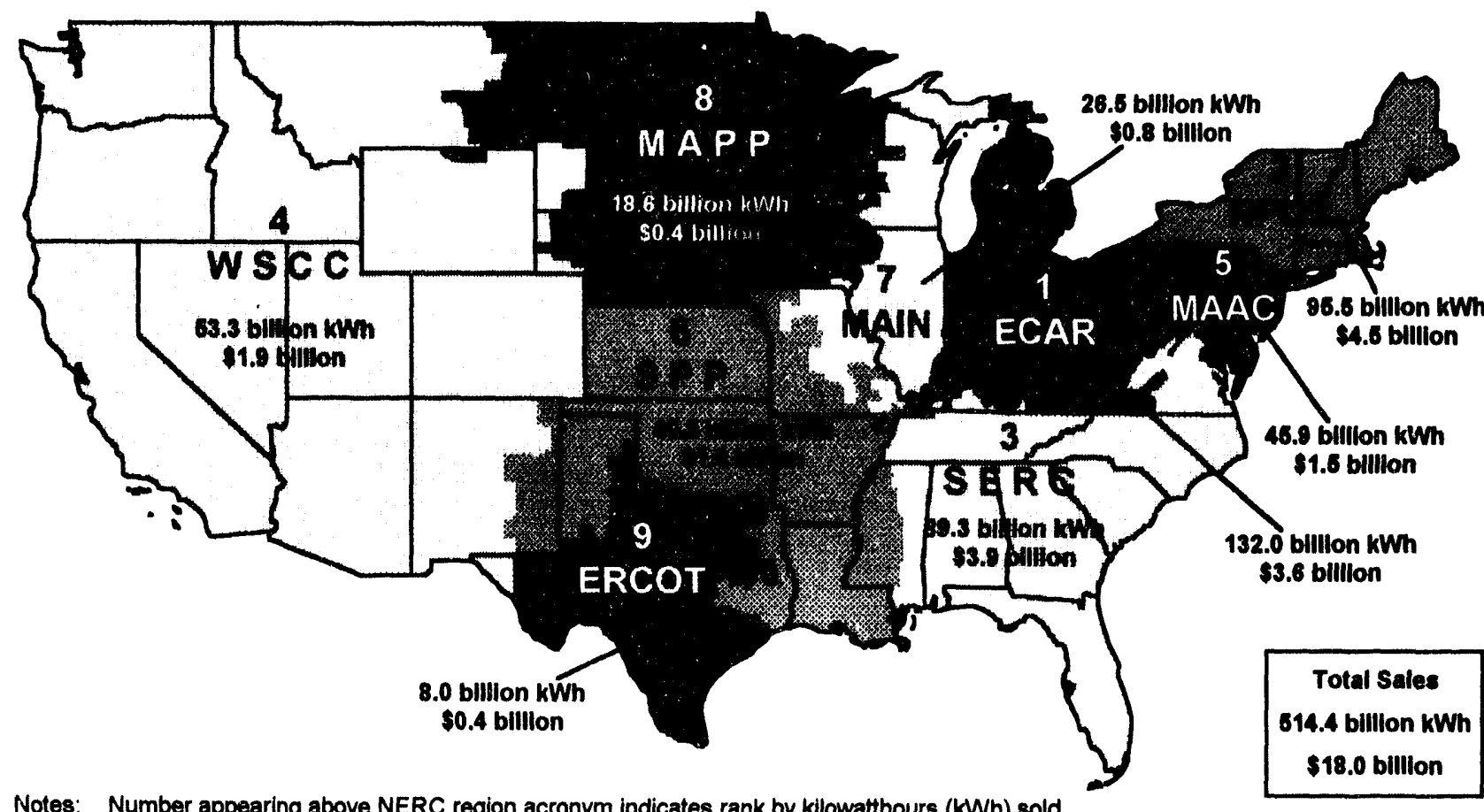

Notes: Number appearing above NERC region acronym indicates rank by kilowatthours (kWh) sold No sales for resale were reported in the ASCC region (Alaska) and Hawall.

Source: Federal Energy Regulatory Administration, FERC Form 1, "Annual Report of Major Electric Utillities, Licensees and Others." 
Table 4. Electric Trade in the United States by NERC Reglon, 1986 Through 1992 (Million Kilowatthours)

\begin{tabular}{|c|c|c|c|c|c|c|c|c|c|c|}
\hline Yoar & $\begin{array}{c}\text { Net } \\
\text { Generation }\end{array}$ & $\begin{array}{l}\text { Percent } \\
\text { Increase } \\
\text { froin } \\
\text { Year } \\
\text { Earlier }\end{array}$ & $\begin{array}{l}\text { Purchases } \\
\text { \& Exchanges } \\
\text { Received' }\end{array}$ & $\begin{array}{l}\text { Percent } \\
\text { Increase } \\
\text { from } \\
\text { Year } \\
\text { Earlier }\end{array}$ & $\begin{array}{c}\text { Purchases \& } \\
\text { Exch. Rec. as } \\
\text { Percent of } \\
\text { Net } \\
\text { Generation' }\end{array}$ & $\begin{array}{l}\text { Sales to } \\
\text { Ultimate } \\
\text { Consumers }\end{array}$ & $\begin{array}{l}\text { Percent } \\
\text { Increase } \\
\text { from } \\
\text { Year } \\
\text { Earlier }\end{array}$ & $\begin{array}{c}\text { Sales } \\
\text { for Resale } \\
\text { \& Exchanges } \\
\text { Delivered }^{2}\end{array}$ & $\begin{array}{c}\text { Percent } \\
\text { Increase } \\
\text { from } \\
\text { Year } \\
\text { Earller }\end{array}$ & $\begin{array}{c}\text { Sales tor } \\
\text { Pesale } \\
\text { \& Exch. Del. } \\
\text { as } \\
\text { Percent of } \\
\text { Sales to } \\
\text { Ultimate } \\
\text { Consumers }\end{array}$ \\
\hline
\end{tabular}

All Utilitios in AsCC

\begin{tabular}{|c|c|c|c|c|c|c|c|c|c|c|}
\hline $\begin{array}{l}1992 \\
1991 \\
1990 \\
1989\end{array}$ & $\begin{array}{l}4,735 \\
4,654 \\
4,680 \\
4,575 \\
4,460 \\
4,412 \\
4,421\end{array}$ & $\begin{array}{r}1.8 \\
-.1 \\
1.8 \\
2.6 \\
1.1 \\
-.2 \\
-\end{array}$ & $\begin{array}{l}2,543 \\
2,363 \\
2,347 \\
2,281 \\
2,183 \\
2,081 \\
2,058\end{array}$ & $\begin{array}{r}7.6 \\
.6 \\
2.9 \\
4.5 \\
4.9 \\
1.1 \\
-\end{array}$ & $\begin{array}{l}53.7 \\
50.8 \\
50.4 \\
49.8 \\
48.9 \\
47.2 \\
46.5\end{array}$ & $\begin{array}{l}4,338 \\
4,255 \\
4,253 \\
4,142 \\
4,045 \\
3,943 \\
4,029\end{array}$ & $\begin{array}{r}2.0 \\
.0 \\
2.7 \\
2.4 \\
2.6 \\
-2.2 \\
-\end{array}$ & $\begin{array}{l}2,542 \\
2,336 \\
2,330 \\
2,244 \\
2,177 \\
2,097 \\
2,068\end{array}$ & \begin{tabular}{r|}
8.8 \\
.2 \\
3.8 \\
3.1 \\
3.8 \\
1.4 \\
-
\end{tabular} & $\begin{array}{l}58.6 \\
54.9 \\
54.8 \\
54.2 \\
53.8 \\
53.2 \\
51.3\end{array}$ \\
\hline
\end{tabular}

\begin{tabular}{|c|c|c|c|c|c|c|c|c|c|c|}
\hline \multirow[b]{2}{*}{ 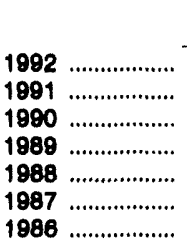 } & \multicolumn{10}{|c|}{ All Utilitios In ECAR } \\
\hline & $\begin{array}{l}483,530 \\
489,102 \\
485,128 \\
487,659 \\
487,040 \\
463,569 \\
438,890\end{array}$ & $\begin{array}{r}-0.9 \\
.6 \\
-.5 \\
.1 \\
5.1 \\
5.6 \\
-\end{array}$ & $\begin{array}{l}158,417 \\
165,313 \\
210,322 \\
184,902 \\
170,991 \\
150,752 \\
176,423\end{array}$ & $\begin{array}{r}-4.2 \\
-21.4 \\
13.8 \\
8.1 \\
13.4 \\
-14.5 \\
-\end{array}$ & $\begin{array}{l}32.8 \\
33.9 \\
43.3 \\
37.9 \\
35.1 \\
32.5 \\
40.2\end{array}$ & $\begin{array}{l}429,591 \\
430,314 \\
418,732 \\
414,977 \\
408,171 \\
389,799 \\
372,808\end{array}$ & $\begin{array}{r}-0.2 \\
2.8 \\
.9 \\
1.7 \\
4.7 \\
4.6 \\
-\end{array}$ & $\begin{array}{l}181,111 \\
191,274 \\
245,897 \\
224,880 \\
217,724 \\
193,641 \\
212,821\end{array}$ & $\begin{array}{r}-5.3 \\
-22.2 \\
9.3 \\
3.3 \\
12.4 \\
-9.0 \\
-\end{array}$ & $\begin{array}{l}42.2 \\
44.4 \\
58.7 \\
54.2 \\
53.3 \\
49.7 \\
57.1\end{array}$ \\
\hline
\end{tabular}

All Utillites in ERCOT

\begin{tabular}{|c|c|c|c|c|c|c|c|c|c|c|}
\hline $\begin{array}{l}1992 \\
i 991 \\
1980 \\
1989\end{array}$ & $\begin{array}{l}190,442 \\
182,000 \\
188,586 \\
185,011 \\
179,442 \\
173,645 \\
173,381\end{array}$ & $\begin{array}{r}-0.8 \\
1.8 \\
1.8 \\
3.1 \\
3.3 \\
-1\end{array}$ & $\begin{array}{r}105,972 \\
106,408 \\
94,932 \\
93,087 \\
93,782 \\
90,193 \\
68,942\end{array}$ & $\begin{array}{r}-0.4 \\
12.1 \\
2.0 \\
-.7 \\
4.0 \\
34.7 \\
-\end{array}$ & $\begin{array}{l}55.6 \\
55.4 \\
50.3 \\
50.3 \\
52.3 \\
51.9 \\
38.6\end{array}$ & $\begin{array}{l}203,206 \\
204,319 \\
202,090 \\
195,648 \\
192,367 \\
184,494 \\
181,746\end{array}$ & $\begin{array}{r}-0.5 \\
1.1 \\
3.3 \\
1.7 \\
4.3 \\
1.5 \\
-\end{array}$ & $\begin{array}{l}78,876 \\
80,853 \\
68,580 \\
67,372 \\
67,269 \\
68,139 \\
45,709\end{array}$ & $\begin{array}{r}-2.4 \\
17.9 \\
1.8 \\
.1 \\
1.7 \\
44.7 \\
-\end{array}$ & $\begin{array}{l}38.8 \\
39.6 \\
33.9 \\
34.4 \\
35.0 \\
35.8 \\
25.1\end{array}$ \\
\hline
\end{tabular}

\begin{tabular}{|c|c|c|c|c|c|c|c|c|c|c|}
\hline \multirow[b]{2}{*}{ 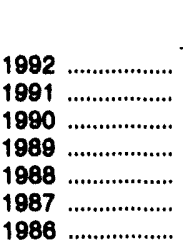 } & \multicolumn{10}{|c|}{ All Utilties in HI } \\
\hline & $\begin{array}{l}6,555 \\
6,991 \\
7,746 \\
7,692 \\
7,499 \\
7,103 \\
6,773\end{array}$ & \begin{tabular}{r|}
-6.2 \\
-9.8 \\
.7 \\
2.6 \\
5.6 \\
4.9 \\
-
\end{tabular} & $\begin{array}{r}2,328 \\
1,605 \\
714 \\
452 \\
497 \\
481 \\
529\end{array}$ & $\begin{array}{r}45.1 \\
124.6 \\
57.9 \\
-9.1 \\
3.3 \\
-9.0 \\
-\end{array}$ & $\begin{array}{r}35.5 \\
22.9 \\
9.2 \\
5.9 \\
6.6 \\
6.8 \\
7.8\end{array}$ & $\begin{array}{l}8,332 \\
8,154 \\
7,968 \\
7,649 \\
7,411 \\
7,028 \\
6,780\end{array}$ & $\begin{array}{r}2.2 \\
2.3 \\
4.2 \\
3.2 \\
5.4 \\
3.7\end{array}$ & $\begin{array}{r}3 \\
5 \\
5 \\
3 \\
89 \\
83 \\
81\end{array}$ & $\begin{array}{r}-39.2 \\
10.4 \\
38.6 \\
-96.3 \\
6.6 \\
2.7 \\
-\end{array}$ & $\begin{array}{r}0.0 \\
.1 \\
.1 \\
.0\end{array}$ \\
\hline
\end{tabular}

All Utilities in MAAC

\begin{tabular}{|c|c|c|c|c|c|c|c|c|c|c|}
\hline \multirow[t]{2}{*}{$\begin{array}{l}1982 \\
1991 \\
1980 \\
1989\end{array}$} & $\begin{array}{l}193,330 \\
197,235 \\
193,393 \\
193,889 \\
191,874 \\
185,54 \\
180,313\end{array}$ & $\begin{array}{r}-2.0 \\
2.0 \\
-.3 \\
1.0 \\
3.4 \\
2.9 \\
--\end{array}$ & $\begin{array}{l}82,808 \\
78,747 \\
73,631 \\
77,882 \\
68,904 \\
64,208 \\
56,672\end{array}$ & \begin{tabular}{r|}
5.2 \\
6.9 \\
-5.5 \\
13.0 \\
7.3 \\
13.3 \\
-
\end{tabular} & $\begin{array}{l}42.8 \\
39.9 \\
38.1 \\
40.2 \\
35.9 \\
34.6 \\
31.4\end{array}$ & $\begin{array}{l}210,799 \\
212,728 \\
207,770 \\
207,325 \\
202,705 \\
191,677 \\
182,720\end{array}$ & $\begin{array}{r}-0.9 \\
2.4 \\
.2 \\
2.3 \\
5.8 \\
4.9 \\
-\end{array}$ & $\begin{array}{l}49,637 \\
45,447 \\
43,847 \\
46,930 \\
40,141 \\
41,120 \\
39,018\end{array}$ & $\begin{array}{r}9.2 \\
3.6 \\
-6.6 \\
16.9 \\
-2.4 \\
-\quad 5.4 \\
-\end{array}$ & $\begin{array}{l}23.5 \\
21.4 \\
21.1 \\
22.6 \\
19.8 \\
21.4 \\
21.3\end{array}$ \\
\hline & \multicolumn{10}{|c|}{ All Utilitios in MAIN } \\
\hline \multirow[t]{2}{*}{$\begin{array}{l}1992 \\
1991 \\
1990 \\
1989 \\
1988 \\
1987\end{array}$} & $\begin{array}{l}200,288 \\
206,906 \\
201,653 \\
200,660 \\
198,666 \\
185,240 \\
180,431\end{array}$ & $\begin{array}{r}-3.2 \\
2.6 \\
.5 \\
1.0 \\
7.3 \\
2.7 \\
-\end{array}$ & $\begin{array}{l}52,321 \\
54,585 \\
68,308 \\
62,575 \\
56,841 \\
54,605 \\
50,478\end{array}$ & $\begin{array}{r}-4.1 \\
-20.1 \\
9.2 \\
10.1 \\
4.1 \\
8.2 \\
-\end{array}$ & $\begin{array}{l}26.1 \\
26.4 \\
33.9 \\
31.2 \\
28.6 \\
29.5 \\
28.0\end{array}$ & $\begin{array}{l}200,571 \\
201,815 \\
193,068 \\
188,425 \\
185,430 \\
176,801 \\
171,735\end{array}$ & $\begin{array}{r}-0.6 \\
4.5 \\
2.5 \\
1.6 \\
4.9 \\
2.9 \\
-\end{array}$ & $\begin{array}{l}37,302 \\
44,527 \\
62,174 \\
59,407 \\
54,957 \\
48,517 \\
45,555\end{array}$ & \begin{tabular}{r|}
-16.2 \\
-28.4 \\
4.7 \\
8.1 \\
13.3 \\
6.5 \\
-
\end{tabular} & $\begin{array}{l}18.6 \\
22.1 \\
32.2 \\
31.5 \\
29.6 \\
27.4 \\
28.5\end{array}$ \\
\hline & \multicolumn{10}{|c|}{ All ytiltios in MAPP } \\
\hline 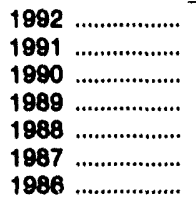 & $\begin{array}{l}120,053 \\
122,991 \\
120,750 \\
118,410 \\
118,238 \\
122,381 \\
113,274\end{array}$ & $\begin{array}{r}-2.4 \\
1.9 \\
3.7 \\
-1.5 \\
-3.4 \\
8.0 \\
\end{array}$ & $\begin{array}{r}113,672 \\
105,175 \\
107,416 \\
105,038 \\
106,850 \\
97,835 \\
108,723\end{array}$ & $\begin{array}{r}8.1 \\
-2.1 \\
2.3 \\
-1.8 \\
9.3 \\
-10.0 \\
-\end{array}$ & $\begin{array}{l}94.7 \\
85.5 \\
89.0 \\
90.2 \\
90.4 \\
79.8 \\
98.0\end{array}$ & $\begin{array}{l}117,283 \\
120,522 \\
115,912 \\
113,692 \\
113,136 \\
104,687 \\
104,573\end{array}$ & $\begin{array}{r}-2.7 \\
4.0 \\
1.9 \\
.5 \\
8.1 \\
-\quad .1\end{array}$ & $\begin{array}{r}105,352 \\
98,084 \\
101,829 \\
97,303 \\
101,686 \\
101,044 \\
107,009\end{array}$ & $\begin{array}{r}7.4 \\
-3.7 \\
4.6 \\
-4.3 \\
.6 \\
-5.6 \\
-\end{array}$ & $\begin{array}{r}89.8 \\
81.4 \\
87.8 \\
85.6 \\
89.9 \\
88.5 \\
102.3\end{array}$ \\
\hline
\end{tabular}

See notes and footnotes at end of table. 
Table 4. Electric Trade In the United Status by NERC Reglon, 1986 Through 1992 (Million Kilowatthours) (Continued)

\begin{tabular}{|c|c|c|c|c|c|c|c|c|c|c|}
\hline Year & $\begin{array}{c}\text { Not } \\
\text { Ceneration }\end{array}$ & $\begin{array}{c}\text { Percent } \\
\text { inorease } \\
\text { trom } \\
\text { Year } \\
\text { Eartier }\end{array}$ & $\begin{array}{l}\text { Purohases } \\
\text { Exohanges } \\
\text { Recolved' }\end{array}$ & $\begin{array}{l}\text { Percent } \\
\text { Inorvase } \\
\text { from } \\
\text { Year } \\
\text { Eerlior }\end{array}$ & $\begin{array}{c}\text { Purcheses a } \\
\text { Exch. Aec. as } \\
\text { Peroent of } \\
\text { Not } \\
\text { Generation' }\end{array}$ & $\begin{array}{l}\text { Sules to } \\
\text { Utimnte } \\
\text { Conoumers }\end{array}$ & $\begin{array}{c}\text { Percent } \\
\text { Inorease } \\
\text { from } \\
\text { Year } \\
\text { Earller }\end{array}$ & $\begin{array}{c}\text { Bales } \\
\text { for Posale } \\
\text { Exchanges } \\
\text { Deltvered" }\end{array}$ & $\begin{array}{l}\text { Peroont } \\
\text { inorease } \\
\text { from } \\
\text { Year } \\
\text { Eariler }\end{array}$ & $\begin{array}{c}\text { Sales for } \\
\text { Aceale } \\
\text { Exoh. Dol. } \\
\text { as } \\
\text { Percent of } \\
\text { 8xles to } \\
\text { Uttimnte } \\
\text { Consumers" }\end{array}$ \\
\hline & \multicolumn{10}{|c|}{ All Utilities in NPCe } \\
\hline \multirow[t]{2}{*}{ 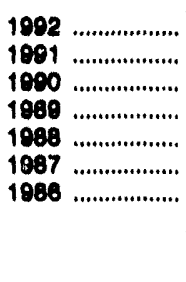 } & $\begin{array}{l}202,078 \\
210,053 \\
227,868 \\
233,024 \\
223,009 \\
212,168 \\
221,331\end{array}$ & $\begin{array}{r}-6.9 \\
-4.3 \\
-2.5 \\
4.3 \\
6.6 \\
-4.1 \\
-\end{array}$ & $\begin{array}{l}169,884 \\
184,208 \\
141,784 \\
141,102 \\
140,209 \\
139,060 \\
139,889\end{array}$ & $\begin{array}{r}8.2 \\
8.8 \\
.5 \\
.6 \\
.0 \\
-.5 \\
-\end{array}$ & $\begin{array}{l}82.2 \\
70.7 \\
62.2 \\
60.4 \\
62.6 \\
66.5 \\
63.1\end{array}$ & $\begin{array}{l}233,308 \\
238,043 \\
234,542 \\
238,673 \\
228,883 \\
216,979 \\
207,686\end{array}$ & $\begin{array}{r}-0.1 \\
-.4 \\
.4 \\
2.0 \\
6.6 \\
4.6 \\
-\end{array}$ & $\begin{array}{l}118,108 \\
120,113 \\
118,104 \\
122,287 \\
116,847 \\
116,181 \\
136,128\end{array}$ & $\begin{array}{r}-1.7 \\
1.6 \\
-3.3 \\
4.8 \\
1.3 \\
-14.8 \\
-\end{array}$ & $\begin{array}{l}50.0 \\
61.4 \\
50.4 \\
52.4 \\
51.0 \\
53.1 \\
66.1\end{array}$ \\
\hline & \multicolumn{10}{|c|}{ All vellthes in senc } \\
\hline 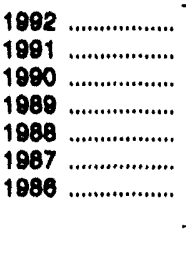 & \multicolumn{10}{|c|}{ All Utiltibe in 8Pp } \\
\hline \multirow[t]{2}{*}{ 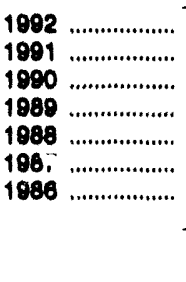 } & $\begin{array}{l}242,514 \\
244,416 \\
244,762 \\
239,032 \\
233,398 \\
231,865 \\
228,130\end{array}$ & $\begin{array}{r}-0.8 \\
-.1 \\
2.4 \\
2.4 \\
.7 \\
1.6 \\
-\quad\end{array}$ & $\begin{array}{l}128,686 \\
127,898 \\
120,683 \\
120,646 \\
110,228 \\
106,872 \\
116,084\end{array}$ & $\begin{array}{r}0.5 \\
6.1 \\
.0 \\
9.4 \\
4.0 \\
-8.7 \\
-\end{array}$ & $\begin{array}{l}53.0 \\
52.3 \\
49.3 \\
50.5 \\
47.2 \\
46.7 \\
50.9\end{array}$ & $\begin{array}{l}235,320 \\
238,328 \\
236,253 \\
223,134 \\
218,281 \\
211,808 \\
208,238\end{array}$ & $\begin{array}{r}-1.3 \\
.9 \\
5.8 \\
2.2 \\
3.1 \\
1.7 \\
-\end{array}$ & $\begin{array}{l}115,001 \\
114,484 \\
109,113 \\
114,760 \\
105,777 \\
107,229 \\
117,038\end{array}$ & $\begin{array}{r}1.2 \\
4.8 \\
-4.8 \\
8.6 \\
-1.3 \\
-8.4 \\
-\end{array}$ & $\begin{array}{l}49.3 \\
48.0 \\
46.2 \\
51.4 \\
48.6 \\
50.6 \\
56.2\end{array}$ \\
\hline & \multicolumn{10}{|c|}{ All Utiltibes in wecc } \\
\hline \multirow[t]{2}{*}{ 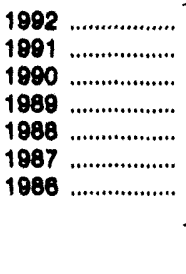 } & $\begin{array}{l}522,863 \\
523,468 \\
535,242 \\
530,205 \\
514,622 \\
486,398 \\
476,586\end{array}$ & $\begin{array}{r}-0.1 \\
-2.2 \\
.9 \\
3.0 \\
5.8 \\
2.1 \\
-\end{array}$ & $\begin{array}{l}361,255 \\
368,894 \\
364,977 \\
321,046 \\
285,580 \\
286,132 \\
283,898\end{array}$ & $\begin{array}{r}-2.1 \\
-1.7 \\
13.7 \\
12.4 \\
-.2 \\
-2.7 \\
--\end{array}$ & $\begin{array}{l}67.2 \\
68.6 \\
86.2 \\
60.5 \\
55.5 \\
58.8 \\
01.7\end{array}$ & $\begin{array}{l}511,362 \\
505,936 \\
503,744 \\
486,675 \\
472,386 \\
447,158 \\
430,304\end{array}$ & $\begin{array}{r}1.1 \\
.4 \\
3.5 \\
3.0 \\
5.6 \\
3.8 \\
-\end{array}$ & $\begin{array}{l}306,540 \\
322,943 \\
338,091 \\
305,522 \\
270,525 \\
268,001 \\
289,726\end{array}$ & $\begin{array}{r}-5.1 \\
-3.8 \\
10.0 \\
12.8 \\
.2 \\
-6.8 \\
-\end{array}$ & $\begin{array}{l}59.8 \\
63.8 \\
66.7 \\
62.8 \\
57.3 \\
60.4 \\
67.3\end{array}$ \\
\hline & \multicolumn{10}{|c|}{ U.8. Total } \\
\hline 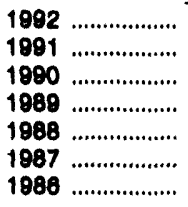 & $\begin{array}{l}2,805,092 \\
2,835,377 \\
2,821,483 \\
2,811,398 \\
2,730,774 \\
2,632,010 \\
2,552,012\end{array}$ & $\begin{array}{r}-1.1 \\
.5 \\
.4 \\
2.0 \\
3.6 \\
3.1 \\
-\end{array}$ & $\begin{array}{l}1,516,264 \\
1,508,408 \\
1,541,310 \\
1,489,852 \\
1,372,433 \\
1,319,796 \\
1,307,501\end{array}$ & $\begin{array}{r}0.5 \\
-2.1 \\
4.8 \\
7.1 \\
4.0 \\
-\quad .0\end{array}$ & $\begin{array}{l}54.0 \\
53.2 \\
54.6 \\
52.3 \\
50.3 \\
50.1 \\
51.2\end{array}$ & $\begin{array}{l}2,763,324 \\
2,762,003 \\
2,712,565 \\
2,646,809 \\
2,578,062 \\
2,457,272 \\
2,368,512\end{array}$ & $\begin{array}{r}0.0 \\
1.8 \\
2.5 \\
2.7 \\
4.8 \\
3.8 \\
-\end{array}$ & $\begin{array}{l}1,327,111 \\
1,386,785 \\
1,427,118 \\
1,384,071 \\
1,296,403 \\
1,270,370 \\
1,284,804\end{array}$ & $\begin{array}{r}-2.2 \\
-4.8 \\
2.4 \\
7.8 \\
2.0 \\
-1.1 \\
-\end{array}$ & $\begin{array}{l}48.0 \\
49.1 \\
52.6 \\
52.7 \\
50.3 \\
51.7 \\
54.3\end{array}$ \\
\hline
\end{tabular}

I Includes all transactions recelved from outside and within the reglon by utlities operating within the region.

- Includes all transactions delivered out of and within the region by utilities operating within the region.

- Not Applicable

Notes: -Aggregate 1992 bulk power data supplied by investor-owned electric utilltes were matched to original filings made to the FEAC. The FEFiC has again required investor-owned electric utilities to refile 1902 bulk power data. These revised data are shown in Tables 5-24 and represent the reclassifi. cation of data among purchased power, exchanges, and sales for resale accounts. The difference between the data series are the result of the reclassifications and adjustments made to the data. •NERC is the North American Electric Reliability Council. See glossary for a list of regions and Figure 2 for a map of the regions. Percentages may not total 100 because of independent rounding.

Source: Energy Information Administration, Form ElA-661, "Annual Electric Utility Report."

The quantity of electricity wheeled differed greatly between the transmission service provided "through the service territory" of investor-owned utilities (wheeling for) and the transmission service of power provided "to the service territory" of investor-owned utilities (wheeling by). In 1992, investor-owned wheeling for other investor-owned utilities decreased by 29 percent from about 90 billion kilowatthours of wheeling received in 1990 to about 64 billion kilowatthours of wheeling received in 1992 (Table 12). Revenue to investor-owned utilities for this service decreased by over 14 percent from 1990 to 1992 or from $\$ 271.2 \mathrm{mil}$ lion to $\$ 232$ million. However, the total wheeling of power by investor-owned utilities remained relatively 
constant with offsetting increases in power wheeled for other classes of ownership. Total revenue for all wheeling in 1992 increased by 10 percent from 1990 levels. Total wheeling of power by all classes to investor-owned service territories increased from over 81 billion kilowatthours received in 1990 to 89.7 billion kilowatthours received in 1992, an increase of 10 percent. The costs for this service to the investor-owned utilities increased from $\$ 403.6$ million in 1990 to $\$ 468.8$ million in 1992, an increase of 16 percent (Table 13).

Cooperative utilities move large amounts of electricity among themselves. Purchases by cooperatives in 1992 (Table 14) remained fairly consistent with purchases reported in 1990. In 1992, cooperative utilities reported purchasing 281 billion kilowatthours at a cost of $\$ 11.6$ billion. This represents an increase of almost 3 percent in power purchased and just over a 4-percent increase in associated costs. Of the 281 billion kilowatthours purchased by cooperatives almost 176 billion kilowatthours ( 62 percent), at a cost of $\$ 7.9$ billion, came from other cooperative utilities. This was over four times as much power as was purchased by cooperative utilities from investor-owned utilities. Much of the power purchased from other cooperatives was generated or purchased by power supply cooperatives.

For receipts of power within a region in 1992 (Table 15), investor-owned utilities in WSCC continued to dominate all other regions as the largest receiver of firm power (83 billion kilowatthours for $\$ 5.1$ billion). However, in 1992, NPCC replaced WSCC as the largest receiver of nonfirm energy $(66.9$ billion kilowatthours for $\$ 3.0$ billion). The regional numbers again indicated that wheeling principally occurs within NERC regions. In 1992, as in 1990, utilities in WSCC received the most wheeling energy with NPCC second for wheeling receipts. WSCC decreased in this category by slightly less than 2 percent over 1990, but still accounted for over 50 percent of the total "Wheeling By Others" reported for all regions.

For investor-owned utility trade within a region (Table 16), utilities in ECAR and NPCC sold the most firm energy, 60 and 52 billion kilowatthours for a revenue of $\$ 1.5$ billion and $\$ 2.9$ billion, respectively. In 1992, ECAR was first in nonfirm energy sales with 45 billion kilowatthours for $\$ 1.2$ billion and SERC was second with 38.9 billion kilowatthours and revenue of $\$ 1.4$ * billion.

Trade between NERC regions was generally small compared with trade within NERC regions. However, SPP and SERC each traded extensively with multiple
NERC regions. Trade alignments that existed in 1990 changed somewhat in 1992. SERC had the most interregional firm purchases in 1992 (6.8 billion kilowatthours from ECAR) and MAAChad the largest nonfirm purchases (8.4 billion kilowatthours from ECAR). Utilities in MAIN purchased more energy outside the region than inside, a departure from normal. SERC received the most exchange energy in 1990, but MAPP led in this category in 1992 (2.7 billion kilowatthours from utilities in WSCC). In 1992, MAPP received over 568 million kilowatthours of wheeled power from utilities located in WSCC (Table 15). Utilities in MAPP sold more energy outside the region than inside. Investor-owned utilities in ECAR wheeled the most power for other utilities (4.1 billion kilowatthours to SERC) (Table 16).

In 1992, the United States purchased considerably more power (71 percent) from Canada than it did in 1990, while trade with Mexico remained stable and relatively small in relation to trade with Canada (Table 18). Power imports from Quebec and Manitoba outpaced the other provinces with increases over 1990 of 208 percent and 184 percent, respectively. Overall exports to Canada since 1990 declined by almost 61 percent. Exports of power to Ontario Province decreased by nearly 91 percent, led by reductions in exports from electric utilities in ECAR. (Figure 5).

\section{Tables}

National and regional aggregates are presented at the end of this chapter for data covering:

- Purchases, sales for resale, and exchanges by investor-owned utilities with all ownership classes (Tables 8 through 10 )

- Exchanges by noninvestor-owned utilities (Table 11)

- Wheeling transactions of investor-owned utilities (Tables 12 and 13)

- Regional purchases by cooperative borrowers (Table 14)

- Trading within and between regions by investorowned utilities (Tables 15 and 16)

- Transactions of investor-owned utilities in Alaska and Hawaii (Table 17)

- U.S. electricity trade with Canada and Mexico (Table 18). 
Table 5. Intra- and Interreglonal Purchases and Sales for Resale by Selected Ownershlp Clacses, 1986, 1988, 1990, and 1992

(Million Kilowatthours)

\begin{tabular}{|c|c|c|c|c|c|c|c|c|c|c|}
\hline \multirow[b]{2}{*}{$\begin{array}{l}\text { Type of Trenescotion } \\
\text { TOwmerehip Clase }\end{array}$} & \multicolumn{5}{|c|}{ Intraregional } & \multicolumn{5}{|c|}{ Interregional } \\
\hline & 1992 & 1990 & 1988 & 1886 & $\begin{array}{c}\text { Total } \\
\text { Percent } \\
\text { Difference } \\
1988-1092\end{array}$ & 1992 & 1990 & 1088 & 1986 & $\begin{array}{c}\text { Total } \\
\text { Percent } \\
\text { Difference } \\
1986-1992\end{array}$ \\
\hline 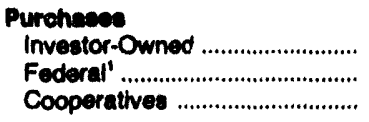 & $\begin{array}{r}598,671 \\
17,848 \\
268,884\end{array}$ & $\begin{array}{r}521,524 \\
8,476 \\
\text { n } 257,467\end{array}$ & $\begin{array}{r}394,994 \\
74,217 \\
254,768\end{array}$ & $\begin{array}{r}380,130 \\
39,037 \\
239,081\end{array}$ & $\begin{array}{r}68.2 \\
-54.0 \\
11.6\end{array}$ & $\begin{array}{r}59,927 \\
1,705 \\
14,234\end{array}$ & $\begin{array}{r}41,627 \\
2,637 \\
15,905\end{array}$ & $\begin{array}{r}20,809 \\
19,644 \\
7,573\end{array}$ & $\begin{array}{l}11,482 \\
10,498 \\
10,885\end{array}$ & $\begin{array}{r}421.8 \\
-83.8 \\
28.8\end{array}$ \\
\hline 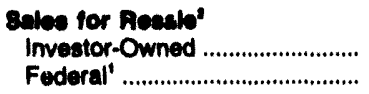 & $\begin{array}{l}452,442 \\
174,156\end{array}$ & $\begin{array}{l}373,897 \\
185,801\end{array}$ & $\begin{array}{l}320,738 \\
174,279\end{array}$ & $\begin{array}{l}320,102 \\
210,731\end{array}$ & $\begin{array}{r}41.7 \\
-17.4\end{array}$ & $\begin{array}{l}61,928 \\
12,973\end{array}$ & $\begin{array}{l}70,284 \\
11,764\end{array}$ & $\begin{array}{r}45,235 \\
2,918\end{array}$ & $\begin{array}{r}26,634 \\
4,111\end{array}$ & $\begin{array}{l}129.5 \\
215.6\end{array}$ \\
\hline
\end{tabular}

1 Federal authorities use different accounting methods for reporting energy recelved from Federal generating assets; the energy may be reported as generation or purchases. Federal date are presented as an agoregated flecal year which ends on September 30 .

- Detailed information for sales for resule by cooperative utilities is collected but not processed in machine ieadable format by the Rural Electriflcation Administration.

i Revised.

Note: The FERC has again required inveator-owned electric utllities to reflle 1992 bulk power data.

Sources: Energy Information Administration, Survey Management Division, calculated from data reported on Federal Energy Regulatory Commission (FERC) Form 1, "Annual Report of Major Electric Utilties, Licensees and Others," FERC Form 1-F. "Annual Report of Nonmajor Public Utilities and Licensees"; Form EIA-412, "Annual Report of Public Electric Utilities"; Rural Electrification Administration, REA Form 7, "Financial and Statistical Report," REA

Forms 12a through 121, "Electric Power Supply Borrowers," and REA Forms 12c through 12g, "Electric Distribution Borrowers with Generating Facilities."

Table 6. Firm and Nonfirm Purchases and Sales for Resale by Investor-Owned Utilities, by NERC Reglon, 1986, 1988, 1990, and 1992 (Million Kilowatthours)

\begin{tabular}{|c|c|c|c|c|c|c|c|c|c|c|}
\hline \multirow[b]{2}{*}{ NEAC Reglon } & \multicolumn{5}{|c|}{ Firm } & \multicolumn{5}{|c|}{ Nonfirm } \\
\hline & 1992 & 1990 & 1988 & 1986 & $\begin{array}{c}\text { Percent } \\
\text { Change } \\
1986-1982 \\
\end{array}$ & 1892 & 1990 & 1888 & 1986 & $\begin{array}{c}\text { Percent } \\
\text { Change } \\
1986-1992 \\
\end{array}$ \\
\hline & \multicolumn{10}{|c|}{ Purchaces by Investor-Owned Utilitioe } \\
\hline $\begin{array}{l}\text { ECAR } \\
\text { ERCOT } \\
\text { MAAC } \\
\text { MAIN } \\
\text { MAPP } \\
\text { NPCC } \\
\text { SERC } \\
\text { SPP } \\
\text { WSCC }\end{array}$ & $\begin{array}{r}46,741 \\
22,716 \\
28,244 \\
11,547 \\
13,652 \\
74,925 \\
57,671 \\
3,941 \\
83,037\end{array}$ & $\begin{array}{r}47,191 \\
22,285 \\
25,925 \\
12,463 \\
6,193 \\
65,349 \\
59,834 \\
6,886 \\
78,397\end{array}$ & $\begin{array}{r}13,777 \\
21,423 \\
23,260 \\
3,910 \\
5,485 \\
73,573 \\
44,418 \\
13,163 \\
49,094\end{array}$ & $\begin{array}{r}15,943 \\
21,046 \\
16,585 \\
4,704 \\
3,146 \\
70,587 \\
22,328 \\
7,035 \\
31,813\end{array}$ & $\begin{array}{r}193.2 \\
7.9 \\
70.3 \\
145.5 \\
334.0 \\
6.1 \\
158.3 \\
-44.0 \\
161.0\end{array}$ & $\begin{array}{r}39,531 \\
5,141 \\
38,994 \\
17,002 \\
12,701 \\
66,934 \\
29,019 \\
44,138 \\
46,206\end{array}$ & $\begin{array}{r}22,106 \\
3,399 \\
14,340 \\
9,307 \\
13,744 \\
38,682 \\
28,642 \\
30,227 \\
59,163\end{array}$ & $\begin{array}{r}10,358 \\
2,819 \\
7,256 \\
4,218 \\
10,117 \\
28,732 \\
8,150 \\
26,777 \\
50,878\end{array}$ & $\begin{array}{r}10,689 \\
1,854 \\
1,918 \\
2,101 \\
12,377 \\
30,558 \\
11,508 \\
19,652 \\
62,641\end{array}$ & $\begin{array}{r}269.8 \\
177.3 \\
1932.7 \\
709.1 \\
2.6 \\
119.0 \\
152.2 \\
124.6 \\
-26.2\end{array}$ \\
\hline \multirow[t]{2}{*}{ 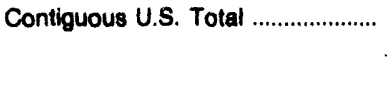 } & 342,472 & 324,524 & 248,104 & 193,185 & 77.3 & 299,666 & 219,700 & 149,405 & 153,299 & 95.5 \\
\hline & \multicolumn{10}{|c|}{ Sales for Resale by Investor-Owned Utilities } \\
\hline $\begin{array}{l}\text { ECAR } \\
\text { ERCOT } \\
\text { MAAC } \\
\text { MAIN } \\
\text { MAPP } \\
\text { NPCC } \\
\text { SERC } \\
\text { SPP } \\
\text { WSCC }\end{array}$ & $\begin{array}{r}78,719 \\
6,589 \\
13,978 \\
16,375 \\
9,828 \\
53,816 \\
42,860 \\
17,139 \\
29,962\end{array}$ & $\begin{array}{r}75,359 \\
8,232 \\
6,372 \\
22,325 \\
6,789 \\
47,578 \\
44,051 \\
15,586 \\
27,516\end{array}$ & $\begin{array}{r}77,391 \\
8,687 \\
6,228 \\
10,890 \\
4,388 \\
49,477 \\
39,759 \\
14,830 \\
19,320\end{array}$ & $\begin{array}{r}50,908 \\
8,019 \\
5,630 \\
13,172 \\
3,871 \\
43,925 \\
33,454 \\
16,682 \\
14,268\end{array}$ & $\begin{array}{r}54.6 \\
-17.7 \\
148.3 \\
24.3 \\
153.9 \\
22.5 \\
28.4 \\
2.9 \\
110.0\end{array}$ & $\begin{array}{r}53,251 \\
1,392 \\
28,411 \\
10,170 \\
8,728 \\
37,864 \\
46,339 \\
27,944 \\
23,210\end{array}$ & $\begin{array}{r}42,054 \\
1,405 \\
9,186 \\
6,884 \\
11,175 \\
19,337 \\
49,429 \\
19,960 \\
23,380\end{array}$ & $\begin{array}{r}22,102 \\
2,065 \\
6,323 \\
1,226 \\
5,002 \\
13,164 \\
36,568 \\
21,377 \\
18,351\end{array}$ & $\begin{array}{r}28,190 \\
2,370 \\
5,861 \\
1,612 \\
2,591 \\
16,564 \\
34,431 \\
19,459 \\
21,788\end{array}$ & \begin{tabular}{r|}
88.9 \\
-41.3 \\
384.7 \\
530.8 \\
236.9 \\
129.2 \\
34.6 \\
43.6 \\
6.5
\end{tabular} \\
\hline 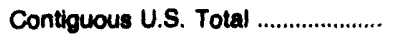 & 269,376 & 253,809 & 230,952 & 189,906 & 41.8 & 237,409 & 182,809 & 127,180 & 132,866 & 78.7 \\
\hline
\end{tabular}

Notes: -Miscellaneous tranesctions for the Purchased Power and Sales for Resale categories have been excluded. See Chapter 2 for a discussion of miscellaneous transactions. -NERC is the North American Electric Rellability Council. The Alaska System Coordinating Council is not included. See Glossary for a list of regions and Figure 2 for a map of the reglons.

Source: Federal Energy Regulatory Commission (FERC) Form 1. "Annual Report of Major Electric Utilities, Licensees and Others," and FERC Form 1-F,

"Annual Peport of Nonmajor Public Utilities and Licensees." 
Figure 5. U.S. Electrlcity Trade with Canada and Mexico by NERC Reglon, 1992

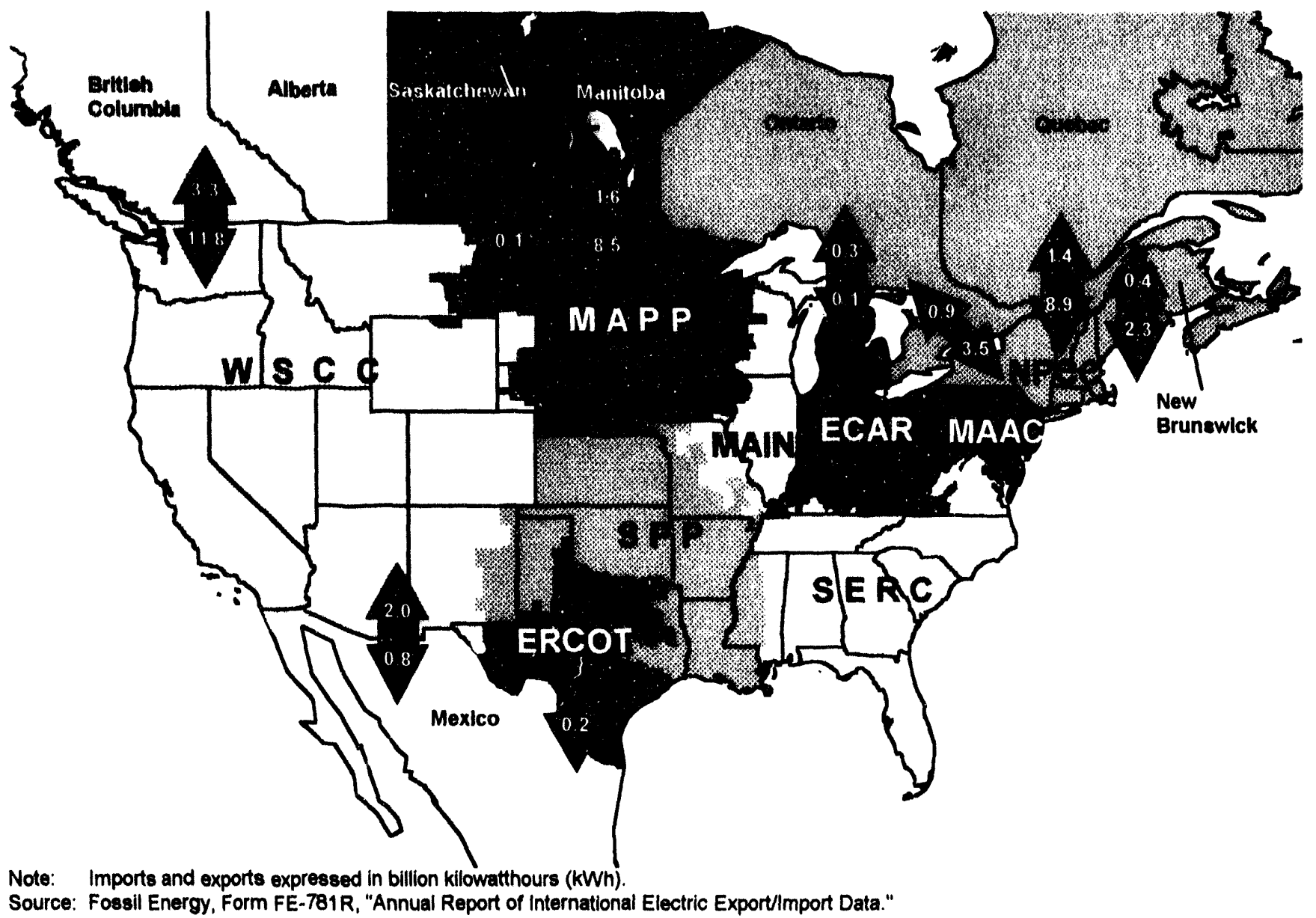

\section{Sources of Data, 1992}

Summary information for the United States are provided in Tables 1 through 4 and are obtained from the Form EIA-861 "Annual Electric Utility Report," which is a mandatory report filed by all U.S. electric utilities. The survey represents the collection of data from the complete universe of electric utilities. All other electric utility surveys cited in this publication represent subsets from the Form EIA-861 universe. Tables 5 through 44 include detailed information developed from direct filings of different surveys by 224 of the 262 investor-owned electric utilities; 484 of the 2,017 State and municipal electric utilities; 915 of the 943 cooperative electric utilities, and 8 of the 10 Federal utilities. ${ }^{4}$ The detailed data represent subsets of the universe data provided in Tables 1 through 4. (The requirements for filing are discussed below.) Of the total number of electric utilities identified, but not required to file a detailed data collection form with a
Federal agency, most have little generating capability and usually purchase part or all of their electrical energy from one or more of the electric utilities who did file. However, some do buy from cogenerators, cooperatives, or other utilities not required to file. The summary quantity of purchases made by these utilities are included in this publication (Tables 22, 31, and 35). These utilities were identified by matching the names of filing utilities and all the utilities listed on these filings with the universe frame of Form EIA-861, "Annual Electric Utility Report." Chapters 2 through 4 provide detailed data on individual electric utilities developed from the direct filings. However, if the direct filing indicates no wholesale trade transactions, then those electric utilities were not shown in the respective tables. Tables 37 through 44 provide information on portions of the physical transmission systems. Data came from the same electric utility filings described above for the FERC Form 1 and Form EIA-412. Information on transmission line statistics covering the public sector has not been published before by EIA nor has the detailed information about transmission-

\footnotetext{
The two Federal utilities that do not file the Form ElA-412 do not sell wholesale power. Electricity produced by these utilities is marketed by one or more of the Federal power marketing administrations.
} 
Table 7. Wheellng Trade In the United States by NERC Reglon, 1986 Through 1992 (Million Kilowatthours)

\begin{tabular}{|c|c|c|c|c|c|c|c|}
\hline Wheelling & 1892 & 1981 & 1990 & 1989 & 1888 & 1887 & 1986 \\
\hline & \multicolumn{7}{|c|}{ All Utiltites in ECAR } \\
\hline \multirow[t]{2}{*}{ 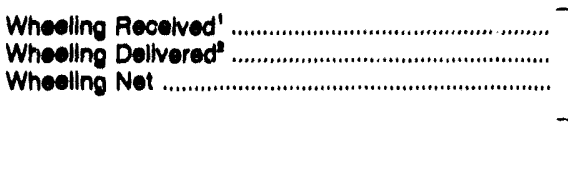 } & $\begin{array}{r}31,803 \\
31,618 \\
186\end{array}$ & $\begin{array}{r}30,293 \\
30,161 \\
132\end{array}$ & $\begin{array}{r}32,841 \\
32,763 \\
88\end{array}$ & $\begin{array}{r}21,098 \\
20,994 \\
104\end{array}$ & $\begin{array}{r}16,121 \\
16,036 \\
85\end{array}$ & $\begin{array}{r}10,347 \\
10,281 \\
65\end{array}$ & $\begin{array}{r}\$ 9,429 \\
18,383 \\
76\end{array}$ \\
\hline & \multicolumn{7}{|c|}{ All Utilitise in Encot } \\
\hline \multirow[t]{2}{*}{ 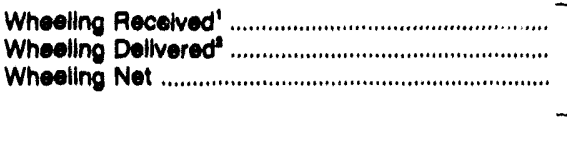 } & $\begin{array}{r}24,077 \\
24,080 \\
-13\end{array}$ & $\begin{array}{r}20,834 \\
20,837 \\
-2\end{array}$ & $\begin{array}{r}16,481 \\
16,498 \\
-14\end{array}$ & $\begin{array}{r}9,414 \\
9,297 \\
117\end{array}$ & $\begin{array}{r}7,861 \\
7,813 \\
-63\end{array}$ & $\begin{array}{r}6,532 \\
6,543 \\
-11\end{array}$ & $\begin{array}{r}2,426 \\
2,425 \\
2\end{array}$ \\
\hline & \multicolumn{7}{|c|}{ All Utilitios in MAAC } \\
\hline \multirow[t]{2}{*}{ 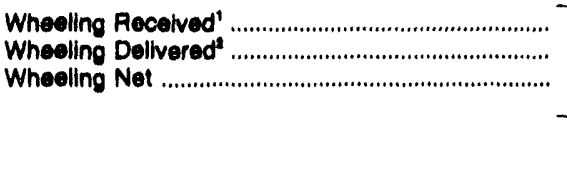 } & $\begin{array}{r}9,868 \\
9,779 \\
88\end{array}$ & $\begin{array}{l}5,842 \\
5,842 \\
-\end{array}$ & $\begin{array}{r}\mathbf{5 , 7 7 4} \\
5,774 \\
\end{array}$ & $\begin{array}{r}8,385 \\
8,360 \\
24\end{array}$ & $\begin{array}{r}7,482 \\
7,465 \\
16\end{array}$ & $\begin{array}{r}6,665 \\
7,226 \\
-560\end{array}$ & $\begin{array}{r}6,832 \\
7,119 \\
-287\end{array}$ \\
\hline & \multicolumn{7}{|c|}{ All Utilitios in MAIN } \\
\hline \multirow[t]{2}{*}{ 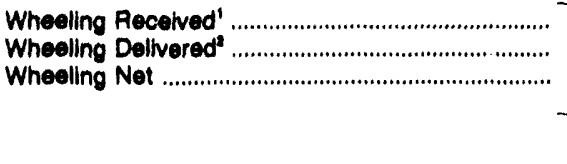 } & $\begin{array}{r}3,489 \\
3,404 \\
85\end{array}$ & $\begin{array}{r}3,819 \\
3,739 \\
80\end{array}$ & $\begin{array}{r}1,451 \\
1,414 \\
37\end{array}$ & $\begin{array}{r}1,215 \\
1,206 \\
10\end{array}$ & $\begin{array}{r}1,815 \\
1,816 \\
10\end{array}$ & $\begin{array}{r}2,071 \\
2,017 \\
54\end{array}$ & $\begin{array}{r}571 \\
561 \\
10\end{array}$ \\
\hline & \multicolumn{7}{|c|}{ All vellitios in MAPP } \\
\hline \multirow[t]{2}{*}{ 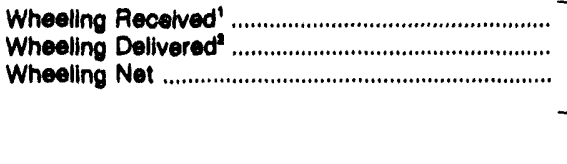 } & $\begin{array}{r}11,681 \\
10,847 \\
814\end{array}$ & $\begin{array}{l}9,484 \\
7,835 \\
1,549\end{array}$ & $\begin{array}{r}8,477 \\
7,856 \\
821\end{array}$ & $\begin{array}{l}9,208 \\
8,187 \\
1,012\end{array}$ & $\begin{array}{l}7,804 \\
6,705 \\
1,098\end{array}$ & $\begin{array}{r}7,374 \\
6,727 \\
647\end{array}$ & $\begin{array}{r}6,095 \\
5,393 \\
702\end{array}$ \\
\hline & \multicolumn{7}{|c|}{ All UtIlitios in NPCC } \\
\hline \multirow[t]{2}{*}{ 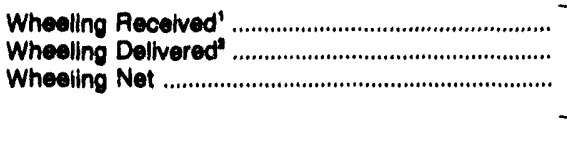 } & $\begin{array}{r}60,687 \\
60,495 \\
191\end{array}$ & $\begin{array}{r}57,173 \\
56,925 \\
248\end{array}$ & $\begin{array}{r}48,613 \\
48,389 \\
214\end{array}$ & $\begin{array}{r}42,897 \\
42,351 \\
546\end{array}$ & $\begin{array}{r}38,365 \\
38,069 \\
276\end{array}$ & $\begin{array}{r}20,486 \\
20,011 \\
475\end{array}$ & $\begin{array}{r}23,615 \\
23,603 \\
212\end{array}$ \\
\hline & \multicolumn{7}{|c|}{ All vetiltioe in BERC } \\
\hline \multirow[t]{2}{*}{ 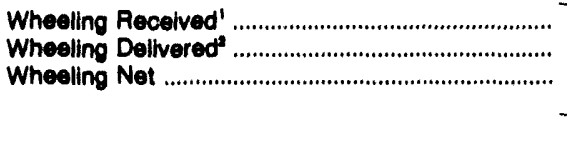 } & $\begin{array}{r}27,211 \\
25,408 \\
1,803\end{array}$ & $\begin{array}{r}28,457 \\
27,447 \\
1,010\end{array}$ & $\begin{array}{r}29,291 \\
28,174 \\
1,117\end{array}$ & $\begin{array}{r}22,726 \\
21,885 \\
841\end{array}$ & $\begin{array}{r}12,354 \\
11,743 \\
611\end{array}$ & $\begin{array}{r}11,038 \\
9,968 \\
1,073\end{array}$ & $\begin{array}{r}12,480 \\
11,238 \\
1,252\end{array}$ \\
\hline & \multicolumn{7}{|c|}{ All Utilitios in SPP } \\
\hline \multirow[t]{2}{*}{ 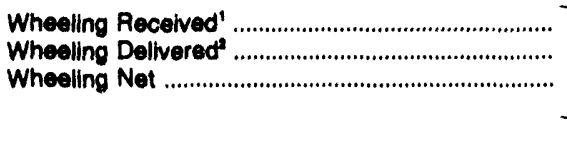 } & $\begin{array}{r}21,749 \\
21,639 \\
110\end{array}$ & $\begin{array}{r}22,150 \\
22,078 \\
72\end{array}$ & $\begin{array}{r}27,091 \\
26,986 \\
106\end{array}$ & $\begin{array}{r}19,591 \\
19,924 \\
-333\end{array}$ & $\begin{array}{r}20,089 \\
18,968 \\
133\end{array}$ & $\begin{array}{r}10,158 \\
9,818 \\
338\end{array}$ & $\begin{array}{r}8,650 \\
8,408 \\
241\end{array}$ \\
\hline & \multicolumn{7}{|c|}{ All Utilitios in wecc } \\
\hline \multirow[t]{2}{*}{ 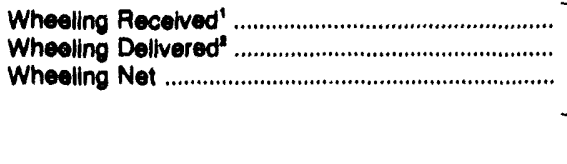 } & $\begin{array}{r}127,514 \\
125,023 \\
2,492\end{array}$ & $\begin{array}{r}144,466 \\
142,592 \\
1,874\end{array}$ & $\begin{array}{r}162,097 \\
148,388 \\
3,708\end{array}$ & $\begin{array}{r}164,824 \\
163,623 \\
1,200\end{array}$ & $\begin{array}{r}157,755 \\
157,682 \\
74\end{array}$ & $\begin{array}{r}118,925 \\
118,995 \\
-69\end{array}$ & $\begin{array}{r}117,607 \\
117,477 \\
330\end{array}$ \\
\hline & \multicolumn{7}{|c|}{ Contiguous U.8. Total } \\
\hline 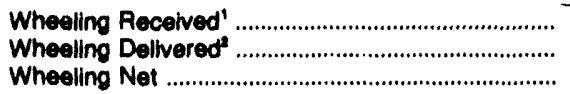 & $\begin{array}{r}318,538 \\
312,782 \\
5,756\end{array}$ & $\begin{array}{r}323,133 \\
318,170 \\
4,963\end{array}$ & $\begin{array}{r}322,667 \\
316,591 \\
6,077\end{array}$ & $\begin{array}{r}299,822 \\
296,297 \\
3,525\end{array}$ & $\begin{array}{r}269,170 \\
268,909 \\
2,262\end{array}$ & $\begin{array}{r}194,049 \\
192,030 \\
2,019\end{array}$ & $\begin{array}{r}195,391 \\
192,844 \\
2,547\end{array}$ \\
\hline
\end{tabular}

1 Includes all transactions received from outside and within the region by utilities operating within the region.

2 Includes all transactions delivered out of and within the region by utilities operating within the region.

- Not Applicable

Notes: - The States of Alaska and Hawail are not included. •A/jgregate 1992 bulk power data supplied by investor-owned electric utilities were matched to original filings made to the FERC. The FERC has again required investor-owned electric utilities to refile 1992 bulk power data. These revised data are shown in Tables 5-24 and represent the reclassification of data among purchased power, exchanges, and sales for resale accounts. The difference between the data series are the result of the reclassifications and adjustments made to the data. ONERC is the North American Electic Reliability Council. See glossary for a list of regions and Figure 2 for a map of the regions. Source: Energy Information Administration, Form EIA-861, "A,inual Electric Utility Report."

lines-added and the associated cost for investor-owned electric utilities. Transmission-lines-added information is not collected for the public sector.
Chapter 1 gives an overview of bulk power trade and provides national and regional electric trade data by all ownership classes. These data come from several forms, as no form was specifically designed to collect electricity trade information from all ownership 
classes. International trade data are from Form FE-781R, "Annual Report of International Electrical Export/Import Data," (Table 18).

Chapter 2 presents data on bulk power transactions of "Major" and "Minor" investor-owred utilities. Major investor-owned electric utilities are defined as those private utilities that have had, in the past 3 consecutive calendar years, sales or transmission services that exceeded one of the following: 1 billion kilowatthours of total annual sales, 100,000 kilowatthours of annual sales for resale, 500,000 kilowatthours of annual gross interchange-out or 500,000 kilowatthours of transmission for others (wheeling). The criteria for classification as a minor utility are: (1) total annual sales of 10 million kilowatthours or more in the previous calendar year, and (2) not being classified as a major utility.

The investor-owned utilities shown in this publication report on FERC Form 1, "Annual Report of Major Electric Utilities, Licensees and Others" and FERC Form 1-F, "Annual Report of Nonmajor Public Utilities and Licen' ses."

The FERC forms are primarily financial reporting forms. The wholesale data are collected on 4 of the approximately 75 survey schedules of the FERC Form 1. The wholesale electricity trade schedules are sales for resale, purchased power, summary of interchange, and transmission of electricity for or by others. These electricity transactions are shown by utility class of ownership in Tables 19 through 24.

The investor-owned electric utilities that fall below the filing criteria for the FERC Forms 1 and 1-F are not required to file. Appendix Table B1 lists the major and minor utilities that file FERC Forms 1 and 1-F.

Data in Chapter 3 cover wholesale electriciry trade for the 492 public utilities that report on Form EIA -412, "Annual Fieport of Public Electric Utilities," which is used to collect data on purchased power and sales for' resale transactions. The public utilities report information by riscal year; therefore, consistent totals cannot be provided for these classes of ownership. Public electric utilities are required to submit Form EIA-412 when their generation, transmission, or distribution of electricity results in 120 million kilowatthours of sales to ultimate consumers and/or 120 million kilowatthours of sales for resale. Data on exchanges and wheeling in this chapter are reported on Form EIA-861, "Annual Electric C'tility Report."

Chapter 4 presents data submitted to the Rural Electrification Administration (REA) of the U.S. Department of Agriculture by cooperative borrowers. The REA collects information from all utilities that have "borrowed" under the REA loan guarantee programs and uses the information to verify and administer its loan programs. Appendix Table B5 lists cooperative borrowers. Data covering electricity trade for cooperative borrowers are reported for a calendar-year basis on several closely related REA forms: REA Form 7, "Financial and Statistical Report," REA Forms 12a through 12i, "Electric Power Supply Borrowers," and REA Forms 12c through 12g, "Electric Distribution Borrowers with Generating Facilities." These forms are used to collect data on purchased power. Sales for resale transactions are not available in a machinereadable format from the REA.

Chapter 5 presents data on the physical transmission systems. The investor-owned data have been shifted from the EIA publication, "Financial Statistics of Selected Investor-Owned Electric Utilities," to this publication. In addition, transmission line statistics are being provided for publicly owned utilities for the first time from data reported on Form EIA-412.

\section{Compllation of Data}

The Federal Government recognizes that wholesaie trade data needs to be consolidated and automated. The EIA examined the data and initiated a process to correct deficiencies with the FERC. Responsibility for correcting errors and making contact with the FERC Form 1 respondents rests with the FERC. Principal among the problems with existing wholesale trade data are: (1) inconsistencies in reporting mutual transactions by buyers and sellers, (2) lack of uniformity and standardization in reporting, (3) lack of uniformity in reporting requirements among various forms including reporting periods, and (4) multiple and sometimes overlapping reporting and aggregation of individual transactions.

Many of these errors are identified automated intraform range, arithmetic and logic checks, and interform consistency edits (for example, crosschecking between submissions of the respondents). The Technical Notes provide more information on data linkages, revisions, and correction procedures used in editing data on wholesale electricity trade. In addition, the corrections to these problems also serve as the foundation for future analytical work to improve reporting on EIA forms.

\section{Limitations of Data Coverage}

Electricity transactions have a wide range of terms and conditions designed to accommodate prevailing circumstances. Because of the lack of standardization in contract terminology, reporting of each transaction is not consistent across the industry. The lack of uniformity, combined with the complexity of the transactions, affects the accuracy of the data. This is further complicated by the accounting of simultaneous energy 
transactions, the receipt and delivery of energy caused by inadvertent flow, and the losses associated with transmission.

The major sources of incompatibility between data collection forms include: (1) different reporting years, (2) different reporting requirements, (3) different reporting of mutual transactions by purchasers and sellers, (4) lack of uniformity and standardization in the definitions of terms used in the industry, and (5) multiple reporting of individual transactions. The single greatest source of incompatibility is the different "reporting year" requirements of Form EIA-412 compared with the other forms. The annual data reported on Form EIA -412 by a public utility are based on that utility's fiscal year. The annual data reported on Form EIA-861, FERC Form 1, REA Form 7, and REA Form 12 are based on the calendar year.

The second largest source of incompatibility is the difference in reporting requirements among the forms. The four types of electric trade transactions and the forms used to collect them are as follows:

- Purchases--FERC Forms 1 and 1-F, Form EIA-412, REA Form 7, REA Form 12

- Sales for Resale--FERC Forms 1 and 1-F, Form EIA-412

- Exchanges--FERC Forms 1 and 1-F

- Wheeling--FERC Forms 1 and 1-F

Form EIA-861 is also used to gather information on all four types of transactions; the data, however, are submitted on energy flows rather than financial performance. EIA-861 data are at an aggregate level and do not identify buyers and sellers. These differences make it impossible to disaggregate the types of electric trade transactions across ownership classes for all forms. This publication provides significantly more complete information on the investor-owned utilities than other ownership classes because of the greater detail collected on FERC Form 1.

The data on wholesale electricity trade are collected on Federal survey data forms that are accountingsystem based. These forms were designed for regulatory purposes to meet administrative and rate case needs of individual utilities as opposed to overall aggregate industry statistical and analytical purposes.

Data for the Commonwealth of Puerto Rico and the U.S. Trust Territories are excluded in this publication. Geographical location and electrical system isolation limit the usefulness of any wholesale trade data from these areas.

\section{Average Unit Cost and Unit Revenue}

This publication presents annual summations of electricity transactions (energy) with the corresponding revenue and cost information. Dividing cost or revenue by the transaction quantity gives the average cost or average revenue per unit of energy. However, it does not necessarily indicate the price specified in contracts.

Wholesale sales are transaction-specific and lose relevance when aggregated to classes of ownership and geographic regions. The price that any one utility charges another for wholesale energy comprises numerous system-specific factors. For example, the price of purchased power can consist of demand charges, energy charges, and facility charges. A demand charge is a fixed charge determined according to the maximum capacity demanded by the purchasing utility during a specified period of time. Demand charges, being fixed, are unaffected by the number of kilowatthours of energy purchased; therefore, the average charge per kilowatthour decreases as the volume of energy sales increases.

In addition to demand charges, wholesale electricity transactions are frequently subject to facility charges that include transmission, generation, and other system operating charges. These charges typically vary by ownership class, system characteristics, and region.

Furthermore, demand charges and energy charges are affected by time of delivery, quantity of energy, and reliability of supply. Typically, energy delivered during peak periods costs more than energy delivered during off-peak periods. Firm power, which can have a level of reliability similar to that given to a supplier's customers, costs more than economy energy. Economy energy is interruptible on short notice and replaces the more expensive firm power energy that is available to the purchaser.

For electric trade transactions, the average revenue or cost per unit of energy, calculated by dividing the revenue or cost by the associated energy, is not a substitute for total price. Electric trade transactions are comprised of various energy transactions of different value. Differences in average revenue or cost per unit of energy may represent a difference in the operating conditions rather than a difference in price structure. The quantity and value numbers presented represent the summation of all trade in that category for a year. Individual prices associated with a particular trade, the impact of seasonal and peak usage, and contract specific conditions cannot be separated from the aggregate. The reader is cautioned against using unit revenue or cost as a substitute for true price. 


\section{Account Adjustments}

Utilities must adjust the current year data to reflect not only the corrections in accounting for physical flows but also any regulatory and court decisions. Rate case rebates, meter adjustments, and inadvertent power flow corrections are examples of these changes.

Both negative and positive adjustments are present in the revenue, cost, and quantity of trade numbers. Negative and positive numbers in the interchange tables represent a direction of flow--positive is a receipt and negative is a delivery. In the purchased power and sales for resale tables, data (either positive or negative) represent either the booking of accounting adjustments or reversal in payments and energy flows.

Transmission of power from generating facilities under joint ownership can be reported in several ways. Typically, joint owners operate with interconnected transmission networks under mutually beneficial service agreements. The actual transfer of energy across transmission systems is usually considered a nonmonetary transaction and not a wholesale transaction. When a joint owner lacks an interconnected path to the generating facility, the actual flow of energy is subject to the transmission service of the wheeling third party, accounted for as a monetary transaction, and reported as "Transmission For Others."

In some of the tables, data categorized as "Other" include power pool data, international trade transactions, and any nonutility generated electricity purchased. This information is often reported in a manner that prevents the identification of the buyer and/or seller.

\section{Appendices}

Appendix $\mathrm{A}$ identifies the regulatory guidance provided by FERC through its Uniform System of Accounts and selected instructions provided on FERC Form 1.

Appendix B contains the location, NERC region, and data source of each utility for the 1992 filings used in this report. The information is presented by the utility forms: (1) FERC Form 1 and 1-F (Investor-Owned); (2) Form EIA-412 (Federal, State and Other Government Utilities, and Municipalities); (3) REA Form 7 and REA Form 12 (Cooperatives); (4) Form EIA-861 (Wheeling and Exchanges for Noninvestor-Owned Utilities); and (5) Energy Marketing (brokering-where ownership of power is transferred) Groups under FERC jurisdictional authority.

Appendix $\mathrm{C}$ contains a description of the fundamentals of electric power transmission, the control and operation of electric systems, and an overview of integrating NUGs into the power grid.

Technical Notes describe the linkages and revisions made to the various data collection systems used in preparing this publication. The Notes also explain data quality and correction procedures used. Copies of the collected data are available to the public. See "Obtaining Copies of the Data" in the Notes for further information.

\section{Glossary}

A glossary of terms has been included to assist nontechnical as well as technical users in understanding the statistical data in this publication. 
Table 8. Electricity Purchases by Investor-Owned Utilitios, by NERC Reglon, 1992

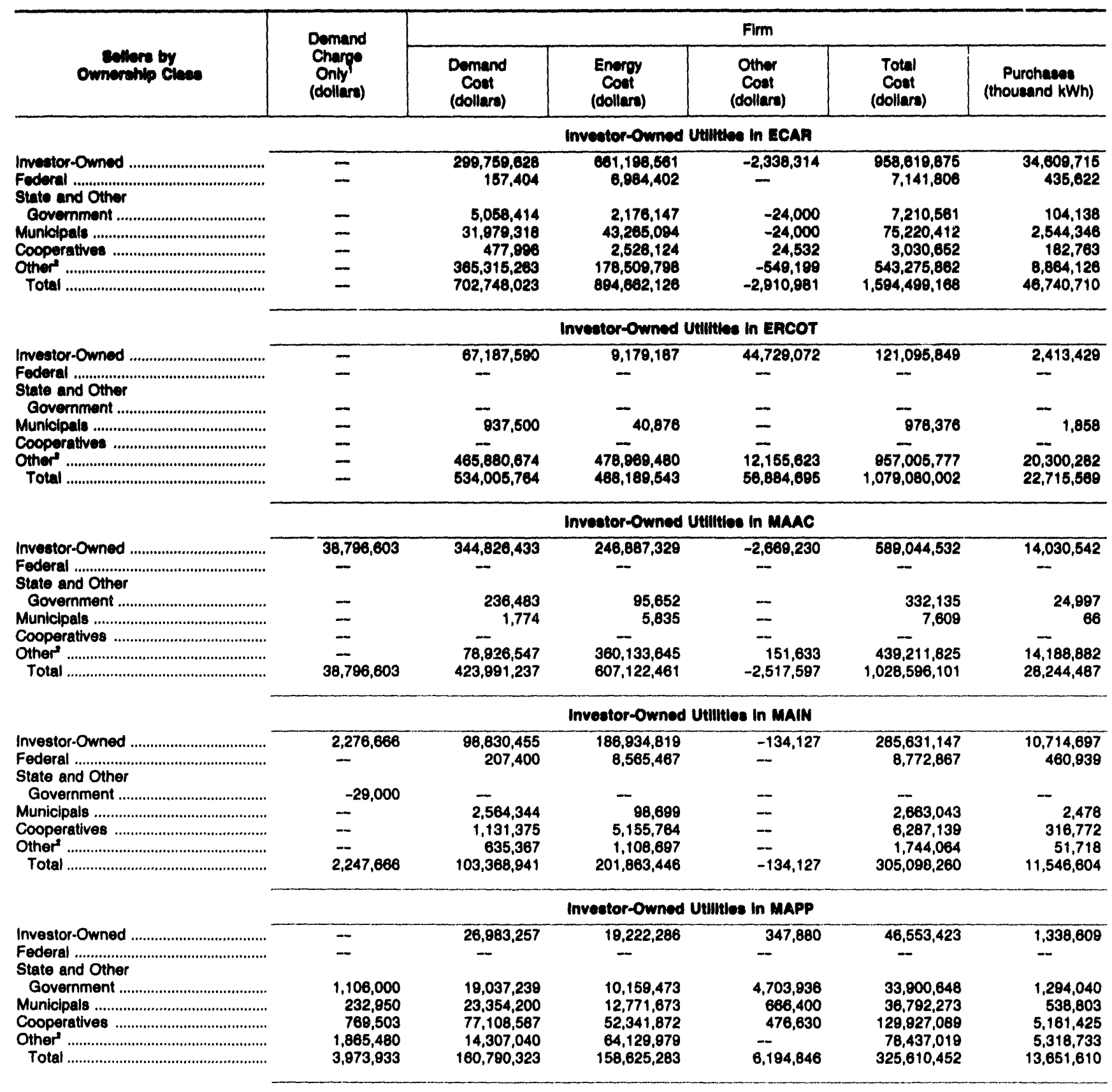

See notes and footnotes at end of table. 
Table 8. Electriclty Purchases by Investor-Owned Utilitios, by NERC Reglon, 1992 (Continuod)

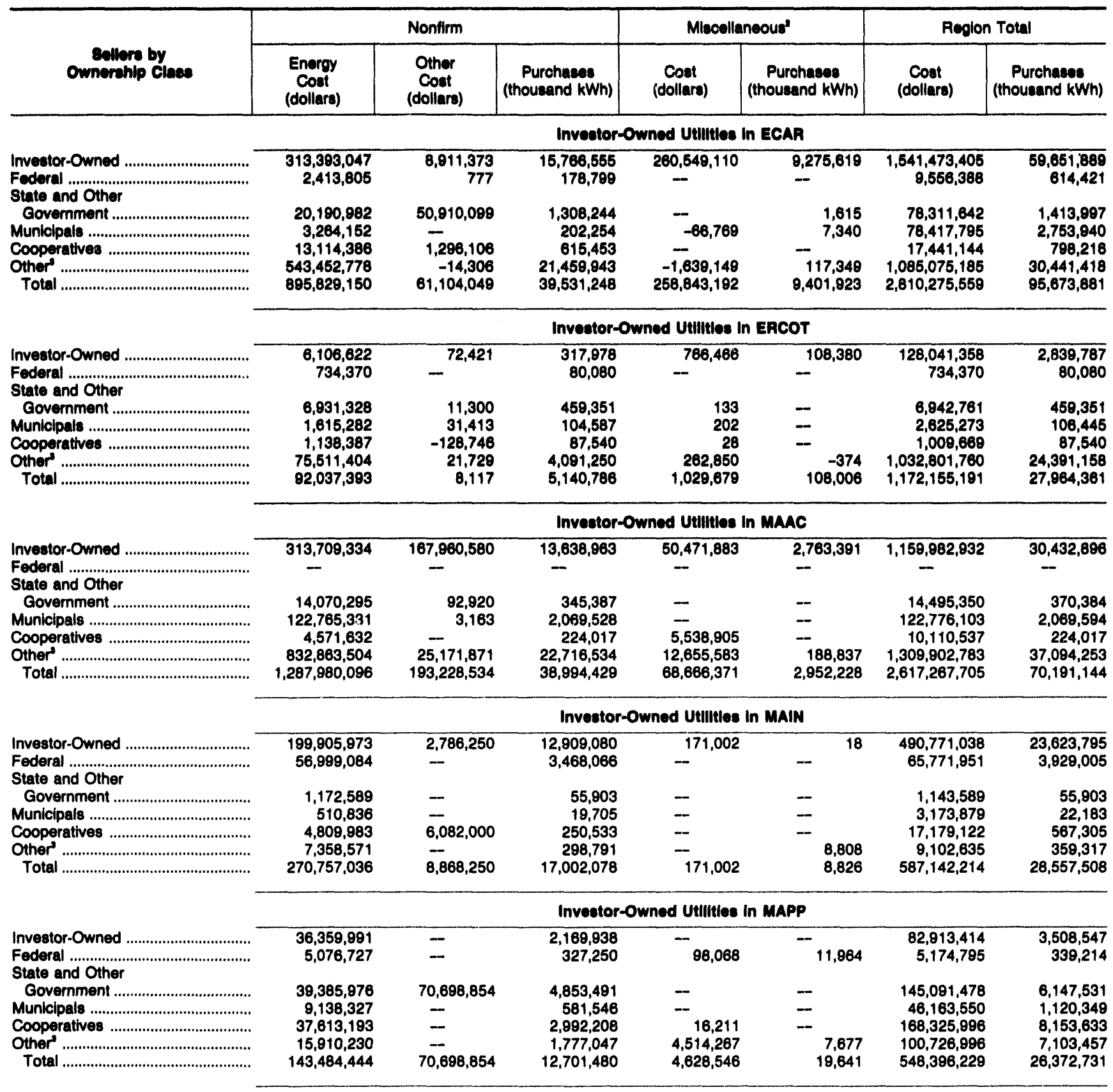

See notes and footnotes at end of table. 
Table 8. Electricity Purchases by Investor-Owned Utilities, by NERC Region, 1992 (Continued)

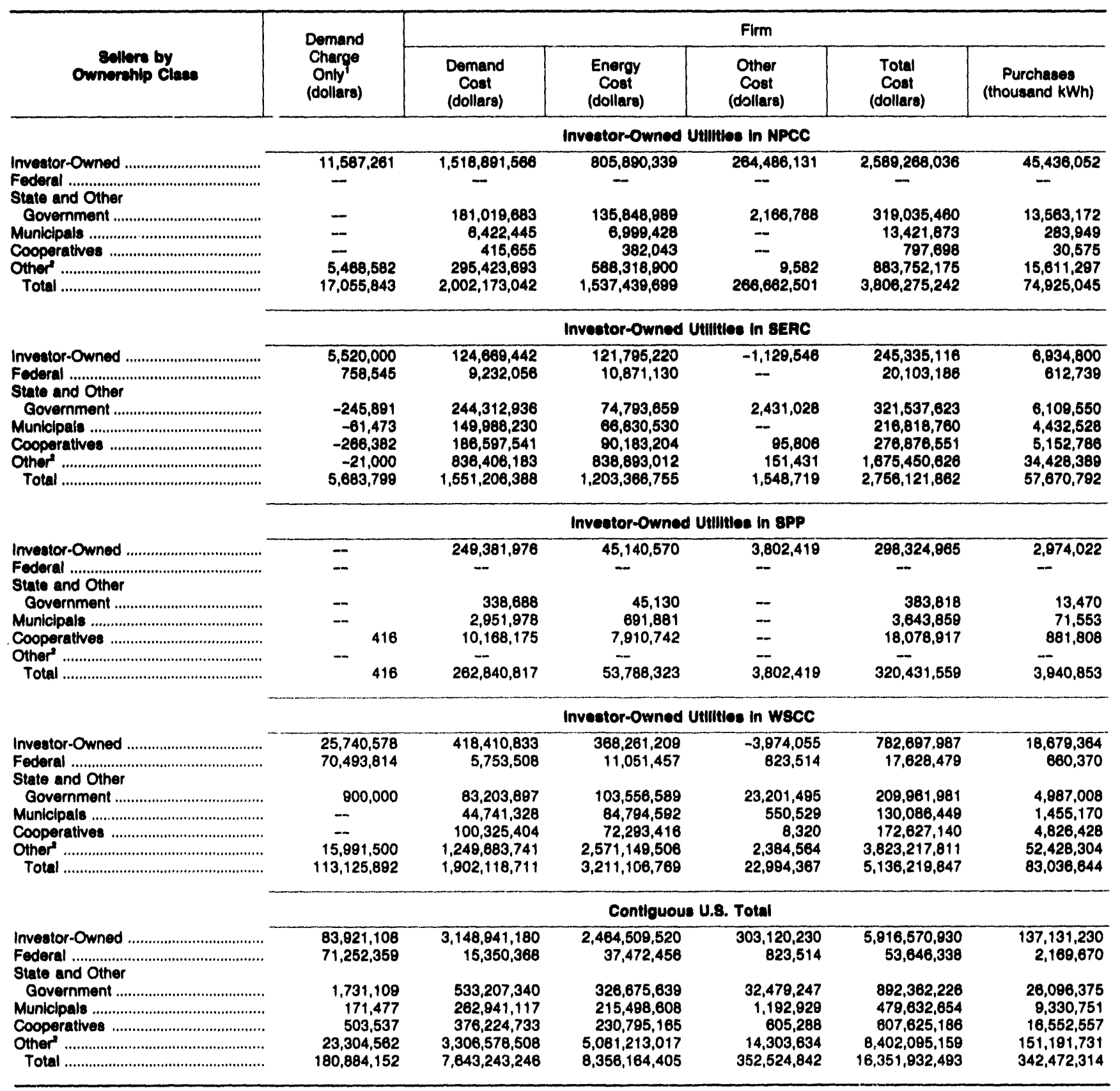

See notes and footnotes at end of table. 
Table 8. Electriclty Purchases by Investor-Owned Utillites, by NERC Reglon, 1992 (Continued)

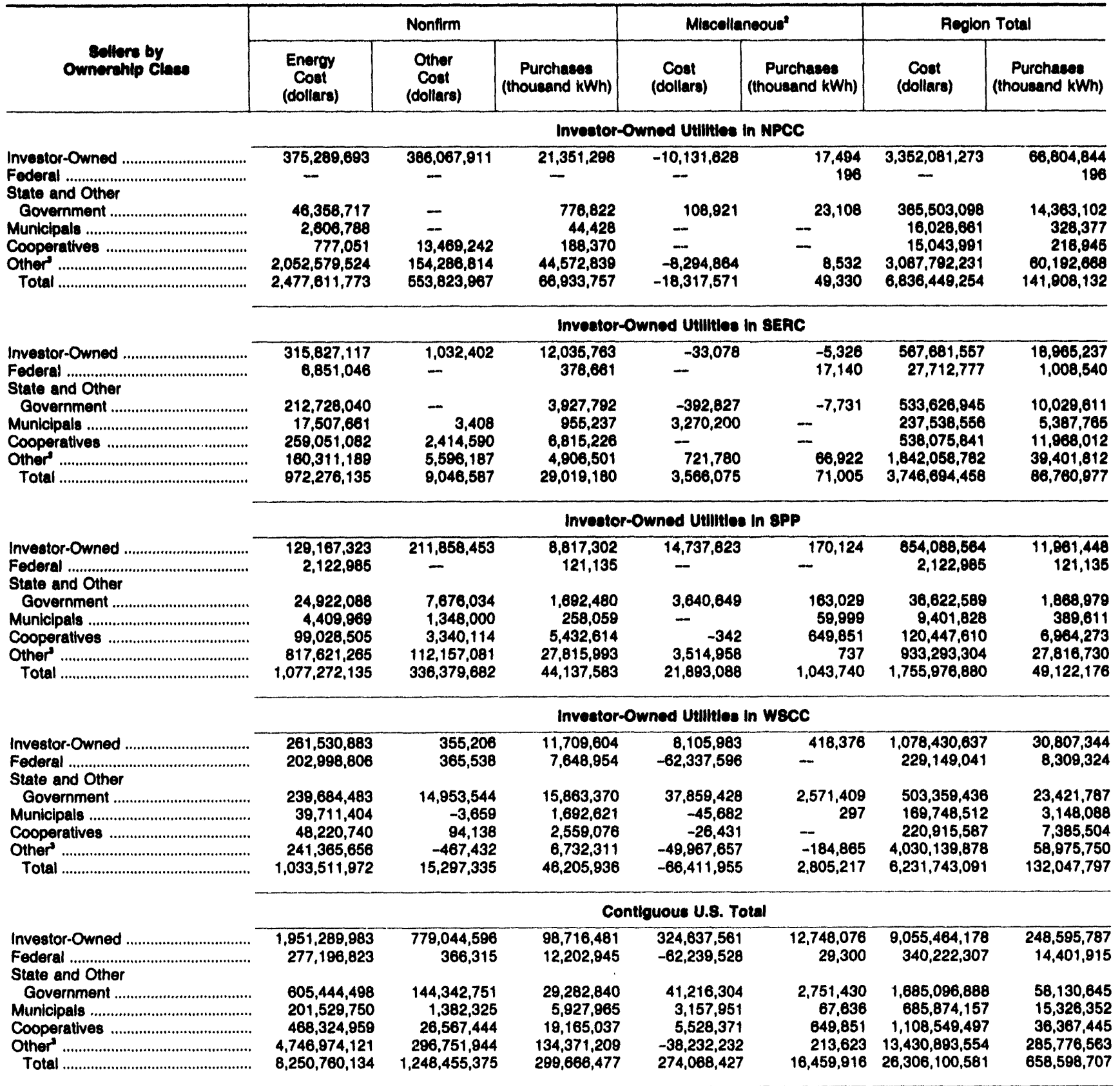

1 Monetary settlements from contracts where electricity is not taken.

2 Includes other transactions involving line and substation rentals, system support charges, and special contract transactions.

- Includes transactions with power pools, utilities in Canada and Mexico, and nonutilities.

- Not Applicable

Notes: -The States of Alaska and Hawaii are not included. •NERC is the North American Electric Reliability Council. The Alaska System Coordinating Council is not included. See glossary for a list of regions and Figure 2 for a map of the regions. -Totals may not equal sum of components because of independent rounding.

Sources: •Federal Energy Regulatory Commission, FERC Form 1, "Annual Report of Major Electric Utilities, Licensees and Others." •FERC Form 1. F. "Annual Report of Nonmajor Public Utilities and Licensees." 
Table 9. Electricity Sales for Resale by Investor-Owned Utilities, by NERC Region, 1992

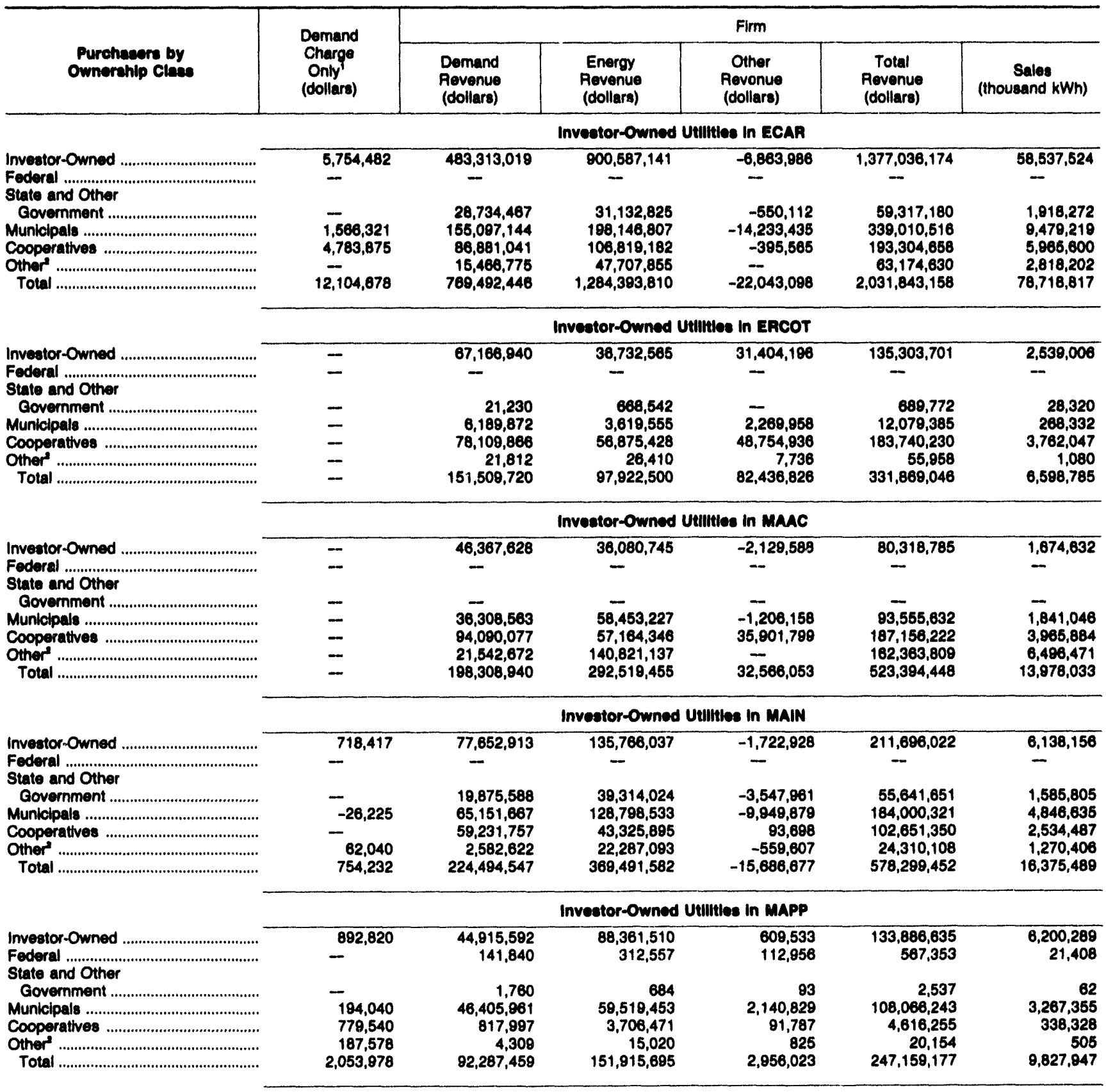

See notes and footnotes at end of table. 
Table 9. Electricity 8ales for Resale by Investor-Owned Utilitios, by NERC Reglon, 1892 (Continued)

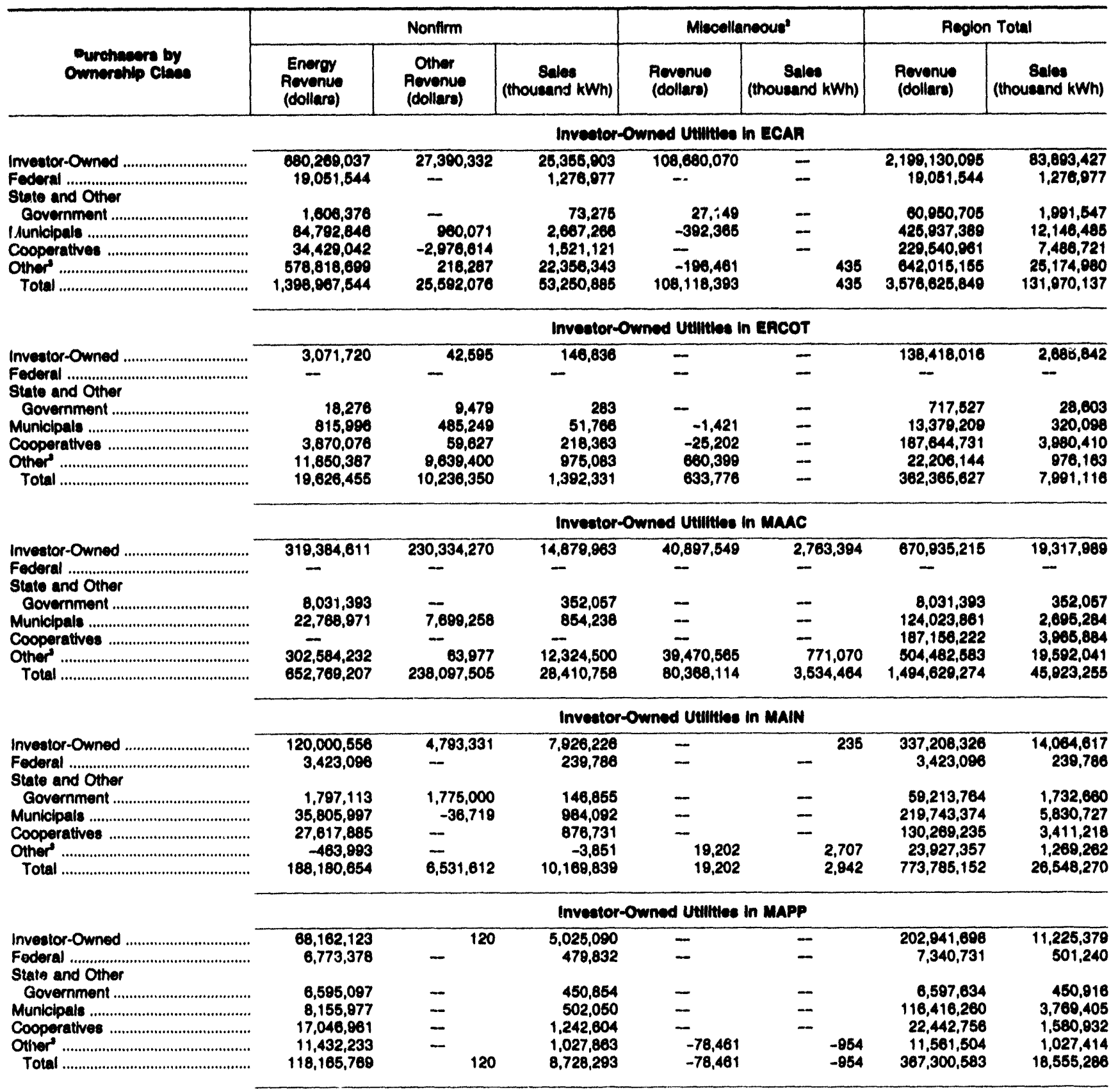

See notes and footnotes at end of table. 
Table 9. Eleotricity 8ales for Resale by Investor-Owned Utilitios, by NERC Reglon, 1992 (Continuod)

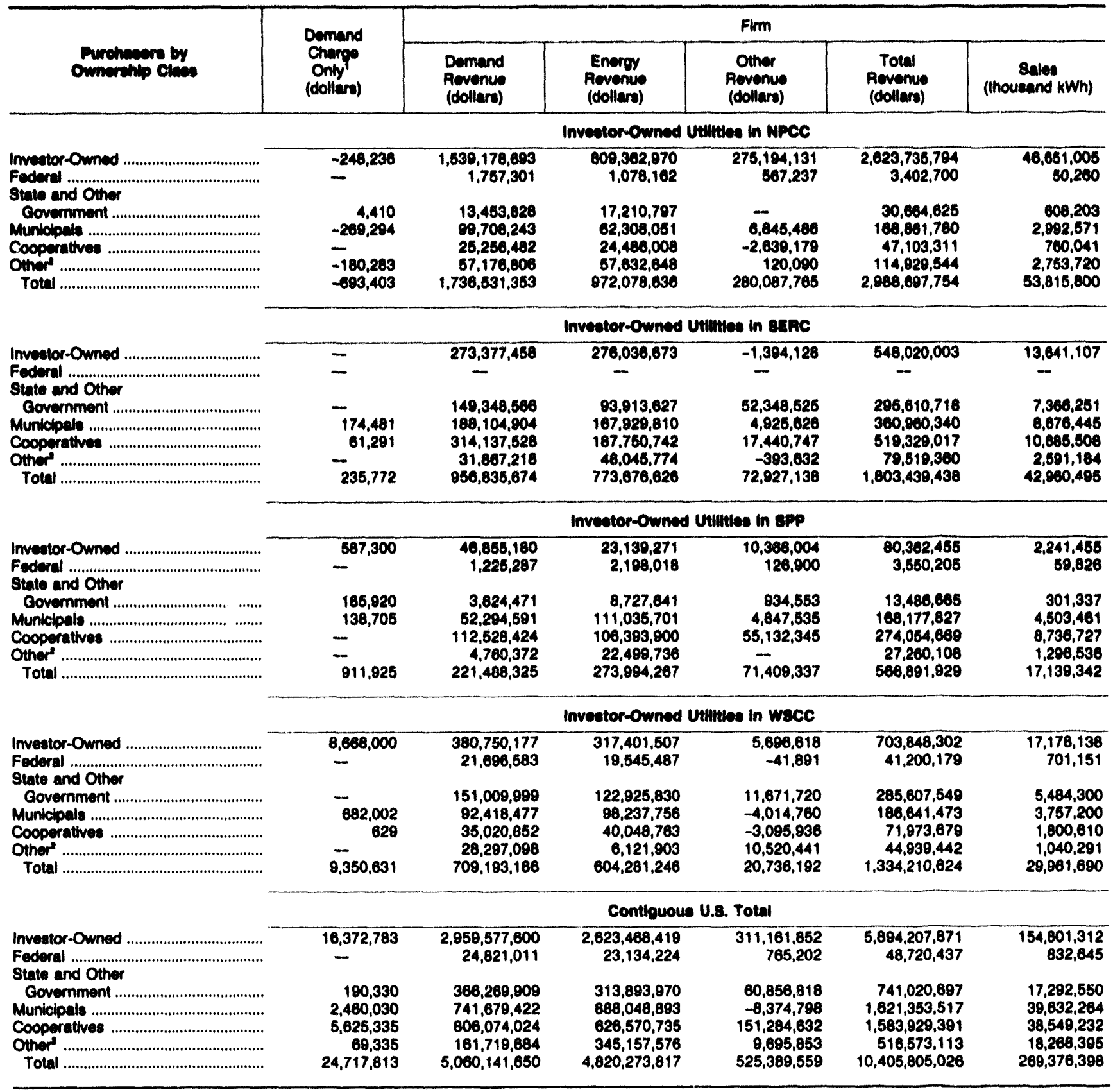

See notes and footnotes at end of table. 
Table 9. Electriolty 8ales for Recalo by Investor-Owned Utilitios, by NERC Rogion, 1902 (Continued)

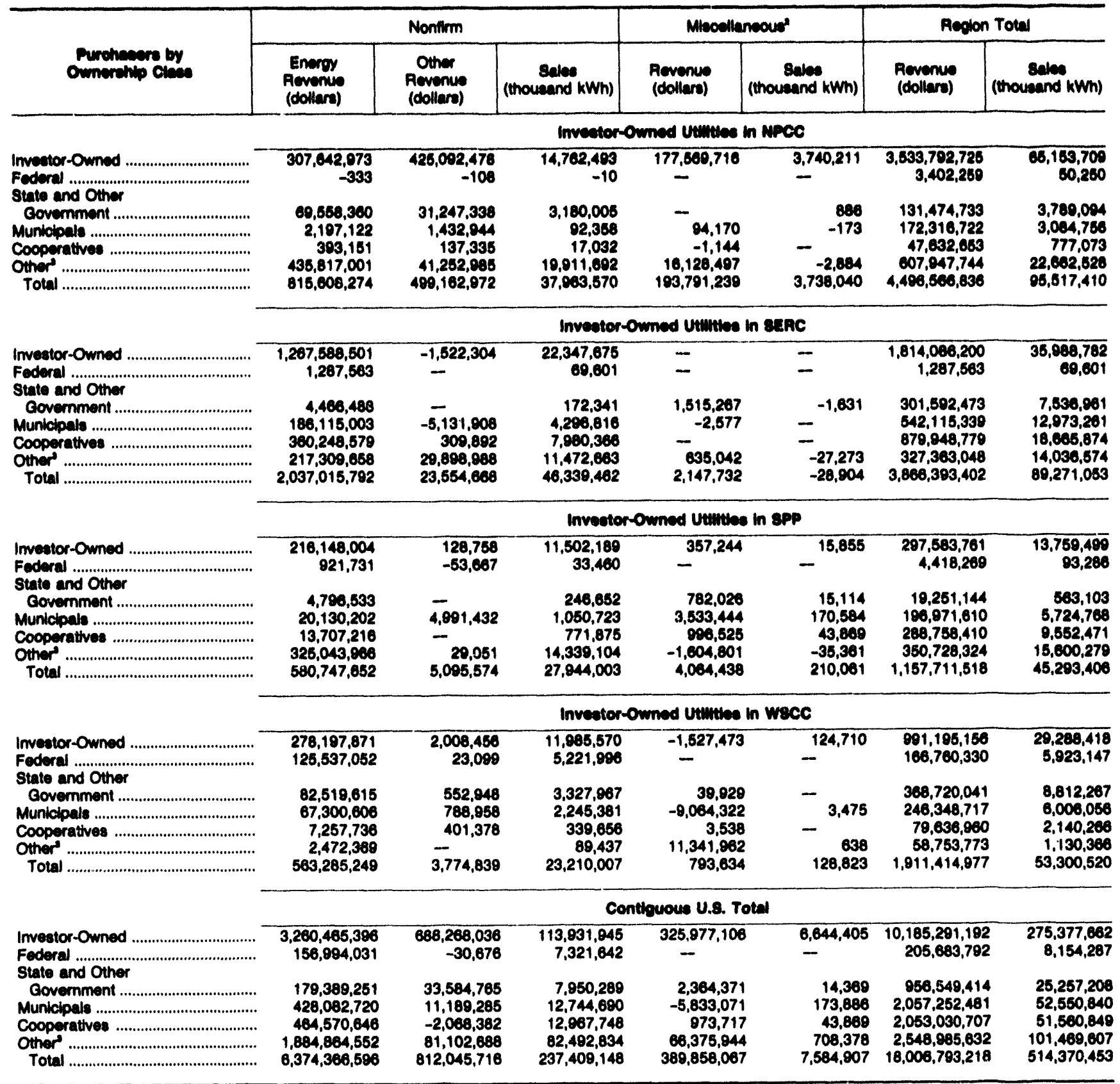

1. Monetary settlements from contracts where electricity is not taken.

- Includes other transactions involving line and substation rentals, system support charges, and special contract transactions.

- Includes transactions with power pools, utilities in Canada and Mexico, and nonutlities.

- Not Applicable

Notes: -The States of Alaska and Hawsil are not included. oNERC is the North American Electric Reliability Council. The Alaska System Coordinating Council is not included. See glossary for a list of regions and Figure 2 for a map of the regions. -Totals may not equal sum of components because of independent rounding.

Sources: -Federal Energy Reoulatory Commission, FERC Form 1, "Annual Report of Major Electric Utilties, Licensees and Others." •FERC Form 1. F. "Annual Report of Nonmajor Public Utilities and Licensees." 
Table 10. Investor-Owned Utility Exchanges of Electriclty with All Ownershlp Classes, by NERC Roglon, 1992

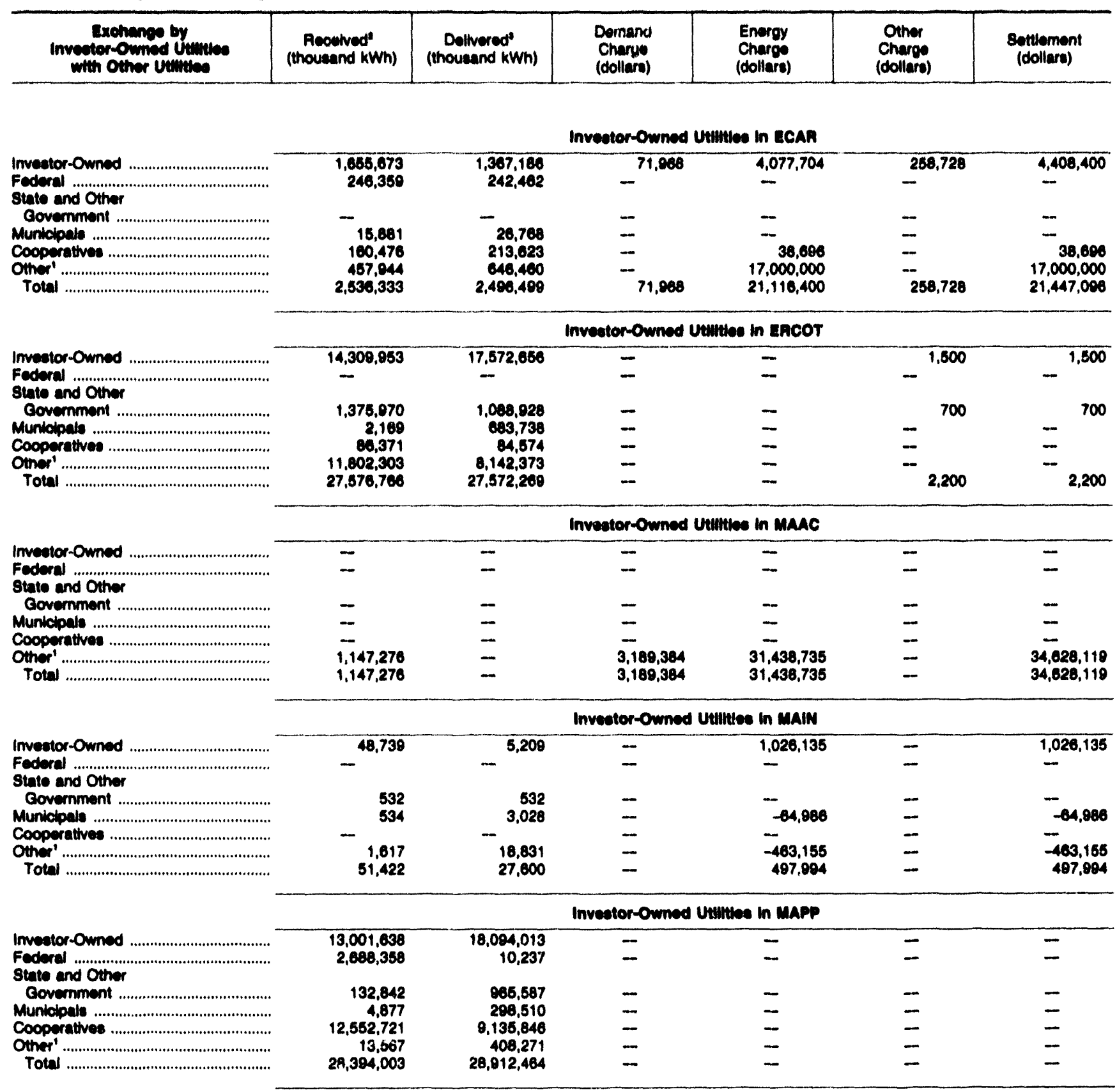

See notes and footnotes at and of table. 
Table 10. Investor-Owned Utility Exchanges of Electricity with All Ownorshlp Classes, by NERC Roglon, 1882 (Continued)

\begin{tabular}{|c|c|c|c|c|c|c|}
\hline 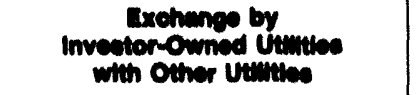 & $\begin{array}{l}\text { Acortved" } \\
\text { (thousand kWh) }\end{array}$ & $\begin{array}{l}\text { Dothered" } \\
\text { (thousand kWh) }\end{array}$ & $\begin{array}{l}\text { Demend } \\
\text { Cheras } \\
\text { (dollars) }\end{array}$ & $\begin{array}{l}\text { Energy } \\
\text { Charge } \\
\text { (dollars) }\end{array}$ & $\begin{array}{l}\text { Other } \\
\text { Charge } \\
\text { (dollery) }\end{array}$ & $\begin{array}{l}\text { Sottioment } \\
\text { (dollers) }\end{array}$ \\
\hline \multirow{3}{*}{ 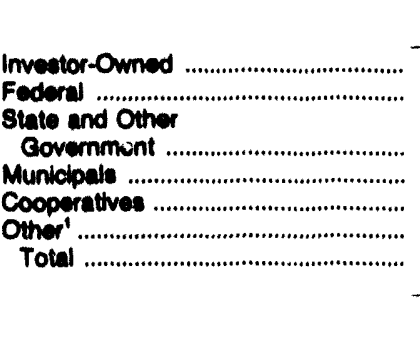 } & \multicolumn{6}{|c|}{ Invector-Owned vitities in NPCC } \\
\hline & $\begin{array}{r}419,467 \\
- \\
305,538 \\
2,320 \\
-\quad 68,396 \\
795,741\end{array}$ & $\begin{array}{l}35,434 \\
- \\
-407,013 \\
- \\
-5,893 \\
448,340\end{array}$ & $\begin{array}{l}5,713,331 \\
- \\
\overline{5,713,331}\end{array}$ & $\begin{array}{l}9,043,862 \\
- \\
335,070 \\
88,146 \\
- \\
- \\
9,467,078\end{array}$ & $\begin{array}{l}- \\
- \\
2,732,400 \\
\overline{-} \\
\overline{2,732,400}\end{array}$ & $\begin{array}{l}0,043,802 \\
- \\
8,780,801 \\
86,146 \\
- \\
17,912,809\end{array}$ \\
\hline & \multicolumn{6}{|c|}{ Investor-Owned Utilitios In senc } \\
\hline 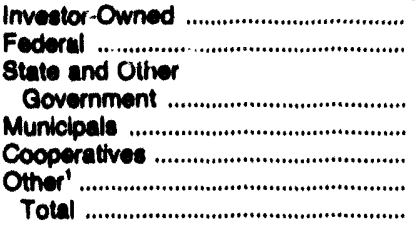 & $\begin{array}{r}28,629 \\
13,914 \\
\\
667,781 \\
210,238 \\
1,573,202 \\
221,753 \\
2,714,517\end{array}$ & $\begin{array}{r}30,381 \\
13,586 \\
583,931 \\
187,063 \\
1,343,071 \\
221,005 \\
2,368,027\end{array}$ & - & $\begin{array}{l}- \\
- \\
919,823 \\
102,497 \\
1,308,236 \\
\overline{2,330,556}\end{array}$ & $\overline{-}$ & $\begin{array}{l}- \\
- \\
910,829 \\
102,497 \\
1,308,236 \\
- \\
2,330,568\end{array}$ \\
\hline & \multicolumn{6}{|c|}{ Investor-ormed Uillities in sPP } \\
\hline 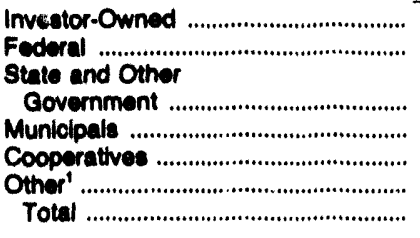 & $\begin{array}{r}531,160 \\
386,107 \\
646,227 \\
-\quad 66,003 \\
1,408,648 \\
3,014,045\end{array}$ & $\begin{array}{r}528,408 \\
347,043 \\
643,771 \\
-\quad 53,479 \\
1,406,989 \\
2,979,688\end{array}$ & $\begin{array}{l}-63,585 \\
- \\
- \\
- \\
63,685\end{array}$ & $\begin{array}{l}700,867 \\
- \\
- \\
- \\
-30,012 \\
- \\
730,879\end{array}$ & $\overline{-}$ & $\begin{array}{r}700,867 \\
63,686 \\
- \\
- \\
-\quad 30,012 \\
-794,464\end{array}$ \\
\hline & \multicolumn{6}{|c|}{ Invostor-Owned Utiltiles in wscc } \\
\hline 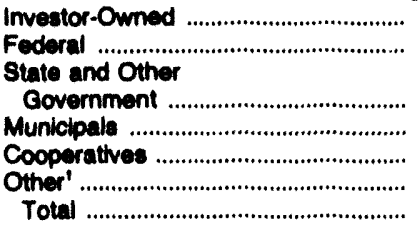 & $\begin{array}{r}\mathbf{5 , 1 8 2 , 8 9 4} \\
27,086,042 \\
\\
3,423,021 \\
587,005 \\
628,115 \\
8,977,123 \\
45,894,290\end{array}$ & $\begin{array}{r}5,130,144 \\
29,371,370 \\
\\
3,000,227 \\
652,664 \\
1,064,507 \\
8,878,693 \\
48,095,605\end{array}$ & 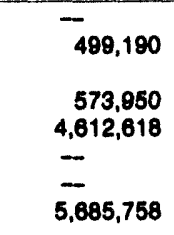 & $\begin{array}{r}-3,589,707 \\
-31,293,244 \\
17,181,896 \\
9,039,552 \\
- \\
-251,413 \\
-8,932,916\end{array}$ & $\begin{array}{r}-138,085 \\
-64,883,469 \\
20,803 \\
193,424 \\
- \\
-64,787,227\end{array}$ & $\begin{array}{r}-3,727,792 \\
-95,657,523 \\
17,758,749 \\
13,845,594 \\
- \\
-251,413 \\
-68,034,385\end{array}$ \\
\hline & \multicolumn{6}{|c|}{ contiquous U.8. Total } \\
\hline 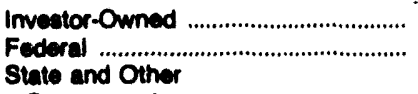 & $\begin{array}{l}35,188,173 \\
30,400,780\end{array}$ & $\begin{array}{l}42,783,400 \\
29,984,708\end{array}$ & $\begin{array}{r}71,968 \\
562,775\end{array}$ & $\begin{array}{r}11,258,861 \\
-31,293,244\end{array}$ & $\begin{array}{r}122,143 \\
-64,863,489\end{array}$ & $\begin{array}{r}11,452,972 \\
-85,593,838\end{array}$ \\
\hline $\begin{array}{l}\text { Government } \\
\text { Muniolpats } \\
\text { Cooperattves } \\
\text { Other' }\end{array}$ & $\begin{array}{r}8,540,911 \\
832,114 \\
15,085,888 \\
24,086,527 \\
112,124,383\end{array}$ & $\begin{array}{r}6,689,989 \\
1,852,671 \\
11,895,100 \\
19,726,525 \\
112,892,402\end{array}$ & $\begin{array}{r}6,287,281 \\
4,612,618 \\
- \\
3,189,384 \\
14,724,026\end{array}$ & $\begin{array}{r}18,416,789 \\
9,165,209 \\
1,376,944 \\
47,724,167 \\
56,648,726\end{array}$ & $\begin{array}{r}2,754,003 \\
193,424 \\
- \\
-61,793,899\end{array}$ & $\begin{array}{r}27,458,073 \\
13,971,261 \\
1,376,944 \\
50,813,551 \\
9,578,853\end{array}$ \\
\hline
\end{tabular}

1 Includes transactions with power pools, utilities in Canada and Mexico, and nonutlities.

- Includes all transactions recelved from outelde and within the reglon by utilties operating within the reglon.

- Includes all transactions delvered out of and within the region by utilities operating within the region.

- Not Appllcable

Notes: -The States of Alaska and Hawali are not included. -The terms of settlement may include transferring and/or exchanging quantities of electricIty with no dollar value aseigned. •NERC is the North American Electric Rellability Council. The Alaska System Coordinating Council is not included. See glossary for a list of reglons and Figure 2 for a map of the Region. -Totais may not equal sum of components because of independent rounding.

Sources: -Federal Energy Reoulatory Commiseion, FERC Form 1, "Annual Report of Major Electric Utilities, Licensees and Others." •FERC Form 1.F. "Annual Report of Nonmajor Public Utilities and Licensees." 
Table 11. Noninvestor-Owned Utility Exchanges of Eloctrlelly, by NERC Reglon, 1992 (Million Kilowatthours)

\begin{tabular}{|c|c|c|c|c|c|c|c|c|c|c|}
\hline \multirow{2}{*}{$\begin{array}{l}\text { Exohengee with } \\
\text { Other Utilites }\end{array}$} & \multicolumn{9}{|c|}{ NERC Region of Noninvestor-Owned Utillty } & \multirow{2}{*}{$\begin{array}{l}\text { Contiguous } \\
\text { U.S. } \\
\text { Total }\end{array}$} \\
\hline & ECAR & EACOT & MAAC & MAIN & MAPP & NPCC & SERC & SPP & wscc & \\
\hline \multicolumn{11}{|l|}{ Federal } \\
\hline Acosived' ............................ & - & - & - & - & - & - & 22,509 & 412 & 16,788 & 38,689 \\
\hline Delivered ............................. & - & - & - & - & - & - & 28,788 & 695 & 22,085 & 49,575 \\
\hline Net & - & - & - & - & - & - & $-4,287$ & -282 & $-6,317$ & $-10,888$ \\
\hline \multicolumn{11}{|l|}{ State and Other } \\
\hline Recetved' ......................... & - & - & - & - & 67 & 1,094 & 39 & 137 & 4,078 & 5,416 \\
\hline Dellvered" ............................ & 1 & - & - & - & & 424 & 46 & 1,440 & 1,546 & 3,467 \\
\hline 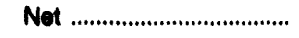 & -1 & - & - & - & 67 & 689 & -6 & $-1,304$ & 2,532 & 1,988 \\
\hline \multicolumn{11}{|l|}{ Municipals } \\
\hline Received' .............................. & 79 & 1,201 & 420 & $-\infty$ & 10 & 1,200 & 498 & 854 & 6,709 & 11,072 \\
\hline Delwered ........................... & 94 & 1,170 & 41 & - & 10 & 493 & 1,187 & 934 & 7,656 & 11,584 \\
\hline 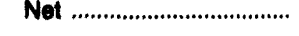 & -14 & & 379 & - & 1 & 706 & -688 & 20 & -947 & -513 \\
\hline \multicolumn{11}{|l|}{ Cooperathves } \\
\hline Aecelved' ........................ & 464 & 17,534 & - & 161 & 1,917 & - & 52 & 422 & 1,914 & 22,463 \\
\hline 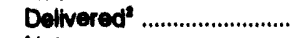 & 298 & 17,835 & - & 48 & 2,129 & - & 39 & 244 & 2,023 & 22,615 \\
\hline Net & 167 & -301 & - & 113 & -212 & - & 13 & 178 & -109 & -151 \\
\hline \multicolumn{11}{|l|}{ Region Total } \\
\hline Recerved' ............................. & 543 & 18,734 & 420 & 161 & 1,994 & 2,293 & 23,100 & 1,824 & 28,469 & 77,639 \\
\hline Delivered ${ }^{2}$............................. & 391 & $\begin{array}{r}19,005 \\
-270\end{array}$ & $\begin{array}{r}41 \\
379\end{array}$ & $\begin{array}{r}48 \\
113\end{array}$ & $\begin{array}{r}2,139 \\
-145\end{array}$ & $\begin{array}{r}918 \\
1376\end{array}$ & 28,068 & $\begin{array}{r}3,313 \\
-1,388\end{array}$ & 33,310 & $\begin{array}{r}87,231 \\
-9,592\end{array}$ \\
\hline Net & 152 & -270 & 379 & 113 & -145 & 1,376 & $-4,888$ & $-1,388$ & $-4,841$ & $-9,582$ \\
\hline
\end{tabular}

1 Includee all transactions recelved from outside and within the region by utlities operating within the region.

- Includes all transactions dellvered out of and within the region by utilities operating within the region.

- Not Applicable

The value of the number is less than 0.5 percent.

Notea: -The States of Alaska and Hawall are not included. oNERC is the North American Electric Rellablity Councill. The Aleska Syatem Coordinating Counct is not included. See glossary for a list of reglons and Figure 2 for a map of the regions. Totals may not equal sum of components because of in. dependent rounding.

Source: Energy Information Administration, Form EIA-861, "Annual Electric Utility Report." 
Table 12. Electricity Wheeling for All Ownership Clasess by Investor-Owned Utilities, by NERC Rogion, 1992

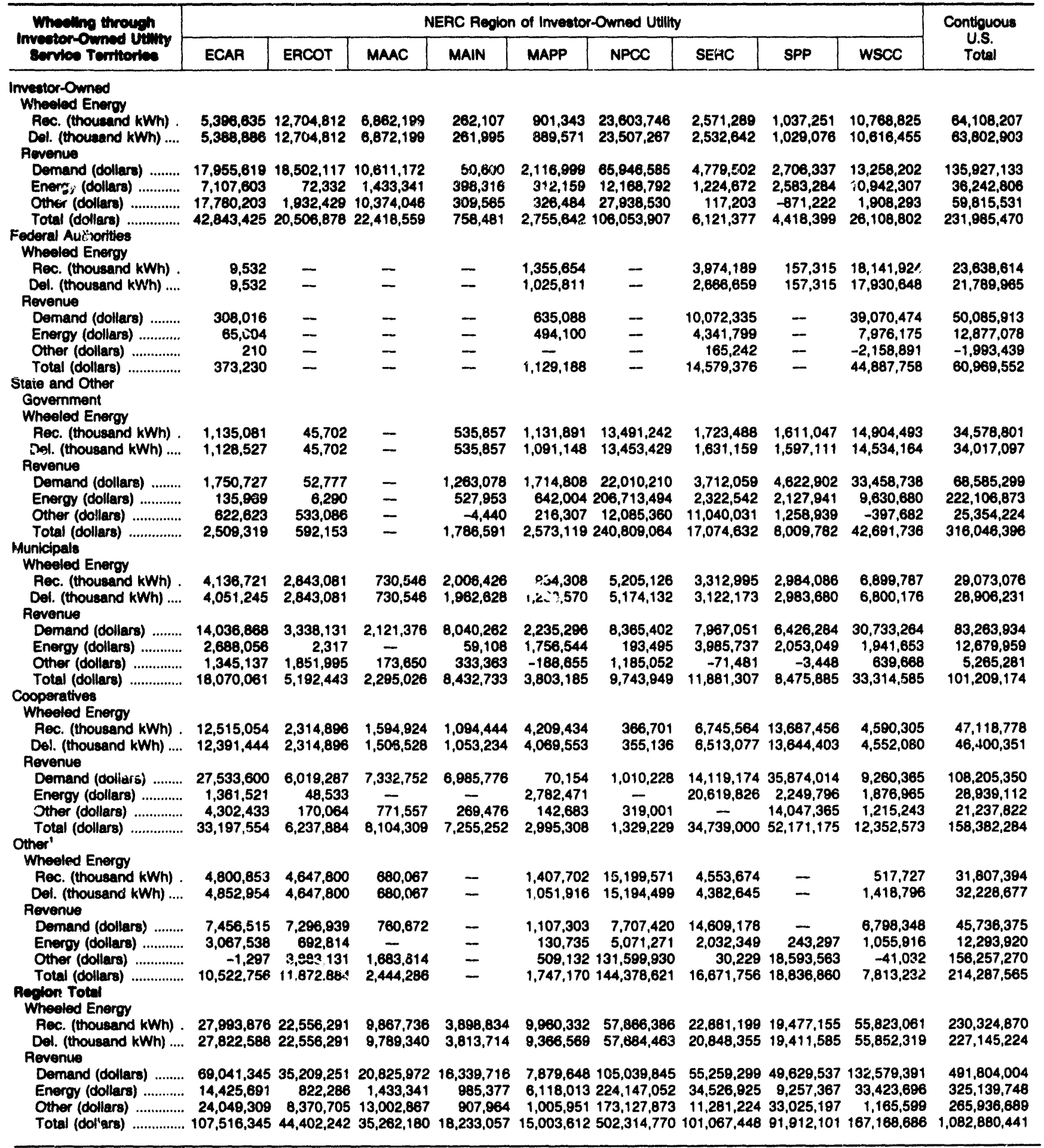

' Includes transactions with power pools, utilhies in Canada and Mexico, and nonutilities.

- Not Applicable

Notes: -Wheeling (transmission services) is reported in account 456. "Transmission For Others," and in account 565, "Transmission By Others," of the FERC Uniform System of Accounts. -The States of Alaska and Hawail are not included. -NERC is the North American Electric Reliability Council. The Alaska System Coordinating Council is not included. See glossary for a list of regions and Figure 2 for a map of the regions. - Totals may not equal sum of components because of independent rounding.

Sources: -Federal Energy Regulatory Commission, FERC Form 1, "Annual Report of Major Electric Utilities, Lizensees and Others." •FERC Form 1.

F, "Annual Report of Nonmajor Public Utilities and Licensees." 
Table 13. Electriclty Wheelling by All Ownershlp Classes to Investor-Owned Utilities, by NERC Reglon, 1992

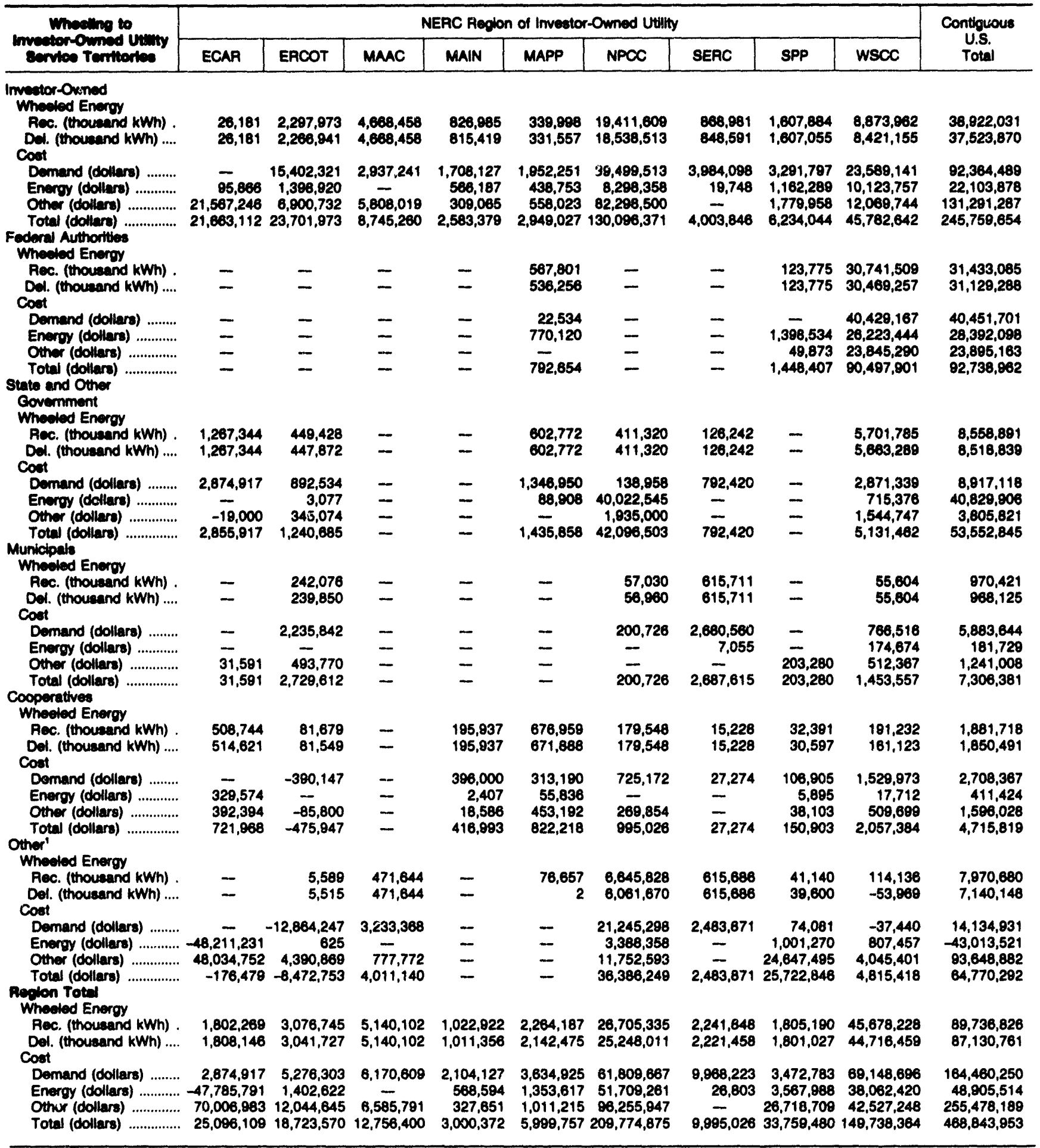

- Includes transactions with power pools, utilities in Canada and Mexico, and nonutillies.

- Not Applicable

Notes: -Wheeling (transmission services) is reported in account 456, "Transmiseion For Others," and in account 565, "Tranemiasion By Others," of the FERC Uniform System of Accounts. -The States of Alaska and Hawail are not included. oNERC is the North American Electric Rellability Council. See gloseary for a list of regions and Figure 2 for a map of the regions. Total quantity of wheeling may not be reported. -Totals may not equal sum of components because of independent rounding.

Sources: -Federal Energy Regulatory Commisaion, FERC Form 1, "Annual Report of Major Electric Utilites, Licensees and Others." -FERC Form 1. F. "Annual Report of Nonmajor Public Utilites and Licensees." 
Table 14. Purchases by Cooperative Borrowore, by NERC Reglon, 1992

\begin{tabular}{|c|c|c|c|c|c|}
\hline \multirow{2}{*}{ ownerentip by } & \multicolumn{5}{|c|}{ NERC Region of Cooperattves } \\
\hline & ECAP & ERCOT & MAAC & MAIN & MAPP \\
\hline 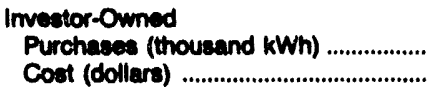 & $\begin{array}{r}2,313,614 \\
73,306,032\end{array}$ & $\begin{array}{r}3,476,707 \\
144,220,608\end{array}$ & $\begin{array}{r}2,874,851 \\
129,872,371\end{array}$ & $\begin{array}{r}2,212,288 \\
9 \$, 161,781\end{array}$ & $\begin{array}{r}647,649 \\
28,473,688\end{array}$ \\
\hline 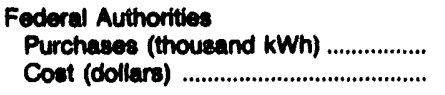 & $\begin{array}{r}2,023,142 \\
55,724,172\end{array}$ & $\begin{array}{r}384,919 \\
3,622,731\end{array}$ & $\overline{-}$ & $\begin{array}{r}42,600 \\
842,821\end{array}$ & $\begin{array}{r}3,398,455 \\
37,272,273\end{array}$ \\
\hline 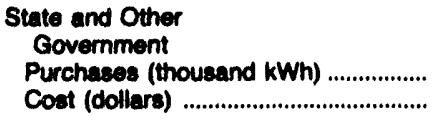 & $\overline{-}$ & $\begin{array}{r}801,387 \\
30,782,764\end{array}$ & $\begin{array}{r}392,890 \\
2,226,724\end{array}$ & - & $\begin{array}{r}2,512,718 \\
83,583,682\end{array}$ \\
\hline 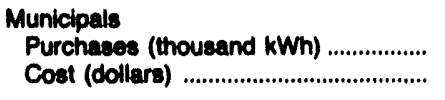 & $\begin{array}{r}1,227,942 \\
28,203,054\end{array}$ & $\begin{array}{r}71,583 \\
464,178\end{array}$ & - & - & $\begin{array}{r}41,085 \\
834,404\end{array}$ \\
\hline 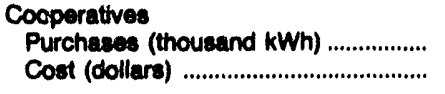 & $\begin{array}{r}28,362,510 \\
1,025,158,939\end{array}$ & $\begin{array}{r}10,540,714 \\
439,394,320\end{array}$ & $\begin{array}{r}3,343,612 \\
185,170,101\end{array}$ & $\begin{array}{r}7,600,281 \\
370,172.596\end{array}$ & $\begin{array}{r}23,054,374 \\
944,481,173\end{array}$ \\
\hline 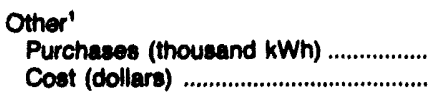 & $-50,046$ & $\begin{array}{r}480,281 \\
13,926,208\end{array}$ & $\begin{array}{r}2,723 \\
85,822\end{array}$ & $\begin{array}{r}150 \\
5,034\end{array}$ & $\begin{array}{r}659,084 \\
9,346,854\end{array}$ \\
\hline 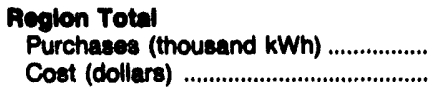 & $\begin{array}{r}31,827,208 \\
1,182,442,243\end{array}$ & $\begin{array}{r}15,875,591 \\
632,410,807\end{array}$ & $\begin{array}{r}6,614,076 \\
327,355,118\end{array}$ & $\begin{array}{r}9,855,318 \\
486,182,232\end{array}$ & $\begin{array}{r}30,313,385 \\
1,103,892,054\end{array}$ \\
\hline & & NERC Region & operatives & & Contiguous \\
\hline & NPCC & SERC & SPP & WsCC & Total \\
\hline 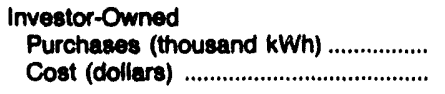 & $\begin{array}{r}787,806 \\
48,392,145\end{array}$ & $\begin{array}{r}16,524,089 \\
777,551,823\end{array}$ & $\begin{array}{r}6,450,925 \\
219,251,493\end{array}$ & $\begin{array}{r}1,789,137 \\
71,416,933\end{array}$ & $\begin{array}{r}37,077,096 \\
1,587,646,872\end{array}$ \\
\hline 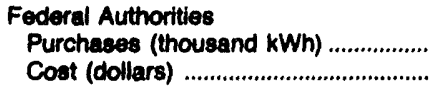 & - & $\begin{array}{r}27,445,580 \\
1,141,882,636\end{array}$ & $\begin{array}{r}3,783,549 \\
56,540,535\end{array}$ & $\begin{array}{r}9,800,456 \\
207,402,438\end{array}$ & $\begin{array}{r}46,888,701 \\
1,503,287,606\end{array}$ \\
\hline $\begin{array}{l}\text { State and Other } \\
\text { Government } \\
\text { Purchases (thousand kWh) } \\
\text { Cost (dollars) }\end{array}$ & $\begin{array}{r}218,497 \\
4,448,883\end{array}$ & $\begin{array}{r}6,342,811 \\
234,789,832\end{array}$ & $\begin{array}{r}3,627,409 \\
88,135,928\end{array}$ & $\begin{array}{r}188,228 \\
3,873,894\end{array}$ & $\begin{array}{r}14,193,940 \\
447,841,687\end{array}$ \\
\hline 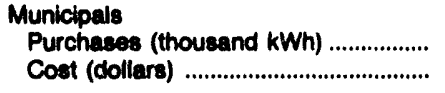 & $\begin{array}{r}3,373 \\
461,625\end{array}$ & $\begin{array}{r}147,712 \\
10,592,745\end{array}$ & $\begin{array}{r}1,252,322 \\
28,138,536\end{array}$ & $\begin{array}{r}18 \\
1,234\end{array}$ & $\begin{array}{r}2,744,035 \\
68,685,776\end{array}$ \\
\hline 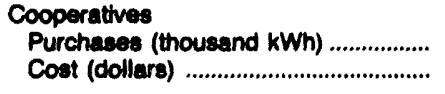 & $\begin{array}{r}108,198 \\
13,835,423\end{array}$ & $\begin{array}{r}56,306,701 \\
2,747,854,810\end{array}$ & $\begin{array}{r}35,530,980 \\
1,553,728,367\end{array}$ & $\begin{array}{r}12,811,092 \\
577,480,052\end{array}$ & $\begin{array}{r}175,659,372 \\
7,867,285,881\end{array}$ \\
\hline 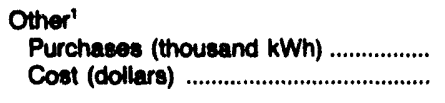 & $\begin{array}{r}100,193 \\
6,360,941\end{array}$ & $\begin{array}{r}527,250 \\
15,818,148\end{array}$ & $\begin{array}{r}313,503 \\
11,208,575\end{array}$ & $\begin{array}{r}2,440,934 \\
89,328,714\end{array}$ & $\begin{array}{r}4,534,118 \\
146,130,442\end{array}$ \\
\hline 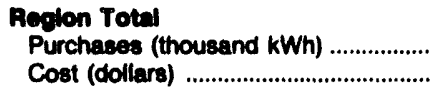 & $\begin{array}{r}1,218,097 \\
73,499,017\end{array}$ & $\begin{array}{r}107,294,143 \\
4,928,490,094\end{array}$ & $\begin{array}{r}50,858,698 \\
1,857,003,434\end{array}$ & $\begin{array}{r}27,040,765 \\
949,513,265\end{array}$ & $\begin{array}{r}281,097,262 \\
11,620,888,264\end{array}$ \\
\hline
\end{tabular}

1 Includes traneactions with power pools, utilities in Canada and Mexico, and nonutllities.

- Not Applicable

Notes: -The States of Alaska and Hawail are not included. -NERC is the North American Electric Reliabllity Council. The Alaska System Coordinating Council is not included. See glossary for a list of regions and Figure 2 for a map of the regions. Totals may not equal sum of components because of independent rounding.

Source: Pural Electrification Administration, AEA Form 7, "Financial And Statistical Report," REA Form 12a through 121, "Electric Power Supply Borrowers," Form 12c through 12g. "Electric Distribution Borrowers with Generating Facilities." 
Table 15. Recelpte by Investor-Owned Utilitles Within and Between NERC Reglone, 1892

\begin{tabular}{|c|c|c|c|c|}
\hline \multirow{3}{*}{$\begin{array}{l}\text { MEnC Recion of } \\
\text { Invector-Owmed Utilltee/ } \\
\text { Type of Truncaction }\end{array}$} & \multicolumn{4}{|c|}{ Source of Electrictly from All Ownerehtip Clasees by NERC Reglon } \\
\hline & \multicolumn{2}{|c|}{ ECAP } & \multicolumn{2}{|c|}{ ERCOT } \\
\hline & $\begin{array}{c}\text { Energy } \\
\text { (thousand kWh) }\end{array}$ & $\begin{array}{c}\text { Cost } \\
\text { (dollare) }\end{array}$ & $\begin{array}{l}\text { Energy } \\
\text { (thousand kWh) }\end{array}$ & $\underset{\text { (dollare) }}{\text { Cost }}$ \\
\hline \multicolumn{5}{|l|}{ ECAR } \\
\hline 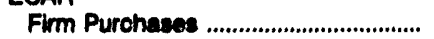 & $46,787,871$ & $1,663,878,664$ & - & - \\
\hline Nonfirm Purchaces ............................. & $38,886,856$ & $848,228,071$ & - & - \\
\hline Mlecellaneous Purchases' .................. & $8,401,023$ & $258,843,182$ & - & - \\
\hline 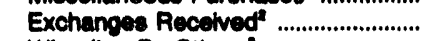 & $2,133,109$ & $21,445,351$ & - & - \\
\hline \multirow{2}{*}{\multicolumn{5}{|c|}{ ERCOT }} \\
\hline & & & & \\
\hline 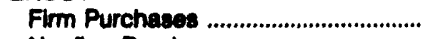 & - & - & $18,886,912$ & $815,212,823$ \\
\hline Nonfirm Purchaces .................................... & -- & - & $4,385,493$ & $78,166,295$ \\
\hline Miscollaneous Purchases' ................... & - & - & 108,006 & 945,987 \\
\hline 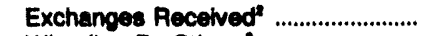 & - & - & $27,559,666$ & 2,200 \\
\hline & - & - & $3,076,745$ & $18,723,570$ \\
\hline \multicolumn{5}{|l|}{ MAAC } \\
\hline 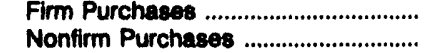 & $\begin{array}{l}5,446,452 \\
8,405,041\end{array}$ & $258,178,010$ & $\overline{-}$ & $\overline{-}$ \\
\hline Miscellaneous Purchases' .................. & - & 51,454 & - & - \\
\hline Exchanges Recelved" ............................. & - & - & - & - \\
\hline \multicolumn{3}{|l|}{$\begin{array}{l}\text { Wheelling By Others }{ }^{3} \text {.............................. } \\
\text { MAIN }\end{array}$} & - & \\
\hline & 442,826 & $7,536,904$ & - & - \\
\hline 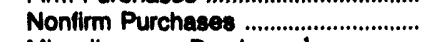 & $1,299,450$ & $23,475,567$ & - & -- \\
\hline Miscellaneous Purchases' ................... & - & - & - & - \\
\hline 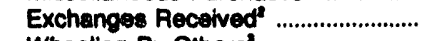 & 1,271 & $-10,292$ & - & - \\
\hline Wheoling By Others' ............................ & - & - & - & - \\
\hline $\begin{array}{l}\text { MAPP } \\
\text { Firm Purchases ....................................... }\end{array}$ & - & - & - & - \\
\hline Nonfirm Purchases ..................................... & - & - & - & - \\
\hline Miscellaneous Purchases' ................. & - & -- & - & - \\
\hline Exctianges Recelved d............................. & - & - & - & -- \\
\hline 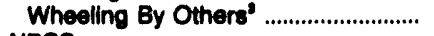 & - & - & - & - \\
\hline \multicolumn{5}{|l|}{ NPCC } \\
\hline & - & - & - & - \\
\hline & $\overline{-}$ & $\bar{z}$ & - & - \\
\hline 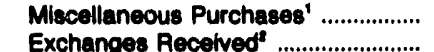 & $\overline{-}$ & - & $\overline{-}$ & $\overline{-}$ \\
\hline & - & $\overline{-}$ & - & $\overline{-}$ \\
\hline \multicolumn{5}{|l|}{ SERC } \\
\hline Firm Purchases .................................. & $6,757,105$ & $265,186,032$ & - & - \\
\hline Nonfirm Purchases ................................. & 315,643 & $7,702,332$ & - & - \\
\hline Miscellaneous Purchases' ................... & & $-33,078$ & - & - \\
\hline 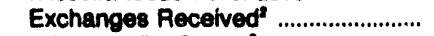 & 21,385 & - & - & - \\
\hline 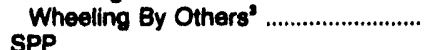 & \multicolumn{4}{|c|}{ SPP } \\
\hline 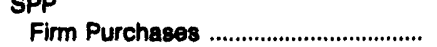 & - & - & - & - \\
\hline 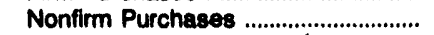 & 43,725 & 826,763 & - & - \\
\hline Miscellaneous Purchases' ................ & - & - & 2,275 & - \\
\hline Exchanges Recolved ${ }^{2}$............................... & - & - & - & \\
\hline Wheeling By Others & - & - & - & $1,468,368$ \\
\hline \multicolumn{5}{|l|}{ wsce } \\
\hline 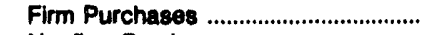 & - & - & 41,069 & $3,077,166$ \\
\hline 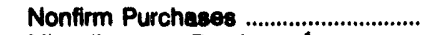 & - & - & - & - \\
\hline Miscellaneous Purchases' .................. & - & - & - & - \\
\hline 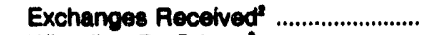 & - & - & - & - \\
\hline \multirow{5}{*}{$\begin{array}{l}\text { Wheelling By Others' .......................... } \\
\text { Reglon Total } \\
\text { Firm Purchases Dellveries ................. } \\
\text { Nonflim Purchases Delveries ......... } \\
\text { Misc Purchases Dellverles' ............. } \\
\text { Exchanges Recelved Dellveries' ..... } \\
\text { Wheeling By Others Dellveries' ....... }\end{array}$} & - & - & - & - \\
\hline & & & $19,937,981$ & \\
\hline & $48,950,815$ & $1,238,410,743$ & $4,385,483$ & $78,168,295$ \\
\hline & $9,401,823$ & $258,861,568$ & 110,281 & 945,987 \\
\hline & $\begin{array}{l}2,155,765 \\
1,833,044\end{array}$ & $\begin{array}{l}21,435,059 \\
21,443,248\end{array}$ & $\begin{array}{r}27,559,666 \\
3,076,746\end{array}$ & $\begin{array}{r}2,200 \\
20,181,938\end{array}$ \\
\hline
\end{tabular}

See notes and footnotes at end of table. 
Table 15. Recelpte by Investor-Owned Utilities Within and Between NERC Reglons, 1992 (Continued)

\begin{tabular}{|c|c|c|c|c|}
\hline \multirow{3}{*}{ 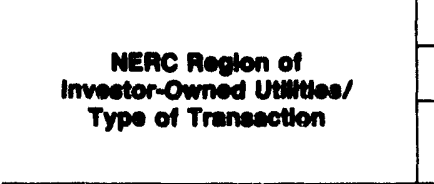 } & \multicolumn{4}{|c|}{ Source of Electricity from All Ownership Clusses by NERC Region } \\
\hline & \multicolumn{2}{|c|}{ MAAC } & \multicolumn{2}{|c|}{ MAIN } \\
\hline & $\begin{array}{l}\text { Energy } \\
\text { (thousand kWh) }\end{array}$ & $\begin{array}{c}\text { Cost } \\
\text { (dollars) }\end{array}$ & $\begin{array}{c}\text { Energy } \\
\text { (thousand } k W h \text { ) }\end{array}$ & $\begin{array}{c}\text { Cost } \\
\text { (dollars) }\end{array}$ \\
\hline \multicolumn{5}{|l|}{ ECAP } \\
\hline 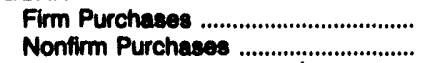 & $\begin{array}{l}308,402 \\
113,869\end{array}$ & $\begin{array}{r}17,187,388 \\
484,924\end{array}$ & $\begin{array}{l}152,997 \\
391,808\end{array}$ & $\begin{array}{l}5,077,406 \\
5,086,629\end{array}$ \\
\hline Miscellaneous Purchases' .................... & - & - & - & - \\
\hline Exchanges Recetved & - & - & 1,726 & 4,200 \\
\hline Wheeling By Others & - & $4,048,442$ & - & - \\
\hline \multicolumn{5}{|l|}{ ERCOT } \\
\hline 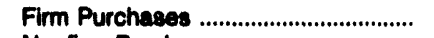 & - & - & - & - \\
\hline 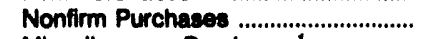 & - & - & - & - \\
\hline Miscellaneous Purchases' ................. & - & - & - & - \\
\hline Exchanges Recelved ${ }^{2}$......................... & - & - & - & - \\
\hline Wheoling By Others' ........................... & - & $\cdots$ & - & - \\
\hline \multicolumn{5}{|l|}{ MAAC } \\
\hline Firm Purchases ........................................ & $22,142,067$ & $880,809,454$ & -- & - \\
\hline 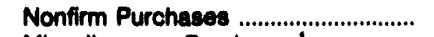 & $30,046,080$ & $1,186,086,922$ & - & - \\
\hline Miscellaneous Purchases' .................. & $2,952,228$ & $68,133,031$ & - & - \\
\hline 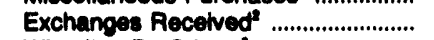 & $1,147,276$ & $34,628,119$ & - & - \\
\hline Wheoling By Others' ................................. & $5,109,327$ & $12,360,819$ & - & - \\
\hline \multicolumn{5}{|l|}{ MAIN } \\
\hline Firm Purchases ....................................... & - & - & $5,371,509$ & $190,367,143$ \\
\hline Nonfirm Purchaees ................................... & - & - & $7,513,414$ & $123,495,033$ \\
\hline Miscollaneous Purchases' .................. & - & - & 8,826 & 171,002 \\
\hline Exchanges Recetved ............................ & - & - & 50,151 & 508,286 \\
\hline Wheeling By Others' ............................. & - & - & 262,107 & 767,803 \\
\hline \multicolumn{5}{|l|}{ MAPP } \\
\hline Firm Purchases ....................................... & - & - & 385,103 & $6,250,425$ \\
\hline Nonfirm Purchases ................................ & - & - & 600,048 & $11,536,081$ \\
\hline Miscellaneous Purchases' .................. & - & - & - & $-\infty$ \\
\hline Exchanges Received ${ }^{2}$............................ & - & - & 839,294 & - \\
\hline Wheeling By Others' .............................. & - & - & 21,025 & 54,342 \\
\hline \multicolumn{5}{|l|}{ NPCC } \\
\hline Firm Purchases & $1,354,126$ & $33,797,242$ & - & - \\
\hline Nontirm Purchases .............................. & $2,395,613$ & $127,211,498$ & - & - \\
\hline Miscellaneous Purchases' ................. & - & - & - & - \\
\hline Exchanges Recelved ${ }^{2}$............................ & - & - & - & -- \\
\hline Wheeling By Others & 115,380 & 263,080 & - & - \\
\hline \multicolumn{5}{|l|}{ SERC } \\
\hline Firm Purchases …................................ & 7,503 & 339,210 & 422,194 & $15,765,340$ \\
\hline Nonfirm Purchases .............................. & 12,322 & 235,355 & 6,301 & 114,457 \\
\hline Miscellaneous Purchases' ................. & 25,685 & - & - & - \\
\hline Exchanges Received ${ }^{2}$......................... & 156,484 & - & - & -- \\
\hline Wheeling By Others ${ }^{3}$............................... & - & - & - & - \\
\hline \multicolumn{5}{|l|}{ SPP } \\
\hline 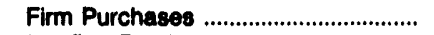 & - & - & 125,350 & $7,422,088$ \\
\hline Nonfirm Purchases .............................. & 56,619 & $1,103,093$ & 287,826 & $5,127,730$ \\
\hline Miscellaneous Purchases' ................ & - & - & - & - \\
\hline 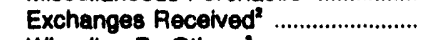 & - & - & - & - \\
\hline Wheeling By Others' .............................. & - & - & - & 18,761 \\
\hline \multicolumn{5}{|l|}{ WSCC } \\
\hline Firm Purchases ................................... & - & - & - & - \\
\hline 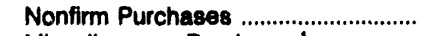 & - & - & - & - \\
\hline Miscellaneous Purchases' ................. & - & - & - & -- \\
\hline Exchanges Received ${ }^{2}$........................... & - & - & - & - \\
\hline Wheeling By Others ${ }^{*}$............................ & - & -- & - & - \\
\hline \multicolumn{5}{|l|}{ Aggion Total } \\
\hline Firm Purchases Deliveries ................. & $23,812,098$ & $942,143,304$ & $6,457,153$ & $224,882,402$ \\
\hline Nonfirm Purchases Deliveries ........... & $32,624,603$ & $1,325,121,793$ & $8,799,495$ & $145,359,930$ \\
\hline Misc Purchases Deliveries' ............... & $2,977,913$ & $68,133,031$ & 8,826 & 171,002 \\
\hline Exchanges Recelved Deliveries ${ }^{2}$..... & $1,303,760$ & $34,628,119$ & 891,171 & 512,486 \\
\hline Wheeling By Others Deliveries" ....... & $5,224,707$ & $16,672,341$ & 283,132 & 841,906 \\
\hline
\end{tabular}

See notes and footnotes at end of table. 
Table 16. Recolpts by Investor-Owned Utilities Within and Botween NERC Reglons, 1992 (Continued)

\begin{tabular}{|c|c|c|c|c|}
\hline \multirow{3}{*}{ 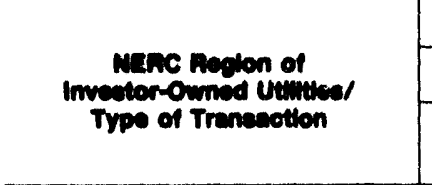 } & \multicolumn{4}{|c|}{ Source of Electricty from All Ownerehip Classes by NERC Region } \\
\hline & \multicolumn{2}{|c|}{ MAPP } & \multicolumn{2}{|c|}{ NPCC } \\
\hline & $\begin{array}{l}\text { Envrgy } \\
\text { (thouseand } k W h \text { ) }\end{array}$ & $\begin{array}{c}\text { Coot } \\
\text { (dollare) }\end{array}$ & $\begin{array}{c}\text { Energy } \\
\text { (thousand kWh) }\end{array}$ & $\begin{array}{c}\text { Cost } \\
\text { (dollare) }\end{array}$ \\
\hline \multicolumn{5}{|l|}{ ECAP } \\
\hline 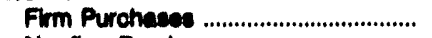 & - & - & - & - \\
\hline Nonfirm Purcheses ............................... & - & - & 93,157 & $1,851,716$ \\
\hline Miscollaneous Purchased' .................. & - & - & - & - \\
\hline Exohanges Recetveof ......................... & - & - & - & - \\
\hline $\begin{array}{l}\text { Wheelling By Othere" .......................... } \\
\text { ERCOT }\end{array}$ & - & - & - & - \\
\hline Firm Purchases ,................................. & - & - & - & - \\
\hline Nonfirm Purchases ................................ & - & - & - & - \\
\hline Miscollaneous Purchaces' ................... & - & - & - & - \\
\hline Exchanges Recelved ${ }^{2}$............................. & -- & - & - & -- \\
\hline $\begin{array}{l}\text { Wheelling By Others" ......................... } \\
\text { MAMC }\end{array}$ & - & - & - & - \\
\hline Firm Purchases & - & - & 164,817 & $5,990,168$ \\
\hline Nonfirm Purchases ................................. & -- & - & 137,804 & $2,486,360$ \\
\hline Miscellaneous Purchases' .................. & - & - & - & 528,088 \\
\hline 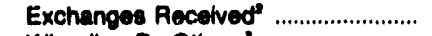 & - & -- & - & - \\
\hline Wheeling By Others' ............................... & -- & - & - & - \\
\hline \multicolumn{5}{|l|}{ MAIN } \\
\hline Firm Purchases & $3,553,232$ & $59,869,255$ & - & - \\
\hline 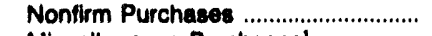 & $4,520,617$ & $72,063,898$ & - & - \\
\hline Miscellaneous Purchases' .................. & - & - & - & - \\
\hline Exchanges Aecetved ${ }^{2}$............................. & -- & - & $\rightarrow$ & - \\
\hline $\begin{array}{l}\text { Wheeling By Others' ................................ } \\
\text { MAPP }\end{array}$ & 431,833 & $1,651,203$ & - & - \\
\hline Firm Purchases ........................................ & $11,557,240$ & $284,080,218$ & - & - \\
\hline 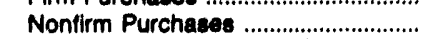 & $11,405,843$ & $191,101,645$ & - & 100,000 \\
\hline Miscelianeous Purchases' .................. & 7,677 & $4,530,478$ & - & - \\
\hline 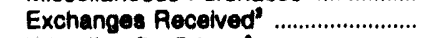 & $24,683,219$ & - & 2,617 & - \\
\hline Wheoling By Others ${ }^{3}$............................ & $1,561,685$ & $5,049,958$ & - & - \\
\hline \multicolumn{5}{|l|}{ NPCC } \\
\hline Firm Purchases & - & -- & $73,570,701$ & $3,768,826,811$ \\
\hline Nonfirm Purchases ................................. & - & - & $64,396,908$ & $2,894,714,822$ \\
\hline Miscellaneous Purchases' .................. & - & - & 49,330 & $-18,317,571$ \\
\hline 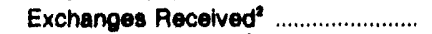 & - & -- & 795,741 & $17,812,808$ \\
\hline Whoeling By Others' ............................ & - & - & $26,589,855$ & $209,511,795$ \\
\hline \multicolumn{5}{|l|}{ SERC } \\
\hline 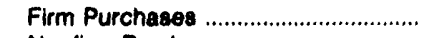 & -- & - & $\sim$ & -- \\
\hline 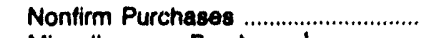 & 2,176 & 43,225 & -- & - \\
\hline Miscellaneous Purchases' .................. & -- & -- & - & -- \\
\hline Exchanges Received ${ }^{2}$............................. & -- & - & - & - \\
\hline Wheeling By Others' ............................ & - & - & - & - \\
\hline \multicolumn{5}{|l|}{ SPP } \\
\hline Firm Purchases & 208,550 & $3,925,028$ & - & - \\
\hline Nonfirm Purchases .............................. & $1,151,779$ & $16,580,524$ & - & -- \\
\hline Miscellaneous Purchases' .................. & - & - & - & - \\
\hline Exchanges Recetved & - & - & $\sim$ & - \\
\hline 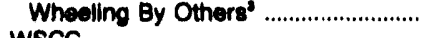 & - & - & - & - \\
\hline \multicolumn{5}{|l|}{ WSCC } \\
\hline Firm Purchases ..................................... & - & - & 170,469 & $13,119,013$ \\
\hline Nonfirm Purchases ............................ & - & - & - & - \\
\hline Miscellaneous Purchases' ................. & - & - & - & - \\
\hline Exchanges Recetved" ........................... & - & - & - & - \\
\hline 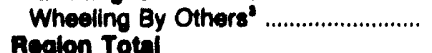 & \multicolumn{4}{|c|}{ Reglon Total } \\
\hline Firm Purchases Deliveries ................ & $15,319,022$ & $347,874,501$ & $73,906,877$ & $3,789,035,890$ \\
\hline Nonfirm Purchases Deliveries ............ & $17,080,515$ & $279,789,292$ & $64,628,029$ & $2,899,262,898$ \\
\hline Misc Purchases Delveries' ............... & 7,677 & $4,530,478$ & 49,330 & $-17,788,583$ \\
\hline Exchanges Recelved Dellveries ${ }^{2}$...... & $24,683,210$ & - & 798,358 & $17,912,809$ \\
\hline Wheeling By Others Deliveries" ........ & $1,893,618$ & $6,701,161$ & $26,589,855$ & $209,511,795$ \\
\hline
\end{tabular}

See notes and footnotes at end of table. 
Table 15. Recelpte by Investor-Owned Utilities Within and Between NERC Reglons, 1992 (Continued)

\begin{tabular}{|c|c|c|c|c|}
\hline \multirow{3}{*}{$\begin{array}{l}\text { NEAC Realen of } \\
\text { Imveotor-Owmed Utivitea/ } \\
\text { Type of Tranesction }\end{array}$} & \multicolumn{4}{|c|}{ Source of Electricity from All Ownership Classes by NERC Region } \\
\hline & \multicolumn{2}{|c|}{ SEAC } & \multicolumn{2}{|c|}{ SPP } \\
\hline & $\begin{array}{c}\text { Energy } \\
\text { (thoueand kWh) }\end{array}$ & $\begin{array}{c}\text { Coot } \\
\text { (dollars) }\end{array}$ & $\begin{array}{c}\text { Energy } \\
\text { (thousand kWh) }\end{array}$ & $\begin{array}{c}\text { Cost } \\
\text { (dollars) }\end{array}$ \\
\hline \multicolumn{5}{|l|}{ ECAR } \\
\hline Firm Purchases ..................................... & 491,440 & $8,344,700$ & - & - \\
\hline Nonfirm Purcheses ................................ & 46,280 & $1,181,858$ & - & - \\
\hline Miscollaneous Purchases' .................. & - & - & - & - \\
\hline Exchanges Recelved ......................... & 401,498 & $-2,455$ & - & - \\
\hline 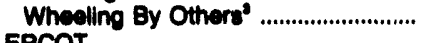 & - & - & - & - \\
\hline \multicolumn{5}{|l|}{ ERCOT } \\
\hline Firm Purchases .......................................... & - & - & $2,818,657$ & $163,867,179$ \\
\hline Nonfirm Purchases ............................... & - & - & 739,123 & $13,579,392$ \\
\hline Mlscellaneous Purchases' .................. & - & - & - & 83,692 \\
\hline Exchanges Recetved .......................... & - & - & 17,100 & - \\
\hline Wheelling By Othere' ............................... & - & - & - & - \\
\hline MAAC & & & & \\
\hline Firm Purchases ......................................... & 491,151 & $11,192,683$ & - & - \\
\hline Nonfirm Purchases ................................ & 387,079 & $22,897,670$ & - & - \\
\hline Miscellaneous Purchases' .................. & - & $-47,102$ & - & - \\
\hline Exchanges Recelved" ......................... & - & - & - & - \\
\hline Wheelling By Others" .............................. & - & - & - & -- \\
\hline \multicolumn{5}{|l|}{ MAIN } \\
\hline Firm Purchases ...................................... & 460,939 & $8,772,867$ & $1,421,623$ & $32,731,454$ \\
\hline Nonfirm Purchases .............................. & $3,465,286$ & $56,984,595$ & 181,598 & $3,240,972$ \\
\hline Miscellaneous Purchases' .................. & - & - & - & - \\
\hline Exchanges Recehved ${ }^{2}$........................... & - & - & - & - \\
\hline Wheeling By Others' .............................. & - & - & 328,882 & 581,366 \\
\hline \multicolumn{5}{|l|}{ MAPP } \\
\hline Firm Purchases ......................................... & - & - & - & - \\
\hline Nonfirm Purchases ................................. & 一 & - & 271,577 & $4,857,803$ \\
\hline Miscellaneous Purchases' .................. & - & - & - & - \\
\hline Exchanges Recelved & - & - & 180,516 & $\cdots$ \\
\hline Wheeling By Others ${ }^{2}$.............................. & - & - & 113,166 & 101,324 \\
\hline \multicolumn{5}{|l|}{ NPCC } \\
\hline Firm Purchases ........................................ & 218 & $2,551,189$ & - & - \\
\hline Nonfirm Purchases ............................. & - & - & - & - \\
\hline Miscellaneous Purchases' .................. & - & - & - & - \\
\hline Exchanges Recelved" ........................... & - & - & -- & - \\
\hline Wheeling By Others ${ }^{2}$.............................. & - & - & - & - \\
\hline \multicolumn{5}{|l|}{ SERC } \\
\hline 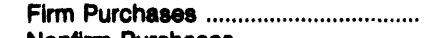 & $50,483,990$ & $2,474,831,280$ & - & - \\
\hline Nonfirm Purchases .............................. & $28,082,186$ & $961,096,302$ & 561,238 & $10,689,334$ \\
\hline Miscellaneous Purchases' ................. & 45,320 & $3,599,153$ & - & - \\
\hline Exchanges Received & $2,536,648$ & $2,330,556$ & - & -- \\
\hline 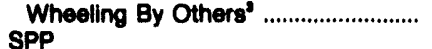 & $2,241,848$ & $9,995,026$ & - & - \\
\hline 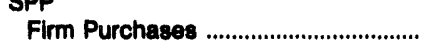 & $2,426,802$ & $277,305,560$ & $1,180,151$ & $31,778,883$ \\
\hline 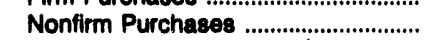 & $2,394,273$ & $226,345,905$ & $40,182,378$ & $1,163,234,068$ \\
\hline Miscellaneous Purchases' ${ }^{1}$................. & - & $11,614,794$ & $1,041,465$ & $10,278,294$ \\
\hline Exchanges Recelved & - & - & $3,011,169$ & 793,597 \\
\hline Wheeling By Others' ............................. & 123,775 & $1,398,534$ & $1,554,850$ & $30,797,677$ \\
\hline \multicolumn{5}{|l|}{ WSCC } \\
\hline Firm Purchases ...................................... & 40,439 & $2,848,582$ & 385,031 & $25,296,893$ \\
\hline Nonfirm Purchases ............................. & - & - & $1,244,041$ & $25,557,691$ \\
\hline Miscellaneous Purchases' ................. & - & - & - & - \\
\hline Exchanges Recelved ${ }^{2}$.......................... & - & - & 1,306 & - \\
\hline Wheeling By Others' ................................ & - & - & 565 & 244,893 \\
\hline Firm Purchases Deliveries ................. & $54,394,979$ & $2,785,846,861$ & & \\
\hline Nonfirm Purchases Deliveries ............ & $34,374,084$ & $1,268,506,331$ & $43,179,955$ & $1,221,258,258$ \\
\hline Misc Purchases Deliveries' ............... & 45,320 & $15,168,845$ & $1,041,465$ & $10,361,886$ \\
\hline Exchanges Recelved Deliveries" ..... & $2,838,146$ & $2,328,101$ & $3,210,090$ & 793,597 \\
\hline Wheeling By Others Deliveries ${ }^{2}$........ & $2,365,623$ & $11,393,560$ & $1,997,463$ & $31,725,360$ \\
\hline
\end{tabular}

See notes and footnotes at end of table. 
Table 15. Reculpte by Investor-Owned Utilities Within and Botween NERC Reglons, 1992 (Continued)

\begin{tabular}{|c|c|c|c|c|}
\hline \multirow{3}{*}{$\begin{array}{l}\text { NEAC Reglon of } \\
\text { Invector-Owned Utillied } \\
\text { Type of Traneaction }\end{array}$} & \multicolumn{4}{|c|}{ Source of Electricity from All Ownership Classes by NERC Region } \\
\hline & \multicolumn{2}{|c|}{ wscc } & \multicolumn{2}{|c|}{ Contiguous U.S. Total } \\
\hline & $\begin{array}{c}\text { Energy } \\
\text { (thousand kWh) }\end{array}$ & $\begin{array}{c}\text { Cost } \\
\text { (dollars) }\end{array}$ & $\begin{array}{c}\text { Energy } \\
\text { (thousand } \mathrm{kWh} \text { ) }\end{array}$ & $\begin{array}{c}\text { Cost } \\
\text { (dollars) }\end{array}$ \\
\hline \multicolumn{5}{|l|}{ ECAR } \\
\hline Firm Purchases & - & - & $46,740,710$ & $1,594,499,168$ \\
\hline Nonfirm Purchases ............................ & - & - & $39,531,248$ & $956,932,189$ \\
\hline Miscellan rus Purchases' ................. & -- & - & $9,401,923$ & $258,843,192$ \\
\hline Exchanges Recelved ........................ & - & - & $2,536,333$ & $21,447,096$ \\
\hline $\begin{array}{l}\text { Wheeling By Others" ........................... } \\
\text { ERCOT }\end{array}$ & -- & - & $1,802,269$ & $25,096,109$ \\
\hline 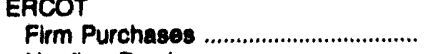 & - & - & $22,715,569$ & $1,079,080,002$ \\
\hline 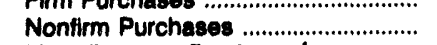 & 16,170 & 289,823 & $5,140,786$ & $92,045,510$ \\
\hline Miscellaneous Purchases' ................. & - & - & 108,008 & $1,029,679$ \\
\hline Exchanges Received ${ }^{2}$............................ & - & - & $27,576,768$ & 2,200 \\
\hline Wheelling By Others" ................................ & - & - & $3,076,745$ & $18,723,570$ \\
\hline \multicolumn{4}{|l|}{ MAAC } & \\
\hline 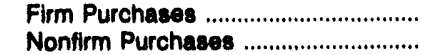 & 18,265 & $\overline{1559688}$ & $28,244,487$ & $1,028,598,101$ \\
\hline $\begin{array}{l}\text { Nonfirm Purchases ................................ } \\
\text { Miscellaneous Purchases' ............... }\end{array}$ & - & $1,559,688$ & $38,994,429$ & $1,481,208,630$ \\
\hline $\begin{array}{l}\text { Miscellaneous Purchases' .................. } \\
\text { Exchanges Pecelved" ......................... }\end{array}$ & - & - & $2,852,228$ & $68,686,371$ \\
\hline 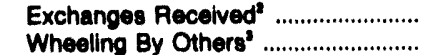 & - & - & $1,147,276$ & $34,628,119$ \\
\hline \multicolumn{2}{|l|}{ MAIN } & - & $5,140,102$ & $12,756,400$ \\
\hline Firm Purchases ..................................... & 296,475 & $5,820,637$ & $11,546,604$ & $305,098,260$ \\
\hline Nonfirm Purchases ............................... & 21,713 & 365,221 & $17,002,078$ & $279,625,286$ \\
\hline Miscellaneous Purchases' .................. & -- & - & 8,826 & 171,002 \\
\hline Exchanges Recelved ........................... & - & - & 51,422 & 497,894 \\
\hline Wheeling By Others" ............................... & - & - & $1,022,922$ & $3,000,372$ \\
\hline MAPP & & & & \\
\hline $\begin{array}{l}\text { Firm Purchases } \\
\text { Nonfirm Purchases }\end{array}$ & $\begin{array}{r}1,709,267 \\
423,812\end{array}$ & $\begin{array}{r}35,279,809 \\
6,487,769\end{array}$ & $13,651,610$ & $325,610,452$ \\
\hline & $\begin{array}{r}423,812 \\
11,964\end{array}$ & $\begin{array}{r}6,487,769 \\
98,068\end{array}$ & $12,701,480$ & $214,183,288$ \\
\hline & $\begin{array}{r}11,964 \\
2,688,358\end{array}$ & ${ }_{-}^{98,068}$ & 18,641 & $4,628,546$ \\
\hline 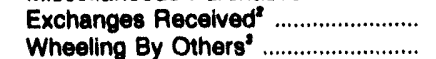 & $\begin{array}{r}2,688,358 \\
568,311\end{array}$ & $=$ & $28,384,003$ & - \\
\hline \multirow{2}{*}{\multicolumn{5}{|c|}{ NPCC }} \\
\hline & & & & \\
\hline & $-\overline{141,236}$ & - & $74,925,045$ & $3,806,275,242$ \\
\hline & 141,236 & $9,509,419$ & $66,933,757$ & $3,031,435,740$ \\
\hline Miscellaneous Purchases' .................. & - & - & 49,330 & $-18,317,571$ \\
\hline Exchanges Received ${ }^{2}$........................ & - & - & 795,741 & $17,912,809$ \\
\hline Wheeling By Others ${ }^{2}$.............................. & -- & - & $26,705,335$ & $209,774,875$ \\
\hline \multicolumn{5}{|l|}{ SERC } \\
\hline 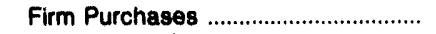 & - & -- & $57,670,792$ & $2,756,121,862$ \\
\hline Nonfirm Purchases ............................... & 39,314 & $1,441,717$ & $29,019,180$ & $881,322,722$ \\
\hline Miscellaneous Purchases' ................. & - & - & 71,005 & $3,566,075$ \\
\hline 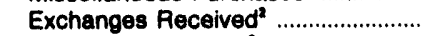 & - & - & $2,714,517$ & $2,330,556$ \\
\hline 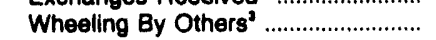 & - & - & $2,241,848$ & $9,995,026$ \\
\hline \multicolumn{5}{|l|}{ SPP } \\
\hline 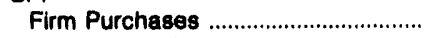 & - & - & $3,940,853$ & $320,431,559$ \\
\hline 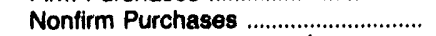 & 20,983 & 433,736 & $44,137,583$ & $1,413,651,817$ \\
\hline Miscellaneous Purchases' ................. & & & $1,043,740$ & $21,893,088$ \\
\hline 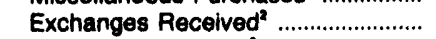 & 2,876 & 867 & $3,014,045$ & 794,464 \\
\hline 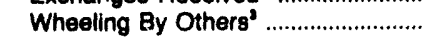 & 126,565 & 75,140 & $1,805,190$ & $33,759,480$ \\
\hline \multicolumn{5}{|l|}{ WSCC } \\
\hline Firm Purchases ....................................... & $82,389,646$ & $5,091,878,193$ & $83,036,644$ & $5,136,219,847$ \\
\hline Nonfirm Purchases ............................. & $44,961,895$ & $1,023,251,616$ & $46,205,936$ & $1,048,809,307$ \\
\hline Miscellaneous Purchases' ................. & $2,805,217$ & $-66,411,955$ & $2,805,217$ & $-66,411,955$ \\
\hline Exchanges Received ${ }^{2}$.......................... & $45,892,984$ & $-68,034,383$ & $45,894,290$ & $-68,034,383$ \\
\hline Wheeling By Others" .......................... & $45,677,663$ & $149,493,371$ & $45,678,228$ & $149,738,364$ \\
\hline \multicolumn{5}{|l|}{$\begin{array}{l}\text { Wheelling by Uners ............................ } \\
\text { Reglon Total }\end{array}$} \\
\hline Firm Purchases Deliveries ................ & $84,405,388$ & $5,132,978,639$ & $342,472,314$ & $16,351,932,493$ \\
\hline Nontirm Purchases Deliveries ........... & $45,643,488$ & $1,043,348,969$ & $299,666,477$ & $9,499,215,509$ \\
\hline Misc Purchases Deliveries' ............... & $2,817,181$ & $-66,313,887$ & $16,459,916$ & $274,068,427$ \\
\hline Exchanges Received Deliveries ${ }^{2}$...... & $48,584,218$ & $-68,033,516$ & $112,124,393$ & $9,578,855$ \\
\hline Wheeling By Others Deliveries" ....... & $46,372,539$ & $150,362,644$ & $89,736,826$ & $468,843,953$ \\
\hline
\end{tabular}

1 Miscellaneous purchases include other transactions involving line and substation rentals, system support charges, and special contract transactions.

2 Reported as Power Exchange in account 555, "Purchased Power Transactions," of the FERC Uniform System of Accounts.

- Reported as transmission services in account 456, "Transmission For Others," and in account 565, "Transmission By Others," of the FERC Uniform System of Accounts. Total quantity of wheeling may not be reported.

- Not Applicable

Notes: The States of Alaska and Hawail are not included. NERC is the North American Electric Reliablity Council. The Alaska System Coordinating Council is not included. See glossary for a list of regions and Figure 2 for a map of the regions. $\bullet 7$ ne isolated service territories of the same utility are assigned one NERC Region identification. Totals may not equal sum of components because of independent rounding.

Sources: -Federal Energy Regulatory Commission, FERC Form 1. "Annual Report of Major Electric Utilities, Licensees and Others."

-FERC Form 1-F, "Annual Report of Nonmajor Public Utilities and Licensees." 
Table 16. Dellverles by Investor-Owned Utilities Within and Between NERC Reglons, 1992

\begin{tabular}{|c|c|c|c|c|}
\hline \multirow{3}{*}{ 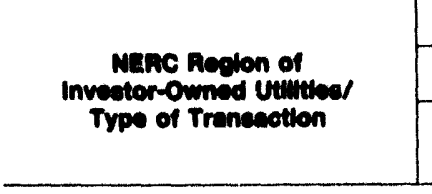 } & \multicolumn{4}{|c|}{ Destination of Electricity to All Ownership Clasees by NERC Reglon } \\
\hline & \multicolumn{2}{|c|}{ ECAR } & \multicolumn{2}{|c|}{ EACOT } \\
\hline & $\begin{array}{c}\text { Energy } \\
\text { (thousand kWh) }\end{array}$ & $\begin{array}{l}\text { Revenus } \\
\text { (dollare) }\end{array}$ & $\begin{array}{c}\text { Enorgy } \\
\text { (thousand kWh) }\end{array}$ & $\begin{array}{l}\text { Aevonue } \\
\text { (dollars) }\end{array}$ \\
\hline \multicolumn{5}{|l|}{ ECAR } \\
\hline 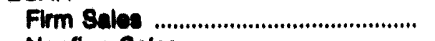 & $59,813,050$ & $1,813,809,338$ & - & - \\
\hline Nonfirm Saloe ............................................ & $44,734,528$ & $1,208,804,446$ & - & - \\
\hline 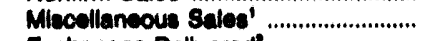 & 436 & $108,118,398$ & - & - \\
\hline Exchanges Deltvered" .............................. & & $-\overline{-}$ & - & - \\
\hline 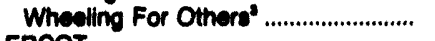 & $23,674,317$ & $75,763,803$ & - & - \\
\hline $\begin{array}{l}\text { EACOT } \\
\text { Firm Sales }\end{array}$ & - & - & $6,472,014$ & $328.344,779$ \\
\hline 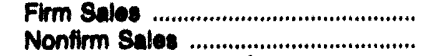 & $\overline{-}$ & $\overline{-}$ & $1,392,331$ & $29,802,805$ \\
\hline 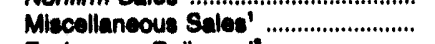 & - & - & - & 633,776 \\
\hline Exchanges Dellvered" .......................... & - & - & $27,572,269$ & $-\overline{x e s} \operatorname{sos}$ \\
\hline 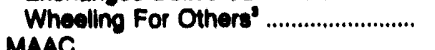 & - & - & $22,556,291$ & $44,251,395$ \\
\hline $\begin{array}{l}\text { MAAC } \\
\text { Firm Sales }\end{array}$ & & 320.716 & & \\
\hline 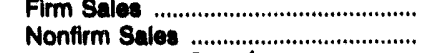 & $\begin{array}{l}6,479 \\
3,786\end{array}$ & $1,367,539$ & $\bar{z}$ & $\overline{-}$ \\
\hline Mlecellaneous Sales' ................................ & - & - & - & - \\
\hline Exchanges Dellvered" ............................... & - & - & - & - \\
\hline Wheeling For Others' & $1,770,230$ & $6,878,284$ & - & - \\
\hline \multicolumn{5}{|l|}{ MAIN } \\
\hline $\begin{array}{l}\text { Firm Sales } \\
\text { Nonfirm Sales }\end{array}$ & $\begin{array}{l}333,975 \\
298,773\end{array}$ & $\begin{array}{r}11,198,834 \\
2,985,831\end{array}$ & $\overline{-}$ & - \\
\hline Miscellaneous Sales' ........................ & - & - & - & - \\
\hline Exchanges Delivered" ................................ & 1,726 & -- & - & - \\
\hline 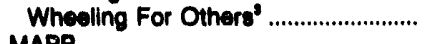 & - & -- & - & - \\
\hline \multicolumn{3}{|l|}{$\begin{array}{l}\text { MAPP } \\
\text { Firm Sales }\end{array}$} & - & - \\
\hline 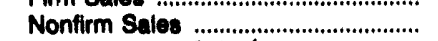 & - & - & - & - \\
\hline Miacellaneous Sales' .............................. & - & - & - & - \\
\hline 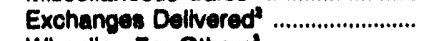 & - & - & - & - \\
\hline \multirow{2}{*}{\multicolumn{5}{|c|}{ NPCC }} \\
\hline & & & & \\
\hline $\begin{array}{l}\text { Firm Sales } \\
\text { Nonfirm Sales }\end{array}$ & 4,000 & 110,400 & - & - \\
\hline 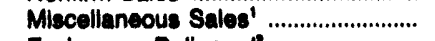 & - & - & - & - \\
\hline 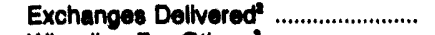 & - & - & - & - \\
\hline 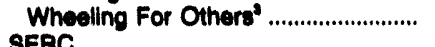 & - & - & - & - \\
\hline & 716,633 & - & \\
\hline 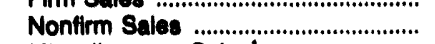 & 10,788 & 282,433 & - & - \\
\hline Miscellaneous Sales' ............................. & - & - & - & - \\
\hline Exchanges Dellvered f ............................... & 21,433 & - & -- & - \\
\hline $\begin{array}{l}\text { Wheeling For Others' ................................. } \\
\text { SPP }\end{array}$ & - & - & - & - \\
\hline 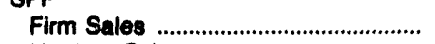 & - & - & 818,444 & $28,986,882$ \\
\hline 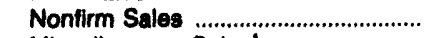 & - & - & - & - \\
\hline 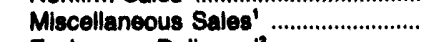 & - & - & - & - \\
\hline Exchanges Delivered" ........................... & 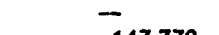 & - & - & - \\
\hline 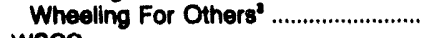 & 147,770 & 329,425 & $\mathbf{3 3 , 5 2 7}$ & 63,021 \\
\hline \multicolumn{5}{|l|}{ WSCC } \\
\hline Firm Sales & $\bar{z}$ & $\overline{-}$ & $-31,557$ & $\overline{1,514,732}$ \\
\hline 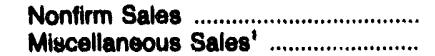 & $\bar{z}$ & $\overline{-}$ & $-{ }^{31,557}$ & $1,514,132$ \\
\hline 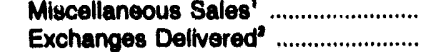 & $\bar{z}$ & $\overline{-}$ & $\overline{-}$ & $\overline{-}$ \\
\hline 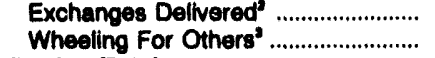 & $\overline{-}$ & - & - & $\overline{-}$ \\
\hline \multirow{5}{*}{ 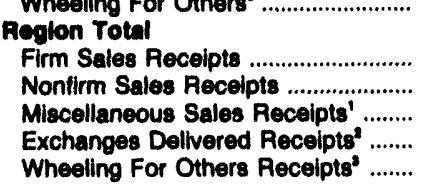 } & & & & \\
\hline & $60,171,945$ & $1,526,045,621$ & $\begin{array}{l}7,290,458 \\
1,423,888\end{array}$ & $\begin{array}{r}355,331,761 \\
31,377,537\end{array}$ \\
\hline & $\begin{array}{r}45,051,855 \\
435\end{array}$ & $\begin{array}{r}1,213,570,649 \\
108,118,393\end{array}$ & $1,423,888$ & $\begin{array}{r}31,377,537 \\
633,776\end{array}$ \\
\hline & $2,120,649$ & $108,118,393$ & $27 \overline{572,289}$ & - \\
\hline & $25,482,317$ & $82,971,512$ & $22,589,818$ & $44,314,416$ \\
\hline
\end{tabular}

See notes and footnotes at ond of table. 
Table 18. Dellverles by Investor-Owned Utilties Within and Botween NERC Roglons, 1902 (Continued)

\begin{tabular}{|c|c|c|c|c|}
\hline \multirow{3}{*}{ 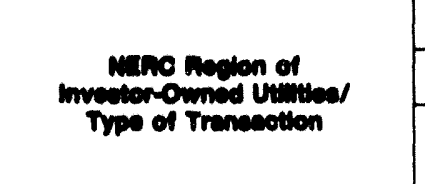 } & \multicolumn{4}{|c|}{ Deotination of Electrility to All Ownerehip Clasece by NERC Acolon } \\
\hline & \multicolumn{2}{|c|}{ MAC } & \multicolumn{2}{|c|}{ MAIN } \\
\hline & $\begin{array}{c}\text { Enwoy } \\
\text { (thousend kWh) }\end{array}$ & $\begin{array}{l}\text { Rovenus } \\
\text { (dollers) }\end{array}$ & $\begin{array}{l}\text { Eneroy } \\
\text { (thousand kWh) }\end{array}$ & $\begin{array}{l}\text { Revenus } \\
\text { (dollars) }\end{array}$ \\
\hline \multicolumn{5}{|l|}{ ECAR } \\
\hline 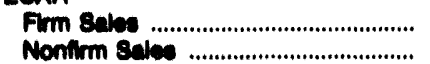 & $\begin{array}{r}14,041,762 \\
010,007\end{array}$ & $\begin{array}{r}380,480,047 \\
18,700,732\end{array}$ & $\begin{array}{r}1,004,760 \\
780,319\end{array}$ & $\begin{array}{l}10,412,360 \\
14,730,134\end{array}$ \\
\hline Muscoltaneous Bales'............................ & - & - & - & - \\
\hline Exchanges Detwered .......................... & - & - & 1,271 & - \\
\hline Wheeling For Othere' ............................ & 16,702 & $13,683,942$ & - & - \\
\hline ERCOT & & & & \\
\hline Firm sales .............................................. & - & - & - & - \\
\hline Nonilim Sales ......................................... & - & - & - & - \\
\hline Mhecelleneous sales' ........................... & - & - & - & - \\
\hline Exohanges Dollverede ............................ & - & - & - & - \\
\hline Whoeling For Others' ............................. & - & - & - & - \\
\hline 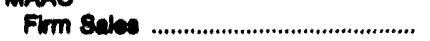 & $13,298,004$ & $300,628,658$ & - & - \\
\hline Nonfirm Sales ...................................... & $26,709,027$ & $830,300,216$ & - & - \\
\hline Mheollaneous sales' .......................... & $3,634,464$ & $80,388,114$ & - & - \\
\hline Exohangess Dellvered ............................ & - & - & - & - \\
\hline Wheeling For Others" .............................. & $7,580,387$ & $27,200,081$ & - & - \\
\hline \multicolumn{5}{|l|}{ MAIN } \\
\hline 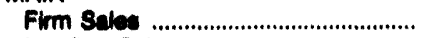 & - & - & $14,348,128$ & $523,776,201$ \\
\hline Nonfirm Sabes ..................................... & - & - & $7,685,850$ & $135,294,462$ \\
\hline Miecellansous sales' .......................... & - & - & 2,042 & 19,202 \\
\hline Exchanges Delivered" .......................... & - & - & 25,874 & - \\
\hline Wheelling For Othere' ............................. & - & - & $3,643,336$ & $17,688,267$ \\
\hline MAPP & & & & \\
\hline 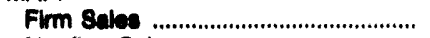 & - & - & $4,876,638$ & $86,318,934$ \\
\hline Nonfirm Sales ...................................... & - & - & $3,461,267$ & $46,371,645$ \\
\hline Miscellaneous sales' .......................... & - & - & - & - \\
\hline 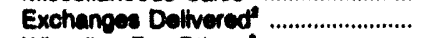 & - & - & $4,403,708$ & - \\
\hline Wheeling For Othere' ........................... & - & - & 372,788 & $2,712,366$ \\
\hline \multicolumn{5}{|l|}{ NPCC } \\
\hline Firm Sales & $1,328,686$ & $68,263,266$ & - & - \\
\hline Nonfirm Sales ....................................... & 20,396 & $1,475,101$ & - & - \\
\hline Miscollaneous Sales' ............................ & - & - & -- & - \\
\hline Exchanges Dellveredt ........................... & -- & - & - & - \\
\hline Wheeling For Others' & 57,762 & 813,723 & - & - \\
\hline \multicolumn{5}{|l|}{ SERC } \\
\hline 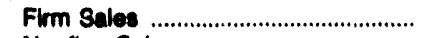 & 534,808 & $12,105,771$ & - & - \\
\hline 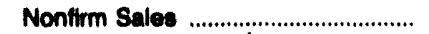 & 14,724 & 280,170 & - & - \\
\hline Miscellaneous Sales' ......................... & - & - & - & - \\
\hline Exchanges Dellvered" ............................ & 156,348 & - & - & - \\
\hline Wheeling For Others" ........................... & 8,408 & 32,029 & - & - \\
\hline \multicolumn{5}{|l|}{ SPP } \\
\hline 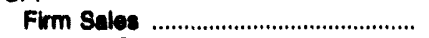 & - & - & 682,252 & $23,644,232$ \\
\hline 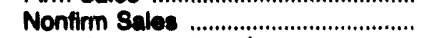 & - & - & 775,030 & $10,494,474$ \\
\hline Mbcallaneous Sales' .......................... & - & - & - & - \\
\hline 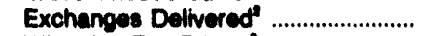 & - & - & - & - \\
\hline Wheeling For Others' & - & - & 155,890 & 586,817 \\
\hline \multicolumn{5}{|l|}{ WsCC } \\
\hline 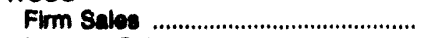 & - & - & - & - \\
\hline 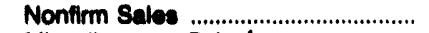 & - & - & -- & - \\
\hline 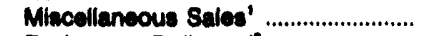 & - & - & - & - \\
\hline Exchanges Deltvered ,............................ & - & - & - & - \\
\hline Wheeling For Others" ........................... & - & - & - & - \\
\hline Firm Sales Recelpts ......................... & $29,801,327$ & $975,634,542$ & $20,871,977$ & $653,152,727$ \\
\hline Nonfirm Sales Recelpts ....................... & $26,753,814$ & $850,826,218$ & $12,688,468$ & $205,890,715$ \\
\hline Miscollaneous Sales Recelpts' ......... & $3,534,464$ & $80,388,114$ & 2,942 & 18,202 \\
\hline Exchanges Deltvered Receipts' ........ & 156,348 & - & $4,430,853$ & - \\
\hline Wheeling For Others Receiptes ........ & $7,632,297$ & $41,700,655$ & $4,172,013$ & $20,987,430$ \\
\hline
\end{tabular}

See notes and footnotes at end of table. 
Table 16. Dollverlee by Inveotor-Owned Utilities Within and Betweon NEAC Reglone, 1992 (Continued)

\begin{tabular}{|c|c|c|c|c|}
\hline \multirow{3}{*}{ 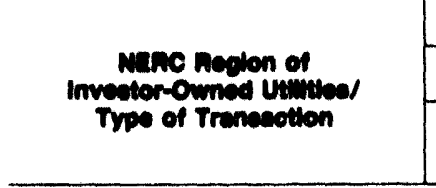 } & \multicolumn{4}{|c|}{ Destination of Electricty to All Ownerahlo Claseces by NERC Recion } \\
\hline & \multicolumn{2}{|c|}{ MAPP } & \multicolumn{2}{|c|}{ NPCC } \\
\hline & $\begin{array}{c}\text { Energy } \\
\text { (thowsand kWh) }\end{array}$ & $\begin{array}{l}\text { Rovenus } \\
\text { (dottars) }\end{array}$ & $\begin{array}{c}\text { Energy } \\
\text { (thoweand kWh) }\end{array}$ & $\begin{array}{l}\text { Aevenue } \\
\text { (dollare) }\end{array}$ \\
\hline \multicolumn{5}{|l|}{ ECAR } \\
\hline 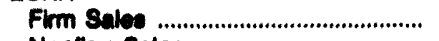 & - & - & 297,109 & $0,804,017$ \\
\hline 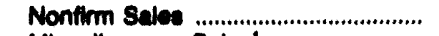 & - & - & 110,086 & $2,707,486$ \\
\hline Mtecellaneous sales' ............................... & - & - & - & - \\
\hline $\begin{array}{l}\text { Exohanges Dellwered" ............................ } \\
\text { Wheeling For Othere }\end{array}$ & - & - & - & - \\
\hline \multirow{2}{*}{\multicolumn{5}{|c|}{$\begin{array}{l}\text { ERCOT } \\
\text { Firm Sales }\end{array}$}} \\
\hline & - & - & - & - \\
\hline Nonfirm 8ales ...................................... & - & - & - & - \\
\hline 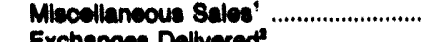 & - & $\overline{-}$ & $\overline{-}$ & $\overline{-}$ \\
\hline Exchanges Dellvereo" ........................ & - & $\bar{E}$ & $\bar{z}$ & $\overline{-}$ \\
\hline $\begin{array}{l}\text { Wheolling For Othere .............................. } \\
\text { MAMC }\end{array}$ & - & - & - & - \\
\hline 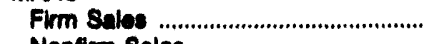 & - & - & 687.073 & $15,805,465$ \\
\hline Nonfirm Seles & - & - & $2,607,946$ & 69,198,058 \\
\hline Miscollancous Salea', .......................... & - & - & - & - \\
\hline 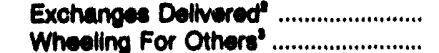 & $\overline{-}$ & $\overline{-}$ & $-\overline{198} 743$ & $\overrightarrow{118} 39$ \\
\hline \multicolumn{5}{|l|}{ MAIN } \\
\hline 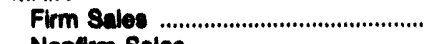 & 524,064 & $18,725,639$ & - & - \\
\hline 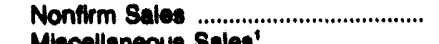 & 408,239 & $8,186,286$ & - & - \\
\hline 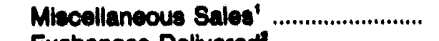 & - & - & - & - \\
\hline 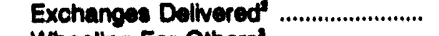 & - & - & - & - \\
\hline 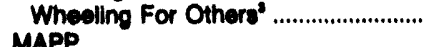 & - & - & - & - \\
\hline $\begin{array}{l}\text { MAPP } \\
\text { Firm Sales }\end{array}$ & $4,108,644$ & $144,151,321$ & - & - \\
\hline Nonfirm Sales ..................................... & $3,593,804$ & $51,134,616$ & 44,710 & 623,263 \\
\hline 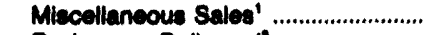 & -954 & $-78,461$ & - & - \\
\hline 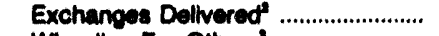 & $23,152,901$ & - & 16,693 & - \\
\hline 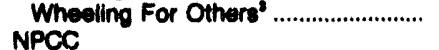 & $7,521,116$ & $11,032,968$ & - & - \\
\hline & $2,820,434,488$ \\
\hline 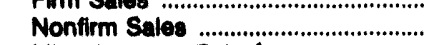 & - & - & $37,839,174$ & $1,313,185,745$ \\
\hline 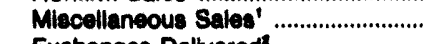 & - & - & $3,738,040$ & $193,791,239$ \\
\hline Exchanges Dellvered" ........................... & - & - & 448,340 & - \\
\hline 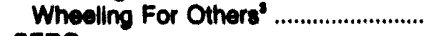 & 153,530 & 210.791 & $57,473,165$ & $501,290,256$ \\
\hline \multicolumn{5}{|l|}{ SERC } \\
\hline 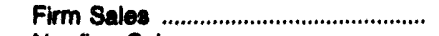 & - & - & - & - \\
\hline 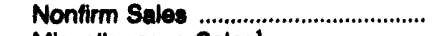 & - & - & - & - \\
\hline Miecellaneous Sales' ............................ & - & - & - & - \\
\hline 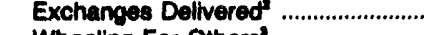 & - & - & - & - \\
\hline 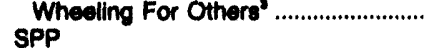 & - & - & - & - \\
\hline & - & - & - \\
\hline Nonfirm Sales & 377.520 & $6,560,154$ & - & - \\
\hline 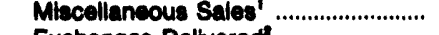 & - & - & - & - \\
\hline 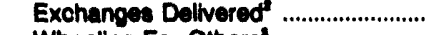 & - & - & - & - \\
\hline 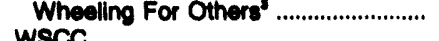 & - & 256,844 & - & - \\
\hline \multicolumn{5}{|l|}{ WSCC } \\
\hline 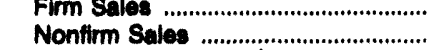 & $\begin{array}{r}275,156 \\
565\end{array}$ & $\begin{array}{r}8,223,941 \\
9,864\end{array}$ & - & $\overline{-}$ \\
\hline Miscellaneous Sales' .............................. & - & - & - & - \\
\hline Exchanges Delivered & $-7=0$ & - & - & - \\
\hline 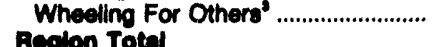 & 7,507 & $1,027,987$ & 8,423 & 50,233 \\
\hline Firm Sales Receipts ................................ & $4,807,864$ & $172,100,801$ & $53,452,217$ & $2,943,144,890$ \\
\hline Nonfirm Sales Recelpts ...................... & $4,380,128$ & $65,800,920$ & $40,701,925$ & $1,375,715,451$ \\
\hline Miscellaneous Sales Recelpts'........ & 23.152901 & $-_{-}^{-78,461}$ & $\begin{array}{r}3,738,040 \\
465,033\end{array}$ & $193,781,239$ \\
\hline Wheelling For Others Recelptes ......... & $\begin{array}{r}23,102,801 \\
7,682,159\end{array}$ & $\overline{12,528,590}$ & $58,055,706$ & $502,813,479$ \\
\hline
\end{tabular}

See notes and footnotes at end of table. 
Table 16. Dellverles by Investor-Owned Utilkles Withln and Botween NERC Reglons, 1992 (Continued)

\begin{tabular}{|c|c|c|c|c|}
\hline \multirow{3}{*}{ 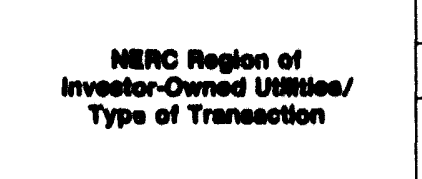 } & \multicolumn{4}{|c|}{ Destination of Electrictly to All Ownerahlp Clasese by NEAC Region } \\
\hline & \multicolumn{2}{|c|}{ SERC } & \multicolumn{2}{|c|}{ 8PP } \\
\hline & $\begin{array}{c}\text { Enmery } \\
\text { (thousand kWh) }\end{array}$ & $\begin{array}{l}\text { Rowenus } \\
\text { (dollars) }\end{array}$ & $\begin{array}{c}\text { Energy } \\
\text { (thousand kWh) }\end{array}$ & $\begin{array}{l}\text { Revenus } \\
\text { (dollara) }\end{array}$ \\
\hline \multicolumn{5}{|l|}{ ECAR } \\
\hline Firm Bake ................................................... & $2,001,547$ & $103,379,890$ & - & - \\
\hline Nonfirm Sales ......................................... & $6,700,276$ & $170,526,823$ & - & - \\
\hline .......................... & - & - & - & - \\
\hline Delivered" ............................ & 397,738 & - & - & - \\
\hline $\begin{array}{l}\text { Whealing For Othere' ............................ } \\
\text { EFCOT }\end{array}$ & $4,127,134$ & $17,708,546$ & - & - \\
\hline Firm sales .............................................. & - & - & 128,771 & $5,524,287$ \\
\hline Nonfirm Sales ...................................... & - & - & - & $-\infty$ \\
\hline Mheallaneous Balee' .............................. & - & - & - & - \\
\hline Exchanges Dellwered .......................... & - & - & - & - \\
\hline Wheeling For Others" ........................... & - & - & - & 150,847 \\
\hline Firm Saled .......................................... & 7.577 & 339,689 & - & - \\
\hline Nonfirm Sales ....................................... & - & - & - & - \\
\hline Miscallancous sales' ............................. & - & - & - & - \\
\hline Exchanges Dellvered" ............................ & - & - & - & - \\
\hline 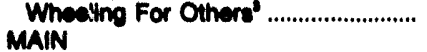 & - & - & - & - \\
\hline & - & - & $1,169,322$ & $24,598,678$ \\
\hline 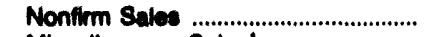 & 239,780 & $3,423,086$ & $1,557,181$ & $44,602,591$ \\
\hline Macellaneous Sales' ............................ & - & - & - & - \\
\hline Exchanges Detheredt .............................. & - & - & - & - \\
\hline Whesling For Othere "........................... & - & - & 170,379 & 544,800 \\
\hline Firm sales ........................................... & - & - & 842,465 & $16,687,022$ \\
\hline Nonfirm Salee ..................................... & - & - & $1,148,297$ & $14,280,465$ \\
\hline Miecalleneous Sales' ........................... & - & - & - & - \\
\hline Exchainges Deltweredt .............................. & - & - & 115,370 & - \\
\hline Whecling For Othere' ............................. & - & - & 5,367 & 16,784 \\
\hline \multicolumn{5}{|l|}{ NPCC } \\
\hline Firm Sales .............................................. & - & - & - & - \\
\hline Nonfirm Sales ....................................... & - & - & - & - \\
\hline Miscollaneous Sales' .............................. & - & - & - & - \\
\hline Exchanges Delvered" ............................. & - & - & - & - \\
\hline Wheolling For Othere' ........................... & - & - & - & - \\
\hline \multicolumn{5}{|l|}{ SEAC } \\
\hline Firm Sales ........................................... & $42,407,748$ & $1,700,617,034$ & - & - \\
\hline Nonfirm Sales ........................................ & $38,021,993$ & $1,336,481,384$ & $7,391,977$ & $724,536,473$ \\
\hline Miscellaneous Sales' ............................ & $-28,904$ & $2,147,732$ & - & 一 \\
\hline Exchanges Dellveredt .......................... & $2,182,146$ & - & - & - \\
\hline $\begin{array}{l}\text { Whealing For Others' ................................ } \\
\text { SPP }\end{array}$ & $17,080,228$ & $86,308,908$ & 104,459 & 520.110 \\
\hline 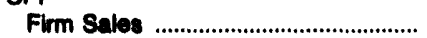 & 64,825 & $5,701,894$ & $14,826,321$ & $464,445,033$ \\
\hline Nonfirm Sales & 97,450 & $3,380,431$ & $25,254,336$ & $541,033,545$ \\
\hline 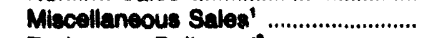 & - & - & 210,061 & $4,064,438$ \\
\hline Exchanges Delivered ............................ & - & - & $2,976,938$ & - \\
\hline Wheeling For Others: .......................... & $2,254,268$ & $6,942,110$ & $16,819,565$ & $83,732,189$ \\
\hline \multicolumn{5}{|l|}{ Wscc } \\
\hline Firm Sales & -- & - & - & - \\
\hline 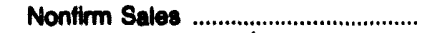 & - & - & 24,123 & 485,382 \\
\hline 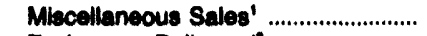 & - & - & - & -- \\
\hline Exchanges Delivered ......................... & - & - & 1,144 & - \\
\hline Wheelling For Others' ........................... & - & - & 166,415 & 757,881 \\
\hline \multirow{5}{*}{$\begin{array}{l}\text { Region Total } \\
\text { Firm Sales Recelpts .......................... } \\
\text { Nonfirm Sales Recelpts .................... } \\
\text { Miscellaneous Sales Recelpts'...... } \\
\text { Exchanges Delivered Recelpts' ....... } \\
\text { Wheelling For Others Fecelpts' ....... }\end{array}$} & & & & \\
\hline & $\begin{array}{l}45,381,697 \\
45,959,504\end{array}$ & $\begin{array}{l}1,800,038,213 \\
1,521,821,734\end{array}$ & $\begin{array}{l}16,864,879 \\
35,375,924\end{array}$ & $\begin{array}{r}511,255,800 \\
1,325,108,436\end{array}$ \\
\hline & $\begin{array}{r}40,800,004 \\
-28,004\end{array}$ & $2,147,732$ & $\begin{array}{r}210,061 \\
210,04\end{array}$ & $\begin{array}{r}4,064,438 \\
\end{array}$ \\
\hline & $2,579,884$ & - & $3,093,452$ & - \\
\hline & $23,471,631$ & $110,959,563$ & $17,266,185$ & $85,722,711$ \\
\hline
\end{tabular}

See notes and footnotes at end of table. 
Table 16. Dollveries by Investor-Owned Utilitios Within and Botween NERC Roglona, 1992 (Continued)

\begin{tabular}{|c|c|c|c|c|}
\hline \multirow{3}{*}{ 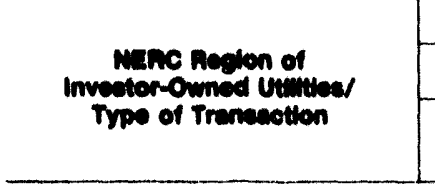 } & \multicolumn{4}{|c|}{ Destination of Electrictly to All Ownerehtp Claseses by NERC Aleglon } \\
\hline & \multicolumn{2}{|c|}{ WscC } & \multicolumn{2}{|c|}{ Contiguous U.S. Total } \\
\hline & $\begin{array}{c}\text { Energy } \\
\text { (thousand kWh) }\end{array}$ & $\begin{array}{l}\text { Revenus } \\
\text { (dollars) }\end{array}$ & $\begin{array}{c}\text { Energy } \\
\text { (thousand kWh) }\end{array}$ & $\begin{array}{l}\text { Revenus } \\
\text { (dollare) } \\
\end{array}$ \\
\hline \multicolumn{5}{|l|}{ ECAP } \\
\hline 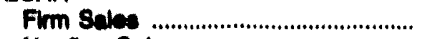 & - & - & $78,718,817$ & $2,031,843,158$ \\
\hline Nonfirm Salos ……............................... & - & - & $53,260,805$ & $1,424,660,620$ \\
\hline Miscollancous sales' .......................... & - & - & 436 & $108,118,393$ \\
\hline 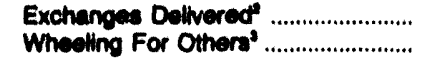 & - & - & $2,486,490$ & - \\
\hline 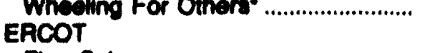 & & - & $27,822,688$ & $107,516,345$ \\
\hline 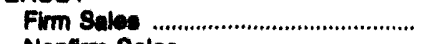 & - & - & $6,608,785$ & $331,869,046$ \\
\hline Nonitrm Sales …............................. & - & - & $1,392,331$ & $29,862,805$ \\
\hline 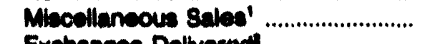 & - & - & - & 633,776 \\
\hline Exchengee Dolivered" ............................. & - & - & $27,572,269$ & - \\
\hline 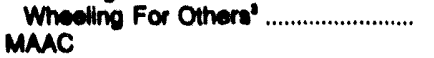 & - & - & $22,566,291$ & $44,402,242$ \\
\hline 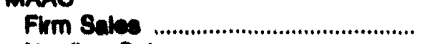 & - & - & $13,078,033$ & $523,394,448$ \\
\hline Nonfirm Salee ........................................... & - & - & $28,410,780$ & $890,868,712$ \\
\hline Mbocellaneous Sales' ............................ & - & - & $3,534,464$ & $80,368,114$ \\
\hline 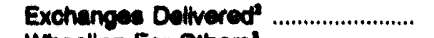 & - & - & - & - \\
\hline $\begin{array}{l}\text { Wheeling For Others' ........................................ } \\
\text { MAIN }\end{array}$ & - & - & $9,789,340$ & $35,282,180$ \\
\hline 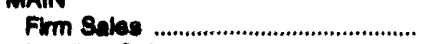 & - & - & $16,375,480$ & $578,299,452$ \\
\hline Nontirm Sales ......................................... & - & - & $10,189,838$ & $194,712,266$ \\
\hline Mtecellaneous Sales' ................................ & - & - & 2,942 & 19,202 \\
\hline Exchanges Dellvereot ............................. & - & - & 27,600 & - \\
\hline 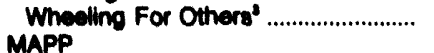 & - & - & $3,813,714$ & $18,233,057$ \\
\hline 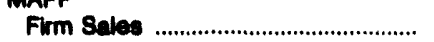 & - & - & $9,827,947$ & MAPP \\
\hline Nonfirm Sales ........................................ & 480,215 & $6,785,800$ & $8,728,293$ & $118,165,889$ \\
\hline Mbocellaneous Sales' ............................. & - & - & -954 & $-78,461$ \\
\hline 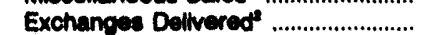 & $1,223,792$ & - & $28,912,464$ & - \\
\hline Wheeling For Othere & $1,467,298$ & $1,241,504$ & $8,368,569$ & $15,003,612$ \\
\hline \multicolumn{5}{|l|}{ NPCC } \\
\hline 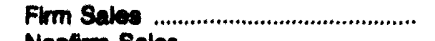 & - & - & $53,815,800$ & $2,988,697,754$ \\
\hline Nonfirm Sales ......................................... & - & - & $37,869,570$ & $1,314,771,246$ \\
\hline Miscellaneous Sales' ............................ & - & - & $3,738,040$ & $193,791,239$ \\
\hline Exchanges Dellvered" ............................ & - & - & 448,340 & - \\
\hline 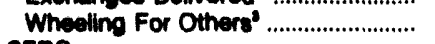 & - & - & $57,684,463$ & $502,314,770$ \\
\hline \multicolumn{5}{|l|}{ SERC } \\
\hline Firm Sales & - & - & $42,960,495$ & $1,803,439,438$ \\
\hline Nonfirm Sales ......................................... & - & - & $46,339,462$ & $2,060,570,460$ \\
\hline Mtacellaneous Sales' ................................ & - & - & $-28,904$ & $2,147,732$ \\
\hline Exchanges Delivered" .......................... & - & - & $2,359,927$ & - \\
\hline $\begin{array}{l}\text { Wheeling For Othere' ....................................... } \\
\text { SpP }\end{array}$ & $3,645,261$ & $14,206,401$ & $20,848,355$ & $101,007,448$ \\
\hline 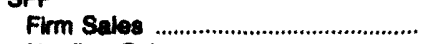 & 747,500 & $44,113,788$ & $17,139,342$ & $566,891,929$ \\
\hline Nonfirm Seles ............................................ & $1,439,667$ & $24,364,622$ & $27,944,003$ & $585,843,228$ \\
\hline 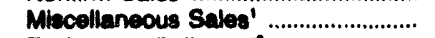 & - & - & 210,061 & $4,064,438$ \\
\hline Exchanges Delivereot ................................ & 2.760 & - & $2,979,698$ & - \\
\hline \multirow{2}{*}{\multicolumn{5}{|c|}{ WSCC }} \\
\hline & & & & \\
\hline Nonfirm Sales ..................................... & $23,153,762$ & $565,050,130$ & $23,210,007$ & $567,060,088$ \\
\hline 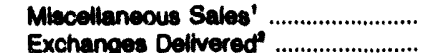 & $\begin{array}{r}128,823 \\
48,094,461\end{array}$ & ${ }^{793,634}$ & $\begin{array}{r}128,823 \\
48,095,605\end{array}$ & $\underline{7}^{793,634}$ \\
\hline 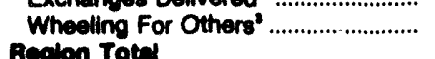 & $55,689,874$ & $165,332,485$ & $55,852,319$ & $167,168,686$ \\
\hline \multirow{4}{*}{ 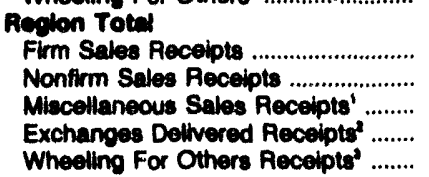 } & & & & 10.405805026 \\
\hline & $\begin{array}{l}30,434,034 \\
25,073,644\end{array}$ & $\begin{array}{r}1,369,100,471 \\
596,200,652\end{array}$ & $\begin{array}{l}269,376,398 \\
237,409,148\end{array}$ & $\begin{array}{r}7,486,412,312 \\
\end{array}$ \\
\hline & $\begin{array}{r}128,823 \\
49,321,013\end{array}$ & $\begin{array}{l}793,634 \\
-\end{array}$ & $\begin{array}{r}7,584,907 \\
112,892,402\end{array}$ & $389,858,067$ \\
\hline & $60,783,098$ & $180,782,085$ & $227,145,224$ & $1,082,880,441$ \\
\hline
\end{tabular}
tions.

"Reported as Power Exchange in account 555, "Purchased Power Transactions," of the FERC Uniform System of Accounts.

- Reported as tranamiselon senvices in account 458. "Tranamiasion For Others," and in account 585, "Tranemiseion By Others," of the FERC Uniform System of Accounts. Total quantity of wheeling may not be reported.

- Not Applicable

Notea: -The States of Alaska and Hawail are not included. -NERC is the North American Electric Reliability Council. The Alaska System Coondinating Council is not included. See gloseary for a list of regions and Figure 2 for a map of the regions. -The isolated service teritories of the same utility are assioned one NERC Region identification. - Totals may not equal sum of components because of independent rounding.

Sources: -Federal Energy Reoulatory Commiasion, FERC Form 1, "Annual Report of Major Electric Utilities, Licensees and Others."

-FEAC Form 1.F. "Annual Report of Nonmajor Public Utilities and Licensees." 
Table 17. Electriolty Traneactione by Inveotor-Owned Utilities In Alacka and Hawall, 1992

\begin{tabular}{|c|c|c|c|c|}
\hline \multirow{2}{*}{ Troe of Truncaction } & \multicolumn{2}{|c|}{ Alacka } & \multicolumn{2}{|c|}{ Hawall } \\
\hline & $\begin{array}{c}\text { Energy } \\
\text { (thowatand } k W h \text { ) }\end{array}$ & $\begin{array}{c}\text { Cont } \\
\text { (dollars) }\end{array}$ & $\begin{array}{l}\text { Energy } \\
\text { (thousand } k W h \text { ) }\end{array}$ & $\begin{array}{c}\text { Coot } \\
\text { (dollaro) }\end{array}$ \\
\hline \multicolumn{5}{|l|}{ necentreat } \\
\hline \multirow[t]{3}{*}{ 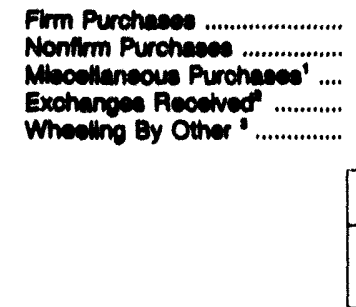 } & $\begin{array}{l}- \\
- \\
=\end{array}$ & $\begin{array}{l}\overline{6}, 026,202 \\
\overline{-}\end{array}$ & $\begin{array}{r}100,076 \\
2,123,012 \\
-\quad 3,046 \\
-\quad\end{array}$ & $\begin{array}{l}7,060,878 \\
184,850,861 \\
= \\
=\end{array}$ \\
\hline & \multicolumn{2}{|c|}{ Alakka } & \multicolumn{2}{|c|}{ Hawall } \\
\hline & $\begin{array}{l}\text { Energy } \\
\text { (thousand kWh) }\end{array}$ & $\begin{array}{l}\text { Revenuse } \\
\text { (dollars) }\end{array}$ & $\begin{array}{l}\text { Energy } \\
\text { (thousand kWh) }\end{array}$ & $\begin{array}{l}\text { Rovenue } \\
\text { (dollare) }\end{array}$ \\
\hline \multicolumn{5}{|l|}{ Dellvered } \\
\hline 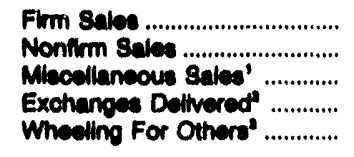 & $\bar{z}$ & $\bar{z}$ & $\begin{array}{l}\overline{-} \\
-\quad 3,054\end{array}$ & $\begin{array}{l}\overline{-} \\
- \\
-\end{array}$ \\
\hline
\end{tabular}


Table 18. U.S. Electrictty Trade with Canada and Mexico by NERC Reglon, 1992

\begin{tabular}{|c|c|c|c|c|c|c|}
\hline \multirow{2}{*}{$\begin{array}{l}\text { Province, Country' } \\
\text { Type of Truneaction }\end{array}$} & \multicolumn{5}{|c|}{ U.S. NERC Reglon } & \multirow{2}{*}{ Region Total } \\
\hline & NPCC & ECAR & ERCOT & MAPP & wsce & \\
\hline 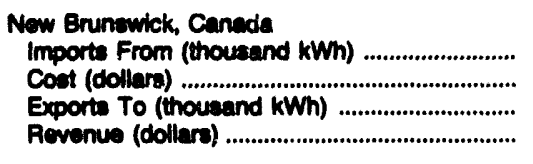 & $\begin{array}{r}2,309,388 \\
70,825,376 \\
384,870 \\
\mathbf{8 2 1 , 7 8 2}\end{array}$ & $\bar{z}$ & $\bar{z}$ & $\bar{z}$ & $\bar{z}$ & $\begin{array}{r}2,309,309 \\
70,825,376 \\
384,870 \\
821,782\end{array}$ \\
\hline 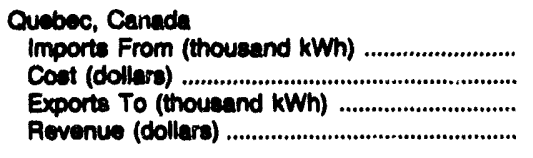 & $\begin{array}{r}6,858,410 \\
186,782,613 \\
1,360,573 \\
41,672,619\end{array}$ & $\bar{z}$ & $\bar{z}$ & $\bar{z}$ & $\bar{z}$ & $\begin{array}{r}8,868,418 \\
196,782,613 \\
1,380,573 \\
41,672,610\end{array}$ \\
\hline 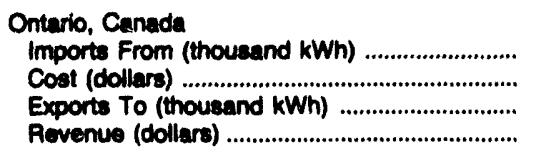 & $\begin{array}{r}3,531,850 \\
74,225,351 \\
900,288 \\
22,868,056\end{array}$ & $\begin{array}{r}82,151 \\
1,281,476 \\
314,118 \\
7,844,584\end{array}$ & $\bar{z}$ & $\begin{array}{r}96 \\
2,597 \\
3,345 \\
87,822\end{array}$ & $\ddot{m}$ & $\begin{array}{r}3,614,097 \\
75,519,424 \\
1,217,751 \\
31,000,462\end{array}$ \\
\hline 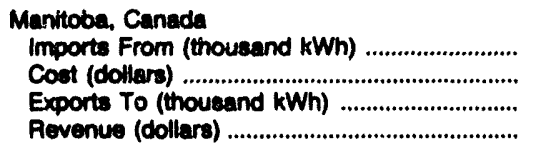 & $=$ & $\bar{m}$ & $\bar{m}$ & $\begin{array}{r}8,471,271 \\
83,400,811 \\
1,600,607 \\
783,881\end{array}$ & $\bar{z}$ & $\begin{array}{r}8,471,271 \\
83,400,811 \\
1,600,607 \\
793,981\end{array}$ \\
\hline 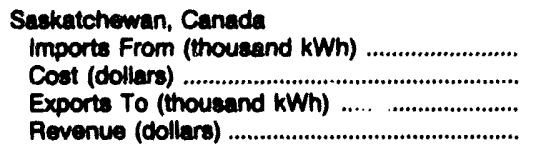 & $\begin{array}{l}\ddot{-} \\
\ddot{-}\end{array}$ & $\bar{z}$ & $\bar{z}$ & $\begin{array}{r}102,285 \\
1,357,172 \\
47,800 \\
1,391,214\end{array}$ & $\bar{m}$ & $\begin{array}{r}102,285 \\
1,357,172 \\
47,800 \\
1,391,214\end{array}$ \\
\hline 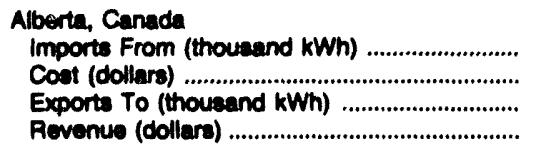 & $\bar{z}$ & $\bar{m}$ & $\bar{m}$ & $\bar{z}$ & $\begin{array}{r}-\overline{1} \\
100 \\
5,838\end{array}$ & $\begin{array}{r}\overline{-} \\
100 \\
5,838\end{array}$ \\
\hline 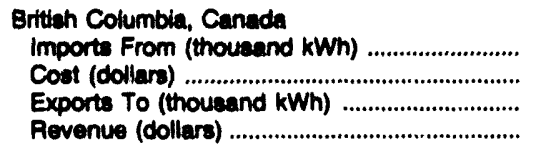 & $\bar{z}$ & $\bar{z}$ & $\overline{-}$ & $\bar{z}$ & $\begin{array}{r}11,826,316 \\
156,452,544 \\
3,254,289 \\
129,153\end{array}$ & $\begin{array}{r}11,828,316 \\
156,452,544 \\
3,254,289 \\
129,153\end{array}$ \\
\hline 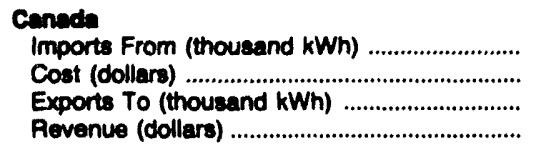 & $\begin{array}{r}14,698,638 \\
341,833,340 \\
2,645,731 \\
65,462,457\end{array}$ & $\begin{array}{r}82,151 \\
1,291,476 \\
314,118 \\
7,944,584\end{array}$ & $\bar{m}$ & $\begin{array}{r}8,573,652 \\
84,760,580 \\
1,651,752 \\
2,273,017\end{array}$ & $\begin{array}{r}11,826,316 \\
158,452,544 \\
3,254,389 \\
134,991\end{array}$ & $\begin{array}{r}35,181,757 \\
584,337,950 \\
7,865,980 \\
75,815,049\end{array}$ \\
\hline 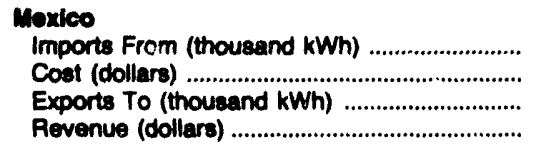 & $\overline{-}$ & $\bar{m}$ & $\begin{array}{r}-\overline{-} \\
169,14 \overline{2} \\
5,383,922\end{array}$ & $\bar{m}$ & $\begin{array}{r}2,022,419 \\
84,515,228 \\
820,745 \\
34,777,252\end{array}$ & $\begin{array}{r}2,022,419 \\
84,515,228 \\
989,887 \\
40,161,174\end{array}$ \\
\hline 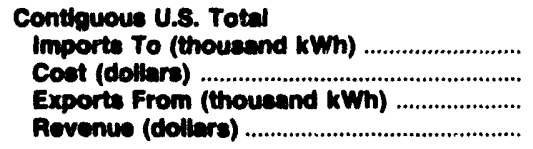 & $\begin{array}{r}14,690,638 \\
341,853,340 \\
2,645,731 \\
65,462,457\end{array}$ & $\begin{array}{r}82,151 \\
1,201,476 \\
314,118 \\
7,044,624\end{array}$ & $\begin{array}{r}- \\
169,1 \overline{2} \\
5,383,922\end{array}$ & $\begin{array}{r}8,573,652 \\
84,760,500 \\
1,651,752 \\
2,273,017\end{array}$ & $\begin{array}{r}13,049,735 \\
240,097,772 \\
4,075,134 \\
34,012,243\end{array}$ & $\begin{array}{r}37,204 ; 176 \\
606,853,176 \\
8,855,077 \\
115,076,223\end{array}$ \\
\hline
\end{tabular}

- Not Applicable

Notes: -The values do not reflect a large portion of U.S.-Canadian electricity trade that occurs on an exchange basis; I.e., energy imported is returned in kind with no monetary conaideration. This "zero-cost" of imported and exported energy diatorts the price-per-kllowatthour calculation. -The States of Alaska and Hawall are not included. •NERC is the North American Electric Reliability Council. The Alaska System Coordinating Council is not included.

See gloseary for a list of regions and Figure 2 for a map of the regions. Totals may not equal sum of components because of independent rounding. Source: Form FE-781R "Annual Report of International Electrical Export/Import Data." 


\section{Bulk Power Transactions by Investor-Owned Utilities}

\section{Overview}

This chapter presents data for individual investorowned utilities on purchased power, sales for resale, exchanges, and wheeling. Sales for resale are generally sales to other investor-owned utilities, public power municipals, and cooperatives under obligations to support wholesale requirement customers. These sales represent one of the most active parts of the electricity trade market. Purchases are generally made from other investor-owned utilities and Federal power marketing authorities. These purchases are made in order to take advantage of cheaper rates generally coming from hydroelectric generation, to supplement or replace internal power supplies, and for handling longer term energy emergencies. Exchanges cover in-kind transfers, and wheeling (transmission for and by others) describes transmission services provided by utilities to other utilities. The tables present data for individual utilities aggregated to show trade with different classes of ownership.

Data are from the FERC Form 1, which is the only data source that identifies individual utilities involved in exchanges and wheeling. ${ }^{5}$

\section{Electrical System}

In 1992, electric trade transactions were affected by a variety of local and regional industry related problems. The North American Electric Reliability Council (NERC) in its summer and winter assessments described the overall system reliability of the bulk electric supply. ${ }^{6}$ These three reviews look at all the projected electrical operations for 1992. Some of the events described influenced trade and will help in the interpretation of regional trade.

NERC indicated that generating capacity was adequate for all areas of the United States (excluding the State of Hawaii which is not covered by NERC). Some re- gions were still suffering from the lingering drought, and transmission loading issues were affecting several regions.

For ECAR, portions of the transmission system were projected to carry heavy loading because of scheduled electricity transfers. Facility upgrades were made to improve overall system voltage and reliability during heavy west-to-east transfers for the ECAR, MAAC and the northern portion of SERC. However, the transfers would be lower than in the prior year because of the affects of changes in generation dispatch and higher base transfer among ECAR, MAAC and SERC. For MAIN, the region depended more on operating procedures than before. These procedures were implemented in order to achieve and protect the established transfer capability for utilities operating in that region.

MAPP was projected to operate its hydroelectric energy production at about 30 percent below normal. For WSCC, both the Northwest Power Pool (NWPP) and the Rocky Mountain Power Area (RMPA) were faced with deteriorated hydro capability. The NWPP anticipated reduced nonfirm energy sales outside the region and below-normal filling of Columbia River system reservoirs. The RMPA expected to offset its lost hydro generation by purchases of other generation sources and increased inter-area transfers. The California-Southern Nevada Power Area was also looking at the sixth consecutive year of below normal precipitation.

Going into the winter of 1992, SERC was affected by two significant decreased regional transmission transfer capability changes. A transmission line in SPP was derated, reducing the SPP-to-SERC transfer capability by 2,750 megawatts. The other was a drop of 600 megawatts capability between the Tennessee Valley Authority and the Southern area because of several reasons: increased electricity flow across that interconnection caused by demand growth in northern Georgia, new generation schedules, and wheeling transactions. NERC also noted that for the $1992 / 1993$ winter, generating utilities heavily dependent on natural gas in

\footnotetext{
${ }^{5}$ This is not the case for other data collected on exchanges and wheeling. Only aggregated data are collected on exchanges and wheeling on Form EIA-861, "Annual Electric Utility Report" and Form ElA-412, "Annual Report of Public Electric Utilities" starting in 1989.

${ }^{6}$ North American Electric Reliability Council, 1992 Summer Assessment(Princeton, New Jersey, May 1992), pp. 4-9; 1991/92 Winter Assessment (Princeton, New Jersey, November 1991), pp. 4-9, and the 1992/93 Winter Assessment (Princeton, New Jersey, November 1992), pp. 4-9
} 


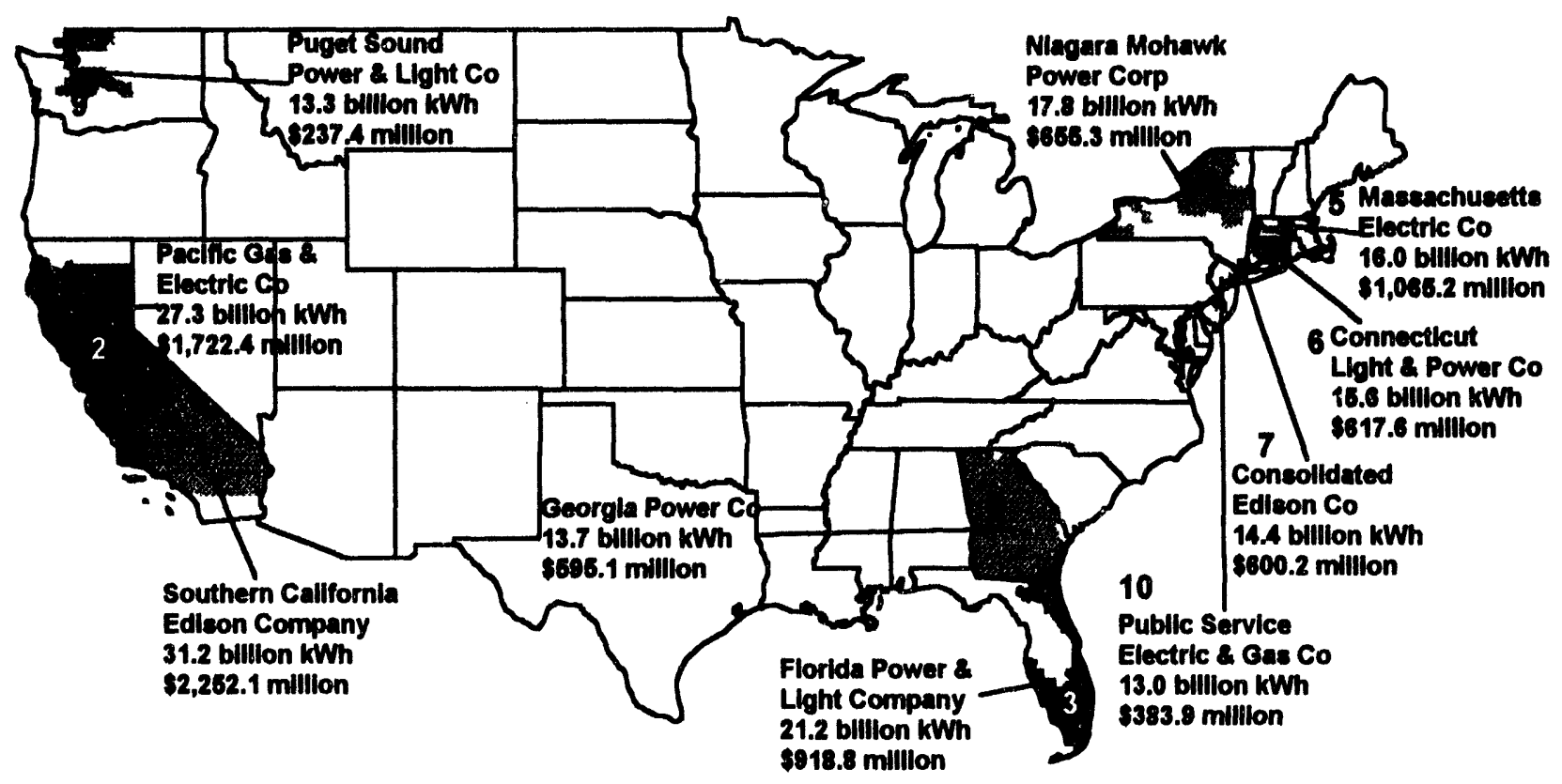

Notes: Numbers indicate rank by kllowatthours (KWh) purchased. Shaded areas show the service territories of the indicated utilities. Source: Federal Energy Regulatory Commission, FERC Form 1, "Annual Report of Major Electric Utilities, Licensees and Others."

Texas experienced some curtailment of supplies in the spot market, but these utilities maintained fuel-oil inventories to cover the disruptions. A 230-kilovolt line in MAAC was delayed from being energized in 1992 because of questions about electromagnetic field exposures.

NERC noted in both of its winter assessments that extreme weather and equipment problems in any region can combine to strain reserve margins. This in turn will affect wholesale electric trade by changing the mix of generation, the dispatch patterns of electrical generation units, the electrical system support requirements for reliability, and economic and firm electricity transfers.

\section{Tables}

This chapter presents data on:

- Energy purchases and cost, with cost data shown for demand, energy, and other (Table 19).

- Sales for resale and revenue, with revenue data shown for demand, energy, and other (Table 20).

- Exchanges of power by ownership class, identifying quantities transferred with any monetary settlement (Table 21). Nonmonetary settlements that may have occurred are not shown. Exchanges are transactions where electricity provided to one utility is returned in kind at a later date. In these transactions, the value of the electricity may or may not have been priced at the same level. Additional money or electricity could have been involved in the exchanges, but the details of the transactions are not collected on the FERC forms.

- Electricity purchases by investor-owned utilities not listed elsewhere (Table 22). (These utilities are not listed elsewhere because of their small size and/or their power was purchased from cooperatives.)

- Wheeling through and to service territories of investor-owned utilities (Tables 23 and 24).

In each table, the investor-owned utilities are listed by State according to their corporate mailing addresses.

\section{Highlights}

In 1992, as in 1990, Southern California Edison led all investor-owned utilities with almost 31.2 billion kilowatthours in purchases for which it paid nearly 


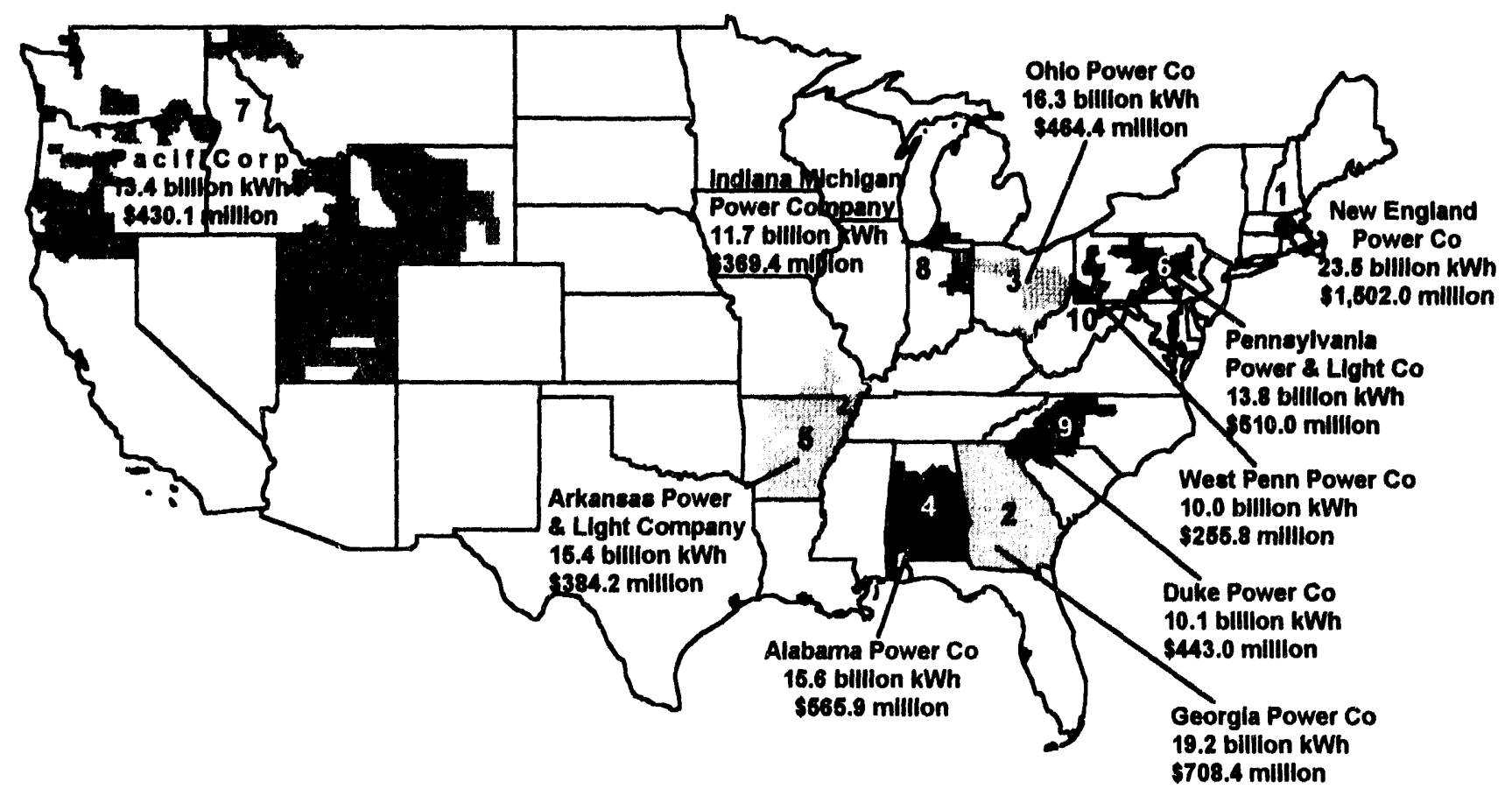

Notes: Numbers indicate rank by kilowatthours (KWh) sold. Shaded areas show the service territories of the indicated utilities. Source: Federal Energy Regulatory Commission, FERC Form 1, "Annual Report of Major Electric Utilities, Licensees and Others."

$\$ 2.3$ billion. Of the next four largest investor-owned utilities, ranked according to the amount of energy purchased, Pacific Gas \& Electric of California, Florida Power \& Light, Niagara Mohawk Power Corp. of New York, and the Massachusetts Electric Company each paid at least $\$ 655$ million for $27.3,21.2,17.8$ and 16 bilition kilowatthours, respectively. These top five investor-owned purchasers purchased 113.5 billion kilowatthours for $\$ 6.6$ billion (Figure 6). In comparing 1992 activity with the aggregated top five purchasers of 1990 ( 120.3 billion kilowatthours for $\$ 6.2$ billion), there was a decrease in the total amount of power purchased from 1990 to 1992 (5.6 percent). However, the total cost increased by 6.5 percent.

For wholesale transactions covered by the sales for resale category, New England Power of Massachusetts sold 23.5 billion kilowatthours for revenue of $\$ 1.5$ billion to lead all investor-owned utilities. Following New England Power in volume of sales for resale were Georgia Power, Ohio Power, Alabama Power, and Arkansas Power \& Light. Each of these utilities sold more than 15.4 billion kilowatthours. The associated revenues ranged from $\$ 384.2$ million for Arkansas Power \& Light's 15.4 billion kilowatthours to $\$ 708.4$ million for Georgia Power's 19.2 billion kilowatthours (Figure 7).

Each of the first 10 investor-owned utilities, ranked by energy exchanged, had receipts totaling more than 1.4 billion kilowatthours. PacifiCorp, headquartered in Oregon, led all investor-owned companies in both receipts (22.1 billion kilowatthours) and deliveries (21.9 billion kilowatthours). Northern States Power of Minnesota was second in receipts with 16.9 billion kilowatthours and also second in deliveries with 21.8 billion kilowatthours. Of the utilities east of the Mississippi River, only Duke Power of North Carolina exchanged enough power to be included in the top 10 ranking. This utility had exchanges of 2.5 billion kilowatthours in receipts and 2.2 bilition kilowatthours in deliveries. Investor-owned utilities west of the Mississippi River were the most active in this category of electric power transactions. In 1990, the aggregated receipts of the top 10 utilities (ranked by receipts) was 115.8 billion kilowatthours. In 1992, the total for the top 10 was down by over 13 percent to 100.3 billion kilowatthours.

Wheeling data reported by investor-owned utilities on FERC Form 1 do not always include the quantity of electricity received and/or delivered. This results because utilities frequently buy and sell capacity on a transmission line or system for a specified time period. Rather than providing individual transmission services, a utility leases portions of its transmission system capability to other utilities. The operation of the transmission lines (e.g., loading, metering, and maintenance) remains the responsibility of the owner of the transmission lines, but FERC Form 1 record keeping of the 
quantity of electricity wheeled (megawatthours) is not necessary since charges are fixed or based on the maximum capacity used (megawatts). For this reason, the discussion below focuses on wheeling costs and revenue instead of receipts and deliveries of wheeled electricity.

Investor-owned utilities also provided transmission services to other utilities for a fee. The company receiving the most money for wheeling power through its system was the Consolidated Edison Company of New York. It received $\$ 213.8$ million for wheeling 13.2 billion kilowatthours. Again, as in 1990, most power wheeled by Consolidated Edison came from upstate New York and other parts of the NPCC region and went to utilities on Long Island. The other utilities in the top 10 received from $\$ 19.3$ million (Connecticut Light and Power) to $\$ 66.7$ million (Niagara Mohawk of New York) for wheeling services. Six of the top 10 utilities (by revenue) are located in New England, and two others are located in California. Of those utilities reporting energy wheeled, Pacific Gas \& Electric of California wheeled the most -- 16.5 billion kilowatthours for which it received $\$ 56.5$ million, substantially less than Consolidated Edison.

Again, Long Island Lighting of New York paid the most for wheeling services in 1992 ( $\$ 45.8$ million), followed by Pacificorp of Oregon, New England Power in Massachusetts, Columbus Southern Power of Ohio, and Puget Sound Power \& Light of Washington State (these utilities paid other utility transmission service charges of \$42.8 million, \$31.9 million, \$29.9 million, and $\$ 26.9$ million, respectively).

\section{Summary}

Differences are present in all wholesale trade categories. Some are based on the diversity of the cost of energy coming from the individual mix of generating facilities that developed over time as the electric utility industry met local and regional changes in load. This includes development of inexpensive local natural resources. In other cases, differences can be explained because some electric utilities are requirement customers of others and lack generation capability so they cannot sell electricity.

The data indicate a great diversity in wholesale electricity trade and a complex market for electricity. The diversity represent differences in the influence of geographical location; the ability to access multiple parts of the transmission grid; the support of reliability and stability standards; and the load requirements coming from varied customer compositions of service territories. 
Table 19. Electriclty Purchases by Investor-Owned Utilitles, by State, 1992

\begin{tabular}{|c|c|c|c|c|c|c|c|}
\hline $\begin{array}{c}\text { souros of } \\
\text { Eicetricity by } \\
\text { owneratip claces }\end{array}$ & $\begin{array}{l}\text { Alabama } \\
\text { Alabama } \\
\text { Power } \\
\text { Co }\end{array}$ & $\begin{array}{l}\text { Alabama } \\
\text { Southern } \\
\text { Electric } \\
\text { Generating } \\
\text { Co' }^{\circ}\end{array}$ & $\begin{array}{l}\text { Alacka } \\
\text { Alaaka } \\
\text { Electric } \\
\text { Light \& } \\
\text { Power }\end{array}$ & $\begin{array}{l}\text { Arizona } \\
\text { Ajo } \\
\text { Improvement } \\
\text { Co: }\end{array}$ & $\begin{array}{l}\text { Artzona } \\
\text { Arizona } \\
\text { Public } \\
\text { Service } \\
\text { Co }\end{array}$ & $\begin{array}{l}\text { Arizona } \\
\text { Century } \\
\text { Power } \\
\text { Corp' }\end{array}$ & $\begin{array}{l}\text { Arizona } \\
\text { Citizens } \\
\text { Utillities } \\
\text { Co }\end{array}$ \\
\hline \multicolumn{8}{|l|}{ Invedtor-Owned } \\
\hline & $2,869,111$ & - & - & - & 497,940 & - & $\begin{array}{r}848,257 \\
40,043,997\end{array}$ \\
\hline 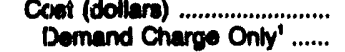 & $77,081,128$ & $\overline{-}$ & $\overline{-}$ & $\bar{z}$ & $21,613,403$ & $\overline{-}$ & 40,$043 ; 997$ \\
\hline 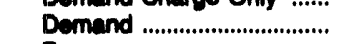 & - & - & - & - & $11,452,772$ & - & $21,790,801$ \\
\hline 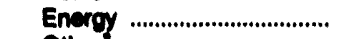 & $77,081,126$ & - & - & -- & $10,160,631$ & - & $18,131,978$ \\
\hline 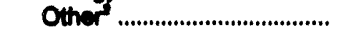 & - & - & - & - & - & - & 121,220 \\
\hline \multicolumn{8}{|l|}{ Fedoral } \\
\hline Purchaees (thousand KWh) & 80,321 & - & 222,165 & - & 2,770 & - & - \\
\hline 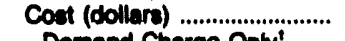 & $1,60 /, 334$ & - & $6,928,262$ & 一 & 43,280 & - & - \\
\hline Demand Charge Only' ....... & - & - & - & - & - & - & - \\
\hline 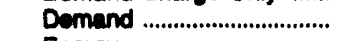 & - & - & - & - & - & - & - \\
\hline 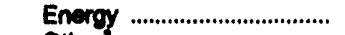 & $1,607,334$ & - & $6,928,262$ & $=$ & 43,260 & - & $\overline{-}$ \\
\hline 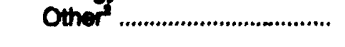 & - & - & - & - & - & - & - \\
\hline \multicolumn{8}{|l|}{$\begin{array}{l}\text { State and Other } \\
\text { Government }\end{array}$} \\
\hline Purchases (thoussand kWh) & 1,894 & - & - & - & 809,095 & - & 10,172 \\
\hline & 40,760 & - & - & - & $35,174,309$ & - & 164,324 \\
\hline Demand Charge Only' ....... & - & - & - & - & - & - & - \\
\hline Demand & & - & - & - & $15,666,793$ & - & 14,821 \\
\hline 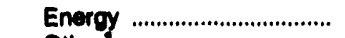 & 40,760 & - & - & - & $19,466,364$ & - & 149,403 \\
\hline 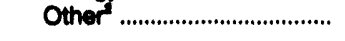 & - & - & - & - & 41,152 & - & - \\
\hline \multicolumn{8}{|l|}{ Munictpal } \\
\hline Purchases (thousand KWh) & 93 & - & - & - & 75 & - & - \\
\hline $\begin{array}{l}\text { Cost (dollarg) ...................... } \\
\text { Demand Charge Only' }\end{array}$ & 2,890 & - & - & - & 2,063 & - & - \\
\hline Demand Charge Only' ...... & - & - & - & - & -- & - & - \\
\hline Demand .................................. & - & - & - & - & - 2003 & $\overline{-}$ & $\overline{-}$ \\
\hline $\begin{array}{l}\text { Energy } \\
\text { Other }\end{array}$ & $-\quad 2,890$ & $\bar{z}$ & $\overline{-}$ & $\overline{-}$ & $-{ }^{2,063}$ & $\overline{-}$ & $\overline{-}$ \\
\hline \multicolumn{8}{|l|}{ Cooperative } \\
\hline Purcheseses (thousand kWh) & 182,970 & - & - & - & 1,270 & - & \\
\hline Cost (dollars) & $7,366,193$ & - & - & - & 27,864 & - & 5,681 \\
\hline Demand Charge Only' ....... & - & - & - & - & - & - & - \\
\hline 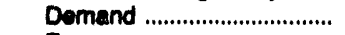 & - & - & - & - & - & - & - \\
\hline 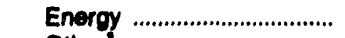 & $4,950,603$ & - & - & -- & 27,864 & - & 5,681 \\
\hline 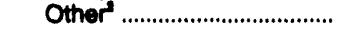 & $2,414,580$ & - & - & - & - & - & - \\
\hline \multicolumn{8}{|l|}{ Other" } \\
\hline Purchases (thousand kWh) & $1,555,116$ & - & - & - & 1,479 & - & - \\
\hline $\begin{array}{l}\text { Cost (dollars) } \\
\text { Demand Charge Only' ........ }\end{array}$ & $35,374,908$ & $\overline{-}$ & $\bar{z}$ & $\overline{-}$ & ${ }_{-}^{146,535}$ & $\overline{-}$ & $\ldots$ \\
\hline 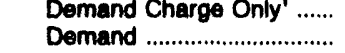 & $\bar{z}$ & $\overline{-}$ & $\overline{-}$ & $\overline{-}$ & $\overline{-}$ & $\bar{z}$ & $\overline{-}$ \\
\hline Energy .................................... & $29,869,685$ & - & - & -- & 3,535 & - & - \\
\hline 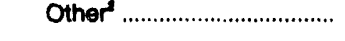 & $5,505,223$ & - & - & - & 143,000 & - & 732 \\
\hline \multicolumn{8}{|l|}{ Total } \\
\hline Purchases (thousand kWh) & $4,688,605$ & - & 222,165 & - & $1,312,629$ & - & 858,499 \\
\hline Cost (dollars) ........................... & $121,472,211$ & - & $6,928,262$ & - & $57,007,434$ & -- & $40,214,744$ \\
\hline Demand Charge Only' ....... & - & - & - & - & - & - & - \\
\hline 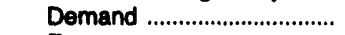 & - & - & - & - & $27,119,565$ & -- & $21,805,722$ \\
\hline 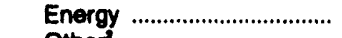 & $113,552,398$ & - & $6,928,262$ & - & $29,703,717$ & - & $\begin{array}{r}18,287,070 \\
121,952\end{array}$ \\
\hline 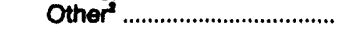 & $7,919,813$ & - & - & - & 184,152 & - & 121,852 \\
\hline
\end{tabular}

See notes and footnotes at end of table. 
Table 19. Electricity Purchases by Investor-Owned Utilities, by State, 1992 (Continued)

\begin{tabular}{|c|c|c|c|c|c|c|c|}
\hline $\begin{array}{c}\text { Eource of } \\
\text { Eleotricity by } \\
\text { Owmerehlp claces }\end{array}$ & $\begin{array}{l}\text { Arizona } \\
\text { Tuceon } \\
\text { Electric } \\
\text { Power } \\
\text { Co }\end{array}$ & $\begin{array}{l}\text { Arkaneas } \\
\text { Arkaneas } \\
\text { Power } \\
\& \\
\text { Light } \\
\text { Co }\end{array}$ & $\begin{array}{l}\text { Arkansas } \\
\text { Entergy } \\
\text { Power } \\
\text { Co }\end{array}$ & $\begin{array}{l}\text { California } \\
\text { Pacific } \\
\text { Gas \& } \\
\text { Electric } \\
\text { Co }\end{array}$ & $\begin{array}{l}\text { Calliornia } \\
\text { San Diego } \\
\text { Gas \& } \\
\text { Electric } \\
\text { Co }\end{array}$ & $\begin{array}{l}\text { California } \\
\text { Southern } \\
\text { California } \\
\text { Edison } \\
\text { Co }\end{array}$ & $\begin{array}{l}\text { Colorado } \\
\text { Publio } \\
\text { Service } \\
\text { Co of } \\
\text { Colorado }\end{array}$ \\
\hline 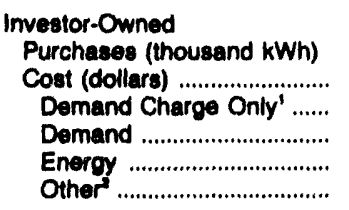 & $\begin{array}{r}2,313,959 \\
147,307,047 \\
- \\
88,201,336 \\
59,105,711 \\
-\end{array}$ & $\begin{array}{r}2,636,539 \\
48,955,358 \\
\overline{1,043,250} \\
47,912,108 \\
-\end{array}$ & $\begin{array}{r}44,293 \\
760,531 \\
- \\
-760,531 \\
-\end{array}$ & $\begin{array}{r}1,556,394 \\
51,505,378 \\
-7,083,390 \\
44,421,988 \\
-\end{array}$ & $\begin{array}{r}4,932,814 \\
165,533,871 \\
-\overline{71,020,768} \\
94,513,103 \\
-\end{array}$ & $\begin{array}{r}2,188,509 \\
80,206,639 \\
16,952,613 \\
24,671,111 \\
38,924,029 \\
-341,114\end{array}$ & $\begin{array}{r}873,876 \\
36,106,857 \\
-- \\
22,241,973 \\
13,649,174 \\
215,710\end{array}$ \\
\hline 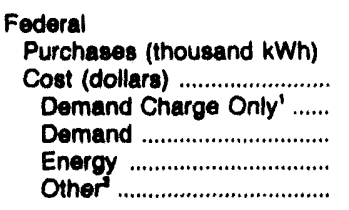 & $\begin{array}{l}\quad 455 \\
-\quad 10,525 \\
-\quad 10,525\end{array}$ & $\begin{array}{l}\overline{-} \\
\overline{-} \\
\overline{-}\end{array}$ & $\begin{array}{l}\overline{-} \\
\overline{-} \\
\overline{-}\end{array}$ & $\begin{array}{l}271,144 \\
4,495,966 \\
-- \\
- \\
4,495,966 \\
--\end{array}$ & $\begin{array}{l}671,668 \\
11,242,026 \\
- \\
\overline{11,242,026}\end{array}$ & $\begin{array}{r}1,119,470 \\
33,676,619 \\
-- \\
3,489,324 \\
29,560,672 \\
626,623\end{array}$ & $\begin{array}{r}860 \\
18,120 \\
-\quad \\
-\quad 18,530 \\
-1,401\end{array}$ \\
\hline 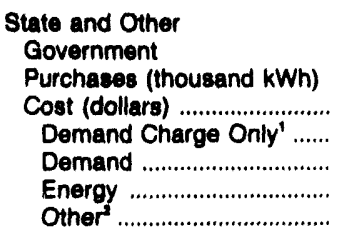 & $\begin{array}{r}11,787 \\
214,189 \\
-\quad \\
86,748 \\
127,451 \\
-\end{array}$ & $\begin{array}{l}- \\
\overline{-} \\
\overline{-} \\
-\end{array}$ & $\begin{array}{l}\overline{-} \\
\overline{-} \\
\bar{m}\end{array}$ & $\begin{array}{r}2,113,297 \\
58,556,980 \\
- \\
961,873 \\
57,595,107 \\
--\end{array}$ & $\begin{array}{l}488,916 \\
8,536,193 \\
- \\
-- \\
6,536,193 \\
--\end{array}$ & $\begin{array}{r}484,617 \\
26,477,969 \\
900,000 \\
5,634,122 \\
18,869,347 \\
74,500\end{array}$ & $\begin{array}{r}1,268,638 \\
74,079,598 \\
- \\
49,339,950 \\
24,739,649 \\
--\end{array}$ \\
\hline 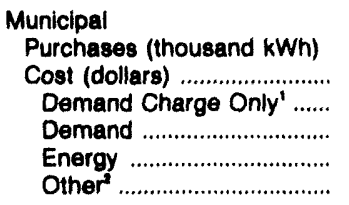 & $\begin{array}{r}11,948 \\
290,377 \\
- \\
- \\
- \\
-\end{array}$ & $\begin{array}{l}129,770 \\
3,320,013 \\
- \\
- \\
\overline{3,320,013}\end{array}$ & $\begin{array}{l}\overline{-} \\
\overline{-} \\
\overline{-} \\
\overline{-}\end{array}$ & $\begin{array}{r}159,856 \\
11,198,284 \\
- \\
2,630,078 \\
8,568,208 \\
--\end{array}$ & $\begin{array}{r}638 \\
14,536 \\
-\sim \\
-\quad 14,536\end{array}$ & $\begin{array}{r}1,367,730 \\
98,072,915 \\
\overline{17,910,817} \\
79,825,478 \\
236,620\end{array}$ & $\begin{array}{r}133,845 \\
4,397,332 \\
-\sim \\
2,310,551 \\
1,534,336 \\
552,445\end{array}$ \\
\hline 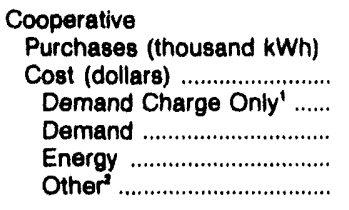 & $\begin{array}{l}75,131 \\
1,568,418 \\
-- \\
-- \\
1,568,418 \\
--\end{array}$ & $\begin{array}{r}3,467,421 \\
66,191,037 \\
416 \\
-\quad \\
66,190,621 \\
--\end{array}$ & $\begin{array}{l}43,725 \\
826,763 \\
-- \\
- \\
-\end{array}$ & $\begin{array}{l}191,297 \\
5,906,514 \\
-- \\
5,906,514 \\
--\end{array}$ & $\begin{array}{r}8,929 \\
193,486 \\
-- \\
- \\
193,486\end{array}$ & $\begin{array}{r}391,684 \\
6,812,638 \\
\overline{-} \\
\overline{6,719,694} \\
92,944\end{array}$ & $\begin{array}{r}3,824,732 \\
142,384,050 \\
- \\
90,313,905 \\
52,115,163 \\
-45,018\end{array}$ \\
\hline 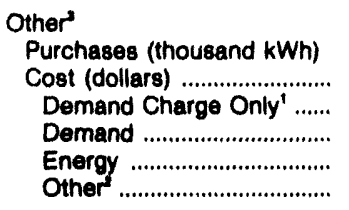 & $\begin{array}{l}53,458 \\
2,795,507 \\
- \\
- \\
1,141,992 \\
1,853,515\end{array}$ & $\begin{array}{c}4,998,010 \\
298,633,321 \\
-\cdots \\
-- \\
298,633,321 \\
-\end{array}$ & $\begin{array}{l}\overline{-} \\
\bar{m} \\
\bar{m}\end{array}$ & $\begin{array}{r}22,972,433 \\
1,590,753,208 \\
-- \\
481,385,767 \\
1,108,367,441 \\
--\end{array}$ & $\begin{array}{r}2,432,987 \\
125,526,223 \\
-- \\
63,460,840 \\
62,065,383 \\
-\end{array}$ & $\begin{array}{r}25,602,392 \\
2,006,847,327 \\
- \\
625,734,244 \\
1,379,005,909 \\
2,107,174\end{array}$ & $\begin{array}{r}1,384,373 \\
72,786,676 \\
- \\
57,618,038 \\
15,050,954 \\
117,684\end{array}$ \\
\hline 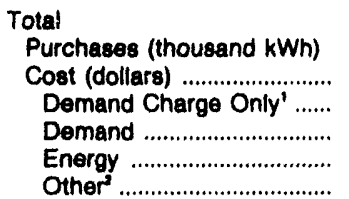 & $\begin{array}{r}2,466,738 \\
152,186,073 \\
- \\
88,288,084 \\
62,244,474 \\
1,653,515\end{array}$ & $\begin{array}{r}11,231,740 \\
417,089,729 \\
416 \\
1,043,250 \\
416,056,063 \\
--\end{array}$ & $\begin{array}{l}88,018 \\
1,587,294 \\
- \\
- \\
1,587,294\end{array}$ & $\begin{array}{r}27,264,421 \\
1,722,416,330 \\
-- \\
492,061,106 \\
1,230,355,224\end{array}$ & $\begin{array}{r}8,536,052 \\
311,046,335 \\
-- \\
134,481,608 \\
176,564,727 \\
--\end{array}$ & $\begin{array}{r}31,154,412 \\
2,252,094,107 \\
17,852,813 \\
677,439,618 \\
1,554,005,129 \\
2,796,747\end{array}$ & $\begin{array}{r}7,486,324 \\
329,772,843 \\
- \\
221,824,417 \\
107,108,806 \\
839,420\end{array}$ \\
\hline
\end{tabular}

See notes and footnotes at end of table. 
Table 19. Electrlelty Purchaces by Investor-Owned Utilitlos, by State, 1992 (Continued)

\begin{tabular}{|c|c|c|c|c|c|c|c|}
\hline $\begin{array}{c}\text { source of } \\
\text { Eisotrithy by } \\
\text { Owmerahip Claces }\end{array}$ & $\begin{array}{l}\text { Colorado } \\
\text { Utillkorp } \\
\text { United } \\
\text { Inc }\end{array}$ & $\begin{array}{l}\text { Conneotlcut } \\
\text { Connecticut } \\
\text { Light \& } \\
\text { Power } \\
\text { Co }\end{array}$ & $\begin{array}{l}\text { Connectiout } \\
\text { Connectiout } \\
\text { Yankee } \\
\text { Atomic Power } \\
\text { Co }\end{array}$ & $\begin{array}{l}\text { Connecticut } \\
\text { United } \\
\text { Illuminating } \\
\text { Co }\end{array}$ & $\begin{array}{l}\text { Delaware } \\
\text { Delmarva } \\
\text { Power } \\
\text { L Light } \\
\text { Co }\end{array}$ & $\begin{array}{l}\text { Dlatrict of } \\
\text { Columbia } \\
\text { Potomac } \\
\text { Electric } \\
\text { Power Co }\end{array}$ & $\begin{array}{l}\text { Florida } \\
\text { Florida } \\
\text { Power } 8 \\
\text { Light } \\
\text { Co }\end{array}$ \\
\hline \multicolumn{8}{|l|}{ Investor-Owned } \\
\hline $\begin{array}{l}\text { Purchases (thousend kWh) } \\
\text { Cost (dollare) ............................. }\end{array}$ & $\begin{array}{r}1,434,334 \\
53,900,625\end{array}$ & $\begin{array}{r}7,404,918 \\
260,434,972\end{array}$ & $\overline{-}$ & $\begin{array}{r}462,718 \\
23,622,811\end{array}$ & $\begin{array}{r}2,649,870 \\
58,870,095\end{array}$ & $\begin{array}{r}3,354,880 \\
150,511,897\end{array}$ & $\begin{array}{r}1,036,770 \\
18,868,829\end{array}$ \\
\hline Demand Charge Only' ....... & 610 & $200,404,012$ & - & $20,022,011$ & - & - & - \\
\hline 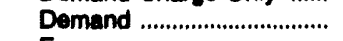 & $29,980,317$ & - & - & $18,321,000$ & - & $4,023,311$ & - \\
\hline $\begin{array}{l}\text { Energy } \\
\text { Other }\end{array}$ & $\begin{array}{l}26,672,062 \\
-2,752,364\end{array}$ & $\begin{array}{l}150,819,946 \\
109,615,026\end{array}$ & $\overline{-}$ & $5,301,811$ & $\begin{array}{l}58,870,095 \\
-\end{array}$ & $\begin{array}{l}54,769,028 \\
91,719,558\end{array}$ & $\begin{array}{r}17,861,858 \\
1,004,871\end{array}$ \\
\hline \multicolumn{8}{|l|}{ Federal } \\
\hline Purchases (thousand kWh) & - & - & - & - & - & - & - \\
\hline 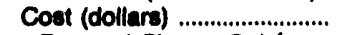 & 200 & - & - & - & - & - & -- \\
\hline Demand Charge Only' ....... & 200 & - & - & - & - & - & - \\
\hline 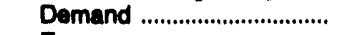 & - & - & - & - & - & - & - \\
\hline 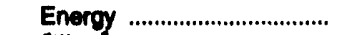 & - & - & - & - & - & - & - \\
\hline 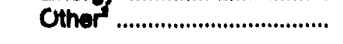 & - & - & - & - & - & -- & - \\
\hline \multicolumn{8}{|l|}{$\begin{array}{l}\text { State and Other } \\
\text { Government }\end{array}$} \\
\hline & - & 454,669 & - & 5,816 & 1,859 & 10,105 & 438,090 \\
\hline Cost (dollare) ........... & - & $34,664,409$ & - & 34,833 & 36,417 & 376,496 & $15,422,269$ \\
\hline & - & -- & - & - & - & - & $=-$ \\
\hline & - & - & - & - & - & - & $7,026,527$ \\
\hline 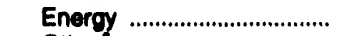 & - & $34,555,488$ & -- & 34,833 & 36,417 & 283,576 & $8,395,742$ \\
\hline 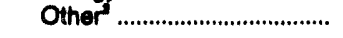 & - & 108,821 & - & - & - & 82,820 & - \\
\hline \multicolumn{8}{|l|}{ Municipal } \\
\hline Purchases (thousand kWh) & 1,187 & 1,548 & - & - & -- & - & $3,435,013$ \\
\hline 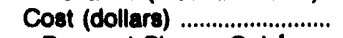 & 30,002 & 132,653 & $\ldots$ & - & - & - & $143,418,948$ \\
\hline Demand Charge Only' ....... & - & - & - & -- & - & - & -- \\
\hline 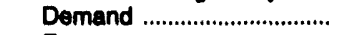 & - & - & - & - & - & - & $85,382,289$ \\
\hline 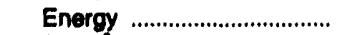 & 30,002 & 132,653 & - & - & - & - & $54,763,061$ \\
\hline Other & - & - & - & - & - & - & $3,273,598$ \\
\hline \multicolumn{8}{|l|}{ Cooperattive } \\
\hline Purchases (thousand kWh) & - & - & - & -- & - & - & 802,651 \\
\hline 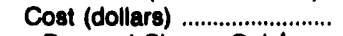 & -- & - & - & - & - & $5,538,905$ & $13,674,278$ \\
\hline Demand Charge Only' ....... & - & - & - & - & -- & - & - \\
\hline 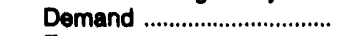 & - & - & - & -- & - & - & - \\
\hline 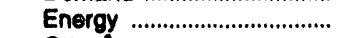 & - & - & - & - & - & - & $13,674,278$ \\
\hline Other & - & - & - & - & -- & $5,538,905$ & - \\
\hline \multicolumn{8}{|l|}{ Other" } \\
\hline Purchases (thousand kWh) & -- & $7,706,969$ & - & 915,523 & 178,480 & $4,913,183$ & $15,533,738$ \\
\hline 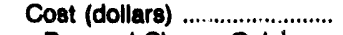 & - & $322,335,452$ & - & $44,010,604$ & $4,049,590$ & $105,259,505$ & $727,409,488$ \\
\hline Demand Charge Only' ...... & -- & - & - & - & - & -- & - \\
\hline Demand & - & - & - & $25,239,647$ & 533,070 & - & $411,377,355$ \\
\hline 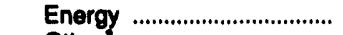 & - & $274,967,619$ & - & $18,770,857$ & $3,632,803$ & $95,987,485$ & $316,131,380$ \\
\hline 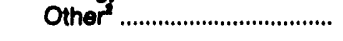 & - & $47,367,833$ & - & - & $-116,383$ & $9,272,020$ & $-89,247$ \\
\hline \multicolumn{8}{|l|}{ Total } \\
\hline Purchases (thousand kWh) & $1,435,521$ & $15,568,104$ & - & $1,384,057$ & $2,830,209$ & $8,278,178$ & $21,245,262$ \\
\hline $\begin{array}{l}\text { Cost (doliars) ......... } \\
\text { Demand Chare Only' }\end{array}$ & $\begin{array}{r}53,930,827 \\
810\end{array}$ & $617,567,486$ & $=$ & $67,688,248$ & $62,956,102$ & $261,686,803$ & $918,791,812$ \\
\hline $\begin{array}{l}\text { Demand Charge Only' } \\
\text { Demand }\end{array}$ & $29,980,317$ & $\overline{-}$ & $\overline{-}$ & $\overline{43,560,647}$ & 533,070 & $4,023,311$ & $503,786,171$ \\
\hline 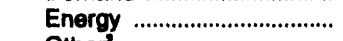 & $26,702,064$ & $460,475,706$ & - & $24,107,601$ & $62,539,415$ & $151,040,089$ & $410,826,319$ \\
\hline Other & $-2,752,364$ & $157,091,780$ & - & 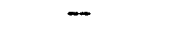 & $-116,383$ & $106,623,403$ & $4,179,322$ \\
\hline
\end{tabular}

See notes and footnotes at end of table. 
Table 19. Eloctricity Purchases by Investor-Owned Utilities, by State, 1992 (Continued)

\begin{tabular}{|c|c|c|c|c|c|c|c|}
\hline $\begin{array}{c}\text { source of } \\
\text { Eleotritity by } \\
\text { Ownerehip claces }\end{array}$ & $\begin{array}{l}\text { Florida } \\
\text { Florlda } \\
\text { Power } \\
\text { Corp }\end{array}$ & $\begin{array}{l}\text { Flonde } \\
\text { Qull } \\
\text { Power } \\
\text { Co }\end{array}$ & $\begin{array}{c}\text { Florida } \\
\text { Tampa } \\
\text { Electric } \\
\text { Co }\end{array}$ & $\begin{array}{c}\text { Georgia } \\
\text { Georgia } \\
\text { Power } \\
\text { Co }\end{array}$ & $\begin{array}{l}\text { Georgia } \\
\text { Savannah } \\
\text { Electric } \\
\text { Power } \\
\text { Co }\end{array}$ & $\begin{array}{c}\text { Hawall } \\
\text { Cittzens } \\
\text { Utilitios } \\
\text { Co }^{4}\end{array}$ & $\begin{array}{l}\text { Hawall } \\
\text { Hawallan } \\
\text { Electric } \\
\text { Co inc }\end{array}$ \\
\hline 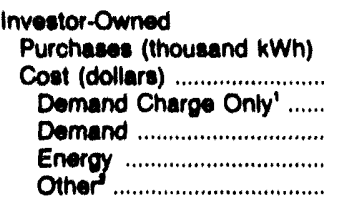 & $\begin{array}{r}1,110,686 \\
37,898,361 \\
- \\
168,698 \\
37,831,683 \\
-\end{array}$ & $\begin{array}{l}43,209 \\
842,649 \\
- \\
- \\
-\end{array}$ & $\begin{array}{r}55,828 \\
1,087,025 \\
-\quad \\
105,451 \\
1,849,350 \\
32,215\end{array}$ & $\begin{array}{l}2,934,294 \\
80,835,802 \\
\overline{-} \\
\overline{80,835,802}\end{array}$ & $\begin{array}{l}19,528 \\
176,690 \\
- \\
- \\
-176,690\end{array}$ & $\bar{m}=$ & 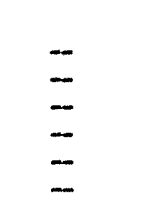 \\
\hline 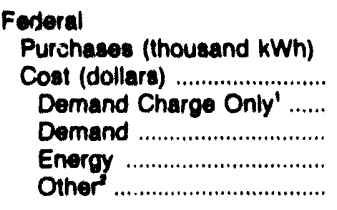 & $\begin{array}{r}33,683 \\
424,615 \\
- \\
- \\
-424,815 \\
-\end{array}$ & $\begin{array}{l}33,266 \\
322,713 \\
-- \\
- \\
- \\
-\end{array}$ & $\begin{array}{l}\overline{-} \\
\bar{z} \\
\bar{m}\end{array}$ & $\begin{array}{l}\overline{-} \\
\overline{-} \\
\overline{-}\end{array}$ & $\begin{array}{r}6,062 \\
121,315 \\
- \\
- \\
-121,315 \\
--\end{array}$ & $\begin{array}{l}\overline{-} \\
\bar{z} \\
\overline{-}\end{array}$ & $\begin{array}{l}\overline{-} \\
\bar{m} \\
\bar{m}\end{array}$ \\
\hline 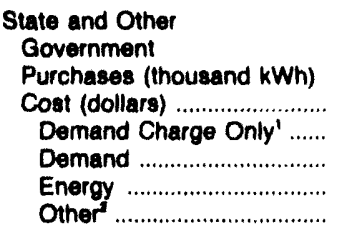 & $\begin{array}{r}813,760 \\
27,586,111 \\
-- \\
5,681,556 \\
21,904,555 \\
-\end{array}$ & $\begin{array}{r}401 \\
-\quad 8,184 \\
-\quad 8,184\end{array}$ & $\begin{array}{r}215,121 \\
7,275,689 \\
- \\
3,165,030 \\
4,037,321 \\
73,348\end{array}$ & $\begin{array}{r}3,210,642 \\
182,818,606 \\
\overline{-} \\
182,816,606\end{array}$ & $\begin{array}{r}151 \\
3,077 \\
-\quad \\
-\quad 3,077 \\
-\quad\end{array}$ & $\begin{array}{l}\bar{z} \\
\bar{z}\end{array}$ & $\begin{array}{l}\overline{-} \\
\overline{-} \\
\bar{m}\end{array}$ \\
\hline 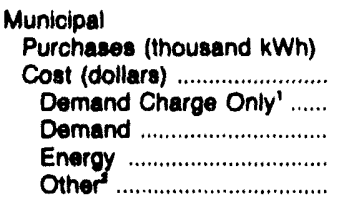 & $\begin{array}{r}252,256 \\
9,489,061 \\
- \\
55,258 \\
8,433,803\end{array}$ & $\begin{array}{r}18 \\
-\quad 545 \\
-\quad \\
-\quad 545\end{array}$ & $\begin{array}{r}20,709 \\
889,493 \\
-\quad \\
11,820 \\
877,563 \\
10\end{array}$ & $\begin{array}{r}50,654 \\
903,393 \\
- \\
- \\
- \\
-\end{array}$ & $\begin{array}{r}7 \\
-\quad 205 \\
-\quad \\
-\quad 205\end{array}$ & $\begin{array}{l}\overline{-} \\
\bar{z} \\
\bar{m}\end{array}$ & $\begin{array}{l}\overline{-} \\
\bar{z} \\
\overline{-}\end{array}$ \\
\hline 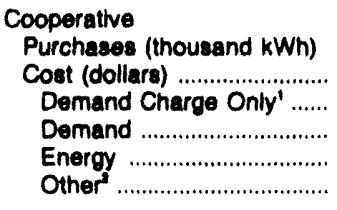 & $\begin{array}{l}241,145 \\
5,448,817 \\
- \\
- \\
5,448,817 \\
--\end{array}$ & $\begin{array}{l}16,817 \\
183,069 \\
- \\
- \\
-183,069\end{array}$ & $\begin{array}{l}14,763 \\
366,158 \\
- \\
- \\
- \\
-\end{array}$ & $\begin{array}{l}5,542,644 \\
234,240,882 \\
\overline{-} \\
\overline{234,240,882} \\
--\end{array}$ & $\begin{array}{r}2,631 \\
-\quad 42,143 \\
-\quad \\
-\quad 42,143\end{array}$ & $\begin{array}{l}-- \\
\overline{-} \\
\overline{-} \\
-\end{array}$ & $\begin{array}{l}\overline{-} \\
\bar{m} \\
\bar{m}\end{array}$ \\
\hline 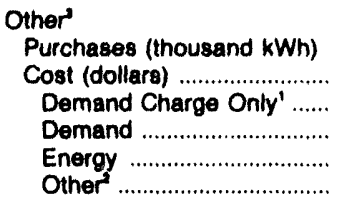 & $\begin{array}{r}1,211,897 \\
59,446,074 \\
- \\
27,187,537 \\
32,258,537 \\
-\end{array}$ & $\begin{array}{r}1,281,630 \\
26,824,398 \\
-- \\
3,807,748 \\
23,016,650 \\
-\end{array}$ & $\begin{array}{r}320,727 \\
9,152,826 \\
- \\
2,786,988 \\
6,206,162 \\
158,676\end{array}$ & $\begin{array}{l}1,856,294 \\
86,243,474 \\
-- \\
-- \\
86,243,474\end{array}$ & $\begin{array}{r}3,172,195 \\
56,642,829 \\
- \\
2,424,430 \\
54,218,489 \\
--\end{array}$ & $\begin{array}{l}80,561 \\
4,327,252 \\
- \\
- \\
4,327,252 \\
--\end{array}$ & $\begin{array}{r}2,022,742 \\
148,889,000 \\
- \\
- \\
91,270,000 \\
57,619,000\end{array}$ \\
\hline 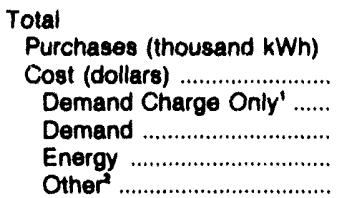 & $\begin{array}{r}3,663,639 \\
140,393,338 \\
-33,091,049 \\
107,302,290\end{array}$ & $\begin{array}{r}1,375,341 \\
28,181,558 \\
- \\
3,807,748 \\
24,373,810 \\
-\end{array}$ & $\begin{array}{r}627,148 \\
19,671,201 \\
-6,069,389 \\
13,336,563 \\
265,249\end{array}$ & $\begin{array}{c}13,694,528 \\
595,140,157 \\
\overline{-} \\
595,140,157 \\
-\end{array}$ & $\begin{array}{r}3,200,574 \\
56,986,359 \\
- \\
2,424,430 \\
54,561,929 \\
-\end{array}$ & $\begin{array}{l}80,561 \\
4,327,252 \\
- \\
\overline{4,327,252}\end{array}$ & $\begin{array}{r}2,022,742 \\
148,889,000 \\
\overline{-} \\
91,270,000 \\
57,619,000\end{array}$ \\
\hline
\end{tabular}

See notes and tootnotes at end of table. 
Table 19. Electrleity Purchases by Inveator-Owned Utilities, by 8tate, 1992 (Continued)

\begin{tabular}{|c|c|c|c|c|c|c|c|}
\hline of & $\begin{array}{l}\text { Howll } \\
\text { Mout } \\
\text { Elootilo } \\
\text { Co Lit' }\end{array}$ & $\begin{array}{l}\text { Idaho } \\
\text { Clibens } \\
\text { Uilitive } \\
\text { Co" }\end{array}$ & $\begin{array}{l}\text { Idaho } \\
\text { Idaho } \\
\text { Powner } \\
\text { Co }\end{array}$ & $\begin{array}{l}\text { Illinois } \\
\text { Central } \\
\text { Illinols } \\
\text { Light } \\
\text { Co }\end{array}$ & $\begin{array}{l}\text { Illinole } \\
\text { Central } \\
\text { litinoit } \\
\text { Publle service } \\
\text { Co }\end{array}$ & $\begin{array}{c}\text { Ilinois } \\
\text { Commommealth } \\
\text { Edison } \\
\text { Co }\end{array}$ & $\begin{array}{l}\text { Illinols } \\
\text { Eleotrio } \\
\text { Eneroy } \\
\text { Ino }\end{array}$ \\
\hline 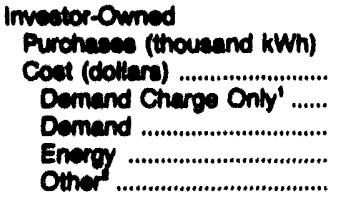 & $\bar{z}$ & $\begin{array}{r}27,638 \\
887,677 \\
-\quad \\
120,775 \\
768,102 \\
-\end{array}$ & $\begin{array}{r}1,280,180 \\
32,197,000 \\
- \\
1,160,800 \\
31,047,272 \\
137\end{array}$ & $\begin{array}{r}180,631 \\
4,130,762 \\
\overline{1,872,000} \\
2,268,762 \\
-\end{array}$ & $\begin{array}{r}1,468,681 \\
18,034,086 \\
- \\
3,086,734 \\
16,097,002 \\
-\end{array}$ & $\begin{array}{r}3,027,818 \\
112,877,016 \\
- \\
22,423,305 \\
90,454,650 \\
-\end{array}$ & $\begin{array}{c}4,298,039 \\
70,416,528 \\
- \\
- \\
70,416,528 \\
-\end{array}$ \\
\hline 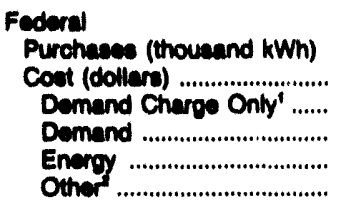 & $\bar{m} \overline{-}$ & $\begin{array}{l}\overline{-} \\
\bar{m}\end{array}$ & $\begin{array}{l}202,090 \\
3,021,422 \\
- \\
- \\
3,021,422\end{array}$ & $\begin{array}{l}\overline{-} \\
\bar{z} \\
\overline{-}\end{array}$ & 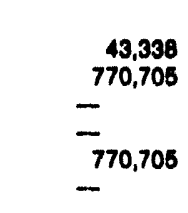 & $\bar{z}=$ & $\begin{array}{l}3,177,691 \\
52,005,480 \\
- \\
\overline{52,005,480} \\
-\end{array}$ \\
\hline 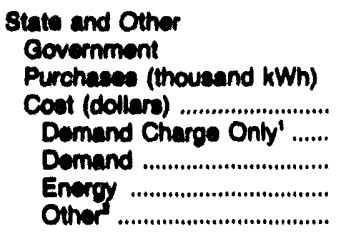 & 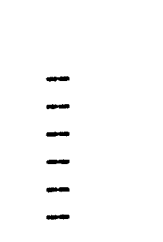 & $\bar{z}=$ & 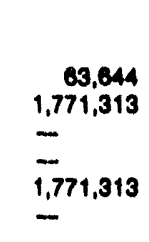 & $\begin{array}{l}- \\
\overline{-} \\
\bar{m}\end{array}$ & $\begin{array}{l}= \\
= \\
=\end{array}$ & $\bar{m}=$ & $\begin{array}{l}\overline{-} \\
\bar{m} \\
=\end{array}$ \\
\hline 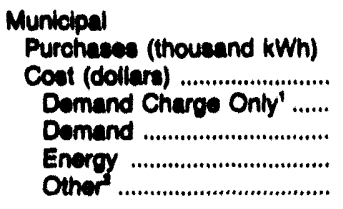 & $\bar{z}=$ & $\begin{array}{l}\overline{-} \\
\overline{-}\end{array}$ & $\begin{array}{r}54,072 \\
090,031 \\
- \\
- \\
1,293,403 \\
-293,472\end{array}$ & $\begin{array}{r}856 \\
-\quad 13,110 \\
-\quad 13,110\end{array}$ & $\begin{array}{r}2,008 \\
-\quad 32,052 \\
-\quad 32,052\end{array}$ & $=$ & $\bar{z}=$ \\
\hline 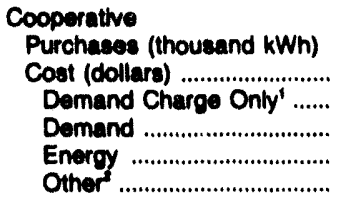 & 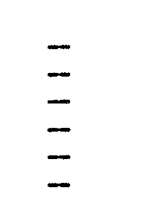 & $\bar{m}=$ & $\begin{array}{r}1,818 \\
-45,508 \\
-\quad 45,598\end{array}$ & $\begin{array}{l}\bar{z} \\
\bar{z} \\
\overline{-}\end{array}$ & $\begin{array}{r}6,858 \\
296,688 \\
-\quad \\
-\quad 296,698 \\
-\end{array}$ & $\begin{array}{l}- \\
\overline{-} \\
\overline{-}\end{array}$ & 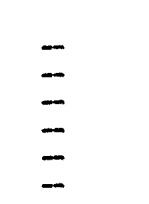 \\
\hline 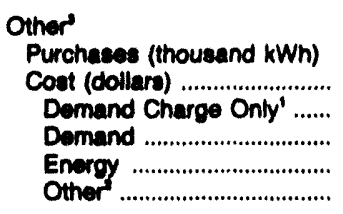 & $\begin{array}{l}120,684 \\
9,294,474 \\
- \\
2,062,637 \\
7,231,837 \\
-\end{array}$ & $=$ & $\begin{array}{r}574,110 \\
19,749,105 \\
- \\
5,071,449 \\
18,269,784 \\
-3,582,128\end{array}$ & $\begin{array}{r}9,859 \\
228,901 \\
-\quad \\
53,167 \\
175,734 \\
-\quad\end{array}$ & $\begin{array}{r}2,051 \\
-\quad 51,878 \\
-\quad 51,078\end{array}$ & $\begin{array}{l}137,210 \\
2,250,851 \\
- \\
- \\
\overline{2,250,951} \\
-\end{array}$ & 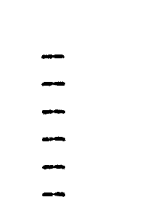 \\
\hline 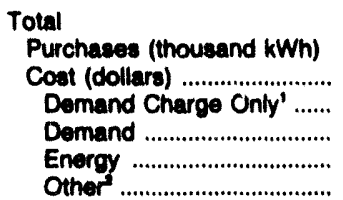 & $\begin{array}{r}120,684 \\
8,294,474 \\
- \\
2,062,637 \\
7,231,837 \\
-\end{array}$ & $\begin{array}{r}27,553 \\
887,877 \\
-\quad \\
129,775 \\
758,102 \\
\end{array}$ & $\begin{array}{r}2,155,629 \\
57,785,276 \\
- \\
6,221,949 \\
55,448,790 \\
-3,885,463\end{array}$ & $\begin{array}{r}191,246 \\
4,372,763 \\
- \\
1,925,167 \\
2,447,596 \\
-\end{array}$ & $\begin{array}{r}1,512,936 \\
21,086,129 \\
- \\
3,036,734 \\
18,049,395\end{array}$ & $\begin{array}{r}3,165,028 \\
115,128,866 \\
- \\
22,423,385 \\
92,705,501 \\
-\end{array}$ & $\begin{array}{r}7,475,630 \\
122,422,008 \\
- \\
- \\
122,422,008 \\
-\end{array}$ \\
\hline
\end{tabular}

See notes and footnotes at end of table. 
Table 19. Electricity Purchases by Investor-Owned Utilitios, by 8tato, 1992 (Continued)

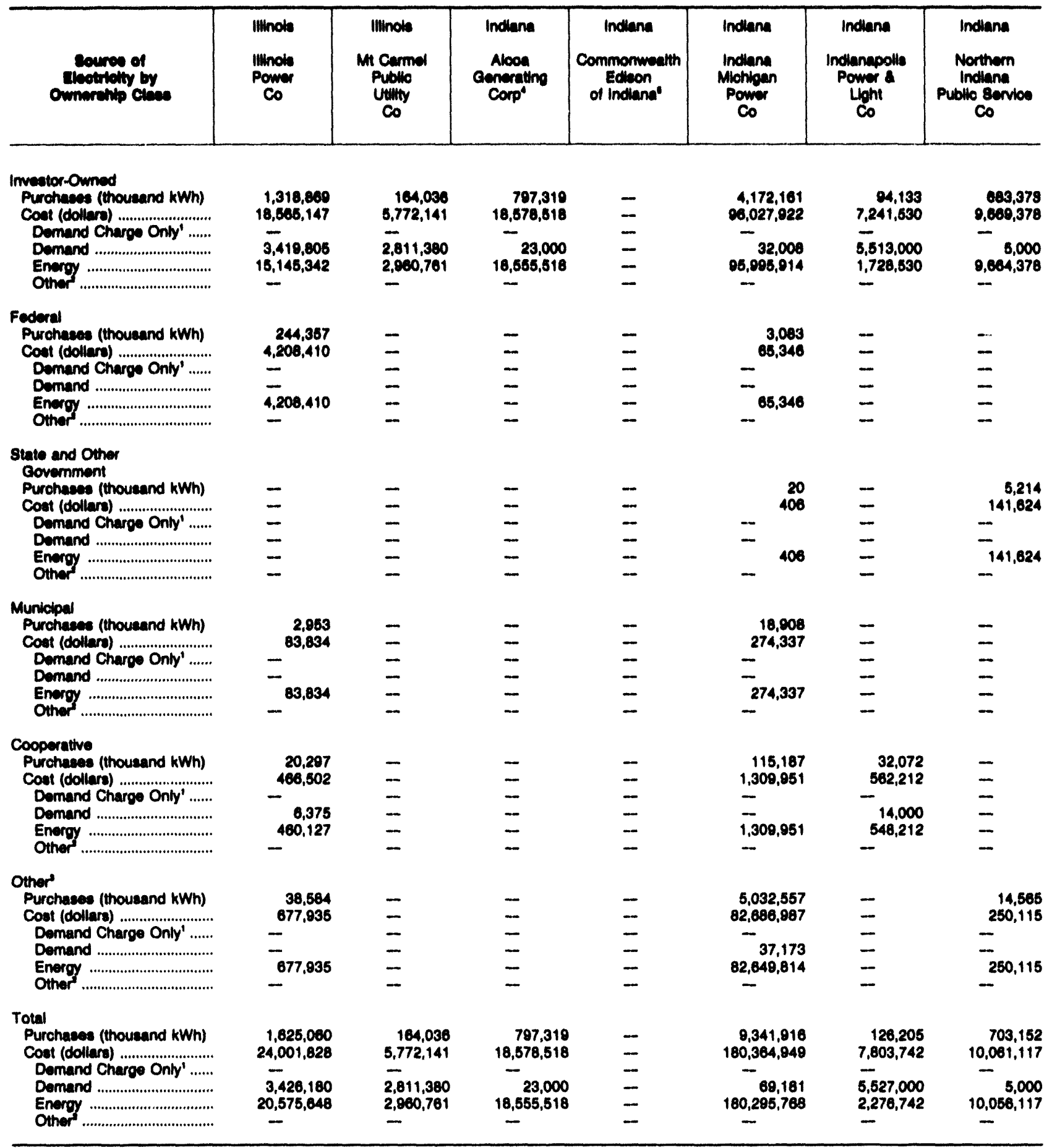

See notes and footnotes at end of table. 
Table 19. Electriclty Purchases by Inveator-Owned Utilitios, by 8tate, 1902 (Continued)

\begin{tabular}{|c|c|c|c|c|c|c|c|}
\hline $\begin{array}{l}\text { Coures of } \\
\text { Eveotrothy by } \\
\text { owmerehtip claces }\end{array}$ & $\begin{array}{l}\text { Indiana } \\
\text { P8I } \\
\text { Eneroy } \\
\text { Ino }\end{array}$ & $\begin{array}{l}\text { Indiana } \\
\text { Southem } \\
\text { indiana Gase } \\
\text { a Electric } \\
\text { Co }\end{array}$ & $\begin{array}{l}\text { lowa } \\
\text { Interntate } \\
\text { Power } \\
\text { Co }\end{array}$ & $\begin{array}{l}\text { lowa } \\
\text { lown } \\
\text { Eleotrlo } \\
\text { Loght } \\
\text { Power co }\end{array}$ & $\begin{array}{l}\text { lown } \\
\text { lown } \\
\text { Southern } \\
\text { Utilties } \\
\text { Co }\end{array}$ & $\begin{array}{l}\text { lown } \\
\text { lowa. } \\
\text { llinole } \\
\text { Gase } \\
\text { Electric } \\
\text { Co }\end{array}$ & $\begin{array}{l}\text { lown } \\
\text { Mlowed } \\
\text { Power } \\
\text { Syetems } \\
\text { ino }\end{array}$ \\
\hline 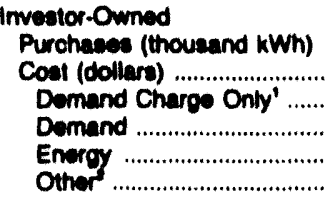 & $\begin{array}{r}851,058 \\
12,385,700 \\
\overline{1,052,609} \\
11,313,091 \\
--\end{array}$ & $\begin{array}{r}149,747 \\
2,176,128 \\
-\quad 17,393 \\
2,158,735 \\
-\end{array}$ & $\begin{array}{r}615,075 \\
17,808,307 \\
- \\
8,750,000 \\
8,410,427 \\
347,880\end{array}$ & $\begin{array}{l}784,700 \\
12,324,955 \\
\overline{-} \\
\overline{12,324,965} \\
-\end{array}$ & $\begin{array}{l}178,660 \\
2,302,126 \\
- \\
- \\
2,302,126\end{array}$ & $\begin{array}{l}54,732 \\
841,051 \\
- \\
- \\
-\end{array}$ & $\begin{array}{r}\$ 30,001 \\
2,446,070 \\
- \\
201,000 \\
2,244,076 \\
-\end{array}$ \\
\hline $\begin{array}{l}\text { Federal } \\
\text { Purchaces (thousand kWh) } \\
\text { Cost (dollars) ............ } \\
\text { Domend Charge Only' .... } \\
\text { Demand } \\
\text { Energy } \\
\text { Other }\end{array}$ & $\begin{array}{l}\overline{-} \\
\bar{m} \\
\bar{m}\end{array}$ & $\begin{array}{l}\bar{m} \\
\bar{m} \\
=\end{array}$ & $\begin{array}{r}20,436 \\
311,635 \\
- \\
- \\
-\end{array}$ & $\begin{array}{r}31,094 \\
486,862 \\
- \\
- \\
-466,602\end{array}$ & $\begin{array}{r}15,095 \\
217,560 \\
- \\
- \\
-\end{array}$ & $\begin{array}{l}\bar{z} \\
\bar{z}\end{array}$ & $\begin{array}{r}57,600 \\
804,516 \\
- \\
- \\
-\end{array}$ \\
\hline 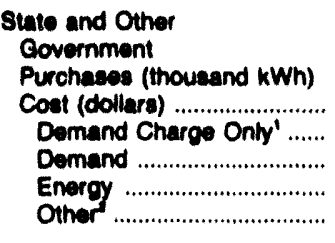 & $\begin{array}{r}-47,270 \\
-49,391 \\
-2,121 \\
-\end{array}$ & $\begin{array}{l}= \\
\overline{-} \\
=\end{array}$ & $\begin{array}{l}161,850 \\
2,320,385 \\
- \\
- \\
2,320,365\end{array}$ & $\begin{array}{l}89,522 \\
1,128,873 \\
- \\
- \\
1,128,873\end{array}$ & $\begin{array}{l}279,235 \\
3,350,686 \\
- \\
\overline{3,350,686} \\
=-\end{array}$ & $\begin{array}{r}3,107 \\
359,348 \\
-\quad \\
300,000 \\
59,348\end{array}$ & $\begin{array}{r}3,687,774 \\
101,286,167 \\
1,106,000 \\
7,000,000 \\
17,777,367 \\
75,402,780\end{array}$ \\
\hline 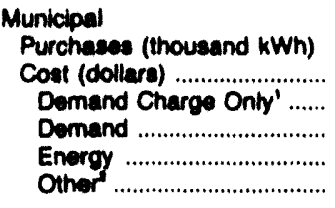 & $\begin{array}{l}\bar{z} \\
\bar{z} \\
\bar{z}\end{array}$ & $\begin{array}{l}- \\
\bar{z} \\
\overline{-}\end{array}$ & $\begin{array}{r}2,710 \\
67,524 \\
30,000 \\
-\quad 37,524\end{array}$ & $\begin{array}{r}531,680 \\
35,722,618 \\
- \\
22,195,741 \\
12,860,600 \\
657,278\end{array}$ & $\begin{array}{l}74,422 \\
1,619,973 \\
-- \\
\overline{1,619,973} \\
-\end{array}$ & 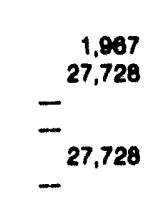 & $\begin{array}{r}92,931 \\
1,608,743 \\
180,000 \\
368,000 \\
1,141,621 \\
9,122\end{array}$ \\
\hline 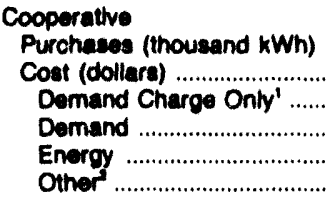 & $\begin{array}{r}73,217 \\
1,276,042 \\
-\quad \\
2,427 \\
1,273,615 \\
-\end{array}$ & $\begin{array}{r}44,046 \\
738,275 \\
-\quad \\
18,000 \\
720,275 \\
-\end{array}$ & $\begin{array}{r}1,098,346 \\
25,289,235 \\
-- \\
9,747,000 \\
15,085,605 \\
476,630\end{array}$ & $\begin{array}{r}627,670 \\
7,417,225 \\
15,303 \\
-\quad \\
7,401,742 \\
180\end{array}$ & $\begin{array}{l}341,203 \\
4,113,979 \\
- \\
\overline{4,113,979} \\
-\end{array}$ & $\begin{array}{r}40,630 \\
568,640 \\
- \\
-568,649 \\
-\end{array}$ & $\begin{array}{r}535,148 \\
7,327,721 \\
754,200 \\
65,586 \\
6,507,955 \\
-\quad\end{array}$ \\
\hline 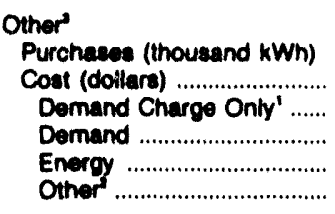 & $\begin{array}{ll}- & 2 \\
- & \\
- & 2\end{array}$ & $\begin{array}{l}\overline{-} \\
\bar{z} \\
\overline{-}\end{array}$ & $\begin{array}{l}\bar{z} \\
\bar{m}\end{array}$ & $\begin{array}{r}158,847 \\
3,553,456 \\
1,860,480 \\
-\quad \\
1,692,876 \\
-\end{array}$ & $\begin{array}{l}196,529 \\
1,862,324 \\
\overline{-} \\
\overline{1,862,324} \\
=\end{array}$ & $\begin{array}{l}170,634 \\
1,787,730 \\
- \\
\overline{1,797,730} \\
-\end{array}$ & $\begin{array}{r}848,628 \\
9,226,517 \\
5,000 \\
125,000 \\
4,582,250 \\
4,514,267\end{array}$ \\
\hline 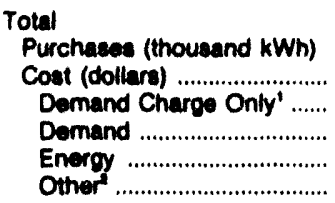 & $\begin{array}{r}926,175 \\
13,389,014 \\
- \\
1,104,427 \\
12,584,687 \\
-\end{array}$ & $\begin{array}{r}193,783 \\
2,914,403 \\
-\quad 35,393 \\
2,879,010 \\
-\end{array}$ & $\begin{array}{r}1,899,026 \\
45,497,066 \\
30,000 \\
18,497,000 \\
26,145,556 \\
824,510\end{array}$ & $\begin{array}{r}2,224,313 \\
60,634,990 \\
1,875,783 \\
22,195,741 \\
35,806,008 \\
657,468\end{array}$ & $\begin{array}{l}1,085,944 \\
13,466,648 \\
- \\
\overline{13,466,648} \\
-\end{array}$ & $\begin{array}{r}271,270 \\
3,594,506 \\
-\quad 300,000 \\
3,294,508 \\
-\end{array}$ & $\begin{array}{r}5,322,082 \\
122,888,730 \\
2,045,200 \\
7,758,568 \\
33,157,785 \\
79,926,179\end{array}$ \\
\hline
\end{tabular}

See noles and footnotes at end of table. 
Table 19. Electriclty Purchases by Inveator-Owned Utilities, by state, 1992 (Continued)

\begin{tabular}{|c|c|c|c|c|c|c|c|}
\hline 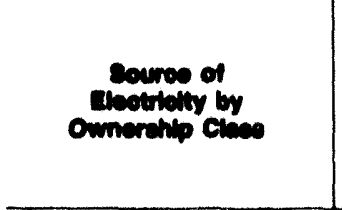 & $\begin{array}{l}\text { Kanaes } \\
\text { Kanaes } \\
\text { Gase } \\
\text { Eleotric } \\
\text { Co }\end{array}$ & $\begin{array}{l}\text { Kaneas } \\
\text { Weatern } \\
\text { Recources } \\
\text { Inc }\end{array}$ & $\begin{array}{l}\text { Kentucky } \\
\text { Kentucky } \\
\text { Power } \\
\text { Co }\end{array}$ & $\begin{array}{l}\text { Kentucky } \\
\text { Kentucky } \\
\text { Utillikes } \\
\text { Co }\end{array}$ & $\begin{array}{l}\text { Kentucky } \\
\text { Louloville } \\
\text { Cass a } \\
\text { Electric } \\
\text { Co }\end{array}$ & $\begin{array}{l}\text { Kentucky } \\
\text { Union } \\
\text { Light } \\
\text { Heat a } \\
\text { Power } \\
\text { Co }\end{array}$ & $\begin{array}{l}\text { Loulalane } \\
\text { Catalyat } \\
\text { Odd } \\
\text { Alver } \\
\text { Hydro" }\end{array}$ \\
\hline 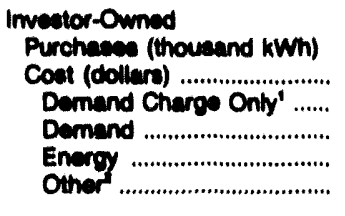 & $\begin{array}{l}628,328 \\
8,436,800 \\
- \\
\overline{8,438,800} \\
-\end{array}$ & $\begin{array}{l}183,205 \\
2,529,335 \\
- \\
2,529,335\end{array}$ & $\begin{array}{r}2,688,374 \\
73,689,025 \\
-\quad 24,713 \\
73,645,212 \\
-\end{array}$ & $\begin{array}{r}227,095 \\
5,660,382 \\
- \\
3,041,810 \\
2,602,800 \\
11,873\end{array}$ & $\begin{array}{r}277,965 \\
2,008,508 \\
-\quad 20 \\
576,264 \\
2,330,231\end{array}$ & $\begin{array}{r}2,935,181 \\
126,754,074 \\
-- \\
56,821,706 \\
78,585,644 \\
-8,633,276\end{array}$ & $\begin{array}{l}\bar{z} \\
\bar{z}\end{array}$ \\
\hline 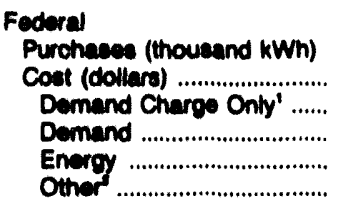 & $\bar{z}$ & $\begin{array}{l}\bar{z} \\
\bar{m}\end{array}$ & $\begin{array}{r}1,108 \\
-\quad 23,438 \\
-\quad 23,438\end{array}$ & $\begin{array}{r}\quad 6,389 \\
140,175 \\
- \\
-140,175\end{array}$ & $\begin{array}{r}435,622 \\
7,141,806 \\
- \\
157,404 \\
6,884,402 \\
-\end{array}$ & $\begin{array}{l}\bar{z} \\
\bar{z} \\
\overline{-}\end{array}$ & $\begin{array}{l}\overline{-} \\
\overline{-} \\
\overline{-}\end{array}$ \\
\hline 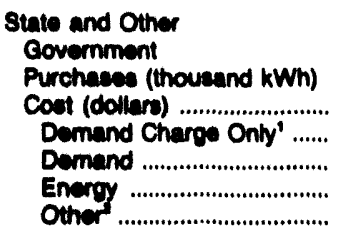 & $\begin{array}{r}\quad 3,286 \\
-\quad 41,002 \\
-\quad 41,002\end{array}$ & $\begin{array}{l}470,000 \\
5,202,370 \\
- \\
\overline{5,202,370}\end{array}$ & $\begin{array}{l}\overline{-} \\
\bar{z} \\
\overline{-}\end{array}$ & $\begin{array}{l}\overline{-} \\
\overline{-}\end{array}$ & 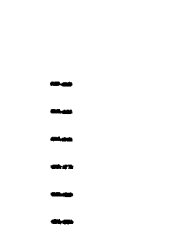 & $\begin{array}{l}\bar{z} \\
\bar{z}\end{array}$ & $\begin{array}{l}\bar{z} \\
\bar{m}\end{array}$ \\
\hline 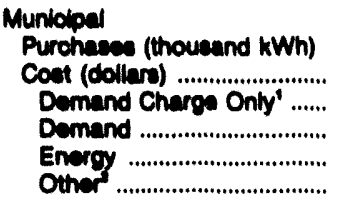 & 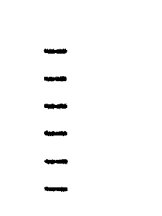 & $\begin{array}{r}10,220 \\
1,023,700 \\
-\quad 1,686,007 \\
327,803 \\
-\end{array}$ & $\begin{array}{r}8,086 \\
-\quad 97,801 \\
-\quad 07,801 \\
-\end{array}$ & $\begin{array}{r}1,768,610 \\
28,385,361 \\
\overline{4,482,800} \\
21,002,542 \\
-\end{array}$ & $\begin{array}{l}- \\
= \\
=\end{array}$ & $\begin{array}{l}\bar{z} \\
\bar{z}\end{array}$ & $\begin{array}{l}\bar{z} \\
\bar{z}\end{array}$ \\
\hline 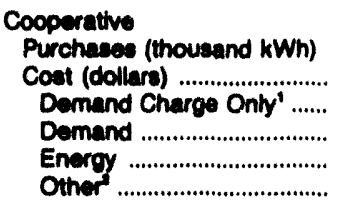 & $\begin{array}{l}16,590 \\
370,553 \\
- \\
- \\
-\end{array}$ & $\begin{array}{l}\bar{z} \\
\bar{m}\end{array}$ & $\begin{array}{r}8,216 \\
116,049 \\
-\quad \\
-\quad 116,049\end{array}$ & $\begin{array}{r}34,207 \\
571,483 \\
-\quad 24,000 \\
547,483 \\
-\end{array}$ & $\begin{array}{r}115,480 \\
1,993,637 \\
- \\
3991,129 \\
1,602,508 \\
-\end{array}$ & $\begin{array}{l}\bar{z} \\
\bar{z}\end{array}$ & $\begin{array}{l}\bar{z} \\
\bar{z}\end{array}$ \\
\hline 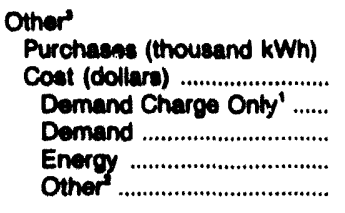 & $\begin{array}{r}27 \\
-\quad 619 \\
-\quad \\
-\quad 684 \\
-145\end{array}$ & $\begin{array}{r}10,066 \\
185,818 \\
-\quad \\
-\quad 187,173 \\
-1,354\end{array}$ & $\begin{array}{l}711,408 \\
17,880,043 \\
\overline{-} \\
\overline{17,880,043}\end{array}$ & 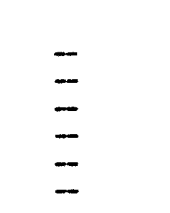 & $\begin{array}{l}-338 \\
\overline{-} \\
\overline{-}\end{array}$ & $\begin{array}{l}\bar{z} \\
\bar{z}\end{array}$ & $\begin{array}{l}\overline{-} \\
\bar{z} \\
\bar{z}\end{array}$ \\
\hline 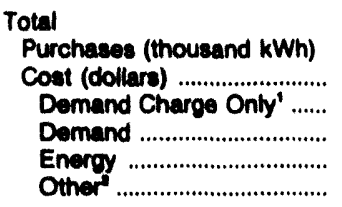 & $\begin{array}{r}545,230 \\
8,849,843 \\
- \\
- \\
8,849,888 \\
-145\end{array}$ & $\begin{array}{r}673,650 \\
9,861,314 \\
- \\
1,595,907 \\
8,256,761 \\
-1,354\end{array}$ & $\begin{array}{r}3,418,087 \\
91,787,256 \\
-\quad 24,713 \\
91,762,543 \\
-\end{array}$ & $\begin{array}{r}2,032,110 \\
32,753,391 \\
\overline{7,548,719} \\
25,192,799 \\
11,873\end{array}$ & $\begin{array}{r}828,729 \\
12,043,948 \\
-548,553 \\
9,165,164 \\
2,330,231\end{array}$ & $\begin{array}{r}2,935,181 \\
126,754,074 \\
- \\
56,821,706 \\
78,565,644 \\
-8,633,276\end{array}$ & $\begin{array}{l}\bar{z} \\
\bar{z}\end{array}$ \\
\hline
\end{tabular}

See notes and footnotes at end of table. 
Table 19. Electrictty Purchases by Inveetor-Ownod Utilities, by state, 1992 (Continued)

\begin{tabular}{|c|c|c|c|c|c|c|c|}
\hline 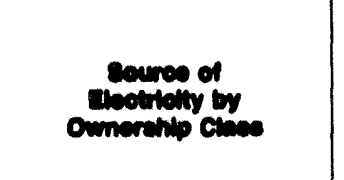 & $\begin{array}{l}\text { Louleinana } \\
\text { Central } \\
\text { Louidiana } \\
\text { Electric } \\
\text { Co Ino }\end{array}$ & $\begin{array}{l}\text { Louladana } \\
\text { Louldiana } \\
\text { Power } 8 \\
\text { Ught } \\
\text { Co }\end{array}$ & $\begin{array}{l}\text { Louldiana } \\
\text { New Orleans } \\
\text { Publlo } \\
\text { Servios } \\
\text { Inc }\end{array}$ & $\begin{array}{l}\text { Louluiana } \\
\text { Southweatern } \\
\text { Eloctric } \\
\text { Pown } \\
\text { Co }\end{array}$ & $\begin{array}{l}\text { Maine } \\
\text { Bangor } \\
\text { Hydro- } \\
\text { Electirlo } \\
\text { Co }\end{array}$ & $\begin{array}{l}\text { Maine } \\
\text { Contral } \\
\text { Moine } \\
\text { Power } \\
\text { Co }\end{array}$ & $\begin{array}{l}\text { Maine } \\
\text { Maine } \\
\text { Eleotric } \\
\text { Power } \\
\text { Co inc }\end{array}$ \\
\hline 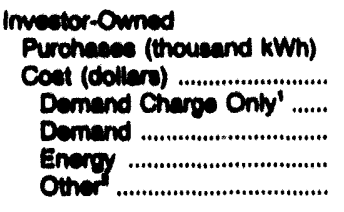 & $\begin{array}{l}14,207 \\
277,000 \\
- \\
- \\
-\end{array}$ & $\begin{array}{c}1,020,540 \\
101,277,303 \\
\overline{-} \\
7,716,416 \\
03,601,040\end{array}$ & $\begin{array}{c}1,260,166 \\
122,970,085 \\
\overline{-} \\
9,368,718 \\
113,010,037\end{array}$ & $\begin{array}{l}123,382 \\
2,002,671 \\
= \\
\overline{1,067,069} \\
44,012\end{array}$ & $\begin{array}{r}946,139 \\
27,262,562 \\
- \\
11,300,307 \\
18,762,166 \\
-\end{array}$ & $\begin{array}{l}2,637,200 \\
90,126,740 \\
\overline{-} \\
10,211,001 \\
70,016,130\end{array}$ & $\begin{array}{r}17,730 \\
249,380 \\
-\quad \\
-\quad 249,380 \\
-\end{array}$ \\
\hline 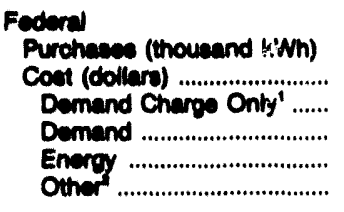 & $\bar{z}$ & 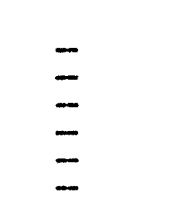 & 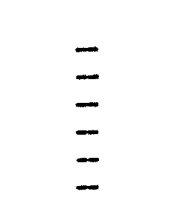 & $\bar{z}$ & 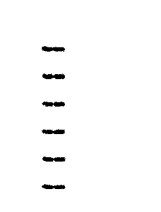 & $\begin{array}{l}\bar{z} \\
\bar{m}\end{array}$ & $\begin{array}{l}\bar{z} \\
\bar{z}\end{array}$ \\
\hline $\begin{array}{l}\text { State and Other } \\
\text { Covernment } \\
\text { Purcheses (thousand kWh) } \\
\text { Coet (dollerg) ..................... } \\
\text { Demand Charge Only' ...... } \\
\text { Demand . } \\
\text { Energy } \\
\text { Other }\end{array}$ & $\begin{array}{l}78,987 \\
1,822,725 \\
\overline{-} \\
\overline{1,822,725}\end{array}$ & $\begin{array}{r}78,687 \\
1,835,446 \\
- \\
- \\
1,718,280 \\
117,168\end{array}$ & $\begin{array}{l}\bar{z} \\
\bar{z}\end{array}$ & $\begin{array}{l}11,171 \\
101,729 \\
- \\
- \\
-\end{array}$ & $\begin{array}{l}\bar{z} \\
\bar{z}\end{array}$ & $\begin{array}{r}\quad 5,852 \\
421,837 \\
-\quad \\
-\quad 421,837\end{array}$ & $\begin{array}{l}\bar{z} \\
\bar{z}\end{array}$ \\
\hline 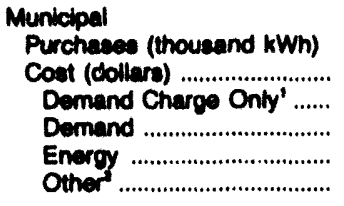 & $\begin{array}{l}\bar{z} \\
\bar{z}\end{array}$ & $\begin{array}{l}\bar{z} \\
\bar{z}\end{array}$ & $\begin{array}{l}\bar{z} \\
\bar{z}\end{array}$ & $\begin{array}{l}59,909 \\
= \\
= \\
=\end{array}$ & $\begin{array}{l}- \\
\overline{-} \\
-\end{array}$ & $\begin{array}{r}1,417 \\
34,760 \\
-\quad 34,760\end{array}$ & $\begin{array}{l}\bar{z} \\
\bar{z}\end{array}$ \\
\hline 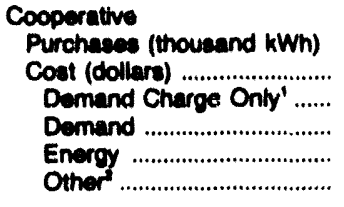 & $\begin{array}{r}59,800 \\
803,107 \\
-\quad \\
-\quad 003,107\end{array}$ & $\begin{array}{l}\overline{-} \\
\overline{-} \\
\bar{m}\end{array}$ & 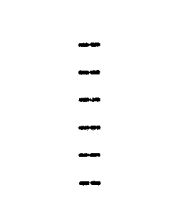 & $\begin{array}{r}1,186,295 \\
1,835,683 \\
-\quad \\
15,806 \\
1,819,777 \\
-\end{array}$ & $\begin{array}{l}\bar{z} \\
\bar{z}\end{array}$ & $\begin{array}{l}\bar{z} \\
\bar{z}\end{array}$ & $\begin{array}{l}\bar{z} \\
\bar{z}\end{array}$ \\
\hline 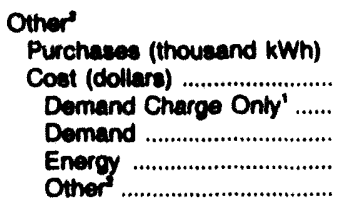 & $\begin{array}{l}370,480 \\
6,125,145 \\
- \\
- \\
6,643,572 \\
-518,427\end{array}$ & $\begin{array}{r}10,184,013 \\
232,637,144 \\
\overline{-} \\
232,637,144\end{array}$ & $\begin{array}{l}2,390,759 \\
47,723,032 \\
- \\
-\overline{47,723,032} \\
-\end{array}$ & $\begin{array}{l}142,008 \\
2,680,107 \\
- \\
- \\
2,680,107\end{array}$ & $\begin{array}{r}603,635 \\
72,062,159 \\
- \\
1,003,800 \\
71,058,359 \\
-\end{array}$ & $\begin{array}{l}4,835,356 \\
386,360,256 \\
\overline{-} \\
368,364,700 \\
-24,444\end{array}$ & $\begin{array}{r}323,881 \\
8,162,117 \\
\overline{-} \\
\overline{8,158,807} \\
3,310\end{array}$ \\
\hline 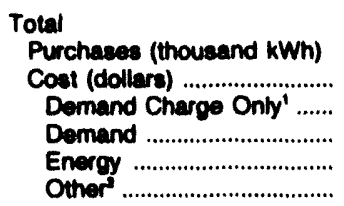 & $\begin{array}{l}523,644 \\
9,128,070 \\
-- \\
\overline{9,648,487} \\
-518,427\end{array}$ & $\begin{array}{r}11,292,746 \\
335,749,953 \\
\overline{2} \\
242,070,839 \\
93,678,114\end{array}$ & $\begin{array}{r}3,640,925 \\
170,702,687 \\
\overline{-} \\
57,091,750 \\
113,610,837\end{array}$ & $\begin{array}{r}1,522,825 \\
6,620,000 \\
-\quad 15,806 \\
6,558,272 \\
44,812\end{array}$ & $\begin{array}{l}1,549,774 \\
89,314,711 \\
- \\
12,504,197 \\
86,810,514 \\
-\end{array}$ & $\begin{array}{l}7,479,825 \\
465,943,593 \\
\overline{-} \\
386,052,898 \\
79,890,895\end{array}$ & $\begin{array}{r}341,711 \\
8,411,467 \\
- \\
-\quad \\
8,408,157 \\
3,310\end{array}$ \\
\hline
\end{tabular}

See notes and footnotes at end of table. 
Table 19. Electricity Purchases by Investor-Owned Utilitles, by State, 1992 (Continued)

\begin{tabular}{|c|c|c|c|c|c|c|c|}
\hline 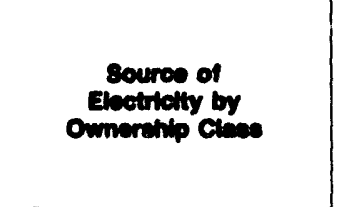 & $\begin{array}{l}\text { Maine } \\
\text { Maine } \\
\text { Public } \\
\text { Sorvice } \\
\text { Co }\end{array}$ & $\begin{array}{l}\text { Maine } \\
\text { Maine } \\
\text { Yankee } \\
\text { Alomic } \\
\text { Power } \\
\text { Co' }\end{array}$ & $\begin{array}{l}\text { Maryland } \\
\text { Allegheny } \\
\text { Generating } \\
\text { Co" }\end{array}$ & $\begin{array}{c}\text { Maryiand } \\
\text { Baltimore } \\
\text { Cass } \\
\text { Electric } \\
\text { Co }\end{array}$ & $\begin{array}{l}\text { Maryland } \\
\text { Conowingo } \\
\text { Power } \\
\text { Co }\end{array}$ & $\begin{array}{l}\text { Maryland } \\
\text { Potomac } \\
\text { Edison } \\
\text { Co }\end{array}$ & $\begin{array}{l}\text { Massachusetts } \\
\text { Boston } \\
\text { Edison } \\
\text { Co }\end{array}$ \\
\hline \multicolumn{8}{|l|}{ Investor-Omned } \\
\hline Purchases (thousand kWh) & 276,352 & - & - & $2,308,580$ & 748,967 & $6,860,569$ & $3,007,454$ \\
\hline 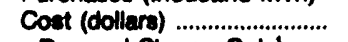 & $9,242,514$ & - & - & $81,686,416$ & $51,365,674$ & $200,986,408$ & $126,462,834$ \\
\hline Demand Charge Only' ....... & - & - & - & - & - & - & $-2,054,227$ \\
\hline 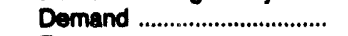 & - & - & - & $50,550,368$ & $39,731,604$ & $41,735,396$ & $83,940,807$ \\
\hline 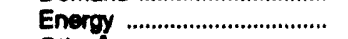 & $9,242,514$ & - & - & $21,568,271$ & $14,056,596$ & $100,890,641$ & $44,576,354$ \\
\hline Other & - & - & - & $19,567,777$ & $-2,422,526$ & $58,360,369$ & - \\
\hline \multicolumn{8}{|l|}{ Federal } \\
\hline Purchases (thousand kWh) & - & - & - & - & - & - & - \\
\hline 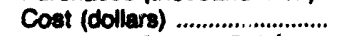 & - & - & - & - & - & - & - \\
\hline Demand Charge Only' ...... & - & - & - & - & - & - & -- \\
\hline Demand ................................. & - & -- & - & - & - & - & - \\
\hline 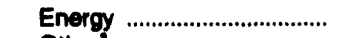 & - & - & - & - & - & - & - \\
\hline Other .............................. & - & - & - & - & - & - & - \\
\hline \multicolumn{8}{|l|}{$\begin{array}{l}\text { State and Other } \\
\text { Government }\end{array}$} \\
\hline Purchases (thousand kWh) & - & - & - & - & -- & - & 6,402 \\
\hline Cost (dollars) & - & - & - & - & - & - & 125,272 \\
\hline Demand Charge Only' ....... & - & - & - & - & - & - & - \\
\hline 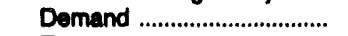 & - & - & - & - & - & - & - \\
\hline 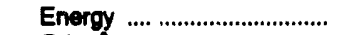 & - & - & - & - & - & - & 125,272 \\
\hline Other & - & - & - & - & - & - & - \\
\hline \multicolumn{8}{|l|}{ Municipal } \\
\hline Purchuses (thousand kWh) & - & - & - & - & - & - & - \\
\hline Cost (dollars) ..................... & - & - & - & - & - & - & - \\
\hline Demand Charge Only' ....... & - & - & -- & - & - & - & - \\
\hline 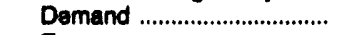 & - & - & - & - & - & -- & - \\
\hline 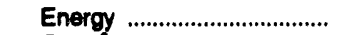 & - & - & - & - & - & - & - \\
\hline Other & - & - & - & - & - & - & - \\
\hline \multicolumn{8}{|l|}{ Cooperative } \\
\hline Purchases (thousand kWh) & - & - & -- & - & - & - & -- \\
\hline Cost (dollars) ........................... & - & - & -- & - & - & - & - \\
\hline Demand Charge Only' ....... & - & - & - & - & - & - & - \\
\hline 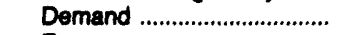 & - & - & - & - & - & - & -- \\
\hline 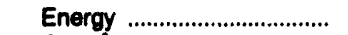 & - & - & - & - & - & - & - \\
\hline Other & - & - & - & - & - & - & - \\
\hline \multicolumn{8}{|l|}{ Other" } \\
\hline Purchases (thousand kWh) & 386,745 & - & - & 867,097 & 730 & 68,075 & $3,367,492$ \\
\hline Cost (dollars) ....... & $19,878,725$ & - & -- & $25,510,100$ & 37,439 & 37,075 & $204,918,679$ \\
\hline Demand Charge Only' ...... & - & - & - & - & - & - & $-1,046,256$ \\
\hline 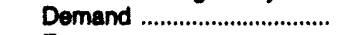 & - & - & - & $8,720,773$ & - & 213 & $80,829,825$ \\
\hline 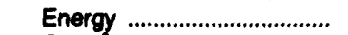 & $19,878,725$ & - & -- & $15,257,340$ & 37,439 & 36,862 & $145,135,110$ \\
\hline Other & - & - & - & $1,531,998$ & - & - & - \\
\hline \multicolumn{8}{|l|}{ Total } \\
\hline Purchases (thousand kWh) & 663,097 & - & - & $3,175,687$ & 749,697 & $6,828,644$ & $6,381,348$ \\
\hline 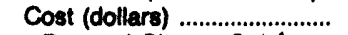 & $29,121,239$ & - & - & $117,196,525$ & $51,403,113$ & $201,023,481$ & $331,506,885$ \\
\hline Domand Charge Only' ....... & - & - & - & - & - & - & $-3,100,483$ \\
\hline 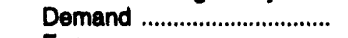 & - & - & - & $59,271,141$ & $39,731,604$ & $41,735,609$ & $144,770,632$ \\
\hline 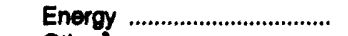 & $29,121,23 \varepsilon$ & $-\infty$ & - & $36,825,611$ & $14,094,035$ & $100,827,503$ & $189,836,736$ \\
\hline Other & - & - & - & $21,099,773$ & $-2,422,526$ & $58,360,369$ & - \\
\hline
\end{tabular}

See notes and footnotes at end of table. 
Table 19. Electricity Purchises by Investor-Owned Utilities, by State, 1992 (Continued)

\begin{tabular}{|c|c|c|c|c|c|c|c|}
\hline $\begin{array}{c}\text { Exurce of } \\
\text { Ebetrictly by } \\
\text { owneretip Claces }\end{array}$ & $\begin{array}{l}\text { Masasachusetts } \\
\text { Cambridge } \\
\text { Electric } \\
\text { Light } \\
\text { Co }\end{array}$ & $\begin{array}{c}\text { Messachusetts } \\
\text { Canal } \\
\text { Electric } \\
\text { Co }\end{array}$ & $\begin{array}{c}\text { Meseachusetts } \\
\text { Commonwealth } \\
\text { Electric } \\
\text { Co }\end{array}$ & $\begin{array}{c}\text { Messachusetts } \\
\text { Eastern } \\
\text { Edison } \\
\text { Co' }^{4}\end{array}$ & $\begin{array}{c}\text { Maseachusetts } \\
\text { Fhchburg } \\
\text { Gas \& } \\
\text { Electric } \\
\text { Lght } \\
\text { Co }\end{array}$ & $\begin{array}{c}\text { Massachusetts } \\
\text { Great } \\
\text { Bay } \\
\text { Power } \\
\text { Co' }\end{array}$ & $\begin{array}{l}\text { Massachusetts } \\
\text { Holyoke } \\
\text { Power \& } \\
\text { Electric } \\
\text { Co }\end{array}$ \\
\hline \multicolumn{8}{|l|}{ Imvestor-Owned } \\
\hline Purchases (thousand kWh) & $1,061,117$ & 311,843 & $2,782,277$ & $2,560,920$ & 154,584 & - & $1,049,515$ \\
\hline 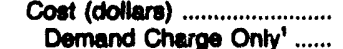 & $\begin{array}{r}52,680,029 \\
-434,751\end{array}$ & $12,157,606$ & $\begin{array}{r}143,840,071 \\
1,813,980\end{array}$ & $180,415,155$ & $5,440,456$ & $\overline{-}$ & $27,188,838$ \\
\hline Demand Charge Only ....... & $38,523,887$ & $8,847,522$ & $99,831,984$ & $139,404,554$ & $3,370,369$ & $\overline{-}$ & $\overline{-}$ \\
\hline 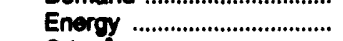 & $14,600,893$ & $3,387,084$ & $42,094,107$ & $41,010,601$ & $2,070,087$ & - & $18,468,563$ \\
\hline 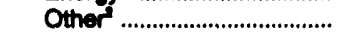 & - & $-77,000$ & - & - & - & - & $8,720,275$ \\
\hline \multicolumn{8}{|l|}{ Federal } \\
\hline Purchases (thousand kWh) & - & - & - & - & - & - & - \\
\hline 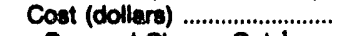 & - & - & - & - & -- & - & - \\
\hline Demand Charge Only' ...... & - & - & - & - & - & - & - \\
\hline 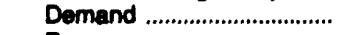 & - & - & - & - & - & - & - \\
\hline 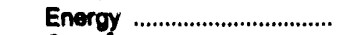 & - & - & - & - & - & - & - \\
\hline 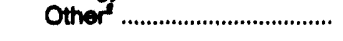 & - & - & - & - & - & - & - \\
\hline \multicolumn{8}{|l|}{ State and Other } \\
\hline $\begin{array}{l}\text { Government } \\
\text { Purchases (thousand kWh) }\end{array}$ & - & - & - & - & - & - & - \\
\hline Cost (dollars) & - & - & - & - & - & - & - \\
\hline Domand Charge Only' ...... & - & - & - & - & - & - & - \\
\hline 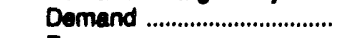 & - & - & - & - & - & - & -- \\
\hline 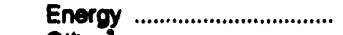 & - & - & - & - & - & - & - \\
\hline 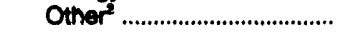 & - & - & - & - & - & - & - \\
\hline \multicolumn{8}{|l|}{ Municipal } \\
\hline Purchaees (thousand kWh) & - & - & - & 214 & - & - & - \\
\hline 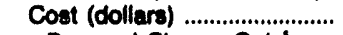 & - & - & - & 22,715 & - & - & - \\
\hline Demand Charge Only' ...... & - & - & - & - & - & - & - \\
\hline 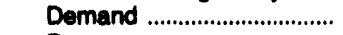 & - & - & - & 17,954 & - & - & - \\
\hline 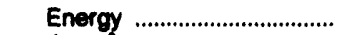 & - & - & - & 4,781 & -- & - & - \\
\hline 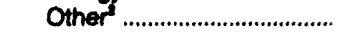 & - & - & - & - & - & - & - \\
\hline \multicolumn{8}{|l|}{ Cooperative } \\
\hline Purchases (thousand kWh) & - & - & - & - & - & - & - \\
\hline 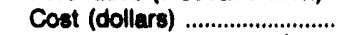 & - & - & - & - & - & -- & - \\
\hline Demand Charge Only' ....... & - & - & - & - & - & - & -- \\
\hline 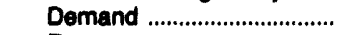 & - & - & -- & - & - & - & -- \\
\hline Energy ......................................... & - & - & - & - & - & - & - \\
\hline Other & - & -- & - & - & - & - & - \\
\hline \multicolumn{8}{|l|}{ Other" } \\
\hline Purchases (thousend kWh) & 429,271 & $149,01 ?$ & $1,660,403$ & 214 & 167,062 & - & - \\
\hline 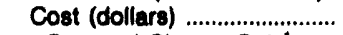 & $11,441,48 \theta$ & $14,354,535$ & $109,890,549$ & $5,663,850$ & $10,362,196$ & - & - \\
\hline Demand Charge Only ${ }^{*} . . . .$. & - & - & - & - & - & -- & - \\
\hline Demand & 165,328 & $11,351,473$ & $35,712,316$ & - & $7,017,007$ & - & - \\
\hline 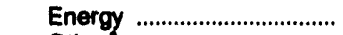 & $12,060,344$ & $2,888,804$ & $74,849,567$ & 4,183 & $3,345,189$ & - & - \\
\hline 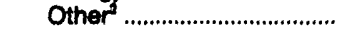 & $-784,173$ & 4,258 & $-571,334$ & $5,659,676$ & - & - & - \\
\hline \multicolumn{8}{|l|}{ Total } \\
\hline Purchases (thousand kWh) & $1,490,388$ & 460,855 & $4,422,680$ & $2,561,348$ & 322,546 & - & $1,049,515$ \\
\hline 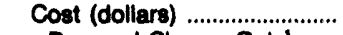 & $64,131,528$ & $26,512,141$ & $253,830,620$ & $186,101,729$ & $15,802,652$ & - & $27,188,838$ \\
\hline Demand Charge Only' ....... & $-434,751$ & - & $1,813,880$ & - & - & -. & - \\
\hline 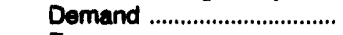 & $38,689,215$ & $20,198,995$ & $135,644,300$ & $139,422,508$ & $10,387,376$ & - & - \\
\hline 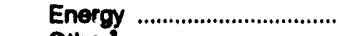 & $26,661,237$ & $6,385,688$ & $116,843,674$ & $41,018,545$ & $5,415,276$ & - & $18,468,563$ \\
\hline Other & $-784,173$ & $-72,742$ & $-571,334$ & $5,659,676$ & - & - & $8,720,275$ \\
\hline
\end{tabular}

See notes and footnotes at end of table. 
Table 19. Electricity Purchases by Investor-Owned Utilities, by State, 1992 (Continued)

\begin{tabular}{|c|c|c|c|c|c|c|c|}
\hline $\begin{array}{c}\text { soures of } \\
\text { Eicetrithy by } \\
\text { Owmerehlp cleces }\end{array}$ & $\begin{array}{l}\text { Mesaechuselts } \\
\text { Holyoke } \\
\text { Water } \\
\text { Power } \\
\text { Co }\end{array}$ & $\begin{array}{l}\text { Maseachusetts } \\
\text { Massechusetts } \\
\text { Electric } \\
\text { Co }\end{array}$ & $\begin{array}{l}\text { Masaschusetts } \\
\text { Montaup } \\
\text { Electrlc } \\
\text { Co }\end{array}$ & $\begin{array}{l}\text { Maseachusetts } \\
\text { New } \\
\text { England } \\
\text { Hydro } \\
\text { Trans Elec } \\
\text { Co' }\end{array}$ & $\begin{array}{l}\text { Masuachusetts } \\
\text { New } \\
\text { England } \\
\text { Power } \\
\text { Co }\end{array}$ & $\begin{array}{l}\text { Masaschusetts } \\
\text { Western } \\
\text { Masaschusetts } \\
\text { Electric } \\
\text { Co }\end{array}$ & $\begin{array}{l}\text { Maseachusetts } \\
\text { Yankee } \\
\text { Atomic } \\
\text { Electric } \\
\text { Co }^{\circ}\end{array}$ \\
\hline 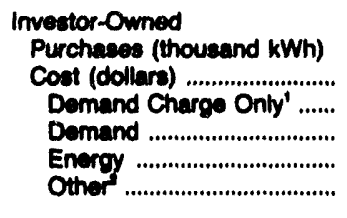 & $\begin{array}{c}658,117 \\
16,562,636 \\
\overline{-} \\
10,792,083 \\
5,760,553\end{array}$ & $\begin{array}{c}16,016,696 \\
1,085,115,888 \\
- \\
543,073,080 \\
349,860,658 \\
172,082,150\end{array}$ & $\begin{array}{r}2,720,395 \\
130,101,836 \\
-\overline{101,224,547} \\
28,807,389 \\
-\end{array}$ & $\begin{array}{l}\overline{-} \\
\bar{z}\end{array}$ & $\begin{array}{r}3,534,818 \\
159,848,672 \\
-\overline{4,863,272} \\
38,069,562 \\
116,815,838\end{array}$ & $\begin{array}{l}1,245,808 \\
51,142,313 \\
\overline{-} \\
20,108,362 \\
31,033,951\end{array}$ & $\begin{array}{l}= \\
= \\
=\end{array}$ \\
\hline 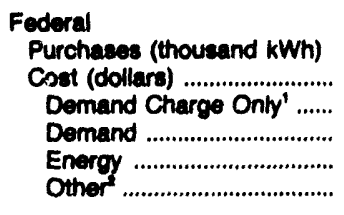 & $\begin{array}{l}\bar{z} \\
\bar{z}\end{array}$ & $\begin{array}{l}\bar{z} \\
\bar{z}\end{array}$ & $\begin{array}{l}\bar{z} \\
\bar{z} \\
\overline{-}\end{array}$ & $\begin{array}{l}\overline{-} \\
\bar{m} \\
\bar{m}\end{array}$ & $\begin{array}{l}- \\
\bar{m} \\
\bar{m}\end{array}$ & $\begin{array}{l}\bar{z} \\
\bar{z} \\
\bar{z}\end{array}$ & $\begin{array}{l}\bar{z} \\
\bar{m} \\
\bar{m}\end{array}$ \\
\hline 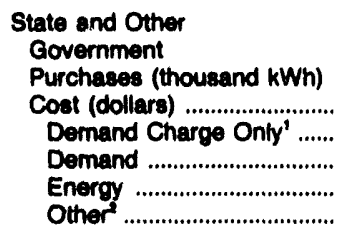 & $\begin{array}{l}\overline{-} \\
\bar{z} \\
\bar{z}\end{array}$ & $\begin{array}{l}\bar{z} \\
\bar{z} \\
\overline{-}\end{array}$ & $\begin{array}{r}2,569 \\
885,517 \\
-\quad \\
860,290 \\
125,227 \\
-\end{array}$ & $\begin{array}{l}\bar{z} \\
\bar{z}\end{array}$ & $\begin{array}{l}19,916 \\
451,945 \\
- \\
- \\
- \\
-\end{array}$ & $\begin{array}{l}\bar{z} \\
\bar{z}\end{array}$ & $\begin{array}{l}\bar{z} \\
\bar{z}\end{array}$ \\
\hline 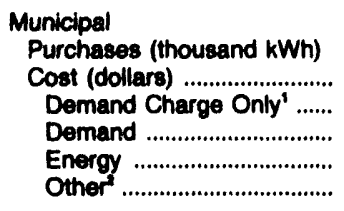 & $\begin{array}{l}\bar{z} \\
\bar{z}\end{array}$ & $\begin{array}{r}321 \\
-\quad 31,282 \\
-\quad 31,282\end{array}$ & $\begin{array}{r}38,333 \\
2,045,764 \\
- \\
888,000 \\
1,157,764 \\
-\end{array}$ & $\begin{array}{l}\bar{z} \\
\bar{z}\end{array}$ & $\begin{array}{l}\bar{z} \\
\bar{z}\end{array}$ & $\begin{array}{r}149 \\
-\quad 4,233 \\
-\quad 4,233\end{array}$ & $\begin{array}{l}\overline{-} \\
\bar{z} \\
\bar{m}\end{array}$ \\
\hline 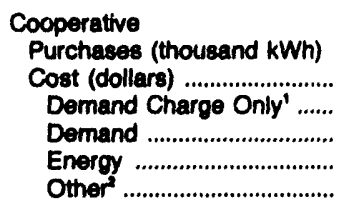 & $\begin{array}{l}\bar{z} \\
\bar{z}\end{array}$ & $\begin{array}{l}- \\
\bar{z} \\
\overline{-}\end{array}$ & $\begin{array}{l}\bar{z} \\
\bar{z} \\
\bar{z}\end{array}$ & $\begin{array}{l}\bar{m} \\
\bar{m} \\
\bar{m}\end{array}$ & $\begin{array}{l}- \\
\overline{-} \\
\overline{-}\end{array}$ & $\begin{array}{l}\overline{-} \\
\overline{-} \\
\overline{-}\end{array}$ & $\begin{array}{l}\bar{z} \\
\bar{z} \\
\bar{z}\end{array}$ \\
\hline 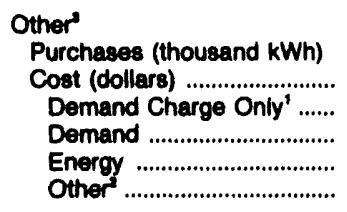 & $\begin{array}{r}52,888 \\
804,569 \\
- \\
- \\
1,009,756 \\
-205,187\end{array}$ & $\begin{array}{r}1,987 \\
-74,733 \\
-\quad 74,733 \\
-\end{array}$ & $\begin{array}{r}360,009 \\
29,860,637 \\
6,509,700 \\
15,536,879 \\
7,814,058 \\
-\end{array}$ & $\begin{array}{l}\overline{-} \\
\bar{z} \\
\bar{m}\end{array}$ & $\begin{array}{r}5,845,606 \\
347,053,817 \\
-\quad \\
45,629,069 \\
269,511,589 \\
31,813,159\end{array}$ & $\begin{array}{r}799,064 \\
16,546,291 \\
- \\
- \\
29,652,132 \\
-13,105,841\end{array}$ & $\begin{array}{l}\bar{z} \\
\bar{z} \\
\bar{z}\end{array}$ \\
\hline 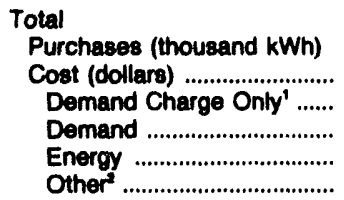 & $\begin{array}{r}711,005 \\
17,357,205 \\
- \\
\overline{11,801,839} \\
5,555,366\end{array}$ & $\begin{array}{r}16,019,004 \\
1,065,221,003 \\
-\overline{543,073,080} \\
350,066,673 \\
172,082,150\end{array}$ & $\begin{array}{r}3,121,306 \\
162,993,854 \\
6,509,700 \\
118,579,716 \\
37,904,438 \\
-\end{array}$ & $\begin{array}{l}\overline{-} \\
\bar{z} \\
\bar{z}\end{array}$ & $\begin{array}{r}9,500,340 \\
507,354,434 \\
- \\
50,592,341 \\
308,033,096 \\
148,728,997\end{array}$ & $\begin{array}{r}2,045,021 \\
67,692,837 \\
- \\
\overline{-} \\
4 \overline{9,764,727} \\
17,928,110\end{array}$ & $\begin{array}{l}\bar{z} \\
\bar{z}\end{array}$ \\
\hline
\end{tabular}

See notes and footnotes at end of table. 
Table 19. Electricity Purchases by Investor-Owned Utilities, by State, 1992 (Continued)

\begin{tabular}{|c|c|c|c|c|c|c|c|}
\hline $\begin{array}{l}\text { source of } \\
\text { Evectrietty by } \\
\text { owmerehip claces }\end{array}$ & $\begin{array}{l}\text { Michloan } \\
\text { Consumers } \\
\text { Power } \\
\text { Co }\end{array}$ & $\begin{array}{l}\text { Michigan } \\
\text { Detrolt } \\
\text { Edison } \\
\text { Co }\end{array}$ & $\begin{array}{l}\text { Michigan } \\
\text { Edicon } \\
\text { Sault } \\
\text { Electric } \\
\text { Co }\end{array}$ & $\begin{array}{l}\text { Michigan } \\
\text { Upper } \\
\text { Penineula } \\
\text { Power } \\
\text { Co }\end{array}$ & $\begin{array}{l}\text { Michigan } \\
\text { Wolverine } \\
\text { Hydroelectric } \\
\text { Corp" }\end{array}$ & $\begin{array}{l}\text { Minnesota } \\
\text { Minnesota } \\
\text { Power \& } \\
\text { Light } \\
\text { Co }\end{array}$ & $\begin{array}{l}\text { Minnesota } \\
\text { Northern } \\
\text { States } \\
\text { Power } \\
\text { Co }\end{array}$ \\
\hline \multicolumn{8}{|l|}{ Imeetor-Owned } \\
\hline 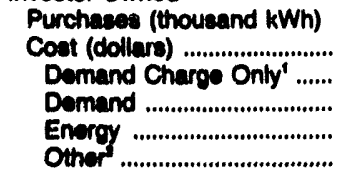 & $\begin{array}{r}3,694,258 \\
64,016,630 \\
-\quad 147,473 \\
67,879,326 \\
-10,169\end{array}$ & $\begin{array}{r}1,056,174 \\
21,589,138 \\
\overline{2,080,936} \\
19,488,200 \\
-\end{array}$ & $\begin{array}{r}337,851 \\
12,849,713 \\
4,828,305 \\
7,503,313 \\
718,095\end{array}$ & $\begin{array}{r}567,661 \\
18,732,384 \\
\overline{5,519,618} \\
11,127,176 \\
85,601\end{array}$ & $\begin{array}{l}\bar{m} \\
\bar{m}\end{array}$ & $\begin{array}{r}238,693 \\
4,543,113 \\
- \\
446,626 \\
4,096,487 \\
-\end{array}$ & $\begin{array}{r}571,509 \\
14,641,256 \\
- \\
4,271,810 \\
10,369,446 \\
-\end{array}$ \\
\hline \multicolumn{8}{|l|}{ Federal } \\
\hline $\begin{array}{l}\text { Purchases (thousand kWh) } \\
\text { Coot (dollars) }\end{array}$ & - & - & 155,362 & - & - & 71,077 & $\begin{array}{r}111,701 \\
1807,198\end{array}$ \\
\hline Demand Charge Onity'....... & $\overline{-}$ & $\overline{-}$ & $\underline{1,81}$ & $\overline{-}$ & $\overline{-}$ & $1,114,088$ & $1,807,198$ \\
\hline Demand ................................ & - & - & -- & - & - & - & - \\
\hline 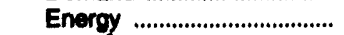 & - & - & $1,914,014$ & - & - & $1,114,080$ & $1,807,198$ \\
\hline 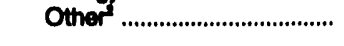 & - & - & - & - & - & $\sim$ & - \\
\hline \multicolumn{8}{|l|}{$\begin{array}{l}\text { State and Other } \\
\text { Government }\end{array}$} \\
\hline $\begin{array}{l}\text { Purchases (thousand kWh) } \\
\text { Cost (dollars) }\end{array}$ & $\begin{array}{r}107,569 \\
7,194,151\end{array}$ & $\begin{array}{r}1,267,445 \\
69,291,511\end{array}$ & $\overline{-}$ & $\overline{-}$ & $\overline{-}$ & $\begin{array}{r}571,018 \\
8,689,276\end{array}$ & $\begin{array}{r}857,028 \\
22,197,839\end{array}$ \\
\hline Demand Charge Only' ${ }^{\prime} . . . . .$. & & - & - & - & - & - & $20,100,000$ \\
\hline Demand ................................ & $5,009,023$ & - & - & - & - & $3,039,752$ & $\begin{array}{r}8,697,487 \\
\end{array}$ \\
\hline 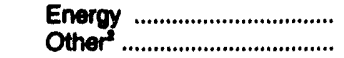 & $\begin{array}{r}2,209,308 \\
-24,180\end{array}$ & $\begin{array}{l}18,381,232 \\
50,810,27 \theta\end{array}$ & $\overline{-}$ & $\overline{-}$ & $\overline{-}$ & $5,649,524$ & ${ }^{13,500,352}$ \\
\hline \multicolumn{8}{|l|}{ Municipal } \\
\hline $\begin{array}{l}\text { Purchases (thousand kWh) } \\
\text { Cost (dollars) }\end{array}$ & $\begin{array}{r}527,585 \\
28,711,057\end{array}$ & $\begin{array}{l}2,115 \\
1,108\end{array}$ & - & $\begin{array}{r}868 \\
2,190032\end{array}$ & $\overline{-}$ & $\begin{array}{r}10,830 \\
290,507\end{array}$ & $\begin{array}{r}307,045 \\
5,281,036\end{array}$ \\
\hline Demand Charge Only' ...... & $20,111,001$ & $-1,108$ & $\overline{-}$ & $2,180,032$ & $=$ & 22,950 & - \\
\hline 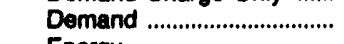 & $17,594,606$ & - & - & $2,154,904$ & - & - & 790,459 \\
\hline 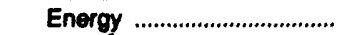 & $11,140,451$ & 1,108 & - & 35,128 & - & 267,557 & $4,490,577$ \\
\hline 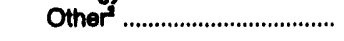 & $-24,000$ & - & - & - & - & - & - \\
\hline \multicolumn{8}{|l|}{ Cooperative } \\
\hline $\begin{array}{l}\text { Purchases (thousand kWh) } \\
\text { Cost (dollers) }\end{array}$ & - & - & 205 & - & - & $2,903,501$ & $1,953,964$ \\
\hline 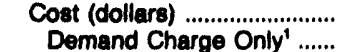 & $\overline{-}$ & $\overline{-}$ & ${ }_{-}^{7,445}$ & $\overline{-}$ & $\overline{-}$ & $61,781,4$ & 45,122, \\
\hline 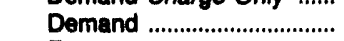 & - & - & - & - & - & $39,867,044$ & $19,267,093$ \\
\hline Energy ................................. & - & - & 7,445 & -- & - & $21,914,407$ & $25,855,866$ \\
\hline Other & - & - & - & - & - & - & - \\
\hline \multicolumn{8}{|l|}{ Others } \\
\hline $\begin{array}{l}\text { Purchases (thousand kWh) } \\
\text { Cost (dollarg) }\end{array}$ & $\begin{array}{r}7,449,699 \\
472,029,700\end{array}$ & $\begin{array}{r}380,155 \\
13,939,964\end{array}$ & $=$ & $\overline{-}$ & $\overline{-}$ & $\begin{array}{r}699,686 \\
9,008,500\end{array}$ & $\begin{array}{r}4,466,889 \\
65,825,180\end{array}$ \\
\hline Demand Charge Only' ....... & $-71,020,100$ & - & - & - & - & - & - \\
\hline Demand & $318,394,496$ & - & - & - & - & $1,220,420$ & $11,918,494$ \\
\hline 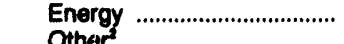 & $154,201,109$ & $11,547,026$ & - & - & - & $7,788,080$ & $53,806,686$ \\
\hline 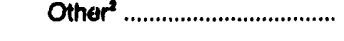 & $-565,805$ & $2,392,938$ & - & - & - & - & - \\
\hline \multicolumn{8}{|l|}{ Total } \\
\hline Purchases (thousand kWh) & $11,779,111$ & $2,704,889$ & 493,418 & 558,517 & -- & $4,494,805$ & $8,368,236$ \\
\hline $\begin{array}{l}\text { Cost (dollars) ............................. } \\
\text { Demand Charge Ony ..... }\end{array}$ & $571,951,538$ & $104,821,719$ & $14,771,172$ & $18,822,426$ & $\bar{z}$ & $\begin{array}{r}85,426,935 \\
22,950\end{array}$ & $154,875,468$ \\
\hline 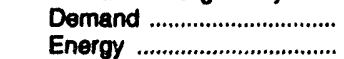 & $\begin{array}{l}347,145,598 \\
225,430,194\end{array}$ & $\begin{array}{r}2,090,936 \\
48,427,566\end{array}$ & $\begin{array}{l}4,628,305 \\
9,424,772\end{array}$ & $\begin{array}{r}7,674,522 \\
11,162,303\end{array}$ & $\overline{-}$ & $\begin{array}{l}44,573,842 \\
40,830,143\end{array}$ & $\begin{array}{r}44,945,343 \\
109,930,125\end{array}$ \\
\hline Other $r^{2}$ & $-624,254$ & $53,303,217$ & 718,095 & 85,601 & - & - & -- \\
\hline
\end{tabular}

See notes and footnotes at end of table. 
Table 19. Eloctricity Purchases by Investor-Owned Utilitles, by State, 1992 (Continued)

\begin{tabular}{|c|c|c|c|c|c|c|c|}
\hline $\begin{array}{l}\text { Eouros of } \\
\text { Electrictly by } \\
\text { owmerchlp clese }\end{array}$ & $\begin{array}{c}\text { Minnesota } \\
\text { Otter } \\
\text { Tall } \\
\text { Power } \\
\text { Co }\end{array}$ & $\begin{array}{l}\text { Misoledippi } \\
\text { Miseledppl } \\
\text { Power \& } \\
\text { Light } \\
\text { Co }\end{array}$ & $\begin{array}{l}\text { Miselseippi } \\
\text { Miselseippi } \\
\text { Power } \\
\text { Co }\end{array}$ & $\begin{array}{l}\text { Mississippi } \\
\text { Systems } \\
\text { Energy } \\
\text { Resources } \\
\text { Inc" }\end{array}$ & $\begin{array}{l}\text { Missouri } \\
\text { Empire } \\
\text { Diatrict } \\
\text { Electric } \\
\text { Co }\end{array}$ & $\begin{array}{l}\text { Miseourl } \\
\text { Kansas Clty } \\
\text { Power } 8 \\
\text { Light } \\
\text { Co }\end{array}$ & $\begin{array}{l}\text { Miseourt } \\
\text { St Joseph } \\
\text { Light \& } \\
\text { Power } \\
\text { Co }\end{array}$ \\
\hline \multicolumn{8}{|l|}{ Investor-Owned } \\
\hline $\begin{array}{l}\text { Purchases (thousand kWh) } \\
\text { Cost (dollare) }\end{array}$ & $\begin{array}{r}31,836 \\
444,750\end{array}$ & $\begin{array}{r}3,071,014 \\
287,053,177\end{array}$ & $\begin{array}{r}69,882 \\
857,465\end{array}$ & - & $\begin{array}{r}468,390 \\
7,647,421\end{array}$ & $\begin{array}{r}573,283 \\
11,214,336\end{array}$ & $\begin{array}{r}267,896 \\
4,891,586\end{array}$ \\
\hline Demand Charge Only' ...... & - & - & - & - & - & - & - \\
\hline 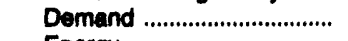 & - & $237,408,928$ & - & - & 595,000 & - & $1,263,118$ \\
\hline 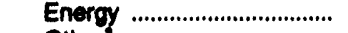 & 444,750 & $49,646,249$ & 857,465 & - & $7,052,421$ & $9,285,336$ & $3,628,467$ \\
\hline Other & - & - & - & - & - & $1,929,000$ & - \\
\hline \multicolumn{8}{|l|}{ Federal } \\
\hline Purchases (thousand kWh) & - & 114,539 & $16,46 i$ & - & 6,585 & - & - \\
\hline 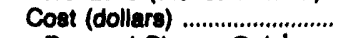 & - & $2,088,555$ & 329,336 & - & 34,242 & - & - \\
\hline Demand Charge Only' ...... & - & - & - & - & - & - & - \\
\hline Demand ................................ & - & - & - & - & - & - & - \\
\hline 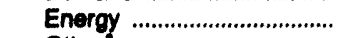 & - & $2,088,555$ & 329,336 & - & 34,242 & - & - \\
\hline 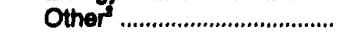 & - & - & - & - & - & - & - \\
\hline \multicolumn{8}{|l|}{$\begin{array}{l}\text { State and Other } \\
\text { Government }\end{array}$} \\
\hline $\begin{array}{l}\text { Purchases (thousand kWh) } \\
\text { Cost (dollars) }\end{array}$ & $\begin{array}{r}388,923 \\
5,262,502\end{array}$ & - & $\begin{array}{r}409 \\
8,352\end{array}$ & - & $\begin{array}{r}168,768 \\
2,575,380\end{array}$ & $\begin{array}{r}95,203 \\
1,292,892\end{array}$ & $\begin{array}{r}206,782 \\
2,794,446\end{array}$ \\
\hline Demand Charge Only" ...... & - & - & - & - & - & $-\infty$ & - \\
\hline Demand ................................ & - & - & - & - & 338,688 & - & - \\
\hline 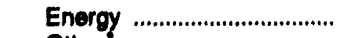 & $5,262,502$ & - & 6,362 & - & $2,236,672$ & $1,292,892$ & $2,794,446$ \\
\hline 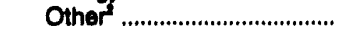 & - & - & - & - & - & - & - \\
\hline \multicolumn{8}{|l|}{ Municipal } \\
\hline $\begin{array}{l}\text { Purchases (thousand kWh) } \\
\text { Cost (dollars) }\end{array}$ & $\begin{array}{r}94,120 \\
1,405,826\end{array}$ & - & $\begin{array}{r}19 \\
556\end{array}$ & - & $\begin{array}{r}144,801 \\
1,954,755\end{array}$ & $\begin{array}{r}31,764 \\
1,982,588\end{array}$ & $\begin{array}{r}1,895 \\
28,157\end{array}$ \\
\hline Demand Charge Only' ....... & - & - & $-\infty$ & - & - & - & $-\infty$ \\
\hline Demand ................................ & - & - & - & -- & $1,315,440$ & 40,631 & - \\
\hline 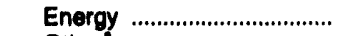 & $1,405,826$ & - & 556 & - & 639,315 & 593,957 & 28,157 \\
\hline Other & - & - & - & - & - & $1,348,000$ & - \\
\hline \multicolumn{8}{|l|}{ Cooperative } \\
\hline $\begin{array}{l}\text { Purchases (thousand kWh) } \\
\text { Cost (dollars) }\end{array}$ & $\begin{array}{r}286,836 \\
6,015,386\end{array}$ & $\begin{array}{r}465,612 \\
7,672,778\end{array}$ & $\begin{array}{r}11,580 \\
141,423\end{array}$ & -- & $\begin{array}{r}309,821 \\
9,792,447\end{array}$ & $\begin{array}{r}221,715 \\
7,345,318\end{array}$ & $\begin{array}{r}13,553 \\
249,183\end{array}$ \\
\hline Demand Charge Only' ....... & - & - & - & - & - & - & - \\
\hline 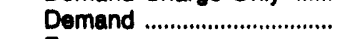 & $1,187,170$ & - & - & - & $4,530,240$ & - & - \\
\hline 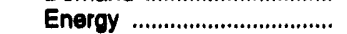 & $4,828,226$ & $7,672,778$ & 141,423 & - & $5,262,207$ & $4,001,568$ & 249,193 \\
\hline Other & - & - & - & - & -- & $3,343,750$ & - \\
\hline \multicolumn{8}{|l|}{ Other' } \\
\hline Purchases (thousand kWh) & 562,144 & $2,179,743$ & $3,475,892$ & - & 20,679 & -336 & 381 \\
\hline 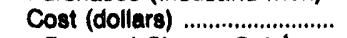 & $8,453,28 \theta$ & $43,677,772$ & $60,689,125$ & - & 529,158 & 159 & - \\
\hline Demand Charge Only' ....... & - & - & - & - & -- & - & - \\
\hline Demand ................................... & $1,043,126$ & - & 215,009 & - & - & - & - \\
\hline 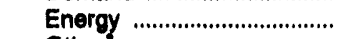 & $8,410,163$ & $43,677,772$ & $60,292,150$ & - & 484,312 & 159 & - \\
\hline 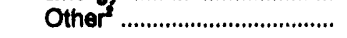 & - & - & 181,966 & - & 44,846 & - & - \\
\hline \multicolumn{8}{|l|}{ Total } \\
\hline $\begin{array}{l}\text { Purchases (thousand kWh) } \\
\text { Cost (dollars) ........................ }\end{array}$ & $\begin{array}{r}1,363,858 \\
22,581,763\end{array}$ & $\begin{array}{r}5,830,908 \\
340,492,282\end{array}$ & $\begin{array}{r}3,574,339 \\
62,026,257\end{array}$ & - & $\begin{array}{r}1,119,044 \\
22,533,383\end{array}$ & $\begin{array}{r}921,629 \\
21,835,293\end{array}$ & $\begin{array}{r}490,617 \\
7,963,382\end{array}$ \\
\hline Demand Charge Only' ...... & - & - & - & - & - & - & - \\
\hline 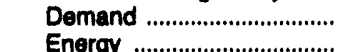 & $\begin{array}{r}2,230,296 \\
20,351,467\end{array}$ & $\begin{array}{l}237,406,028 \\
103,085,354\end{array}$ & $\begin{array}{r}215,009 \\
61,629,282\end{array}$ & $\overline{-}$ & $\begin{array}{r}6,779,368 \\
15,709,169\end{array}$ & $\begin{array}{r}40,631 \\
15,173,912\end{array}$ & $\begin{array}{l}1,263,119 \\
6,700,263\end{array}$ \\
\hline 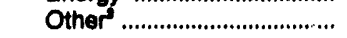 & - & - & 181,966 & - & 44,846 & $6,620,750$ & - \\
\hline
\end{tabular}

See notes and footnotes at end of table. 
Table 19. Electricity Purchases by Investor-Owned Utilities, by State, 1992 (Continued)

\begin{tabular}{|c|c|c|c|c|c|c|c|}
\hline $\begin{array}{c}\text { source of } \\
\text { Evectriofty by } \\
\text { Owmerehip Clases }\end{array}$ & $\begin{array}{c}\text { Missouri } \\
\text { Union } \\
\text { Eloctric } \\
\text { Co }\end{array}$ & $\begin{array}{l}\text { Missour } \\
\text { UtiliCorp } \\
\text { United } \\
\text { Inc }\end{array}$ & $\begin{array}{l}\text { Montana } \\
\text { Montana } \\
\text { Power } \\
\text { Co }\end{array}$ & $\begin{array}{l}\text { Nevada } \\
\text { Nevada } \\
\text { Power } \\
\text { Co }\end{array}$ & $\begin{array}{l}\text { Novada } \\
\text { Slerra } \\
\text { Pacific } \\
\text { Power } \\
\text { Co }\end{array}$ & $\begin{array}{c}\text { Now Hampshire } \\
\text { Concord } \\
\text { Electric } \\
\text { Co' }\end{array}$ & $\begin{array}{l}\text { New Hampshire } \\
\text { Connecticut } \\
\text { Valley } \\
\text { Electric } \\
\mathrm{Co}^{4}\end{array}$ \\
\hline 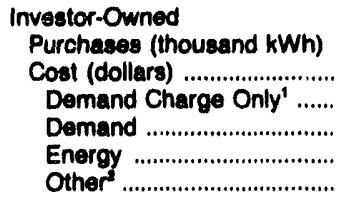 & $\begin{array}{r}6,452,894 \\
107,806,491 \\
-\overline{27,189,184} \\
80,617,307 \\
-\end{array}$ & $\begin{array}{r}354,492 \\
9,904,534 \\
\\
4,557,113 \\
5,359,261 \\
-11,840\end{array}$ & $\begin{array}{l}567,800 \\
13,256,141 \\
-- \\
\overline{13,256,141} \\
--\end{array}$ & $\begin{array}{r}2,289,449 \\
70,771,206 \\
- \\
19,497,550 \\
51,113,790 \\
159,866\end{array}$ & $\begin{array}{r}2,326,440 \\
66,308,835 \\
- \\
22,554,645 \\
43,221,884 \\
532,306\end{array}$ & $\begin{array}{r}455,015 \\
34,448,413 \\
- \\
15,430,480 \\
8,541,107 \\
10,476,828\end{array}$ & $\begin{array}{r}122,844 \\
8,526,339 \\
-- \\
7,372,512 \\
1,778,988 \\
-625,161\end{array}$ \\
\hline 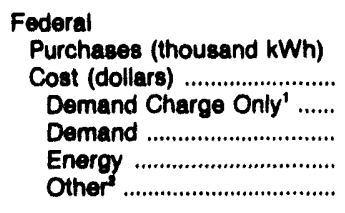 & $\begin{array}{r}463,719 \\
8,787,356 \\
-- \\
207,400 \\
8,579,956 \\
--\end{array}$ & $\begin{array}{l}\bar{z} \\
\bar{z} \\
\overline{-}\end{array}$ & $\begin{array}{r}962,018 \\
33,332,055 \\
900,000 \\
-- \\
32,581,293 \\
-149,238\end{array}$ & $\begin{array}{r}689,945 \\
15,942,023 \\
- \\
1,708,100 \\
13,931,539 \\
402,384\end{array}$ & $\begin{array}{r}\quad 4,962 \\
-\quad 90,791 \\
-\quad 90,791 \\
-\quad\end{array}$ & $\begin{array}{l}= \\
= \\
= \\
=\end{array}$ & $\begin{array}{l}\overline{-} \\
\overline{-} \\
\overline{-} \\
\overline{-}\end{array}$ \\
\hline 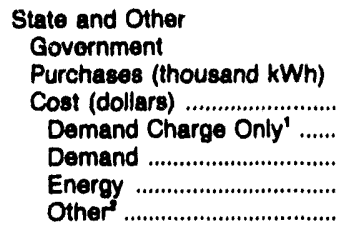 & $\begin{array}{l}= \\
\overline{-} \\
= \\
-\end{array}$ & $\begin{array}{l}\overline{-} \\
\overline{-} \\
\overline{-}\end{array}$ & $\begin{array}{r}3,148 \\
-46,591 \\
-\quad 4 \\
-\quad 46,591\end{array}$ & $\begin{array}{r}1,419,870 \\
29,277,355 \\
\overline{4,166,082} \\
24,227,281 \\
883,992\end{array}$ & $\begin{array}{l}12,483 \\
363,536 \\
- \\
- \\
- \\
-\end{array}$ & $\begin{array}{l}- \\
- \\
= \\
-\end{array}$ & $\begin{array}{l}\overline{-} \\
\overline{-} \\
\overline{-}\end{array}$ \\
\hline 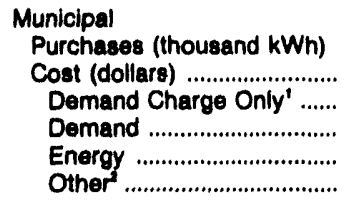 & $\begin{array}{l}= \\
= \\
= \\
=\end{array}$ & $\begin{array}{r}10,879 \\
187,382 \\
-\quad \\
- \\
-187,382\end{array}$ & $\begin{array}{r}2,343 \\
44,033 \\
-\quad \\
-\quad 571 \\
45,102 \\
-1,640\end{array}$ & $\begin{array}{r}85,132 \\
3,086,634 \\
- \\
900,000 \\
2,196,634 \\
--\end{array}$ & $\begin{array}{l}11,026 \\
251,483 \\
- \\
- \\
-251,483\end{array}$ & $\begin{array}{l}= \\
\overline{-} \\
= \\
-\end{array}$ & $\begin{array}{l}- \\
\overline{-} \\
= \\
- \\
-\end{array}$ \\
\hline 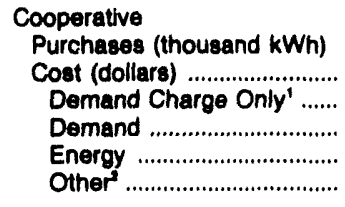 & $\begin{array}{r}52,842 \\
875,835 \\
- \\
- \\
875,835\end{array}$ & $\begin{array}{r}591,876 \\
14,983,860 \\
\overline{5,210,000} \\
9,773,860 \\
--\end{array}$ & $\begin{array}{l}1,076,463 \\
19,532,653 \\
-- \\
-- \\
19,532,656 \\
--\end{array}$ & $\begin{array}{l}55,020 \\
1,250,358 \\
-- \\
-- \\
1,250,358 \\
-\end{array}$ & $\begin{array}{r}166,072 \\
4,718,156 \\
1,845,498 \\
2,872,657 \\
--\end{array}$ & $\begin{array}{l}- \\
= \\
= \\
=\end{array}$ & $\begin{array}{l}- \\
\overline{-} \\
\overline{-} \\
-\end{array}$ \\
\hline 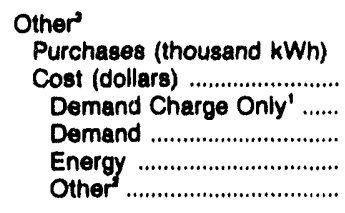 & $\begin{array}{r}55,352 \\
1,840,290 \\
- \\
82,200 \\
1,758,090 \\
-\end{array}$ & $\begin{array}{l}= \\
\because \\
\because \\
-\end{array}$ & $\begin{array}{r}368,058 \\
21,096,788 \\
\overline{3,490,793} \\
14,486,551 \\
3,118,444\end{array}$ & $\begin{array}{r}1,254,408 \\
80,015,290 \\
15,981,500 \\
- \\
64,023,790 \\
-\end{array}$ & $\begin{array}{r}571,569 \\
28,048,803 \\
-- \\
2,814,883 \\
26,602,352 \\
-467,432\end{array}$ & $\begin{array}{l}= \\
= \\
= \\
-\end{array}$ & $\begin{array}{r}40,647 \\
3,806,879 \\
--\quad \\
7,802 \\
3,799,077 \\
--\end{array}$ \\
\hline 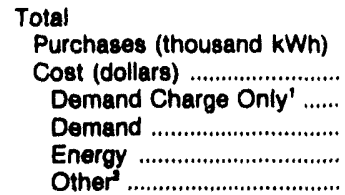 & $\begin{array}{r}7,024,907 \\
119,309,972 \\
- \\
27,478,784 \\
91,831,188\end{array}$ & $\begin{array}{r}957,247 \\
25,075, i, 35 \\
-\quad \\
9,767,113 \\
15,320,503 \\
-11,840\end{array}$ & $\begin{array}{r}2,980,830 \\
87,308,264 \\
900,000 \\
3,491,364 \\
79,948,334 \\
2,968,566\end{array}$ & $\begin{array}{r}5,773,824 \\
200,352,866 \\
15,981,500 \\
26,271,732 \\
156,643,392 \\
1,446,242\end{array}$ & $\begin{array}{r}3,092,552 \\
100,782,604 \\
-- \\
27,315,027 \\
73,402,703 \\
64,874\end{array}$ & $\begin{array}{r}455,015 \\
34,448,413 \\
-- \\
15,430,480 \\
8,541,107 \\
10,478,826\end{array}$ & $\begin{array}{r}163,491 \\
12,333,218 \\
-- \\
7,380,314 \\
5,578,065 \\
-625,161\end{array}$ \\
\hline
\end{tabular}

See notes and footnotes at end of table. 
Table 19. Electriclty Purchases by Investor-Owned Utilitios, by State, 1992 (Continued)

\begin{tabular}{|c|c|c|c|c|c|c|c|}
\hline $\begin{array}{c}\text { soures of } \\
\text { Eleotrlelty by } \\
\text { Ownerehlp Claes }\end{array}$ & $\begin{array}{c}\text { Now Hampshire } \\
\text { Exeter \& } \\
\text { Hampton } \\
\text { Electric } \\
\text { Co' }\end{array}$ & \begin{tabular}{|} 
New Hampshire \\
Granite \\
State \\
Electric \\
Co
\end{tabular} & $\begin{array}{l}\text { New Hampshire } \\
\text { New } \\
\text { England } \\
\text { Trans } \\
\text { Corp }\end{array}$ & $\begin{array}{l}\text { New Hampehire } \\
\text { Now } \\
\text { England } \\
\text { Hydro-Trans } \\
\text { Corp" }\end{array}$ & $\begin{array}{l}\text { Now Hampehire } \\
\text { North } \\
\text { Atlantle } \\
\text { Energy } \\
\text { Corp }\end{array}$ & $\begin{array}{c}\text { New Hampenire } \\
\text { Public } \\
\text { Service } \\
\text { Co of NH }\end{array}$ & $\begin{array}{c}\text { Now Hampenire } \\
\text { Unitil } \\
\text { Power } \\
\text { Corp }\end{array}$ \\
\hline 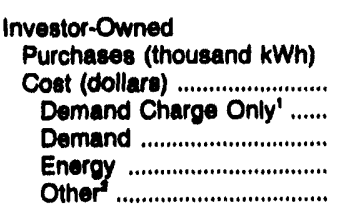 & $\begin{array}{r}491,032 \\
36,794,863 \\
\overline{16,272,445} \\
9,220,017 \\
11,302,401\end{array}$ & $\begin{array}{r}679,420 \\
43,822,059 \\
- \\
21,416,825 \\
15,162,344 \\
7,242,790\end{array}$ & $\begin{array}{l}\bar{z} \\
\bar{z} \\
\overline{-}\end{array}$ & $\bar{I}$ & $\begin{array}{l}\bar{z} \\
\bar{z}\end{array}$ & $\begin{array}{c}1,137,407 \\
52,428,693 \\
- \\
\overline{18,827,331} \\
33,601,282\end{array}$ & $\begin{array}{r}509,579 \\
31,476,244 \\
-- \\
21,064,361 \\
10,420,883 \\
-\end{array}$ \\
\hline 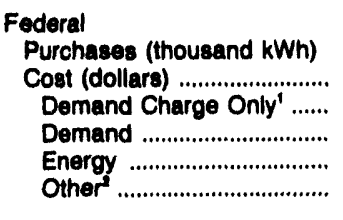 & $\bar{z}$ & $\begin{array}{l}- \\
= \\
= \\
=\end{array}$ & $\begin{array}{l}\overline{-} \\
\bar{z} \\
\overline{-}\end{array}$ & 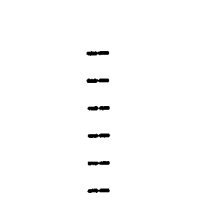 & $\begin{array}{l}\overline{-} \\
\overline{-} \\
\overline{-}\end{array}$ & $\begin{array}{l}\overline{-} \\
\overline{-} \\
\overline{-}\end{array}$ & $\begin{array}{l}- \\
\overline{-} \\
-\end{array}$ \\
\hline 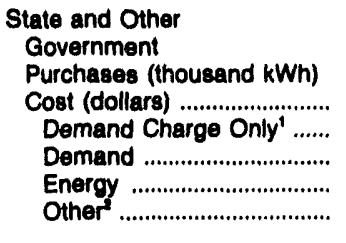 & $\begin{array}{l}\overline{-} \\
\bar{z} \\
\overline{-}\end{array}$ & $\begin{array}{l}= \\
\overline{-} \\
\overline{-} \\
=\end{array}$ & $\begin{array}{l}- \\
\overline{-} \\
\overline{-} \\
-\end{array}$ & $\begin{array}{l}\overline{-} \\
\bar{I} \\
\overline{-}\end{array}$ & $\begin{array}{l}\overline{-} \\
\overline{-} \\
\overline{-}\end{array}$ & $\begin{array}{l}- \\
\overline{-} \\
\overline{-} \\
-\end{array}$ & $\begin{array}{l}\overline{-} \\
\overline{-} \\
=\end{array}$ \\
\hline 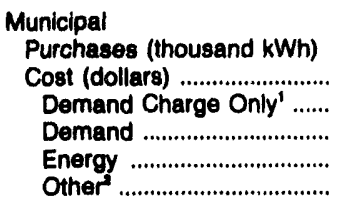 & $\begin{array}{l}- \\
\overline{-} \\
\overline{-} \\
-\end{array}$ & $\begin{array}{l}= \\
\overline{-} \\
= \\
-\end{array}$ & $\begin{array}{l}= \\
\bar{z} \\
= \\
=\end{array}$ & $\begin{array}{l}\overline{-} \\
\bar{z} \\
\overline{-}\end{array}$ & $\begin{array}{l}\overline{-} \\
\overline{-} \\
\overline{-}\end{array}$ & $\begin{array}{r}84 \\
-\quad 2,404 \\
-\quad \\
-\quad 2,404\end{array}$ & $\begin{array}{l}\ddot{-} \\
\overline{-} \\
\ddot{-}\end{array}$ \\
\hline 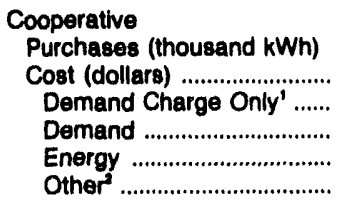 & $\begin{array}{l}- \\
\overline{-} \\
\overline{-} \\
-\end{array}$ & $\begin{array}{l}- \\
\overline{-} \\
\overline{-} \\
\overline{-}\end{array}$ & $\begin{array}{l}- \\
\overline{-} \\
= \\
-\end{array}$ & $\begin{array}{l}= \\
= \\
= \\
=\end{array}$ & $\begin{array}{l}\overline{-} \\
\bar{z} \\
\overline{-}\end{array}$ & $\begin{array}{r}167,271 \\
13,726,557 \\
- \\
- \\
257,315 \\
13,469,242\end{array}$ & $\begin{array}{l}= \\
= \\
= \\
=\end{array}$ \\
\hline 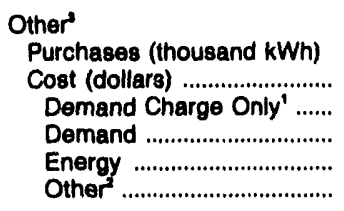 & $\begin{array}{l}- \\
\overline{-} \\
\overline{-} \\
-\end{array}$ & $\begin{array}{r}717 \\
20,134 \\
-\quad \\
-\quad 20,134\end{array}$ & $\begin{array}{l}\bar{z} \\
\bar{z} \\
\bar{m}\end{array}$ & $\begin{array}{l}\overline{-} \\
\bar{m} \\
\bar{m}\end{array}$ & $\begin{array}{l}\overline{-} \\
\bar{z} \\
\overline{-}\end{array}$ & $\begin{array}{r}4,432,328 \\
204,105,580 \\
- \\
\overline{-} \\
116,402,285 \\
87,703,285\end{array}$ & $\begin{array}{r}434,778 \\
30,393,241 \\
- \\
19,233,292 \\
11,159,949 \\
--\end{array}$ \\
\hline 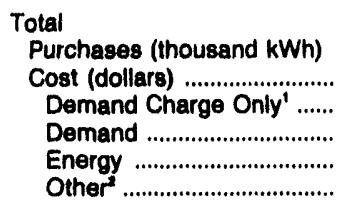 & $\begin{array}{r}491,032 \\
36,794,863 \\
\overline{16,272,445} \\
9,220,017 \\
11,302,401\end{array}$ & $\begin{array}{r}680,137 \\
43,842,193 \\
- \\
21,416,925 \\
15,182,478 \\
7,242,790\end{array}$ & $\begin{array}{l}- \\
\overline{-} \\
\overline{-} \\
-\end{array}$ & $\bar{z}$ & $\begin{array}{l}\overline{-} \\
\bar{z} \\
\overline{-}\end{array}$ & $\begin{array}{r}5,737,090 \\
270,263,134 \\
- \\
\overline{135,489,345} \\
134,773,789\end{array}$ & $\begin{array}{r}944,357 \\
61,868,485 \\
- \\
40,287,653 \\
21,580,832 \\
-\end{array}$ \\
\hline
\end{tabular}

See notes and footnotes at end of table. 
Table 19. Electricity Purchases by Investor-Owned Utilitles, by State, 1992 (Continued)

\begin{tabular}{|c|c|c|c|c|c|c|c|}
\hline 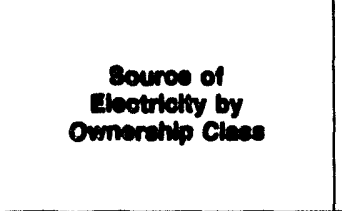 & $\begin{array}{l}\text { Now Jersey } \\
\text { Atlantic City } \\
\text { Electric } \\
\text { Co }\end{array}$ & $\begin{array}{l}\text { New Jereey } \\
\text { Jereoy } \\
\text { Contral } \\
\text { Power \& } \\
\text { Light } \\
\text { Co }\end{array}$ & $\begin{array}{l}\text { New Jerey } \\
\text { Public } \\
\text { Service } \\
\text { Electric \& } \\
\text { Gas } \\
\text { Co }\end{array}$ & $\begin{array}{l}\text { New Jersey } \\
\text { Rockland } \\
\text { Electric } \\
\text { Co' }^{4}\end{array}$ & $\begin{array}{c}\text { Now Mexico } \\
\text { Publlc } \\
\text { Service } \\
\text { Co of NM }\end{array}$ & $\begin{array}{l}\text { New Mexico } \\
\text { Texas- } \\
\text { Now Mexico } \\
\text { Power } \\
\text { Co4 }\end{array}$ & $\begin{array}{l}\text { Now York } \\
\text { Contral } \\
\text { Hudson } \\
\text { Gas \& } \\
\text { Electric } \\
\text { Corp }\end{array}$ \\
\hline 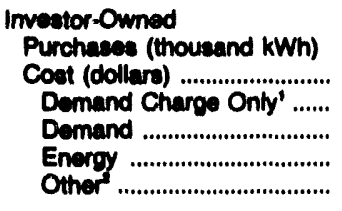 & $\begin{array}{c}3,043,797 \\
129,043,386 \\
- \\
\overline{50,249,345} \\
78,794,041\end{array}$ & $\begin{array}{r}6,356,865 \\
287,980,764 \\
21,733,271 \\
169,674,926 \\
96,714,792 \\
-142,225\end{array}$ & $\begin{array}{l}3,267,243 \\
73,134,490 \\
\overline{-} \\
\overline{73,134,490}\end{array}$ & $\begin{array}{r}1,281,443 \\
65,902,651 \\
- \\
21,108,010 \\
44,794,541 \\
-\end{array}$ & $\begin{array}{r}275,686 \\
12,176,746 \\
\overline{7,020,000} \\
5,151,803 \\
4,943\end{array}$ & $\begin{array}{r}1,180,575 \\
41,003,482 \\
-\overline{21,495,600} \\
14,481,680 \\
5,026,202\end{array}$ & $\begin{array}{l}142,765 \\
3,883,241 \\
- \\
- \\
3,883,241 \\
-\end{array}$ \\
\hline 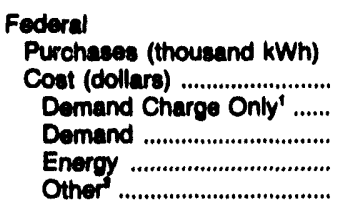 & 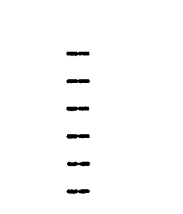 & $\begin{array}{l}\overline{-} \\
\bar{z}\end{array}$ & $\begin{array}{l}\bar{z} \\
\bar{z}\end{array}$ & $\begin{array}{l}\overline{-} \\
\bar{z} \\
\overline{-}\end{array}$ & $\begin{array}{r}1,685 \\
-\quad 34,945 \\
-\quad \\
-\quad 36,727 \\
-1,782\end{array}$ & $\begin{array}{l}\bar{z} \\
\bar{z} \\
\bar{m}\end{array}$ & $\begin{array}{l}\overline{-} \\
\bar{z} \\
\overline{-}\end{array}$ \\
\hline 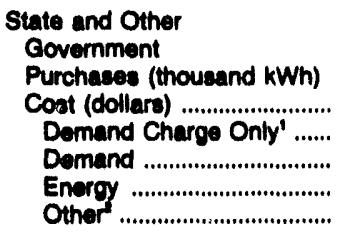 & 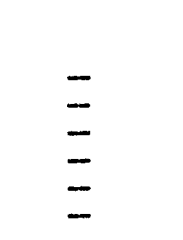 & $\begin{array}{l}\overline{-} \\
\bar{z} \\
\overline{-}\end{array}$ & $\begin{array}{l}55,839 \\
660,244 \\
-\quad \\
- \\
-\quad 680,244\end{array}$ & $\begin{array}{l}- \\
\overline{-} \\
\overline{-} \\
-\end{array}$ & $\begin{array}{r}618,861 \\
40,500,967 \\
\overline{5,937,644} \\
12,313,062 \\
22,250,261\end{array}$ & $\begin{array}{l}\overline{-} \\
\bar{z} \\
\bar{z}\end{array}$ & $\begin{array}{l}153,232 \\
6,862,883 \\
-- \\
1,870,371 \\
4,082,612 \\
--\end{array}$ \\
\hline 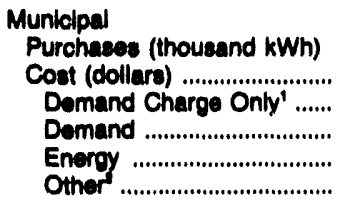 & $\begin{array}{r}1,700 \\
70,703 \\
-\quad \\
-\quad \\
67,630 \\
3,163\end{array}$ & $\begin{array}{r}2,035,219 \\
120,713,313 \\
-\quad 1,774 \\
120,711,539 \\
-\end{array}$ & 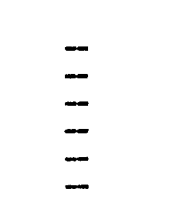 & $\begin{array}{l}-- \\
\overline{-} \\
\overline{-} \\
-\end{array}$ & $\begin{array}{ll}- & -773 \\
- & \\
- & \\
- & -773\end{array}$ & $\begin{array}{r}297 \\
-\quad 8,020 \\
-\quad \\
-\quad \\
-\quad 8,020\end{array}$ & $\begin{array}{l}\overline{-} \\
\overline{-} \\
\bar{m}\end{array}$ \\
\hline 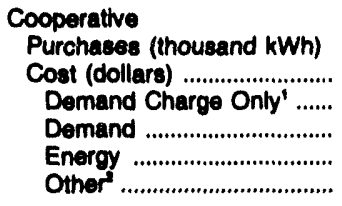 & $\begin{array}{l}\overline{-} \\
\bar{m}\end{array}$ & $\begin{array}{l}\overline{-} \\
\bar{m} \\
\bar{m}\end{array}$ & $\begin{array}{l}160,217 \\
3,605,264 \\
- \\
- \\
3,605,264 \\
-\end{array}$ & $\begin{array}{l}- \\
\overline{-} \\
m \\
m\end{array}$ & $\begin{array}{r}6,368 \\
112,321 \\
-\quad \\
-- \\
114,895 \\
-2,574\end{array}$ & $\begin{array}{l}\overline{-} \\
\overline{-} \\
\overline{-} \\
\overline{-}\end{array}$ & $\begin{array}{l}\overline{-} \\
\overline{-} \\
\overline{-}\end{array}$ \\
\hline 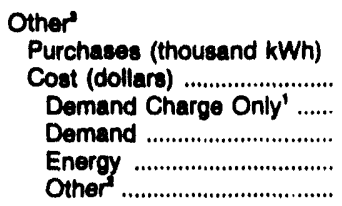 & $\begin{array}{l}1,253,712 \\
64,464,459 \\
- \\
- \\
39,456,569 \\
25,007,890\end{array}$ & $\begin{array}{r}4,000,034 \\
232,004,661 \\
-\quad 12,020,010 \\
218,396,683 \\
1,587,968\end{array}$ & $\begin{array}{r}9,542,826 \\
306,512,893 \\
\overline{-} \\
19,816,510 \\
286,771,451 \\
-75,068\end{array}$ & $\begin{array}{l}\overline{1,166,258} \\
\overline{-} \\
\overline{1} \\
1,166,258\end{array}$ & $\begin{array}{l}-144,599 \\
-2,049,842 \\
- \\
- \\
-2,056,472\end{array}$ & $\begin{array}{l}\overline{-} \\
\overline{-} \\
\overline{-} \\
\overline{-}\end{array}$ & $\begin{array}{r}385,690 \\
12,925,627 \\
- \\
478,247 \\
11,795,794 \\
651,586\end{array}$ \\
\hline 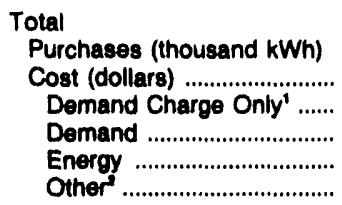 & $\begin{array}{r}4,299,299 \\
193,578,638 \\
- \\
\overline{89,773,544} \\
103,805,094\end{array}$ & $\begin{array}{r}12,392,118 \\
640,698,738 \\
21,733,271 \\
181,696,710 \\
435,823,014 \\
1,445,743\end{array}$ & $\begin{array}{r}13,026,225 \\
383,812,891 \\
- \\
19,816,510 \\
364,171,449 \\
-75,068\end{array}$ & $\begin{array}{r}1,281,443 \\
67,068,809 \\
-\overline{21,108,010} \\
44,794,541 \\
1,166,258\end{array}$ & $\begin{array}{r}758,201 \\
50,774,364 \\
-\overline{12,957,644} \\
17,623,117 \\
20,193,603\end{array}$ & $\begin{array}{r}1,160,872 \\
41,011,502 \\
-- \\
21,495,600 \\
14,481,680 \\
5,034,222\end{array}$ & $\begin{array}{r}681,687 \\
23,671,851 \\
\overline{2,348,618} \\
20,671,647 \\
651,586\end{array}$ \\
\hline
\end{tabular}

See notes and footnotes at end of table. 
Table 19. Eloctrlcity Purchaces by Investor-Owned Utilitiea, by State, 1982 (Continued)

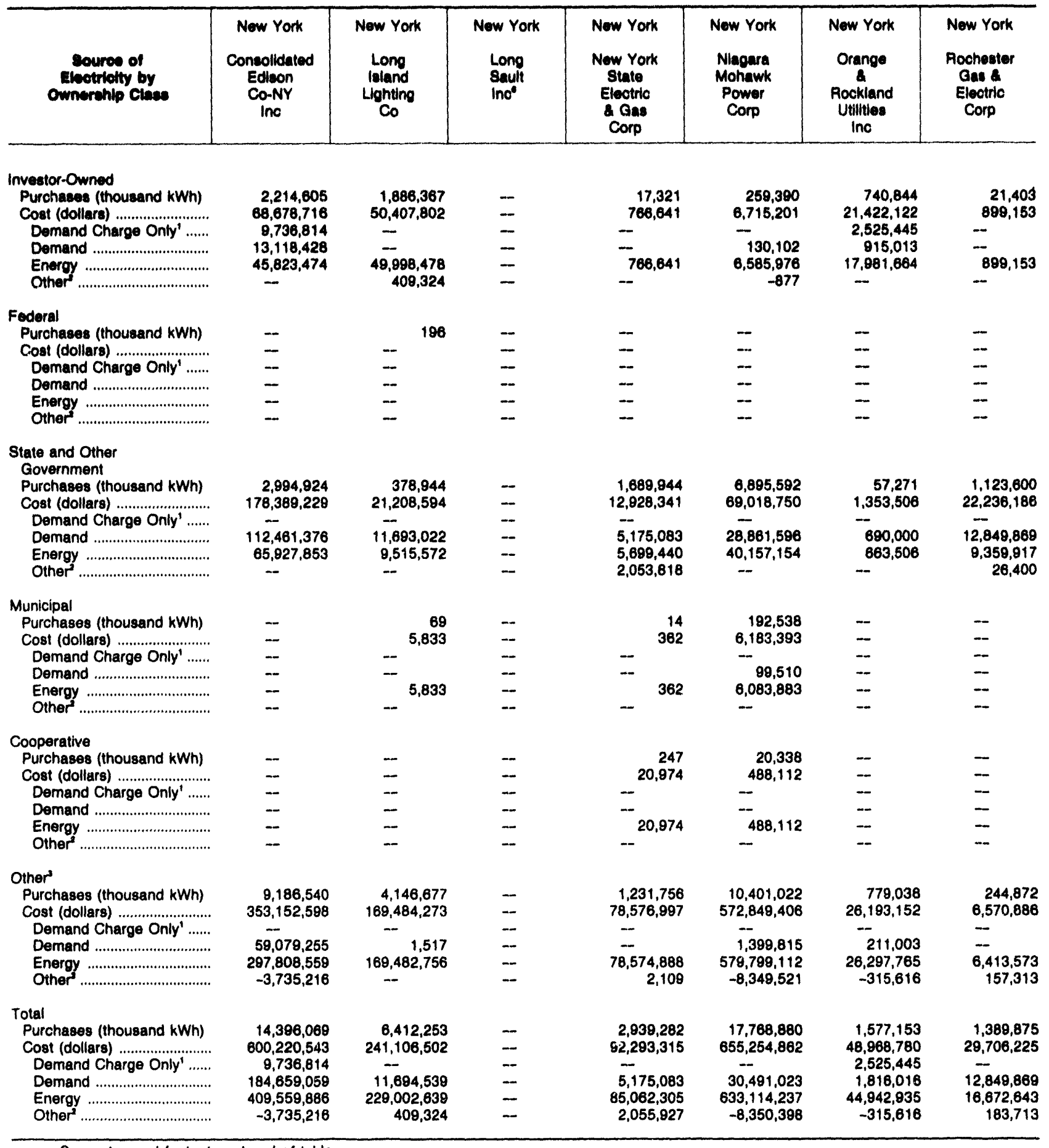

\footnotetext{
See notes and footnotes at end of table.
} 
Table 19. Eloctrleity Purchases by Investor-Owned Utilities, by State, 1992 (Continued)

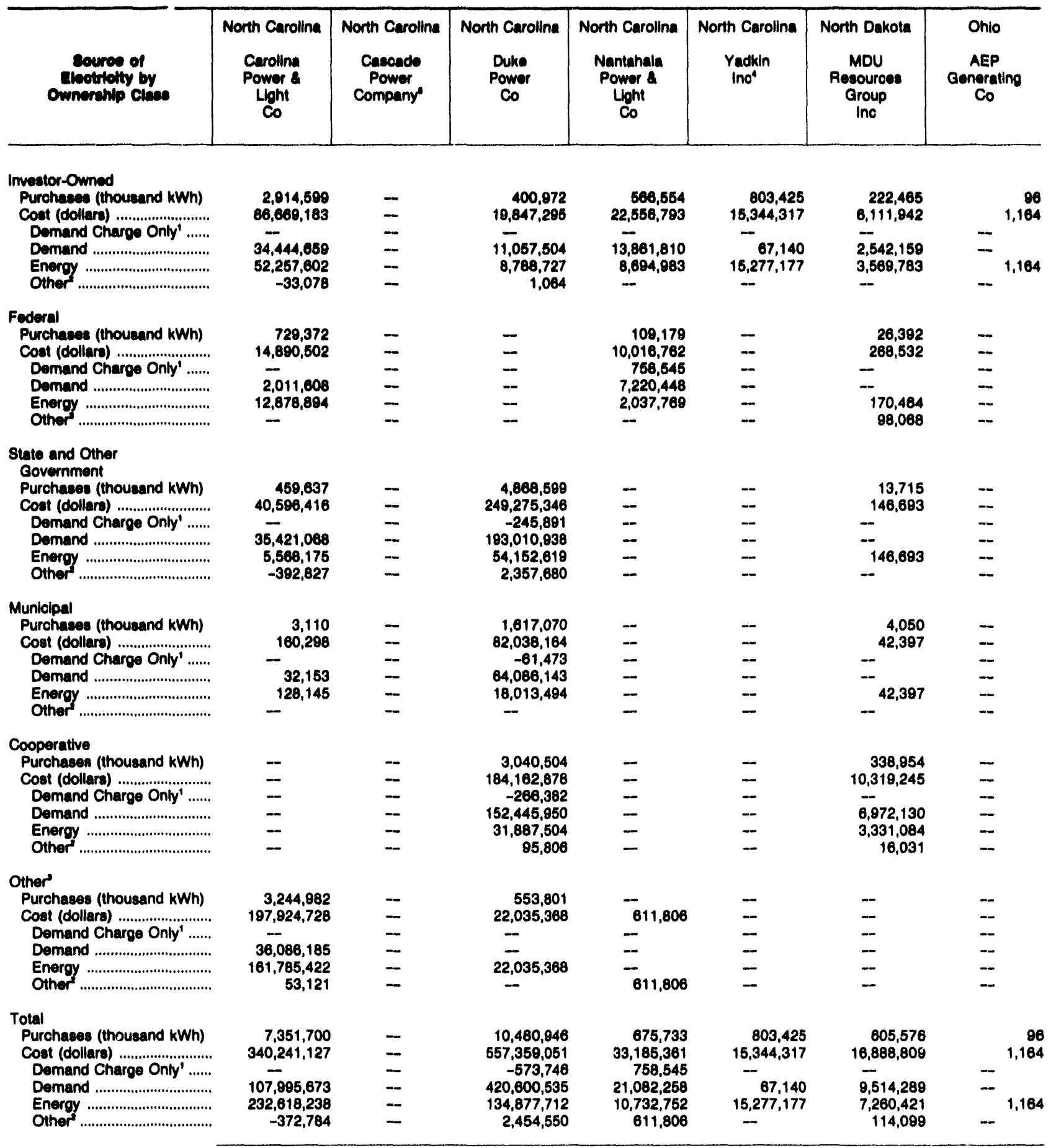

See notes and footnotes at end of table. 
Table 19. Electricity Pui shases by Inveotor-Owned Utilities, by 8tate, 1092 (Continued)

\begin{tabular}{|c|c|c|c|c|c|c|c|}
\hline $\begin{array}{l}\text { source of } \\
\text { Eleotrilety by } \\
\text { ownerentp Clases }\end{array}$ & $\begin{array}{l}\text { Ohio } \\
\text { Cincinnat } \\
\text { Oat a } \\
\text { Electrlo } \\
\text { Co }\end{array}$ & $\begin{array}{c}\text { Ohio } \\
\text { Cleveland } \\
\text { Electrio } \\
\text { Illuminating } \\
\text { Co }\end{array}$ & $\begin{array}{l}\text { Onio } \\
\text { Columbus } \\
\text { Southern } \\
\text { Power Co }\end{array}$ & $\begin{array}{l}\text { Ohio } \\
\text { Dayton } \\
\text { Power } \\
\text { Ught } \\
\text { Co }\end{array}$ & $\begin{array}{l}\text { Ohlo } \\
\text { Inclianu. } \\
\text { Konluoky } \\
\text { Eloctric } \\
\text { Corp" }\end{array}$ & $\begin{array}{l}\text { Onlo } \\
\text { Ohio } \\
\text { Edieon } \\
\text { Co }\end{array}$ & $\begin{array}{l}\text { Ohio } \\
\text { Ohlo } \\
\text { Power } \\
\text { Co }\end{array}$ \\
\hline 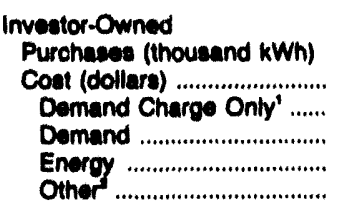 & $\begin{array}{r}1,170,466 \\
17,385,722 \\
- \\
2,410,580 \\
11,823,161 \\
3,131,971\end{array}$ & $\begin{array}{r}1,613,647 \\
129,787,260 \\
-\quad 13,526,086 \\
116,183,211 \\
77,963\end{array}$ & $\begin{array}{r}390,181 \\
6,398,122 \\
-- \\
27,017 \\
6,370,205 \\
-\cdots\end{array}$ & $\begin{array}{r}1,480,710 \\
26,185,732 \\
\overline{4,455,750} \\
20,723,626 \\
6,366\end{array}$ & $\begin{array}{l}\bar{m} \\
\bar{m} \\
\bar{m}\end{array}$ & $\begin{array}{r}1,624,844 \\
38,627,134 \\
- \\
8,900,963 \\
29,853,764 \\
72,417\end{array}$ & $\begin{array}{r}1,466,714 \\
26,171,060 \\
- \\
1,494,780 \\
24,677,180 \\
-\end{array}$ \\
\hline 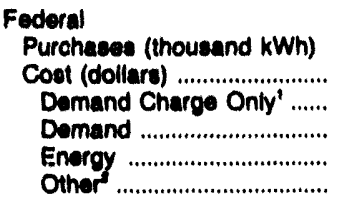 & $\begin{array}{r}210 \\
-\quad 3,046 \\
-\quad 2,268 \\
777\end{array}$ & $\begin{array}{l}\overline{-} \\
\bar{m} \\
\bar{m}\end{array}$ & 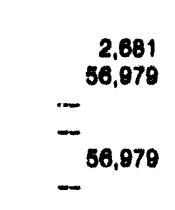 & $\begin{array}{l}\overline{-} \\
\overline{-} \\
\overline{-} \\
\overline{-}\end{array}$ & $\begin{array}{l}\overline{-} \\
\overline{-} \\
\overline{-}\end{array}$ & $\begin{array}{l}= \\
\overline{-} \\
=\end{array}$ & $\begin{array}{r}\quad 4,629 \\
-\quad 88,323 \\
-\quad \\
-\quad 88,323 \\
--\end{array}$ \\
\hline 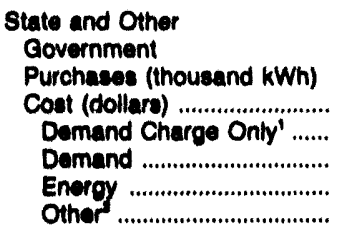 & $\begin{array}{l}\bar{m} \\
\bar{m}\end{array}$ & $\begin{array}{l}\bar{z} \\
\bar{z}\end{array}$ & $\begin{array}{l}\overline{-} \\
\bar{z} \\
\bar{m}\end{array}$ & $\begin{array}{l}33,749 \\
1,638,680 \\
- \\
\overline{1,636,680} \\
-\end{array}$ & $\begin{array}{l}- \\
\overline{-} \\
=\end{array}$ & $\begin{array}{l}\bar{z} \\
\bar{m}\end{array}$ & $\begin{array}{l}\overline{-} \\
\bar{m} \\
\bar{m}\end{array}$ \\
\hline 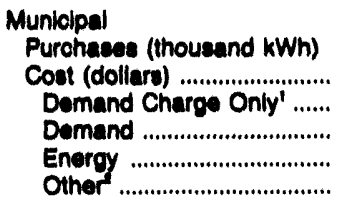 & $\begin{array}{l}\bar{z} \\
\bar{z} \\
\bar{m}\end{array}$ & $\begin{array}{r}61,674 \\
455,615 \\
-\quad \\
-\quad 423,692 \\
32,123\end{array}$ & $\begin{array}{l}17,038 \\
243,064 \\
- \\
- \\
- \\
-\end{array}$ & $\begin{array}{l}\overline{-} \\
\overline{-} \\
\bar{m}\end{array}$ & $\begin{array}{l}\bar{z} \\
\bar{z} \\
\bar{z}\end{array}$ & $\begin{array}{l}\bar{z} \\
\overline{-} \\
\overline{-}\end{array}$ & $\begin{array}{r}30,385 \\
410,093 \\
- \\
- \\
-\end{array}$ \\
\hline 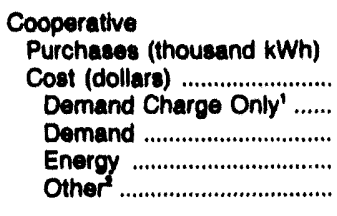 & $\begin{array}{r}307,890 \\
4,747,058 \\
- \\
28,440 \\
3,397,880 \\
1,320,638\end{array}$ & $\begin{array}{l}\bar{z} \\
\bar{z} \\
\bar{m}\end{array}$ & $\begin{aligned} & 11,726 \\
& 282,220 \\
&- \\
&- \\
&-\end{aligned}$ & $\begin{array}{l}\overline{-} \\
\bar{z} \\
\bar{m}\end{array}$ & $\begin{array}{l}\overline{-} \\
\bar{z} \\
\bar{m}\end{array}$ & $\begin{array}{l}\overline{-} \\
\overline{-} \\
\overline{-}\end{array}$ & $\begin{array}{l}26,268 \\
5,276,021 \\
- \\
- \\
5,276,021 \\
--\end{array}$ \\
\hline 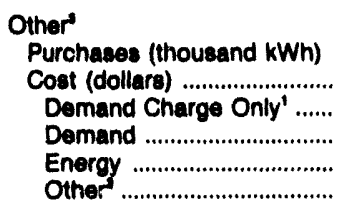 & 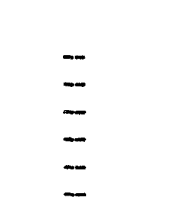 & $\begin{array}{r}50 \\
-\quad 248 \\
-\quad 248\end{array}$ & $\begin{array}{r}3,151,310 \\
130,737,342 \\
-\quad 32,194 \\
130,705,148 \\
-\end{array}$ & $\bar{z}$ & 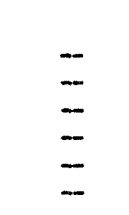 & $\begin{array}{l}= \\
\bar{z} \\
\bar{m}\end{array}$ & $\begin{array}{l}1,854,331 \\
29,099,993 \\
- \\
\overline{29,009,993}\end{array}$ \\
\hline 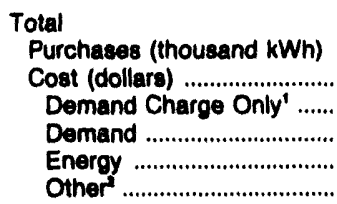 & $\begin{array}{r}1,484,556 \\
22,115,825 \\
- \\
2,439,030 \\
15,223,409 \\
4,453,386\end{array}$ & $\begin{array}{r}1,675,371 \\
130,243,323 \\
- \\
13,526,086 \\
116,607,151 \\
110,086\end{array}$ & $\begin{array}{r}3,579,836 \\
137,717,747 \\
-\quad \\
60,111 \\
137,657,636 \\
-\end{array}$ & $\begin{array}{r}1,514,549 \\
26,822,412 \\
-\quad \\
4,455,750 \\
22,360,306 \\
6,356\end{array}$ & $\begin{array}{l}\bar{z} \\
\bar{z} \\
\bar{z}\end{array}$ & $\begin{array}{r}1,624,814 \\
38,527,134 \\
-\quad \\
6,900,853 \\
29,553,764 \\
72,417\end{array}$ & $\begin{array}{r}3,372,327 \\
61,057,290 \\
- \\
1,494,780 \\
58,562,510 \\
-\end{array}$ \\
\hline
\end{tabular}

See notes and footnotes at end of table. 
Table 19. Electricity Purchaces by Inveator-Owned Utilities, by 8tate, 1902 (Continued)

\begin{tabular}{|c|c|c|c|c|c|c|c|}
\hline 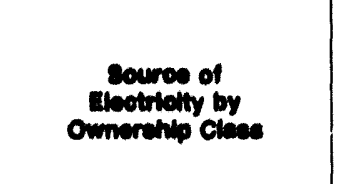 & $\begin{array}{l}\text { Onio } \\
\text { Onio } \\
\text { Valloy } \\
\text { Electric } \\
\text { Corp }\end{array}$ & $\begin{array}{l}\text { Ohio } \\
\text { Toledo } \\
\text { Edieon } \\
\text { Co }\end{array}$ & $\begin{array}{l}\text { Oklahoma } \\
\text { Oklahoma } \\
\text { Gas } 8 \\
\text { Electrio } \\
\text { Co }\end{array}$ & $\begin{array}{l}\text { Oklahoma } \\
\text { Public } \\
\text { Service } \\
\text { Co of } \\
\text { Oklahoma }\end{array}$ & $\begin{array}{l}\text { Oregon } \\
\text { Pucificorp }\end{array}$ & $\begin{array}{l}\text { Oregon } \\
\text { Portiand } \\
\text { General } \\
\text { Eleotric } \\
\text { Co }\end{array}$ & $\begin{array}{l}\text { Pennoytuania } \\
\text { Cltizens } \\
\text { Eloctric } \\
\text { Co }^{4}\end{array}$ \\
\hline 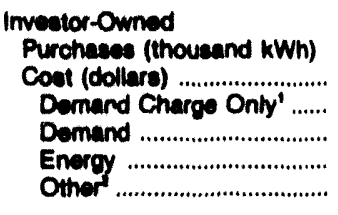 & $\begin{array}{c}9,254,087 \\
141,779,033 \\
= \\
= \\
141,779,833\end{array}$ & $\begin{array}{r}186,614 \\
3,087,626 \\
-\quad 8,912 \\
3,004,969 \\
53,644\end{array}$ & 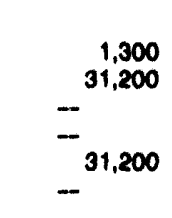 & $\begin{array}{l}780,795 \\
16,143,862 \\
- \\
\overline{15,143,862}\end{array}$ & $\begin{array}{r}1,842,629 \\
47,177,823 \\
219,356 \\
8,248,480 \\
37,225,804 \\
1,483,184\end{array}$ & $\begin{array}{r}1,236,716 \\
38,900,241 \\
7,968,000 \\
731,936 \\
28,200,305 \\
--\end{array}$ & $\begin{array}{r}152,757 \\
6,813,082 \\
- \\
1,581,585 \\
5,581,400 \\
-328,013\end{array}$ \\
\hline 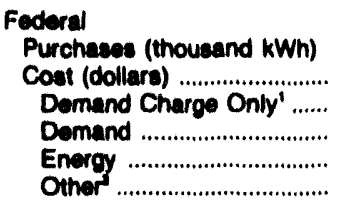 & $\begin{array}{l}\overline{-} \\
\bar{m} \\
\bar{m}\end{array}$ & $\begin{array}{l}- \\
\overline{-} \\
\overline{-}\end{array}$ & $\begin{array}{l}- \\
\overline{-} \\
\overline{-}\end{array}$ & $\begin{array}{l}\overline{-} \\
\overline{-} \\
\overline{-}\end{array}$ & $\begin{array}{r}384,173 \\
71,738,582 \\
68,793,614 \\
\overline{4,944,688} \\
--\end{array}$ & $\begin{array}{r}323,084 \\
-57,699,472 \\
2,800,000 \\
- \\
5,535,043 \\
-68,034,515\end{array}$ & $\begin{array}{l}= \\
= \\
= \\
-\end{array}$ \\
\hline 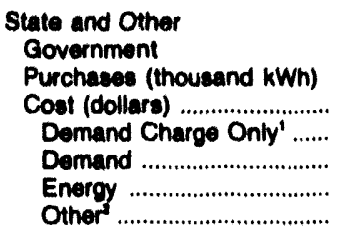 & $\begin{array}{l}\overline{-} \\
\overline{-} \\
\overline{-}\end{array}$ & $\begin{array}{l}\overline{-} \\
\overline{-} \\
\overline{-}\end{array}$ & $\begin{array}{l}\bar{z} \\
\bar{z} \\
\overline{-}\end{array}$ & $\begin{array}{l}76,942 \\
1,267,476 \\
- \\
- \\
1,267,476 \\
--\end{array}$ & $\begin{array}{r}3,127,811 \\
52,366,896 \\
- \\
7866,014 \\
13,926,754 \\
37,654,128\end{array}$ & $\begin{array}{l}4,439,861 \\
82,724,127 \\
- \\
\overline{67,620,915} \\
15,103,212\end{array}$ & $\begin{array}{l}\overline{-} \\
\overline{-} \\
\bar{m}\end{array}$ \\
\hline 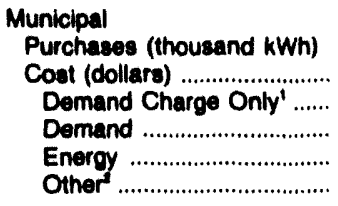 & $\begin{array}{l}\bar{z} \\
\bar{z} \\
\bar{m}\end{array}$ & $\begin{array}{l}- \\
- \\
- \\
- \\
- \\
-98,89,892\end{array}$ & $\begin{array}{l}\overline{-} \\
\overline{-} \\
\overline{-}\end{array}$ & $\begin{array}{l}\overline{-} \\
\overline{-} \\
\overline{-} \\
\overline{-}\end{array}$ & $\begin{array}{r}481,150 \\
30,052,607 \\
-- \\
20,889,313 \\
9,067,229 \\
-3,935\end{array}$ & $\begin{array}{l}452,321 \\
12,563,003 \\
- \\
-\overline{12,563,063} \\
--\end{array}$ & $\begin{array}{l}- \\
\overline{-} \\
\overline{-} \\
-\end{array}$ \\
\hline 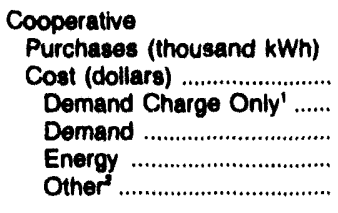 & $\begin{array}{l}- \\
\overline{-} \\
\overline{-} \\
\overline{-}\end{array}$ & $\begin{array}{l}-- \\
\overline{-} \\
\overline{-} \\
-\end{array}$ & $\begin{array}{r}30,795 \\
1,090,162 \\
- \\
412,029 \\
678,133 \\
-\end{array}$ & $\begin{array}{r}61,188 \\
1,113,720 \\
-- \\
-- \\
1,114,062 \\
-342\end{array}$ & $\begin{array}{r}1,544,324 \\
37,503,480 \\
-- \\
8,166,000 \\
29,337,480 \\
--\end{array}$ & $\begin{array}{r}2,250 \\
62,616 \\
-\quad \\
-\quad 50,622 \\
11,994\end{array}$ & $\begin{array}{l}m \\
\overline{-} \\
\overline{-} \\
-\end{array}$ \\
\hline 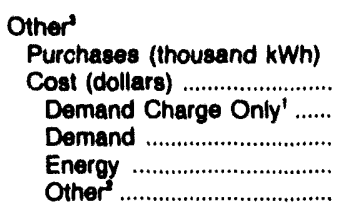 & $\begin{array}{l}\overline{-} \\
\overline{-} \\
\overline{-}\end{array}$ & $\begin{array}{r}9,240 \\
386,499 \\
-\quad \\
64,839 \\
321,650 \\
-\end{array}$ & $\begin{array}{r}2,682,274 \\
181,108,566 \\
- \\
- \\
75,121,913 \\
105,886,653\end{array}$ & $\begin{array}{r}1,047,396 \\
25,545,327 \\
- \\
- \\
19,374,899 \\
6,170,428\end{array}$ & $\begin{array}{r}770,132 \\
35,864,132 \\
\overline{7} \\
7,614,286 \\
26,819,604 \\
1,430,222\end{array}$ & $\begin{array}{r}1,632,498 \\
35,265,601 \\
2,154,732 \\
30,364,143 \\
2,746,726\end{array}$ & $\begin{array}{l}\overline{-} \\
\overline{-} \\
\overline{-}\end{array}$ \\
\hline 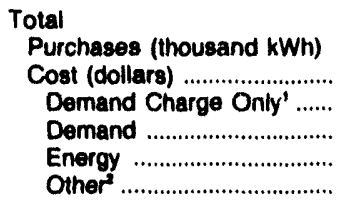 & $\begin{array}{l}9,254,087 \\
141,779,833 \\
\overline{-} \\
\overline{141,779,833}\end{array}$ & $\begin{array}{r}195,854 \\
3,355,122 \\
-\quad \\
73,751 \\
3,326,619 \\
-45,248\end{array}$ & $\begin{array}{r}2,724,369 \\
182,229,928 \\
-- \\
412,029 \\
75,831,246 \\
105,986,653\end{array}$ & $\begin{array}{r}1,976,321 \\
43,070,385 \\
- \\
- \\
36,900,299 \\
6,170,086\end{array}$ & $\begin{array}{r}8,150,219 \\
274,703,510 \\
67,012,969 \\
45,805,103 \\
121,321,839 \\
40,563,599\end{array}$ & $\begin{array}{r}8,086,730 \\
109,816,176 \\
10,768,000 \\
2,886,688 \\
144,334,091 \\
-48,172,583\end{array}$ & $\begin{array}{r}152,757 \\
6,813,082 \\
-- \\
1,581,595 \\
5,561,400 \\
-329,913\end{array}$ \\
\hline
\end{tabular}

See noles and lootnotes at end of table. 
Table 19. Eloctrielty Purchases by Investor-Owned Utilitios, by 8tate, 1902 (Conilnued)

\begin{tabular}{|c|c|c|c|c|c|c|c|}
\hline 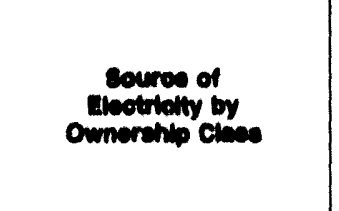 & $\begin{array}{l}\text { Penneytvania } \\
\text { Duqueano } \\
\text { Lbent } \\
\text { Co }\end{array}$ & $\begin{array}{l}\text { Pennoyivanla } \\
\text { Motropolttan } \\
\text { Ediaon } \\
\text { Co }\end{array}$ & $\begin{array}{l}\text { Pennoyivania } \\
\text { Pennoyivania } \\
\text { Electrio } \\
\text { Co }\end{array}$ & $\begin{array}{l}\text { Pennoytvania } \\
\text { Pennaytvania } \\
\text { Power a } \\
\text { Light } \\
\text { Co }\end{array}$ & $\begin{array}{l}\text { Pennoyivania } \\
\text { Pennoyivania } \\
\text { Power } \\
\text { Co }\end{array}$ & $\begin{array}{l}\text { Pennayivania } \\
\text { Philadolphia } \\
\text { Electric } \\
\text { Co }\end{array}$ & $\begin{array}{l}\text { Pennoytvanita } \\
\text { Pike } \\
\text { County } \\
\text { Lipht \& } \\
\text { Power } \\
\text { Cot }\end{array}$ \\
\hline 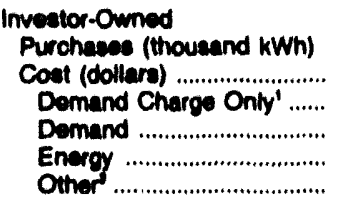 & $\begin{array}{l}417,113 \\
6,703,085 \\
- \\
- \\
-\end{array}$ & $\begin{array}{r}2,882,042 \\
92,768,286 \\
13,362,760 \\
35,798,104 \\
43,638,423 \\
-\end{array}$ & $\begin{array}{r}1,194,271 \\
31,176,115 \\
3,710,573 \\
8,004,157 \\
18,280,464 \\
80,921\end{array}$ & $\begin{array}{r}1,360,534 \\
74,982,707 \\
-\quad 14,400 \\
65,845,333 \\
8,432,974\end{array}$ & $\begin{array}{l}797,430 \\
14,230,182 \\
- \\
\overline{14,230,182} \\
-\end{array}$ & $\begin{array}{r}1,609,747 \\
19,097,581 \\
- \\
- \\
19,052,0626\end{array}$ & $\begin{array}{r}47,326 \\
2,367,792 \\
- \\
608,216 \\
1,698,676 \\
-\end{array}$ \\
\hline 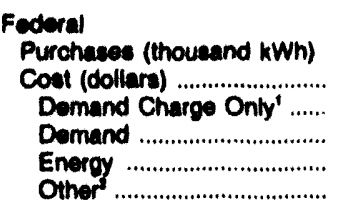 & $\begin{array}{l}- \\
\overline{-} \\
\overline{-}\end{array}$ & $\begin{array}{l}\overline{-} \\
\overline{-} \\
\overline{-}\end{array}$ & $\bar{m}=$ & 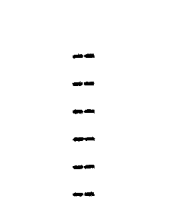 & $\begin{array}{l}\overline{-} \\
\overline{-} \\
\overline{-}\end{array}$ & $\begin{array}{l}\bar{m} \\
\bar{m} \\
\bar{m}\end{array}$ & $\begin{array}{l}\overline{-} \\
\overline{-} \\
\overline{-}\end{array}$ \\
\hline 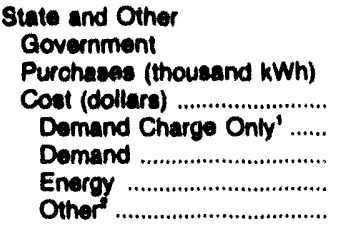 & $\begin{array}{l}\bar{z} \\
\bar{z}\end{array}$ & $\begin{array}{r}218,617 \\
12,157,341 \\
- \\
118,078 \\
12,039,263 \\
-\end{array}$ & $\begin{array}{r}14,626 \\
173,007 \\
-\quad \\
118,405 \\
55,502 \\
-\end{array}$ & $\begin{array}{r}28,674 \\
468,375 \\
- \\
- \\
-\end{array}$ & $\begin{array}{l}\overline{-} \\
\overline{-} \\
\bar{m}\end{array}$ & $\begin{array}{r}40,464 \\
622,570 \\
- \\
- \\
-622,570\end{array}$ & $\begin{array}{l}\overline{-} \\
\bar{m} \\
\bar{m}\end{array}$ \\
\hline 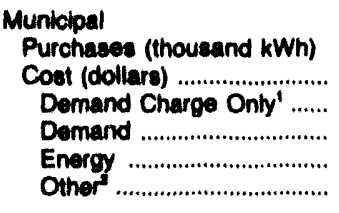 & $\begin{array}{l}18,280 \\
1,088,800 \\
- \\
- \\
1,086,800\end{array}$ & $\begin{array}{l}1,684 \\
103,607 \\
-- \\
- \\
-\end{array}$ & 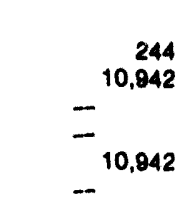 & $\begin{array}{r}30,657 \\
1,877,448 \\
= \\
- \\
1,877,448\end{array}$ & $\begin{array}{l}m \\
m \\
m \\
m\end{array}$ & $\begin{array}{l}\bar{m} \\
\bar{m} \\
\bar{m}\end{array}$ & $\begin{array}{l}\overline{-} \\
\overline{-} \\
\overline{-}\end{array}$ \\
\hline 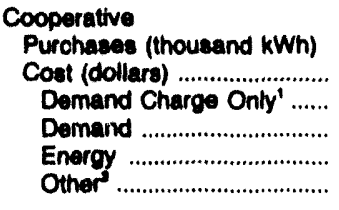 & $\begin{array}{l}\overline{-} \\
\overline{-} \\
\bar{m}\end{array}$ & $\begin{array}{l}- \\
\overline{-} \\
\overline{-} \\
\overline{-}\end{array}$ & $\begin{array}{r}28,593 \\
299,437 \\
- \\
- \\
299,437\end{array}$ & $\begin{array}{r}35,207 \\
666,931 \\
- \\
- \\
-\end{array}$ & $\begin{array}{l}\overline{-} \\
\bar{m} \\
\bar{m}\end{array}$ & $\begin{array}{l}= \\
\overline{-} \\
= \\
-\end{array}$ & $\begin{array}{l}\overline{-} \\
\overline{-} \\
\bar{m}\end{array}$ \\
\hline 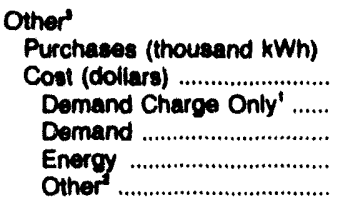 & $\begin{array}{l}156,170 \\
3,481,036 \\
\overline{-} \\
\overline{3,481,036}\end{array}$ & $\begin{array}{r}1,870,615 \\
82,093,374 \\
\overline{1,167,267} \\
80,924,118 \\
1,889\end{array}$ & $\begin{array}{r}1,942,391 \\
86,750,378 \\
-- \\
9,852,329 \\
87,699,156 \\
-801,107\end{array}$ & $\begin{array}{r}3,891,770 \\
197,483,440 \\
- \\
2,760,093 \\
194,522,917 \\
210,430\end{array}$ & $\begin{array}{r}771 \\
12,151 \\
-\quad \\
-\quad 12,151 \\
-\end{array}$ & $\begin{array}{r}8,633,305 \\
194,459,288 \\
\overline{24,056,495} \\
170,311,088 \\
91,705\end{array}$ & $\begin{array}{l}-101,389 \\
- \\
- \\
- \\
101,389\end{array}$ \\
\hline 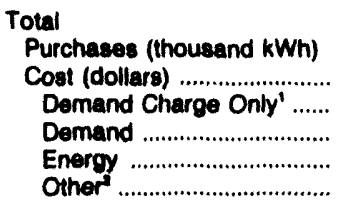 & $\begin{array}{r}581,563 \\
11,280,901 \\
\overline{-} \\
\overline{11,280,901}\end{array}$ & $\begin{array}{r}4,672,958 \\
187,143,808 \\
13,352,759 \\
37,083,449 \\
136,705,411 \\
1,889\end{array}$ & $\begin{array}{r}3,180,125 \\
128,410,779 \\
3,710,573 \\
18,064,891 \\
107,345,501 \\
-710,186\end{array}$ & $\begin{array}{r}5,347,042 \\
275,498,001 \\
-\quad \\
2,774,493 \\
263,081,004 \\
9,643,404\end{array}$ & $\begin{array}{l}798,201 \\
14,242,333 \\
\overline{-} \\
14,242,333\end{array}$ & $\begin{array}{r}10,343,516 \\
214,178,439 \\
-7,056,495 \\
24,056,485 \\
170,978,613 \\
19,144,331\end{array}$ & $\begin{array}{r}47,326 \\
2,469,181 \\
668,216 \\
1,699,576 \\
101,389\end{array}$ \\
\hline
\end{tabular}

See notes and footnotes at end of table. 
Table 19. Eleotriclty Purchases by Inveotor-Owned Utilities, by 8 tato, 1992 (Continued)

\begin{tabular}{|c|c|c|c|c|c|c|c|}
\hline $\begin{array}{c}\text { soures of } \\
\text { ateotrloity by } \\
\text { owmerehtip claces }\end{array}$ & $\begin{array}{l}\text { Pennoytvania } \\
\text { Sale Hartor } \\
\text { Water } \\
\text { Power } \\
\text { Corp' }\end{array}$ & $\begin{array}{l}\text { Pennaytvania } \\
\text { Suequehanna } \\
\text { Electric } \\
\text { Co }^{\circ}\end{array}$ & $\begin{array}{l}\text { Pennoytvania } \\
\text { Uail } \\
\text { Corp }\end{array}$ & $\begin{array}{l}\text { Pennoytvania } \\
\text { Weat } \\
\text { Pann } \\
\text { Power } \\
\text { Co }\end{array}$ & $\begin{array}{c}\text { Penns itvania } \\
\text { y xk } \\
\text { Hi ven } \\
\text { Po ver } \\
\text { Co' }\end{array}$ & $\begin{array}{l}\text { Ahode laland } \\
\text { Blackstone } \\
\text { Valley } \\
\text { Electric } \\
\text { Co' }\end{array}$ & $\begin{array}{l}\text { Ahode laland } \\
\text { Narraganeett } \\
\text { Eloctrle } \\
\text { Co }\end{array}$ \\
\hline \multicolumn{8}{|l|}{ Inveotor-Owned } \\
\hline $\begin{array}{l}\text { Purchases (thousand KWh) } \\
\text { Cost (dollerg) }\end{array}$ & $\bar{z}$ & $\overline{-}$ & $\begin{array}{r}414,504 \\
\end{array}$ & $\begin{array}{r}7,087.268 \\
207380.410\end{array}$ & - & $\begin{array}{r}1,274,074 \\
05,043,530\end{array}$ & $\begin{array}{r}4,730,680 \\
288,384,287\end{array}$ \\
\hline Demand Charge Only' ....... & $\overline{-}$ & $\bar{z}$ & $24,201,000$ & $201,380,410$ & $\overline{-}$ & $\begin{array}{l}80,043,030 \\
-\end{array}$ & $200,384,281$ \\
\hline 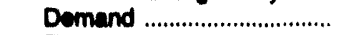 & - & -- & $13,881,742$ & $61,079,398$ & - & $74,264,316$ & $140,483,483$ \\
\hline Energy n................................. & - & - & $10,669,354$ & $102,465,289$ & - & $17,781,714$ & $84,468,731$ \\
\hline 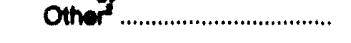 & - & - & - & $43,835,723$ & - & $2,897,500$ & $51,444,073$ \\
\hline \multicolumn{8}{|l|}{ Federal } \\
\hline Purchases (thousand kWh) & - & - & - & - & - & - & - \\
\hline Coot (dollars) ......................... & - & - & - & - & - & - & - \\
\hline Demand Charge Only' ...... & - & - & - & -- & -- & - & - \\
\hline 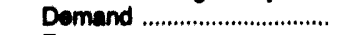 & - & - & $\cdots$ & -- & - & - & - \\
\hline 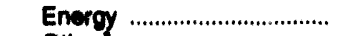 & - & - & - & -- & - & - & - \\
\hline 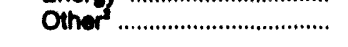 & - & - & - & - & - & - & - \\
\hline \multicolumn{8}{|l|}{$\begin{array}{l}\text { State and Other } \\
\text { Government }\end{array}$} \\
\hline Purchases (thousand kWh) & - & - & -- & - & - & - & - \\
\hline Cost (dollara) ............................... & - & - & - & - & - & - & - \\
\hline Demand Charge Only' ...... & - & - & - & - & - & - & - \\
\hline 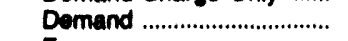 & - & - & - & - & - & - & - \\
\hline 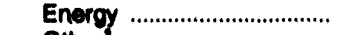 & - & - & -- & - & - & - & - \\
\hline 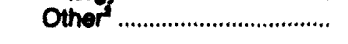 & - & - & - & - & - & - & - \\
\hline \multicolumn{8}{|l|}{ Municipal } \\
\hline & - & - & - & - & -- & - & -- \\
\hline Cost (dollars) ........................ & - & - & - & - & - & - & -- \\
\hline & - & - & - & - & -- & - & - \\
\hline Demand & - & - & - & - & - & - & - \\
\hline Energy .................................. & - & - & - & - & - & - & - \\
\hline Other ........................................... & - & - & -- & - & - & - & - \\
\hline \multicolumn{8}{|l|}{ Cooperative } \\
\hline Purchases (thousand kWh) & - & - & - & - & - & - & -- \\
\hline Cost (dollars) ......................... & - & - & - & - & - & - & - \\
\hline Demand Charge Only' ....... & - & - & - & - & - & - & -- \\
\hline 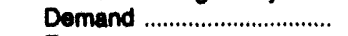 & - & - & -- & -- & - & -- & - \\
\hline 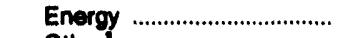 & - & - & - & - & -- & $\cdots$ & - \\
\hline 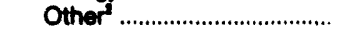 & - & - & - & - & - & - & - \\
\hline \multicolumn{8}{|l|}{ Other" } \\
\hline Purchases (thousand kWh) & - & - & - & $1,060,733$ & -- & 1,755 & 1,302 \\
\hline 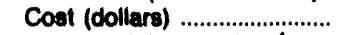 & $\ldots .$. & - & - & $56,554,434$ & - & 29,453 & 99,293 \\
\hline Demand Charge Only' ....... & - & - & - & - & - & - & -- \\
\hline 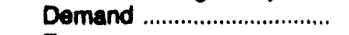 & - & - & - & $36,449,252$ & - & - & - \\
\hline Energy & - & - & - & $20,105,182$ & - & 29,453 & 99,293 \\
\hline 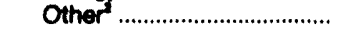 & - & - & - & - & - & - & - \\
\hline \multicolumn{8}{|l|}{ Total } \\
\hline Purchases (thousand kWh) & - & -- & 414,564 & $8,128,001$ & -- & $1,275,829$ & 4,731,982 \\
\hline 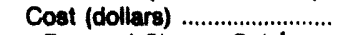 & - & - & $24,251,096$ & $263,834,844$ & - & $95,072,983$ & $286,483,580$ \\
\hline Demand Charge Only' ....... & - & - & - & - & - & - & - \\
\hline 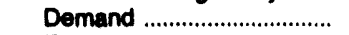 & - & - & $13,581,742$ & $97,528,650$ & - & $74,264,316$ & $140,483,483$ \\
\hline 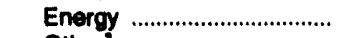 & - & - & $10,669,354$ & $122,570,471$ & - & $17,811,167$ & $94,556,024$ \\
\hline Other" & - & - & - & $43,835,723$ & - & $2,997,500$ & $51,444,073$ \\
\hline
\end{tabular}

See notes and footnotes at end of table. 
Table 19. Electricity Purchases by Investor-Owned Utilities, by State, 1992 (Continued)

\begin{tabular}{|c|c|c|c|c|c|c|c|}
\hline $\begin{array}{c}\text { Source of } \\
\text { Electriclty by } \\
\text { Ownorahip clases }\end{array}$ & $\begin{array}{l}\text { Rhode Island } \\
\text { Newport } \\
\text { Electric } \\
\text { Corp }\end{array}$ & $\begin{array}{l}\text { Rhode Island } \\
\text { Ocean } \\
\text { State } \\
\text { Power } \\
\text { ' } 2^{8}\end{array}$ & $\begin{array}{l}\text { Rhode island } \\
\text { Ocean } \\
\text { State } \\
\text { Power } \\
\text { Co" }\end{array}$ & $\begin{array}{l}\text { South Carolina } \\
\text { Lockhart } \\
\text { Power } \\
\text { Co }\end{array}$ & $\begin{array}{l}\text { South Carolina } \\
\text { South Carolina } \\
\text { Electric \& } \\
\text { Gas } \\
\text { Co }\end{array}$ & $\begin{array}{l}\text { South Carolina } \\
\text { South Carolina } \\
\text { Generating } \\
\text { Co Inc }\end{array}$ & $\begin{array}{l}\text { South Dakota } \\
\text { Black Hills } \\
\text { Corp }\end{array}$ \\
\hline \multicolumn{8}{|l|}{ Investor-Owned } \\
\hline $\begin{array}{l}\text { Purchases (thousand kWh) } \\
\text { Cost (dollars) }\end{array}$ & 557,661 & - & $\overline{-}$ & 229,752 & $3,019,127$ & - & 368,222 \\
\hline 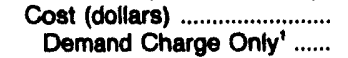 & $33,935,806$ & $\overline{-}$ & $\overline{-}$ & $11,350,311$ & $78,353,624$ & $\overline{-}$ & $23,349,774$ \\
\hline & $\overline{27,303,519}$ & - & $\overline{-}$ & $6,044,252$ & $\overline{-}$ & - & $\overline{18,698,489}$ \\
\hline 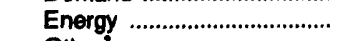 & $5,735,887$ & - & - & $6,441,453$ & $78,353,624$ & - & $4,642,183$ \\
\hline Other & 896,400 & - & - & $-1,135,394$ & - & - & 9,102 \\
\hline \multicolumn{8}{|l|}{ Federal } \\
\hline Purchases (thousand kWh) & - & - & - & - & - & - & - \\
\hline Cost (dollars) & - & - & - & - & - & - & 87,232 \\
\hline Demand Charge Only' ...... & - & - & - & 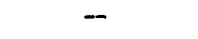 & - & - & - \\
\hline 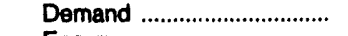 & - & - & - & - & -- & - & - \\
\hline 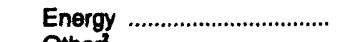 & - & - & - & - & - & - & -- \\
\hline 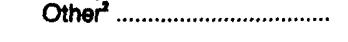 & - & - & - & - & - & - & 87,232 \\
\hline \multicolumn{8}{|l|}{$\begin{array}{l}\text { State and Other } \\
\text { Government }\end{array}$} \\
\hline 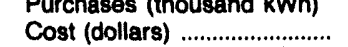 & $\overline{-}$ & -- & - & - & 365,199 & - & $\begin{array}{r}17,974 \\
462,765\end{array}$ \\
\hline Demand Charge Only' ....... & - & - & - & - & - & - & $-\infty 0<, 100$ \\
\hline 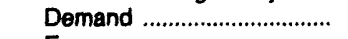 & - & - & - & - & - & - & - \\
\hline 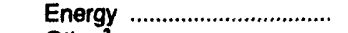 & - & - & - & -- & 365,199 & - & 462,765 \\
\hline Other & - & - & - & - & - & - & - \\
\hline \multicolumn{8}{|l|}{ Municipal } \\
\hline Purchases (thousand kWh) & 23,828 & - & - & - & - & -- & - \\
\hline Cost (dollars) ................................ & $3,911,380$ & -- & - & - & - & - & - \\
\hline Demand Charge Only' ....... & - & - & - & - & - & - & - \\
\hline 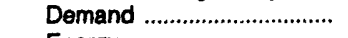 & $3,064,872$ & -- & - & - & - & - & - \\
\hline 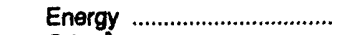 & 846,508 & - & - & - & - & -- & - \\
\hline 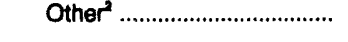 & - & - & - & - & - & - & - \\
\hline \multicolumn{8}{|l|}{ Cooperative } \\
\hline Purchases (thousand kWh) & -- & - & - & -- & 25 & - & 10,345 \\
\hline 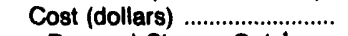 & -- & -- & -- & - & 3,609 & - & 213,402 \\
\hline Demand Charge Only' ...... & - & -- & - & -- & -- & - & - \\
\hline 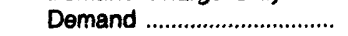 & - & - & - & -- & -- & - & - \\
\hline 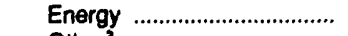 & - & - & - & - & 3,609 & -- & 213,402 \\
\hline 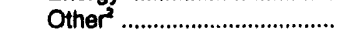 & - & - & - & - & - & - & - \\
\hline \multicolumn{8}{|l|}{ Other' } \\
\hline Purchases (thousand kWh) & 86,036 & - & - & 3,710 & 61,765 & - & - \\
\hline 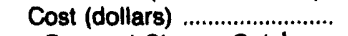 & $2,558,565$ & - & - & 142,308 & $1,708,523$ & - & - \\
\hline Demand Charge Only' ...... & -- & - & - & - & - & - & - \\
\hline 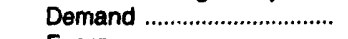 & $1,597,797$ & - & - & - & $-\overline{7}$ & - & - \\
\hline 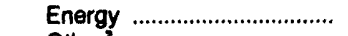 & $1,455,268$ & - & - & 85.455 & $1,708,523$ & -- & -- \\
\hline Other & $-494,500$ & - & - & 56,853 & - & -- & - \\
\hline \multicolumn{8}{|l|}{ Total } \\
\hline Purchases (thousand kWh) & 667,525 & - & - & 233,462 & $3,099,745$ & - & 396,541 \\
\hline 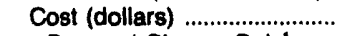 & $40,405,751$ & - & - & $11,492,619$ & $80,430,955$ & - & $24,113,173$ \\
\hline Demand Charge Only' ....... & - & - & - & - & - & - & - \\
\hline Demand & $31,966,188$ & - & - & $6,044,252$ & - & - & $18,698,489$ \\
\hline 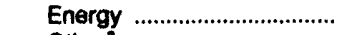 & $8,037,663$ & - & - & $6,526,908$ & $80,430,955$ & - & $5,318,350$ \\
\hline Other $r^{2}$ & 401,900 & - & - & $-1,078,541$ & - & - & 96,334 \\
\hline
\end{tabular}

See notes and footnotes at end of table. 
Table 19. Electricity Purchases by Investor-Owned Utilitles, by State, 1992 (Continued)

\begin{tabular}{|c|c|c|c|c|c|c|c|}
\hline $\begin{array}{c}\text { source of } \\
\text { Electrictly by } \\
\text { Ownerahip Clates }\end{array}$ & $\begin{array}{l}\text { South Dakota } \\
\text { Northwestern } \\
\text { Public } \\
\text { Service } \\
\text { Co }\end{array}$ & $\begin{array}{l}\text { Tennessee } \\
\text { Kingsport } \\
\text { Power } \\
\text { Co4 }\end{array}$ & $\begin{array}{c}\text { Tennessee } \\
\text { Tapoco } \\
\text { Inc }^{\circ}\end{array}$ & $\begin{array}{l}\text { Texas } \\
\text { Central } \\
\text { Power \& } \\
\text { Light } \\
\text { Co }\end{array}$ & $\begin{array}{c}\text { Texas } \\
\text { El Paso } \\
\text { Electric } \\
\text { Co }\end{array}$ & $\begin{array}{c}\text { Texas } \\
\text { Gult } \\
\text { States } \\
\text { Utillities } \\
\text { Co }\end{array}$ & $\begin{array}{l}\text { Texas } \\
\text { Houston } \\
\text { Lighting } \\
\text { \& Power } \\
\text { Co }\end{array}$ \\
\hline \multicolumn{8}{|l|}{ Investor-Owned } \\
\hline $\begin{array}{l}\text { Purchases (thousand kWh) } \\
\text { Cost (dollars) }\end{array}$ & $\begin{array}{r}3,079 \\
50,880\end{array}$ & $\begin{array}{r}1,871,580 \\
58,909,992\end{array}$ & - & $\overline{-}$ & $\begin{array}{r}606,015 \\
15,856,253\end{array}$ & $\begin{array}{r}246,830 \\
18,980,361\end{array}$ & $\begin{array}{r}191,217 \\
3,552,762\end{array}$ \\
\hline Demand Charge Only' ....... & $-{ }^{50,800}$ & - & $\overline{-}$ & $\overline{-}$ & $15,800,253$ & $18,080,361$ & $3,052,162$ \\
\hline 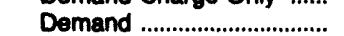 & & $24,910,907$ & - & - & - & - & - \\
\hline 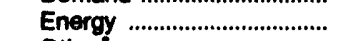 & 50,880 & $33,228,229$ & - & - & $15,840,308$ & $4,608,999$ & $3,552,762$ \\
\hline Other & - & 770,856 & - & - & 15,945 & $14,371,362$ & - \\
\hline \multicolumn{8}{|l|}{ Federal } \\
\hline Purchases (thousand kWh) & 4,119 & - & - & 80,080 & - & - & - \\
\hline Cost (dollars) …............................. & 64,404 & - & -- & 734,370 & 24,707 & -- & - \\
\hline Demand Charge Only' ....... & - & - & - & - & - & - & - \\
\hline Demand ................................. & - & - & - & - & - & - & - \\
\hline 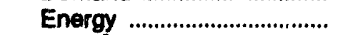 & 64,404 & - & - & 734,370 & -- & - & -- \\
\hline Other & - & - & - & $-\infty$ & 24,707 & - & - \\
\hline \multicolumn{8}{|l|}{$\begin{array}{l}\text { State and Other } \\
\text { Government }\end{array}$} \\
\hline Purchases (thousand kWh) & 25,359 & - & - & 37,200 & 18,180 & 677,712 & 251,465 \\
\hline 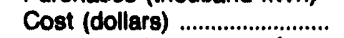 & 348,739 & - & - & 528,360 & 398,017 & $19,658,886$ & $3,919,159$ \\
\hline Demand Charge Only' ...... & - & - & - & - & - & - & - \\
\hline 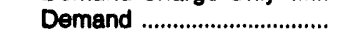 & - & - & - & - & - & - & - \\
\hline Energy ………........................... & 348,739 & -- & -- & 528,360 & 390,795 & $8,459,369$ & $3,919,159$ \\
\hline 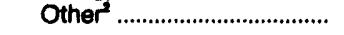 & - & -- & - & - & 7,222 & $11,199,517$ & - \\
\hline \multicolumn{8}{|l|}{ Municipal } \\
\hline Purchases (thousand kWh) & 585 & - & - & -- & -- & 23 & 54,604 \\
\hline Cost (dollars) & 7,197 & - & - & - & 3,923 & 805 & 898,671 \\
\hline Demand Charge Only' ....... & - & -- & - & - & - & - & - \\
\hline Demand ………………........... & -- & - & -- & - & -- & -- & -- \\
\hline 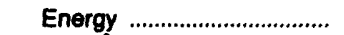 & 7,197 & - & - & - & -- & 805 & 888,671 \\
\hline Other & -- & -- & - & - & 3,923 & -- & - \\
\hline \multicolumn{8}{|l|}{ Cooperative } \\
\hline Purchases (thousand kWh) & 27,181 & - & - & - & 29,621 & 493,690 & 14,376 \\
\hline Cost (dollars) ................................ & 370,136 & - & -- & -- & 575,570 & $8,065,993$ & 185,486 \\
\hline Demand Charge Only' ...... & - & - & -- & -- & -- & $\ldots$ & - \\
\hline 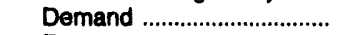 & 2,584 & - & - & - & -- & -- & - \\
\hline 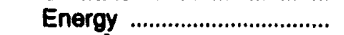 & 367,552 & - & -- & -- & 556,889 & $8,069,629$ & 185,496 \\
\hline Other ${ }^{2}$.................................. & - & - & - & - & 18,681 & $-3,636$ & - \\
\hline \multicolumn{8}{|l|}{ Other' } \\
\hline Purchases (thousand kWh) & - & -- & - & 926,967 & $-2,426$ & $3,517,214$ & $11,026,310$ \\
\hline Cost (dollars) ……....................... & - & -- & - & $15,894,824$ & -- & $90,010,258$ & $477,857,681$ \\
\hline Demand Charge Only'...... & - & - & - & - & - & - & - \\
\hline 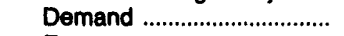 & - & -- & - & - & -- & - & $183,351,855$ \\
\hline Energy ............................... & - & - & - & $15,631,974$ & -- & $86,014,970$ & $284,505,826$ \\
\hline Other & - & -- & - & 262,850 & - & $3,995,288$ & - \\
\hline \multicolumn{8}{|l|}{ Total } \\
\hline Purchases (thousand kWh) & 60,323 & $1,871,580$ & - & $1,044,247$ & 651,390 & $4,935,469$ & $11,537,972$ \\
\hline Cost (dollars) .............................. & 841,356 & $58,909,982$ & - & $17,157,554$ & $16,858,470$ & $136,716,303$ & $486,413,759$ \\
\hline Demand Charge Only' ...... & & - & - & - & - & - & - \\
\hline Demand ................................ & 2,534 & $24,910,907$ & - & - & - & -- & $193,351,855$ \\
\hline 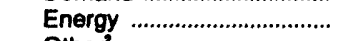 & 838,772 & $33,228,229$ & - & $16,894,704$ & $16,787,992$ & $107,153,772$ & $293,061,904$ \\
\hline 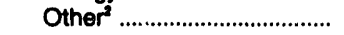 & - & 770,856 & - & 262,850 & 70,478 & $29,562,531$ & - \\
\hline
\end{tabular}

Soe notes and footnotes at end of table. 
Table 19. Electricity Purchases by Investor-Owned Utilities, by State, 1992 (Continued)

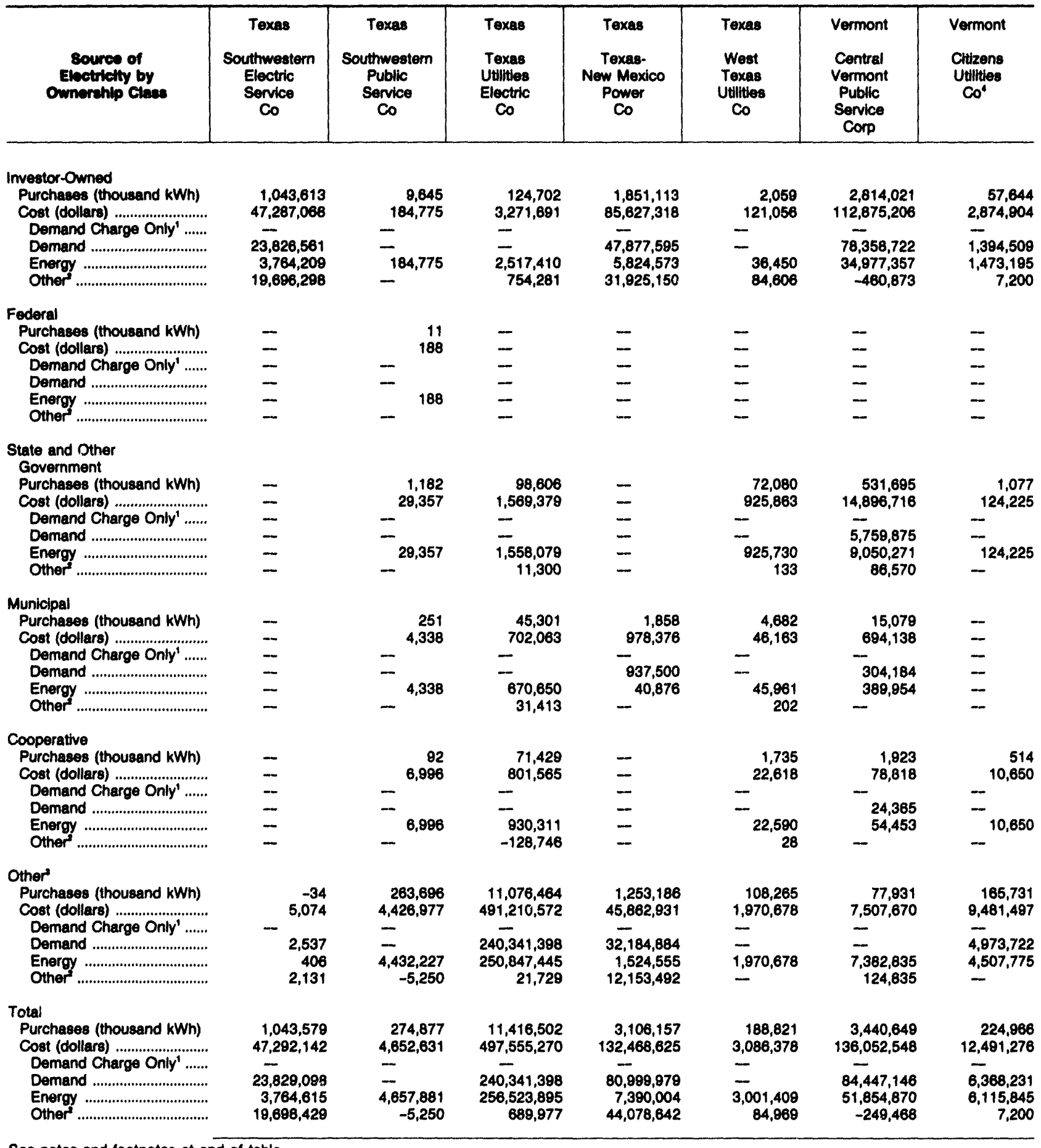

See notes and footnotes at end of table. 
Table 19. Electricity Purchases by Investor-Owned Utilities, by State, 1992 (Continued)

\begin{tabular}{|c|c|c|c|c|c|c|c|}
\hline $\begin{array}{c}\text { source of } \\
\text { Eloctriclty by } \\
\text { Owmerahip Clases }\end{array}$ & $\begin{array}{l}\text { Vermont } \\
\text { Green } \\
\text { Mountain } \\
\text { Power } \\
\text { Corp }\end{array}$ & $\begin{array}{l}\text { Vermont } \\
\text { Vermont } \\
\text { Electric } \\
\text { Power } \\
\text { Co Inc }\end{array}$ & $\begin{array}{l}\text { Vermont } \\
\text { Vermont } \\
\text { Electric } \\
\text { Trans } \\
\mathrm{Co}^{\circ}\end{array}$ & $\begin{array}{l}\text { Vermont } \\
\text { Vermont } \\
\text { Marble } \\
\text { Co }^{*}\end{array}$ & $\begin{array}{l}\text { Vermont } \\
\text { Vermont } \\
\text { Yankes } \\
\text { Nuclear } \\
\text { Power } \\
\text { Corp" }\end{array}$ & $\begin{array}{l}\text { Virginia } \\
\text { Appalachian } \\
\text { Power } \\
\text { Co }\end{array}$ & $\begin{array}{l}\text { Virginia } \\
\text { Virginia } \\
\text { Electric } \\
\text { Power } \\
\text { Co }\end{array}$ \\
\hline \multicolumn{8}{|l|}{ Investor-Owned } \\
\hline $\begin{array}{l}\text { Purchases (thousand kWh) } \\
\text { Cost (dollars) }\end{array}$ & $1,005,654$ & 858,517 & - & - & $\overline{-}$ & $\begin{array}{r}1,302,473 \\
\end{array}$ & $\begin{array}{r}2,882,488 \\
\end{array}$ \\
\hline 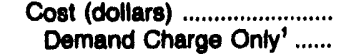 & $39,471,861$ & 30,341,819 & $\overline{-}$ & $\overline{-}$ & $\overline{-}$ & $20,381,208$ & $\begin{array}{r}14,814,087 \\
5,520,000\end{array}$ \\
\hline 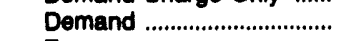 & $29,895,545$ & $18,309,787$ & - & - & - & $3,105,601$ & $58,921,928$ \\
\hline 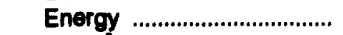 & $11,098,425$ & $11,244,306$ & - & - & -- & $17,275,607$ & $50,472,159$ \\
\hline 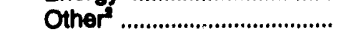 & $-1,522,109$ & 787,726 & - & - & - & - & - \\
\hline \multicolumn{8}{|l|}{ Foderal } \\
\hline Purchases (thousend kWh) & - & - & - & - & - & 5,339 & - \\
\hline 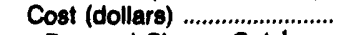 & - & - & - & - & - & 113,262 & - \\
\hline Demand Charge Only' ...... & - & - & - & - & - & - & - \\
\hline 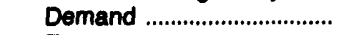 & - & - & - & -- & - & - & - \\
\hline 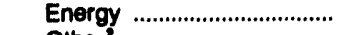 & - & - & - & - & - & 113,262 & - \\
\hline 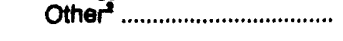 & - & - & - & - & -- & - & - \\
\hline \multicolumn{8}{|l|}{$\begin{array}{l}\text { State and Other } \\
\text { Government }\end{array}$} \\
\hline Purchases (thousand kWh) & 41,599 & - & - & - & - & - & 1,979 \\
\hline 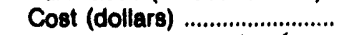 & $1,800,755$ & -- & - & - & - & - & 128,826 \\
\hline Demand Charge Only' ....... & - & - & - & - & - & - & - \\
\hline 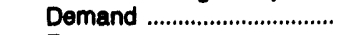 & 798,201 & - & - & - & - & - & 7,817 \\
\hline 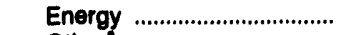 & $1,002,554$ & - & - & - & -- & - & 121,109 \\
\hline Other & - & - & -- & - & - & -- & - \\
\hline \multicolumn{8}{|l|}{ Municipal } \\
\hline Purchases (thousand kWh) & 54,783 & - & -- & - & - & 36,190 & 8,816 \\
\hline 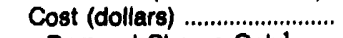 & $2,959,744$ & -- & - & - & -- & 472,515 & 635,003 \\
\hline Demand Charge Only' ...... & - & - & - & -- & - & - & - \\
\hline Demand & $2,047,925$ & - & - & - & -- & - & 420,467 \\
\hline 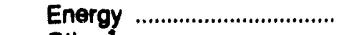 & 911,819 & - & - & -- & -- & 472,515 & 214,536 \\
\hline Other & - & - & - & - & - & - & - \\
\hline \multicolumn{8}{|l|}{ Cooperative } \\
\hline Purchases (thousand kWh) & 28,652 & - & 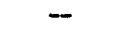 & - & - & 29,692 & $2,112,282$ \\
\hline 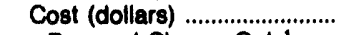 & 718,880 & -- & - & - & - & 560,751 & $92,447,291$ \\
\hline Demand Charge Only' ....... & -- & - & - & - & - & -- & - \\
\hline 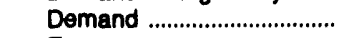 & 391,290 & -- & -- & - & - & - & $34,151,591$ \\
\hline Energy & 327,590 & - & - & - & - & 560,751 & $58,295,700$ \\
\hline Other & - & - & - & -- & -- & - & - \\
\hline \multicolumn{8}{|l|}{ Other" } \\
\hline Purchases (thousand kWh) & 868,731 & - & - & - & - & $\begin{array}{r}10,067,552 \\
\end{array}$ & $\begin{array}{r}7,029,965 \\
\end{array}$ \\
\hline 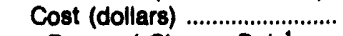 & $26,156,303$ & - & $\overline{-}$ & $=$ & $\overline{-}$ & $264,911,528$ & $557,852,827$ \\
\hline Demand Charge Only' ....... & 5,138 & - & - & - & - & - & $-21,000$ \\
\hline 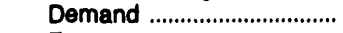 & $5,955,899$ & - & - & - & - & -- & $352,520,931$ \\
\hline 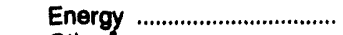 & $20,195,266$ & - & - & -- & -- & $264,911,528$ & $205,352,896$ \\
\hline 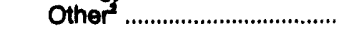 & - & - & - & - & - & -- & - \\
\hline \multicolumn{8}{|l|}{ Total } \\
\hline Purchases (thousand kWh) & $2,000,419$ & 853,517 & -- & - & - & $11,441,246$ & $12,045,530$ \\
\hline 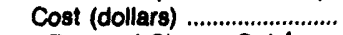 & $71,107,543$ & $30,341,819$ & - & - & -- & $286,439,264$ & $765,978,134$ \\
\hline Demand Charge Only' ...... & 5,138 & - & - & - & - & - & $5,489,000$ \\
\hline Demand & $39,083,860$ & 18,309787 & - & - & - & $3,105,601$ & $446,022,734$ \\
\hline 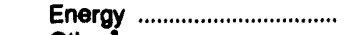 & $33,535,654$ & $11,244,306$ & - & - & - & $283,333,663$ & $314,456,400$ \\
\hline Other & $-1,522,108$ & 787,726 & -- & - & -- & - & - \\
\hline
\end{tabular}

See notes and footnotes at end of table. 
Table 19. Electricity Purchases by Investor-Owned Utilities, by State, 1992 (Continued)

\begin{tabular}{|c|c|c|c|c|c|c|c|}
\hline $\begin{array}{c}\text { Source of } \\
\text { Electricity by } \\
\text { Ownerahip Clase }\end{array}$ & $\begin{array}{c}\text { Washington } \\
\text { Puget } \\
\text { Sound } \\
\text { Power \& } \\
\text { Light } \\
\text { Co }\end{array}$ & $\begin{array}{l}\text { Washington } \\
\text { Washington } \\
\text { Water } \\
\text { Power } \\
\text { Co }\end{array}$ & $\begin{array}{c}\text { West Virginia } \\
\text { Kanawha } \\
\text { Valley } \\
\text { Power } \\
\text { Co" }\end{array}$ & $\begin{array}{l}\text { West Virginia } \\
\text { Monongahela } \\
\text { Power } \\
\text { Co }\end{array}$ & $\begin{array}{c}\text { West Virginia } \\
\text { UtillCorp } \\
\text { United } \\
\text { Inc4 }\end{array}$ & $\begin{array}{l}\text { Weat Virginia } \\
\text { Wheeling } \\
\text { Power } \\
\text { Co4 }\end{array}$ & $\begin{array}{l}\text { Wisconsin } \\
\text { Consolidated } \\
\text { Water } \\
\text { Power } \\
\text { Co }\end{array}$ \\
\hline \multicolumn{8}{|l|}{ Investor-Owned } \\
\hline 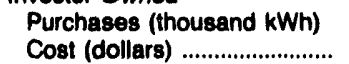 & $\begin{array}{r}2,570,645 \\
82,665,434\end{array}$ & $\begin{array}{r}914,746 \\
18,912,330\end{array}$ & - & $\begin{array}{r}3,939,415 \\
115,542,450\end{array}$ & $\begin{array}{r}379,960 \\
13,456,215\end{array}$ & $\begin{array}{r}1,861,253 \\
62,095,484\end{array}$ & $\begin{array}{r}1,013,146 \\
27,517,787\end{array}$ \\
\hline Demand Charge Only' ....... & 300,000 & 300,000 & - & - & - & - & - \\
\hline Demand & $\begin{array}{l}35,023,764 \\
47,341,670\end{array}$ & $\begin{array}{r}425,000 \\
18,167,330\end{array}$ & - & $\begin{array}{l}33,598,194 \\
57,038,887\end{array}$ & $\begin{array}{l}6,473,001 \\
7,274,300\end{array}$ & $\begin{array}{l}18,634,070 \\
43,461,414\end{array}$ & $\begin{array}{r}8,351,816 \\
19,165,971\end{array}$ \\
\hline 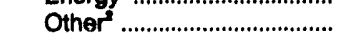 & - & - & - & $24,907,369$ & $-291,086$ & - & - \\
\hline \multicolumn{8}{|l|}{ Federal } \\
\hline Purchases (thousand kWh) & $2,709,624$ & 984,570 & - & - & - & - & -- \\
\hline 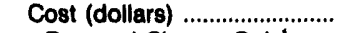 & $77,007,988$ & $36,082,043$ & - & - & - & - & - \\
\hline Demand Charge Only' ...... & - & -- & - & - & - & - & -- \\
\hline 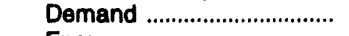 & 556,084 & -- & - & -- & - & - & - \\
\hline 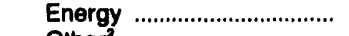 & $72,711,415$ & $35,925,086$ & - & - & - & - & -- \\
\hline Other ${ }^{2}$ & $3,740,489$ & 156,957 & - & - & - & - & - \\
\hline \multicolumn{8}{|l|}{$\begin{array}{l}\text { State and Other } \\
\text { Government }\end{array}$} \\
\hline $\begin{array}{l}\text { Government } \\
\text { Purchases (thousand kWh) }\end{array}$ & $7,000,784$ & $1,512,649$ & - & - & - & - & $-\infty$ \\
\hline 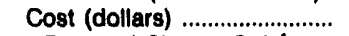 & $79,714,042$ & $12,530,254$ & - & - & - & - & - \\
\hline Demand Charge Only' ....... & - & -- & - & - & - & - & - \\
\hline 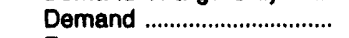 & 609,750 & - & - & - & - & - & - \\
\hline 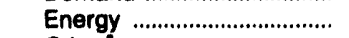 & $79,104,292$ & $12,530,254$ & - & - & - & - & - \\
\hline Other & - & - & - & - & - & - & - \\
\hline \multicolumn{8}{|l|}{ Municipal } \\
\hline Purchases (thousand kWh) & 275,301 & 111,167 & - & 268,361 & - & - & - \\
\hline 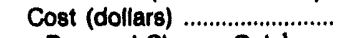 & $5,997,344$ & $2,726,738$ & - & $20,367,826$ & - & - & - \\
\hline Demand Charge Only' ...... & - & - & - & - & - & - & - \\
\hline 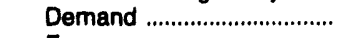 & - & - & - & $9,901,903$ & - & - & - \\
\hline 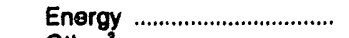 & $5,997,344$ & $2,726,738$ & - & $10,465,923$ & - & - & - \\
\hline 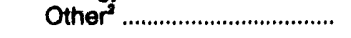 & -- & - & - & -- & - & -- & - \\
\hline \multicolumn{8}{|l|}{ Cooperative } \\
\hline Purchases (thousand kWh) & - & - & - & - & - & - & - \\
\hline 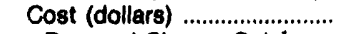 & - & 2,771 & - & - & -- & - & - \\
\hline Demand Charge Only' ...... & - & - & -- & - & -- & - & -- \\
\hline Demand .................................... & - & - & - & - & - & -- & - \\
\hline Energy .................................. & - & 2,771 & - & -- & - & -- & - \\
\hline Other $r^{2}$ & - & - & - & - & - & - & - \\
\hline \multicolumn{8}{|l|}{ Other' } \\
\hline Purchases (thousand kWh) & 776,658 & 727,220 & -- & 485,052 & -- & - & - \\
\hline 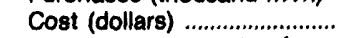 & $-8,029,611$ & $20,322,414$ & - & $17,097,765$ & - & $-4,029,687$ & -- \\
\hline Demand Charge Only' ...... & - & - & - & $\ldots$ & -- & -- & - \\
\hline 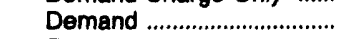 & 238,699 & - & - & $10,337,098$ & - & - & - \\
\hline 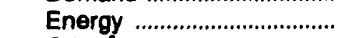 & $45,026,932$ & $20,280,162$ & - & $6,760,669$ & - & - & -- \\
\hline Other & $-53,295,242$ & 42,252 & - & - & - & $-4,029,687$ & - \\
\hline \multicolumn{8}{|l|}{ Total } \\
\hline Purchases (thousand kWh) & $13,333,012$ & $4,250,352$ & - & $4,692,828$ & 379,960 & $1,861,253$ & $\begin{array}{r}1,013,146 \\
27517,787\end{array}$ \\
\hline Cost (dollars) & $237,355,197$ & $90,576,550$ & - & $153,008,041$ & $13,456,215$ & $58,065,797$ & $27,517,787$ \\
\hline Demand Charge Only' ${ }^{\top} . . . .$. & 300,000 & 300,000 & - & $\therefore$ & - & - & - \\
\hline 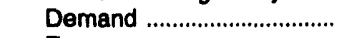 & $36,428,297$ & 425,000 & - & $53,837,193$ & $6,473,001$ & $18,634,070$ & $8,351,816$ \\
\hline 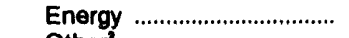 & $250,181,653$ & $89,652,341$ & - & $74,263,479$ & $7,274,300$ & $43,461,414$ & $19,165,971$ \\
\hline Other & $-49,554,753$ & 199,209 & - & $24,907,369$ & $-291,086$ & $-4,029,687$ & - \\
\hline
\end{tabular}

See notes and footnotes at end of table. 
Table 19. Electricity Purchases by Investor-Owned Utilitles, by State, 1992 (Continued)

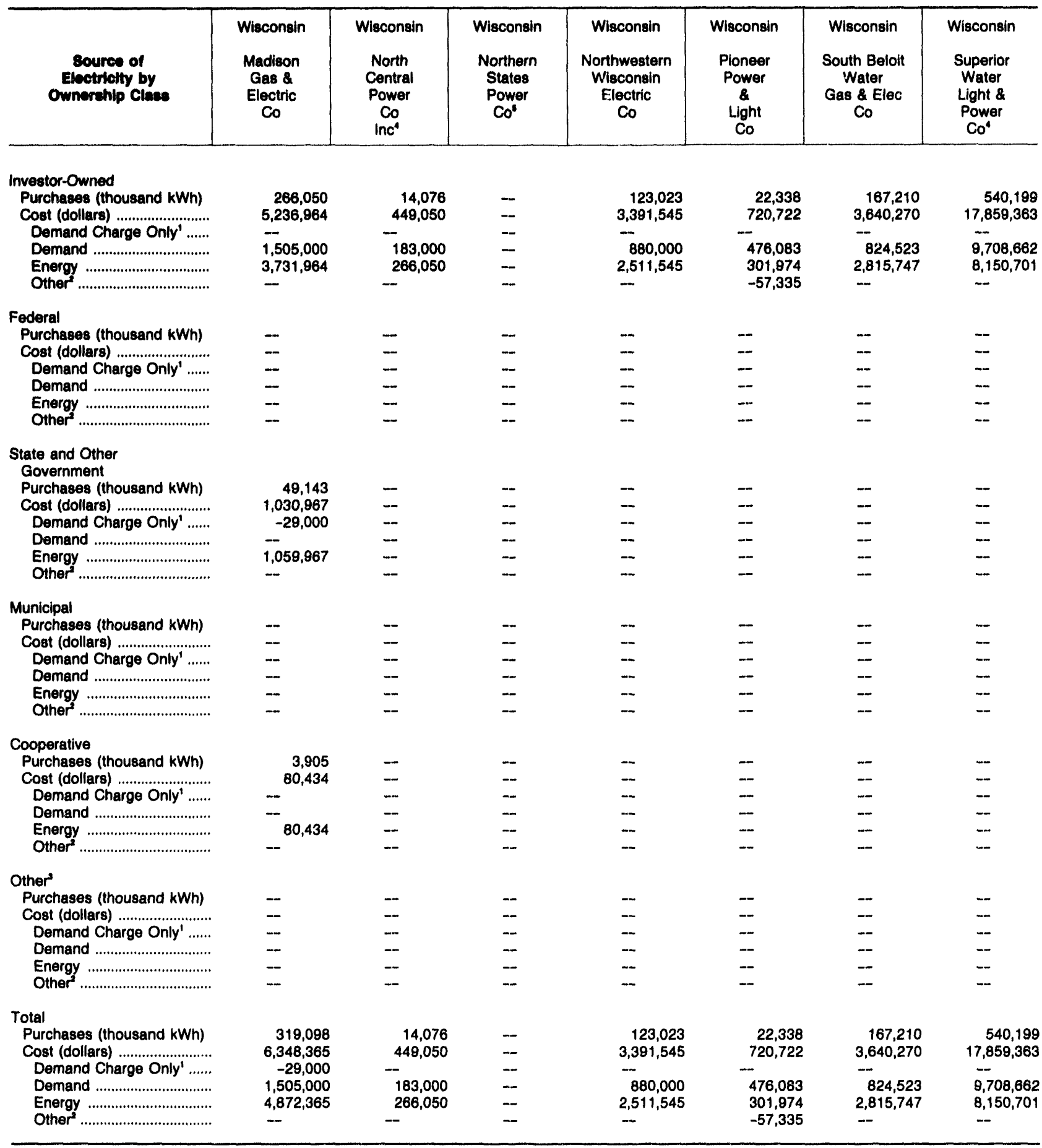

See notes and footnotes at end of table. 
Table 19. Electricity Purchases by Investor-Owned Utilities, by State, 1992 (Continued)

\begin{tabular}{|c|c|c|c|c|c|}
\hline $\begin{array}{c}\text { Source of } \\
\text { Electrlelty by } \\
\text { Ownerchlp clases }\end{array}$ & $\begin{array}{l}\text { Wisconsin } \\
\text { Wisconsin } \\
\text { Electric } \\
\text { Power } \\
\text { Co }\end{array}$ & $\begin{array}{l}\text { Wisconsin } \\
\text { Wisconsin } \\
\text { Power \& } \\
\text { Light } \\
\text { Co }\end{array}$ & $\begin{array}{l}\text { Wisconsin } \\
\text { Wisconsin } \\
\text { Public } \\
\text { Senvice } \\
\text { Corp }\end{array}$ & $\begin{array}{l}\text { Wisconsin } \\
\text { Wisconsin } \\
\text { Alver } \\
\text { Power } \\
\text { Co' }\end{array}$ & $\begin{array}{l}\text { Wyoming } \\
\text { Cheyenne } \\
\text { Light } \\
\text { Fuel \& } \\
\text { Power Co4 }\end{array}$ \\
\hline \multicolumn{6}{|l|}{ Investor-Owned } \\
\hline 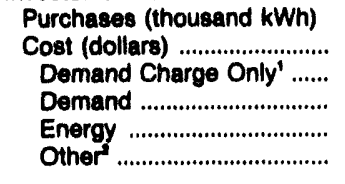 & $\begin{array}{r}2,137,733 \\
54,026,712 \\
716,667 \\
21,377,085 \\
31,924,351 \\
8,609\end{array}$ & $\begin{array}{r}961,428 \\
15,118,523 \\
-\quad \\
23,862 \\
12,308,411 \\
2,786,250\end{array}$ & $\begin{array}{r}1,597,471 \\
28,273,996 \\
1,559,999 \\
-- \\
26,713,997\end{array}$ & $\begin{array}{l}- \\
- \\
- \\
-\end{array}$ & $\begin{array}{r}715,496 \\
20,748,769 \\
\overline{6,991,626} \\
13,745,146 \\
11,997\end{array}$ \\
\hline \multicolumn{6}{|l|}{ Federal } \\
\hline Purchases (thousand kWh) & - & - & - & - & - \\
\hline 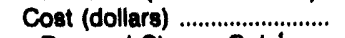 & - & - & - & - & - \\
\hline Demand Charge Only' & - & - & - & - & - \\
\hline 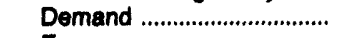 & - & - & - & - & - \\
\hline 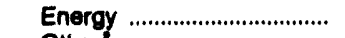 & - & - & - & - & - \\
\hline 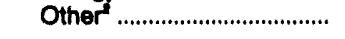 & - & - & - & - & - \\
\hline \multicolumn{6}{|l|}{ State and Other } \\
\hline Purchases (thousand kWh) & 2,525 & 1,775 & 2,460 & - & - \\
\hline 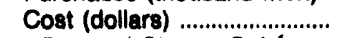 & 42,156 & 29,210 & 41,256 & - & - \\
\hline Demand Charge Only' ...... & - & - & $-\infty$ & - & - \\
\hline 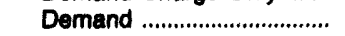 & - & - & -- & - & -- \\
\hline 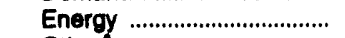 & 42,156 & 29,210 & 41,256 & -- & - \\
\hline 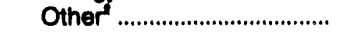 & - & - & - & - & - \\
\hline \multicolumn{6}{|l|}{ Municipal } \\
\hline Purchases (thousand kWh) & 15,501 & - & - & -- & - \\
\hline Cost (dollars) & 854,851 & - & - & - & - \\
\hline Demand Charge Only' ...... & - & - & - & -- & - \\
\hline 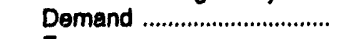 & 409,440 & - & -- & - & - \\
\hline 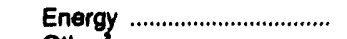 & 445,411 & - & -- & -- & - \\
\hline 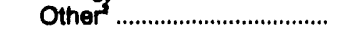 & - & - & - & - & - \\
\hline \multicolumn{6}{|l|}{ Cooperative } \\
\hline Purchases (thousand kWh) & 318,188 & 165,015 & -- & - & - \\
\hline 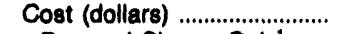 & $6,185,858$ & $9,273,795$ & - & -- & - \\
\hline Demand Charge Only' ...... & - & - & -- & - & - \\
\hline Demand ..................................... & $1,125,000$ & - & - & - & - \\
\hline 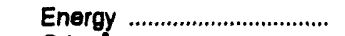 & $5,060,858$ & $3,191,795$ & -- & -- & - \\
\hline Other & - & $6,082,000$ & - & - & - \\
\hline \multicolumn{6}{|l|}{ Other' } \\
\hline Purchases (thousand kWh) & 88,509 & 2,981 & 24,771 & -- & - \\
\hline 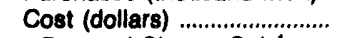 & $2,635,131$ & 139,096 & $1,278,353$ & - & - \\
\hline Demand Charge Only' ....... & - & - & - & - & - \\
\hline 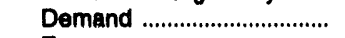 & - & - & $500,00 n$ & - & - \\
\hline 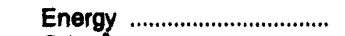 & $2,635,131$ & 139,096 & $778,35 \mathrm{~J}$ & - & - \\
\hline 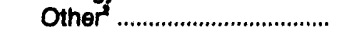 & - & - & - & - & - \\
\hline \multicolumn{6}{|l|}{ Total } \\
\hline Purchases (thousand kWh) & $2,562,456$ & $1,131,199$ & $1,624,702$ & - & 715,496 \\
\hline 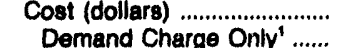 & $\begin{array}{r}63,744,708 \\
716,667\end{array}$ & $24,560,624$ & $\begin{array}{r}29,593,605 \\
1,559,999\end{array}$ & - & $\begin{array}{l}20,748,789 \\
-\end{array}$ \\
\hline 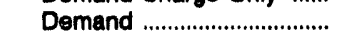 & $22,911,525$ & 23,862 & 500,000 & - & $6,991,626$ \\
\hline 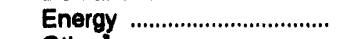 & $40,107,907$ & $15,668,512$ & $27,533,606$ & - & $13,745,146$ \\
\hline Other & 8,609 & $8,868,250$ & - & - & 11,997 \\
\hline \multicolumn{6}{|c|}{ 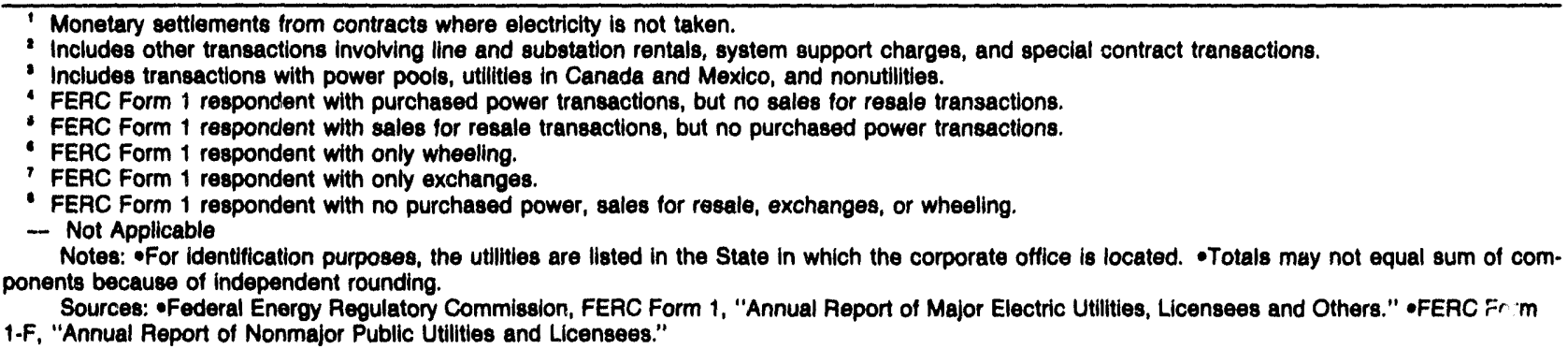 } \\
\hline
\end{tabular}


Table 20. Electriclty Sales for Resale by Investor-Owned Utilities, by State, 1992

\begin{tabular}{|c|c|c|c|c|c|c|c|}
\hline $\begin{array}{l}\text { Purchaeer of } \\
\text { Electrictity by } \\
\text { Ownerahip Clases }\end{array}$ & $\begin{array}{c}\text { Alabama } \\
\text { Alabama } \\
\text { Power } \\
\text { Co }\end{array}$ & $\begin{array}{l}\text { Alabama } \\
\text { Southern } \\
\text { Electric } \\
\text { Generating } \\
\text { Co' }^{\circ}\end{array}$ & $\begin{array}{l}\text { Alaska } \\
\text { Alaska } \\
\text { Electric } \\
\text { Light \& } \\
\text { Power' }\end{array}$ & $\begin{array}{l}\text { Arizona } \\
\text { Ajo } \\
\text { Improvement } \\
\text { Co }\end{array}$ & $\begin{array}{l}\text { Arizona } \\
\text { Arizona } \\
\text { Public } \\
\text { Service } \\
\text { Co }\end{array}$ & $\begin{array}{l}\text { Arizona } \\
\text { Century } \\
\text { Power } \\
\text { Corps }\end{array}$ & $\begin{array}{c}\text { Arizona } \\
\text { Citizens } \\
\text { Utillities } \\
\text { Co }\end{array}$ \\
\hline 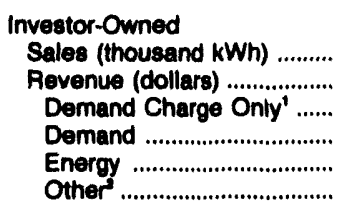 & $\begin{array}{l}4,894,604 \\
263,143,122 \\
- \\
- \\
263,143,122\end{array}$ & $\begin{array}{r}5,328,171 \\
146,545,420 \\
- \\
\overline{146,545,420} \\
-\end{array}$ & $\begin{array}{l}\bar{z} \\
\bar{z} \\
\overline{-}\end{array}$ & $\begin{array}{l}\overline{-} \\
\overline{-} \\
\bar{m}\end{array}$ & $\begin{array}{r}3,289,330 \\
93,085,650 \\
\overline{30,941,836} \\
62,143,814 \\
--\end{array}$ & $\begin{array}{r}3,047,598 \\
177,863,284 \\
\overline{-} \\
101,361,938 \\
76,398,273 \\
103,073\end{array}$ & $\begin{array}{l}\overline{-} \\
\overline{-} \\
\overline{-} \\
\overline{-}\end{array}$ \\
\hline 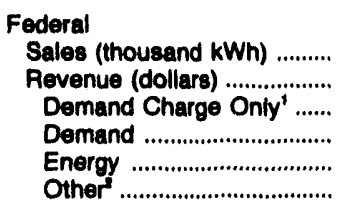 & $\begin{array}{r}\quad 3,324 \\
-\quad 87,424 \\
-\quad 87,424 \\
-\end{array}$ & $\begin{array}{l}- \\
\overline{-} \\
\overline{-} \\
-\end{array}$ & $\begin{array}{l}\overline{-} \\
\overline{-} \\
\overline{-}\end{array}$ & $\begin{array}{l}-- \\
\overline{-} \\
\overline{-} \\
-\end{array}$ & $\begin{array}{r}251,941 \\
10,289,501 \\
- \\
5,013,044 \\
5,276,457 \\
-\end{array}$ & $\begin{array}{l}= \\
\overline{-} \\
= \\
=\end{array}$ & $\begin{array}{l}\overline{-} \\
\bar{m} \\
\bar{m}\end{array}$ \\
\hline 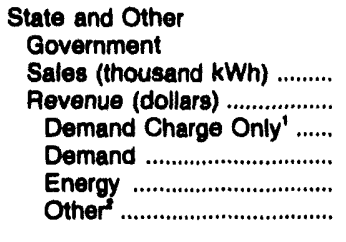 & $\begin{array}{l}16,903 \\
461,649 \\
- \\
- \\
-\end{array}$ & $\begin{array}{l}\bar{z} \\
\bar{z} \\
\overline{-}\end{array}$ & $\begin{array}{l}- \\
\overline{-} \\
\overline{-} \\
-\end{array}$ & $\begin{array}{l}\bar{z} \\
\bar{z}\end{array}$ & $\begin{array}{r}745,226 \\
28,405,130 \\
-- \\
17,453,253 \\
10,951,877 \\
--\end{array}$ & $\begin{array}{r}32,104 \\
1,303,441 \\
-\quad \\
566,186 \\
737,255 \\
-\end{array}$ & $\begin{array}{l}\overline{-} \\
\bar{m} \\
\bar{m}\end{array}$ \\
\hline 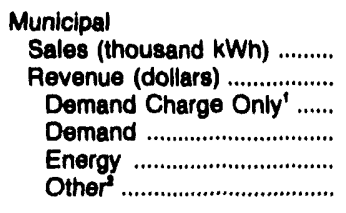 & $\begin{array}{r}3,128,199 \\
131,913,572 \\
\overline{46,345,542} \\
88,990,637 \\
-3,422,607\end{array}$ & $\begin{array}{l}= \\
\overline{-} \\
\overline{-} \\
=\end{array}$ & $\begin{array}{l}\overline{-} \\
\bar{z} \\
\overline{-}\end{array}$ & $\begin{array}{l}\overline{-} \\
\bar{z} \\
\bar{m}\end{array}$ & $\begin{array}{r}205,613 \\
3,437,089 \\
- \\
204,990 \\
3,232,099 \\
--\end{array}$ & $\begin{array}{l}\bar{z} \\
\bar{z} \\
\bar{m}\end{array}$ & $\begin{array}{l}\bar{z} \\
\bar{m} \\
\bar{m}\end{array}$ \\
\hline 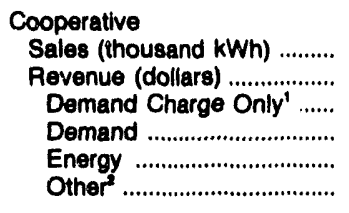 & $\begin{array}{r}338,563 \\
12,385,065 \\
- \\
6,020,705 \\
7,310,872 \\
-946,512\end{array}$ & $\begin{array}{l}- \\
\overline{-} \\
- \\
-\end{array}$ & $\begin{array}{l}\overline{-} \\
\overline{-} \\
\bar{m}\end{array}$ & $\begin{array}{l}\overline{-} \\
\overline{-} \\
\overline{-} \\
\overline{-}\end{array}$ & $\begin{array}{r}30,064 \\
613,595 \\
-\quad \\
164,205 \\
449,390 \\
-\end{array}$ & $\begin{array}{l}\overline{-} \\
\bar{z} \\
\bar{m}\end{array}$ & $\begin{array}{l}- \\
\overline{-} \\
\overline{-}\end{array}$ \\
\hline 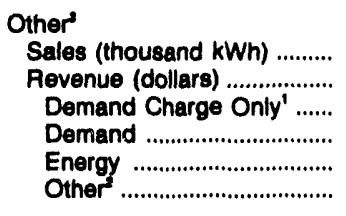 & $\begin{array}{r}7,211,675 \\
157,887,424 \\
\overline{-} \\
127,680,253 \\
30,207,171\end{array}$ & $\begin{array}{l}\overline{-} \\
\overline{-} \\
\overline{-} \\
\overline{-}\end{array}$ & $\begin{array}{l}\overline{-} \\
\bar{z} \\
\bar{z}\end{array}$ & $\begin{array}{l}\bar{z} \\
\bar{z} \\
\overline{-}\end{array}$ & $\begin{array}{r}5,898 \\
279,300 \\
-\quad 79,100 \\
200,200 \\
-\end{array}$ & 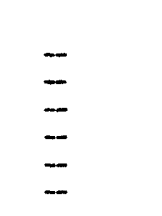 & $\begin{array}{r}1,214 \\
115,303 \\
- \\
-61,398 \\
-53,905\end{array}$ \\
\hline 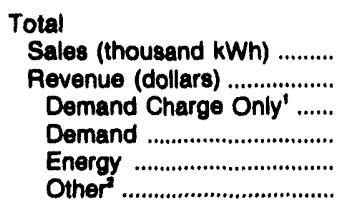 & $\begin{array}{r}15,593,268 \\
565,878,256 \\
-\quad \\
52,366,247 \\
487,673,957 \\
25,838,052\end{array}$ & $\begin{array}{r}5,328,171 \\
146,545,420 \\
- \\
- \\
146,545,420 \\
-\end{array}$ & $\begin{array}{l}\overline{-} \\
\bar{z} \\
\bar{m}\end{array}$ & $\begin{array}{l}\overline{-} \\
\bar{z} \\
\overline{-}\end{array}$ & $\begin{array}{r}4,528,172 \\
136,110,265 \\
-- \\
53,856,428 \\
82,253,837 \\
--\end{array}$ & $\begin{array}{r}3,079,702 \\
179,166,725 \\
- \\
101,928,124 \\
77,135,528 \\
103,073\end{array}$ & $\begin{array}{r}1,214 \\
115,303 \\
-- \\
61,398 \\
53,905\end{array}$ \\
\hline
\end{tabular}

See notes and footnotes at end of table. 
Table 20. Electriclty Sales for Resale by Investor-Owned Utilitles, by State, 1992 (Continued)

\begin{tabular}{|c|c|c|c|c|c|c|c|}
\hline $\begin{array}{l}\text { Purchaeer of } \\
\text { Eloctrictly by } \\
\text { Ownerehlp Clases }\end{array}$ & $\begin{array}{l}\text { Arizona } \\
\text { Tucson } \\
\text { Electric } \\
\text { Power } \\
\text { Co }\end{array}$ & $\begin{array}{c}\text { Arkansas } \\
\text { Arkansas } \\
\text { Power } \\
\mathbf{z} \\
\text { Light } \\
\text { Co } \\
\end{array}$ & $\begin{array}{c}\text { Arkansas } \\
\text { Entergy } \\
\text { Power } \\
\text { Co }\end{array}$ & $\begin{array}{c}\text { Calliornia } \\
\text { Pacific } \\
\text { Gas \& } \\
\text { Electric } \\
\text { Co }\end{array}$ & $\begin{array}{c}\text { Callifornia } \\
\text { San Diogo } \\
\text { Gas \& } \\
\text { Eloctric } \\
\text { Co }\end{array}$ & $\begin{array}{c}\text { California } \\
\text { Southern } \\
\text { California } \\
\text { Edison } \\
\text { Co }\end{array}$ & $\begin{array}{c}\text { Jolorado } \\
\text { Public } \\
\text { Service } \\
\text { Co of } \\
\text { Colorado }\end{array}$ \\
\hline \multicolumn{8}{|l|}{ Investor-Owned } \\
\hline Sales (thousand kWh) .......... & $1,326,064$ & $1,155,366$ & $2,599,914$ & 167,515 & 273,307 & 776,987 & $1,076,869$ \\
\hline Revenue (dollars) ................. & $34,766,067$ & $32,172,609$ & $47,364,684$ & $5,250,690$ & $3,986,454$ & $19,524,0.27$ & $37,282,333$ \\
\hline Demand Charge Only' ....... & - & -- & - & - & 300,000 & - & - \\
\hline Demand & $9,186,000$ & $16,468,405$ & $\overline{-}$ & $1,252,933$ & 428,220 & $\left.2,736,21^{\prime}\right)$ & $18,516,400$ \\
\hline 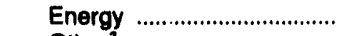 & $25,580,067$ & $15,704,204$ & $47,364,684$ & $3,812,789$ & $3,258,234$ & $15,18 i, 7 \mathrm{d3}$ & $21,456,447$ \\
\hline 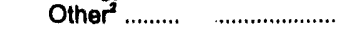 & - & - & -- & 184,968 & - & $1,586,034$ & $-2,690,514$ \\
\hline \multicolumn{8}{|l|}{ Federal } \\
\hline Sales (thousand kWh) .......... & 62,006 & - & 17,000 & 5,600 & 17,247 & 146,201 & 44,189 \\
\hline Revenue (dollars) ...................... & $1,370,914$ & -- & $1,287,267$ & 100,800 & 324,882 & $2,785,456$ & 819,396 \\
\hline Demand Charge Only' ....... & -- & -- & - & - & - & - & -- \\
\hline 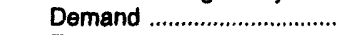 & -- & -- & 951,532 & - & - & - & - \\
\hline 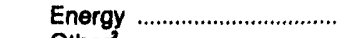 & $1,370,914$ & - & 335,735 & 100,800 & 324,882 & $2,785,456$ & 819,396 \\
\hline Other & - & -- & -- & - & -- & - & -- \\
\hline \multirow{2}{*}{\multicolumn{8}{|c|}{$\begin{array}{l}\text { State and Other } \\
\text { Governinent }\end{array}$}} \\
\hline $\begin{array}{l}\text { Governinent } \\
\text { Sales (thousand kWh) .......... }\end{array}$ & 502,865 & - & - & & & & \\
\hline 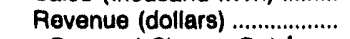 & $28,512,007$ & 185,920 & -- & $125,488,233$ & $6,638,464$ & $42,879,941$ & 56,768 \\
\hline Demand Charge Only' ...... & - & 185,920 & -- & - & - & - & - \\
\hline Demand & $18,000,000$ & -- & - & $51,895,281$ & $1,655,807$ & $18,473,230$ & - \\
\hline Energy ................................ & $10,512,007$ & -- & -- & $73,312,314$ & $4,982,657$ & $23,668,757$ & 56,768 \\
\hline Other $r^{2}$ & -- & -- & - & 280,638 & - & 737,954 & - \\
\hline \multicolumn{8}{|l|}{ Municipal } \\
\hline Sales (thousand kWh) ........ & 200,994 & $1,358,019$ & - & $1,170,020$ & 132,077 & $1,510,247$ & 68,874 \\
\hline 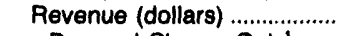 & $3,639,364$ & $68,778,829$ & -- & $54,714,954$ & $4,054,997$ & $57,995,842$ & $2,226,755$ \\
\hline Demand Charge Only' ....... & - & 138,705 & -- & - & - & - & - \\
\hline 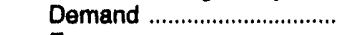 & - & $19,390,160$ & -- & $24,849,705$ & 854,678 & $23,419,670$ & 854,523 \\
\hline 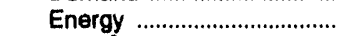 & $3,639,364$ & $49,249,964$ & -- & $38,886,691$ & $3,200,319$ & $38,754,830$ & $1,486,440$ \\
\hline Other $r^{2}$ & - & -- & -- & $-9,021,442$ & - & $-4,178,658$ & $-114,208$ \\
\hline \multicolumn{8}{|l|}{ Cooperative } \\
\hline Sales (thousand kWh) ........... & 18,766 & $1,536,913$ & $\begin{array}{r}145,275 \\
\end{array}$ & - & -- & - & 961,381 \\
\hline Revenue (dollars) ...................... & 419,099 & $44,483,789$ & $7,805,058$ & -- & -- & -- & $39,804,976$ \\
\hline Demand Charge Only' ....... & -- & - & -- & - & -- & - & -- \\
\hline Demand & - & $13,739,354$ & $3,480,704$ & -- & $\cdots$ & - & $23,221,081$ \\
\hline 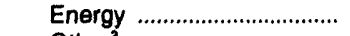 & 419,099 & $30,744,435$ & $4,324,354$ & - & -- & -- & $19,851,479$ \\
\hline 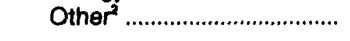 & -- & - & - & - & -- & - & $-3,167,594$ \\
\hline \multicolumn{8}{|l|}{ Others } \\
\hline Sales (thousand kWh) .......... & 10,550 & $11,363,070$ & -- & -- & 25,311 & - & -- \\
\hline 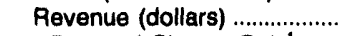 & 204,750 & $238,548,490$ & -- & - & 788,861 & -- & -- \\
\hline Demand Charge Only' ....... & -- & - & -- & -- & - & -- & - \\
\hline Demand & - & - & - & - & 14,600 & - & -- \\
\hline 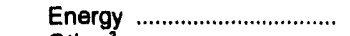 & 204,750 & $238,548,490$ & -- & - & 774,261 & -- & -- \\
\hline Other $r^{2}$ & - & - & - & - & - & - & - \\
\hline \multicolumn{8}{|l|}{ Total } \\
\hline Sales (thousand kWh) .......... & $2,121,245$ & $15,413,368$ & $2,762,189$ & $3,634,102$ & 646,871 & $3,252,182$ & $2,154,775$ \\
\hline 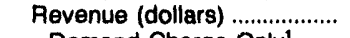 & $68,912,201$ & $384,169,637$ & $56,457,009$ & $185,554,677$ & $15,793,658$ & $123,185,266$ & $80,290,228$ \\
\hline Demand Charge Only' ....... & -- & 324,625 & -- & $-\overline{-}$ & 300,000 & - & $-\overline{-}$ \\
\hline 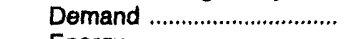 & $27,186,000$ & $49,597,919$ & 4,432,236 & $77,997,919$ & $2,953,305$ & $44,629,110$ & $42,592,014$ \\
\hline 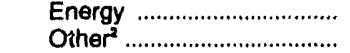 & $41,726,201$ & $\begin{array}{l}334,247,093 \\
--\end{array}$ & $52,024,773$ & $\begin{array}{r}116,112,594 \\
-8,555,836\end{array}$ & $\stackrel{12,540,353}{-}$ & $\begin{array}{l}80,400,826 \\
-1,844,670\end{array}$ & $\begin{array}{l}43,670,530 \\
-5,972,316\end{array}$ \\
\hline & & & & & & & \\
\hline
\end{tabular}

See notes and footnotes at end of table. 
Table 20. Electriclty Sales for Resale by Investor-Owned Utilities, by State, 1992 (Continued)

\begin{tabular}{|c|c|c|c|c|c|c|c|}
\hline $\begin{array}{l}\text { Purchacer of } \\
\text { Elbotrifty by } \\
\text { Ownerahip Clace }\end{array}$ & $\begin{array}{l}\text { Colorado } \\
\text { Utillcorp } \\
\text { United } \\
\text { Inc }\end{array}$ & $\begin{array}{l}\text { Connecticut } \\
\text { Connecticut } \\
\text { Light \& } \\
\text { Power } \\
\text { Co }\end{array}$ & $\begin{array}{c}\text { Connecticut } \\
\text { Connecticut } \\
\text { Yankee } \\
\text { Atomic Power } \\
\text { Co }\end{array}$ & $\begin{array}{l}\text { Connecticut } \\
\text { United } \\
\text { Illuminating } \\
\text { Co }\end{array}$ & $\begin{array}{c}\text { Delaware } \\
\text { Delmarva } \\
\text { Power } \\
\text { \& Light } \\
\text { Co }\end{array}$ & $\begin{array}{l}\text { District of } \\
\text { Columbia } \\
\text { Potomac } \\
\text { Electric } \\
\text { Power Co }\end{array}$ & $\begin{array}{l}\text { Florida } \\
\text { Florida } \\
\text { Power \& } \\
\text { Light } \\
\text { Co }\end{array}$ \\
\hline \multicolumn{8}{|l|}{ Investor-Owned } \\
\hline 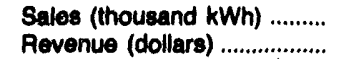 & $\begin{array}{r}15,238 \\
317,404\end{array}$ & $\begin{array}{r}2,968,937 \\
181,159,329\end{array}$ & $\begin{array}{r}3,891,764 \\
206,834,516\end{array}$ & $\begin{array}{r}1,244,040 \\
46,242,383\end{array}$ & $\begin{array}{r}595 \\
29,577\end{array}$ & $\begin{array}{r}7,577 \\
339,689\end{array}$ & $\begin{array}{r}580,931 \\
25,581,250\end{array}$ \\
\hline Demand Charge Only' ....... & - & - & - & -- & $-{ }^{20,011}$ & - & - \\
\hline 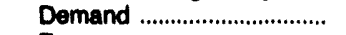 & - & - & - & $19,445,536$ & - & 169,766 & 179,046 \\
\hline 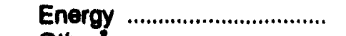 & 317,404 & $50,704,566$ & $31,366,495$ & $26,796,847$ & 29,577 & 169,923 & $25,324,793$ \\
\hline 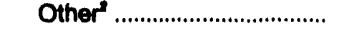 & - & $130,454,763$ & $175,468,021$ & -- & - & -- & 77,411 \\
\hline \multicolumn{8}{|l|}{ Foderal } \\
\hline Sales (thousand kWh) ......... & - & - & -- & -- & - & -- & - \\
\hline 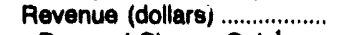 & - & - & -- & - & - & -- & - \\
\hline Demand Charge Only' ....... & - & -- & - & -- & -- & -- & - \\
\hline 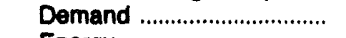 & - & - & -- & -- & - & -- & -- \\
\hline 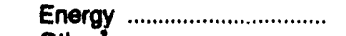 & - & - & - & $-\infty$ & - & -- & -- \\
\hline 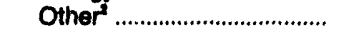 & - & - & -- & - & - & -- & - \\
\hline \multicolumn{8}{|l|}{$\begin{array}{l}\text { State and Other } \\
\text { Government }\end{array}$} \\
\hline Sales (thousand kWh) .......... & - & $1,259,640$ & - & 50,650 & -- & -- & 11,915 \\
\hline 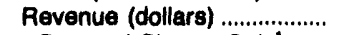 & -- & $54,101,841$ & -- & $1,497,143$ & -- & - & 552,599 \\
\hline Demand Charge Only' ...... & - & - & -- & - & - & -- & -- \\
\hline 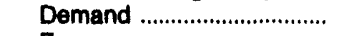 & -- & - & -- & 256,515 & -- & -- & 346,841 \\
\hline 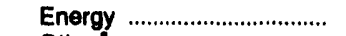 & - & $24,330,301$ & - & $1,240,628$ & - & -. & -- \\
\hline Other $r^{2}$ & - & $29,771,540$ & - & - & - & -- & 205,758 \\
\hline \multicolumn{8}{|l|}{ Municipal } \\
\hline Sales (thousand kWh) ......... & 657 & 534,265 & -- & 7,410 & $1,102,283$ & - & $1,110,042$ \\
\hline Revenue (dollars) ..................... & 27,760 & $33,500,925$ & -- & 200,366 & $43,615,767$ & -- & $30,236,120$ \\
\hline Demand Charge Only' ...... & & - & -- & -- & -- & -- & 5,556 \\
\hline 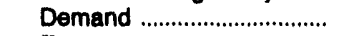 & 2,520 & $14,820,414$ & - & 43,971 & $14,526,167$ & -- & $10,846,860$ \\
\hline 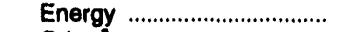 & 6,193 & $15,745,061$ & - & 156,395 & $30,155,303$ & -- & $17,151,728$ \\
\hline Other" & 19,056 & $2,935,450$ & -- & -- & $-1,065,703$ & -- & $2,231,976$ \\
\hline \multicolumn{8}{|l|}{ Cooperative } \\
\hline Sales (thousand kWh) .......... & - & 1,601 & -- & - & $1,309,331$ & $2,122,121$ & 636,861 \\
\hline Revenue (dollars) ...................... & -- & 181,371 & -- & - & $67,441,151$ & $95,010,412$ & $41,851,633$ \\
\hline Demand Charge Only' ....... & - & - & -- & -- & -- & - & - \\
\hline 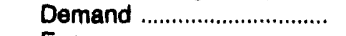 & - & -- & - & - & $38,035,663$ & $40,938,504$ & $26,685,226$ \\
\hline 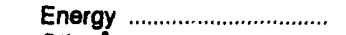 & -- & 46,963 & -- & - & $31,058,858$ & $14,704,487$ & $3,164,110$ \\
\hline Other ${ }^{2}$ & - & 134,408 & - & - & $-1,653,370$ & $39,367,421$ & $12,002,297$ \\
\hline \multicolumn{8}{|l|}{ Other' } \\
\hline Sales (thousand kWh) .......... & - & 679,100 & - & $1,223,280$ & 573,863 & 770,742 & 5,417 \\
\hline Revenue (dollars) ...................... & -- & $6,098,899$ & - & $27,544,204$ & $16,539,874$ & $39,390,460$ & 156,966 \\
\hline Demand Charge Only' ...... & -- & - & -- & $\ldots$ & - & - & - \\
\hline 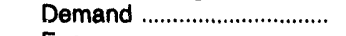 & -- & -- & -- & 569,340 & 1,424 & -- & $\sim$ \\
\hline 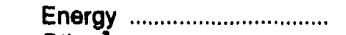 & -- & $14,370,047$ & -- & $26,974,864$ & $16,536,751$ & - & 156,966 \\
\hline Other ${ }^{2} \ldots \ldots$ & - & $-8,271,148$ & - & - & 1,699 & $39,390,460$ & -- \\
\hline \multicolumn{8}{|l|}{ Total } \\
\hline Sales (thousand kWh) .......... & 15,895 & $5,443,543$ & $3,891,764$ & $2,525,380$ & $2,986,072$ & $2,900,440$ & $2,345,166$ \\
\hline Pevenue (dollars) ....................... & 345,173 & $275,042,365$ & $206,834,516$ & $75,484,096$ & $127,626,369$ & $134,740,561$ & $\begin{array}{r}98,378,568 \\
5,556\end{array}$ \\
\hline Demand Charge Only' ....... & $-{ }^{-} 2520$ & $\overline{14.820,414}$ & -- & $\overline{20.315362}$ & $\overline{52563254}$ & $\overline{41.108,270}$ & $\begin{array}{r}5,556 \\
38,057,973\end{array}$ \\
\hline 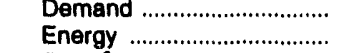 & $\begin{array}{r}2,520 \\
323,597\end{array}$ & $\begin{array}{r}14,820,414 \\
105,196,938\end{array}$ & $\overline{31,366,495}$ & $\begin{array}{l}20,315,362 \\
55,168,734\end{array}$ & $\begin{array}{l}52,563,254 \\
77,780,489\end{array}$ & $\begin{array}{l}41,108,270 \\
14,874,410\end{array}$ & $\begin{array}{l}38,057,973 \\
45,797,597\end{array}$ \\
\hline Other ${ }^{2}$ & 19,056 & $155,025,013$ & $175,468,021$ & - & $-2,717,374$ & $78,757,881$ & $14,517,442$ \\
\hline
\end{tabular}

See notes and footnotes at end of table. 
Table 20. Electricity Sales for Resalo by Investor-Owned Utilities, by State, 1992 (Continued)

\begin{tabular}{|c|c|c|c|c|c|c|c|}
\hline $\begin{array}{l}\text { Purchacer of } \\
\text { Eleotrloty by } \\
\text { Ownenhlp clees }\end{array}$ & $\begin{array}{c}\text { Florida } \\
\text { Floria: } \\
\text { Power } \\
\text { Corp }\end{array}$ & $\begin{array}{l}\text { Florida } \\
\text { Culf } \\
\text { Power } \\
\text { Co }\end{array}$ & $\begin{array}{l}\text { Florida } \\
\text { Tampa } \\
\text { Electric } \\
\text { Co }\end{array}$ & $\begin{array}{c}\text { Ceorgla } \\
\text { Georgla } \\
\text { Power } \\
\text { Co }\end{array}$ & $\begin{array}{l}\text { Goorgla } \\
\text { Savannah } \\
\text { Electric } \\
\text { Power } \\
\text { Co }\end{array}$ & $\begin{array}{c}\text { Hawell } \\
\text { Chtzens } \\
\text { Utilties } \\
\text { Có }^{4}\end{array}$ & $\begin{array}{l}\text { Hawall } \\
\text { Hawallan } \\
\text { Eleotrio } \\
\text { Co Ino' }\end{array}$ \\
\hline \multicolumn{8}{|l|}{ Investor-Owned } \\
\hline Sales (thousand kWh) ......... & 335,252 & $1,265,325$ & $1,136,452$ & $7,457,289$ & 334,092 & - & - \\
\hline 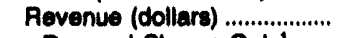 & $5,634,322$ & $59,890,195$ & $24,529,974$ & $355,858,698$ & $7,131,590$ & - & - \\
\hline Demand Charge Only' ...... & - & - & - & - & - & - & - \\
\hline 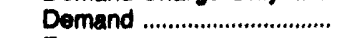 & - & $32,297,893$ & $4,398,071$ & $202,367,060$ & 540,309 & - & - \\
\hline Energy .................................... & $5,634,322$ & $29,727,424$ & $20,131,803$ & $153,601,636$ & $6,591,281$ & - & - \\
\hline 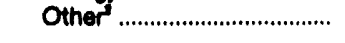 & - & $-2,035,122$ & - & - & - & - & - \\
\hline \multicolumn{8}{|l|}{ Federal } \\
\hline Sales (thousand kWh) ......... & 14,601 & 667 & - & 4,852 & 251 & - & - \\
\hline Revenue (dollars) ................... & 346,585 & 17,553 & - & 127,601 & 6,598 & - & - \\
\hline Demand Charge Only' ....... & - & - & - & - & - & - & -- \\
\hline 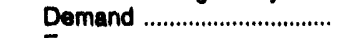 & - & - & - & - & - & - & - \\
\hline 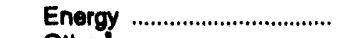 & 346,585 & 17,553 & - & 127,601 & 6,598 & - & - \\
\hline 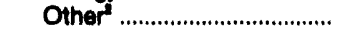 & - & - & - & - & - & - & - \\
\hline \multicolumn{8}{|l|}{$\begin{array}{l}\text { State and Other } \\
\text { Government }\end{array}$} \\
\hline Sales (thousand kWh) ......... & - & 3,394 & - & $1,473,720$ & 1,276 & - & - \\
\hline Revenue (dollars) ................... & - & 92,688 & - & $27,610,325$ & 34,844 & - & - \\
\hline Demand Charge Only' ...... & - & - & - & - & -- & - & - \\
\hline Demand .................................. & - & - & - & $-2,336,985$ & - & - & - \\
\hline 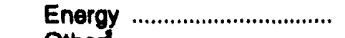 & - & 92,688 & - & $29,947,310$ & 34,844 & - & - \\
\hline 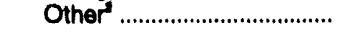 & - & - & - & - & - & - & - \\
\hline \multicolumn{8}{|l|}{ Municipal } \\
\hline Sales (thousand kWh) .......... & $1,240,271$ & 167,370 & $1,213,532$ & $1,263,245$ & 31,276 & $\rightarrow$ & - \\
\hline Revenue (dollars) ................... & $60,883,540$ & $9,868,877$ & $36,809,894$ & $81,180,806$ & 643,055 & - & - \\
\hline Demand Charge Only' ...... & 168,925 & - & - & - & - & - & - \\
\hline 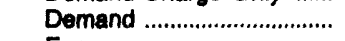 & $28,372,724$ & $6,208,462$ & $10,527,131$ & $37,001,082$ & 2,444 & - & - \\
\hline 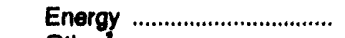 & $25,080,899$ & $3,880,19 \theta$ & $25,005,636$ & $24,178,724$ & 640,611 & - & - \\
\hline 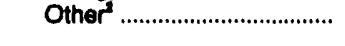 & $7,260,992$ & $-219,784$ & $1,277,127$ & - & - & - & - \\
\hline \multicolumn{8}{|l|}{ Cooperative } \\
\hline Sales (thousand $k W h$ ) ......... & 370,205 & 2,210 & 360,054 & $5,460,092$ & & - & - \\
\hline 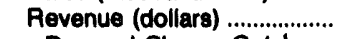 & $33,741,786$ & 53,327 & $11,616,778$ & $185,673,033$ & 1,334 & - & - \\
\hline Demand Charge Only' ...... & - & - & 61,291 & - & - & - & - \\
\hline 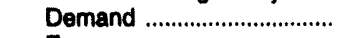 & $16,063,776$ & - & $2,932,247$ & $83,438,510$ & - & - & -- \\
\hline 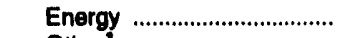 & $10,379,724$ & 53,327 & $8,535,347$ & $112,234,523$ & 1,334 & - & - \\
\hline Other & $7,298,286$ & - & 87,893 & - & - & - & - \\
\hline \multicolumn{8}{|l|}{ Other" } \\
\hline Sales (thousand $k W h$ ) ......... & 1,171 & $1,210,713$ & - & $3,531,085$ & 37,753 & - & - \\
\hline 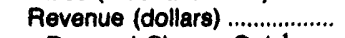 & $-225,395$ & $24,130,072$ & - & $67,896,327$ & $1,508,294$ & - & - \\
\hline Demand Charge Only' ...... & - & - & - & - & - & - & - \\
\hline 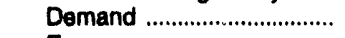 & - & $2,153,315$ & - & - & 723,872 & - & - \\
\hline 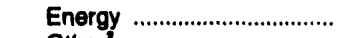 & 10,305 & $21,976,757$ & - & $67,896,327$ & 757,032 & - & - \\
\hline 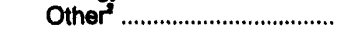 & $-235,700$ & $-\infty$ & -- & - & 26,480 & - & - \\
\hline \multicolumn{8}{|l|}{ Total } \\
\hline Sales (thousand kWh) .......... & $1,961,500$ & $2,639,679$ & $2,710,038$ & $19,190,283$ & 404,698 & - & - \\
\hline 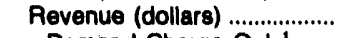 & $100,380,838$ & $94,152,712$ & $72,956,646$ & $708,446,788$ & $9,325,715$ & - & - \\
\hline Demand Charge Only' ...... & 168,925 & - & 61,291 & - & - & - & - \\
\hline Demand ................................. & $44,436,500$ & $40,659,670$ & $17,857,449$ & $320,459,667$ & $1,266,625$ & - & - \\
\hline 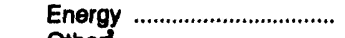 & $41,451,835$ & $55,747,948$ & $53,672,866$ & $387,987,121$ & $8,032,600$ & - & - \\
\hline 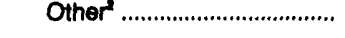 & $14,323,578$ & $-2,254,906$ & $1,365,020$ & - & 26,490 & - & - \\
\hline
\end{tabular}

See notes and footnotes at end of table. 
Table 20. Electricity Sales for Resale by Investor-Owned Utilities, by State, 1992 (Continued)

\begin{tabular}{|c|c|c|c|c|c|c|c|}
\hline 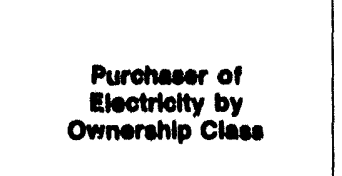 & $\begin{array}{l}\text { Hawall } \\
\text { Maui } \\
\text { Electrlc } \\
\text { Co Lid" }\end{array}$ & $\begin{array}{l}\text { Idaho } \\
\text { Citizens } \\
\text { Utillites } \\
\text { Co' }^{\circ}\end{array}$ & $\begin{array}{l}\text { Idaho } \\
\text { Idaho } \\
\text { Power } \\
\text { Co }\end{array}$ & $\begin{array}{l}\text { Illinols } \\
\text { Central } \\
\text { Illinois } \\
\text { Light } \\
\text { Co }\end{array}$ & $\begin{array}{c}\text { Illinole } \\
\text { Central } \\
\text { Illinole } \\
\text { Public Service } \\
\text { Co }\end{array}$ & $\begin{array}{c}\text { Illinols } \\
\text { Commonwealth } \\
\text { Edieon } \\
\text { Co }\end{array}$ & $\begin{array}{l}\text { Illinols } \\
\text { Electric } \\
\text { Energy } \\
\text { Ino }\end{array}$ \\
\hline \multicolumn{8}{|l|}{ Investor-Owned } \\
\hline $\begin{array}{l}\text { Sales (thousand kWh) } \\
\text { Revenue (dollars) ..................... }\end{array}$ & - & - & $\begin{array}{r}1,145,638 \\
29,866,124\end{array}$ & $\begin{array}{r}422,695 \\
7,795,521\end{array}$ & $\begin{array}{r}1,685,850 \\
34,177,576\end{array}$ & $\begin{array}{r}3,096,324 \\
45,316,722\end{array}$ & $\begin{array}{r}1,297,056 \\
30,227,038\end{array}$ \\
\hline Demand Charge Only' ...... & - & - & - & $-1,00,01$ & $\begin{array}{l}34,171,010 \\
-\end{array}$ & $\begin{array}{r}40,310,122 \\
1,750\end{array}$ & $30,221,038$ \\
\hline Demand .................................. & - & - & $5,028,780$ & 64,000 & $5,074,240$ & $11,158,750$ & $14,953,812$ \\
\hline $\begin{array}{l}\text { Energy } \\
\text { Other }\end{array}$ & - & $\overline{-}$ & $24,837,344$ & $\begin{array}{l}7,731,521 \\
-\end{array}$ & $\begin{array}{r}27,528,492 \\
1,573,844\end{array}$ & $34,156,222$ & $\begin{array}{l}15,273,226 \\
-\end{array}$ \\
\hline \multicolumn{8}{|l|}{ Federal } \\
\hline Sales (thousand kWh) .......... & - & - & 32,015 & - & 62,696 & - & - \\
\hline Revenue (dollars) .................... & -- & - & 691,685 & - & 852,174 & -- & $\sim$ \\
\hline Demand Charge Only' ...... & - & - & - & - & - & - & - \\
\hline Demand ................................. & - & - & - & - & - & - & - \\
\hline 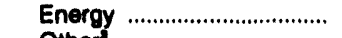 & -- & - & 691,665 & - & 852,174 & -- & - \\
\hline Other & - & - & - & - & - & - & - \\
\hline \multicolumn{8}{|l|}{ State and Other } \\
\hline Sales (thousand kWh) ......... & - & - & 298,682 & - & - & 114,546 & - \\
\hline 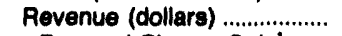 & - & -- & $7,374,189$ & - & - & $1,339,063$ & $-\infty$ \\
\hline Demand Charge Only' ...... & - & -- & $-\rightarrow$ & - & - & $-\infty$ & - \\
\hline Demand ............................... & - & - & $2,950,500$ & $m$ & -- & 150,000 & - \\
\hline 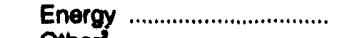 & - & - & $4,423,689$ & - & -- & $1,189,063$ & - \\
\hline Other & - & - & - & - & - & $\cdots$ & - \\
\hline \multicolumn{8}{|l|}{ Municipal } \\
\hline Sales (thousand kWh) .......... & -- & - & 74,483 & 19,494 & 621,634 & $1,402,955$ & - \\
\hline Revenue (dollars) ................... & - & - & $2,071,849$ & 628,856 & $20,327,498$ & $66,947,332$ & $\cdots$ \\
\hline Demand Charge Only' ...... & - & - & - & -- & - & $\bar{\varepsilon}$ & - \\
\hline Demand ................................ & -- & - & 850,739 & 168,126 & $9,093,995$ & $15,804,827$ & - \\
\hline 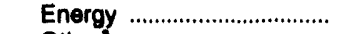 & - & - & $1,220,720$ & 212,409 & $10,901,857$ & $51,137,753$ & - \\
\hline Other & - & - & 390 & 249,321 & 331,646 & 4,752 & - \\
\hline \multicolumn{8}{|l|}{ Cooperative } \\
\hline Sales (thousand kWh) .......... & - & - & 23,808 & $-\infty$ & 636,369 & - & - \\
\hline Revenue (dollars) ................... & -- & - & 540,198 & - & $38,206,004$ & -- & - \\
\hline Demand Charge Only' ...... & - & - & - & - & - & - & - \\
\hline Demand ................................. & -- & - & 40,230 & $\cdots$ & $28,308,820$ & - & - \\
\hline Energy .................................. & -- & - & 499,968 & - & $7,577,769$ & - & - \\
\hline 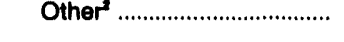 & -- & - & - & $\sim$ & $2,319,415$ & -- & - \\
\hline \multicolumn{8}{|l|}{ Other } \\
\hline Sales (thousand kWh) ......... & - & -- & 49,073 & 150 & 1,846 & - & - \\
\hline Revenue (dollars) ................... & -- & - & $1,355,953$ & 7,265 & 39,539 & - & $-\infty$ \\
\hline Demand Charge Only' ...... & $-\infty$ & - & - & - & - & - & -- \\
\hline Demand ............................... & -- & - & - & - & 1,200 & - & - \\
\hline Energy .................................. & -- & - & $1,355,953$ & $-7 \operatorname{sac}$ & 38,339 & - & $-\cdots$ \\
\hline Other ${ }^{2}$ & -- & - & $-\cdots$ & 7,265 & - & - & - \\
\hline \multicolumn{8}{|l|}{ Total } \\
\hline Sales (thousand kWh) .......... & - & -- & $1,623,890$ & 442,348 & $3,018,395$ & $4,613,825$ & $1,297,056$ \\
\hline Revenue (dollars) ..................... & -- & -- & $41,999,978$ & $8,432,642$ & $93,602,791$ & $113,603,117$ & $30,227,038$ \\
\hline Demand Charge Only' ...... & - & - & 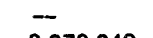 & - & - & 1,750 & - \\
\hline Demand .................................. & - & -- & $8,870,249$ & 232,126 & $42,478,255$ & $27,113,577$ & $14,953,812$ \\
\hline 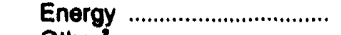 & - & -- & $33,1<f, 339$ & $7,943,930$ & $46,899,631$ & $86,483,038$ & $15,273,226$ \\
\hline Other & - & - & 390 & 256,586 & $4,224,905$ & 4,752 & - \\
\hline
\end{tabular}

See notes and footnotes at end of table. 
Table 20. Electricity Sales for Resale by Investor-Owned Utilities, by State, 1992 (Continued)

\begin{tabular}{|c|c|c|c|c|c|c|c|}
\hline $\begin{array}{l}\text { Purchaeer of } \\
\text { Electriclty by } \\
\text { Ownerahlp Claes }\end{array}$ & $\begin{array}{l}\text { Illinols } \\
\text { Illinols } \\
\text { Power } \\
\text { Co }\end{array}$ & $\begin{array}{l}\text { Illinois } \\
\text { Mit Carmel } \\
\text { Public } \\
\text { Utility } \\
\text { Co }\end{array}$ & $\begin{array}{l}\text { Indiana } \\
\text { Alcoa } \\
\text { Generating } \\
\text { Corp }\end{array}$ & $\begin{array}{c}\text { Indiana } \\
\text { Commonwealti } \\
\text { Edison } \\
\text { of Indiana" }\end{array}$ & $\begin{array}{l}\text { Indiana } \\
\text { Indiana } \\
\text { Michigan } \\
\text { Power } \\
\text { Co }\end{array}$ & $\begin{array}{l}\text { Indiana } \\
\text { Indianapolis } \\
\text { Power } 8 \\
\text { Light } \\
\text { Co }\end{array}$ & $\begin{array}{l}\text { Indiana } \\
\text { Northern } \\
\text { Indiana } \\
\text { Public Service } \\
\text { Co }\end{array}$ \\
\hline \multicolumn{8}{|l|}{ Investor-Owned } \\
\hline $\begin{array}{l}\text { Sales (thousand kWh) ......... } \\
\text { Revenue (dollars) ................. }\end{array}$ & $\begin{array}{r}1,323,991 \\
24,392,778\end{array}$ & - & - & $\begin{array}{r}610,185 \\
69,549,486\end{array}$ & $\begin{array}{r}3,564,033 \\
93,631,683\end{array}$ & $\begin{array}{r}77,388 \\
1,170,370\end{array}$ & $\begin{array}{r}705,654 \\
14,108,695\end{array}$ \\
\hline Demand Charge Only' ...... & - & - & - & - & - & $-\infty$ & - \\
\hline 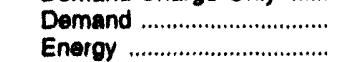 & $\begin{array}{r}2,410,822 \\
21,981,958\end{array}$ & - & - & $\begin{array}{l}22,423,365 \\
47,128,121\end{array}$ & $\begin{array}{r}4,113,166 \\
88,518,517\end{array}$ & $\begin{array}{r}2,000 \\
1,168,370\end{array}$ & $\begin{array}{r}113,860 \\
14,084,835\end{array}$ \\
\hline Other & - & - & - & - & - & - & - \\
\hline \multicolumn{8}{|l|}{ Federal } \\
\hline Sales (thousand kWh) ......... & 80,648 & - & -- & - & 11,135 & - & - \\
\hline Revenue (dollars) ................... & $1,063,322$ & - & - & - & 275,878 & - & -- \\
\hline Demand Charge Only' ...... & - & - & -- & - & -- & - & -- \\
\hline Demand .................................. & - & - & -- & - & - & -- & - \\
\hline 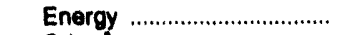 & $1,063,322$ & - & -- & -- & 275,878 & - & -- \\
\hline Other & -- & - & - & - & - & -- & -- \\
\hline \multicolumn{8}{|l|}{$\begin{array}{l}\text { State and Other } \\
\text { Government }\end{array}$} \\
\hline Sales (thousand kWh) ......... & - & - & $-\infty$ & - & 493,410 & - & - \\
\hline Revenue (dollars) ..................... & -- & - & - & - & $20,541,592$ & - & 29,054 \\
\hline Demand Charge Only' ...... & - & - & - & - & - & - & - \\
\hline 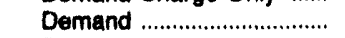 & - & - & -- & - & $13,394,160$ & - & - \\
\hline 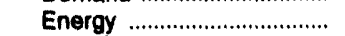 & -- & - & -- & - & $7,133,752$ & - & 29,054 \\
\hline Other & - & - & - & - & 13,680 & -- & -- \\
\hline \multicolumn{8}{|l|}{ Municipal } \\
\hline Sales (thousand kWh) ......... & 162,887 & 2,882 & - & - & $1,918,928$ & -- & 210,804 \\
\hline Revenue (dollars) ..................... & $6,804,647$ & 114,870 & - & - & $76,735,493$ & - & $8,915,834$ \\
\hline Demand Charge Only' ...... & - & - & - & $\sim$ & - & -- & $1,566,321$ \\
\hline 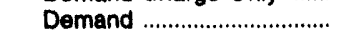 & $3,089,948$ & 53,360 & - & - & $45,916,658$ & - & $1,092,834$ \\
\hline 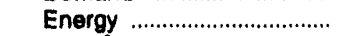 & $3,714,699$ & 44,091 & - & -- & $27,721,534$ & - & $7,272,753$ \\
\hline Other & $\ldots$ & 17,419 & - & -- & $3,097,301$ & - & $-1,816,174$ \\
\hline \multicolumn{8}{|l|}{ Cooperative } \\
\hline Sales (thousand kWh) ......... & $1,402,000$ & - & - & -- & 618,447 & 114,922 & 165,498 \\
\hline Revenue (dollars) ..................... & $47,507,572$ & - & -- & - & $21,743,144$ & $2,090,230$ & $6,552,240$ \\
\hline Demand Charge Only' ...... & - & - & - & - & - & - & $4,050,000$ \\
\hline Demand ................................. & $23,719,716$ & - & - & - & $12,432,095$ & 487,251 & - \\
\hline Energy & $23,787,856$ & -- & - & -- & $8,717,631$ & $1,689,354$ & $2,502,240$ \\
\hline Other & - & -- & - & - & 593,418 & $-86,375$ & $-\infty$ \\
\hline \multicolumn{8}{|l|}{ Other' } \\
\hline Sales (thousand $k$ Wh) ......... & $-2,712$ & - & - & - & $5,125,486$ & - & 49 \\
\hline 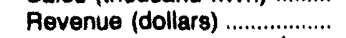 & $-489,572$ & - & - & - & $156,451,209$ & - & 683 \\
\hline Demand Charge Only' ...... & -- & - & - & - & - & - & - \\
\hline Demand & -- & - & -- & - & - & -- & - \\
\hline 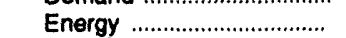 & - & -- & - & - & $156,451,209$ & -- & 683 \\
\hline Other & $-489,572$ & -- & - & - & - & -- & - \\
\hline \multicolumn{8}{|l|}{ Total } \\
\hline $\begin{array}{l}\text { Sales (thousand kWh) ......... } \\
\text { Revenue (dolla;s) }\end{array}$ & $\begin{array}{r}2,966,8 / 4 \\
79,278,747\end{array}$ & $\begin{array}{r}2,882 \\
114,870\end{array}$ & - & $\begin{array}{r}610,165 \\
69,549,486\end{array}$ & $\begin{array}{r}11,731,440 \\
369,378,999\end{array}$ & $\begin{array}{r}192,310 \\
3,260,600\end{array}$ & $\begin{array}{r}1,162,005 \\
29,696,506\end{array}$ \\
\hline Demand Charge Only'....... & - & $-\infty$ & - & - & - & - & $5,616,321$ \\
\hline 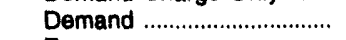 & $29,220,486$ & 53,360 & - & $22,423,365$ & $\begin{array}{r}75,856,079 \\
289,818,521\end{array}$ & $\begin{array}{r}489,251 \\
2,857,724\end{array}$ & $\begin{array}{r}2,106,794 \\
23,889,565\end{array}$ \\
\hline Energy & $\begin{array}{r}50,547,833 \\
-489,572\end{array}$ & $\begin{array}{l}44,091 \\
17,419\end{array}$ & -- & $\begin{array}{l}47,126,121 \\
-\end{array}$ & $\begin{array}{r}289,818,521 \\
3,704,399\end{array}$ & $\begin{array}{r}2,857,724 \\
-86,375\end{array}$ & $\begin{array}{r}23,889,565 \\
-1,916,174\end{array}$ \\
\hline
\end{tabular}

See notes and footnotes at end of table. 
Table 20. Electriclty 8ales for Resale by Investor-Owned Utilitios, by State, 1992 (Continued)

\begin{tabular}{|c|c|c|c|c|c|c|c|}
\hline 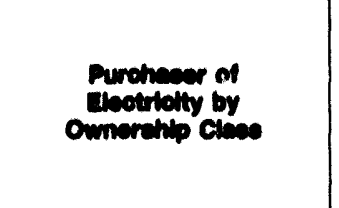 & $\begin{array}{l}\text { Indlane } \\
\text { PSI } \\
\text { Enmoy } \\
\text { Inc }\end{array}$ & $\begin{array}{l}\text { Indiana } \\
\text { Southem } \\
\text { Ind I Gas } \\
\text { a Electric } \\
\text { Co }\end{array}$ & $\begin{array}{l}\text { Iowa } \\
\text { Interatate } \\
\text { Power } \\
\text { Co }\end{array}$ & $\begin{array}{l}\text { lowa } \\
\text { lowa } \\
\text { Eleotrlo } \\
\text { Lloht a } \\
\text { Power Co }\end{array}$ & $\begin{array}{l}\text { lowa } \\
\text { lowa } \\
\text { Southern } \\
\text { Utillites } \\
\text { Co }\end{array}$ & $\begin{array}{l}\text { lowa } \\
\text { lowa- } \\
\text { lilinois } \\
\text { Gas a } \\
\text { Eleotito } \\
\text { Co }\end{array}$ & $\begin{array}{l}\text { lowa } \\
\text { Midwest } \\
\text { Power } \\
\text { Systems } \\
\text { Inc }\end{array}$ \\
\hline 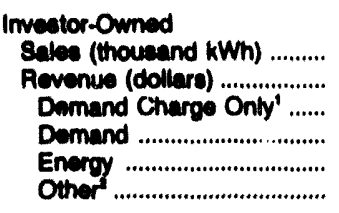 & $\begin{array}{r}3,081,687 \\
49,405,900 \\
- \\
2,023,772 \\
46,782,218 \\
-\end{array}$ & $\begin{array}{r}805,402 \\
18,728,905 \\
- \\
37,000 \\
18,430,763 \\
269,142\end{array}$ & $\begin{array}{r}41,868 \\
888,288 \\
278,000 \\
-710,268 \\
-\end{array}$ & $\begin{array}{r}724,979 \\
10,708,430 \\
438,320 \\
1,331,911 \\
8,520,480 \\
414,709\end{array}$ & $\begin{array}{l}344,788 \\
3,724,440 \\
- \\
\overline{3,724,440} \\
-\end{array}$ & $\begin{array}{r}976,229 \\
16,281,093 \\
- \\
3,016,929 \\
13,264,164 \\
-\end{array}$ & $\begin{array}{r}3,185,682 \\
54,464,309 \\
103,600 \\
13,816,069 \\
40,445,740 \\
-\end{array}$ \\
\hline 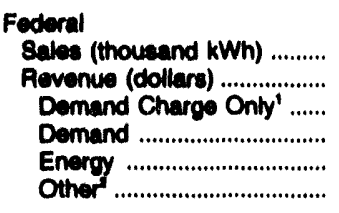 & $\bar{m}=$ & 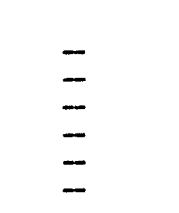 & $\begin{array}{r}15 \\
369 \\
369\end{array}$ & $\begin{array}{r}24,210 \\
289,686 \\
- \\
- \\
289,666\end{array}$ & $\begin{array}{r}29,810 \\
682,480 \\
- \\
141,840 \\
427,684 \\
112,956\end{array}$ & 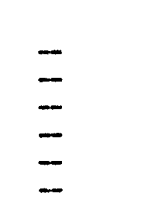 & $\begin{array}{l}87,365 \\
1,260,396 \\
-- \\
\overline{1,260,396} \\
-\end{array}$ \\
\hline 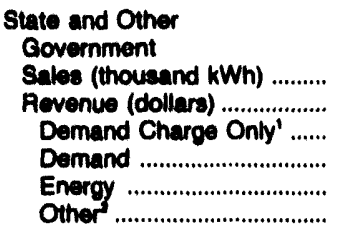 & $\begin{array}{r}1,191,802 \\
33,784,890 \\
\overline{14,506,757} \\
19,841,834 \\
-583,792\end{array}$ & $\begin{array}{l}- \\
\overline{-} \\
\overline{-}\end{array}$ & $\begin{array}{r}1,066 \\
-\quad 25,389 \\
-\quad 25,389\end{array}$ & $\begin{array}{r}\quad 3,629 \\
-\quad 59,287 \\
-\quad 59,287 \\
-\end{array}$ & $\begin{array}{r}6,197 \\
102,650 \\
-\quad \\
-102,650\end{array}$ & $\begin{array}{r}2,936 \\
-\quad 44,711 \\
-\quad 44,711\end{array}$ & $\begin{array}{r}18,861 \\
276,867 \\
-\quad \\
-276,967 \\
-\end{array}$ \\
\hline 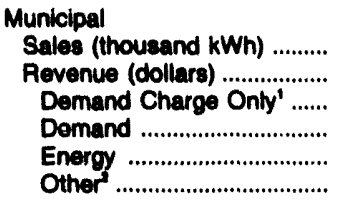 & $\begin{array}{r}541,600 \\
15,788,476 \\
- \\
6,602,930 \\
9,571,476 \\
-385,931\end{array}$ & $\begin{array}{r}459,506 \\
21,440,703 \\
-\overline{10,209,600} \\
10,175,883 \\
1,055,220\end{array}$ & $\begin{array}{r}225,537 \\
8,138,817 \\
- \\
2,806,418 \\
5,332,399 \\
-\end{array}$ & $\begin{array}{r}280,263 \\
10,668,313 \\
184,040 \\
4,385,606 \\
4,560,824 \\
1,527,843\end{array}$ & $\begin{array}{r}154,732 \\
4,201,500 \\
- \\
974,591 \\
2,563,140 \\
663,768\end{array}$ & $\begin{array}{r}57,781 \\
1,574,785 \\
-\quad \\
788,286 \\
759,756 \\
26,740\end{array}$ & $\begin{array}{r}905,023 \\
20,564,122 \\
- \\
8,379,983 \\
12,114,134 \\
70,005\end{array}$ \\
\hline 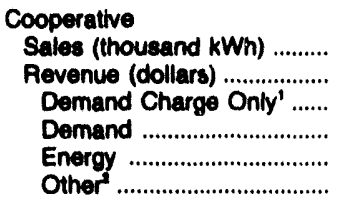 & $\begin{array}{r}1,475,241 \\
47,612,975 \\
- \\
22,067,300 \\
25,675,451 \\
-129,776\end{array}$ & $\begin{array}{r}386 \\
747,883 \\
733,875 \\
-\quad 14,008\end{array}$ & $\begin{array}{r}844 \\
675,222 \\
652,500 \\
-\quad 22,722 \\
-\quad\end{array}$ & $\begin{array}{r}198,372 \\
4,552,631 \\
82,800 \\
244,291 \\
4,133,753 \\
91,787\end{array}$ & $\begin{array}{l}793,593 \\
9,382,369 \\
- \\
\overline{9,382,368} \\
--\end{array}$ & $\begin{array}{r}27,314 \\
329,987 \\
- \\
-\quad 329,887 \\
-\end{array}$ & $\begin{array}{r}415,361 \\
4,773,962 \\
44,240 \\
153,706 \\
4,576,016 \\
-\quad\end{array}$ \\
\hline 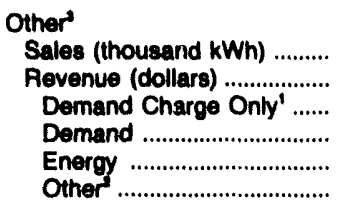 & $\begin{array}{l}- \\
-85,167 \\
- \\
- \\
- \\
-85,167\end{array}$ & $\begin{array}{l}- \\
\overline{-} \\
\overline{-}\end{array}$ & $\begin{array}{l}\overline{-} \\
\overline{-} \\
\overline{-}\end{array}$ & $\begin{array}{l}168,292 \\
1,738,154 \\
-- \\
- \\
1,738,154 \\
-\end{array}$ & $\begin{array}{r}80,405 \\
889,186 \\
-\quad \\
4,309 \\
884,062 \\
825\end{array}$ & $\begin{array}{l}237,189 \\
2,518,725 \\
- \\
-- \\
2,518,725 \\
-\end{array}$ & $\begin{array}{r}471,744 \\
4,883,467 \\
187,578 \\
-\quad \\
4,695,889 \\
-\end{array}$ \\
\hline 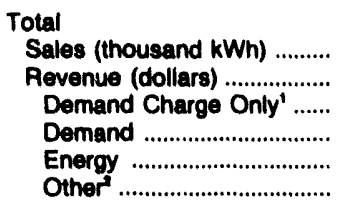 & $\begin{array}{r}6,290,210 \\
146,507,172 \\
- \\
45,800,759 \\
101,871,079 \\
-1,164,666\end{array}$ & $\begin{array}{r}1,265,294 \\
40,915,491 \\
733,875 \\
10,246,600 \\
28,620,654 \\
1,314,362\end{array}$ & $\begin{array}{r}269,417 \\
9,828,065 \\
930,500 \\
2,806,418 \\
6,091,147 \\
-\end{array}$ & $\begin{array}{r}1,397,745 \\
28,014,481 \\
716,160 \\
5,961,808 \\
19,302,174 \\
2,034,339\end{array}$ & $\begin{array}{r}1,409,525 \\
18,982,634 \\
- \\
1,120,740 \\
17,084,344 \\
777,550\end{array}$ & $\begin{array}{r}1,301,449 \\
20,749,301 \\
\overline{3,805,215} \\
16,917,346 \\
26,740\end{array}$ & $\begin{array}{r}5,085,036 \\
86,223,223 \\
335,318 \\
22,448,758 \\
63,369,142 \\
70,005\end{array}$ \\
\hline
\end{tabular}

So"' notes and footnotes at end of table. 

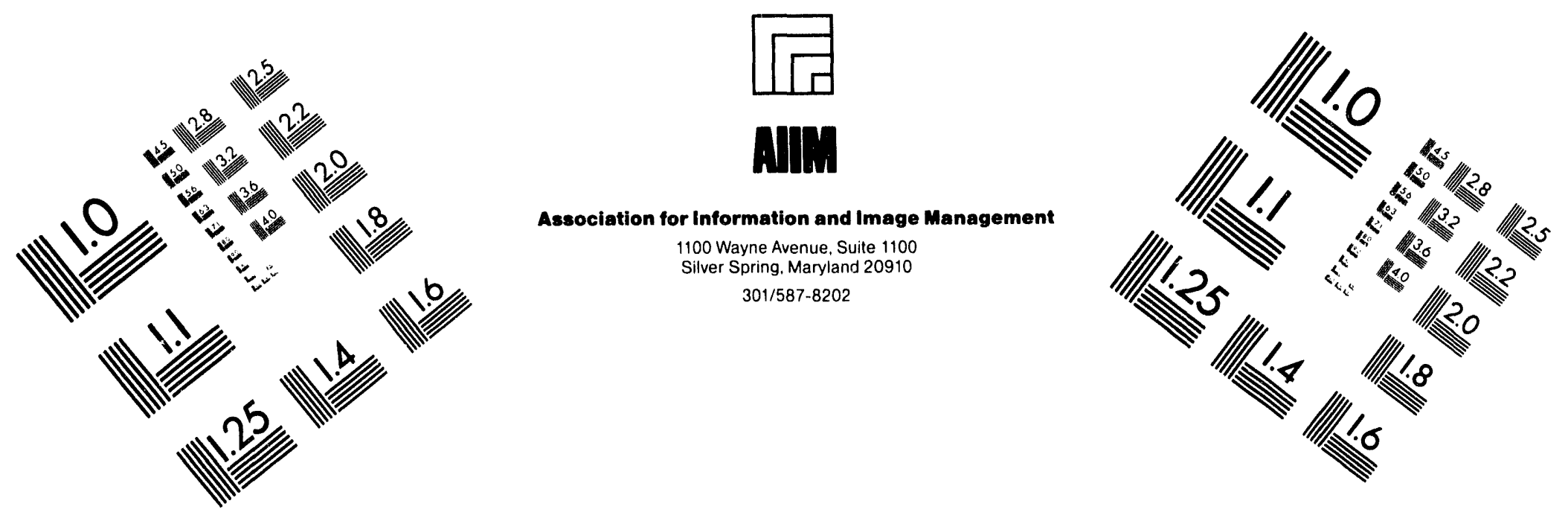

\section{Centimeter}

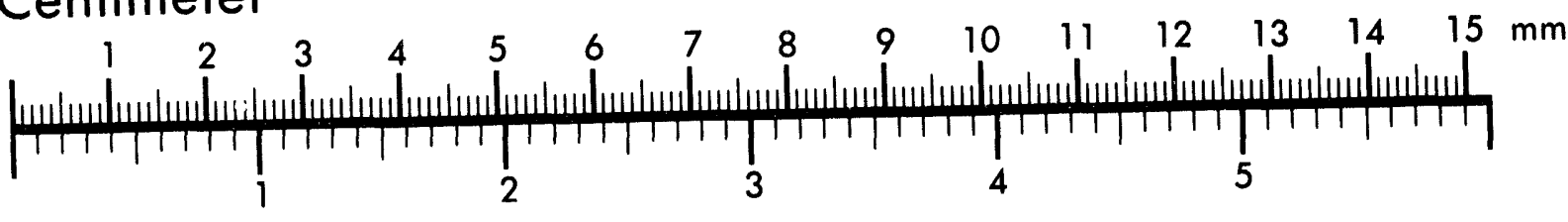
Inches
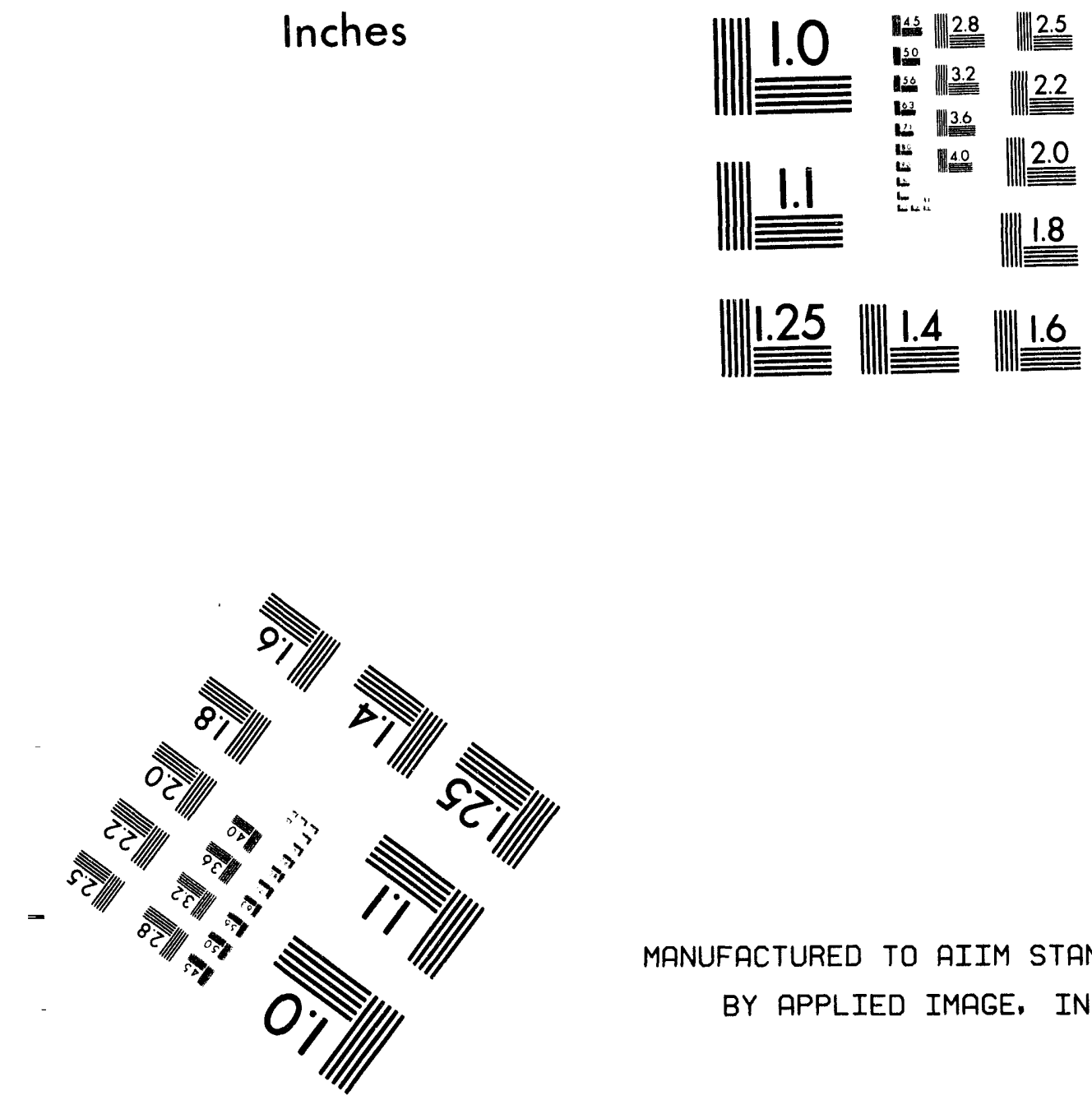

MANUFACTURED TO AIIM STANDARDS

BY APPLIED IMAGE, INC.

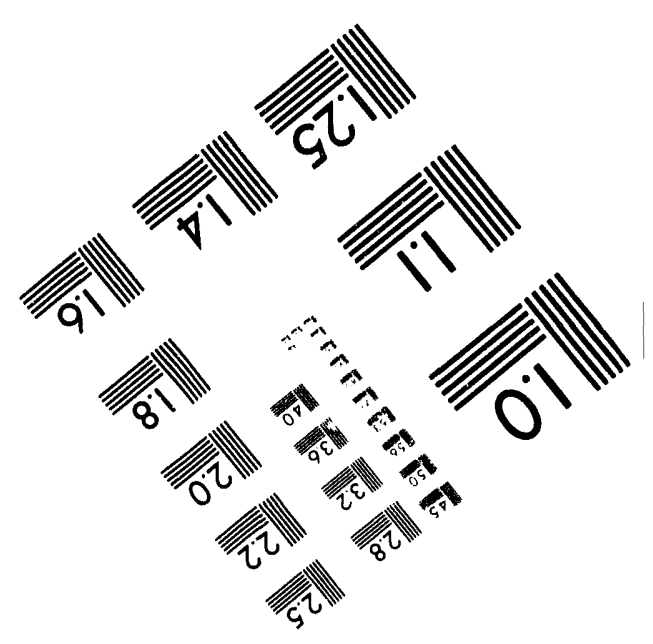



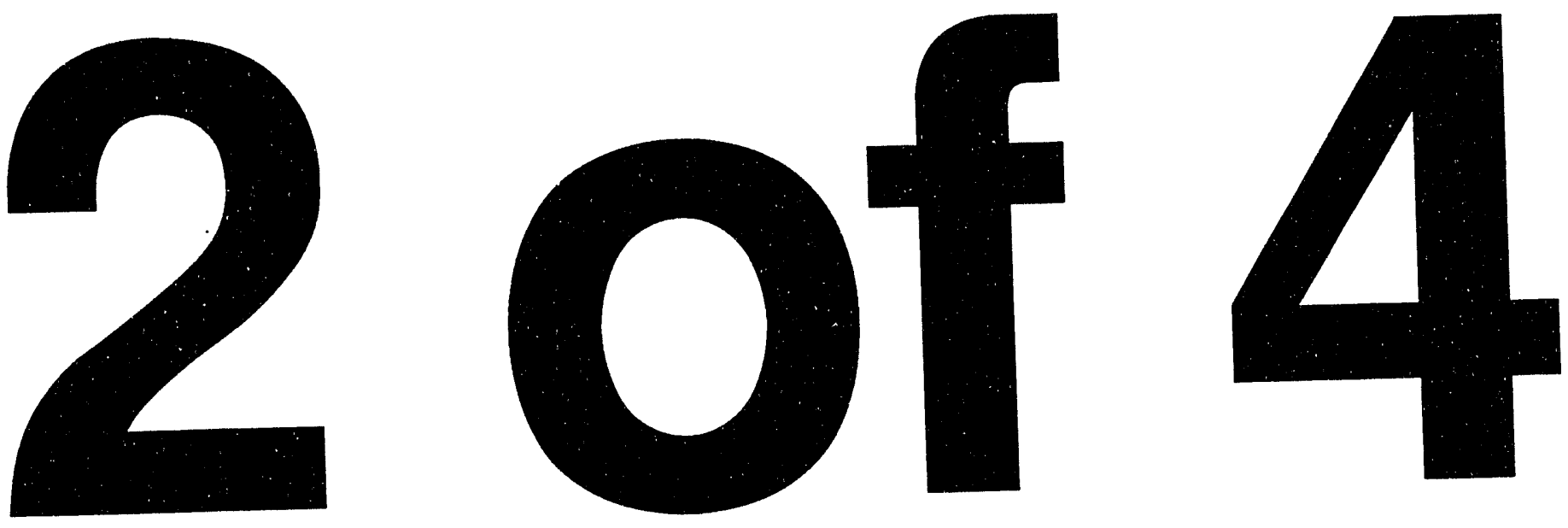
Table 20. Electricity Sales for Resale by Investor-Owned Utilities, by State, 1992 (Continued)

\begin{tabular}{|c|c|c|c|c|c|c|c|}
\hline $\begin{array}{l}\text { Purcheser of } \\
\text { Electrictly by } \\
\text { Owmerahlp Claes }\end{array}$ & $\begin{array}{c}\text { Kansas } \\
\text { Kansas } \\
\text { Gas \& } \\
\text { Electric } \\
\text { Co }\end{array}$ & $\begin{array}{l}\text { Kanses } \\
\text { Westem } \\
\text { Resources } \\
\text { Inc }\end{array}$ & $\begin{array}{l}\text { Kentucky } \\
\text { Kentucky } \\
\text { Power } \\
\text { Co }\end{array}$ & $\begin{array}{l}\text { Kentucky } \\
\text { Kentucky } \\
\text { Utilities } \\
\text { Co }\end{array}$ & $\begin{array}{c}\text { Kentucky } \\
\text { Louisville } \\
\text { Gas \& } \\
\text { Electric } \\
\text { Co }\end{array}$ & $\begin{array}{c}\text { Kentucky } \\
\text { Union } \\
\text { Light } \\
\text { Heat \& } \\
\text { Power } \\
\text { Co }\end{array}$ & $\begin{array}{l}\text { Loulaiana } \\
\text { Catalyst } \\
\text { Old } \\
\text { River } \\
\text { Hydro" }\end{array}$ \\
\hline \multicolumn{8}{|l|}{ Imvestor-Owned } \\
\hline Sales (thousand kWh) ......... & 208,354 & 306,684 & $\begin{array}{r}533,487 \\
\end{array}$ & 852,682 & $1,076,842$ & - & $\begin{array}{r}601,495 \\
99097,175\end{array}$ \\
\hline $\begin{array}{l}\text { Revenue (dollars) ................. } \\
\text { Demand Charge Only' ...... }\end{array}$ & $\begin{array}{l}4,479,815 \\
-\end{array}$ & $16,591,967$ & $\begin{array}{r}13,382,300 \\
461,897\end{array}$ & $16,115,474$ & $16,523,884$ & $\overline{-}$ & $\begin{array}{l}39,097,175 \\
-\end{array}$ \\
\hline 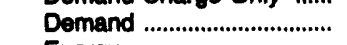 & 836,144 & $11,505,000$ & $1,488,875$ & $1,583,973$ & $1,379,974$ & - & $\overline{30007} 175$ \\
\hline 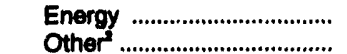 & $\begin{array}{r}3,618,049 \\
25,6 ? ?\end{array}$ & $\underline{5,086,867}$ & $11,451,528$ & $\begin{array}{r}15,306,055 \\
-774,554\end{array}$ & $15,143,910$ & - & $39,097,175$ \\
\hline & & & & & & & \\
\hline \multicolumn{8}{|l|}{ Federal } \\
\hline Sales (thousand kWh) .......... & - & - & 3,988 & 130,523 & $1,085,903$ & - & - \\
\hline 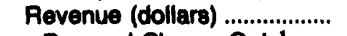 & - & - & 98,773 & $2,270,139$ & $15,278,378$ & - & - \\
\hline Demand Charge Only' ...... & - & - & - & - & - & - & - \\
\hline 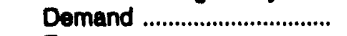 & - & - & - & - & - & - & - \\
\hline 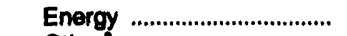 & - & - & 98,773 & $2,270,139$ & $15,278,378$ & - & - \\
\hline Other & - & - & - & - & - & - & - \\
\hline \multicolumn{8}{|l|}{ State and Other } \\
\hline $\begin{array}{l}\text { Government } \\
\text { Sales (thousand kWh) ......... }\end{array}$ & 41,409 & 28,663 & - & - & 67,924 & - & - \\
\hline Revenue (dollars) ................... & $\begin{array}{r}41,409 \\
2,156,343\end{array}$ & 552,174 & $\overline{-}$ & $\overline{-}$ & $1,496,452$ & $\overline{-}$ & $\overline{-}$ \\
\hline Demand Charge Only' ...... & - & - & - & - & - & - & - \\
\hline Demand .................................. & $1,236,000$ & - & - & - & 292,800 & - & - \\
\hline 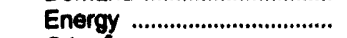 & 920,343 & 552,174 & - & - & $1,203,652$ & - & - \\
\hline Other & - & - & - & - & - & - & - \\
\hline \multicolumn{8}{|l|}{ Munisipal } \\
\hline Sales (thousand kWh) .......... & 628,340 & 889,354 & 110,899 & $1,318,067$ & 66,431 & 43,108 & 38,382 \\
\hline Revenue (dollars) ................. & $16,183,447$ & $27,555,464$ & $3,924,738$ & $40,489,755$ & $1,019,878$ & $1,636,347$ & $2,495,480$ \\
\hline 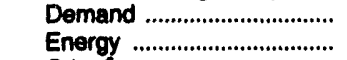 & $\begin{array}{r}1,457,509 \\
14,513,955\end{array}$ & $17,239,886$ & $2,797,481$ & & $\overline{1,019,878}$ & $\begin{array}{r}612,544 \\
1,163,912\end{array}$ & $\overline{2,495,480}$ \\
\hline Other & 211,983 & $-69,667$ & 8,196 & $-9,873,309$ & - & $-140,109$ & - \\
\hline \multicolumn{8}{|l|}{ Cooperative } \\
\hline Sales (thousand kWh) .......... & 368,710 & 812,730 & & 5,820 & 937,658 & - & - \\
\hline Reverive (dollars) ................... & $5,359,213$ & $30,575,749$ & 44,203 & 103,385 & $15,778,024$ & - & - \\
\hline Demand Charge Only' ....... & - & - & - & - & - & - & - \\
\hline 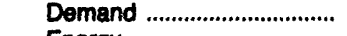 & $3,228,421$ & $14,242,505$ & 43,762 & - & $1,208,316$ & - & - \\
\hline 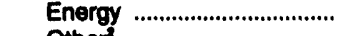 & $1,821,327$ & $16,371,837$ & 441 & 103,385 & $14,569,708$ & - & - \\
\hline Other & 309,465 & $-38,593$ & - & - & - & - & - \\
\hline \multicolumn{8}{|l|}{ Other' } \\
\hline Sales (thousand kWh) .......... & 2,300 & - & $3,456,382$ & - & - & - & - \\
\hline Revenue (dollars) ................... & 19,788 & 106,791 & $43,910,056$ & - & $-4,398,129$ & - & - \\
\hline Demand Charge Only' ...... & - & - & - & - & - & - & - \\
\hline 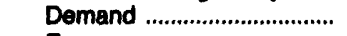 & - & - & - & - & - & - & - \\
\hline 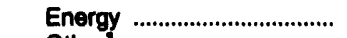 & 45,060 & - & $43,810,056$ & - & $-4,398,129$ & - & - \\
\hline Other & $-25,262$ & 106,791 & - & - & - & - & - \\
\hline \multicolumn{8}{|l|}{ Total } \\
\hline 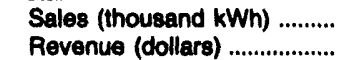 & $\begin{array}{r}1,249,113 \\
28,198,616\end{array}$ & $\begin{array}{r}2,037,431 \\
75,382,145\end{array}$ & $\begin{array}{r}4,104,779 \\
61,360,070\end{array}$ & $\begin{array}{r}2,307,172 \\
58,978,753\end{array}$ & $\begin{array}{r}3,234,758 \\
45,698,487\end{array}$ & $\begin{array}{r}43,108 \\
1,636,347\end{array}$ & $\begin{array}{r}639,887 \\
41,592,655\end{array}$ \\
\hline Demand Charge Only' ....... & - & - & 461,897 & - & - & - & - \\
\hline 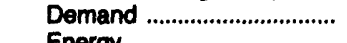 & $6,758,074$ & $36,132,750$ & $\begin{array}{r}2,631,698 \\
58,258\end{array}$ & $21,160,625$ & $2,881,090$ & 612,544 & $\overline{1592} 655$ \\
\hline $\begin{array}{l}\text { Energy } \\
\text { Other }\end{array}$ & $\begin{array}{r}20,918,734 \\
521,808\end{array}$ & $\begin{array}{r}39,250,864 \\
-1,469\end{array}$ & $\begin{array}{r}58,258,279 \\
8,196\end{array}$ & $\begin{array}{r}48,565,991 \\
-10,747,860\end{array}$ & $\begin{array}{l}42,817,397 \\
-\end{array}$ & $\begin{array}{r}4,163,812 \\
-140,109\end{array}$ & 41,592,655 \\
\hline
\end{tabular}

See notes and footnotes at end of table. 
Table 20. Electricity Sales for Resale by Investor-Owned Utilities, by State, 1992 (Continued)

\begin{tabular}{|c|c|c|c|c|c|c|c|}
\hline $\begin{array}{l}\text { Purcheser of } \\
\text { Eloctrictly by } \\
\text { Ownerahlp Claes }\end{array}$ & $\begin{array}{l}\text { Louisiana } \\
\text { Contral } \\
\text { Loulsiana } \\
\text { Electric } \\
\text { Co Inc }\end{array}$ & $\begin{array}{l}\text { Louisiana } \\
\text { Louisiana } \\
\text { Power \& } \\
\text { Light } \\
\text { Co }\end{array}$ & $\begin{array}{l}\text { Louisiana } \\
\text { Now Orleans } \\
\text { Public } \\
\text { Sorvice } \\
\text { Inc }\end{array}$ & $\begin{array}{l}\text { Louisiana } \\
\text { Southwestern } \\
\text { Electric } \\
\text { Power } \\
\text { Co }\end{array}$ & $\begin{array}{l}\text { Maine } \\
\text { Bangor } \\
\text { Hydro- } \\
\text { Electric } \\
\text { Co }\end{array}$ & $\begin{array}{l}\text { Maine } \\
\text { Central } \\
\text { Maine } \\
\text { Power } \\
\text { Co }\end{array}$ & $\begin{array}{l}\text { Maine } \\
\text { Meine } \\
\text { Electric } \\
\text { Power } \\
\text { Co inc }\end{array}$ \\
\hline \multicolumn{8}{|l|}{ Investor-Owned } \\
\hline 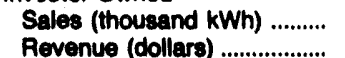 & $\begin{array}{r}15,855 \\
312,332\end{array}$ & $\overline{-}$ & - & $\begin{array}{r}872,309 \\
17,244,511\end{array}$ & $\begin{array}{r}13,192 \\
533,439\end{array}$ & $\begin{array}{r}55,820 \\
975,497\end{array}$ & $\begin{array}{r}276,572 \\
6,936,720\end{array}$ \\
\hline Demand Charge Only' ........ & - & - & - & 587,300 & $-000,400$ & - & $0,-\infty 0,1<0$ \\
\hline 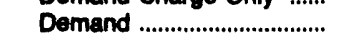 & - & - & - & 308,550 & 156,813 & - & - \\
\hline 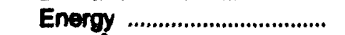 & & - & - & $16,348,661$ & 376,626 & 975,497 & $6,936,720$ \\
\hline Other & 312,332 & - & - & - & - & - & - \\
\hline \multicolumn{8}{|l|}{ Federal } \\
\hline Sales (thousand kWh) .......... & - & - & - & - & - & - & - \\
\hline Revenue (dollars) .................. & - & - & - & - & - & - & - \\
\hline Demand Charge Only' ...... & - & - & - & - & - & - & - \\
\hline 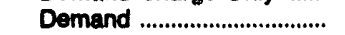 & - & - & - & - & - & - & - \\
\hline 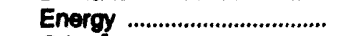 & - & - & - & - & - & - & - \\
\hline Other & - & - & - & - & - & - & - \\
\hline \multicolumn{8}{|l|}{$\begin{array}{l}\text { State and Other } \\
\text { Government }\end{array}$} \\
\hline Sales (thousand kWh) .......... & 15,114 & - & - & - & - & - & - \\
\hline 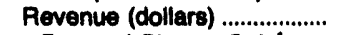 & 388,447 & - & - & - & - & - & - \\
\hline Demand Charge Only' ....... & - & - & - & - & - & - & - \\
\hline 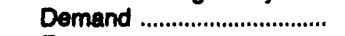 & - & - & - & - & - & - & - \\
\hline 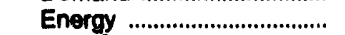 & - & - & - & - & - & - & - \\
\hline Other $r^{2}$ & 388,447 & - & - & - & - & - & - \\
\hline \multicolumn{8}{|l|}{ Municipal } \\
\hline Sales (thousand kWh) .......... & 180,988 & 8 & - & 422,525 & -- & 110,916 & - \\
\hline Revenue (dollars) .................. & $4,182,880$ & 287 & - & $13,552,324$ & - & $7,498,890$ & - \\
\hline Demand Charge Only' ....... & - & - & - & - & - & - & - \\
\hline 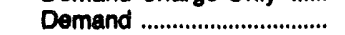 & 253,906 & - & - & $4,784,241$ & - & $2,457,234$ & - \\
\hline 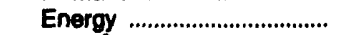 & 12,693 & 287 & - & $8,768,083$ & - & $5,002,222$ & - \\
\hline Other & $3,916,281$ & - & - & - & - & 39,434 & - \\
\hline \multicolumn{8}{|l|}{ Cooperative } \\
\hline Sales (thousand kWh) .......... & 13,856 & - & - & $2,014,308$ & 10,310 & 7,759 & - \\
\hline Revenue (dollars) ..................... & 304,816 & - & - & $37,832,301$ & 881,227 & 569,079 & - \\
\hline Demand Charge Only' ...... & - & - & - & - & - & - & - \\
\hline 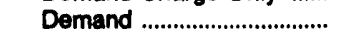 & - & - & - & $15,370,021$ & 288,432 & 212,996 & - \\
\hline 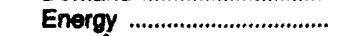 & - & - & -- & $22,372,514$ & 592,795 & 342,509 & - \\
\hline 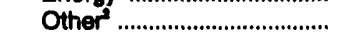 & 304,916 & - & - & 89,766 & - & 13,574 & -- \\
\hline \multicolumn{8}{|l|}{ Other } \\
\hline $\begin{array}{l}\text { Sales (thousand kWh) .......... } \\
\text { Revenue (dollars) }\end{array}$ & $\begin{array}{r}8,785 \\
175,339\end{array}$ & $\begin{array}{r}1,305,059 \\
38,631,891\end{array}$ & $\begin{array}{r}404,823 \\
10,319,873\end{array}$ & $\begin{array}{r}545,041 \\
10,761,294\end{array}$ & $\begin{array}{r}99,387 \\
99,396\end{array}$ & $\begin{array}{r}263,676 \\
263,707\end{array}$ & $\begin{array}{r}65,139 \\
1,474,747\end{array}$ \\
\hline Demand Charge Only' ....... & ${ }_{-}^{1 / 2,338}$ & 38,6 & 10,31 & $\stackrel{10,76}{-}$ & 13,24 & - & ${ }^{1,4 / 4,147}$ \\
\hline 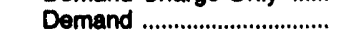 & - & - & - & 37,500 & $-24,167$ & - & - \\
\hline Energy ........................................... & 1,834 & $38,631,891$ & $10,319,973$ & $10,723,794$ & $13,264,463$ & $10,382,707$ & $1,471,437$ \\
\hline 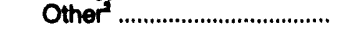 & 173,505 & - & 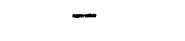 & - & - & - & 3,310 \\
\hline \multicolumn{8}{|l|}{ Total } \\
\hline Sales (thousand kWh) .......... & 234,598 & $\begin{array}{r}1,305,067 \\
\end{array}$ & 404,823 & $3,854,183$ & 122,889 & $\begin{array}{r}438,171 \\
\end{array}$ & $\begin{array}{r}341,711 \\
8,411,467\end{array}$ \\
\hline $\begin{array}{l}\text { Revenue (dollars) ............... } \\
\text { Demand Charge Only' ...... }\end{array}$ & $5,363,914$ & $38,632,178$ & $10,319,873$ & $\begin{array}{r}79,390,430 \\
587,300\end{array}$ & $14,654,962$ & $19,426,173$ & $8,411,467$ \\
\hline 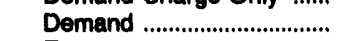 & 253,906 & - & - & $20,500,312$ & 421,078 & $2,670,230$ & - \\
\hline 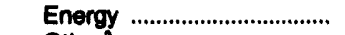 & 14,527 & $38,632,178$ & $10,319,973$ & $58,213,052$ & $14,233,884$ & $16,702,935$ & $8,408,157$ \\
\hline Other & $5,095,481$ & - & - & 89,766 & - & 53,008 & 3,310 \\
\hline
\end{tabular}

See notes and footnotes at end of table. 
Table 20. Electriclty Sales for Resale by Investor-Owned Utilities, by State, 1992 (Continued)

\begin{tabular}{|c|c|c|c|c|c|c|c|}
\hline $\begin{array}{l}\text { Purchaser of } \\
\text { Electrlelty by } \\
\text { Ownorehip clases }\end{array}$ & $\begin{array}{l}\text { Maine } \\
\text { Maine } \\
\text { Public } \\
\text { Service } \\
\text { Co }\end{array}$ & $\begin{array}{l}\text { Maine } \\
\text { Maine } \\
\text { Yankee } \\
\text { Alol.tic } \\
\text { Power } \\
\text { Co" }\end{array}$ & $\begin{array}{l}\text { Maryland } \\
\text { Allegheny } \\
\text { Generating } \\
\text { Co: }\end{array}$ & $\begin{array}{c}\text { Maryland } \\
\text { Baltimore } \\
\text { Gas \& } \\
\text { Electric } \\
\text { Co }\end{array}$ & $\begin{array}{l}\text { Maryland } \\
\text { Conowingo } \\
\text { Power } \\
\text { Co }\end{array}$ & $\begin{array}{l}\text { Maryland } \\
\text { Potomac } \\
\text { Edison } \\
\text { Co }\end{array}$ & $\begin{array}{l}\text { Massachusetts } \\
\text { Boston } \\
\text { Edison } \\
\text { Co }\end{array}$ \\
\hline \multicolumn{8}{|l|}{ Investor-Owned } \\
\hline 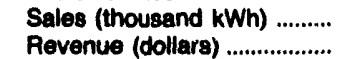 & $\begin{array}{r}1,127 \\
1,1999\end{array}$ & $5,006,997$ & $9 \overline{4} 406000$ & $\overline{-}$ & $\begin{array}{r}122 \\
11865\end{array}$ & $\begin{array}{r}4,415,756 \\
115,370727\end{array}$ & $\begin{array}{r}1,051,260 \\
\end{array}$ \\
\hline $\begin{array}{l}\text { Revenue (dollarg) ............. } \\
\text { Demand Charge Only' ..... }\end{array}$ & ${ }_{-}^{181,828}$ & $1 / 0,480,401$ & $97,405,000$ & $\bar{z}$ & $--^{11,865}$ & $110,510,126$ & $-178,552$ \\
\hline 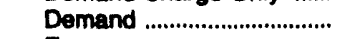 & 149,039 & $151,423,771$ & - & - & - & $47,666,876$ & $42,118,559$ \\
\hline 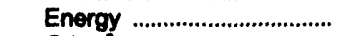 & 32,789 & $24,066,710$ & - & - & 11,865 & $67,558,263$ & $15,537,464$ \\
\hline Other & - & - & $97,406,000$ & - & - & 145,588 & $-1,457,543$ \\
\hline \multicolumn{8}{|l|}{ Federal } \\
\hline Sales (thousand kWh) .......... & - & - & - & - & - & - & - \\
\hline Revenue (dollars) ................... & - & - & - & - & - & - & - \\
\hline Demand Charge Only' ....... & - & - & - & - & - & - & - \\
\hline 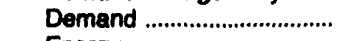 & - & - & - & - & $\overline{-}$ & - & $\overline{-}$ \\
\hline 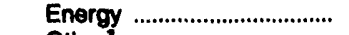 & - & - & - & - & - & - & - \\
\hline Other & - & - & - & - & - & - & - \\
\hline \multicolumn{8}{|l|}{$\begin{array}{l}\text { State and Other } \\
\text { Government }\end{array}$} \\
\hline Sales (thousand kWh) .......... & - & 25,143 & - & - & - & - & 139,350 \\
\hline Revenue (dollars) ................. & - & 881,242 & - & - & - & - & $3,823,060$ \\
\hline Demand Charge Only' ....... & - & - & - & - & - & - & - \\
\hline 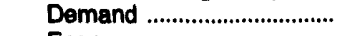 & - & 760,389 & - & - & - & - & 784,490 \\
\hline 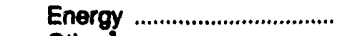 & - & 120,853 & - & - & - & - & $3,038,570$ \\
\hline Other & - & - & - & - & - & - & - \\
\hline \multicolumn{8}{|l|}{ Municipal } \\
\hline $\begin{array}{l}\text { Sales (thousand kWh) .......... } \\
\text { Rovenue (dollars) }\end{array}$ & $\begin{array}{r}84,111 \\
6,089,924\end{array}$ & 254,849 & $\overline{-}$ & $\overline{-}$ & 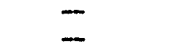 & $\begin{array}{r}522,515 \\
\end{array}$ & 597,621 \\
\hline Revenue (dollars) .................. & $6,089,924$ & $8,932,262$ & $\overline{-}$ & $\overline{-}$ & $\bar{z}$ & $18,845,795$ & $37,089,617$ \\
\hline Demand Charge Only' ....... & - & $=$ & - & $=$ & - & - & $-272,234$ \\
\hline Demand & $3,021,433$ & $7,707,294$ & - & - & - & $9,657,073$ & $28,073,775$ \\
\hline 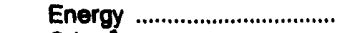 & 706,537 & $1,224,968$ & - & - & - & $8,124,281$ & $11,720,148$ \\
\hline Other & $2,361,954$ & - & - & - & $\sim$ & $1,064,441$ & $-2,432,072$ \\
\hline \multicolumn{8}{|l|}{ Cooperative } \\
\hline Sales (thousand kWh) .......... & 11,451 & 55,784 & - & - & - & $\begin{array}{r}684,029 \\
21,43,692\end{array}$ & - \\
\hline Revenue (dollars) ............. & 856,534 & $1,955,173$ & $\overline{-}$ & $\overline{-}$ & $\overline{-}$ & $21,483,692$ & $\because$ \\
\hline & 445,832 & $\overline{1,687,041}$ & - & - & - & $\overline{9,300,322}$ & $\overline{-}$ \\
\hline 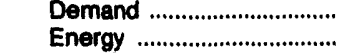 & $\begin{array}{r}440,002 \\
81,843\end{array}$ & $\begin{array}{r}1,68 /, 041 \\
268,132\end{array}$ & - & - & - & $\begin{array}{r}9,300,322 \\
12,117,021\end{array}$ & $\overline{-}$ \\
\hline Other & 318,859 & - & - & - & - & 66,349 & - \\
\hline \multicolumn{8}{|l|}{ Other } \\
\hline Sales (thousand kWh) .......... & $\begin{array}{r}36,213 \\
568,016\end{array}$ & $\overline{-}$ & $\bar{z}$ & $3,179,408$ & - & $\begin{array}{r}366,299 \\
\end{array}$ & $2,627,076$ \\
\hline Revenue (dollars) ............... & 526,216 & $\overline{-}$ & $\bar{z}$ & $64,322,817$ & - & $7,440,821$ & $85,651,410$ \\
\hline Demand Charge Only' ....... & - & - & - & - & - & - & $-180,283$ \\
\hline Demand & 9,213 & - & - & $\overline{-}$ & - & $1,111,521$ & $35,610,066$ \\
\hline 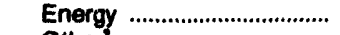 & 394,702 & - & - & $64,322,817$ & - & $6,329,400$ & $50,221,627$ \\
\hline Other ${ }^{2}$ & 122,301 & - & - & - & - & - & - \\
\hline \multicolumn{8}{|l|}{ Total } \\
\hline 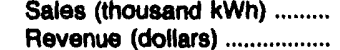 & $\begin{array}{r}132,902 \\
7,654,502\end{array}$ & $\begin{array}{r}5,342,773 \\
187,259,158\end{array}$ & $\overline{97,406,000}$ & $\begin{array}{r}3,179,408 \\
64,322,817\end{array}$ & $\begin{array}{r}122 \\
11,865\end{array}$ & $\begin{array}{r}5,988,599 \\
183,141,135\end{array}$ & $\begin{array}{r}4,415,307 \\
182,584,015\end{array}$ \\
\hline Demand Charge Only' ...... & - & - & - & - & - & $100,121,100$ & $\begin{array}{r}102,004,010 \\
-631,069\end{array}$ \\
\hline 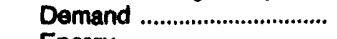 & $3,625,517$ & $161,578,495$ & - & - & - & $67,735,792$ & $106,586,890$ \\
\hline Energy ......................... & $1,225,871$ & $25,680,663$ & $9 \overline{7} 406,000$ & $64,322,817$ & $-{ }^{11,865}$ & $\begin{array}{r}94,128,965 \\
1,276,378\end{array}$ & $\begin{array}{l}80,517,800 \\
-3,889,615\end{array}$ \\
\hline (.)................... & $2,608,774$ & - & & - & 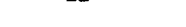 & $1,260,010$ & $-0,000,010$ \\
\hline
\end{tabular}

See notes and footnotes at end of table. 
Table 20. Electricity Sales for Resale by Investor-Owned Utilities, by State, 1992 (Continued)

\begin{tabular}{|c|c|c|c|c|c|c|c|}
\hline $\begin{array}{l}\text { Purchacer of } \\
\text { Electriclty by } \\
\text { Owmerehlp clases }\end{array}$ & $\begin{array}{l}\text { Massachusetts } \\
\text { Cambridge } \\
\text { Electric } \\
\text { Light } \\
\text { Co }\end{array}$ & $\begin{array}{c}\text { Massachusetts } \\
\text { Canal } \\
\text { Electric } \\
\text { Co }\end{array}$ & $\begin{array}{c}\text { Massachusetts } \\
\text { Commonwealth } \\
\text { Electric } \\
\mathrm{Co}\end{array}$ & $\begin{array}{l}\text { Massachusetts } \\
\text { Eastern } \\
\text { Edison } \\
\text { Co' }\end{array}$ & $\begin{array}{l}\text { Massachusetts } \\
\text { Fitchburg } \\
\text { Gas \& } \\
\text { Electric } \\
\text { Lght } \\
\text { Co }\end{array}$ & $\begin{array}{l}\text { Massachusetts } \\
\text { Great } \\
\text { Bay } \\
\text { Power } \\
\text { Co' }\end{array}$ & $\begin{array}{l}\text { Massachusetts } \\
\text { Holyoke } \\
\text { Power \& } \\
\text { Electric } \\
\text { Co }\end{array}$ \\
\hline \multicolumn{8}{|l|}{ Investor-Owned } \\
\hline 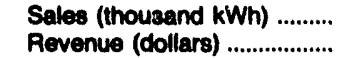 & $=$ & $\begin{array}{r}5,499,090 \\
216,932,055\end{array}$ & $\begin{array}{r}5,910 \\
51,755\end{array}$ & $=$ & $\begin{array}{r}295 \\
-48.061\end{array}$ & $\begin{array}{r}146,370 \\
3,608,477\end{array}$ & $\begin{array}{r}1,034,897 \\
25,971,545\end{array}$ \\
\hline Demand Charge Only' ....... & $\overline{-}$ & $\begin{array}{r}210,002,680 \\
-69,684\end{array}$ & $-101,100$ & - & - & & $20,01,090$ \\
\hline 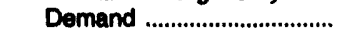 & - & $113,212,264$ & 27,390 & - & 6,334 & $2,253,515$ & - \\
\hline 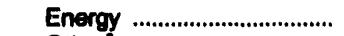 & - & $102,389,903$ & 124,365 & - & 18,070 & $1,354,962$ & $16,875,263$ \\
\hline Other & - & $1,399,572$ & - & - & $-72,465$ & - & $9,096,282$ \\
\hline \multicolumn{8}{|l|}{ Federal } \\
\hline Sales (thousand $\mathrm{kWh}$ ) ........... & - & - & - & - & -- & - & $\overline{-}$ \\
\hline 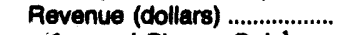 & - & - & - & - & - & - & - \\
\hline Demand Charge Only' ....... & - & - & - & - & - & - & - \\
\hline Jemand & - & - & - & - & - & - & - \\
\hline 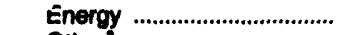 & - & - & - & - & - & - & - \\
\hline Other & - & - & - & - & - & - & - \\
\hline \multicolumn{8}{|l|}{$\begin{array}{l}\text { State and Other } \\
\text { Government }\end{array}$} \\
\hline Sales (thousand kWh) ......... & - & - & - & - & - & 24,719 & - \\
\hline 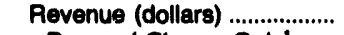 & - & - & - & - & - & 581,418 & - \\
\hline Demand Charge Only' ....... & - & - & - & - & - & - & - \\
\hline Demand & - & - & - & - & - & 366,014 & - \\
\hline 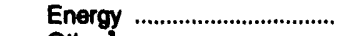 & - & - & - & - & - & 215,404 & - \\
\hline Other & - & - & - & - & - & - & - \\
\hline \multicolumn{8}{|l|}{ Municipal } \\
\hline Sales (thousand $k W h$ ) .......... & 99,330 & - & - & - & $\overline{-}$ & $\overline{-}$ & $\begin{array}{r}14,618 \\
1318,160\end{array}$ \\
\hline 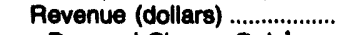 & $6,068,841$ & - & - & - & - & - & $1,318,160$ \\
\hline Demand Charge Only' ....... & - & - & - & - & - & - & - \\
\hline Demand & $4,215,962$ & - & - & - & - & - & 897,600 \\
\hline 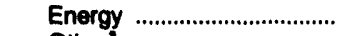 & $1,806,433$ & - & - & - & - & - & 303,541 \\
\hline Other & 46,446 & - & - & - & - & - & 117,018 \\
\hline \multicolumn{8}{|l|}{ Cooperative } \\
\hline Sales (thousand $\mathrm{kWh}$ ) .......... & - & - & - & - & - & - & - \\
\hline Revenue (dollars) .................. & - & - & - & - & $-1,144$ & - & - \\
\hline Demand Charge Only' ....... & - & - & - & - & - & - & - \\
\hline Demand & - & - & - & - & - & -- & - \\
\hline Energy & - & - & - & - & - & -- & - \\
\hline Other & - & - & - & - & $-1,144$ & - & - \\
\hline \multicolumn{8}{|l|}{ Other' } \\
\hline Sales (thousand $\mathbf{k W h}$ ) .......... & 155,018 & - & 986,044 & - & 13,677 & 783,541 & - \\
\hline 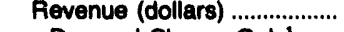 & $2,783,202$ & - & $22,099,869$ & - & 438,340 & $18,762,699$ & - \\
\hline Demand Charge Only' ....... & - & - & - & - & - & - & - \\
\hline 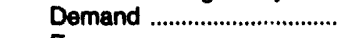 & - & - & 424,103 & - & - & $11,728,933$ & - \\
\hline 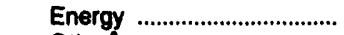 & $2,783,202$ & - & $21,675,766$ & - & 438,340 & $7,033,766$ & - \\
\hline 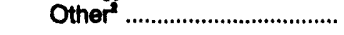 & - & - & - & - & - & - & - \\
\hline \multicolumn{8}{|l|}{ Total } \\
\hline $\begin{array}{l}\text { Sales (thousand kWh) } \\
\text { Revenue (dollars) }\end{array}$ & $\begin{array}{r}254,348 \\
8,852,043\end{array}$ & $\begin{array}{r}5,499,090 \\
216,932,055\end{array}$ & $\begin{array}{r}991,954 \\
22,251,624\end{array}$ & $\overline{-}$ & $\begin{array}{r}13,972 \\
389,135\end{array}$ & $\begin{array}{r}954,630 \\
22,952,594\end{array}$ & $\begin{array}{r}1,049,515 \\
27,289,705\end{array}$ \\
\hline Demand Charge Only' ....... & & $\begin{array}{r}210,932,000 \\
-69,684\end{array}$ & $22,251,624$ & - & - & $22,952,594$ & - \\
\hline 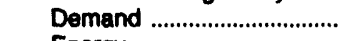 & $4,215,962$ & $113,212,264$ & 451,483 & - & 6,334 & $14,348,462$ & 897,600 \\
\hline 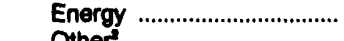 & $4,589,635$ & $102,389,903$ & $21,800,131$ & - & 456,410 & $8,604,132$ & $17,178,804$ \\
\hline Other & 46,446 & $1,399,572$ & - & - & $-73,609$ & - & $9,213,301$ \\
\hline
\end{tabular}

See notes and footnotes at ond of table. 
Table 20. Electricity Sales for Resale by Investor-Owned Utilities, by State, 1992 (Continued)

\begin{tabular}{|c|c|c|c|c|c|c|c|}
\hline $\begin{array}{l}\text { Purchaser of } \\
\text { Electrietty by } \\
\text { Owmerchlp clase }\end{array}$ & $\begin{array}{l}\text { Massachusetts } \\
\text { Holyoke } \\
\text { Water } \\
\text { Power } \\
\text { Co }\end{array}$ & $\begin{array}{l}\text { Massachusetts } \\
\text { Massachusetts } \\
\text { Electric } \\
\text { Co }\end{array}$ & $\begin{array}{l}\text { Massachusetts } \\
\text { Montaup } \\
\text { Electric } \\
\text { Co }\end{array}$ & $\begin{array}{c}\text { Maseachusetts } \\
\text { New } \\
\text { England } \\
\text { Hydro } \\
\text { Trans Eloc } \\
\text { Co }^{\circ}\end{array}$ & $\begin{array}{l}\text { Massachusetts } \\
\text { Now } \\
\text { England } \\
\text { Power } \\
\text { Co }\end{array}$ & $\begin{array}{l}\text { Massachusetts } \\
\text { Western } \\
\text { Massachusetts } \\
\text { Electric } \\
\text { Co }\end{array}$ & $\begin{array}{c}\text { Massachusetts } \\
\text { Yankee } \\
\text { Atomic } \\
\text { Electric } \\
\text { Co' }\end{array}$ \\
\hline \multicolumn{8}{|l|}{ Investor-Owned } \\
\hline $\begin{array}{l}\text { Sales (thousand kWh) .......... } \\
\text { Revenue (dollara) ................. }\end{array}$ & $\begin{array}{r}1,093,265 \\
27,915,940\end{array}$ & $\begin{array}{r}3,676 \\
270,869\end{array}$ & $\begin{array}{r}4,576,633 \\
317,162,144\end{array}$ & - & $\begin{array}{r}21,747,398 \\
1,415,327,501\end{array}$ & $\begin{array}{r}98,268 \\
4,190,666\end{array}$ & $\overline{74,414,340}$ \\
\hline Demand Charge Only' ....... & - & - & - & - & - & - & - \\
\hline 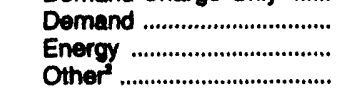 & $\begin{array}{r}\overline{18,181,759} \\
9,734,181\end{array}$ & $\begin{array}{r}50,374 \\
122,263 \\
98,232\end{array}$ & $\begin{array}{r}238,590,559 \\
64,911,153 \\
13,660,432\end{array}$ & $\overline{-}$ & $\begin{array}{l}705,125,248 \\
465,316,749 \\
244,885,504\end{array}$ & $\begin{array}{r}696,643 \\
2,438,831 \\
1,055,192\end{array}$ & $\overline{74,414,340}$ \\
\hline \multicolumn{8}{|l|}{ Federal } \\
\hline Sales (thousand kWh) .......... & - & - & - & - & 50,250 & - & - \\
\hline Revenuo (dollare) .................... & - & - & - & - & $3,402,259$ & - & - \\
\hline Demand Charge Only' ...... & - & - & - & - & - & - & - \\
\hline 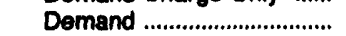 & - & - & - & - & $1,757,301$ & - & - \\
\hline 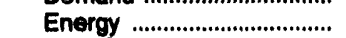 & - & - & - & - & $1,077,829$ & - & - \\
\hline Other & - & - & - & - & 567,129 & - & - \\
\hline \multicolumn{8}{|l|}{$\begin{array}{l}\text { State and Other } \\
\text { Government }\end{array}$} \\
\hline Sales (thousand kWh) ......... & - & - & 229,219 & - & 9,889 & 1,818 & - \\
\hline Revenue (dollars) ................... & - & - & $6,649,941$ & - & 292,325 & 77,602 & - \\
\hline Demand Charge Only' ...... & - & - & - & - & - & - & - \\
\hline 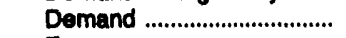 & - & - & $2,106,463$ & - & - & - & - \\
\hline 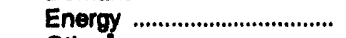 & - & - & $4,453,478$ & - & 174,054 & 52,075 & - \\
\hline 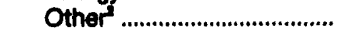 & - & - & - & - & 118,271 & 25,527 & - \\
\hline \multicolumn{8}{|l|}{ Municipal } \\
\hline Sales (thousand kWh) .......... & 159,009 & 71 & 281,217 & - & 415,659 & - & - \\
\hline 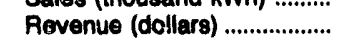 & $8,715,751$ & 7,191 & $10,243,311$ & - & $26,888,042$ & - & - \\
\hline Demand Charge Only" ....... & - & - & -60 & - & - & - & - \\
\hline 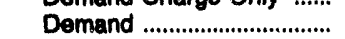 & $4,842,500$ & - & $5,460,531$ & - & $13,080,119$ & - & - \\
\hline 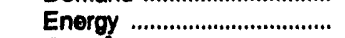 & $3,363,332$ & 5,193 & $4,782,840$ & - & $9,110,630$ & - & - \\
\hline Other & 519,919 & 1,898 & - & - & $4,697,293$ & - & - \\
\hline \multicolumn{8}{|l|}{ Cooperative } \\
\hline Sales (thousand kWh) .......... & - & - & 2,295 & - & 7,353 & - & - \\
\hline 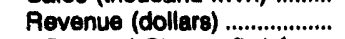 & - & - & 60,383 & - & 434,188 & - & - \\
\hline Demand Charge Only' ...... & - & - & - & - & - & - & - \\
\hline 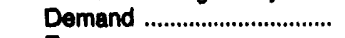 & - & - & $-1,107$ & - & 182,292 & - & - \\
\hline 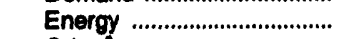 & - & - & 61,490 & - & 164,541 & - & - \\
\hline Other & - & - & - & - & 77,365 & - & - \\
\hline \multicolumn{8}{|l|}{ Other } \\
\hline Sales (thousand kWh) ......... & 601,743 & - & $1,049,825$ & - & $1,304,941$ & 453,584 & - \\
\hline 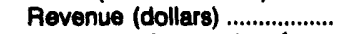 & $8,175,574$ & - & $21,510,187$ & - & $55,648,174$ & $15,739,825$ & - \\
\hline Demand Charge Only' ...... & - & - & - & - & - & - & - \\
\hline 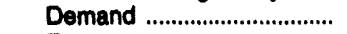 & - & - & 286,612 & - & - & - & - \\
\hline Energy .................................... & $5,123,826$ & $\overline{-}$ & $21,223,575$ & - & $38,613,786$ & $-24,015,216$ & $\overline{-}$ \\
\hline \multirow{2}{*}{\multicolumn{8}{|c|}{ Total }} \\
\hline & & & & & & & \\
\hline $\begin{array}{l}\text { Sales (thousand kWh) .......... } \\
\text { Revenue (dollars) .................. }\end{array}$ & $\begin{array}{r}1,854,017 \\
44,807,265\end{array}$ & $\begin{array}{r}3,747 \\
278,060\end{array}$ & $\begin{array}{r}8,119,289 \\
355,625,966\end{array}$ & - & $\begin{array}{r}23,536,490 \\
1,501,992,499\end{array}$ & $\begin{array}{r}553,670 \\
20,008,093\end{array}$ & $\overline{74,414,340}$ \\
\hline Demand Charge Only' ...... & - & - & -60 & - & - & - & - \\
\hline Demand ................................ & $\begin{array}{r}4,842,500 \\
26,659,017\end{array}$ & $\begin{array}{r}50,374 \\
127,456\end{array}$ & $\begin{array}{r}246,533,058 \\
95,432,536\end{array}$ & $\overline{-}$ & $\begin{array}{l}720,154,960 \\
514,457,589\end{array}$ & $\begin{array}{r}696,643 \\
-21,524,310\end{array}$ & $\overline{74,414,340}$ \\
\hline $\begin{array}{l}\text { Energy } \\
\text { Other }\end{array}$ & $\begin{array}{l}26,659,017 \\
13,305,748\end{array}$ & 100,230 & $\begin{array}{l}80,432,030 \\
13,660,432\end{array}$ & - & $267,379,950$ & $40,835,760$ & - \\
\hline
\end{tabular}

See notes and footnotes at end of table. 
Table 20. Electriclty Sales for Resale by Investor-Owned Utilities, by State, 1992 (Continued)

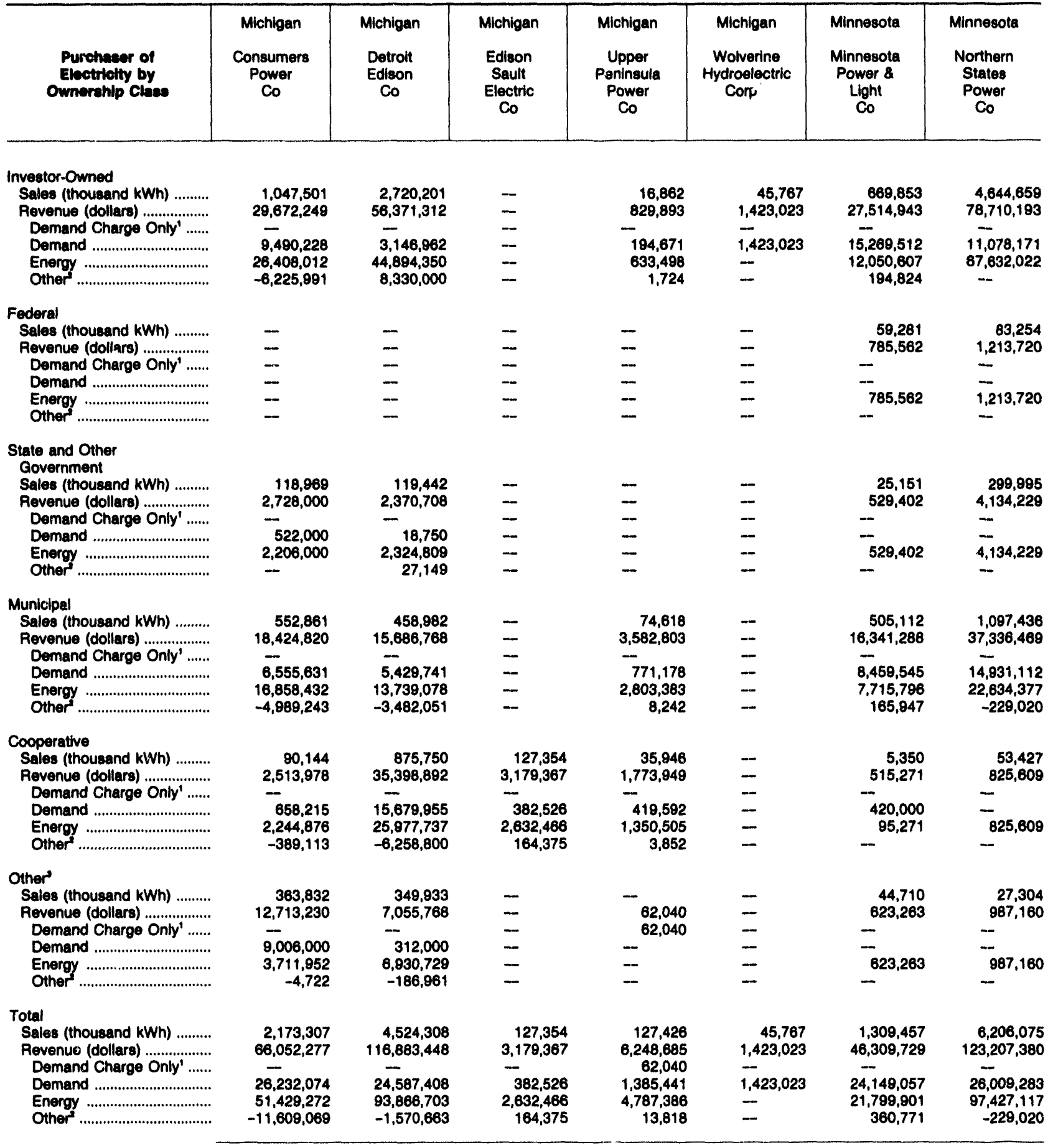

See notes and footnotes at end of table. 
Table 20. Electricity Sales for Resale by Investor-Owned Utilities, by State, 1992 (Continued)

\begin{tabular}{|c|c|c|c|c|c|c|c|}
\hline $\begin{array}{l}\text { Purchaser of } \\
\text { Electrlefty by } \\
\text { Ownerahlp Claes }\end{array}$ & $\begin{array}{c}\text { Minnesota } \\
\text { Otter } \\
\text { Tail } \\
\text { Power } \\
\text { Co }\end{array}$ & $\begin{array}{l}\text { Mississippi } \\
\text { Mississippi } \\
\text { Power \& } \\
\text { Light } \\
\text { Co }\end{array}$ & $\begin{array}{l}\text { Mississippi } \\
\text { Mississippi } \\
\text { Power } \\
\text { Co }\end{array}$ & $\begin{array}{l}\text { Mississippi } \\
\text { Systems } \\
\text { Energy } \\
\text { Resources } \\
\text { Inc }^{4}\end{array}$ & $\begin{array}{c}\text { Missouri } \\
\text { Empire } \\
\text { District } \\
\text { Electric } \\
\text { Co }\end{array}$ & $\begin{array}{l}\text { Missouri } \\
\text { Kansas City } \\
\text { Power \& } \\
\text { Light } \\
\text { Co }\end{array}$ & $\begin{array}{l}\text { Missourl } \\
\text { St Joseph } \\
\text { Light \& } \\
\text { Power } \\
\text { Co }\end{array}$ \\
\hline \multicolumn{8}{|l|}{ investor-Owned } \\
\hline 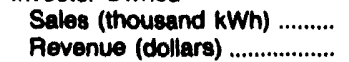 & $\begin{array}{r}411,868 \\
6,847,051\end{array}$ & $\overline{-}$ & $\begin{array}{r}926,400 \\
21,647,423\end{array}$ & $\begin{array}{r}7,353,922 \\
723,409,732\end{array}$ & $\begin{array}{r}314,106 \\
5,245,834\end{array}$ & $\begin{array}{r}1,664,144 \\
23,683,685\end{array}$ & $\begin{array}{r}12,859 \\
194,775\end{array}$ \\
\hline Demand Charge Only' ....... & & $\ddot{-}$ & $21,041,423$ & 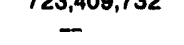 & - & $-\infty, 0,000$ & - \\
\hline 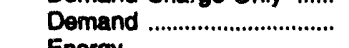 & 240,000 & - & $3,349,834$ & $70-$ & $1,991,832$ & 62,683 & - \\
\hline $\begin{array}{l}\text { Energy } \\
\text { Other }\end{array}$ & $6,707,051$ & $\overline{-}$ & $18,297,589$ & $723,409,732$ & $3,226,657$ & $23,541,103$ & $\underbrace{194,775}_{-}$ \\
\hline & & & & & & & \\
\hline \multicolumn{8}{|l|}{ Federal } \\
\hline Sales (thousand kWh) .......... & - & - & 681 & - & - & - & - \\
\hline Revenue (dollars) .................... & - & - & 17,813 & - & - & - & - \\
\hline Demand Charge Only' ....... & - & - & - & - & - & -- & - \\
\hline 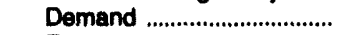 & - & - & - & - & - & - & - \\
\hline 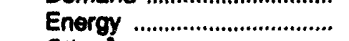 & - & - & 17,913 & - & - & - & - \\
\hline Other & - & - & - & - & 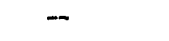 & - & - \\
\hline \multicolumn{8}{|l|}{$\begin{array}{l}\text { State and Other } \\
\text { Government }\end{array}$} \\
\hline Sales (thousand kWh) .......... & 73,227 & - & 3,463 & - & 45,820 & 61,300 & 4,251 \\
\hline Revenue (dollars) ....................... & $1,146,062$ & - & 94,589 & - & 746,317 & $1,078,586$ & 91,096 \\
\hline Demand Charge Only' ...... & - & - & - & - & - & - & - \\
\hline 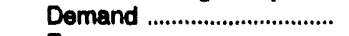 & - & - & - & - & - & - & - \\
\hline Energy ..................................... & $1,146,060$ & - & 94,589 & - & 746,317 & $1,078,586$ & 91,096 \\
\hline Other & - & - & - & - & - & - & - \\
\hline \multicolumn{8}{|l|}{ Municipal } \\
\hline Sales (thousand kWh) .......... & 133,328 & - & 116,409 & - & 208,683 & 909,663 & 130 \\
\hline 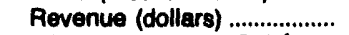 & $2,250,338$ & - & $3,038,490$ & - & $6,448,907$ & $21,108,892$ & 4,521 \\
\hline Demand Charge Only' ....... & & - & - & - & - & - & - \\
\hline Demand ................................. & 197,153 & - & 238,352 & - & $2,409,794$ & $1,277,497$ & - \\
\hline 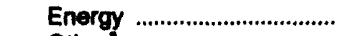 & $2,061,197$ & - & $2,784,781$ & - & $4,039,113$ & $15,096,684$ & 4,521 \\
\hline Other & $-8,012$ & - & 15,357 & - & - & $4,734,711$ & - \\
\hline \multicolumn{8}{|l|}{ Cooperative } \\
\hline Sales (thousand $k W h$ ) .......... & 47,531 & - & $1,583,511$ & - & 12,558 & 408,769 & 10,115 \\
\hline 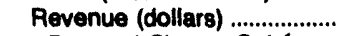 & 782,912 & - & $52,564,036$ & - & 392,714 & $6,506,025$ & 122,011 \\
\hline Demand Charge Only' ...... & - & - & - & - & - & - & -- \\
\hline Demand .................................. & - & - & 400,000 & - & 214,567 & 643,285 & - \\
\hline 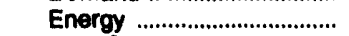 & 782,912 & - & $51,409,315$ & - & 178,147 & $5,985,514$ & 122,011 \\
\hline Other & - & - & 754,721 & - & - & $-122,754$ & - \\
\hline \multicolumn{8}{|l|}{ Other? } \\
\hline Sales (thousand $\mathbf{k W h}$ ) .... ... & - & $1,189,580$ & 280,443 & - & - & - & -- \\
\hline Revenue (dollars) ................... & - & $37,982,637$ & $10,054,709$ & - & - & - & - \\
\hline Demand Charge Only' ....... & - & - & - & - & - & - & - \\
\hline 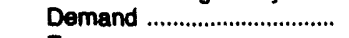 & - & $4,722,872$ & $5,023,104$ & - & - & - & - \\
\hline 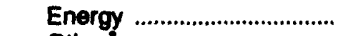 & - & $33,259,765$ & $5,031,605$ & - & - & - & - \\
\hline 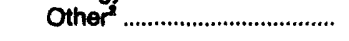 & - & - & - & - & - & - & - \\
\hline \multicolumn{8}{|l|}{ Total } \\
\hline Sales (thousand kWh) .......... & 665,954 & $\begin{array}{r}1,189,580 \\
\end{array}$ & $2,910,907$ & $\begin{array}{r}7,353,922 \\
7,922\end{array}$ & $\begin{array}{r}581,167 \\
583,770\end{array}$ & $\begin{array}{r}3,043,876 \\
5,077,88\end{array}$ & 27,355 \\
\hline $\begin{array}{l}\text { Revenue (dollars) ........... } \\
\text { Demand Charge Only' }\end{array}$ & $11,126,361$ & $37,982,637$ & $87,417,160$ & $723,409,732$ & $12,833,772$ & $52,377,188$ & $ـ_{-}^{412,403}$ \\
\hline Demand Charge Only' ...... & & $\overline{4722872}$ & $\overline{9.011290}$ & $\overline{-}$ & $\overline{4.316 .193}$ & $\overline{1.983,445}$ & $=$ \\
\hline 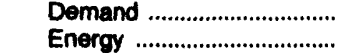 & $\begin{array}{r}437,153 \\
10,697,220\end{array}$ & $\begin{array}{r}4,722,872 \\
33,259,765\end{array}$ & $\begin{array}{r}9,011,290 \\
77,635,792\end{array}$ & $72 \overline{3}, 409,732$ & $\begin{array}{l}4,316,193 \\
8,190,234\end{array}$ & $\begin{array}{r}1,983,445 \\
45,701,887\end{array}$ & 412,403 \\
\hline 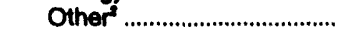 & $-8,012$ & - & 770,078 & - & 27,345 & $4,691,856$ & - \\
\hline
\end{tabular}

See notes and footnotes at end of table. 
Table 20. Electricity Sales for Resale by Investor-Owned Utilitles, by State, 1992 (Continued)

\begin{tabular}{|c|c|c|c|c|c|c|c|}
\hline $\begin{array}{l}\text { Purchacer of } \\
\text { Eleotricthy by } \\
\text { Owmerehlp clase }\end{array}$ & $\begin{array}{l}\text { Mlesouri } \\
\text { Union } \\
\text { Electric } \\
\text { Co }\end{array}$ & $\begin{array}{l}\text { Missourl } \\
\text { Utilicorp } \\
\text { United } \\
\text { Inc }\end{array}$ & $\begin{array}{l}\text { Montana } \\
\text { Montana } \\
\text { Power } \\
\text { Co }\end{array}$ & $\begin{array}{l}\text { Nevada } \\
\text { Nevada } \\
\text { Power } \\
\text { Co }\end{array}$ & $\begin{array}{l}\text { Nevada } \\
\text { Sierra } \\
\text { Pacific } \\
\text { Power } \\
\text { Co }\end{array}$ & $\begin{array}{c}\text { New Hampshire } \\
\text { Concord } \\
\text { Electric } \\
\mathrm{Co}^{4}\end{array}$ & $\begin{array}{c}\text { Now Hampshire } \\
\text { Connecticut } \\
\text { Valley } \\
\text { Electric } \\
\mathrm{Co}^{4}\end{array}$ \\
\hline \multicolumn{8}{|l|}{ Imvestor-Owned } \\
\hline Sales (thousand kWh) .......... & $3,262,227$ & 25,616 & $1,489,164$ & - & 27,777 & -- & - \\
\hline 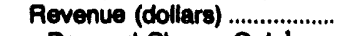 & $59,387,424$ & 480,835 & $44,030,840$ & - & 797,750 & -- & - \\
\hline Demand Charge Only' ....... & - & - & - & - & - & - & - \\
\hline 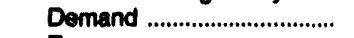 & $5,902,918$ & - & - & - & 132,562 & - & - \\
\hline 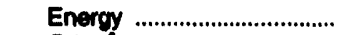 & $53,542,088$ & 480,835 & $44,030,840$ & - & 649,329 & - & -- \\
\hline Other & $-57,582$ & - & - & - & 15,859 & -- & - \\
\hline \multicolumn{8}{|l|}{ Federal } \\
\hline Sales (thousand kWh) .......... & 96,442 & - & $1,540,202$ & - & 8,766 & - & - \\
\hline Revenue (dollars) ...................... & $1,507,600$ & - & $34,705,807$ & - & 534,716 & - & - \\
\hline Demand Charge Only' ...... & - & - & - & - & -- & - & -- \\
\hline 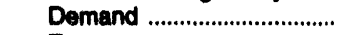 & - & - & - & - & 183,539 & -- & -- \\
\hline 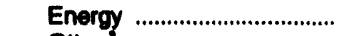 & $1,507,600$ & - & $34,705,807$ & - & 393,068 & - & - \\
\hline Other & - & - & - & - & $-41,891$ & - & - \\
\hline \multicolumn{8}{|l|}{$\begin{array}{l}\text { State and Other } \\
\text { Government }\end{array}$} \\
\hline Sales (thousand kWh) .......... & - & -- & 88,959 & 268,195 & 140,472 & - & - \\
\hline 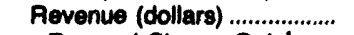 & - & - & $1,624,704$ & $8,149,232$ & $6,396,370$ & - & - \\
\hline Demand Charge Only' ...... & - & - & - & - & - & - & - \\
\hline 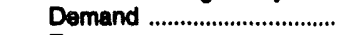 & - & - & - & 417,560 & $1,782,671$ & - & -- \\
\hline 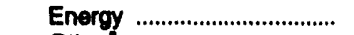 & - & - & $1,624,704$ & $7,651,576$ & $5,239,553$ & - & - \\
\hline Other & - & - & -- & 80,096 & $-625,854$ & - & - \\
\hline \multicolumn{8}{|l|}{ Municipal } \\
\hline Sales (thousand kWh) .......... & $1,073,932$ & 363,714 & 928,579 & 50,515 & 51,816 & - & - \\
\hline Revenus (dollars) ..................... & $37,661,093$ & $12,429,057$ & $42,829,253$ & $2,217,710$ & 2,926,962 & - & - \\
\hline Demand Charge Only' ....... & - & - & - & - & - & - & -- \\
\hline 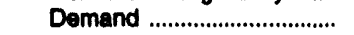 & $1,235,496$ & $4,138,305$ & - & $1,060,655$ & 910,882 & - & - \\
\hline 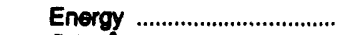 & $36,462,316$ & $8,960,878$ & $42,829,253$ & $1,044,306$ & $2,323,430$ & - & - \\
\hline Other & $-36,719$ & $-670,126$ & - & 112,749 & $-307,350$ & - & - \\
\hline \multicolumn{8}{|l|}{ Cooperative } \\
\hline Sales (thousand kWh) .......... & 869,995 & 104,045 & 407,243 & 154,590 & - & - & - \\
\hline Revenue (dollars) .................. & $27,466,607$ & $4,526,029$ & $14,014,721$ & $5,226,059$ & - & - & - \\
\hline Demand Charge Only' ...... & - & - & - & - & - & - & - \\
\hline 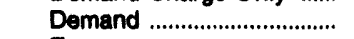 & - & $2,575,271$ & $4,379,014$ & $1,247,455$ & - & - & - \\
\hline 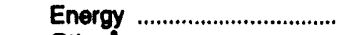 & $27,466,607$ & $1,957,630$ & $9,635,707$ & $3,955,836$ & -- & - & -- \\
\hline Other & - & $-6,872$ & - & 22,768 & - & - & -- \\
\hline \multicolumn{8}{|l|}{ Other" } \\
\hline Sales (thousand kWh) .......... & $1,025,372$ & $-1,262$ & $-1,792$ & - & - & - & -- \\
\hline Revenue (dollars) ...................... & $16,107,622$ & $-6,153$ & $9,088,211$ & - & - & - & - \\
\hline Demand Charge Only' ....... & - & -- & - & - & - & - & -- \\
\hline 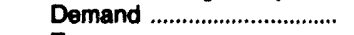 & 32,400 & - & - & - & - & - & - \\
\hline 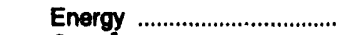 & $16,075,222$ & $-5,253$ & - & - & - & - & - \\
\hline Other' & - & -900 & $9,088,211$ & - & - & - & - \\
\hline \multicolumn{8}{|l|}{ Total } \\
\hline Sales (thousand kWh) .......... & & 492,113 & $4,453,355$ & 473,300 & 228,831 & - & -- \\
\hline Revenue (dollars) ..................... & $142,130,346$ & $17,429,768$ & $146,293,536$ & $15,593,001$ & $10,655,798$ & - & - \\
\hline Demand Charge Only' ....... & - & - & - & - & - & - & - \\
\hline 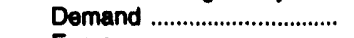 & $7,170,814$ & $6,713,576$ & $4,379,014$ & $2,725,670$ & $3,009,654$ & - & - \\
\hline Energy .................................. & $135,053,833$ & $11,394,090$ & $132,826,311$ & $12,651,718$ & $8,605,380$ & - & - \\
\hline 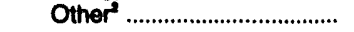 & $-94,301$ & $-677,898$ & $9,088,211$ & 215,613 & $-959,236$ & - & -- \\
\hline
\end{tabular}

See notes and footnotes at end of table. 
Table 20. Electricity Sales for Resale by Investor-Owned Utilities, by State, 1992 (Continued)

\begin{tabular}{|c|c|c|c|c|c|c|c|}
\hline $\begin{array}{l}\text { Purchaeer of } \\
\text { Eloctrictly by } \\
\text { Owmorehlp Claes }\end{array}$ & $\begin{array}{c}\text { New Hampshire } \\
\text { Exeter \& } \\
\text { Hampton } \\
\text { Eloctric } \\
\mathrm{Co}^{4}\end{array}$ & \begin{tabular}{|c|} 
New Hampshire \\
Granite \\
State \\
Electric \\
Co $^{4}$
\end{tabular} & $\begin{array}{c}\text { Now Hampshire } \\
\text { Now } \\
\text { England } \\
\text { Trans } \\
\text { Corp" }\end{array}$ & $\begin{array}{c}\text { Now Hampshire } \\
\text { Now } \\
\text { England } \\
\text { Hydro-Trans } \\
\text { Corp" }\end{array}$ & $\begin{array}{c}\text { Now Hampshire } \\
\text { North } \\
\text { Attantic } \\
\text { Energy } \\
\text { Corp }\end{array}$ & $\begin{array}{c}\text { Now Hampshire } \\
\text { Public } \\
\text { Service } \\
\text { Co of NH }\end{array}$ & $\begin{array}{c}\text { Now Hampshire } \\
\text { Unitil } \\
\text { Power } \\
\text { Corp }\end{array}$ \\
\hline \multicolumn{8}{|l|}{ Investor-Owned } \\
\hline Sales (thousand $k W h$ ) .......... & - & - & - & - & $1,268,123$ & 823,435 & 946,047 \\
\hline 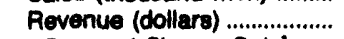 & - & - & - & - & $78,443,879$ & $32,841,712$ & $70,383,994$ \\
\hline Demand Charge Only' ....... & - & - & - & - & - & - & - \\
\hline 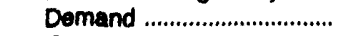 & - & - & - & - & - & 61,905 & $31,702,925$ \\
\hline 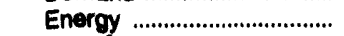 & - & - & - & - & $1,687,971$ & $12,106,000$ & $17,761,124$ \\
\hline Other & - & - & - & - & $76,755,908$ & $20,673,717$ & $20,819,045$ \\
\hline \multicolumn{8}{|l|}{ Federal } \\
\hline Sales (thousand kWh) .......... & - & - & - & - & - & - & - \\
\hline Revenue (dollars) ....................... & - & - & - & - & - & - & - \\
\hline Demand Charge Only' ....... & - & - & - & - & - & - & - \\
\hline 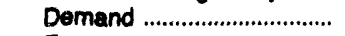 & - & - & - & - & - & - & - \\
\hline 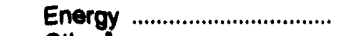 & - & - & - & - & - & - & - \\
\hline 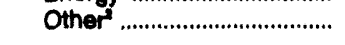 & - & - & - & - & - & - & - \\
\hline \multicolumn{8}{|l|}{$\begin{array}{l}\text { State and Other } \\
\text { Government }\end{array}$} \\
\hline $\begin{array}{l}\text { Government } \\
\text { Sales (thousand kWh) ......... }\end{array}$ & - & - & - & - & - & 113,220 & - \\
\hline 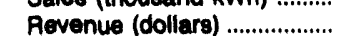 & - & - & - & - & - & $3,331,800$ & - \\
\hline Demand Charge Only' ...... & - & - & - & - & - & - & - \\
\hline Demand ................. & - & - & - & - & - & - & - \\
\hline 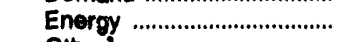 & - & - & - & - & - & $1,999,800$ & - \\
\hline 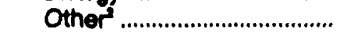 & - & - & - & - & - & $1,332,000$ & - \\
\hline \multicolumn{8}{|l|}{ Municipal } \\
\hline Sales (thousand kWh) .......... & - & - & - & - & - & 82,685 & - \\
\hline 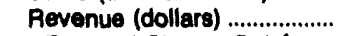 & - & - & - & - & - & $5,615,657$ & - \\
\hline Demand Charge Only' ....... & - & - & - & - & - & - & - \\
\hline Demand & - & - & - & - & - & $1,797,396$ & - \\
\hline 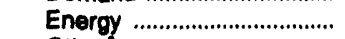 & - & - & - & - & -- & $3,793,753$ & - \\
\hline 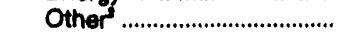 & - & - & - & - & - & 24,508 & - \\
\hline \multicolumn{8}{|l|}{ Cooperative } \\
\hline Sales (thousand kWh) .......... & - & - & - & - & - & 581,442 & - \\
\hline Revenue (dollars) ...................... & - & - & - & - & - & $38,089,320$ & - \\
\hline Demand Charge Only'...... & - & - & - & - & - & - & - \\
\hline Demand & - & - & - & - & - & $19,297,778$ & - \\
\hline 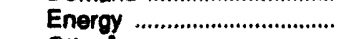 & - & - & - & - & - & $21,951,202$ & - \\
\hline 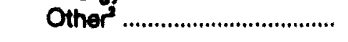 & - & - & - & - & - & $-3,159,660$ & - \\
\hline \multicolumn{8}{|l|}{ Other' } \\
\hline Sales (thousand kWh) ......... & - & - & - & - & - & $\begin{array}{r}4,486,057 \\
\end{array}$ & $\begin{array}{r}1,245 \\
\end{array}$ \\
\hline 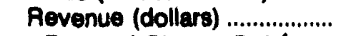 & - & - & - & - & - & $106,554,980$ & $1,155,385$ \\
\hline Demand Charge Only' ...... & - & - & - & - & - & - & - \\
\hline 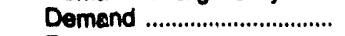 & - & - & - & - & - & - & - \\
\hline 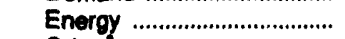 & - & - & - & - & - & $100,830,928$ & $1,155,365$ \\
\hline Other ................................... & - & - & - & - & - & $5,624,052$ & - \\
\hline \multicolumn{8}{|l|}{ Total } \\
\hline Sales (thousand kWh) .......... & - & - & - & - & $1,268,123$ & $6,086,839$ & $\begin{array}{r}947,292 \\
\end{array}$ \\
\hline Revenue (dollars) ...................... & - & - & - & - & $78,443,879$ & $186,433,469$ & $71,539,359$ \\
\hline Demand Charge Only' ....... & - & - & - & - & - & - & - \\
\hline Demand & - & - & - & - & - & $21,157,078$ & $31,702,925$ \\
\hline Energy ................................. & - & - & - & - & $1,687,971$ & $140,781,773$ & $18,916,489$ \\
\hline Other & - & - & - & - & $76,755,908$ & $24,494,617$ & $20,919,845$ \\
\hline
\end{tabular}

See notes and footnotes at end of table. 
Table 20. Electricity Sales for Resale by Investor-Owned Utilities, by State, 1992 (ContInued)

\begin{tabular}{|c|c|c|c|c|c|c|c|}
\hline $\begin{array}{l}\text { Purchaear of } \\
\text { Eleotrithy by } \\
\text { Ownerentp clases }\end{array}$ & $\begin{array}{l}\text { Now Jersoy } \\
\text { Atlantic City } \\
\text { Electric } \\
\text { Co }\end{array}$ & $\begin{array}{l}\text { New Jersey } \\
\text { Jersey } \\
\text { Contral } \\
\text { Power \& } \\
\text { Light } \\
\text { Co }\end{array}$ & $\begin{array}{l}\text { New Jersey } \\
\text { Public } \\
\text { Service } \\
\text { Electric \& } \\
\text { Gas } \\
\text { Co }\end{array}$ & $\begin{array}{l}\text { Now Jersey } \\
\text { Pockland } \\
\text { Electric } \\
\text { Co' }\end{array}$ & $\begin{array}{l}\text { New Mexico } \\
\text { Public } \\
\text { Service } \\
\text { Co of NM }\end{array}$ & $\begin{array}{l}\text { New Mexico } \\
\text { Texas- } \\
\text { Now Mexico } \\
\text { Power } \\
\text { Co" }\end{array}$ & $\begin{array}{l}\text { Now York } \\
\text { Contral } \\
\text { Hudson } \\
\text { Gas \& } \\
\text { Electric } \\
\text { Corp }\end{array}$ \\
\hline 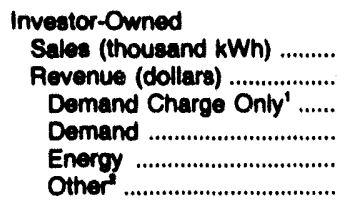 & $\begin{array}{l}\bar{z} \\
\bar{z}\end{array}$ & $\begin{array}{r}83,283 \\
3,883,829 \\
-\quad 889,284 \\
3,094,545 \\
-\end{array}$ & $\begin{array}{r}1,051,242 \\
23,213,398 \\
-\quad \\
419,895 \\
22,794,048 \\
-545\end{array}$ & $\begin{array}{l}\overline{-} \\
\bar{z} \\
\overline{-}\end{array}$ & $\begin{array}{r}2,181,497 \\
72,560,156 \\
- \\
30,811,651 \\
41,332,302 \\
416,203\end{array}$ & $\begin{array}{l}\overline{-} \\
\overline{-} \\
\overline{-}\end{array}$ & $\begin{array}{r}48,220 \\
2,495,868 \\
- \\
915,000 \\
1,580,688 \\
-\end{array}$ \\
\hline 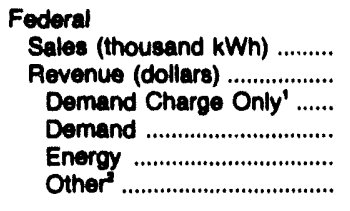 & $\begin{array}{l}\bar{z} \\
\bar{z} \\
\overline{-}\end{array}$ & $\bar{z}$ & $\begin{array}{l}\bar{z} \\
\bar{m} \\
\bar{m}\end{array}$ & $\begin{array}{l}\bar{z} \\
\bar{z} \\
\bar{m}\end{array}$ & $\begin{array}{l}125,674 \\
2,648,784 \\
- \\
- \\
2,648,784 \\
-\end{array}$ & $\begin{array}{l}\overline{-} \\
\bar{m} \\
\overline{-}\end{array}$ & $\begin{array}{l}- \\
\overline{-} \\
\overline{-} \\
-\end{array}$ \\
\hline 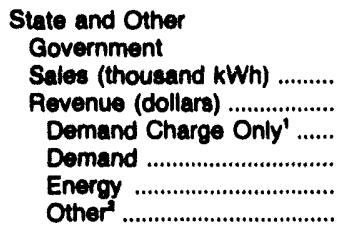 & $\begin{array}{l}\overline{-} \\
\bar{z} \\
\overline{-}\end{array}$ & $\begin{array}{l}\overline{-} \\
\bar{m} \\
\bar{m}\end{array}$ & $\begin{array}{l}152,100 \\
4,166,700 \\
- \\
\overline{4,166,700}\end{array}$ & $\begin{array}{l}\overline{-} \\
\bar{z} \\
\overline{-}\end{array}$ & $\begin{array}{r}488,592 \\
14,783,359 \\
- \\
5,878,442 \\
8,702,220 \\
202,697\end{array}$ & $\begin{array}{l}- \\
\overline{-} \\
\overline{-} \\
-\end{array}$ & $\begin{array}{l}-4,410 \\
-\quad 4,410 \\
- \\
-\end{array}$ \\
\hline 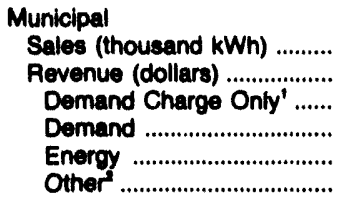 & $\begin{array}{l}351,729 \\
15,847,719 \\
- \\
\overline{7,948,461} \\
7,689,258\end{array}$ & $\begin{array}{r}268,228 \\
16,978,363 \\
- \\
10,859,804 \\
6,118,559 \\
-\end{array}$ & $\begin{array}{r}125,833 \\
7,494,737 \\
2,482,783 \\
5,248,581 \\
-236,627\end{array}$ & $\begin{array}{l}\bar{z} \\
\bar{m} \\
\bar{m}\end{array}$ & $\begin{array}{r}474,723 \\
16,329,786 \\
- \\
7,019,529 \\
8,472,273 \\
837,884\end{array}$ & $\begin{array}{l}\overline{-} \\
\bar{z} \\
\overline{-}\end{array}$ & $\begin{array}{l}\overline{-} \\
\bar{z} \\
\overline{-}\end{array}$ \\
\hline 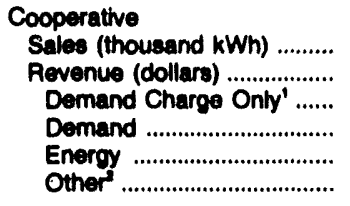 & $\begin{array}{l}\bar{z} \\
\bar{z}\end{array}$ & $\begin{array}{r}44,525 \\
3,878,410 \\
- \\
2,882,791 \\
995,619 \\
-\end{array}$ & $\begin{array}{l}\bar{z} \\
\bar{z} \\
\bar{m}\end{array}$ & $\begin{array}{l}\bar{z} \\
\bar{z} \\
\bar{m}\end{array}$ & $\begin{array}{r}293,830 \\
10,561,770 \\
- \\
5,006,991 \\
5,502,351 \\
52,428\end{array}$ & $\begin{array}{l}\overline{-} \\
\bar{z} \\
\bar{m}\end{array}$ & $\begin{array}{l}\overline{-} \\
\overline{-} \\
\overline{-}\end{array}$ \\
\hline 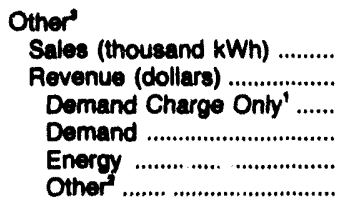 & $\begin{array}{r}701,352 \\
20,127,109 \\
- \\
\overline{20,064,831} \\
62,278\end{array}$ & $\begin{array}{r}1,915,574 \\
49,308,217 \\
\overline{4,610,708} \\
44,697,509 \\
-\end{array}$ & $\begin{array}{r}922,848 \\
24,443,387 \\
- \\
- \\
24,401,038 \\
42,349\end{array}$ & $\begin{array}{l}\bar{z} \\
\bar{z}\end{array}$ & $\begin{array}{r}121,102 \\
6,407,145 \\
- \\
4,345,724 \\
2,060,449 \\
972\end{array}$ & $\begin{array}{l}\overline{-} \\
\overline{-} \\
\overline{-}\end{array}$ & $\begin{array}{l}521,635 \\
15,973,451 \\
-- \\
\overline{15,973,451} \\
--\end{array}$ \\
\hline 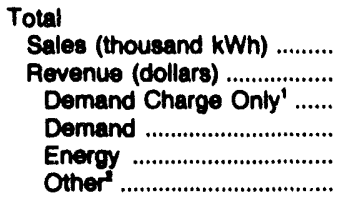 & $\begin{array}{r}1,053,081 \\
35,774,828 \\
\overline{-} \\
28,013,292 \\
7,761,536\end{array}$ & $\begin{array}{r}2,311,610 \\
74,148,819 \\
- \\
19,242,587 \\
54,906,232 \\
-\end{array}$ & $\begin{array}{r}2,252,023 \\
59,318,222 \\
\overline{2,802,678} \\
56,610,367 \\
-194,823\end{array}$ & $\begin{array}{l}\overline{-} \\
\bar{z} \\
\overline{-}\end{array}$ & $\begin{array}{r}3,685,418 \\
123,291,000 \\
- \\
53,062,337 \\
68,718,379 \\
1,510,284\end{array}$ & $\begin{array}{l}\overline{-} \\
\bar{z} \\
\bar{m}\end{array}$ & $\begin{array}{r}570,855 \\
18,473,529 \\
4,410 \\
915,000 \\
17,554,119 \\
-\end{array}$ \\
\hline
\end{tabular}

See notes and footnotes at end of table. 
Table 20. Electricity Sales for Resale by Investor-Owned Utilitles, by State, 1992 (Continued)

\begin{tabular}{|c|c|c|c|c|c|c|c|}
\hline $\begin{array}{l}\text { Purcheser of } \\
\text { Electrictly by } \\
\text { Ownership Claese }\end{array}$ & $\begin{array}{l}\text { Now York } \\
\text { Consolidated } \\
\text { Edison } \\
\text { Co-NY } \\
\text { inc }\end{array}$ & $\begin{array}{c}\text { Now York } \\
\text { Long } \\
\text { lsland } \\
\text { Lighting } \\
\text { Co }\end{array}$ & $\begin{array}{l}\text { Now York } \\
\text { Long } \\
\text { Sautt } \\
\text { Inc }^{\circ}\end{array}$ & $\begin{array}{l}\text { New York } \\
\text { New York } \\
\text { State } \\
\text { Electric } \\
8 \text { Gas } \\
\text { Corp }\end{array}$ & $\begin{array}{c}\text { Now York } \\
\text { Nlagara } \\
\text { Mohawk } \\
\text { Power } \\
\text { Corp }\end{array}$ & $\begin{array}{c}\text { New York } \\
\text { Orange } \\
\& \\
\text { Aockland } \\
\text { Utilities } \\
\text { Inc }\end{array}$ & $\begin{array}{l}\text { New York } \\
\text { Rochester } \\
\text { Gas \& } \\
\text { Electric } \\
\text { Corp }\end{array}$ \\
\hline \multicolumn{8}{|l|}{ Investor-Owned } \\
\hline Sales (thousand kWh) & - & - & - & $1,862,900$ & 264,450 & $1,344,428$ & 6,851 \\
\hline 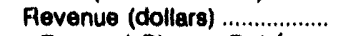 & $-6,115$ & -- & - & $53,001,400$ & $14,833,684$ & $68,564,041$ & 587,007 \\
\hline Demand Charge Only' ...... & - & - & - & - & - & - & - \\
\hline 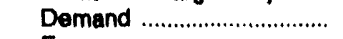 & $\cdots$ & - & -- & 27,491 & - & $21,807,675$ & - \\
\hline 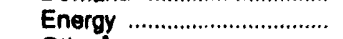 & - & - & - & $52,973,278$ & $14,933,664$ & $46,756,366$ & 587,007 \\
\hline Other & $-6,115$ & -- & - & 631 & - & - & $-\infty$ \\
\hline \multicolumn{8}{|l|}{ Federal } \\
\hline Sales (thousand kWh) .......... & - & - & - & - & - & - & - \\
\hline Revenue (dollars) & - & - & - & - & - & - & - \\
\hline Demand Chargo Only' ...... & - & - & - & -- & - & - & - \\
\hline Demand ................................ & - & - & - & - & -- & -- & $\cdots$ \\
\hline Energy ................................. & $\cdots$ & - & - & -- & -- & $-\infty$ & -- \\
\hline Other & - & - & - & - & - & - & $\cdots$ \\
\hline \multicolumn{8}{|l|}{$\begin{array}{l}\text { State and Other } \\
\text { Government }\end{array}$} \\
\hline Sales (thousand kWh) ......... & 78,173 & - & - & $1,146,119$ & 542,580 & 886 & 14,633 \\
\hline Revenue (dollara) .................. & $14,846,633$ & - & - & $27,002,870$ & $12,959,996$ & - & 730,557 \\
\hline Demand Charge Only' ...... & - & - & - & - & - & - & -- \\
\hline Demand ............................... & $8,262,559$ & - & - & - & - & -- & - \\
\hline 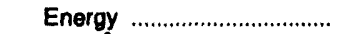 & $6,584,074$ & - & - & $27,002,870$ & $12,959,996$ & -- & 730,557 \\
\hline Other & - & - & - & - & -- & - & -- \\
\hline \multicolumn{8}{|l|}{ Municipal } \\
\hline Sales (thousand kWh) ......... & - & - & - & - & -- & - & $-\cdots$ \\
\hline Revenue (dollars) ..................... & - & - & - & - & 879 & - & - \\
\hline Demand Charge Only' ...... & - & $-m$ & - & - & - & - & -- \\
\hline Demand & - & - & - & - & - & - & - \\
\hline 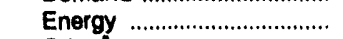 & - & - & - & - & 879 & - & -- \\
\hline Other & - & - & - & - & -- & - & - \\
\hline \multicolumn{8}{|l|}{ Cooperative } \\
\hline Sales (thousand kWh) ......... & - & - & - & - & - & - & -- \\
\hline 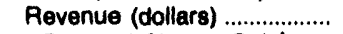 & - & - & - & - & - & - & - \\
\hline Demand Charge Only' ...... & - & - & - & - & - & - & -- \\
\hline 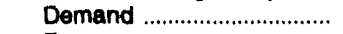 & - & - & -- & - & - & - & -- \\
\hline 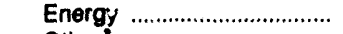 & - & - & - & - & - & - & -- \\
\hline Other & - & - & - & - & - & - & -- \\
\hline \multicolumn{8}{|l|}{ Other' } \\
\hline $\begin{array}{l}\text { Sales (thousand kWh) .......... } \\
\text { Revenue (dollars) ....................... }\end{array}$ & $\begin{array}{r}365,459 \\
13,504,066\end{array}$ & $\begin{array}{r}227,482 \\
9,996,783\end{array}$ & - & $\begin{array}{r}2,893,787 \\
63,409,314\end{array}$ & $\begin{array}{r}2,045,380 \\
55,938,618\end{array}$ & $\begin{array}{r}236,641 \\
6,664,049\end{array}$ & $\begin{array}{r}1,041,454 \\
24,224,028\end{array}$ \\
\hline Demand Charge Only' ...... & $-\infty$ & - & - & - & - & $-\infty$ & -- \\
\hline 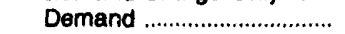 & $1,297,818$ & - & - & - & - & -- & -- \\
\hline 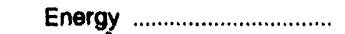 & $12,138,853$ & $9,996,783$ & - & $63,409,314$ & $55,938,618$ & $6,549,790$ & $24,224,028$ \\
\hline Other & 67,395 & - & -- & - & - & 114,259 & - \\
\hline \multicolumn{8}{|l|}{ Total } \\
\hline Sales (thousand kWh) ......... & 443,632 & 227,482 & - & $6,002,806$ & $2,852,410$ & $1,581,955$ & $1,062,738$ \\
\hline 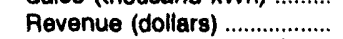 & $28,344,584$ & $9,996,783$ & $m$ & $143,413,584$ & $83,833,157$ & $75,228,090$ & $25,541,592$ \\
\hline Demand Charge Only' ...... & 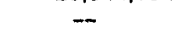 & $\cdots$ & - & - & - & - & - \\
\hline 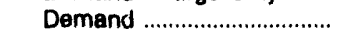 & $9,560,377$ & -- & -- & 27,491 & - & $21,807,675$ & - \\
\hline 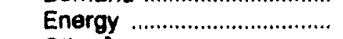 & $18,722,927$ & $9,996,783$ & $-\therefore$ & $143,385,462$ & $83,833,157$ & $53,30 e .156$ & $25,541,592$ \\
\hline Other & 61,280 & - & - & 631 & - & 114,259 & - \\
\hline
\end{tabular}

See notes and footnotes at end of table. 
Table 20. Electricity Sales for Resale by Investor-Owned Utilities, by State, 1992 (Continued)

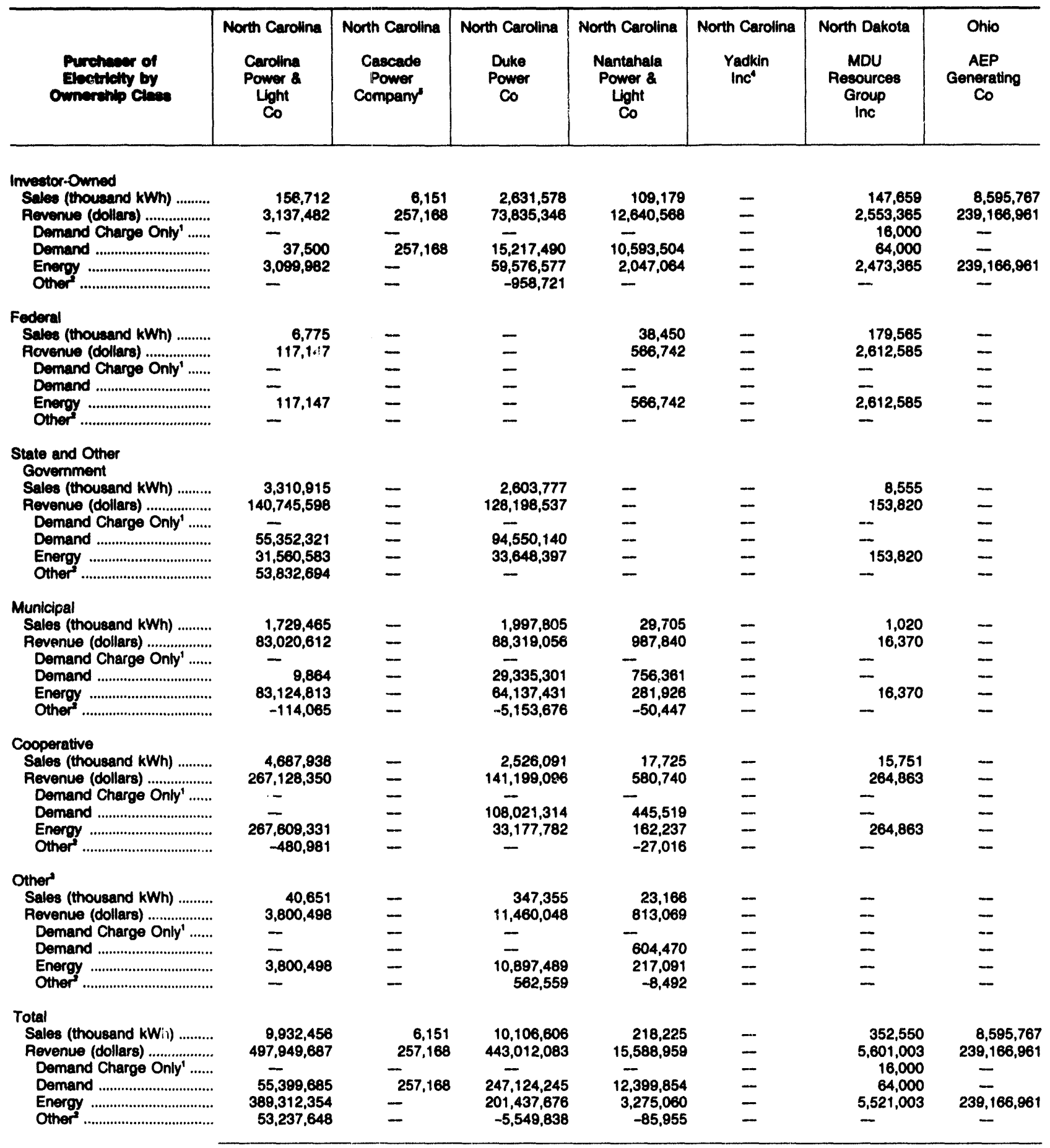

See notes and footnotes at end of table. 
Table 20. Electriclty Sales for Resale by Investor-Owned Utilities, by State, 1992 (Continued)

\begin{tabular}{|c|c|c|c|c|c|c|c|}
\hline $\begin{array}{l}\text { Purcheser of } \\
\text { Electuletty by } \\
\text { Owmorchip clases }\end{array}$ & $\begin{array}{c}\text { Ohio } \\
\text { Cincinnat } \\
\text { Cas \& } \\
\text { Electric } \\
\text { Co }\end{array}$ & $\begin{array}{c}\text { Ohio } \\
\text { Cloveland } \\
\text { Electric } \\
\text { Illuminating } \\
\text { Co }\end{array}$ & $\begin{array}{c}\text { Onio } \\
\text { Columbus } \\
\text { Southern } \\
\text { Power Co }\end{array}$ & $\begin{array}{l}\text { Ohio } \\
\text { Dayton } \\
\text { Power } 8 \\
\text { Light } \\
\text { Co }\end{array}$ & $\begin{array}{l}\text { Ohio } \\
\text { Indiana- } \\
\text { Kentucky } \\
\text { Electric } \\
\text { Corp' }\end{array}$ & $\begin{array}{l}\text { Onio } \\
\text { Ohio } \\
\text { Edison } \\
\text { Co }\end{array}$ & $\begin{array}{l}\text { Ohio } \\
\text { Ohio } \\
\text { Power } \\
\text { Co }\end{array}$ \\
\hline 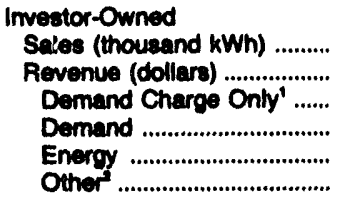 & $\begin{array}{r}4,613,776 \\
155,926,258 \\
- \\
61,234,112 \\
97,691,670 \\
-2,999,524\end{array}$ & $\begin{array}{r}341,239 \\
5,031,792 \\
- \\
130,356 \\
4,647,761 \\
53,675\end{array}$ & $\begin{array}{r}381,028 \\
14,465,044 \\
1,124,046 \\
3,814,529 \\
9,526,469 \\
-\end{array}$ & $\begin{array}{r}1,056,182 \\
18,348,453 \\
- \\
3,919,680 \\
14,482,183 \\
-52,410\end{array}$ & $\begin{array}{r}9,185,404 \\
140,703,815 \\
- \\
40,071,030 \\
100,632,785 \\
-\end{array}$ & $\begin{array}{r}7,784,188 \\
214,823,156 \\
- \\
8 \overline{7}, 788,072 \\
128,815,468 \\
2,219,616\end{array}$ & $\begin{array}{r}4,638,502 \\
130,248,240 \\
1,936,881 \\
26,172,681 \\
102,138,677 \\
1\end{array}$ \\
\hline 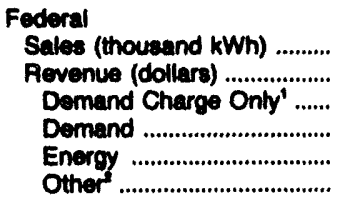 & $\begin{array}{l}\bar{z} \\
\bar{z}\end{array}$ & $\begin{array}{l}\bar{z} \\
\bar{z} \\
\overline{-}\end{array}$ & $\begin{array}{r}9,634 \\
239,489 \\
-\quad \\
-239,499\end{array}$ & $\begin{array}{l}- \\
\bar{z} \\
=\end{array}$ & $\begin{array}{l}\overline{-} \\
\overline{-} \\
\bar{m}\end{array}$ & $\begin{array}{l}\overline{-} \\
\overline{-}\end{array}$ & $\begin{array}{l}16,564 \\
411,519 \\
- \\
- \\
-\end{array}$ \\
\hline 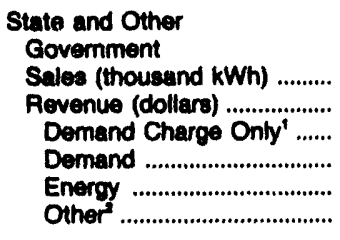 & $\begin{array}{l}\overline{-} \\
\bar{m}\end{array}$ & 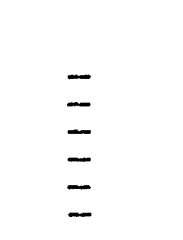 & $\begin{array}{l}\bar{z} \\
\bar{z}\end{array}$ & $\begin{array}{l}= \\
= \\
=\end{array}$ & 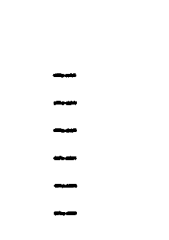 & 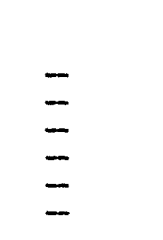 & 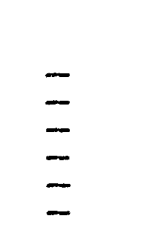 \\
\hline 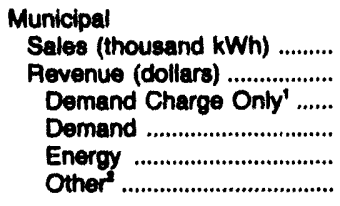 & $\begin{array}{r}238,169 \\
8,815,418 \\
3,022,049 \\
6,414,486 \\
-621,117\end{array}$ & $\begin{array}{r}147,696 \\
4,147,684 \\
1,061,738 \\
3,038,036 \\
46,890\end{array}$ & $\begin{array}{r}557,444 \\
18,019,516 \\
- \\
4,431,990 \\
13,587,526 \\
-\end{array}$ & $\begin{array}{r}582,626 \\
16,824,412 \\
- \\
5,890,021 \\
12,130,104 \\
-1,195,713\end{array}$ & $\begin{array}{l}\bar{z} \\
\bar{z}\end{array}$ & $\begin{array}{r}86,084 \\
5,652,717 \\
-\quad \\
4,542,615 \\
967,722 \\
142,380\end{array}$ & $\begin{array}{r}1,055,841 \\
34,096,311 \\
- \\
9,835,301 \\
24,525,525 \\
-364,515\end{array}$ \\
\hline 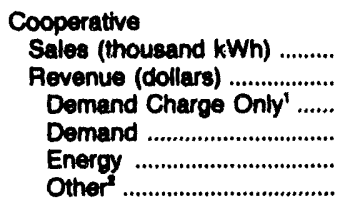 & $\begin{array}{r}35,278 \\
834,688 \\
-\quad \\
183,750 \\
499,597 \\
151,321\end{array}$ & $\begin{array}{l}\overline{-} \\
\overline{-}\end{array}$ & $\begin{array}{r}60 \\
108,697 \\
-\quad \\
107,611 \\
-\quad 1,086 \\
-\quad\end{array}$ & $\begin{array}{l}- \\
\overline{-} \\
\overline{-}\end{array}$ & $\begin{array}{l}\overline{-} \\
\bar{z}\end{array}$ & 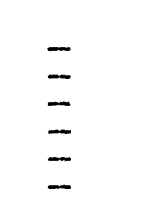 & $\begin{array}{r}413,460 \\
7,713,931 \\
- \\
184,068 \\
7,529,863 \\
-\end{array}$ \\
\hline 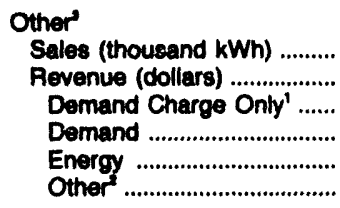 & $\begin{array}{l}-1,370 \\
-52,889 \\
- \\
- \\
-52,889\end{array}$ & $\begin{array}{r}1,489,711 \\
54,329,036 \\
- \\
2,533,895 \\
51,611,708 \\
183,433\end{array}$ & $\begin{array}{l}1,759,722 \\
31,761,018 \\
- \\
- \\
31,761,018 \\
-\end{array}$ & 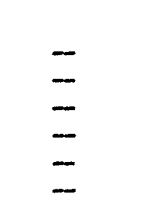 & 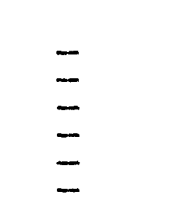 & $\overline{-}$ & $\begin{array}{r}10,151,505 \\
291,881,080 \\
- \\
- \\
291,881,080 \\
-\end{array}$ \\
\hline 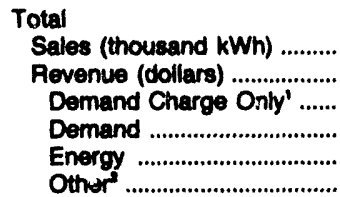 & $\begin{array}{r}4,865,853 \\
165,523,455 \\
- \\
64,439,811 \\
104,605,753 \\
-3,522,209\end{array}$ & $\begin{array}{r}1,988,646 \\
63,508,492 \\
\overline{3} \\
3,725,989 \\
59,488,505 \\
283,998\end{array}$ & $\begin{array}{r}2,707,688 \\
64,583,774 \\
1,124,046 \\
8,354,130 \\
55,115,598 \\
\end{array}$ & $\begin{array}{r}1,648,818 \\
35,173,865 \\
- \\
9,809,701 \\
26,612,287 \\
-1,248,123\end{array}$ & $\begin{array}{r}9,185,404 \\
140,703,815 \\
- \\
40,071,030 \\
100,632,785 \\
-\end{array}$ & $\begin{array}{r}7,850,252 \\
220,475,873 \\
- \\
90,330,687 \\
127,783,190 \\
2,361,996\end{array}$ & $\begin{array}{r}16,275,872 \\
464,351,081 \\
1,936,881 \\
36,292,050 \\
426,486,664 \\
-364,514\end{array}$ \\
\hline
\end{tabular}

See notes and footnotes at end of table. 
Table 20. Electricity Sales for Resale by Investor-Owned Utilities, by State, 1992 (Continued)

\begin{tabular}{|c|c|c|c|c|c|c|c|}
\hline $\begin{array}{l}\text { Purcherer of } \\
\text { Electivicty by } \\
\text { onnerchip Claes }\end{array}$ & $\begin{array}{l}\text { Onio } \\
\text { Onio } \\
\text { Valley } \\
\text { Electric } \\
\text { Corp }\end{array}$ & $\begin{array}{l}\text { Onlo } \\
\text { Toledo } \\
\text { Edison } \\
\text { Co }\end{array}$ & $\begin{array}{l}\text { Oklahoma } \\
\text { Oklahoma } \\
\text { Gas \& } \\
\text { Electric } \\
\text { Co }\end{array}$ & $\begin{array}{l}\text { Oklahoma } \\
\text { Public } \\
\text { Service } \\
\text { Co of } \\
\text { Oklahoma }\end{array}$ & $\begin{array}{l}\text { Oregon } \\
\text { Paciflécorp }\end{array}$ & $\begin{array}{l}\text { Oregon } \\
\text { Portland } \\
\text { General } \\
\text { Electric } \\
\text { Co }\end{array}$ & $\begin{array}{c}\text { Pennoylvania } \\
\text { Cltizens } \\
\text { Electric } \\
\text { Co }\end{array}$ \\
\hline 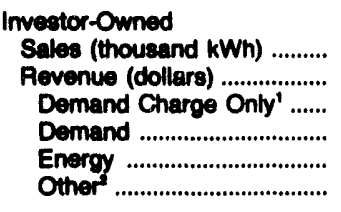 & $\begin{array}{r}2,564,453 \\
29,434,017 \\
- \\
-562,410 \\
29,996,427 \\
-\end{array}$ & $\begin{array}{r}2,100,093 \\
136,837,134 \\
- \\
13,638,884 \\
123,022,748 \\
175,502\end{array}$ & $\begin{array}{l}4,112,843 \\
61,497,324 \\
- \\
\overline{61,497,324} \\
-\end{array}$ & $\begin{array}{r}41,557 \\
804,710 \\
-\quad \\
-\quad 721,040 \\
83,670\end{array}$ & $\begin{array}{r}9,384,034 \\
303,702,883 \\
- \\
133,886,263 \\
171,890,886 \\
-2,074,276\end{array}$ & $\begin{array}{r}1,123,071 \\
46,793,609 \\
- \\
21,220,000 \\
25,460,260 \\
113,349\end{array}$ & $\begin{array}{l}\overline{-} \\
\bar{z} \\
\overline{-}\end{array}$ \\
\hline 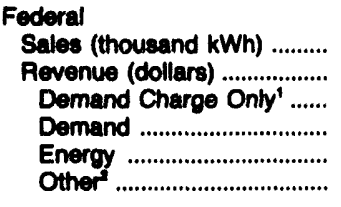 & $\begin{array}{l}\overline{-} \\
\overline{-} \\
\overline{-}\end{array}$ & $\begin{array}{l}\bar{z} \\
\bar{z} \\
\overline{-}\end{array}$ & $\begin{array}{r}9,171 \\
318,486 \\
-\quad \\
-\quad 372,153 \\
-53,667\end{array}$ & $\begin{array}{r}42,826 \\
2,262,938 \\
-\quad \\
273,755 \\
1,862,283 \\
126,900\end{array}$ & $\begin{array}{l}2,319,415 \\
59,899,288 \\
- \\
\overline{59,899,288} \\
-\end{array}$ & $\begin{array}{r}822,645 \\
40,143,617 \\
- \\
16,500,000 \\
23,643,617 \\
--\end{array}$ & $\begin{array}{l}- \\
\overline{-} \\
\overline{-}\end{array}$ \\
\hline 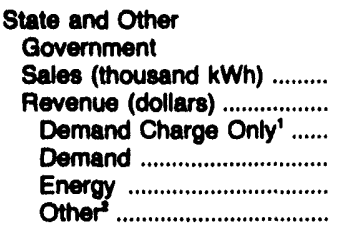 & $\begin{array}{l}- \\
- \\
-\end{array}$ & $\begin{array}{l}- \\
- \\
-\end{array}$ & $\begin{array}{r}277,387 \\
7,677,560 \\
- \\
1,942,138 \\
7,759,688 \\
-2,024,266\end{array}$ & $\begin{array}{r}24,632 \\
4,682,682 \\
-396,000 \\
934,294 \\
3,352,398\end{array}$ & $\begin{array}{r}1,067,079 \\
31,827,310 \\
-\quad \\
197,936 \\
31,587,256 \\
42,118\end{array}$ & $\begin{array}{l}654,583 \\
14,214,367 \\
- \\
- \\
14,214,367 \\
-\end{array}$ & 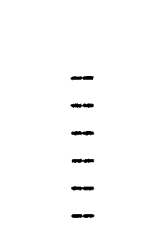 \\
\hline 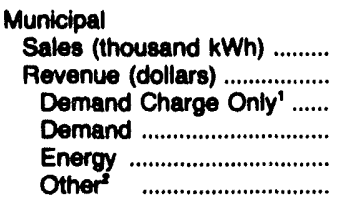 & $\begin{array}{l}- \\
\overline{-} \\
\overline{-}\end{array}$ & $\begin{array}{r}492,302 \\
17,750,513 \\
8,892,198 \\
8,837,558 \\
20,757\end{array}$ & $\begin{array}{r}122,240 \\
5,192,300 \\
-- \\
2,055,523 \\
3,706,028 \\
-568,251\end{array}$ & $\begin{array}{r}4,568 \\
-35,061 \\
-\quad \\
- \\
-138,939 \\
-174,000\end{array}$ & $\begin{array}{r}509,179 \\
29,742,303 \\
- \\
22,445,378 \\
7,294,220 \\
2,705\end{array}$ & $\begin{array}{r}138,903 \\
7,641,456 \\
- \\
4,720,000 \\
2,921,456 \\
-\end{array}$ & $\begin{array}{l}- \\
\overline{-} \\
\overline{-}\end{array}$ \\
\hline 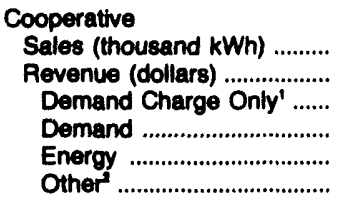 & $\begin{array}{l}- \\
\overline{-} \\
\overline{-}\end{array}$ & 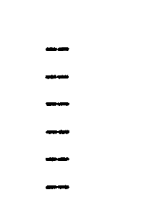 & $\begin{array}{r}540,056 \\
20,843,111 \\
- \\
7,686,700 \\
16,151,631 \\
-2,975,220\end{array}$ & $\begin{array}{r}1,084 \\
47,587 \\
-\quad \ldots \\
-\quad \ldots \\
46,219 \\
1,368\end{array}$ & $\begin{array}{r}15,428 \\
476,585 \\
629 \\
56,500 \\
419,456 \\
-\quad\end{array}$ & $\begin{array}{l}- \\
\overline{-} \\
\overline{-} \\
-\end{array}$ & $\begin{array}{l}- \\
\overline{-} \\
-\end{array}$ \\
\hline 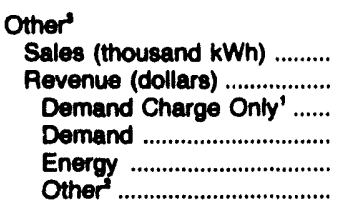 & $\begin{array}{l}- \\
\overline{-} \\
\overline{-}\end{array}$ & $\begin{array}{r}160,537 \\
2,986,896 \\
- \\
107,121 \\
2,879,775 \\
-\end{array}$ & $\begin{array}{l}- \\
\overline{-} \\
-\end{array}$ & $\begin{array}{r}550,156 \\
10,019,620 \\
- \\
- \\
9,989,213 \\
30,407\end{array}$ & $\begin{array}{r}124,777 \\
4,404,922 \\
-\quad \\
2,268,588 \\
2,137,445 \\
-1,111\end{array}$ & $\begin{array}{l}- \\
\overline{-} \\
\overline{-} \\
-\end{array}$ & $\begin{array}{l}- \\
\bar{m} \\
\bar{m}\end{array}$ \\
\hline 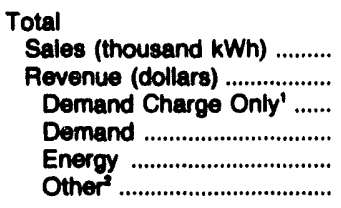 & $\begin{array}{r}2,564,453 \\
29,434,017 \\
- \\
-562,410 \\
29,996,427 \\
-\end{array}$ & $\begin{array}{r}2,752,932 \\
157,574,543 \\
- \\
22,638,203 \\
134,740,081 \\
196,259\end{array}$ & $\begin{array}{r}5,061,697 \\
95,528,781 \\
- \\
11,664,361 \\
89,486,824 \\
-5,622,404\end{array}$ & $\begin{array}{r}664,823 \\
17,782,486 \\
- \\
669,755 \\
13,691,988 \\
3,420,743\end{array}$ & $\begin{array}{r}13,419,912 \\
430,053,291 \\
629 \\
158,854,665 \\
273,228,561 \\
-2,030,564\end{array}$ & $\begin{array}{r}2,739,212 \\
108,793,049 \\
- \\
42,440,000 \\
66,239,700 \\
113,349\end{array}$ & $\begin{array}{l}- \\
- \\
- \\
-\end{array}$ \\
\hline
\end{tabular}

See notes and footnotes at end of table. 
Table 20. Electriclty Sales for Resale by Investor-Owned Utilities, by State, 1992 (Continued)

\begin{tabular}{|c|c|c|c|c|c|c|c|}
\hline $\begin{array}{l}\text { Purchacer of } \\
\text { Ebeetrictly by } \\
\text { Ownorchlp clases }\end{array}$ & $\begin{array}{l}\text { Pennsytvania } \\
\text { Duqueane } \\
\text { Light } \\
\text { Co }\end{array}$ & $\begin{array}{l}\text { Pennoylvania } \\
\text { Metropolitan } \\
\text { Edison } \\
\text { Co }\end{array}$ & $\begin{array}{l}\text { Pennoytvania } \\
\text { Pennsytvania } \\
\text { Electric } \\
\text { Co }\end{array}$ & $\begin{array}{l}\text { Pennsylvania } \\
\text { Pennsytvania } \\
\text { Power \& } \\
\text { Light } \\
\text { Co }\end{array}$ & $\begin{array}{l}\text { Pennsylvania } \\
\text { Pennsylvania } \\
\text { Power } \\
\text { Co }\end{array}$ & $\begin{array}{l}\text { Penneytvania } \\
\text { Philadelphia } \\
\text { Electric } \\
\text { Co }\end{array}$ & $\begin{array}{l}\text { Pennsylvania } \\
\text { Pike } \\
\text { County } \\
\text { Light \& } \\
\text { Power } \\
\text { Co' }\end{array}$ \\
\hline 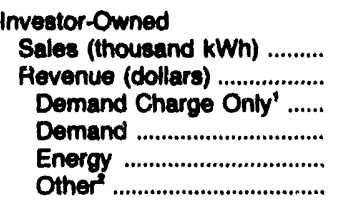 & $\begin{array}{r}4,059,989 \\
72,439,458 \\
\overline{18,218,771} \\
54,220,685 \\
-\end{array}$ & $\begin{array}{r}182,249 \\
4,223,746 \\
- \\
406,395 \\
3,813,948 \\
3,403\end{array}$ & $\begin{array}{r}500,763 \\
12,687,019 \\
-\quad 1,49,085 \\
9,223,762 \\
1,014,172\end{array}$ & $\begin{array}{r}7,564,085 \\
351,259,503 \\
\overline{1,360,804} \\
125,411,047 \\
224,487,652\end{array}$ & $\begin{array}{r}1,691,731 \\
41,383,492 \\
\overline{-} \\
13,420,448 \\
27,963,044 \\
-\end{array}$ & $\begin{array}{r}6,903,464 \\
215,587,457 \\
-- \\
33,617,052 \\
184,009,924 \\
-2,039,519\end{array}$ & $\begin{array}{l}\bar{z} \\
\bar{z}\end{array}$ \\
\hline 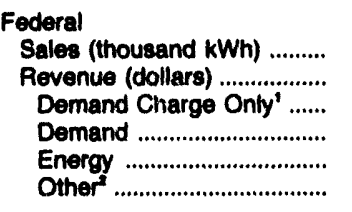 & $\begin{array}{l}\bar{z} \\
\bar{z} \\
\bar{z}\end{array}$ & $\begin{array}{l}\bar{z} \\
\bar{z}\end{array}$ & $\begin{array}{l}\bar{z} \\
\bar{z}\end{array}$ & $\begin{array}{l}\overline{-} \\
\overline{-}\end{array}$ & $\begin{array}{l}\overline{-} \\
\overline{-}\end{array}$ & $\begin{array}{l}\bar{z} \\
\bar{z} \\
\bar{z}\end{array}$ & $\begin{array}{l}\overline{-} \\
\bar{z} \\
\overline{-}\end{array}$ \\
\hline 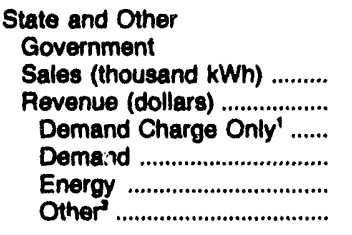 & $\begin{array}{l}\bar{z} \\
\bar{z}\end{array}$ & $\begin{array}{l}\bar{z} \\
\bar{z}\end{array}$ & $\begin{array}{l}\bar{z} \\
\bar{z} \\
\bar{z}\end{array}$ & $\begin{array}{l}199,957 \\
3,864,693 \\
- \\
\overline{3,864,693}\end{array}$ & $\bar{z}$ & $\begin{array}{l}\bar{z} \\
\bar{z}\end{array}$ & $\begin{array}{l}\bar{z} \\
\bar{z} \\
\bar{z}\end{array}$ \\
\hline 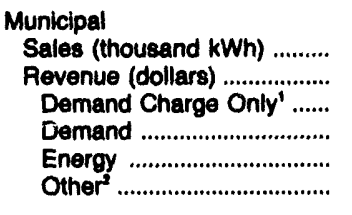 & $\begin{array}{r}11,780 \\
796,032 \\
-\quad \\
426,023 \\
353,400 \\
16,609\end{array}$ & $\begin{array}{r}86,991 \\
1,257,377 \\
-264,800 \\
979,695 \\
12,882\end{array}$ & $\begin{array}{r}72,623 \\
3,110,564 \\
- \\
1,574,831 \\
1,452,443 \\
83,290\end{array}$ & $\begin{array}{r}687,597 \\
35,919,334 \\
\overline{6,600,178} \\
29,319,156 \\
-\end{array}$ & $\begin{array}{l}131,226 \\
7,049,990 \\
-- \\
2,676,166 \\
4,373,824 \\
-\end{array}$ & $\begin{array}{l}\bar{z} \\
\bar{z}\end{array}$ & $\begin{array}{l}\bar{z} \\
\bar{z}\end{array}$ \\
\hline 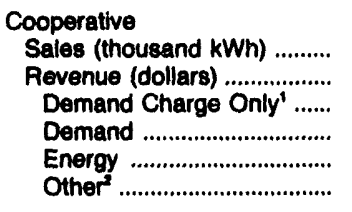 & $\begin{array}{l}\overline{-} \\
\bar{z} \\
\overline{-}\end{array}$ & $\begin{array}{r}100,990 \\
6,359,376 \\
- \\
4,082,980 \\
2,071,669 \\
204,727\end{array}$ & $\begin{array}{r}370,737 \\
13,532,780 \\
- \\
7,998,754 \\
7,551,005 \\
-2,016,979\end{array}$ & $\begin{array}{r}18,180 \\
934,093 \\
- \\
151,385 \\
782,708 \\
-\end{array}$ & $\begin{array}{l}\bar{z} \\
\bar{z} \\
=\end{array}$ & $\begin{array}{l}\bar{z} \\
\bar{z} \\
\bar{z}\end{array}$ & $\begin{array}{l}\bar{z} \\
\bar{z} \\
\bar{z}\end{array}$ \\
\hline 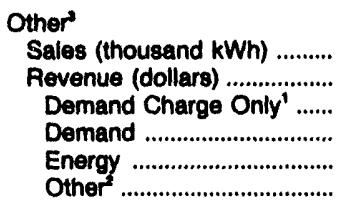 & $\begin{array}{l}\bar{z} \\
\bar{z}\end{array}$ & $\begin{array}{r}2,201,564 \\
58,996,266 \\
- \\
11,119,103 \\
47,877,163 \\
-\end{array}$ & $\begin{array}{r}2,375,668 \\
53,972,822 \\
\overline{5,811,437} \\
48,161,485 \\
-\end{array}$ & $\begin{array}{r}5,294,779 \\
117,996,506 \\
\overline{-} \\
117,958,840 \\
37,756\end{array}$ & $\begin{array}{l}\bar{z} \\
\bar{z} \\
\overline{-}\end{array}$ & $\begin{array}{l}1,656,243 \\
59,384,935 \\
- \\
\overline{59,384,935} \\
-\end{array}$ & $\begin{array}{l}\bar{z} \\
\bar{z}\end{array}$ \\
\hline 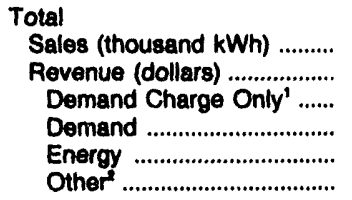 & $\begin{array}{r}4,071,769 \\
73,235,488 \\
-\quad \\
18,644,794 \\
54,574,085 \\
16,609\end{array}$ & $\begin{array}{r}2,571,794 \\
70,836,765 \\
-\quad \\
15,873,278 \\
54,742,475 \\
221,012\end{array}$ & $\begin{array}{r}3,319,791 \\
83,303,285 \\
\overline{17,834,107} \\
66,388,695 \\
-919,517\end{array}$ & $\begin{array}{r}13,764,598 \\
509,974,219 \\
- \\
8,112,367 \\
277,336,444 \\
224,525,408\end{array}$ & $\begin{array}{r}1,822,957 \\
48,433,482 \\
\overline{16,096,614} \\
32,336,868 \\
-\end{array}$ & $\begin{array}{r}8,559,707 \\
274,972,392 \\
- \\
33,617,052 \\
243,394,859 \\
-2,039,519\end{array}$ & $\begin{array}{l}\bar{z} \\
\bar{z} \\
\overline{-}\end{array}$ \\
\hline
\end{tabular}

See notes and footnotes at end of table. 
Table 20. Electricity Sales for Resale by Investor-Owned Utilities, by State, 1992 (Continued)

\begin{tabular}{|c|c|c|c|c|c|c|c|}
\hline 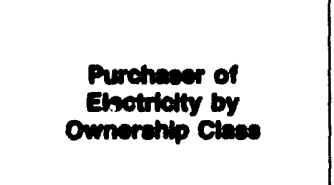 & $\begin{array}{l}\text { Pennoyivania } \\
\text { Safe Harbor } \\
\text { Water } \\
\text { Power } \\
\text { Corp" }\end{array}$ & $\begin{array}{l}\text { Pennoyivania } \\
\text { Susquohanna } \\
\text { Electric } \\
\text { Co" }\end{array}$ & $\begin{array}{c}\text { Penneylvania } \\
\text { UGI } \\
\text { Corp }\end{array}$ & $\begin{array}{l}\text { Pennsyivania } \\
\text { West } \\
\text { Penn } \\
\text { Power } \\
\text { Co }\end{array}$ & $\begin{array}{l}\text { Penneylvania } \\
\text { York } \\
\text { Haven } \\
\text { Power } \\
\text { Cos }\end{array}$ & $\begin{array}{l}\text { Ahode Island } \\
\text { Blackstone } \\
\text { Valley } \\
\text { Electric } \\
\mathrm{Co}^{4}\end{array}$ & $\begin{array}{c}\text { Rhode loland } \\
\text { Narragansett } \\
\text { Electric } \\
\text { Co }\end{array}$ \\
\hline 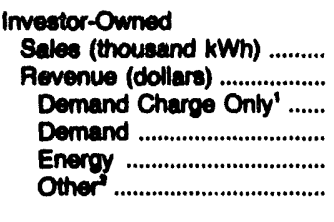 & $\begin{array}{l}1,090,380 \\
28,244,563 \\
\overline{-} \\
\overline{28,244,563}\end{array}$ & $\begin{array}{r}1,788,123 \\
27,010,385 \\
- \\
7,055,347 \\
2,562,513 \\
17,382,505\end{array}$ & $\begin{array}{r}552 \\
-\quad 8,386 \\
-\quad 8,386\end{array}$ & $\begin{array}{r}9,394,285 \\
205,904,379 \\
- \\
70,089,212 \\
127,171,893 \\
8,643,274\end{array}$ & $\begin{array}{l}141,544 \\
4,335,608 \\
= \\
\overline{-} \\
\overline{-}\end{array}$ & $\begin{array}{l}\bar{z} \\
\overline{-}\end{array}$ & $\begin{array}{r}569 \\
-\quad 64,904 \\
-\quad \\
\quad 58,205 \\
6,689\end{array}$ \\
\hline 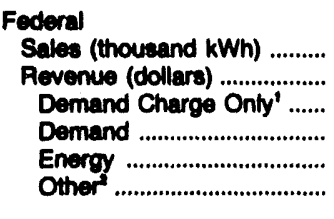 & $\begin{array}{l}\bar{z} \\
\bar{z}\end{array}$ & $\begin{array}{l}\bar{z} \\
\bar{z}\end{array}$ & $\begin{array}{l}\overline{-} \\
\bar{z} \\
\overline{-}\end{array}$ & $\begin{array}{l}= \\
= \\
=\end{array}$ & $\begin{array}{l}\bar{z} \\
\bar{z} \\
\overline{-}\end{array}$ & $\begin{array}{l}\bar{z} \\
\bar{z} \\
\overline{-}\end{array}$ & $\begin{array}{l}\overline{-} \\
\bar{I}\end{array}$ \\
\hline 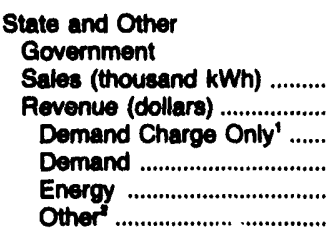 & $\begin{array}{l}\bar{z} \\
\bar{z}\end{array}$ & $\begin{array}{l}\bar{z} \\
\bar{z}\end{array}$ & $\begin{array}{l}\overline{-} \\
\bar{z} \\
\overline{-}\end{array}$ & $\begin{array}{l}\bar{z} \\
\bar{m} \\
\overline{-}\end{array}$ & 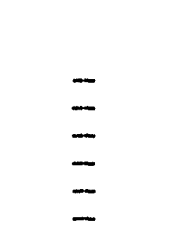 & $\begin{array}{l}\overline{-} \\
\overline{-} \\
\overline{-}\end{array}$ & $\begin{array}{l}\bar{z} \\
\bar{z} \\
\overline{-}\end{array}$ \\
\hline 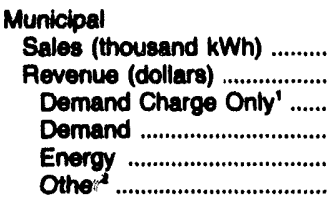 & $\begin{array}{l}\bar{z} \\
\bar{z}\end{array}$ & 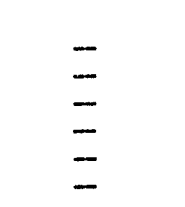 & $\begin{array}{l}\bar{z} \\
\bar{m} \\
\bar{m}\end{array}$ & $\begin{array}{r}268,153 \\
9,153,603 \\
- \\
3,628,009 \\
2,864,258 \\
2,661,336\end{array}$ & $\begin{array}{l}\bar{z} \\
\bar{m}\end{array}$ & $\begin{array}{l}\bar{z} \\
\bar{z} \\
\bar{z}\end{array}$ & $\begin{array}{l}\overline{-} \\
\overline{-} \\
\overline{-}\end{array}$ \\
\hline 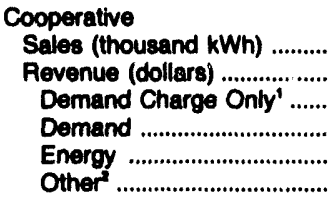 & $\begin{array}{l}\bar{z} \\
\bar{m}\end{array}$ & $\begin{array}{l}\overline{-} \\
\bar{z} \\
\overline{-}\end{array}$ & $\begin{array}{l}\overline{-} \\
\overline{-} \\
\overline{-}\end{array}$ & $\begin{array}{r}1,067,218 \\
35,620,100 \\
- \\
16,130,714 \\
16,956,968 \\
2,532,418\end{array}$ & $\begin{array}{l}\overline{-} \\
\overline{-} \\
-\end{array}$ & $\begin{array}{l}\bar{z} \\
\bar{z}\end{array}$ & $\begin{array}{l}\bar{z} \\
\bar{z}\end{array}$ \\
\hline 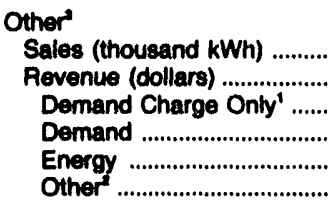 & $\begin{array}{l}\bar{z} \\
\bar{z} \\
\overline{-}\end{array}$ & $\begin{array}{l}\bar{z} \\
\bar{z} \\
\overline{-}\end{array}$ & $\begin{array}{l}\overline{-} \\
\bar{z} \\
\overline{-}\end{array}$ & $\begin{array}{r}245,923 \\
5,157,222 \\
- \\
1,487,404 \\
3,669,818 \\
-\end{array}$ & $\begin{array}{l}\overline{-} \\
\bar{z} \\
\overline{-}\end{array}$ & $\begin{array}{l}\bar{z} \\
\bar{z} \\
\bar{z}\end{array}$ & $\begin{array}{r}30 \\
3,454 \\
-\quad \\
-\quad 3,128 \\
326\end{array}$ \\
\hline 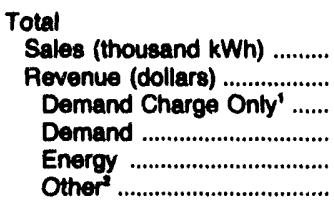 & $\begin{array}{l}1,094,390 \\
28,244,563 \\
\bar{z} \\
\overline{28,244,563}\end{array}$ & $\begin{array}{r}1,788,123 \\
27,010,365 \\
-7 \\
7,055,347 \\
2,562,513 \\
17,392,505\end{array}$ & $\begin{array}{l}{ }^{852} \\
-\quad 896 \\
-\quad 8,396\end{array}$ & $\begin{array}{r}9,975,579 \\
255,835,304 \\
-\overline{91,335,339} \\
150,662,937 \\
13,837,028\end{array}$ & $\begin{array}{l}141,544 \\
4,335,808 \\
\overline{4} \\
\overline{4}, 335,808\end{array}$ & $\begin{array}{l}\bar{z} \\
\bar{z}\end{array}$ & 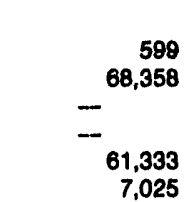 \\
\hline
\end{tabular}

See notes and footnotes at end of table. 
Table 20. Electricity Sales for Resale by Investor-Owned Utilities, by State, 1992 (Continued)

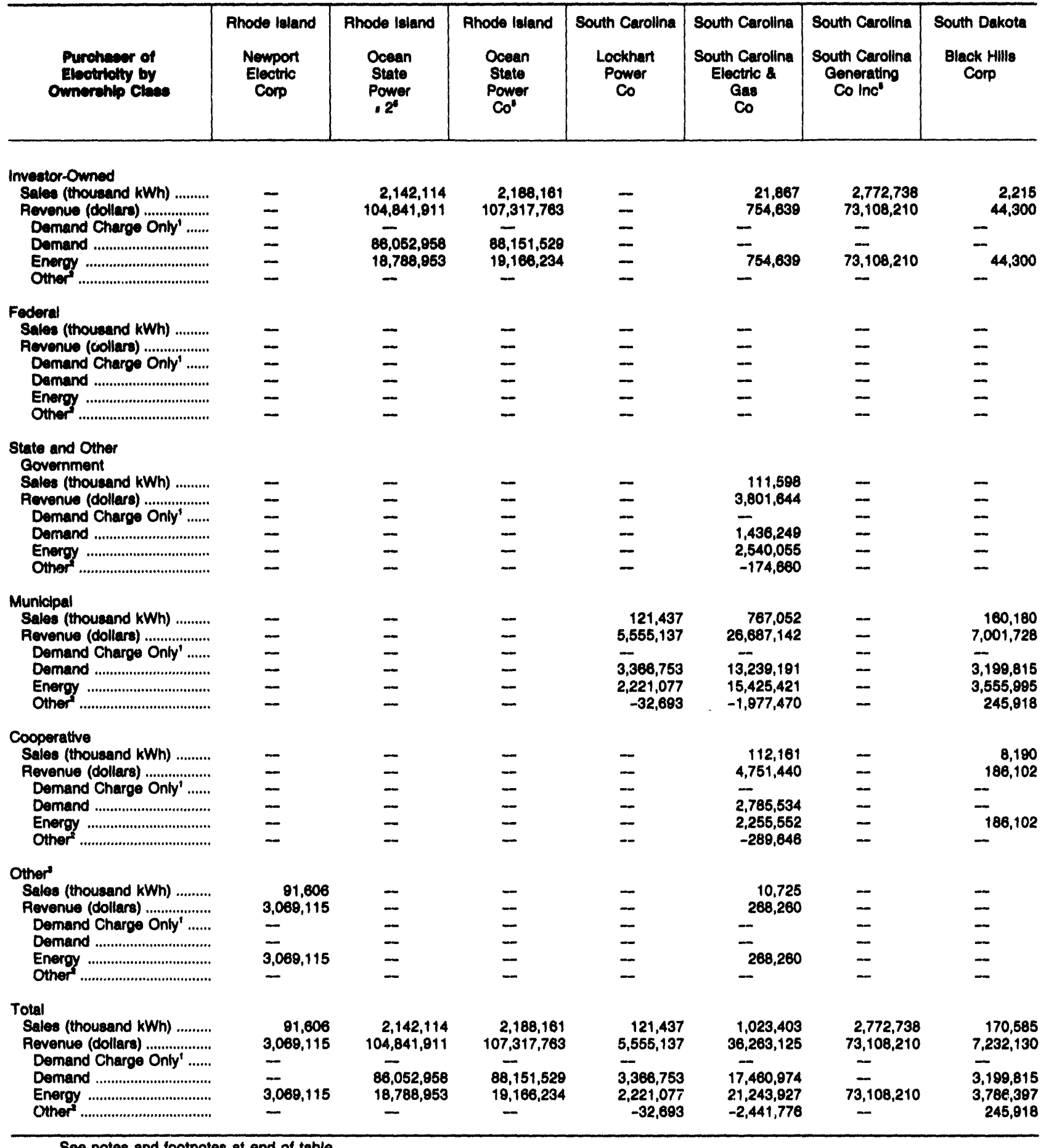


Table 20. Electricity Sales for Resale by Investor-Owned Utilitios, by State, 1992 (Continued)

\begin{tabular}{|c|c|c|c|c|c|c|c|}
\hline $\begin{array}{l}\text { Purchecer of } \\
\text { Eleotrlothy by } \\
\text { Ownerehip Clases }\end{array}$ & $\begin{array}{l}\text { South Dakota } \\
\text { Northweatern } \\
\text { Publlc } \\
\text { Service } \\
\text { Co }\end{array}$ & $\begin{array}{l}\text { Tennessee } \\
\text { KIngeport } \\
\text { Power } \\
\text { Co }\end{array}$ & $\begin{array}{c}\text { Tennessee } \\
\text { Tapoco } \\
\text { inc }\end{array}$ & $\begin{array}{l}\text { Texas } \\
\text { Central } \\
\text { Power \& } \\
\text { Light } \\
\text { Co }\end{array}$ & $\begin{array}{c}\text { Texas } \\
\text { El Paso } \\
\text { Eloctric } \\
\text { Co }\end{array}$ & $\begin{array}{l}\text { Texas } \\
\text { Gulf } \\
\text { States } \\
\text { Utillites } \\
\text { Co }\end{array}$ & $\begin{array}{l}\text { Texas } \\
\text { Houston } \\
\text { Lighting } \\
\text { a Power } \\
\text { Co }\end{array}$ \\
\hline 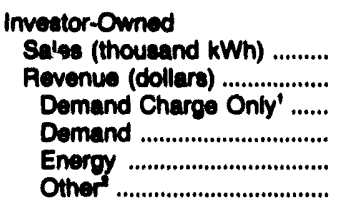 & $\begin{array}{r}77,807 \\
1,051,608 \\
56,000 \\
-\quad \\
995,486 \\
120\end{array}$ & 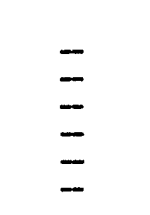 & $\begin{array}{l}\bar{z} \\
\bar{z}\end{array}$ & $\bar{z}$ & $\begin{array}{r}890,364 \\
31,896,726 \\
\overline{17,316,000} \\
6,132,998 \\
8,447,728\end{array}$ & $\begin{array}{r}2,723 \\
-\quad 61,979 \\
-\quad 61,979 \\
-\end{array}$ & $\begin{array}{r}287,126 \\
27,797,744 \\
-- \\
22,109,108 \\
5,688,638 \\
-\end{array}$ \\
\hline 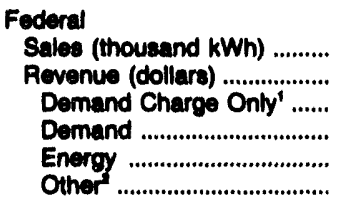 & $\begin{array}{l}37,740 \\
495,953 \\
- \\
- \\
-\end{array}$ & $\begin{array}{l}\overline{-} \\
\bar{m} \\
\bar{m}\end{array}$ & $\begin{array}{l}\bar{z} \\
\bar{I}\end{array}$ & $\begin{array}{l}\bar{z} \\
\bar{z}\end{array}$ & $\bar{z}$ & $\begin{array}{l}\bar{z} \\
\bar{z} \\
\overline{-}\end{array}$ & $\begin{array}{l}\bar{z} \\
\bar{z} \\
\overline{-}\end{array}$ \\
\hline 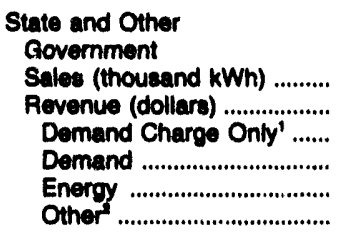 & $\begin{array}{r}10,199 \\
125,119 \\
-\quad 1,760 \\
123,268 \\
93\end{array}$ & $\begin{array}{l}\bar{z} \\
\bar{z}\end{array}$ & $\begin{array}{l}\bar{z} \\
\bar{z}\end{array}$ & $\begin{array}{l}\bar{z} \\
\bar{z}\end{array}$ & $\begin{array}{r}902,438 \\
46,311,301 \\
- \\
31,238,000 \\
3,688,121 \\
11,605,180\end{array}$ & $\begin{array}{r}54,177 \\
1,433,809 \\
- \\
250,333 \\
1,183,576 \\
-\end{array}$ & $\begin{array}{r}28,320 \\
689,772 \\
-\quad \\
21,230 \\
668,542 \\
-\end{array}$ \\
\hline 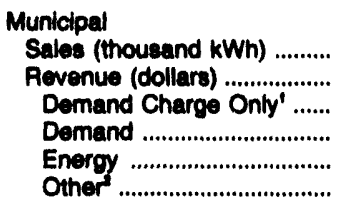 & $\begin{array}{r}8,650 \\
298,608 \\
-\quad \\
191,637 \\
96,764 \\
10,207\end{array}$ & $\begin{array}{l}\overline{-} \\
\bar{z} \\
\overline{-}\end{array}$ & $\begin{array}{l}\bar{z} \\
\bar{z}\end{array}$ & $\begin{array}{r}135,395 \\
6,750,006 \\
-- \\
3,695,136 \\
582,162 \\
2,472,708\end{array}$ & $\begin{array}{r}6,039 \\
-\quad 90,570 \\
-\quad 90,570 \\
-\end{array}$ & $\begin{array}{l}159,716 \\
6,702,183 \\
-- \\
3,131,371 \\
3,570,812 \\
--\end{array}$ & $\begin{array}{r}24,070 \\
495,077 \\
- \\
- \\
-\end{array}$ \\
\hline 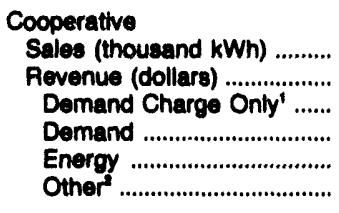 & $\begin{array}{l}25,389 \\
339,931 \\
- \\
- \\
-\end{array}$ & $\begin{array}{l}\bar{z} \\
\bar{z} \\
\bar{z}\end{array}$ & $\begin{array}{l}- \\
\bar{z} \\
-\end{array}$ & $\begin{array}{r}598,039 \\
28,663,886 \\
- \\
14,756,374 \\
2,695,675 \\
11,211,937\end{array}$ & $\begin{array}{r}33,749 \\
1,567,343 \\
- \\
- \\
1,165,965 \\
401,378\end{array}$ & $\begin{array}{r}324,676 \\
16,488,619 \\
- \\
9,238,574 \\
6,902,235 \\
347,810\end{array}$ & $\begin{array}{l}175,642 \\
3,097,296 \\
- \\
- \\
3,097,296 \\
-\end{array}$ \\
\hline 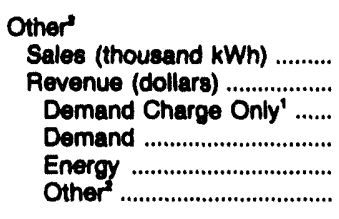 & $\begin{array}{l}\bar{z} \\
\bar{z} \\
\overline{-}\end{array}$ & $\begin{array}{l}\bar{z} \\
\bar{z}\end{array}$ & $\begin{array}{l}\bar{z} \\
\bar{z} \\
\bar{z}\end{array}$ & $\begin{array}{r}636,780 \\
14,928,107 \\
- \\
\overline{5,354,341} \\
9,573,766\end{array}$ & $\begin{array}{r}793,268 \\
36,281,601 \\
- \\
21,527,688 \\
1,795,952 \\
12,957,961\end{array}$ & $\begin{array}{l}-1,006 \\
-201,705 \\
- \\
- \\
566,624 \\
-768,329\end{array}$ & $\begin{array}{l}49,711 \\
1,117,785 \\
- \\
- \\
1,117,795 \\
-\end{array}$ \\
\hline 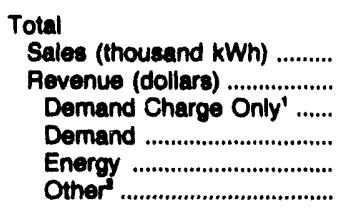 & $\begin{array}{r}159,785 \\
2,311,217 \\
56,000 \\
193,397 \\
2,051,400 \\
10,420\end{array}$ & $\begin{array}{l}- \\
\bar{z} \\
\overline{-}\end{array}$ & $\begin{array}{l}\bar{z} \\
\bar{z}\end{array}$ & $\begin{array}{r}1,370,214 \\
50,342,099 \\
- \\
18,451,510 \\
8,632,178 \\
23,258,411\end{array}$ & $\begin{array}{r}2,625,858 \\
116,147,541 \\
- \\
70,081,688 \\
12,753,606 \\
33,312,247\end{array}$ & $\begin{array}{r}540,286 \\
24,484,985 \\
-\quad \\
12,620,278 \\
12,285,226 \\
-420,519\end{array}$ & $\begin{array}{r}585,989 \\
33,197,684 \\
- \\
22,130,338 \\
11,067,348 \\
-\end{array}$ \\
\hline
\end{tabular}

See notes and footnotes at end of tabie. 
Table 20. Electricity Sales for Resale by Investor-Owned Utilities, by State, 1992 (Continued)

\begin{tabular}{|c|c|c|c|c|c|c|c|}
\hline $\begin{array}{l}\text { Purchaes of } \\
\text { Electrieity by } \\
\text { Owmerahtp slace }\end{array}$ & $\begin{array}{l}\text { Texas } \\
\text { Southwestern } \\
\text { Electric } \\
\text { Senvice } \\
\text { Co }\end{array}$ & $\begin{array}{l}\text { Texas } \\
\text { Soutmuestern } \\
\text { Publlc } \\
\text { Service } \\
\text { Co }\end{array}$ & $\begin{array}{c}\text { Texas } \\
\text { Texas } \\
\text { Utilities } \\
\text { Electric } \\
\text { Co }\end{array}$ & $\begin{array}{l}\text { Texas } \\
\text { Texas- } \\
\text { New Moxico } \\
\text { Power } \\
\text { Co }\end{array}$ & $\begin{array}{c}\text { Texas } \\
\text { West } \\
\text { Texas } \\
\text { Utilities } \\
\text { Co }\end{array}$ & $\begin{array}{l}\text { Vermont } \\
\text { Central } \\
\text { Vermont } \\
\text { Public } \\
\text { Service } \\
\text { Corp }\end{array}$ & $\begin{array}{c}\text { Vermont } \\
\text { Clizens } \\
\text { Utillties } \\
\text { Co }^{4}\end{array}$ \\
\hline 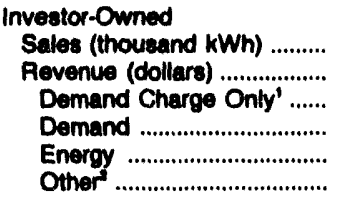 & $\begin{array}{l}\bar{z} \\
\bar{z}\end{array}$ & $\begin{array}{r}1,825,674 \\
48,351,626 \\
- \\
15,682,566 \\
22,343,822 \\
10,325,138\end{array}$ & $\begin{array}{r}2,181,905 \\
104,301,849 \\
- \\
43,382,024 \\
29,530,986 \\
31,408,839\end{array}$ & $\begin{array}{l}\bar{m} \\
\bar{m}\end{array}$ & $\begin{array}{r}216,811 \\
6,318,423 \\
- \\
1,685,810 \\
4,584,681 \\
37,852\end{array}$ & $\begin{array}{r}963,326 \\
38,035,842 \\
- \\
24,606,135 \\
14,071,949 \\
-642,242\end{array}$ & $\begin{array}{l}- \\
\overline{-} \\
\overline{-} \\
-\end{array}$ \\
\hline 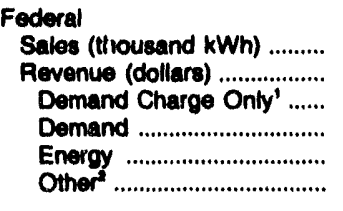 & $\begin{array}{l}\bar{z} \\
\bar{z} \\
\overline{-}\end{array}$ & $\begin{array}{l}24,289 \\
549,578 \\
- \\
- \\
549,578 \\
-\end{array}$ & $\bar{m}=$ & $\begin{array}{l}\bar{z} \\
\bar{z} \\
\bar{m}\end{array}$ & $\begin{array}{l}\bar{z} \\
\bar{z} \\
\overline{-}\end{array}$ & $\begin{array}{l}- \\
\bar{m} \\
-\end{array}$ & $\begin{array}{l}\overline{-} \\
\overline{-} \\
\overline{-}\end{array}$ \\
\hline $\begin{array}{l}\text { State and Other } \\
\text { Government } \\
\text { Sales (thousand kWh) ......... } \\
\text { Revenue (doliars) ................ } \\
\text { Demand Charge Only' ....... } \\
\text { Demand ........................ } \\
\text { Energy } \\
\text { Other }\end{array}$ & $\begin{array}{l}\overline{-} \\
\bar{z} \\
\overline{-}\end{array}$ & $\begin{array}{l}10,350 \\
258,100 \\
-\quad \\
- \\
-\quad 258,100\end{array}$ & $\begin{array}{r}150 \\
-\quad 5,118 \\
-\quad \\
-\quad 5,118\end{array}$ & $\bar{m}=$ & $\begin{array}{r}133 \\
22,637 \\
-\quad \\
-\quad 13,158 \\
9,479\end{array}$ & $\begin{array}{r}122,510 \\
3,587,216 \\
-545,685 \\
3,041,531 \\
-\end{array}$ & $\bar{m}=$ \\
\hline 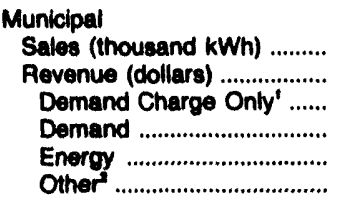 & $\begin{array}{l}- \\
- \\
- \\
-\end{array}$ & $\begin{array}{r}438,428 \\
12,372,100 \\
- \\
3,011,040 \\
3,368,580 \\
5,992,480\end{array}$ & $\begin{array}{r}67,596 \\
3,597,433 \\
- \\
2,059,136 \\
1,259,194 \\
279,103\end{array}$ & 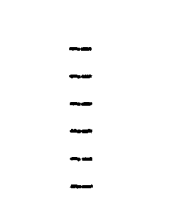 & $\begin{array}{r}92,137 \\
2,536,693 \\
- \\
435,600 \\
2,099,118 \\
1,975\end{array}$ & $\begin{array}{r}103,421 \\
4,441,526 \\
2,914,743 \\
1,812,209 \\
-285,426\end{array}$ & $\begin{array}{l}- \\
\overline{-} \\
-\end{array}$ \\
\hline 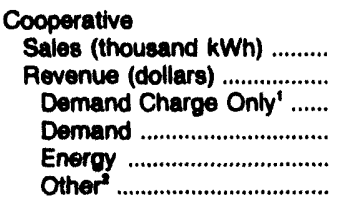 & $\begin{array}{r}93,368 \\
4,505,093 \\
- \\
1,486,346 \\
2,549,870 \\
458,877\end{array}$ & $\begin{array}{r}3,259,376 \\
113,471,288 \\
- \\
42,129,042 \\
13,123,262 \\
58,218,984\end{array}$ & $\begin{array}{r}1,750,072 \\
105,141,108 \\
- \\
46,224,935 \\
21,916,582 \\
36,999,591\end{array}$ & $\begin{array}{r}3,174 \\
244,849 \\
-\quad \\
108,832 \\
106,049 \\
29,968\end{array}$ & $\begin{array}{r}1,359,915 \\
45,892,399 \\
- \\
15,523,379 \\
30,380,032 \\
88,988\end{array}$ & $\begin{array}{r}3 \\
2,543 \\
-\quad \\
2,344 \\
-\quad 189\end{array}$ & $\begin{array}{l}- \\
\overline{-} \\
\overline{-}\end{array}$ \\
\hline 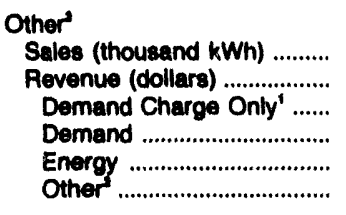 & $\begin{array}{r}1,080 \\
55,858 \\
-\quad \\
21,812 \\
26,410 \\
7,736\end{array}$ & $\begin{array}{r}233,733 \\
4,370,349 \\
- \\
\overline{5}, 462,311 \\
-1,091,962\end{array}$ & 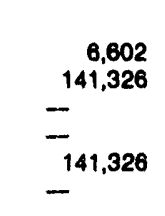 & $\bar{z}$ & $\begin{array}{l}281,980 \\
5,862,958 \\
- \\
\overline{5,236,925} \\
726,033\end{array}$ & $\begin{array}{r}408,368 \\
17,353,472 \\
\overline{7,274,888} \\
10,078,584 \\
-\end{array}$ & $\begin{array}{l}- \\
\bar{z} \\
\overline{-}\end{array}$ \\
\hline 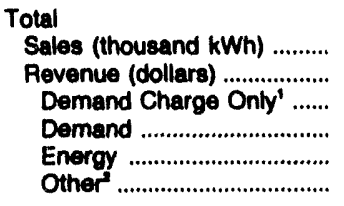 & $\begin{array}{r}94,448 \\
4,561,051 \\
- \\
1,518,158 \\
2,576,280 \\
466,613\end{array}$ & $\begin{array}{r}5,791,850 \\
179,372,941 \\
- \\
60,822,648 \\
45,105,653 \\
73,444,640\end{array}$ & $\begin{array}{r}4,006,325 \\
213,186,834 \\
- \\
91,646,095 \\
52,853,206 \\
68,687,533\end{array}$ & $\begin{array}{r}3,174 \\
244,849 \\
-\quad \\
108,832 \\
106,049 \\
29,968\end{array}$ & $\begin{array}{r}1,850,986 \\
60,833,110 \\
- \\
17,654,789 \\
42,313,894 \\
864,427\end{array}$ & $\begin{array}{r}1,597,628 \\
63,420,589 \\
- \\
35,343,795 \\
29,004,472 \\
-927,668\end{array}$ & $\begin{array}{l}- \\
\overline{-} \\
\overline{-} \\
-\end{array}$ \\
\hline
\end{tabular}

See notes and footnotes at end of table. 
Table 20. Electricity Sales for Recale by Investor-Owned Utilities, by State, 1992 (Continued)

\begin{tabular}{|c|c|c|c|c|c|c|c|}
\hline 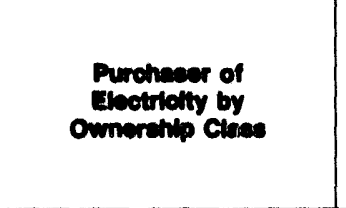 & $\begin{array}{l}\text { Vermoit } \\
\text { Green } \\
\text { Mountain } \\
\text { Power } \\
\text { Corp }\end{array}$ & $\begin{array}{l}\text { Vermont } \\
\text { Vermont } \\
\text { Electric } \\
\text { Power } \\
\text { Co inc }\end{array}$ & $\begin{array}{l}\text { Vermont } \\
\text { Vermont } \\
\text { Electric } \\
\text { Trans } \\
\mathrm{Co}^{\circ}\end{array}$ & $\begin{array}{l}\text { Vermont } \\
\text { Vermont } \\
\text { Marble } \\
\text { Co }^{\circ}\end{array}$ & $\begin{array}{l}\text { Vermont } \\
\text { Vermont } \\
\text { Yankee } \\
\text { Nuclear } \\
\text { Power } \\
\text { Corp" }\end{array}$ & $\begin{array}{l}\text { Virginia } \\
\text { Appalachian } \\
\text { Power } \\
\text { Co }\end{array}$ & $\begin{array}{l}\text { Virginia } \\
\text { Virginia } \\
\text { Electric } \\
\text { Power } \\
\text { Co }\end{array}$ \\
\hline 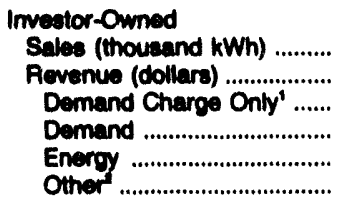 & $\begin{array}{r}271,586 \\
11,808,124 \\
- \\
6,215,853 \\
5,804,809 \\
-112,538\end{array}$ & $\begin{array}{r}472,593 \\
14,188,835 \\
- \\
6,381,177 \\
7,788,253 \\
18,405\end{array}$ & $\begin{array}{l}\bar{z} \\
\bar{z}\end{array}$ & $\begin{array}{l}\bar{z} \\
\bar{z}\end{array}$ & $\begin{array}{l}3,734,595 \\
175,918,744 \\
\overline{-} \\
\overline{175,918,744}\end{array}$ & $\begin{array}{r}4,920,323 \\
141,329,374 \\
2,231,658 \\
32,136,286 \\
105,823,757 \\
1,137,673\end{array}$ & $\begin{array}{r}688,119 \\
16,781,063 \\
- \\
4,149,583 \\
12,631,480 \\
-\end{array}$ \\
\hline 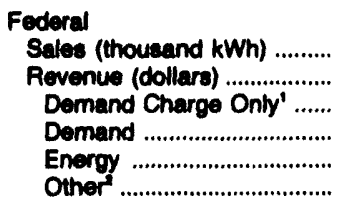 & $\begin{array}{l}\bar{z} \\
\bar{z}\end{array}$ & $\bar{z}$ & $\begin{array}{l}\bar{z} \\
\bar{z}\end{array}$ & $\begin{array}{l}\bar{z} \\
\bar{z}\end{array}$ & $\begin{array}{l}\bar{z} \\
\bar{z} \\
\bar{z}\end{array}$ & 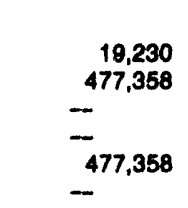 & $\begin{array}{l}\bar{z} \\
\bar{z}\end{array}$ \\
\hline 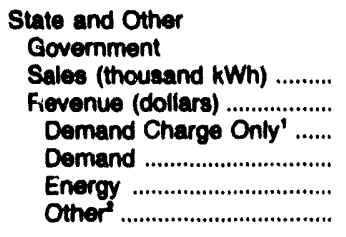 & $\begin{array}{r}30,527 \\
1,105,826 \\
- \\
281,713 \\
824,213 \\
-\end{array}$ & $\begin{array}{r}18 \\
-\quad 753 \\
-\quad 753\end{array}$ & $\begin{array}{l}- \\
\bar{z} \\
\overline{-}\end{array}$ & $\begin{array}{l}\bar{z} \\
\bar{z}\end{array}$ & $\begin{array}{l}\bar{z} \\
\bar{z} \\
\bar{z}\end{array}$ & $\begin{array}{l}\overline{-} \\
\overline{-} \\
\overline{-}\end{array}$ & $\begin{array}{l}\bar{z} \\
\bar{z}\end{array}$ \\
\hline 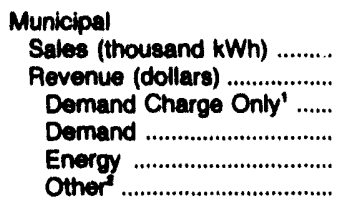 & $\begin{array}{r}53,540 \\
3,007,122 \\
3,000 \\
1,078,188 \\
2,171,058 \\
-245,124\end{array}$ & $\begin{array}{r}306,034 \\
12,698,258 \\
- \\
9,297,083 \\
2,809,974 \\
591,201\end{array}$ & $\begin{array}{l}- \\
\bar{m} \\
-\end{array}$ & 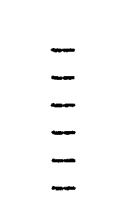 & $\begin{array}{l}\bar{z} \\
\bar{z}\end{array}$ & $\begin{array}{r}2,300,460 \\
77,984,609 \\
\overline{1,826,845} \\
74,728,478 \\
1,429,286\end{array}$ & $\begin{array}{r}57,453 \\
2,971,198 \\
- \\
1,854,837 \\
1,139,830 \\
-23,569\end{array}$ \\
\hline 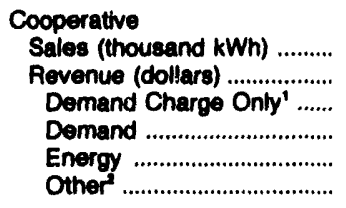 & $\begin{array}{r}19,203 \\
1,149,980 \\
- \\
4999,347 \\
714,159 \\
-63,510\end{array}$ & $\begin{array}{r}79,872 \\
3,453,973 \\
- \\
2,631,527 \\
645,326 \\
177,120\end{array}$ & $\begin{array}{l}\bar{z} \\
\bar{z}\end{array}$ & $\begin{array}{l}\bar{z} \\
\bar{z}\end{array}$ & $\begin{array}{l}\bar{z} \\
\bar{z} \\
\bar{z}\end{array}$ & $\begin{array}{r}166,069 \\
6,594,875 \\
- \\
211,491 \\
6,251,487 \\
131,897\end{array}$ & $\begin{array}{r}2,570,413 \\
118,402,161 \\
- \\
67,344,697 \\
51,705,867 \\
-648,403\end{array}$ \\
\hline 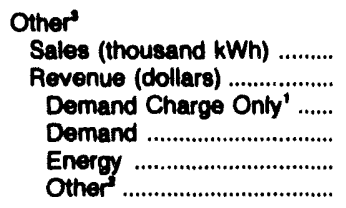 & $\begin{array}{r}1,040 \\
24,700 \\
-\quad 24,700\end{array}$ & $\begin{array}{l}\bar{z} \\
\bar{z}\end{array}$ & $\begin{array}{l}\overline{-} \\
\bar{z}\end{array}$ & $\begin{array}{l}\bar{z} \\
\bar{z} \\
\overline{-}\end{array}$ & $\begin{array}{l}\bar{z} \\
\bar{z}\end{array}$ & $\begin{array}{r}1,394,500 \\
26,724,591 \\
= \\
26,556,459 \\
168,132\end{array}$ & $\begin{array}{r}1,336,420 \\
49,612,776 \\
-\quad \\
23,362,457 \\
26,661,949 \\
-411,630\end{array}$ \\
\hline 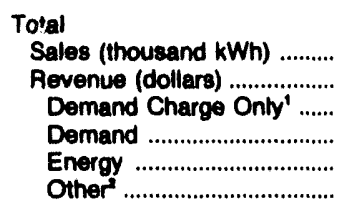 & $\begin{array}{r}375,896 \\
17,195,868 \\
3,000 \\
8,075,101 \\
9,538,939 \\
-421,172\end{array}$ & $\begin{array}{r}858,517 \\
30,341,819 \\
- \\
18,308,787 \\
11,244,306 \\
787,726\end{array}$ & $\begin{array}{l}\bar{z} \\
\bar{z}\end{array}$ & $\begin{array}{l}\bar{z} \\
\bar{z} \\
\bar{z}\end{array}$ & $\begin{array}{c}3,734,595 \\
175,018,744 \\
= \\
= \\
175,918,744\end{array}$ & $\begin{array}{r}8,800,582 \\
253,110,807 \\
2,231,668 \\
34,174,622 \\
213,837,539 \\
2,866,888\end{array}$ & $\begin{array}{r}4,652,405 \\
187,767,198 \\
- \\
96,711,574 \\
92,139,228 \\
-1,083,602\end{array}$ \\
\hline
\end{tabular}

See notes and footnotes at end of table. 
Table 20. Eloctricity Sales for Resale by Investor-Owned Utiltilee, by State, 1992 (Continued)

\begin{tabular}{|c|c|c|c|c|c|c|c|}
\hline $\begin{array}{l}\text { Purchecer of } \\
\text { beotriolty by } \\
\text { Owmerehip clees }\end{array}$ & $\begin{array}{l}\text { Waahington } \\
\text { Puget } \\
\text { Sound } \\
\text { Power a } \\
\text { Loht } \\
\text { Co }\end{array}$ & $\begin{array}{l}\text { Waahington } \\
\text { Wachington } \\
\text { Water } \\
\text { Power } \\
\text { Co }\end{array}$ & $\begin{array}{l}\text { Weat Virginie } \\
\text { Kanawhe } \\
\text { Valley } \\
\text { Power } \\
\text { Co" }\end{array}$ & $\begin{array}{l}\text { Weat Virginia } \\
\text { Mononganela } \\
\text { Powier } \\
\text { Co }\end{array}$ & $\begin{array}{l}\text { Weat Virginia } \\
\text { Utillcorp } \\
\text { United } \\
\text { Inct }\end{array}$ & $\begin{array}{l}\text { Weat Virginis } \\
\text { Wheeling } \\
\text { Power } \\
\text { Co' }\end{array}$ & $\begin{array}{l}\text { Wieconein } \\
\text { coneolldated } \\
\text { Water } \\
\text { Power } \\
\text { Co }\end{array}$ \\
\hline 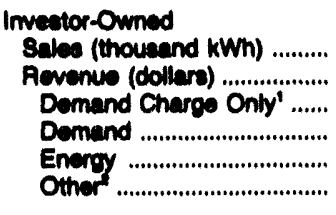 & $\begin{array}{r}849,026 \\
19,440,075 \\
\overline{-} \\
18,374,806 \\
85,177\end{array}$ & $\begin{array}{r}2,222,024 \\
69,886,784 \\
8,388,000 \\
7,031,384 \\
53,687,400 \\
-\end{array}$ & $\begin{array}{l}276,073 \\
4,273,080 \\
- \\
2,886,186 \\
1,286,804 \\
-\end{array}$ & $\begin{array}{r}4,284,124 \\
128,416,822 \\
- \\
39,205,468 \\
65,321,836 \\
20,888,424\end{array}$ & $\begin{array}{l}\bar{z} \\
\bar{m}\end{array}$ & $\bar{z}$ & $\begin{array}{r}1,100 \\
-\quad 6,024 \\
-\quad 6,024 \\
-\quad\end{array}$ \\
\hline 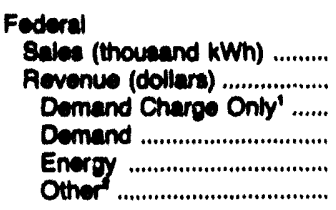 & $\begin{array}{r}168,247 \\
4,292,772 \\
- \\
\overline{4,260,673} \\
23,090\end{array}$ & $\begin{array}{l}368,009 \\
6,152,732 \\
- \\
\overline{8,152,732} \\
-\end{array}$ & $\begin{array}{l}\overline{-} \\
\bar{z}\end{array}$ & $\begin{array}{l}\bar{z} \\
\bar{z}\end{array}$ & 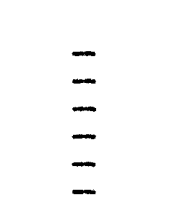 & 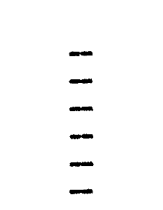 & $\bar{z}$ \\
\hline 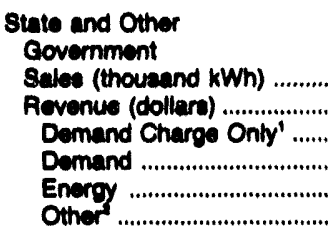 & $\begin{array}{r}63,047 \\
1,311,673 \\
-\quad \\
20,633 \\
1,249,172 \\
41,768\end{array}$ & $\begin{array}{r}246,610 \\
3,443,662 \\
- \\
480,600 \\
2,863,152 \\
\end{array}$ & $\begin{array}{l}\bar{z} \\
\bar{z}\end{array}$ & 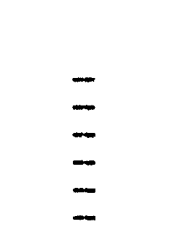 & $\begin{array}{l}\bar{z} \\
\bar{z}\end{array}$ & $\bar{z}$ & $\begin{array}{l}\bar{z} \\
\bar{z}\end{array}$ \\
\hline 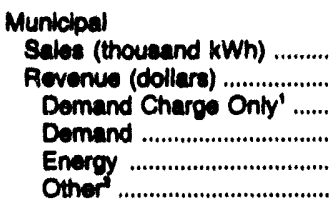 & $\begin{array}{r}212,325 \\
5,047,206 \\
- \\
1,206,069 \\
3,689,405 \\
112,732\end{array}$ & $\begin{array}{r}109,832 \\
4,353,124 \\
682,002 \\
700,324 \\
2,810,798 \\
-\end{array}$ & 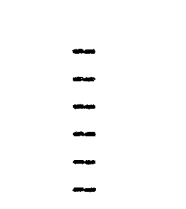 & $\begin{array}{r}81,022 \\
2,737,088 \\
- \\
1,091,386 \\
1,786,616 \\
-139,083\end{array}$ & $\begin{array}{l}\bar{z} \\
= \\
=\end{array}$ & $\bar{z}$ & $\begin{array}{l}\bar{z} \\
\bar{z}\end{array}$ \\
\hline 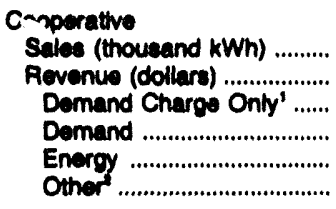 & $\begin{array}{l}\bar{z} \\
\bar{z}\end{array}$ & $\begin{array}{r}193,217 \\
6,128,512 \\
- \\
805,386 \\
5,221,146\end{array}$ & $\begin{array}{l}\bar{z} \\
\bar{z} \\
\bar{z}\end{array}$ & $\begin{array}{r}709,264 \\
21,420,677 \\
- \\
7,803,685 \\
13,764,805 \\
-147,893\end{array}$ & $\begin{array}{l}\bar{z} \\
\bar{m}\end{array}$ & $\begin{array}{l}\bar{z} \\
\bar{z}\end{array}$ & $\begin{array}{l}\bar{z} \\
\bar{z}\end{array}$ \\
\hline 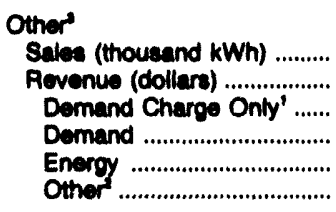 & $\bar{z}$ & $\begin{array}{l}885 \\
-172,273 \\
-\quad \\
-\quad 11,357 \\
-183,630\end{array}$ & $\begin{array}{l}\bar{z} \\
\bar{z}\end{array}$ & $\begin{array}{r}302,471 \\
6,139,630 \\
- \\
908,834 \\
5,230,796 \\
-\end{array}$ & $\begin{array}{l}\overline{-} \\
\bar{z}\end{array}$ & $\begin{array}{l}\bar{z} \\
\bar{z}\end{array}$ & $\begin{array}{l}\bar{z} \\
\bar{z}\end{array}$ \\
\hline 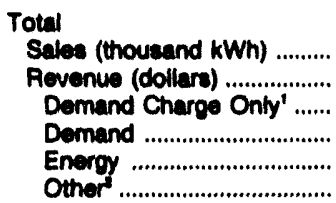 & $\begin{array}{r}1,283,546 \\
30,091,626 \\
1,285,702 \\
28,563,148 \\
242,776\end{array}$ & $\begin{array}{r}3,162,447 \\
91,790,431 \\
9,050,002 \\
10,077,574 \\
72,846,585 \\
-183,630\end{array}$ & $\begin{array}{r}276,073 \\
4,273,090 \\
2,886,196 \\
1,286,894\end{array}$ & $\begin{array}{r}5,376,881 \\
155,714,127 \\
- \\
49,009,327 \\
88,104,252 \\
20,600,548\end{array}$ & 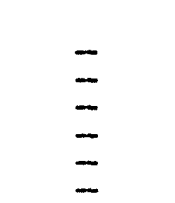 & $\begin{array}{l}\bar{z} \\
\bar{z}\end{array}$ & 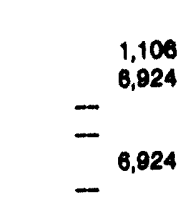 \\
\hline
\end{tabular}

See notes and footnotes at end of table. 
Table 20. Electricity Sales for Resale by Investor-Owned Utilities, by 8tate, 1982 (Continued)

\begin{tabular}{|c|c|c|c|c|c|c|c|}
\hline $\begin{array}{l}\text { Purohecer of } \\
\text { elcotrifity by } \\
\text { owmerehip claces }\end{array}$ & $\begin{array}{l}\text { Wheoonein } \\
\text { Madison } \\
\text { Gas \& } \\
\text { Eleotric } \\
\text { Co }\end{array}$ & $\begin{array}{l}\text { Wheoonaln } \\
\text { North } \\
\text { Contral } \\
\text { Powrer } \\
\text { Co } \\
\text { Ine4 }\end{array}$ & $\begin{array}{l}\text { Wisconein } \\
\text { Northern } \\
\text { Stites } \\
\text { Power } \\
\text { Co' }\end{array}$ & $\begin{array}{l}\text { Wisconain } \\
\text { Northwestern } \\
\text { Wiscondin } \\
\text { Electric } \\
\text { Co }\end{array}$ & $\begin{array}{l}\text { Wisconsin } \\
\text { Plonear } \\
\text { Power } \\
\text { A } \\
\text { Light } \\
\text { Co }\end{array}$ & $\begin{array}{l}\text { Wieonnsin } \\
\text { South Beloit } \\
\text { Water } \\
\text { Gas \& Elec } \\
\text { Co }\end{array}$ & $\begin{array}{l}\text { Wieconain } \\
\text { Superior } \\
\text { Water } \\
\text { Light \& } \\
\text { Power } \\
\text { Co4 }\end{array}$ \\
\hline 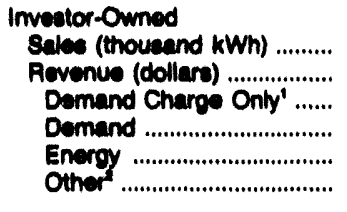 & $\begin{array}{l}28,770 \\
572,571 \\
- \\
- \\
-\end{array}$ & $\begin{array}{l}- \\
= \\
=\end{array}$ & 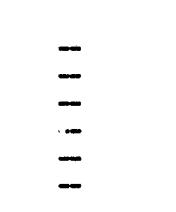 & $\begin{array}{l}\overline{-} \\
\bar{m} \\
\overline{-}\end{array}$ & $\begin{array}{r}9,678 \\
375,306 \\
-\quad \\
117,396 \\
309,384 \\
-51,415\end{array}$ & $\begin{array}{r}6,632 \\
134,169 \\
-\quad 23,862 \\
110,307 \\
-\end{array}$ & $\bar{m}=$ \\
\hline 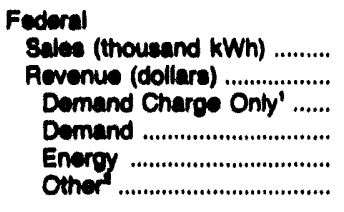 & $\begin{array}{l}\bar{z} \\
\bar{z}\end{array}$ & $\begin{array}{l}\bar{I} \\
\bar{m}\end{array}$ & $\bar{z}$ & $\begin{array}{l}\bar{z} \\
\bar{z}\end{array}$ & $\begin{array}{l}\overline{-} \\
\bar{z} \\
\overline{-}\end{array}$ & $\begin{array}{l}= \\
= \\
=\end{array}$ & $\begin{array}{l}\bar{z} \\
\bar{z}\end{array}$ \\
\hline 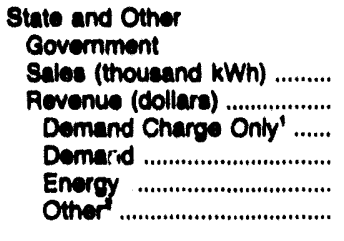 & $\begin{array}{r}1,906 \\
-\quad 28,492 \\
-\quad 29,492\end{array}$ & $\begin{array}{l}\bar{z} \\
\bar{z}\end{array}$ & 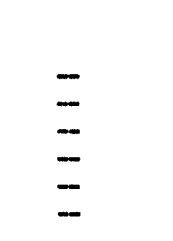 & $\begin{array}{l}\bar{z} \\
\bar{z}\end{array}$ & $\begin{array}{l}\overline{-} \\
\bar{z} \\
=\end{array}$ & $\begin{array}{l}\bar{z} \\
\bar{z}\end{array}$ & $\begin{array}{l}\bar{z} \\
\bar{z}\end{array}$ \\
\hline 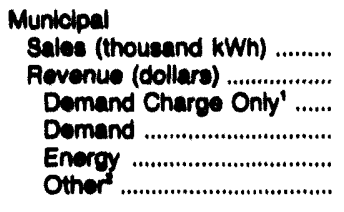 & $\bar{z}$ & $\begin{array}{l}\bar{z} \\
\bar{z}\end{array}$ & $\begin{array}{r}398,040 \\
14,833,792 \\
5,239,335 \\
9,876,331 \\
-81,874\end{array}$ & $\begin{array}{r}4,483 \\
191,858 \\
-\quad \\
52,296 \\
144,339 \\
-4,776\end{array}$ & $\begin{array}{l}\overline{-} \\
\bar{z} \\
\overline{-}\end{array}$ & $\begin{array}{l}\bar{z} \\
\bar{z} \\
\overline{-}\end{array}$ & $\begin{array}{l}\bar{z} \\
\overline{=}\end{array}$ \\
\hline 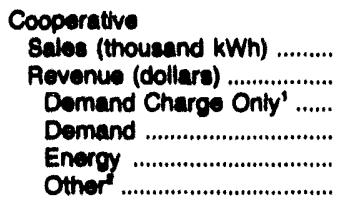 & 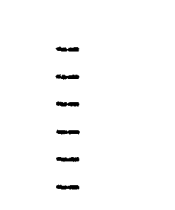 & 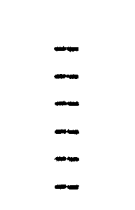 & $\bar{z}$ & $\begin{array}{l}\bar{z} \\
\bar{m}\end{array}$ & $\begin{array}{l}\bar{z} \\
\bar{m}\end{array}$ & $\begin{array}{l}\bar{z} \\
\bar{m}\end{array}$ & $\begin{array}{l}\bar{z} \\
\bar{m}\end{array}$ \\
\hline 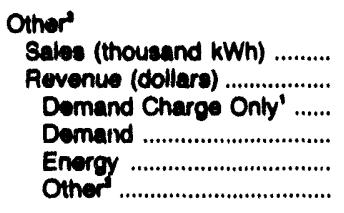 & 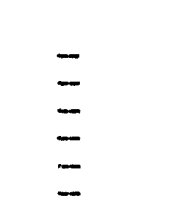 & $\begin{array}{l}\bar{z} \\
\bar{z}\end{array}$ & $\begin{array}{l}-2,230 \\
-78,461 \\
- \\
- \\
- \\
-78,461\end{array}$ & $\begin{array}{l}\bar{z} \\
\bar{z} \\
\overline{-}\end{array}$ & $\begin{array}{l}\bar{z} \\
\bar{z} \\
\overline{-}\end{array}$ & $\bar{z}$ & $\begin{array}{l}\bar{m} \\
\bar{m} \\
\bar{m}\end{array}$ \\
\hline 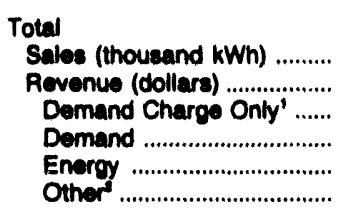 & $\begin{array}{r}30,765 \\
602,063 \\
- \\
- \\
- \\
-\end{array}$ & $\begin{array}{l}\overline{-} \\
\bar{z} \\
\bar{z}\end{array}$ & $\begin{array}{r}393,810 \\
14,755,331 \\
- \\
5,239,335 \\
9,676,331 \\
-160,335\end{array}$ & $\begin{array}{r}4,483 \\
191,858 \\
-\quad 52,295 \\
144,339 \\
-4,776\end{array}$ & $\begin{array}{r}9,578 \\
375,365 \\
-- \\
117,386 \\
309,384 \\
-51,415\end{array}$ & $\begin{array}{r}6,532 \\
134,169 \\
-\quad 23,862 \\
110,307 \\
-\end{array}$ & $\begin{array}{l}\overline{-} \\
\bar{m}\end{array}$ \\
\hline
\end{tabular}

See notes and footnotes at end of table. 
Table 20. Electricity 8ales for Resalo by Investor-Owned Utilities, by state, 1992 (Continued)

\begin{tabular}{|c|c|c|c|c|c|}
\hline 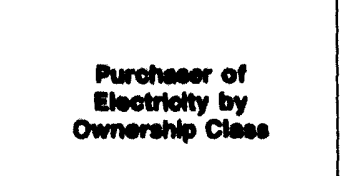 & $\begin{array}{l}\text { Whecondin } \\
\text { Wieconein } \\
\text { Electric } \\
\text { Power } \\
\text { Co }\end{array}$ & $\begin{array}{l}\text { Wieconaln } \\
\text { Wieconaln } \\
\text { Power a } \\
\text { Light } \\
\text { Co }\end{array}$ & $\begin{array}{l}\text { Wieconsin } \\
\text { Wieconein } \\
\text { Fublic } \\
\text { Service } \\
\text { Corp }\end{array}$ & $\begin{array}{l}\text { Wieconein } \\
\text { Wiaconein } \\
\text { Plver } \\
\text { Power } \\
\text { Co' }\end{array}$ & $\begin{array}{l}\text { Wyoming } \\
\text { Cheyenne } \\
\text { Light } \\
\text { Fuel } \\
\text { Power Co' }\end{array}$ \\
\hline 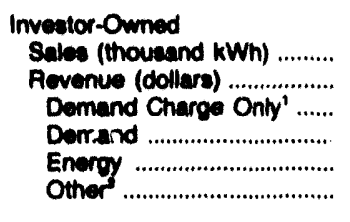 & $\begin{array}{r}680,001 \\
18,984,120 \\
- \\
5,461,587 \\
13,581,045 \\
-59,412\end{array}$ & $\begin{array}{r}365,501 \\
12,143,425 \\
-1,126,500 \\
6,337,968 \\
4,678,857\end{array}$ & $\begin{array}{r}1,018,609 \\
27,700,480 \\
716,687 \\
8,740,990 \\
21,268,646 \\
-3,015,713\end{array}$ & $\begin{array}{l}229,290 \\
5,614,624 \\
- \\
\overline{5,614,824} \\
-\end{array}$ & $\begin{array}{l}= \\
\bar{z} \\
=\end{array}$ \\
\hline 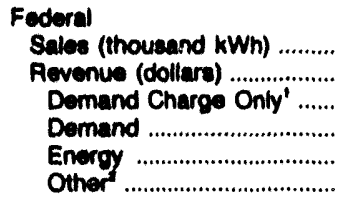 & $\begin{array}{l}\bar{z} \\
\bar{z}\end{array}$ & $\begin{array}{l}\bar{z} \\
\bar{z}\end{array}$ & $\begin{array}{l}m \\
= \\
m\end{array}$ & $\bar{z}$ & $\begin{array}{l}\overline{-} \\
\overline{-} \\
\bar{m}\end{array}$ \\
\hline 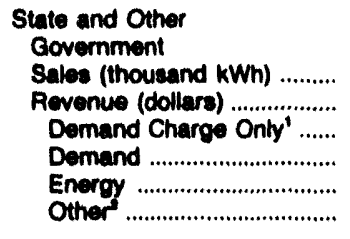 & $\begin{array}{r}1,179,589 \\
41,805,826 \\
-- \\
13,878,787 \\
29,632,430 \\
-1,705,371\end{array}$ & $\begin{array}{r}436,485 \\
16,038,606 \\
- \\
5,846,821 \\
10,259,465 \\
-67,590\end{array}$ & $\begin{array}{r}36 \\
-\quad 687 \\
-\quad 687\end{array}$ & $\begin{array}{l}\bar{z} \\
\overline{-}\end{array}$ & $\begin{array}{l}\overline{-} \\
\overline{-} \\
\overline{-}\end{array}$ \\
\hline 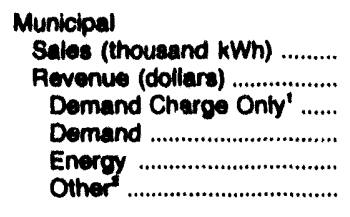 & $\begin{array}{r}322,211 \\
11,454,680 \\
-26,226 \\
4,157,290 \\
7,784,271 \\
-400,756\end{array}$ & $\begin{array}{r}1,133,294 \\
38,436,887 \\
\overline{16,512,416} \\
27,506,682 \\
-6,582,091\end{array}$ & $\begin{array}{r}1016,820 \\
33,783,708 \\
-\quad 14,265,031 \\
24,037,089 \\
-4,518,412\end{array}$ & $\begin{array}{l}\bar{z} \\
\bar{z}\end{array}$ & $\begin{array}{l}\bar{z} \\
\bar{z} \\
\overline{-}\end{array}$ \\
\hline 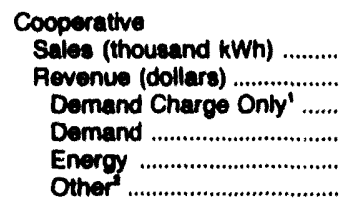 & $\begin{array}{r}22,822 \\
923,580 \\
-\quad \\
392,692 \\
546,135 \\
-16,247\end{array}$ & $\begin{array}{r}434,557 \\
14,004,515 \\
- \\
6,210,804 \\
9,970,739 \\
-2,176,828\end{array}$ & $\begin{array}{r}9,629 \\
387,008 \\
-\quad 180,333 \\
244,169 \\
-37,494\end{array}$ & $\begin{array}{l}- \\
\overline{-} \\
\overline{-}\end{array}$ & $\begin{array}{l}\overline{-} \\
\overline{-} \\
\overline{-}\end{array}$ \\
\hline 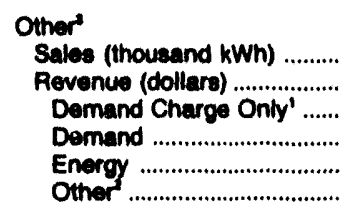 & $\begin{array}{r}239,337 \\
7,688,954 \\
- \\
2,549,022 \\
5,709,539 \\
-559,607\end{array}$ & $\begin{array}{l}5,260 \\
501,509 \\
-\quad \\
- \\
-501,509\end{array}$ & $\begin{array}{l}\bar{z} \\
\bar{z} \\
\bar{z}\end{array}$ & 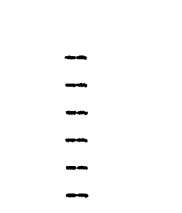 & $\begin{array}{l}\bar{z} \\
\bar{z}\end{array}$ \\
\hline 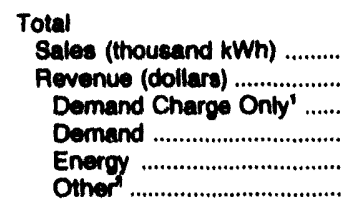 & $\begin{array}{r}2,444,030 \\
80,867,000 \\
-26,225 \\
26,439,358 \\
57,254,320 \\
-2,800,393\end{array}$ & $\begin{array}{r}2,375,097 \\
81,125,132 \\
- \\
29,696,341 \\
54,074,834 \\
-2,646,043\end{array}$ & $\begin{array}{r}2,044,993 \\
61,671,893 \\
716,667 \\
23,186,354 \\
45,540,491 \\
-7,571,619\end{array}$ & $\begin{array}{l}229,280 \\
5,614,824 \\
- \\
\overline{5,614,824} \\
-\end{array}$ & $\begin{array}{l}\bar{z} \\
\bar{z}\end{array}$ \\
\hline
\end{tabular}

Monetary settlements from contracts where electricity is not taken.

- Includes other transactions involving line and substation rentals, syetem support charges, and special contract transactions.

- Includes transactions with power pools, utilties in Canada and Mexico, and nonutilities.

- FERC Form 1 respondent with purchased power transactions, but no sales for resale transactions.

- FERC Form 1 respondent with sales for resale transactions, but no purchased power transactions.

- FERC Form 1 respondent with only wheeling.

- FERC Form 1 respondent with only exchanges.

- FERC Form 1 respondent with no purchased power, sales for resale, exchanges, or wheeling.

- Not Applicable

Notes: -For identification purposes, the utilities are listed in the State in which the corporate office is located. -Totals may not equal sum of components beceuse of independent rounding.

Sources: •Federal Energy Regulatory Commisaion, FERC Form 1, "Annual Report of Major Electric Utilties, Licensees and Others." •FERC Form 1.F. "Annual Peport of Nonmajor Public Utilties and Licensees." 
Table 21. Electricity Exchanges by Investor-Owned Utilities, by State, 1992

\begin{tabular}{|c|c|c|c|c|c|c|c|}
\hline \multirow{2}{*}{$\begin{array}{l}\text { State / } \\
\text { Utulty }\end{array}$} & \multicolumn{3}{|c|}{ Investor-Owned } & \multicolumn{3}{|c|}{ Federal } & \multirow{2}{*}{$\begin{array}{c}\text { State and Other } \\
\text { Government } \\
\begin{array}{c}\text { Recelved } \\
\text { (thousand } \mathrm{kWh} \text { ) }\end{array}\end{array}$} \\
\hline & $\begin{array}{c}\text { Pecelved } \\
\text { (thousand kWh) }\end{array}$ & $\begin{array}{c}\text { Dellvered } \\
\text { (thousand } \mathrm{kWh} \text { ) }\end{array}$ & $\begin{array}{l}\text { Settlement } \\
\text { (dollars) }\end{array}$ & $\begin{array}{c}\text { Peceived } \\
\text { (thousand kWh) }\end{array}$ & $\begin{array}{c}\text { Dellvered } \\
\text { (thousand kWh) }\end{array}$ & $\begin{array}{c}\text { Settlement } \\
\text { (dollars) }\end{array}$ & \\
\hline $\begin{array}{l}\text { Alabama } \\
\text { Alabama Power Company ...................... } \\
\text { Artzona }\end{array}$ & - & -- & - & 1,205 & 1,717 & -- & -- \\
\hline Arizona Public Service Company .......... & - & - & - & - & -- & - & 19,832 \\
\hline $\begin{array}{l}\text { Tuceon Electric Power Company ........... } \\
\text { Artkanases }\end{array}$ & 186,209 & 139,964 & -- & - & -- & - & - \\
\hline $\begin{array}{l}\text { Arkansas Power \& Light Company ...... } \\
\text { Calliomia }\end{array}$ & - & - & - & 243,947 & 223,200 & -- & - \\
\hline $\begin{array}{l}\text { Pacific Gas \& Electric Company ........... } \\
\text { San Dlego Gas \& Electric Co............. } \\
\text { Southern Callitornia Edison Co ............. } \\
\text { Coloredo }\end{array}$ & $\begin{array}{r}644,628 \\
46,345 \\
149,306\end{array}$ & $\begin{array}{r}630,192 \\
42,445 \\
224,177\end{array}$ & $\begin{array}{r}-586,594 \\
-1,874,567\end{array}$ & $\begin{array}{l}791,632 \\
116,963 \\
265,000\end{array}$ & $\begin{array}{r}3,817,346 \\
148,575 \\
304,750\end{array}$ & $\begin{array}{r}-29,338,646 \\
-\overline{-319,664}\end{array}$ & $\begin{array}{r}468,659 \\
10,209 \\
--\end{array}$ \\
\hline 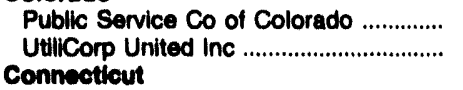 & $\begin{array}{r}21,383 \\
4,221\end{array}$ & $\begin{array}{r}76,432 \\
4,21 \theta\end{array}$ & -210 & $4,512,639$ & $3,638,552$ & $--^{1,106}$ & $1,216,063$ \\
\hline $\begin{array}{l}\text { United Illuminating Company ................... } \\
\text { Florlda }\end{array}$ & 348,237 & - & $8,682,259$ & - & - & -- & 11,228 \\
\hline $\begin{array}{l}\text { Florida Power \& Light Company ............ } \\
\text { Hawwll }\end{array}$ & - & -- & - & - & - & -- & -- \\
\hline $\begin{array}{l}\text { Maui Electric Company Lid ................................. } \\
\text { Iddaho }\end{array}$ & - & - & - & - & - & - & -- \\
\hline $\begin{array}{c}\text { Idaho Power Company ............................... } \\
\text { innots }\end{array}$ & 111,323 & 111,213 & 3,540 & 294,685 & 329,616 & 707,308 & $-\infty$ \\
\hline $\begin{array}{l}\text { Central Illinois Light Company ............... } \\
\text { Contral IIIinois Pub Sorv Co ................. } \\
\text { Indlana }\end{array}$ & $\begin{array}{r}50 \\
2,191\end{array}$ & $\begin{array}{r}920 \\
1,794\end{array}$ & $\begin{array}{r}-20,886 \\
8,880\end{array}$ & $\overline{-}$ & $\overline{-}$ & $\overline{-}$ & - \\
\hline Alcoa Generating Corporation ................ & 150,652 & 151,046 & -- & - & - & - & -- \\
\hline Indianapolis Power \& Light Co .............. & 175 & 857 & - & -- & $\cdots$ & - & - \\
\hline Northern Indiana Pub Serv Co ............. & 1,114 & 946 & - & - & - & - & - \\
\hline 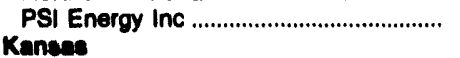 & 13,839 & 13,733 & 1,484 & - & -- & - & - \\
\hline 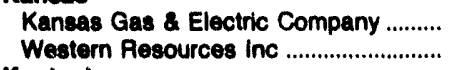 & $\begin{array}{r}21,621 \\
472,409\end{array}$ & $\begin{array}{r}469,533 \\
? 4,381\end{array}$ & $\overline{700,867}$ & - & $\overline{-}$ & $\overline{-}$ & $\overline{-}$ \\
\hline Kentucky & & & & & & & \\
\hline 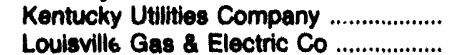 & 3,672 & 1,850 & - & 246,359 & 242,462 & - & -- \\
\hline $\begin{array}{l}\text { Louisville Gas \& Electric Co ................... } \\
\text { Malno }\end{array}$ & 18,042 & 19,980 & - & - & - & -- & - \\
\hline $\begin{array}{l}\text { Maine Public Service Company .............. } \\
\text { Maryland }\end{array}$ & - & - & - & - & - & - & - \\
\hline Baltimore Gas \& Eloctric Co ..................... & - & - & - & - & - & - & - \\
\hline 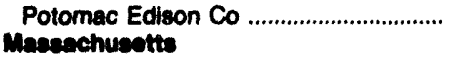 & 203,888 & 195,495 & 196,896 & - & - & -- & - \\
\hline $\begin{array}{l}\text { New England Power Company ............... } \\
\text { michigan }\end{array}$ & 9,506 & 7,014 & - & - & - & - & - \\
\hline Detrott Edison Company ............................. & 281,751 & 223,979 & - & - & - & - & - \\
\hline $\begin{array}{l}\text { Upper Peninsula Power Company ....... } \\
\text { Minnesola }\end{array}$ & 46,232 & 1,728 & $1,038,041$ & - & - & -- & - \\
\hline $\begin{array}{l}\text { Northern States Power Company ......... } \\
\text { miseourt }\end{array}$ & $4,853,133$ & $13,728,804$ & - & $2,671,992$ & - & -- & 132,842 \\
\hline Empire District Electric Co ....................... & 6,783 & 4,145 & - & - & - & - & - \\
\hline Kansas City Power \& Light Co .............. & - & - & - & - & - & - & - \\
\hline 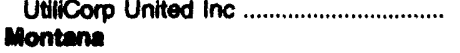 & - & - & - & - & - & - & - \\
\hline $\begin{array}{l}\text { Montana Power Company ........................ } \\
\text { New Moxleo }\end{array}$ & 606,989 & 628,657 & 20,083 & 656,213 & 848,730 & $-1,396,566$ & 13,232 \\
\hline $\begin{array}{l}\text { Public Service Company of NM ............ } \\
\text { Now York }\end{array}$ & 185,216 & 232,618 & - & 14,987 & 4,102 & - & 5,710 \\
\hline Central Hudson Gas \& Elec Corp ......... & - & - & - & - & - & - & - \\
\hline $\begin{array}{l}\text { Consolidated Edison Co-NY Inc............ } \\
\text { New York State Elec \& Gas Corp ........ }\end{array}$ & $\bar{z}$ & - & - & - & - & - & 175,990 \\
\hline $\begin{array}{l}\text { New York Stale Elec \& Gas Corp ....... } \\
\text { North Carollina }\end{array}$ & - & - & - & - & - & - & 110,135 \\
\hline $\begin{array}{l}\text { Duke Power Company } \\
\text { Ygokin Inc }\end{array}$ & 5,013 & 6,815 & - & - & - & - & 657,781 \\
\hline 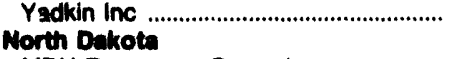 & 2,231 & 2,113 & - & 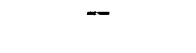 & - & - & - \\
\hline $\begin{array}{l}\text { MDU Resources Group Inc ........................ } \\
\text { Ohto }\end{array}$ & - & - & - & 16,366 & 10,237 & - & - \\
\hline Cincinnati Gas \& Electric Co .................... & 3,036 & 1,980 & - & - & - & - & - \\
\hline 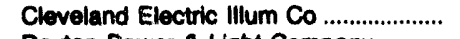 & 209,918 & 236,684 & $1,067,490$ & - & - & - & - \\
\hline Power \& Light Company ........... & 22,617 & 8,787 & 258,728 & - & - & - & - \\
\hline 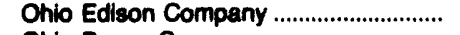 & 366,849 & 76,550 & $4,350,452$ & - & - & - & - \\
\hline 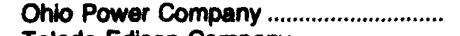 & 51,685 & $\begin{array}{r}2,757 \\
\end{array}$ & $\bar{x}$ & $\overline{-}$ & $\overline{-}$ & - & $\overline{-}$ \\
\hline Edison Company & 61,401 & 155,394 & $-2,003,898$ & - & - & - & - \\
\hline
\end{tabular}

See notes and footnotes at end of table. 
Table 21. Electriclty Exchanges by Investor-Owned Utilities, by State, 1992 (Sontinued)

\begin{tabular}{|c|c|c|c|c|c|c|c|}
\hline \multirow{2}{*}{ State ' } & \multicolumn{2}{|c|}{$\begin{array}{l}\text { State and Other } \\
\text { Government }\end{array}$} & \multicolumn{3}{|c|}{ Municipal } & \multicolumn{2}{|c|}{ Cooperative } \\
\hline & $\begin{array}{c}\text { Dellvered } \\
\text { (thousand kWh) }\end{array}$ & $\begin{array}{l}\text { Sottlement } \\
\text { (dollars) }\end{array}$ & $\begin{array}{c}\text { Recotved } \\
\text { (thousand kWh) }\end{array}$ & $\begin{array}{c}\text { Dellvered } \\
\text { (thousand } \mathrm{kWh} \text { ) }\end{array}$ & $\begin{array}{l}\text { Settloment } \\
\text { (dollars) }\end{array}$ & $\begin{array}{c}\text { Recelved } \\
\text { (thousend kWh) }\end{array}$ & $\begin{array}{l}\text { Dellvered } \\
\text { (thousand kWh) }\end{array}$ \\
\hline $\begin{array}{l}\text { Alebama } \\
\text { Alabama Power Company ...................... } \\
\text { Anteona }\end{array}$ & - & - & - & - & - & - & - \\
\hline Arizona Publle Sorvice Company .......... & 21,043 & - & - & - & - & 4,748 & $13,6,47$ \\
\hline $\begin{array}{l}\text { Tuceon Electric Power Company .......... } \\
\text { Artuneas }\end{array}$ & - & - & - & - & - & - & - \\
\hline $\begin{array}{l}\text { Arkaneas Power \& Light Company...... } \\
\text { Callfomb }\end{array}$ & - & - & - & - & - & - & - \\
\hline 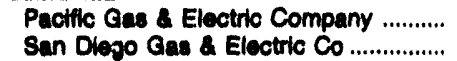 & $-{ }_{11,561}$ & $17,286,408$ & $\overline{-}$ & $\bar{z}$ & $\overline{-}$ & $\overline{-}$ & $\overline{-}$ \\
\hline $\begin{array}{l}\text { Southern Calfiornia Edison Co ............... } \\
\text { colorado }\end{array}$ & - & - & 28,220 & - & 800,865 & - & - \\
\hline Public Sonvice Co of Colorado ............... & $1,214,833$ & - & 369,784 & 384,441 & $13,842,080$ & 72,924 & 524,688 \\
\hline 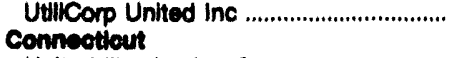 & - & - & - & - & - & - & - \\
\hline $\begin{array}{l}\text { United Illuminating Company ................... } \\
\text { Fiorlda }\end{array}$ & 15,374 & - & - & - & - & - & - \\
\hline $\begin{array}{l}\text { Florlda Power \& Light Company ........... } \\
\text { Hawall }\end{array}$ & - & - & - & - & - & 4,009 & 3,177 \\
\hline $\begin{array}{c}\text { Maul Electric Company LId ............................ } \\
\text { Idsho }\end{array}$ & - & - & - & - & - & - & - \\
\hline 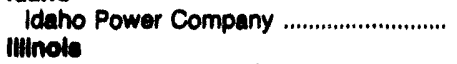 & - & - & 108,000 & 126,000 & - & - & - \\
\hline $\begin{array}{l}\text { Contral Illinols Llght Company ................ } \\
\text { Contral Illinois Pub Sorv Co .................. } \\
\text { Indiana }\end{array}$ & $\overline{-}$ & $\overline{-}$ & $\begin{array}{l}314 \\
220\end{array}$ & $\begin{array}{r}2,718 \\
290\end{array}$ & $\begin{array}{r}-57,236 \\
-1,583\end{array}$ & $\overline{-}$ & $\overline{-}$ \\
\hline $\begin{array}{l}\text { Indiana } \\
\text { Alco Gonerating Corporation ................. }\end{array}$ & - & - & - & - & - & - & - \\
\hline Indianapolls Power \& Light Co ............... & - & - & - & - & - & - & 543 \\
\hline $\begin{array}{l}\text { Northern Indlana Pub Sorv Co ................ } \\
\text { PSI Eneroy Inc }\end{array}$ & - & - & - & - & - & - & - \\
\hline 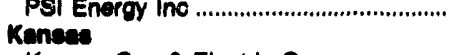 & - & - & - & - & - & 8,138 & 5,374 \\
\hline $\begin{array}{l}\text { Kansase Gas \& Electric Company ........... } \\
\text { Western Resources Inc ............................ }\end{array}$ & $\overline{-}$ & $\overline{-}$ & $\overline{-}$ & - & - & - & - \\
\hline Kentucky & - & - & - & - & - & - & - \\
\hline $\begin{array}{l}\text { Kentucky Utilities Company ...................... } \\
\text { Louieville Gas \& Electric Co .................. }\end{array}$ & $\overline{-}$ & $\overline{-}$ & $\overline{-}$ & $\overline{-}$ & $\overline{-}$ & $\begin{array}{r}149,290 \\
2,814\end{array}$ & $\begin{array}{r}151,718 \\
7,614\end{array}$ \\
\hline $\begin{array}{l}\text { Malno } \\
\text { Maine Public Service Company ............. }\end{array}$ & - & - & - & - & - & 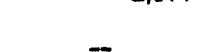 & 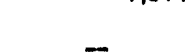 \\
\hline Marrtand & - & - & - & - & - & - & - \\
\hline 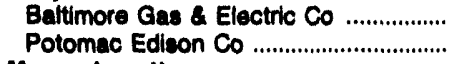 & $\overline{-}$ & $\overline{-}$ & $\overline{-}$ & $\overline{-}$ & $\overline{-}$ & $\overline{-}$ & $\overline{-}$ \\
\hline $\begin{array}{l}\text { Maseachusotte } \\
\text { New England Power Company .............. } \\
\text { michigen }\end{array}$ & - & - & -- & - & - & - & - \\
\hline $\begin{array}{l}\text { Dotrott Edison Company ....................... } \\
\text { Upper Penineula Power Company ....... } \\
\text { Minnecota }\end{array}$ & $\overline{-}$ & $\overline{-}$ & $\overline{-}$ & - & $-6,168$ & $\overline{-}$ & $\overline{-}$ \\
\hline $\begin{array}{l}\text { Northern States Power Company ........ } \\
\text { Mleseurt }\end{array}$ & 730,801 & - & 2,260 & 281,817 & - & $8,220,634$ & $6,688,153$ \\
\hline Empire District Electric Co ........................ & - & - & - & - & - & - & - \\
\hline 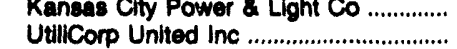 & $\overline{-}$ & $\overline{-}$ & $\overline{-}$ & $\overline{-}$ & $\overline{-}$ & $\begin{array}{l}10,045 \\
54,958\end{array}$ & $\begin{array}{r}7,567 \\
45,812\end{array}$ \\
\hline Montuna & 29,827 & $-101,426$ & 20,683 & 45,241 & $-491,820$ & - & - \\
\hline $\begin{array}{l}\text { Public Servicie Company of NM ........... } \\
\text { New York }\end{array}$ & 5,848 & - & 8,610 & 8,448 & - & 62,528 & 72,563 \\
\hline Central Hudson Gas \& Elec Corp ......... & - & $\overline{-}$ & - & - & - & - & - \\
\hline $\begin{array}{l}\text { Consollidated Edison Co-NY Inc ............ } \\
\text { New York Stute Elec \& Gas Corp ...... }\end{array}$ & $\begin{array}{l}211,686 \\
165,457\end{array}$ & $\begin{array}{l}6,601,596 \\
2,732,400\end{array}$ & $\overline{-}$ & $\overline{-}$ & - & $\overline{-}$ & $\overline{-}$ \\
\hline 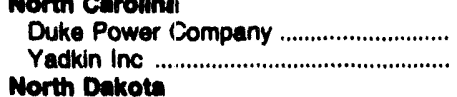 & $-563,831$ & $5,19,823$ & $-_{-}^{219,238}$ & $-^{187,963}$ & 102,497 & $1,569,193$ & $\begin{array}{l}1,339,894 \\
--\end{array}$ \\
\hline $\begin{array}{l}\text { MDU Resources Group inc ......................... } \\
\text { Ohlo }\end{array}$ & - & - & - & - & - & 3,413 & 2,411 \\
\hline Cincinnati Gas \& Electric Co .................. & - & - & - & 10,712 & - & 225 & 48,474 \\
\hline $\begin{array}{l}\text { Electric Illum Co ..................... } \\
\text { ower \& Light Company. }\end{array}$ & - & $\overline{-}$ & $\overline{-}$ & $\overline{-}$ & 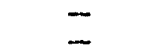 & - & - \\
\hline 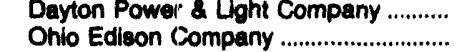 & $\overline{-}$ & $\overline{-}$ & $\overline{-}$ & 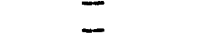 & 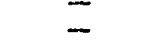 & $\bar{z}$ & $\overline{-}$ \\
\hline Ohio Power Clompany .................................... & - & - & 15,881 & 16,056 & - & - & - \\
\hline odo Edisoll Company ............................ & - & - & - & - & - & -- & - \\
\hline
\end{tabular}


Table 21. Electricity Exchanges by Investor-Owned Utilitios, by State, 1992 (Continued)

\begin{tabular}{|c|c|c|c|c|c|c|c|}
\hline \multirow{2}{*}{$\begin{array}{l}\text { Etate / } \\
\text { Utanly }\end{array}$} & \multirow{2}{*}{$\begin{array}{c}\text { Cooperative } \\
\begin{array}{c}\text { Seltlement } \\
\text { (dollare) }\end{array}\end{array}$} & \multicolumn{3}{|c|}{ Other' } & \multicolumn{3}{|c|}{ Total } \\
\hline & & $\begin{array}{c}\text { Received } \\
\text { (thousend kWh) }\end{array}$ & $\begin{array}{c}\text { Dellwered } \\
\text { (thousand kWh) }\end{array}$ & $\begin{array}{c}\text { Settlement } \\
\text { (dollern) }\end{array}$ & $\begin{array}{c}\text { Recelved } \\
\text { (thousand } \mathrm{kWh} \text { ) }\end{array}$ & $\begin{array}{c}\text { Deilvered } \\
\text { (thousand kWh) }\end{array}$ & $\begin{array}{l}\text { Seltlement } \\
\text { (dollara) }\end{array}$ \\
\hline 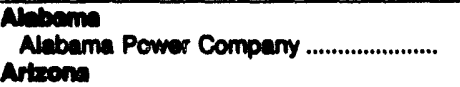 & - & - & - & - & 1,205 & 1,717 & - \\
\hline $\begin{array}{l}\text { Artzona Publlc Service Company .......... } \\
\text { Tucion Electric Power Company ......... } \\
\text { Artemens }\end{array}$ & $\overline{-}$ & -708 & $-{ }^{2,792}$ & $\overline{-}$ & $\begin{array}{r}25,288 \\
168,209\end{array}$ & $\begin{array}{r}37,482 \\
138,964\end{array}$ & $\overline{-}$ \\
\hline $\begin{array}{l}\text { Arkanaas Power \& Light Company ...... } \\
\text { Cestromia }\end{array}$ & - & - & - & - & 243,947 & 223,200 & - \\
\hline $\begin{array}{l}\text { Pacific Gas \& Electric Company ........... } \\
\text { San Diego Gas \& Electrle Co ................ } \\
\text { Southern Calliomi Edison Co .............. } \\
\text { Coloredo }\end{array}$ & $\bar{z}$ & $\overline{-}^{56,269}$ & $\overline{-}^{62,195}$ & $\begin{array}{l}-251,413 \\
=\end{array}$ & $\begin{array}{r}1,960,168 \\
173,517 \\
442,526\end{array}$ & $\begin{array}{r}4,509,733 \\
202,581 \\
528,927\end{array}$ & $\begin{array}{l}-12,880,245 \\
-1,393,368\end{array}$ \\
\hline 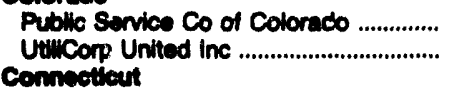 & $\overline{-}$ & $-^{129,831}$ & $-22,249$ & $\underline{-}$ & $\begin{array}{r}6,322,624 \\
4,221\end{array}$ & $\begin{array}{r}5,861,105 \\
4,219\end{array}$ & $\begin{array}{r}13,843,188 \\
210\end{array}$ \\
\hline $\begin{array}{l}\text { United Illuminating Company .................. } \\
\text { Florice }\end{array}$ & - & - & - & - & 359,465 & 15,374 & $8,882,259$ \\
\hline Forida Power \& Light Company ........... & - & - & - & - & 4,000 & 3,177 & - \\
\hline Maui Electric Company Lid ......................... & - & 3,645 & 3,054 & 14,876 & 3,645 & 3,054 & 14,876 \\
\hline Idaho Power Company ................................ & - & - & - & - & 514,008 & 566,829 & 710,848 \\
\hline $\begin{array}{l}\text { Central IIInole Light Company ................ } \\
\text { Central Winole Pub serv Co .................. } \\
\text { indiene }\end{array}$ & $\overline{-}$ & $\overline{-}$ & $\overline{-}$ & $\overline{-}$ & $\begin{array}{r}384 \\
2,411\end{array}$ & $\begin{array}{l}3,638 \\
2,084\end{array}$ & $\begin{array}{r}-78,12: \\
7,397\end{array}$ \\
\hline 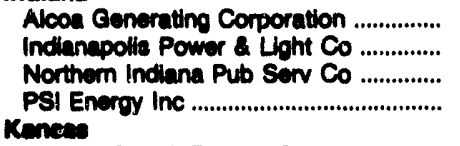 & $\bar{z}$ & $\begin{array}{r}-2,271 \\
-\quad 62,710\end{array}$ & $\begin{array}{r}-1,488 \\
-\quad 65,823\end{array}$ & $\bar{z}$ & $\begin{array}{r}150,652 \\
2,446 \\
1,114 \\
84,687\end{array}$ & $\begin{array}{r}151,046 \\
2,888 \\
846 \\
84,830\end{array}$ & $\bar{I}=$ \\
\hline $\begin{array}{l}\text { Kanasas Gas \& Electric Company ........... } \\
\text { Weetern: Resources inc .......................... } \\
\text { Kenturely }\end{array}$ & $\overline{-}$ & $\overline{-}$ & $\overline{-}$ & $\overline{-}$ & $\begin{array}{r}21,621 \\
472,400\end{array}$ & $\begin{array}{r}469,533 \\
24,381\end{array}$ & $\overline{700,867}$ \\
\hline $\begin{array}{l}\text { Kentucky Utillities Company ................... } \\
\text { Louloville Gas \& Electric Co ................. } \\
\text { Malns }\end{array}$ & - & 83 & $\overline{-}$ & $\overline{-}$ & $\begin{array}{r}398,423 \\
20,856\end{array}$ & $\begin{array}{r}386,030 \\
27,494\end{array}$ & $\overline{-}$ \\
\hline $\begin{array}{l}\text { Meine Public Service Company .............. } \\
\text { mentend }\end{array}$ & - & - & 2,232 & - & - & 2,232 & - \\
\hline $\begin{array}{l}\text { Baltimore Gas \& Electric Co ................... } \\
\text { Potomac Edison Co ................................. }\end{array}$ & $\overline{-}$ & $1,147,276$ & $\overline{-}$ & $34,628,118$ & $\begin{array}{r}1,147,276 \\
203,888\end{array}$ & $-\overline{1}_{185,485}$ & $\begin{array}{r}34,62 \varepsilon, 119 \\
196,896\end{array}$ \\
\hline $\begin{array}{l}\text { Now England Power Company ................ } \\
\text { methloen }\end{array}$ & - & - & - & - & 9,506 & 7,014 & - \\
\hline $\begin{array}{l}\text { Edieon Company ......................... } \\
\text { Penineilla Power Company ....... } \\
\text { tu }\end{array}$ & $\overline{-}$ & $-1,617$ & $-{ }_{18,831}$ & $-\overline{463,156}$ & $\begin{array}{r}281,7 \notin 1 \\
47,849\end{array}$ & $\begin{array}{r}223,979 \\
20,579\end{array}$ & $\overline{588,718}$ \\
\hline $\begin{array}{l}\text { Northern States Power Company ........ } \\
\text { miscourt }\end{array}$ & - & 1 & 408,271 & - & $16,880,862$ & $21,837,946$ & - \\
\hline 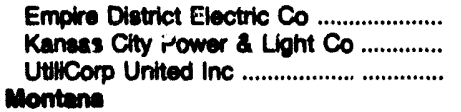 & $\begin{array}{l}\overline{32,214} \\
-2,202\end{array}$ & $\overline{1,406,548}$ & $\overline{-}$ & $\overline{-}$ & $\begin{array}{r}6,783 \\
10,045 \\
1,461,506\end{array}$ & $\begin{array}{r}4,145 \\
7,567 \\
1,452,911\end{array}$ & $\begin{array}{l}\overline{32,214} \\
-2,202\end{array}$ \\
\hline $\begin{array}{l}\text { Montana Power Company ........................ } \\
\text { New Hoxlece }\end{array}$ & - & $8,785,423$ & $8,785,447$ & - & $10,082,520$ & $10,338,002$ & $-1,869,729$ \\
\hline $\begin{array}{l}\text { Public Service Company of NM ............. } \\
\text { New York }\end{array}$ & - & 4,292 & 2,393 & - & 281,343 & 326,072 & - \\
\hline $\begin{array}{l}\text { Contral Hudson Gas \& Elec Corp ........ } \\
\text { Consolidated Edieon Co-NY Inc ........ } \\
\text { New York State Elec \& Gas Corp ....... }\end{array}$ & $\bar{z}$ & ${ }_{-}^{68,386}$ & $-^{3,681}$ & $\overline{-}$ & $\begin{array}{r}68,396 \\
175,990 \\
110,135\end{array}$ & $\begin{array}{r}3,68 i \\
211,686 \\
165,457\end{array}$ & $\begin{array}{c}\overline{6,601,586} \\
2,732,400\end{array}$ \\
\hline 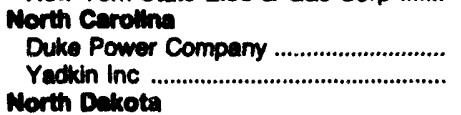 & $1,308,236$ & $-{ }^{65,289}$ & 64,657 & $\overline{-}$ & $\begin{array}{r}2,516,494 \\
2,231\end{array}$ & $\begin{array}{r}2,163,260 \\
2,113\end{array}$ & $2,330,556$ \\
\hline $\begin{array}{l}\text { MDU Rgeources Group Inc .................... } \\
\text { Onto }\end{array}$ & - & - & - & - & 19,779 & 12,648 & - \\
\hline $\begin{array}{l}\text { Cincinnat Ges \& Electric Co ................. } \\
\text { Cleveland Electric Illum Co ................. } \\
\text { Dayton Power \& Light Company ........... } \\
\text { Ohio Edison Company ............................ } \\
\text { Ohio Power Compary ........................... } \\
\text { Toledo Edieon Company ........................ }\end{array}$ & $\begin{array}{l}\bar{z} \\
\bar{z}\end{array}$ & $\begin{array}{l}\overline{-} \\
\bar{z} \\
380,854\end{array}$ & $\begin{array}{l}\overline{-} \\
\overline{575,003}\end{array}$ & $\begin{array}{l}\bar{z} \\
\bar{z} \\
17, \overline{000,000}\end{array}$ & $\begin{array}{r}3,261 \\
209,918 \\
22,617 \\
366,849 \\
67,566 \\
452,255\end{array}$ & $\begin{array}{r}61,203 \\
236,684 \\
8,787 \\
78,550 \\
18,813 \\
730,387\end{array}$ & $\begin{array}{r}\overline{1,087,480} \\
258,728 \\
4,350,452 \\
14, \overline{998,102}\end{array}$ \\
\hline
\end{tabular}

See notes and footnotes at end of table. 
Table 21. Electricity Exchanges by Investor-Owned Utillties, by State, 1992 (Continued)

\begin{tabular}{|c|c|c|c|c|c|c|c|}
\hline \multirow{2}{*}{$\begin{array}{l}\text { State / } \\
\text { Utullty }\end{array}$} & \multicolumn{3}{|c|}{ Investor-Owned } & \multicolumn{3}{|c|}{ Federal } & \multirow{2}{*}{\begin{tabular}{|c}
$\begin{array}{c}\text { State and Other } \\
\text { Government }\end{array}$ \\
$\begin{array}{c}\text { Recelved } \\
\text { (thousand } \mathrm{kWh} \text { ) }\end{array}$
\end{tabular}} \\
\hline & $\begin{array}{c}\text { Recelved } \\
\text { (thousand kWh) }\end{array}$ & $\begin{array}{c}\text { Delivered } \\
\text { (thousand kWh) }\end{array}$ & $\begin{array}{c}\text { Settlement } \\
\text { (dollars) }\end{array}$ & $\begin{array}{c}\text { Received } \\
\text { (thousend kWh) }\end{array}$ & $\begin{array}{c}\text { Delivered } \\
\text { (thousand } \mathrm{kWh} \text { ) }\end{array}$ & $\begin{array}{l}\text { Settlement } \\
\text { (dollars) }\end{array}$ & \\
\hline $\begin{array}{l}\text { Orlahoma } \\
\text { Public Service Co of Oklahoma ............ } \\
\text { Oregon }\end{array}$ & 30,347 & 30,347 & - & 122,160 & 123,843 & 63,585 & 645,227 \\
\hline 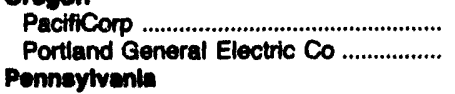 & $\begin{array}{r}1,381,881 \\
559,868\end{array}$ & $\begin{array}{r}1,232,386 \\
467,395\end{array}$ & $\begin{array}{l}-74,616 \\
-98,814\end{array}$ & $\begin{array}{r}18,783,093 \\
580,639\end{array}$ & $\begin{array}{r}18,647,473 \\
566,255\end{array}$ & $\begin{array}{r}-63,832,403 \\
-1,060,458\end{array}$ & $\begin{array}{r}1,468,399 \\
59,680\end{array}$ \\
\hline $\begin{array}{l}\text { Duquesne Light Company ...................... } \\
\text { West Penn Power Company ................. }\end{array}$ & $\begin{array}{r}155,897 \\
70,782\end{array}$ & $\begin{array}{r}189,996 \\
59,287\end{array}$ & $\overline{273,134}$ & $\overline{-}$ & $\overline{-}$ & $\underline{-}$ & $\overline{-}$ \\
\hline $\begin{array}{l}\text { South Dakota } \\
\text { Black Hills Corporation ............................. } \\
\text { Taxas }\end{array}$ & 7,514 & 8,321 & - & 9,248 & 11,640 & - & - \\
\hline $\begin{array}{l}\text { Contral Power \& Light Company ........... } \\
\text { EI Paso Electric Company }\end{array}$ & $\begin{array}{l}33,629 \\
17,568\end{array}$ & $\begin{array}{l}31,345 \\
69,380\end{array}$ & $-^{1,500}$ & $\overline{-}$ & $\overline{-}$ & $\overline{-}$ & 32,844 \\
\hline 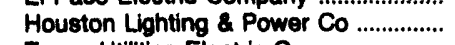 & $2,804,985$ & $10,272,882$ & - & - & - & - & 13,110 \\
\hline $\begin{array}{l}\text { Texas Utillites Electric Co .......................... } \\
\text { Vermont }\end{array}$ & $11,471,339$ & $7,268,429$ & - & - & - & - & $1,330,016$ \\
\hline $\begin{array}{c}\text { Citizens Utilities Company ................................. } \\
\text { Vilroinita }\end{array}$ & 61,744 & 28,420 & 361,603 & - & - & - & 8,185 \\
\hline $\begin{array}{l}\text { Virginia Electric \& Power Co .................. } \\
\text { Weshington }\end{array}$ & 21,385 & 21,433 & - & 12,709 & 11,879 & - & - \\
\hline $\begin{array}{l}\text { Puget Sound Power \& Light Co ......... } \\
\text { Washington Water Power Company .... } \\
\text { Weat Virglnta }\end{array}$ & $\begin{array}{l}452,347 \\
818,096\end{array}$ & $\begin{array}{l}448,972 \\
813,793\end{array}$ & $\begin{array}{r}11,710 \\
-1,128,743\end{array}$ & $\begin{array}{l}735,488 \\
345,455\end{array}$ & $\begin{array}{l}779,628 \\
274,703\end{array}$ & $\begin{array}{r}-2,118,808 \\
1,700,609\end{array}$ & $\begin{array}{r}25,246 \\
135,991\end{array}$ \\
\hline $\begin{array}{l}\text { Monongahela Power Company ............. } \\
\text { Whecondin }\end{array}$ & 40,355 & 27,765 & 264,114 & - & - & - & - \\
\hline North Central Power Co Inc .................... & $\begin{array}{r}2,617 \\
8,145888\end{array}$ & $\begin{array}{r}2,617 \\
4,362,592\end{array}$ & $\overline{-}$ & $=$ & $\overline{-}$ & $\overline{-}$ & $\overline{-}$ \\
\hline $\begin{array}{l}\text { Northern States Power Company ......... } \\
\text { Wisconsin Power \& Light Co ................ }\end{array}$ & $\begin{array}{r}8,745,868 \\
31\end{array}$ & $\begin{array}{r}4,502,582 \\
532\end{array}$ & - & - & - & $\overline{-}$ & - \\
\hline Public Service Corp ............. & 235 & 235 & - & - & - & - & 532 \\
\hline
\end{tabular}

Oxtahoms

Oregon

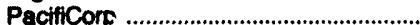

Portiand General Electric Co ................

Pennoytuanls

Duquesne Light Company

West Penn Power Company

south Dakota

Black Hills Corporation ...........................

Toxas

Central Power \& Light Company .........

El Paso Electric Company

Houston Lighting \& Power $\mathrm{Co}$.................

Texas Utilties Electric $\mathrm{Co}$...

Vermont

Citizens Utilities Company .......................

Viroint

Virginia Electric \& Power Co ................

Weshington

Puget Sound Power \& Light Co ..........

Washington Water Power Company ...

Weat Virginia

Monongahela Power Company ............

Weconsin

North Central Power Co Inc ..................

Northern States Power Company ........

Wisconsin Power \& Light Co.

Wisconsin Public Service Corp

See notes and footnotes at end of table.

\begin{tabular}{|c|c|c|c|c|c|c|}
\hline \multicolumn{2}{|c|}{$\begin{array}{c}\text { State and Other } \\
\text { Government }\end{array}$} & \multicolumn{3}{|c|}{ Municipal } & \multicolumn{3}{c|}{ Cooperative } \\
\hline $\begin{array}{c}\text { Delivered } \\
\text { (thousand } \mathrm{kWh} \text { ) }\end{array}$ & $\begin{array}{c}\text { Settlement } \\
\text { (dollars) }\end{array}$ & $\begin{array}{c}\text { Received } \\
\text { (thousand } \mathrm{kWh} \text { ) }\end{array}$ & $\begin{array}{c}\text { Delivered } \\
\text { (thousand } \mathrm{kWh} \text { ) }\end{array}$ & $\begin{array}{c}\text { Settlement } \\
\text { (dollars) }\end{array}$ & $\begin{array}{c}\text { Received } \\
\text { (thousand } \mathrm{kWh} \text { ) }\end{array}$ & $\begin{array}{c}\text { Delivered } \\
\text { (thousand } \mathrm{kWh} \text { ) }\end{array}$ \\
\hline
\end{tabular}

\begin{tabular}{|c|c|c|c|c|c|c|}
\hline 643,771 & - & - & - & - & - & - \\
\hline $\begin{array}{r}1,502,144 \\
64,942\end{array}$ & $\begin{array}{r}-261,728 \\
5,280\end{array}$ & $\begin{array}{l}27,831 \\
22,377\end{array}$ & $\begin{array}{l}31,578 \\
28,239\end{array}$ & $\begin{array}{r}-304,990 \\
-3,940\end{array}$ & $\begin{array}{l}487,915 \\
-\end{array}$ & $\begin{array}{l}453,698 \\
-\end{array}$ \\
\hline- & - & - & - & - & - & - \\
\hline- & - & - & - & - & - & - \\
\hline- & - & - & - & - & - & - \\
\hline 33,550 & 700 & - & - & - & 86,371 & 84,574 \\
\hline 10,310 & - & - & - & - & - & - \\
\hline 25,643 & - & 2,169 & 683,738 & - & - & - \\
\hline $1,029,735$ & - & - & - & - & - & - \\
\hline 14,496 & $-553,195$ & 2,320 & - & 88,146 & - & - \\
\hline- & - & - & - & - & - & - \\
\hline$\overline{139,519}^{-}$ & $\begin{array}{l}277,706 \\
556,969\end{array}$ & $\begin{array}{r}1,080 \\
530\end{array}$ & $\begin{array}{r}28,397 \\
320\end{array}$ & $\begin{array}{r}-360 \\
3,759\end{array}$ & $\overline{-}$ & $\overline{-}$ \\
\hline- & - & - & - & - & - & - \\
\hline - & - & - & - & - & - & - \\
\hline 234,686 & - & 2,617 & 16,693 & - & $3,328,674$ & $2,445,282$ \\
\hline - & - & - & - & - & - & - \\
\hline 532 & - & - & - & - & - & - \\
\hline
\end{tabular}


Table 21. Electricity Exchanges by Investor-Owned Utilities, by State, 1992 (Continued)

\begin{tabular}{|c|c|c|c|c|c|c|c|}
\hline \multirow{2}{*}{$\begin{array}{l}\text { 8tate I } \\
\text { Uutity }\end{array}$} & \multirow{2}{*}{$\begin{array}{c}\text { Cooperattive } \\
\begin{array}{c}\text { Settlement } \\
\text { (dollars) }\end{array}\end{array}$} & \multicolumn{3}{|c|}{ Other' } & \multicolumn{3}{|c|}{ Total } \\
\hline & & $\begin{array}{c}\text { Pecelved } \\
\text { (thousand kWh) }\end{array}$ & $\begin{array}{c}\text { Dellvered } \\
\text { (thousand kWh) }\end{array}$ & $\begin{array}{l}\text { Settlement } \\
\text { (dollars) }\end{array}$ & $\begin{array}{c}\text { Recelved } \\
\text { (thousand kWh) }\end{array}$ & $\begin{array}{c}\text { Delivered } \\
\text { (thousand kWh) }\end{array}$ & $\begin{array}{l}\text { Settlement } \\
\text { (dollars) }\end{array}$ \\
\hline $\begin{array}{l}\text { Oddehoma } \\
\text { Public Senvice Co of Oklahoma ........... } \\
\text { Oregen }\end{array}$ & - & - & - & - & 797,734 & 797,961 & 63,585 \\
\hline 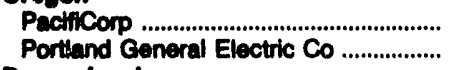 & - & 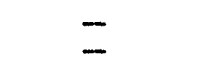 & $\overline{-}$ & $\overline{-}$ & $\begin{array}{r}22,149,119 \\
1,202,564\end{array}$ & $\begin{array}{r}21,867,280 \\
1,126,831\end{array}$ & $\begin{array}{r}-64,473,737 \\
-1,157,932\end{array}$ \\
\hline $\begin{array}{l}\text { Pennoytventa } \\
\text { Duquesene Light Company ...................... } \\
\text { Weet Penn Power Company ................ }\end{array}$ & $\overline{-}$ & $-2,016$ & $-\quad 4,129$ & $\overline{-}$ & $\begin{array}{r}157,913 \\
70,782\end{array}$ & $\begin{array}{r}194,125 \\
59,287\end{array}$ & $\overline{273,134}$ \\
\hline $\begin{array}{l}\text { South Dakota } \\
\text { Black Hills Corporation .............................. }\end{array}$ & - & - & - & - & 16,762 & 19,961 & - \\
\hline $\begin{array}{l}\text { Central Power \& Light Company ............ } \\
\text { El Paso Electric Company .................. } \\
\text { Houston Lighting \& Power Co ............ } \\
\text { Texas Utilities Electric Co ..................... } \\
\text { Vermont }\end{array}$ & $\bar{z}$ & $\begin{array}{l}21,237 \\
- \\
8,265,134 \\
3,515,932\end{array}$ & $\begin{array}{r}21,448 \\
- \\
102,551 \\
8,018,374\end{array}$ & $\bar{z}$ & $\begin{array}{r}174,081 \\
17,568 \\
11,085,398 \\
16,317,287\end{array}$ & $\begin{array}{r}170,917 \\
79,670 \\
11,084,814 \\
16,316,538\end{array}$ & $\begin{array}{l}2,200 \\
- \\
=\end{array}$ \\
\hline $\begin{array}{c}\text { Cltizens Utillties Company ......................... } \\
\text { Virginla }\end{array}$ & - & - & - & - & 72,249 & 42,916 & $-103,446$ \\
\hline $\begin{array}{l}\text { Virginia Electric \& Power Co .................. } \\
\text { Weahington }\end{array}$ & - & 156,484 & 158,348 & - & 190,578 & 189,660 & - \\
\hline $\begin{array}{l}\text { Puget Sound Power \& Light Co ........... } \\
\text { Weshington Water Power Company .... } \\
\text { Weat Virginla }\end{array}$ & $\overline{-}$ & $-1,600$ & $-{ }_{1,617}$ & $\overline{-}$ & $\begin{array}{l}1,214,161 \\
1,301,672\end{array}$ & $\begin{array}{l}1,256,997 \\
1,229,952\end{array}$ & $\begin{array}{r}-1,829,752 \\
1,132,594\end{array}$ \\
\hline $\begin{array}{l}\text { Monongahela Power Company ............. } \\
\text { weconaln }\end{array}$ & - & - & - & - & 40,355 & 27,765 & 264,114 \\
\hline North Central Power Co Inc ................... & - & - & - & - & 2,617 & 2,617 & - \\
\hline States Power Company & $\overline{-}$ & 13,566 & $\overline{-}$ & $\overline{-}$ & $11,490,745$ & $7,059,253$ & - \\
\hline $\begin{array}{l}\text { n Power \& Ligh } \\
\text { in Public Servic }\end{array}$ & $\overline{-}$ & $\bar{z}$ & $\overline{-}$ & $\overline{-}$ & $\begin{array}{r}31 \\
767\end{array}$ & $\begin{array}{l}532 \\
767\end{array}$ & $\overline{-}$ \\
\hline ublic Service Cc & & & & & 168 & $16 r$ & - \\
\hline
\end{tabular}

Includes transactions with power pools, utilities in Canada and Mexico, and nonutilities.

- Not Applicable

Notes: - The terms of settlement may include transferring and/or exchanging quantities of electricity with no dollar value assigned. -For identification purposes, the utilities are listed in the State in which the corporate office is located. - Totals may not equal sum of components because of independent rounding.

Sources: •Federal Energy Regulatory Commission, FERC Form 1, "Annual Report of Major Electric Utilities, Licensees and Others." •FERC Form 1.F. "Annual Report of Nonmajor Public Utilities and Licensees." 
Table 22. Electriclty Purchases by Invostor-Owned Utilitios Not Roported to the Fodoral Enorgy Regulatory Commiselon, by state, 1992

\begin{tabular}{|c|c|c|c|c|c|c|c|}
\hline \multirow{2}{*}{ Seate I' } & \multicolumn{7}{|c|}{$\begin{array}{l}\text { Purchases } \\
\text { (Thousand Kllowatthours) }\end{array}$} \\
\hline & $\begin{array}{l}\text { Invertor- } \\
\text { Owned }\end{array}$ & Federal & $\begin{array}{l}\text { State and } \\
\text { Other } \\
\text { Government }\end{array}$ & Municipal & Cooperative & Other' & Total \\
\hline 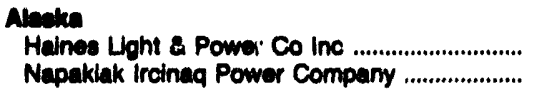 & -570 & $\overline{-}$ & $\overline{-}$ & $\overline{-}$ & $\overline{-}$ & $--^{1,056}$ & $\begin{array}{r}1.056 \\
570\end{array}$ \\
\hline $\begin{array}{l}\text { Arbon } \\
\text { Morenct Water \& Electric Co ................................. }\end{array}$ & - & - & - & - & 25,948 & - & 25,848 \\
\hline 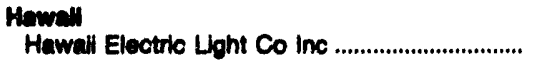 & - & - & - & - & - & 181,033 & 181,033 \\
\hline $\begin{array}{l}\text { Idaho } \\
\text { Adlants Power Company Inc .................................. }\end{array}$ & - & - & - & - & - & 175 & 175 \\
\hline $\begin{array}{l}\text { Phodo leland } \\
\text { Block Ialand Power Company .............................. }\end{array}$ & - & - & - & - & - & 6 & 6 \\
\hline $\begin{array}{l}\text { Utah } \\
\text { Panace Power \& Light Company ....................... }\end{array}$ & - & - & 5,728 & - & - & - & $5,72 B$ \\
\hline
\end{tabular}


Table 23. Electricity Wheelling by Investor-Owned Utilities, by State, 1992

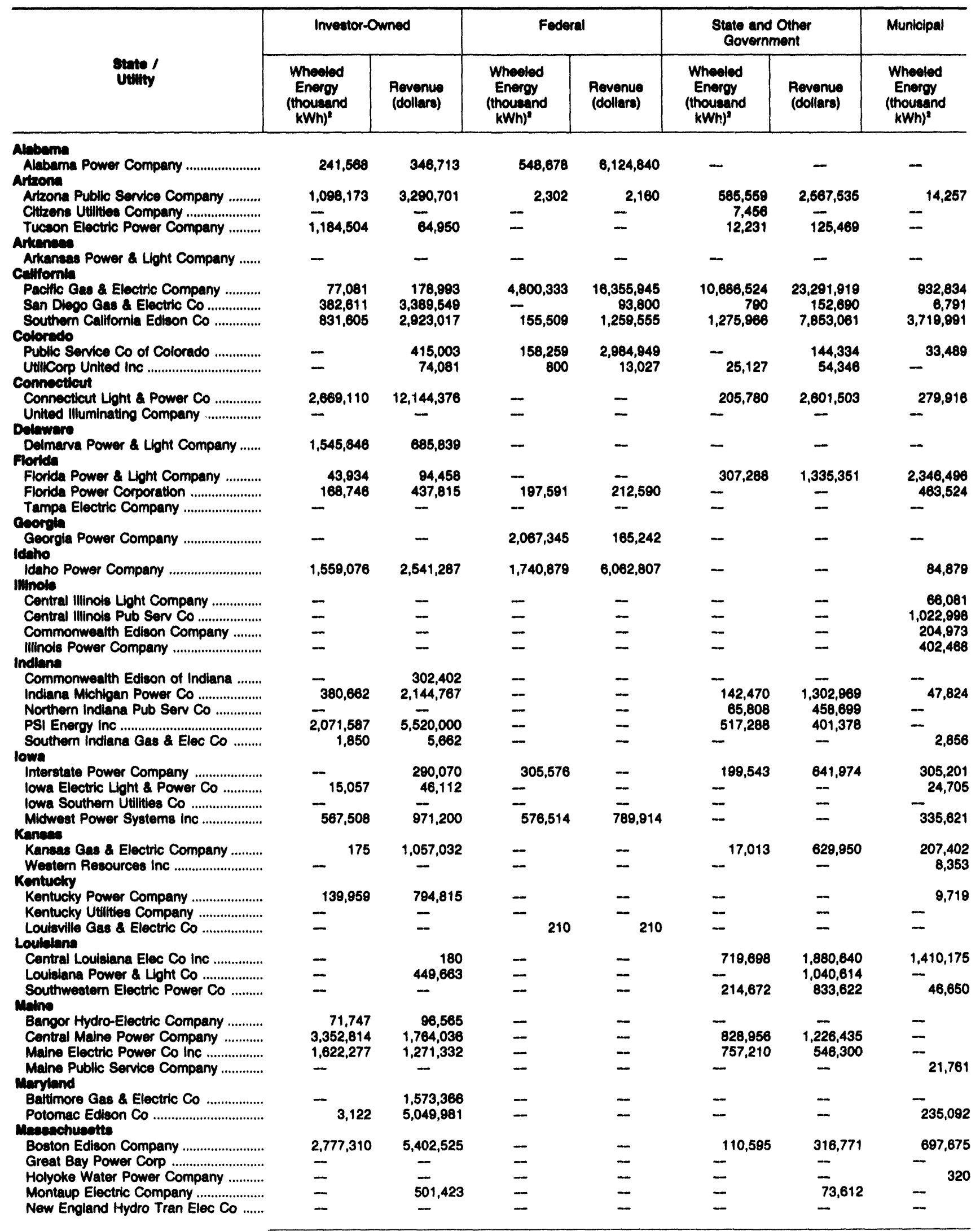

See notes and footnotes at end of table. 
Table 23. Electrlcity Wheeling by Investor-Owned Utilitios, by State, 1992 (Continued)

\begin{tabular}{|c|c|c|c|c|c|c|c|}
\hline \multirow[b]{2}{*}{$\begin{array}{l}\text { State } / \\
\text { Utility }\end{array}$} & \multirow{2}{*}{$\begin{array}{c}\text { Municipal } \\
\begin{array}{c}\text { Revenue } \\
\text { (dollars) }\end{array}\end{array}$} & \multicolumn{2}{|c|}{ Cooperative } & \multicolumn{2}{|l|}{ Other' } & \multicolumn{2}{|l|}{ Total } \\
\hline & & $\begin{array}{c}\text { Whealed } \\
\text { Energy } \\
\text { (thousand } \\
k W h)^{2}\end{array}$ & $\begin{array}{l}\text { Revenue } \\
\text { (dollars) }\end{array}$ & $\begin{array}{l}\text { Wheeled } \\
\text { Energy } \\
\text { (thousand } \\
\text { kWh) }\end{array}$ & $\begin{array}{l}\text { Revenue } \\
\text { (dollars) }\end{array}$ & $\begin{array}{l}\text { Whoeled } \\
\text { Energy } \\
\text { (thousand } \\
\text { kWh })^{2}\end{array}$ & $\begin{array}{l}\text { Revenue } \\
\text { (dollars) }\end{array}$ \\
\hline \multicolumn{8}{|l|}{ Alabama } \\
\hline $\begin{array}{l}\text { Alabama Power Company ...................... } \\
\text { Artzona }\end{array}$ & - & $1,509,283$ & $6,369,698$ & - & - & $2,299,529$ & $12,841,251$ \\
\hline 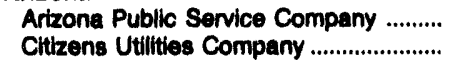 & 108,475 & 185,304 & $1,187,863$ & $-{ }^{90,859}$ & 521,286 & $\begin{array}{r}1,956,454 \\
7,456\end{array}$ & $7,658,120$ \\
\hline $\begin{array}{l}\text { Tucson Electric Power Company .......... } \\
\text { Arkanaad }\end{array}$ & - & $1,180,695$ & 923,520 & - & - & $2,377,430$ & $1,113,939$ \\
\hline $\begin{array}{l}\text { Arkansas Power \& Light Company ...... } \\
\text { Celffornla }\end{array}$ & - & - & 27,676 & - & $14,820,480$ & - & $14,848,166$ \\
\hline Pacific Gas \& Electric Company ............ & $\begin{array}{r}10,894,958 \\
11,495\end{array}$ & $\overline{-}$ & $\overline{151836}$ & $-51,346$ & $\underline{5,781,247}$ & $\begin{array}{r}16,548,118 \\
380,192\end{array}$ & $\begin{array}{r}66,503,062 \\
3,647,534\end{array}$ \\
\hline $\begin{array}{l}\text { Southern California Edison Co ............... } \\
\text { Colorado }\end{array}$ & $16,884,235$ & - & 154,836 & 909,986 & - & $6,893,057$ & $29,074,704$ \\
\hline 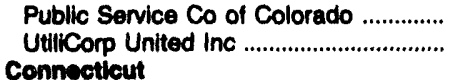 & 86,183 & $\begin{array}{r}443,405 \\
51,851\end{array}$ & $\begin{array}{l}136,607 \\
163,958\end{array}$ & $\begin{array}{r}257,355 \\
11,452\end{array}$ & $\begin{array}{r}641,222 \\
45,229\end{array}$ & $\begin{array}{r}892,508 \\
89,330\end{array}$ & $\begin{array}{r}4,408,298 \\
350,641\end{array}$ \\
\hline \\
\hline $\begin{array}{l}\text { Connecticut Light \& Power Co ............... } \\
\text { United llluminating Company ................. } \\
\text { Delaware }\end{array}$ & - & - & $-23,181$ & $-28,155$ & $\begin{array}{r}3,769,405 \\
519,096\end{array}$ & $\begin{array}{r}3,154,997 \\
28,155\end{array}$ & $\begin{array}{r}19,260,323 \\
519,098\end{array}$ \\
\hline $\begin{array}{l}\text { Delmarva Power \& Light Company ...... } \\
\text { Florlda }\end{array}$ & - & - & - & - & - & $1,545,646$ & 685,839 \\
\hline $\begin{array}{l}\text { Florida Power \& Light Company ........... } \\
\text { Florida Power Corporation ...................... } \\
\text { Tampa Electric Company .................... } \\
\text { Ceorgla }\end{array}$ & $\begin{array}{l}7,416,240 \\
3,685,046 \\
-\end{array}$ & $-16,013$ & $10, \overline{154,927}$ & $\begin{array}{r}3,789,013 \\
513,052 \\
59,012\end{array}$ & $\begin{array}{r}14,206,401 \\
1,832,948 \\
206,883\end{array}$ & $\begin{array}{r}6,486,731 \\
1,358,926 \\
59,012\end{array}$ & $\begin{array}{r}23,052,450 \\
16,303,326 \\
208,883\end{array}$ \\
\hline 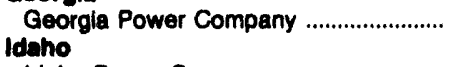 & - & - & - & - & - & $2,067,345$ & 165,242 \\
\hline $\begin{array}{l}\text { Idaho Power Company ............................... } \\
\text { Iilinols }\end{array}$ & 742,839 & - & - & $\mathbf{9 , 3 4 4}$ & 15,137 & $3,394,178$ & $9,362,070$ \\
\hline Central Illinois Light Company ................ & 226,025 & 21,288 & 58,623 & - & - & 87,369 & 284,648 \\
\hline Central Illinois Pub Sen Co ................... & $4,700,745$ & 956,984 & $7,196,629$ & - & - & $1,979,982$ & $11,897,374$ \\
\hline Commonwealth Edison Company ......... & $1,068,348$ & - & - & - & - & 204,973 & $1,068,348$ \\
\hline $\begin{array}{l}\text { Illinois Power Company .................................. } \\
\text { Indiana }\end{array}$ & $1,191,117$ & - & - & - & - & 402,468 & $1,191,117$ \\
\hline Commonwealth Edison of Indiana ........ & - & - & - & - & - & - & 302,402 \\
\hline Indiana Michigan Power Co .................... & 213,678 & 739,746 & $6,278,097$ & $-52,101$ & - & $1,258,601$ & $9,939,511$ \\
\hline Northern Indiana Pub Serv Co ............... & - & 886,192 & $8,160,785$ & - & - & 952,000 & $8,619,484$ \\
\hline 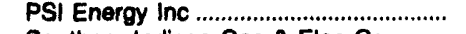 & - & $2,539,833$ & $3,864,130$ & - & - & $5,128,808$ & $9,785,508$ \\
\hline $\begin{array}{l}\text { Southern Indiana Gas \& Elec Co ........ } \\
\text { lowa }\end{array}$ & 428 & 32,173 & 64,559 & - & - & 36,879 & 70,649 \\
\hline Interstate Power Company ...................... & $1,239,380$ & 482,435 & $1,203,631$ & - & - & $1,292,755$ & $3,375,055$ \\
\hline lowa Electric Light \& Power Co ............ & 23,449 & - & - & - & - & 39,762 & 69,581 \\
\hline lowa Southern Utilities Co .......................... & - & - & - & 204,841 & 101,324 & 204,941 & 101,324 \\
\hline $\begin{array}{l}\text { Midwest Power Systems Inc ................... } \\
\text { Kanaas }\end{array}$ & 162,865 & 101,853 & 70,154 & 97,840 & 7,650 & $1,679,336$ & $2,001,783$ \\
\hline Kansas Gas \& Eloctric Company .......... & 625,525 & 878 & $2,892,107$ & - & - & 225,466 & $5,204,614$ \\
\hline $\begin{array}{l}\text { Western Resources Inc ................................ } \\
\text { Kentucky }\end{array}$ & 46,585 & 240,824 & $2,264,238$ & - & $\rightarrow$ & 249,277 & $2,310,823$ \\
\hline Kentucky Power Company ......................... & 46,004 & 39,460 & 59,192 & - & - & 189,138 & 800,011 \\
\hline Kentucky Utillities Company ..................... & - & 893,952 & 897,378 & - & - & 893,952 & 897,378 \\
\hline Louisville Gas \& Electric Co ....................... & - & 165,160 & 165,160 & - & - & 165,370 & 165,370 \\
\hline Loulclana & & & & & & & \\
\hline Central Louisiana Elec Co Inc ................. & $3,765,730$ & $1,009,165$ & $5,106,265$ & - & - & $3,139,038$ & $10,752,815$ \\
\hline Louisiana Power \& Light Co ................... & - & - & $5,564,522$ & - & - & - & $7,054,799$ \\
\hline $\begin{array}{l}\text { Southwestern Electric Power Co .......... } \\
\text { Maine }\end{array}$ & 165,682 & 364,107 & $1,313,179$ & - & - & 625,429 & $2,312,483$ \\
\hline Bangor Hydro-Electric Company ............ & - & - & - & - & - & 71,747 & 96,565 \\
\hline Central Maine Power Company ............ & - & - & - & 389,589 & 955,715 & $4,571,359$ & $3,946,186$ \\
\hline Maine Electric Power Co inc ................. & - & - & - & 47,409 & $1,334,427$ & $2,426,896$ & $3,152,050$ \\
\hline $\begin{array}{l}\text { Maine Public Service Company ............. } \\
\text { Maryland }\end{array}$ & 58,951 & - & $\sim$ & 247,495 & $1,089,891$ & 269,256 & $1,148,642$ \\
\hline Baltimore Gas \& Electric Co ..................... & - & - & - & - & $1,425,132$ & - & $2,998,498$ \\
\hline $\begin{array}{l}\text { Potomac Edison Co ..................................... } \\
\text { Masasechuestts }\end{array}$ & 930,515 & - & - & - & - & 238,214 & $5,880,496$ \\
\hline 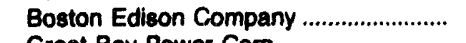 & $1,689,980$ & - & - & $1,211,723$ & $3,002,922$ & $4,797,303$ & $10,412,188$ \\
\hline Great Bay Power Corp .............................. & - & - & - & - & 74,258 & & 74,256 \\
\hline Holyoke Water Power Company ........... & 2,134 & - & - & - & 3,483 & 320 & 5,617 \\
\hline Montaup Electric Company ....................... & 173,048 & - & - & - & 912,439 & - & $1,660,522$ \\
\hline New England Hydro Tran Elec Co ....... & - & - & - & - & $45,025,000$ & - & $45,025,000$ \\
\hline
\end{tabular}

See notes and footnotes at end of table. 
Table 23. Electriclty Wheelling by Investor-Owned Utilities, by State, 1992 (Continued)

\begin{tabular}{|c|c|c|c|c|c|c|c|}
\hline \multirow[b]{2}{*}{$\begin{array}{l}\text { 8tate I } \\
\text { Utillity }\end{array}$} & \multicolumn{2}{|c|}{ Investor-Owned } & \multicolumn{2}{|c|}{ Federal } & \multicolumn{2}{|c|}{$\begin{array}{l}\text { State and Other } \\
\text { Government }\end{array}$} & \multirow{2}{*}{$\begin{array}{l}\text { Municipal } \\
\text { Wheeled } \\
\text { Energy } \\
\text { (thousand } \\
\text { kWh)' }\end{array}$} \\
\hline & $\begin{array}{l}\text { Wheoled } \\
\text { Eneroy } \\
\text { (thouaand } \\
\text { kWh) }\end{array}$ & $\begin{array}{l}\text { Revenue } \\
\text { (dollars) }\end{array}$ & $\begin{array}{l}\text { Whoeled } \\
\text { Energy } \\
\text { (thousand } \\
\text { kWh) }\end{array}$ & $\begin{array}{l}\text { Revenue } \\
\text { (dollars) }\end{array}$ & $\begin{array}{l}\text { Wheeled } \\
\text { Energy } \\
\text { (thousand } \\
\text { kWh)" }\end{array}$ & $\begin{array}{l}\text { Revenue } \\
\text { (dollars) }\end{array}$ & \\
\hline \\
\hline $\begin{array}{l}\text { Now England Power Company ............. } \\
\text { Weatern Massachusetts Elec Co ......... } \\
\text { milohigan }\end{array}$ & $\begin{array}{r}3,873,771 \\
329,304\end{array}$ & $\begin{array}{l}7,527,119 \\
3,526,516\end{array}$ & $\overline{-}$ & $\overline{-}$ & $\begin{array}{r}167,308 \\
28,726\end{array}$ & $\begin{array}{l}948,881 \\
775,606\end{array}$ & $\begin{array}{r}2,967,717 \\
47,603\end{array}$ \\
\hline Consumers Power Company ................... & 1,008 & 3,648 & - & - & 409,515 & 346,273 & 33,571 \\
\hline $\begin{array}{l}\text { Detrot Edieon Company ........................... } \\
\text { Minneeots }\end{array}$ & 1,008 & 4,226 & - & - & - & - & 2,781 \\
\hline Minnesota Power \& Llght Co .................. & - & - & - & - & - & - & 84,204 \\
\hline Northern States Power Company ......... & 318,773 & $1,433,779$ & - & - & 932,348 & $1,931,145$ & 507,855 \\
\hline 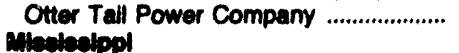 & - & - & - & - & - & - & 6,680 \\
\hline Misalesippl Power \& Lught Co ................ & - & - & 157,315 & - & - & - & 701,854 \\
\hline $\begin{array}{l}\text { Misalsaippl Power Company .................... } \\
\text { Miseour }\end{array}$ & $1,249,284$ & $1,107,516$ & 143,373 & 875,152 & - & - & - \\
\hline Empire District Electric Co & - & - & - & - & - & - & - \\
\hline Kanses City Power \& Light Co ............. & $1,028,336$ & $2,588,597$ & - & - & - & - & 581,259 \\
\hline St Joseph Light \& Power Co ................. & 8,175 & 256,844 & - & - & - & - & - \\
\hline 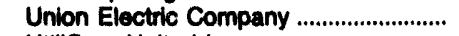 & - & - & - & - & - & - & 309,906 \\
\hline 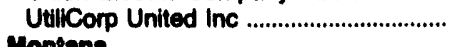 & - & - & - & - & - & - & 40,148 \\
\hline $\begin{array}{l}\text { Montana } \\
\text { Montana Power Company ....................... }\end{array}$ & 733,276 & 69,448 & $1,844,693$ & $2,390,397$ & 46,827 & 179,362 & 32,298 \\
\hline Noveda & & & & & & & \\
\hline Novada Power Company ............................. & 2,343 & 7,029 & - & - & 991,753 & $1,530,766$ & 23,571 \\
\hline $\begin{array}{l}\text { Slerra Pactic Power Company .............. } \\
\text { Now Hampehire }\end{array}$ & - & - & 396,238 & $2,212,124$ & - & - & - \\
\hline New England Elect Trans Corp ............ & -- & - & - & - & - & - & - \\
\hline New England Hydro-Trans Corp ........... & - & - & - & - & - & - & - \\
\hline Public Service Company of NH............. & 361,514 & $2,852,013$ & - & - & 757,210 & 425,311 & 3,613 \\
\hline UNITIL Power Corp ......................................... & 17,162 & 40,887 & - & - & - & - & - \\
\hline Now Jeresy & & & & & & & \\
\hline & - & $\overline{315} 519$ & $\overline{-}$ & - & - & - & 18,252 \\
\hline $\begin{array}{l}\text { Jersey Central Power\&Light Co ............ } \\
\text { Public Sorvice Eloctric\&Gas Co ........... }\end{array}$ & $\begin{array}{r}105,173 \\
2,558,429\end{array}$ & $\begin{array}{r}315,519 \\
4,829,969\end{array}$ & - & $\overline{-}$ & - & - & 25,833 \\
\hline Now Moxico & & & & & & & \\
\hline $\begin{array}{l}\text { Public Service Company of NM ............ } \\
\text { Texas-New Mexico Power Company }\end{array}$ & $1,179,966$ & $5,246,765$ & 738,363 & $2,816,066$ & 243,244 & 783,474 & 587,516 \\
\hline $\begin{array}{l}\text { Texas-Now Mexico Power Company .. } \\
\text { Now York }\end{array}$ & - & 64233 & - & - & - & - & - \\
\hline Contral Hudson Gas \& Elec Corp ......... & 775,660 & 409,257 & - & - & 2,857 & 172,836 & - \\
\hline Consolidated Edison Co-NY Inc ............. & $4,105,08 B$ & $11,696,930$ & - & - & $8,961,066$ & $197,090,383$ & - \\
\hline Long Island Lighting Company ............... & - & - & - & - & 708,787 & $4,324,941$ & - \\
\hline 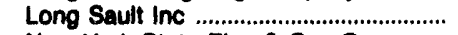 & - & -- & - & - & - & - & - \\
\hline Now York State Elec \& Gas Corp ........ & - & $\overline{-}$ & - & - & 756,270 & $11,165,498$ & - \\
\hline Nlagare Mohawk Power Corp ................. & - & $40,806,639$ & - & - & 80,311 & $19,711,609$ & 10,717 \\
\hline $\begin{array}{l}\text { Orange \& Rockland Utils Inc ................. } \\
\text { North Carollns }\end{array}$ & - & - & - & - & 128.166 & 89,716 & - \\
\hline Carolina Power \& Light Company ......... & 80,677 & 117,203 & 166,075 & $1,426,941$ & 77,203 & $11,040,031$ & - \\
\hline $\begin{array}{l}\text { Duke Power Company ......................................... } \\
\text { North Dakota }\end{array}$ & 854,193 & $3,984,098$ & 414,497 & $2,781,663$ & 657,781 & $2,376,708$ & 502,975 \\
\hline MDU Resources Group Inc ........................ & - & - & 425,314 & 286,234 & - & - & - \\
\hline Ohlo & & & & & & & \\
\hline $\begin{array}{l}\text { Cinuinnati Gas \& Electric Co } \\
\text { Cloveland Eloctric Illum Co }\end{array}$ & 208,928 & 297.034 & $\overline{-}$ & - & - & - & 304,142 \\
\hline $\begin{array}{l}\text { Cleveland Electric Illum Co ....................... } \\
\text { Columbus Southern Power Co .............. }\end{array}$ & $\begin{array}{r}24,191 \\
323,624\end{array}$ & $\begin{array}{r}-10,347 \\
1860966\end{array}$ & $\bar{z}$ & $\overline{-}$ & $\overline{-}$ & - & 407,515 \\
\hline $\begin{array}{l}\text { Columbus Southern Power Co ............. } \\
\text { Dayton Power \& Light Company .......... }\end{array}$ & $-323,624$ & $1,860,968$ & - & $=$ & $\overline{-}$ & $\overline{-}$ & 79,872 \\
\hline & -887789 & $\overline{700}$ & $\overline{-}$ & - & - & - & 483,376 \\
\hline 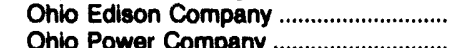 & 887,789 & $1,705,757$ & $\overline{-}$ & $\overline{-}$ & - & - & $1,541,386$ \\
\hline 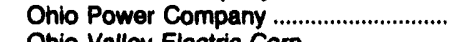 & 557,750 & $3,206,798$ & - & - & - & - & 370,708 \\
\hline 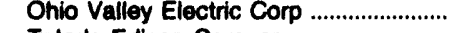 & 103 & 232 & - & - & - & - & \\
\hline 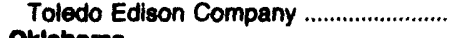 & 4,144 & $-8,713$ & - & - & - & - & 307 \\
\hline $\begin{array}{l}\text { Oklahoma } \\
\text { Public Service Co of Oklahoma }\end{array}$ & - & - & - & - & - & 218.325 & - \\
\hline $\begin{array}{l}\text { Public Service Co of Oklahoma ............ } \\
\text { Oregon }\end{array}$ & - & - & - & - & - & 210,320 & - \\
\hline 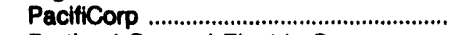 & $1,173,708$ & $2,835,211$ & $6,584,091$ & $5,480,809$ & 506,002 & $4,795,999$ & - \\
\hline $\begin{array}{l}\text { Portland General Electric Co ..................... } \\
\text { Pennaylvania }\end{array}$ & 635 & 315,558 & 216,913 & 550,553 & - & - & - \\
\hline Duquesne Light Company ........................ & - & - & - & - & - & - & - \\
\hline Metropolitan Edison Company ............... & 51,276 & $6,220,433$ & - & - & - & - & 43,938 \\
\hline nia Eloctric Company ............. & $1,748,417$ & $6,430,893$ & - & - & - & - & 601,779 \\
\hline Pennsylvania Power \& Light Co ........... & 862,258 & $2,362,540$ & - & - & - & - & 25,642 \\
\hline insylvania Power Company ............... & 141,531 & 271,654 & - & - & - & - & 9,104 \\
\hline
\end{tabular}

See notes and footnotes at end of table. 
Table 23. Electricity Wheeling by Investor-Owned Utilities, by 8tate, 1992 (Continued)

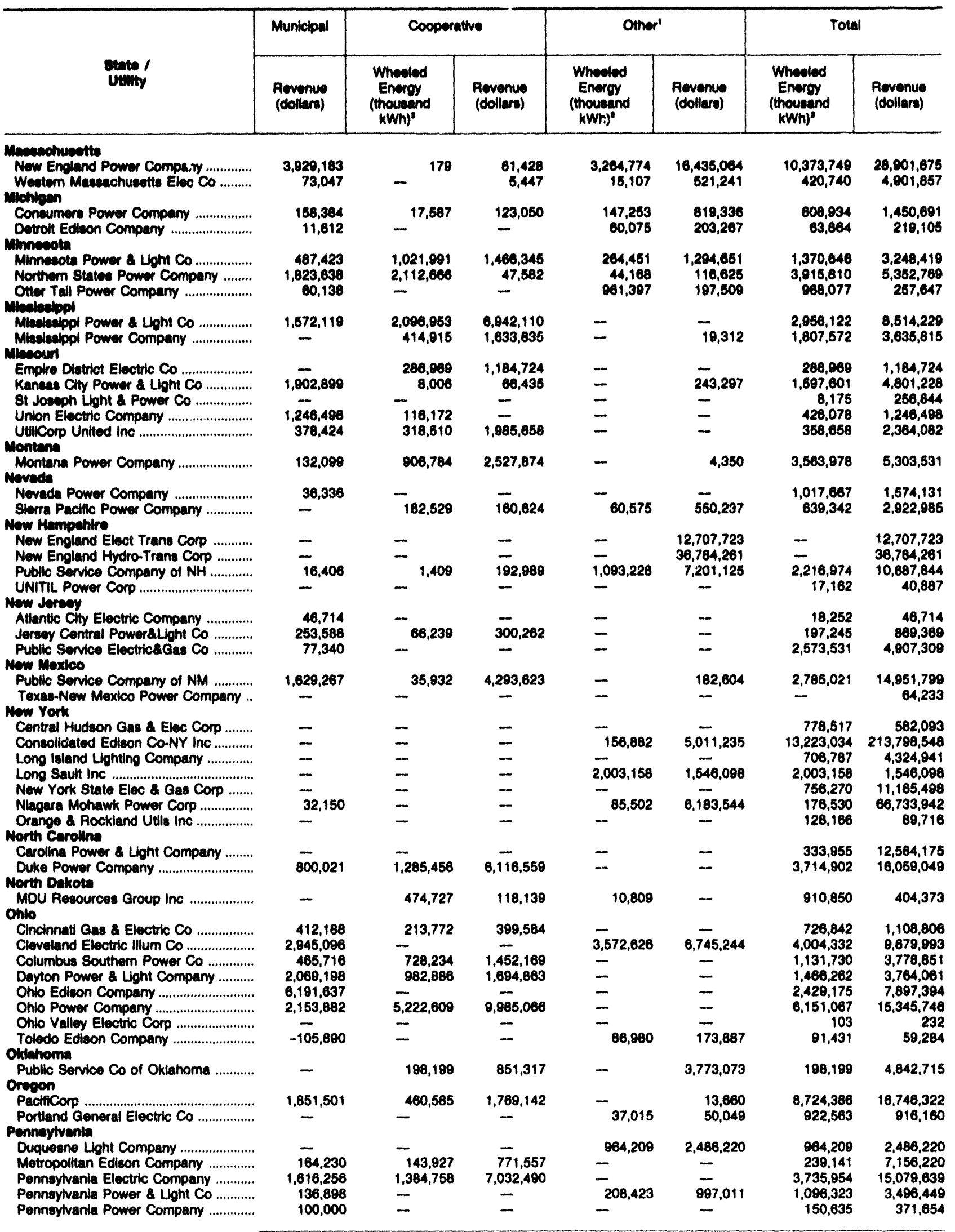

See notes and footnotes at end of table. 
Table 23. Electriclty Wheelling by Investor-Owned UtIIIties, by State, 1992 (Continued)

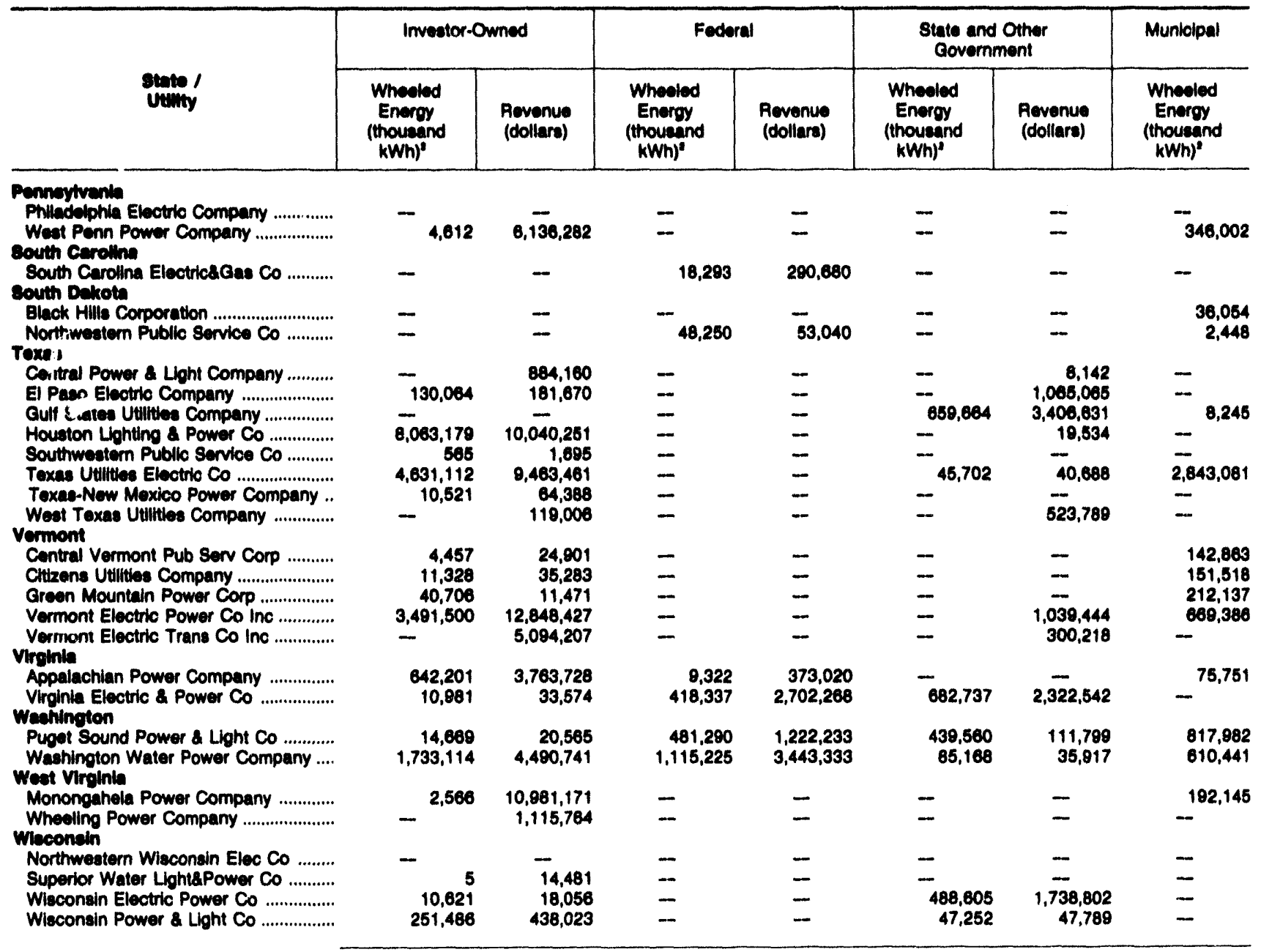

See notes and footnotes at end of table. 
Table 23. Electricity Wheelling by Inveator-Owned Utilitlea, by state, 1992 (Continued)

\begin{tabular}{|c|c|c|c|c|c|c|c|}
\hline \multirow[b]{2}{*}{ suin 1} & \multirow{2}{*}{$\begin{array}{l}\text { Municipal } \\
\begin{array}{l}\text { Revenue } \\
\text { (dollars) }\end{array}\end{array}$} & \multicolumn{2}{|c|}{ Cooperative } & \multicolumn{2}{|l|}{ Other' } & \multicolumn{2}{|l|}{ Total } \\
\hline & & $\begin{array}{l}\text { Whecled } \\
\text { Enerey } \\
\text { (thousend } \\
\text { kWh) }\end{array}$ & $\begin{array}{l}\text { Revenue } \\
\text { (dollars) }\end{array}$ & $\begin{array}{l}\text { Whesled } \\
\text { Energy } \\
\text { (thousend } \\
\text { kWh })^{2}\end{array}$ & $\begin{array}{l}\text { Revenus } \\
\text { (dollare) }\end{array}$ & $\begin{array}{l}\text { Wheeled } \\
\text { Eneroy } \\
\text { (thousand } \\
\text { KWh') }\end{array}$ & $\begin{array}{l}\text { Revenus } \\
\text { (dollars) }\end{array}$ \\
\hline \multicolumn{8}{|l|}{ Pernorituminam } \\
\hline Philadelphia Electric Company .............. & - & - & - & 471,644 & 22,143 & 471,644 & 22,143 \\
\hline $\begin{array}{l}\text { Weet Pern Power Company ................... } \\
\text { south Cerreine }\end{array}$ & $1,369,501$ & - & - & - & - & 350,614 & $7,808,783$ \\
\hline $\begin{array}{l}\text { South Carollne Electricsicas Co ........... } \\
\text { south Devrota }\end{array}$ & - & - & - & - & - & 18,293 & 280,680 \\
\hline $\begin{array}{l}\text { Bleck Hill Corporation ......................... } \\
\text { Northweatern Publlc Senvice Co .......... }\end{array}$ & $\begin{array}{r}41,146 \\
6,292\end{array}$ & $\begin{array}{r}1,163,080 \\
2,105\end{array}$ & $\begin{array}{r}1,062,422 \\
7,014\end{array}$ & $-14,182$ & $\overline{20,411}$ & $\begin{array}{r}1,199,134 \\
68,085\end{array}$ & $\begin{array}{r}1,493,697 \\
96,757\end{array}$ \\
\hline $\begin{array}{l}\text { Toxes } \\
\text { Central Power \& Light Company ........... }\end{array}$ & $1,807,016$ & - & 128,591 & - & 19,980 & - & $2,647,689$ \\
\hline $\begin{array}{l}\text { El Paso Electric Company ..................... } \\
\text { Gult states Uullties Company ............... }\end{array}$ & $\overline{18,021}$ & $\begin{array}{r}2,004 \\
0,163,747\end{array}$ & $\begin{array}{r}2,004 \\
23,972,044\end{array}$ & - & - & $\begin{array}{r}132,068 \\
9,831,686\end{array}$ & $\begin{array}{r}1,248,739 \\
27,399,498\end{array}$ \\
\hline $\begin{array}{l}\text { Houton Lighting \& Power Co ................ } \\
\text { Southwestern Public Service Co .......... }\end{array}$ & 404,685 & & 881,008 & $1,374,690$ & $1,439,603$ & $\begin{array}{r}9,437,869 \\
665\end{array}$ & $\begin{array}{r}12,865,681 \\
1,605\end{array}$ \\
\hline $\begin{array}{l}\text { Toxas Utilties Electric Co ................... } \\
\text { Texas-New Moxico Power Company... } \\
\text { West Toxas Utilties Company .............. } \\
\text { Vemont }\end{array}$ & $\begin{array}{r}2,938,429 \\
342,313\end{array}$ & $\begin{array}{r}2,307,404 \\
7,492 \\
-\quad\end{array}$ & $\begin{array}{r}5,072,911 \\
27,383 \\
47,391\end{array}$ & $\begin{array}{r}176,072 \\
3,096,138 \\
-\end{array}$ & $\begin{array}{r}212,216 \\
6,552,772 \\
3,648,314\end{array}$ & $\begin{array}{r}10,004,271 \\
3,114,151 \\
-\end{array}$ & $\begin{array}{r}17,727,704 \\
6,644,643 \\
4,680,813\end{array}$ \\
\hline Central Vermont Pub Serv Corp ........... & 397,680 & 92,522 & 392,768 & 106,724 & - & 346,586 & 815,327 \\
\hline $\begin{array}{l}\text { Cltzens Utllties Company ...................... } \\
\text { Green Mountain Power Corp ................ }\end{array}$ & $\begin{array}{l}464,381 \\
267,881\end{array}$ & 88,351 & $\overline{108,185}$ & - & $-\overline{294,778}$ & $\begin{array}{l}102,840 \\
341,184\end{array}$ & $\begin{array}{r}499,674 \\
02,859\end{array}$ \\
\hline 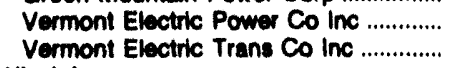 & $\begin{array}{r}1,869,661 \\
47,515\end{array}$ & $-184,049$ & 545,217 & $\overline{6,549,825}$ & $\begin{array}{r}26,504 \\
1,567,970\end{array}$ & $\begin{array}{l}4,344,935 \\
6,548,826\end{array}$ & $\begin{array}{r}16,331,253 \\
7,009,910\end{array}$ \\
\hline Vireinia & & & & & & & \\
\hline $\begin{array}{l}\text { Appalachlan Power Company ................ } \\
\text { Virginia Electric \& Power Co ................ }\end{array}$ & 347,618 & $\overline{3,518,897}$ & $10, \overline{463,881}$ & $\begin{array}{r}21,811 \\
192,597\end{array}$ & $\begin{array}{r}94,802 \\
408,212\end{array}$ & $\begin{array}{r}749,085 \\
4,824,549\end{array}$ & $\begin{array}{r}4,579,168 \\
15,828,577\end{array}$ \\
\hline $\begin{array}{l}\text { Weahingten } \\
\text { Pugat Sound Power \& Light Co ........... } \\
\text { Weahington Water Power Company .... } \\
\text { Wcat Virginia }\end{array}$ & $\begin{array}{l}188,264 \\
307,798\end{array}$ & $\overline{-}$ & - & $\overline{-}$ & $-8,211$ & $\begin{array}{l}1,753,501 \\
3,543,948\end{array}$ & $\begin{array}{l}1,542,851 \\
8,286,000\end{array}$ \\
\hline Monongahela Power Company ............. & 760,494 & 53,350 & 53,521 & - & - & 248,081 & $11,795,186$ \\
\hline $\begin{array}{l}\text { Whoeltng Power Company ....................... } \\
\text { whoond' }\end{array}$ & - & - & - & - & - & - & $1,115,764$ \\
\hline Northweatem Wisconsin Elec Co ......... & - & 13,647 & 52,122 & - & - & 13,647 & 52,122 \\
\hline Superior Water LightsPower Co ........... & - & 10 & 30,321 & - & - & 15 & 44,802 \\
\hline Wheconsin Electric Power Co ................ & - & - & - & - & - & 499,226 & $1,758,858$ \\
\hline Whoconain Power \& Light Co ................. & - & - & - & - & - & 298,738 & 485,812 \\
\hline
\end{tabular}

Includes traneactions with power pools, utilties in Canada and Mexico, and nonutilities.

- Value presented is the greater of the recelved or delivered quantity.

- Nol Applicable

Notes: "Wheeling (tranamiseion services) b reported in account 458, "Transmission For Others," and in account 585, "Tranamiasion By Others," of the FERC Uniform System of Accounts. -For identification purposes, the utilities are listed in the State in which the corporate office is located. -To. tals may not equal aum of components because of independent rounding.

Sources: -Federal Eneroy Regulatory Commiseion, FERC Form 1. "Annual Report of Major Electric Utilities, Licensees and Others." •FERC

Form 1.F, "Annual Report of Nonmajor Public Utilities and Licensees." 
Table 24. Electriolity Wheolling to Investor-Owned Utillties, by 8tate, 1992

\begin{tabular}{|c|c|c|c|c|c|c|c|}
\hline \multirow[b]{2}{*}{ Eume ' } & \multicolumn{2}{|c|}{ Inventor-Owned } & \multicolumn{2}{|c|}{ Federal } & \multicolumn{2}{|c|}{$\begin{array}{l}\text { State and Other } \\
\text { Government }\end{array}$} & \multirow{2}{*}{$\begin{array}{l}\text { Munlolpal } \\
\text { Wheeled } \\
\text { Energy } \\
\text { (thoueand } \\
\text { kWh)! }\end{array}$} \\
\hline & $\begin{array}{l}\text { Whooled } \\
\text { Enerey } \\
\text { (thoungnd } \\
\text { kWh })^{2}\end{array}$ & $\begin{array}{c}\text { Coot } \\
\text { (dollars) }\end{array}$ & $\begin{array}{l}\text { Whecled } \\
\text { Eneroy } \\
\text { (thouagnd } \\
\text { kWh') }\end{array}$ & $\begin{array}{c}\text { Cont } \\
\text { (dollars) }\end{array}$ & $\begin{array}{l}\text { Whecled } \\
\text { Eneroy } \\
\text { (thousand } \\
\text { kWh't }\end{array}$ & $\begin{array}{c}\text { Coot } \\
\text { (dollars) }\end{array}$ & \\
\hline \multicolumn{8}{|l|}{ Artaen } \\
\hline $\begin{array}{l}\text { Avteona Public Service Company .......... } \\
\text { Century Power Corp }\end{array}$ & 30,001 & 87,260 & $2,431,684$ & $2,448,332$ & $1,040,403$ & 787,356 & - \\
\hline Contury Power Corp & - & - & & - & 32,314 & 120,740 & - \\
\hline 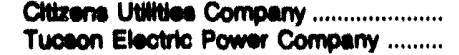 & $\overline{1,834,976}$ & $1, \overline{002,217}$ & $\begin{array}{l}071,638 \\
400,334\end{array}$ & $\begin{array}{r}1,891,029 \\
429,438\end{array}$ & $-72,800$ & $\overline{-}$ & $\overline{-}$ \\
\hline Artenemes & & & & & & & \\
\hline $\begin{array}{l}\text { Attenase Power L Light Company ....... } \\
\text { Entergy Power inc }\end{array}$ & - & 10,701 & - & 49,873 & - & - & - \\
\hline 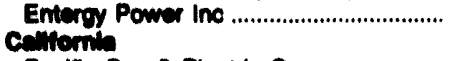 & 27,620 & 867,780 & 123,778 & $1,398,534$ & - & - & - \\
\hline $\begin{array}{l}\text { Pactilo aes a Eleotric Company ........... } \\
\text { San Diego Gas \& Electric Co .............. }\end{array}$ & $\begin{array}{l}189,681 \\
300,655\end{array}$ & $1,277,160$ & $\overline{-}$ & $\begin{array}{r}364,244 \\
03,304\end{array}$ & $-\overline{668,604}$ & $\begin{array}{l}191,123 \\
330,282\end{array}$ & - \\
\hline 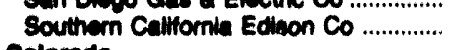 & & 781,270 & - & 397,890 & & 65,000 & 3,759 \\
\hline $\begin{array}{l}\text { Colorido } \\
\text { Publle Service Co of Colorado .............. }\end{array}$ & - & - & 206,094 & 871,057 & 180,608 & 107,673 & -- \\
\hline 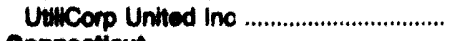 & - & 243,298 & - & - & - & - & - \\
\hline $\begin{array}{l}\text { Conneotiout } \\
\text { Connectiout Loht \& Power Co .............. }\end{array}$ & 73,825 & $23,461.044$ & - & - & -- & - & - \\
\hline Unted Illuminating Company ......................... & 717,056 & $2,175,805$ & - & - & 5,816 & 32,920 & - \\
\hline $\begin{array}{l}\text { Dioturit of Columbin } \\
\text { Potomac Electric Power Company ...... }\end{array}$ & 30775 & $205 \mathrm{Eg1}$ & 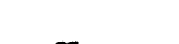 & - & - & 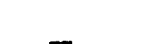 & $\pi$ \\
\hline Plorke & $30,7 / 6$ & 300,001 & - & - & - & - & $\cdots$ \\
\hline $\begin{array}{l}\text { Florida Power \& Light Company ........... } \\
\text { Tamo Electric Company }\end{array}$ & 4,227 & 6,604 & - & - & -- & - & 615,711 \\
\hline $\begin{array}{l}\text { Tarmpa Electric Company .......................... } \\
\text { coorela }\end{array}$ & 20,682 & 14.120 & - & - & - & - & - \\
\hline Georgla Power Company .......................... & - & - & - & - & - & - & - \\
\hline 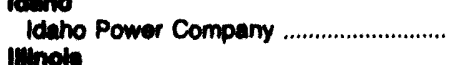 & 232,019 & 377.894 & 759,032 & $1,688,757$ & - & - & 19,217 \\
\hline 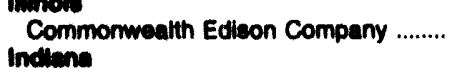 & - & 302,402 & - & - & - & - & - \\
\hline 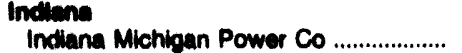 & & - & & & & & \\
\hline Northern Indiana Pub Serv Co ................ & $\overline{-}$ & $\overline{207,612}$ & $\overline{-}$ & $\bar{z}$ & $\overline{-}$ & $\bar{z}$ & $\overline{-}$ \\
\hline 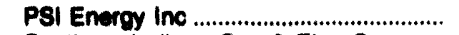 & - & - & - & - & - & $-19,000$ & - \\
\hline Southern indiana Gas \& Eloc Co ......... & - & 7,307 & - & - & - & - & - \\
\hline $\begin{array}{l}\text { lowe } \\
\text { Interstate Power Company ..................... }\end{array}$ & - & 1002211 & & & & & \\
\hline lowa Electric Light \& Power Co ............ & 45,307 & 943,920 & $\overline{-}$ & $\overline{-}$ & $\overline{-}$ & $\overline{-}$ & $\overline{-}$ \\
\hline lowa Southern Utilties Co ........................ & 140,261 & - & - & - & - & - & - \\
\hline Midwest Power Systems Inc .................... & 44,701 & 6,120 & - & - & 88,808 & 88,808 & - \\
\hline 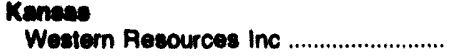 & -- & 1.057 .032 & - & - & - & - & - \\
\hline Kentucky & & & & & & & \\
\hline Kentucky Power Company ......................... & - & - & - & - & - & -- & - \\
\hline $\begin{array}{l}\text { Kentucky Utillties Company .................... } \\
\text { Louldalena }\end{array}$ & - & - & - & - & - & - & - \\
\hline ana Power \& Light Co ................... & - & - & - & - & - & - & - \\
\hline Now Orleans Publlc Service Inc ............ & - & - & - & - & - & - & - \\
\hline Southweatern Electric Power Co .......... & - & $1,468,368$ & - & - & - & - & - \\
\hline $\begin{array}{l}\text { Malno } \\
\text { Bangor Hydro-Electric Company .......... }\end{array}$ & 756,968 & $1,172,426$ & - & - & - & - & - \\
\hline Central Maine Power Company ........... & 847,176 & 927,079 & - & - & - & - & - \\
\hline $\begin{array}{l}\text { Maine Public Service Company ............. } \\
\text { Merylend }\end{array}$ & 565,828 & $\mathbf{9 6 8 , 8 2 7}$ & - & - & - & - & - \\
\hline 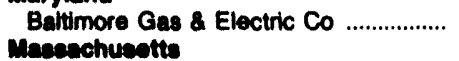 & - & 3,976 & - & - & - & - & - \\
\hline Edison Company .............................. & - & & & & & & \\
\hline 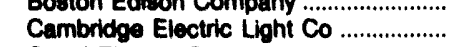 & 885,969 & $\begin{array}{r}296,820 \\
7,466,721\end{array}$ & $\overline{-}$ & $\overline{-}$ & $\overline{-}$ & $\overline{-}$ & $\overline{-}$ \\
\hline Canal Electric Company .............................. & 311,843 & $5,008,300$ & - & - & - & - & - \\
\hline Commonwealth Electric Company ........ & $2,781,758$ & $5,083,641$ & - & - & - & - & -- \\
\hline Fitchburg Gas \& Elec Light Co ............. & 244.183 & 471,504 & - & - & - & - & - \\
\hline 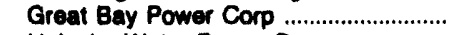 & - & - & - & - & - & -- & - \\
\hline Holyoke Water Power Company ........... & - & 317,110 & - & - & - & - & - \\
\hline Montaup Eloctric Company ..................... & $=-10$ & 339,257 & - & - & - & - & - \\
\hline Now England Power Company .............. & $4,051,647$ & $22,344,933$ & - & - & - & - & - \\
\hline Western Massachusetts Elec Co ......... & 12,837 & $5,219,560$ & - & - & - & - & - \\
\hline $\begin{array}{l}\text { Mlohlgan } \\
\text { Detroit Edison Company ........................... }\end{array}$ & - & - & - & - & $1,287,344$ & $2,874,817$ & - \\
\hline
\end{tabular}

See notes and footnotes at end of table. 
Table 24. Elootrloity Wheollng to Investor-Owned Utilitios, by 8tate, 1992 (Continued)

\begin{tabular}{|c|c|c|c|c|c|c|c|}
\hline \multirow[b]{2}{*}{ vinisy } & \multirow{2}{*}{$\begin{array}{c}\text { Muniolpel } \\
\text { Coet } \\
\text { (dollent) }\end{array}$} & \multicolumn{2}{|c|}{ Cooperative } & \multicolumn{2}{|l|}{ Other' } & \multicolumn{2}{|c|}{ Total } \\
\hline & & $\begin{array}{l}\text { Wholed } \\
\text { Energy } \\
\text { (thousend } \\
\text { KWh })^{*}\end{array}$ & $\begin{array}{c}\text { Coat } \\
\text { (dollare) }\end{array}$ & $\begin{array}{l}\text { Wheeled } \\
\text { Enerey } \\
\text { (thoueand } \\
\text { kWh)" }\end{array}$ & $\begin{array}{c}\text { Coot } \\
\text { (dollara) }\end{array}$ & $\begin{array}{l}\text { Whecled } \\
\text { Eneroy } \\
\text { (thouseand } \\
\text { KWh') }\end{array}$ & $\begin{array}{c}\text { Coot } \\
\text { (dollari) }\end{array}$ \\
\hline \multicolumn{8}{|l|}{ Antens } \\
\hline Atrona Publlo senvice Company .......... & - & - & - & - & 86,020 & $4,402,289$ & $3,388,873$ \\
\hline Century Power Corp ................................. & - & - & - & - & - & 32,314 & 120,740 \\
\hline Citzen Uithice Company ....................... & - & - & - & - & - & 871,688 & $1,881,029$ \\
\hline Tuceon Electric Power Company .......... & - & - & -- & - & - & $2,308,276$ & $2,331,686$ \\
\hline Nhanas Pown L Loh Company ...... & - & 30,730 & 00,001 & - & - & 30,736 & 160,236 \\
\hline Entergy Powes ine ....................................... & - & - & - & 40,040 & 007,070 & 191,486 & $3,264,208$ \\
\hline Gas a Electrio Company ............ & 302,710 & - & - & 142,147 & $4,897,364$ & 326,628 & $0,012,009$ \\
\hline Yopo cas a Electrio Co .................. & - & - & - & - & - & 907,310 & $8,880,800$ \\
\hline diteon Co ................. & 784.078 & - & - & - & - & & $2,010,060$ \\
\hline Io Eervios Co of Colorado ............... & - & - & - & - & - & 306,000 & 078,080 \\
\hline 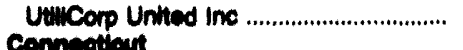 & - & - & - & - & - & - & 243,200 \\
\hline Conneoticut Lipht a Power Co .............. & - & - & - & - & - & 73,825 & $23,401,044$ \\
\hline Unived lluminating Company .................. & - & - & - & 93,000 & $8,008,000$ & 816,771 & $10,214,523$ \\
\hline Potomac Elsotric Power Company ...... & - & - & - & - & - & 30,776 & 305,681 \\
\hline Florida Power \& Lont Company ........... & $2,687,616$ & - & - & 018,686 & $2,483,871$ & $1,236,624$ & $8,177,090$ \\
\hline Tempa Electrio Company ........................ & - & - & - & - & - & 20,682 & 14,120 \\
\hline orita Power Company ........................ & - & - & - & - & - & - & - \\
\hline Power Company .............................. & $\infty 0,571$ & 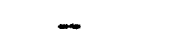 & - & - & - & $1,010,268$ & $2,086,222$ \\
\hline Commonmealth Edison Company ......... & - & - & - & - & - & - & 302,402 \\
\hline in Michigen Power Co .................... & - & - & - & - & $-48,211,231$ & - & $-48,211,231$ \\
\hline Northem Indlana Pub Serv Co ............... & $\overline{2}$ & - & - & - & - & - & 207,012 \\
\hline 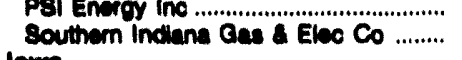 & $\underline{31,601}$ & - & 322,380 & $\overline{-}$ & -765 & - & $\begin{array}{r}336,702 \\
7,307\end{array}$ \\
\hline rotate Power Company ..................... & - & - & 330,174 & - & - & - & $1,338,386$ \\
\hline - Eleotric Light a Powier Co ............ & - & 42,090 & 42,342 & - & - & 88,303 & 000,202 \\
\hline 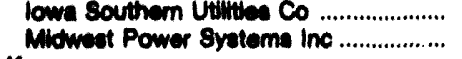 & - & $\begin{array}{r}81,050 \\
101,709\end{array}$ & $\begin{array}{l}101,324 \\
241,382\end{array}$ & $68,076^{2}$ & - & $\begin{array}{l}221,322 \\
303,304\end{array}$ & $\begin{array}{l}101,324 \\
338,380\end{array}$ \\
\hline & & & & & & & \\
\hline $\begin{array}{l}\text { Weotem Rosources Inc ............................... } \\
\text { Kentuciny }\end{array}$ & - & - & - & - & - & - & $1,057,032$ \\
\hline Kentucky Power Company ........................ & - & - & 70,038 & - & $-4,164,213$ & - & $-4,094,175$ \\
\hline $\begin{array}{l}\text { Kentucky Utiltien Company ..................... } \\
\text { Leciletene }\end{array}$ & - & 659,148 & 329,574 & - & - & 689,148 & 329,574 \\
\hline Ulelana Power \& Ught Co .................. & - & - & - & - & $14,079,545$ & - & $14,070,848$ \\
\hline w Orbans Public Senvice inc ............ & - & - & - & - & $6,413,070$ & - & $6,413,679$ \\
\hline Withmestern Electric Power Co .......... & - & - & - & - & $2,796,376$ & - & $4,204,744$ \\
\hline noor Hydro-Electric Company ........... & - & - & - & 388,327 & $1,534,639$ & $1,145,295$ & $2,706,906$ \\
\hline ntral Maine Power Compeny ............ & - & - & - & - & 375,982 & 847,176 & $1,303,081$ \\
\hline Maine Public Service Company .............. & - & - & - & 538,310 & 989,041 & $1,104,147$ & $1,857,868$ \\
\hline 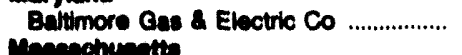 & - & - & - & - & 755,020 & - & 750,605 \\
\hline n Edieon Company .......................... & - & - & - & 529,328 & $3,040,062$ & 529,326 & $3,330,892$ \\
\hline abridge Electric Light Co ................... & - & - & - & 604,418 & 455,468 & $1,400,388$ & $7,822,188$ \\
\hline Cand Electric Company ............................. & - & - & - & 149,012 & 785,136 & 460,058 & $8,791,486$ \\
\hline Commomwealth Electric Company ....... & - & - & - & 151,467 & 141,255 & $2,913,226$ & $5,224,898$ \\
\hline Fitchburg Gas \& Elec Light Co ............... & - & - & - & 247,844 & 501,372 & 492,027 & 972,876 \\
\hline Great Bay Power Corp ............................. & - & - & - & - & $-39,700$ & - & $-39,700$ \\
\hline Holyoke Water Power Company ........... & - & - & - & - & - & - & 317,110 \\
\hline Montaup Electric Company ..................... & - & - & - & - & $1,437,822$ & - & $1,777,079$ \\
\hline Now England Power Company ............... & - & - & $\overline{-}$ & 341,353 & $9,587,342$ & $4,393,000$ & $31,932,278$ \\
\hline $\begin{array}{l}\text { Western Massachusetts Elec Co .......... } \\
\text { ulohlean }\end{array}$ & - & - & 263,733 & - & $-107,428$ & 12,837 & $5,375,865$ \\
\hline Detrot Ediaon Company ............................. & - & - & - & - & - & $1,267,344$ & $2,874,817$ \\
\hline
\end{tabular}

See notes and lootnotes at end of table. 
Table 24. Eloetriclty Wheelling to Investor-Owned Utilitios, by State, 1992 (Continued)

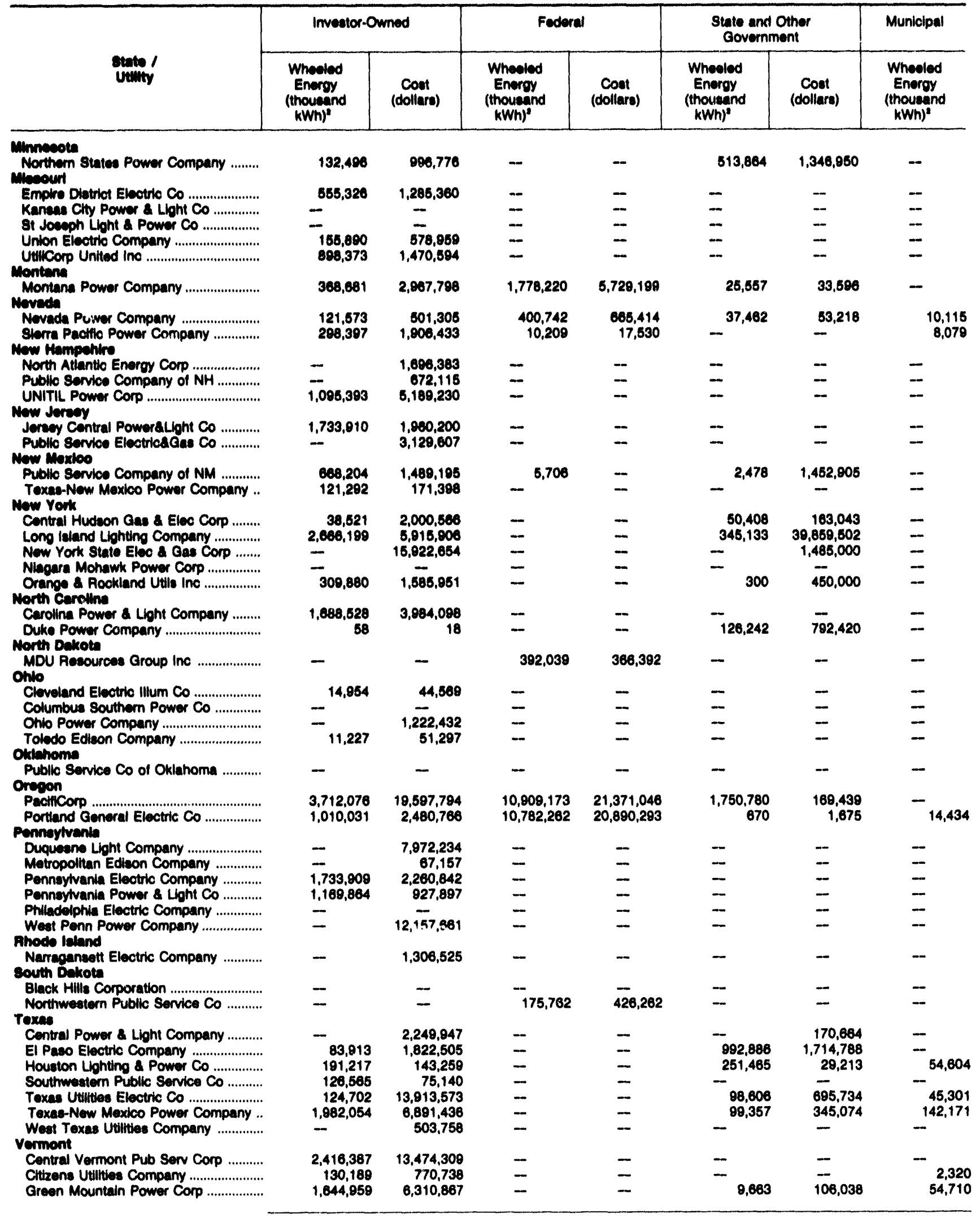

See notes and footnotes at end of table. 
Table 24. Electriclity Whoelling to Investor-Owned Utilities, by State, 1992 (Continued)

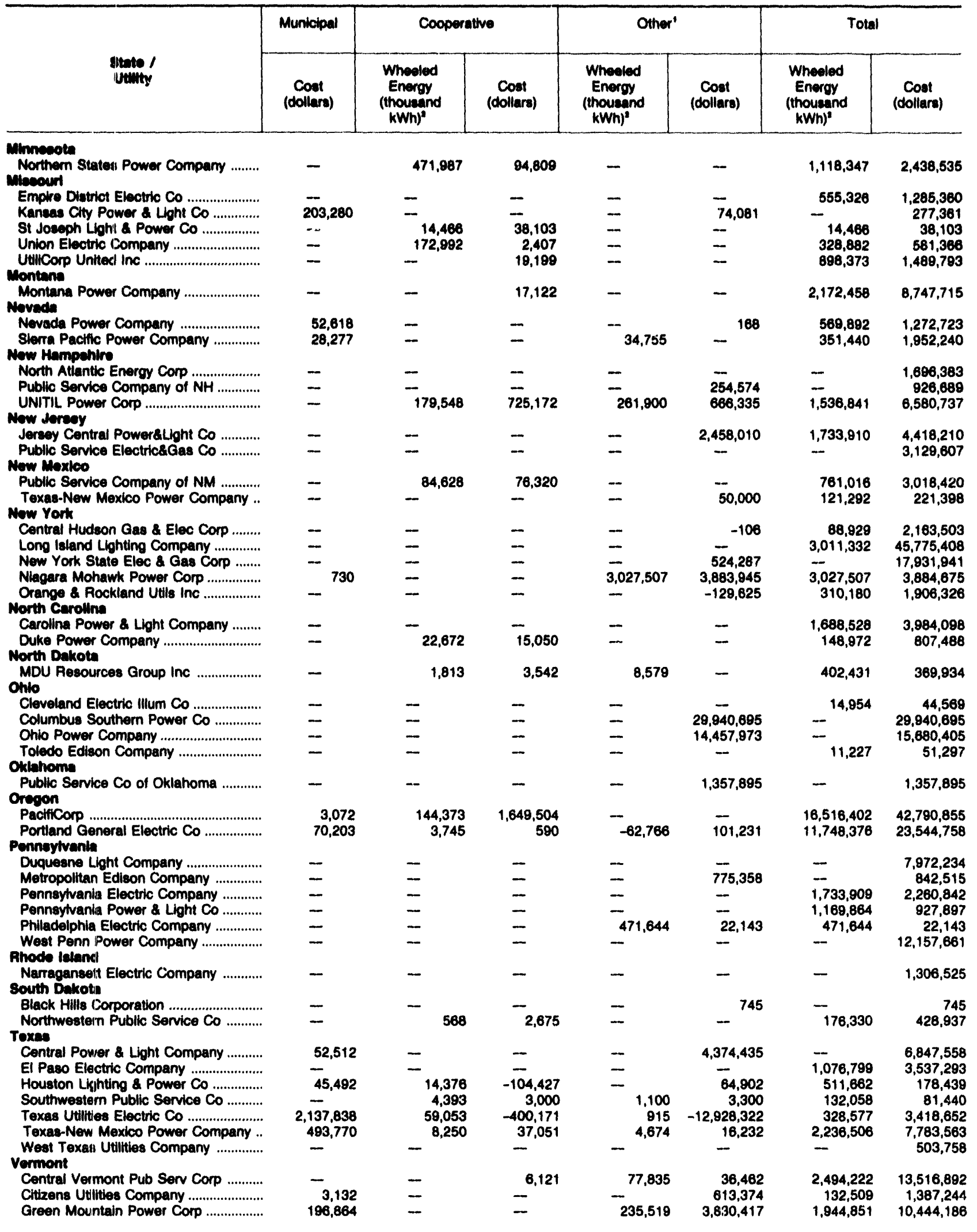

See notes and footnotes at end of table. 
Table 24. Electricity Wheeling to Investor-Owned Utilities, by State, 1992 (Continued)

\begin{tabular}{|c|c|c|c|c|c|c|c|}
\hline \multirow[b]{2}{*}{$\begin{array}{l}\text { State / } \\
\text { Ucinity }\end{array}$} & \multicolumn{2}{|c|}{ Imvestor-Owned } & \multicolumn{2}{|c|}{ Federal } & \multicolumn{2}{|c|}{$\begin{array}{l}\text { State and Other } \\
\text { Government }\end{array}$} & \multirow{2}{*}{$\begin{array}{c}\text { Municipal } \\
\text { Wheoled } \\
\text { Energy } \\
\text { (thousend } \\
\text { kWh }^{2}\end{array}$} \\
\hline & $\begin{array}{l}\text { Wheoled } \\
\text { Energy } \\
\text { (thousand } \\
\text { kWh })^{2}\end{array}$ & $\begin{array}{c}\text { Cost } \\
\text { (dollars) }\end{array}$ & $\begin{array}{l}\text { Whoeled } \\
\text { Energy } \\
\text { (thouseand } \\
\text { kWh })^{2}\end{array}$ & $\begin{array}{c}\text { Cost } \\
\text { (dollars) }\end{array}$ & $\begin{array}{l}\text { Wheoled } \\
\text { Energy } \\
\text { (thousand } \\
\text { (WWh't }\end{array}$ & $\begin{array}{c}\text { Cost } \\
\text { (dollars) }\end{array}$ & \\
\hline \multicolumn{8}{|l|}{ Vinglin } \\
\hline Appelachian Power Company ............... & 一 & - & - & - & - & - & - \\
\hline $\begin{array}{l}\text { Virginia Electric \& Power Co ................. } \\
\text { Wethington }\end{array}$ & - & - & - & - & - & - & $\rightarrow$ \\
\hline $\begin{array}{l}\text { Puget Sound Power \& Light Co ........... } \\
\text { Weshingten Wator Power Company .... } \\
\text { Wheconets }\end{array}$ & $\begin{array}{r}16,85 E \\
185,222\end{array}$ & $\begin{array}{r}1,662,842 \\
89,528\end{array}$ & $\begin{array}{r}1,456,925 \\
745,061\end{array}$ & $\begin{array}{r}25,151,939 \\
8,630,420\end{array}$ & - & $\begin{array}{l}93,859 \\
-\end{array}$ & - \\
\hline Madison Gas a Electric Company ....... & 242,326 & 426,043 & - & - & - & - & $-\infty$ \\
\hline Wisconsin Flectric Power Co ................ & 318,188 & 719,337 & - & - & - & - & - \\
\hline Wisconsin Power \& Light Co ................. & 90,800 & 517,280 & - & - & - & - & - \\
\hline Wisconein Public Service Corp ............. & 19,781 & 39,358 & - & - & - & - & - \\
\hline
\end{tabular}

\begin{tabular}{|c|c|c|c|c|c|c|}
\hline Municipal & \multicolumn{2}{|c|}{ Cooperative } & \multicolumn{2}{|c|}{ Other' } & \multicolumn{2}{|c|}{ Total } \\
\hline $\begin{array}{c}\text { Cost } \\
\text { (dollars) }\end{array}$ & $\begin{array}{c}\text { Wheeled } \\
\text { Energy } \\
\text { (thousand } \\
\mathrm{kWh})^{2}\end{array}$ & $\begin{array}{c}\text { Cost } \\
\text { (dollars) }\end{array}$ & $\begin{array}{c}\text { Wheoled } \\
\text { Energy } \\
(\text { thousand } \\
\mathrm{kWh})^{2}\end{array}$ & $\begin{array}{c}\text { Cost } \\
\text { (dollars) } \\
\text { (thousand } \\
\mathrm{kWh})^{2}\end{array}$ & $\begin{array}{c}\text { Cost } \\
\text { (dollars) }\end{array}$ \\
\hline
\end{tabular}

Vireinia
Appalachian Power Company .............
Virginia Electric \& Power Co ..............

Includes transactions with power pools, utilities in Canada and Mexico, and nonutilities

- Value presented is the groater of the received or delivered quantity.

- Not Applicable

Notes: -Whesling (transmission services) ie reported in account 456, "Transmission For Others," and in account 565, "Transmission By Others," of the FERC Uniform System of Accountc. PFor identification purposes, the utilities are listed in the State in which the corporate office is located. -Totals may not equal sum of components because of independent rounding.

Sources: -Federal Energy Regulatory Commission, FERC Form 1, "Annual Report of Major Electric Utilitios, Licensees and Others." •FERC Form 1-F, "Annual Report of Nonmajor Public Utilities and Licensees." 


\section{Bulk Power Transactions by Federal and Other Publicly Owned Utilities}

\section{Overview}

This chapter presents electricity trade data for publicly owned utilities that report on Form EIA-412, "Annual Report of Public Electric Utilities." These data are separated into three types of public ownership: Federal, State and Other Government Utilities, and Municipalities. Summary totals by calendar year (except for Federal) cannot be provided because filings by utilities were based on different fiscal years and ending months.
Individual transactions by public utilities with other utilities by class of ownership are identified for purchased power and sales for resale data. Form EIA-412 collects only aggregate information (on a sample basis) on exchanges and wheeling in the "Energy Account Balance" schedule. Summary data from Form EIA-861, "Annual Electric Utility Report," are used to provide complete the coverage for the public utilities because it is collected from all electric utilities. However, Form EIA-861 does not identify individual transaction partners.

Figure 8. Electricity Purchases and Costs by Federal Utilitles, Fiscal Year 1992

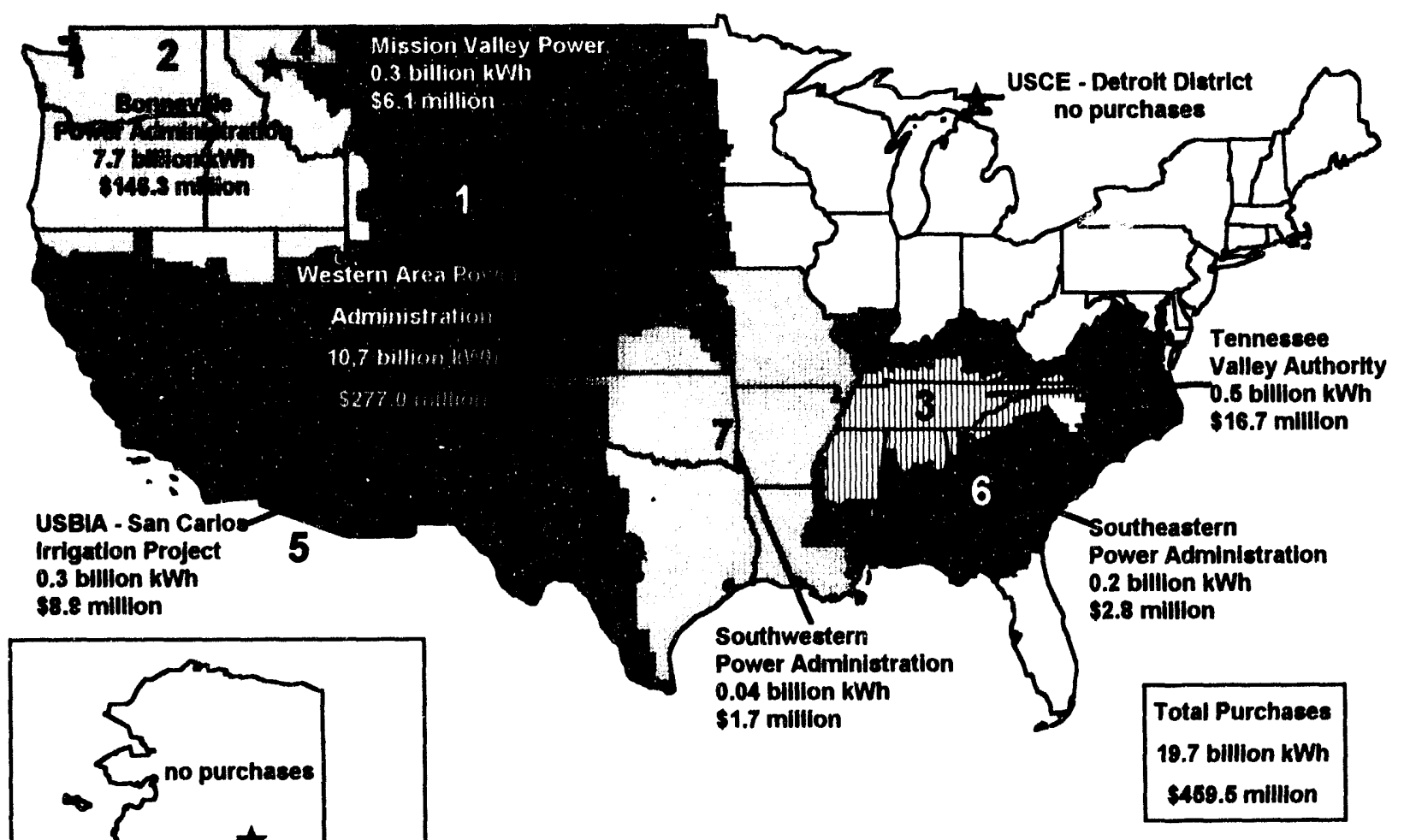

Notes. Numbers indicate rank by kilowatthours (kWh) purchased.

USCE $=$ U.S. Army Corps of Engineers. USBIA $=$ U.S. Bureau of Indian Affairs. Shaded areas show the service territories of the indicated utilities.

Source: Energy Information Administration, Form ElA-412, "Annual Report of Public Electric Utilities." 


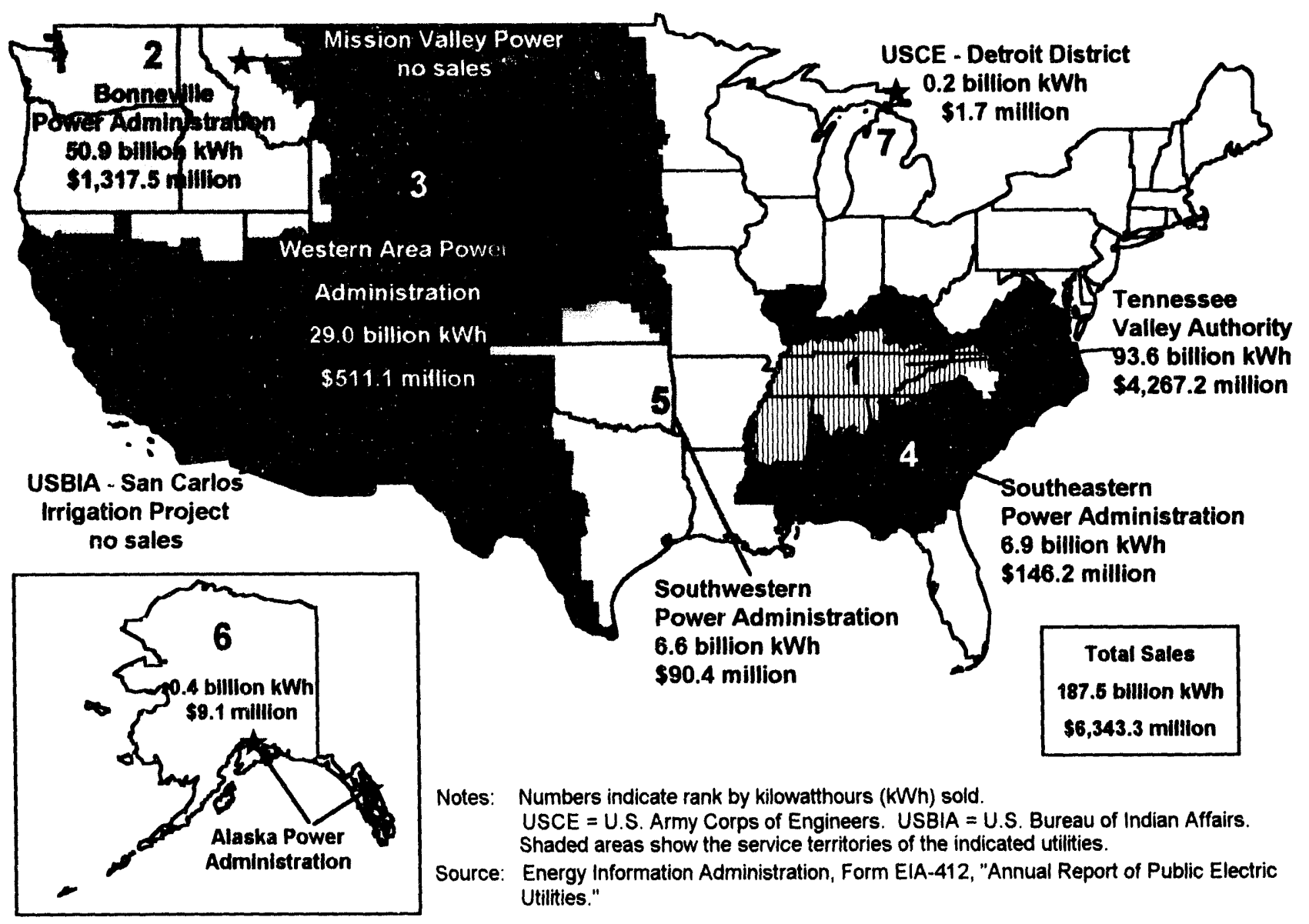

\section{Tables}

This chapter presents cost, revenue, and quantity data on purchased power and sales for resale for the following types of public utilities.

- Federal utilities (Tables 25 and 26)

- State and other government utilities (Tables 27 and 28)

- Municipalities (Tables 29 and 30)

- Electricity purchases by publicly owned utilities not listed elsewhere (Table 31).

Tables 25 through 30 provide information on the total cost and revenue associated with the total quantity of purchased power and sales for resale. Other utilities which do not report on the Form EIA-412 because of their small size are shown in Table 31 . Table 32 provides information on exchanges and wheeling for public electric utilities. In 1990, Form EIA-412 was updated to collect data on the different categories of power and energy and the associated value of electric utility trade. However, the value of the categories were not subdivided into Demand, Energy, and Other for revenues and costs.

The public utilities are listed by State according to the mailing addresses of their administrative offices. The fiscal-year ending date for each public utility is also included in the tables. Federal utilities are listed by major project, office, or administrative reporting level.

\section{Highlights}

In 1992, four Federal power marketing agencies and the Tennessee Valley Authority (which operates primarily in a four-state region centered in Tennessee), reported purchases of 19.1 billion kilowatthours at a combined cost of $\$ 444.6$ million. Power purchases by the Bonneville Power Administration (BPA), which in 1990 accounted for less than 4 percent of total power marketing agency activity, appeared to increase dra- 


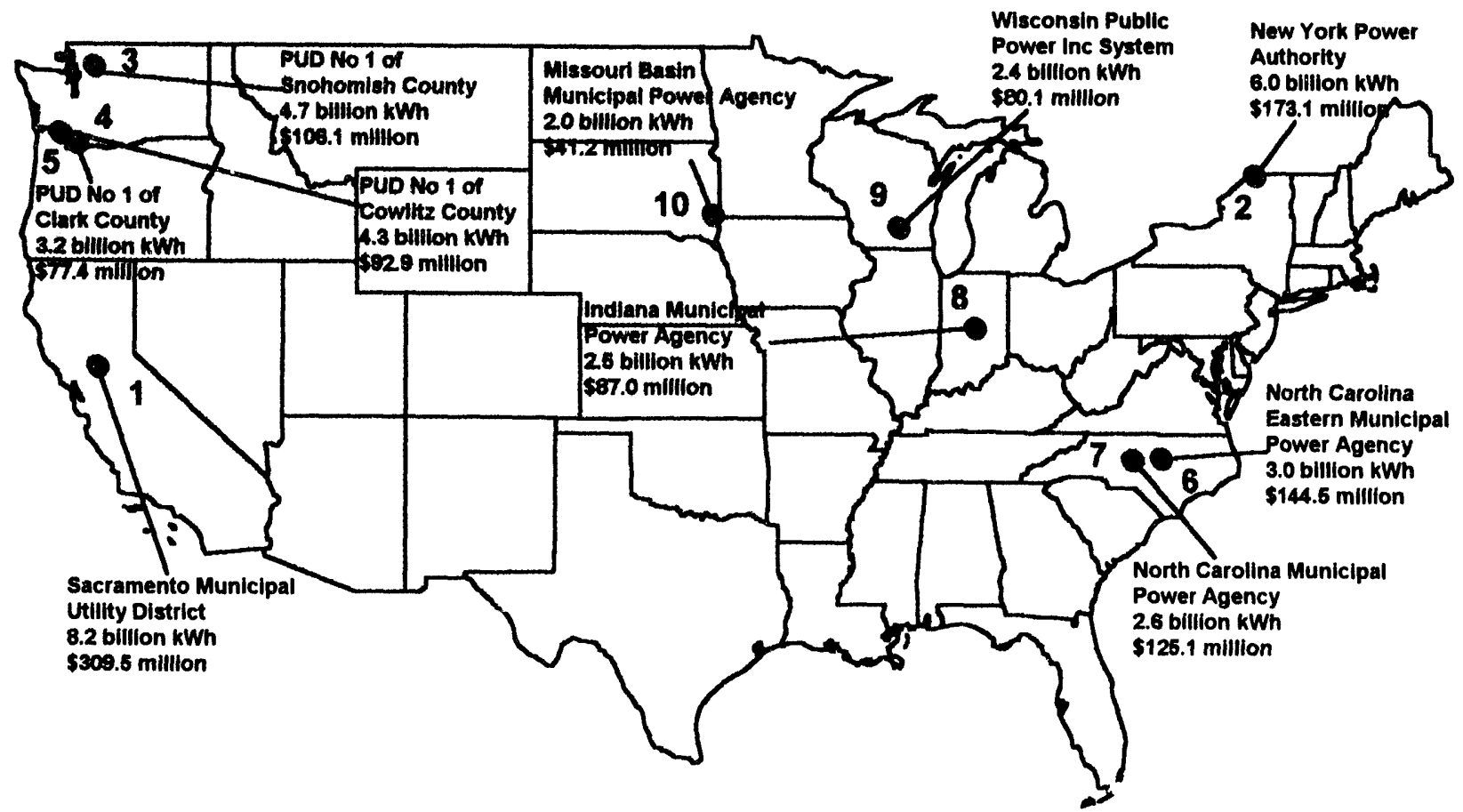

Notes: Numbers indicate rank by kilowatthours $(\mathrm{kWh})$ purchased. PUD $=$ Public Utility District.

Source: Energy Information Administration, Form EIA-412, "Annual Report of Public Electric Utilities."

matically in 1992 to almost 40 percent of total power. This increase was primarily caused by lower water levels in the Pacific Northwest resulting in reduced generation from Bonneville's hydroelectric assets. These conditions forced Bonneville Power to resort to increased purchases of electricity to satisfy its wholesale and retail consumer loads. ${ }^{7}$ The largest Federal purchaser of electricity was the Western Area Power Administration (WAPA) which purchased 10.7 billion kilowatthours for $\$ 277$ million. WAPA purchases amounted to 54.5 percent of the total 19.7 billion kilowatthours purchased by all Federal utilities (including facilities identified by the Bureau of Indian Affairs as operating utilities). The Tennessee Valley Authority (TVA), which provides full requirements support for approximately 110 municipal utilities and 50 cooperative electric utilities, reduced purchases substantially from 1990 to about 507 million kilowat thours for which it paid \$16.7 million (Figure 8).
Federal utilities were much more active as energy sellers (Table 26). By far the largest wholesaler of electric power in the United States, including all ownership classes was (in 1992 as in 1990) the TVA, which sold just over 93.6 billion kilowatthours for revenue of $\$ 4.3$ billion. The second largest seller in 1992 was the Bonneville Power Administration which sold 50.9 billion kilowatthours for revenue of $\$ 1.3$ billion. WAPA was third, selling almost 29 billion kilowatthours for revenue of $\$ 511.1$ million. All other Federal utilities sold substantially less power (Figure 9).

The 10 largest purchases of electricity by State and other government utilities in 1992 ranged from almost 2.0 billion kilowatthours (Missouri Basin Municipal Power District) for a cost of $\$ 41.2$ million, to 8.2 billion kilowatthours (Sacramento Municipal Utility District of California) for $\$ 309.5$ million. The largest (Sacramento) purchaser's quantity was 37 percent greater than the second ranked purchaser, the New York Power Authority (NYPA). NYPA bought almost

\footnotetext{
${ }^{7}$ With the drought conditions present in 1992, BPA had little nonfirm energy available after the Northwest power markets were met. (By law, the BPA is required to serve the Northwest first.) Revenues fell mai...y because BPA's power rates for the aluminum companies (direct service customers) went down after its variable industrial rates dropped to the minimum level for most of the year. These rates are linked to the price of aluminum and allow aluminum companies to stay in production when the market is soft. A worldwide recession cut the demand for aluminum and large amounts of aluminum were exported by former republics of the Soviet Union in order to earn hard currency. Bonneville Power Administration Annual Report 1992 , DOE/BP-2030 (January 1993), pp. 18-19.
} 
Figure 11. Electricity Sales for Resale and Revenue by the Top Ten State and Other Government Utilites, Fiscal Year 1992

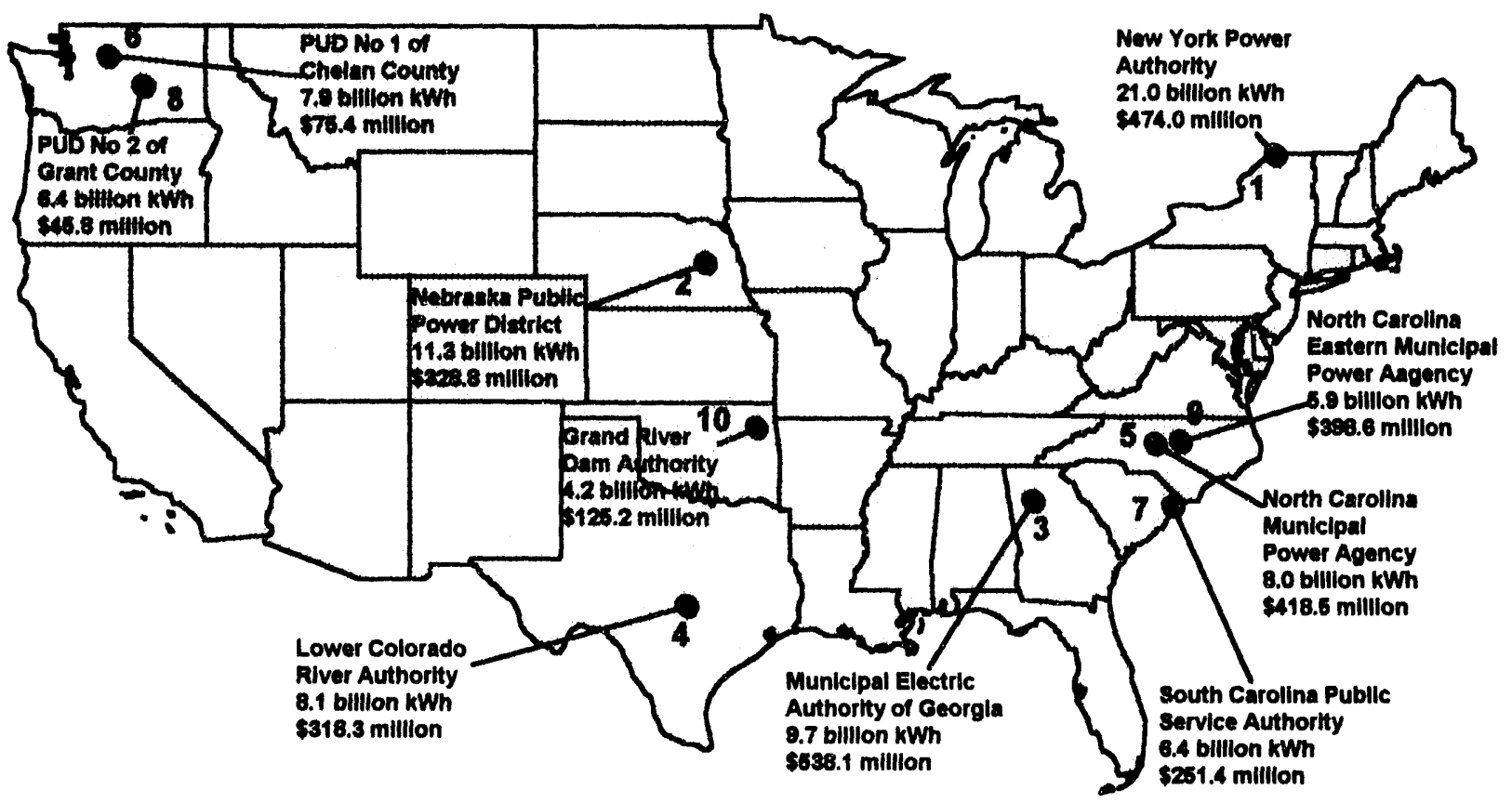

Notes : Numbers indicate rank by kilowatthours $(\mathrm{kWh})$ sold. PUD $=$ Public Utility District.

Source: Energy Information Administration, Form EIA-412, "Annual Report of Public Electric Utilities."

6 billion kilowatthours for $\$ 173.1$ million. Five of the remaining top ten are located in two States (Washington State has three and two are in North Carolina). Each of the top 10 purchasers, regardless of geographic region, paid more than $\$ 41.2$ million for the electric power they purchased in 1992 (Figure 10). The top 10 purchasers among the State and other government utilities generally bought less than the top 10 municipals and the State and other government utility costs were generally less (Tables 27 and 29).

The largest seller of electric power among State and other government utilities was the New York Power Authority (NYPA), which sold nearly 21 billion kilowatthours for $\$ 474$ million. This was over 85 percent more than the sales of the second largest seller, the Nebraska Public Power District, which sold 11.3 billion kilowatthours for revenue of $\$ 328.8$ million. NYPA sales for resale were more than any utility in the Nation with the exceptions of the TVA, BPA, WAPA, and the New England Power Company. The Municipal Electric Authority of Georgia (MEAG), which was second largest seller in 1990, was the third largest among State and other government utilities in 1992, selling 9.7 billion kilowatthours for revenue of $\$ 538.1$ million. Several of the public utilities in the Southeastern States of North Carolina, South Carolina, and Georgia were active as sellers, accounting for 4 of the top 10. Public Utility Districts (PUDs) in the State of Washington accounted for 2 of the remaining top 10 sellers (Figure 11).

Revenue for the sales varied widely. While MEAG sold only $\mathbf{4 6}$ percent as much power as NYPA, nevertheless it received nearly 14 percent more revenue. The lowest total revenue received by any of the top 10 sellers was the PUD No. 2 of Grant County of Washington State, which received $\$ 45.8$ million for 6.4 billion kilowatthours. In contrast, the North Carolina Eastern Municipal Power Agency and the Grand River Dam Authority of Oklahoma, ranking 9 and 10 in power sales, received \$398.6 million and \$125.2 million in revenues, respectively (Table 28 ).

The 10 largest total purchases by municipal utilities ranged from 2.4 billion kilowatthours at a cost of $\$ 43$ million by the City of Eugene, Oregon to 11.8 billion kilowatthours for $\$ 498.8$ million by the City of Memphis, Tennessee (which relies upon the Tennessee $\mathrm{Val}$ ley Authority for its system requirements). The City of Los Angeles, California, which has substantial generation assets of its own, was the second largest purchaser of power among municipal utilities with 11.4 billion kilowatthours at a cost of $\$ 660.3$ million. Of the top 10 purchasers of electricity among municipal utilities (Figure 12), 5 are supported wholly by the Ten- 


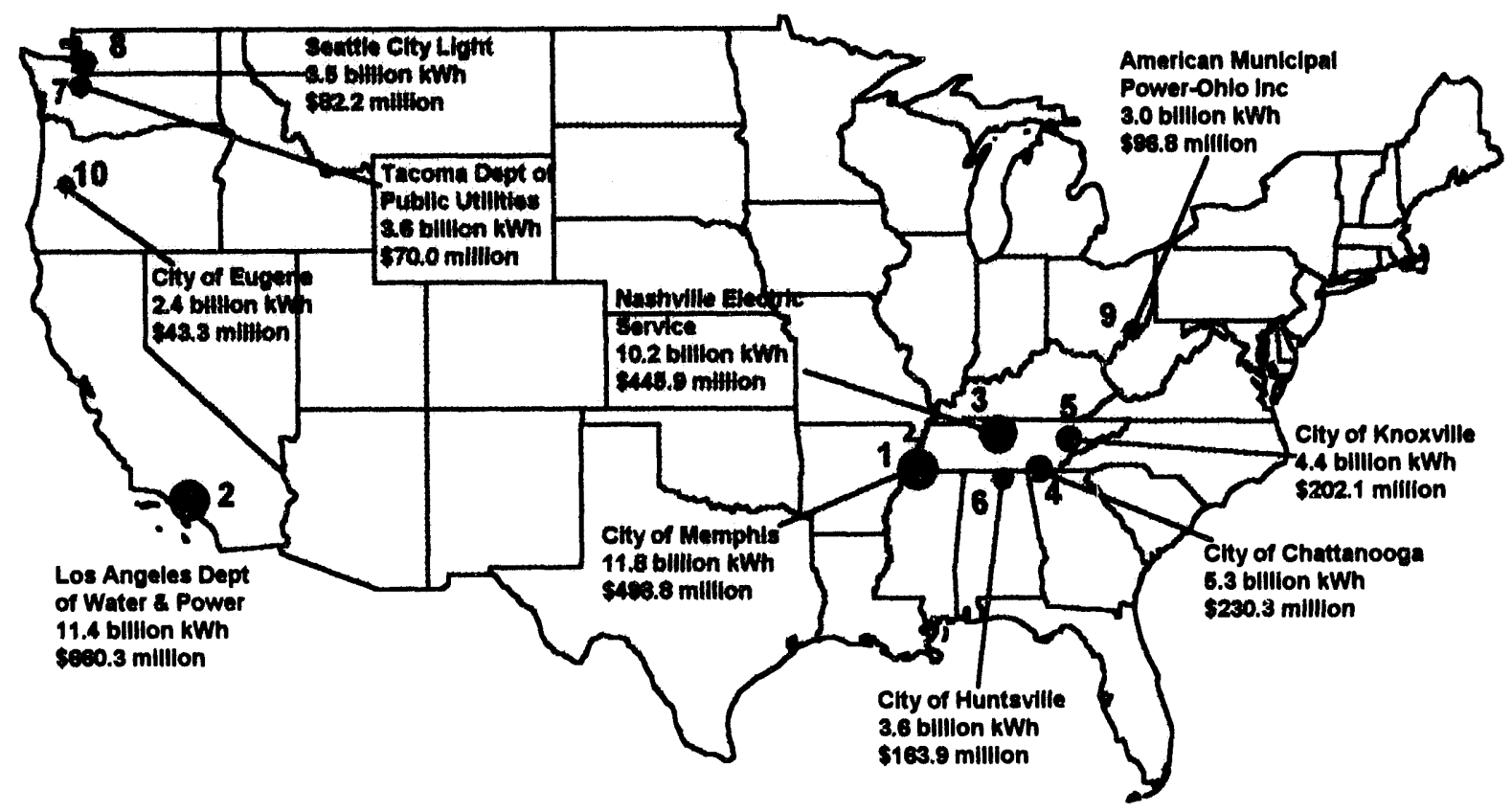

Note: Numbers and symbol sizes indicate rank by kilowatthours (kWh) purchased.

Source: Energy Information Administration, Form EIA-412, "Annual Report of Public Electric Utilities."

nessee Valley Authority. Of the 5, 4 are in Tennessee--Memphis (first), Nashville (third), Chattanooga (fourth), and Knoxville (fifth)--and the other is Huntsville, Alabama (ranked six). Overall, each of the top 10 municipal purchasers bought more than 2.4 billion kilowatthours and collectively spent almost $\$ 2.5$ billion (Table 29).

As a group, municipal utilities are not active as sellers of power. However, the 10 largest municipal wholesale sellers (Figure 13) of electric power (which includes several organizations that act as agents for groups of municipal utilities) each sold at least 1.4 billion kilowatthours. Revenue from power sold for the 10 largest, ranged from $\$ 23.8$ million received by Owensboro Municipal Utilities of Kentucky, which sold 1.5 billion kilowatthours, to $\$ 648.6$ million to the Intermountain Power Agency (a municipal agent) located in Utah for the sale of 11.4 billion kilowatthours. Seven of the top 10 municipal sellers, American Municipal Power of Ohio (second), Florida Municipal Power (third), Piedmont Municipal Power of South Carolina (fourth), Texas Municipal Power (fifth), Alabama Municipal Electric Authority (sixth), and Western Minnesota Municipal Power (seventh), are actually buying and selling wholesale electric power on behalf of groups of municipalities. Intermountain Power is in the top 10 (first), but it generates rather than purchases the power it sells. Overall, these municipal selling organizations are reducing costs to their wholesale customers through large joint purchases, which provide better economies of scale (Table 30 ).

Although public sector utilities do not customarily participate in exchange or wheeling transactions (Table 32), the Tennessee Valley Authority exchanged 26.8 billion kilowatthours for 22.5 billion kilowatthours. The Bonneville Power Administration exchanged 20.8 billion kilowatthours for 13.9 billion kilowatthours and wheeled about 39.6 billion kilowatthours. The City of Los Angeles was by far the largest provider of transmission services among both municipal and State and other government utilities, wheeling about 12.8 billion kilowatthours.

\section{Summary}

In 1992, as in prior years, the Federal utilities dominated electric trade by publicly owned utilities. Much of this trade exists because of authority given and restrictions placed in the Federal laws that guide these Federal power authorities. Many of these differences are due to Federal flood prevention and irrigation requirements 


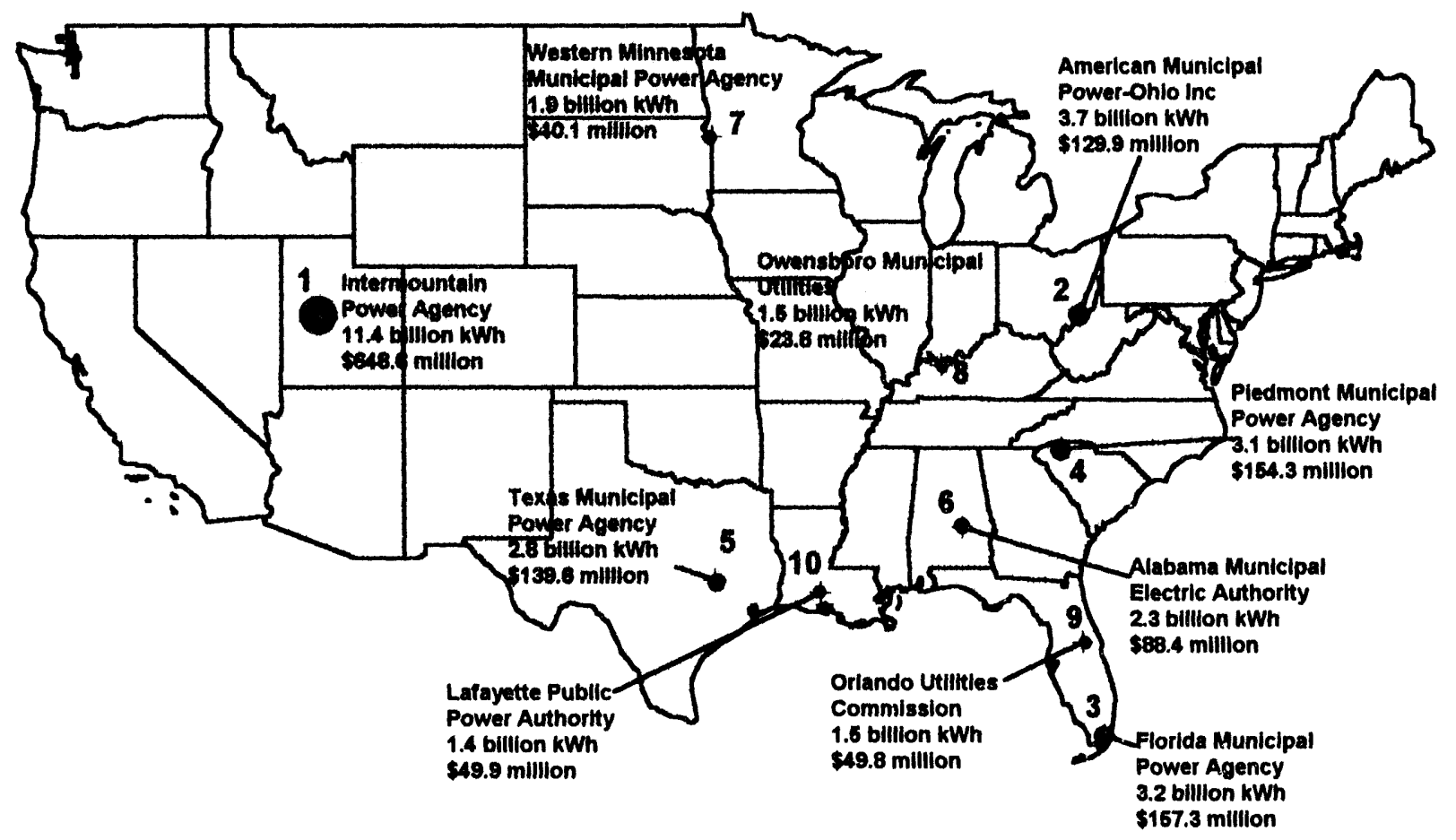

Note: Numbers and symbol sizes indicate rank by kilowatthours (kWh) sold.

Source: Energy Information Administration, Form EIA-412, "Annual Report of Public Electric Utilities."

at hydroelectric projects or the limitations of corresponding transmission systems operated by the power marketing authorities.

Geographically, the Western Area Power Administration (WAPA) and the Bonneville Power Administration are the two largest Federal power marketing administrations and both operate Federally owned transmission lines. The Tennessee Valley Authority collects the most revenue of any Federal utility, but its legal authority differs from the power marketing administrations. Other Federal authorities, like the Southeastern Power Administration, market power from a much smaller group of Federally owned generating facilities and transmit the power over a non-Federal transmission system. The Alaska Power Administration is the smallest power marketing administration. The International Boundary and Water Commission, United States and Mexico, facilities are the smallest in terms of size and 1992 trade (as reported by WAPA).

Many State power agencies and other public authorities were established to purchase large quantities of electricity for resale to their member electric utilities. Some of these State organizations have been set up to acquire power for special in-State or regional projects, such as water transfer for irrigation and/or public consumption. In other cases. States have established power brokering agencies to make bulk power purchases for redistribution to municipalities and cooperatives, which reduces the rates to the members.

Municipalities purchase far more power than they sell for resale as the electricity is almost always redistributed to the ultimate consumers. The municipal utilities involved in sales for resale do have generating capability, frequently including hydroelectric facilities and/ or jointly owned thermal generators.

If Federal and State marketing authorities operate nearby, municipals will probably have a preferential authorization which will allow them to purchase any available power first, thereby reducing the overall cost of purchased power. State municipal power agencies and authorities are becoming an increasingly important source of electric power for municipal utilities. Many States have municipal power agencies or their equivalent, and membership in these organizations is growing. By aggregating the power requirements of their members, municipal power agencies are able to purchase power at the most favorable rates available and pass these savings on to their members. 
Table 25. Electriclty Purchases by Fedoral Utilities, Flecal Year Ending Soptombor 30, 1992

\begin{tabular}{|c|c|c|c|c|c|c|c|}
\hline \multirow{3}{*}{ Sute I } & \multicolumn{7}{|c|}{ Source of Electrictly } \\
\hline & \multicolumn{2}{|c|}{ Imveator-Owned } & \multicolumn{2}{|c|}{ Fecleral } & \multicolumn{2}{|c|}{$\begin{array}{l}\text { State and Other } \\
\text { Government }\end{array}$} & \multirow{2}{*}{ 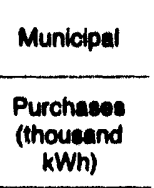 } \\
\hline & $\begin{array}{c}\text { Purchases } \\
\text { (thousand } \\
\text { kWh) }\end{array}$ & $\begin{array}{c}\text { Coot } \\
\text { (dollars) }\end{array}$ & $\begin{array}{l}\text { Purchases } \\
\text { (thouseand } \\
\text { kWh) }\end{array}$ & $\begin{array}{c}\text { Cost } \\
\text { (dollars) }\end{array}$ & $\begin{array}{c}\text { Purchases } \\
\text { (thousand } \\
\text { kWh) }\end{array}$ & $\begin{array}{c}\text { Cost } \\
\text { (dollars) }\end{array}$ & \\
\hline $\begin{array}{l}\text { Arteona } \\
\text { USBIA-San Carlos Imig Project ......... }\end{array}$ & 183,068 & $7,327,056$ & 87,136 & 955,320 & 3,136 & 570,089 & - \\
\hline $\begin{array}{l}\text { Colorado } \\
\text { Weoterr area Power Admin .............. }\end{array}$ & $6,070,263$ & $165,585,863$ & 138,364 & $2,299,000$ & 523,718 & $9,538,283$ & 462,923 \\
\hline $\begin{array}{l}\text { Cesorgta } \\
\text { Southeastern Power Admin ............... }\end{array}$ & 17,780 & 420,876 & - & - & 32,346 & 439,791 & - \\
\hline $\begin{array}{l}\text { Montena } \\
\text { Mitedion Valley Power ........................... }\end{array}$ & 84,761 & $1,213,830$ & 201,633 & $4,636,141$ & 801 & 21,035 & - \\
\hline $\begin{array}{l}\text { Oklwhoma } \\
\text { Southwestern Power Admin ............... }\end{array}$ & 36,781 & $1,688,965$ & - & - & - & - & - \\
\hline $\begin{array}{l}\text { Orecon } \\
\text { Bonneville Power Admin ...................... }\end{array}$ & $2,702,108$ & $56,695,650$ & - & - & 360,116 & $6,216,324$ & 274,022 \\
\hline 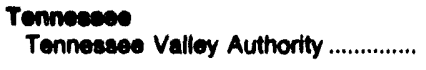 & - & - & - & - & 1,160 & 34,807 & - \\
\hline 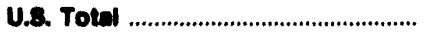 & $9,093,751$ & $232,812,340$ & 427,033 & $8,090,461$ & 921,377 & $16,818,429$ & 736,945 \\
\hline
\end{tabular}

\begin{tabular}{|c|c|c|c|c|c|c|}
\hline Source of Electricity \\
\hline Municipal & \multicolumn{2}{|c|}{ Cooperattve } & \multicolumn{2}{|c|}{ Other' } & \multicolumn{2}{|c|}{ Total } \\
\hline $\begin{array}{c}\text { Cost } \\
\text { (dollars) }\end{array}$ & $\begin{array}{c}\text { Purchases } \\
\text { (thousand } \\
\mathrm{kWh})\end{array}$ & $\begin{array}{c}\text { Cost } \\
\text { (dollars) }\end{array}$ & $\begin{array}{c}\text { Purchases } \\
\text { (thousand } \\
\mathrm{kWh})\end{array}$ & $\begin{array}{c}\text { Cost } \\
\text { (dollars) }\end{array}$ & $\begin{array}{c}\text { Purchases } \\
\text { (thousand } \\
\mathrm{kWh})\end{array}$ & $\begin{array}{c}\text { Cost } \\
\text { (dollars) }\end{array}$ \\
\hline
\end{tabular}

Arizona

\begin{tabular}{|c|c|c|c|c|c|c|c|}
\hline USBIA-San Carlos Irrig Project ......... & - & - & - & - & - & 273,330 & $8,852,465$ \\
\hline Western Araa Power Admin .............. & $26,672,501$ & $2,898,307$ & $50,895,772$ & 620,412 & $21,965,284$ & $10,713,887$ & $277,034,703$ \\
\hline $\begin{array}{l}\text { Deorgla } \\
\text { Southesstern Power Admin ............... }\end{array}$ & - & 106,718 & $1,825,440$ & - & - & 156,845 & $2,788,207$ \\
\hline $\begin{array}{l}\text { Montana } \\
\text { Mision Valley Power ............................ }\end{array}$ & - & - & - & - & - & 287,185 & $6,071,000$ \\
\hline Southwestern Power Admin ............... & - & - & - & - & - & 35,781 & $1,688,865$ \\
\hline Bonneville Power Admin .................... & $5,131,500$ & 931,917 & $19,104,354$ & $3,410,014$ & $59,162,017$ & $7,678,177$ & $146,309,845$ \\
\hline Tenneseee Valley Authority ................ & - & - & - & 506,205 & $16,710,305$ & 507,365 & $16,745,212$ \\
\hline 8. Total. & $31,804,001$ & $3,836,943$ & $72,025,686$ & $4,536,631$ & $97,837,606$ & $18,652,680$ & $459,488,403$ \\
\hline
\end{tabular}

Includes transactions with power pools, utilties in Canada and Mexico, and nonutlittes.

- Not Applicable

Notes: -Federal authorties use different accounting methods for reporting energy recelved from Federal generating assets; the energy may be reported as generation or purchases. -For identification purposes, the public utlities are liated in the state in vahich the administrative office is located. - Totals may not equal sum of components because of independent rounding.

Source: Energy Information Administration, Form EIA-412, "Annual Report of Publlc Electric Utilties." 
Table 26. Electricity 8ales for Resale by Fedoral Utilitios, Flecal Year Ending Soptember 30, 1992

\begin{tabular}{|c|c|c|c|c|c|c|c|}
\hline \multirow{3}{*}{ Stape I } & \multicolumn{7}{|c|}{ Purchaser of Electriotty } \\
\hline & \multicolumn{2}{|c|}{ Investor-Owned } & \multicolumn{2}{|c|}{ Federal } & \multicolumn{2}{|c|}{$\begin{array}{l}\text { State and Other } \\
\text { Covernment }\end{array}$} & \multirow{2}{*}{$\begin{array}{c}\text { Municipal } \\
\text { Sules for } \\
\text { Rosale } \\
\text { (thousand } \\
\text { kWh) }\end{array}$} \\
\hline & $\begin{array}{l}\text { Sales for } \\
\text { Reacle } \\
\text { (thousand } \\
\text { KWh) }\end{array}$ & $\begin{array}{l}\text { Rovenue } \\
\text { (dollare) }\end{array}$ & $\begin{array}{l}\text { Sales for } \\
\text { Realale } \\
\text { (thousand } \\
\text { kWh) }\end{array}$ & $\begin{array}{l}\text { Rovenue } \\
\text { (dollars) }\end{array}$ & $\begin{array}{l}\text { Sales for } \\
\text { Resale } \\
\text { (thousand } \\
\text { kWh) }\end{array}$ & $\begin{array}{l}\text { Rovenue } \\
\text { (dollars) }\end{array}$ & \\
\hline \multicolumn{8}{|l|}{ Alackn } \\
\hline Alacka Power Adminiatration ............ & 213,830 & $6,693,878$ & - & - & - & - & 70,622 \\
\hline \multicolumn{8}{|l|}{ Colorndo } \\
\hline Weatem Area Power Admin .............. & $2,516,002$ & $46,693,182$ & 88,108 & 850,464 & $8,048,616$ & $151,360,680$ & $9,274,184$ \\
\hline \multicolumn{7}{|l|}{ corrin } & $1,315,662$ \\
\hline \multicolumn{8}{|l|}{ Mohlon } \\
\hline USCE-Detrolt Diatrict ............................. & 162,696 & $1,749,885$ & - & - & - & - & - \\
\hline \multicolumn{8}{|l|}{ Oxlenema } \\
\hline Southweatern Power Admin ............... & 3,516 & 17,813 & 159,649 & $3,635,295$ & 383,423 & $4,429,715$ & $1,744,346$ \\
\hline \multicolumn{8}{|l|}{ Oregon } \\
\hline Bonneville Power Admin ...................... & $8,776,446$ & $349,567,000$ & 54,084 & $2,167,000$ & $21,655,850$ & $505,872,000$ & $11,114,189$ \\
\hline $\begin{array}{l}\text { Tennesece } \\
\text { Tennessed Valley Authority ................ }\end{array}$ & - & $1,000,886$ & - & - & - & - & $67,184,073$ \\
\hline 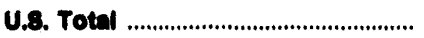 & $11,712,546$ & $408,224,698$ & $2,651,645$ & $27,119,302$ & $30,295,160$ & $687,118,373$ & $80,703,076$ \\
\hline
\end{tabular}

\begin{tabular}{|c|c|c|c|c|c|c|}
\hline Municipal & \multicolumn{2}{|c|}{ Cooperative } & \multicolumn{2}{|c|}{ Other' } & \multicolumn{2}{|c|}{ Total } \\
\hline $\begin{array}{c}\text { Revenue } \\
\text { (dollars) }\end{array}$ & $\begin{array}{c}\text { Sales for } \\
\text { Resale } \\
\text { (thousand } \\
\mathrm{kWh} \text { ) }\end{array}$ & $\begin{array}{c}\text { Revenue } \\
\text { (dollars) }\end{array}$ & $\begin{array}{c}\text { Sales for } \\
\text { Resale } \\
\text { (thousand } \\
\mathrm{kWh}\end{array}$ & $\begin{array}{c}\text { Revenue } \\
\text { (dollars) }\end{array}$ & $\begin{array}{c}\text { Sales for } \\
\text { Resale } \\
\text { (thousand } \\
\mathrm{kWh}\end{array}$ & $\begin{array}{c}\text { Revenue } \\
\text { (dollars) }\end{array}$ \\
\hline
\end{tabular}

\begin{tabular}{|c|c|c|c|c|c|c|c|}
\hline Alaska Power Administration ............. & $1,200,574$ & 73,742 & $1,249,883$ & - & - & 358,194 & $9,144,245$ \\
\hline \multicolumn{8}{|l|}{ Colorado } \\
\hline Western Area Power Admin ............... & $174,496,523$ & $7,472,642$ & $108,260,211$ & $1,579,520$ & $29,453,201$ & $28,979,129$ & $511,114,271$ \\
\hline \multicolumn{8}{|l|}{ Coorgla } \\
\hline Southeastern Power Admin ................ & $40,603,193$ & $2,876,389$ & $79,184,601$ & - & - & $6,889,227$ & $146,212,257$ \\
\hline \multicolumn{8}{|l|}{ Michlenen } \\
\hline USCE-Detrolt District ............................... & - & -- & - & - & - & 162,695 & $1,749,885$ \\
\hline \multicolumn{8}{|l|}{ Oklahoma } \\
\hline Southwestern Power Admin .............. & $21,486,680$ & $4,315,200$ & $60,812,685$ & - & - & $6,606,133$ & $90,382,208$ \\
\hline \multicolumn{8}{|l|}{ Oregon } \\
\hline Bonneville Power Admin ..................... & $252,938,000$ & $9,258,254$ & $206,725,000$ & 11,412 & 268,000 & $50,670,335$ & $1,317,537,000$ \\
\hline \multicolumn{8}{|l|}{ Tenneares } \\
\hline Tenneseed Valley Authority ................ & $3,060,688,860$ & $26,437,803$ & $1,205,478,036$ & - & - & $93,621,976$ & $4,267,165,791$ \\
\hline 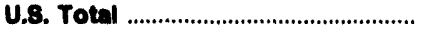 & $3,551,411,849$ & $50,534,130$ & $1,661,710,236$ & $1,580,832$ & $29,721,201$ & $187,487,689$ & $6,343,305,657$ \\
\hline
\end{tabular}

\footnotetext{
1 Includes transactions with power pools, utilities in Canada and Mexico, and nonutilities.

- Not Applicable

Notes: -For identification purposes, the public utilities are listed in the State in which the administrative office is located. - Totals may not equal sum of components because of independent rounding.

Source: Energy information Administration, Form EIA-412, "Annual Report of Public Electric Utilities."
} 
Table 27. Elootriolty Purchases by 8tate and Other Covernment Utilitios, Flecal Year 1902

\begin{tabular}{|c|c|c|c|c|c|c|}
\hline \multirow{3}{*}{ 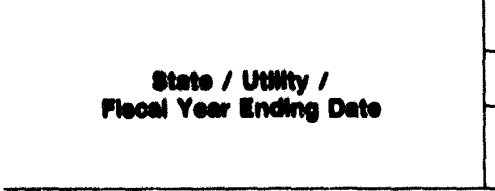 } & \multicolumn{6}{|c|}{ Sources of Electritity } \\
\hline & \multicolumn{2}{|c|}{ Invector-Owned } & \multicolumn{2}{|c|}{ Federal } & \multicolumn{2}{|c|}{ Munioipal } \\
\hline & $\begin{array}{l}\text { Purchages } \\
\text { (thousand kWh) }\end{array}$ & $\begin{array}{c}\text { Cost } \\
\text { (dollars) }\end{array}$ & $\begin{array}{l}\text { Purchease } \\
\text { (thousand kWh) }\end{array}$ & $\begin{array}{c}\text { Coot } \\
\text { (dollarn) }\end{array}$ & $\begin{array}{c}\text { Purchaces } \\
\text { (thousand kWh) }\end{array}$ & $\begin{array}{c}\text { Cont } \\
\text { (dollars) }\end{array}$ \\
\hline \multicolumn{7}{|l|}{$\begin{array}{l}\text { Artiona } \\
\text { Arizona Power Authortty/Jun } 30\end{array}$} \\
\hline 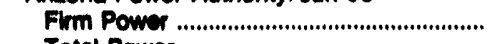 & 87 & 274,536 & 786,292 & $14,800,203$ & - & - \\
\hline Total Power & 67 & 274,830 & 758,202 & $14,800,203$ & - & - \\
\hline 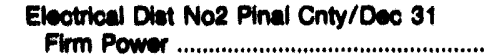 & & & & & & \\
\hline $\begin{array}{l}\text { Firm Power } \\
\text { Total Power }\end{array}$ & - & - & - & - & - & - \\
\hline Navalo Tribal Utillty Auth/Dec 31 & - & - & - & - & - & - \\
\hline 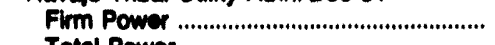 & 449,709 & $17,763,332$ & 112,783 & $1,810,710$ & - & - \\
\hline $\begin{array}{l}\text { Total Power ...tin............................. } \\
\text { Papago Tribal Utilty }\end{array}$ & 449.700 & $17,763,332$ & 112.783 & $1,810,710$ & - & - \\
\hline 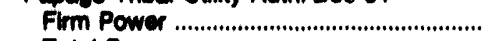 & 172,073 & $6,771,733$ & 13,644 & 126,572 & - & - \\
\hline 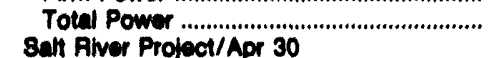 & 172,073 & $6,771,733$ & 13,644 & 126,572 & - & - \\
\hline Firm Power .......................................... & 204,686 & $22,070,090$ & 832,312 & $7,485,130$ & 34,089 & 787,367 \\
\hline Nonfirm Power ................................................. & 73,684 & $1,009,860$ & 72,021 & $1,011,440$ & 502 & 0,354 \\
\hline 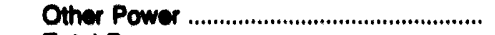 & & $-117,341$ & 83,383 & $-19,680$ & & \\
\hline Total Power ........... & 293,080 & $23,031,609$ & 667,716 & $8,476,899$ & 35,681 & 785,760 \\
\hline Welton-Mohawk ImaDrain Dist/Dac 31' & & & & & & \\
\hline 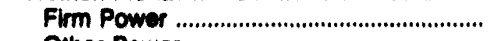 & - & - & - & - & - & - \\
\hline 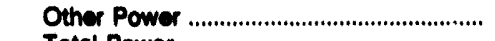 & 20,636 & $1,097,378$ & 1.146 & 23,460 & - & - \\
\hline Total Power ....................................... & 20,636 & $1,097,378$ & 1.146 & 23,460 & - & - \\
\hline \multicolumn{7}{|l|}{$\begin{array}{l}\text { Callformla } \\
\text { Calfiornia Dept Wtr Resources/Dec } 31\end{array}$} \\
\hline 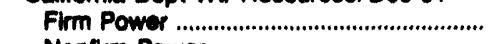 & 625,017 & $23,639,400$ & - & - & 162,399 & $7,008,460$ \\
\hline Nontirm Power .............................................. & 37,851 & 829,606 & 13,764 & 176,368 & 5,115 & 107,430 \\
\hline 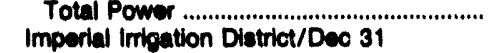 & 683,768 & $24,469,000$ & 13,754 & 175,368 & 167,514 & $7,205,890$ \\
\hline 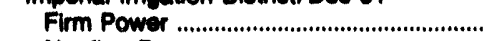 & $1,303,683$ & $62,802,619$ & 156,303 & $1,472,404$ & - & - \\
\hline Nontirm Power ………………………………........ & 52,923 & $1,300,245$ & 23,600 & 551,365 & - & - \\
\hline 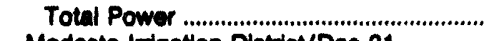 & $1,356,500$ & $64,202,864$ & 178,803 & $2,023,769$ & - & - \\
\hline \multicolumn{6}{|l|}{ Modeato Imbation Diatrict/Dec 31} & 18.428 .954 \\
\hline 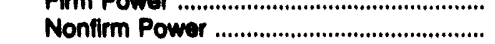 & $\begin{array}{r}548,382 \\
46,504\end{array}$ & $\begin{array}{r}26,006,373 \\
847,313\end{array}$ & $\begin{array}{l}62,111 \\
13,224\end{array}$ & $\begin{array}{r}1,516,000 \\
202,552\end{array}$ & $-008,187$ & $18,428,864$ \\
\hline Other Power ...................................................... & - & - & - & - & - & - \\
\hline 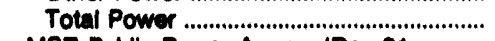 & 593,886 & $27,010,686$ & 75,365 & $1,719,211$ & 559,797 & $18,428,954$ \\
\hline $\begin{array}{l}\text { MSR Publle Power Agency/Dec } 31 \\
\text { Nonfirm Power }\end{array}$ & 1,897 & 62,233 & $=$ & - & -- & - \\
\hline 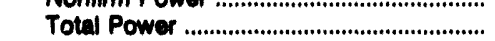 & 1,887 & 62,233 & - & - & -- & $\overline{-}$ \\
\hline \multicolumn{7}{|l|}{ 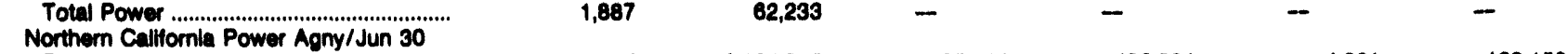 } \\
\hline 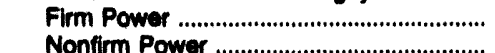 & 183,387 & $6,127,745$ & 25,124 & 402,204 & 4,861 & 160,152 \\
\hline $\begin{array}{l}\text { Nonfirm Power } \\
\text { Other Power }\end{array}$ & -41.176 & $\begin{array}{r}982,129 \\
15,512\end{array}$ & - & $\overline{-}$ & $-\quad 4,949$ & 84,600 \\
\hline 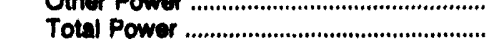 & 224,543 & $7,125,386$ & 25,124 & 402,204 & 8,810 & $\overline{244,752}$ \\
\hline \multicolumn{7}{|l|}{ Sacramento Municipal Util Dist/Dec 31} \\
\hline 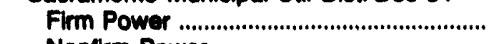 & $2,360,510$ & $156,479,529$ & $2,082,893$ & $57,482,205$ & -- & - \\
\hline 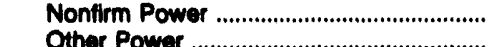 & $1,468,672$ & $30,984,957$ & $-111,803$ & $2,366,318$ & $\overline{-}$ & $\overline{-}$ \\
\hline $\begin{array}{l}\text { Other Power } \\
\text { Total Power }\end{array}$ & $\overline{3,827,182}$ & $187,464,486$ & $\overline{2,174,890}$ & $59,838,523$ & $\overline{-}$ & $\overline{-}$ \\
\hline \\
\hline & - & - & - & - & - & - \\
\hline \\
\hline & & & & & & \\
\hline 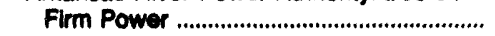 & - & - & 83,694 & $1,643,966$ & 116,108 & $3,670,167$ \\
\hline Nonfirm Power ....................................................... & - & - & 29,832 & 568,800 & $-1,931$ & 80,142 \\
\hline \multirow{2}{*}{\multicolumn{7}{|c|}{ Platte Plver Power Authority/Dec 31}} \\
\hline & & & & & & \\
\hline 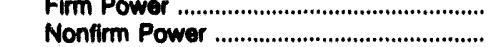 & 268 & 6,256 & $\begin{array}{r}727,365 \\
10\end{array}$ & $\begin{array}{r}12,167,581 \\
593\end{array}$ & 3,408 & $-73,791$ \\
\hline 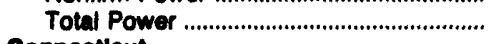 & 268 & 6,256 & 727,384 & $12,168,174$ & 3,406 & 73,701 \\
\hline \multirow{2}{*}{\multicolumn{7}{|c|}{$\begin{array}{l}\text { Conneetlout } \\
\text { Mun Electric Engy Coop/Dec } 31\end{array}$}} \\
\hline Firm Power & 609.521 & 38.547 .988 & _- & & & \\
\hline Other Power . & -165 & 181,712 & - & $\overline{-}$ & - & $1,373,100$ \\
\hline Total Power .............................................. & 609,356 & $38,729,710$ & - & - & 814 & $1,373,105$ \\
\hline
\end{tabular}

See notes and tootnotes at end of table. 
Table 27. Elootrlelty Purchaees by 8tate and Other Covernment Uillitios, Flecal Year 1902 (Continued)

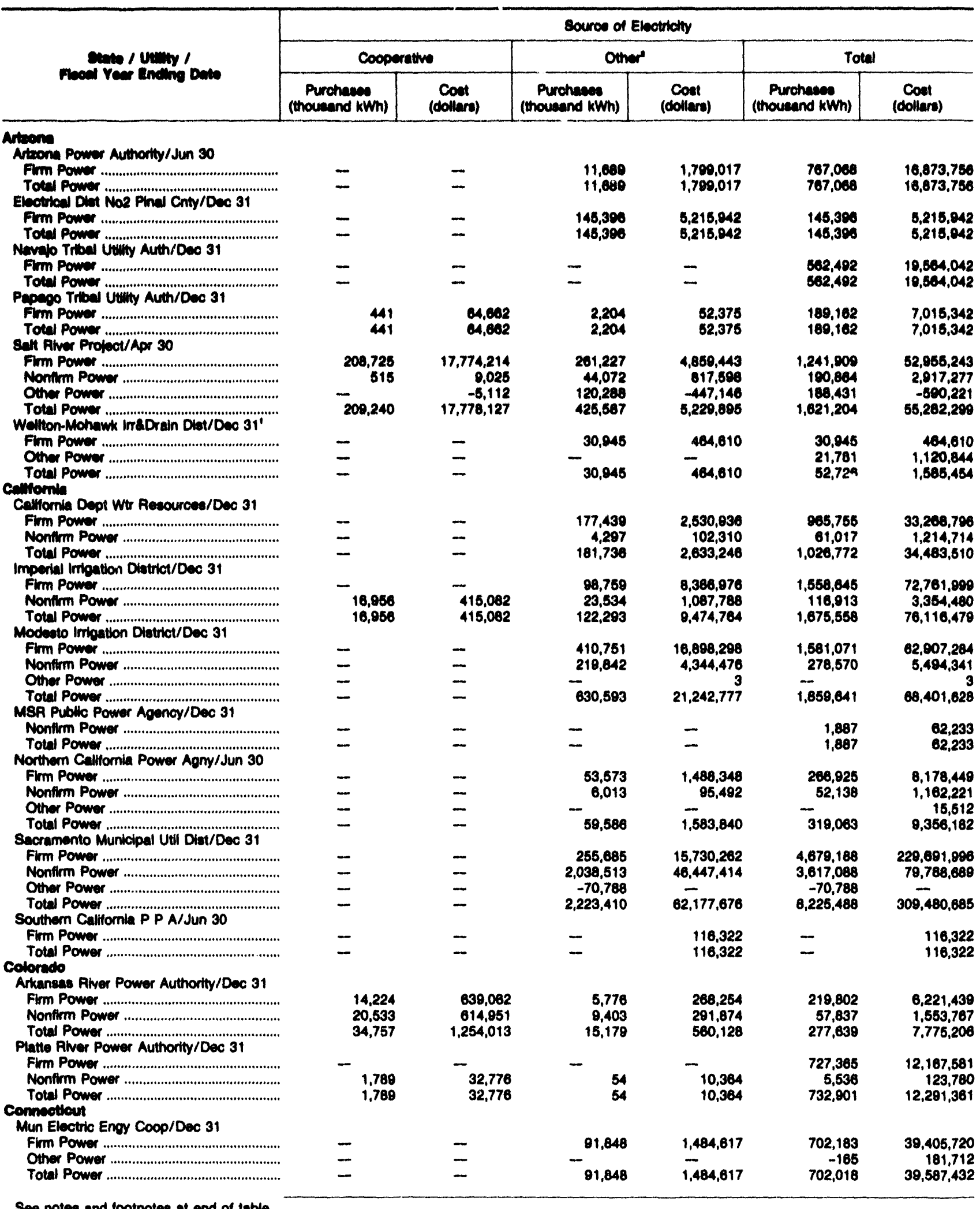

See notes and footnotes at and of table. 
Tablo 27. Eloctriolty Purchaces by state and Other Qovernment Utilitiea, Fiecal Year 1992 (Continued)

\begin{tabular}{|c|c|c|c|c|c|c|}
\hline \multirow{3}{*}{ 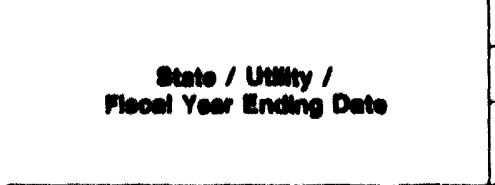 } & \multicolumn{6}{|c|}{ souros of Electratly } \\
\hline & \multicolumn{2}{|c|}{ Inventor-Omned } & \multicolumn{2}{|c|}{ Fecderal } & \multicolumn{2}{|c|}{ Municipal } \\
\hline & $\begin{array}{l}\text { Purchesese } \\
\text { (thousand kWh) }\end{array}$ & $\begin{array}{c}\text { Cont } \\
\text { (dollere) }\end{array}$ & $\begin{array}{l}\text { Purohases } \\
\text { (thousend } \mathrm{kWh} \text { ) }\end{array}$ & $\begin{array}{c}\text { Coot } \\
\text { (dollars) }\end{array}$ & $\begin{array}{l}\text { Purohases } \\
\text { (thousand kWh) }\end{array}$ & $\begin{array}{c}\text { Cost } \\
\text { (dollars) }\end{array}$ \\
\hline \multicolumn{7}{|l|}{$\begin{array}{l}\text { Qcoorein } \\
\text { Critep County Power Commisedion/Dec } 31\end{array}$} \\
\hline Firm Power . & - & - & 32,894 & $1,101,161$ & - & - \\
\hline Other Power ............................................. & 17,284 & 508,178 & - & - & - & - \\
\hline 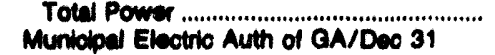 & 17,234 & 596,178 & 32,894 & $1.101,161$ & - & - \\
\hline Firm Power .................................................. & $1,491,775$ & $40,680,000$ & - & - & - & - \\
\hline 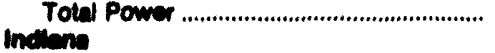 & $1,481,775$ & $40,860,000$ & - & - & - & - \\
\hline \multicolumn{7}{|l|}{ Indindiana Municipal Power Agenoy/Dec 31} \\
\hline 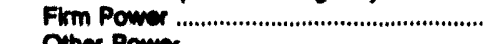 & $1,850,143$ & $60,216,420$ & - & - & 502,000 & $26,270,008$ \\
\hline Other Power ..................................................... & 67,916 & $1,667,017$ & - & - & - & - \\
\hline \multirow{2}{*}{\multicolumn{7}{|c|}{ Kenaes }} \\
\hline & & & & & & \\
\hline 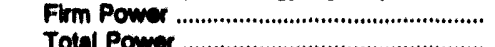 & - & - & 30,371 & 408,000 & 284,600 & $8,727,000$ \\
\hline 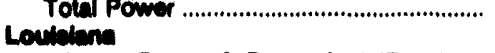 & - & - & 36,371 & $\$ 08,000$ & 204,500 & $8,727,000$ \\
\hline \multicolumn{7}{|l|}{ Loulaiana Energy a Power Auth/Dec 31} \\
\hline 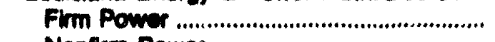 & - & - & 97,237 & $1,386,052$ & 470,400 & $18,092,015$ \\
\hline 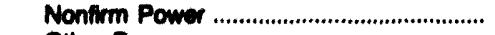 & 20,435 & 511,684 & - & - & 38,392 & 803,018 \\
\hline Other Power ............................................ & - & - & - & $=$ & - & 684,097 \\
\hline 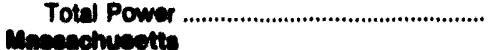 & 20,435 & 511,034 & 97,237 & $1,386,052$ & 608,882 & $19,654,130$ \\
\hline \multicolumn{7}{|l|}{ Maseanchusetts Mun Wholes EI/Dec 31} \\
\hline 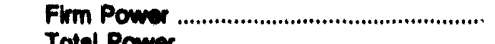 & 241,674 & $7,100,086$ & - & - & 63,238 & $3,352,057$ \\
\hline Total Power .................................... & 241,674 & $7,100,086$ & - & - & 63,236 & $3,352,857$ \\
\hline \multicolumn{7}{|l|}{ Michigan Public Power Agoncy/Dec 31} \\
\hline Firm Power . & 102,477 & $2,192,306$ & - & - & 136,192 & $4,108,807$ \\
\hline Nonfirm Power & 39,859 & $1,040,322$ & - & - & 1,562 & 39,230 \\
\hline $\begin{array}{l}\text { Tolal Power } \\
\text { Michioan South Contral Pw Aoy/Jun } 30\end{array}$ & 142,336 & $\mathbf{3 , 2 3 2 , \mathbf { 6 2 8 }}$ & - & - & 137.744 & $4,145,203$ \\
\hline $\begin{array}{l}\text { Michigan South Central Pwr Agy/Jun } 30 \\
\text { Firm Power }\end{array}$ & 157,794 & $3,841,544$ & - & - & 34,446 & $1,135,874$ \\
\hline 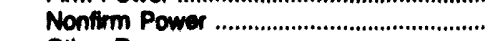 & 75,345 & $1,578,463$ & - & - & 6,153 & 140,843 \\
\hline 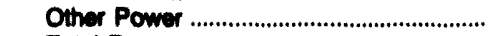 & - & - & - & - & - & - \\
\hline Total Power .................................................... & 233,139 & $5,420,007$ & - & - & 40,599 & $1,276,717$ \\
\hline \multicolumn{7}{|l|}{$\begin{array}{l}\text { Minnecota } \\
\text { Southern Minnesota Mun P Agny/Dec } 31\end{array}$} \\
\hline 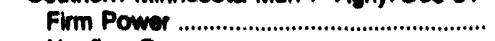 & 6,092 & 106,224 & - & - & 200,402 & $11,812,333$ \\
\hline Nonfirm Power ............................................... & 14,004 & 221,053 & 7,025 & 209,659 & 1,616 & 28,279 \\
\hline 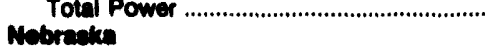 & & 327,277 & 7,025 & 209,659 & 211,018 & $11,840,612$ \\
\hline \multicolumn{7}{|l|}{$\begin{array}{l}\text { Nebraake } \\
\text { Burt County Public Power Diat/Dec 31' }\end{array}$} \\
\hline 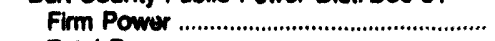 & - & - & - & - & - & - \\
\hline 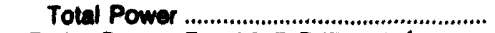 & - & - & - & - & - & - \\
\hline \multicolumn{7}{|l|}{ Butter County Rural P P D/Doc $31^{\prime}$} \\
\hline 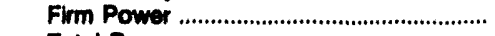 & - & - & - & - & - & - \\
\hline 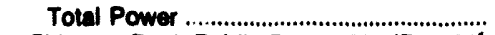 & - & - & - & - & - & - \\
\hline \multicolumn{7}{|l|}{ Chimney Rock Public Power Dist/Dec 31' } \\
\hline 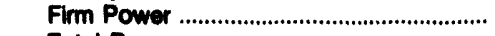 & - & - & - & - & - & - \\
\hline Total Power & - & - & - & - & - & - \\
\hline $\begin{array}{l}\text { Comhusker Public Power Dist/Dec } 31 \\
\text { Firm Power }\end{array}$ & - & - & - & - & - & - \\
\hline 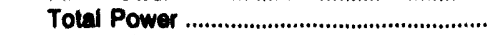 & - & - & - & - & - & - \\
\hline \multicolumn{7}{|l|}{ Dawson County Public Pwr Dist/Dec 31} \\
\hline 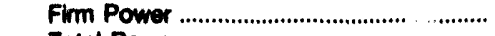 & - & - & - & - & - & - \\
\hline $\begin{array}{l}\text { Total Power . } \\
\text { KaR Rur Public Power Dist/Dec } 311 \text {. }\end{array}$ & - & - & - & - & - & - \\
\hline 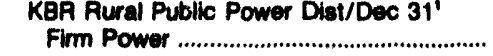 & - & - & - & - & - & - \\
\hline 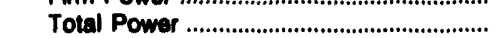 & - & - & - & - & - & - \\
\hline Loup River Public Power Dist/Dec 31 & & & & & & \\
\hline Firm Power & - & - & - & - & - & - \\
\hline or & - & - & - & - & - & - \\
\hline Municipal Energy Agency of NE/Mar 31 & & & & & & \\
\hline or & - & - & 114,360 & $2,171,360$ & 94,595 & $4,691,228$ \\
\hline ower & - & - & 6,138 & 111,274 & 42,513 & 287,017 \\
\hline 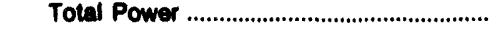 & - & - & 120,498 & $2,282,634$ & 137,108 & $4,978,245$ \\
\hline
\end{tabular}

See notes and footnotes at end of table. 
Table 27. Eleotriolty Purohaces by 8tate and Other Government Utilities, Flocal Year 1992 (Continued)

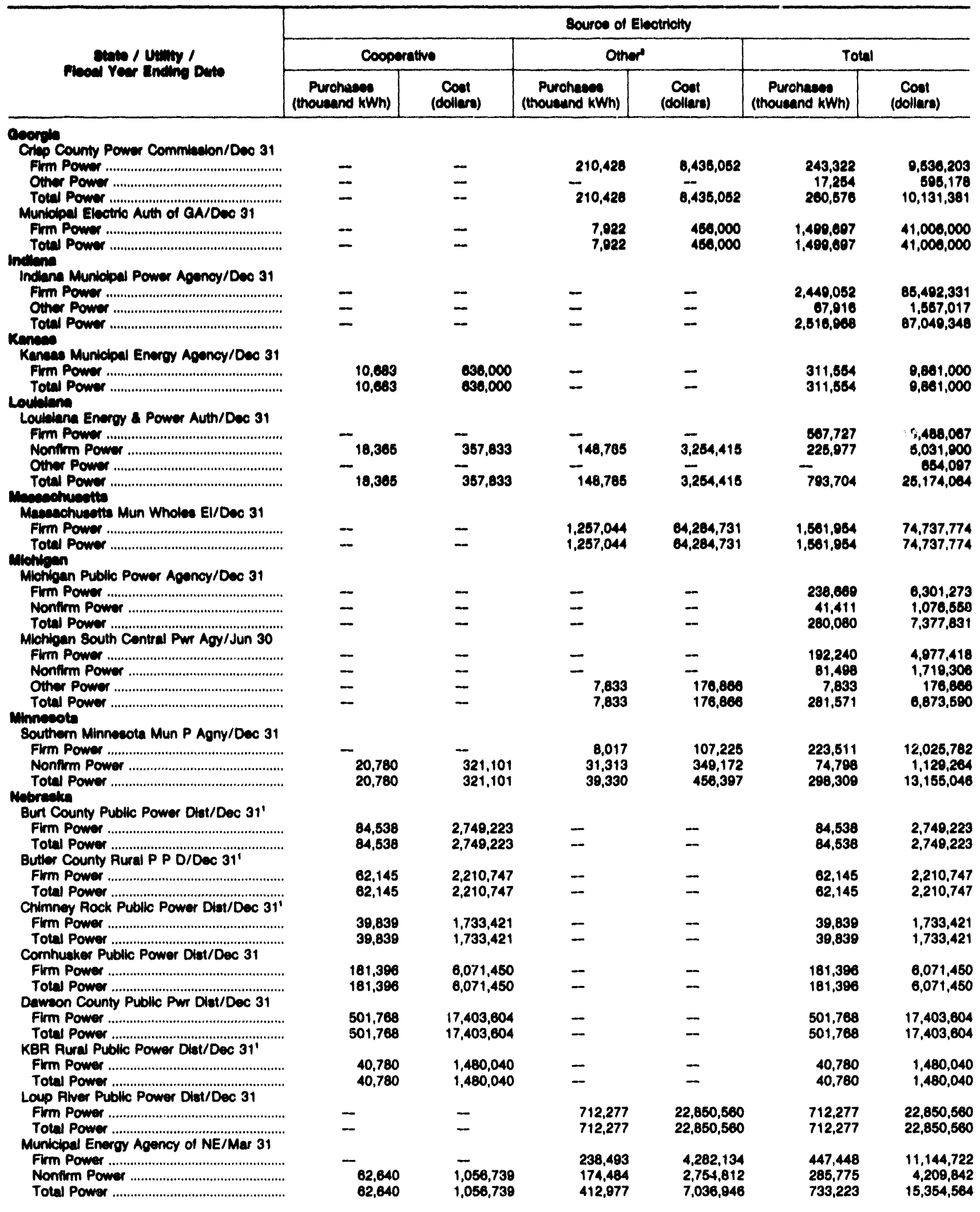

See notes and footnotes at end of table. 
Table 27. Electrlelty Purchases by State and Othor Covornmont Utilitios, Flecal Year 1902 (Continuod)

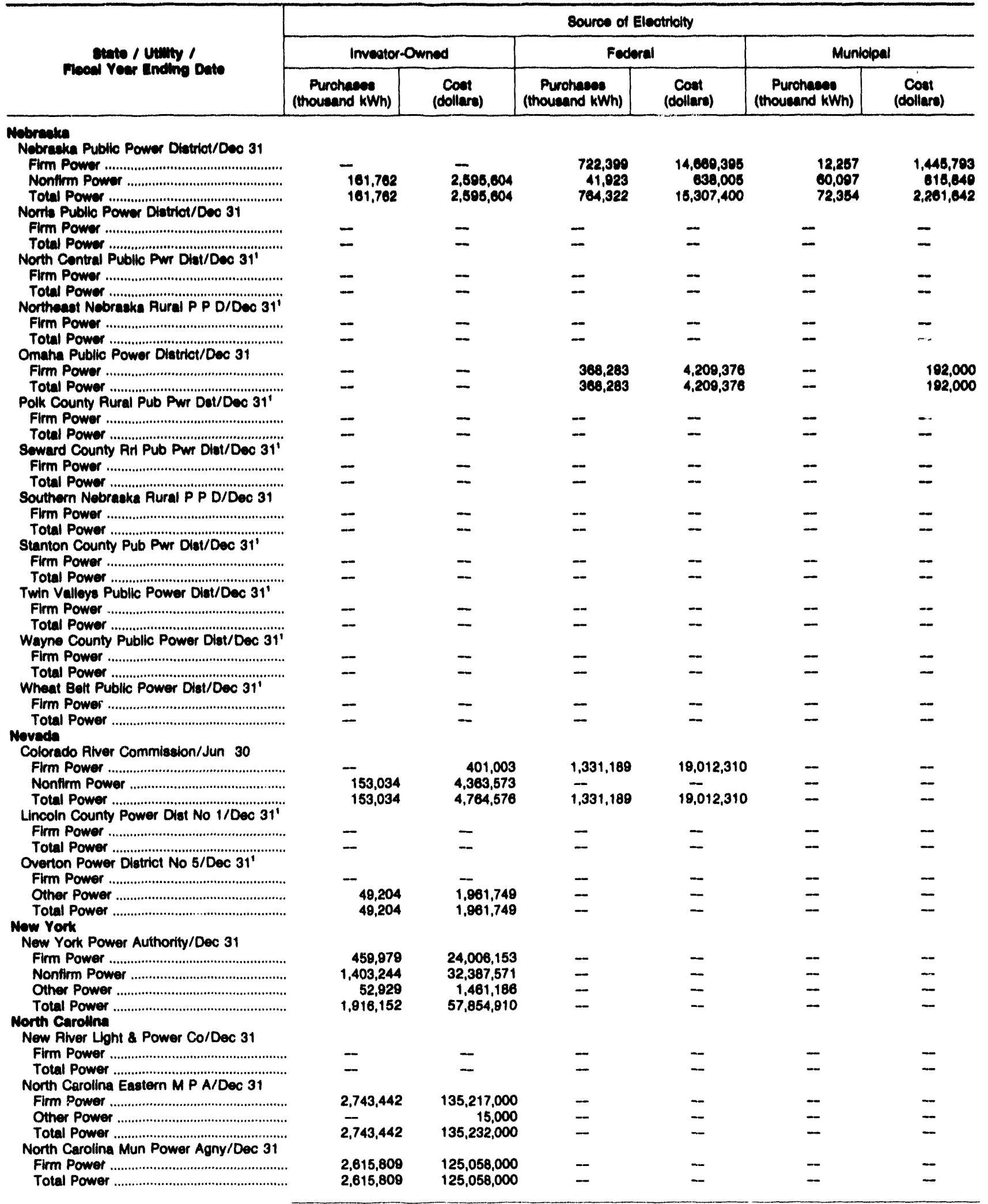

See notes and footnotes at end of table. 
Table 27. Electrictly Purchases by State and Other Covernment Utilties, Fiscal Year 1992 (Continued)

\begin{tabular}{|c|c|c|c|c|c|c|}
\hline \multirow{3}{*}{ 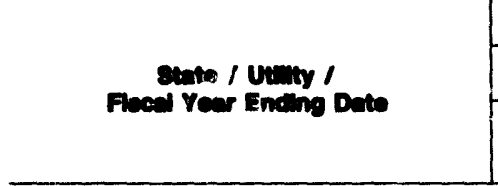 } & \multicolumn{6}{|c|}{ Source of Electrictly } \\
\hline & \multicolumn{2}{|c|}{ Cooperative } & \multicolumn{2}{|c|}{ Other" } & \multicolumn{2}{|c|}{ Total } \\
\hline & $\begin{array}{c}\text { Purchases } \\
\text { (thousand } \mathrm{KWh} \text { ) }\end{array}$ & $\begin{array}{c}\text { Cost } \\
\text { (dollers) }\end{array}$ & $\begin{array}{l}\text { Purchases } \\
\text { (thousand kWh) }\end{array}$ & $\begin{array}{c}\text { Cost } \\
\text { (doltars) }\end{array}$ & $\begin{array}{c}\text { Purchases } \\
\text { (thousand kWh) }\end{array}$ & $\begin{array}{c}\text { Cost } \\
\text { (dollars) }\end{array}$ \\
\hline \multicolumn{7}{|l|}{ Nebranka } \\
\hline 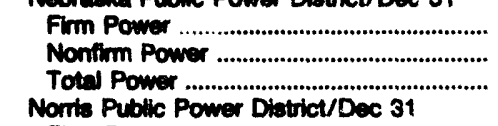 & $\begin{array}{l}- \\
227,175 \\
227,175\end{array}$ & $\begin{array}{l}-\overline{3,417,593} \\
3,417,593\end{array}$ & $\begin{array}{r}403,276 \\
17,005 \\
421,181\end{array}$ & $\begin{array}{r}12,468,198 \\
223,131 \\
12,686,329\end{array}$ & $\begin{array}{r}1,137,832 \\
508,862 \\
1,646,794\end{array}$ & $\begin{array}{r}28,578,386 \\
7,690,182 \\
38,268,568\end{array}$ \\
\hline 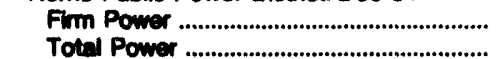 & $\overline{-}$ & $\overline{-}$ & $\begin{array}{l}400,140 \\
400,140\end{array}$ & $\begin{array}{l}12,428,143 \\
12,428,143\end{array}$ & $\begin{array}{l}400,140 \\
400,140\end{array}$ & $\begin{array}{l}12,428,143 \\
12,428,143\end{array}$ \\
\hline \multicolumn{7}{|l|}{ North Central Public Pw Diat/Dec 31' } \\
\hline Fim Power & $\begin{array}{l}60,253 \\
60,253\end{array}$ & $\begin{array}{l}2,271,124 \\
2,271,124\end{array}$ & $\overline{-}$ & $\overline{-}$ & $\begin{array}{l}60,253 \\
80,253\end{array}$ & $\begin{array}{l}2,271,124 \\
2,271,124\end{array}$ \\
\hline 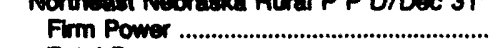 & 67,290 & $2,106,800$ & - & - & 67,290 & $2,106,800$ \\
\hline \multicolumn{6}{|l|}{ Omaha Public Power District/Dec 31} & $2,106,800$ \\
\hline 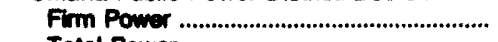 & $2,2,0$ & 313,280 & - & - & 370,503 & $4,714,666$ \\
\hline \multicolumn{7}{|l|}{ Polk County Rural Pub Pwr Dst/Dec $31^{1}$} \\
\hline 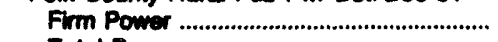 & 49.761 & $1,671,755$ & - & - & 48,761 & $1,671,755$ \\
\hline \multicolumn{7}{|l|}{ Sewerd County RAt Pub Pw Dtot/Dec 31' } \\
\hline 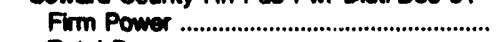 & 49,452 & $1,724,437$ & - & - & 49,452 & $1,724,437$ \\
\hline 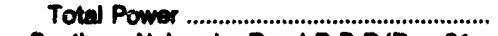 & 49,452 & $1,724,437$ & - & - & 49,452 & $1,724,437$ \\
\hline \multicolumn{7}{|l|}{ Southern Notreakta Pural P P D/Dec 31} \\
\hline $\begin{array}{l}\text { Firm Power } \\
\text { Total Power }\end{array}$ & $\begin{array}{l}379,750 \\
379,750\end{array}$ & $\begin{array}{l}14,424,916 \\
14,424,916\end{array}$ & $\overline{-}$ & $\overline{-}$ & $\begin{array}{l}379,750 \\
379,750\end{array}$ & $\begin{array}{l}14,424,916 \\
14,424,816\end{array}$ \\
\hline \multicolumn{7}{|l|}{ Stamton County Pub Pwr Dist/Dec 31' } \\
\hline & 42,569 & $1,424,689$ & - & - & 42,569 & $1,424,699$ \\
\hline \multicolumn{7}{|l|}{$\begin{array}{l}\text { Total Power ........ } \\
\text { Twin Valleys Public Power Dist/Dec } 31^{1}\end{array}$} \\
\hline 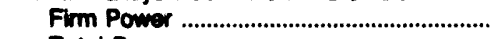 & 57,397 & $1,887,122$ & - & - & 57,397 & $1,887,122$ \\
\hline Total Power ... & 57,387 & $1,887,122$ & - & - & 57,397 & $1,887,122$ \\
\hline \multicolumn{7}{|l|}{ Weyne County Public Power Dist/Dec 31' } \\
\hline 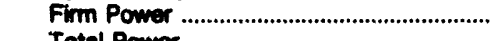 & 53,292 & $1,850,370$ & - & - & 53,292 & $1,850,370$ \\
\hline \multicolumn{7}{|l|}{ Wheat Belt Public Power Dist/Dec 31' } \\
\hline 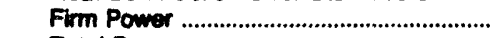 & 110,448 & $5,016,621$ & - & - & 110,448 & $5,016,621$ \\
\hline \multirow{3}{*}{\multicolumn{7}{|c|}{$\begin{array}{l}\text { Movada } \\
\text { Colorado River Commiseion/Jun } 30\end{array}$}} \\
\hline & & & & & & \\
\hline 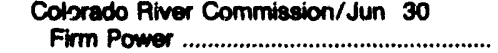 & - & & & & & \\
\hline 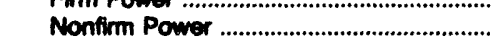 & - & $\overline{-}$ & $\overline{-}$ & $\bar{z}$ & $\begin{array}{r}1,331,189 \\
153,034\end{array}$ & $\begin{array}{r}19,413,313 \\
4,363,573\end{array}$ \\
\hline 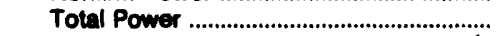 & - & - & - & - & $1,484,223$ & $23,776,886$ \\
\hline \multicolumn{7}{|l|}{ Lincoln County Power Dist No 1/Dec 31' } \\
\hline Firm Power ................................................. & - & - & 66,263 & 776.157 & 66,263 & 776,157 \\
\hline $\begin{array}{l}\text { Total Power . } \\
\text { Overton Power District No 5/Dec } 31\end{array}$ & - & - & 66,283 & 776,157 & 66,263 & 776.157 \\
\hline 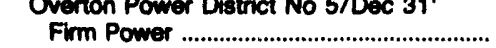 & - & _ & 104,838 & $1,628,422$ & 104,836 & $1,628,422$ \\
\hline Other Power .................................................... & - & - & & & 49,204 & $1,861,749$ \\
\hline 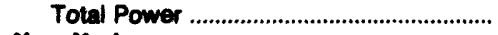 & - & - & 104,838 & $1,628,422$ & 154,042 & $3,590,171$ \\
\hline $\begin{array}{l}\text { Now York } \\
\text { Now York Power Authority/Dec 31 }\end{array}$ & & & & & & \\
\hline 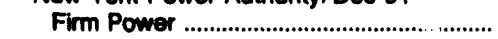 & - & - & $1,985,939$ & $65,806,009$ & $2,455,918$ & $89,812,162$ \\
\hline 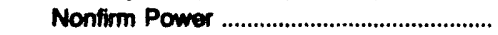 & $\ldots .$. & - & $2,047,511$ & $47,869,373$ & $3,450,755$ & $80,356,944$ \\
\hline 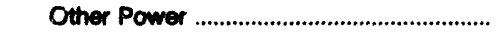 & - & - & 28,839 & $1,421,882$ & 81,768 & $2,863,168$ \\
\hline Total Power ..................................................... & - & - & $4,072,289$ & $115,197,364$ & $5,888,441$ & $173,052,274$ \\
\hline North Caroline & & & & & & \\
\hline New River Light \& Power Co/Dec 31 & & & & & & \\
\hline Firm Power ........ & 165,753 & $8,377,884$ & - & - & 165,753 & $8,377,684$ \\
\hline $\begin{array}{l}\text { Total Power } \\
\text { North Caroline Eastern M P A/Dec } 31\end{array}$ & & 8,377, & - & - & 165,753 & $8,377,684$ \\
\hline 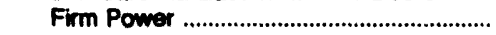 & 277,372 & $9,233,000$ & - & - & $3,020,814$ & $144,450,000$ \\
\hline 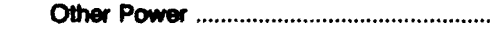 & - & - & - & - & & 15,000 \\
\hline Total Power. & 277,372 & $8,233,000$ & - & - & $3,020,814$ & $144,465,000$ \\
\hline North Carolina Mun Power Agny/Dec 31 & & & & & & \\
\hline 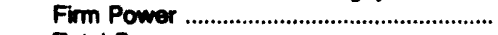 & - & - & - & - & $2,615,809$ & $125,058,000$ \\
\hline 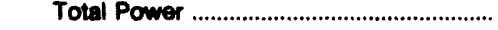 & - & - & - & - & $2,615,809$ & $125,058,000$ \\
\hline
\end{tabular}

See notes and tootnotes at end of table. 
Table 27. Electricity Purchases by State and Other Government Utilities, Fiscal Year 1992 (Continued)

\begin{tabular}{|c|c|c|c|c|c|c|}
\hline \multirow{3}{*}{$\begin{array}{l}\text { State / Utinty I } \\
\text { Floced Year Ending Date }\end{array}$} & \multicolumn{6}{|c|}{ Source of Electricity } \\
\hline & \multicolumn{2}{|c|}{ Investor-Owned } & \multicolumn{2}{|c|}{ Federal } & \multicolumn{2}{|c|}{ Municipal } \\
\hline & $\begin{array}{l}\text { Purchases } \\
\text { (thouseand kWh) }\end{array}$ & $\begin{array}{c}\text { Cost } \\
\text { (dollars) }\end{array}$ & $\begin{array}{c}\text { Purchases } \\
\text { (thousand kWh) }\end{array}$ & $\begin{array}{c}\text { Cost } \\
\text { (dollars) }\end{array}$ & $\begin{array}{c}\text { Purchases } \\
\text { (thousand kWh) }\end{array}$ & $\begin{array}{c}\text { Cost } \\
\text { (dollars) }\end{array}$ \\
\hline \multicolumn{7}{|l|}{ 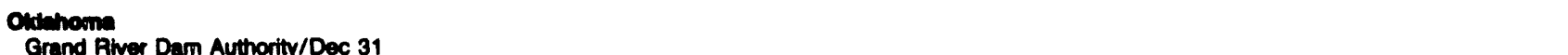 } \\
\hline Nonfirm Power ....................................... & 58,488 & 883,534 & 6,335 & 32,842 & - & - \\
\hline Total Power ....................................... & 58,488 & 983,534 & 6,335 & 32,942 & - & - \\
\hline \multicolumn{7}{|l|}{ Oklahoma Municipal Power Auth/Dec 31} \\
\hline 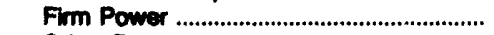 & 312,183 & $10,277,863$ & 13,938 & 290,732 & 19,225 & $2,564,115$ \\
\hline Other Power .......................................................... & - & - & - & - & - & - \\
\hline Total Power ............................................................ & 312,163 & $10,277,863$ & 13,938 & 290,732 & 19,225 & $2,564,115$ \\
\hline \multicolumn{7}{|l|}{ Oreqen } \\
\hline 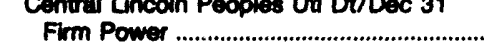 & - & - & $1,344,901$ & $31,742,333$ & - & - \\
\hline Total Power ……........................................... & - & - & $1,344,901$ & $31,742,333$ & - & - \\
\hline \multicolumn{7}{|l|}{ Clatokanio Peoples Util Dist/Dec 31} \\
\hline 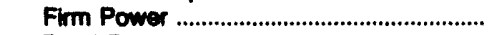 & - & - & 883,763 & $20,164,953$ & - & - \\
\hline \multicolumn{7}{|l|}{ Columbia River Peoples Ut Dist/Dec 31} \\
\hline 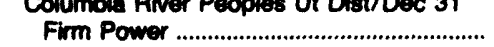 & - & - & 274,491 & $6,320,832$ & - & - \\
\hline Other Power ...................................................... & - & - & - & - & - & - \\
\hline 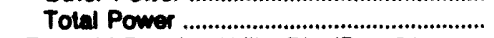 & - & - & 274,481 & $6,320,832$ & - & - \\
\hline \multicolumn{7}{|l|}{ Emerald Peoples Utility Dist/Dec 31} \\
\hline 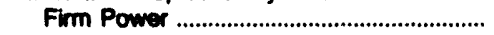 & 103 & 2,750 & 377,054 & $8,702,944$ & - & - \\
\hline 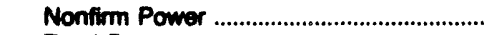 & & & - & - & - & - \\
\hline 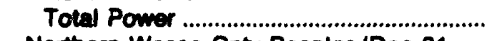 & 103 & 2,750 & 377,054 & $8,702,944$ & - & - \\
\hline \multirow{2}{*}{\multicolumn{7}{|c|}{$\begin{array}{l}\text { Northern Wasco Cnty Peoples/Dec } 31 \\
\text { Firm Power }\end{array}$}} \\
\hline & - & - & 238,532 & $5,976,792$ & - & - \\
\hline \multirow{2}{*}{\multicolumn{3}{|c|}{ Tillamook Peoples Utility Dist/Dec 31}} & & $5,976,792$ & - & - \\
\hline & - & - & 342,137 & $8,059,425$ & - & - \\
\hline Nonfirm Power ................................................... & - & - & - & - & - & -- \\
\hline \multirow{2}{*}{\multicolumn{7}{|c|}{$\begin{array}{l}\text { South Carollna } \\
\text { South Carolina Pub Serv Auth/Dec } 31\end{array}$}} \\
\hline & & & & & & \\
\hline Firm Power & _ & - & 234,867 & $5,118,201$ & 192,324 & $2,590,622$ \\
\hline 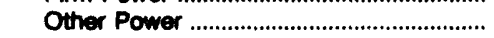 & 109,149 & $1,463,886$ & - & - & - & - \\
\hline \multirow{3}{*}{\multicolumn{7}{|c|}{$\begin{array}{l}\text { South Danota } \\
\text { Heartland Consumers Power Dist/Dec } 31\end{array}$}} \\
\hline & & & & & & \\
\hline & & - & 630 & $\mathbf{9 , 9 9 9}$ & - & - \\
\hline Other Power .......................................................... & - & - & - & 132,430 & - & 416,048 \\
\hline Total Power & - & - & 630 & 142,429 & - & 416,048 \\
\hline Missouri Basin Mun Power Agny/Dec 31 & & & & & & \\
\hline 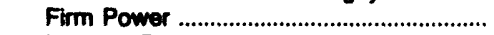 & - & - & - & - & $1,948,458$ & $40,093,645$ \\
\hline Nonfirm Power ............................................... & 4,387 & 54,889 & 4.248 & 63,975 & & 3,885 \\
\hline 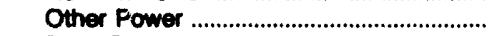 & - & - & & 685,960 & - & - \\
\hline Total Power ........................................................ & 4,387 & 54,889 & 4,248 & 749,935 & $1,948,658$ & $40,097,530$ \\
\hline Toxes & & & & & & \\
\hline Lower Colorado River Authority/Jun 30 & & & & & & \\
\hline Nontirm Power ............................................. & 2,484 & 40,011 & - & - & - & - \\
\hline 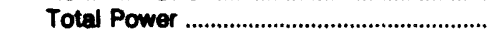 & 2,484 & 40,011 & - & - & - & - \\
\hline Sam Rayburn Mun Pwr Agny/Sep 30 & & & & & & \\
\hline 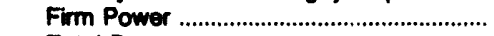 & - & - & - & - & - & - \\
\hline 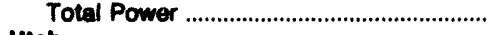 & - & - & - & - & - & - \\
\hline Utan & & & & & & \\
\hline Utah Associated Mun Power Sys/Mar 31 & & & & & & \\
\hline 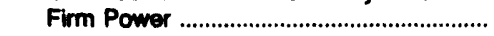 & 358,205 & $8,993,164$ & - & - & - & - \\
\hline Nonfirm Power .......................................... & 17,745 & 406,225 & - & - & 5,454 & 91,518 \\
\hline 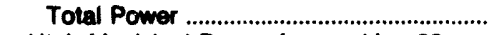 & 375,950 & $9,399,389$ & - & - & 5,454 & 91,518 \\
\hline Utah Municipal Power Agency/Jun 30 & & & & & & \\
\hline 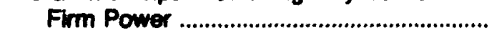 & 8,887 & 307,335 & 358,175 & $5,977,696$ & - & - \\
\hline 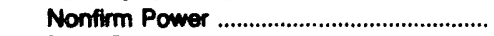 & 2,333 & 57,991 & - & & - & - \\
\hline 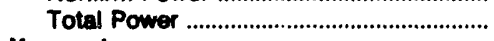 & 11,220 & 365,326 & 358,175 & $5,977,696$ & - & - \\
\hline $\begin{array}{l}\text { Vermont } \\
\text { Vermont Public Pwr Supply Auth/Dec } 31\end{array}$ & & & & & & \\
\hline 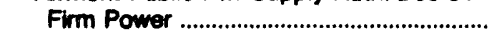 & 3 & 20,311 & - & - & 40 & 80,607 \\
\hline 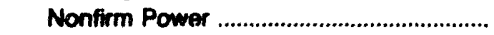 & 139 & 4,699 & - & - & 35 & 884 \\
\hline 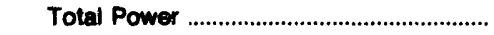 & 142 & 25,010 & - & - & 75 & 81,501 \\
\hline
\end{tabular}


Table 27. Electricity Purchaces by State and Other Government Utilties, Flecal Year 1992 (Continued)

\begin{tabular}{|c|c|c|c|c|c|c|}
\hline \multirow{3}{*}{$\begin{array}{l}\text { Etete / Uaitly I } \\
\text { Placel Yeer Ending Dote }\end{array}$} & \multicolumn{6}{|c|}{ Source of Electricity } \\
\hline & \multicolumn{2}{|c|}{ Cooperative } & \multicolumn{2}{|c|}{ Other' } & \multicolumn{2}{|c|}{ Total } \\
\hline & $\begin{array}{l}\text { Purchases } \\
\text { (thousand } \mathrm{kWh} \text { ) }\end{array}$ & $\begin{array}{l}\text { Cost } \\
\text { (dollara) }\end{array}$ & $\begin{array}{l}\text { Purchases } \\
\text { (thousand kWh) }\end{array}$ & $\begin{array}{c}\text { Cost } \\
\text { (dollars) }\end{array}$ & $\begin{array}{c}\text { Purchases } \\
\text { (thoueand } \mathrm{KW} / \mathrm{h} \text { ) }\end{array}$ & $\begin{array}{c}\text { Cost } \\
\text { (dollars) }\end{array}$ \\
\hline \multicolumn{7}{|l|}{ Oudemoma } \\
\hline 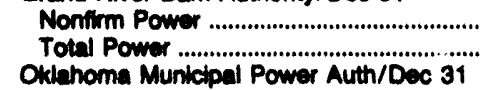 & $\begin{array}{l}31,251 \\
31,251\end{array}$ & $\begin{array}{l}651,743 \\
551,743\end{array}$ & - & - & $\begin{array}{l}96,074 \\
86,074\end{array}$ & $\begin{array}{l}1,588,219 \\
1,568,218\end{array}$ \\
\hline 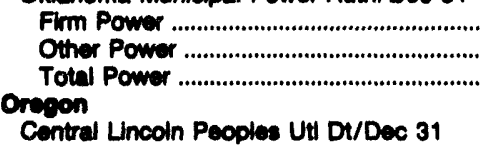 & $\begin{array}{r}156,701 \\
-158,701\end{array}$ & $\begin{array}{l}5,755,840 \\
5,755,840\end{array}$ & $\frac{142,084}{142,084}$ & $\begin{array}{r}4,736,889 \\
140,252 \\
4,877,141\end{array}$ & $\frac{644,109}{644,109}$ & $\begin{array}{r}23,625,539 \\
140,252 \\
23,785,791\end{array}$ \\
\hline 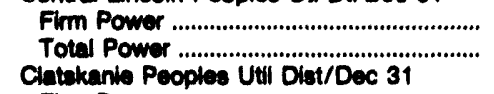 & $\begin{array}{l}66 \\
68\end{array}$ & $\begin{array}{l}1,793 \\
1,793\end{array}$ & $\begin{array}{l}26,043 \\
28,043\end{array}$ & $\begin{array}{l}109,046 \\
109,046\end{array}$ & $\begin{array}{l}1,371,010 \\
1,371,010\end{array}$ & $\begin{array}{l}31,853,172 \\
31,853,172\end{array}$ \\
\hline 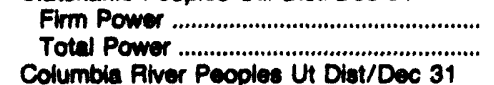 & - & - & $\overline{-}$ & $\overline{-}$ & $\begin{array}{l}883,763 \\
883,763\end{array}$ & $\begin{array}{l}20,164,953 \\
20,164,953\end{array}$ \\
\hline 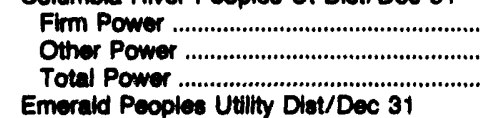 & $\overline{-}$ & $\overline{-}$ & $\overline{-}$ & $\begin{array}{l}303 \\
303\end{array}$ & $\begin{array}{l}274,491 \\
-274,481\end{array}$ & $\begin{array}{r}6,320,832 \\
303 \\
6,321,135\end{array}$ \\
\hline 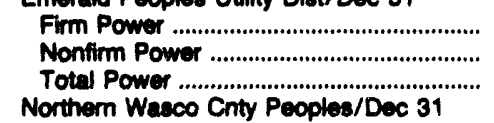 & $\bar{z}$ & $\overline{-}$ & $\begin{array}{l}100 \\
100\end{array}$ & $\begin{array}{r}- \\
2,457 \\
2,457\end{array}$ & $\begin{array}{r}377,157 \\
100 \\
377,257\end{array}$ & $\begin{array}{r}8,705,694 \\
2,457 \\
8,708,151\end{array}$ \\
\hline 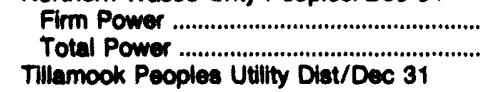 & $\overline{-}$ & - & $\overline{-}$ & $\overline{-}$ & $\begin{array}{l}238,532 \\
238,532\end{array}$ & $\begin{array}{l}5,976,792 \\
5,976,792\end{array}$ \\
\hline 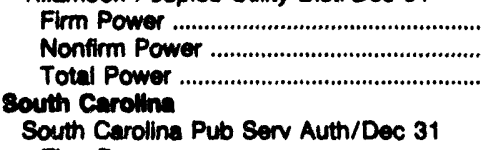 & $\overline{-}$ & - & $\begin{array}{r}13,020 \\
77 \\
13,097\end{array}$ & $\begin{array}{r}54,613 \\
1,201 \\
55,814\end{array}$ & $\begin{array}{r}365,157 \\
77 \\
355,234\end{array}$ & $\begin{array}{r}8,114,038 \\
1,201 \\
8,115,238\end{array}$ \\
\hline 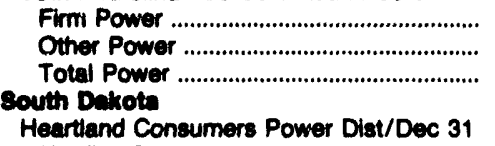 & - & $\overline{-}$ & $\begin{array}{r}1,782 \\
-81,442 \\
-79,650\end{array}$ & $\begin{array}{r}14,336 \\
1,237,709 \\
1,252,045\end{array}$ & $\begin{array}{r}428,883 \\
27,707 \\
458,680\end{array}$ & $\begin{array}{r}7,723,158 \\
2,701,595 \\
10,424,754\end{array}$ \\
\hline 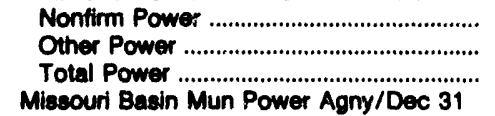 & $\begin{array}{r}6,030 \\
6,030\end{array}$ & $\begin{array}{l}102,600 \\
102,600\end{array}$ & $\begin{array}{l}13,287 \\
12,240 \\
25,527\end{array}$ & $\begin{array}{l}179,144 \\
227,000 \\
408,144\end{array}$ & $\begin{array}{l}13,917 \\
18,270 \\
32,187\end{array}$ & $\begin{array}{r}189,143 \\
878,078 \\
1,067,221\end{array}$ \\
\hline 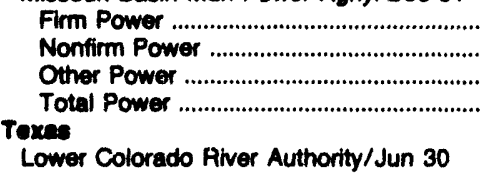 & $\begin{array}{r}-21,586 \\
-21,586\end{array}$ & $\begin{array}{l}-254,124 \\
- \\
254,124\end{array}$ & $\begin{array}{r}-4,408 \\
-\quad 4,408\end{array}$ & $\begin{array}{r}-54,035 \\
2,543 \\
56,578\end{array}$ & $\begin{array}{r}1,948,458 \\
34,829 \\
-\quad \\
1,983,287\end{array}$ & $\begin{array}{r}40,093,645 \\
430,908 \\
688,503 \\
41,213,056\end{array}$ \\
\hline $\begin{array}{l}\text { Nonfirm Power } \\
\text { Total Power } \\
\text { Sam Rayburn Mun Pw Agny/Sep } 30\end{array}$ & - & - & - & - & $\begin{array}{l}2,484 \\
2,484\end{array}$ & $\begin{array}{l}40,011 \\
40,011\end{array}$ \\
\hline $\begin{array}{l}\text { Firm Power } \\
\text { Total Power } \\
\text { Uth } \\
\text { Utah Associated Mun Power Sys/Mar } 31\end{array}$ & $\begin{array}{l}87,716 \\
87,716\end{array}$ & $\begin{array}{l}2,514,673 \\
2,514,673\end{array}$ & - & $\overline{-}$ & $\begin{array}{l}87,716 \\
87,716\end{array}$ & $\begin{array}{l}2,514,673 \\
2,514,673\end{array}$ \\
\hline 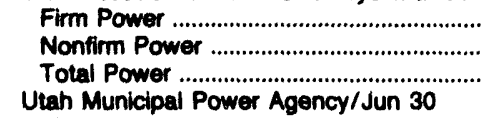 & $\begin{array}{l}- \\
218,065 \\
218,065\end{array}$ & $\begin{array}{l}\overrightarrow{3,819,794} \\
3,819,794\end{array}$ & $\begin{array}{r}- \\
52,728 \\
52,728\end{array}$ & $\begin{array}{l}\overline{967,942} \\
967,942\end{array}$ & $\begin{array}{l}358,205 \\
293,992 \\
652,197\end{array}$ & $\begin{array}{r}8,993,164 \\
5,285,479 \\
14,278,643\end{array}$ \\
\hline 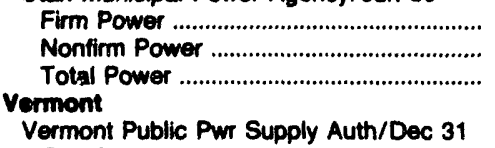 & $\begin{array}{r}5,580 \\
5,590\end{array}$ & $\begin{array}{r}91,478 \\
91,478\end{array}$ & $\begin{array}{l}6,021 \\
6,021\end{array}$ & $\begin{array}{r}- \\
98,778 \\
98,778\end{array}$ & $\begin{array}{r}367,062 \\
13,944 \\
381,006\end{array}$ & $\begin{array}{r}6,285,031 \\
248,247 \\
6,533,278\end{array}$ \\
\hline 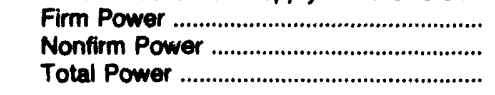 & $\overline{-}$ & - & $\begin{array}{r}99,411 \\
421 \\
99,832\end{array}$ & $\begin{array}{r}2,586,615 \\
12,080 \\
2,588,695\end{array}$ & $\begin{array}{r}99,454 \\
595 \\
100,049\end{array}$ & $\begin{array}{r}2,687,533 \\
17,673 \\
2,705,206\end{array}$ \\
\hline
\end{tabular}

See notes and footnotes at end of table. 
Table 27. Electriclty Purchases by State and Other Government Utilities, Flecal Year 1992 (Continued)

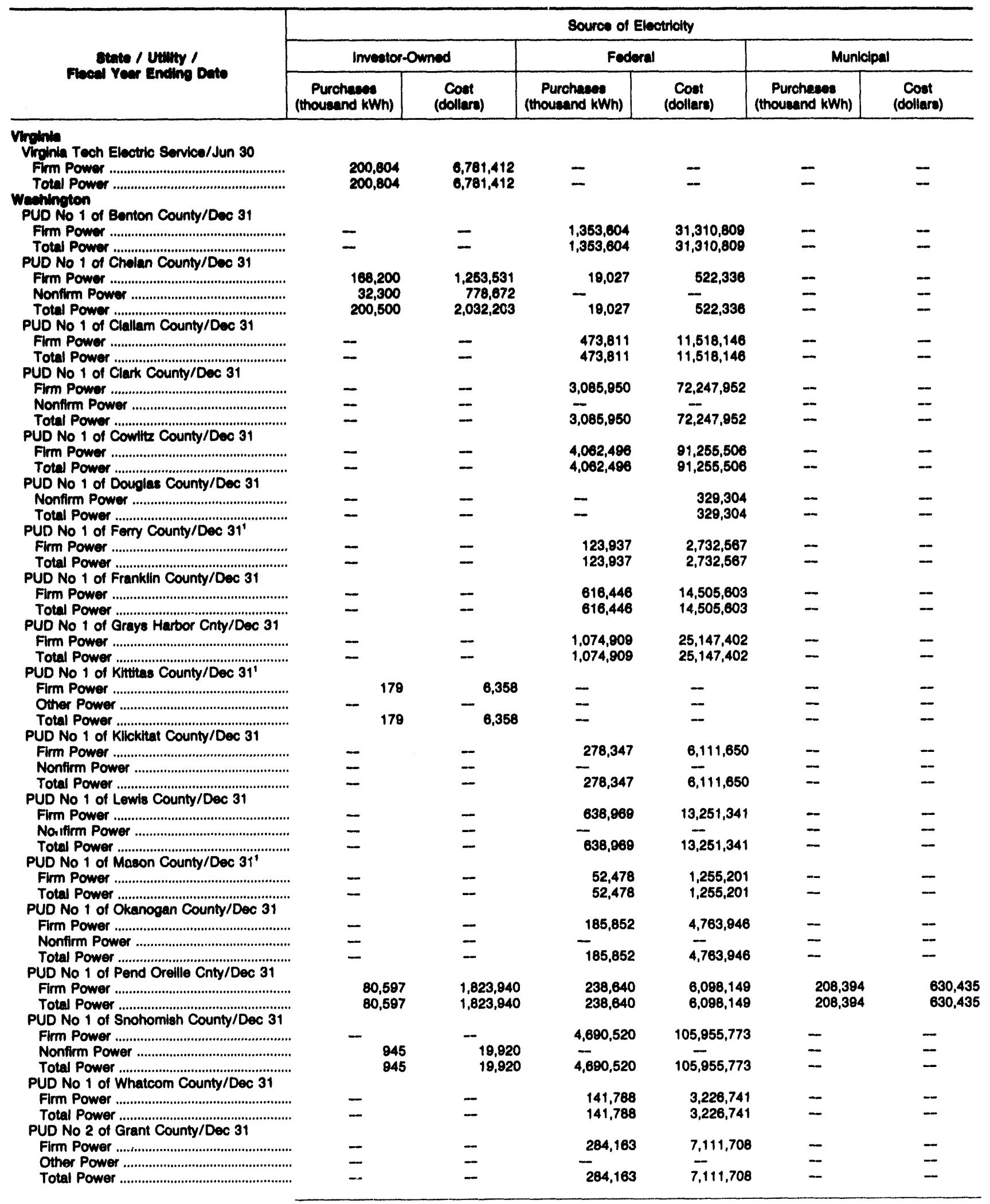

See notes and footnotes at end of table. 
Table 27. Electricity Purchases by State and Other Government Utilities, Fiscal Year 1992 (Continued)

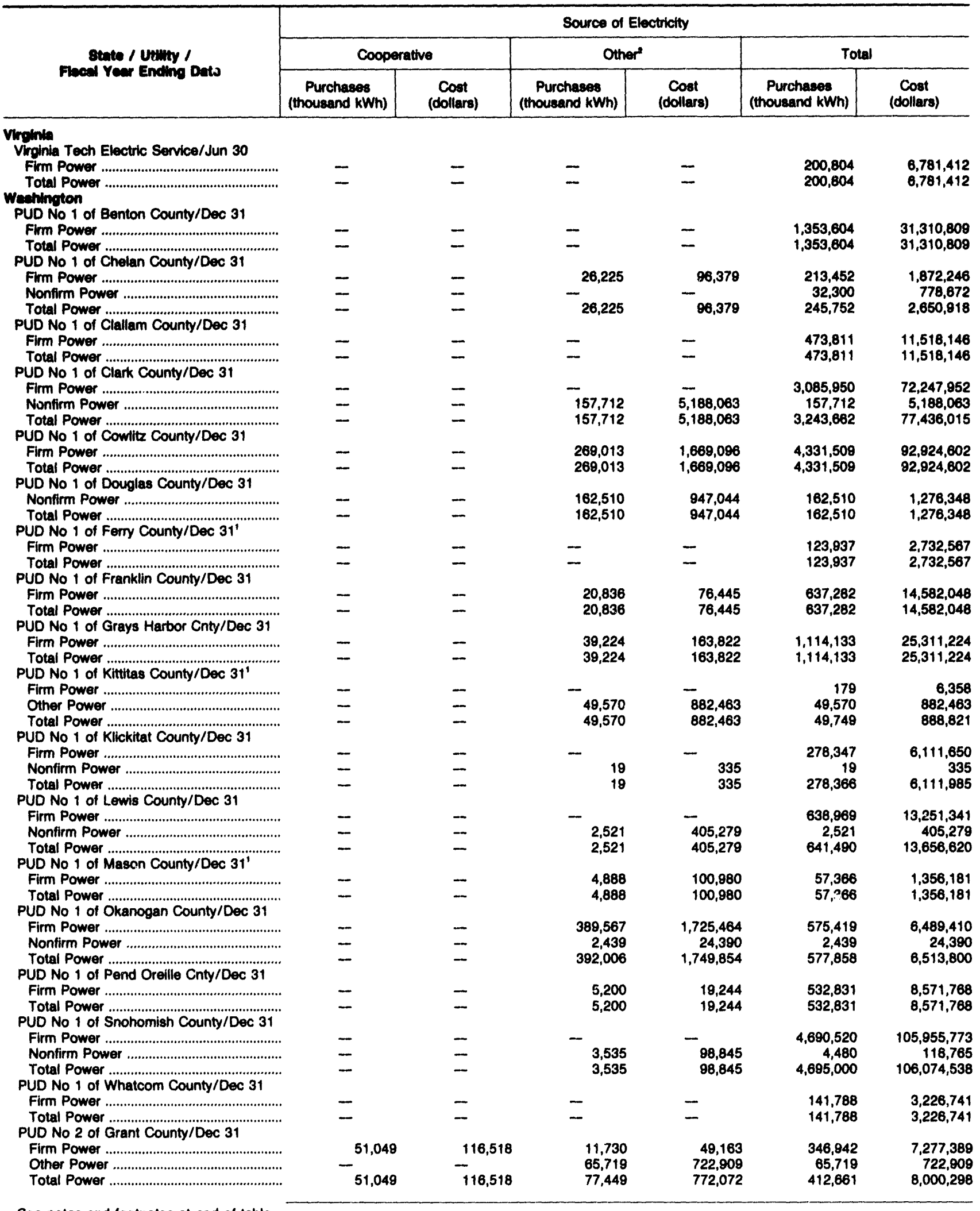

See notes and footnotes at end of table. 
Table 27. Electricity Purchases by State and Other Government Utilities, Fiscal Year 1992 (Continued)

\begin{tabular}{|c|c|c|c|c|c|c|}
\hline \multirow{3}{*}{$\begin{array}{l}\text { Btate / Unilty / } \\
\text { Flecel Year Ending Date }\end{array}$} & \multicolumn{6}{|c|}{ Source of Electricity } \\
\hline & \multicolumn{2}{|c|}{ Investor-Owned } & \multicolumn{2}{|c|}{ Federal } & \multicolumn{2}{|c|}{ Munioipal } \\
\hline & $\begin{array}{l}\text { Purchases } \\
\text { (thousand kWh) }\end{array}$ & $\begin{array}{c}\text { Cost } \\
\text { (dollars) }\end{array}$ & $\begin{array}{l}\text { Purchases } \\
\text { (thousand kWh) }\end{array}$ & $\begin{array}{c}\text { Cost } \\
\text { (dollars) }\end{array}$ & $\begin{array}{c}\text { Purchases } \\
\text { (thousand kWh) }\end{array}$ & $\begin{array}{c}\text { Cost } \\
\text { (dollars) }\end{array}$ \\
\hline \multicolumn{7}{|l|}{$\begin{array}{l}\text { Weehingten } \\
\text { PUD No } 2 \text { of Pactic County/Dec } 31\end{array}$} \\
\hline 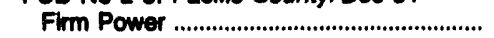 & - & - & 255,242 & $6,516,258$ & - & - \\
\hline $\begin{array}{l}\text { Total Power ............. } \\
\text { PUD No } 3 \text { of Mason County/Dec } 31\end{array}$ & - & - & 255,242 & $6,516,258$ & - & - \\
\hline 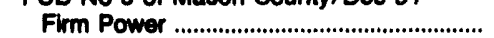 & - & - & 476,074 & $12,105,895$ & - & - \\
\hline $\begin{array}{l}\text { Total Power ..................................... } \\
\text { Vera Imigation District } \$ 15 / \text { Dec } 31\end{array}$ & - & - & 476,074 & $12,105,885$ & - & - \\
\hline 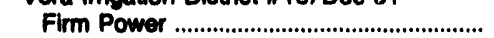 & - & - & 164,812 & $4,004,745$ & - & - \\
\hline 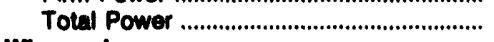 & - & - & 164,812 & $4,004,745$ & - & - \\
\hline \multicolumn{7}{|l|}{ Wisconsin Public Power Inc Sys/Dec 31} \\
\hline 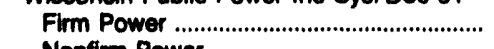 & $1,955,341$ & $71,387,009$ & - & - & 14,474 & $3,027,889$ \\
\hline 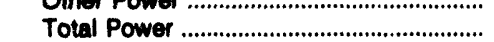 & $2,281,770$ & $76,009,294$ & 3,398 & 45,217 & 14,534 & $3,029,090$ \\
\hline \multicolumn{7}{|l|}{ Wyoming Municipal Pwr Agcy/Dec 31} \\
\hline 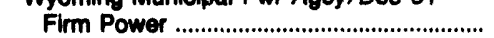 & - & - & 56,471 & $1,169,434$ & - & - \\
\hline 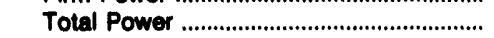 & - & - & 56,471 & $1,169,434$ & - & - \\
\hline
\end{tabular}

\begin{tabular}{|c|c|c|c|c|c|}
\hline \multicolumn{6}{|c|}{ Source of Electricity } \\
\hline \multicolumn{2}{|c|}{ Cooperative } & \multicolumn{2}{|c|}{ Other ${ }^{2}$} & \multicolumn{2}{|c|}{ Total } \\
\hline $\begin{array}{c}\text { Purchases } \\
\text { (thousand kWh) }\end{array}$ & $\begin{array}{c}\text { Cost } \\
\text { (dollars) }\end{array}$ & $\begin{array}{c}\text { Purchases } \\
\text { (thousand kWh) }\end{array}$ & $\begin{array}{c}\text { Cost } \\
\text { (dollars) }\end{array}$ & $\begin{array}{c}\text { Purchases } \\
\text { (thousand kWh) }\end{array}$ & $\begin{array}{c}\text { Cost } \\
\text { (dollars) }\end{array}$ \\
\hline
\end{tabular}

Weshington

PUD No 2 of Pacific County/Dec 31

Firm Power

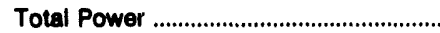

PUD No 3 of Mason County/Dec 31

Firm Power

Total Power

Vera Imigation District \#15/Dec 31

Firm Power

Total Power

Moconein

Wisconsin Public Power Inc Sys/Dec 31

Firm Power...

Nonfirm Power

Other Power

Total Power

Wyoming

Wyorning Municipal Pwr Agcy/Dec 31

Firm Power

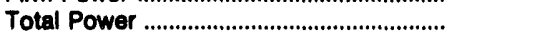

$\begin{array}{ll} & \\ & \end{array}$

$=$

$\overline{-}$

$\overline{-}$

$255,242 \quad 6,516,258$

$=$

$-\quad \overline{-}$

$6,516,258$

$\begin{array}{rrr}-52,152 & -563,692 \\ -52,152 & -563,692\end{array}$

$-$

476,074

Source of data is Rural Electrification Administration, REA Form 7. "Financial and Statistical Report."

2 Includes transactions with State and other government utilities, power pools, utilities in Canada and Mexico, and nonutlities.

- Not Applicable

Notes: •For identification purposes, the public utilities are listed in the State in which the administrative office is located. $\bullet$ Totals may not equal sum of components because of independent rounding.

Source: Energy Information Administration, Form EIA-412, "Annual Report of Public Electric Utilities," except where footnoted. 
Table 28. Electricity Sales for Resale by State and Other Government Utilities, Flecal Year 1992

\begin{tabular}{|c|c|c|c|c|c|c|}
\hline \multirow{3}{*}{$\begin{array}{l}\text { State / Utility ' } \\
\text { Fiecal Year Ending Dato }\end{array}$} & \multicolumn{6}{|c|}{ Purchaeer of Electricity } \\
\hline & \multicolumn{2}{|c|}{ Investor-Owned } & \multicolumn{2}{|c|}{ Foderal } & \multicolumn{2}{|c|}{ Municipal } \\
\hline & $\begin{array}{c}\text { Sales for } \\
\text { Pesale } \\
\text { (thousand kWh) }\end{array}$ & $\begin{array}{l}\text { Revenue } \\
\text { (dollare) }\end{array}$ & $\begin{array}{c}\text { Sales for } \\
\text { Reesale } \\
\text { (thousand } \mathrm{kWh} \text { ) }\end{array}$ & $\begin{array}{l}\text { Revenue } \\
\text { (dollare) }\end{array}$ & $\begin{array}{c}\text { Salos for } \\
\text { Recale } \\
\text { (thousand kWh) }\end{array}$ & $\begin{array}{l}\text { Revenue } \\
\text { (dollars) }\end{array}$ \\
\hline \multicolumn{7}{|l|}{$\begin{array}{l}\text { Aleaka } \\
\text { Aleakn Energy Authority/Jun } 30\end{array}$} \\
\hline 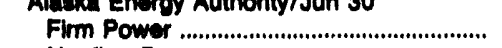 & - & - & - & - & 192,365 & $6,800,056$ \\
\hline Nonfirm Power ............................................. & - & - & - & - & 15,537 & 159,087 \\
\hline 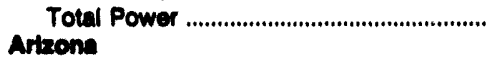 & - & - & - & - & 207,902 & $6,959,123$ \\
\hline \multicolumn{7}{|l|}{$\begin{array}{l}\text { Artzona } \\
\text { Artzons Power Authorty/Jun } 30\end{array}$} \\
\hline 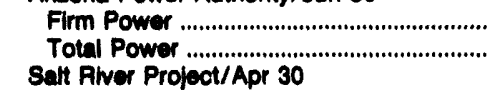 & $\begin{array}{l}33,243 \\
33,243\end{array}$ & $\begin{array}{l}811,261 \\
811,261\end{array}$ & $\begin{array}{l}2,423 \\
2,423\end{array}$ & $\begin{array}{l}57,854 \\
57,854\end{array}$ & $\begin{array}{l}26,260 \\
26,260\end{array}$ & $\begin{array}{l}665,629 \\
665,629\end{array}$ \\
\hline 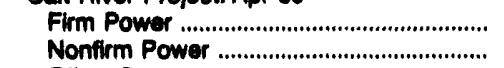 & $\begin{array}{r}1,645,419 \\
908,233\end{array}$ & $\begin{array}{l}53,863,269 \\
15,436,778\end{array}$ & $\begin{array}{l}4,041 \\
3,040\end{array}$ & $\begin{array}{r}556,458 \\
41,365\end{array}$ & $\begin{array}{r}24,501 \\
622,445\end{array}$ & $\begin{array}{r}2,481,672 \\
11,046,089\end{array}$ \\
\hline 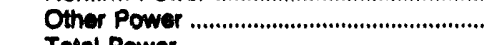 & & - & - & & - & \\
\hline 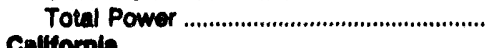 & $2,554,652$ & $68,298,047$ & 7,081 & 597,813 & 646,846 & $13,527,771$ \\
\hline \multicolumn{7}{|l|}{$\begin{array}{l}\text { Calfiomla } \\
\text { Cellitornia Dept Wtr Resources/Dec } 31\end{array}$} \\
\hline Firm Power & $\begin{array}{l}239,530 \\
332,462\end{array}$ & $\begin{array}{l}7,227,794 \\
6,898,781\end{array}$ & $-\overline{117,294}$ & $\overline{2,405,248}$ & $\begin{array}{l}294,308 \\
148,726\end{array}$ & $\begin{array}{r}18,079,905 \\
3,207,883\end{array}$ \\
\hline Other Power ……………………………..... & - & - & - & $-2,40,240$ & - $100,1<0$ & 663,600 \\
\hline $\begin{array}{l}\text { Total Power .......................................... } \\
\text { Imperial Imrloation Dlatrict/Doc } 31\end{array}$ & 571,882 & $14,126,575$ & 117,294 & $2,405,246$ & 444,034 & $21,951,388$ \\
\hline 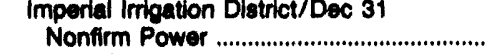 & - & - & - & - & - & - \\
\hline 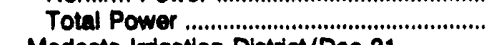 & - & - & - & - & - & - \\
\hline \multicolumn{2}{|l|}{$\begin{array}{l}\text { Modeato Irrigation District/Dec } 31 \\
\text { Nonfirm Power }\end{array}$} & & - & - & 35,161 & 892,512 \\
\hline 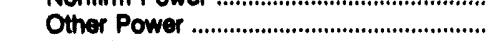 & 685 & 27,249 & - & - & - & - \\
\hline \multirow{2}{*}{\multicolumn{6}{|c|}{ 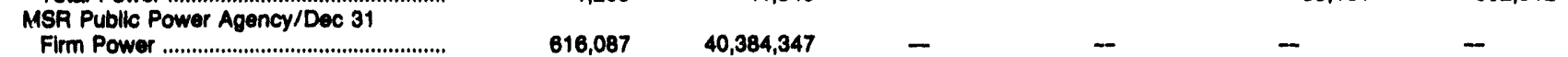 }} & 892,512 \\
\hline & 616,087 & $40,384,347$ & - & - & - & - \\
\hline 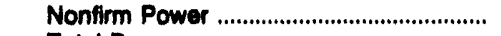 & 178,892 & $3,642,243$ & - & - & - & - \\
\hline \multicolumn{6}{|l|}{$\begin{array}{l}\text { Total Power .............................. } \\
\text { Northern Calfornia Power Agny/Jun } 30\end{array}$} & \\
\hline 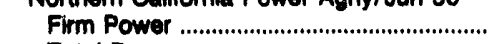 & - & - & - & - & $1,720,766$ & $153,089,000$ \\
\hline $\begin{array}{l}\text { Total Power ................................... } \\
\text { Oakdale \& South San Joaun/Dec }\end{array}$ & - & - & - & - & $1,720,766$ & $153,089,000$ \\
\hline 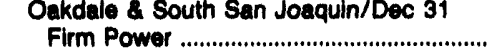 & 283,319 & $5,275,131$ & - & - & -- & - \\
\hline 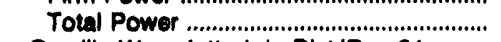 & 283,310 & $5,275,131$ & - & - & - & - \\
\hline \multicolumn{7}{|l|}{ Oroville-Wyandotte Irrig Diat/Dec 31} \\
\hline 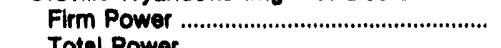 & 346,201 & $8,300,000$ & - & - & - & - \\
\hline \multicolumn{7}{|l|}{ Placer County Water Agency/Dec 31} \\
\hline 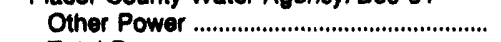 & 322,314 & $7,688,330$ & - & - & - & - \\
\hline \multicolumn{7}{|l|}{ Southern Callifornia P P A/Jun 30} \\
\hline 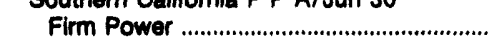 & - & - & - & - & $1,340,821$ & $115,136,445$ \\
\hline \multirow{2}{*}{\multicolumn{7}{|c|}{ 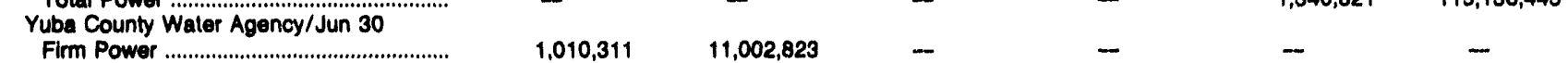 }} \\
\hline & & & & & & \\
\hline 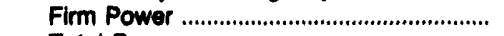 & $1,010,311$ & $11,002,823$ & - & - & - & - \\
\hline 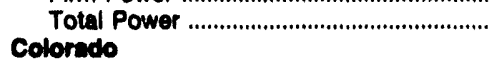 & $1,010,311$ & $11,002,823$ & - & -- & - & - \\
\hline \multicolumn{7}{|l|}{$\begin{array}{l}\text { Colorado } \\
\text { Arkanses River Power Authority/Dec } 31\end{array}$} \\
\hline 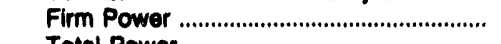 & - & - & - & - & 265,274 & $9,602,654$ \\
\hline $\begin{array}{l}\text { Total Power } \\
\text { Platte River Power Authority/Dec } 31\end{array}$ & - & - & - & - & 265,274 & 9,602 \\
\hline 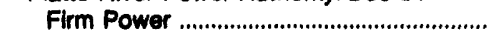 & $1,180,132$ & $69,448,455$ & - & - & $1,745,144$ & $50,589,809$ \\
\hline Nonfirm Power ........................................... & 108,882 & $1,600,711$ & 19,299 & 313,088 & 10,526 & 171,291 \\
\hline 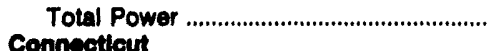 & $1,289,014$ & $71,048,166$ & 19,299 & 313,088 & $1,755,670$ & $59,761,100$ \\
\hline \multicolumn{7}{|l|}{$\begin{array}{l}\text { Connectlout } \\
\text { Mun Electric Engy Coop/Dec } 31\end{array}$} \\
\hline 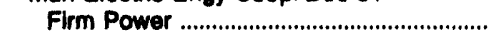 & - & - & - & - & $1,070,571$ & $59,814,365$ \\
\hline 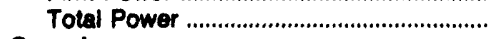 & - & - & - & - & $1,070,571$ & $59,814,365$ \\
\hline \multicolumn{7}{|l|}{$\begin{array}{l}\text { Coorgla } \\
\text { Municipal Electric Auth of GA/Dec } 31\end{array}$} \\
\hline 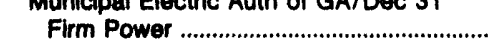 & - & _- & _- & - & $6,190,691$ & $319,544,755$ \\
\hline Nonfirm Power ........................................ & $3,280,181$ & $210,048,000$ & - & - & & - \\
\hline Total Power ……………………………...... & $3,280,181$ & $210,048,000$ & - & - & $6,190,691$ & $319,544,755$ \\
\hline
\end{tabular}

See notes and footnotes at end of table. 
Table 28. Electricity Sales for Reaale by State and Other Government Utilities, Flecal Year 1992 (Continued)

\begin{tabular}{|c|c|c|c|c|c|c|}
\hline \multirow{3}{*}{$\begin{array}{l}\text { State / Utllty I } \\
\text { Fiecal Year Ending Date }\end{array}$} & \multicolumn{6}{|c|}{ Purchaeer of Electricity } \\
\hline & \multicolumn{2}{|c|}{ Cooperatve } & \multicolumn{2}{|c|}{ Other' } & \multicolumn{2}{|c|}{ Total } \\
\hline & $\begin{array}{c}\text { Sales for } \\
\text { Resale } \\
\text { (thousand kWh) }\end{array}$ & $\begin{array}{l}\text { Revenue } \\
\text { (dollars) }\end{array}$ & $\begin{array}{c}\text { Sales for } \\
\text { Pesale } \\
\text { (thousend kWh) }\end{array}$ & $\begin{array}{l}\text { Rovenue } \\
\text { (dollars) }\end{array}$ & $\begin{array}{c}\text { Sales for } \\
\text { Resale } \\
\text { (thousand kWh) }\end{array}$ & $\begin{array}{l}\text { Revenue } \\
\text { (dollars) }\end{array}$ \\
\hline \multicolumn{7}{|l|}{ Alacka } \\
\hline $\begin{array}{l}\text { Alacka Energy Authority/Jun } 30 \\
\text { Firm Power }\end{array}$ & 412,864 & $10,679,015$ & - & - & 605,229 & $17,479,071$ \\
\hline 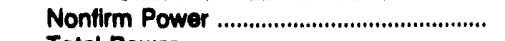 & - & - & - & - & 15,537 & 169,067 \\
\hline 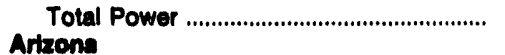 & 412,864 & $10,679,015$ & - & - & 620,768 & $17,638,138$ \\
\hline \\
\hline Firm Power & $\begin{array}{l}80,467 \\
80,467\end{array}$ & $\begin{array}{l}2,026,146 \\
2,026,146\end{array}$ & $\begin{array}{l}598,112 \\
598,112\end{array}$ & $\begin{array}{l}13,406,522 \\
13,406,522\end{array}$ & $\begin{array}{l}740,505 \\
740,506\end{array}$ & $\begin{array}{l}16,967,412 \\
16,967,412\end{array}$ \\
\hline Salt Piver Projoct/Apr 30 & & & & & & \\
\hline 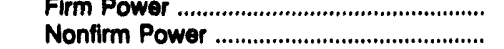 & $\begin{array}{r}135 \\
12,083\end{array}$ & $\begin{array}{r}3,035 \\
223,914\end{array}$ & $\begin{array}{r}69,773 \\
302,692\end{array}$ & $\begin{array}{l}1,653,840 \\
5,705,217\end{array}$ & $\begin{array}{l}1,743,869 \\
1,849,473\end{array}$ & $\begin{array}{l}58,558,274 \\
32,452,363\end{array}$ \\
\hline 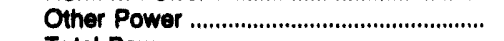 & & & & $1,115,189$ & - & $1,115,180$ \\
\hline 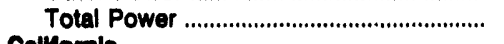 & 12,188 & 228,949 & 372,465 & $8,474,246$ & $3,593,342$ & $92,125,826$ \\
\hline \multicolumn{7}{|l|}{$\begin{array}{l}\text { Callormla } \\
\text { Calfornia Dept Wtr Resources/Dec } 31\end{array}$} \\
\hline 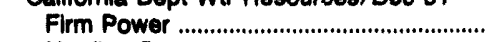 & - & - & $1,333,788$ & $34,343,862$ & $1,867,636$ & $58,651,561$ \\
\hline 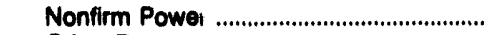 & - & - & - & - & 599,472 & $12,511,910$ \\
\hline 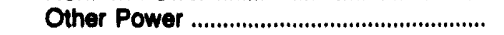 & - & - & - & - & - & 663,600 \\
\hline 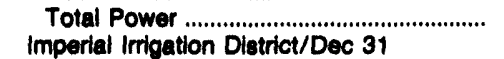 & - & - & $1,333,798$ & $34,343,862$ & $2,467,108$ & $72,827,071$ \\
\hline 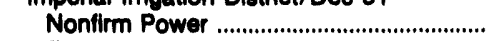 & - & - & 210 & 4,830 & 210 & 4,830 \\
\hline 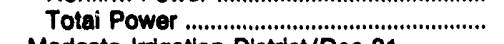 & - & - & 210 & 4,830 & 210 & 4,830 \\
\hline \multicolumn{7}{|l|}{ Modesto Irrigation District/Dec 31} \\
\hline 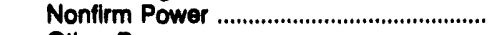 & - & - & 7,367 & 198,909 & 43,048 & $1,105,721$ \\
\hline 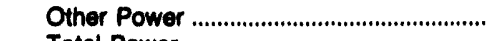 & - & - & - & & 685 & 27,249 \\
\hline 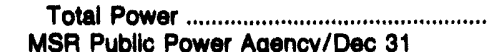 & - & - & 7,367 & 188,909 & 43,733 & $1,132,970$ \\
\hline 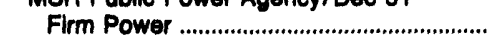 & - & - & - & - & 616.087 & 40.384 .347 \\
\hline 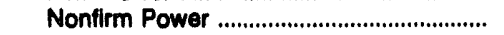 & - & - & 1,887 & 73,027 & 180,778 & $3,715,270$ \\
\hline 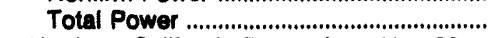 & - & - & 1,887 & 73,027 & 796,866 & $44,099,617$ \\
\hline \multicolumn{7}{|l|}{ Northern Calfornia Power Agny/Jun 30} \\
\hline 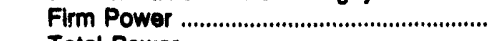 & 13,346 & 422,000 & - & -- & $1,734,112$ & $153,521,000$ \\
\hline \multicolumn{7}{|l|}{ Oakdale \& South San Joaquin/Dec 31} \\
\hline 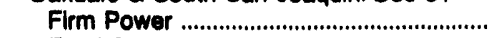 & - & - & -- & - & 283,319 & $5,275,131$ \\
\hline \multirow{2}{*}{\multicolumn{7}{|c|}{ 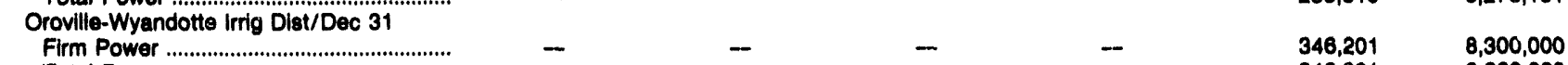 }} \\
\hline & - & -- & - & & & \\
\hline 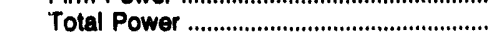 & - & - & - & $\overline{-}$ & 346,201 & $8,300,000$ \\
\hline \multicolumn{7}{|l|}{ Total Power . } \\
\hline 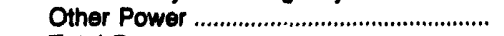 & & - & & - & 322,314 & $7,668,330$ \\
\hline 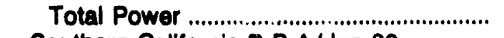 & - & - & - & - & 322,314 & $7,668,330$ \\
\hline \multicolumn{7}{|l|}{ Southern Calfornia P P A/Jun 30} \\
\hline Firm Power ........................................................ & - & -- & 93,212 & $7,885,504$ & $1,434,033$ & $123,031,949$ \\
\hline \multirow{2}{*}{\multicolumn{7}{|c|}{ Yuba County Wator Agency/Jun 30}} \\
\hline & & & & & & \\
\hline 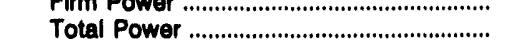 & $\overline{-}$ & - & $\overline{-}$ & $\overline{-}$ & $\begin{array}{l}1,010,311 \\
1,010,311\end{array}$ & $\begin{array}{l}11,002,823 \\
11,002,823\end{array}$ \\
\hline \multirow{2}{*}{\multicolumn{7}{|c|}{$\begin{array}{l}\text { Colorado } \\
\text { Arkansas Rlver Power Authority/Dec } 31\end{array}$}} \\
\hline & & - & & & & \\
\hline $\begin{array}{l}\text { Firm Power } \\
\text { Total Power }\end{array}$ & - & $\overline{-}$ & $\overline{-}$ & - & 265,274 & $8,602,654$ \\
\hline $\begin{array}{l}\text { Total Power } \\
\text { Platte Alver Pover Authority/Dec } 31\end{array}$ & & & & - & 274 & \\
\hline 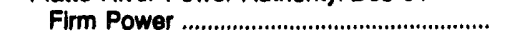 & - & -- & - & - & $2,925,276$ & $129,038,264$ \\
\hline 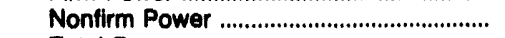 & 18,251 & 272,873 & 208,577 & $3,741,990$ & 366,535 & $6,099,953$ \\
\hline 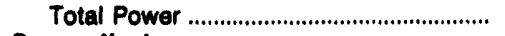 & 19,251 & 272,873 & 208,577 & $3,741,890$ & $3,291,811$ & $135,138,217$ \\
\hline $\begin{array}{l}\text { Connectlcut } \\
\text { Mun Electric Engy Coop/Dec } 31\end{array}$ & & & & & & \\
\hline $\begin{array}{l}\text { Mun Electrlc Engy Coop/Dec } 31 \\
\text { Firm Power }\end{array}$ & - & -- & -. & - & $1,070,571$ & $59,814,365$ \\
\hline 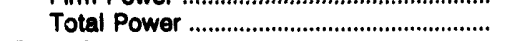 & - & -- & - & - & $1,070,571$ & $59,814,365$ \\
\hline Goorgla & & & & & & \\
\hline $\begin{array}{l}\text { Municipal Electric Auth of GA/Dec } 31 \\
\text { Firm Power }\end{array}$ & & & & & & 328.048724 \\
\hline $\begin{array}{l}\text { Firm Power } \\
\text { Nonfirm Puwer }\end{array}$ & $\overline{-}$ & $\overline{-}$ & $-212,550$ & - & $\begin{array}{l}6,403,241 \\
3,280,181\end{array}$ & $\begin{array}{l}328,048,724 \\
210,048,000\end{array}$ \\
\hline $\begin{array}{l}\text { Nonfirm Puwer } \\
\text { Total Power }\end{array}$ & -- & - & 212,550 & $8,503,969$ & $9,683,422$ & $538,096,724$ \\
\hline & & & & & & \\
\hline
\end{tabular}

See notes and footnotes at end of table. 
Table 28. Electricity Sales for Resale by State and Other Govornment Utilitles, Flecal Year 1992 (Continued)

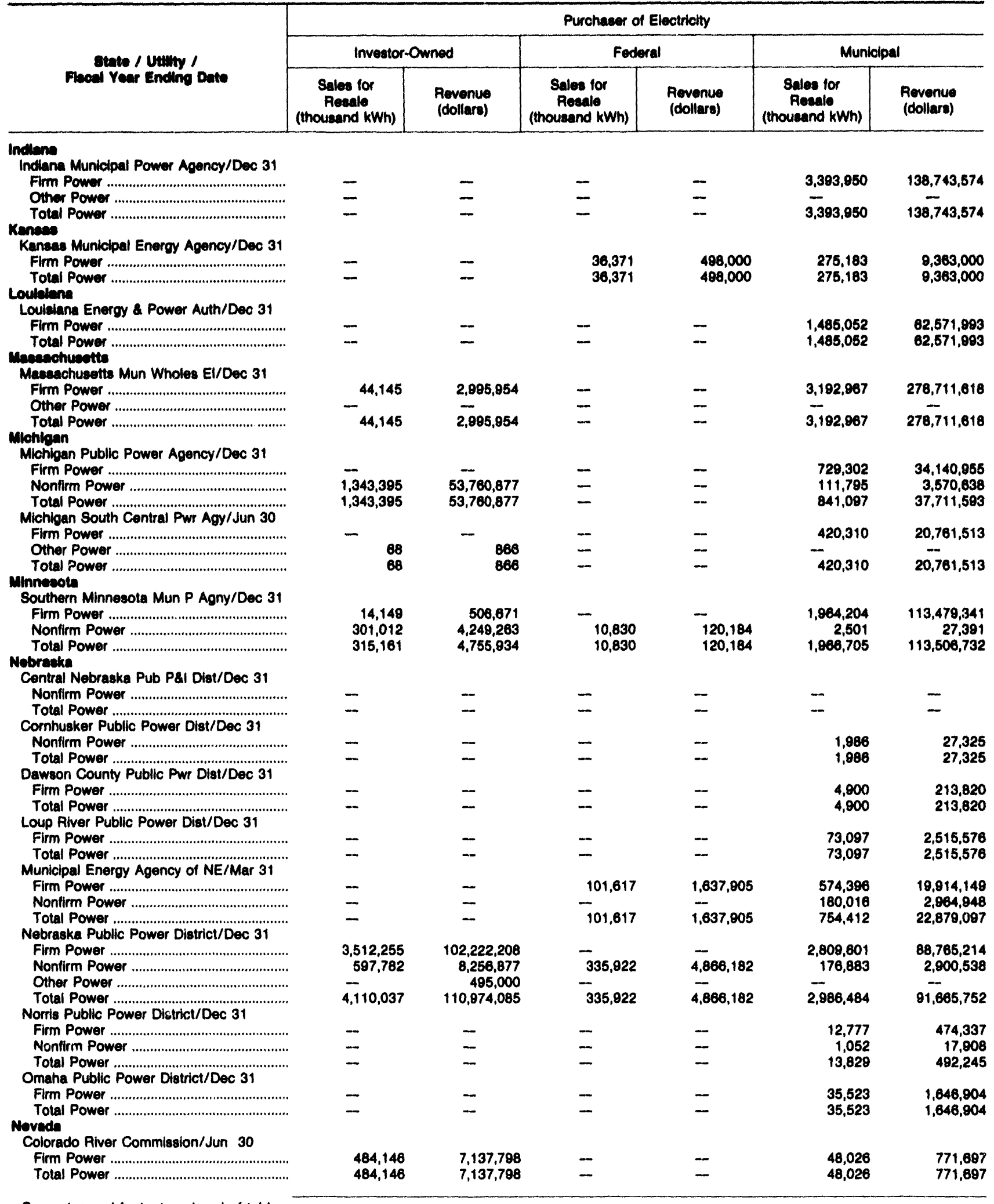


Table 28. Electriclity 8ales for Recalo by State and Other Government Utilites, Flecal Year 1992 (Continued)

\begin{tabular}{|c|c|c|c|c|c|c|}
\hline \multirow{3}{*}{$\begin{array}{l}\text { Stets / Utilty I } \\
\text { Fiecal Year Ending Date }\end{array}$} & \multicolumn{6}{|c|}{ Purchaser of Eleotriclty } \\
\hline & \multicolumn{2}{|c|}{ Cooperative } & \multicolumn{2}{|c|}{ Other' } & \multicolumn{2}{|c|}{ Total } \\
\hline & $\begin{array}{c}\text { Sales for } \\
\text { Reanlo } \\
\text { (thousand KWh) }\end{array}$ & $\begin{array}{l}\text { Rovenus } \\
\text { (dollars) }\end{array}$ & $\begin{array}{c}\text { 8ales for } \\
\text { Peaslo } \\
\text { (thousand kWh) }\end{array}$ & $\begin{array}{l}\text { Revenue } \\
\text { (dollars) }\end{array}$ & $\begin{array}{c}\text { Sales for } \\
\text { Resale } \\
\text { (thousand kWh) }\end{array}$ & $\begin{array}{l}\text { Rovenue } \\
\text { (dollars) }\end{array}$ \\
\hline \multicolumn{7}{|l|}{$\begin{array}{l}\text { Incliena } \\
\text { Indiana Municipal Power Agency/Dec } 31\end{array}$} \\
\hline 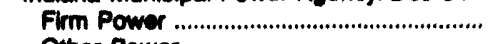 & -- & - & - & - & $3,393,950$ & $138,743,674$ \\
\hline 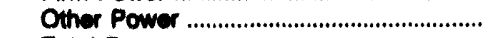 & - & - & - & $3,009,335$ & - & $3,000,336$ \\
\hline 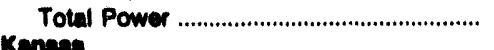 & - & - & - & $3,009,335$ & $3,303,850$ & $141,752,009$ \\
\hline \multicolumn{7}{|l|}{$\begin{array}{l}\text { Kenease } \\
\text { Kaneas Municipal Energy Agency/Dec } 31\end{array}$} \\
\hline Firm Power ......................................... & - & - & - & - & 311,554 & $8,801,000$ \\
\hline Tolal Power ................................................. & - & - & - & - & 311,564 & $8,861,000$ \\
\hline \multicolumn{7}{|l|}{$\begin{array}{l}\text { Loulalena } \\
\text { Loulaiana Energy \& Power Auth/Dec } 31\end{array}$} \\
\hline Firm Power ................................. & - & - & - & - & $1,486,052$ & $62,671,903$ \\
\hline Total Power & - & - & - & - & $1,485,052$ & $62,571,993$ \\
\hline \multicolumn{7}{|l|}{$\begin{array}{l}\text { Meseachueette } \\
\text { Massachusetts Mun Wholes El/Dec } 31\end{array}$} \\
\hline 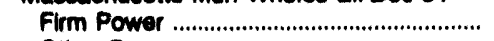 & - & - & 252 & 7.114 & $3,237,384$ & $281,714,686$ \\
\hline 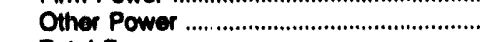 & - & - & - & $-8,268,090$ & - & $-9,266,090$ \\
\hline 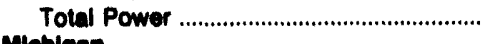 & - & - & 252 & $-9,258,978$ & $3,237,364$ & $272,446,586$ \\
\hline \multicolumn{7}{|l|}{$\begin{array}{l}\text { Mlohigan } \\
\text { Michigan Public Power Agency/Dec } 31\end{array}$} \\
\hline 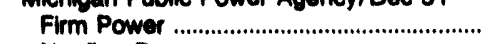 & - & - & - & - & 729,302 & $34,140,956$ \\
\hline $\begin{array}{l}\text { Nonfirm Power } \\
\text { Total Power }\end{array}$ & 28,412 & 818,758 & - & - & $1,483,602$ & $58,160,273$ \\
\hline \multicolumn{7}{|l|}{ Michigan South Central Pwr Agy/Jun 30} \\
\hline 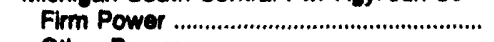 & - & - & - & - & 420,310 & $20,761,513$ \\
\hline 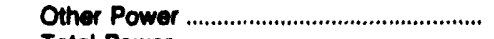 & - & - & - & - & & 868 \\
\hline 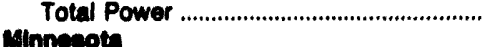 & - & - & - & - & 420,378 & $20,762,379$ \\
\hline \multicolumn{7}{|l|}{$\begin{array}{l}\text { Minnecotin } \\
\text { Southern Minnesota Mun P Aany/Dec } 31\end{array}$} \\
\hline 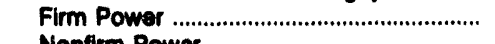 & - & - & 12,228 & 181,305 & $1,890,581$ & $114,167,317$ \\
\hline $\begin{array}{l}\text { Nonfirm Power } \\
\text { Total Power }\end{array}$ & 65,694 & 852,894 & 23,891 & 301,238 & 403,828 & $5,550,870$ \\
\hline \multirow{2}{*}{\multicolumn{7}{|c|}{$\begin{array}{l}\text { Nobracka } \\
\text { Contral Nebraska Pub P\&I Dist/Dec } 31\end{array}$}} \\
\hline & & & & & & \\
\hline Nonfirm Power .............................................. & - & - & 253,110 & $8,032,997$ & 253,110 & $8,032,897$ \\
\hline $\begin{array}{l}\text { Total Power } \\
\text { Cornhuaker Public Power Diat/Dec } 31\end{array}$ & - & - & 253,110 & $8,032,997$ & 253,110 & $8,032,897$ \\
\hline 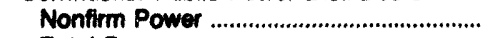 & - & - & - & - & 1,986 & 27,325 \\
\hline 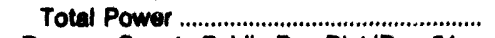 & - & - & - & - & 1,886 & 27,326 \\
\hline \multicolumn{7}{|l|}{ Dawson Cuunty Publlc Pwr Diat/Dec 31} \\
\hline 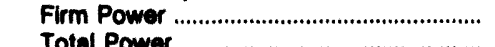 & $\overline{-}$ & - & - & - & 4,800 & 213,820 \\
\hline \multicolumn{7}{|l|}{ Loup River Public Power Diat/Dec 31} \\
\hline 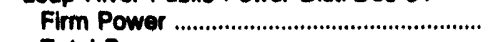 & 181,398 & $6,058,956$ & 144,070 & $3,800,861$ & 398,563 & $12,476,393$ \\
\hline \multirow{2}{*}{\multicolumn{7}{|c|}{ Municipal Energy Agency of NE/Mar 31}} \\
\hline & & & & & & \\
\hline Firm Power ...................................... & - & - & - & - & 676,013 & $21,552,054$ \\
\hline 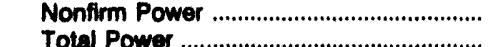 & 17,202 & 236,978 & - & - & 197,218 & $3,201,826$ \\
\hline \multicolumn{7}{|l|}{ 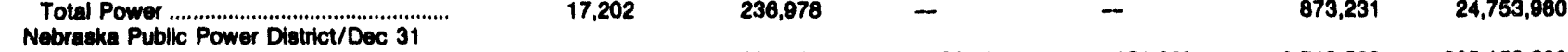 } \\
\hline 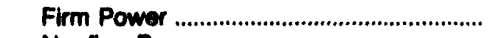 & 82,622 & $3,009,167$ & $3,309,127$ & $111,161,620$ & $9,713,605$ & $305,158,209$ \\
\hline 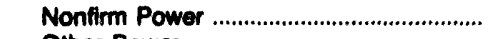 & 360,184 & $5,011,843$ & 125,118 & $1,827,256$ & $1,585,889$ & $22,862,686$ \\
\hline 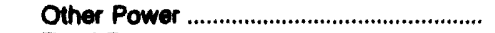 & & 237,000 & & - & & 732,000 \\
\hline Total Power .................................. & 442,806 & $8,258,010$ & $3,434,245$ & $112,888,878$ & $11,309,494$ & $328,752,905$ \\
\hline \multicolumn{7}{|l|}{ Norris Publlc Power District/Dec 31} \\
\hline 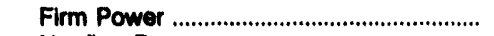 & - & - & - & - & 12,777 & 474,337 \\
\hline 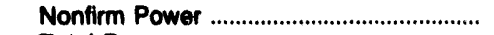 & - & - & - & - & 1,052 & 17,908 \\
\hline Total Power . & - & - & - & - & 13,829 & 492,245 \\
\hline $\begin{array}{l}\text { Omaha Public Power District/Dec } 31 \\
\text { Firm Power }\end{array}$ & & & & & & \\
\hline $\begin{array}{l}\text { Firm Power } \\
\text { Total Power }\end{array}$ & - & - & 103,338 & $1,398,965$ & 138,862 & $3,045,858$ \\
\hline $\begin{array}{l}\text { Total Power .................................................... } \\
\text { Novada }\end{array}$ & - & - & 103,339 & 355 & 138,862 & ,859 \\
\hline Colorado River Commiasion/Jun 30 & & & & & & \\
\hline Firm Power & 121,297 & $3,663,287$ & 172,557 & $2,838,354$ & 828,028 & $14,411,136$ \\
\hline 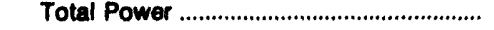 & 121,297 & $3,683,287$ & 172,557 & $2,838,354$ & 826,026 & $14,411,136$ \\
\hline
\end{tabular}

See notes and footnotes at end of table. 
Table 28. Elootriclty 8ales for Rocale by 8tate and Othor Covernmont Utilities, Flecal Year 1992 (Continued)

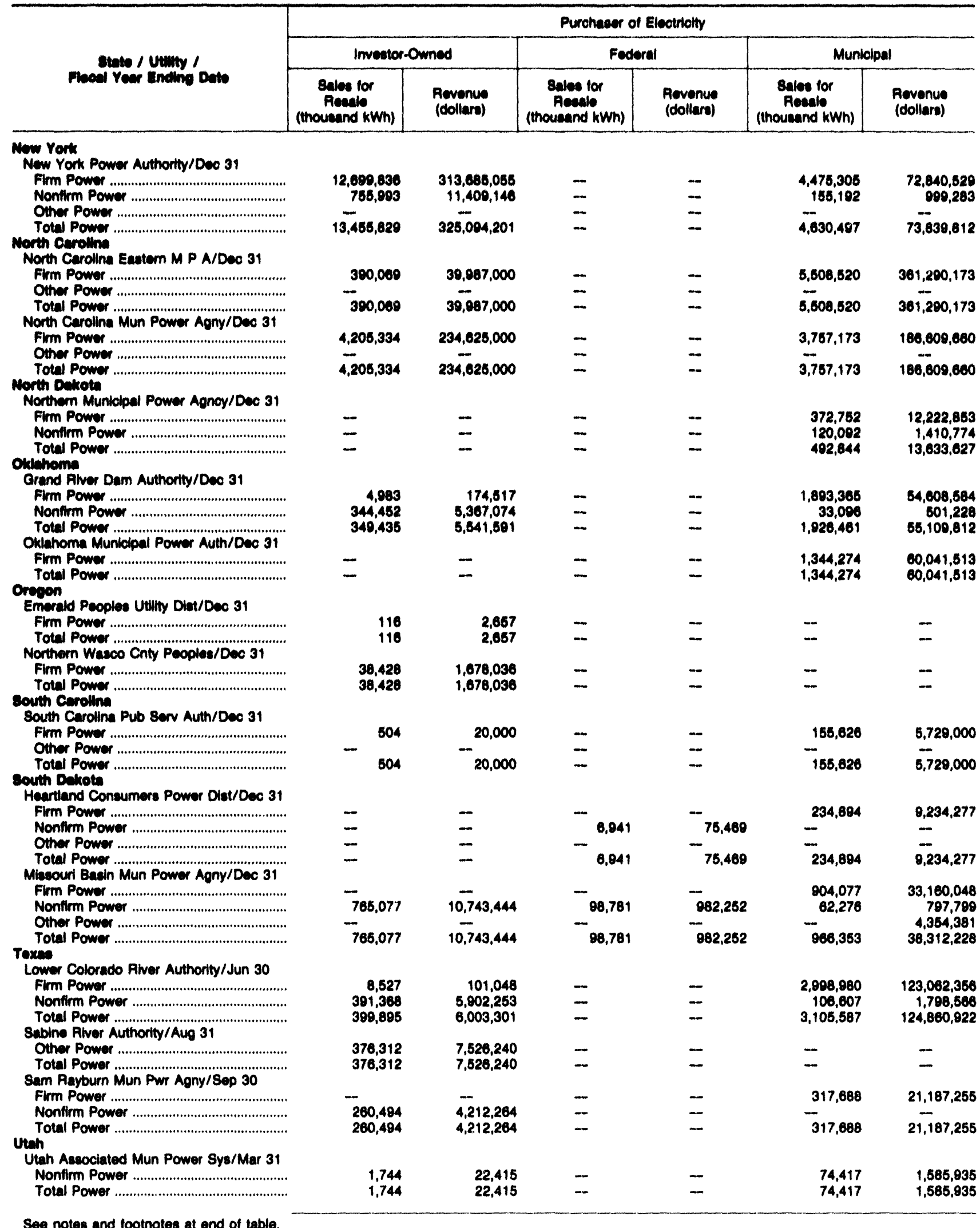

See notes and footnotes at end of table. 
Table 28. Eleotriolty sales for Resale by 8tate and Othor Covornmont Uillition, Flecal Year 1902 (Continued)

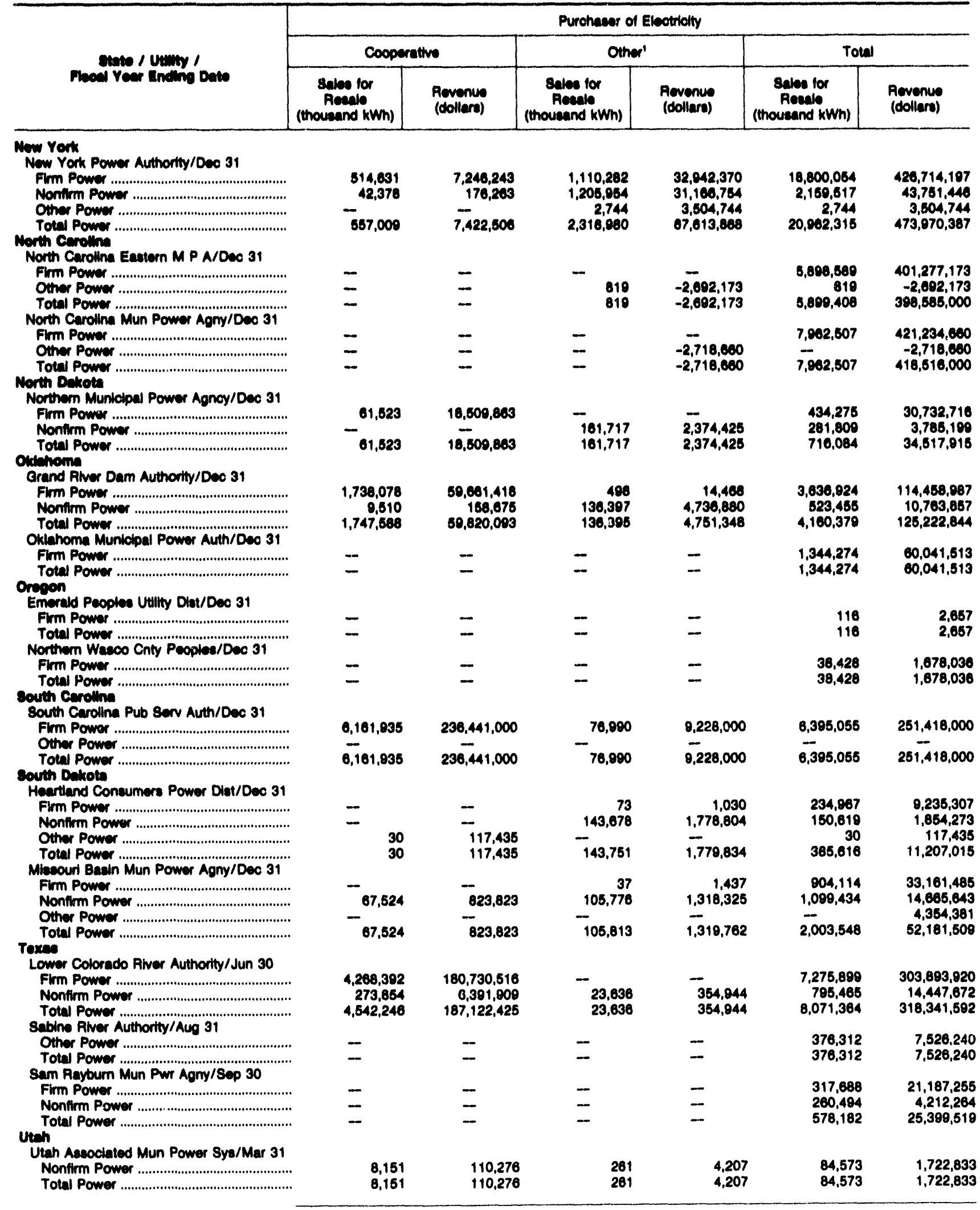

See notes and footnotes at end of table. 
Table 28. Elsotrletty 8ales for Rosale by 8tate and Other Covornment Utilities, Flecal Year 1902 (Continued)

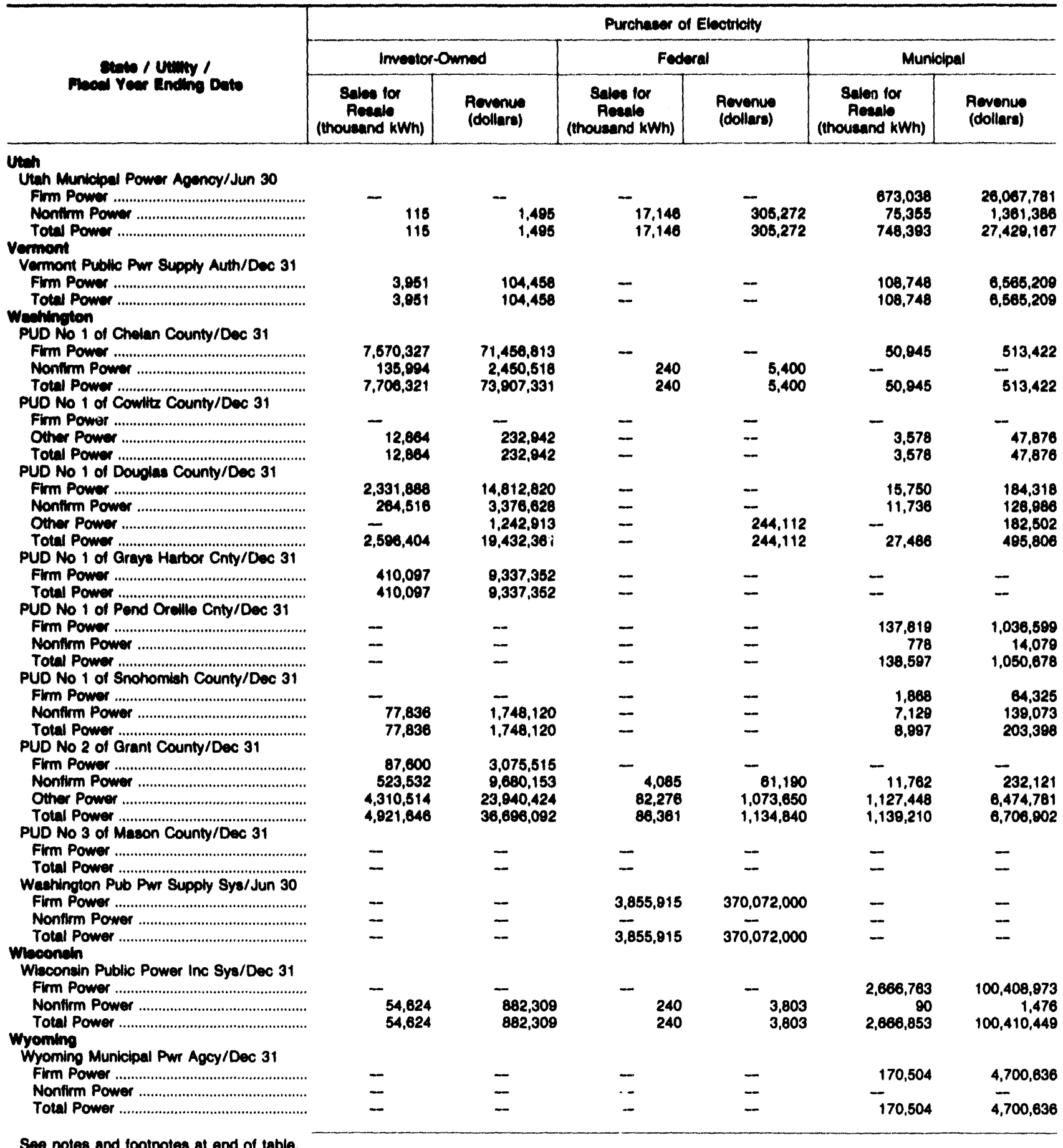


Table 28. Electricity 8ales for Rosale by 8tate and Other Covernment Utilities, Flecal Yoar 1902 (Continued)

\begin{tabular}{|c|c|c|c|c|c|c|}
\hline \multirow{3}{*}{$\begin{array}{l}\text { state / Uuminy I } \\
\text { Froed Yeer Ending Dats }\end{array}$} & \multicolumn{6}{|c|}{ Purohacer of Electricity } \\
\hline & \multicolumn{2}{|c|}{ Cooperative } & \multicolumn{2}{|c|}{ Other' } & \multicolumn{2}{|c|}{ Total } \\
\hline & $\begin{array}{c}\text { Salos for } \\
\text { Reealo } \\
\text { (thousand kWh) }\end{array}$ & $\begin{array}{l}\text { Aovenus } \\
\text { (dolluers) }\end{array}$ & $\begin{array}{c}\text { Salee tor } \\
\text { Roeale } \\
\text { (thousand KWh) }\end{array}$ & $\begin{array}{l}\text { Revenue } \\
\text { (dollare) }\end{array}$ & $\begin{array}{c}\text { Sales for } \\
\text { Reacalo } \\
\text { (thouseand KWh) }\end{array}$ & $\begin{array}{l}\text { Revenue } \\
\text { (tollare) }\end{array}$ \\
\hline \multicolumn{7}{|l|}{ Utah } \\
\hline 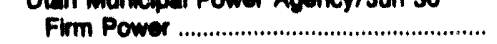 & - & - & - & -- & 673,038 & $26,087,781$ \\
\hline Nonfirm Power .................................................. & - & - & 12 & 236 & 92,628 & $1,606,389$ \\
\hline $\begin{array}{l}\text { Total Power ................................................ } \\
\text { Vermolnt }\end{array}$ & - & - & 12 & 236 & 765,686 & $27,736,170$ \\
\hline Vermont Public Pwr Supply Auth/Dec 31 & & & & & & \\
\hline 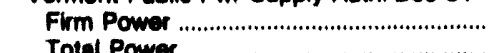 & 17,057 & 813,020 & - & -- & 129,756 & $7,482,687$ \\
\hline \multirow{2}{*}{\multicolumn{7}{|c|}{$\begin{array}{l}\text { Weahington } \\
\text { PUD No } 1 \text { of Chelen County/Dec } 31\end{array}$}} \\
\hline & & & & & & \\
\hline $\begin{array}{l}\text { Firm Power } \\
\text { Nonfirm Power }\end{array}$ & - & - & 157,250 & 827,833 & $7,778,522$ & $72,898,008$ \\
\hline $\begin{array}{l}\text { Nonfirm Power } \\
\text { Total Power }\end{array}$ & $\overline{-}$ & $\overline{-}$ & $-157,250$ & $\overline{927,833}$ & $\begin{array}{r}136,234 \\
7,914,756\end{array}$ & $\begin{array}{r}2,455,918 \\
75,353,986\end{array}$ \\
\hline \multicolumn{7}{|l|}{ PUD No 1 of Cowlitz County/Dec 31} \\
\hline 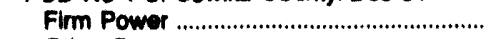 & - & - & 1,529 & 43,100 & 1,529 & 43.100 \\
\hline 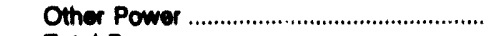 & - & - & - & & 16,442 & 280,818 \\
\hline & \multicolumn{3}{|c|}{ PUD No 1 of Douglas County/Doc 31} & 43,100 & 17,971 & 323,918 \\
\hline 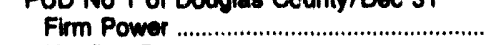 & - & - & 441,360 & $3,890,276$ & $2,788,998$ & $18,687,414$ \\
\hline Nonfirm Power ................................................... & - & - & 1,847 & 22,697 & 278,090 & $3,528,311$ \\
\hline 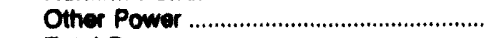 & - & - & & 200,573 & & $1,870,100$ \\
\hline \multicolumn{7}{|l|}{ PUD No 1 of Grave Harbor Cnty/Dec 31} \\
\hline Firm Power ............................................ & - & - & - & - & 410,097 & $9,337,352$ \\
\hline \multicolumn{7}{|l|}{ PUD No 1 of Pend Oreille Cnty/Dec 31} \\
\hline Firm Power ........................................ & - & - & - & - & 137,819 & $1,036,590$ \\
\hline 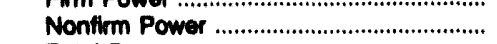 & - & - & - & - & 778 & 14,079 \\
\hline $\begin{array}{l}\text { Total Power ............................ } \\
\text { PUD No } 1 \text { of Snomomish }\end{array}$ & - & - & - & - & 138,597 & $1,050,678$ \\
\hline Firm Power ............................................ & - & - & - & - & 1,868 & 64,325 \\
\hline Nonfirm Power ............................................... & - & - & - & - & 84,905 & $1,887,183$ \\
\hline \multirow{2}{*}{\multicolumn{5}{|c|}{ PUD No 2 of Grant County/Doc 31}} & & \\
\hline & - & - & - & - & 87,600 & $3,075,515$ \\
\hline Nonfirm Power ............................................ & - & - & -- & - & 539,379 & $0,973,464$ \\
\hline 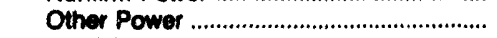 & - & - & 220.444 & $1,221,350$ & $5,740,682$ & $32,710,205$ \\
\hline 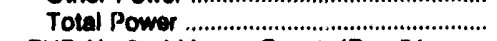 & - & - & 220.444 & $1,221,350$ & $6,387,681$ & $45,759,184$ \\
\hline \multicolumn{7}{|l|}{ PUD No 3 of Mason County/Dec 31} \\
\hline 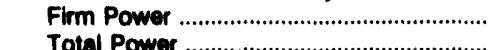 & $\overline{-}$ & - & 1,269 & $\begin{array}{l}38,202 \\
38,202\end{array}$ & $\begin{array}{l}1,269 \\
1,269\end{array}$ & 38,202 \\
\hline \multicolumn{7}{|l|}{$\begin{array}{l}\text { Total Power } \\
\text { Washington Pub Pw Supply Sys/Jun } 30\end{array}$} \\
\hline 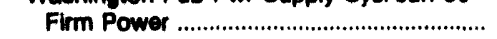 & - & - & 4,659 & $2,307,866$ & $3,860,574$ & $372,378,966$ \\
\hline Nonfirm Power .................................................. & - & - & 21,437 & 400,872 & 21,437 & 400,872 \\
\hline 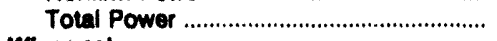 & - & - & 26,096 & $2,708,838$ & $3,882,011$ & $372,780,838$ \\
\hline \multicolumn{7}{|l|}{$\begin{array}{l}\text { Whoconain } \\
\text { Wisconsin Public Power Inc Sys/Dec } 31\end{array}$} \\
\hline $\begin{array}{l}\text { Wisconsin Public Power Inc Sys/Dec } 31 \\
\text { Firm Power }\end{array}$ & _- & - & - & - & $2,686,763$ & \\
\hline Nonfirm Power ........................................ & 1.717 & 27,782 & 167 & 2,854 & 56,838 & 918,234 \\
\hline \multirow{2}{*}{\multicolumn{7}{|c|}{$\begin{array}{l}\text { Wyoming } \\
\text { Wyoming Municipal Pwr Agcy/Dec } 31\end{array}$}} \\
\hline & & & & & & \\
\hline 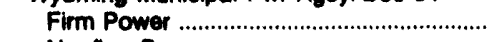 & 368 & 13,416 & - & - & 170,872 & $4,714,052$ \\
\hline 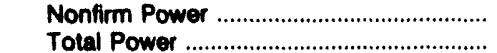 & $\begin{array}{l}29,282 \\
28,650\end{array}$ & $\begin{array}{l}552,337 \\
565,753\end{array}$ & $\overline{-}$ & $=$ & $\begin{array}{r}29,282 \\
200,154\end{array}$ & $\begin{array}{r}552,337 \\
5,268,389\end{array}$ \\
\hline
\end{tabular}

' Includes transactions with State and other government utilities, power pools, utilities in Canada and Mexico, and nonutilities.

- Not Applicable

Notes: •For identification purposes, the public utilities are listed in the State in which the administrative office is located. $\bullet$ Totals may not equal sum of components because of independent rounding.

Source: Energy Information Administration, Form ElA-412, "Annual Report of Public Electric Utilties," except where footnoted. 
Table 29. Electriclity Purchases by Municlpal Utilities, Fiscal Year 1992

\begin{tabular}{|c|c|c|c|c|c|c|}
\hline \multirow{3}{*}{$\begin{array}{l}\text { State / Uuthy I } \\
\text { Foeed Yoe Encing Date }\end{array}$} & \multicolumn{6}{|c|}{ Source of Electrictily } \\
\hline & \multicolumn{2}{|c|}{ Inveator-Owned } & \multicolumn{2}{|c|}{ Federal } & \multicolumn{2}{|c|}{$\begin{array}{l}\text { State and Other } \\
\text { Government }\end{array}$} \\
\hline & $\begin{array}{c}\text { Purchases } \\
\text { (thoueand } k W h \text { ) }\end{array}$ & $\begin{array}{c}\text { Cost } \\
\text { (dollara) }\end{array}$ & $\begin{array}{l}\text { Purchaeces } \\
\text { (thousand kWh) }\end{array}$ & $\begin{array}{c}\text { Coot } \\
\text { (dollars) }\end{array}$ & $\begin{array}{l}\text { Purchasess } \\
\text { (thousand kWh) }\end{array}$ & $\begin{array}{c}\text { Coot } \\
\text { (dollars) }\end{array}$ \\
\hline \multicolumn{7}{|l|}{ Noboema } \\
\hline 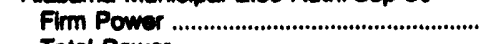 & $2,252,355$ & $68,878,822$ & - & - & - & - \\
\hline 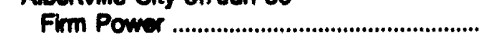 & - & - & 428,744 & $16,628,249$ & - & - \\
\hline \multirow{2}{*}{\multicolumn{7}{|c|}{ Andaluala City of $/ \operatorname{Sep} 30$}} \\
\hline & & & & & & \\
\hline 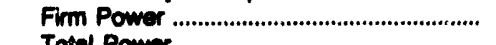 & - & - & - & - & - & - \\
\hline \multicolumn{7}{|l|}{ Athens Chy of/Dec 31} \\
\hline 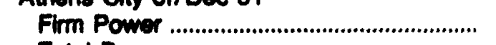 & - & - & 648,620 & $29,351,808$ & - & - \\
\hline \multirow{2}{*}{\multicolumn{4}{|c|}{ Beveemer Chy of/Jun 30}} & & - & - \\
\hline & - & - & 316,785 & $12,870,823$ & - & - \\
\hline 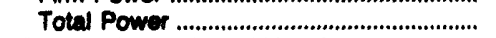 & - & - & 316,785 & $12,870,823$ & - & $\overline{-}$ \\
\hline \multicolumn{7}{|l|}{ Cullman Power Board/Jun 30} \\
\hline 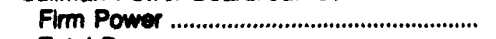 & - & - & 289,834 & $13,946,398$ & - & - \\
\hline \multicolumn{7}{|l|}{ Docatur Utiltios/Jun 30} \\
\hline 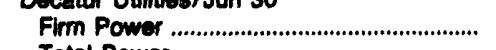 & - & - & $1,386,397$ & $54,447,261$ & - & - \\
\hline \multicolumn{5}{|l|}{ Dothan Electrical Department/Sep 30} & - & - \\
\hline 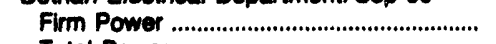 & - & - & 80,441 & $3,069,384$ & - & - \\
\hline \multirow{2}{*}{\multicolumn{7}{|c|}{ Florence Cty ot/Jun 30}} \\
\hline & & & & & & \\
\hline Firm Power .................................................. & - & - & $1,004,216$ & $44,608,525$ & - & - \\
\hline \multirow{2}{*}{\multicolumn{5}{|c|}{$\begin{array}{l}\text { Fort Payne Improvement Auth/Jun } 30 \\
\text { Firm Power }\end{array}$}} & - & - \\
\hline & - & - & 290,484 & $13,441,089$ & - & - \\
\hline \multirow{2}{*}{\multicolumn{6}{|c|}{ Guntersville Electric Board/Jun 30}} & - \\
\hline & - & - & 218,360 & $8,608,456$ & - & - \\
\hline Total Power .................................................... & - & - & 218,380 & $9,606,456$ & - & - \\
\hline \multicolumn{7}{|l|}{ Hunteville Cty of/Sep 30} \\
\hline 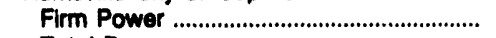 & - & - & $3,644,300$ & $163,883,280$ & - & - \\
\hline $\begin{array}{l}\text { Total Power ................................... } \\
\text { Muscle Shoals Electric Board/Jun }\end{array}$ & - & - & $3,644,300$ & $163,883,280$ & - & -- \\
\hline 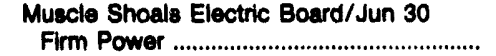 & - & - & 190,351 & $8,739,121$ & - & - \\
\hline 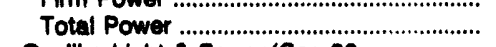 & - & - & 190,351 & $8,738,121$ & - & - \\
\hline Opelika Light \& Power/Sep 30 & & & & & & \\
\hline 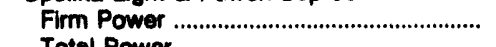 & 291,264 & $11,249,978$ & 30,581 & $1,220,572$ & - & - \\
\hline 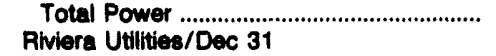 & 291,264 & $11,249,978$ & 30,581 & $1,220,572$ & - & - \\
\hline 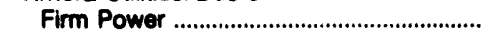 & - & - & 38,308 & $1,300,872$ & - & - \\
\hline $\begin{array}{l}\text { Total Power } \\
\text { Scottsboro City of/Jun } 30\end{array}$ & - & - & 38,308 & $1,300,972$ & - & - \\
\hline 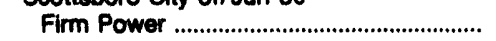 & - & - & 338,307 & $13,521,495$ & - & - \\
\hline 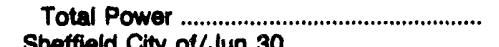 & - & - & 338,307 & $13,521,495$ & - & - \\
\hline 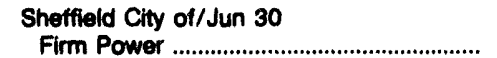 & - & - & 416,327 & $18,268,268$ & - & - \\
\hline 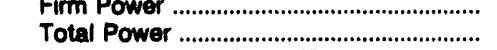 & - & - & 416,327 & $18,268,268$ & - & $\overline{-}$ \\
\hline Sylacauga Utilities Board/Sep 30 & & & & & & \\
\hline 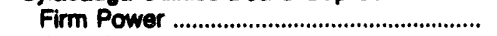 & - & - & 26,358 & 898,713 & - & - \\
\hline 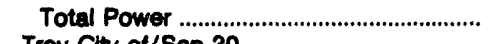 & -- & - & 26,358 & 898,713 & - & - \\
\hline $\begin{array}{l}\text { Troy City of/Sep } 30 \\
\text { Firm Power }\end{array}$ & 201,607 & $7,302,118$ & 18,395 & 624,731 & - & - \\
\hline $\begin{array}{l}\text { Total Power } \\
\text { Tuskegee City ot/Sep } 30\end{array}$ & 201,607 & $7,302,118$ & 18,395 & 624,731 & - & - \\
\hline 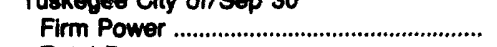 & - & - & 18,307 & 686,202 & - & - \\
\hline 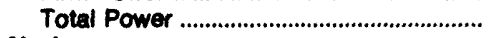 & - & - & 18,307 & 686,202 & - & - \\
\hline Alacka & & & & & & \\
\hline $\begin{array}{l}\text { Anchorage Mun Light and Power/Dec } 31 \\
\text { Firm Power .......................................... }\end{array}$ & & & & & 83,262 & $3,700,586$ \\
\hline Firm Power .......................................... & $\overline{-}$ & $\overline{-}$ & $-58,303$ & $\underbrace{801,151}$ & $-83,262$ & $3,700,586$ \\
\hline $\begin{array}{l}\text { Nonfirm Power } \\
\text { Other Power }\end{array}$ & - & - & - & 57,830 & - & $=$ \\
\hline Total Power & - & - & 58,303 & $1,048,981$ & 83,262 & $3,700,566$ \\
\hline
\end{tabular}


Table 29. Electricity Furchases by Munlcipal Utilities, Fiecal Year 1992 (Continued)

\begin{tabular}{|c|c|c|c|c|c|c|}
\hline \multirow{3}{*}{$\begin{array}{l}\text { Stute / Uullty I } \\
\text { Floed Year Ending Dote }\end{array}$} & \multicolumn{6}{|c|}{ Source of Electrility } \\
\hline & \multicolumn{2}{|c|}{ Cooperative } & \multicolumn{2}{|c|}{ Other' } & \multicolumn{2}{|c|}{ Total } \\
\hline & $\begin{array}{l}\text { Purchaves } \\
\text { (thousand kWh) }\end{array}$ & $\begin{array}{c}\text { Cost } \\
\text { (dollara) }\end{array}$ & $\begin{array}{l}\text { Purchaeses } \\
\text { (thousand kWh) }\end{array}$ & $\begin{array}{c}\text { Cost } \\
\text { (dollara) }\end{array}$ & $\begin{array}{l}\text { Purchases } \\
\text { (thousand kWh) }\end{array}$ & $\begin{array}{c}\text { Coot } \\
\text { (dollare) }\end{array}$ \\
\hline \multicolumn{7}{|l|}{$\begin{array}{l}\text { Aleberma } \\
\text { Alebema Municipal Elec Auth/Sep } 30\end{array}$} \\
\hline 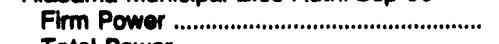 & - & - & - & - & $2,252,355$ & $68,078,822$ \\
\hline \multicolumn{5}{|l|}{ Albertville City of/Jun 30} & $2,252,355$ & $68,078,822$ \\
\hline 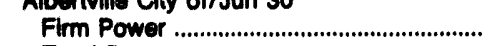 & - & - & - & - & 426,744 & $16,628,249$ \\
\hline \multicolumn{7}{|l|}{ Andaluala Clty of/Sep 30} \\
\hline 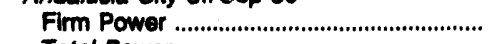 & 305,070 & $11,329,600$ & - & - & 305,070 & $11,328,600$ \\
\hline \multicolumn{5}{|l|}{ Athens Clty of/Dec 31} & & \\
\hline $\begin{array}{l}\text { Firm Power } \\
\text { Total Power }\end{array}$ & $\bar{z}$ & $\overline{-}$ & $\overline{-}$ & $\bar{z}$ & 648,620 & $\begin{array}{l}29,361,808 \\
29,351,808\end{array}$ \\
\hline \multicolumn{7}{|l|}{ Bescemer Clty of/Jun 30} \\
\hline 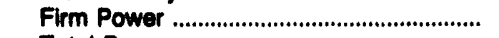 & - & - & - & - & 316,705 & $12,870,823$ \\
\hline 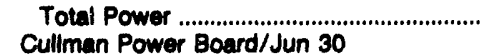 & - & - & - & - & 316,785 & $12,870,823$ \\
\hline & - & - & - & - & 289,934 & $13,946,389$ \\
\hline 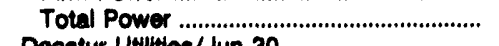 & - & - & - & - & 290,034 & $13,946,389$ \\
\hline & - & - & - & - & $1,386,397$ & $54,447,261$ \\
\hline \multirow{2}{*}{\multicolumn{7}{|c|}{ Dothan Electrical Department/Sep 30}} \\
\hline & - & - & 808,411 & $34,642,440$ & & \\
\hline $\begin{array}{l}\text { Firm Power } \\
\text { Total Power }\end{array}$ & $\overline{-}$ & - & 808,411 & $34,642,440$ & $\begin{array}{l}888,852 \\
988,852\end{array}$ & $\begin{array}{l}37,711,824 \\
37,711,824\end{array}$ \\
\hline \multicolumn{7}{|l|}{ Florence Clty of/Jun 30} \\
\hline 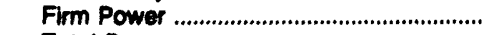 & - & - & - & - & $1,004,216$ & $44,608,625$ \\
\hline 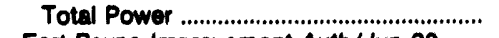 & - & - & - & - & $1,004,216$ & $44,608,525$ \\
\hline \multicolumn{6}{|l|}{$\begin{array}{l}\text { Fort Payne Improvement Auth/Jun } 30 \\
\text { Firm Power }\end{array}$} & \\
\hline 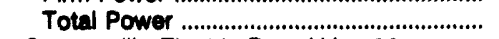 & - & - & - & - & 280,484 & $13,441,089$ \\
\hline \multirow{2}{*}{\multicolumn{7}{|c|}{ 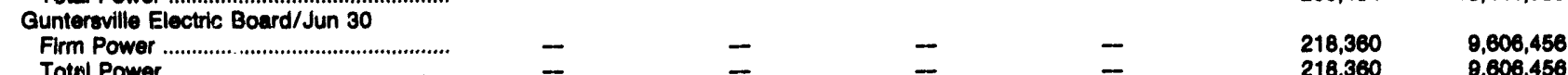 }} \\
\hline & - & - & - & - & 218,360 & $9,806,456$ \\
\hline & - & - & - & - & 218,360 & $8,606,456$ \\
\hline 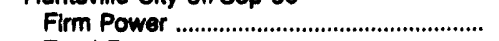 & - & - & - & - & $3,644,300$ & \\
\hline 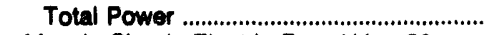 & - & - & - & - & $3,644,300$ & $\begin{array}{l}163,883,280 \\
163,883,280\end{array}$ \\
\hline Muscle Shoels Electric Board/Jun 30 & & & & & & \\
\hline 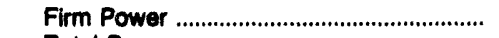 & - & - & - & - & 190,351 & $8,739,121$ \\
\hline 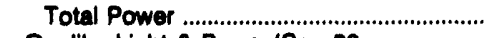 & - & - & - & - & 100,351 & $8,739,121$ \\
\hline Opelika Light \& Power/Sep 30 & & & & & & \\
\hline 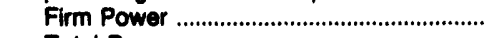 & - & - & - & - & 321,845 & $12,470,550$ \\
\hline Total Power & - & - & - & - & 321,845 & $12,470,560$ \\
\hline Riviera Utillties/Dec 31 & & & & & & \\
\hline 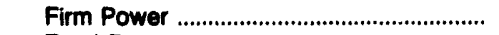 & - & - & 425,737 & $17,999,177$ & 464,045 & $19,300,149$ \\
\hline 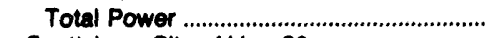 & - & - & 425,737 & $17,999,177$ & 464,045 & $18,300,14 \theta$ \\
\hline Scottsboro City of/Jun 30 & & & & & & \\
\hline 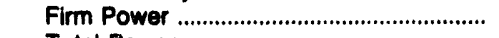 & - & - & - & - & 338,307 & $13,621,496$ \\
\hline 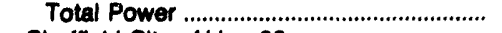 & - & - & - & - & 338,307 & $13,521,495$ \\
\hline Sheffield City of/Jun 30 & & & & & & \\
\hline 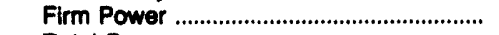 & - & - & - & - & 416,327 & $18,269,268$ \\
\hline Total Power & - & - & - & - & 416,327 & $18,269,268$ \\
\hline Sylacauga Utilities Board/Sep 30 & & & & & & \\
\hline 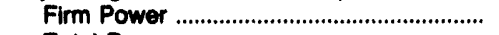 & - & - & 139,978 & $5,488,008$ & 166,336 & $6,386,721$ \\
\hline Total Power & - & - & 139,978 & $5,488,008$ & 168,336 & 6,386,721 \\
\hline Troy City of/Sep 30 & & & & & & \\
\hline 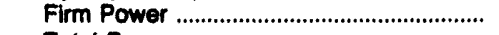 & - & - & - & - & 220,002 & $7,826,849$ \\
\hline 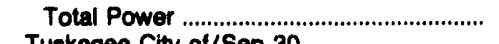 & - & - & - & - & 220,002 & $7,926,849$ \\
\hline $\begin{array}{l}\text { Tuskegee City of/Sep } 30 \\
\text { Firm Power }\end{array}$ & - & - & 125,656 & $5,012,342$ & 143,063 & $5,698,544$ \\
\hline 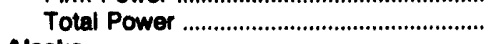 & - & - & 125,656 & $5,012,342$ & 143,963 & $5,698,544$ \\
\hline Alacke & & & & & & \\
\hline Anchorage Mun Light and Power/Dec 31 & & & & & & \\
\hline 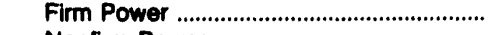 & - & - & - & - & 141,565 & $4,601,717$ \\
\hline 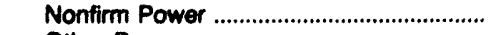 & 2,269 & 41,843 & - & - & 2,268 & 41,843 \\
\hline 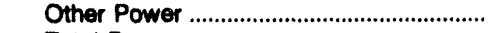 & - & - & - & - & - & 57,830 \\
\hline 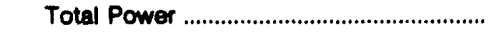 & 2,269 & 41,843 & - & - & 143,834 & $4,791,390$ \\
\hline
\end{tabular}

See notes and footnotes at end of table. 
Table 29. Electricity Purchases by Municipal Utilties, Fiscal Year 1992 (Continued)

\begin{tabular}{|c|c|c|c|c|c|c|}
\hline \multirow{3}{*}{ 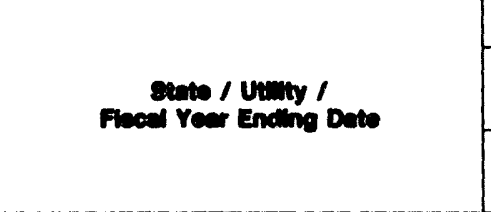 } & \multicolumn{6}{|c|}{ Source of Electricty } \\
\hline & \multicolumn{2}{|c|}{ Investor-Owned } & \multicolumn{2}{|c|}{ Federal } & \multicolumn{2}{|c|}{$\begin{array}{l}\text { State and Other } \\
\text { Government }\end{array}$} \\
\hline & $\begin{array}{l}\text { Purchases } \\
\text { (thousand kWh) }\end{array}$ & $\begin{array}{c}\text { Cost } \\
\text { (dollars) }\end{array}$ & $\begin{array}{l}\text { Purchases } \\
\text { (thoussand kWh) }\end{array}$ & $\begin{array}{c}\text { Cost } \\
\text { (dollars) }\end{array}$ & $\begin{array}{c}\text { Purchases } \\
\text { (thousand kWh) }\end{array}$ & $\begin{array}{c}\text { Cost } \\
\text { (dollars) }\end{array}$ \\
\hline \multicolumn{7}{|l|}{ Nomber } \\
\hline Nonfirm Power ............................................... & - & - & - & - & - & - \\
\hline 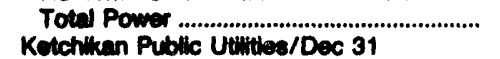 & - & - & - & - & - & - \\
\hline 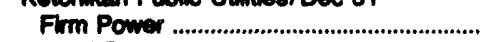 & - & - & - & - & 80,085 & $4,234,954$ \\
\hline 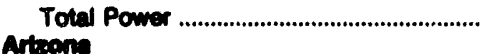 & - & - & - & - & 80,085 & $4,234,954$ \\
\hline \multicolumn{7}{|l|}{$\begin{array}{l}\text { Artroma } \\
\text { Mesa Ututios/Jun } 30\end{array}$} \\
\hline 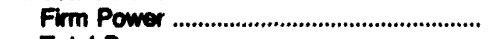 & - & - & - & - & - & - \\
\hline 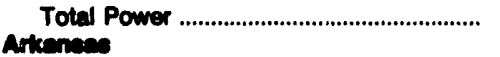 & - & - & - & - & - & - \\
\hline \multicolumn{7}{|l|}{ 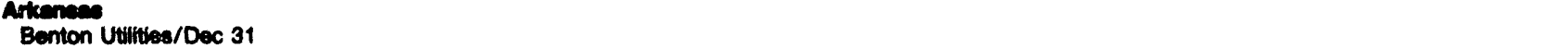 } \\
\hline 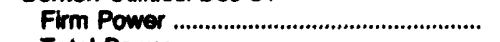 & 144,883 & $6,952,534$ & - & - & - & - \\
\hline $\begin{array}{l}\text { Total Power ... } \\
\text { Bentonville Cty of Dec } 31\end{array}$ & \multicolumn{6}{|c|}{ Bentonville City of/Dec 31} \\
\hline 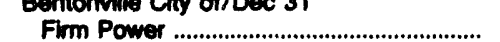 & 142,483 & $5,589,261$ & 59,639 & 801,255 & - & $\ldots$ \\
\hline 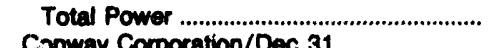 & 142,493 & $5,589,261$ & 59,639 & 801,255 & - & - \\
\hline \multicolumn{6}{|l|}{ Conway Corporation/Dec 31} & \\
\hline $\begin{array}{l}\text { Firm Power } \\
\text { Total Power }\end{array}$ & $\begin{array}{l}104,854 \\
104,854\end{array}$ & $\begin{array}{l}5,704,832 \\
5,704,832\end{array}$ & - & - & - & - \\
\hline \multicolumn{7}{|l|}{$\begin{array}{l}\text { Total Power } \\
\text { Hope Water \& Light Plant/Dec } 31\end{array}$} \\
\hline 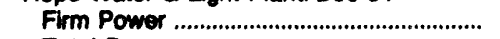 & 176,000 & $6,989,695$ & - & - & - & - \\
\hline 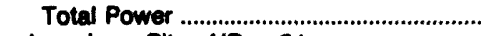 & 176,000 & $6,989,695$ & - & - & - & - \\
\hline \multicolumn{7}{|l|}{ Joneaboro City of/Dec 31} \\
\hline 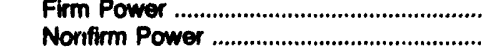 & $\overline{-}$ & $\overline{-}$ & 264,310 & $2,738,134$ & 30,345 & $\overline{514,273}$ \\
\hline 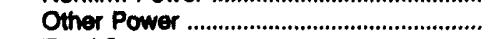 & - & $1,801,120$ & - & - & - & -- \\
\hline 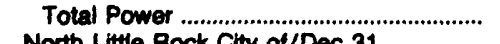 & - & $1,801,120$ & 264,310 & $2,739,134$ & 30,345 & 514,273 \\
\hline \multicolumn{3}{|l|}{ North Little Rock City of/Dec 31} & - & & - & - \\
\hline 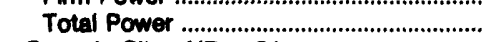 & 575,002 & $29,110,189$ & - & - & - & $\overline{-}$ \\
\hline \multicolumn{7}{|l|}{ Osceola City of/Dec 31} \\
\hline Firm Power ......... & 180,644 & $6,270,0: 5$ & - & - & - & - \\
\hline \multicolumn{7}{|l|}{ Paragould Light \& Water Comm/Dec 31} \\
\hline 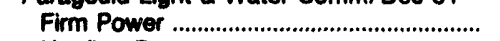 & - & - & 163,565 & $2,028,184$ & 161,645 & $3,404,998$ \\
\hline 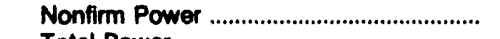 & - & - & - & - & - & \\
\hline \multicolumn{7}{|l|}{$\begin{array}{l}\text { Total Power ....1.1. } \\
\text { Siloam Springs City ot/Dec } 31\end{array}$} \\
\hline 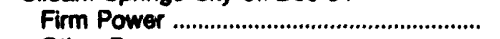 & - & - & - & - & 173,435 & $6,034,931$ \\
\hline 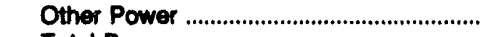 & - & - & - & - & - & - \\
\hline \multicolumn{7}{|l|}{ West Memphis Utility Dept/Dec 31} \\
\hline 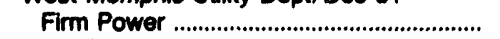 & 130,050 & $11,816,970$ & - & - & - & - \\
\hline 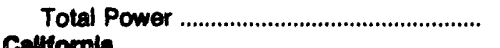 & 130,050 & $11,816,970$ & - & - & - & - \\
\hline $\begin{array}{l}\text { Callitomia } \\
\text { Alameda City of/Jun } 30\end{array}$ & & & & & & \\
\hline 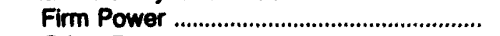 & - & - & 171,043 & $4,589,473$ & 329,557 & $26,091,096$ \\
\hline 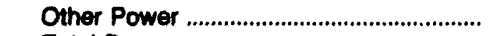 & - & - & - & - & - & - \\
\hline $\begin{array}{l}\text { Total Power .................................... } \\
\text { Anaheim Cty of/Jun } 30\end{array}$ & - & - & 171,043 & $4,599,473$ & 329,557 & $26,091,096$ \\
\hline 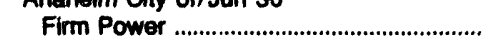 & 167,941 & $8,892,000$ & 40,528 & 689,000 & - & - \\
\hline Nonfirm Power ..................................................... & 17,401 & 397,000 & 4,253 & 84,000 & 16,526 & 310,000 \\
\hline 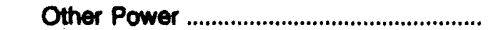 & 17,381 & 268,000 & - & 580,000 & - & - \\
\hline 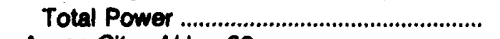 & 202,723 & $9,557,000$ & 44,781 & $1,353,000$ & 16,526 & 310,000 \\
\hline Azusa City of/Jun 30 & & & & & & \\
\hline 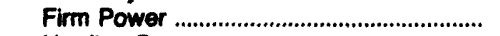 & 72,221 & $3,883,311$ & - & - & 18,583 & $1,739,235$ \\
\hline 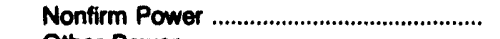 & - & - & - & - & - & - \\
\hline 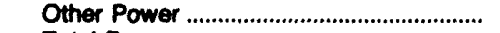 & 17,665 & 524,916 & - & - & - & \\
\hline ver ................................. & 89,886 & $4,408,227$ & - & - & 18,583 & $1,739,235$ \\
\hline Burbank Public Service Dept/Jun 30 & & & & & & \\
\hline 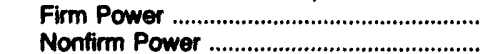 & $\begin{array}{l}81,190 \\
99,381\end{array}$ & $\begin{array}{l}7,753,325 \\
2,391,528\end{array}$ & $\begin{array}{l}22,169 \\
91,624\end{array}$ & $\begin{array}{r}428,610 \\
1,478,314\end{array}$ & $-35,122$ & $\overline{703.763}$ \\
\hline 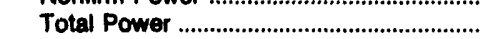 & 180,571 & $10,144,853$ & 113,793 & $1,806,924$ & 35,122 & 703,763 \\
\hline Colton Electric Utility Dept/Jun 30 & & & & & & \\
\hline 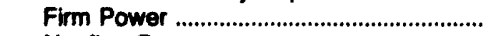 & 110,726 & $6,145,895$ & 3,346 & 149,012 & - & - \\
\hline 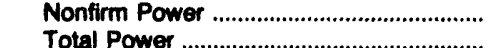 & $\begin{array}{r}821 \\
111547\end{array}$ & $\begin{array}{r}16,238 \\
6,162,133\end{array}$ & $-_{3,346}$ & $-\overline{149012}$ & $\begin{array}{l}754 \\
754\end{array}$ & $\begin{array}{l}15,941 \\
15,941\end{array}$ \\
\hline & & & & & & \\
\hline
\end{tabular}


Table 29. Electricity Purchases by Municipal Utilities, Flecal Year 1992 (Continued)

\begin{tabular}{|c|c|c|c|c|c|c|}
\hline \multirow{3}{*}{$\begin{array}{l}\text { State / Uillty I } \\
\text { Fiecal Year Endling Date }\end{array}$} & \multicolumn{6}{|c|}{ Source of Electricity } \\
\hline & \multicolumn{2}{|c|}{ Cooperative } & \multicolumn{2}{|c|}{ Other' } & \multicolumn{2}{|c|}{ Total } \\
\hline & $\begin{array}{c}\text { Purchases } \\
\text { (thousand kWh) }\end{array}$ & $\underset{\text { (dollars) }}{\text { Cost }}$ & $\begin{array}{c}\text { Purchases } \\
\text { (thousand kWh) }\end{array}$ & $\underset{\text { (dollars) }}{\text { Cost }}$ & $\begin{array}{c}\text { Purchases } \\
\text { (thousend kWh) }\end{array}$ & $\begin{array}{c}\text { Cost } \\
\text { (dollars) }\end{array}$ \\
\hline \multicolumn{7}{|l|}{ Alacka } \\
\hline 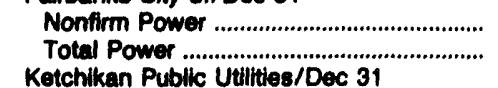 & $\begin{array}{l}13,942 \\
13,842\end{array}$ & $\begin{array}{l}922,100 \\
922,100\end{array}$ & $\begin{array}{l}10,840 \\
10,840\end{array}$ & $\begin{array}{l}426,734 \\
426,734\end{array}$ & $\begin{array}{l}24,782 \\
24,782\end{array}$ & $\begin{array}{l}1,348,834 \\
1,348,834\end{array}$ \\
\hline Firm Power . & - & - & - & - & 80,085 & $4,234,954$ \\
\hline 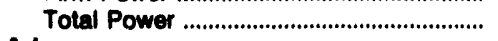 & -- & -- & - & - & 80,085 & $4,234,954$ \\
\hline \multicolumn{7}{|l|}{$\begin{array}{l}\text { Artzona } \\
\text { Mera Utilitios/Jun } 30\end{array}$} \\
\hline 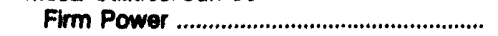 & - & - & 322,026 & $13,263,975$ & 322,026 & $13,263,975$ \\
\hline \multirow{2}{*}{\multicolumn{7}{|c|}{$\begin{array}{l}\text { Arkansas } \\
\text { Benton Utilitites/Dec } 31\end{array}$}} \\
\hline & & & & & & \\
\hline 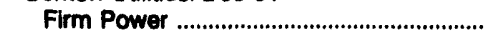 & - & - & - & -- & 144,883 & $6,952,534$ \\
\hline \multicolumn{7}{|l|}{ Bentonville City of/Dec 31} \\
\hline 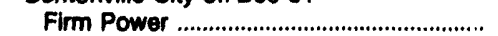 & -- & - & - & - & 202,132 & $6,380,516$ \\
\hline \multicolumn{7}{|l|}{ Conway Corporation/Dec 31} \\
\hline 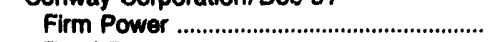 & - & -- & - & - & 104,854 & $5,704,832$ \\
\hline \multicolumn{7}{|l|}{ Hope Water \& Uight Plant/Dec 31} \\
\hline Firm Power ....................................... & - & - & - & -- & 176,000 & $6,989,695$ \\
\hline 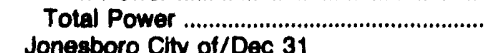 & - & - & - & -- & 176,000 & $6,989,695$ \\
\hline 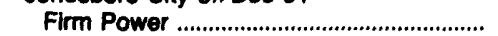 & - & -- & - & -- & 264,310 & $2,739,134$ \\
\hline Nonfirm Power ................................................. & - & - & 291,801 & $4,993,741$ & 322,146 & $5,508,014$ \\
\hline 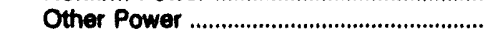 & -- & - & - & - & - & $1,801,120$ \\
\hline \multirow{2}{*}{\multicolumn{7}{|c|}{ North Little Rock City of/Dec 31}} \\
\hline & & & & & & \\
\hline 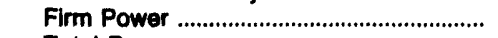 & - & - & -- & - & 575,002 & $28,110,183$ \\
\hline 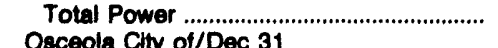 & - & - & - & -- & 575,002 & $29,110,183$ \\
\hline Firm Power ................................................. & - & -- & -- & -- & 180,644 & $6,270,055$ \\
\hline Total Power .................................................... & - & - & - & - & 180,644 & $6,270,055$ \\
\hline Paragould Light \& Water Comm/Dec 31 & & & & & & \\
\hline Firm Power .............................................. & -- & - & - & 502,600 & 325,210 & $5,933,782$ \\
\hline 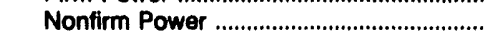 & - & -- & 3,795 & 69,704 & 3,795 & 69,704 \\
\hline 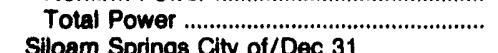 & - & -- & 3,795 & 572,304 & 329,005 & $6,003,486$ \\
\hline & & & & & & \\
\hline 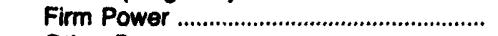 & - & -- & -- & - & 173,435 & $6,034,931$ \\
\hline 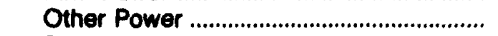 & - & -. & - & $-639,668$ & - & $-639,668$ \\
\hline 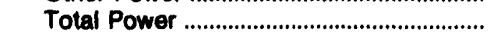 & -_ & -- & - & $-639,668$ & 173,435 & $5,395,263$ \\
\hline West Memphis Utility Dept/Dec 31 & & & & & & \\
\hline 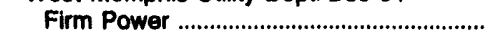 & - & - & - & -- & 130,050 & $11,816,970$ \\
\hline 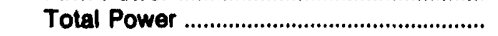 & - & -- & -. & -- & 130,050 & $11,816,970$ \\
\hline $\begin{array}{l}\text { Callfornia } \\
\text { Alameda City of / Jun } 30\end{array}$ & & & & & & \\
\hline 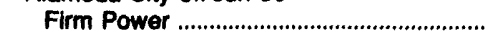 & - & - & -- & - & 500,600 & $30,690,569$ \\
\hline 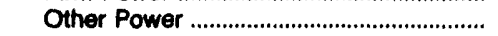 & - & -- & -- & $-2,105,843$ & - & $-2,105,843$ \\
\hline 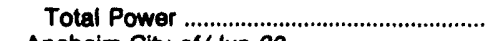 & - & - & - & $-2,105,843$ & 500,600 & $28,584,726$ \\
\hline Anaheim City of/Jun 30 & & & & & & \\
\hline 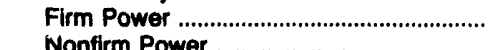 & 571,184 & $21,684,000$ & $1,550,687$ & $86,962,000$ & $2,330,340$ & $118,227,000$ \\
\hline & 3,369 & 72,000 & - & - & 41,549 & 863,000 \\
\hline Other Power & - & -- & -_ & -. & 17,381 & 848,000 \\
\hline 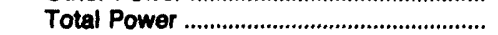 & 574,553 & $21,756,000$ & $1,550,687$ & $86,962,000$ & $2,389,270$ & $119,938,000$ \\
\hline Azusa City of/Jun 30 & & & & & & \\
\hline 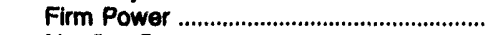 & - & - & 8,137 & $1,816,167$ & 98,941 & $7,438,713$ \\
\hline 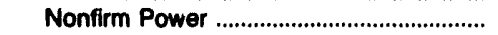 & - & - & 48,013 & 854,590 & 48,013 & 854,590 \\
\hline 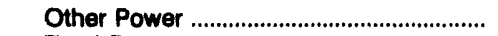 & - & - & 41,700 & 842,558 & 59,374 & $1,367,474$ \\
\hline 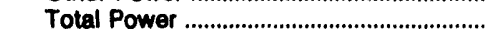 & -- & - & 97,859 & $3,513,315$ & 206,328 & $0,680,777$ \\
\hline Burbank Public Service Dept/Jun 30 & & & & & & \\
\hline 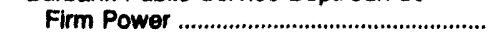 & 4,101 & 138,939 & 512,076 & $30,208,938$ & 619,536 & $38,529,812$ \\
\hline 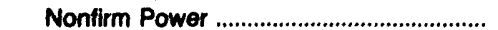 & 83,018 & $1,358,439$ & 6,336 & 564,682 & 315,481 & $6,496,726$ \\
\hline 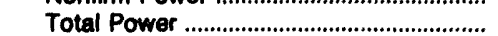 & 87,119 & $1,497,378$ & 518,412 & $30,773,620$ & 935,017 & $45,026,538$ \\
\hline Colton Electric Utility Dept/Jun 30 & & & & & & \\
\hline 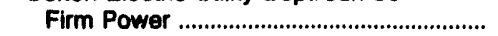 & - & - & 7,777 & $1,363,939$ & $121,84 \theta$ & $7,658,846$ \\
\hline Nonfirm Power ..................................................... & 37,243 & 728,666 & 54,762 & $1,086,520$ & 93,580 & $1,847,365$ \\
\hline 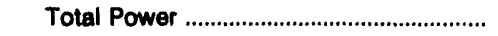 & 37,243 & 728,666 & 62,539 & $2,450,450$ & 215,429 & $9,506,211$ \\
\hline
\end{tabular}

See notes and footnotes at end of table. 
Table 29. Electriclty Purchases by Munlcipal Utilitles, Flecal Year 1992 (Continued)

\begin{tabular}{|c|c|c|c|c|c|c|}
\hline \multirow{3}{*}{$\begin{array}{l}\text { State / Uuthy I } \\
\text { Fisoal Yoar Ending Date }\end{array}$} & \multicolumn{6}{|c|}{ Source of Electricity } \\
\hline & \multicolumn{2}{|c|}{ Investor-Owned } & \multicolumn{2}{|c|}{ Federal } & \multicolumn{2}{|c|}{$\begin{array}{l}\text { State and Other } \\
\text { Government }\end{array}$} \\
\hline & $\begin{array}{l}\text { Purchases } \\
\text { (thousand kWh) }\end{array}$ & $\begin{array}{c}\text { Cost } \\
\text { (dollars) }\end{array}$ & $\begin{array}{l}\text { Purchases } \\
\text { (thousand kWh) }\end{array}$ & $\begin{array}{c}\text { Cost } \\
\text { (dollars) }\end{array}$ & $\begin{array}{l}\text { Purchases } \\
\text { (thousand kWh) }\end{array}$ & $\begin{array}{c}\text { Cost } \\
\text { (dollars) }\end{array}$ \\
\hline \multicolumn{7}{|l|}{ Calfromia } \\
\hline 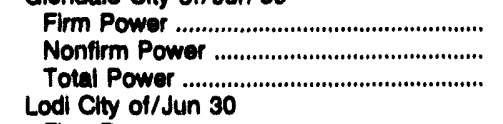 & $\begin{array}{r}97,232 \\
103,219 \\
200,451\end{array}$ & $\begin{array}{r}5,177,511 \\
6,636,069 \\
11,813,580\end{array}$ & $\begin{array}{r}160,849 \\
60,191 \\
241,040\end{array}$ & $\begin{array}{r}4,197,019 \\
704,981 \\
4,902,010\end{array}$ & $\begin{array}{r}75,340 \\
110,221 \\
185,561\end{array}$ & $\begin{array}{l}1,361,110 \\
2,068,108 \\
3,427,227\end{array}$ \\
\hline $\begin{array}{l}\text { Firm Power } \\
\text { Total Power } \\
\text { Los Angeles Dept of Wtr \& PWr/Jun } 30\end{array}$ & - & - & $\begin{array}{l}111,918 \\
111,918\end{array}$ & $\begin{array}{l}3,011,022 \\
3,011,022\end{array}$ & $\begin{array}{l}260,878 \\
260,878\end{array}$ & $\begin{array}{l}23,591,332 \\
23,591,332\end{array}$ \\
\hline 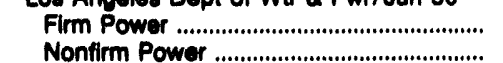 & $\begin{array}{r}1,252,970 \\
536,030\end{array}$ & $\begin{array}{l}57,514,098 \\
10,374,741\end{array}$ & $\begin{array}{l}542,891 \\
304,671\end{array}$ & $\begin{array}{r}10,634,643 \\
3,479,213\end{array}$ & $\begin{array}{l}962,203 \\
149,353\end{array}$ & $\begin{array}{r}87,856,621 \\
2,729,345\end{array}$ \\
\hline 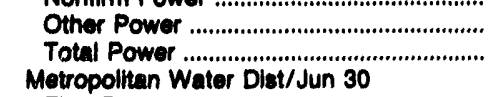 & $\overline{1,789,000}$ & $6 \overline{7,888,839}$ & $-847,562$ & $14,113,856$ & $-\overline{1,111,556}$ & $\overline{80,585,868}$ \\
\hline 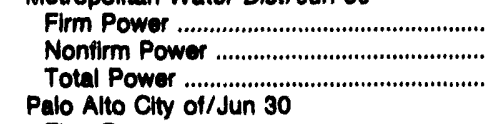 & $\begin{array}{l}123,822 \\
197,585 \\
321,407\end{array}$ & $\begin{array}{l}2,049,162 \\
3,418,892 \\
5,468,054\end{array}$ & $\begin{array}{l}- \\
242,075 \\
242,075\end{array}$ & $\begin{array}{l}\overline{3,426,092} \\
3,426,092\end{array}$ & $\begin{array}{l}329,797 \\
328,797\end{array}$ & $\begin{array}{l}- \\
5,448,083 \\
5,448,063\end{array}$ \\
\hline 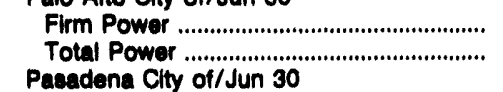 & - & - & $\begin{array}{l}969,268 \\
989,258\end{array}$ & $\begin{array}{l}26,384,000 \\
26,384,000\end{array}$ & $\begin{array}{l}132,172 \\
132,172\end{array}$ & $\begin{array}{l}2,013,000 \\
2,013,000\end{array}$ \\
\hline 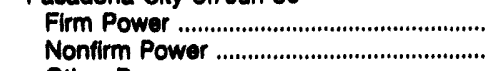 & $\begin{array}{r}545 \\
18,860\end{array}$ & $\begin{array}{r}4,762 \\
689,673\end{array}$ & $-52,287$ & 641,920 & $\begin{array}{l}63,141 \\
56,913\end{array}$ & $\begin{array}{l}5,504,346 \\
1,249,650\end{array}$ \\
\hline 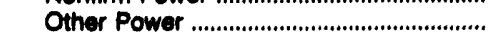 & - & - & - & - & - & - \\
\hline $\begin{array}{l}\text { Total Power .................................... } \\
\text { Redding Clty of/Jun } 30\end{array}$ & 18,405 & 694,435 & 52,287 & 641,820 & 120,054 & $6,753,996$ \\
\hline 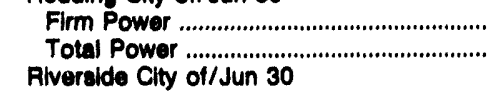 & $\begin{array}{l}102,985 \\
102,985\end{array}$ & $\begin{array}{l}7,123,523 \\
7,123,523\end{array}$ & $\begin{array}{l}584,013 \\
584,013\end{array}$ & $\begin{array}{l}18,667,620 \\
18,667,620\end{array}$ & - & - \\
\hline 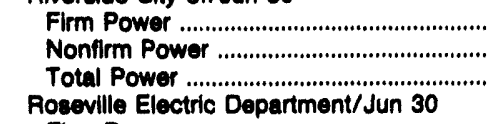 & $\begin{array}{r}126,450 \\
19,694 \\
146,144\end{array}$ & $\begin{array}{r}12,731,760 \\
420,109 \\
13,151,869\end{array}$ & $\begin{array}{l}32,645 \\
10,974 \\
43,619\end{array}$ & $\begin{array}{l}589,510 \\
176,587 \\
788,097\end{array}$ & $\begin{array}{l}78,892 \\
15,096 \\
93,988\end{array}$ & $\begin{array}{r}7,403,280 \\
322,816 \\
7,726,196\end{array}$ \\
\hline 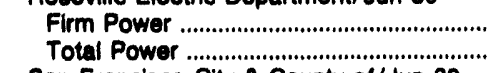 & - & - & $\begin{array}{l}493,067 \\
493,057\end{array}$ & $\begin{array}{l}12,880,495 \\
12,880,495\end{array}$ & $\begin{array}{l}57,932 \\
57,932\end{array}$ & $\begin{array}{l}13,253,729 \\
13,253,729\end{array}$ \\
\hline San Francisco City \& County of/Jun 30 & & & & & & \\
\hline 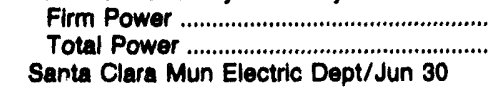 & $\begin{array}{l}285,517 \\
285,517\end{array}$ & $\begin{array}{l}27,899,203 \\
27,899,203\end{array}$ & $\overline{-}$ & $\overline{-}$ & $\begin{array}{l}15,690 \\
15,690\end{array}$ & $\begin{array}{l}374,820 \\
374,920\end{array}$ \\
\hline 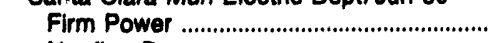 & 487,443 & $23,799,187$ & $1,221,098$ & $12,798,853$ & - & - \\
\hline Nonfirm Power .................................................. & 5,730 & 91,160 & 4,733 & 52,682 & - & - \\
\hline 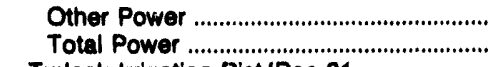 & $\begin{array}{r}1,244 \\
484,417\end{array}$ & $\begin{array}{l}16,836,738 \\
40,827,095\end{array}$ & & $\overline{12,851,535}$ & $\begin{array}{l}628,945 \\
628,945\end{array}$ & $\begin{array}{l}59,445,550 \\
59,446,550\end{array}$ \\
\hline Turlock Irrigation Dist/Dec 31 & & & & & & \\
\hline 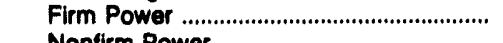 & 316,322 & $14,342,631$ & 34,119 & 756,976 & 288,493 & $18,783,880$ \\
\hline $\begin{array}{l}\text { Nonfirm Power } \\
\text { Total Power }\end{array}$ & $\begin{array}{r}744 \\
316,066\end{array}$ & $\begin{array}{r}16,140 \\
14,358,771\end{array}$ & & & $\begin{array}{r}90,427 \\
378,920\end{array}$ & $\begin{array}{r}2,903,195 \\
22,687,075\end{array}$ \\
\hline Vernon City of/Jun 30 & & & & & & \\
\hline 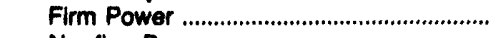 & 716,144 & $18,733,460$ & 19,427 & 437,493 & 298,259 & $20,041,287$ \\
\hline 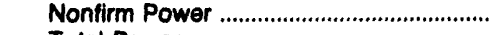 & - & - & - & - & 29 & 657 \\
\hline $\begin{array}{l}\text { Total Power } \\
\text { Colorado }\end{array}$ & 716,144 & $18,733,460$ & 19,427 & 437,493 & 298,288 & $20,041,944$ \\
\hline Colorado Springs Utilities/Dec 31 & & & & & & \\
\hline 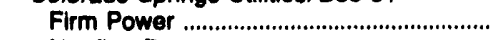 & - & - & 376,021 & $7,268,030$ & - & - \\
\hline 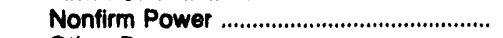 & 55,568 & $1,318,002$ & 1,515 & 44,115 & 10,526 & 171,291 \\
\hline 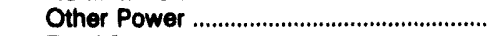 & - & 13,287 & - & 71,380 & - & - \\
\hline $\begin{array}{l}\text { Total Power ... } \\
\text { Fort Collins Lat \& Pur Utillity/Dec } 31\end{array}$ & 55,568 & $1,332,289$ & 377,536 & $7,383,525$ & 10,526 & 171,291 \\
\hline 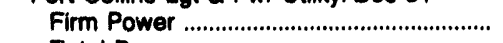 & - & - & - & - & 848,241 & $29,161,983$ \\
\hline $\begin{array}{l}\text { Total Power ........................................... } \\
\text { Longmont City of/Dec } 31\end{array}$ & - & - & - & - & 848,241 & $29,161,963$ \\
\hline 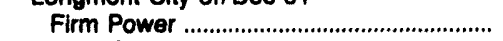 & - & - & - & - & 440,084 & $15,068,981$ \\
\hline $\begin{array}{l}\text { Total Power .......................................... } \\
\text { Loveland Electric Division/Dec } 31\end{array}$ & - & - & - & - & 440,084 & $15,068,981$ \\
\hline 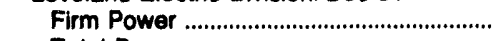 & - & - & - & - & 368,505 & $12,205,681$ \\
\hline 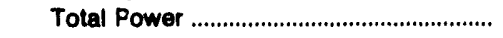 & - & - & - & - & 369,505 & $12,205,681$ \\
\hline
\end{tabular}

See notes and footnotes at end of table. 
Table 29. Electricity Purchases by Municipal Utilties, Fiscal Year 1992 (Continued)

\begin{tabular}{|c|c|c|c|c|c|c|}
\hline \multirow{3}{*}{$\begin{array}{l}\text { Btate / Utilly I } \\
\text { Flecel Year Ending Date }\end{array}$} & \multicolumn{6}{|c|}{ Source of Electricity } \\
\hline & \multicolumn{2}{|c|}{ Cooperattve } & \multicolumn{2}{|c|}{ Other' } & \multicolumn{2}{|c|}{ Total } \\
\hline & $\begin{array}{c}\text { Purchases } \\
\text { (thousand kWh) }\end{array}$ & $\begin{array}{c}\text { Coot } \\
\text { (dollars) }\end{array}$ & $\begin{array}{c}\text { Purchases } \\
\text { (thousand } k W h \text { ) }\end{array}$ & $\begin{array}{c}\text { Coont } \\
\text { (dollare) }\end{array}$ & $\begin{array}{c}\text { Purchases } \\
\text { (thoueand kWh) }\end{array}$ & $\begin{array}{c}\text { Coost } \\
\text { (dollars) }\end{array}$ \\
\hline \multicolumn{7}{|l|}{$\begin{array}{l}\text { Calliomia } \\
\text { Glendale } \mathrm{Cl}\end{array}$} \\
\hline 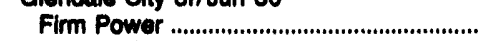 & - & - & - & - & 353,421 & $10,735,648$ \\
\hline 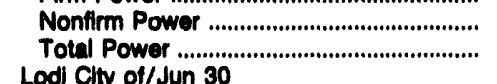 & $\begin{array}{l}117 \\
117\end{array}$ & $\begin{array}{l}2,034 \\
2,034\end{array}$ & $\begin{array}{l}312,697 \\
312,597\end{array}$ & $\begin{array}{l}16,475,698 \\
16,475,598\end{array}$ & $\begin{array}{l}586,345 \\
939,766\end{array}$ & $\begin{array}{l}25,884,600 \\
36,620,449\end{array}$ \\
\hline $\begin{array}{l}\text { Lodl Clty of/Jun } 30 \\
\text { Firm Power .................... }\end{array}$ & - & - & - & - & 372,796 & $26,802,364$ \\
\hline 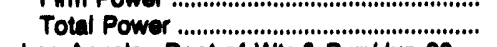 & - & - & - & - & 372,796 & $26,602,364$ \\
\hline $\begin{array}{l}\text { Los Angeles Dept of Wir \& Pwr/Jun } 30 \\
\text { Firm Power }\end{array}$ & 308,461 & $9,429,296$ & $7,134,124$ & $431,869,407$ & $10,200,639$ & $597,304,065$ \\
\hline 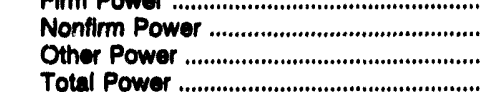 & $\begin{array}{r}168,477 \\
- \\
476,928\end{array}$ & $\begin{array}{r}2,246,332 \\
-\overline{11,674,628}\end{array}$ & $\begin{array}{r}71,579 \\
7,205,703\end{array}$ & $\begin{array}{r}1,922,185 \\
42,290,286 \\
476,081,858\end{array}$ & $\begin{array}{r}1,230,110 \\
11,430,749\end{array}$ & $\begin{array}{r}20,750,796 \\
42,290,286 \\
680,345,147\end{array}$ \\
\hline Metropolltan Water Diet/Jun 30 & & & & & & \\
\hline 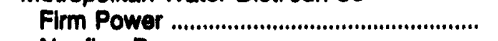 & - & - & - & - & 123,822 & $2,049,162$ \\
\hline 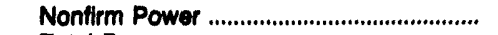 & - & - & - & - & 769,457 & $12,293,047$ \\
\hline Total Power ....................................................... & - & - & - & - & 893,279 & $14,342,209$ \\
\hline $\begin{array}{l}\text { Paln Alto City of/Jun } 30 \\
\text { Firm Power ........................... }\end{array}$ & - & - & - & - & $1,101,430$ & $28,397,000$ \\
\hline 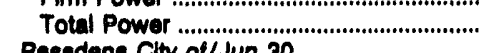 & - & - & - & - & $1,101,430$ & $28,397,000$ \\
\hline $\begin{array}{l}\text { Pacadena Clty of/Jun } 30 \\
\text { Firm Power }\end{array}$ & - & - & 716,772 & $42,074,853$ & 832,745 & $48,226,881$ \\
\hline Nontirm Power ........................................ & 2,600 & 42,480 & 9,971 & $1,881,968$ & 88,344 & $3,863,779$ \\
\hline $\begin{array}{l}\text { Other Power } \\
\text { Total Power }\end{array}$ & $-2,600$ & $-42,490$ & $\begin{array}{r}554 \\
727,297\end{array}$ & $\begin{array}{r}31,481 \\
43,888,300\end{array}$ & $\begin{array}{r}554 \\
021,643\end{array}$ & $\begin{array}{r}31,481 \\
52,121,141\end{array}$ \\
\hline Redding City of/Jun 30 & & & & & & \\
\hline Firm Power ........................................... & - & - & - & - & 686,998 & $25,791,143$ \\
\hline $\begin{array}{l}\text { Total Power ................................................ } \\
\text { Phveralde City of /Jun } 30\end{array}$ & - & - & - & - & 686,998 & $25,791,143$ \\
\hline Firm Power ................................................ & 250,636 & $11,122,430$ & 895,643 & $44,601,368$ & $1,384,285$ & $76,448,346$ \\
\hline Nonfirm Power .......................................................... & 107,576 & $1,682,835$ & 2,159 & 34,084 & 155,489 & $2,636,531$ \\
\hline 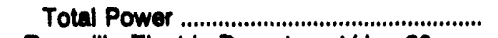 & 368,211 & $12,805,285$ & 887,802 & $44,635,450$ & $1,539,764$ & $79,084,877$ \\
\hline Roseville Electric Department/Jun 30 & & & & & & \\
\hline 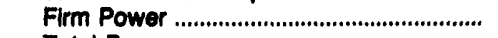 & - & - & - & - & 550,889 & $26,134,224$ \\
\hline $\begin{array}{l}\text { Total Power } \\
\text { San Francisco City \& County of/Jun } 30\end{array}$ & - & - & - & - & 550,989 & $26.134,224$ \\
\hline 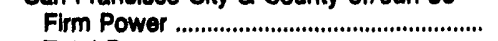 & - & - & - & - & 301,207 & $28,274,123$ \\
\hline Total Power & - & - & - & - & 301,207 & $28,274,123$ \\
\hline Santa Clara Mun Electric Dept/Jun 30 & & & & & & \\
\hline 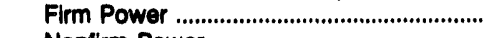 & - & - & - & - & $1,708,541$ & $36,598,050$ \\
\hline 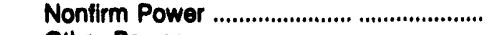 & - & - & $\overline{-}$ & 7002040 & $\begin{array}{r}10,463 \\
\end{array}$ & $\begin{array}{r}143,842 \\
\end{array}$ \\
\hline Other Power & - & - & - & $7,923,649$ & 630,189 & $84,305,937$ \\
\hline 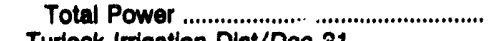 & - & - & - & $7,923,649$ & $2,348,183$ & $121,047,829$ \\
\hline $\begin{array}{l}\text { Turiock Imigation Dist/Dec } \mathbf{3 1} \\
\text { Firm Power }\end{array}$ & & & & $11,629,676$ & 987.416 & $46,513,163$ \\
\hline 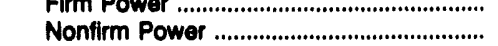 & $\overline{-}$ & $\overline{-}$ & $\begin{array}{r}040,406 \\
440\end{array}$ & $\begin{array}{r}11,0<8,490 \\
9,490\end{array}$ & $\begin{array}{r}88,410 \\
81,611\end{array}$ & $2,928,825$ \\
\hline 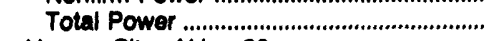 & - & - & 349,922 & $11,639,168$ & $1,078,027$ & $49,441,988$ \\
\hline Vernon City of/Jun 30 & & & & & & \\
\hline 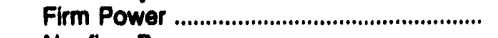 & - & - & - & -- & $1,033,830$ & $39,212,240$ \\
\hline 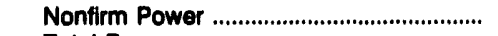 & - & - & - & - & & \\
\hline 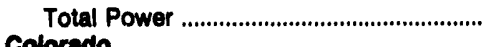 & - & - & - & -- & $1,033,859$ & $38,212,897$ \\
\hline $\begin{array}{l}\text { Colorado } \\
\text { Colorado Springs Utilities/Dec } 31\end{array}$ & & & & & & \\
\hline 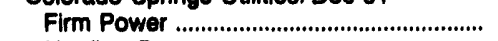 & - & - & -- & - & 376,021 & $7,268,030$ \\
\hline Nontirm Power ....................................................... & 2,615 & 54,460 & - & - & 70,224 & $1,588,868$ \\
\hline 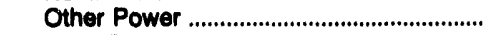 & & 16,200 & - & - & & 100,867 \\
\hline 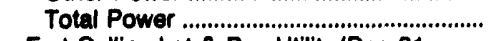 & 2,615 & 70,660 & - & - & 446,245 & $8,957,765$ \\
\hline Fort Collins Lgt \& Pwr Utillty/Dec 31 & & & & & & \\
\hline Firm Power & - & - & - & - & 848,241 & $29,161,963$ \\
\hline $\begin{array}{l}\text { Total Power } \\
\text { Lonamont City of/Dec } 31\end{array}$ & - & - & - & - & 848,241 & $29,161,963$ \\
\hline 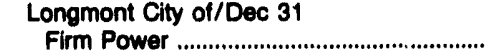 & - & - & - & -- & 440,084 & $15,088,881$ \\
\hline Total Power & - & - & - & - & 440,084 & $15,088,981$ \\
\hline ctric Division/Dec 31 & & & & & & \\
\hline$r$ & - & - & - & - & 369,505 & $12,205,681$ \\
\hline Total Power & - & - & - & - & 369,505 & $12,205,681$ \\
\hline
\end{tabular}

Seo notes and footnotes at end of table. 
Table 29. Electricity Purchases by Municlpal Utilitles, Fiscal Year 1992 (Continued)

\begin{tabular}{|c|c|c|c|c|c|c|}
\hline \multirow{3}{*}{$\begin{array}{l}\text { 8tate / Ualty / } \\
\text { Freal Year Ending Dato }\end{array}$} & \multicolumn{6}{|c|}{ Source of Electricity } \\
\hline & \multicolumn{2}{|c|}{ Investor-Owned } & \multicolumn{2}{|c|}{ Federal } & \multicolumn{2}{|c|}{$\begin{array}{l}\text { State and Other } \\
\text { Government }\end{array}$} \\
\hline & $\begin{array}{c}\text { Purchases } \\
\text { (thousand kWh) }\end{array}$ & $\begin{array}{c}\text { Cost } \\
\text { (dollars) }\end{array}$ & $\begin{array}{c}\text { Purchases } \\
\text { (thousand kWh) }\end{array}$ & $\begin{array}{c}\text { Cost } \\
\text { (dollars) }\end{array}$ & $\begin{array}{c}\text { Purchases } \\
\text { (thousand kWh) }\end{array}$ & $\begin{array}{c}\text { Cost } \\
\text { (dollars) }\end{array}$ \\
\hline \multicolumn{7}{|l|}{$\begin{array}{l}\text { Connoctlout } \\
\text { Groton Clty of Dept of Utils/Jun } 30\end{array}$} \\
\hline 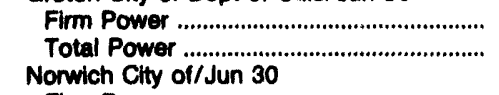 & - & - & - & - & $\begin{array}{l}344,861 \\
644,861\end{array}$ & $\begin{array}{l}35,657,778 \\
35,657,778\end{array}$ \\
\hline 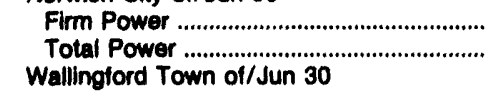 & - & - & - & $\overline{-}$ & $\begin{array}{l}272,600 \\
272,600\end{array}$ & $\begin{array}{l}15,015,137 \\
15,015,137\end{array}$ \\
\hline $\begin{array}{l}\text { Firm Power } \\
\text { Total Power } \\
\text { Delawars } \\
\text { Dover City of/Jun } 30\end{array}$ & $\begin{array}{l}515,842 \\
515,842\end{array}$ & $\begin{array}{l}33,139,305 \\
33,139,305\end{array}$ & - & - & $\begin{array}{l}26,564 \\
26,564\end{array}$ & $\begin{array}{l}315,872 \\
315,872\end{array}$ \\
\hline 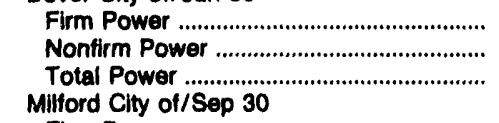 & $\begin{array}{l}- \\
204,921 \\
204,921\end{array}$ & $\begin{array}{l}\overline{6,210,962} \\
6,210,862\end{array}$ & - & - & $\overline{-}$ & - \\
\hline 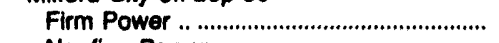 & 131,372 & $5,717,989$ & - & -- & - & - \\
\hline 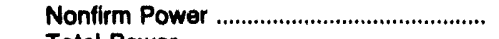 & - & - & - & - & - & - \\
\hline $\begin{array}{l}\text { Total Power } \\
\text { Newark City of /Dec } 31\end{array}$ & 131,372 & $5,717,989$ & - & - & - & - \\
\hline 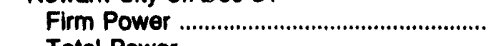 & 281,513 & $12,750,734$ & - & - & - & - \\
\hline $\begin{array}{l}\text { Total Power } \\
\text { Florida } \\
\text { Bartow City of } / \text { Sep } 30\end{array}$ & 281,513 & $12,750,734$ & -- & - & - & - \\
\hline 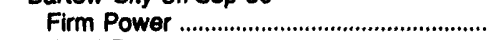 & 248,647 & $10,834,514$ & - & - & - & - \\
\hline 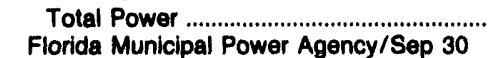 & 248,847 & $10,934,514$ & - & - & - & - \\
\hline 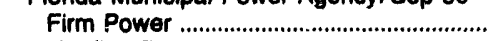 & 706,077 & $30,674,582$ & - & - & - & - \\
\hline Nonfirm Power ............................................. & - & - & - & - & - & -- \\
\hline $\begin{array}{l}\text { Total Power ............................................ } \\
\text { Fort Pierce Utities Au/Sep } 30\end{array}$ & 706,077 & $30,674,582$ & - & - & - & - \\
\hline Firm Power & 10,232 & 872,885 & - & - & - & - \\
\hline 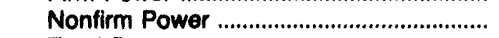 & 23,858 & 634,888 & - & - & - & - \\
\hline $\begin{array}{l}\text { Total Power ................................... } \\
\text { Gainesville City of/Sep } 30\end{array}$ & 34,090 & $1,507,773$ & - & - & -- & - \\
\hline Firm Power & - & - & - & - & - & - \\
\hline 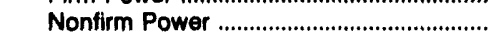 & 28,750 & 669,742 & - & - & - & - \\
\hline 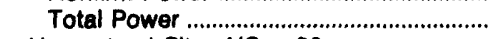 & 28,750 & 669,742 & - & - & - & - \\
\hline Homestead City of/Sep 30 & & & & & & \\
\hline 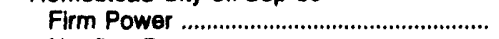 & 9,706 & 901,506 & - & -- & - & - \\
\hline 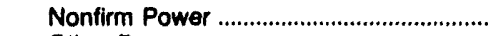 & 4,672 & 107,322 & - & -- & - & - \\
\hline 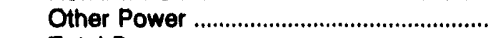 & - & -- & - & - & - & - \\
\hline $\begin{array}{l}\text { Total Power ................................................ } \\
\text { Jacksonville Beach City/Sep } 30\end{array}$ & 14,378 & $1,008,828$ & - & - & - & - \\
\hline 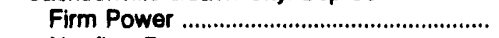 & 37,736 & $2,514,610$ & - & - & - & -- \\
\hline Nonfirm Power ............................................ & $-\infty$ & - & - & -- & - & - \\
\hline $\begin{array}{l}\text { Total Power ......................................... } \\
\text { Jacksonville Elec Auth/Sep }\end{array}$ & 37,736 & $2,514,610$ & - & - & - & - \\
\hline 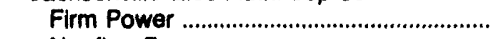 & - & - & -- & -- & - & - \\
\hline 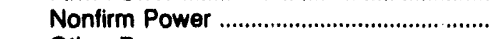 & 21,565 & 772,500 & - & - & - & - \\
\hline 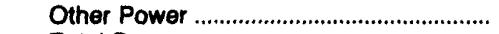 & 1,392 & 59,177 & - & - & - & - \\
\hline Total Power .......................................... & 22,957 & 831,677 & - & - & - & - \\
\hline Key West Utility Board of/Sep 30 & & & & & & \\
\hline 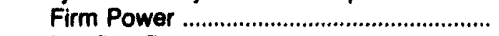 & 230,262 & $6,958,473$ & - & - & - & - \\
\hline 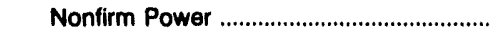 & 25,656 & 995,612 & -- & - & - & - \\
\hline $\begin{array}{l}\text { Total Power } \\
\text { Kissimmee Utility Auth/Sep } 30\end{array}$ & 255,918 & $7,954,085$ & - & - & - & - \\
\hline $\begin{array}{l}\text { Kissimmee Utility Auth/sep } 30 \\
\text { Firm Power }\end{array}$ & 221,975 & $8,576,718$ & -- & - & - & - \\
\hline Nonfirm Power ............................................. & 52,051 & $1,347,580$ & - & - & - & - \\
\hline 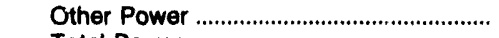 & -- & - & - & - & - & - \\
\hline $\begin{array}{l}\text { Total Power .............................................. } \\
\text { Lake Worth City of/Sep } 30\end{array}$ & 274,026 & $9,924,298$ & - & - & - & - \\
\hline Firm Power & - & - & - & - & - & - \\
\hline 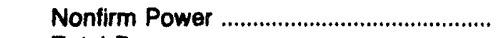 & 13,106 & 415,914 & - & - & - & - \\
\hline $\begin{array}{l}\text { Total Power } \\
\text { Lakeland City of/Sep } 30\end{array}$ & 13,106 & 415,914 & - & - & - & - \\
\hline 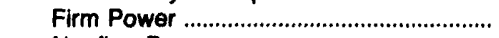 & 143,837 & $3,118,102$ & - & - & - & - \\
\hline 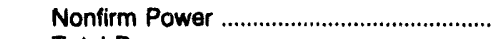 & 5,385 & 236,353 & - & - & - & - \\
\hline 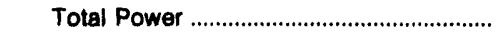 & 149,322 & $3,354,455$ & - & - & - & - \\
\hline
\end{tabular}

See notes and footnotes at end of table. 
Table 29. Electricity Purchases by Municipal Utilities, Fiscal Year 1992 (Continued)

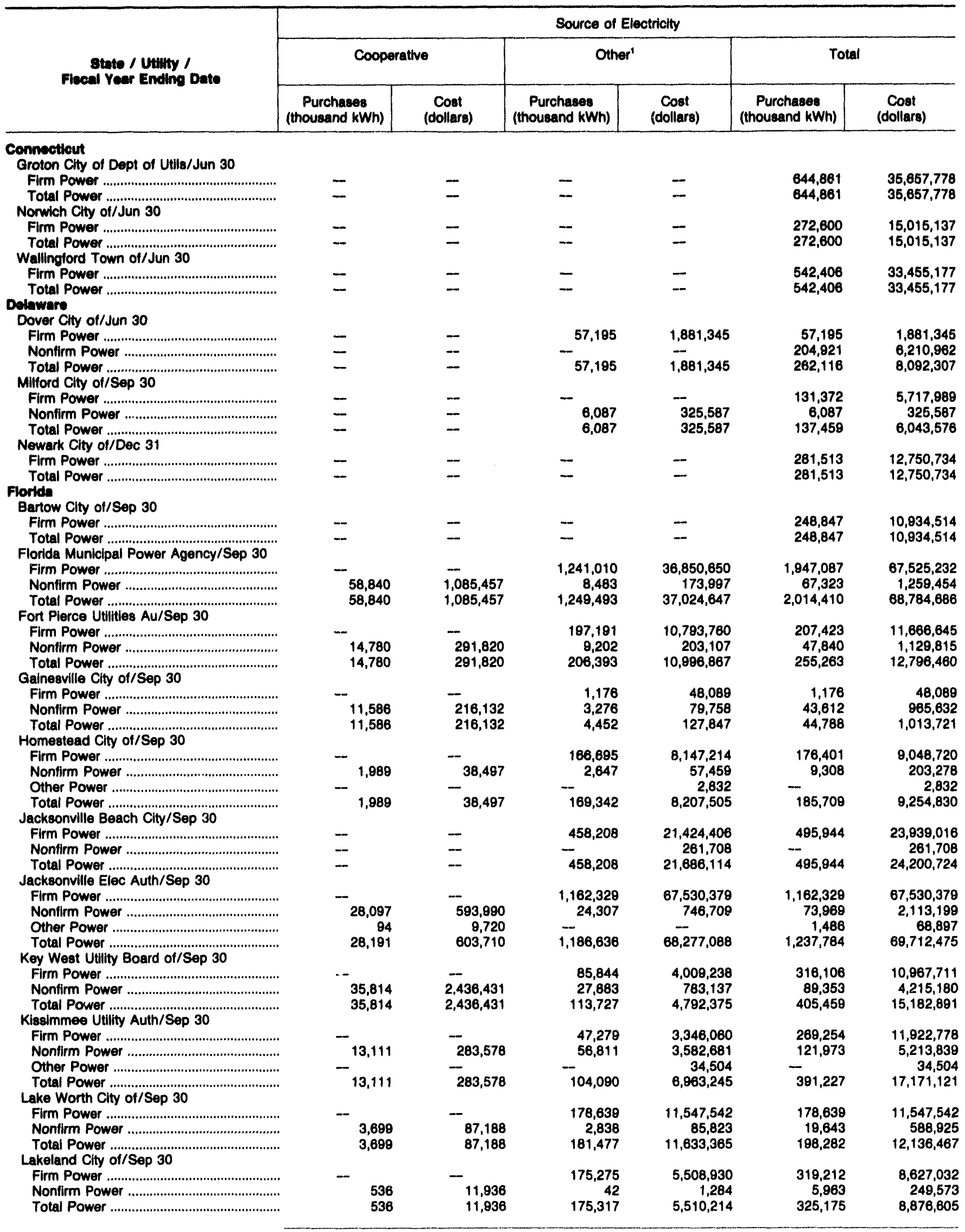

See notes and footnotes at end of table. 
Table 29. Eleotriclty Purchases by Muniolpal Utilitiee, Fleoal Year 1902 (Continued)

\begin{tabular}{|c|c|c|c|c|c|c|}
\hline \multirow{3}{*}{$\begin{array}{l}\text { State / Uunity I } \\
\text { Fleod Yoer Ending Dats }\end{array}$} & \multicolumn{6}{|c|}{ souros of Electriclity } \\
\hline & \multicolumn{2}{|c|}{ Investor-Owned } & \multicolumn{2}{|c|}{ Federal } & \multicolumn{2}{|c|}{$\begin{array}{l}\text { 8tate and Other } \\
\text { Government }\end{array}$} \\
\hline & $\begin{array}{l}\text { Purchases } \\
\text { (thoucand kWh) }\end{array}$ & $\begin{array}{l}\text { Coat } \\
\text { (dollary) }\end{array}$ & $\begin{array}{l}\text { Purchaces } \\
\text { (thousand kWh) }\end{array}$ & $\begin{array}{c}\text { Coot } \\
\text { (doliars) }\end{array}$ & $\begin{array}{l}\text { Purchaces } \\
\text { (thouseand kWh) }\end{array}$ & $\underset{\text { Cootlara) }}{\text { (dollat }}$ \\
\hline \multicolumn{7}{|l|}{$\begin{array}{l}\text { Plordde } \\
\text { Leeaburg Electric Department/8ep } 30\end{array}$} \\
\hline Firm Power ................................................. & $\overline{-}$ & - & - & - & - & - \\
\hline 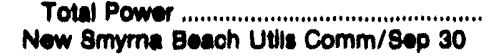 & - & - & - & - & - & - \\
\hline 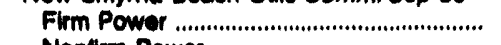 & 140,476 & $6,293,674$ & - & - & - & - \\
\hline Nontirm Power ................................................ & & 2,616 & - & - & - & - \\
\hline 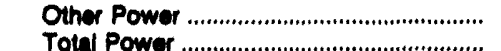 & 63,629 & $2,592,693$ & $=$ & - & - & - \\
\hline Ocala Clty of/Sep 30 & & & & - & - & - \\
\hline Firm Power ..................................................... & - & - & - & -- & - & - \\
\hline \multirow{2}{*}{\multicolumn{6}{|c|}{ Orlando Utilltioe Commiselon/8ep 30}} & - \\
\hline 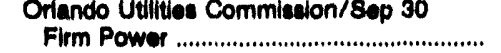 & & & & & & -- \\
\hline Nonftrm Power ............................................... & 32,164 & 686,726 & - & - & -- & $\cdots$ \\
\hline \multirow{2}{*}{\multicolumn{7}{|c|}{ Quinoy Eloctrlc Department/sep 30}} \\
\hline & & & & & & \\
\hline 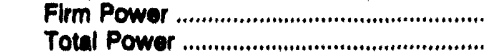 & $\begin{array}{l}93,000 \\
93,000\end{array}$ & $\begin{array}{l}3,099,946 \\
3,099,046\end{array}$ & $\begin{array}{l}47,000 \\
47,000\end{array}$ & $\begin{array}{l}1,039,131 \\
1,030,131\end{array}$ & $\overline{-}$ & $\overline{-}$ \\
\hline \multicolumn{7}{|l|}{ Reedy Creek Improvement Diat/Sep 30} \\
\hline 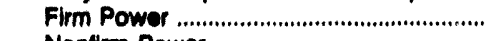 & 391,329 & $13,616,654$ & - & - & - & -- \\
\hline 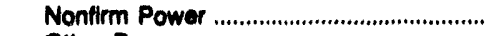 & 6,463 & 141,067 & - & - & - & - \\
\hline 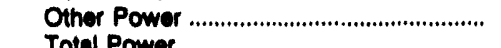 & 1 & $1,019,164$ & - & - & - & - \\
\hline \multicolumn{7}{|l|}{ Sobring Utilties Commiadion/Sop 30} \\
\hline 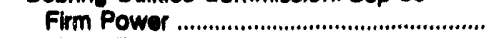 & 173,478 & $0,330,422$ & - & - & - & - \\
\hline 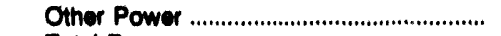 & & 27,494 & - & -- & - & -- \\
\hline \multicolumn{7}{|l|}{ si Cloud City ot/sep 30} \\
\hline 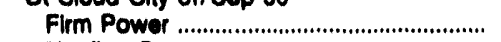 & 151,104 & $6,863,863$ & - & - & - & - \\
\hline Nonfirm Power ............................................. & 8,846 & 391,767 & - & -- & - & - \\
\hline 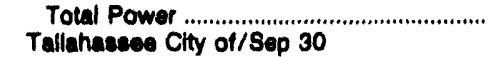 & 169,950 & $7,245,730$ & - & -- & - & - \\
\hline 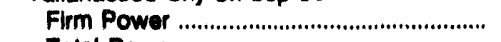 & 21,670 & 408,476 & - & -- & - & - \\
\hline \multicolumn{7}{|l|}{$\begin{array}{l}\text { Total Power ...he } \\
\text { Vero Beach Clty of/Sep } 30\end{array}$} \\
\hline 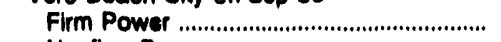 & 3,578 & 365,764 & - & -- & - & - \\
\hline Nonfirm Power .............................................. & 31,655 & 734,828 & - & - & - & - \\
\hline 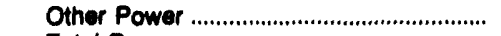 & & 5,557 & - & -- & - & - \\
\hline \multirow{2}{*}{\multicolumn{7}{|c|}{$\begin{array}{l}\text { Cocorgla } \\
\text { Albany Water Gas Light/Jun } 30\end{array}$}} \\
\hline & & & & & & \\
\hline 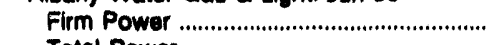 & - & - & 97,007 & $2,337,346$ & 777,110 & $40,063,305$ \\
\hline $\begin{array}{l}\text { Total Power . } \\
\text { Calhoun Light Department/Jun } 30\end{array}$ & \multicolumn{6}{|c|}{ Calhoun Light Department/Jun 30} \\
\hline 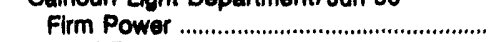 & -- & - & - & -- & 302,674 & $14,026,711$ \\
\hline Total Power & \multicolumn{6}{|c|}{ Carterovillo Electric Systom/Jun 30} \\
\hline 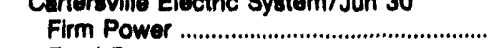 & -- & - & 27,552 & 660,292 & 323,878 & $16,157,020$ \\
\hline \multicolumn{7}{|l|}{ College Park City of/Jun 30} \\
\hline 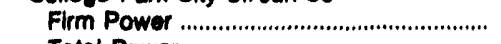 & - & - & 27,683 & 273,644 & 216,056 & $12,401,720$ \\
\hline \multicolumn{7}{|l|}{ Covington City of/Dec 31} \\
\hline Firm Power ......................................... & - & - & 16,936 & 666,428 & 188,515 & $9,757,901$ \\
\hline \multicolumn{7}{|l|}{ Dalton City of/Nov 30} \\
\hline 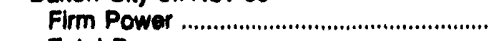 & 324,019 & $9,807,085$ & 73,578 & $1,839,840$ & - & - \\
\hline $\begin{array}{l}\text { Total Power } \\
\text { Douglas City of/Jun } 30\end{array}$ & 324,918 & $9,807,085$ & 73,678 & $1,839,840$ & - & - \\
\hline 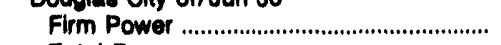 & - & - & 16,394 & 395,027 & 179,272 & $9,223,218$ \\
\hline $\begin{array}{l}\text { Total Power } \\
\text { East Point Clty of/Jun } 30\end{array}$ & - & - & 16,394 & 395,027 & 179,272 & $9,223,218$ \\
\hline 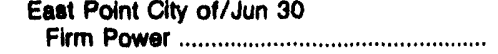 & - & - & 52,800 & $1,398,245$ & 282,300 & $13,308,183$ \\
\hline 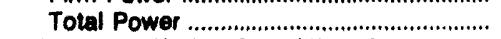 & -- & - & 52,800 & $1,398,245$ & 282,300 & $13,306,183$ \\
\hline Fitzgerald Wir Lgt \& Bnd/Dec 31 & & & & & & \\
\hline 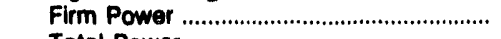 & - & - & 17,681 & 372,646 & 150,300 & $7,863,068$ \\
\hline 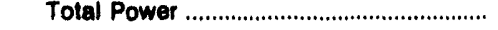 & - & - & 17,681 & 372,648 & 150,300 & $7,863,068$ \\
\hline
\end{tabular}

See notes and footnotes at end of table. 
Table 29. Eloctriolty Purchases by Munlolpal Utilitles, Flecal Year 1992 (Contlnued)

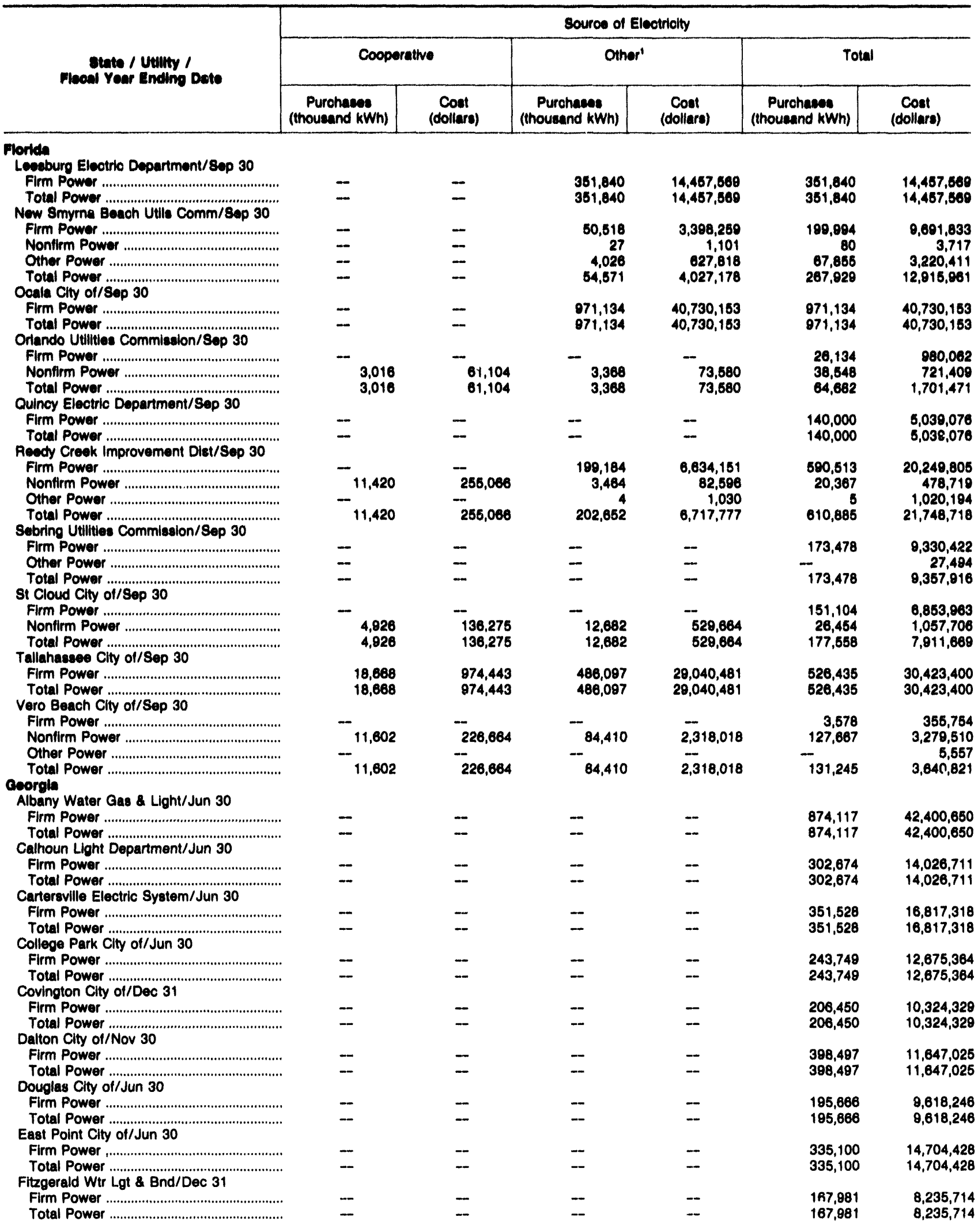

See notes and footnotes at end of table. 
Table 29. Electriclty Purchases by Municlpal Utilitlea, Flocal Yoar 1992 (Continued)

\begin{tabular}{|c|c|c|c|c|c|c|}
\hline \multirow{3}{*}{ 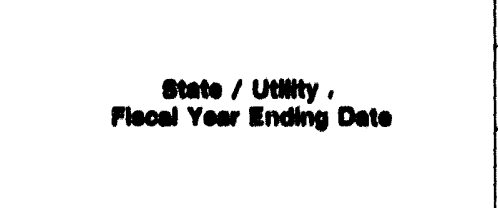 } & \multicolumn{6}{|c|}{ Souroe of Eloctricity } \\
\hline & \multicolumn{2}{|c|}{ Invettor-Owned } & \multicolumn{2}{|c|}{ Federal } & \multicolumn{2}{|c|}{$\begin{array}{l}\text { State and Other } \\
\text { Government }\end{array}$} \\
\hline & $\begin{array}{c}\text { Purchases } \\
\text { (thousand } k W h \text { ) }\end{array}$ & $\begin{array}{c}\text { Cont } \\
\text { (dollars) }\end{array}$ & $\begin{array}{l}\text { Purchases } \\
\text { (thousend } k W h \text { ) }\end{array}$ & $\begin{array}{l}\text { Coat } \\
\text { (dollars) }\end{array}$ & $\begin{array}{l}\text { Purchaves } \\
\text { (thousand } k W h \text { ) }\end{array}$ & $\begin{array}{c}\text { Coot } \\
\text { (dollara) }\end{array}$ \\
\hline \multicolumn{7}{|l|}{$\begin{array}{l}\text { Georgle } \\
\text { Grttin Electric Depertment/Jun } 30\end{array}$} \\
\hline 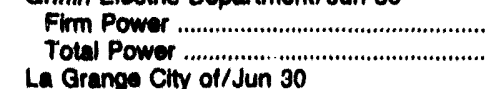 & $\check{-}$ & $\overline{-}$ & $\begin{array}{l}29,094 \\
28,094\end{array}$ & $\begin{array}{l}701,043 \\
701,043\end{array}$ & $\begin{array}{l}288,213 \\
258,213\end{array}$ & $\begin{array}{l}13,500,537 \\
13,500,537\end{array}$ \\
\hline Firm Power & $\overline{-}$ & $\overline{-}$ & $\ddot{-}$ & $\overline{-}$ & $\begin{array}{l}563,601 \\
553,601\end{array}$ & $\begin{array}{l}25,576,832 \\
25,576,832\end{array}$ \\
\hline Frm Power ............................................ & - & - & - & - & 173,767 & $9,873,340$ \\
\hline 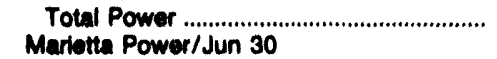 & - & - & - & - & 173,787 & $\mathbf{9}, 873,340$ \\
\hline Firm Power & $\overline{-}$ & $\overline{-}$ & $\begin{array}{l}66,309 \\
68,308\end{array}$ & $\begin{array}{l}1,586,026 \\
1,586,026\end{array}$ & $\begin{array}{l}739,974 \\
739,974\end{array}$ & $\begin{array}{l}42,325,622 \\
42,325,622\end{array}$ \\
\hline Firm Power & $\overline{-}$ & $\overline{-}$ & $\begin{array}{l}24,137 \\
24,137\end{array}$ & $\begin{array}{l}\theta 12,801 \\
612,801\end{array}$ & $\begin{array}{l}141,849 \\
141,840\end{array}$ & $\begin{array}{l}7,812,400 \\
7,812,408\end{array}$ \\
\hline 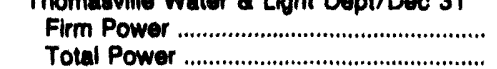 & $\overline{-}$ & $\overline{-}$ & $\begin{array}{l}46,401 \\
45,491\end{array}$ & $\begin{array}{l}1,074,420 \\
1,074,420\end{array}$ & $\begin{array}{l}351,281 \\
351,281\end{array}$ & $\begin{array}{l}17,203,298 \\
17,203,298\end{array}$ \\
\hline $\begin{array}{l}\text { Idaho } \\
\text { Idaho Falls Clty of/Sep } 30\end{array}$ & & & & & & \\
\hline 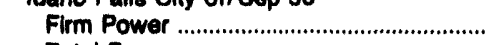 & - & - & 538,886 & $13,331,433$ & -_ & _ \\
\hline 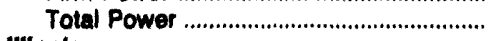 & - & - & 538,886 & $13,331,433$ & -- & - \\
\hline $\begin{array}{l}\text { Ullinols } \\
\text { Betevis City of Electric Util/Dec } 31\end{array}$ & & & & & & \\
\hline 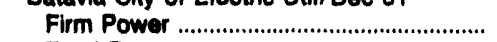 & 191,800 & $10,549,173$ & - & - & - & - \\
\hline $\begin{array}{l}\text { Total Power ... } \\
\text { Geneva Electric Department/Apr } 30\end{array}$ & 191,800 & $10,549,173$ & - & -- & - & - \\
\hline 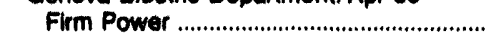 & 138,462 & $5,303,086$ & - & - & - & - \\
\hline 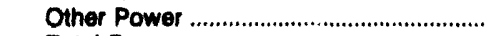 & - & 771,484 & - & - & - & - \\
\hline Total Power ........................... & 138,462 & $6,074,570$ & - & - & - & - \\
\hline $\begin{array}{l}\text { IIInois Municipal Elec Agency/Apr } 30 \\
\text { Firm Power }\end{array}$ & 857,927 & $33,834,663$ & - & - & - & - \\
\hline Nontirm Power …………………...................... & 74,834 & $1,182,710$ & - & -_ & - & - \\
\hline $\begin{array}{l}\text { Total Power } \\
\text { Napervilie Clty of/Apr } 30\end{array}$ & 932,861 & $35,017,373$ & - & - & - & - \\
\hline 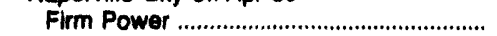 & 679,039 & $35,255,495$ & - & - & - & - \\
\hline Other Power ................................................. & - & - & - & - & - & - \\
\hline Total Power & 679,038 & $35,255,495$ & - & - & - & - \\
\hline 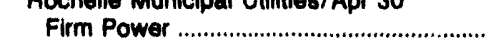 & 133,375 & $4,128,943$ & - & - & -_ & -. \\
\hline 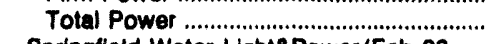 & 133,375 & $4,128,943$ & - & - & - & - \\
\hline $\begin{array}{l}\text { Springfield Water Light\&Power/Fob } 28 \\
\text { Nonfirm Power }\end{array}$ & 1,180 & 62.216 & - & - & -- & - \\
\hline 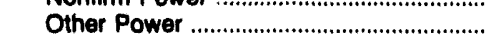 & 6,330 & 83,580 & - & - & - & - \\
\hline 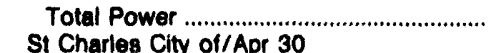 & 7,510 & 135,796 & - & - & - & - \\
\hline 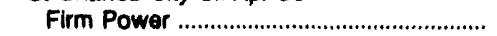 & 352,224 & $18,062,618$ & - & - & -- & - \\
\hline Total Power ...................................................... & 352,224 & $18,062,618$ & - & - & - & - \\
\hline $\begin{array}{l}\text { Indiana } \\
\text { Anderson Mun Light and Water/Dec } 31\end{array}$ & & & & & & \\
\hline 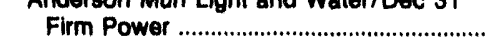 & - & - & -_ & _- & $618,45 n$ & $27,803,718$ \\
\hline 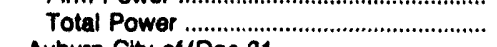 & - & - & - & - & 619,455 & $27,803,718$ \\
\hline Auburn Clty of/Dec 31 & & & & & & \\
\hline 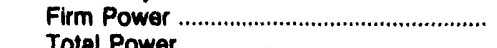 & 310,566 & $12,207,761$ & - & - & - & - \\
\hline $\begin{array}{l}\text { Total Power ....................................... } \\
\text { Bluffton City of/Dec } 31\end{array}$ & 310,566 & $12,207,761$ & - & - & -- & - \\
\hline 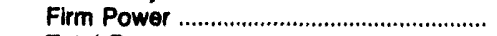 & 148,142 & $6,018,448$ & - & - & - & - \\
\hline 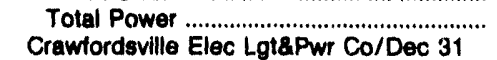 & 148,142 & $6,018,448$ & -- & - & - & - \\
\hline Firm Power & -- & - & -- & - & 330,063 & $12,392,737$ \\
\hline $\begin{array}{l}\text { Total Power . } \\
\text { Frankfort Light \& Power/Dec } 31\end{array}$ & - & - & - & - & 330,063 & $12,382,737$ \\
\hline 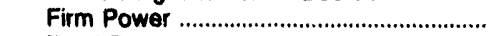 & - & -- & -- & -- & 279,065 & $10,433,011$ \\
\hline $\begin{array}{l}\text { Total Power } \\
\text { Greenfield Municipal Utility/Dec } 31\end{array}$ & - & - & - & - & 279,065 & $10,433,011$ \\
\hline 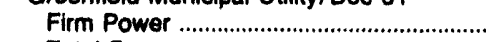 & - & $\sim$ & - & - & 150,635 & $5,903,062$ \\
\hline 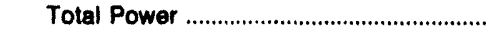 & - & - & - & - & 150,635 & $5,903,062$ \\
\hline
\end{tabular}

See notes and footnotes at end of table. 
Table 29. Electrielty Purchases by Municipal Utilitlos, Fiscal Yoar 1992 (Continued)

\begin{tabular}{|c|c|c|c|c|c|c|}
\hline \multirow{3}{*}{$\begin{array}{l}\text { stete / Uumly I } \\
\text { Fisod Year Ending Date }\end{array}$} & \multicolumn{6}{|c|}{ Source of Eleotricity } \\
\hline & \multicolumn{2}{|c|}{ Cooperative } & \multicolumn{2}{|c|}{ Other' } & \multicolumn{2}{|c|}{ Total } \\
\hline & $\begin{array}{l}\text { Purchases } \\
\text { (thousand kWh) }\end{array}$ & $\begin{array}{c}\text { Cost } \\
\text { (dollers) }\end{array}$ & $\begin{array}{l}\text { Purchacee } \\
\text { (thousand kWh) }\end{array}$ & $\begin{array}{c}\text { Cool } \\
\text { (dollars) }\end{array}$ & $\begin{array}{c}\text { Purchases } \\
\text { (thousand kWh) }\end{array}$ & $\underset{\text { (dollars) }}{\text { Cost }}$ \\
\hline \multicolumn{7}{|l|}{$\begin{array}{l}\text { Cecorgle } \\
\text { Gritin Electric Depertment/Jun } 30\end{array}$} \\
\hline 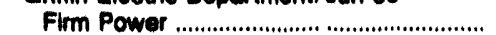 & - & - & - & - & 287,307 & $14,201,580$ \\
\hline 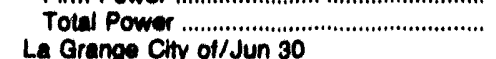 & - & - & - & - & 287,307 & $14,201,580$ \\
\hline 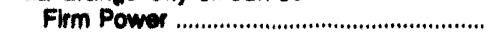 & - & - & - & - & 553,601 & $25,576,932$ \\
\hline $\begin{array}{l}\text { Total Power ................................ } \\
\text { Lawrenceville Electrc Dept/Ag } 31\end{array}$ & - & - & - & - & 553,601 & $25,678,832$ \\
\hline 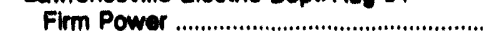 & - & - & - & - & 173,707 & $8,873,340$ \\
\hline $\begin{array}{l}\text { Total Power................................................... } \\
\text { Marletta Power/Jun } 30\end{array}$ & - & - & - & - & 173,787 & $8,873,340$ \\
\hline Firm Power ......................................... & - & - & -- & - & 806,283 & $43,890,548$ \\
\hline 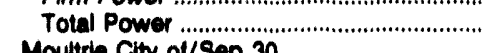 & - & - & - & - & 806,283 & $43,890,548$ \\
\hline $\begin{array}{l}\text { Moultrie Clity of /Sop } 30 \\
\text { Firm Power }\end{array}$ & - & - & - & - & 165,980 & $8.425,309$ \\
\hline Total Power .. & - & - & - & - & 185,886 & $8,425,309$ \\
\hline 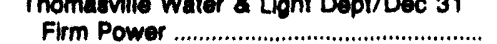 & - & - & - & $\ldots$ & 396,772 & 18.277718 \\
\hline \multirow{2}{*}{\multicolumn{7}{|c|}{ Idaho }} \\
\hline & & & & & & \\
\hline Firm Power . & - & - & - & $-\ldots$ & 538,886 & $13,331,433$ \\
\hline Total Power ……………………………. & - & - & - & -- & 538,886 & $13,331,433$ \\
\hline \multicolumn{7}{|l|}{$\begin{array}{l}\text { IIInolo } \\
\text { Batavia City of Electric UtII/Dec } 31\end{array}$} \\
\hline 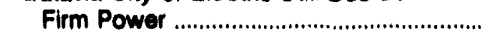 & - & - & -- & -- & 191,800 & $10,548,173$ \\
\hline $\begin{array}{l}\text { Total Power .............................. } \\
\text { Geneva Electric Department/Aor }\end{array}$ & - & - & - & - & 191,800 & $10,549,173$ \\
\hline 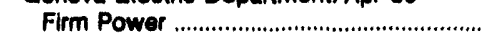 & -- & -- & 19,145 & 526,634 & 157,607 & $5,829,720$ \\
\hline 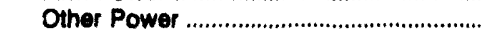 & - & - & $\ldots$ & - & - & 771,484 \\
\hline Total Power .............................................. & - & -- & 19,145 & 526,634 & 157.607 & $6,601,204$ \\
\hline \multicolumn{7}{|l|}{ Illincis Municipal Elec Agency/Apr 30} \\
\hline 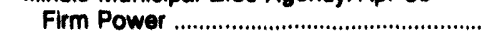 & - & -- & 68,088 & $2,084,264$ & 926,015 & $35,818,847$ \\
\hline 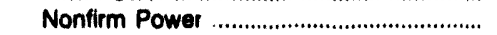 & -- & -- & - & - & 74,834 & $1,182,710$ \\
\hline 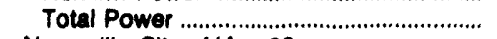 & - & - & 68,088 & $2,084,284$ & $1,000,849$ & $37,101,657$ \\
\hline \multicolumn{7}{|l|}{ Naperville City of/Apr 30} \\
\hline 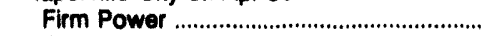 & -- & - & - & - & 679,039 & $35,255,495$ \\
\hline 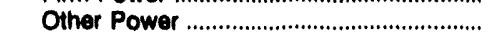 & - & - & 3,103 & 24,531 & 3,183 & 24,531 \\
\hline \multicolumn{7}{|l|}{ Rochelle Municipal Utilities/Apr 30} \\
\hline 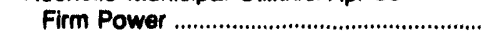 & - & -- & - & - & 133,375 & $4,128,843$ \\
\hline Total Power ................................... & - & - & - & -- & 133,375 & $4,128,843$ \\
\hline \multicolumn{7}{|l|}{ Springtield Water Light\&Power/Feb 28} \\
\hline $\begin{array}{l}\text { Nonfirm Power .............................................. } \\
\text { Other Power }\end{array}$ & - & -- & - & - & 1,180 & 52,216 \\
\hline 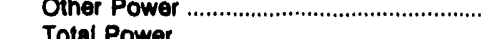 & - & - & -- & -- & 6,330 & 83,580 \\
\hline \multicolumn{7}{|l|}{ St Charles City of/Apr 30} \\
\hline 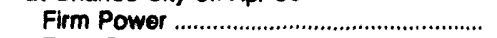 & -- & - & -- & -- & 352,224 & $18,062,618$ \\
\hline Total Power ……………………………....... & - & - & - & - & 352,224 & $18,062,618$ \\
\hline \multirow{2}{*}{\multicolumn{7}{|c|}{ Indiana }} \\
\hline \multicolumn{2}{|l|}{ Anderson Mun Light and Water/Dec 31} & & & & & \\
\hline 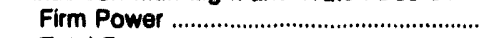 & - & - & -- & - & 619,455 & $27,803,718$ \\
\hline \multirow{2}{*}{\multicolumn{7}{|c|}{ Auburn City of/Dec 31}} \\
\hline & & & & & & \\
\hline 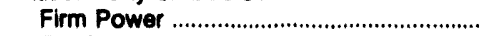 & - & - & - & - & 310,560 & $12,207,761$ \\
\hline Total Power & - & - & - & - & 310,566 & $12,207,761$ \\
\hline Bluftton City of/Dec 31 & & & & & & \\
\hline 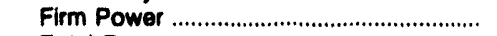 & - & - & - & - & 148,142 & $8,019,448$ \\
\hline $\begin{array}{l}\text { Total Power . } \\
\text { Crawordsville Elec Lots Pwr Co/Dec } 31\end{array}$ & - & - & - & - & 148,142 & $6,018,448$ \\
\hline $\begin{array}{l}\text { Crawordsville Elec Lgiar-wr Co/Dec } 31 \\
\text { Firm Power }\end{array}$ & - & - & - & -_ & 330,063 & \\
\hline Total Power & - & -- & - & 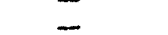 & $\begin{array}{l}330,063 \\
330,063\end{array}$ & $\begin{array}{l}12,392,737 \\
12,392,737\end{array}$ \\
\hline Frankfort Light \& Power/Dec 31 & & & & & & \\
\hline 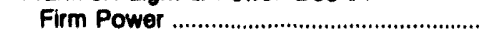 & - & - & - & - & 279,065 & $10,433,011$ \\
\hline 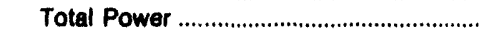 & - & - & -- & - & 279,065 & $10,433,011$ \\
\hline Greenfield Municipal Utility/Dec 31 & & & & & & \\
\hline 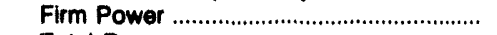 & - & - & - & - & 150,635 & $5,903,062$ \\
\hline 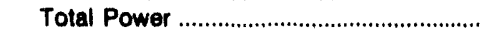 & - & -- & - & - & 150,635 & $5,903,062$ \\
\hline
\end{tabular}

See notes and footnotes at end of table. 
Table 29. Eleotriclty Purchases by Municipal Utilities, Fiscal Year 1902 (Continued)

\begin{tabular}{|c|c|c|c|c|c|c|}
\hline \multirow{3}{*}{ 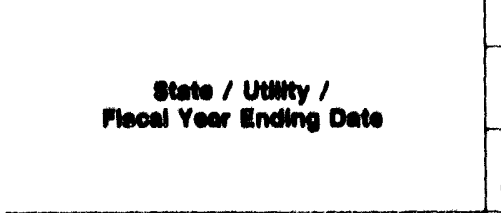 } & \multicolumn{6}{|c|}{ Source of Electrioly } \\
\hline & \multicolumn{2}{|c|}{ Invertor-Owned } & \multicolumn{2}{|c|}{ Fedoral } & \multicolumn{2}{|c|}{$\begin{array}{l}\text { State and Other } \\
\text { Covernment }\end{array}$} \\
\hline & $\begin{array}{l}\text { Purcheses } \\
\text { (thourand kWh) }\end{array}$ & $\begin{array}{c}\text { Cost } \\
\text { (dollara) }\end{array}$ & $\begin{array}{l}\text { Purchases } \\
\text { (thousund kWh) }\end{array}$ & $\begin{array}{c}\text { Cout } \\
\text { (dollars) }\end{array}$ & $\begin{array}{c}\text { Purchases } \\
\text { (thoueand kWh) }\end{array}$ & $\begin{array}{c}\text { Cost } \\
\text { (dollare) }\end{array}$ \\
\hline \multicolumn{7}{|l|}{$\begin{array}{l}\text { Inclene } \\
\text { Jasper Municipal Electric UtI/Dec } 31\end{array}$} \\
\hline 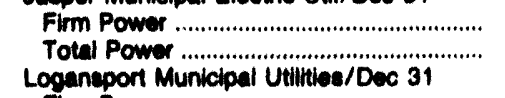 & $\begin{array}{l}187,314 \\
187,314\end{array}$ & $\begin{array}{l}8,869,055 \\
8,869,056\end{array}$ & $\overline{-}$ & - & - & - \\
\hline 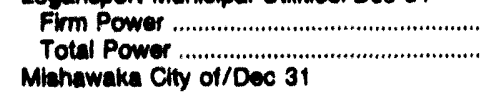 & $\begin{array}{l}228,255 \\
226,255\end{array}$ & $\begin{array}{l}6,622,804 \\
6,622,804\end{array}$ & - & - & $\overline{-}$ & - \\
\hline 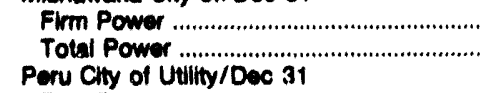 & $\begin{array}{l}419,850 \\
419,850\end{array}$ & $\begin{array}{l}18,851,741 \\
18,851,741\end{array}$ & - & - & - & - \\
\hline 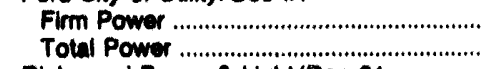 & - & - & - & - & $\begin{array}{l}188,456 \\
188,456\end{array}$ & $\begin{array}{l}5,027,736 \\
5,027,736\end{array}$ \\
\hline Alchmond Power \& Light/Dec 31 & & & & & & \\
\hline 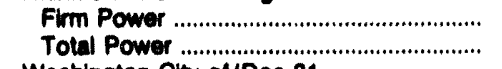 & - & - & $\overline{-}$ & - & $\begin{array}{l}172,706 \\
172,706\end{array}$ & $\begin{array}{l}10,778,352 \\
10,778,352\end{array}$ \\
\hline Weshington Clty of/Dec 31 & & & & & & \\
\hline 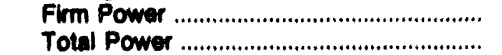 & $\overline{-}$ & $\overline{-}$ & - & - & $\begin{array}{l}158,226 \\
156,226\end{array}$ & $\begin{array}{l}6,089,370 \\
6,089,370\end{array}$ \\
\hline Iown Ames Clty of/Jun 30 & & & & & & \\
\hline 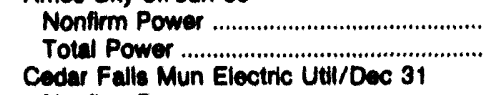 & $\begin{array}{l}89,431 \\
89,431\end{array}$ & $\begin{array}{l}1,323,303 \\
1,323,303\end{array}$ & - & - & - & - \\
\hline Nonfirm Power & 63,364 & 918,745 & - & - & - & - \\
\hline $\begin{array}{l}\text { Total Power ................................. } \\
\text { Muscatine Power and Water/Dec } 31\end{array}$ & 63,364 & 918,745 & - & - & - & - \\
\hline 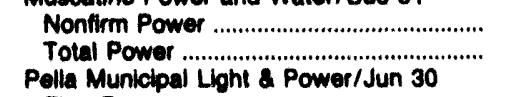 & $\begin{array}{l}13,739 \\
13,739\end{array}$ & $\begin{array}{l}170,784 \\
170,784\end{array}$ & $\begin{array}{l}700 \\
700\end{array}$ & $\begin{array}{l}10,827 \\
10,927\end{array}$ & $\begin{array}{l}60,978 \\
60,978\end{array}$ & $\begin{array}{l}743,723 \\
743,723\end{array}$ \\
\hline 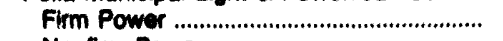 & 19,469 & 418,044 & -- & - & - & - \\
\hline 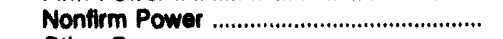 & 53,313 & 954,843 & - & - & - & - \\
\hline 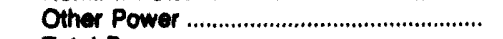 & 10,287 & 111,602 & - & - & - & - \\
\hline Total Power ................................................... & 83,069 & $1,485,089$ & - & - & - & - \\
\hline $\begin{array}{l}\text { Kanase } \\
\text { Chanute Mun Electric Utility/Dec } 31\end{array}$ & & & & & & \\
\hline 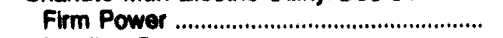 & - & - & 3,645 & 94,879 & - & - \\
\hline 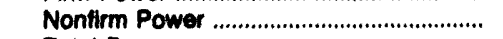 & 127,837 & $3,120,650$ & - & - & - & - \\
\hline $\begin{array}{l}\text { Total Power ........................................... } \\
\text { Cofteyville Clty of } 31\end{array}$ & 127,837 & $3,120,850$ & 3,645 & 94,879 & - & - \\
\hline 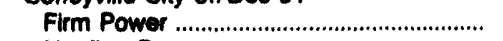 & - & - & 6,190 & 142,206 & - & - \\
\hline 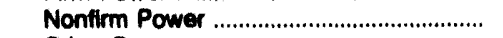 & 103,470 & $2,438,639$ & - & - & - & - \\
\hline 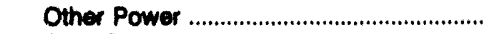 & - & - & - & - & - & - \\
\hline $\begin{array}{l}\text { Total Power .............................................. } \\
\text { Garden Clty City of/Dec } 31\end{array}$ & 103,470 & $2,438,539$ & 6,180 & 142,206 & - & - \\
\hline 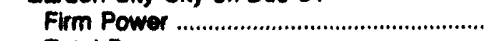 & - & - & - & - & - & - \\
\hline 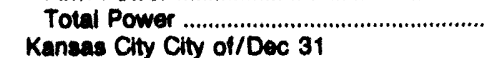 & - & - & - & - & - & -- \\
\hline 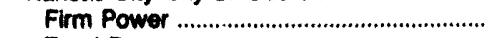 & 139,073 & $2,333,330$ & 128,412 & $2,637,087$ & - & - \\
\hline Total Power & 139,073 & $2,333,330$ & 128,412 & $2,637,007$ & - & - \\
\hline McPherson Board of Pub Utils/Dec 31 & & & & & & \\
\hline 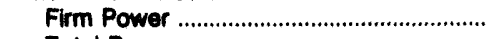 & 457,369 & $12,247,341$ & - & - & - & - \\
\hline & 457,369 & $12,247,34 t$ & - & - & - & $-\infty$ \\
\hline 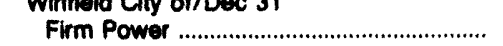 & - & - & - & - & 108,735 & $3,727,289$ \\
\hline 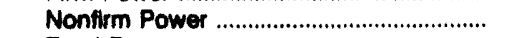 & 79,015 & $2,534,683$ & - & - & - & - \\
\hline 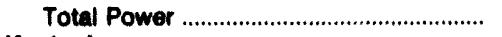 & 79,015 & $2,534,683$ & - & - & 108,735 & $3,727,299$ \\
\hline $\begin{array}{l}\text { Kentucky } \\
\text { Bowling Green Municipal Utils/Jun } 30\end{array}$ & & & & & & \\
\hline 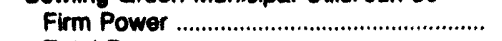 & - & - & 654,459 & $29,497,990$ & - & - \\
\hline 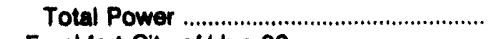 & - & - & 654,458 & $29,497,990$ & - & - \\
\hline Frankiort City of/Jun 30 & & & & & & \\
\hline 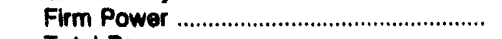 & 579,208 & $18,135,342$ & - & - & - & - \\
\hline $\begin{array}{l}\text { Total Power } \\
\text { Franklin Clty of/Jun } 30\end{array}$ & 579,208 & $18,135,342$ & - & - & - & - \\
\hline 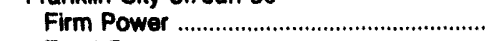 & - & - & 187,714 & $7,827,832$ & - & - \\
\hline 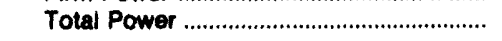 & - & - & 187.714 & $7,827,832$ & - & - \\
\hline
\end{tabular}

See notes and footnotes at end of table. 
Table 29. Eloctrlalty Purchases by Munlolpal Utilities, Flocal Year 1992 (Continued)

\begin{tabular}{|c|c|c|c|c|c|c|}
\hline \multirow{3}{*}{$\begin{array}{l}\text { Etate / Uuilty I } \\
\text { Fiead Year Ending Date }\end{array}$} & \multicolumn{6}{|c|}{ Source of Electricity } \\
\hline & \multicolumn{2}{|c|}{ Cooperative } & \multicolumn{2}{|c|}{ Other' } & \multicolumn{2}{|c|}{ Total } \\
\hline & $\begin{array}{c}\text { Purchases } \\
\text { (thousend kWh) }\end{array}$ & $\begin{array}{c}\text { Cost } \\
\text { (dollarn) }\end{array}$ & $\begin{array}{c}\text { Purchasese } \\
\text { (thousand kWh) }\end{array}$ & $\begin{array}{c}\text { Cont } \\
\text { (oollara) }\end{array}$ & $\begin{array}{c}\text { Purchases } \\
\text { (thousand kWh) }\end{array}$ & $\begin{array}{c}\text { Coot } \\
\text { (dollare) }\end{array}$ \\
\hline \multicolumn{7}{|l|}{ Indlana } \\
\hline Firm Power ............................................. & - & - & - & -- & 187,314 & $8,869,055$ \\
\hline $\begin{array}{l}\text { Total Power ... } \\
\text { Logeneport Municipal Uillitiea/Dec } 31\end{array}$ & - & - & - & - & 187,314 & $8,869,055$ \\
\hline 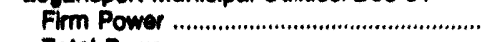 & - & - & - & -- & 226,265 & $6,622,604$ \\
\hline \multicolumn{7}{|l|}{ Mishawaka City of/Dec 31} \\
\hline 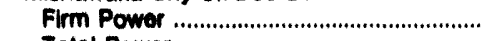 & - & - & - & - & 418,850 & $18,851,741$ \\
\hline \multicolumn{7}{|l|}{ Peru City of Utilly /Dec 31} \\
\hline Firm Power ............................................. & - & - & - & - & 186,465 & $5,027,736$ \\
\hline \multicolumn{6}{|l|}{ Alchmond Power \& Light/Dec 31} & $5,027,736$ \\
\hline 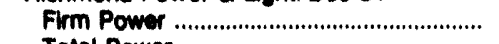 & - & - & - & - & 172,706 & $10,778,352$ \\
\hline $\begin{array}{l}\text { Total Power } \\
\text { Washington City of /Dec } 31\end{array}$ & - & - & - & - & 172,708 & $10,778,352$ \\
\hline 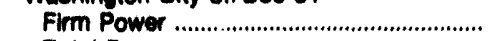 & - & - & - & - & 156,226 & $6,080,370$ \\
\hline 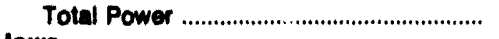 & - & - & - & - & 156,226 & $6,089,370$ \\
\hline \multicolumn{7}{|l|}{$\begin{array}{l}\text { lowe } \\
\text { Ames City of/Jun } 30\end{array}$} \\
\hline 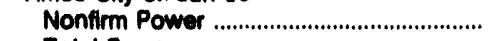 & - & - & - & - & $\mathbf{9 0 , 4 3 1}$ & $1,323,303$ \\
\hline \multirow{2}{*}{\multicolumn{5}{|c|}{ Cedar Falla Mun Eloctric Util/Doc 31}} & 89,431 & $1,323,303$ \\
\hline 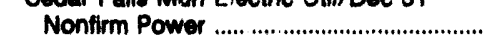 & -- & - & - & -- & 63,364 & \\
\hline Total Power .......... & - & - & - & - & 63,364 & 918,745 \\
\hline & & \\
\hline & $1,074,995$ \\
\hline 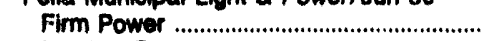 & - & - & 2,364 & 114,874 & 21,833 & 533,518 \\
\hline 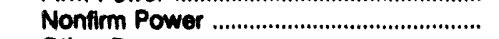 & - & - & - & - & 53,313 & 854,943 \\
\hline 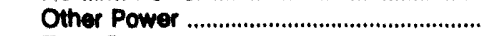 & -- & - & - & - & 10,287 & 111,502 \\
\hline 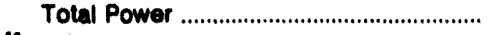 & - & - & 2,364 & 114,874 & 85,433 & $1,589,963$ \\
\hline \multirow{2}{*}{\multicolumn{7}{|c|}{$\begin{array}{l}\text { Kansad } \\
\text { Chanute ML: Electric Utility/Dec } 31\end{array}$}} \\
\hline & - & - & - & - & 3,645 & 94,879 \\
\hline 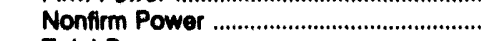 & - & - & - & -- & 127,837 & $3,120,650$ \\
\hline \multicolumn{7}{|l|}{ Coffeyville City of / Dec 31} \\
\hline 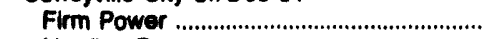 & -- & - & - & - & 6,190 & 142,206 \\
\hline Nontirm Power ..................................................... & - & - & - & -- & 103,470 & $2,438,538$ \\
\hline 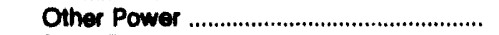 & - & - & - & 540,000 & - & 540,000 \\
\hline \multicolumn{6}{|l|}{ Garden City City of/Dec 31} & $3,120,746$ \\
\hline 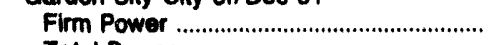 & 159,805 & $8,522,810$ & - & - & 159,805 & $8,522,810$ \\
\hline 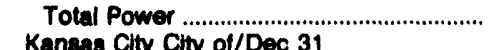 & 159,805 & $8,522,810$ & - & - & 159,805 & $8,522,810$ \\
\hline \multicolumn{4}{|l|}{ Kaneas City City of/Dec 31} & -- & 267,485 & $4,870,427$ \\
\hline 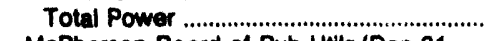 & -- & - & - & - & 267,485 & $4,970,427$ \\
\hline \multicolumn{7}{|l|}{ McPherson Board of Pub Utils/Dec 31} \\
\hline 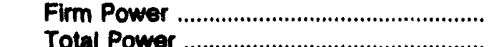 & - & - & $\overline{-}$ & - & 457,369 & $12,247,341$ \\
\hline $\begin{array}{l}\text { Total Power ... } \\
\text { Wintiold City of/Dec } 31\end{array}$ & - & - & - & - & 457,369 & $12,247,341$ \\
\hline 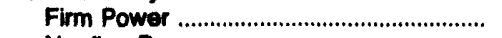 & - & - & - & - & 108,735 & $3,727,299$ \\
\hline 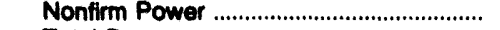 & - & - & - & - & 79,015 & $2,534,683$ \\
\hline 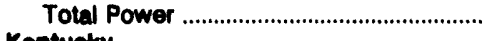 & - & - & - & - & 187,750 & $6,261,982$ \\
\hline Kentucky & & & & & & \\
\hline 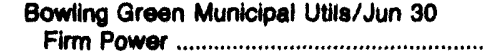 & & & & & & \\
\hline $\begin{array}{l}\text { Firm Power } \\
\text { Total Power }\end{array}$ & $\overline{-}$ & $\overline{-}$ & $\overline{-}$ & $\overline{-}$ & $\begin{array}{l}654,459 \\
654,459\end{array}$ & $\begin{array}{l}29,497,890 \\
29,497,990\end{array}$ \\
\hline Frankfort City of/Jun 30 & & & & & & \\
\hline 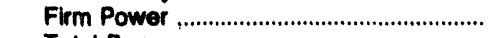 & - & - & - & - & 579,208 & $18,135,342$ \\
\hline $\begin{array}{l}\text { Total Power ........................................ } \\
\text { Franklin City of/Jun } 30\end{array}$ & - & - & - & - & 578,208 & $18,135,342$ \\
\hline 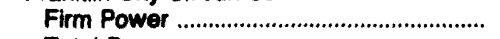 & - & - & - & - & 187,714 & $7,927,832$ \\
\hline 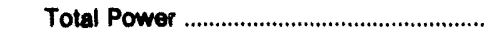 & - & - & - & - & 187,714 & $7,827,832$ \\
\hline
\end{tabular}

See notes and tootnotes at end of table. 
Table 29. Electricity Purchases by Municipal Utilities, Fiscal Year 1992 (Continued)

\begin{tabular}{|c|c|c|c|c|c|c|}
\hline \multirow{3}{*}{$\begin{array}{l}\text { State / Utility / } \\
\text { Flecal Yesr Ending Date }\end{array}$} & \multicolumn{6}{|c|}{ Source of Electricity } \\
\hline & \multicolumn{2}{|c|}{ Investor-Owned } & \multicolumn{2}{|c|}{ Federal } & \multicolumn{2}{|c|}{$\begin{array}{l}\text { State and Other } \\
\text { Government }\end{array}$} \\
\hline & $\begin{array}{l}\text { Purchases } \\
\text { (thousand kWh) }\end{array}$ & $\begin{array}{c}\text { Cost } \\
\text { (dollars) }\end{array}$ & $\begin{array}{l}\text { Purchases } \\
\text { (thousand kWh) }\end{array}$ & $\begin{array}{c}\text { Cost } \\
\text { (dollars) }\end{array}$ & $\begin{array}{l}\text { Purchases } \\
\text { (thousand kWh) }\end{array}$ & $\begin{array}{c}\text { Cost } \\
\text { (dollars) }\end{array}$ \\
\hline \multicolumn{7}{|l|}{ Komtucky } \\
\hline 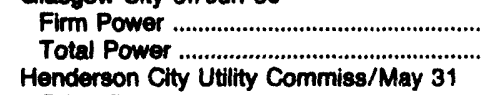 & - & - & $\begin{array}{l}270,681 \\
270,681\end{array}$ & $\begin{array}{l}11,857,859 \\
11,857,859\end{array}$ & $\overline{-}$ & $\overline{-}$ \\
\hline 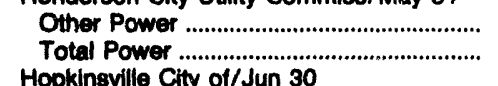 & - & - & $\begin{array}{l}18,000 \\
18,000\end{array}$ & $\begin{array}{l}359,622 \\
359,622\end{array}$ & - & - \\
\hline 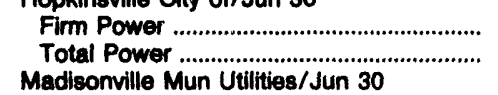 & $\overline{-}$ & - & $\begin{array}{l}394,779 \\
394,779\end{array}$ & $\begin{array}{l}16,295,612 \\
16,295,612\end{array}$ & $\overline{-}$ & $\overline{-}$ \\
\hline 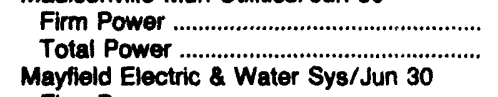 & $\begin{array}{l}258,954 \\
258,954\end{array}$ & $\begin{array}{l}8,267,961 \\
8,267,961\end{array}$ & - & - & - & - \\
\hline 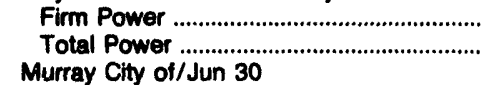 & $\overline{-}$ & $\overline{-}$ & $\begin{array}{l}137,719 \\
137,719\end{array}$ & $\begin{array}{l}6,341,241 \\
6,341,241\end{array}$ & $\overline{-}$ & - \\
\hline 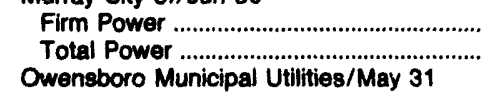 & - & - & $\begin{array}{l}228,933 \\
228,933\end{array}$ & $\begin{array}{l}10,385,779 \\
10,385,779\end{array}$ & - & $\overline{-}$ \\
\hline 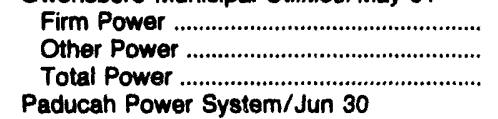 & $-\begin{array}{r}28,631 \\
-28,631\end{array}$ & $\begin{array}{r}458,555 \\
-458,555\end{array}$ & - & - & - & $\overline{-}$ \\
\hline Firm Power & - & - & $\begin{array}{l}525,689 \\
525,689\end{array}$ & $\begin{array}{l}23,241,853 \\
23,241,853\end{array}$ & - & - \\
\hline $\begin{array}{l}\text { Louldelana } \\
\text { Alexandria City of/Apr } 30\end{array}$ & & & & & & \\
\hline 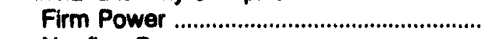 & - & - & - & - & 434,418 & $16,670,275$ \\
\hline $\begin{array}{l}\text { Nonfirm Power } \\
\text { Total Power . } \\
\text { Lafayette City of/Oct } 31\end{array}$ & $\begin{array}{l}129,510 \\
129,510\end{array}$ & $\begin{array}{l}2,686,607 \\
2,686,607\end{array}$ & $\overline{-}$ & $\overline{-}$ & $-434,418$ & $1 \overline{16,670,275}$ \\
\hline 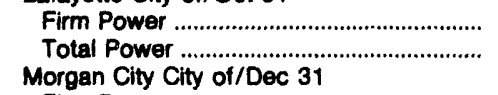 & $\begin{array}{l}138,668 \\
138,668\end{array}$ & $\begin{array}{l}2,808,504 \\
2,808,504\end{array}$ & - & - & - & - \\
\hline Firm Power & - & - & - & - & $\begin{array}{l}155,200 \\
155,200\end{array}$ & $\begin{array}{l}6,876,600 \\
6,876,600\end{array}$ \\
\hline Natchitoches City of/May 31 & & & & & & \\
\hline 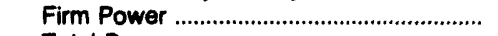 & -- & - & - & - & - & - \\
\hline $\begin{array}{l}\text { Total Power } \\
\text { Ruston City of/Sep } 30\end{array}$ & - & $\cdots$ & - & - & - & - \\
\hline 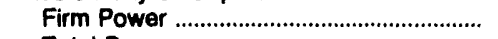 & - & - & 20,817 & 367,338 & - & - \\
\hline $\begin{array}{l}\text { Total Power } \\
\text { Terrebonne Parish Consol Govt/Dec } 31\end{array}$ & -- & - & 20,817 & 367,338 & -- & - \\
\hline 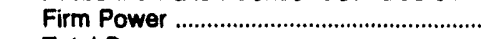 & - & - & - & - & 228,676 & $9,949,681$ \\
\hline 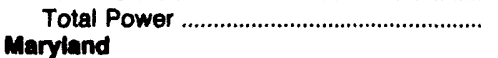 & - & - & - & - & 228,676 & $9,949,681$ \\
\hline Easton Utilities Commission/Jun 30 & & & & & & \\
\hline 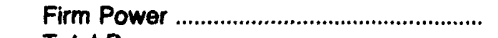 & 172,620 & $4,732,332$ & - & - & - & - \\
\hline $\begin{array}{l}\text { Total Power } \\
\text { Hagerstown City of /Jun } 30\end{array}$ & 172,620 & $4,732,332$ & - & - & - & - \\
\hline 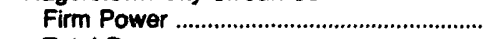 & 302,208 & $10,946,453$ & - & - & - & - \\
\hline $\begin{array}{l}\text { Total Power } \\
\text { Masesachusetts }\end{array}$ & 302,208 & $10,946,453$ & - & - & - & - \\
\hline Braintree Town of/Dec 31 & & & & & & \\
\hline 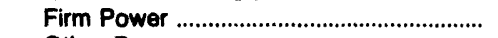 & 203,709 & $6,648,675$ & - & - & 144,411 & $8,961,320$ \\
\hline 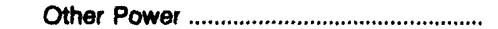 & - & - & - & - & - & - \\
\hline $\begin{array}{l}\text { Total Power } \\
\text { Chicopee City of/Dec } 31\end{array}$ & 203,709 & $6,648,675$ & - & - & 144,411 & $8,961,320$ \\
\hline 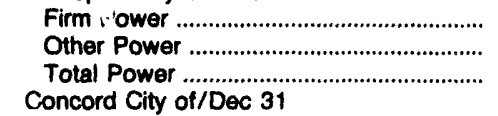 & $\begin{array}{r}162,590 \\
50,958 \\
213,548\end{array}$ & $\begin{array}{r}8,892,173 \\
1,851,891 \\
10,844,064\end{array}$ & - & - & $\begin{array}{r}35,971 \\
2,623 \\
38,504\end{array}$ & $\begin{array}{r}427,231 \\
61,811 \\
489,042\end{array}$ \\
\hline 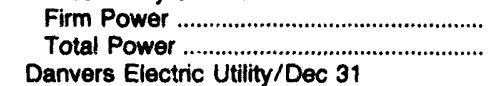 & $\begin{array}{l}136,691 \\
136,681\end{array}$ & $\begin{array}{l}8,706,576 \\
8,706,576\end{array}$ & - & - & $\begin{array}{l}10,342 \\
10,342\end{array}$ & $\begin{array}{l}175,475 \\
175,475\end{array}$ \\
\hline Firm Power & $\begin{array}{l}20,207 \\
20,207\end{array}$ & $\begin{array}{l}644,047 \\
644,047\end{array}$ & - & - & $\begin{array}{l}260,492 \\
260,492\end{array}$ & $\begin{array}{l}22,666,878 \\
22,666,878\end{array}$ \\
\hline
\end{tabular}

See notes and footnotes at end of table. 
Table 29. Electricity Purchases by Municipal Utilities, Fiscal Year 1992 (Continued)

\begin{tabular}{|c|c|c|c|c|c|c|}
\hline \multirow{3}{*}{$\begin{array}{l}\text { State / Utilty / } \\
\text { Fiecal Year Ending Date }\end{array}$} & \multicolumn{6}{|c|}{ Source of Electricity } \\
\hline & \multicolumn{2}{|c|}{ Cooperative } & \multicolumn{2}{|c|}{ Other' } & \multicolumn{2}{|c|}{ Total } \\
\hline & $\begin{array}{c}\text { Purchases } \\
\text { (thousand kWh) }\end{array}$ & $\begin{array}{c}\text { Cost } \\
\text { (dollars) }\end{array}$ & $\begin{array}{c}\text { Purchases } \\
\text { (thousand kWh) }\end{array}$ & $\begin{array}{c}\text { Cost } \\
\text { (dollars) }\end{array}$ & $\begin{array}{c}\text { Purchases } \\
\text { (thousand kWh) }\end{array}$ & $\begin{array}{c}\text { Cost } \\
\text { (dollars) }\end{array}$ \\
\hline \multicolumn{7}{|l|}{ Kontucky } \\
\hline \multicolumn{4}{|l|}{ Glasgow City of/Jun 30} & - & & \\
\hline 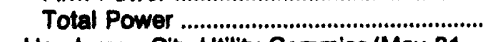 & - & - & - & - & 270,681 & $11,857,859$ \\
\hline & 467,244 \\
\hline $\begin{array}{l}\text { Total Power ........................................... } \\
\text { Hopkinsville City ofJun }\end{array}$ & 5,549 & 107,622 & - & $\overline{-}$ & 23,549 & 467,244 \\
\hline 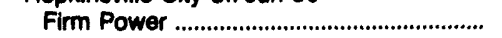 & - & - & - & - & 394,779 & $16,295,612$ \\
\hline 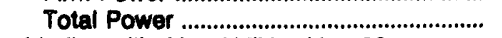 & - & - & - & - & 394,779 & $16,295,612$ \\
\hline \multicolumn{7}{|l|}{ Madisonville Mun Utilities/Jun 30} \\
\hline 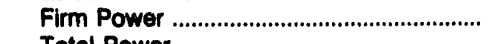 & - & - & - & - & 258,854 & 8,267,961 \\
\hline $\begin{array}{l}\text { Total Power ... } \\
\text { Mavfield Eloctric \& Water Sys/Jun } 30\end{array}$ & - & - & - & - & 258,854 & $8,267,961$ \\
\hline & - & - & - & - & 137,719 & $6,341,241$ \\
\hline 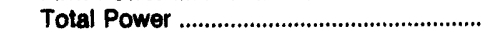 & - & -- & - & - & 137,719 & $6,341,241$ \\
\hline \multicolumn{7}{|l|}{ Murray City of/Jun 30} \\
\hline 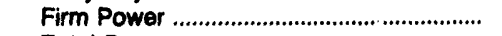 & - & -- & - & - & 228,933 & $10,385,779$ \\
\hline Total Power .... & - & - & - & - & 228,933 & $10,385,779$ \\
\hline \multicolumn{2}{|l|}{ Owensboro Municipal Utilities/May 31} & -- & - & - & 28,631 & 458,555 \\
\hline 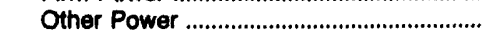 & - & -- & - & $-8,888$ & - & $-8,888$ \\
\hline 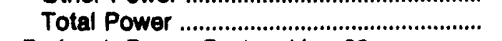 & - & -- & - & $-8,888$ & 28,631 & 449,667 \\
\hline Paducah Power System/Jun 30 & & & & & & \\
\hline 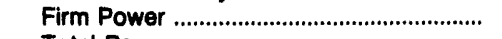 & - & - & - & - & 525,689 & $23,241,853$ \\
\hline Total Power ....................................................... & - & - & - & - & 525,689 & $23,241,853$ \\
\hline \multirow{2}{*}{\multicolumn{7}{|c|}{$\begin{array}{l}\text { Loulelana } \\
\text { Alexandria City of/Apr } 30\end{array}$}} \\
\hline \multirow{2}{*}{\multicolumn{7}{|c|}{ 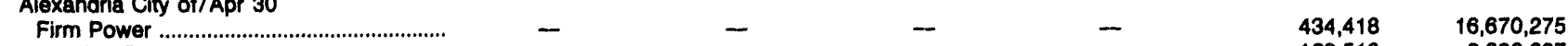 }} \\
\hline & $\bar{z}$ & - & - & & & \\
\hline & $\overline{-}$ & - & - & $\overline{-}$ & $\begin{array}{l}129,510 \\
563,928\end{array}$ & $\begin{array}{r}2,686,607 \\
19,356,882\end{array}$ \\
\hline $\begin{array}{l}\text { Total Power ... } \\
\text { Lafayette Clty of/Oct } 31\end{array}$ & & & & & & \\
\hline Firm Power & 2,515 & 50,937 & $1,462,704$ & $50,245,858$ & $1,603,887$ & $53,105,299$ \\
\hline 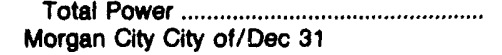 & 2,515 & 50,937 & $1,462,704$ & $50,245,858$ & $1,603,887$ & $53,105,299$ \\
\hline 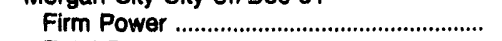 & - & - & - & - & 155,200 & $6,876,600$ \\
\hline 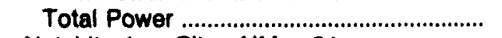 & - & - & - & - & 155,200 & $6,876,600$ \\
\hline Natchitoches City of/May 31 & & & & & & \\
\hline 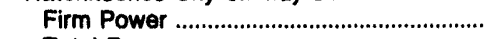 & - & - & 196,067 & $7,715,901$ & 196,067 & $7,715,901$ \\
\hline 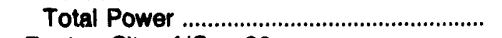 & - & - & 196,067 & $7,715,901$ & 196,067 & $7,715,901$ \\
\hline Ruston City of/Sep 30 & & & & & & \\
\hline 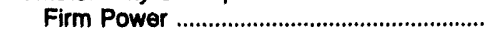 & - & - & 29,345 & 623,190 & 50,162 & 890,528 \\
\hline Total Power .................................... & - & - & 29,345 & 623,190 & 50,162 & 990,528 \\
\hline Terrebonne Parish Consol Govt/Dec 31 & & & & & & \\
\hline 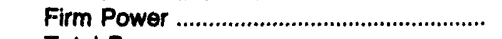 & - & - & - & -- & 228,676 & $9,948,681$ \\
\hline 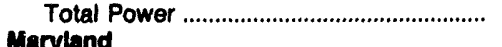 & - & - & - & - & 228,676 & $9,949,681$ \\
\hline $\begin{array}{l}\text { Maryland } \\
\text { Easton Utilities Commission/Jun } 30\end{array}$ & & & & & & \\
\hline $\begin{array}{l}\text { Easton Utilities Commission/Jun } 30 \\
\text { Firm Power . }\end{array}$ & & & & & & \\
\hline $\begin{array}{l}\text { Firm Power } \\
\text { Total Power }\end{array}$ & $\overline{-}$ & $\overline{-}$ & $=$ & - & $\begin{array}{l}172,620 \\
172,620\end{array}$ & $\begin{array}{l}4,732,332 \\
4,732,332\end{array}$ \\
\hline Hagerstown City of/Jun 30 & & & & & & \\
\hline 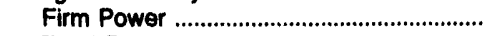 & - & - & - & - & 302,208 & $10,946,453$ \\
\hline 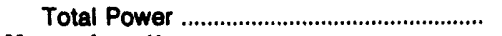 & -- & - & - & - & 302,208 & $10,946,453$ \\
\hline Maesachueatte & & & & & & \\
\hline Braintree Town of/Dec 31 & & & & & & \\
\hline 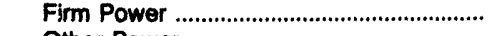 & - & - & 8,457 & $1,027,280$ & 356,577 & $16,637,275$ \\
\hline 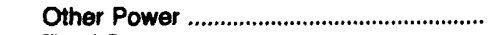 & - & - & -173 & 20,833 & -173 & 20,833 \\
\hline Total Power & - & - & 8,284 & $1,048,113$ & 356,404 & $16,658,108$ \\
\hline Chicopee City ot/Dec 31 & & & & & & \\
\hline 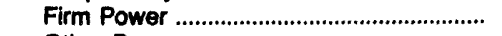 & - & -- & - & $=-$ & 198,561 & $9,419,404$ \\
\hline Other Power & - & - & 17,189 & $2,431,411$ & 70,770 & $4,345,113$ \\
\hline 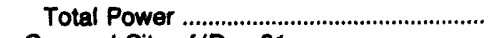 & - & - & 17,189 & $2,431,411$ & 269,331 & $13,764,517$ \\
\hline Concord City of/Dec 31 & & & & & & \\
\hline 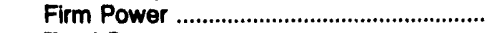 & - & - & - & - & 147,033 & $8,882,051$ \\
\hline 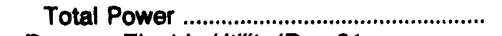 & - & - & - & - & 147,033 & $8,882,051$ \\
\hline Danvers Electric Utility/Dec 31 & & & & & & \\
\hline 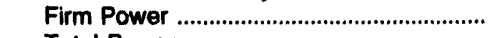 & - & - & 21,798 & $1,066,599$ & 302,497 & $24,377,524$ \\
\hline 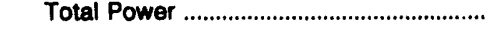 & - & - & 21,798 & $1,066,599$ & 302,497 & $24,377,524$ \\
\hline
\end{tabular}

See notes and footnotes at end of table. 
Table 29. Electricity Purchases by Municipal Utilities, Fiscal Year 1992 (Continued)

\begin{tabular}{|c|c|c|c|c|c|c|}
\hline \multirow{3}{*}{$\begin{array}{l}\text { 8tate / Uellity / } \\
\text { Fiecal Year Ending Date }\end{array}$} & \multicolumn{6}{|c|}{ Source of Electricity } \\
\hline & \multicolumn{2}{|c|}{ Investor-Owned } & \multicolumn{2}{|c|}{ Federal } & \multicolumn{2}{|c|}{$\begin{array}{c}\text { State and Other } \\
\text { Government }\end{array}$} \\
\hline & $\begin{array}{c}\text { Purchases } \\
\text { (thousand kWh) }\end{array}$ & $\begin{array}{c}\text { Cost } \\
\text { (dollars) }\end{array}$ & $\begin{array}{c}\text { Purchases } \\
\text { (thousand kWh) }\end{array}$ & $\begin{array}{c}\text { Cost } \\
\text { (dollars) }\end{array}$ & $\begin{array}{c}\text { Purchuses } \\
\text { (thousand kWh) }\end{array}$ & $\begin{array}{c}\text { Cost } \\
\text { (dollars) }\end{array}$ \\
\hline \multicolumn{7}{|l|}{$\begin{array}{l}\text { Maseachusette } \\
\text { Hingham Municipal Lighting Pla/Dec } 31\end{array}$} \\
\hline 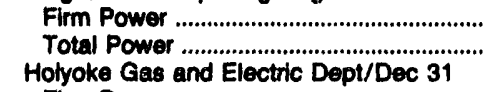 & $\begin{array}{l}13,425 \\
13,425\end{array}$ & $\begin{array}{l}574,992 \\
574,992\end{array}$ & $\overline{-}$ & - & $\begin{array}{l}108,754 \\
108,764\end{array}$ & $\begin{array}{l}8,736,439 \\
8,736,439\end{array}$ \\
\hline 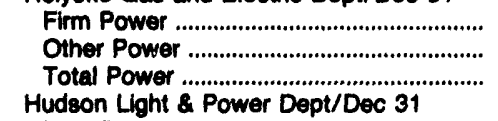 & $\begin{array}{l}- \\
125,730 \\
125,730\end{array}$ & $\begin{array}{l}- \\
6,399,762 \\
6,399,762\end{array}$ & - & - & $\begin{array}{r}22,231 \\
161,459 \\
183,690\end{array}$ & $\begin{array}{r}287,765 \\
10,851,610 \\
11,139,375\end{array}$ \\
\hline 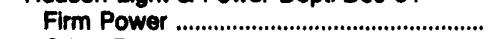 & 32,582 & $1,901,697$ & - & - & 162,117 & $17,927,040$ \\
\hline 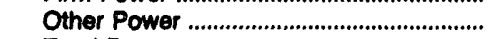 & - & - & - & - & - & - \\
\hline $\begin{array}{l}\text { Total Power ............................................ } \\
\text { Littleton Town of/Dec } 31\end{array}$ & 32,582 & $1,901,697$ & - & - & 162,117 & $17,927,040$ \\
\hline 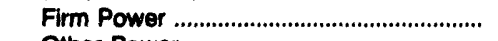 & 10,539 & 612,191 & - & - & 8,274 & 53,233 \\
\hline 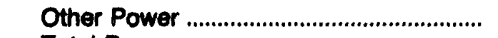 & - & - & - & - & 82,467 & $5,641,621$ \\
\hline $\begin{array}{l}\text { Total Power ................................. } \\
\text { Mansfleld Electric Light Dept/Dec } 31\end{array}$ & 10,539 & 612,191 & - & - & 90,741 & $5,684,854$ \\
\hline Other Power & - & - & - & - & 158,294 & $15,331,013$ \\
\hline $\begin{array}{l}\text { Total Power ........................................ } \\
\text { Middleborough Town of/Dec } 31\end{array}$ & - & - & - & - & 158,294 & $15,331,013$ \\
\hline Firm Power & $\begin{array}{l}67,536 \\
67,536\end{array}$ & $\begin{array}{l}4,217,103 \\
4,217,103\end{array}$ & $\overline{-}$ & - & $\begin{array}{l}101,766 \\
101,766\end{array}$ & $\begin{array}{l}8,494,272 \\
8,484,272\end{array}$ \\
\hline North Attleborough Elec Dept/Dec 31 & & & & & & \\
\hline Other Power & $\begin{array}{l}14,033 \\
14,033\end{array}$ & $\begin{array}{l}598,351 \\
598,351\end{array}$ & $\overline{-}$ & $\overline{-}$ & $\begin{array}{l}80,384 \\
80,384\end{array}$ & $\begin{array}{l}7,093,231 \\
7,093,231\end{array}$ \\
\hline Norwood City of/Jun 30 & & & & & & \\
\hline $\begin{array}{l}\text { Firm Power } \\
\text { Total Power }\end{array}$ & 327,305 & $21,186,801$ & - & - & - & - \\
\hline $\begin{array}{l}\text { Total Power .................................. } \\
\text { Peabody Municipal Light Plant/Dec } 31\end{array}$ & 327,305 & $21,188,901$ & - & - & - & - \\
\hline 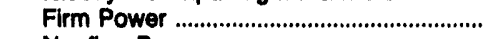 & 42,412 & $2,061,293$ & - & - & 88,565 & $5,025,515$ \\
\hline 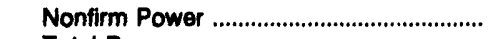 & - & - & - & - & - & - \\
\hline $\begin{array}{l}\text { Total Power .............................................. } \\
\text { Reading City of/Dec } 31\end{array}$ & 42,412 & $2,081,293$ & - & - & 88,565 & $5,025,515$ \\
\hline 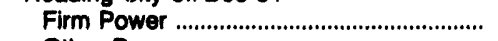 & 231,720 & $15,307,816$ & - & - & 297,440 & $22,659,376$ \\
\hline 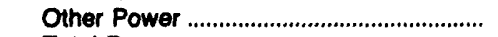 & - & - & - & - & - & - \\
\hline $\begin{array}{l}\text { Total Power .............................................. } \\
\text { Shrewsbury Town of/Dec } 31\end{array}$ & 231,720 & $15,307,916$ & - & - & 297,440 & $22,658,376$ \\
\hline $\begin{array}{l}\text { Firm Power } \\
\text { Total Power ................................................ } \\
\text { Taunton Municipal Light Plant/Dec }\end{array}$ & $\begin{array}{l}43,039 \\
43,039\end{array}$ & $\begin{array}{l}2,288,756 \\
2,288,756\end{array}$ & - & - & $\begin{array}{l}223,508 \\
223,508\end{array}$ & $\begin{array}{l}13,608,884 \\
13,608,894\end{array}$ \\
\hline $\begin{array}{l}\text { Firm Power ............................................ } \\
\text { Total Power ..................................... } \\
\text { Wakefield Town of/Dec } 31\end{array}$ & $\begin{array}{l}106,470 \\
106,470\end{array}$ & $\begin{array}{l}2,897,574 \\
2,897,574\end{array}$ & $\overline{-}$ & $\overline{-}$ & $\begin{array}{l}35,381 \\
35,381\end{array}$ & $\begin{array}{l}566,289 \\
568,289\end{array}$ \\
\hline Firm Power & $-23,650$ & $\begin{array}{l}907,945 \\
347,986\end{array}$ & - & $\overline{-}$ & $\begin{array}{r}133,022 \\
138\end{array}$ & $\begin{array}{r}10,088,462 \\
105,139\end{array}$ \\
\hline $\begin{array}{l}\text { Total Power .............................................. } \\
\text { Wellesley Town of/Dec } 31\end{array}$ & 23,650 & $1,255,931$ & - & - & 133,160 & $10,193,601$ \\
\hline 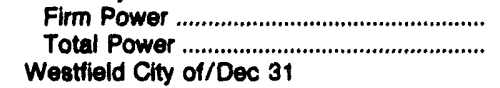 & $\begin{array}{l}183,900 \\
183,900\end{array}$ & $\begin{array}{l}12,351,087 \\
12,351,087\end{array}$ & $\overline{-}$ & $\overline{-}$ & $\begin{array}{l}15,400 \\
15,400\end{array}$ & $\begin{array}{l}258,903 \\
258,803\end{array}$ \\
\hline 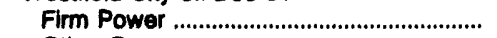 & - & - & - & - & - & - \\
\hline $\begin{array}{l}\text { Other Power } \\
\text { Total Power }\end{array}$ & $\begin{array}{l}36,567 \\
36,567\end{array}$ & $\begin{array}{l}1,832,368 \\
1,832,368\end{array}$ & $\overline{-}$ & - & $\begin{array}{l}193,098 \\
193,098\end{array}$ & $\begin{array}{l}18,757,970 \\
18,757,870\end{array}$ \\
\hline $\begin{array}{l}\text { Mlehlgan } \\
\text { Bay City Electric Light\&Power/Jun } 30\end{array}$ & & & & & & \\
\hline 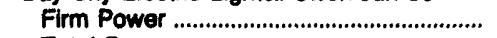 & 253,159 & $8,665,842$ & - & - & - & - \\
\hline $\begin{array}{l}\text { Total Power ........................................ } \\
\text { Coldwater Board of Public Util/Jun }\end{array}$ & 253,159 & $8,665,942$ & - & - & - & - \\
\hline 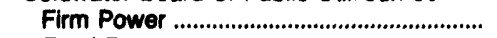 & - & - & - & - & 135,407 & $7,643,385$ \\
\hline $\begin{array}{l}\text { Total Power ..................................... } \\
\text { Detrolt Public Lighting Dept/Jun } 30\end{array}$ & - & - & - & - & 135,407 & $7,643,385$ \\
\hline 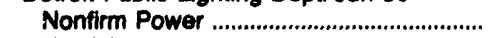 & 419,470 & $11,122,363$ & - & - & - & - \\
\hline $\begin{array}{l}\text { Total Power ............................................ } \\
\text { Grand Haven City of/Jun } 30\end{array}$ & 419,470 & $11,122,363$ & - & - & - & - \\
\hline 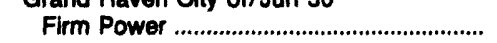 & - & - & - & - & - & - \\
\hline 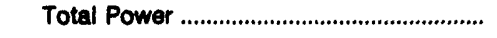 & - & - & - & - & - & - \\
\hline
\end{tabular}


Table 29. Electricity Purchases by Municipal Utilitles, Fiscal Year 1992 (Continued)

\begin{tabular}{|c|c|c|c|c|c|c|}
\hline \multirow{3}{*}{$\begin{array}{l}\text { State / Utilty / } \\
\text { Flecel Year Ending Date }\end{array}$} & \multicolumn{6}{|c|}{ Source of Electricity } \\
\hline & \multicolumn{2}{|c|}{ Cooperative } & \multicolumn{2}{|c|}{ Other' } & \multicolumn{2}{|c|}{ Total } \\
\hline & $\begin{array}{c}\text { Purchases } \\
\text { (thousand kWh) }\end{array}$ & $\begin{array}{c}\text { Cost } \\
\text { (dollars) }\end{array}$ & $\begin{array}{c}\text { Purchases } \\
\text { (thousand KWh) }\end{array}$ & $\begin{array}{c}\text { Cost } \\
\text { (dollars) }\end{array}$ & $\begin{array}{c}\text { Purchases } \\
\text { (thousand kWh) }\end{array}$ & $\begin{array}{c}\text { Cost } \\
\text { (dollars) }\end{array}$ \\
\hline \multicolumn{7}{|l|}{$\begin{array}{l}\text { Masaschusetta } \\
\text { Hingham Municipal Lighting Pla/Doc } 31\end{array}$} \\
\hline 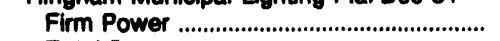 & - & - & 17,818 & 831,881 & 139,998 & $10,243,312$ \\
\hline $\begin{array}{l}\text { Total Power ................................. } \\
\text { Holyoke Gas Eept/Dec } 31\end{array}$ & - & - & 17,818 & 931,881 & 139,898 & $10,243,312$ \\
\hline Firm Power ........................................... & - & - & - & - & 22,231 & 287,765 \\
\hline 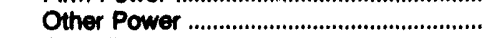 & - & - & - & - & 287,189 & $17,251,372$ \\
\hline 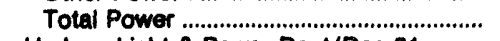 & - & - & - & - & 309,420 & $17,539,137$ \\
\hline \multicolumn{7}{|l|}{ Hudson Light \& Power Dept/Dec 31} \\
\hline 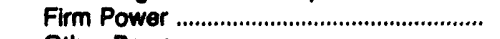 & - & - & 68,924 & $3,983,421$ & 263,623 & $23,812,158$ \\
\hline 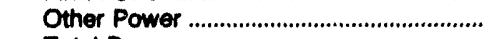 & - & - & $-1,446$ & $-42,239$ & $-1,446$ & $-42,239$ \\
\hline \multirow{2}{*}{\multicolumn{7}{|c|}{ Littieton Town of/Dec 31}} \\
\hline & - & & & & & \\
\hline 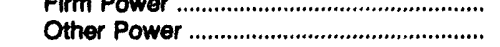 & - & - & $\begin{array}{r}9,938 \\
19,041\end{array}$ & $\begin{array}{r}754,264 \\
1,934,030\end{array}$ & $\begin{array}{r}28,751 \\
101,508\end{array}$ & $\begin{array}{l}\mathbf{1 , 4 1 9 , 6 8 8} \\
\mathbf{7 , 5 7 5 , 6 5 1}\end{array}$ \\
\hline 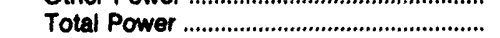 & - & - & 28,979 & $2,688,294$ & 130,259 & $8,895,339$ \\
\hline \multicolumn{7}{|l|}{ Mansfleld Electric Light Dept/Dec 31} \\
\hline Other Power & - & - & - & -- & 158,294 & $15,331,013$ \\
\hline Total Power & - & - & - & - & 158,294 & $15,331,013$ \\
\hline \multicolumn{7}{|l|}{ Middleborough Town of/Dec 31} \\
\hline 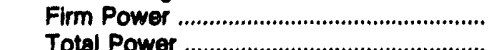 & - & - & - & - & 169,302 & \\
\hline \multicolumn{6}{|l|}{ North Attleborough Elec Dept/Doc 31} & $12,711,375$ \\
\hline Other Power . & - & - & 25,657 & $2,310,196$ & 120,074 & $10,001,778$ \\
\hline 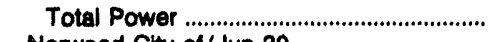 & - & - & 25,657 & $2,310,196$ & 120,074 & $10,001,778$ \\
\hline \multicolumn{7}{|l|}{ Norwood Clty of/Jun 30} \\
\hline 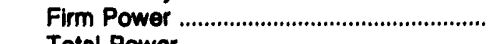 & - & - & - & -- & 327,305 & $21,188,901$ \\
\hline \multicolumn{7}{|l|}{ Peabody Municipal Light Plant/Doc 31} \\
\hline 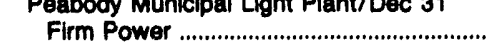 & - & - & 255,078 & $22,807,432$ & 386,055 & $29,894,240$ \\
\hline 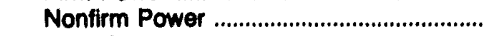 & - & - & 1,232 & 21,724 & 1,232 & 21,724 \\
\hline 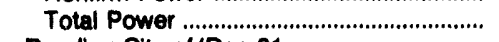 & - & -- & 256,310 & $22,829,156$ & 387,287 & $29,815,964$ \\
\hline \multicolumn{7}{|l|}{ Reading City of/Dec 31} \\
\hline 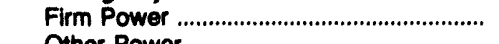 & - & - & 59,482 & $2,370,362$ & 588,642 & $\begin{array}{r}40,337,654 \\
-669,698\end{array}$ \\
\hline 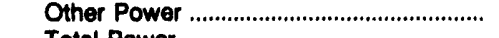 & - & - & -50490 & $-689,698$ & -509810 & $\begin{array}{r}-669,698 \\
39,667,956\end{array}$ \\
\hline $\begin{array}{l}\text { Total Power ... } \\
\text { Shrewsbury Town of/Dec } 31\end{array}$ & \multicolumn{5}{|c|}{ Shrewsbury Town of/Dec 31} & \\
\hline 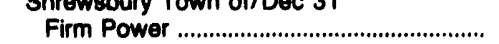 & - & - & - & - & 266,547 & $15,897,650$ \\
\hline Total Power ..................................... & - & - & - & - & 266,547 & $15,897,650$ \\
\hline Taunton Municipal Light Plant/Dec 31 & & & & & & \\
\hline Firm Power & - & - & 297,002 & $8,609,059$ & 438,853 & $12,072,822$ \\
\hline Total Power & - & - & 297,002 & $8,608,059$ & 438,853 & $12,072,822$ \\
\hline Wakefield Town of/Dec 31 & - & -- & & & & \\
\hline 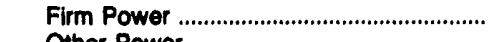 & $\overline{-}$ & - & $-\quad 477$ & $-{ }^{39,622}$ & $\begin{array}{r}157,149 \\
138\end{array}$ & $11,036,029$ \\
\hline Other Power & & & - & & & 453,125 \\
\hline 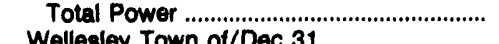 & - & - & 477 & 39,622 & 157,287 & $11,480,154$ \\
\hline $\begin{array}{l}\text { Wellesley Town of/Dec } 31 \\
\text { Firm Power }\end{array}$ & - & - & - & & & \\
\hline $\begin{array}{l}\text { Firm Power } \\
\text { Total Power }\end{array}$ & - & - & $\overline{-}$ & $\overline{-}$ & $\begin{array}{l}200,300 \\
209,300\end{array}$ & $\begin{array}{l}12,609,990 \\
12,609,990\end{array}$ \\
\hline Westfield City of/Dec 31 & & & & & & \\
\hline Firm Power ........................................................ & - & - & 14,617 & $1,281,708$ & 14,617 & $1,281,708$ \\
\hline Other Power .............................................. & - & - & - & & 228,665 & $20,590,338$ \\
\hline 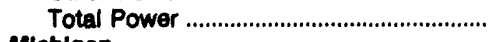 & - & - & 14,617 & $1,281,708$ & 244,282 & $21,872,046$ \\
\hline Michlgan & & & & & & \\
\hline $\begin{array}{l}\text { Bay City Electric Light8Power/Jun } 30 \\
\text { Firm Power . }\end{array}$ & - & - & & & 253,159 & \\
\hline $\begin{array}{l}\text { Firm Power } \\
\text { Total Power }\end{array}$ & - & - & $\overline{-}$ & $\overline{-}$ & 253,158 & $\begin{array}{l}8,665,942 \\
8,665,842\end{array}$ \\
\hline Coldwater Board of Public Uetl/Jun 30 & & & & & & \\
\hline 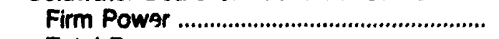 & - & - & - & - & 135,407 & $7,643,385$ \\
\hline Total Power & - & - & - & - & 135,407 & $7,643,385$ \\
\hline Detroit Public Lighting Dept/Jun 30 & & & & & & \\
\hline 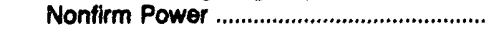 & - & - & - & - & 419,470 & $11,122,363$ \\
\hline 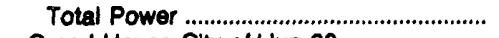 & - & - & - & - & 419,470 & $11,122,363$ \\
\hline Grand Haven City of/Jun 30 & & & & & & \\
\hline 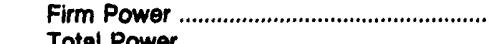 & $\begin{array}{l}31,234 \\
31,234\end{array}$ & $\begin{array}{l}879,423 \\
879,423\end{array}$ & $\bar{z}$ & $\overline{-}$ & $\begin{array}{l}31,234 \\
31,234\end{array}$ & $\begin{array}{l}879,423 \\
879,423\end{array}$ \\
\hline 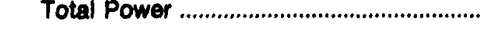 & 31,234 & 879,423 & & & & 878,423 \\
\hline
\end{tabular}

See notes and footnotes at end of table. 
Table 29. Electricity Purchases by Municipal Utilities, Flacal Year 1992 (Continued)

\begin{tabular}{|c|c|c|c|c|c|c|}
\hline \multirow{3}{*}{$\begin{array}{l}\text { 8tate / Uutlity / } \\
\text { Flecal Year Ending Dato }\end{array}$} & \multicolumn{6}{|c|}{ Source of Electricity } \\
\hline & \multicolumn{2}{|c|}{ Investor-Owned } & \multicolumn{2}{|c|}{ Federal } & \multicolumn{2}{|c|}{$\begin{array}{l}\text { State and Other } \\
\text { Government }\end{array}$} \\
\hline & $\begin{array}{l}\text { Purchases } \\
\text { (thousand } \mathrm{kWh} \text { ) }\end{array}$ & $\begin{array}{c}\text { Cost } \\
\text { (dollars) }\end{array}$ & $\begin{array}{c}\text { Purchases } \\
\text { (thousand } \mathrm{kWh} \text { ) }\end{array}$ & $\begin{array}{c}\text { Cost } \\
\text { (dollars) }\end{array}$ & $\begin{array}{c}\text { Purchases } \\
\text { (thousand } \mathrm{kWh} \text { ) }\end{array}$ & $\begin{array}{c}\text { Cost } \\
\text { (dollars) }\end{array}$ \\
\hline \multicolumn{7}{|l|}{ Mlehlean } \\
\hline 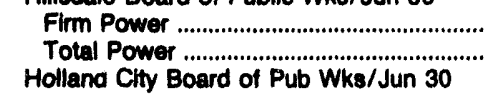 & $\overline{-}$ & - & - & $\overline{-}$ & $\begin{array}{l}120,736 \\
120,736\end{array}$ & $\begin{array}{l}6,021,085 \\
6,021,085\end{array}$ \\
\hline 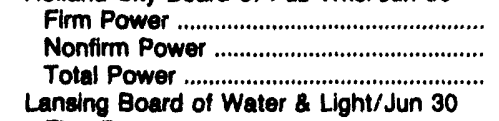 & $\begin{array}{r}122,210 \\
2,335 \\
124,545\end{array}$ & $\begin{array}{r}3,726,235 \\
118,827 \\
3,845,062\end{array}$ & - & - & $\begin{array}{r}211,360 \\
-211,360\end{array}$ & $\begin{array}{l}8,075,121 \\
- \\
8,075,121\end{array}$ \\
\hline $\begin{array}{l}\text { Firm Power } \\
\text { Total Power } \\
\text { Marquette City ol/Jun } 30\end{array}$ & $\begin{array}{l}1,667 \\
1,667\end{array}$ & $\begin{array}{l}71,457 \\
71,457\end{array}$ & - & - & $\begin{array}{l}163,711 \\
163,711\end{array}$ & $\begin{array}{l}9,340,362 \\
9,340,362\end{array}$ \\
\hline 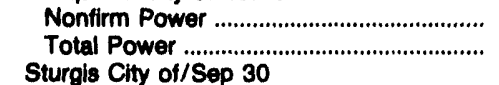 & $\begin{array}{l}16,150 \\
16,150\end{array}$ & $\begin{array}{l}454,788 \\
454,788\end{array}$ & - & - & - & - \\
\hline $\begin{array}{l}\text { Firm Power } \\
\text { Total Power } \\
\text { Traverse City City of/Jun } 30\end{array}$ & $\begin{array}{l}173,016 \\
173,016\end{array}$ & $\begin{array}{l}6,620,211 \\
6,620,211\end{array}$ & - & - & - & - \\
\hline 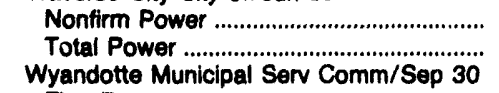 & $\begin{array}{l}31,306 \\
31,308\end{array}$ & $\begin{array}{l}830,831 \\
830,831\end{array}$ & - & - & $\begin{array}{l}99,691 \\
99,691\end{array}$ & $\begin{array}{l}3,425,036 \\
3,425,036\end{array}$ \\
\hline $\begin{array}{l}\text { Firm Power } \\
\text { Total Power }\end{array}$ & $\begin{array}{l}12,904 \\
12,904\end{array}$ & $\begin{array}{l}525,895 \\
525,895\end{array}$ & -- & $\overline{-}$ & - & 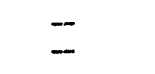 \\
\hline Alexandria City of/Dec 31 & & & & & & \\
\hline $\begin{array}{l}\text { Firm Power } \\
\text { Total Power ...... } \\
\text { Anoka City of/Dec } 31\end{array}$ & $\overline{-}$ & - & $\begin{array}{l}93,775 \\
93,775\end{array}$ & $\begin{array}{l}986,006 \\
986,006\end{array}$ & $\begin{array}{l}79,080 \\
79,080\end{array}$ & $\begin{array}{l}2,902,813 \\
2,902,813\end{array}$ \\
\hline 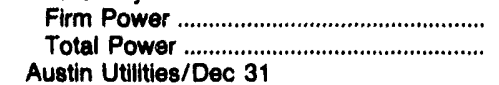 & $\begin{array}{l}185,064 \\
185,064\end{array}$ & $\begin{array}{l}8,294,077 \\
8,294,077\end{array}$ & - & -- & -- & - \\
\hline Firm Power . & - & - & - & - & $\begin{array}{l}241,559 \\
241,559\end{array}$ & $\begin{array}{l}13,272,151 \\
13,272,151\end{array}$ \\
\hline 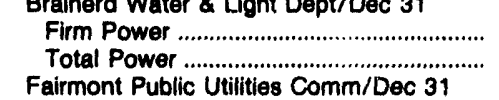 & $\begin{array}{l}140,657 \\
140,657\end{array}$ & $\begin{array}{l}5,299,862 \\
5,299,862\end{array}$ & - & - & - & $\overline{-}$ \\
\hline 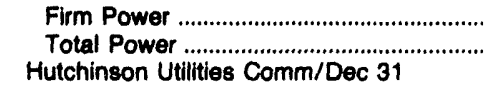 & - & - & - & - & $\begin{array}{l}152,055 \\
152,055\end{array}$ & $\begin{array}{l}8,916,830 \\
8,916,830\end{array}$ \\
\hline 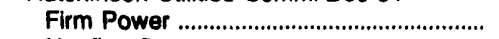 & - & - & - & - & - & - \\
\hline 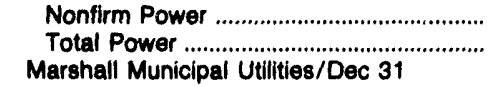 & $\begin{array}{l}103,665 \\
103,665\end{array}$ & $\begin{array}{l}1,572,488 \\
1,572,488\end{array}$ & - & - & $\begin{array}{l}672 \\
672\end{array}$ & $\begin{array}{l}9,099 \\
9,099\end{array}$ \\
\hline 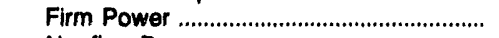 & - & - & 131,654 & $1,344,389$ & 178,479 & $6,116,912$ \\
\hline 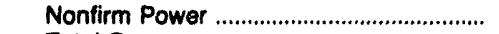 & - & - & - & -- & -- & - \\
\hline $\begin{array}{l}\text { Total Power ............................................. } \\
\text { Moorhead City of /Dec } 31\end{array}$ & - & - & 131,654 & $1,344,389$ & 178,479 & $6,116,912$ \\
\hline 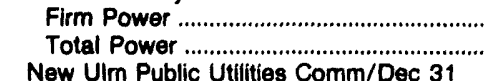 & - & - & $\begin{array}{l}223,424 \\
223,424\end{array}$ & $\begin{array}{l}2,567,903 \\
2,567,903\end{array}$ & $\begin{array}{l}106,982 \\
106,982\end{array}$ & $\begin{array}{l}3,910,753 \\
3,910,753\end{array}$ \\
\hline 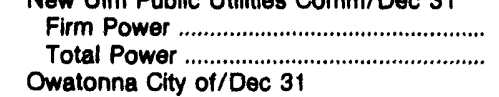 & $\begin{array}{l}103,546 \\
103,546\end{array}$ & $\begin{array}{l}2,183,332 \\
2,183,332\end{array}$ & - & - & $\overline{--}$ & - \\
\hline 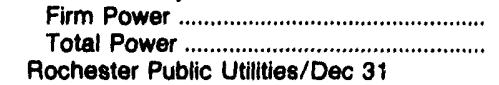 & $\overline{-}$ & - & $\overline{-}$ & - & $\begin{array}{l}225,828 \\
225,828\end{array}$ & $\begin{array}{l}12,981,992 \\
12,981,992\end{array}$ \\
\hline 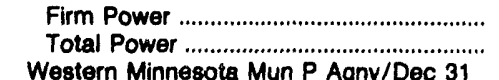 & - & - & $\overline{-}$ & - & $\begin{array}{l}873,060 \\
873,060\end{array}$ & $\begin{array}{l}49,286,681 \\
49,286,681\end{array}$ \\
\hline Nonfirm Power .......................................... & $\sim$ & - & - & - & 168 & 6,106 \\
\hline $\begin{array}{l}\text { Total Power .............................. } \\
\text { Willmar Municipal Utils Comm/Dec } 31\end{array}$ & - & - & - & - & 168 & 6,106 \\
\hline 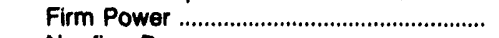 & - & - & 32,094 & 358,380 & - & - \\
\hline 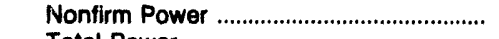 & - & - & - & - & - & - \\
\hline 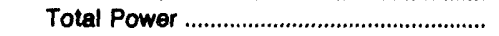 & - & - & 32,094 & 358,380 & - & - \\
\hline
\end{tabular}

See notes and footnotes at end of table. 
Table 29. Electricity Purchases by Municipal UtIIItles, Fiscal Year 1992 (Continued)

\begin{tabular}{|c|c|c|c|c|c|c|}
\hline \multirow{3}{*}{$\begin{array}{l}\text { Stato / Utility I } \\
\text { Flecal Year Ending Date }\end{array}$} & \multicolumn{6}{|c|}{ Source of Electricity } \\
\hline & \multicolumn{2}{|c|}{ Cooperative } & \multicolumn{2}{|c|}{ Other' } & \multicolumn{2}{|c|}{ Total } \\
\hline & $\begin{array}{c}\text { Purchases } \\
\text { (thousand } k W h \text { ) }\end{array}$ & $\begin{array}{c}\text { Cost } \\
\text { (dollars) }\end{array}$ & $\begin{array}{c}\text { Purchases } \\
\text { (thousand KWh) }\end{array}$ & $\begin{array}{c}\text { Cost } \\
\text { (dollars) }\end{array}$ & $\begin{array}{c}\text { Purchases } \\
\text { (thousand kWh) }\end{array}$ & $\begin{array}{c}\text { Cost } \\
\text { (dollars) }\end{array}$ \\
\hline \multicolumn{7}{|l|}{$\begin{array}{l}\text { Michlgan } \\
\text { Hillsdale Board of Public Wks/Jun } 30\end{array}$} \\
\hline 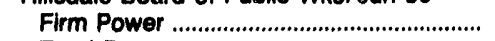 & - & - & - & - & 120,736 & $6,021,085$ \\
\hline 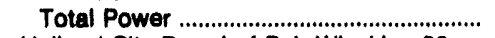 & -- & - & - & - & 120,736 & $6,021,085$ \\
\hline \multicolumn{7}{|l|}{ Holland City Board of Pub Wks/Jun 30} \\
\hline 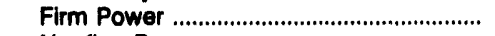 & - & - & - & -- & 333,570 & $11,801,356$ \\
\hline Nonfirm Power ................................................ & - & - & - & -- & 2,335 & 118,827 \\
\hline \multicolumn{7}{|l|}{ Lansing Board of Water \& Light/Jun 30} \\
\hline 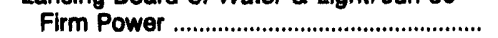 & -- & -- & - & - & 165,378 & $9,411,819$ \\
\hline $\begin{array}{l}\text { Total Power ................................... } \\
\text { Marquette Clty of/Jun }\end{array}$ & & - & - & - & 165,378 & $9,411,819$ \\
\hline Nonfirm Power ............................................. & -- & -- & -- & - & 16,150 & 454,788 \\
\hline 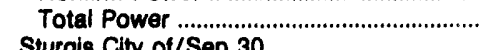 & - & -- & - & - & 16,150 & 454,788 \\
\hline Sturgis City of /Sep 30 & -- & - & 3,893 & 122,769 & 176,909 & $6,742,980$ \\
\hline $\begin{array}{l}\text { Total Power } \\
\text { Traverse City City of /Jun } 30\end{array}$ & - & - & 3,893 & 122,769 & 176,909 & $6,742,880$ \\
\hline 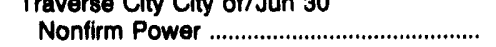 & - & - & 1,922 & 53,289 & 132,819 & $4,309,156$ \\
\hline Total Power .................................................... & - & -- & 1,022 & 53,289 & 132,819 & $4,309,156$ \\
\hline \multicolumn{7}{|l|}{ Wyandotte Municipal Serv Comm/Sep 30} \\
\hline 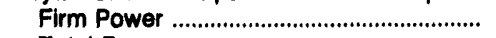 & -- & -- & - & - & 12,804 & 525,895 \\
\hline 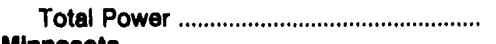 & -- & - & - & - & 12,904 & 525,895 \\
\hline \multicolumn{7}{|l|}{ Minnecota } \\
\hline 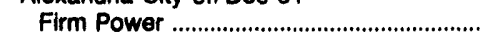 & -_ & - & - & -- & 172,855 & $3,888,819$ \\
\hline 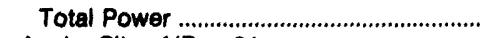 & -- & -- & - & -- & 172,855 & $3,888,819$ \\
\hline \multicolumn{7}{|l|}{ Anoka City of/Dec 31} \\
\hline 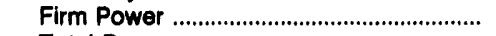 & - & - & - & -- & 185,064 & $8,294,077$ \\
\hline 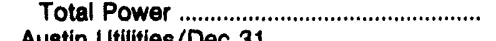 & - & - & -- & - & 185,064 & $8,294,077$ \\
\hline Austin Utilities/Dec 31 & & & & & & \\
\hline 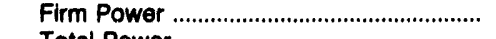 & - & - & - & - & 241,559 & $13,272,151$ \\
\hline $\begin{array}{l}\text { Total Power ... } \\
\text { Brainerd Water \& Light Dept/Dec } 31\end{array}$ & - & - & - & - & 241,559 & $13,272,151$ \\
\hline Firm Power ........................................... & - & -- & -- & - & 140,657 & $5,299,862$ \\
\hline 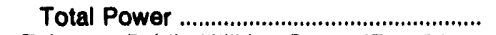 & - & - & - & - & 140,657 & $5,299,862$ \\
\hline Fairmont Public Utilities Comm/Dec 31 & & & & & & \\
\hline 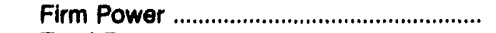 & -- & - & -- & -- & 152,055 & $8,916,830$ \\
\hline 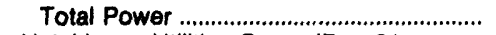 & - & -- & -- & -- & 152,055 & $8,916,830$ \\
\hline Hutchinson Utilities Comm/Dec 31 & & & & & & \\
\hline 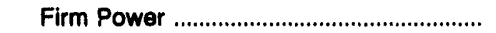 & 30,776 & $2,021,374$ & - & - & 30,776 & $2,021,374$ \\
\hline 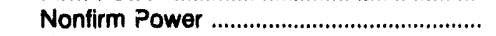 & 91,496 & $1,127,424$ & - & -- & 195,833 & $2,709,011$ \\
\hline 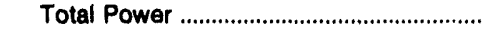 & 122,272 & $3,148,798$ & - & -- & 226,609 & $4,730,385$ \\
\hline Marshall Municipal Utlities/Dec 31 & & & & & & \\
\hline 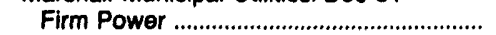 & -- & - & -- & -- & 310,133 & $7,461,301$ \\
\hline Nonfirm Power ................................................. & -- & - & 302 & 14,030 & 302 & 14,030 \\
\hline 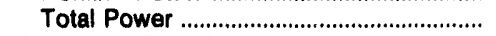 & -- & - & 302 & 14,030 & 310,435 & $7,475,331$ \\
\hline Moorhead City of/Dec 31 & & & & & & \\
\hline 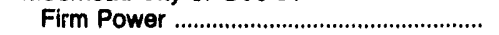 & -- & -- & -- & -- & 330,406 & $6,478,656$ \\
\hline 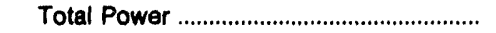 & -- & - & - & -- & 330,406 & $6,478,656$ \\
\hline New UIm Public Utilities Comm/Dec 31 & & & & & & \\
\hline 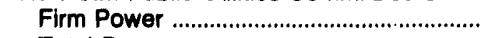 & -- & - & -- & -- & 103,546 & $2,183,332$ \\
\hline $\begin{array}{l}\text { Total Power ......................................... } \\
\text { Owatonna City of/Dec }\end{array}$ & - & -- & - & -- & 103,546 & $2,183,332$ \\
\hline Firm Power ........................................... & - & - & -- & -- & 225,828 & $12,981,892$ \\
\hline 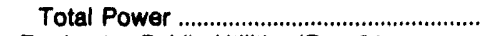 & -- & -- & -- & -- & 225,828 & $12,981,892$ \\
\hline Rochester Public Utilities/Dec 31 & & & & & & \\
\hline 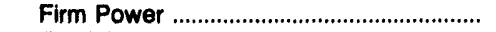 & - & -- & - & -- & 873,060 & $49,286,681$ \\
\hline 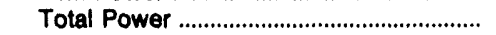 & -- & - & -- & -- & 873,060 & $49,286,681$ \\
\hline Western Minnesota Mun P Agny/Dec 31 & & & & & & \\
\hline 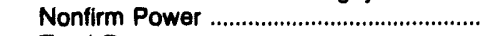 & - & - & -- & - & 168 & 6,106 \\
\hline 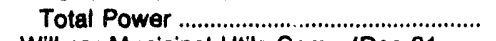 & - & - & - & - & 168 & 6,106 \\
\hline Willmar Municipal Utils Comm/Dec 31 & & & & & & \\
\hline 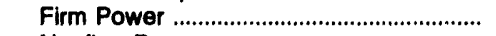 & 102,974 & $2,822,330$ & - & - & 135,068 & $3,180,710$ \\
\hline 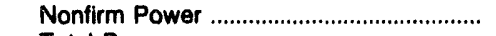 & 36,287 & 698,572 & -- & - & 36,287 & 698,572 \\
\hline 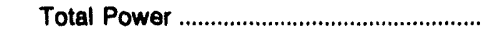 & 139,261 & $3,520,802$ & - & -- & 171,355 & $3,879,282$ \\
\hline
\end{tabular}

See notes and footnotes at end of table. 
Table 29. Electricity Purchases by Municipal Utilities, Fiscal Year 1992 (Continued)

\begin{tabular}{|c|c|c|c|c|c|c|}
\hline \multirow{3}{*}{$\begin{array}{l}\text { State / Utilty / } \\
\text { Flecal Year Ending Date }\end{array}$} & \multicolumn{6}{|c|}{ Source of Electricity } \\
\hline & \multicolumn{2}{|c|}{ Investor-Owned } & \multicolumn{2}{|c|}{ Federal } & \multicolumn{2}{|c|}{$\begin{array}{l}\text { State and Other } \\
\text { Government }\end{array}$} \\
\hline & $\begin{array}{c}\text { Purchases } \\
\text { (thousand kWh) }\end{array}$ & $\begin{array}{c}\text { Cost } \\
\text { (dollars) }\end{array}$ & $\begin{array}{c}\text { Purchases } \\
\text { (thousand } k: A / h \text { ) }\end{array}$ & $\begin{array}{c}\text { Cost } \\
\text { (dollars) }\end{array}$ & $\begin{array}{c}\text { Purchases } \\
\text { (thousand } \mathrm{kWh} \text { ) }\end{array}$ & $\begin{array}{c}\text { Cost } \\
\text { (dollars) }\end{array}$ \\
\hline \multicolumn{7}{|l|}{ Miseledppi } \\
\hline 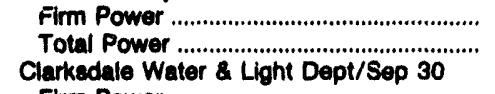 & $\overline{-}$ & - & $\begin{array}{l}157,479 \\
157,479\end{array}$ & $\begin{array}{l}6,939,013 \\
6,939,013\end{array}$ & - & $\overline{-}$ \\
\hline 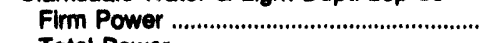 & - & - & - & - & - & - \\
\hline $\begin{array}{l}\text { Total Power } \\
\text { Columbus City of /Jun } 30\end{array}$ & - & - & - & - & - & -- \\
\hline 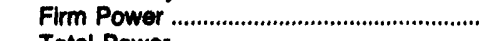 & - & - & 444,087 & $21,020,520$ & -- & -- \\
\hline $\begin{array}{l}\text { Total Power ............................................... } \\
\text { Greenwood Utitios Commion }\end{array}$ & - & - & 444,087 & $21,020,520$ & - & -- \\
\hline 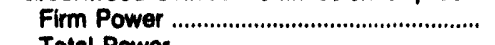 & -- & - & - & - & -- & - \\
\hline $\begin{array}{l}\text { Total Power .......................................... } \\
\text { Holly Springs Electric Dept/Jun } 30\end{array}$ & - & - & - & - & - & - \\
\hline $\begin{array}{l}\text { Firm Power } \\
\text { Total Power }\end{array}$ & - & - & 204,445 & $8,777,062$ & - & - \\
\hline $\begin{array}{l}\text { Total Power } \\
\text { Louleville Electric System/Jun } 30\end{array}$ & - & - & 204,445 & $8,777,062$ & - & -- \\
\hline 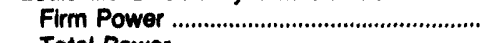 & - & - & 155,117 & $7,027,161$ & - & - \\
\hline $\begin{array}{l}\text { Total Power } \\
\text { Municipal Energy Agncy of MS/Sep } 30\end{array}$ & - & - & 155,117 & $7,027,161$ & - & - \\
\hline 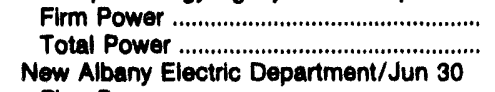 & $\begin{array}{l}639,156 \\
639,156\end{array}$ & $\begin{array}{l}12,619,494 \\
12,619,494\end{array}$ & $\begin{array}{l}43,511 \\
43,511\end{array}$ & $\begin{array}{l}899,055 \\
899,055\end{array}$ & $\begin{array}{l}7 \\
7\end{array}$ & $\begin{array}{l}276,778 \\
276,778\end{array}$ \\
\hline 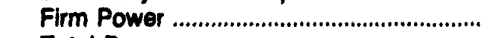 & - & - & 221,626 & $10,096,872$ & - & - \\
\hline $\begin{array}{l}\text { Total Power ...................................... } \\
\text { Oxford Electric Department/Jun } 30\end{array}$ & - & - & 221,626 & $10,096,872$ & - & -- \\
\hline 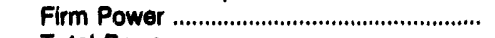 & - & - & 142,141 & $6,502,095$ & - & -- \\
\hline $\begin{array}{l}\text { Total Power } \\
\text { Starkville City of/Jun } 30\end{array}$ & - & - & 142,141 & $6,502,095$ & - & -- \\
\hline 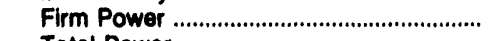 & - & - & 312,833 & $13,633,068$ & - & - \\
\hline $\begin{array}{l}\text { Total Power .............................................. } \\
\text { Tupelo City of } 30\end{array}$ & - & - & 312,833 & $13,633,068$ & - & - \\
\hline Firm Power & - & - & 601,344 & $27,544,481$ & - & - \\
\hline Total Power ................................................... & - & - & 601,344 & $27,544,481$ & - & $-\infty$ \\
\hline Firm Power .................................................. & - & - & 180,529 & $8,395,726$ & - & - \\
\hline $\begin{array}{l}\text { Total Power } \\
\text { Mlesourt }\end{array}$ & - & - & 180,529 & $8,395,726$ & - & $\sim$ \\
\hline Carthage City of/Jun 30 & & & & & & \\
\hline 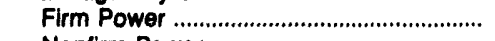 & - & - & 18,802 & 438,371 & - & - \\
\hline Nonfirm Power .............................................. & - & - & - & - & 9,606 & 178,979 \\
\hline 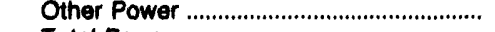 & - & - & - & - & - & - \\
\hline $\begin{array}{l}\text { Total Power .................................... } \\
\text { Columbia City of Water \& Light/Sep } 30\end{array}$ & - & - & 18,802 & 438,371 & 9,606 & 178,979 \\
\hline 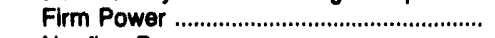 & 266,845 & $5,926,068$ & - & - & 154,165 & $4,959,408$ \\
\hline Nonfirm Power ........................................... & - & - & - & - & 128 & 6,429 \\
\hline 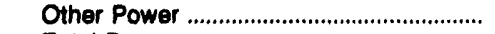 & - & - & - & - & - & - \\
\hline $\begin{array}{l}\text { Total Power .................................. } \\
\text { Hannibal Board of Public Works/Jun } 30\end{array}$ & 266,845 & $5,926,068$ & - & -- & 154,293 & $4,985,837$ \\
\hline 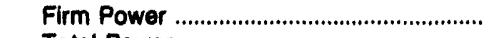 & 177,768 & $7,253,570$ & - & - & - & - \\
\hline $\begin{array}{l}\text { Total Power } \\
\text { Independence Clty of/Jun } 30\end{array}$ & 177.768 & $7,253,570$ & - & - & - & - \\
\hline Firm Power & 353,931 & $8,210,066$ & - & - & - & - \\
\hline Nonfirm Power ............................................ & 32,183 & 442,024 & - & - & - & - \\
\hline 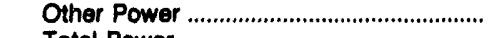 & 378,941 & $10,179,408$ & - & -- & - & - \\
\hline 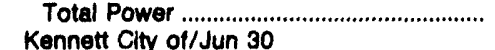 & 765,055 & $18,831,498$ & - & - & - & - \\
\hline $\begin{array}{l}\text { Kennett Clty of/Jun } 30 \\
\text { Firm Power }\end{array}$ & -- & - & 28,018 & 561,861 & -- & - \\
\hline Nonfirm Power ........................................... & - & - & - & - & - & - \\
\hline Total Power .................................... & - & - & 28,018 & 561,861 & -- & - \\
\hline Kirkwood Clty of/Mar 31 & & & & & & \\
\hline 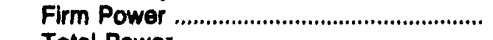 & 184,813 & $7,781,856$ & - & - & -- & - \\
\hline $\begin{array}{l}\text { Total Power .......................................... } \\
\text { Lebanon Clty oft } 31\end{array}$ & 184,813 & $7,781,856$ & - & - & -- & $\sim$ \\
\hline 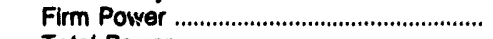 & - & - & - & -- & -- & - \\
\hline 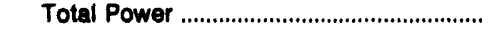 & - & - & - & - & - & - \\
\hline
\end{tabular}


Table 29. Electricity Purchases by Municipal Utilities, Flscal Year 1982 (Continued)

\begin{tabular}{|c|c|c|c|c|c|c|}
\hline \multirow{3}{*}{$\begin{array}{l}\text { 8tate / Utility / } \\
\text { Fiacal Year Ending Date }\end{array}$} & \multicolumn{6}{|c|}{ Source of Electricity } \\
\hline & \multicolumn{2}{|c|}{ Cooperative } & \multicolumn{2}{|c|}{ Other' } & \multicolumn{2}{|c|}{ Total } \\
\hline & $\begin{array}{c}\text { Purchases } \\
\text { (thousand kWh) }\end{array}$ & $\begin{array}{c}\text { Cost } \\
\text { (dollars) }\end{array}$ & $\begin{array}{c}\text { Purchases } \\
\text { (thousend kWh) }\end{array}$ & $\begin{array}{c}\text { Cost } \\
\text { (dollar8) }\end{array}$ & $\begin{array}{c}\text { Purchases } \\
\text { (thousand kWh) }\end{array}$ & $\begin{array}{c}\text { Cost } \\
\text { (dollars) }\end{array}$ \\
\hline \multicolumn{7}{|l|}{ Miselealppi } \\
\hline Firm Power & -- & - & - & - & 157,479 & $6,838,013$ \\
\hline Total Power & - & - & - & - & 157,479 & $6,839,013$ \\
\hline \multicolumn{7}{|l|}{ Clarksdale Water \& Light Dept/Sep 30} \\
\hline 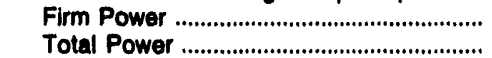 & $\overline{-}$ & $\overline{-}$ & $\begin{array}{l}178,476 \\
178,476\end{array}$ & $\begin{array}{l}5,752,990 \\
5,752,990\end{array}$ & $\begin{array}{l}178,478 \\
178,476\end{array}$ & $\begin{array}{l}5,752,990 \\
5,752,990\end{array}$ \\
\hline \multicolumn{7}{|l|}{ Columbus City of/Jun 30} \\
\hline Firm Power & - & - & - & - & 444,087 & $21,020,520$ \\
\hline \multicolumn{6}{|l|}{ Groenwood Utilties Commission/Sep 30} & $21,020,520$ \\
\hline 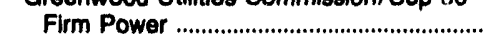 & - & - & 272,234 & $9,012,355$ & 272,234 & $9,012,355$ \\
\hline $\begin{array}{l}\text { Total Power ........................... } \\
\text { Holly Springs Electric Dept/Jun } 30\end{array}$ & \multicolumn{3}{|c|}{ Holly Springs Electric Dept/Jun 30} & $9,012,356$ & 272,234 & $9,012,355$ \\
\hline 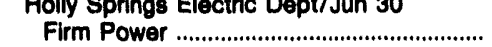 & - & - & - & -- & 204,446 & $8,777,062$ \\
\hline Total Power & - & - & - & - & 204,445 & $8,777,062$ \\
\hline Louisville Electric System/Jun 30 & - & - & - & - & 155,117 & $7,027,161$ \\
\hline 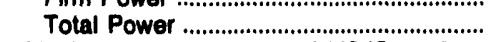 & - & - & - & - & 155,117 & $7,027,161$ \\
\hline \multicolumn{7}{|l|}{ Municipal Energy Agncy of MS/Sep 30} \\
\hline 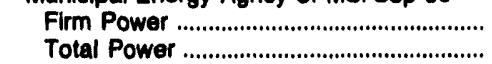 & $\overline{-}$ & $\begin{array}{l}7,484,400 \\
7,484,400\end{array}$ & $\begin{array}{l}89,819 \\
89,819\end{array}$ & $\begin{array}{l}3,235,614 \\
3,235,614\end{array}$ & $\begin{array}{l}772,493 \\
772,493\end{array}$ & $\begin{array}{l}24,515,341 \\
24,515,341\end{array}$ \\
\hline \multicolumn{7}{|l|}{ New Albany Electric Department/Jun 30} \\
\hline Firm Power .................................................... & - & - & - & - & 221,628 & $10,096,872$ \\
\hline \multicolumn{7}{|l|}{ Oxtord Electric Department/Jun 30} \\
\hline 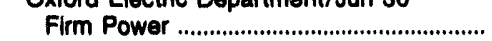 & - & - & - & -- & 142,141 & $6,502,095$ \\
\hline $\begin{array}{l}\text { Total Power } \\
\text { Starkville Clty of/Jun } 30\end{array}$ & - & -- & -- & -- & 142,141 & $6,502,095$ \\
\hline 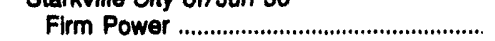 & - & - & - & - & 312,833 & $13,633,068$ \\
\hline Total Power ........................................... & - & - & -- & -- & 312,833 & $13,633,068$ \\
\hline Tupelo City of/Jun 30 & - & -- & -. & -- & 601,344 & $27,544,481$ \\
\hline Total Power & - & - & - & - & 601,344 & $27,544,481$ \\
\hline \multicolumn{6}{|l|}{ West Point Electric System/Jun 30} & \\
\hline 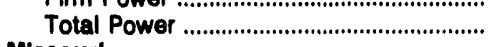 & - & - & - & - & 180,528 & $8,395,726$ \\
\hline Miesourl & & & & & & \\
\hline 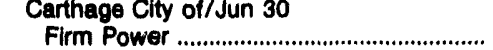 & - & - & 147,806 & $6,824,079$ & 166,608 & $7,262,450$ \\
\hline Nonfirm Power ........................................ & 8,691 & 166,636 & - & & 18,297 & 345,615 \\
\hline 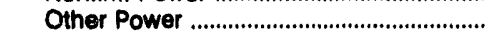 & - & - & - & $-1,094,820$ & -- & $-1,094,820$ \\
\hline Total Power ................................ & 8,691 & 166,636 & 147,800 & $5,729,259$ & 184,905 & $6,513,245$ \\
\hline Columbia City of Water \& Light/Sep 30 & -- & - & 244,659 & $12,984,091$ & 665,669 & 23.869 .567 \\
\hline $\begin{array}{l}\text { Firm Power } \\
\text { Nonfirm Power }\end{array}$ & 683 & 15,576 & - & $12,804,081$ & 811 & $\begin{array}{r}23,869,567 \\
22,004\end{array}$ \\
\hline 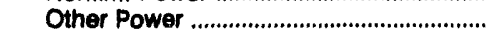 & -- & 22,969 & -- & 5,174 & -- & 28,143 \\
\hline 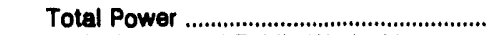 & 683 & 38,544 & 244,659 & $12,889,265$ & 666,480 & $23,819,714$ \\
\hline Hannibal Board of Public Works/Jun 30 & & & & & & \\
\hline 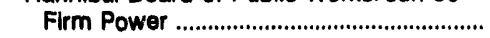 & - & -- & -- & -- & 177,768 & $7,253,570$ \\
\hline $\begin{array}{l}\text { Total Power ..................................... } \\
\text { Independence City on }\end{array}$ & - & - & - & - & 177,768 & $7,253,570$ \\
\hline 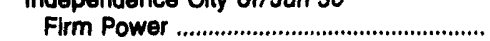 & -- & -- & -- & - & 353,831 & $8,210,066$ \\
\hline Nonfirm Power .................................................. & -- & - & - & - & 32,183 & 442,024 \\
\hline Other Power ....................................................... & - & -- & $\ldots$ & -- & 378,941 & $10,179,408$ \\
\hline 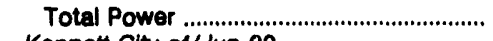 & -- & - & - & - & 765,055 & $18,831,498$ \\
\hline Kennett City of/Jun 30 & & & & & & \\
\hline 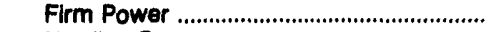 & - & - & - & - & 28,018 & 561,861 \\
\hline 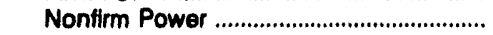 & - & - & 117,262 & $2,147,644$ & 117,262 & $2,147,644$ \\
\hline Total Power ……………………………….... & - & - & 117,262 & $2,147,644$ & 145,280 & $2,709,505$ \\
\hline Kirkwood City of/Mar 31 & & & & & & \\
\hline 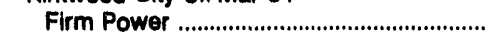 & - & - & -- & -- & 184,813 & $7,781,856$ \\
\hline 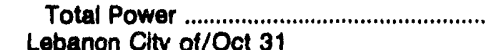 & - & - & - & - & 184,813 & $7,781,856$ \\
\hline $\begin{array}{l}\text { Lebanon City of/Oct } 31 \\
\text { Firm Power }\end{array}$ & 151,000 & $6,043,589$ & -- & 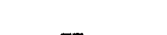 & & \\
\hline $\begin{array}{l}\text { Firm Power } \\
\text { Total Power }\end{array}$ & $\begin{array}{l}151,000 \\
151,000\end{array}$ & $6,043,589$ & - & - & $\begin{array}{l}151,000 \\
151,000\end{array}$ & $\begin{array}{l}6,043,589 \\
6,043,589\end{array}$ \\
\hline
\end{tabular}

See notes and footnotes at end of table. 
Table 29. Electricity Purchases by Munlclpal Utilities, Fiscal Year 1992 (Continued)

\begin{tabular}{|c|c|c|c|c|c|c|}
\hline \multirow{3}{*}{$\begin{array}{l}\text { 8tate / Utllity / } \\
\text { Flecal Year Ending Date }\end{array}$} & \multicolumn{6}{|c|}{ Source of Electricity } \\
\hline & \multicolumn{2}{|c|}{ Invertor-Owned } & \multicolumn{2}{|c|}{ Federal } & \multicolumn{2}{|c|}{$\begin{array}{l}\text { State and Other } \\
\text { Government }\end{array}$} \\
\hline & $\begin{array}{c}\text { Purchases } \\
\text { (thousand kWh) }\end{array}$ & $\begin{array}{c}\text { Cost } \\
\text { (dollars) }\end{array}$ & $\begin{array}{c}\text { Purchases } \\
\text { (thousand kWh) }\end{array}$ & $\begin{array}{c}\text { Cost } \\
\text { (dollars) }\end{array}$ & $\begin{array}{c}\text { Purchases } \\
\text { (thousand '.Wh) }\end{array}$ & $\begin{array}{c}\text { Cost } \\
\text { (dollars) }\end{array}$ \\
\hline \multicolumn{7}{|l|}{ Mtecourt } \\
\hline 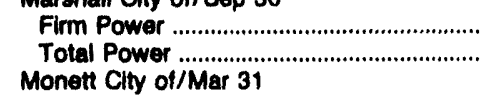 & $\begin{array}{l}71,302 \\
71,302\end{array}$ & $\begin{array}{l}1,419,502 \\
1,419,00 ?\end{array}$ & - & - & - & - \\
\hline 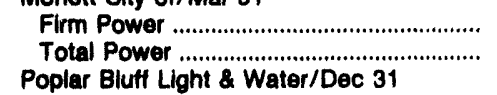 & $\begin{array}{l}149,947 \\
149,947\end{array}$ & $\begin{array}{l}4,459,009 \\
4,459,009\end{array}$ & - & - & - & - \\
\hline 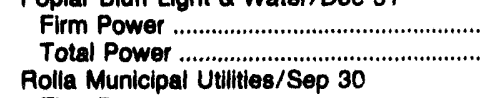 & - & - & $\begin{array}{l}127,536 \\
127,536\end{array}$ & $\begin{array}{l}1,271.026 \\
1,271,026\end{array}$ & $\begin{array}{l}118,931 \\
118,931\end{array}$ & $\begin{array}{l}2,631,535 \\
2,631,535\end{array}$ \\
\hline 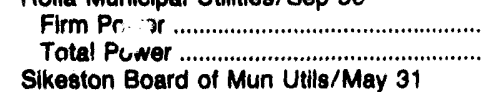 & $\begin{array}{l}194,076 \\
194,076\end{array}$ & $\begin{array}{l}7,696,113 \\
7,696,113\end{array}$ & $\cdots$ & $\overline{--}$ & - & $\overline{-}$ \\
\hline 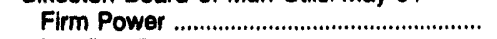 & - & -- & 83,352 & 957,311 & - & - \\
\hline 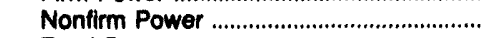 & - & -- & - & - & -- & - \\
\hline $\begin{array}{l}\text { Total Power .......................................... } \\
\text { Springfield City of } 30\end{array}$ & - & -- & 83,352 & 957,311 & -- & - \\
\hline 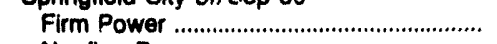 & - & $-\infty$ & 168,567 & $2,290,000$ & 77,875 & $1,376,000$ \\
\hline $\begin{array}{l}\text { Nonfirm Power } \\
\text { Total Power }\end{array}$ & $\begin{array}{l}10,530 \\
10,530\end{array}$ & $\begin{array}{l}154,000 \\
154,000\end{array}$ & $-\overline{168,567}$ & $\overline{2,290,000}$ & 77,875 & $\overline{1,376,000}$ \\
\hline \multicolumn{7}{|l|}{$\begin{array}{l}\text { Nebraakn } \\
\text { Fremont City of/Jul } 31\end{array}$} \\
\hline 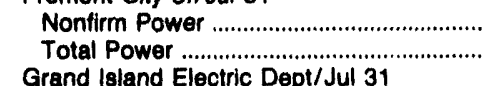 & - & $\overline{-}$ & $\begin{array}{l}24,870 \\
24,870\end{array}$ & $\begin{array}{l}342,822 \\
342,822\end{array}$ & $\begin{array}{l}34,422 \\
34,422\end{array}$ & $\begin{array}{l}420,832 \\
420,832\end{array}$ \\
\hline $\begin{array}{l}\text { Grand Island Electric Dept/Jul } 31 \\
\text { Firm Power }\end{array}$ & -- & - & 34,918 & 368,156 & 14,431 & Grand Island Electric Dept/Jul 31 \\
\hline 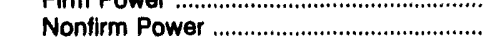 & - & - & -- & - & 7,078 & $\begin{array}{l}480,303 \\
115,905\end{array}$ \\
\hline $\begin{array}{l}\text { Totai Fower ............................................... } \\
\text { Hastings Utilities/Dec } 31\end{array}$ & - & - & 34,918 & 368,156 & 21,509 & 609,268 \\
\hline 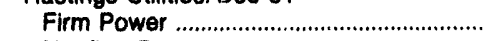 & - & - & 44,522 & 673,460 & - & - \\
\hline 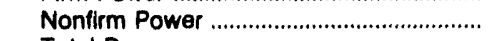 & - & -- & $-\infty$ & $-\infty$ & 54,341 & 860,604 \\
\hline \multicolumn{7}{|l|}{ Lincoln Electric System/Dec 31} \\
\hline 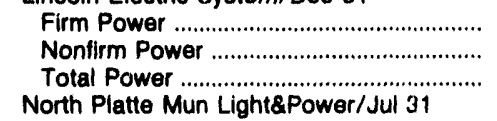 & $\begin{array}{r}7,280 \\
84,643 \\
91,923\end{array}$ & $\begin{array}{r}179,397 \\
1,220,153 \\
1,399,550\end{array}$ & $\begin{array}{r}139,725 \\
8,382 \\
148,107\end{array}$ & $\begin{array}{r}3,189,338 \\
119,505 \\
3,308,843\end{array}$ & $\begin{array}{r}1,491,323 \\
10,813 \\
1,502,136\end{array}$ & $\begin{array}{r}42,670,443 \\
129,237 \\
42,799,680\end{array}$ \\
\hline 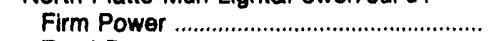 & - & - & - & - & 211,581 & $7,364,566$ \\
\hline 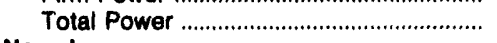 & -- & - & -- & - & 211,581 & $7,364,566$ \\
\hline \multicolumn{7}{|l|}{$\begin{array}{l}\text { Now Jersey } \\
\text { Vineland City of/Jun } 30\end{array}$} \\
\hline 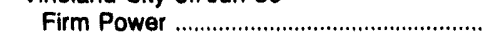 & 158,625 & $9,773,964$ & -- & - & 17,905 & 435,610 \\
\hline 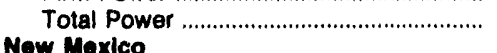 & 158,625 & $9,773,964$ & - & - & 17,905 & 435,610 \\
\hline Farmington City of/Jun 30 & \multicolumn{6}{|c|}{ New Mexico } \\
\hline 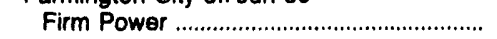 & 4,464 & 204,130 & 85,763 & $1,505,025$ & - & - \\
\hline Nonfirm Power ............................................. & -- & - & -- & - & $\rightarrow$ & - \\
\hline 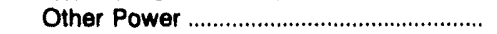 & 5,722 & 134,418 & - & - & -- & - \\
\hline \multicolumn{7}{|l|}{ Gallup Electric Utility/Jun 30} \\
\hline 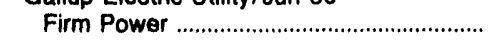 & 155,065 & $9,813,846$ & 16,790 & 261,814 & - & - \\
\hline Total Power ........................................... & 155,065 & $9,813,846$ & Los Alamos County of/Jun 30 & 261,814 & - & - \\
\hline 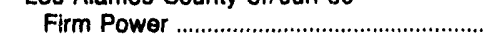 & -- & - & 6,217 & 96,862 & -- & -- \\
\hline 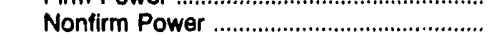 & 52,702 & $1,008,439$ & - & $-\infty$ & - & -- \\
\hline 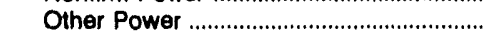 & - & - & -- & $\ldots$ & -- & - \\
\hline 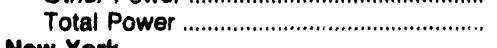 & 52,702 & $1,008,439$ & 6,217 & 96,862 & - & - \\
\hline \multicolumn{7}{|l|}{$\begin{array}{l}\text { Now York } \\
\text { Fairport Village of/May } 31\end{array}$} \\
\hline 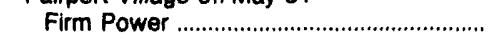 & - & - & - & - & 350,327 & $6,052,189$ \\
\hline 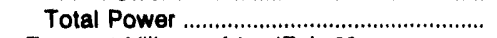 & -- & - & - & - & 350,327 & $6,052,189$ \\
\hline Freeport Village of Inc/Feb 28 & & & & & & \\
\hline 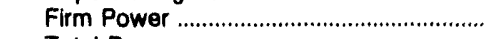 & 2,657 & 224,686 & - & - & 208,473 & $4,567,492$ \\
\hline $\begin{array}{l}\text { Total Power .............................................. } \\
\text { Jamestown Clty of/Dec }\end{array}$ & 2,657 & 224,686 & - & - & 208,473 & $4,567,482$ \\
\hline 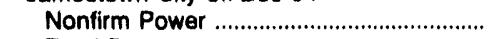 & 454,268 & $5,022,228$ & - & - & - & - \\
\hline 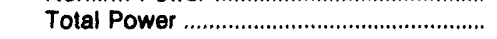 & 454,268 & $5,022,229$ & - & - & - & - \\
\hline
\end{tabular}

See notes and footnotes at end of table. 
Table 29. Electricity Purchases by Municlpal Utilitles, Flecal Yoar 1992 (Continued)

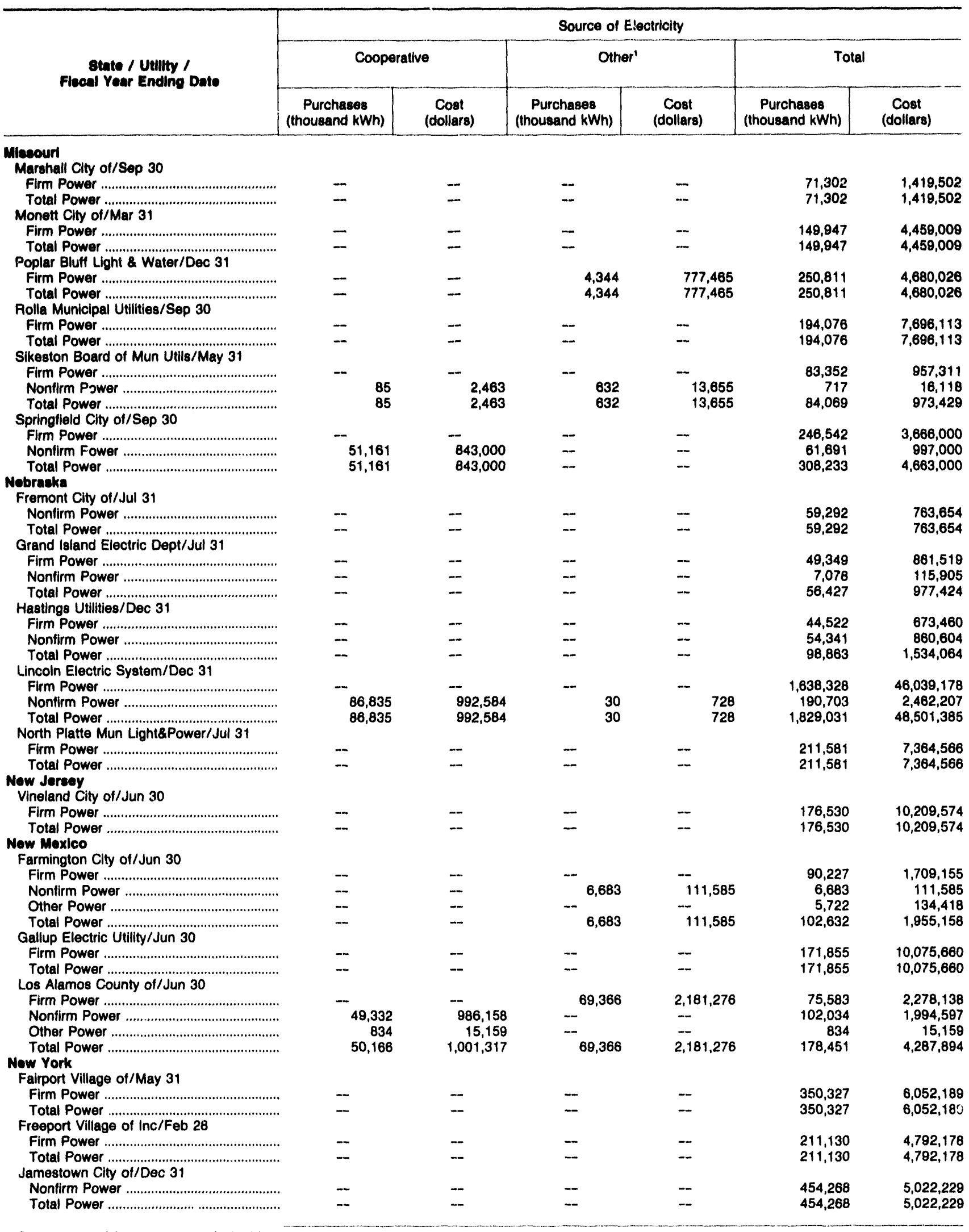

See notes and footnotes at ind of table. 
Table 29. Electriclty Purchases by Municipal Utilities, Fiscal Year 1992 (Continued)

\begin{tabular}{|c|c|c|c|c|c|c|}
\hline \multirow{3}{*}{$\begin{array}{l}\text { State / Utillty I } \\
\text { Flecal Year Ending Date }\end{array}$} & \multicolumn{6}{|c|}{ Source of Electricity } \\
\hline & \multicolumn{2}{|c|}{ Investor-Owned } & \multicolumn{2}{|c|}{ Federal } & \multicolumn{2}{|c|}{$\begin{array}{c}\text { State and Other } \\
\text { Government }\end{array}$} \\
\hline & $\begin{array}{l}\text { Purchases } \\
\text { (thousand } k W h \text { ) }\end{array}$ & $\begin{array}{c}\text { Cost } \\
\text { (dollara) }\end{array}$ & $\begin{array}{c}\text { Purchases } \\
\text { (thousand kWh) }\end{array}$ & $\begin{array}{c}\text { Coost } \\
\text { (dollara) }\end{array}$ & $\begin{array}{c}\text { Purchases } \\
\text { (thousand kWh) }\end{array}$ & $\begin{array}{c}\text { Coot } \\
\text { (dollars) }\end{array}$ \\
\hline \multicolumn{7}{|l|}{$\begin{array}{l}\text { Mow York } \\
\text { Massena Electric Department/Dec } 31\end{array}$} \\
\hline 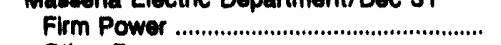 & 1,100 & 40,720 & - & -- & 151,303 & $2,241,703$ \\
\hline 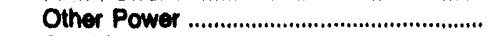 & & 142,003 & - & - & - & \\
\hline 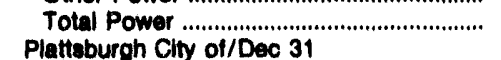 & 1,100 & 182,803 & - & - & 151,303 & $2,241,703$ \\
\hline 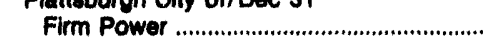 & - & - & - & - & 635,143 & $5,791,187$ \\
\hline $\begin{array}{l}\text { Total Power .... } \\
\text { Rockville Centre Village of/May } 31\end{array}$ & - & - & - & - & 535,143 & $5,791,187$ \\
\hline 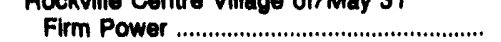 & - & - & - & - & 134,749 & $2,744,028$ \\
\hline 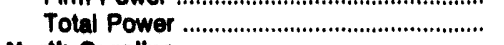 & - & - & - & - & 134,748 & $2,744,028$ \\
\hline \multicolumn{7}{|l|}{$\begin{array}{l}\text { North Carollina } \\
\text { Albemarle City of/Jun } 30\end{array}$} \\
\hline 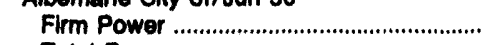 & - & - & - & - & 258,061 & $12,856,398$ \\
\hline 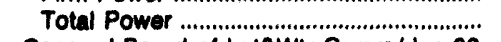 & - & - & - & - & 258,061 & $12,856,399$ \\
\hline Concord Board of LgteWtr Comm/Jun 30 & & & & & & \\
\hline Firm Power ..................................................... & 371,141 & $16,648,394$ & 3,726 & 290,138 & - & - \\
\hline \multicolumn{7}{|l|}{$\begin{array}{l}\text { Total Power } \\
\text { Ellizabeth City City of/Jun } 30\end{array}$} \\
\hline 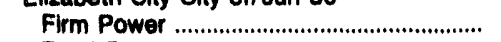 & - & - & 1,581 & 104,590 & 229,628 & $14,394,178$ \\
\hline Total Fower ........................................ & - & - & 1,581 & 104,590 & 229,628 & $14,394,178$ \\
\hline \multicolumn{7}{|l|}{ Fayetteville Public Works Comm/Jun 30} \\
\hline 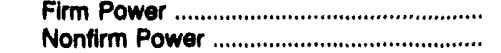 & 1,300,010 & - & 3,866 & 204,057 & $\overline{-}$ & $\overline{-}$ \\
\hline 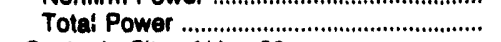 & $1,366,578$ & $61,270,792$ & 7,954 & 437,472 & - & - \\
\hline \multicolumn{7}{|l|}{ Gastonia Clty ot/Jun 30} \\
\hline 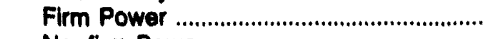 & - & - & - & - & 590,269 & $27,079,491$ \\
\hline Nontirm Power .................................................... & -- & - & 7,210 & 562,190 & - & - \\
\hline Total Power ........................... & - & - & 7,210 & 582,190 & 590,268 & $27,079,491$ \\
\hline \multicolumn{7}{|l|}{ Greenville Utilties Comm/Jun 30} \\
\hline $\begin{array}{l}\text { Firm Power } \\
\text { Total Power }\end{array}$ & - & $\overline{-}$ & $\overline{-}$ & $\overline{-}$ & $\begin{array}{l}1,047,000 \\
1,047,000\end{array}$ & $68,967,737$ \\
\hline \multirow{2}{*}{\multicolumn{7}{|c|}{$\begin{array}{l}\text { High Point Town ot/Jun } 30 \\
\text { Firm Power }\end{array}$}} \\
\hline & - & - & - & - & 805,481 & \\
\hline & $38,605,677$ \\
\hline 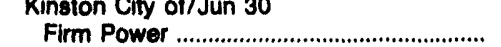 & & - & 1,103 & 62,892 & & \\
\hline 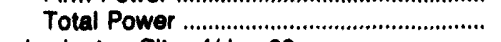 & - & - & 1,103 & 62,992 & $\begin{array}{l}415,767 \\
415,767\end{array}$ & $\begin{array}{l}26,788,557 \\
26,788,557\end{array}$ \\
\hline \multicolumn{7}{|l|}{ Lexington City of/Jun 30} \\
\hline 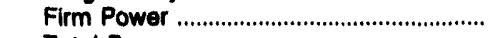 & - & -- & - & - & 424,081 & $22,492,777$ \\
\hline Total Power . & - & - & - & - & 424,081 & $22,492,777$ \\
\hline \multicolumn{7}{|l|}{ Lumberton Electric Utils Dept/Jun 30} \\
\hline 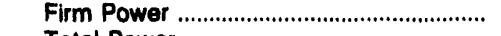 & - & - & 673 & 38,467 & 265,946 & $17,486,003$ \\
\hline Total Power ................................... & - & - & 873 & $\mathbf{3 8 , 4 6 7}$ & 265,946 & $17,486,003$ \\
\hline \multicolumn{6}{|l|}{ Monroe Clty of/Jun 30} & \\
\hline 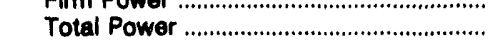 & 397,706 & $18,453,532$ & $\begin{array}{l}3,474 \\
3,474\end{array}$ & $\begin{array}{l}270,820 \\
270,820\end{array}$ & - & $=$ \\
\hline Morganton City of/Jun 30 & & & & & & \\
\hline 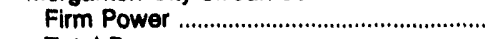 & -- & - & 15,717 & 462,266 & 256,552 & $11,509,145$ \\
\hline 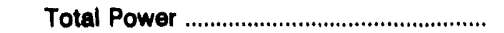 & - & - & 15,717 & 462,266 & 256,552 & $11,500,145$ \\
\hline Murphy Electric Power Board/Jun 30 & & & & & & \\
\hline 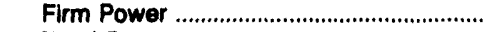 & - & - & 146,259 & $6,769,541$ & - & - \\
\hline 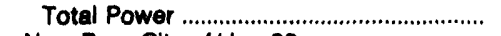 & - & - & 146,259 & $6,769,541$ & - & $-\infty$ \\
\hline New Bern City ot/Jun 30 & & & & & & \\
\hline 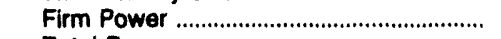 & - & -- & 913 & 51,754 & 361,581 & $24,730,148$ \\
\hline $\begin{array}{l}\text { Total Power } \\
\text { Rocky Mount City of/Jun } 30\end{array}$ & - & - & 813 & 51,754 & 361,581 & $24,730,148$ \\
\hline 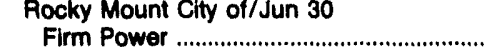 & - & - & - & - & 772,015 & $47,111,291$ \\
\hline 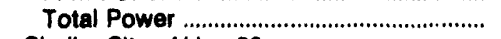 & - & - & - & - & 772,015 & $47,111,291$ \\
\hline Shelby City of/Jun 30 & & & & & & \\
\hline 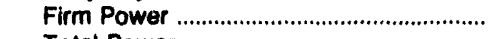 & - & - & 2,660 & 207,398 & 165,187 & $7,571,999$ \\
\hline $\begin{array}{l}\text { Total Power } \\
\text { Statesville City of/Jun } 30\end{array}$ & - & - & 2,660 & 207,398 & 165,187 & $7,571,999$ \\
\hline $\begin{array}{l}\text { Statesville City of/Jun } 30 \\
\text { Firm Power }\end{array}$ & - & - & 4,382 & 341,679 & 355,498 & $16,829,312$ \\
\hline $\begin{array}{l}\text { Total Power ............................................... } \\
\text { Tarboro Town of/Jun }\end{array}$ & -- & - & 4,382 & 341,679 & 355,488 & $16,829,312$ \\
\hline Firm Power ..................................................... & - & - & - & - & 242,329 & $15,747,908$ \\
\hline 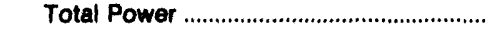 & - & -- & - & - & 242,329 & $15,747,908$ \\
\hline
\end{tabular}

See notes and footnotes at end of table. 
Table 29. Electriclty Purchases by Municlpal Utilities, Fiecal Year 1992 (Continued)

\begin{tabular}{|c|c|c|c|c|c|c|}
\hline \multirow{3}{*}{$\begin{array}{l}\text { State / Utility / } \\
\text { Fisoul Year Ending Date }\end{array}$} & \multicolumn{6}{|c|}{ Source of Electricity } \\
\hline & \multicolumn{2}{|c|}{ Cooperative } & \multicolumn{2}{|c|}{ Other' } & \multicolumn{2}{|c|}{ Total } \\
\hline & $\begin{array}{c}\text { Purchases } \\
\text { (thousand } k W h \text { ) }\end{array}$ & $\begin{array}{c}\text { Coot } \\
\text { (dollarg) }\end{array}$ & $\begin{array}{c}\text { Purchases } \\
\text { (thousand } k W h \text { ) }\end{array}$ & $\begin{array}{c}\text { Cost } \\
\text { (dollars) }\end{array}$ & $\begin{array}{c}\text { Purchases } \\
\text { (thousand kWh) }\end{array}$ & $\begin{array}{c}\text { Coot } \\
\text { (dollars) }\end{array}$ \\
\hline \multicolumn{7}{|l|}{$\begin{array}{l}\text { Now York } \\
\text { Maesens Electric Department/Dec } 31\end{array}$} \\
\hline 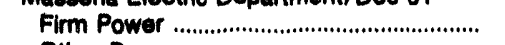 & - & - & - & -- & 152,403 & $2,282,423$ \\
\hline 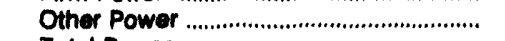 & - & - & - & -- & - & 142,083 \\
\hline 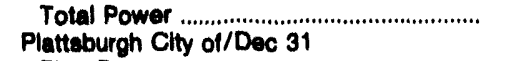 & - & -- & - & - & 152,403 & $2,424,506$ \\
\hline 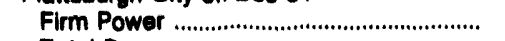 & - & - & - & - & 535,143 & $5,791,187$ \\
\hline $\begin{array}{l}\text { Total Power ... } \\
\text { Rockville Centre Villeage of /May } 31\end{array}$ & - & - & - & - & 535,143 & $5,791,187$ \\
\hline 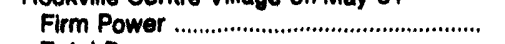 & - & - & - & - & 134,749 & $2,744,028$ \\
\hline 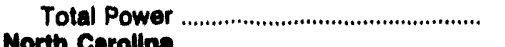 & - & - & - & - & 134,740 & $2,744,028$ \\
\hline \multicolumn{7}{|l|}{$\begin{array}{l}\text { North Carollina } \\
\text { Albemarle City of/Jun } 30\end{array}$} \\
\hline Firm Power ................................................. & - & - & - & -- & 268,061 & $12,856,389$ \\
\hline $\begin{array}{l}\text { Total Power } \\
\text { Concord Board of Lotswtr Comm/Jun } 30\end{array}$ & - & - & - & - & 258,061 & $12,866,398$ \\
\hline 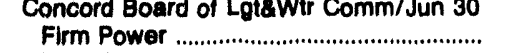 & - & - & - & -- & 374,867 & $16,838,532$ \\
\hline 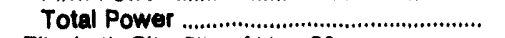 & -- & -- & - & -- & 374,887 & $16,938,532$ \\
\hline \multicolumn{7}{|l|}{ Elizabeth City City of/Jun 30} \\
\hline 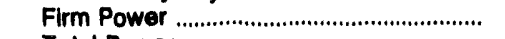 & - & - & -- & - & 231,209 & $14,498,768$ \\
\hline $\begin{array}{l}\text { Total Power } \\
\text { Fayetteville Public Works Comm/Jun } 30\end{array}$ & - & -- & - & - & 231,208 & $14,498,768$ \\
\hline 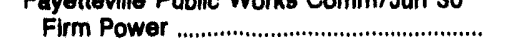 & - & - & - & - & $1,370,666$ & $61,504,207$ \\
\hline 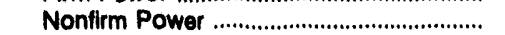 & - & - & -- & - & 3,866 & 204,057 \\
\hline Total Power & -- & -- & - & - & $1,374,532$ & $61,708,264$ \\
\hline \multicolumn{7}{|l|}{ Gastonia City of/Jun 30} \\
\hline 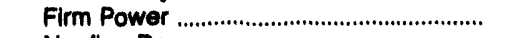 & -- & - & - & - & 590,269 & $27,079,481$ \\
\hline 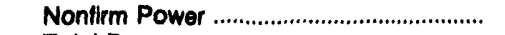 & - & - & - & -- & 7,210 & 562,180 \\
\hline 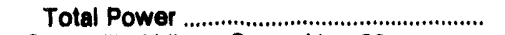 & - & -- & -- & - & 597,479 & $27,641,681$ \\
\hline \multicolumn{7}{|l|}{ Greenville Utilities Comm/Jun 30} \\
\hline 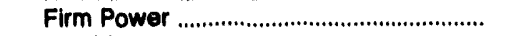 & - & - & - & - & $1,047,000$ & $68,967,737$ \\
\hline 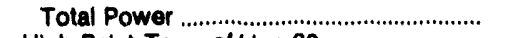 & - & - & - & - & $1,047,000$ & $68,867,737$ \\
\hline \multicolumn{7}{|l|}{ High Point Town of/Jun 30} \\
\hline 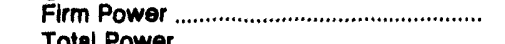 & - & - & - & - & 605,481 & $39,605,677$ \\
\hline \multicolumn{5}{|l|}{ Kinston City of/Jun 30} & 805,481 & $39,605,677$ \\
\hline 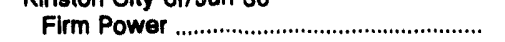 & - & - & - & - & 416,870 & $26,851,549$ \\
\hline 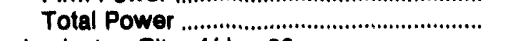 & -- & - & - & - & 416,870 & $26,851,549$ \\
\hline \multicolumn{7}{|l|}{ Lexington City of/Jun 30} \\
\hline 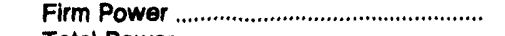 & - & - & - & - & 424,061 & $22,492,777$ \\
\hline Total Power .... & - & - & - & - & 424,061 & $22,482,777$ \\
\hline \multicolumn{6}{|l|}{ Lumberton Electric Utils Dept/Jun 30} & $17,524,470$ \\
\hline $\begin{array}{l}\text { Firm Power } \\
\text { Total Power }\end{array}$ & $\overline{-}$ & $\overline{-}$ & - & $\overline{-}$ & 266,619 & $\begin{array}{l}17,524,470 \\
17,524,470\end{array}$ \\
\hline \multicolumn{7}{|l|}{ Monroe Clty of/Jun 30} \\
\hline 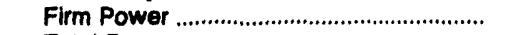 & - & - & - & - & 401,180 & $18,724,352$ \\
\hline 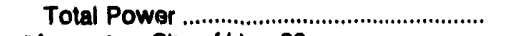 & - & - & -- & -- & 401,180 & $18,724,352$ \\
\hline Morganton City of/Jun 30 & & & & & & \\
\hline Firm Power & - & - & - & - & 272,269 & $11,971,411$ \\
\hline Total Power & - & - & - & -- & 272,269 & $11,971,411$ \\
\hline Murphy Electric Power Board/Jun 30 & & & & & & \\
\hline 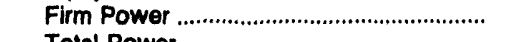 & - & - & - & - & 146,250 & $6,769,541$ \\
\hline Total Power & - & - & - & - & 146,259 & $6,769,541$ \\
\hline New Bern City of/Jun 30 & & & & & & \\
\hline 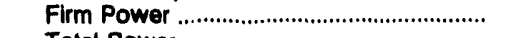 & -- & - & -- & $-\infty$ & 362,494 & $24,781,902$ \\
\hline & - & - & - & - & 362,494 & $24,781,902$ \\
\hline Rocky Mount City of/Jun 30 & & & & & & \\
\hline 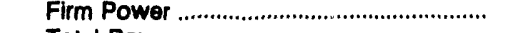 & - & - & - & - & 772,015 & $47,111,291$ \\
\hline 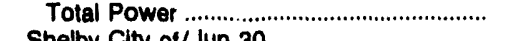 & - & - & - & - & 772,015 & $47,111,291$ \\
\hline Shelby City of/Jun 30 & & & & & & \\
\hline 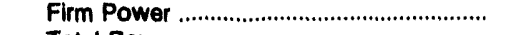 & - & $\bar{z}$ & -- & - & $\begin{array}{l}167,847 \\
167,847\end{array}$ & $\begin{array}{l}7,779,397 \\
7779,397\end{array}$ \\
\hline 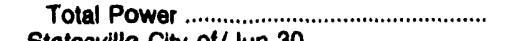 & - & - & - & -- & 167,847 & $7,779,397$ \\
\hline Statesville Clty of/Jun 30 & - & - & - & -- & & $17,170,991$ \\
\hline Firm Power ........................................... & - & - & -- & -- & 359,880 & $\begin{array}{l}17,170,991 \\
17,170,991\end{array}$ \\
\hline $\begin{array}{l}\text { Total Power } \\
\text { Tarboro Town of/Jun } 30\end{array}$ & & & & & & \\
\hline 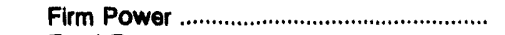 & -- & - & - & - & 242,329 & $15,747,908$ \\
\hline 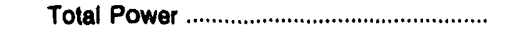 & - & - & - & - & 242,329 & $15,747,908$ \\
\hline
\end{tabular}

See notes and footnotes at end of table. 
Table 29. Electricity Purchases by Municipal Utilitios, Flecal Year 1992 (Continued)

\begin{tabular}{|c|c|c|c|c|c|c|}
\hline \multirow{3}{*}{$\begin{array}{l}\text { State / Uumly / } \\
\text { Fleod Year Ending Dato }\end{array}$} & \multicolumn{6}{|c|}{ Source of Electritity } \\
\hline & \multicolumn{2}{|c|}{ Investor-Owned } & \multicolumn{2}{|c|}{ Federal } & \multicolumn{2}{|c|}{$\begin{array}{l}\text { State and Other } \\
\text { Government }\end{array}$} \\
\hline & $\begin{array}{c}\text { Purchases } \\
\text { (thousand } \mathrm{kWh} \text { ) }\end{array}$ & $\begin{array}{c}\text { Cost } \\
\text { (dollars) }\end{array}$ & $\begin{array}{l}\text { Purchaces } \\
\text { (thousand kWh) }\end{array}$ & $\begin{array}{c}\text { Cost } \\
\text { (dollara) }\end{array}$ & $\begin{array}{c}\text { Purchases } \\
\text { (thousand kWh) }\end{array}$ & $\begin{array}{c}\text { Cost } \\
\text { (dollare) }\end{array}$ \\
\hline \multicolumn{7}{|l|}{ North Coroling } \\
\hline 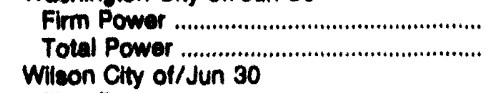 & - & - & $\begin{array}{l}2,034 \\
2,034\end{array}$ & $\begin{array}{l}116,184 \\
116,184\end{array}$ & $\begin{array}{l}229,072 \\
229,072\end{array}$ & $\begin{array}{l}15,261,862 \\
15,261,862\end{array}$ \\
\hline 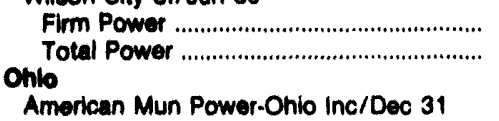 & - & - & $\begin{array}{l}2,220 \\
2,220\end{array}$ & $\begin{array}{l}126,771 \\
126,771\end{array}$ & $\begin{array}{l}962,229 \\
962,229\end{array}$ & $\begin{array}{l}60,395,094 \\
60,395,094\end{array}$ \\
\hline 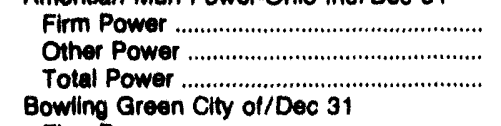 & $\frac{2,286,155}{2,286,155}$ & $\begin{array}{r}83,740,866 \\
1,611,550 \\
85,352,516\end{array}$ & $\ddot{--}$ & - & $\begin{array}{r}462,717 \\
- \\
452,717\end{array}$ & $\begin{array}{l}3,529,473 \\
3,529,473\end{array}$ \\
\hline 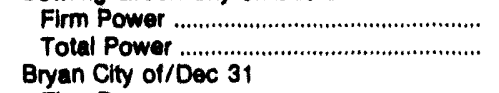 & $\overline{-}$ & - & $\overline{-}$ & - & $\overline{-}$ & $\overline{-}$ \\
\hline 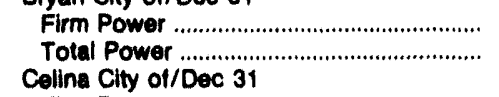 & $\begin{array}{l}188,037 \\
188,037\end{array}$ & $\begin{array}{l}6,002,989 \\
6,002,889\end{array}$ & $\overline{-}$ & - & $\begin{array}{l}5,509 \\
5,509\end{array}$ & $\begin{array}{l}86,188 \\
86,188\end{array}$ \\
\hline Firm Power ................................................... & 146,401 & $5,577,524$ & - & - & - & - \\
\hline 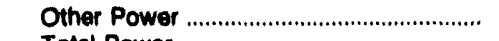 & - & $=-1$ & - & - & -- & - \\
\hline $\begin{array}{l}\text { Total Power } \\
\text { Cleveland City of/Dec } 31\end{array}$ & 146,401 & $5,577,524$ & - & - & - & - \\
\hline Firm Power & 620,840 & $20,854,640$ & - & - & 152,622 & $2,532,570$ \\
\hline 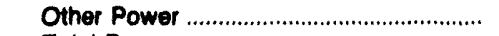 & 2,840 & 107,793 & - & - & - & - \\
\hline 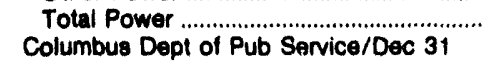 & 623,680 & $20,862,433$ & - & - & 152,622 & $2,532,570$ \\
\hline 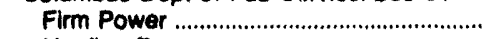 & - & - & - & - & - & - \\
\hline 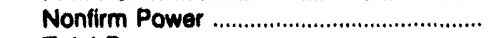 & 1,562 & 167,846 & - & - & - & - \\
\hline $\begin{array}{l}\text { Total Power ......................... } \\
\text { Cuyahoga Falls City of/Dec }\end{array}$ & 1,562 & 167,946 & - & - & - & - \\
\hline Firm Power & - & - & - & - & - & - \\
\hline $\begin{array}{l}\text { Total Power ................................................ } \\
\text { Dover City of }\end{array}$ & - & -- & - & - & - & -- \\
\hline 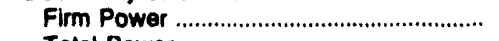 & - & - & - & - & - & - \\
\hline $\begin{array}{l}\text { Total Power ........................ } \\
\text { Hamilton Dept of Public Utis/Dec }\end{array}$ & - & - & -- & - & - & -- \\
\hline 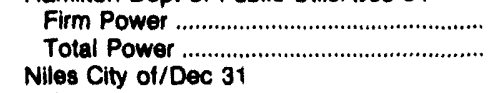 & $\begin{array}{l}276,327 \\
276,327\end{array}$ & $\begin{array}{l}2,121,014 \\
2,121,014\end{array}$ & - & - & $\begin{array}{l}54,122 \\
54,122\end{array}$ & $\begin{array}{l}1,332,459 \\
1,332,459\end{array}$ \\
\hline 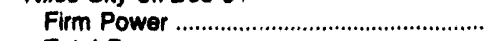 & - & - & - & - & - & - \\
\hline $\begin{array}{l}\text { Total Power .......................................... } \\
\text { Orrville City ol/Dec } 31\end{array}$ & - & - & -- & - & - & - \\
\hline 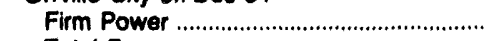 & - & - & - & -- & - & - \\
\hline $\begin{array}{l}\text { Total Power ............................................ } \\
\text { Painesville City of/Dec } 31\end{array}$ & - & - & -- & - & - & - \\
\hline 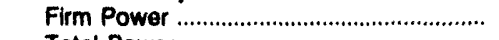 & 59,891 & $1,543,357$ & - & - & - & - \\
\hline 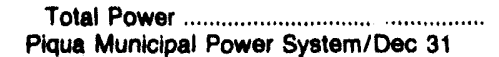 & 59,891 & $1,543,357$ & -- & - & - & - \\
\hline 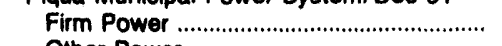 & -- & - & -- & -- & - & -- \\
\hline 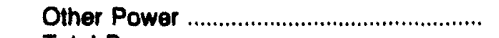 & 135,436 & $3,740,182$ & - & - & - & -- \\
\hline $\begin{array}{l}\text { Total Power .............................................. } \\
\text { St Marys City of/Dec } 31\end{array}$ & 135,436 & $3,740,182$ & - & - & - & - \\
\hline Other Power & - & - & - & - & - & - \\
\hline $\begin{array}{l}\text { Total Power ............................................... } \\
\text { Wadsworth City of/Dec } 31\end{array}$ & - & - & $\cdots$ & -- & - & -- \\
\hline 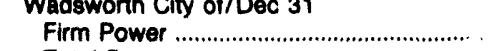 & - & - & -- & - & - & - \\
\hline $\begin{array}{l}\text { Total Power ... } \\
\text { Wapakoneta Electric Light Dept/Dec } 31\end{array}$ & - & - & - & -- & - & - \\
\hline 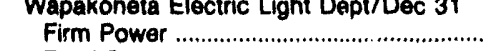 & 145,319 & $4,569,397$ & - & - & - & - \\
\hline $\begin{array}{l}\text { Total Power .................................................. } \\
\text { Westerville City of/Dec } 31\end{array}$ & 145,319 & $4,569,397$ & - & - & -- & - \\
\hline 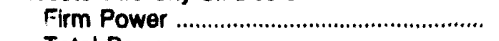 & 301,190 & $9,562,754$ & - & -- & 11,019 & 225,530 \\
\hline $\begin{array}{l}\text { Total Power } \\
\text { Oklahoma }\end{array}$ & 301,190 & $9,562,754$ & - & - & 11,019 & 225,530 \\
\hline Altus City of/Jun 30 & & & & & & \\
\hline Firm Power . & - & - & - & - & 139,382 & $5,841,000$ \\
\hline Total Power ............................................... & - & - & - & - & 139,382 & $5,941,000$ \\
\hline
\end{tabular}

See notes and footnotes at end of table. 
Table 29. Electriclty Purchases by Municipal Utilitios, Flecal Yoar 1992 (Continued)

\begin{tabular}{|c|c|c|c|c|c|c|}
\hline \multirow{3}{*}{$\begin{array}{l}\text { State / Utilhy / } \\
\text { Fical Year Ending Dato }\end{array}$} & \multicolumn{6}{|c|}{ Source of Electricity } \\
\hline & \multicolumn{2}{|c|}{ Cooperative } & \multicolumn{2}{|c|}{ Other' } & \multicolumn{2}{|c|}{ Total } \\
\hline & $\begin{array}{c}\text { Purchases } \\
\text { (thousand } \mathrm{kWh} \text { ) }\end{array}$ & $\begin{array}{c}\text { Cool } \\
\text { (dollara) }\end{array}$ & $\begin{array}{c}\text { Purchases } \\
\text { (thousand kWh) }\end{array}$ & $\begin{array}{c}\text { Cost } \\
\text { (dolliars) }\end{array}$ & $\begin{array}{c}\text { Purchases } \\
\text { (thousand } \mathrm{kWh} \text { ) }\end{array}$ & $\begin{array}{c}\text { Cost } \\
\text { (dollara) }\end{array}$ \\
\hline \multicolumn{7}{|l|}{$\begin{array}{l}\text { North Carollina } \\
\text { Waahington City of/Jun } 30\end{array}$} \\
\hline 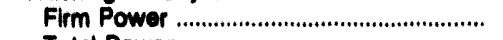 & -. & - & - & - & 231,108 & $15,378,046$ \\
\hline $\begin{array}{l}\text { Total Power } \\
\text { Willeon City of/Jun } 30\end{array}$ & - & - & - & - & 231,106 & $15,378,046$ \\
\hline 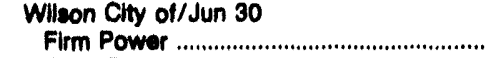 & -- & - & - & -- & 964,449 & $60,521,865$ \\
\hline 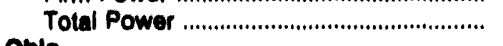 & - & - & -. & - & 964,449 & $60,521,865$ \\
\hline \multicolumn{7}{|l|}{$\begin{array}{l}\text { Ohio } \\
\text { American Mun Power-Ohio Inc/Dec } 31\end{array}$} \\
\hline $\begin{array}{l}\text { Amorican Mun Power-Ohio Inc/Dec } 31 \\
\text { Firm Power }\end{array}$ & 20.541 & 556,273 & 254,774 & $7,339,024$ & $3,014,187$ & $95,165,738$ \\
\hline 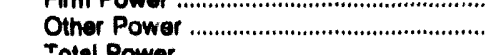 & $-20,04$ & - & - & - & - & $\begin{array}{l}80,100, / 30 \\
1,611,550\end{array}$ \\
\hline $\begin{array}{l}\text { Total Power } \\
\text { Bowling Green City of/Dec } 31\end{array}$ & 20,541 & 556,273 & 254,774 & $7,339,024$ & $3,014,187$ & $96,777,286$ \\
\hline 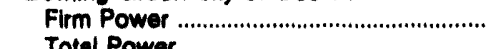 & - & - & 280,308 & $10,831,400$ & 280,308 & $10,831,400$ \\
\hline $\begin{array}{l}\text { Total Power } \\
\text { Bryan Cly of /Dec } 31\end{array}$ & - & - & 280,308 & $10,831,400$ & 280,308 & $10,831,400$ \\
\hline 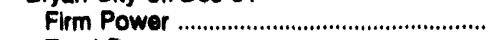 & - & -- & - & - & 193,546 & $6,089,177$ \\
\hline $\begin{array}{l}\text { Tolal Power } \\
\text { Colina Clty of/Doc } 31\end{array}$ & - & - & - & - & 193,546 & $6,089,177$ \\
\hline 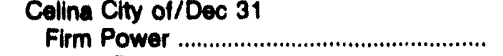 & - & - & - & -- & 146,401 & $5,577,524$ \\
\hline Other Power ..................................................... & - & - & 8.053 & 171,841 & 8,053 & 171,841 \\
\hline \multirow{2}{*}{\multicolumn{7}{|c|}{ Cleveland City of/Dec 31}} \\
\hline & & & & & & \\
\hline 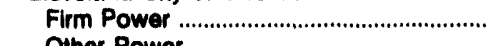 & 81,259 & $2,143,577$ & 87,830 & $2,965,421$ & 942,551 & $26,496,208$ \\
\hline $\begin{array}{l}\text { Other Power } \\
\text { Total Power }\end{array}$ & - & $\overline{0} 11967$ & 416 & $\begin{array}{r}13,684 \\
0\end{array}$ & $\begin{array}{r}3,256 \\
0,507\end{array}$ & 121,477 \\
\hline $\begin{array}{l}\text { Total Power at of Pub Service/Dec } 31 \\
\text { Columbus Dept }\end{array}$ & 81,258 & $2,143,577$ & 88,246 & $2,979,105$ & 945,807 & $28,617,685$ \\
\hline 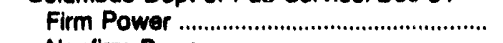 & - & - & 287,435 & $8,687,524$ & 287,435 & $8,687,524$ \\
\hline 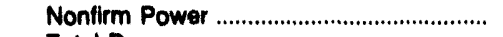 & - & - & - & & 1,562 & 167,946 \\
\hline 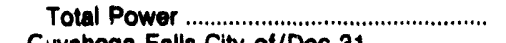 & - & - & 287,435 & $8,687,524$ & 288,997 & $8,855,470$ \\
\hline \multicolumn{7}{|l|}{ Cuyahoga Falls City of/Dec 31} \\
\hline 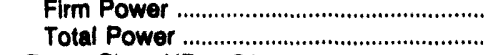 & - & - & 289,208 & $\begin{array}{l}11,818,116 \\
11,818,116\end{array}$ & 289,208 & $\begin{array}{l}11,818,116 \\
11,818,116\end{array}$ \\
\hline \multicolumn{7}{|l|}{ Dover City of/Dec 31} \\
\hline 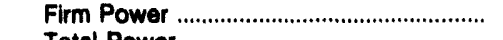 & - & - & 130,152 & $3,750,064$ & 130,152 & $3,750,064$ \\
\hline $\begin{array}{l}\text { Total Power } \\
\text { Hamilton Dept of Public Utils/Dec } 31\end{array}$ & -- & - & 130,152 & $3,750,064$ & 130,152 & $3,750,064$ \\
\hline Firm Power . & - & - & - & - & 330,449 & $3,453,473$ \\
\hline \multirow{2}{*}{\multicolumn{7}{|c|}{ Niles City of/Dec 31}} \\
\hline & & & & & & \\
\hline Firm Power ....................................................... & -- & - & 248,976 & $9,777,850$ & 248,976 & $9,777,850$ \\
\hline \multicolumn{5}{|l|}{ Orville City of/Dec 31} & & $9,777,850$ \\
\hline 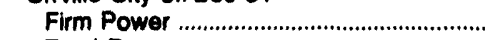 & - & -- & 59,433 & $3,397,000$ & 59,433 & \\
\hline Total Power & - & - & 59,433 & $3,397,000$ & 59,433 & $3,397,000$ \\
\hline \multicolumn{7}{|l|}{ Painesville City of/Dec 31} \\
\hline 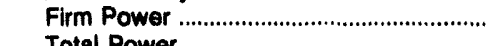 & - & - & 17,767 & 491,424 & 77,658 & $2,034,781$ \\
\hline \multicolumn{4}{|l|}{ Piqua Municipal Power System/Dec 31} & 491,424 & 77,658 & $2,034,781$ \\
\hline 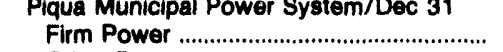 & - & - & 15,624 & 390,368 & 15,624 & 390,368 \\
\hline Other Power ……………………………....... & - & - & - & -- & 135,436 & $3,740,182$ \\
\hline Total Power & - & - & 15,624 & 390,368 & 151,060 & $4,130,550$ \\
\hline \multicolumn{7}{|l|}{ St Marys City ot/Dec 31} \\
\hline Other Power ...................................................... & - & - & 108,551 & $2,953,553$ & 108,551 & $2,953,553$ \\
\hline \multicolumn{4}{|l|}{ Wadsworth City of/Doc 31} & $2,853,553$ & 108,551 & $2,953,553$ \\
\hline 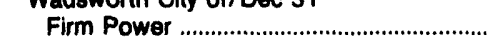 & - & -- & 195,494 & $7,460,236$ & 195,494 & $7,460,236$ \\
\hline 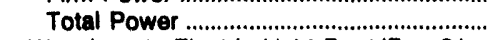 & -- & -- & 195,494 & $7,460,236$ & 195,494 & $7,460,236$ \\
\hline \multicolumn{7}{|l|}{ Wapakoneta Electric Light Dept/Dec 31} \\
\hline 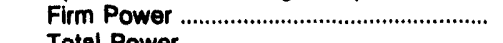 & - & -- & 6,420 & 126,615 & 151,739 & $4,696,012$ \\
\hline 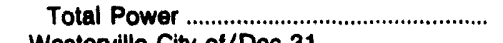 & - & - & 6,420 & 126,615 & 151,739 & $4,696,012$ \\
\hline Westerville City of/Dec 31 & & & & & & \\
\hline $\begin{array}{l}\text { Firm Power } \\
\text { Total Power }\end{array}$ & $\bar{z}$ & $\bar{z}$ & $\overline{-}$ & $=$ & $\begin{array}{l}312,209 \\
312,209\end{array}$ & $\begin{array}{l}9,788,284 \\
9,788,284\end{array}$ \\
\hline 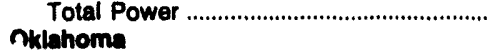 & & & & & 312,209 & $9,788,284$ \\
\hline Altus City oi/Jun 30 & & & & & & \\
\hline 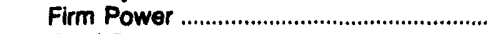 & - & - & - & - & 139,382 & $\begin{array}{l}5,941,000 \\
5,941,000\end{array}$ \\
\hline 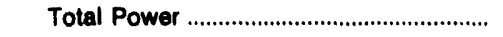 & - & - & - & - & 139,382 & $5,941,000$ \\
\hline
\end{tabular}



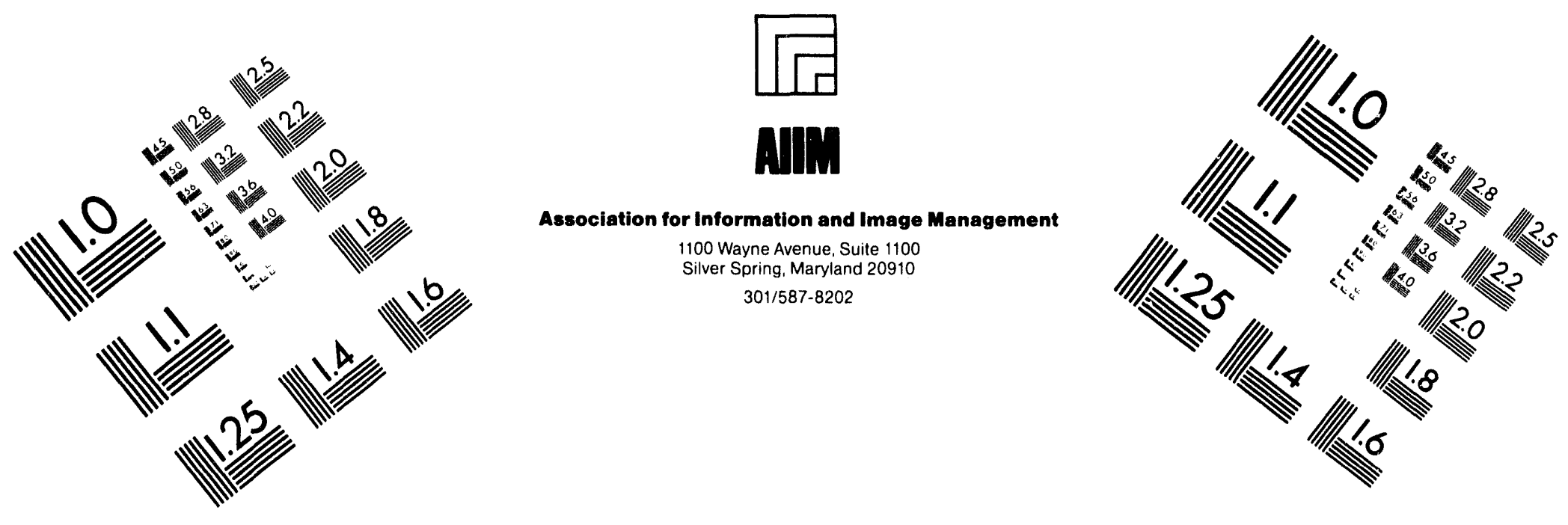

\section{Centimeter}

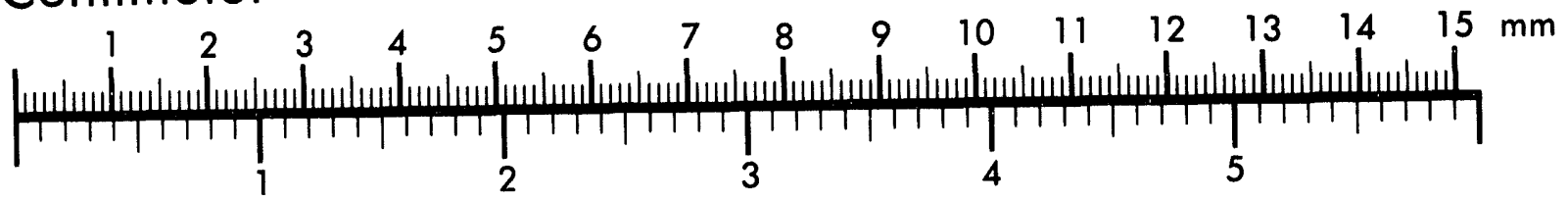

Inches
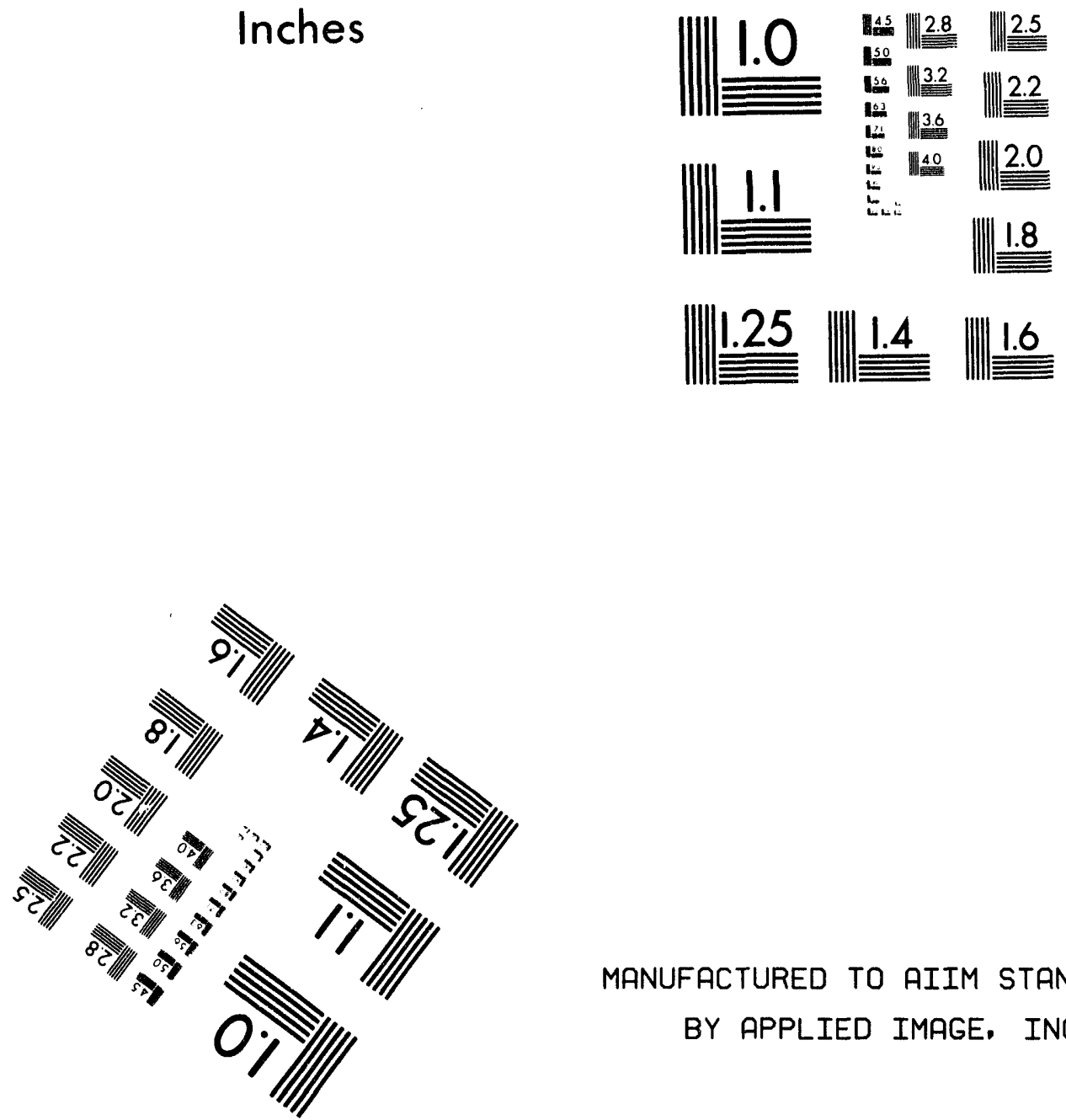

MANUFACTURED TO AIIM STANDARDS

BY APPLIED IMAGE, INC.

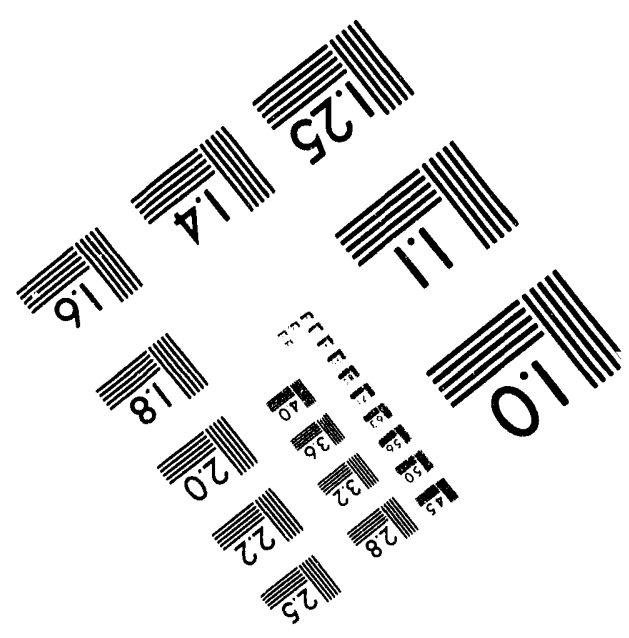



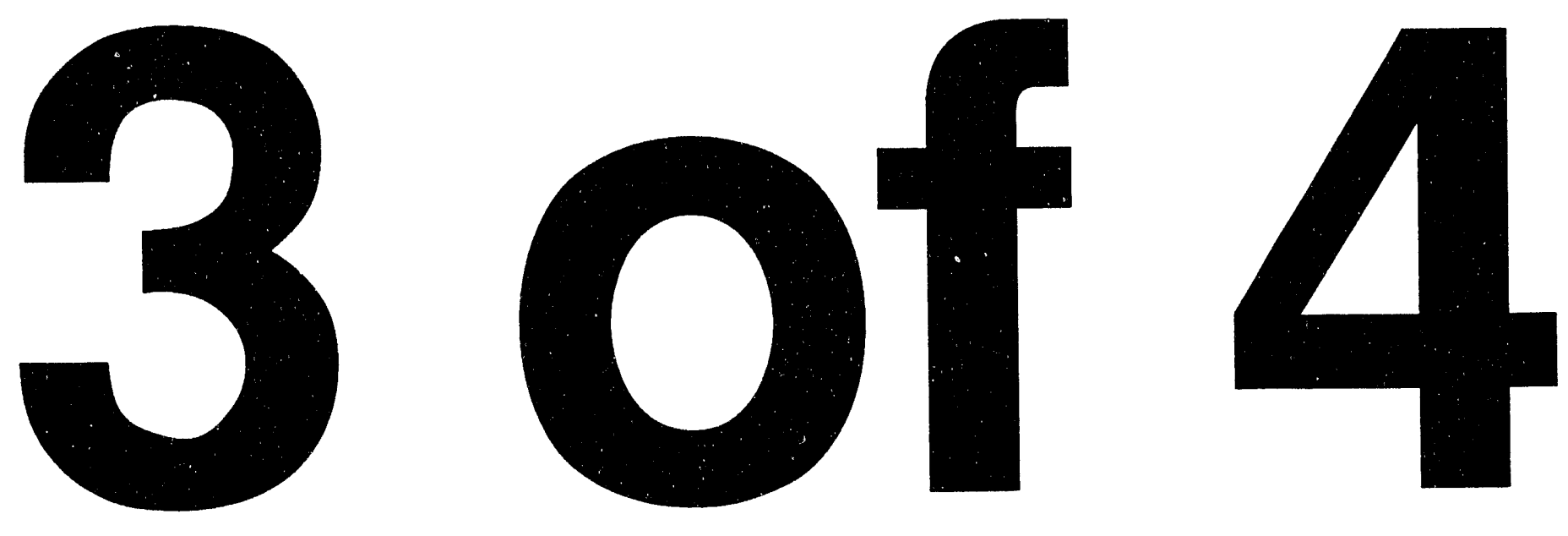
Table 29. Electricity Purchases by Municipal Utilities, Fiscal Year 1992 (Continued)

\begin{tabular}{|c|c|c|c|c|c|c|}
\hline \multirow{3}{*}{$\begin{array}{l}\text { State / Utilly / } \\
\text { Fiecal Year Ending Date }\end{array}$} & \multicolumn{6}{|c|}{ Source of Electricity } \\
\hline & \multicolumn{2}{|c|}{ Investor-Owned } & \multicolumn{2}{|c|}{ Federal } & \multicolumn{2}{|c|}{$\begin{array}{l}\text { State and Other } \\
\text { Government }\end{array}$} \\
\hline & $\begin{array}{c}\text { Purchases } \\
\text { (thousand kWh) }\end{array}$ & $\begin{array}{c}\text { Cost } \\
\text { (dollars) }\end{array}$ & $\begin{array}{c}\text { Purchases } \\
\text { (thousand kWh) }\end{array}$ & $\begin{array}{c}\text { Cost } \\
\text { (dollars) }\end{array}$ & $\begin{array}{c}\text { Purchases } \\
\text { (thousand kWh) }\end{array}$ & $\begin{array}{c}\text { Cost } \\
\text { (dollars) }\end{array}$ \\
\hline \multicolumn{7}{|l|}{$\begin{array}{l}\text { Oklahoma } \\
\text { Claremore City of/Jun } 30\end{array}$} \\
\hline 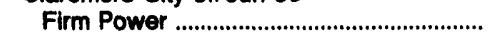 & - & - & - & - & 198,206 & $7,149,162$ \\
\hline \multicolumn{7}{|l|}{ Duncan City of/Jun 30} \\
\hline 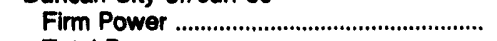 & - & - & - & - & 139,412 & $4,508,302$ \\
\hline & \multicolumn{3}{|c|}{ Edmond Electric Department/Jun 30} & - & 139,412 & $4,508,302$ \\
\hline 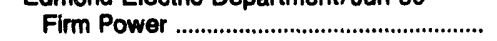 & - & - & - & - & 429,507 & $18,730,818$ \\
\hline 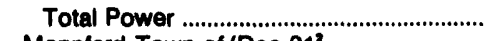 & - & - & - & - & 429,507 & $18,730,818$ \\
\hline \multicolumn{7}{|l|}{ Mannford Town of/Dec $31^{2}$} \\
\hline 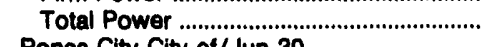 & 24,335 & 901,123 & - & - & $\overline{-}$ & - \\
\hline \multicolumn{7}{|l|}{ Ponca City City of/Jun 30} \\
\hline 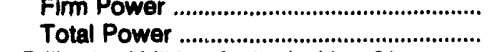 & $\overline{-}$ & - & $\overline{-}$ & $\overline{-}$ & $\begin{array}{l}284,936 \\
284,936\end{array}$ & $\begin{array}{l}11,289,739 \\
11,289,739\end{array}$ \\
\hline \multicolumn{7}{|l|}{ Stillwater Utilities Authority/Jun 30} \\
\hline 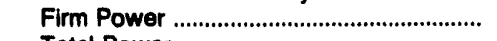 & - & - & - & - & 360,577 & $11,016,126$ \\
\hline 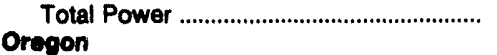 & - & - & - & - & 360,577 & $11,016,126$ \\
\hline \multicolumn{7}{|l|}{$\begin{array}{l}\text { Oregon } \\
\text { Ashland City of/Jun } 30\end{array}$} \\
\hline 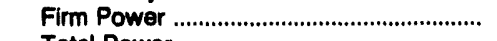 & - & - & 154,796 & $3,926,067$ & - & - \\
\hline \multicolumn{3}{|l|}{ Eugene City of/Dec 31} & 154,796 & $3,926,067$ & - & - \\
\hline 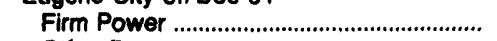 & 5,630 & 148,979 & $2,023,056$ & $39,155,586$ & 175,001 & $1,738,717$ \\
\hline 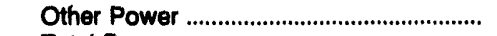 & - & 237,940 & - & - & - & \\
\hline \multirow{2}{*}{\multicolumn{7}{|c|}{ Forest Grove City of/Jun 30}} \\
\hline & & & & & & \\
\hline 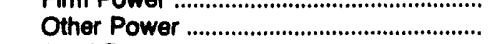 & - & - & - & - & - & $\begin{array}{r}2 / 3,331 \\
-251,854\end{array}$ \\
\hline \multirow{2}{*}{\multicolumn{7}{|c|}{ McMinnville City of/Jun 30}} \\
\hline & & & & & & \\
\hline 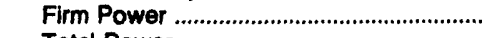 & - & - & 457,466 & $11,863,292$ & 44,571 & 209,876 \\
\hline 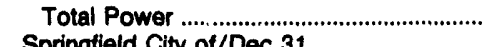 & - & - & 457,466 & $11,863,292$ & 44,571 & 209,876 \\
\hline Springfield City of/Dec 31 & & & & & & \\
\hline 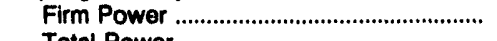 & - & - & 781,674 & $18,644,704$ & - & - \\
\hline 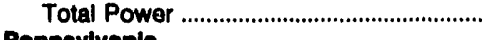 & - & - & 781,674 & $18,644,704$ & - & - \\
\hline $\begin{array}{l}\text { Pennaylvania } \\
\text { Chambersburg Borough of/Dec } 31\end{array}$ & & & & & & \\
\hline 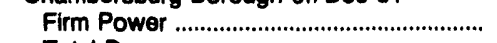 & 227,539 & $8,063,479$ & - & - & - & - \\
\hline 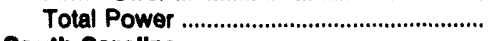 & 227,539 & $8,063,478$ & - & - & - & -- \\
\hline $\begin{array}{l}\text { South Carolina } \\
\text { Camden City of/Jun } 30\end{array}$ & & & & & & \\
\hline 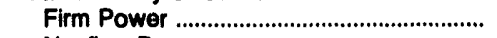 & 151,412 & $8,822,285$ & - & -- & - & - \\
\hline 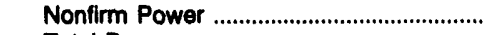 & - & - & - & - & - & - \\
\hline 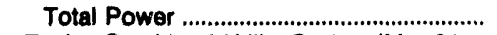 & 151,412 & $8,822,285$ & - & - & - & - \\
\hline Easley Combined Utility System/Mar 31 & & & & & & \\
\hline 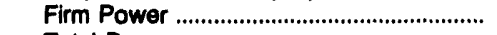 & - & - & 15,629 & 437,603 & - & -- \\
\hline 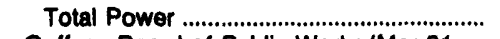 & - & - & 15,629 & 437,603 & -- & - \\
\hline Gaftney Board of Public Works/Mar 31 & & & & & & \\
\hline 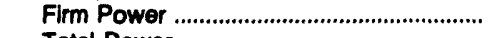 & - & - & 12,673 & 354,833 & -- & - \\
\hline 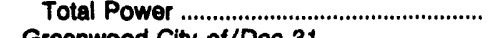 & - & - & 12,673 & 354,833 & - & - \\
\hline Greenwood City of/Dec 31 & & & & & & \\
\hline 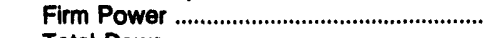 & 214,341 & $9,378,276$ & 22,349 & 607,512 & - & -- \\
\hline Total Power & 214,341 & $9,378,276$ & 22,349 & 607,512 & - & - \\
\hline Greer Commission of Public Wks/Dec 31 & & & & & & \\
\hline 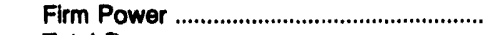 & - & - & 18,326 & 488,154 & - & - \\
\hline Total Power & - & - & 18,326 & 498,154 & - & - \\
\hline Newberry City of/Jun 30 & & & & & & \\
\hline 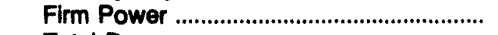 & - & - & 1,479 & 115,338 & - & - \\
\hline 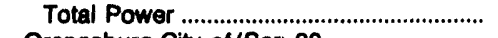 & - & -- & 1,479 & 115,338 & - & - \\
\hline Orangeburg City of/Sep 30 & & & & & & \\
\hline 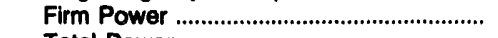 & 689,861 & $24,178,452$ & 7,145 & 634,215 & - & - \\
\hline Total Power & 689,861 & $24,178,452$ & 7,145 & 634,215 & - & - \\
\hline Piedmont Municipal Power Agny/Dec 31 & & & & & & \\
\hline 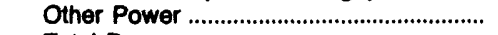 & 993,200 & $43,399,000$ & - & - & - & - \\
\hline 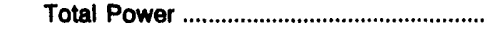 & 993,200 & $43,399,000$ & - & - & -- & - \\
\hline
\end{tabular}

See notes and footnotes at end of table. 
Table 29. Electriclty Purchases by Munlcipal Utilities, Fiscal Year 1992 (Continued)

\begin{tabular}{|c|c|c|c|c|c|c|}
\hline \multirow{3}{*}{$\begin{array}{l}\text { 8tate / Utilly I } \\
\text { Flecal Year Ending Date }\end{array}$} & \multicolumn{6}{|c|}{ Source of Electricity } \\
\hline & \multicolumn{2}{|c|}{ Cooperative } & \multicolumn{2}{|c|}{ Other' } & \multicolumn{2}{|c|}{ Total } \\
\hline & $\begin{array}{c}\text { Purchases } \\
\text { (thousand kWh) }\end{array}$ & $\begin{array}{c}\text { Cost } \\
\text { (dollars) }\end{array}$ & $\begin{array}{c}\text { Purchases } \\
\text { (thousand kWh) }\end{array}$ & $\begin{array}{c}\text { Cost } \\
\text { (dollars) }\end{array}$ & $\begin{array}{c}\text { Purchases } \\
\text { (thousand kWh) }\end{array}$ & $\begin{array}{c}\text { Cost } \\
\text { (dollars) }\end{array}$ \\
\hline \multicolumn{7}{|l|}{ Oklahoma } \\
\hline Firm Power ......................................... & - & - & - & -- & 198,206 & $7,149,162$ \\
\hline 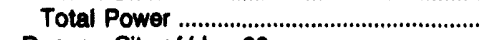 & - & - & - & -- & 198,206 & $7,148,162$ \\
\hline \multicolumn{7}{|l|}{ Duncan City of/Jun 30} \\
\hline 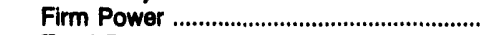 & - & - & - & -- & 139,412 & $4,508,302$ \\
\hline & - & - & - & -- & 139,412 & $4,508,302$ \\
\hline \multicolumn{3}{|l|}{ Edmond Electric Department/Jun 30} & - & -- & & \\
\hline \multicolumn{7}{|l|}{ Mannford Town of/Dec $31^{2}$} \\
\hline 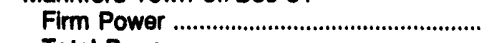 & - & - & - & - & 24,335 & 801,123 \\
\hline $\begin{array}{l}\text { Total Power ......in } 30 \\
\text { Ponca City City of Jun }\end{array}$ & \multicolumn{4}{|c|}{ Ponca City City of/Jun 30} & 24,335 & 901,123 \\
\hline 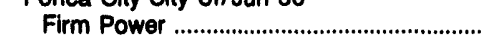 & - & - & -- & - & 284,936 & $11,289,739$ \\
\hline 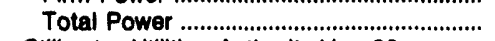 & - & - & - & - & 284,936 & $11,289,739$ \\
\hline \multicolumn{7}{|l|}{ Stillwater Utilities Authority/Jun 30} \\
\hline 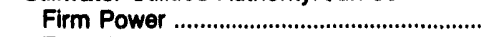 & -- & - & -- & - & 360,577 & $11,016,126$ \\
\hline 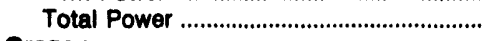 & -- & - & -- & - & 360,577 & $11,016,126$ \\
\hline \multicolumn{7}{|l|}{$\begin{array}{l}\text { Oregon } \\
\text { Ashlend City of/Jun } 30\end{array}$} \\
\hline Firm Power .............................................. & - & - & -- & - & 154,796 & $3,926,067$ \\
\hline Total Power & - & - & - & - & 154,796 & $3,926,067$ \\
\hline \multicolumn{7}{|l|}{ Eugene City of/Dec 31} \\
\hline Other Power & - & - & - & - & $-2,19,102$ & 237,940 \\
\hline 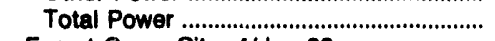 & - & - & 210,495 & $1,976,423$ & $2,414,182$ & $43,257,645$ \\
\hline \multicolumn{7}{|l|}{ Forest Grove City of/Jun 30} \\
\hline 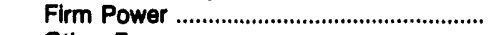 & - & - & 7,984 & 29,767 & 202,380 & $2,126,561$ \\
\hline Other Power .................................................... & - & - & -- & $2,021,370$ & - & $1,768,516$ \\
\hline 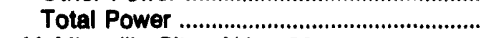 & - & - & 7,984 & $2,051,137$ & 202,380 & $3,896,077$ \\
\hline McMinnville City of/Jun 30 & & & & & & \\
\hline 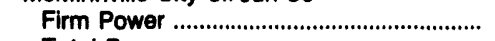 & - & - & 10,630 & 44,950 & 512,667 & $12,118,118$ \\
\hline 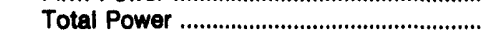 & - & -- & 10,630 & 44,950 & 512,667 & $12,118,118$ \\
\hline Springfield City of/Dec 31 & & & & & & \\
\hline 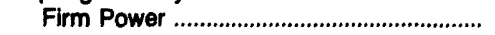 & - & -- & - & - & 781,674 & $18,644,704$ \\
\hline 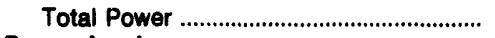 & - & - & - & - & 781,674 & $18,644,704$ \\
\hline $\begin{array}{l}\text { Penneylvanla } \\
\text { Chambersburg Borough of/Dec } 31\end{array}$ & & & & & & \\
\hline 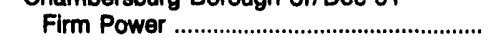 & - & - & - & - & 227,539 & $8,063,479$ \\
\hline 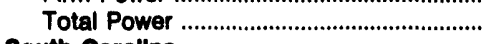 & - & $\cdots$ & - & -- & 227,539 & $8,063,479$ \\
\hline $\begin{array}{l}\text { South Carollna } \\
\text { Camden City of/Jun } 30\end{array}$ & & & & & & \\
\hline 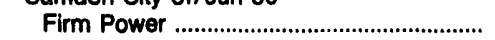 & - & - & - & -- & 151,412 & $8,822,285$ \\
\hline Nonfirm Power ............................................... & - & - & 2 & -- & & \\
\hline 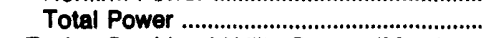 & - & - & 2 & - & 151,414 & $8,822,285$ \\
\hline Easley Combined Utility System/Mar 31 & & & & & & \\
\hline 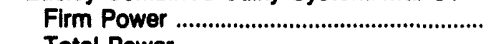 & - & - & 192,914 & $9,442,584$ & 208,543 & $9,880,187$ \\
\hline $\begin{array}{l}\text { Total Power ..... } \\
\text { Gaffney Board of Public Works/Mar } 31\end{array}$ & - & - & 192,914 & $9,442,584$ & 208,543 & $9,880,187$ \\
\hline 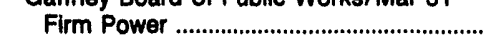 & - & - & 176,542 & $8,327,962$ & 189,215 & $8,682,795$ \\
\hline Total Power .......................................... & - & - & 176,542 & $8,327,962$ & 189,215 & $8,682,795$ \\
\hline Greenwood City of/Dec 31 & & & & & & \\
\hline 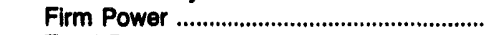 & - & - & - & - & 236,690 & $9,985,788$ \\
\hline 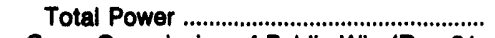 & - & - & - & - & 236,690 & $9,985,788$ \\
\hline Greer Commission of Public Wks/Dec 31 & & & & & & \\
\hline 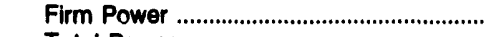 & - & - & 137,598 & $6,636,155$ & 155,924 & $7,134,309$ \\
\hline 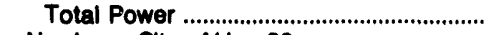 & - & - & 137,598 & $6,636,155$ & 155,924 & $7,134,300$ \\
\hline Newberny City of/Jun 30 & & & & & & \\
\hline 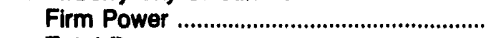 & - & - & 140,079 & $6,855,703$ & 141,558 & $6,971,041$ \\
\hline 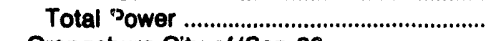 & - & - & 140,079 & $6,855,703$ & 141,558 & $6,971,041$ \\
\hline Orangeburg City of/Sep 30 & & & & & & \\
\hline 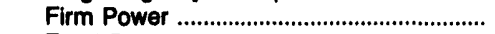 & - & - & - & - & 697,006 & $24,812,667$ \\
\hline 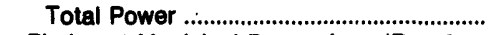 & -- & -- & - & -- & 697,006 & $24,812,667$ \\
\hline Piedmont Municipal Power Agny/Dec 31 & & & & & & \\
\hline 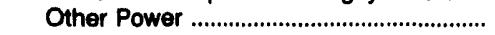 & - & - & 126,130 & $6,378,000$ & $1,119,330$ & $49,777,000$ \\
\hline 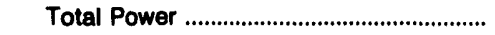 & - & - & 126,130 & $6,378,000$ & $1,119,330$ & $49,777,000$ \\
\hline
\end{tabular}

See notes and footnotes at end of table. 
Table 29. Electricity Purchases by Municipal Utilities, Fiscal Year 1992 (Continued)

\begin{tabular}{|c|c|c|c|c|c|c|}
\hline \multirow{3}{*}{$\begin{array}{l}\text { State / Utility I } \\
\text { Flecal Year Ending Date }\end{array}$} & \multicolumn{6}{|c|}{ Source of Electricity } \\
\hline & \multicolumn{2}{|c|}{ Investor-Owned } & \multicolumn{2}{|c|}{ Federal } & \multicolumn{2}{|c|}{$\begin{array}{l}\text { State and Other } \\
\text { Government }\end{array}$} \\
\hline & $\begin{array}{c}\text { Purchases } \\
\text { (thousand kWh) }\end{array}$ & $\begin{array}{c}\text { Cost } \\
\text { (dollars) }\end{array}$ & $\begin{array}{c}\text { Purchases } \\
\text { (thousand kWh) }\end{array}$ & $\begin{array}{c}\text { Cost } \\
\text { (dollars) }\end{array}$ & $\begin{array}{c}\text { Purchases } \\
\text { (thousand kWh) }\end{array}$ & $\begin{array}{c}\text { Cost } \\
\text { (dollars) }\end{array}$ \\
\hline \multicolumn{7}{|l|}{$\begin{array}{l}\text { South Carollina } \\
\text { Rock Hill City ol/Dec } 31\end{array}$} \\
\hline 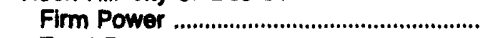 & - & - & 37,071 & $1,007,714$ & - & - \\
\hline 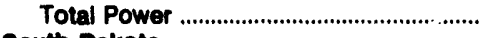 & - & - & 37,071 & $1,007,714$ & -- & - \\
\hline \multicolumn{7}{|l|}{$\begin{array}{l}\text { South Dakota } \\
\text { Brookings City of/Dec } 31\end{array}$} \\
\hline 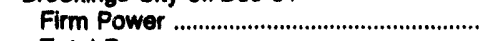 & - & - & 98,640 & $1,136,454$ & 74,874 & $2,711,140$ \\
\hline \multicolumn{6}{|l|}{ Plerre Municipal Electric/Dec 31} & $2,711,140$ \\
\hline 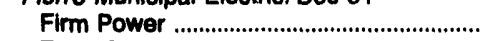 & - & - & 107,229 & $1,349,074$ & 41,530 & $1,577,014$ \\
\hline 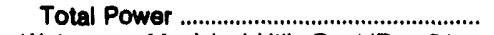 & - & - & 107,229 & $1,349,074$ & 41,530 & $1,577,014$ \\
\hline \multicolumn{7}{|l|}{ Watertown Municipal Utils Dept/Dec 31} \\
\hline 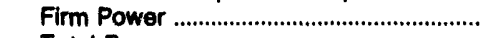 & - & - & 116,367 & $1,304,459$ & 84,652 & $3,149,557$ \\
\hline 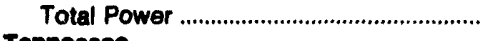 & - & - & 116,367 & $1,304,459$ & 84,652 & $3,149,557$ \\
\hline \multicolumn{7}{|l|}{$\begin{array}{l}\text { Tennesese } \\
\text { Alcos City of Utilities/Jun } 30\end{array}$} \\
\hline 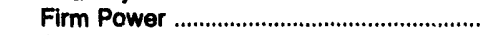 & - & - & 418,443 & $18,518,948$ & - & - \\
\hline \multirow{2}{*}{\multicolumn{7}{|c|}{ Athens Utilities Board/Jun 30}} \\
\hline & & & & & & \\
\hline 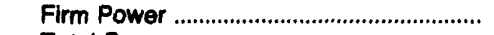 & - & - & 502,014 & $22,104,216$ & - & - \\
\hline \multirow{2}{*}{\multicolumn{7}{|c|}{ Benton County Bd of Pub Uti//Jun 30}} \\
\hline & - & $\cdots$ & 55,348 & $2,595,188$ & - & - \\
\hline 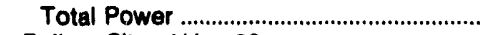 & - & - & 55,348 & $2,595,188$ & - & - \\
\hline \multicolumn{7}{|l|}{ Bolivar City of/Jun 30} \\
\hline 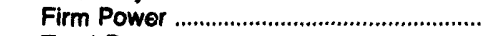 & -- & - & 234,592 & $10,626,696$ & - & - \\
\hline Total Power .................................................... & - & - & 234,592 & $10,626,696$ & -- & - \\
\hline \multicolumn{7}{|l|}{ Bristol City of/Jun 30} \\
\hline 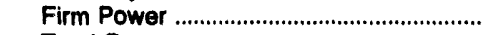 & - & -- & 760,766 & $33,480,086$ & -- & - \\
\hline $\begin{array}{l}\text { Total Power } \\
\text { Brownsville Citv of/Jun } 30\end{array}$ & -- & - & 760,766 & $33,480,086$ & -- & - \\
\hline Firm Power ........................................... & - & -. & 187,443 & $8,655,552$ & -- & -- \\
\hline Total Power ……………………………….. & - & -- & 187,443 & $8,655,552$ & - & - \\
\hline Carroll County/Jun 30 & & & & & & \\
\hline 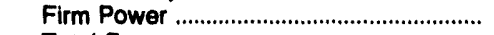 & - & -- & 371,270 & $16,337,891$ & - & - \\
\hline Total Power ..................................................... & - & - & 371,270 & $16,337,891$ & $\cdots$ & $-\infty$ \\
\hline C.iattanooga City of/Jun 30 & & & & & & \\
\hline 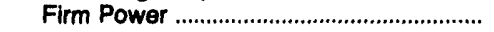 & - & - & $5,318,656$ & $230,268,725$ & -- & - \\
\hline 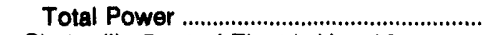 & - & -- & $5,318,656$ & $230,268,725$ & - & -- \\
\hline Clarksville Dept of Electric/Jun 30 & & & & & & \\
\hline 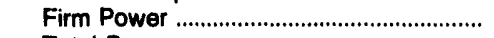 & - & - & 798,059 & $35,453,654$ & - & - \\
\hline Total Power & - & - & 798,059 & $35,453,654$ & - & - \\
\hline Cleveland City of/Jun 30 & & & & & & \\
\hline 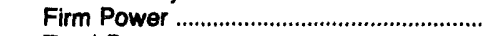 & - & - & 866,082 & $37,523,995$ & - & - \\
\hline 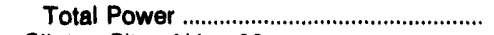 & - & - & 866,082 & $37,523,995$ & - & -- \\
\hline Clinton City of/Jun 30 & & & & & & \\
\hline Firm Power ..................................................... & - & 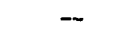 & 592,035 & $26,494,080$ & - & - \\
\hline 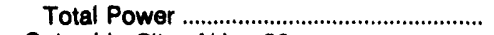 & - & - & 592,035 & $26,494,080$ & - & - \\
\hline Columbia City of/Jun 30 & & & & & & \\
\hline 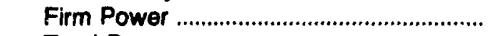 & - & - & 499,827 & $22,383,830$ & - & - \\
\hline Total Power & - & - & 499,827 & $22,383,830$ & - & - \\
\hline Cookeville City of/Jun 30 & & & & & & \\
\hline 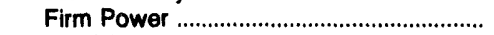 & - & - & 442,517 & $20,595,119$ & - & - \\
\hline Total Power ...................................................... & - & - & 442,517 & $20,595,119$ & - & -- \\
\hline Covington Electric System/Jun 30 & & & & & & \\
\hline Firm Power & - & - & 189,241 & $8,705,444$ & - & - \\
\hline Total Power .................................................... & - & -- & 189,241 & $8,705,444$ & - & - \\
\hline Dayton City of/Jun 30 & & & & & & \\
\hline 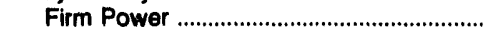 & - & - & 191,009 & $8,573,346$ & - & - \\
\hline Total Power & - & - & 191,009 & $8,573,346$ & - & - \\
\hline Dickson Electric Department/Jun 30 & & & & & & \\
\hline 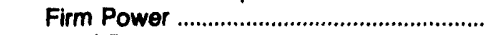 & - & - & 546,251 & $24,340,295$ & - & - \\
\hline 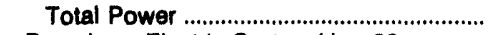 & - & -- & 546,251 & $24,340,295$ & - & - \\
\hline Dyersburg Electric System/Jun 30 & & & & & & \\
\hline 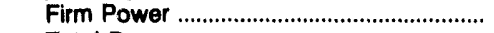 & - & -- & 571,902 & $22,876,350$ & - & - \\
\hline 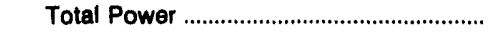 & - & -- & 571,902 & $22,876,350$ & - & - \\
\hline
\end{tabular}

See notes and footnotes at end of table. 
Table 29. Electricity Purchases by Municipal Utilitles, Flecal Year 1992 (Continued)

\begin{tabular}{|c|c|c|c|c|c|c|}
\hline \multirow{3}{*}{$\begin{array}{l}\text { State / Utillty / } \\
\text { Fiecal Year Ending Date }\end{array}$} & \multicolumn{6}{|c|}{ Source of Electricity } \\
\hline & \multicolumn{2}{|c|}{ Cooperative } & \multicolumn{2}{|c|}{ Other' } & \multicolumn{2}{|c|}{ Total } \\
\hline & $\begin{array}{c}\text { Purchases } \\
\text { (thousand kWh) }\end{array}$ & $\begin{array}{c}\text { Cost } \\
\text { (dollars) }\end{array}$ & $\begin{array}{c}\text { Purchases } \\
\text { (thousand kWh) }\end{array}$ & $\begin{array}{c}\text { Cost } \\
\text { (dollars) }\end{array}$ & $\begin{array}{c}\text { Purchases } \\
\text { (thousand kWh) }\end{array}$ & $\begin{array}{c}\text { Cost } \\
\text { (dollars) }\end{array}$ \\
\hline \multicolumn{7}{|l|}{$\begin{array}{l}\text { South Carollna } \\
\text { Rock Hill City of/Dec } 31\end{array}$} \\
\hline Firm Power .............................................. & - & -- & 458,951 & $22,459,223$ & 496,022 & $23,466,937$. \\
\hline & - & - & 458,951 & $22,459,223$ & 496,022 & $23,466,937$ \\
\hline \multicolumn{7}{|l|}{$\begin{array}{l}\text { South Dakota } \\
\text { Brookings City of/Dec } 31\end{array}$} \\
\hline 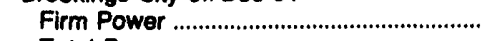 & - & - & - & - & 173,514 & $3,847,594$ \\
\hline \multicolumn{4}{|l|}{ Pierre Municipal Electric/Dec 31} & - & 173,514 & $3,847,594$ \\
\hline 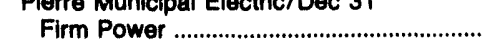 & - & -- & - & - & 148,759 & $2,926,088$ \\
\hline 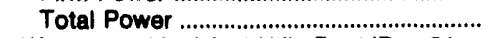 & - & - & - & -- & 148,759 & $2,926,088$ \\
\hline \multicolumn{7}{|l|}{ Watertown Municipal Utils Dept/Dec 31} \\
\hline 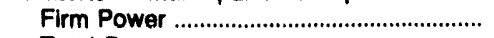 & - & - & -- & - & 201,019 & $\begin{array}{l}4,454,016 \\
4,454,016\end{array}$ \\
\hline $\begin{array}{l}\text { Total Power } \\
\text { Tonnesese }\end{array}$ & $\cdots$ & - & -- & -- & 201,019 & $4,454,016$ \\
\hline $\begin{array}{l}\text { Tonnosese } \\
\text { Alcoa City of Utilities/Jun } 30\end{array}$ & \\
\hline 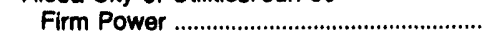 & - & - & - & - & 418,443 & $18,518,948$ \\
\hline 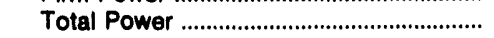 & -- & - & - & - & 418,443 & $18,518,848$ \\
\hline \multicolumn{7}{|l|}{ Athens Utillties Board/Jun 30} \\
\hline & - & -- & - & -- & 502,014 & $22,104,216$ \\
\hline & $22,104,216$ \\
\hline & -- & - & & & & \\
\hline 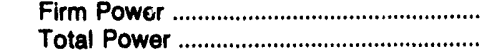 & - & $\overline{-}$ & $=$ & -- & $\begin{array}{l}55,348 \\
55,348\end{array}$ & $\begin{array}{l}2,595,188 \\
2,595,188\end{array}$ \\
\hline \multicolumn{7}{|l|}{ Bolivar City of/Jun 30} \\
\hline 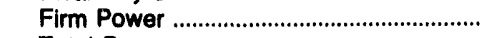 & - & -- & - & -- & 234,592 & $10,626,696$ \\
\hline 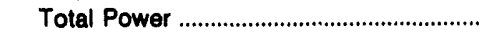 & -- & -- & - & -- & 234,592 & $10,626,696$ \\
\hline Bristol City of/Jun 30 & & & & & & \\
\hline 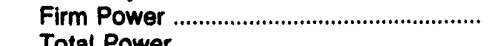 & - & -- & - & -- & 760,766 & $\begin{array}{l}33,480,086 \\
33,480,086\end{array}$ \\
\hline $\begin{array}{l}\text { Total Power } \\
\text { Brownsville City of /Jun } 30\end{array}$ & - & - & - & - & 760,766 & \\
\hline 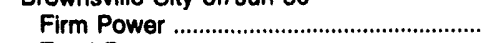 & - & - & -- & - & 187,443 & $8,655,552$ \\
\hline 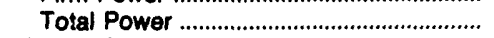 & -- & - & - & -- & 187,443 & $8,655,552$ \\
\hline Carroll County/Jun 30 & & & & & & \\
\hline 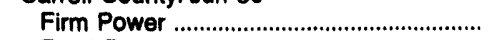 & - & - & -- & - & 371,270 & $16,337,891$ \\
\hline 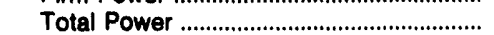 & - & -- & - & -- & 371,270 & $16,337,891$ \\
\hline Chattanooga City of, Jun 30 & & & & & & \\
\hline 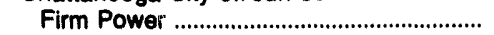 & - & - & - & -- & $5,318,656$ & $230,288,725$ \\
\hline 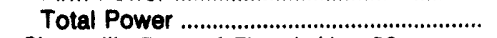 & - & -- & -- & -- & $5,318,656$ & $230,268,725$ \\
\hline Clarksville Dept of Electric/Jun 30 & & & & & & \\
\hline 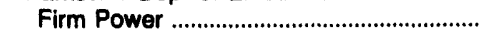 & - & - & - & - & 798,059 & $35,453,654$ \\
\hline 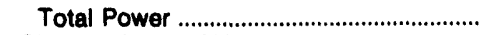 & -- & -- & - & -- & 798,059 & $35,453,654$ \\
\hline Cleveland City of/Jun 30 & & & & & & \\
\hline 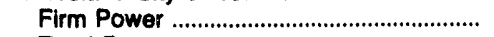 & -- & -- & -- & -- & 866,082 & $37,523,995$ \\
\hline Total Power ......................................... & -- & -. & -- & -- & 866,082 & $37,523,995$ \\
\hline Clinton City of/Jun 30 & & & & & & \\
\hline 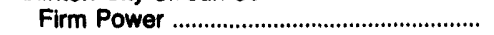 & -- & -- & - & -- & 592,035 & $26,494,080$ \\
\hline 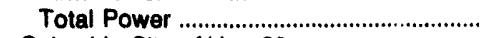 & - & - & - & -- & 592,035 & $26,494,080$ \\
\hline Columbia City of/Jun 30 & & & & & & \\
\hline 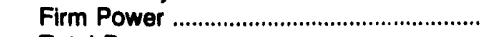 & - & - & -- & -- & 499,827 & $22,383,830$ \\
\hline 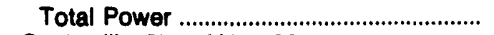 & - & -- & -- & - & 499,827 & $22,383,830$ \\
\hline Cookeville City of/Jun 30 & & & & & & \\
\hline 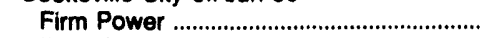 & - & - & - & - & 442,517 & $20,595,119$ \\
\hline Total Power ................................................ & - & - & -- & -- & 442,517 & $20,595,119$ \\
\hline Covington Electric System/Jun 30 & & & & & & \\
\hline 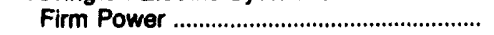 & - & - & - & -- & 189,241 & $8,705,444$ \\
\hline 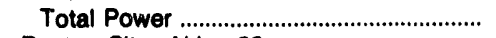 & - & - & - & - & 189,241 & $8,705,444$ \\
\hline Dayton City of/Jun 30 & & & & & & \\
\hline 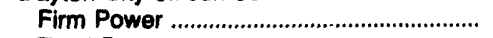 & - & -- & - & - & 191,009 & $8,573,346$ \\
\hline 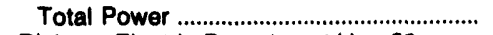 & - & - & - & - & 191,009 & $8,573,346$ \\
\hline Dickson Electric Department/Jun 30 & & & & & & \\
\hline 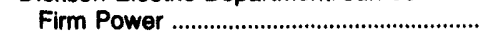 & -- & - & - & - & 546,251 & $24,340,295$ \\
\hline 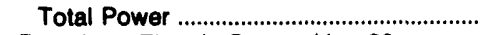 & - & - & - & - & 546,251 & $24,340,295$ \\
\hline Dyersburg Electric System/Jun 30 & & & & & & \\
\hline 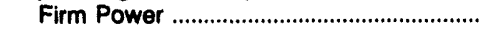 & - & - & - & - & 571,902 & $22,876,350$ \\
\hline 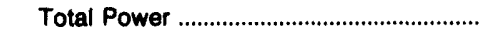 & - & - & - & - & 571,902 & $22,876,350$ \\
\hline
\end{tabular}

See notes and footnotes at end of table. 
Table 29. Electricity Purchases by Municipal Utilitles, Fiscal Year 1992 (Continued)

\begin{tabular}{|c|c|c|c|c|c|c|}
\hline \multirow{3}{*}{$\begin{array}{l}\text { State / Utility / } \\
\text { Flocal Year Ending Date }\end{array}$} & \multicolumn{6}{|c|}{ Source of Electricity } \\
\hline & \multicolumn{2}{|c|}{ Investor-Owned } & \multicolumn{2}{|c|}{ Federal } & \multicolumn{2}{|c|}{$\begin{array}{l}\text { State and Other } \\
\text { Government }\end{array}$} \\
\hline & $\begin{array}{l}\text { Purchases } \\
\text { (thousand kWh) }\end{array}$ & $\begin{array}{c}\text { Cost } \\
\text { (dollars) }\end{array}$ & $\begin{array}{c}\text { Purchases } \\
\text { (thoucand } k W h \text { ) }\end{array}$ & $\begin{array}{c}\text { Cost } \\
\text { (dollars) }\end{array}$ & $\begin{array}{c}\text { Purchases } \\
\text { (thousand kWh) }\end{array}$ & $\begin{array}{c}\text { Cost } \\
\text { (dollars) }\end{array}$ \\
\hline \multicolumn{7}{|l|}{$\begin{array}{l}\text { Tennessee } \\
\text { Elizabethton Electric System/Jun } 30\end{array}$} \\
\hline $\begin{array}{l}\text { Firm Power } \\
\text { Total Power } \\
\text { Enwin City of/Jun } 30\end{array}$ & - & - & $\begin{array}{l}493,331 \\
493,331\end{array}$ & $\begin{array}{l}21,841,498 \\
21,841,498\end{array}$ & - & - \\
\hline 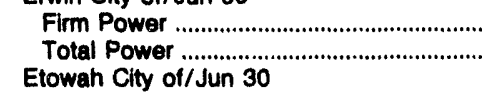 & - & - & $\begin{array}{l}204,090 \\
204,090\end{array}$ & $\begin{array}{l}9,296,831 \\
9,296,831\end{array}$ & - & - \\
\hline 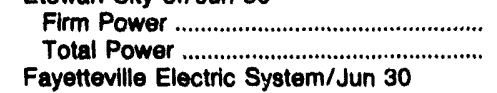 & - & - & $\begin{array}{l}139,024 \\
139,024\end{array}$ & $\begin{array}{l}6,098,344 \\
6,098,344\end{array}$ & - & - \\
\hline 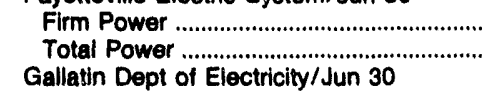 & - & - & $\begin{array}{l}352,020 \\
352,020\end{array}$ & $\begin{array}{l}15,625,127 \\
15,625,127\end{array}$ & - & - \\
\hline 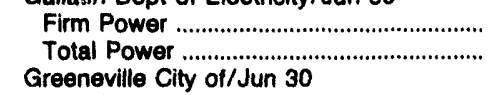 & - & - & $\begin{array}{l}412,411 \\
412,411\end{array}$ & $\begin{array}{l}18,488,652 \\
18,488,652\end{array}$ & -- & - \\
\hline 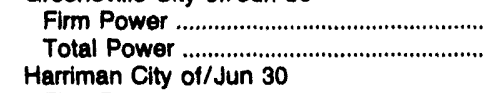 & - & - & $\begin{array}{l}856,100 \\
856,100\end{array}$ & $\begin{array}{l}37,546,127 \\
37,546,127\end{array}$ & - & - \\
\hline 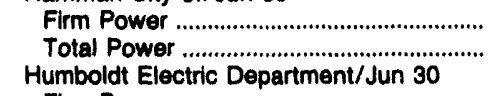 & - & - & $\begin{array}{l}214,947 \\
214,947\end{array}$ & $\begin{array}{l}9,898,888 \\
9,898,888\end{array}$ & - & - \\
\hline 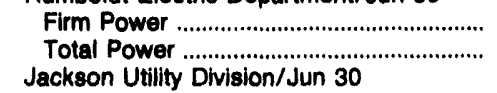 & - & - & $\begin{array}{l}204,945 \\
204,945\end{array}$ & $\begin{array}{l}9,123,402 \\
9,123,402\end{array}$ & - & - \\
\hline 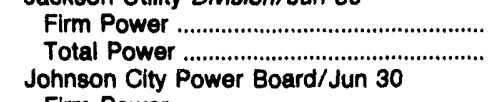 & - & - & $\begin{array}{l}1,115,881 \\
1,115,881\end{array}$ & $\begin{array}{l}48,778,416 \\
48,778,416\end{array}$ & - & - \\
\hline Firm Power & - & - & $\begin{array}{l}1,488,631 \\
1,488,631\end{array}$ & $\begin{array}{l}66,674,557 \\
66,674,557\end{array}$ & - & - \\
\hline Knoxville City of/Jun 30 & & & & & & \\
\hline $\begin{array}{l}\text { Firm Power } \\
\text { Total Power } \\
\text { Lawrenceburg City of/Jun } 30\end{array}$ & - & - & $\begin{array}{l}4,393,079 \\
4,393,079\end{array}$ & $\begin{array}{l}201,972,847 \\
201,972,847\end{array}$ & - & - \\
\hline 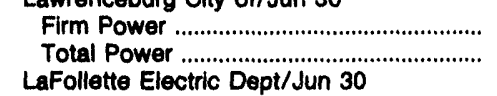 & - & - & $\begin{array}{l}470,419 \\
470,419\end{array}$ & $\begin{array}{l}20,599,719 \\
20,599,719\end{array}$ & - & - \\
\hline 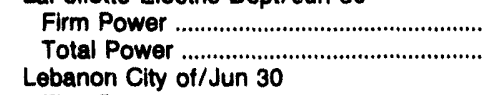 & - & - & $\begin{array}{l}327,446 \\
327,446\end{array}$ & $\begin{array}{l}14,541,253 \\
14,541,253\end{array}$ & - & - \\
\hline 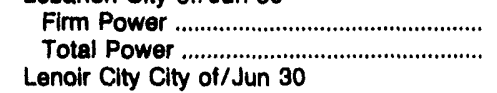 & - & - & $\begin{array}{l}318,631 \\
318,631\end{array}$ & $\begin{array}{l}14,528,713 \\
14,528,713\end{array}$ & - & - \\
\hline 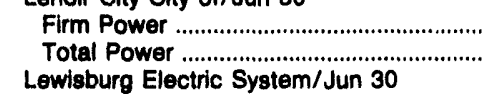 & - & - & $\begin{array}{l}984,481 \\
984,481\end{array}$ & $\begin{array}{l}44,925,261 \\
44,925,261\end{array}$ & - & - \\
\hline 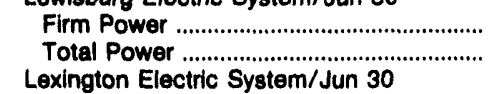 & - & - & $\begin{array}{l}274,731 \\
274,731\end{array}$ & $\begin{array}{l}12,500,766 \\
12,500,766\end{array}$ & - & - \\
\hline $\begin{array}{l}\text { Firm Power } \\
\text { Total Power } \\
\text { Loudon Utilities Board/Jun } 30\end{array}$ & - & - & $\begin{array}{l}364,336 \\
364,336\end{array}$ & $\begin{array}{l}15,711,840 \\
15,711,840\end{array}$ & - & - \\
\hline 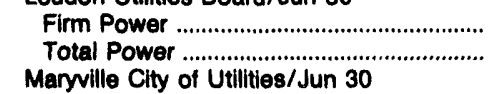 & - & - & $\begin{array}{l}254,233 \\
254,233\end{array}$ & $\begin{array}{l}10,747,609 \\
10,747,609\end{array}$ & - & - \\
\hline $\begin{array}{l}\text { Firm Power } \\
\text { Total Power } \\
\text { McMinnville Electric System/Jun } 30\end{array}$ & - & - & $\begin{array}{l}486,677 \\
486,677\end{array}$ & $\begin{array}{l}20,849,846 \\
20,849,846\end{array}$ & - & - \\
\hline $\begin{array}{l}\text { Firm Power } \\
\text { Total Power } \\
\text { Memphis City of/Jun } 30\end{array}$ & - & - & $\begin{array}{l}209,858 \\
209,858\end{array}$ & $\begin{array}{l}9,309,059 \\
9,309,059\end{array}$ & - & - \\
\hline $\begin{array}{l}\text { Firm Power } \\
\text { Total Power } \\
\text { Morristown Power System/Jun } 30\end{array}$ & - & - & $\begin{array}{l}11,818,703 \\
11,818,703\end{array}$ & $\begin{array}{l}498,793,712 \\
498,793,712\end{array}$ & - & - \\
\hline Firm Power & - & - & $\begin{array}{l}596,111 \\
596,111\end{array}$ & $\begin{array}{l}27,008,874 \\
27,008,874\end{array}$ & - & $\overline{-}$ \\
\hline
\end{tabular}

See notes and footnotes at end of table. 
Table 29. Electriclty Purchases by Municipal Utilities, Flscal Year 1992 (Continued)

\begin{tabular}{|c|c|c|c|c|c|c|}
\hline \multirow{3}{*}{$\begin{array}{l}\text { State / Uttlity / } \\
\text { Flecal Yoar Ending Date }\end{array}$} & \multicolumn{6}{|c|}{ Source of Electricity } \\
\hline & \multicolumn{2}{|c|}{ Cooperative } & \multicolumn{2}{|c|}{ Other' } & \multicolumn{2}{|c|}{ Total } \\
\hline & $\begin{array}{c}\text { Purchases } \\
\text { (thousand kWh) }\end{array}$ & $\begin{array}{c}\text { Cost } \\
\text { (dollars) }\end{array}$ & $\begin{array}{c}\text { Purchases } \\
\text { (thousand kWh) }\end{array}$ & $\begin{array}{c}\text { Cost } \\
\text { (dollars) }\end{array}$ & $\begin{array}{c}\text { Purchases } \\
\text { (thousand kWh) }\end{array}$ & $\begin{array}{c}\text { Cost } \\
\text { (dollars) }\end{array}$ \\
\hline \multicolumn{7}{|l|}{$\begin{array}{l}\text { Tenneesee } \\
\text { Ellzabethton Electric System/Jun } 30\end{array}$} \\
\hline 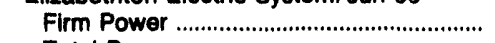 & - & - & -- & - & 493,331 & $21,841,498$ \\
\hline $\begin{array}{l}\text { TC ial Power .............................................. } \\
\text { Enwin City of/Jun } 30\end{array}$ & \multicolumn{6}{|c|}{ Erwin City of/Jun 30} \\
\hline 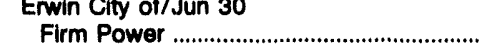 & -- & - & -- & - & 204,090 & $9,296,831$ \\
\hline & $9,296,831$ \\
\hline 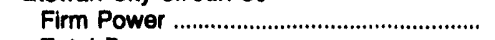 & - & - & -. & -- & 139,024 & $6,098,344$ \\
\hline \multicolumn{7}{|l|}{ Fayetteville Electric System/Jun 30} \\
\hline Firm Power & - & -- & -- & -- & 352,020 & $15,625,127$ \\
\hline Total Power ... & - & - & - & - & 352,020 & $15,625,127$ \\
\hline 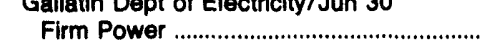 & - & -- & - & - & 412,411 & $18,488,652$ \\
\hline $\begin{array}{l}\text { Total Power } \\
\text { Greeneville City of/Jun } 30\end{array}$ & - & - & - & - & 412,411 & $18,488,652$ \\
\hline 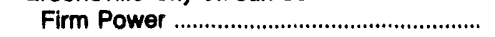 & - & -- & - & - & 856,100 & $37,546,127$ \\
\hline \multicolumn{7}{|l|}{ Harriman City of/Jun 30} \\
\hline 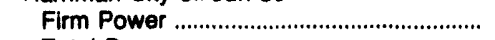 & - & -- & - & -- & 214,947 & $9,898,888$ \\
\hline 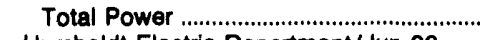 & -- & -- & - & - & 214,947 & $9,808,888$ \\
\hline \multicolumn{7}{|l|}{ Humboldt Electric Department/Jun 30} \\
\hline 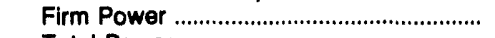 & - & -- & - & -- & 204,945 & $9,123,402$ \\
\hline $\begin{array}{l}\text { Total Power . } \\
\text { Jackson Utility Division/Jun } 30\end{array}$ & - & - & - & - & 204,945 & $9,123,402$ \\
\hline 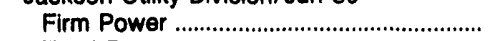 & - & _- & - & -- & $1,115,881$ & $48,778,416$ \\
\hline \multirow{2}{*}{\multicolumn{7}{|c|}{ Johnson City Power Board/Jun 30}} \\
\hline & & & & & & \\
\hline 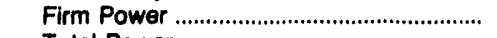 & -- & - & -- & - & $1,488,631$ & $66,674,557$ \\
\hline Total Power & - & - & - & -- & $1,488,631$ & $66,674,557$ \\
\hline \multicolumn{7}{|l|}{ Knoxville City of/Jun 30} \\
\hline 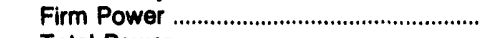 & -- & - & 7,568 & 131,054 & $4,400,647$ & $202,103,901$ \\
\hline 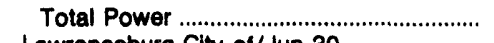 & - & - & 7,568 & 131,054 & $4,400,647$ & $202,103,801$ \\
\hline Lawrenceburg City of/Jun 30 & & & & & & \\
\hline 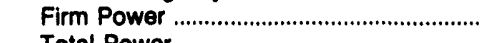 & - & - & -- & -- & 470,419 & $20,599,710$ \\
\hline $\begin{array}{l}\text { Total Power } \\
\text { LaFollette Electric Dept/Jun } 30\end{array}$ & - & - & - & -- & 470,419 & $20,598,719$ \\
\hline 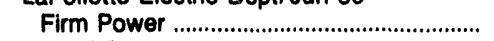 & - & - & -- & - & 327,446 & $14,541,253$ \\
\hline 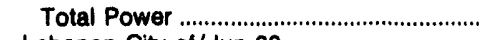 & - & - & - & - & 327,446 & $14,541,253$ \\
\hline Lebanon City of/Jun 30 & & & & & & \\
\hline 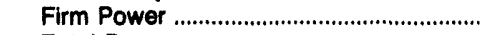 & - & - & - & - & 318,631 & $14,528,713$ \\
\hline 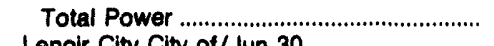 & - & - & - & - & 318,631 & $14,528,713$ \\
\hline $\begin{array}{l}\text { Lenoir City City of/Jun } 30 \\
\text { Firm Power }\end{array}$ & -- & - & - & - & 984,481 & $44,825,261$ \\
\hline 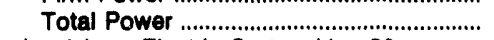 & -- & - & - & - & 984,481 & $44,925,261$ \\
\hline Lewisburg Electric System/Jun 30 & & & & & & \\
\hline 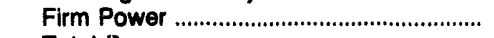 & -- & -- & -- & - & 274,731 & $12,500,786$ \\
\hline 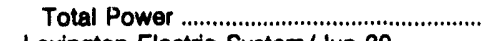 & - & - & - & - & 274,731 & $12,500,766$ \\
\hline Lexington Electric System/Jun 30 & & & & & & \\
\hline 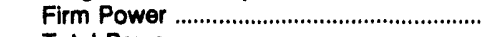 & - & - & - & - & 364,336 & $15,711,840$ \\
\hline 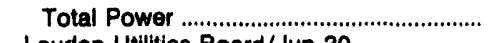 & - & - & - & - & 364,336 & $15,711,840$ \\
\hline Loudon Utilities Board/Jun 30 & & & & & & \\
\hline 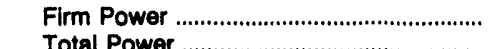 & $\overline{-}$ & $\overline{-}$ & $\overline{-}$ & $\overline{-}$ & $\begin{array}{l}254,233 \\
254,233\end{array}$ & $\begin{array}{l}10,747,609 \\
10,747,609\end{array}$ \\
\hline 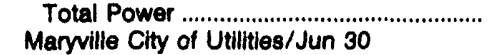 & - & - & & - & 254,233 & $10,747,609$ \\
\hline 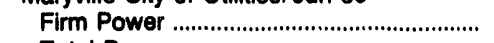 & - & - & - & - & 486,677 & $20,849,846$ \\
\hline 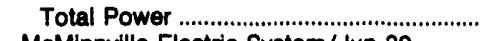 & - & - & -- & - & 486,677 & $20,849,846$ \\
\hline McMinnville Electric System/Jun 30 & & & & & & \\
\hline 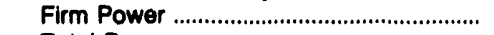 & - & - & - & - & 209,858 & $9,309,059$ \\
\hline 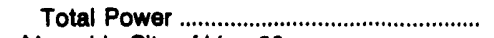 & - & - & -- & -- & 209,858 & $9,309,059$ \\
\hline Memphis City of/Jun 30 & & & & & & \\
\hline 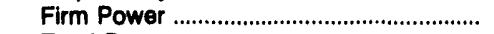 & - & - & - & - & $11,818,703$ & $498,793,712$ \\
\hline 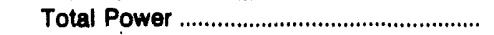 & - & - & - & -- & $11,818,703$ & $498,793,712$ \\
\hline Morristown Power System/Jun 30 & & & & & & \\
\hline 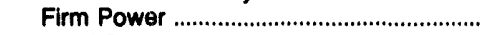 & - & -- & - & -- & 596,111 & $27,008,874$ \\
\hline 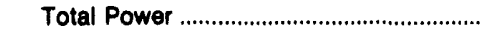 & - & -- & - & - & 596,111 & $27,008,874$ \\
\hline
\end{tabular}

See notes and footnotes at end of table. 
Table 29. Electriclty Purchases by Municịaal Utilitles, Flscal Year 1992 (Continued)

\begin{tabular}{|c|c|c|c|c|c|c|}
\hline \multirow{3}{*}{$\begin{array}{l}\text { State / Utilty / } \\
\text { Fleal Year Ending Date }\end{array}$} & \multicolumn{6}{|c|}{ Source of Electricity } \\
\hline & \multicolumn{2}{|c|}{ Investor-Owned } & \multicolumn{2}{|c|}{ Federal } & \multicolumn{2}{|c|}{$\begin{array}{l}\text { State and Other } \\
\text { Government }\end{array}$} \\
\hline & $\begin{array}{c}\text { Purchases } \\
\text { (thousand kWh) }\end{array}$ & $\begin{array}{c}\text { Cost } \\
\text { (dollars) }\end{array}$ & $\begin{array}{c}\text { Purchases } \\
\text { (thousand kWh) }\end{array}$ & $\begin{array}{c}\text { Cost } \\
\text { (dollars) }\end{array}$ & $\begin{array}{c}\text { Purchases } \\
\text { (thousand kWh) }\end{array}$ & $\begin{array}{c}\text { Cost } \\
\text { (dollars) }\end{array}$ \\
\hline \multicolumn{7}{|l|}{ Tennesese } \\
\hline 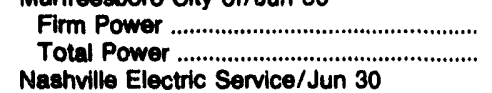 & $\overline{-}$ & $\overline{-}$ & $\begin{array}{l}811,736 \\
811,736\end{array}$ & $\begin{array}{l}35,693,618 \\
35,693,618\end{array}$ & - & $-\overline{-}$ \\
\hline 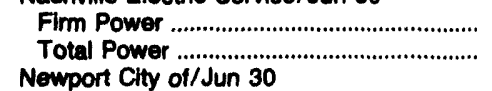 & -- & - & $\begin{array}{l}10,176,010 \\
10,176,010\end{array}$ & $\begin{array}{l}445,923,236 \\
445,923,236\end{array}$ & - & - \\
\hline 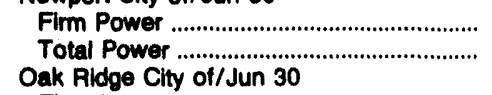 & $\overline{-}$ & - & $\begin{array}{l}421,066 \\
421,066\end{array}$ & $\begin{array}{l}18,345,154 \\
18,345,154\end{array}$ & - & - \\
\hline $\begin{array}{l}\text { Firm Power } \\
\text { Total Power } \\
\text { Okolona Electric Department/Dec } 31\end{array}$ & - & - & $\begin{array}{l}438,613 \\
438,613\end{array}$ & $\begin{array}{l}20,012,141 \\
20,012,141\end{array}$ & - & - \\
\hline 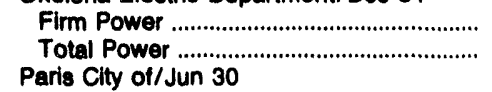 & - & - & $\begin{array}{l}81,662 \\
81,662\end{array}$ & $\begin{array}{l}3,699,635 \\
3,699,635\end{array}$ & - & - \\
\hline 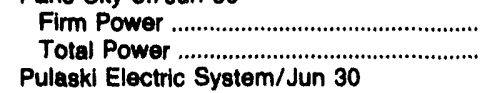 & - & - & $\begin{array}{l}412,982 \\
412,982\end{array}$ & $\begin{array}{l}18,591,100 \\
18,591,100\end{array}$ & $\overline{-}$ & - \\
\hline 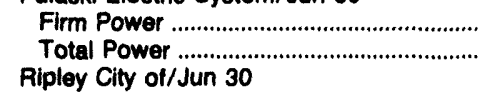 & - & - & $\begin{array}{l}390,643 \\
390,643\end{array}$ & $\begin{array}{l}16,492,086 \\
16,492,086\end{array}$ & - & - \\
\hline 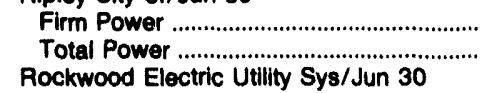 & - & - & $\begin{array}{l}253,117 \\
253,117\end{array}$ & $\begin{array}{l}11,138,702 \\
11,138,702\end{array}$ & - & - \\
\hline 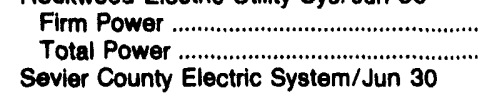 & - & - & $\begin{array}{l}276,945 \\
276,945\end{array}$ & $\begin{array}{l}12,473,391 \\
12,473,391\end{array}$ & $\overline{-}$ & - \\
\hline 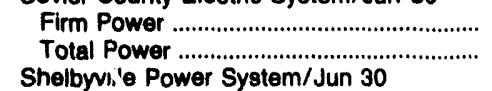 & - & - & $\begin{array}{l}833,612 \\
833,612\end{array}$ & $\begin{array}{l}37,583,286 \\
37,583,286\end{array}$ & - & - \\
\hline 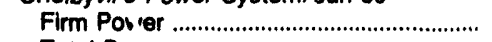 & - & - & 303,390 & $14,090,430$ & - & - \\
\hline $\begin{array}{l}\text { Total Power ................................................. } \\
\text { Springfield City }: \text { Jun } 30\end{array}$ & - & -- & 303,390 & $14,090,430$ & - & -- \\
\hline 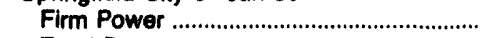 & - & -- & 189,622 & $8,492,457$ & -- & - \\
\hline $\begin{array}{l}\text { Total Power ...................... } \\
\text { Swoetwater Utilities Board/Jun } 30\end{array}$ & - & - & 189,622 & $8,492,457$ & -- & - \\
\hline 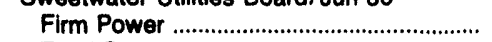 & - & - & 172,813 & $7,924,967$ & - & - \\
\hline $\begin{array}{l}\text { Total Power ........................................ } \\
\text { Tullahoma Power System/Ju }\end{array}$ & - & - & 172,813 & $7,924,967$ & - & -- \\
\hline 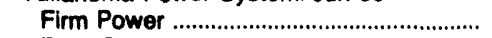 & - & -- & 200,000 & $12,024,757$ & - & - \\
\hline $\begin{array}{l}\text { Total Power ...................... } \\
\text { Union City Electric System/Jun } 30\end{array}$ & -- & - & 200,000 & $12,024,757$ & - & - \\
\hline 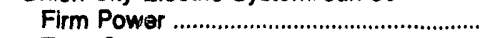 & - & - & 339,089 & $13,079,590$ & - & -- \\
\hline $\begin{array}{l}\text { Total Power .................................. } \\
\text { Weakley County Mun Elec Sys/Jun }\end{array}$ & - & - & 339,089 & $13,079,590$ & -- & -- \\
\hline 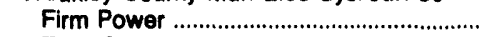 & - & - & 456,136 & $20,443,164$ & - & - \\
\hline 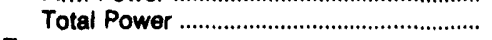 & - & - & 456,136 & $20,443,164$ & - & - \\
\hline $\begin{array}{l}\text { Toxas } \\
\text { Austin City of/Sep } 30\end{array}$ & & & & & & \\
\hline $\begin{array}{l}\text { Nontirm Power } \\
\text { Total Power } \\
\text { Brenham City of/Sep } 30\end{array}$ & $\begin{array}{l}24,887 \\
24,887\end{array}$ & $\begin{array}{l}472,226 \\
472,226\end{array}$ & - & - & $\begin{array}{l}33,707 \\
33,707\end{array}$ & $\begin{array}{l}562,725 \\
562,725\end{array}$ \\
\hline 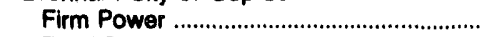 & - & - & - & - & 241,947 & $9,615,007$ \\
\hline $\begin{array}{l}\text { Total Power ........................................... } \\
\text { Brownsville City of/Sep }\end{array}$ & -- & - & -- & - & 241,947 & $9,615,007$ \\
\hline 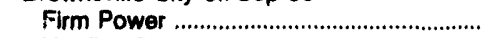 & 99,711 & $4,469,819$ & - & - & -- & - \\
\hline Nonfirm Power ................................................. & 77,304 & $1,033,468$ & - & - & 12,058 & 209,339 \\
\hline $\begin{array}{l}\text { Total Power ........................................ } \\
\text { Bryan Clty of Sep }\end{array}$ & 177,015 & $5,503,287$ & - & - & 12,058 & 209,339 \\
\hline 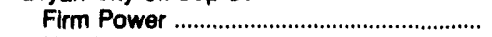 & - & - & - & - & - & - \\
\hline 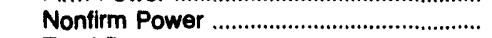 & - & - & -- & - & - & - \\
\hline $\begin{array}{l}\text { Total Power ........... } \\
\text { College Station City of/Sep } 30\end{array}$ & - & - & -- & - & -- & -- \\
\hline 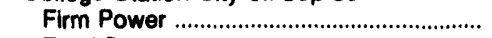 & 131,722 & $6,170,253$ & - & - & - & - \\
\hline 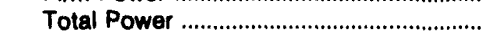 & 131,722 & $6,170,253$ & - & - & - & - \\
\hline
\end{tabular}

See notes and footnotes at end of table. 
Table 29. Electricity Purchases by Municipal Utillies, Fiscal Year 1992 (Continued)

\begin{tabular}{|c|c|c|c|c|c|c|}
\hline \multirow{3}{*}{$\begin{array}{l}\text { State / Utility / } \\
\text { Flecal Year Ending Date }\end{array}$} & \multicolumn{6}{|c|}{ Source of Electricity } \\
\hline & \multicolumn{2}{|c|}{ Cooperative } & \multicolumn{2}{|c|}{ Other' } & \multicolumn{2}{|c|}{ Total } \\
\hline & $\begin{array}{c}\text { Purchases } \\
\text { (thousand kWh) }\end{array}$ & $\begin{array}{c}\text { Cost } \\
\text { (dollars) }\end{array}$ & $\begin{array}{c}\text { Purchases } \\
\text { (thousand kWh) }\end{array}$ & $\begin{array}{c}\text { Cost } \\
\text { (dollars) }\end{array}$ & $\begin{array}{c}\text { Purchases } \\
\text { (thousand kWh) }\end{array}$ & $\begin{array}{c}\text { Cost } \\
\text { (dollars) }\end{array}$ \\
\hline \multicolumn{7}{|l|}{$\begin{array}{l}\text { Tennesese } \\
\text { Murfreesboro City of/Jun } 30\end{array}$} \\
\hline Firm Power & - & - & - & $\cdots$ & 811,736 & $35,693,618$ \\
\hline $\begin{array}{l}\text { Total Power } \\
\text { Nashville Electric Service/Jun } 30\end{array}$ & - & - & - & - & 811,736 & $35,693,618$ \\
\hline \multicolumn{3}{|l|}{ Nashville Electric Service/Jun 30} & - & -- & & \\
\hline 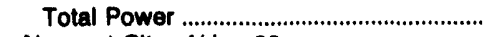 & - & - & - & - & $10,176,010$ & $445,923,236$ \\
\hline \multicolumn{7}{|l|}{ Newport City of/Jun 30} \\
\hline $\begin{array}{l}\text { Firm Power } \\
\text { Total Power }\end{array}$ & - & -- & -- & - & 421,066 & $18,345,154$ \\
\hline \multicolumn{7}{|l|}{ Oak Ridge City of/Jun 30} \\
\hline 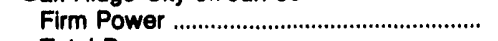 & - & - & - & - & 438,613 & $20,012,141$ \\
\hline Total Power & - & - & -- & - & 438,613 & $20,012,141$ \\
\hline \multicolumn{7}{|l|}{ Okolona Electric Department/Dec 31} \\
\hline 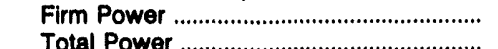 & - & -- & -- & - & 81,662 & $\begin{array}{l}3,699,635 \\
3699935\end{array}$ \\
\hline \multicolumn{7}{|l|}{$\begin{array}{l}\text { Total Power } \\
\text { Paris City of/Jun } 30\end{array}$} \\
\hline 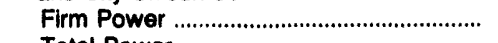 & - & - & - & - & 412,782 & $18,591,100$ \\
\hline \multicolumn{7}{|l|}{ Pulaski Electric System/Jun 30} \\
\hline 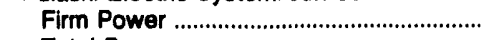 & - & - & -- & -- & 390,643 & $16,492,086$ \\
\hline 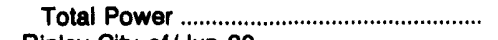 & - & - & - & - & 390,643 & $16,492,086$ \\
\hline \multicolumn{7}{|l|}{ Ripley City ot/Jun 30} \\
\hline 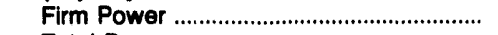 & - & - & -- & - & 253,117 & $11,138,702$ \\
\hline & - & - & 253,117 & $14,138,702$ \\
\hline 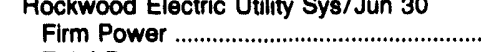 & - & - & 787 & 46,544 & 277,732 & $12,519,935$ \\
\hline 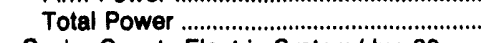 & - & - & 787 & 46,544 & 277,732 & $12,519,935$ \\
\hline Sevier County Electric System/Jun 30 & & & & & & \\
\hline 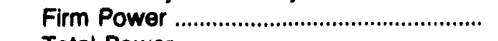 & -- & - & 257 & 8,826 & 833,869 & $37,592,112$ \\
\hline 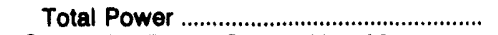 & -- & -- & 257 & 8,826 & 833,869 & $37,592,112$ \\
\hline Shelbyville Power System/Jun 30 & & & & & & \\
\hline Firm Power & - & - & - & -- & 303,390 & $14,090,430$ \\
\hline 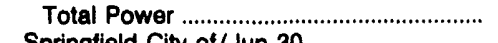 & - & - & - & - & 303,390 & $14,090,430$ \\
\hline Springtield City of/Jun 30 & & & & & & \\
\hline 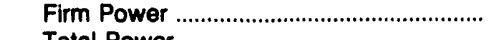 & - & -- & -- & -- & 189,622 & $8,492,457$ \\
\hline $\begin{array}{l}\text { Total Power } \\
\text { Sweetwater Utilities Board/Jun } 30\end{array}$ & - & -- & - & - & 189,622 & $8,492,457$ \\
\hline 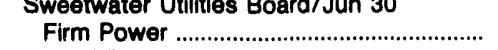 & -- & -- & - & -- & 172,813 & $7,924,967$ \\
\hline 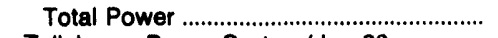 & - & -- & - & - & 172,813 & $7,924,967$ \\
\hline Tullahoma Power System/Jun 30 & & & & & & \\
\hline 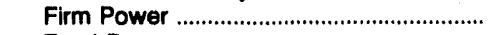 & -- & - & - & -- & 200,000 & $12,024,757$ \\
\hline 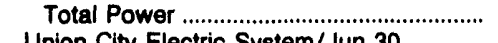 & - & - & -- & - & 200,000 & $12,024,757$ \\
\hline Union City Electric System/Jun 30 & & & & & & \\
\hline 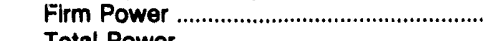 & - & - & - & - & 339,089 & $13,079,590$ \\
\hline 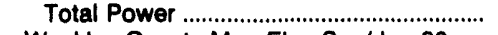 & - & - & - & -- & 339,089 & $13,079,590$ \\
\hline Weakley County Mun Elec Sys/Jun 30 & & & & & & \\
\hline 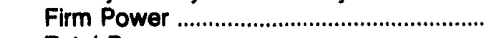 & - & -- & -- & -- & 456,136 & $20,443,164$ \\
\hline 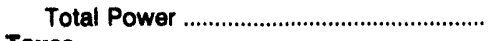 & - & -- & - & -- & 456,136 & $20,443,164$ \\
\hline Toxas & & & & & & \\
\hline Austin City of/Sep 30 & & & & & & \\
\hline 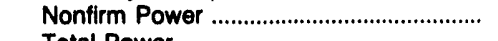 & 4,803 & 65,037 & 1,382 & 23,600 & 64,779 & $1,123,588$ \\
\hline 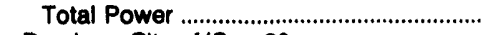 & 4,803 & 65,037 & 1,382 & 23,600 & 64,779 & $1,123,588$ \\
\hline Brenham City of $/$ Sep 30 & & & & & & \\
\hline Firm Power & - & - & -- & - & 241,947 & $9,615,007$ \\
\hline Total Power & - & - & - & - & 241,947 & $9,615,007$ \\
\hline Brownsville City of/Sep 30 & & & & & & \\
\hline 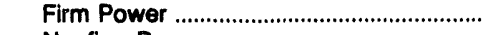 & -- & -- & -- & - & 99,711 & $4,469,819$ \\
\hline 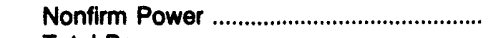 & 3,034 & 38,199 & 1,277 & 20,256 & 93,673 & $1,301,262$ \\
\hline 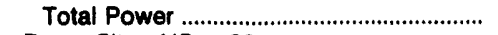 & 3,034 & 38,199 & 1,277 & 20,256 & 193,384 & $5,771,081$ \\
\hline Bryan City of/Sep 30 & & & & & & \\
\hline 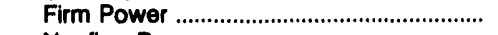 & - & - & 564,079 & $28,377,237$ & 564,079 & $28,377,237$ \\
\hline Nonfirm Power & - & -- & 16,910 & 322,762 & 16,910 & 322,762 \\
\hline 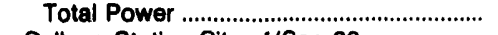 & - & - & 580,989 & $28,699,899$ & 580,989 & $28,699,999$ \\
\hline College Station City of/Sep 30 & & & & & & \\
\hline Firm Power & - & -- & 292,963 & $12,308,108$ & 424,685 & $18,478,361$ \\
\hline 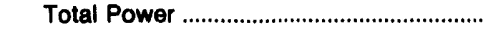 & - & - & 292,963 & $12,308,108$ & 424,685 & $18,478,361$ \\
\hline
\end{tabular}

See notes and footnotes at end of table. 
Table 29. Electricity Purchases by Municipal Utilities, Fiscal Year 1992 (Continued)

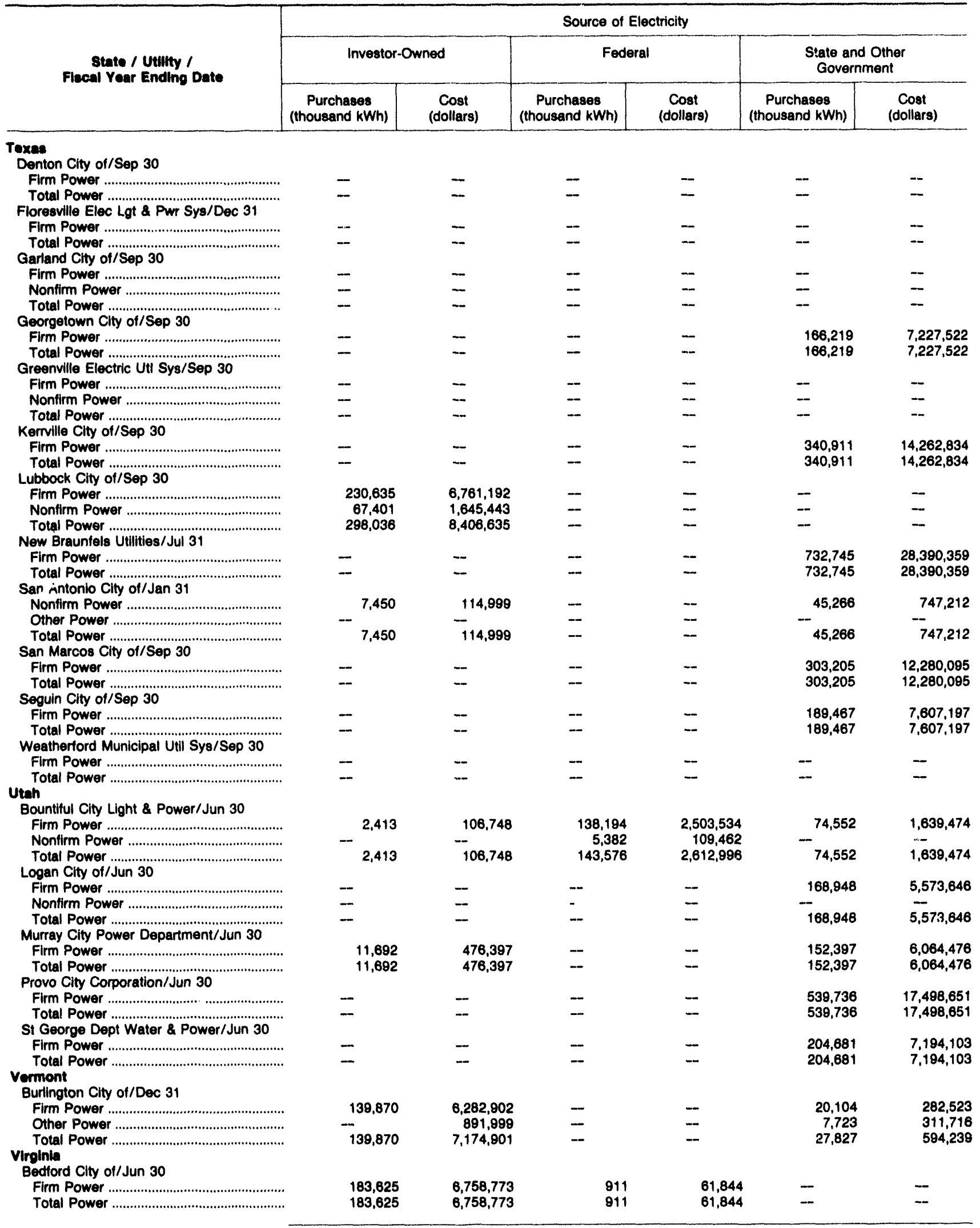

See notes and footnotes at end of table. 
Table 29. Electricity Purchases by Municipal Utilities, Fiscal Year 1992 (Continued)

\begin{tabular}{|c|c|c|c|c|c|c|}
\hline \multirow{3}{*}{$\begin{array}{l}\text { State / Utilty I } \\
\text { Fiscal Year Ending Dato }\end{array}$} & \multicolumn{6}{|c|}{ Source of Electricity } \\
\hline & \multicolumn{2}{|c|}{ Cooperattve } & \multicolumn{2}{|c|}{ Other' } & \multicolumn{2}{|c|}{ Total } \\
\hline & $\begin{array}{c}\text { Purchases } \\
\text { (thousand KWh) }\end{array}$ & $\begin{array}{c}\text { Cost } \\
\text { (dollars) }\end{array}$ & $\begin{array}{c}\text { Purchases } \\
\text { (thousand kWh) }\end{array}$ & $\begin{array}{c}\text { Cost } \\
\text { (dollars) }\end{array}$ & $\begin{array}{c}\text { Purchasers } \\
\text { (thousand KWh) }\end{array}$ & $\begin{array}{c}\text { Cost } \\
\text { (dollars) }\end{array}$ \\
\hline \multicolumn{7}{|l|}{ Toxese } \\
\hline Denton City of/Sep 30 & - & - & 794,900 & $40,518,816$ & 794,990 & $40,518,916$ \\
\hline \multirow{2}{*}{\multicolumn{5}{|c|}{ Fioresville Elec Lgt \& Pwr Sys/Dec 31}} & 794,990 & $40,518,916$ \\
\hline & - & - & 138,763 & & & \\
\hline $\begin{array}{l}\text { Firm Power } \\
\text { Total Power }\end{array}$ & - & - & 138,763 & $5,689,979$ & 138,763 & $\begin{array}{l}5,686,8 / 9 \\
5,698,979\end{array}$ \\
\hline \multicolumn{7}{|l|}{ Garland Clty of/Sep 30} \\
\hline 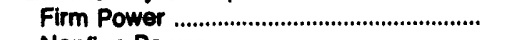 & - & - & $1,338,333$ & $64,468,244$ & $1,338,333$ & $64,468,244$ \\
\hline Nonfirm Power ................................................ & - & - & 75,448 & $1,412,832$ & 75,448 & $1,412,932$ \\
\hline 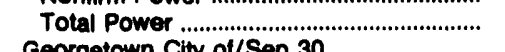 & - & - & $1,413,781$ & $65,681,178$ & $1,413,781$ & $65,881,178$ \\
\hline $\begin{array}{l}\text { Firm Power } \\
\text { Total Power }\end{array}$ & - & - & - & - & 168,219 & $7,227,522$ \\
\hline \multicolumn{7}{|l|}{ Greenville Electric UtI Sys/Sep 30} \\
\hline 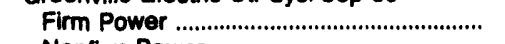 & - & - & 312,596 & $14,493,642$ & 312,596 & $14,493,642$ \\
\hline Nonfirm Power ................................................ & $\begin{array}{l}26,235 \\
26,235\end{array}$ & $\begin{array}{l}507,865 \\
507865\end{array}$ & $\begin{array}{l}103,282 \\
415,878\end{array}$ & $\begin{array}{r}2,131,963 \\
16,625,605\end{array}$ & 129,517 & $\begin{array}{r}2,639,828 \\
17133,470\end{array}$ \\
\hline $\begin{array}{l}\text { Total Power ...is } 30 \\
\text { Kerrville City ot/Sep }\end{array}$ & & & & & & \\
\hline 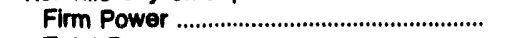 & - & - & - & - & 340,811 & $14,262,834$ \\
\hline 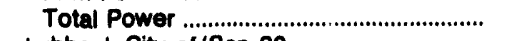 & - & - & - & - & 340,811 & $14,262,834$ \\
\hline \multicolumn{7}{|l|}{ Lubbock City of/Sep 30} \\
\hline & - & - & - & - & 230,635 & $6,761,182$ \\
\hline $\begin{array}{l}\text { Nonfirm Power } \\
\text { Total Power }\end{array}$ & - & - & - & - & 67,401 & $1,645,443$ \\
\hline \multirow{2}{*}{\multicolumn{4}{|c|}{ Now Braunfels Utillites/Jul 31}} & - & 298,036 & $8,406,635$ \\
\hline & & - & - & - & & \\
\hline 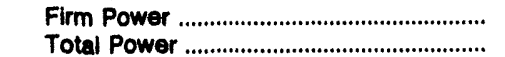 & $\overline{-}$ & - & - & - & $\begin{array}{l}732,745 \\
732,745\end{array}$ & $\begin{array}{l}28,390,359 \\
28,390,359\end{array}$ \\
\hline \multicolumn{7}{|l|}{$\begin{array}{l}\text { Total Power ............................... } \\
\text { San Antonio City of } 31\end{array}$} \\
\hline Nonfirm Power .......................................... & 12,207 & 158,248 & 969 & 18,882 & 65,892 & $1,040,342$ \\
\hline 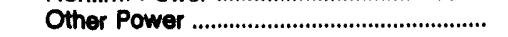 & & - & 226,794 & - & 226,794 & \\
\hline 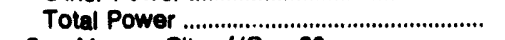 & 12,207 & 158,249 & 227,763 & 19,882 & 282,686 & $1,040,342$ \\
\hline \multicolumn{7}{|l|}{ San Marcos City of/Sep 30} \\
\hline 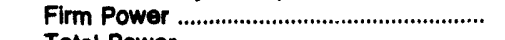 & - & - & - & - & 303,205 & $\begin{array}{l}12,280,095 \\
12,280,095\end{array}$ \\
\hline $\begin{array}{l}\text { Total Power . } \\
\text { Seguin City of /Sep } 30\end{array}$ & - & - & - & - & 303,205 & \\
\hline 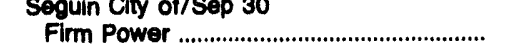 & - & - & - & - & 189,467 & $7,607,197$ \\
\hline 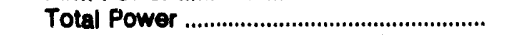 & - & - & - & - & 189,467 & $7,607,197$ \\
\hline Weatherford Municipal Util Sys/Sep 30 & & & & & & \\
\hline & 29,614 & $\begin{array}{l}1,691,529 \\
1,691,529\end{array}$ & $\begin{array}{l}166,308 \\
166,308\end{array}$ & $\begin{array}{l}4,070,057 \\
4,070,057\end{array}$ & $\begin{array}{l}195,922 \\
195,922\end{array}$ & $\begin{array}{l}5,761,588 \\
5,761,586\end{array}$ \\
\hline $\begin{array}{l}\text { Total Power ......................................................... } \\
\text { Utah }\end{array}$ & 29,614 & & & & & \\
\hline Bountful City Light \& Power/Jun 30 & & & & & & \\
\hline 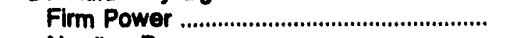 & - & - & - & - & 215,169 & $4,249,766$ \\
\hline 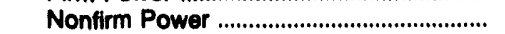 & - & - & - & - & 5,382 & 109,462 \\
\hline 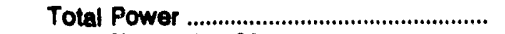 & - & - & - & - & 220,541 & $4,359,218$ \\
\hline !ogan City of/Jun 30 & & & & & & \\
\hline Firm Power & - & $\overline{-}$ & 89,461 & $1,386,823$ & 258,409 & $\begin{array}{r}6,960,469 \\
320,568\end{array}$ \\
\hline 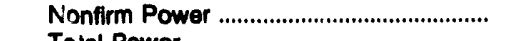 & $\overline{-}$ & $\overline{-}$ & $\begin{array}{r}1,500 \\
90,961\end{array}$ & $\begin{array}{r}320,566 \\
1,707,389\end{array}$ & $\begin{array}{r}1,500 \\
258,809\end{array}$ & $\begin{array}{r}320,568 \\
7,281,035\end{array}$ \\
\hline $\begin{array}{l}\text { To:al Power ............................ } \\
\text { Murray City Popertment/Jun }\end{array}$ & - & & 80,861 & & & \\
\hline 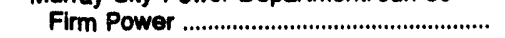 & - & - & 131.167 & $2,356,644$ & 295,256 & $8,897,517$ \\
\hline 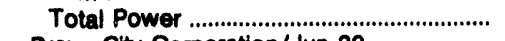 & - & - & 131,167 & $2,356,644$ & 295,256 & $8,897,517$ \\
\hline Provo City Corporation/Jun 30 & & & & & & \\
\hline Firm Power & $\overline{-}$ & - & - & - & 539,736 & $17,498,651$ \\
\hline $\begin{array}{l}\text { Total Power ... W Water \& Power/Jun } 30 \\
\text { St George De }\end{array}$ & - & - & - & - & 539,736 & $17,498,651$ \\
\hline 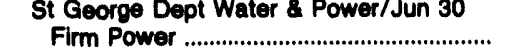 & - & - & 100,726 & $1,883,301$ & 305,407 & $9,077,404$ \\
\hline 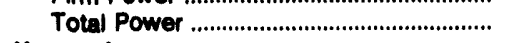 & - & - & 100,726 & $1,883,301$ & 305,407 & $9,077,404$ \\
\hline $\begin{array}{l}\text { Vermont } \\
\text { Burlinoton City of/Dec } 31\end{array}$ & & & & & & \\
\hline 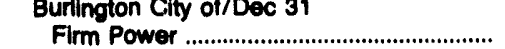 & 38 & 450 & 193,032 & $5,859,899$ & 353,044 & $12,425,774$ \\
\hline 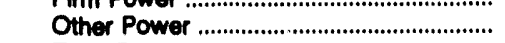 & - & - & & 135,971 & 7,723 & $1,339,686$ \\
\hline 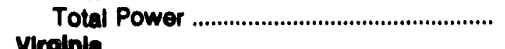 & 38 & 450 & 193,032 & $5,995,870$ & 360,787 & $13,785,460$ \\
\hline $\begin{array}{l}\text { Virolnia } \\
\text { Bedford City of/Jun } 30\end{array}$ & & & & & & \\
\hline 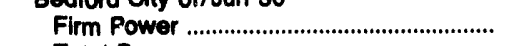 & - & - & - & - & 184,536 & $6,820,617$ \\
\hline 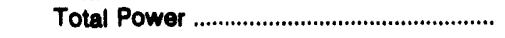 & - & - & - & - & 184,536 & $6,820,617$ \\
\hline
\end{tabular}

See notes and footnotes at end of table. 
Table 29. Electricity Purchases by Municipal Utilities, Fisca! Year 1992 (Continued)

\begin{tabular}{|c|c|c|c|c|c|c|}
\hline \multirow{3}{*}{$\begin{array}{l}\text { State / Utility / } \\
\text { Fiecal Yoar Ending Date }\end{array}$} & \multicolumn{6}{|c|}{ Source of Electricity } \\
\hline & \multicolumn{2}{|c|}{ Investor-Owned } & \multicolumn{2}{|c|}{ Federal } & \multicolumn{2}{|c|}{$\begin{array}{l}\text { State and Other } \\
\text { Government }\end{array}$} \\
\hline & $\begin{array}{c}\text { Purchases } \\
\text { (thousand kWh) }\end{array}$ & $\begin{array}{c}\text { Cost } \\
\text { (dollars) }\end{array}$ & $\begin{array}{c}\text { Purchases } \\
\text { (thousand kWh) }\end{array}$ & $\begin{array}{c}\text { Cost } \\
\text { (dollars) }\end{array}$ & $\begin{array}{c}\text { Purchases } \\
\text { (thousand kWh) }\end{array}$ & $\begin{array}{c}\text { Cost } \\
\text { (dollars) }\end{array}$ \\
\hline \multicolumn{7}{|l|}{ Virginia } \\
\hline 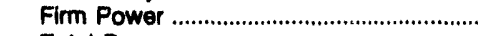 & - & - & 525,176 & $19,841,000$ & -- & - \\
\hline 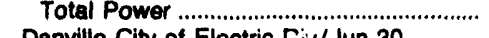 & - & - & 525,176 & $19,841,000$ & - & - \\
\hline \multicolumn{7}{|l|}{ Danville City of Electric Civ/Jun 30} \\
\hline 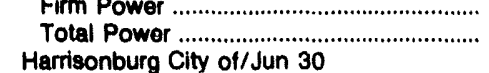 & $\begin{array}{l}768,922 \\
768,922\end{array}$ & $\begin{array}{l}26,242,582 \\
26,242,582\end{array}$ & $\begin{array}{l}7,202 \\
4,202\end{array}$ & $\begin{array}{l}288,606 \\
288,606\end{array}$ & - & - \\
\hline Firm Power & 488,957 & $21,320,249$ & 1,992 & 135,351 & - & - \\
\hline \multicolumn{7}{|l|}{ Manassas City of/Jun 30} \\
\hline 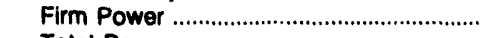 & 264,602 & $11,132,749$ & - & - & - & - \\
\hline 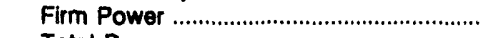 & 202,369 & $6,715,758$ & 1,201 & 82,594 & - & - \\
\hline \multicolumn{6}{|l|}{ Radford City of/Jun 30} & - \\
\hline Firm Power & 283,464 & $9,298,106$ & - & - & - & - \\
\hline $\begin{array}{l}\text { Total Power } \\
\text { Salem City of/Jun } 30\end{array}$ & 283,464 & $9,298,106$ & - & -- & - & - \\
\hline 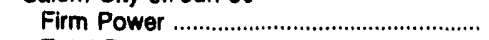 & 323,303 & $10,949,598$ & 1,651 & 113,381 & - & - \\
\hline 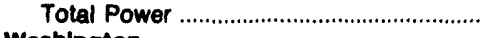 & 323,303 & $10,949,598$ & 1,651 & 113,381 & -- & - \\
\hline \multicolumn{6}{|l|}{ Centralia City of/Dec 31} & \\
\hline Firm Power & -- & - & 162,458 & $4,275,365$ & - & - \\
\hline 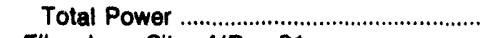 & - & - & 162,458 & $4,275,365$ & - & -- \\
\hline Ellensburg City of/Dec 31 & & & & & & \\
\hline 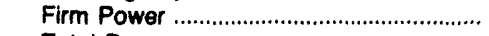 & - & -- & 159,071 & $3,884,018$ & -- & - \\
\hline 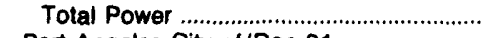 & -- & - & 159,071 & $3,884,018$ & -- & - \\
\hline Port Angeles City of/Dec 31 & & & & & & \\
\hline 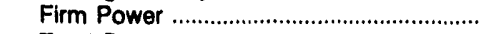 & -- & - & 666,520 & $15,524,000$ & - & - \\
\hline 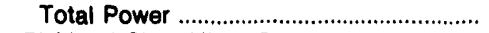 & - & - & 666,520 & $15,524,000$ & -- & - \\
\hline 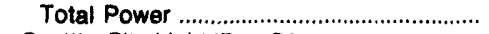 & - & - & 582,257 & $14,471,278$ & - & - \\
\hline Seattle City Light/Dec 31 & & & & & & \\
\hline 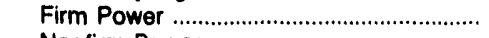 & - & 682,000 & $2,012,628$ & $41,862,509$ & 760,608 & $15,883,514$ \\
\hline 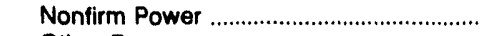 & 79,830 & $-1,539,213$ & $-14,393$ & 114,422 & 15,166 & $-337,190$ \\
\hline 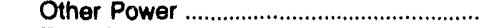 & 27,612 & - & 67,616 & - & 3,102 & $-\infty$ \\
\hline 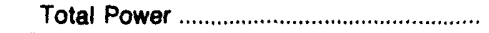 & 107,442 & $-857,213$ & $2,065,851$ & $41,976,931$ & 778,876 & $15,546,324$ \\
\hline Tacoma Dept of Public Utils/Dec 31 & & & & & & \\
\hline 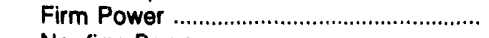 & -- & -- & $2,503,765$ & $54,053,342$ & 599,200 & $11,974,734$ \\
\hline 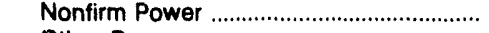 & -- & -- & - & - & - & - \\
\hline 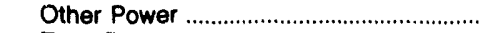 & -- & -- & - & -- & - & - \\
\hline 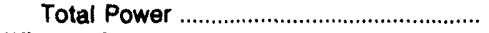 & -- & -- & $2,503,765$ & $54,053,342$ & 599,200 & $11,974,734$ \\
\hline Wisconsin & & & & & & \\
\hline Jefferson Water \& Elec Dept/Dec 31 & & & & & & \\
\hline 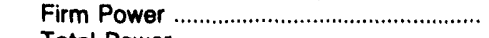 & - & -- & - & -- & 131,000 & $5,084,757$ \\
\hline 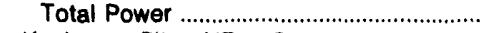 & -- & -- & -- & -- & 131,000 & $5,084,757$ \\
\hline Kaukauna City of/Dec 31 & & & & & & \\
\hline Firm Power ........................................... & - & -- & -- & - & 383,757 & $13,250,034$ \\
\hline Nonfirm Power .............................................. & - & - & -- & -- & 1,430 & 44,343 \\
\hline Total Power ................................................ & - & -- & -- & -- & 385,187 & $13,280,377$ \\
\hline Manitowoc Public Utilities/Dec 31 & & & & & & \\
\hline 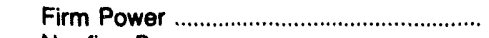 & -- & - & - & - & 73,527 & $2,329,846$ \\
\hline 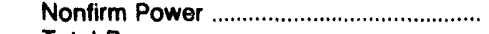 & - & - & -- & -- & 149,912 & $2,729,397$ \\
\hline Total Power ........................................... & - & -- & - & - & 223,439 & $5,059,243$ \\
\hline Marshfield Electric\&Water Dept/Dec 31 & & & & & & \\
\hline 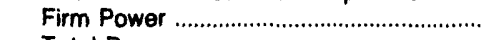 & 299,175 & $10,225,898$ & -- & - & - & - \\
\hline 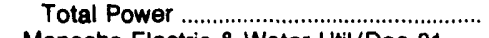 & 299,175 & $10,225,898$ & - & -- & - & - \\
\hline Menasha Electric \& Water Util/Dec 31 & & & & & & \\
\hline 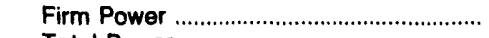 & -- & - & - & - & 513,600 & $17,547,720$ \\
\hline 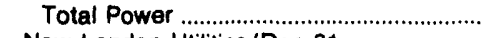 & -- & - & - & - & 513,600 & $17,547,720$ \\
\hline New London Utilities/Dec 31 & & & & & & \\
\hline 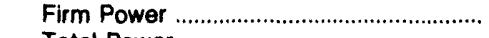 & - & - & - & - & 157,907 & $6,055,230$ \\
\hline 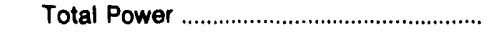 & - & -- & - & - & 157,907 & $6,055,230$ \\
\hline
\end{tabular}

See notes and footnotes at end of table. 
Table 29. Electricity Purchases by Municipal Utilities, Fiscal Year 1992 (Continued)

\begin{tabular}{|c|c|c|c|c|c|c|}
\hline \multirow{3}{*}{$\begin{array}{l}\text { State / Uitily / } \\
\text { Fiecal Yoer Ending Date }\end{array}$} & \multicolumn{6}{|c|}{ Source of Electrictly } \\
\hline & \multicolumn{2}{|c|}{ Cooperative } & \multicolumn{2}{|c|}{ Other' } & \multicolumn{2}{|c|}{ Total } \\
\hline & $\begin{array}{c}\text { Purchaseis } \\
\text { (thousand kWh) }\end{array}$ & $\underset{\text { (dollars) }}{\text { Cost }}$ & $\begin{array}{c}\text { Purchases } \\
\text { (thousand kWh) }\end{array}$ & $\begin{array}{c}\text { Cost } \\
\text { (dollars) }\end{array}$ & $\begin{array}{c}\text { Purchases } \\
\text { (thousand } k W h \text { ) }\end{array}$ & $\begin{array}{l}\text { Cost } \\
\text { (dollars) }\end{array}$ \\
\hline \multicolumn{7}{|l|}{$\begin{array}{l}\text { Virghinda } \\
\text { Bristol Utility Board/Jun } 30\end{array}$} \\
\hline Firm Power ........................... & - & - & - & - & 525,176 & $19,841,000$ \\
\hline \multicolumn{7}{|l|}{ Danville City of Electric Div/Jun 30} \\
\hline 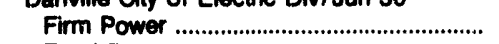 & - & - & - & - & 773,124 & $26,531,188$ \\
\hline $\begin{array}{l}\text { Total Power .................................. } \\
\text { Harrisonburg City of } 30\end{array}$ & Harrisonburg City of/Jun 30 & - & - & - & 773,124 & $26,531,188$ \\
\hline 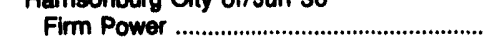 & - & -- & - & - & 490,849 & $21,455,600$ \\
\hline 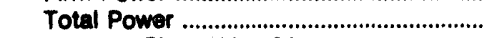 & - & - & -- & - & 490,949 & $21,455,600$ \\
\hline \multicolumn{7}{|l|}{ Manassas City of/Jun 30} \\
\hline 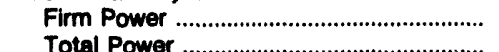 & $-\cdot$ & - & - & - & 264,602 & $11,132,749$ \\
\hline \multicolumn{7}{|l|}{ Martinsville City of/Jun 30} \\
\hline 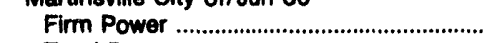 & - & - & - & - & 203,570 & $6,798,352$ \\
\hline & Radford City of/Jun 30 & - & - & - & 203,570 & $6,798,352$ \\
\hline Firm Power .................................................... & - & - & - & -- & 283,464 & $9,298,106$ \\
\hline Total Power ........................................... & - & - & - & - & 283,464 & $9,298,106$ \\
\hline 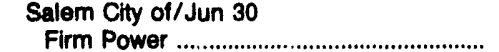 & - & - & - & - & 324,954 & $11,062,879$ \\
\hline Total Power & - & - & - & - & 324,954 & $11,062,879$ \\
\hline \multicolumn{7}{|l|}{ Washington } \\
\hline 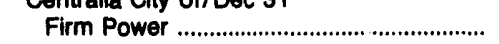 & - & - & - & -- & 162,458 & $4,275,365$ \\
\hline \multirow{2}{*}{\multicolumn{7}{|c|}{ Ellensburg City of/Dec 31}} \\
\hline & - & - & - & - & 159.071 & \\
\hline $\begin{array}{l}\text { Firm Power } \\
\text { Total Power ................ }\end{array}$ & - & - & - & - & 159,071 & $3,884,018$ \\
\hline \multicolumn{7}{|l|}{ Port Angeles City of/Dec 31} \\
\hline 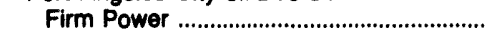 & - & - & 12,321 & - & 678,841 & $15,524,000$ \\
\hline \multicolumn{6}{|l|}{ Richland City of/Dec 31} & $15,524,000$ \\
\hline Firm Power ................................................. & - & -- & 19,261 & 83,281 & 601,518 & $14,554,559$ \\
\hline 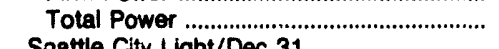 & - & - & 19,261 & 83,281 & 601,518 & $14,554,559$ \\
\hline $\begin{array}{l}\text { Seattle City Light/Dec } 31 \\
\text { Firm Power }\end{array}$ & $\ldots$ & - & 668,598 & $23,691,764$ & $3,442,834$ & $82,119,787$ \\
\hline Nonfirm Power ........................................... & -- & - & $-78,680$ & $1,811,188$ & 1,923 & 48,208 \\
\hline 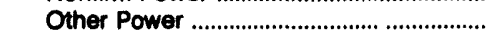 & - & - & $-5,129$ & - & 93,201 & - \\
\hline 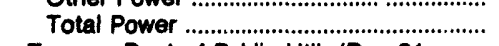 & - & - & 585,789 & $25,502,953$ & $3,537,958$ & $82,168,995$ \\
\hline Tacoma Dept of Public Utils/Dec 31 & & & & & & \\
\hline 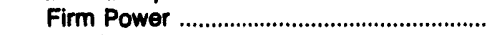 & - & - & 328,448 & $1,363,087$ & $3,431,413$ & $67,391,163$ \\
\hline Nonfirm Power ............................................. & - & - & 136,853 & $1,487,973$ & 136,853 & $1,487,973$ \\
\hline Other Power & - & - & - & $1,133,979$ & -- & $1,133,879$ \\
\hline Total Power ..................................... & -- & - & 465,301 & $3,885,039$ & $3,568,266$ & $70,013,115$ \\
\hline $\begin{array}{l}\text { Wheconsin } \\
\text { Jefferson Water \& Elec Dept/Dec } 31\end{array}$ & & & & & & \\
\hline 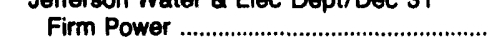 & - & - & - & - & 131,000 & $5,084,757$ \\
\hline Total Power ............................................ & - & - & $\ldots$ & - & 131,000 & $5,084,757$ \\
\hline Kaukauna City of/Dec 31 & & & & & & \\
\hline 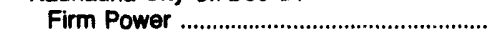 & - & - & - & -- & 383,757 & $13,236,034$ \\
\hline 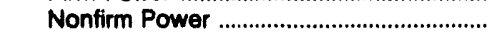 & - & -- & - & - & 1,430 & 44,343 \\
\hline 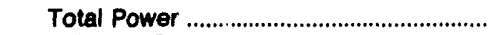 & - & - & - & - & 385,187 & $13,280,377$ \\
\hline Manitowoc Public Utilities/Dec 31 & & & & & & \\
\hline 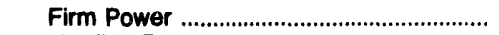 & - & - & - & - & 73,527 & $2,329,846$ \\
\hline 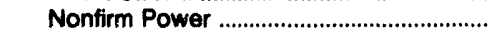 & - & - & - & - & 149,912 & $2,729,397$ \\
\hline Total Power & - & - & - & - & 223,439 & $5,059,243$ \\
\hline Marshfield Electric\&Water Dept/Dec 31 & & & & & & \\
\hline Firm Power .......................................... & - & - & - & - & 299,175 & $10,225,898$ \\
\hline Total Power ....................................................... & - & - & - & - & 299,175 & $10,225,898$ \\
\hline Menasha Electric \& Water Util/Dec 31 & & & & & & \\
\hline 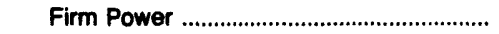 & - & - & - & - & 513,600 & $17,547,720$ \\
\hline 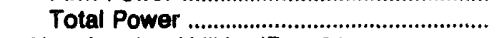 & -- & -- & - & - & 513,600 & $17,547,720$ \\
\hline Now London Utilities/Dec 31 & & & & & & \\
\hline 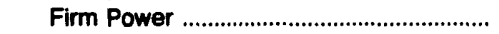 & - & - & - & - & 157,907 & $6,055,230$ \\
\hline 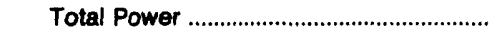 & - & - & - & - & 157,907 & $6,055,230$ \\
\hline
\end{tabular}

See notes and footnotes at end of table. 
Table 29. Electricity Purchasee by Municipal Utilities, Flecal Year 1992 (Continued)

\begin{tabular}{|c|c|c|c|c|c|c|}
\hline \multirow{3}{*}{$\begin{array}{l}\text { State / Uitily ' } \\
\text { Fleed Year Ending Date }\end{array}$} & \multicolumn{6}{|c|}{ Source of Electricity } \\
\hline & \multicolumn{2}{|c|}{ Investor-Owned } & \multicolumn{2}{|c|}{ Federal } & \multicolumn{2}{|c|}{$\begin{array}{l}\text { State and Other } \\
\text { Government }\end{array}$} \\
\hline & $\begin{array}{c}\text { Purchases } \\
\text { (thousand } \mathrm{kWh} \text { ) }\end{array}$ & $\underset{\text { (dollars) }}{\text { Cost }}$ & $\begin{array}{l}\text { Purchases } \\
\text { (thousand kWh) }\end{array}$ & $\begin{array}{c}\text { Cost } \\
\text { (dollars) }\end{array}$ & $\begin{array}{l}\text { Purchases } \\
\text { (thousand kWh) }\end{array}$ & $\begin{array}{c}\text { Cost } \\
\text { (dollars) }\end{array}$ \\
\hline \multicolumn{7}{|l|}{ 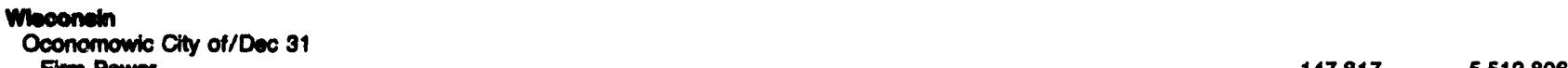 } \\
\hline 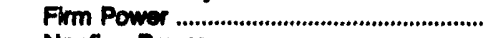 & - & - & - & - & 147,817 & $5,513,806$ \\
\hline 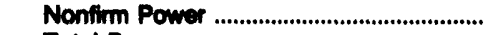 & - & 46,817 & - & - & - & \\
\hline 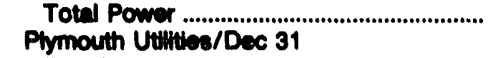 & - & 45,817 & - & - & 147,817 & $5,513,806$ \\
\hline Firm Power & $\begin{array}{l}154,584 \\
154,584\end{array}$ & $\begin{array}{l}\mathbf{5}, 018,108 \\
5,018,108\end{array}$ & $\overline{-}$ & $\overline{-}$ & $\overline{-}$ & $\overline{-}$ \\
\hline $\begin{array}{l}\text { Recodaburg Utilty Commiacion/Dec } 31 \\
\text { Firm Power }\end{array}$ & & & & & & \\
\hline $\begin{array}{l}\text { Firm Power } \\
\text { Total Power } \\
\text { Shawrono Municipel Utilltites/Dec31 }\end{array}$ & $\begin{array}{l}150,120 \\
150,120\end{array}$ & $\begin{array}{l}\mathbf{5 , 1 8 9 , 2 5 9} \\
\mathbf{5 , 1 8 9 , 2 5 9}\end{array}$ & $\overline{-}$ & $\overline{-}$ & $\overline{-}$ & $\overline{-}$ \\
\hline 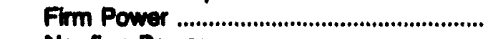 & - & - & - & - & - & - \\
\hline 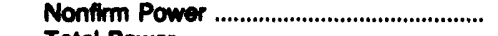 & - & - & - & - & - & - \\
\hline 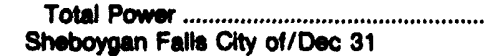 & - & - & - & - & - & - \\
\hline 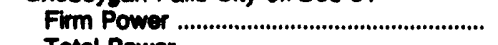 & 175,637 & $5,578,010$ & - & - & - & - \\
\hline $\begin{array}{l}\text { Total Power ................................................ } \\
\text { Sturgeon Bay City of/Dec } 31\end{array}$ & 175,537 & $5,578,010$ & - & - & - & - \\
\hline 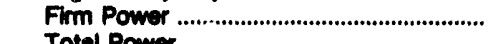 & 132,023 & $5,197,167$ & - & - & - & - \\
\hline $\begin{array}{l}\text { Total Power } \\
\text { Whaconain Rapids W \& L Comm/Dec } 31\end{array}$ & 132,023 & $5,197,167$ & - & - & - & - \\
\hline 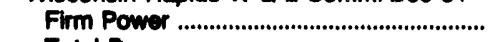 & 192,686 & $7,132,751$ & - & - & - & - \\
\hline 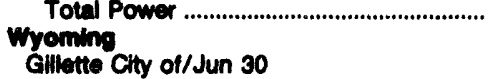 & 182,686 & $7,132,751$ & - & - & - & - \\
\hline 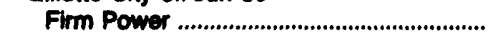 & 150,854 & $7,520,740$ & 26,834 & 374,468 & - & - \\
\hline 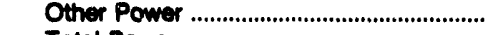 & - & $=$ & - & - & 621 & 20,4 \\
\hline 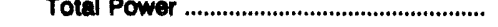 & 150,864 & $7,520,740$ & 26,834 & 374,468 & 621 & 20,493 \\
\hline
\end{tabular}

See notes and footnotes at end of table. 
Table 29. Electriclty Purchases by Municipal Utilitles, Flecal Year 1992 (Continued)

\begin{tabular}{|c|c|c|c|c|c|c|}
\hline \multirow{3}{*}{$\begin{array}{l}\text { State / Uallty I } \\
\text { Fiecal Year Ending Dato }\end{array}$} & \multicolumn{6}{|c|}{ Source of Electricity } \\
\hline & \multicolumn{2}{|c|}{ Cooperative } & \multicolumn{2}{|c|}{ Other' } & \multicolumn{2}{|c|}{ Total } \\
\hline & $\begin{array}{c}\text { Purchases } \\
\text { (thousand kWh) }\end{array}$ & $\begin{array}{c}\text { Cost } \\
\text { (dollars) }\end{array}$ & $\begin{array}{c}\text { Purchases } \\
\text { (thousand kWh) }\end{array}$ & $\begin{array}{l}\text { Cost } \\
\text { (dollars) }\end{array}$ & $\begin{array}{c}\text { Purchases } \\
\text { (thousand kWh) }\end{array}$ & $\begin{array}{c}\text { Cost } \\
\text { (dollars) }\end{array}$ \\
\hline \multicolumn{7}{|l|}{$\begin{array}{l}\text { Wheconaln } \\
\text { Oconomowic City of/Dec } 31\end{array}$} \\
\hline 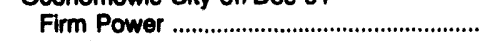 & - & - & - & - & 147,817 & $5,513,806$ \\
\hline Nontirm Power ................................................... & - & - & - & -- & -- & 46,817 \\
\hline $\begin{array}{l}\text { Total Power } \\
\text { Plumouth Utilities/Dec } 31\end{array}$ & - & - & - & - & 147,817 & $5,559,623$ \\
\hline \multicolumn{4}{|l|}{ Plymouth Utilities/Dec 31} & & & \\
\hline 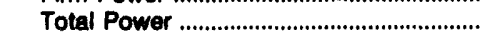 & -- & - & - & $=$ & $\begin{array}{l}154,584 \\
154,584\end{array}$ & $\begin{array}{l}5,018,108 \\
5,018,108\end{array}$ \\
\hline \multicolumn{7}{|l|}{ Roedsburg Utility Commission/Dec 31} \\
\hline 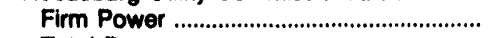 & - & - & - & - & 150,120 & $5,188,259$ \\
\hline Total Power & -- & - & - & -- & 150,120 & $5,189,259$ \\
\hline \multicolumn{7}{|l|}{ Shawano Municipal Utillties/Dec31 } \\
\hline 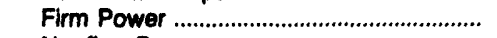 & - & - & 174,369 & $6,195,040$ & 174,369 & $6,195,040$ \\
\hline 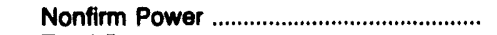 & - & -- & & 126 & & 126 \\
\hline \multirow{2}{*}{\multicolumn{7}{|c|}{ Sheboygan Falls City of/Dec 31}} \\
\hline & & & & & & \\
\hline 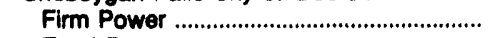 & - & - & - & -- & 175,537 & $5,578,010$ \\
\hline 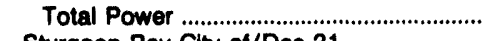 & -- & - & -- & -- & 175,537 & $5,578,010$ \\
\hline \multicolumn{7}{|l|}{ Sturgeon Bay City of/Dec 31} \\
\hline 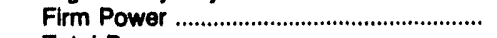 & - & - & - & - & 132,023 & $5,197,167$ \\
\hline 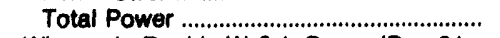 & -- & - & -- & - & 132,023 & $5,197,167$ \\
\hline \multicolumn{7}{|l|}{ Wisconsin Rapids W \& L Comm/Doc 31} \\
\hline 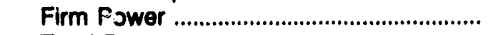 & - & - & -- & -- & 192,666 & $7,132,751$ \\
\hline 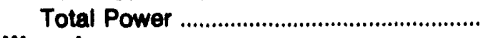 & - & - & -- & -- & 192,666 & $7,132,751$ \\
\hline \multicolumn{7}{|l|}{ Wyoming } \\
\hline 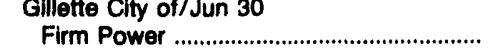 & -- & - & - & -- & 177,788 & $7,895,208$ \\
\hline Other Power & - & - & - & -- & 621 & 20,493 \\
\hline 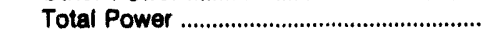 & - & - & - & - & 178,409 & $7,915,701$ \\
\hline
\end{tabular}

1 Includes transactions with municipal utilities, power pools, utilities in Canada and Mexico, and nonutilities.

- S urce of data is Rural Electrification Administration, REA Form 7, "Financial and Statistical Report."

- Not Applicable

Notes: - For identification purposes, the municipal utilities are listed in the State in which the municipality is located. - Totals may not equal sum of components because of independent rounding.

Source: Energy Information Administratiori, Form ElA-412, "Annual Report of Public Electric Utilities," except where footnoted. 
Table 30. Electriclty Sales for Resale by Municlpal Utilities, Fiscal Year 1992

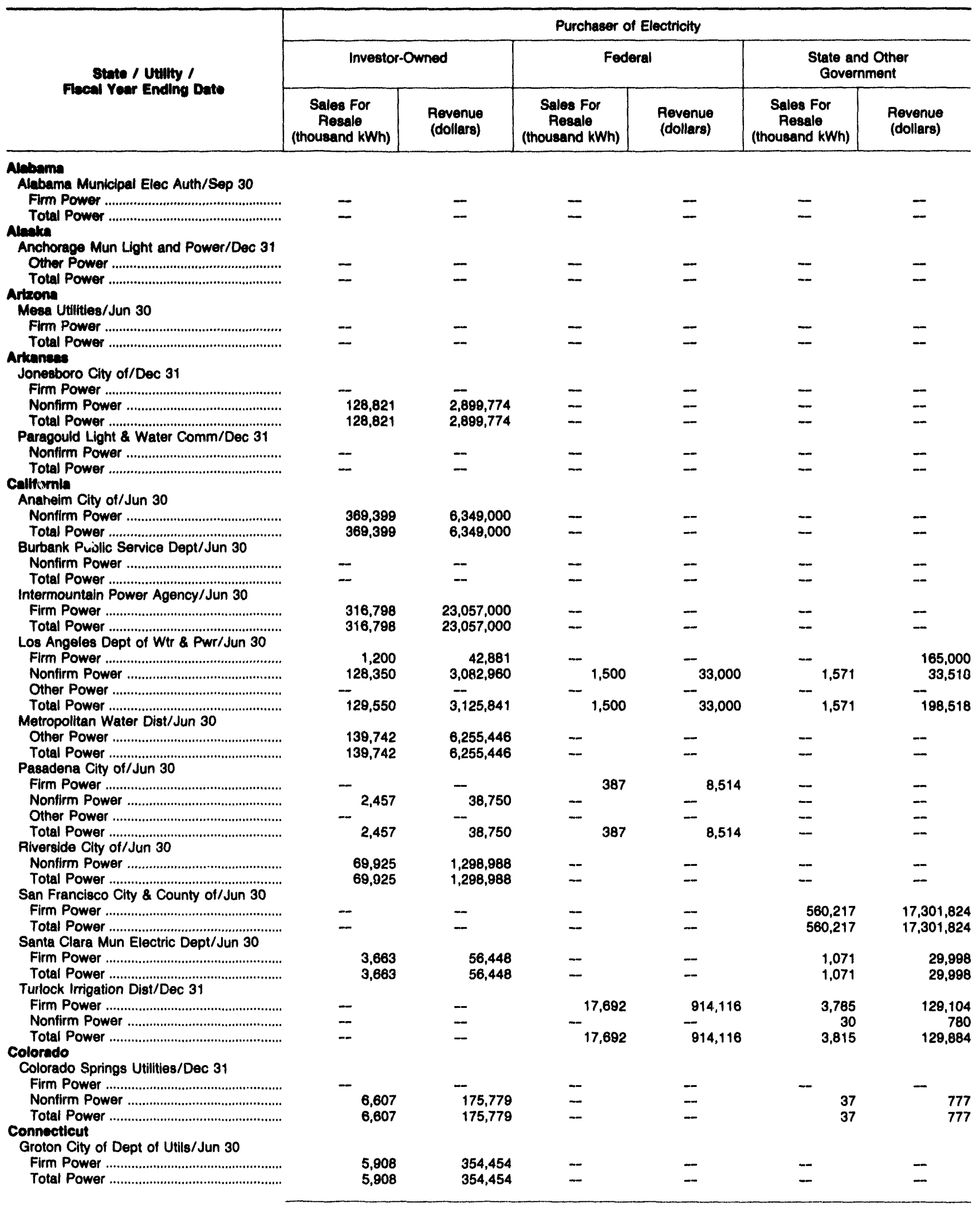


Table 30. Electricity Sales for Resale by Municipal Utilities, Fiscal Year 1992 (Continued)

\begin{tabular}{|c|c|c|c|c|c|c|}
\hline \multirow{3}{*}{$\begin{array}{l}\text { State / Utilty I } \\
\text { Flecal Year Ending Dato }\end{array}$} & \multicolumn{6}{|c|}{ Purchaser of Eloctricity } \\
\hline & \multicolumn{2}{|c|}{ Cooperative } & \multicolumn{2}{|c|}{ Other' } & \multicolumn{2}{|c|}{ Total } \\
\hline & $\begin{array}{c}\text { Sales For } \\
\text { Ressle } \\
\text { (thousand kWh) }\end{array}$ & $\begin{array}{l}\text { Pevenue } \\
\text { (dollars) }\end{array}$ & $\begin{array}{c}\text { Sales For } \\
\text { Resale } \\
\text { (thnusand kWh) }\end{array}$ & $\begin{array}{l}\text { Revenue } \\
\text { (dollars) }\end{array}$ & $\begin{array}{c}\text { Sales For } \\
\text { Resale } \\
\text { (thousand kWh) }\end{array}$ & $\begin{array}{l}\text { Revenue } \\
\text { (dollars) }\end{array}$ \\
\hline \multicolumn{7}{|l|}{ Alabama } \\
\hline 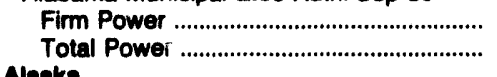 & $\overline{-}$ & $\overline{-}$ & $\begin{array}{l}2,252,354 \\
2,252,354\end{array}$ & $\begin{array}{l}88,399,598 \\
88,399,598\end{array}$ & $\begin{array}{l}2,252,354 \\
2,252,354\end{array}$ & $\begin{array}{l}88,399,598 \\
88,399,598\end{array}$ \\
\hline \multicolumn{7}{|l|}{ Alacka } \\
\hline Other Power & $\begin{array}{l}2,532 \\
2,532\end{array}$ & $\begin{array}{l}81,392 \\
81,392\end{array}$ & $\begin{array}{l}14,593 \\
14,593\end{array}$ & $\begin{array}{l}528,617 \\
528,617\end{array}$ & $\begin{array}{l}17,125 \\
17,125\end{array}$ & $\begin{array}{l}610,009 \\
610,009\end{array}$ \\
\hline \multicolumn{7}{|l|}{$\begin{array}{l}\text { Artzona } \\
\text { Mesa Utilities/Jun } 30\end{array}$} \\
\hline 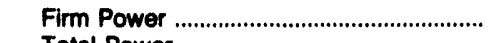 & - & - & 150 & 3,209 & 150 & 3,209 \\
\hline 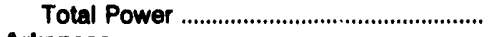 & - & - & 150 & 3,209 & 150 & 3,209 \\
\hline \multicolumn{7}{|l|}{$\begin{array}{l}\text { Arkanaas } \\
\text { Jonesboro City of/Dec } 31\end{array}$} \\
\hline 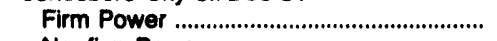 & - & - & 13,905 & 400,580 & 13,905 & 400,580 \\
\hline 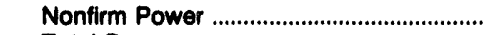 & - & - & 70,614 & $1,543,492$ & 199,435 & $4,443,266$ \\
\hline 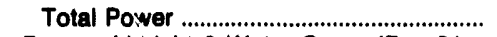 & - & - & 84,518 & $1,944,072$ & 213,340 & $4,843,846$ \\
\hline \multicolumn{7}{|l|}{ Paragould l.ight \& Water Comm/Dec 31} \\
\hline 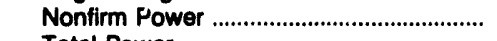 & - & - & 4,872 & 82,824 & 4,872 & 82,824 \\
\hline $\begin{array}{l}\text { Total Power } \\
\text { Calfornta }\end{array}$ & - & - & 4,872 & 82,824 & 4,872 & 82,824 \\
\hline \multicolumn{7}{|l|}{$\begin{array}{l}\text { Calfiomla } \\
\text { Anaheim City of/Jun } 30\end{array}$} \\
\hline Nonfirm Power ................................................. & - & - & - & - & 369,399 & $6,349,000$ \\
\hline \multirow{2}{*}{\multicolumn{7}{|c|}{ Burbank Public Service Dept/Jun 30}} \\
\hline $\begin{array}{l}\text { Burbank Public Service Dept/Jun } 30 \\
\text { Nonfirm Power }\end{array}$ & - & & & & & \\
\hline Total Power & -. & - & $\begin{array}{l}753 \\
753\end{array}$ & $\begin{array}{l}12,823 \\
12,823\end{array}$ & 753 & $\begin{array}{l}12,823 \\
12,823\end{array}$ \\
\hline \multicolumn{7}{|l|}{ Intermountain Power Agency/Jun 30} \\
\hline 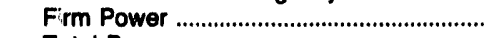 & -- & -- & $11,068,569$ & $625,529,000$ & $11,385,367$ & $648,586,000$ \\
\hline 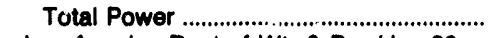 & - & - & $11,068,569$ & $625,529,000$ & $11,385,367$ & $648,586,000$ \\
\hline \multicolumn{7}{|l|}{ Los Angeles Cept of Wtr \& Pwr/Jun 30} \\
\hline 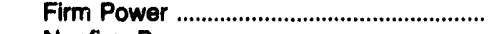 & - & - & - & 60,000 & 1,200 & 267,881 \\
\hline 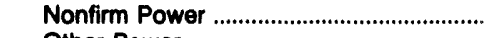 & - & - & 7,868 & 193,168 & 139,289 & $3,342,646$ \\
\hline 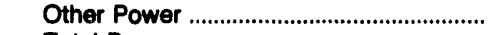 & - & - & - & 73.481 & - & 73,481 \\
\hline 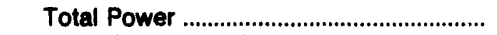 & - & - & 7,868 & 326,649 & 140,489 & $3,684,008$ \\
\hline \multicolumn{7}{|l|}{ Metropolitan Water Dist/Jun 30} \\
\hline 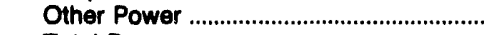 & - & - & - & - & 139,742 & $6,255,446$ \\
\hline 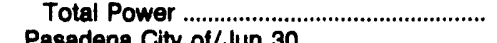 & - & - & - & - & 139,742 & $6,255,446$ \\
\hline Pasadena City of/Jun 30 & & & & & & \\
\hline 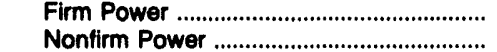 & $\overline{-}$ & $\overline{-}$ & $-41,474$ & $\overline{636,533}$ & $\begin{array}{r}387 \\
43,931\end{array}$ & $\begin{array}{r}8,514 \\
675,283\end{array}$ \\
\hline Other Power ............................................ & - & - & - & $3,216,000$ & -- & $3,216,000$ \\
\hline 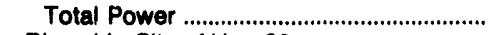 & - & - & 41,474 & $3,852,533$ & 44,318 & $3,888,797$ \\
\hline Riverside City of/Jun 30 & & & & & & \\
\hline 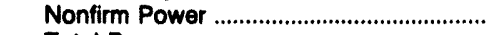 & - & - & - & - & 69,925 & $1,298,988$ \\
\hline Total Power & - & - & - & - & 69,925 & $1,298,988$ \\
\hline San Francisco City \& County of/Jun 30 & & & & & & \\
\hline 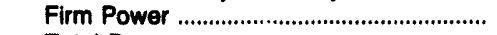 & - & - & 445,206 & $15,517,039$ & $1,005,423$ & $32,818,863$ \\
\hline Total Power & - & - & 445,206 & $15,517,039$ & $1,005,423$ & $32,818,863$ \\
\hline Santa Clara Mun Electric Dept/Jun 30 & & & & & & \\
\hline 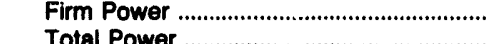 & - & - & - & - & 4.734 & $\begin{array}{l}86,446 \\
86,446\end{array}$ \\
\hline $\begin{array}{l}\text { Total Power } \\
\text { Turlock Irigation Dist/Dec } 31\end{array}$ & - & - & - & - & 4,734 & 86,446 \\
\hline 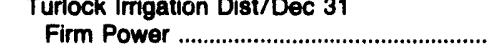 & - & -- & - & - & 21,477 & $1,043,220$ \\
\hline 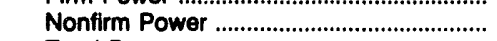 & - & - & - & - & 30 & 780 \\
\hline 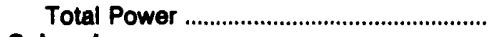 & - & - & - & - & 21,507 & $1,044,000$ \\
\hline Colorado & & & & & & \\
\hline Colorado Springs Utilities/Dec 31 & & & & & & \\
\hline 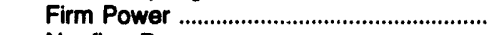 & - & -- & 119,669 & $4,214,260$ & 119,669 & $4,214,260$ \\
\hline 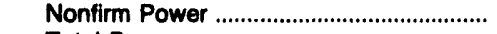 & - & - & - & - & 6,644 & 176,556 \\
\hline Total Power & - & -- & 119,669 & $4,214,260$ & 126,313 & $4,390,816$ \\
\hline $\begin{array}{l}\text { Connecticut } \\
\text { Groton City of Dept of Utils/Jun } 30\end{array}$ & & & & & & \\
\hline 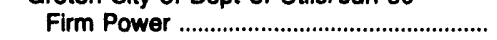 & - & - & - & -- & 5,908 & 354,454 \\
\hline 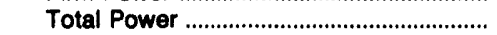 & - & - & - & -- & 5,908 & 354,454 \\
\hline
\end{tabular}

See notes and footnotes at end of table. 
Table 30. Electricity Sales for Resale by Municipal Utilities, Fiscal Year 1992 (Continued)

\begin{tabular}{|c|c|c|c|c|c|c|}
\hline \multirow{3}{*}{$\begin{array}{l}\text { State / Uttilly / } \\
\text { Flecal Yoar Ending Dato }\end{array}$} & \multicolumn{6}{|c|}{ Purchaser of Electricity } \\
\hline & \multicolumn{2}{|c|}{ Investor-Owned } & \multicolumn{2}{|c|}{ Federal } & \multicolumn{2}{|c|}{$\begin{array}{c}\text { State and Other } \\
\text { Government }\end{array}$} \\
\hline & $\begin{array}{c}\text { Sales For } \\
\text { Ressle } \\
\text { (thousand kWh) }\end{array}$ & $\begin{array}{l}\text { Revenue } \\
\text { (dollars) }\end{array}$ & $\begin{array}{c}\text { Sales For } \\
\text { Resale } \\
\text { (thousand } \mathrm{kWh} \text { ) }\end{array}$ & $\begin{array}{l}\text { Revenue } \\
\text { (dollars) }\end{array}$ & $\begin{array}{c}\text { Sales For } \\
\text { Pesale } \\
\text { (thousand kWh) }\end{array}$ & $\begin{array}{l}\text { Revenue } \\
\text { (dollars) }\end{array}$ \\
\hline \multicolumn{7}{|l|}{$\begin{array}{l}\text { Delawware } \\
\text { Dover City of/Jun } 30\end{array}$} \\
\hline Nonfirm Power .......................................... & 38,005 & 941,245 & - & - & - & - \\
\hline 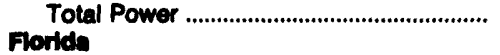 & 38,005 & 941,245 & - & - & - & - \\
\hline \multicolumn{7}{|l|}{$\begin{array}{l}\text { Florlda } \\
\text { Florida Municipal Power Agency/Sep } 30\end{array}$} \\
\hline Firm Power ............................................ & 254,515 & $2,811,418$ & - & - & - & -- \\
\hline 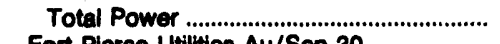 & 254,515 & $2,811,418$ & - & - & -- & - \\
\hline \multicolumn{7}{|l|}{ Fort Pierce Utilities Au/Sep 30} \\
\hline $\begin{array}{l}\text { Firm Power } \\
\text { Nonfirm Power }\end{array}$ & $-4,211$ & 187,482 & $\overline{-}$ & $\bar{z}$ & $\overline{-}$ & $\overline{-}$ \\
\hline 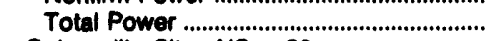 & 4,211 & 187,482 & - & - & - & - \\
\hline \multirow{2}{*}{\multicolumn{7}{|c|}{ 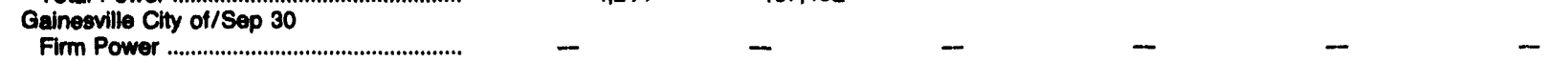 }} \\
\hline & - & - & - & - & - & - \\
\hline Nonfirm Power .................................................. & 187,125 & $4,698,308$ & - & - & - & - \\
\hline \multicolumn{7}{|l|}{ Homestead City of/Sep 30} \\
\hline 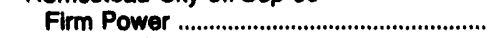 & 295 & 13,059 & - & - & - & - \\
\hline Nonfirm Power .................................................... & 5,985 & 257,319 & - & - & - & -- \\
\hline 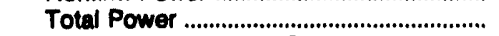 & 6,280 & 270,378 & - & - & - & - \\
\hline \multicolumn{7}{|l|}{ Jacksonville Elec Auth/Sep 30} \\
\hline 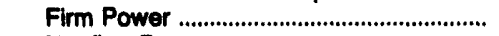 & 291,469 & $16,466,433$ & -- & - & - & - \\
\hline 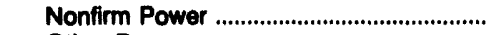 & 131,579 & $3,081,363$ & - & - & - & -- \\
\hline 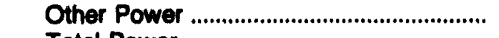 & 195 & 8,067 & - & - & - & - \\
\hline \multirow{2}{*}{\multicolumn{7}{|c|}{ Key West Utility Board of/Sep 30}} \\
\hline & - & - & - & - & - & - \\
\hline 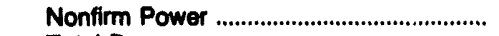 & - & - & - & - & - & - \\
\hline \multirow{2}{*}{\multicolumn{7}{|c|}{ Klssimmee Utility Auth/Sep 30}} \\
\hline & & & & & & \\
\hline 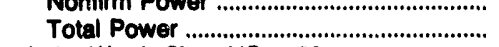 & 4,803 & 204,831 & $\overline{-}$ & - & $\overline{-}$ & $\overline{-}$ \\
\hline \multirow{2}{*}{\multicolumn{7}{|c|}{$\begin{array}{l}\text { Lake Worth City of/Sep } 30 \\
\text { Firm Power }\end{array}$}} \\
\hline & - & - & - & - & - & - \\
\hline 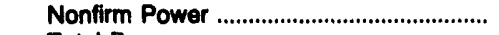 & 13,888 & 323,105 & - & -- & - & - \\
\hline \multicolumn{4}{|l|}{ Lakeland City of/Sep 30} & & & - \\
\hline 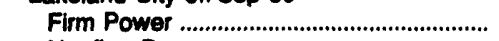 & 877 & 63,500 & - & - & - & - \\
\hline 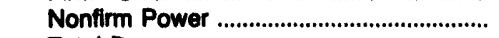 & 6,288 & 358,685 & - & - & - & - \\
\hline 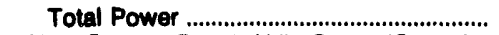 & 7,165 & 422,185 & - & - & -- & - \\
\hline \multicolumn{7}{|l|}{ Now Smyma Beach Utils Comm/Sep 30} \\
\hline 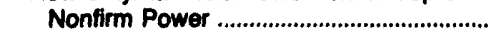 & 215 & 13,099 & - & - & -- & - \\
\hline 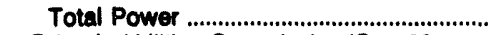 & 215 & 13,099 & - & - & - & - \\
\hline \multicolumn{7}{|l|}{ Orlando Utilities Commission/Sep 30} \\
\hline 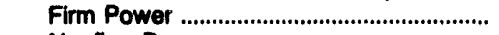 & 83,097 & $2,668,855$ & - & - & -- & - \\
\hline 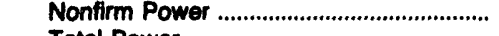 & 30,026 & $1,709,141$ & - & - & - & - \\
\hline $\begin{array}{l}\text { Total Power ......................................... } \\
\text { St Cloud City of Sep } 30\end{array}$ & 113,123 & $4,377,896$ & - & - & - & -- \\
\hline 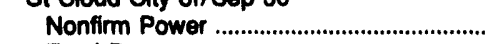 & - & - & - & - & - & - \\
\hline 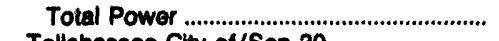 & - & - & - & - & - & - \\
\hline $\begin{array}{l}\text { Tallahassee City of/Sep } 30 \\
\text { Firm Power }\end{array}$ & & $4,076,053$ & - & - & - & - \\
\hline 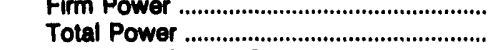 & $\begin{array}{l}135,310 \\
135,310\end{array}$ & $4,076,053$ & - & - & - & $\overline{-}$ \\
\hline Vero Beach City of/Sep 30 & & & & & & \\
\hline 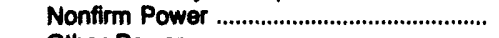 & 3,341 & 147,820 & - & - & - & - \\
\hline 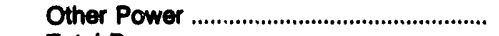 & & - & - & - & - & - \\
\hline 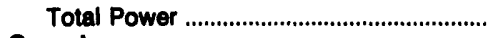 & 3,341 & 147,820 & - & - & - & - \\
\hline $\begin{array}{l}\text { Ceorgla } \\
\text { Dalion City of/Nov } 30\end{array}$ & & & & & & \\
\hline Firm Power ....................................................... & 44,625 & $1,093,234$ & -- & - & -- & - \\
\hline 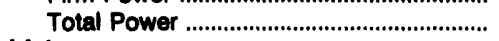 & 44,625 & $1,093,234$ & - & - & - & - \\
\hline $\begin{array}{l}\text { Idaho } \\
\text { Idaho Falls City of/Sep } 30\end{array}$ & & & & & & \\
\hline $\begin{array}{l}\text { Idaho Falls City of/Sep } 30 \\
\text { Firm Power }\end{array}$ & 41,298 & $1,801,802$ & 125,606 & $4,769,712$ & - & - \\
\hline 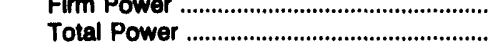 & 41,298 & $1,901,802$ & 125,606 & $4,769,712$ & - & - \\
\hline
\end{tabular}

See notes and footnotes at end of table. 
Table 30. Electricity Sales for Resale by Municipal Utilitles, Fiscal Year 1992 (Continued)

\begin{tabular}{|c|c|c|c|c|c|c|}
\hline \multirow{3}{*}{$\begin{array}{l}\text { State / Utility I } \\
\text { Flecal Yoar Ending Date }\end{array}$} & \multicolumn{6}{|c|}{ Purchaser of Electricity } \\
\hline & \multicolumn{2}{|c|}{ Cooperative } & \multicolumn{2}{|c|}{ Other' } & \multicolumn{2}{|c|}{ Total } \\
\hline & $\begin{array}{c}\text { Sales For } \\
\text { Pesale } \\
\text { (thousand kWh) }\end{array}$ & $\begin{array}{l}\text { Revenue } \\
\text { (dollars) }\end{array}$ & $\begin{array}{c}\text { Sales For } \\
\text { Pesale } \\
\text { (thousand kWh) }\end{array}$ & $\begin{array}{l}\text { Revenue } \\
\text { (dollars) }\end{array}$ & $\begin{array}{c}\text { Seles For } \\
\text { Pesale } \\
\text { (thousand kWh) }\end{array}$ & $\begin{array}{l}\text { Revenue } \\
\text { (dollars) }\end{array}$ \\
\hline \multicolumn{7}{|l|}{ Delaware } \\
\hline 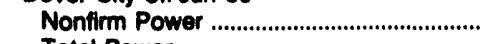 & - & - & - & - & 38,005 & 941,245 \\
\hline $\begin{array}{l}\text { Total Power .......................................................... } \\
\text { Florlda }\end{array}$ & - & - & - & - & 38,005 & 841,245 \\
\hline \multicolumn{7}{|l|}{$\begin{array}{l}\text { Florlda } \\
\text { Florlda Municipal Power Agency/Sep } 30\end{array}$} \\
\hline 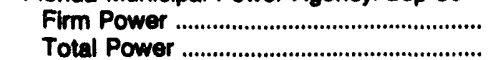 & $\overline{-}$ & $\overline{-}$ & $\begin{array}{l}2,917,046 \\
2,917,046\end{array}$ & $\begin{array}{l}154,382,603 \\
154,382,603\end{array}$ & $\begin{array}{l}3,171,561 \\
3,171,561\end{array}$ & $\begin{array}{l}157,294,021 \\
157,294,021\end{array}$ \\
\hline Fort Plerce Utilities Au/Sep 30 & & & & & & \\
\hline 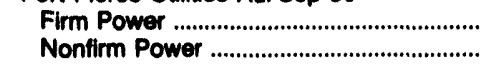 & $-\quad 158$ & $-7,041$ & $\begin{array}{r}73,363 \\
706\end{array}$ & $\begin{array}{r}2,073,335 \\
29,543\end{array}$ & $\begin{array}{r}73,363 \\
5,075\end{array}$ & $\begin{array}{r}2,073,335 \\
224,066\end{array}$ \\
\hline \multicolumn{7}{|l|}{ Gainesville City of/Sep 30} \\
\hline 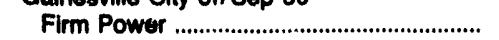 & 49,882 & $2,586,123$ & 293,937 & $10,574,735$ & 343,819 & $13,160,858$ \\
\hline 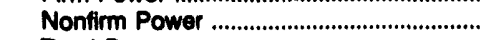 & 13,373 & 758,148 & 35,631 & 820,150 & 236,129 & $6,276,608$ \\
\hline \multirow{2}{*}{\multicolumn{7}{|c|}{ Homestead City of/Sep 30}} \\
\hline & & & & & & \\
\hline 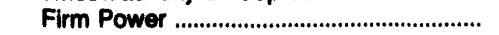 & - & - & 7,902 & 382,989 & 8,197 & 396,048 \\
\hline 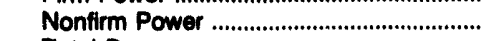 & 309 & 15,751 & 2,201 & 89,378 & 8.495 & 362,448 \\
\hline \multirow{2}{*}{\multicolumn{5}{|c|}{ Jacksonville Elec Auth/Sep 30}} & 16,692 & 758,496 \\
\hline & 35,450 & $1,558,560$ & -- & - & 326,928 & $18,024,993$ \\
\hline 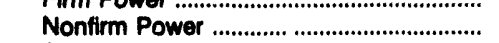 & 16,813 & 550,456 & 30,385 & 682,944 & 178,857 & $\begin{array}{r}18,024,883 \\
4,314,763\end{array}$ \\
\hline 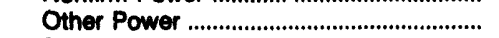 & 24,476 & $3,846,368$ & 268 & 298,441 & 24,939 & $4,153,874$ \\
\hline 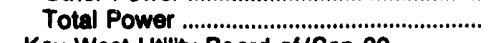 & 76,848 & $5,955,382$ & 30,633 & 882,385 & 530,724 & $26,493,630$ \\
\hline \multicolumn{7}{|l|}{ Key West Utility Board of/Sep 30} \\
\hline 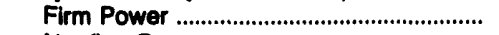 & - & - & 2,735 & 74,936 & 2,735 & 74,936 \\
\hline 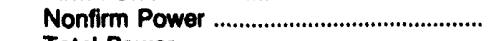 & 1,598 & 137,377 & - & - & 1,598 & 137,377 \\
\hline \multirow{2}{*}{\multicolumn{7}{|c|}{ Kissimmeo Utility Auth/Sep 30}} \\
\hline & & & & & & \\
\hline 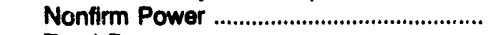 & 170 & 5,267 & 3,080 & 144,827 & 8,053 & 355,025 \\
\hline 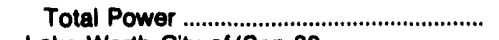 & 170 & 5,267 & 3,080 & 144,827 & 8,053 & 355,025 \\
\hline \multicolumn{7}{|l|}{ Lake Worth City of/Sep 30} \\
\hline 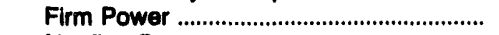 & - & - & 1.800 & 331,953 & 1,900 & 331,953 \\
\hline 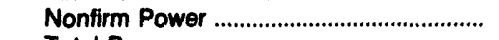 & 173 & 9,108 & 2,944 & 69,346 & 17,005 & 401,559 \\
\hline \multirow{2}{*}{\multicolumn{7}{|c|}{ Lakeland City of/Sep 30}} \\
\hline & & & & & & \\
\hline 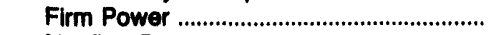 & - & - & $\$, 026$ & 451,695 & 4,803 & 515,195 \\
\hline 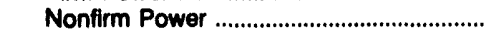 & - & -- & 43,797 & $1,124,422$ & 50,685 & $1,483,107$ \\
\hline 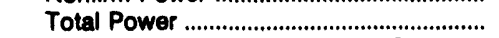 & - & - & 47,823 & $1,576,117$ & 54,988 & $1,998,302$ \\
\hline \multicolumn{7}{|l|}{ New Smyrna Beach Utils Comm/Sep 30} \\
\hline Nonfirm Power .................................................. & 50 & 3,420 & 30 & 1,739 & 295 & 18,258 \\
\hline 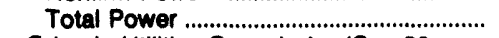 & 50 & 3,420 & 30 & 1,739 & 295 & 18,258 \\
\hline Orlando Utilities Commission/Sep 30 & & & & & & \\
\hline 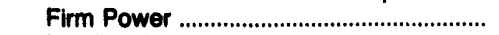 & - & - & 454,838 & $20,273,361$ & 537,935 & $22,942,216$ \\
\hline 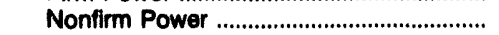 & 1,580 & 72,013 & 909,224 & $25,029,736$ & 940,830 & $26,810,890$ \\
\hline 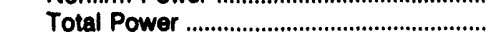 & 1,580 & 72,013 & $1,364,062$ & $45,303,097$ & $1,478,785$ & $49,753,106$ \\
\hline St Cloud City of/Sep 30 & & & & & & \\
\hline 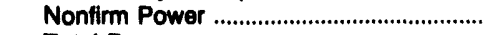 & 3 & 113 & - & - & 3 & 113 \\
\hline 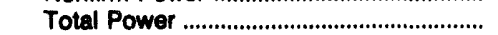 & 3 & 113 & - & - & 3 & 113 \\
\hline Tallahassee City of/Sep 30 & & & & & & \\
\hline 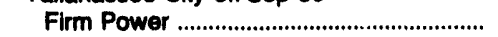 & 48,170 & $2,446,840$ & 11,301 & 296,507 & 194,781 & $6,819,400$ \\
\hline 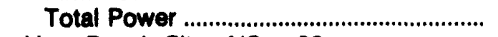 & 48,170 & $2,446,840$ & 11,301 & 296,507 & 194,781 & $6,819,400$ \\
\hline Vero Beach City of/Sep 30 & & & & & & \\
\hline 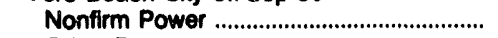 & 264 & 12,912 & 764 & 32,029 & 4,369 & 192,761 \\
\hline 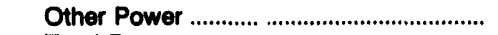 & - & & 230 & 12,178 & 230 & 12,178 \\
\hline 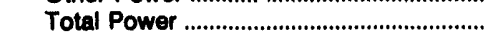 & 264 & 12,912 & 994 & 44,207 & 4,599 & 204,838 \\
\hline Coorgla & & & & & & \\
\hline Dalton City of/Nov 30 & & & & & & \\
\hline 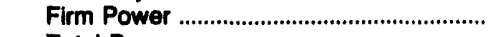 & - & -- & - & - & 44,625 & $1,093,234$ \\
\hline Total Power & - & - & - & -- & 44,625 & $1,083,234$ \\
\hline $\begin{array}{l}\text { Idaho } \\
\text { Idaho Falls City. of/Sep } 30\end{array}$ & & & & & & \\
\hline 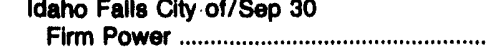 & - & -- & - & - & 166,904 & $6,671,514$ \\
\hline Total Power & - & - & -- & - & 166,904 & $6,671,514$ \\
\hline
\end{tabular}

See notes and footnotes at end of table. 
Table 30. Electriclty Sales for Resale by Municipal Utilities, Flscal Year 1992 (Continued)

\begin{tabular}{|c|c|c|c|c|c|c|}
\hline \multirow{3}{*}{$\begin{array}{l}\text { State / Utillty / } \\
\text { Fiscal Year Ending Dato }\end{array}$} & \multicolumn{6}{|c|}{ Purchaser of Electricity } \\
\hline & \multicolumn{2}{|c|}{ Investor-Owned } & \multicolumn{2}{|c|}{ Foderal } & \multicolumn{2}{|c|}{$\begin{array}{l}\text { State and Other } \\
\text { Government }\end{array}$} \\
\hline & $\begin{array}{c}\text { Sales For } \\
\text { Resale } \\
\text { (thousand kWh) }\end{array}$ & $\begin{array}{l}\text { Revenue } \\
\text { (dollars) }\end{array}$ & $\begin{array}{c}\text { Sales For } \\
\text { Resale } \\
\text { (thousand kWh) }\end{array}$ & $\begin{array}{l}\text { Revenue } \\
\text { (dollars) }\end{array}$ & $\begin{array}{c}\text { Sales For } \\
\text { Pesale } \\
\text { (thousand kWh) }\end{array}$ & $\begin{array}{l}\text { Revenue } \\
\text { (dollars) }\end{array}$ \\
\hline \multicolumn{7}{|l|}{ Iminols } \\
\hline 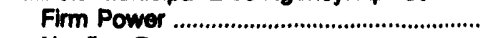 & -- & - & - & - & - & - \\
\hline Nonfirm Power .............................................. & - & - & - & - & -- & - \\
\hline 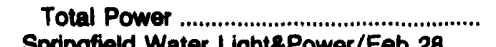 & - & - & - & - & - & - \\
\hline 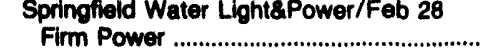 & - & - & - & - & - & - \\
\hline 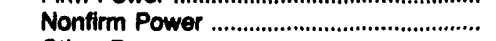 & 5,165 & 105,223 & - & - & - & - \\
\hline 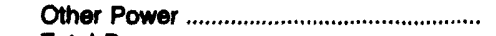 & 20,876 & 375,871 & - & - & -- & - \\
\hline 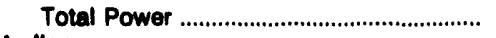 & 26,041 & 481,194 & - & -- & -- & - \\
\hline \multicolumn{7}{|l|}{$\begin{array}{l}\text { Indlans } \\
\text { Crawtordsville Elec Lgt\&Pwr Co/Dec } 31\end{array}$} \\
\hline 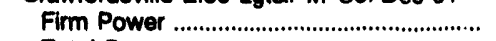 & - & - & - & - & 27.195 & $2,228,361$ \\
\hline \multirow{2}{*}{\multicolumn{7}{|c|}{ lowe }} \\
\hline & & & & & & \\
\hline \multicolumn{7}{|l|}{ Ames City of/Jun 30} \\
\hline 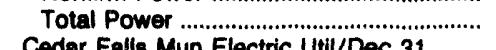 & 6,842 & 124,601 & - & - & - & - \\
\hline \multicolumn{7}{|l|}{ Codar Falls Mun Electric Util/Dec 31} \\
\hline $\begin{array}{l}\text { Firm Power ... } \\
\text { Nontirm Power }\end{array}$ & 90 & 3,607 & - & - & - & - \\
\hline & 17,107 & 251,554 & - & - & - & - \\
\hline \multirow{2}{*}{\multicolumn{7}{|c|}{ Muscatine Power and Water/Dec 31}} \\
\hline & & & & & & \\
\hline 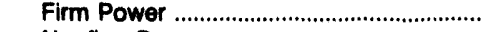 & 515,096 & $35,914,417$ & - & -- & - & -- \\
\hline 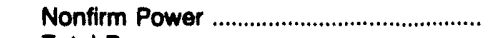 & 11,457 & 309,885 & 180 & 3,897 & 1,828 & 40,442 \\
\hline Total Power & 526,553 & $36,224,302$ & 180 & 3,897 & 1,828 & 40,442 \\
\hline \multirow{2}{*}{\multicolumn{7}{|c|}{$\begin{array}{l}\text { Kanaas } \\
\text { Kansas City City of/Dec } 31\end{array}$}} \\
\hline Kansas City City of/Dec 31 & & & & & & \\
\hline 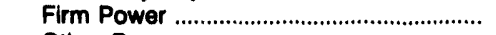 & 1,750 & 33,758 & - & -- & 264,500 & $8,701,892$ \\
\hline 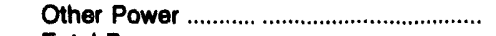 & - & - & - & -- & - & \\
\hline $\begin{array}{l}\text { Total Power } \\
\text { McPherson Board of Pub Utils/Dec } 31\end{array}$ & 1,750 & 33,758 & - & - & 264,500 & $8,701,892$ \\
\hline 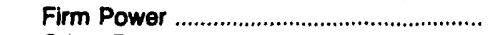 & -- & - & - & -- & - & - \\
\hline Other Power & 8,290 & $1,862,950$ & -- & -- & - & - \\
\hline 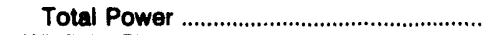 & 8,290 & $1,862,950$ & - & -- & -- & - \\
\hline Winfield City of/Dec 31 & & & & & & \\
\hline 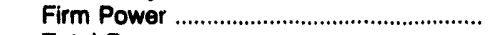 & - & - & -- & - & - & - \\
\hline 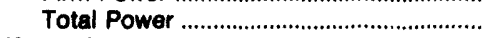 & -- & - & - & -- & - & - \\
\hline \multirow{2}{*}{\multicolumn{7}{|c|}{$\begin{array}{l}\text { Kentucky } \\
\text { Henderson City Utility Commiss/May } 31\end{array}$}} \\
\hline & & & & & & \\
\hline $\begin{array}{l}\text { Other Power } \\
\text { Total Power }\end{array}$ & $\overline{-}$ & - & - & - & - & - \\
\hline \multirow{2}{*}{\multicolumn{7}{|c|}{ Owensboro Munici,al Utilities/May 31}} \\
\hline & & & & & & \\
\hline $\begin{array}{l}\text { Firm Power } \\
\text { Total Power }\end{array}$ & $\begin{array}{l}1,526,274 \\
1,526,274\end{array}$ & $23,780,550$ & $\overline{-}$ & $\overline{-}$ & - & - \\
\hline $\begin{array}{l}\text { Total Power } \\
\text { Loulelana }\end{array}$ & $1,526,274$ & $23,780,550$ & - & - & - & - \\
\hline Lafayette City of/Oct 31 & & & & & & \\
\hline 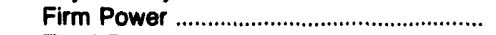 & 23 & 805 & - & -- & 367,981 & $13,946,898$ \\
\hline $\begin{array}{l}\text { Total Power ... } \\
\text { Lafayette Pulic Power Auth/Oct } 31\end{array}$ & 23 & 805 & -- & -- & 367,981 & $13,946,898$ \\
\hline 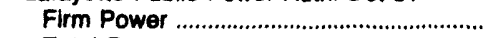 & - & - & - & -- & - & -- \\
\hline Total Power ... & - & - & - & - & -- & - \\
\hline Terrebonne Parish Consol Govt/Dec 31 & & & & & & \\
\hline 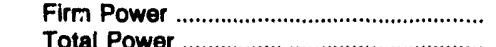 & $\cdots$ & - & - & - & 102,391 & $3,109,413$ \\
\hline 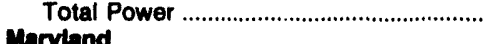 & - & - & - & - & 102,391 & $3,109,413$ \\
\hline $\begin{array}{l}\text { Maryland } \\
\text { Easton Utilities Commission/Jun } 30\end{array}$ & & & & & & \\
\hline Other Power & -- & - & -- & - & - & .- \\
\hline Total Power & - & - & - & - & - & - \\
\hline Maseachusefts & & & & & & \\
\hline Braintree Town of/Dec 31 & & & & & & \\
\hline 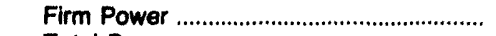 & 25,649 & $1,630,913$ & - & - & - & - \\
\hline Total Power & 25,649 & $1,630,913$ & - & - & - & - \\
\hline Chicopee City of/Dec 31 & & & & & & \\
\hline 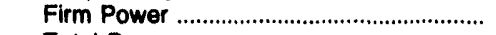 & - & - & - & - & 300 & 7,800 \\
\hline 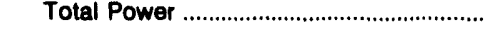 & - & - & - & -- & 300 & 7,800 \\
\hline
\end{tabular}

See notes and footnotes at end of table. 
Table 30. Electricity Sales for Resale by Municipal Utilitles, Flecal Year 1992 (Continued)

\begin{tabular}{|c|c|c|c|c|c|c|}
\hline \multirow{3}{*}{$\begin{array}{l}\text { State / Utility / } \\
\text { Flecal Year Ending Date }\end{array}$} & \multicolumn{6}{|c|}{ Purchaser of Electricity } \\
\hline & \multicolumn{2}{|c|}{ Cooperative } & \multicolumn{2}{|c|}{ Other' } & \multicolumn{2}{|c|}{ Total } \\
\hline & $\begin{array}{c}\text { Sales For } \\
\text { Pesale } \\
\text { (thousand kW/h) }\end{array}$ & $\begin{array}{l}\text { Revenue } \\
\text { (dollars) }\end{array}$ & $\begin{array}{c}\begin{array}{c}\text { Sales For } \\
\text { Resale } \\
\text { (thousand kWh) }\end{array} \\
\end{array}$ & $\begin{array}{l}\text { Revenue } \\
\text { (dollars) }\end{array}$ & $\begin{array}{c}\text { Sales For } \\
\text { Resale } \\
\text { (thousand kWh) }\end{array}$ & $\begin{array}{l}\text { Revenue } \\
\text { (dollars) }\end{array}$ \\
\hline \multicolumn{7}{|l|}{$\begin{array}{l}\text { Illinole } \\
\text { Illinois Municipal E }\end{array}$} \\
\hline 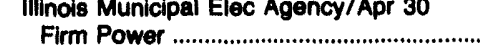 & - & - & $1,281,982$ & $57,601,575$ & $1,281,982$ & $57,601,575$ \\
\hline 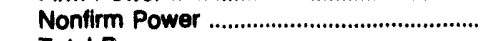 & - & - & 26,292 & 771,466 & 26,292 & 771,466 \\
\hline $\begin{array}{l}\text { Total Power ... } \\
\text { Sorinofield Water Liaht8Power/Feb } 28\end{array}$ & - & - & $1,308,274$ & & & $58,373,041$ \\
\hline 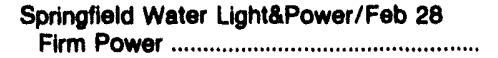 & & & & & & \\
\hline $\begin{array}{l}\text { Firm Power } \\
\text { Nonfirm Power }\end{array}$ & - & - & 94,433 & $3,079,547$ & 94,433 & $3,079,547$ \\
\hline Nonfirm Power ................................................ & - & - & - & - & 5,165 & 105,223 \\
\hline $\begin{array}{l}\text { Other Power } \\
\text { Total Power }\end{array}$ & - & - & - & - & 20,876 & 375,971 \\
\hline Total Power ......................................................... & - & - & 94,433 & $3,079,547$ & 120,474 & $3,560,741$ \\
\hline $\begin{array}{l}\text { Indlana } \\
\text { Crawfordsville Elec Lgt\&PWr Co/Doc } 31\end{array}$ & & & & & & \\
\hline $\begin{array}{l}\text { Crawfordsville Elec Lgt\&Pwr Co/Dec } 31 \\
\text { Firm Power }\end{array}$ & & & & & & \\
\hline $\begin{array}{l}\text { Firm Power } \\
\text { Total Power }\end{array}$ & - & - & - & - & $27.19 \mathrm{E}$ & $2,228,361$ \\
\hline $\begin{array}{l}\text { Total Power ...................................................... } \\
\text { lowa }\end{array}$ & - & -- & -- & -- & 27,195 & $2,228,3^{r}, 1$ \\
\hline \multicolumn{7}{|l|}{$\begin{array}{l}\text { lowa } \\
\text { Ames City of/Jun } 30\end{array}$} \\
\hline 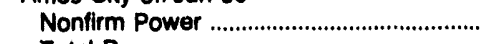 & - & -- & 358 & 7,250 & 7,300 & 131,851 \\
\hline Total Power & - & - & 358 & 7,250 & 7,300 & 131,851 \\
\hline \multicolumn{7}{|l|}{ Cedar Falls Mun Electric Util/Dec 31} \\
\hline 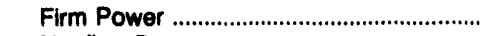 & -- & -- & -- & - & 80 & 3,607 \\
\hline 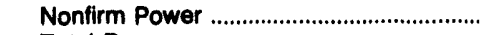 & - & - & -- & - & 17,107 & 251,554 \\
\hline 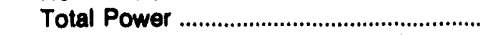 & - & - & -- & - & 17,197 & 255,161 \\
\hline \multicolumn{7}{|l|}{ Muscatine Power and Water/Dec 31} \\
\hline 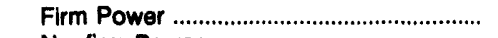 & -- & - & - & - & 515,096 & $35,914,417$ \\
\hline Nonfirm Power & 4,386 & 106,463 & 25 & 675 & 17,876 & 461,362 \\
\hline Total Power & 4,386 & 106,463 & 25 & 675 & 532,972 & $36,375,779$ \\
\hline \multirow{2}{*}{\multicolumn{7}{|c|}{$\begin{array}{l}\text { Kaneass } \\
\text { Kansas City City of/Dec } 31\end{array}$}} \\
\hline & & & & & & \\
\hline 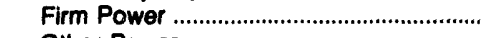 & - & -- & 147,770 & $4,884,127$ & 414,020 & $13,619,777$ \\
\hline 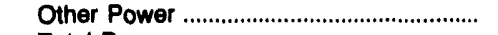 & - & - & - & 38,047 & & 38,047 \\
\hline 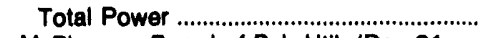 & - & - & 147,770 & $4,822,174$ & 414,020 & $13,657,824$ \\
\hline \multicolumn{7}{|l|}{ McPherson Board of Pub Utils/Dec 31} \\
\hline 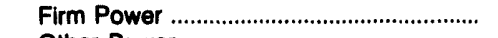 & - & -- & 18,893 & 854,634 & 18,893 & 854,634 \\
\hline 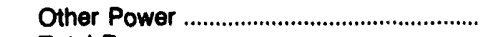 & - & -- & -- & - & 8,290 & $1,862,950$ \\
\hline 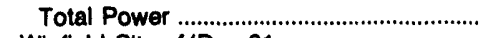 & - & -- & 18,893 & 854,634 & 27,183 & $2,717,584$ \\
\hline \multicolumn{7}{|l|}{ Winfield City of/Dec 31} \\
\hline 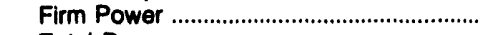 & - & - & 4,243 & 194,400 & 4,243 & 194,400 \\
\hline 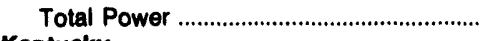 & - & -- & 4,243 & 194,400 & 4,243 & 194,400 \\
\hline Kentucky & & & & & & \\
\hline Henderson City Utility Commiss/May 31 & & & & & & \\
\hline Other Power & $1,411,007$ & $12,428,314$ & - & - & $1,411,007$ & $12,428,314$ \\
\hline Total Power & $1,411,007$ & $12,428,314$ & - & - & $1,411,007$ & $12,428,314$ \\
\hline Owensboro Municipal Utilities/May 31 & & & & & & \\
\hline 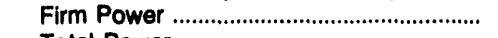 & - & - & - & - & $1,526,274$ & $23,780,550$ \\
\hline 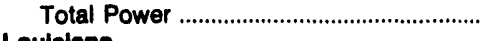 & - & - & - & - & $1,526,274$ & $23,780,550$ \\
\hline $\begin{array}{l}\text { Loulalana } \\
\text { Lafayette City of/Oct } 31\end{array}$ & & & & & & \\
\hline 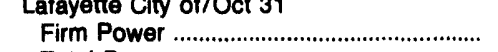 & - & - & 192,647 & $6,454,473$ & 560,651 & $20,402,176$ \\
\hline Total Power & - & - & 192,647 & $6,454,473$ & 560,651 & $20,402,176$ \\
\hline Lafayette Public Power Auth/Oct 31 & & & & & & \\
\hline Firm Power & - & -- & $1,418,646$ & $49,937,278$ & $1,418,646$ & $49,937,278$ \\
\hline Total Power & - & - & $1,418,646$ & $49,937,278$ & $1,418,646$ & $49,937,278$ \\
\hline Terrebonne Parish Consol Govt/Dec 31 & & & & & & \\
\hline 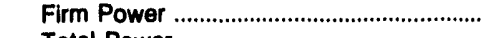 & - & - & - & - & 102,391 & $3,109,413$ \\
\hline 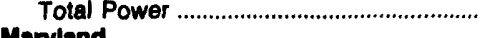 & - & - & - & -- & 102,391 & $3,109,413$ \\
\hline $\begin{array}{l}\text { Maryland } \\
\text { Easton Utilities Commission/Jun } 30\end{array}$ & & & & & & \\
\hline 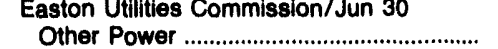 & 2,759 & 290,227 & - & & 2,759 & 290.227 \\
\hline $\begin{array}{l}\text { Other Power } \\
\text { Total Power }\end{array}$ & 2,759 & 290,227 & - & - & 2,759 & $\begin{array}{l}290,227 \\
290,227\end{array}$ \\
\hline $\begin{array}{l}\text { Total Power } \\
\text { Masasachueotts }\end{array}$ & & & & & 2,159 & 280,221 \\
\hline Braintree Town of/Dec 31 & & & & & & \\
\hline 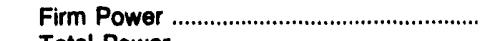 & -- & - & 4,151 & 557,888 & 29,800 & $2,188,801$ \\
\hline Total Power & - & - & 4,151 & 557,888 & 29,800 & $2,188,801$ \\
\hline Chicopee City of/Dec 31 & & & & & & \\
\hline 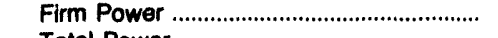 & - & - & 28,640 & $1,797,238$ & 28,940 & $1,805,038$ \\
\hline 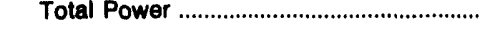 & - & - & 28,640 & $1,797,238$ & 28,940 & $1,805,038$ \\
\hline
\end{tabular}

See notes and footnotes at end of table. 
Table 30. Electriclty Sales for Resale by Municlpal Utilities, Fiscal Year 1992 (Continued)

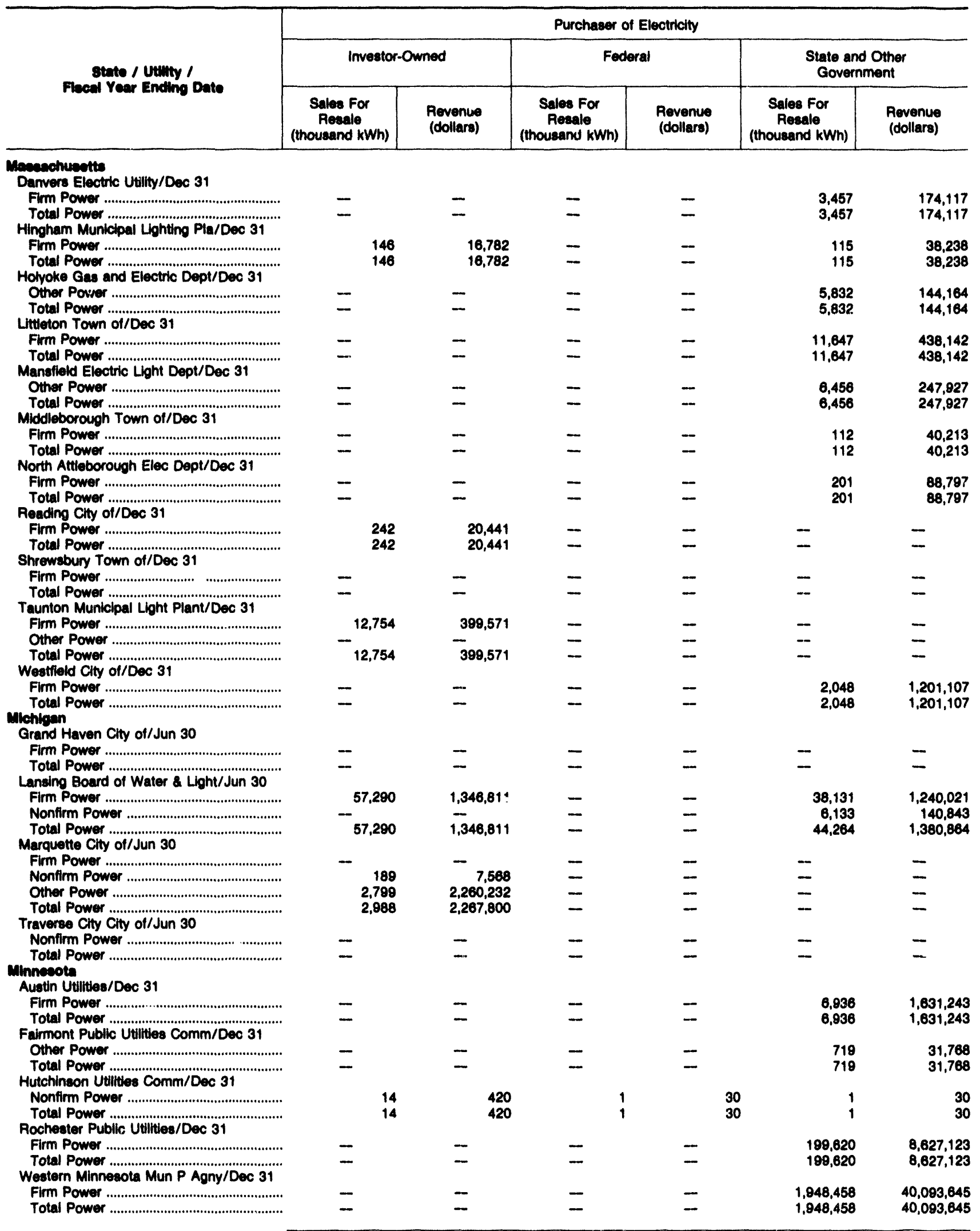

See notes and footnotes at end of table. 
Table 30. Electricity Sales for Resale by Municipal Utilities, Fiscal Year 1992 (Continued)

\begin{tabular}{|c|c|c|c|c|c|c|}
\hline \multirow{3}{*}{$\begin{array}{l}\text { State / Utilly I } \\
\text { Flecal Year Ending Doto }\end{array}$} & \multicolumn{6}{|c|}{ Purchaser of Electricity } \\
\hline & \multicolumn{2}{|c|}{ Cooperattve } & \multicolumn{2}{|c|}{ Other' } & \multicolumn{2}{|c|}{ Total } \\
\hline & $\begin{array}{c}\text { Sales For } \\
\text { Resale } \\
\text { (thousand kWh) }\end{array}$ & $\begin{array}{l}\text { Revenue } \\
\text { (dollare) }\end{array}$ & $\begin{array}{c}\text { Sales For } \\
\text { Reasle } \\
\text { (thousand kWh) }\end{array}$ & $\begin{array}{l}\text { Revenue } \\
\text { (dollars) }\end{array}$ & $\begin{array}{c}\text { Sales For } \\
\text { Peade } \\
\text { (thousand kWh) }\end{array}$ & $\begin{array}{l}\text { Revenue } \\
\text { (dollars) }\end{array}$ \\
\hline \multicolumn{7}{|l|}{$\begin{array}{l}\text { Maseachuseotts } \\
\text { Danvers Electric Utility/Doc } 31\end{array}$} \\
\hline 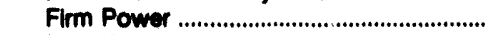 & - & - & - & - & 3,457 & 174,117 \\
\hline 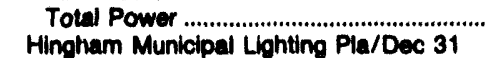 & - & - & - & - & 3,457 & 174,117 \\
\hline 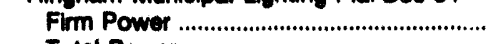 & - & - & - & - & 261 & 56,020 \\
\hline $\begin{array}{l}\text { Total Power ... } \\
\text { Holyoke Ges and Electric Dept/Dec } 31\end{array}$ & - & - & - & - & 281 & 55,020 \\
\hline 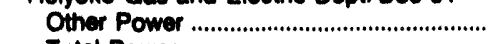 & - & - & - & - & 5,832 & 144,164 \\
\hline $\begin{array}{l}\text { Total Power .................................... } \\
\text { Llttioton Town of/Dec } 31\end{array}$ & - & - & - & - & 5,832 & 144,164 \\
\hline 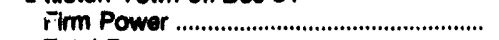 & - & - & - & - & 11,647 & 438,142 \\
\hline $\begin{array}{l}\text { Total Power } \\
\text { Mansfleld Electric Light Dept/Dec } 31\end{array}$ & - & - & - & - & 11,647 & 438,142 \\
\hline 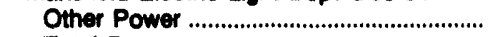 & - & - & - & - & 6,456 & 247,827 \\
\hline 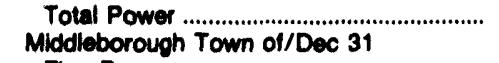 & - & - & - & - & 6,456 & 247,927 \\
\hline 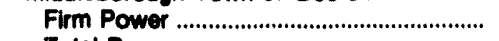 & - & - & - & - & 112 & 40,213 \\
\hline $\begin{array}{l}\text { Total Power .... } \\
\text { North Attleborough Elec Dept/Dec } 31\end{array}$ & - & - & $\sim$ & - & 112 & 40,213 \\
\hline 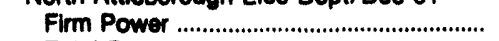 & - & - & - & - & 201 & 88,797 \\
\hline $\begin{array}{l}\text { Total Power ......................................... } \\
\text { Ragading City of/Dec }\end{array}$ & - & - & - & - & 201 & 88,797 \\
\hline 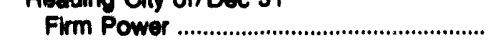 & - & - & 390 & 43,871 & 632 & $6: 412$ \\
\hline $\begin{array}{l}\text { Total Power .................................... } \\
\text { Shrewsbury Town of/Dec } 31\end{array}$ & - & - & 390 & 43,871 & 632 & 64,412 \\
\hline 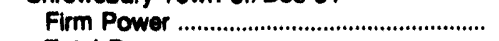 & - & - & 1,329 & 108,073 & 1,329 & 108,073 \\
\hline $\begin{array}{l}\text { Total Power ................................... } \\
\text { Taunton Municipl Liaht Plant/Dec }\end{array}$ & - & - & 1,328 & 108,073 & 1,320 & 108,073 \\
\hline 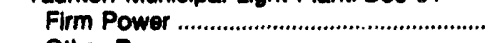 & - & - & 13,987 & 265,588 & 26,741 & 665,159 \\
\hline 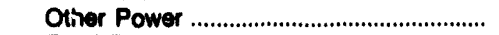 & - & - & 23,680 & $3,280,445$ & 23,680 & $3,280,445$ \\
\hline $\begin{array}{l}\text { Total Power ........................................ } \\
\text { Weatfield City of/Dec } 31\end{array}$ & - & - & 37,667 & $3,546,033$ & 50,421 & $3,945,604$ \\
\hline 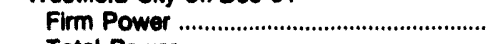 & - & - & 7,321 & 614,541 & 9,369 & $1,815,648$ \\
\hline 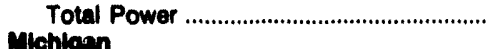 & - & - & 7,321 & 614,541 & 9,369 & $1,815,648$ \\
\hline $\begin{array}{l}\text { Mlohlgan } \\
\text { Grand Haven City of/Jun } 30\end{array}$ & & & & & & \\
\hline 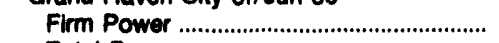 & 131,828 & $3,305,020$ & - & - & 131,828 & $3,305,020$ \\
\hline $\begin{array}{l}\text { Total Power ....................... } \\
\text { Lansing Board of L Light/Jun } 30\end{array}$ & 131,828 & $3,305,020$ & - & - & 131,828 & $3,305,020$ \\
\hline $\begin{array}{l}\text { Lansing Board of Water Light/Jun } 30 \\
\text { Firm Power }\end{array}$ & 5,381 & 308,290 & 80,708 & $2,867,589$ & 181,510 & $5,762,711$ \\
\hline 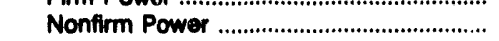 & - & & - & - & 6,133 & 140,843 \\
\hline Total Power & 5,381 & 308,290 & 80,708 & $2,867,589$ & 187,643 & $5,903,554$ \\
\hline Marquette Clity of/Jun 30 & & & & & & \\
\hline 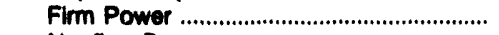 & 10,386 & 636,969 & - & -- & 10,386 & 636,869 \\
\hline 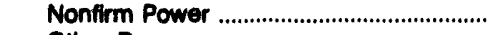 & -- & - & - & - & 189 & 7,568 \\
\hline 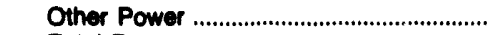 & - & - & - & - & 2,799 & $2,260,232$ \\
\hline $\begin{array}{l}\text { Total Power .................................... } \\
\text { Traverse City City of Jun }\end{array}$ & 10,386 & 636,969 & - & - & 13,374 & $2,804,769$ \\
\hline $\begin{array}{l}\text { Traverse Clty City of/Jun } 30 \\
\text { Nonfirm Power }\end{array}$ & 6,747 & 114,872 & - & - & 6,747 & 114,872 \\
\hline 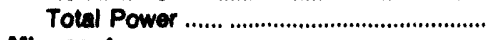 & 6,747 & 114,872 & - & - & 6,747 & 114,872 \\
\hline $\begin{array}{l}\text { Minnesota } \\
\text { Austin Utilities/Dec } 31\end{array}$ & & & & & & \\
\hline 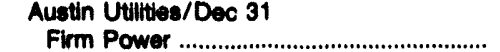 & - & - & - & - & 6,836 & $1,631,243$ \\
\hline Total Power & - & - & - & - & 6,936 & $1,631,243$ \\
\hline Fairmont Public Utilties Comm/Dec 31 & & & & & & \\
\hline 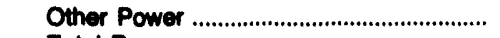 & - & - & - & - & 718 & 31,768 \\
\hline $\begin{array}{l}\text { Total Power . } \\
\text { Hutchineon Utilities Comm/Dec } 31\end{array}$ & - & - & - & - & 718 & 31.788 \\
\hline Nonfirm Power ......................................... & - & - & 1 & 30 & 17 & 510 \\
\hline 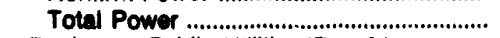 & - & - & 1 & 30 & 17 & 510 \\
\hline Rocheater Public Utilities/Dec 31 & & & & & & \\
\hline 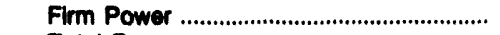 & - & - & - & - & 199,620 & $8,627,123$ \\
\hline Total Power .... & - & - & - & - & 199,620 & $8,627,123$ \\
\hline 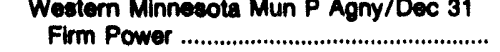 & - & - & - & - & $1,848,458$ & $40,093,645$ \\
\hline 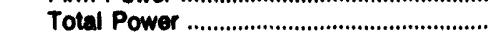 & - & - & - & - & $1,948,458$ & $40,093,646$ \\
\hline
\end{tabular}

See notes and footnotes at end of table. 
Table 30. Electriclty Sales for Resale by Municlpal Utilities, Fiecal Year 1992 (Continued)

\begin{tabular}{|c|c|c|c|c|c|c|}
\hline \multirow{3}{*}{$\begin{array}{l}\text { State / Utulty I } \\
\text { Freed Year Ending Date }\end{array}$} & \multicolumn{6}{|c|}{ Purchaser of Electricity } \\
\hline & \multicolumn{2}{|c|}{ Investor-Owned } & \multicolumn{2}{|c|}{ Federal } & \multicolumn{2}{|c|}{$\begin{array}{l}\text { State and Other } \\
\text { Government }\end{array}$} \\
\hline & $\begin{array}{c}\text { Sales For } \\
\text { Resale } \\
\text { (thousand kWh) }\end{array}$ & $\begin{array}{l}\text { Revenue } \\
\text { (dollars) }\end{array}$ & $\begin{array}{c}\text { Sales For } \\
\text { Resale } \\
\text { (thousand kWh) }\end{array}$ & $\begin{array}{l}\text { Revenue } \\
\text { (dollars) }\end{array}$ & $\begin{array}{c}\text { Sales For } \\
\text { Resale } \\
\text { (thousand } \mathrm{kWh} \text { ) }\end{array}$ & $\begin{array}{l}\text { Revenue } \\
\text { (dollars) }\end{array}$ \\
\hline \multicolumn{7}{|l|}{$\begin{array}{l}\text { Clarkedale Water \& Light Dept/Sep } 30 \\
\text { Shalapol }\end{array}$} \\
\hline 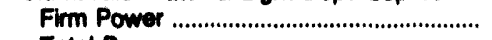 & - & - & - & - & - & - \\
\hline $\begin{array}{l}\text { Total Power ............................................ } \\
\text { Greenwood Utilties Commission/Sep } 30\end{array}$ & - & - & - & - & - & - \\
\hline 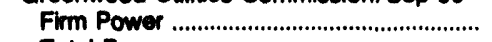 & - & - & - & - & - & - \\
\hline $\begin{array}{l}\text { Total Power ......................................... } \\
\text { Municipal Energy Agnoy of MS/Sep }\end{array}$ & - & - & - & - & - & - \\
\hline 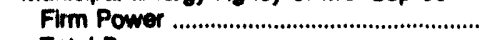 & - & - & - & - & 99,314 & $3,427,491$ \\
\hline $\begin{array}{l}\text { Total Power } \\
\text { Mlesount } \\
\text { Independence City of/Jun } 30\end{array}$ & - & \multicolumn{4}{|c|}{ Independence City of/Jun 30} & $3,427,491$ \\
\hline 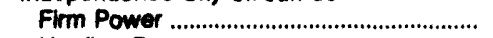 & 1,807 & 59,063 & - & - & - & - \\
\hline 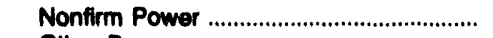 & 10,620 & 182,172 & - & - & - & -- \\
\hline 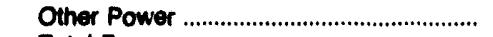 & - & 805,000 & - & - & - & - \\
\hline \multicolumn{7}{|l|}{ Skeston Board of Mun Utils/May 31} \\
\hline 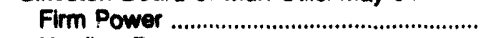 & - & - & - & - & - & $\cdots$ \\
\hline 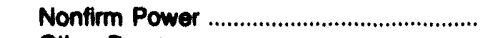 & - & - & - & - & 12,089 & 210,688 \\
\hline 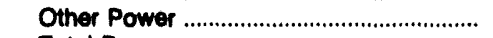 & - & - & - & - & - & - \\
\hline $\begin{array}{l}\text { Total Power ....................... } \\
\text { Springfield City of/Sep } 30\end{array}$ & - & - & - & - & 12,089 & 210,688 \\
\hline 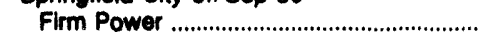 & - & - & - & - & - & - \\
\hline 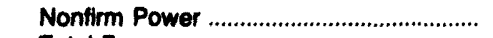 & 80 & 4,000 & - & - & - & - \\
\hline Total Power .......................................... & 90 & 4,000 & - & - & - & - \\
\hline $\begin{array}{l}\text { Nebrecka } \\
\text { Fremont City of/Jul } 31\end{array}$ & & & & & & \\
\hline 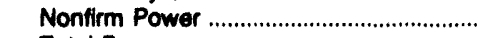 & - & - & - & - & 4,884 & 88,807 \\
\hline \multicolumn{7}{|l|}{ Grand Island Electric Dept/Jul 31} \\
\hline 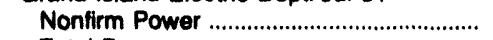 & - & - & - & - & 78,460 & $1,035,559$ \\
\hline 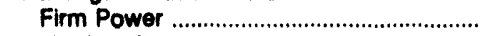 & - & - & - & - & 96,055 & $1,078,911$ \\
\hline 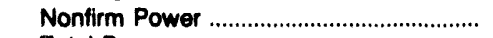 & - & - & - & - & 3,630 & 59,535 \\
\hline 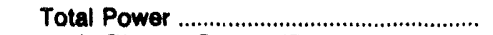 & - & - & - & - & 99,685 & $1,138,446$ \\
\hline \multicolumn{7}{|l|}{ Lincoln Electric System/Dec 31} \\
\hline 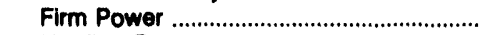 & - & - & - & - & - & - \\
\hline Nonfirm Power & $\begin{array}{l}480,979 \\
480,979\end{array}$ & $\begin{array}{l}6,931,239 \\
6,931,239\end{array}$ & $\begin{array}{l}79,547 \\
79,547\end{array}$ & $\begin{array}{l}1,155,786 \\
1,155,786\end{array}$ & $\begin{array}{l}41,369 \\
41,369\end{array}$ & $\begin{array}{l}590,399 \\
590,399\end{array}$ \\
\hline \multicolumn{7}{|l|}{$\begin{array}{l}\text { Now Mexico } \\
\text { Farmington City of/Jun } 30\end{array}$} \\
\hline Firm Power . & - & - & - & - & - & - \\
\hline 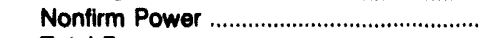 & 265 & 5,950 & 5,931 & 99,961 & -- & - \\
\hline Total Power ..................................... & \multirow{2}{*}{\multicolumn{6}{|c|}{ Los Alamos County of/Jun 30}} \\
\hline 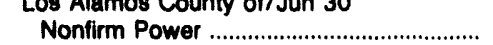 & 19,571 & 358,675 & - & -- & 0,540 & \\
\hline $\begin{array}{l}\text { Total Power ................................................. } \\
\text { Now York }\end{array}$ & 19,571 & 358,675 & - & - & 9,540 & 168,003 \\
\hline Jamestown City of/Dec 31 & & \\
\hline 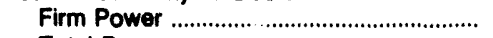 & 165,565 & $4,277,105$ & - & - & - & - \\
\hline 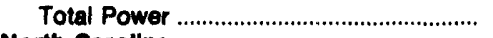 & 165,585 & $4,277,105$ & - & - & - & - \\
\hline \multicolumn{7}{|l|}{$\begin{array}{l}\text { North Carollns } \\
\text { Fayetteville Public Works Comm/Jun } 30\end{array}$} \\
\hline 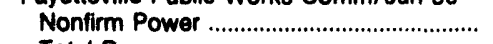 & 843 & 20,635 & - & - & -- & - \\
\hline Total Power .................................. & 843 & 20,635 & - & - & - & - \\
\hline 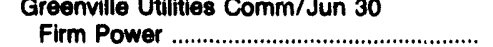 & - & - & - & & -- & - \\
\hline 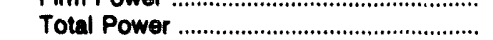 & - & - & - & - & - & $\overline{-}$ \\
\hline Rocky Mount City of/Jun 30 & & & & & & \\
\hline Firm Power . & - & - & - & - & - & - \\
\hline 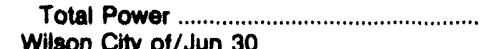 & - & - & - & - & - & - \\
\hline $\begin{array}{l}\text { Wison City of/Jun } 30 \\
\text { Firm Power }\end{array}$ & - & - & - & - & - & - \\
\hline 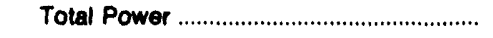 & - & - & - & - & - & - \\
\hline
\end{tabular}

See notes and footnotes at end of table. 
Table 30. Electricity Sales for Resale by Municipal Utilities, Fiscal Year 1992 (Continued)

\begin{tabular}{|c|c|c|c|c|c|c|}
\hline \multirow{3}{*}{$\begin{array}{l}\text { State / Utilty I } \\
\text { Flecal Year Ending Date }\end{array}$} & \multicolumn{6}{|c|}{ Purchaser of Electricity } \\
\hline & \multicolumn{2}{|c|}{ Cooperative } & \multicolumn{2}{|c|}{ Other' } & \multicolumn{2}{|c|}{ Total } \\
\hline & $\begin{array}{c}\text { Sales For } \\
\text { Resale } \\
\text { (thousand kWh) }\end{array}$ & $\begin{array}{l}\text { Revenue } \\
\text { (dollars) }\end{array}$ & $\begin{array}{c}\text { Sales For } \\
\text { Resale } \\
\text { (thousand kWh) }\end{array}$ & $\begin{array}{l}\text { Revenue } \\
\text { (dollars) }\end{array}$ & $\begin{array}{c}\text { Sales For } \\
\text { Resale } \\
\text { (thousand kWh) }\end{array}$ & $\begin{array}{l}\text { Revenue } \\
\text { (dollars) }\end{array}$ \\
\hline \multicolumn{7}{|l|}{$\begin{array}{l}\text { Mlaclasippl } \\
\text { Clarksdale Water \& Light Dept/Sep } 30\end{array}$} \\
\hline Firm Power . & - & -- & 78,035 & $2,302,796$ & 78,035 & $2,302,796$ \\
\hline \multicolumn{5}{|l|}{ Greenwood Utilities Commission/Sep 30} & 78,035 & $2,302,796$ \\
\hline 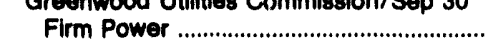 & - & -- & 11,508 & 932,818 & 11,508 & 932,818 \\
\hline & - & 11,508 & 932,818 & 11,508 & 932,818 \\
\hline 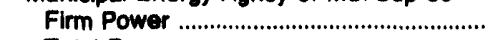 & - & - & 673,120 & $24,040,373$ & 772,434 & $27,467,864$ \\
\hline \\
\hline Independence City of/Jun 30 & & & & & & \\
\hline 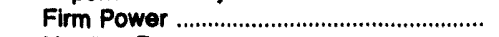 & - & -- & - & -- & 1,807 & 59,063 \\
\hline 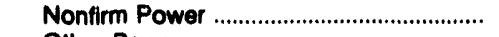 & 120 & 1,901 & - & - & 10,740 & 184,073 \\
\hline 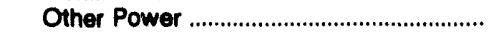 & - & -- & - & - & - & 805,000 \\
\hline $\begin{array}{l}\text { Total Power .................................. } \\
\text { Sikeston Board of Mun Utis/May }\end{array}$ & 20 & 1,901 & - & - & 12,547 & $1,048,136$ \\
\hline 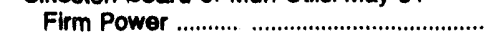 & -- & - & 681,550 & $28,947,452$ & 661,550 & $28,947,452$ \\
\hline 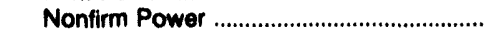 & & - & 533,154 & $9,535,522$ & 545,243 & $9,746,210$ \\
\hline 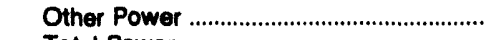 & 9,653 & $2,032,237$ & 74,054 & $2,604,732$ & 83,707 & $4,636,969$ \\
\hline $\begin{array}{l}\text { Total Power . } \\
\text { Springtild City of /Sep } 30\end{array}$ & 9,653 & $2,032,237$ & $1,268,758$ & $41,087,706$ & $1,290,500$ & $43,330,631$ \\
\hline 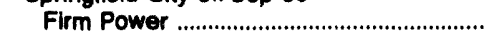 & -- & - & 51,195 & $2,873,000$ & 51,195 & $2,873,000$ \\
\hline 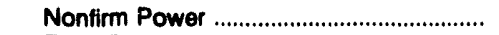 & - & - & - & - & 90 & 4,000 \\
\hline 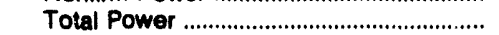 & -- & -- & 51,195 & $2,873,000$ & 51,285 & $2,877,000$ \\
\hline \multicolumn{7}{|l|}{ 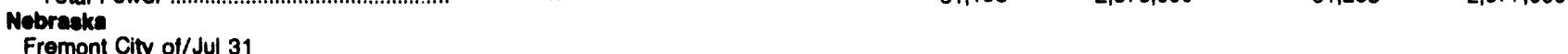 } \\
\hline Nonfirm Power ....................................... & - & -- & - & -- & 4,884 & 88,907 \\
\hline 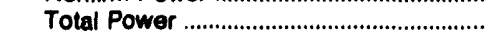 & -- & -- & -- & -- & 4,884 & 88,907 \\
\hline \multicolumn{7}{|l|}{ Grand Island Electric Dept/Jul 31} \\
\hline 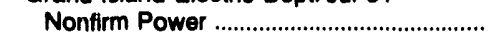 & -- & - & -- & -- & 78,460 & $1,035,559$ \\
\hline \multirow{2}{*}{\multicolumn{7}{|c|}{ Hastings Utilities/Dec 31}} \\
\hline 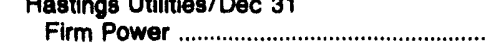 & -- & & & & & \\
\hline 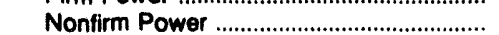 & $\overline{-}$ & $\overline{-}$ & - & - & 96,055 & $1,078,911$ \\
\hline 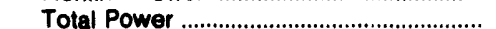 & -- & $\overline{-}$ & - & -- & 3,630 & $\begin{array}{r}59,535 \\
138,446\end{array}$ \\
\hline \multicolumn{7}{|l|}{ Lincoln Electric System/Dec 31} \\
\hline 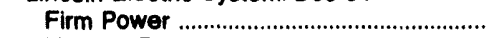 & -- & - & 80,261 & $2,151,386$ & 80,261 & $2,151,386$ \\
\hline Nonfirm Power & 336,826 & $3,996,954$ & 625 & 8,740 & 939,346 & $12,683,118$ \\
\hline 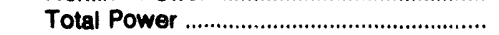 & 336,826 & $3,996,954$ & 80,886 & $2,160,126$ & $1,019,607$ & $14,834,504$ \\
\hline \multicolumn{7}{|l|}{$\begin{array}{l}\text { Now Moxico } \\
\text { Farmington }\end{array}$} \\
\hline Firm Power ......................................... & - & - & 15,692 & 906,016 & 15,692 & 906,016 \\
\hline 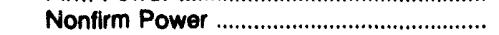 & - & -- & - & - & 6,196 & 105,911 \\
\hline 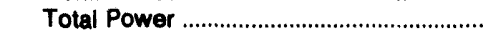 & - & - & 15,692 & 906,016 & 21,888 & $1,011,927$ \\
\hline \multicolumn{7}{|l|}{ Los Alamos County of/Jun 30} \\
\hline 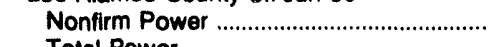 & 250 & 3,525 & 6,673 & 111,585 & 36,034 & 641,788 \\
\hline $\begin{array}{l}\text { Total Power ............................................. } \\
\text { Now York }\end{array}$ & 250 & 3,525 & 6,673 & 111,585 & 36,034 & 641,788 \\
\hline \multicolumn{7}{|l|}{$\begin{array}{l}\text { Now York } \\
\text { Jamestown City of/Dec } 31\end{array}$} \\
\hline 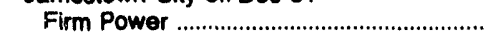 & -_ & -- & - & -. & 165,565 & $4,277,105$ \\
\hline 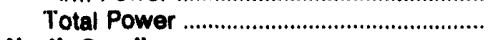 & - & -- & -- & $\ldots$ & 165,565 & $4,277,105$ \\
\hline \multicolumn{7}{|l|}{$\begin{array}{l}\text { North Carollina } \\
\text { Fayetteville Public Works Comm/Jun } 30\end{array}$} \\
\hline 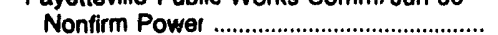 & -- & -- & - & -- & 843 & 20,635 \\
\hline 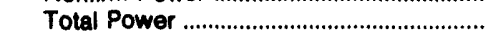 & -- & -- & -- & -- & 843 & 20,635 \\
\hline \multicolumn{7}{|l|}{ Greenville Utilities Comm/Jun 30} \\
\hline 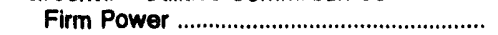 & -- & -- & 23,700 & $1,801,962$ & 23,700 & $1,801,962$ \\
\hline $\begin{array}{l}\text { Rocky Mount City of/Jun } 30 \\
\text { Firm Power }\end{array}$ & & & & & & \\
\hline $\begin{array}{l}\text { Firm Power } \\
\text { Total Power }\end{array}$ & $\overline{-}$ & - & $\begin{array}{l}15,872 \\
15,872\end{array}$ & 988,941 & 15,872 & 988,941 \\
\hline Wilson City of/Jun 30 & & & & & & \\
\hline 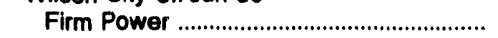 & -- & -- & 56,166 & $4,387,091$ & 56,166 & $4,387,091$ \\
\hline 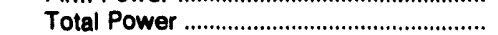 & -- & -- & 56,166 & $4,387,091$ & 56,166 & $4,387,091$ \\
\hline
\end{tabular}

See notes and footnotes at end of table. 
Table 30. Electriclty Sales for Reaale by Municlpal UtIlitles, Fiscal Year 1992 (Continued)

\begin{tabular}{|c|c|c|c|c|c|c|}
\hline \multirow{3}{*}{$\begin{array}{l}\text { State / Utilty I } \\
\text { Flecal Year Ending Date }\end{array}$} & \multicolumn{6}{|c|}{ Purchaser of Electricity } \\
\hline & \multicolumn{2}{|c|}{ Investor-Owned } & \multicolumn{2}{|c|}{ Federal } & \multicolumn{2}{|c|}{$\begin{array}{l}\text { State and Other } \\
\text { Government }\end{array}$} \\
\hline & $\begin{array}{c}\text { Sales For } \\
\text { Resale } \\
\text { (thousand kWh) }\end{array}$ & $\begin{array}{l}\text { Revenue } \\
\text { (dollars) }\end{array}$ & $\begin{array}{c}\text { Sales For } \\
\text { Resale } \\
\text { (thousand kWh) }\end{array}$ & $\begin{array}{l}\text { Revenue } \\
\text { (dollars) }\end{array}$ & $\begin{array}{c}\text { Sales For } \\
\text { Resale } \\
\text { (thousand kWh) }\end{array}$ & $\begin{array}{l}\text { Revenue } \\
\text { (dollars) }\end{array}$ \\
\hline \multicolumn{7}{|l|}{$\begin{array}{l}\text { Ohlo } \\
\text { American Mun Power-Ohio Inc/D.c } 31\end{array}$} \\
\hline 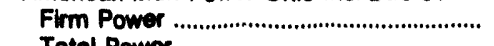 & - & - & - & - & - & - \\
\hline $\begin{array}{l}\text { Total Power } \\
\text { Bowling Green City of/Dec } 31\end{array}$ & - & - & - & - & - & - \\
\hline 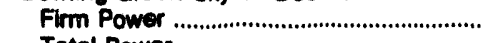 & - & - & - & - & - & - \\
\hline $\begin{array}{l}\text { Total Power ... } \\
\text { Columbus Dopt of Pub Service/Dec } 31\end{array}$ & - & - & - & - & - & - \\
\hline 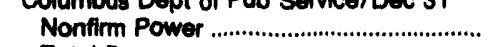 & 988 & 5,911 & - & - & - & - \\
\hline $\begin{array}{l}\text { Total Power ............ivilio/Dec } 31 \\
\text { Hamilton Dept Public.... }\end{array}$ & 988 & 5,911 & - & - & - & -- \\
\hline 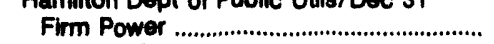 & - & - & - & - & - & -- \\
\hline Nonftrm Power ............................................... & 38,046 & 233,690 & - & - & - & - \\
\hline $\begin{array}{l}\text { Total Power } \\
\text { Orville City of/Dec } 31\end{array}$ & 38,046 & 233,690 & - & - & - & - \\
\hline 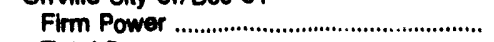 & - & - & - & - & - & - \\
\hline 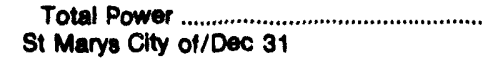 & - & - & - & - & - & - \\
\hline Other Power & - & - & - & - & - & - \\
\hline 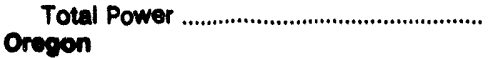 & - & - & - & - & - & -- \\
\hline \multicolumn{7}{|l|}{$\begin{array}{l}\text { Oregon } \\
\text { Ashland City of/Jun } 30\end{array}$} \\
\hline 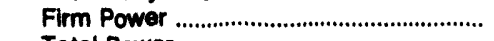 & 1,562 & 47,601 & - & - & - & - \\
\hline $\begin{array}{l}\text { Total Power ......................................... } \\
\text { Eugene City of/De }\end{array}$ & 1,562 & 47,601 & - & - & - & - \\
\hline 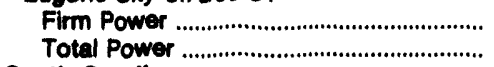 & $\begin{array}{l}138,571 \\
138,571\end{array}$ & $\begin{array}{l}3,624,441 \\
3,624,441\end{array}$ & $\begin{array}{l}72,700 \\
72,700\end{array}$ & $\begin{array}{l}1,996,606 \\
1,996,606\end{array}$ & $\begin{array}{l}29,777 \\
29,777\end{array}$ & $\begin{array}{l}707,670 \\
707,870\end{array}$ \\
\hline \multicolumn{7}{|l|}{$\begin{array}{l}\text { South Carollna } \\
\text { Piedmont Municipal Power Agny/Doc } 31\end{array}$} \\
\hline 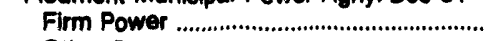 & - & - & - & - & - & - \\
\hline 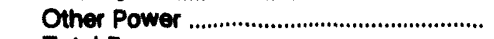 & $1,606,944$ & $81,608,000$ & - & - & - & - \\
\hline 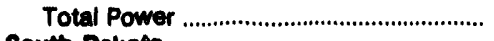 & $1,606,944$ & $81,608,000$ & - & -- & - & -- \\
\hline \multicolumn{7}{|l|}{$\begin{array}{l}\text { South Dakota } \\
\text { Pierre Municipal Electric/Dec } 31\end{array}$} \\
\hline 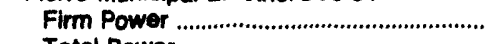 & - & - & - & - & 12,182 & 219,542 \\
\hline 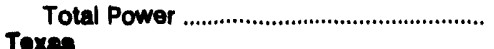 & - & - & - & - & 12,182 & 219,542 \\
\hline \multirow{2}{*}{\multicolumn{7}{|c|}{$\begin{array}{l}\text { Toxes } \\
\text { Austin City of/Sep } 30\end{array}$}} \\
\hline & & & & & & \\
\hline 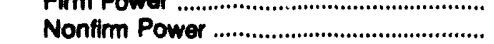 & 91,116 & $1,087,979$ & - & $\overline{-}$ & $\bar{z}$ & $\overline{-}$ \\
\hline 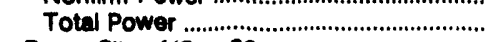 & 91,116 & $1,087,979$ & - & - & - & - \\
\hline \multicolumn{7}{|l|}{ Bryan City of/Sep 30} \\
\hline 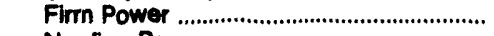 & - & - & - & - & - & - \\
\hline Nonfirm Power .................................................. & - & -- & - & - & - & - \\
\hline Total Power ..................................................... & - & - & - & -- & -- & - \\
\hline \multicolumn{7}{|l|}{ Denton City of/Sep 30} \\
\hline $\begin{array}{l}\text { Firm Power } \\
\text { Total Power }\end{array}$ & $\overline{-}$ & $\bar{z}$ & $\overline{-}$ & $\bar{z}$ & $\overline{-}$ & - \\
\hline \multicolumn{7}{|l|}{ Garland City of/Sep 30} \\
\hline Firm Power & - & - & - & - & - & - \\
\hline Nonfirm Power ................................................. & - & - & - & - & - & - \\
\hline Total Power & - & - & - & - & - & - \\
\hline \multicolumn{7}{|l|}{ Greenville Electric UtI Sys/Sep 30} \\
\hline 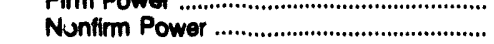 & $\therefore$ & -- & - & - & - & $\overline{-}$ \\
\hline \multirow{2}{*}{\multicolumn{7}{|c|}{ San Antonio City of/Jan 31}} \\
\hline & & & & & & \\
\hline 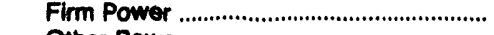 & $-\infty$ & $-\bar{x}+10$. & - & - & - & - \\
\hline 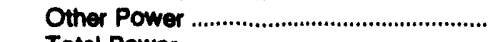 & 93,127 & $1,714,941$ & - & - & 6,567 & 113,236 \\
\hline \multirow{2}{*}{\multicolumn{7}{|c|}{ Texas Municipal Power Agency/Sep 30}} \\
\hline & & & & & & \\
\hline $\begin{array}{l}\text { Firm Power } \\
\text { Total Power }\end{array}$ & - & - & - & - & - & - \\
\hline \multirow{4}{*}{ 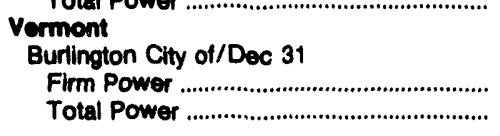 } & - & - & - & - & - & - \\
\hline & & & & & & \\
\hline & $\begin{array}{l}24,048 \\
24,048\end{array}$ & $\begin{array}{l}4,165,449 \\
4,165,449\end{array}$ & $\overline{-}$ & $\overline{-}$ & $\begin{array}{l}19,293 \\
19,293\end{array}$ & $\begin{array}{l}1,224,030 \\
1,224,030\end{array}$ \\
\hline & & & & & & $1,224,030$ \\
\hline
\end{tabular}

See notes and footnotes at end of table. 
Table 30. Electriclty Sales for Resale by Municipal Utilities, Fiscal Year 1992 (Continued)

\begin{tabular}{|c|c|c|c|c|c|c|}
\hline \multirow{3}{*}{$\begin{array}{l}\text { State / Utilty I } \\
\text { Fineal Yeer Ending Dato }\end{array}$} & \multicolumn{6}{|c|}{ Purchaser of Electricity } \\
\hline & \multicolumn{2}{|c|}{ Cooperative } & \multicolumn{2}{|c|}{ Other' } & \multicolumn{2}{|c|}{ Total } \\
\hline & $\begin{array}{c}\text { Seles For } \\
\text { Resele } \\
\text { (thouseand kWh) }\end{array}$ & $\begin{array}{l}\text { Revenue } \\
\text { (dollars) }\end{array}$ & $\begin{array}{c}\text { Sales For } \\
\text { Resale } \\
\text { (thousand kWh) }\end{array}$ & $\begin{array}{l}\text { Revenue } \\
\text { (dollars) }\end{array}$ & $\begin{array}{c}\text { Sales For } \\
\text { Resale } \\
\text { (thouseand kWh) }\end{array}$ & $\begin{array}{l}\text { Revenue } \\
\text { (dollars) }\end{array}$ \\
\hline \multicolumn{7}{|l|}{ Onio } \\
\hline 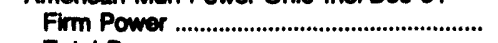 & - & - & $3,730,681$ & $129,859,996$ & $3,730,661$ & $129,859,996$ \\
\hline \multicolumn{5}{|l|}{ Bowling Green City of/Dec 31} & $3,730,681$ & $129,858,986$ \\
\hline 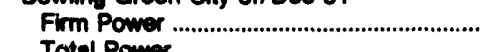 & - & - & 3,454 & 151,506 & 3,454 & 151,506 \\
\hline \multicolumn{7}{|l|}{ Columbus Dept of Pub Service/Dec 31} \\
\hline 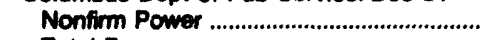 & - & - & - & - & 988 & 5,811 \\
\hline \multicolumn{6}{|l|}{ Hamilton Dept of Public Utile/Dec 31} & 5,811 \\
\hline 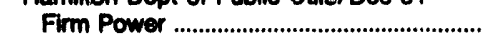 & - & - & 44,812 & $1,878,270$ & 44,812 & $1,878,270$ \\
\hline Nonfirm Power ................................................ & - & - & - & - & 38,046 & 233,690 \\
\hline \multicolumn{2}{|l|}{ Orville City of/Dec 31} & - & 44,812 & & 82,858 & $2,111,960$ \\
\hline 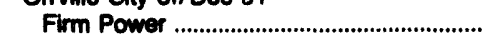 & - & - & 115,279 & $2,841,760$ & 115,279 & $2,841,760$ \\
\hline 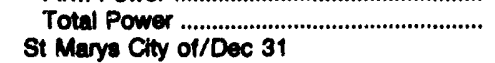 & St Marys City of/Dec 31 & - & 115,279 & $2,841,760$ & 115,279 & $2,841,760$ \\
\hline Other Power ......................................... & - & - & 4,463 & 164,610 & 4,463 & 164,610 \\
\hline \multicolumn{7}{|l|}{$\begin{array}{l}\text { Total Power ................................................ } \\
\text { Oregon } \\
\text { Ashland City of/Jur } 30\end{array}$} \\
\hline 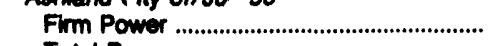 & - & - & - & - & 1,562 & 47,601 \\
\hline $\begin{array}{l}\text { Total Power ................................... } \\
\text { Eugene City of/Dec } 31 \text {. }\end{array}$ & - & - & - & - & 1,562 & 47,601 \\
\hline Firm Power ........................................... & - & - & 4,738 & 116,163 & 245,786 & $6,444,880$ \\
\hline 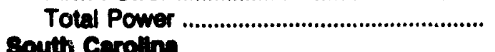 & - & - & 4,738 & 116,163 & 245,786 & $6,444,880$ \\
\hline \multicolumn{7}{|l|}{$\begin{array}{l}\text { South Carollna } \\
\text { Piedmont Municipal Power Agny/7ec } 31\end{array}$} \\
\hline 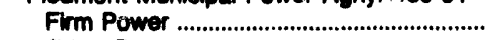 & - & - & $1,487,316$ & $72,652,000$ & $1,487,316$ & $72,652,000$ \\
\hline 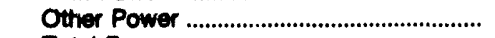 & - & - & - & - & $1,606,944$ & $81,608,000$ \\
\hline & - & - & $1,487,316$ & $72,652,000$ & $3,094,260$ & $154,260,000$ \\
\hline \multicolumn{7}{|l|}{$\begin{array}{l}\text { South Dakota } \\
\text { Pierre Municipal Electric/Dec } 31\end{array}$} \\
\hline 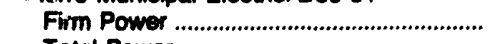 & - & - & - & - & 12,182 & 219,542 \\
\hline 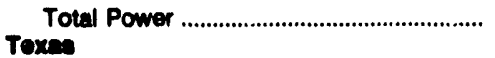 & - & - & - & - & 12,182 & 219,542 \\
\hline \multicolumn{7}{|l|}{$\begin{array}{l}\text { Toxes } \\
\text { Austin City of / Sop } 30\end{array}$} \\
\hline 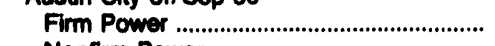 & - & - & 168,533 & $3,583,983$ & 166,533 & $3,583,993$ \\
\hline 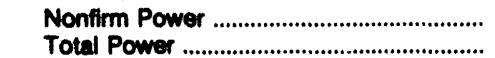 & 6,316 & 97,649 & 13,893 & 192,263 & 111,325 & $1,377,891$ \\
\hline \multicolumn{7}{|l|}{ Bryan Clty of/Sep 30} \\
\hline 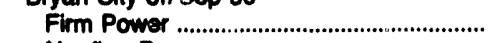 & - & - & 392,842 & $16,733,959$ & 392,842 & $16,733,959$ \\
\hline 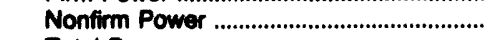 & - & - & 156,447 & $3,757,439$ & $i 56,447$ & $3,757,439$ \\
\hline \multicolumn{7}{|l|}{ Denton City of/Sep 30} \\
\hline 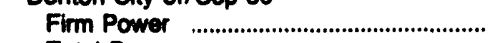 & - & - & 104,358 & $3,489,210$ & 104,358 & $3,489,210$ \\
\hline $\begin{array}{l}\text { Total Power ............................................ } \\
\text { Gerland City of/Sep } 30\end{array}$ & \multicolumn{6}{|c|}{ Garland City of/Sep 30} \\
\hline 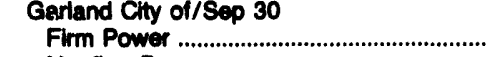 & 233,667 & $4,461,871$ & 204,718 & $7,259,072$ & 438,385 & $11,720,943$ \\
\hline 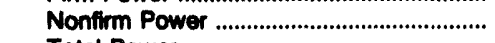 & & & 145,190 & $3,831,380$ & 145,190 & $3,831,380$ \\
\hline 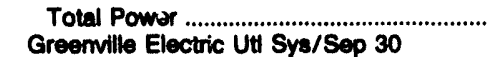 & 233,667 & $4,461,871$ & 349,908 & $11,090,452$ & 583,575 & $15,552,323$ \\
\hline 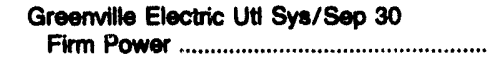 & & & & & & \\
\hline $\begin{array}{l}\text { Firm Power } \\
\text { Nonfirm Power }\end{array}$ & $-14,543$ & $-548,614$ & $\begin{array}{r}32,294 \\
2,382\end{array}$ & $\begin{array}{r}1,745,217 \\
39,101\end{array}$ & $\begin{array}{r}46,837 \\
2,382\end{array}$ & $\begin{array}{r}2,377,915 \\
39,101\end{array}$ \\
\hline 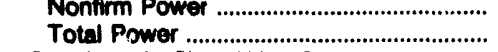 & $-14,543$ & 548,614 & 34,676 & $\begin{array}{r}39,101 \\
1,784,318\end{array}$ & $\begin{array}{r}2,382 \\
49,219\end{array}$ & $2,417,016$ \\
\hline San Antonio Clty of/Jan 31 & & & & & & \\
\hline 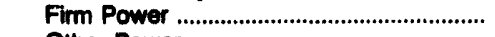 & - & - & 205,758 & $8,545,895$ & 205,758 & $8,545,895$ \\
\hline $\begin{array}{l}\text { Other Power } \\
\text { Total Power }\end{array}$ & $\begin{array}{l}19,034 \\
19,034\end{array}$ & $\begin{array}{l}359,180 \\
359,180\end{array}$ & $\begin{array}{r}4,061 \\
209,819\end{array}$ & $\begin{array}{r}71,563 \\
8,617,458\end{array}$ & $\begin{array}{l}122,789 \\
328,547\end{array}$ & $\begin{array}{r}2,258,920 \\
10,804,815\end{array}$ \\
\hline Texas Municipal Fower Agency/Sep 30 & & & & & & \\
\hline 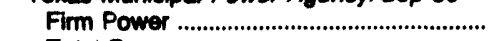 & - & - & $2,818,728$ & $139,6<7,301$ & $2,818,728$ & $139,627,30$ \\
\hline 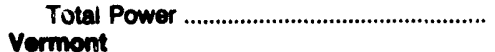 & - & - & $2,818,728$ & $139,627,301$ & $2,818,728$ & $139,627,3$ \\
\hline $\begin{array}{l}\text { Vermont } \\
\text { Burtington City of/Dec } 31\end{array}$ & & & & & & \\
\hline $\begin{array}{l}\text { Firm Power } \\
\text { Total Power }\end{array}$ & $\begin{array}{l}128 \\
128\end{array}$ & $\begin{array}{l}4,745 \\
4.745\end{array}$ & $\begin{array}{l}48,444 \\
48,444\end{array}$ & $\begin{array}{l}944,449 \\
944,449\end{array}$ & $\begin{array}{l}81,813 \\
91,913\end{array}$ & $\begin{array}{l}6,338,673 \\
6,338,673\end{array}$ \\
\hline ......... & & & & & & \\
\hline
\end{tabular}


Table 30. Electricity Sales for Resale by Municipal Utilities, Fiscal Year 1992 (Continued)

\begin{tabular}{|c|c|c|c|c|c|c|}
\hline \multirow{3}{*}{$\begin{array}{l}\text { State / Uumby / } \\
\text { Flacel Year Ending Dato }\end{array}$} & \multicolumn{6}{|c|}{ Purchaser of Electricity } \\
\hline & \multicolumn{2}{|c|}{ Investor-Owned } & \multicolumn{2}{|c|}{ Federal } & \multicolumn{2}{|c|}{$\begin{array}{l}\text { State and Other } \\
\text { Government }\end{array}$} \\
\hline & $\begin{array}{c}\text { Sales For } \\
\text { Resale } \\
\text { (thousand kWh) }\end{array}$ & $\begin{array}{l}\text { Revenue } \\
\text { (dollars) }\end{array}$ & $\begin{array}{c}\text { Sales For } \\
\text { Resale } \\
\text { (thousand kWh) }\end{array}$ & $\begin{array}{l}\text { Revenue } \\
\text { (dollars) }\end{array}$ & $\begin{array}{c}\text { Sales For } \\
\text { Resale } \\
\text { (thousand kWh) }\end{array}$ & $\begin{array}{l}\text { Revenue } \\
\text { (dollars) }\end{array}$ \\
\hline \multicolumn{7}{|l|}{$\begin{array}{l}\text { Weahington } \\
\text { Seattle City Light/Dec } 31\end{array}$} \\
\hline 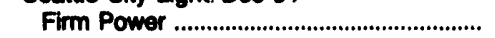 & - & - & - & - & 209,730 & 637,962 \\
\hline $\begin{array}{l}\text { Nonfirm Power } \\
\text { Total Power . } \\
\text { Tacoma Dept of Public Utils/Dec } 31\end{array}$ & $\begin{array}{l}110,900 \\
110,900\end{array}$ & $\begin{array}{l}2,034,513 \\
2,034,513\end{array}$ & $\begin{array}{l}32,467 \\
32,467\end{array}$ & $\begin{array}{l}829,213 \\
829,213\end{array}$ & $\begin{array}{r}30,930 \\
240,660\end{array}$ & $\begin{array}{r}634,824 \\
1,272,786\end{array}$ \\
\hline 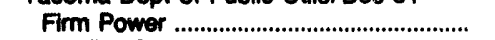 & - & - & 370,426 & $15,207,856$ & - & - \\
\hline 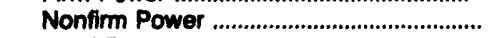 & - & - & - & - & - & - \\
\hline 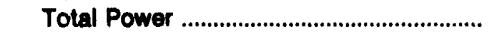 & - & - & 370,426 & $15,207,856$ & - & - \\
\hline
\end{tabular}

\begin{tabular}{|c|c|c|c|c|c|}
\hline \multicolumn{2}{|c|}{ Purchaser of Electricity } \\
\hline \multicolumn{2}{|c|}{ Cooperative } & \multicolumn{2}{|c|}{ Other' } & \multicolumn{2}{c}{ Total } \\
\hline $\begin{array}{c}\text { Sales For } \\
\text { Resale } \\
\text { (thousand kWh) }\end{array}$ & $\begin{array}{c}\text { Revenue } \\
\text { (dollars) }\end{array}$ & $\begin{array}{c}\text { Sales For } \\
\text { Resale } \\
\text { (thousand kWh) }\end{array}$ & $\begin{array}{c}\text { Revenue } \\
\text { (dollars) }\end{array}$ & $\begin{array}{c}\text { Sales For } \\
\text { Resale } \\
\text { (thousand kWh) }\end{array}$ & $\begin{array}{c}\text { Revenue } \\
\text { (dollars) }\end{array}$ \\
\hline
\end{tabular}

\begin{tabular}{|c|c|c|c|c|c|c|}
\hline \multicolumn{7}{|l|}{ Whington } \\
\hline \multicolumn{7}{|l|}{$\begin{array}{l}\text { Washington } \\
\text { Seattie City Light/Dec } 31\end{array}$} \\
\hline 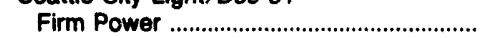 & - & - & - & $-\infty$ & 209,730 & 637,962 \\
\hline 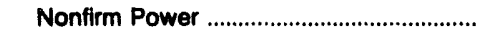 & - & - & 7,520 & 139,788 & 181,817 & $3,638,338$ \\
\hline 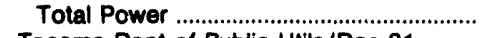 & - & - & 7,520 & 139,788 & 391,547 & $4,276,300$ \\
\hline Tacoma Dept of Public Utils/Dec 31 & & & & & & \\
\hline 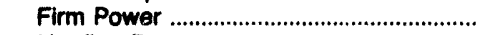 & - & - & 6,617 & 141,355 & 377,043 & $15,349,211$ \\
\hline 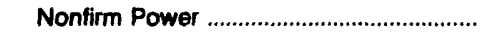 & - & - & 405,467 & $7,420,893$ & 405,467 & $7,420,893$ \\
\hline 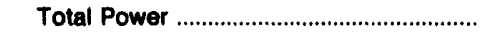 & - & - & 412,084 & $7,562,248$ & 782,510 & $22,770,104$ \\
\hline
\end{tabular}

Includes transactions with municipal utilities, power pools, utilities in Canada and Mexico, and nonutilties.

- Not Applicable

Notes: •For identification purposes, the municipal utilities are listed in the State in which the municipality is located. -Totals may not equal sum of components because of independent rounding.

Source: Energy Information Administration, Form ElA-412, "Annual Report of Public Electric Utilities." 
Table 31. Electricity Purchases by Publicly Owned Utilities Not Reported on Form ElA-412, by State, 1992

\begin{tabular}{|c|c|c|c|c|c|c|c|}
\hline \multirow{2}{*}{$\begin{array}{l}\text { State I } \\
\text { Utillity }\end{array}$} & \multicolumn{7}{|c|}{$\begin{array}{l}\text { Source of Electricity } \\
\text { (Purchases in thousand kWh) }\end{array}$} \\
\hline & $\begin{array}{l}\text { Investor- } \\
\text { Owned }\end{array}$ & Foderal & $\begin{array}{l}\text { State and } \\
\text { Other } \\
\text { Government }\end{array}$ & Municipal & Cooperative & Other' & Total \\
\hline \multicolumn{8}{|l|}{ Alabama } \\
\hline 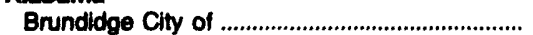 & - & -- & - & - & 25,000 & - & 25,000 \\
\hline 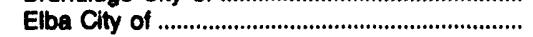 & - & - & - & - & 47,443 & - & 47,443 \\
\hline 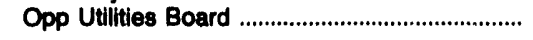 & - & - & - & - & 71,407 & - & 71,407 \\
\hline \multicolumn{8}{|l|}{ Alacka } \\
\hline 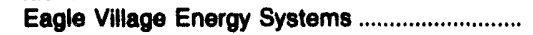 & - & - & \multirow{3}{*}{73} & - & \multirow[t]{2}{*}{-} & \multirow[t]{2}{*}{57} & 57 \\
\hline Kokhanok Village Council ....................................... & - & - & & - & & & 73 \\
\hline 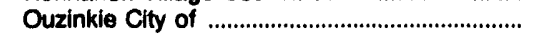 & - & - & & - & - & 355 & 355 \\
\hline \multicolumn{8}{|l|}{ Colorado } \\
\hline 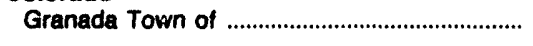 & - & - & - & 2,514 & - & - & 2,514 \\
\hline \multicolumn{8}{|l|}{ Illinois } \\
\hline 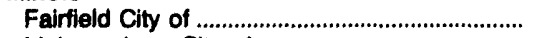 & -- & - & - & - & 67,891 & - & 67,891 \\
\hline McLeansboro City of & - & - & - & - & 19,193 & -- & 19,193 \\
\hline 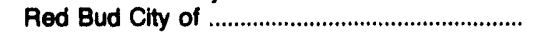 & - & - & - & - & 32,347 & - & 32,347 \\
\hline \multicolumn{8}{|l|}{ Indlana } \\
\hline Cannelton City of & - & -- & - & 18,461 & - & - & 18,461 \\
\hline 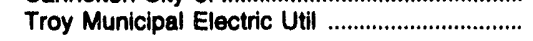 & - & - & - & 9,667 & - & -- & 9,667 \\
\hline \multicolumn{8}{|l|}{ lowa } \\
\hline Aplington City of & 5,176 & - & - & - & - & - & 5,176 \\
\hline 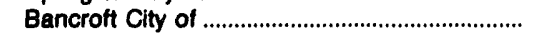 & -- & - & -- & -- & 9,784 & - & $\mathbf{9 , 7 8 4}$ \\
\hline Brooklyn City of & -- & - & - & -- & 10,032 & -- & 10,032 \\
\hline Cascade Municipal Utilities ...................................... & - & - & - & -- & 12,839 & -- & 12,839 \\
\hline 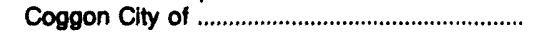 & - & - & -- & - & 3,307 & -- & 3,307 \\
\hline 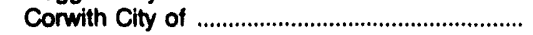 & - & - & - & - & 2,343 & -- & 2,343 \\
\hline Dayton City of & - & -- & - & -- & 5,071 & -. & 5,071 \\
\hline 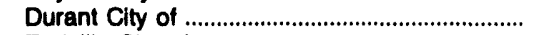 & -- & -- & - & - & 12,382 & -- & 12,382 \\
\hline 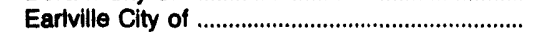 & -. & - & $\ldots$ & -- & 3,920 & - & 3,920 \\
\hline Ellsworth Municipal Electric ................................ & - & -- & - & -- & 5,699 & -- & 5,699 \\
\hline 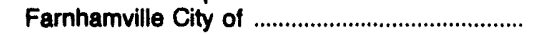 & -- & -- & -- & -- & 4,543 & -- & 4,543 \\
\hline 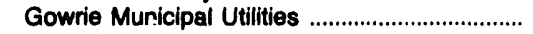 & - & - & -- & - & 6,693 & - & 6,693 \\
\hline 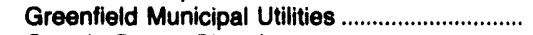 & -- & -- & - & -- & 23,781 & -- & 23,781 \\
\hline 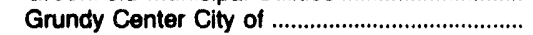 & -- & -- & - & -- & 22,965 & -- & 22,965 \\
\hline Indianola Mun Wtr \& Elec Utils .......................... & - & -- & 82,523 & - & -- & - & 82,523 \\
\hline 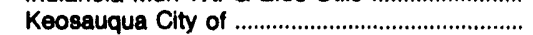 & - & -- & - & -- & $\mathbf{9 , 4 4 0}$ & -- & 9,440 \\
\hline 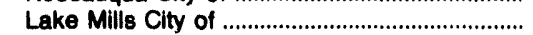 & -- & -- & - & -- & 28,272 & - & 28,272 \\
\hline 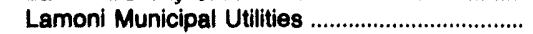 & -- & -- & -- & -- & 17,377 & -- & 17,377 \\
\hline Larchwood City of ............................................ & - & -- & - & - & 4,244 & -- & 4,244 \\
\hline 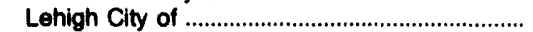 & -- & -- & -- & -- & 2,142 & -. & 2,142 \\
\hline New Hampton Mun Light Plant ............................. & - & - & - & -- & 53,467 & - & 53,467 \\
\hline Paton Electrical Department ........................... & - & - & -- & -- & 1,986 & - & 1,986 \\
\hline Renwick City of .................. & -- & -- & - & -- & 2,925 & -- & 2,925 \\
\hline 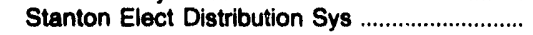 & -- & - & -- & -- & 6,282 & - & 6,282 \\
\hline 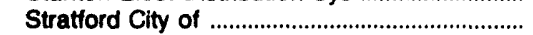 & -- & -- & -- & -- & 4,674 & -- & 4,674 \\
\hline Sumner Municipal Light Plant ............................... & - & - & -. & - & 14,584 & - & 14,584 \\
\hline 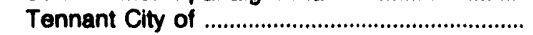 & - & - & -- & 298 & -- & -- & 298 \\
\hline 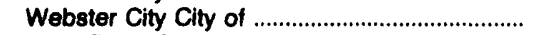 & - & - & -- & -- & 115,200 & - & 115,200 \\
\hline 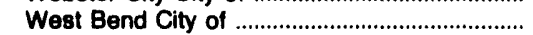 & -- & -- & -- & -- & 12,598 & -- & 12,598 \\
\hline 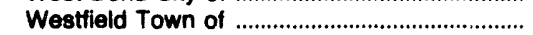 & -- & -- & -- & -- & 682 & -- & 682 \\
\hline Witton City of & -- & - & -- & - & 19,252 & -- & 19,252 \\
\hline Winterset City of ......................................... & -- & -- & -- & - & 32,875 & -- & 32,875 \\
\hline Woolstock City of & -- & - & -- & 1,460 & -- & - & 1,460 \\
\hline \multicolumn{8}{|l|}{ Kaneas } \\
\hline 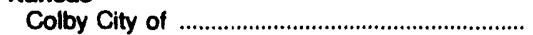 & -- & -- & -- & -- & 47,716 & -- & 47,716 \\
\hline 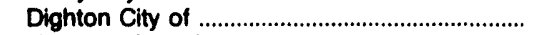 & - & - & -- & -. & 9,101 & - & 9,101 \\
\hline Herndon City of ................................................. & - & -- & - & -- & 1,050 & - & 1,050 \\
\hline 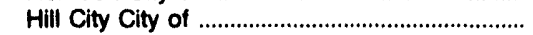 & - & -- & -- & - & 12,917 & - & 12,917 \\
\hline 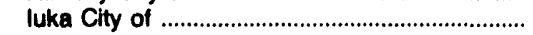 & - & -- & -- & 1,663 & -- & - & 1,663 \\
\hline Jetmore City of & -- & - & -- & -- & 6,574 & - & 6,574 \\
\hline 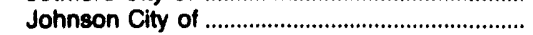 & - & -- & - & -- & 8,793 & - & 9,793 \\
\hline 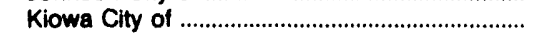 & - & - & -. & -- & 9,222 & -- & 9,222 \\
\hline 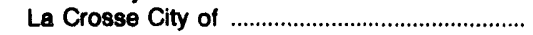 & - & -- & - & - & 9,200 & -- & 9,200 \\
\hline
\end{tabular}

See notes and footnotes at end of table. 
Table 31. Electriclty Purchases by Publicly Owned Utilities Not Reported on Form EIA-412, by State, 1992 (Continued)

\begin{tabular}{|c|c|c|c|c|c|c|c|}
\hline \multirow{2}{*}{$\begin{array}{l}\text { Btate I } \\
\text { Utilty }\end{array}$} & \multicolumn{7}{|c|}{$\begin{array}{l}\text { Source of Electrictly } \\
\text { (Purchases in thousand } \mathrm{kWh} \text { ) }\end{array}$} \\
\hline & $\begin{array}{l}\text { Investor- } \\
\text { Owned }\end{array}$ & Federal & $\begin{array}{l}\text { State and } \\
\text { Other } \\
\text { Government }\end{array}$ & Municipal & Cooperative & Other' & Total \\
\hline \multicolumn{8}{|l|}{ Kaneas } \\
\hline 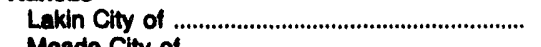 & - & - & - & - & 5,584 & - & 5,584 \\
\hline 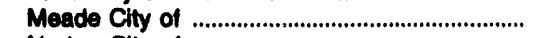 & - & - & - & - & 5,608 & - & 5,608 \\
\hline 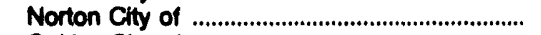 & - & - & - & - & 24,058 & - & 24,058 \\
\hline Oakley City of & - & - & - & - & 17,970 & - & 17,970 \\
\hline Radium City of & - & - & - & - & 703 & - & 703 \\
\hline Seward Clity of & - & - & - & - & 385 & - & 385 \\
\hline \multicolumn{8}{|l|}{ Minnesota } \\
\hline 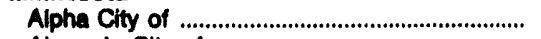 & - & - & - & - & 1,052 & - & 1.052 \\
\hline Alvarado Clty of & - & - & - & - & 3,166 & - & 3,166 \\
\hline 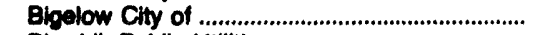 & - & - & - & 1,782 & - & - & 1,782 \\
\hline 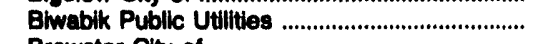 & - & - & - & -- & 6,174 & - & 6,174 \\
\hline 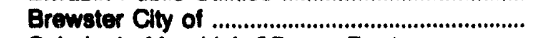 & -- & 1,57 & 1,575 & - & - & - & 3,150 \\
\hline Caledonla Mun Light\&Power Dept ..................... & - & - & - & - & 17,334 & - & 17,334 \\
\hline 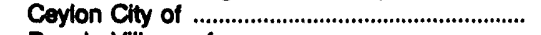 & - & - & - & - & 2,607 & - & 2,607 \\
\hline 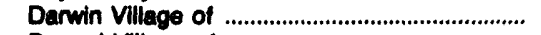 & - & - & -- & 1,880 & -- & - & 1,880 \\
\hline 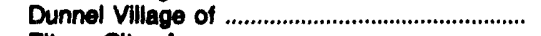 & - & -- & - & - & 1,008 & - & 1,008 \\
\hline 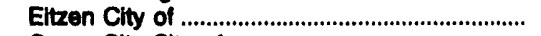 & - & - & - & - & 2,153 & - & 2,153 \\
\hline 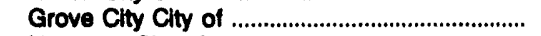 & - & - & - & 5.715 & - & - & 5,715 \\
\hline Harmony Clty of & - & - & - & - & 6,936 & - & 6,836 \\
\hline 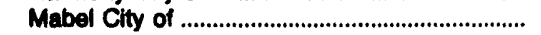 & - & - & - & - & 4,122 & - & 4,122 \\
\hline Nashwauk Public Utillitios Dept ............................... & - & - & - & - & 5,890 & - & 5,890 \\
\hline Peterson Electric System ........................................... & - & - & - & - & 1,371 & - & 1,371 \\
\hline Proctor Public Utilities Comm ..................................... & 15,791 & - & - & - & - & - & 15,791 \\
\hline 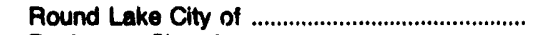 & - & - & - & - & 7,510 & - & 7,510 \\
\hline 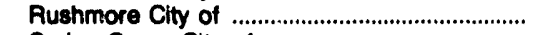 & - & - & - & 1,845 & - & - & 1,846 \\
\hline 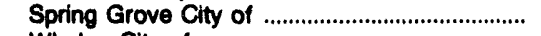 & - & -- & - & - & 14,200 & -- & 14,200 \\
\hline Whalan City of & - & - & - & - & 416 & - & 416 \\
\hline \multicolumn{8}{|l|}{ Mtacourt } \\
\hline 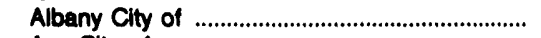 & - & - & - & 3,963 & 10,000 & - & 13,863 \\
\hline 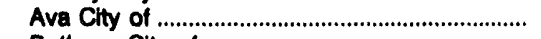 & - & - & - & - & 36,263 & - & 36,263 \\
\hline 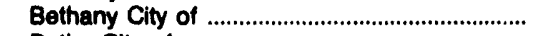 & - & - & - & - & 27,922 & - & 27,822 \\
\hline 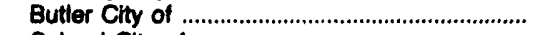 & - & - & - & - & 24,923 & - & 24,823 \\
\hline Cabool City of & - & - & - & - & 31,487 & - & 31,487 \\
\hline 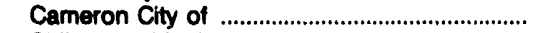 & - & - & - & - & 38,137 & - & 38,137 \\
\hline Chillicothe Municipal Utils ....................................... & - & - & - & - & 80,303 & - & 80,303 \\
\hline 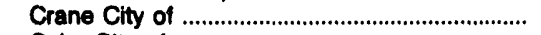 & - & - & - & - & 12,000 & -- & 12,000 \\
\hline 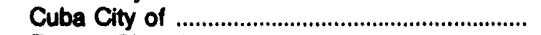 & - & - & - & -- & 38,120 & - & 38,120 \\
\hline 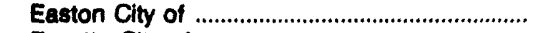 & - & - & - & - & 1,316 & - & 1,316 \\
\hline 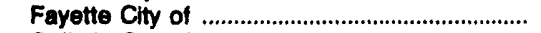 & - & - & - & - & 17,581 & - & 17,581 \\
\hline 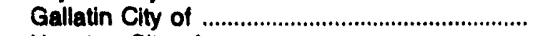 & - & - & - & 7,327 & 10,000 & - & 17,327 \\
\hline 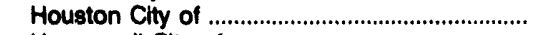 & - & - & - & - & 27,300 & - & 27,300 \\
\hline 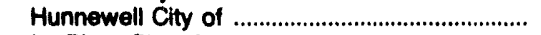 & - & - & - & - & 879 & - & 878 \\
\hline La Plata City of & - & - & - & - & 22,261 & -- & 22,261 \\
\hline 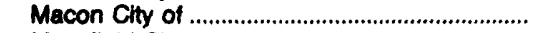 & - & - & - & - & 74,952 & - & 74,852 \\
\hline 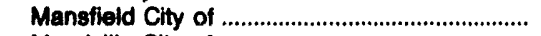 & - & - & - & - & 23,787 & - & 23,787 \\
\hline 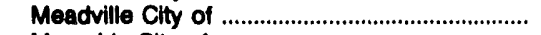 & - & - & - & - & 2,000 & - & 2,000 \\
\hline 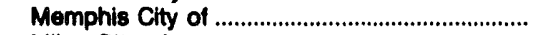 & - & - & - & 14,881 & - & - & 14,881 \\
\hline 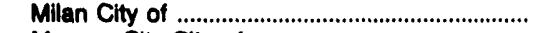 & - & - & - & - & 27,666 & - & 27,686 \\
\hline 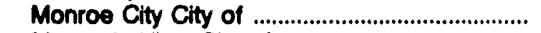 & - & - & - & - & 49,545 & - & 49,545 \\
\hline 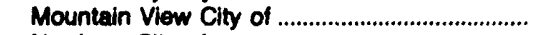 & - & - & - & - & 29,525 & - & 29,525 \\
\hline 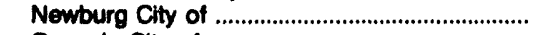 & - & - & - & - & 3,725 & - & 3,725 \\
\hline 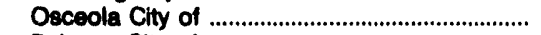 & - & - & - & - & 5,320 & - & 5,320 \\
\hline 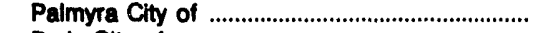 & - & - & - & - & 29,689 & - & 29,689 \\
\hline 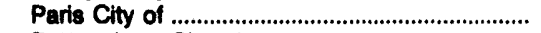 & - & - & - & - & 11,110 & - & 11,110 \\
\hline 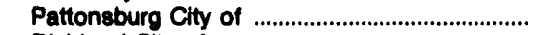 & - & - & - & - & 3,424 & - & 3,424 \\
\hline 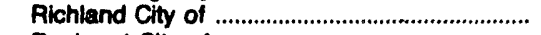 & - & - & - & - & 16,700 & - & 16,700 \\
\hline 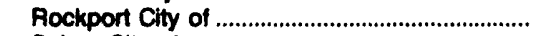 & - & - & - & - & 10,821 & - & 10,921 \\
\hline Salern City of & - & - & - & - & 47,300 & - & 47,300 \\
\hline 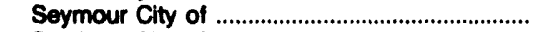 & - & - & - & - & 19,056 & - & 19,056 \\
\hline 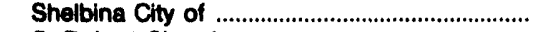 & - & - & - & - & 24,589 & - & 24,589 \\
\hline 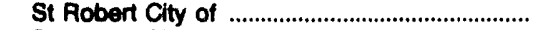 & - & - & - & - & 30,381 & -- & 30,381 \\
\hline 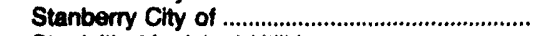 & - & - & - & - & 9,383 & - & 9,383 \\
\hline 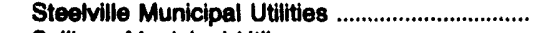 & - & - & - & - & 16,841 & - & 16,841 \\
\hline 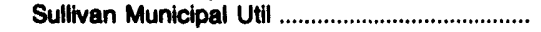 & - & - & - & - & 74,883 & - & 74,883 \\
\hline
\end{tabular}

See notes and footnotes at end of table. 
Table 31. Electricity Purchases by Publlcly Owned Utilitios Not Reported on Form ElA-412, by State, 1992 (Continued)

\begin{tabular}{|c|c|c|c|c|c|c|c|}
\hline \multirow{2}{*}{$\begin{array}{l}\text { State / } \\
\text { Utilty' }\end{array}$} & \multicolumn{7}{|c|}{$\begin{array}{l}\text { Source of Electricity } \\
\text { (Purcheses in thousand kWh) }\end{array}$} \\
\hline & $\begin{array}{l}\text { Investor- } \\
\text { Owned }\end{array}$ & Federal & $\begin{array}{l}\text { State and } \\
\text { Other } \\
\text { Government }\end{array}$ & Municipal & Cooperative & Other' & Total \\
\hline \multicolumn{8}{|l|}{ miseourt } \\
\hline 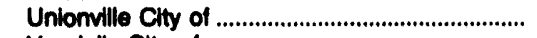 & - & - & - & 14,689 & - & - & 14,689 \\
\hline 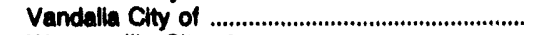 & - & - & - & 7,879 & 7,879 & - & 15,758 \\
\hline 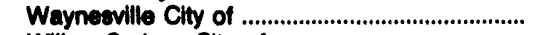 & - & - & - & - & 26,628 & - & 26,628 \\
\hline 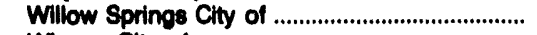 & - & - & - & - & 24,087 & - & 24,087 \\
\hline 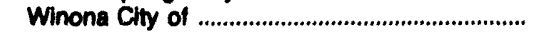 & - & & - & & 8,476 & - & 0,476 \\
\hline \multicolumn{8}{|l|}{ Mebracke } \\
\hline 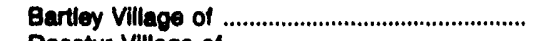 & - & - & 1,718 & - & - & - & 1,718 \\
\hline 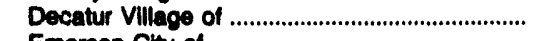 & - & - & 4,622 & - & -- & - & 4,622 \\
\hline Emerson City of & - & - & 4,894 & - & - & - & 4,894 \\
\hline 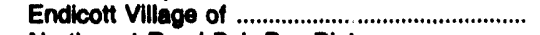 & - & - & - & 647 & - & - & 647 \\
\hline Northwest Rural Pub Pwr Dist ............................ & - & - & - & & 50,300 & - & 60,300 \\
\hline 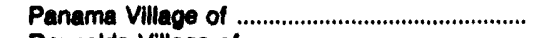 & - & - & - & 1,293 & - & - & 1,293 \\
\hline 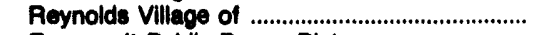 & - & - & - & 580 & - & - & 580 \\
\hline Roosevelt Public Power Dist ................................ & - & - & - & - & 42,880 & -- & 42,880 \\
\hline 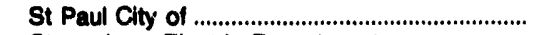 & - & - & 14,793 & - & - & - & 14,793 \\
\hline Stromsburg Electric Department ......................... & - & - & 10,004 & -- & - & - & 10,004 \\
\hline Talmage Viliage of ................................................. & - & - & - & 2,400 & - & - & 2,400 \\
\hline \multicolumn{8}{|l|}{ Novade } \\
\hline Alamo Power District No $3^{2}$ & - & - & 10,747 & - & - & - & 10,747 \\
\hline 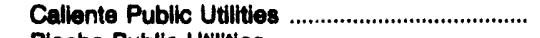 & - & - & 13,157 & - & - & - & 13,157 \\
\hline 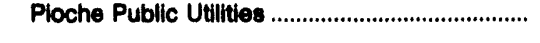 & - & & 6,445 & - & & - & 6,445 \\
\hline \multicolumn{8}{|l|}{ Now Moxico } \\
\hline 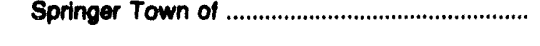 & - & - & - & - & 4,898 & - & 4,898 \\
\hline \multicolumn{8}{|l|}{ North Carollina } \\
\hline 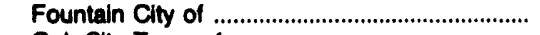 & - & - & - & 6,778 & -- & - & 6,778 \\
\hline 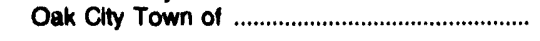 & - & - & -- & - & $\mathbf{3 , 4 8 5}$ & - & 3,485 \\
\hline \multicolumn{8}{|l|}{ Onlo } \\
\hline Woodsfield Board of Public Aff ............................. & - & - & - & - & 21,111 & -- & 21,111 \\
\hline \multicolumn{8}{|l|}{ Oklahoma } \\
\hline 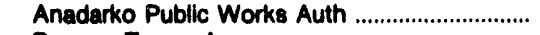 & - & - & - & - & 56,702 & - & 56,702 \\
\hline 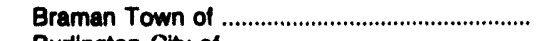 & - & - & - & 1,615 & - & - & 1,615 \\
\hline Burlington City of & - & - & - & - & 1,439 & $\cdots$ & 1,439 \\
\hline 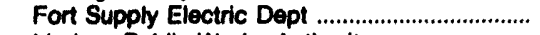 & - & - & -- & - & 2,255 & - & 2,255 \\
\hline 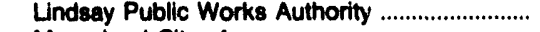 & - & - & - & - & 22,354 & - & 22,354 \\
\hline 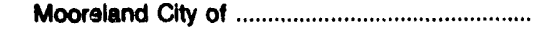 & - & - & - & - & 8,388 & - & 8,398 \\
\hline \multicolumn{8}{|l|}{ South Dakota } \\
\hline 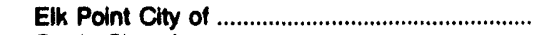 & - & -- & -- & - & 11,199 & - & 11,189 \\
\hline 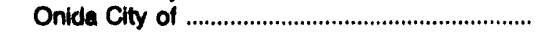 & - & - & - & - & 6,159 & -- & 6,159 \\
\hline \multicolumn{8}{|l|}{ Toxas } \\
\hline Electra City of & - & - & - & - & 19,059 & - & 19,059 \\
\hline Garrison Eloctric Department ............................. & - & - & - & - & 8,295 & -- & 8,295 \\
\hline 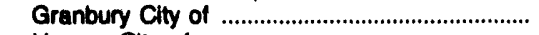 & - & - & - & - & 60,896 & - & 60,996 \\
\hline & - & - & - & - & 40,718 & - & 40,719 \\
\hline 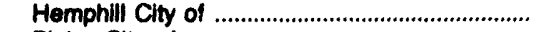 & - & - & - & - & 15,839 & - & $15,83 \theta$ \\
\hline 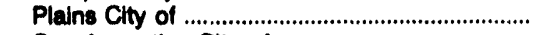 & - & - & - & - & 5,874 & - & 5,874 \\
\hline 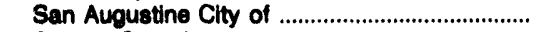 & - & - & - & - & 21,362 & - & 21,362 \\
\hline 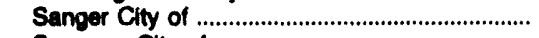 & - & - & - & - & 27,000 & -- & 27,000 \\
\hline Soymour City of & - & - & - & - & 26,359 & - & 26,350 \\
\hline Timpson City of & - & - & - & - & 9,153 & - & 9,153 \\
\hline \multicolumn{8}{|l|}{ Utah } \\
\hline 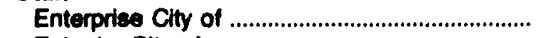 & - & -- & 6,918 & - & - & - & 6,918 \\
\hline 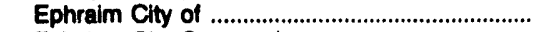 & - & - & - & - & -- & 15,986 & 15,986 \\
\hline Fairview City Corporation ...................................... & - & - & - & - & - & 5,134 & 5,134 \\
\hline Heber Light \& Power Company .......................... & - & 43,895 & - & - & -- & - & 43,995 \\
\hline 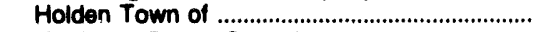 & - & $\mathbf{3 , 4 4 2}$ & - & - & - & - & 3,442 \\
\hline Hurricane Power Committee .................................. & - & - & 26,802 & - & - & - & 26,802 \\
\hline 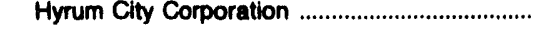 & - & 11,483 & 45,105 & - & - & - & 56,598 \\
\hline
\end{tabular}

See notes and footnotes at end of table. 
Table 31. Electricity Purchases by Publicly Owned Utilities Not Reported on Form EIA-412, by State, 1992 (Continued)

\begin{tabular}{|c|c|c|c|c|c|c|c|}
\hline \multirow{2}{*}{$\begin{array}{l}\text { State / } \\
\text { Utillity }\end{array}$} & \multicolumn{7}{|c|}{$\begin{array}{c}\text { Source of Electricity } \\
\text { (Purchases in thousand kWh) }\end{array}$} \\
\hline & $\begin{array}{l}\text { Investor- } \\
\text { Owned }\end{array}$ & Fodoral & $\begin{array}{l}\text { State and } \\
\text { Other } \\
\text { Government }\end{array}$ & Municipal & Cooperative & Other' & Total \\
\hline \multicolumn{8}{|l|}{ Utah } \\
\hline 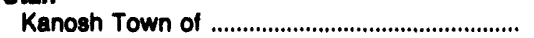 & - & - & 1,892 & - & - & - & 1,892 \\
\hline Kaysville Clty Corp Elec Dept .............................. & - & - & - & -- & - & 25,606 & 25,606 \\
\hline Lehi City City of & - & - & -- & -- & - & 30,684 & 30,684 \\
\hline Meadow Town Corporation ........................................ & - & - & 1,454 & - & - & 700 & 2,154 \\
\hline 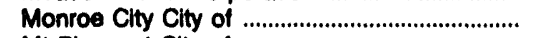 & - & - & - & - & -- & 6,130 & 6,130 \\
\hline 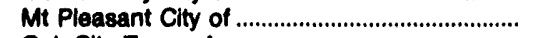 & - & - & 5,201 & - & -- & 6,180 & 11,381 \\
\hline 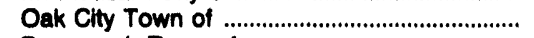 & - & - & 2,650 & - & - & 294 & 2,944 \\
\hline 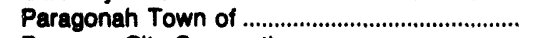 & - & - & 521 & - & - & 1,054 & 1,575 \\
\hline 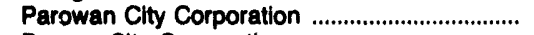 & - & 6,184 & 4,073 & -- & - & -- & 10,257 \\
\hline Payson City Corporation & - & - & 10,000 & - & - & 36,807 & 46,807 \\
\hline 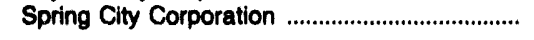 & 700 & 803 & -- & - & -- & - & 1,503 \\
\hline \multicolumn{8}{|l|}{ Wyoming } \\
\hline Basin Town of & - & - & - & - & 8,742 & - & 8,742 \\
\hline 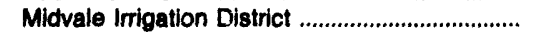 & - & - & - & - & 237 & - & 237 \\
\hline
\end{tabular}

I Includes transactions with power pools, utilities in Canada and Mexico, and nonutilities.

" Source of data is Rural Electrification Administration, REA Form 7, "Financial and Statistical Report."

- Not Applicable

Notes: -For identification purposes, the municipalities are listed in the State in which the municipality is located. - Totals may not equal sum of components because of independent rounding.

Source: -Energy Information Administration Form EIA-861, "Annual Electric Utility Report," except where footnoted. 
Table 32. Electriclity Exchanges and Wheeling by Publicly Owned Utilities, 1992 (Thousand Kilowatthours)

\begin{tabular}{|c|c|c|c|c|}
\hline \multirow{2}{*}{$\begin{array}{l}\text { Publle Authortty / } \\
\text { State / Utilty }\end{array}$} & \multicolumn{2}{|c|}{ Exchanges } & \multicolumn{2}{|c|}{ Wheeling } \\
\hline & Recelved & Dellvered & Received & Delivered \\
\hline \multicolumn{5}{|l|}{ Foderal } \\
\hline \multicolumn{5}{|l|}{ Alane } \\
\hline $\begin{array}{l}\text { Alaska Power Administration } \\
\text { Color...................................... }\end{array}$ & - & - & 303,522 & 303,522 \\
\hline $\begin{array}{l}\text { Western Area Power Admin } \\
\text { Ceorgla }\end{array}$ & $1,841,000$ & $1,269,000$ & $6,144,038$ & $6,144,038$ \\
\hline $\begin{array}{l}\text { Southeastern Power Admin } \\
\text { Oklahoma }\end{array}$ & 185 & - & $4,154,527$ & $4,154,527$ \\
\hline 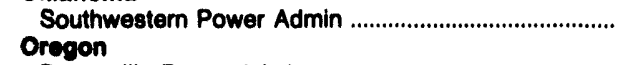 & 412,210 & 694,502 & - & - \\
\hline 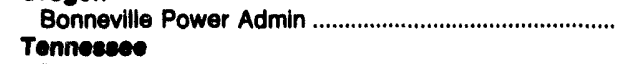 & $13,926,757$ & $20,815,831$ & $39,611,596$ & $37,213,924$ \\
\hline 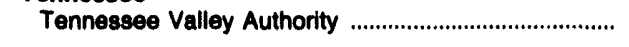 & $22,509,076$ & $26,795,816$ & $1,611,167$ & $1,565,429$ \\
\hline \multicolumn{5}{|l|}{ State and Other Covernment } \\
\hline \multicolumn{5}{|l|}{ Calliomia } \\
\hline 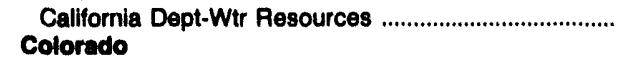 & $4,038,007$ & $1,470,477$ & - & -- \\
\hline $\begin{array}{l}\text { Platte River Power Authority } \\
\text { Connecticut }\end{array}$ & 12,865 & 56,973 & -- & -- \\
\hline $\begin{array}{l}\text { Connecticut Mun Elec Engy Coop .................................... } \\
\text { Georgia }\end{array}$ & 318,764 & 21,781 & -- & - \\
\hline 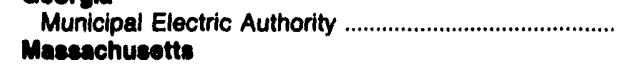 & - & - & 822,455 & 777,220 \\
\hline 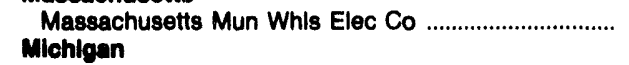 & 774,820 & 402,384 & - & - \\
\hline $\begin{array}{l}\text { Michigan South Central Pwr Agy ............................................ } \\
\text { Oklahoma }\end{array}$ & - & 854 & -- & - \\
\hline $\begin{array}{l}\text { Grand River Dam Authority .................................................... } \\
\text { South Carollna }\end{array}$ & 135,726 & $1,439,228$ & 1,045 & 1,045 \\
\hline $\begin{array}{l}\text { South Carolina Pub Serv Auth } \\
\text { South Dakota }\end{array}$ & 39,388 & 45,864 & 445,716 & 439,327 \\
\hline $\begin{array}{l}\text { Missouri Basin Mun Power Agny .................................... } \\
\text { Texas }\end{array}$ & 67,222 & 198 & - & -- \\
\hline $\begin{array}{l}\text { Toledo Bend Project Joint Oper .................................... } \\
\text { Utah }\end{array}$ & 908 & 908 & -- & - \\
\hline 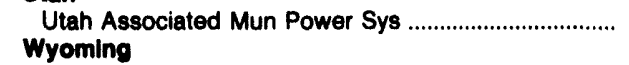 & 23,649 & 16,419 & - & -- \\
\hline 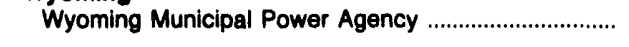 & 3,912 & 2,268 & - & - \\
\hline \multicolumn{5}{|l|}{ Municlpal } \\
\hline \multicolumn{5}{|l|}{ Alanka } \\
\hline 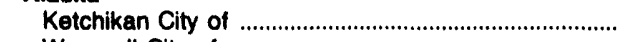 & 81 & - & - & - \\
\hline $\begin{array}{l}\text { Wrangell City of } \\
\text { Callfornia }\end{array}$ & 1,468 & 3,352 & - & -- \\
\hline Anaheim City of & 18,013 & 47,795 & -- & -- \\
\hline Burbank City of & 23,295 & 56,706 & -- & - \\
\hline 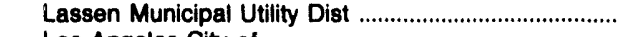 & - & -- & 196,722 & 196,722 \\
\hline 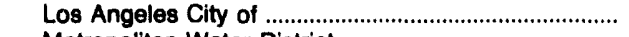 & 438,555 & $1,074,855$ & $12,814,463$ & $12,765,840$ \\
\hline 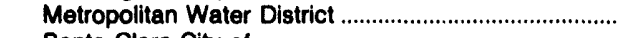 & - & 28,220 & - & - \\
\hline 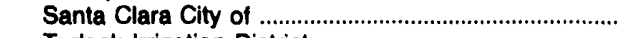 & - & -- & 214,246 & 211,161 \\
\hline 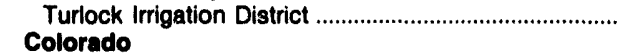 & 1,401 & 1,192 & 69 & 1,107 \\
\hline 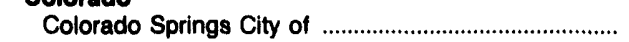 & 2,326 & 15,805 & 27,149 & 27,149 \\
\hline 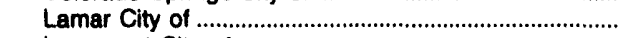 & $-\infty$ & - & 7,911 & 7,911 \\
\hline Longmont Clty of & - & - & 6,648 & 6,644 \\
\hline 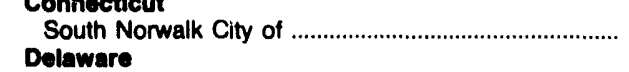 & 1,120 & 104 & -- & - \\
\hline $\begin{array}{l}\text { Dover City of } \\
\text { Florlda }\end{array}$ & 240,638 & 40,244 & - & - \\
\hline Lakeland City of & 266,948 & 968,000 & -- & -- \\
\hline 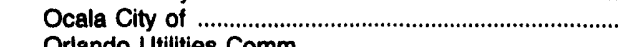 & - & - & 17,096 & 17,096 \\
\hline 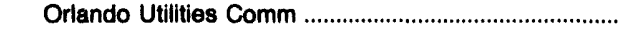 & - & - & 333,524 & 325,343 \\
\hline
\end{tabular}

See notes and footnotes at end of table. 
Table 32. Electricity Exchanges and Wheeling by Publicly Owned Utilities, 1992 (Therusand Kilowatthours) (Continued)

\begin{tabular}{|c|c|c|c|c|}
\hline \multirow{2}{*}{$\begin{array}{l}\text { Publl: Authority I } \\
\text { State / Utilly }\end{array}$} & \multicolumn{2}{|c|}{ Exchanges } & \multicolumn{2}{|c|}{ Wheoling } \\
\hline & Received & Dellvered & Recelved & Delvered \\
\hline \multicolumn{5}{|l|}{ Municipal } \\
\hline \multicolumn{5}{|l|}{ lown } \\
\hline 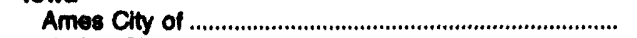 & 907 & 719 & - & - \\
\hline 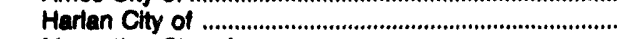 & 2,502 & 1,748 & - & - \\
\hline 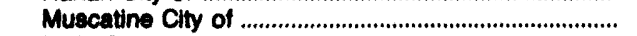 & - & - & 145 & 397 \\
\hline 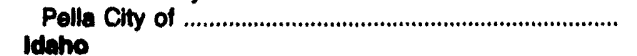 & 5,700 & 5,900 & - & - \\
\hline 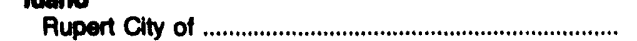 & -- & - & 126 & 126 \\
\hline Kanaas & & & 保 & 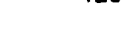 \\
\hline 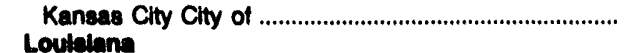 & 37 & 11,333 & - & - \\
\hline Lafayette City of & 26,238 & 26,226 & - & - \\
\hline 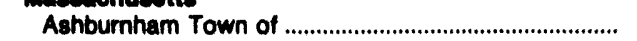 & 5,767 & 5,008 & - & - \\
\hline 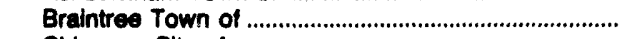 & 49,456 & 63,481 & -- & - \\
\hline 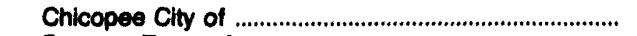 & 67,011 & 2,266 & - & - \\
\hline 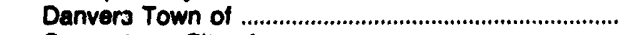 & 78,075 & 56,669 & - & - \\
\hline 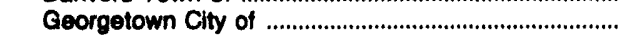 & 4,123 & 408 & - & - \\
\hline Groton City of & 11,162 & 4,689 & - & - \\
\hline 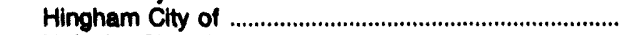 & 16,549 & 2,538 & - & - \\
\hline Holyoke City of & 14,636 & 51,912 & - & - \\
\hline 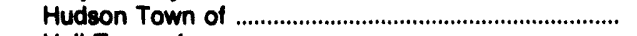 & 37,801 & 25,484 & - & -- \\
\hline 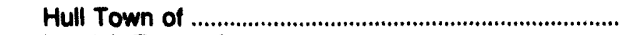 & 7,093 & 11,893 & -- & -- \\
\hline 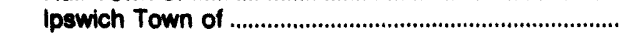 & 36,029 & 4,645 & - & - \\
\hline 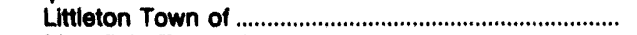 & 83,549 & 11,776 & - & - \\
\hline 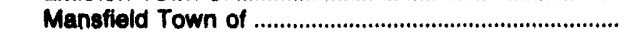 & 70,387 & 28,111 & - & - \\
\hline 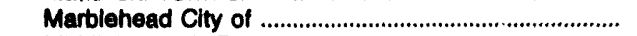 & 15,061 & 614 & - & - \\
\hline 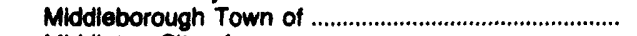 & - & 2,278 & - & - \\
\hline Middleton City of & 7,599 & 2,029 & - & - \\
\hline 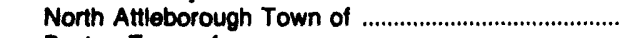 & 80,748 & 21,721 & - & - \\
\hline Paxton Town of & 5,611 & 4,250 & - & - \\
\hline Peabody City of & 133,804 & 81,689 & - & -- \\
\hline 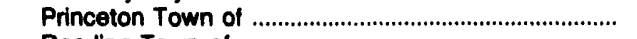 & - & - & 3,274 & 2,855 \\
\hline 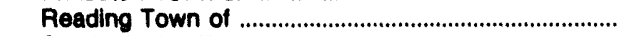 & 128,583 & 69,203 & - & - \\
\hline 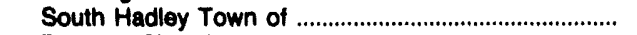 & 25,096 & 13,224 & -- & - \\
\hline Taunton City of & 297,002 & 13,987 & - & - \\
\hline 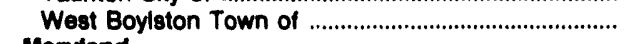 & 14,803 & 8,270 & - & -- \\
\hline Maryland & & & & \\
\hline $\begin{array}{c}\text { Easton Utilities Comm } \\
\text { Mlchigan }\end{array}$ & 179,057 & 308 & - & - \\
\hline 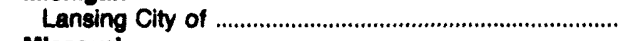 & 65,778 & 65,919 & - & -- \\
\hline $\begin{array}{l}\text { Mlacourt } \\
\text { Bethany City of }\end{array}$ & - & - & 536 & \\
\hline 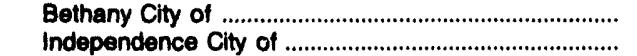 & $\overline{744,200}$ & 743,838 & $\ldots$ & -343 \\
\hline 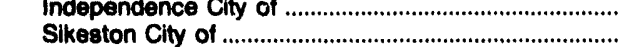 & 141,855 & 64,077 & $\ldots$ & $\overline{-}$ \\
\hline $\begin{array}{l}\text { Sikeston City of } \\
\text { Springtield City of }\end{array}$ & 41,541 & 88,829 & - & - \\
\hline North Carollna & & & & \\
\hline 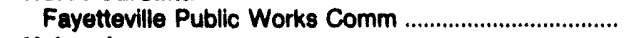 & 44,164 & 135 & - & - \\
\hline Nebrecka & & & & \\
\hline 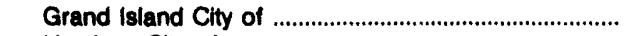 & 1,099 & 1,032 & - & - \\
\hline 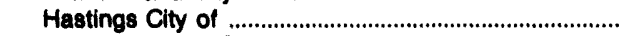 & - & - & 5,228 & 5,228 \\
\hline 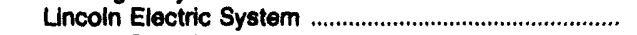 & - & 128 & 105,494 & 105,494 \\
\hline 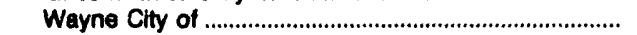 & - & - & 1,892 & 1,778 \\
\hline Now Hampohire & & & & \\
\hline 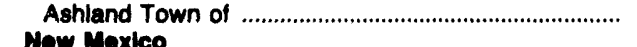 & -- & - & 617 & 617 \\
\hline $\begin{array}{l}\text { Now Moxlco } \\
\text { Farminoton City of }\end{array}$ & & & 9,921 & \\
\hline $\begin{array}{l}\text { Farmington City of } \\
\text { Los Alamos County }\end{array}$ & 65,606 & $\begin{array}{r}1,093 \\
47,290\end{array}$ & 3,921 & 9,921 \\
\hline 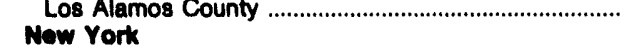 & & & & \\
\hline 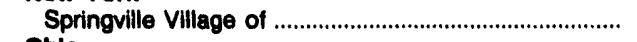 & - & - & 55,740 & 55,183 \\
\hline Ohio & & & & \\
\hline American Mun Power-Ohio Inc .............................................. & - & -- & 149,389 & 149,389 \\
\hline 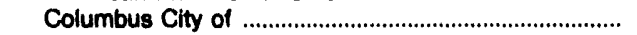 & - & - & 298,376 & 298,376 \\
\hline 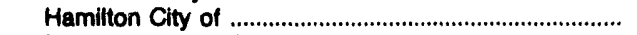 & 10,712 & 18,695 & - & - \\
\hline 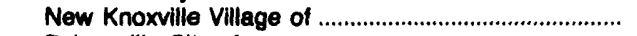 & - & - & 1,511 & 1,511 \\
\hline 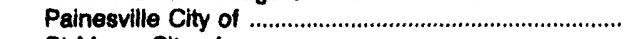 & 1,705 & 7,464 & - & - \\
\hline 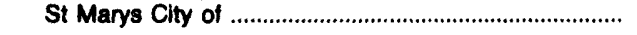 & 1,168 & 1,448 & - & - \\
\hline
\end{tabular}

See notes and footnotes at end of table. 
Table 32. Electriclty Exchanges and Whoeling by Publlcly Owned Utillties, 1992 (Thousand Kilowatthours) (Continued)

\begin{tabular}{|c|c|c|c|c|}
\hline \multirow{2}{*}{$\begin{array}{l}\text { Publle Authority / } \\
\text { state / Utilly }\end{array}$} & \multicolumn{2}{|c|}{ Exchanges } & \multicolumn{2}{|c|}{ Wheeling } \\
\hline & Received & Dellvered & Recelved & Dellvered \\
\hline \multicolumn{5}{|l|}{ Mu iclpal } \\
\hline 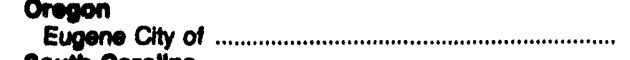 & 117,803 & 319,005 & - & - \\
\hline $\begin{array}{l}\text { Pledmont Municipal Power Agny ........................................ } \\
\text { Toxes }\end{array}$ & 187,883 & 219,238 & - & - \\
\hline 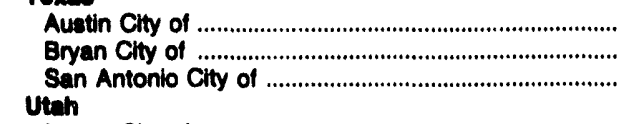 & $\begin{array}{r}59,580 \\
184,336 \\
956,905\end{array}$ & $\begin{array}{r}46,104 \\
185,587 \\
937,971\end{array}$ & $\begin{array}{l}24,514 \\
- \\
-\end{array}$ & $\begin{array}{l}22,885 \\
-\end{array}$ \\
\hline 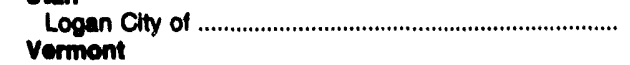 & - & - & 5,601 & 5,601 \\
\hline 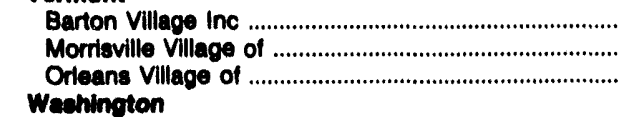 & $\begin{array}{r}758 \\
7,550 \\
301\end{array}$ & $\begin{array}{r}758 \\
5,121 \\
1,315\end{array}$ & - & - \\
\hline Seactle City of & $\begin{array}{r}5,932,411 \\
95,676\end{array}$ & $\begin{array}{r}5,935,697 \\
128,221\end{array}$ & $\begin{array}{r}51,381 \\
1,227,049\end{array}$ & $\begin{array}{r}51,381 \\
1,227,048\end{array}$ \\
\hline
\end{tabular}

- Not Applicable

Source: Energy Information Administration, Form ElA-861, "Annual Electric Utility Report." 


\section{Bulk Power Transactions by Cooperative Borrowers}

\section{Overview}

This chapter presents electric trade data for participants in the U. S. Department of Agriculture's Rural Electrification Administration (REA) Cooperative Borrowers program. The data are separated and presented for the Power Supply Borrowers and Distribution Borrowers. Power Supply Borrowers generate and purchase electricity for wholesale transactions with other electric utilities, while most Distribution Borrowers do not own generators and provide only distribution services to the final consumer. Coopera- tive borrowers are required to provide REA with financial and operational data to support the REA loan management program. The cooperative borrowers represent nearly all the cooperatives in the country. ${ }^{8}$

There are many types of cooperative associations, but five basic principles or features distinguish cooperatives from other types of private enterprise businesses.

- Ownership is held by member-users.

- Control is on the basis of one vote per member, or on volume provided.

- Operations have an at-cost (nonprofit) objective.

Figure 14. Electricity Purchases by Cooperative Borrowers by State, 1992

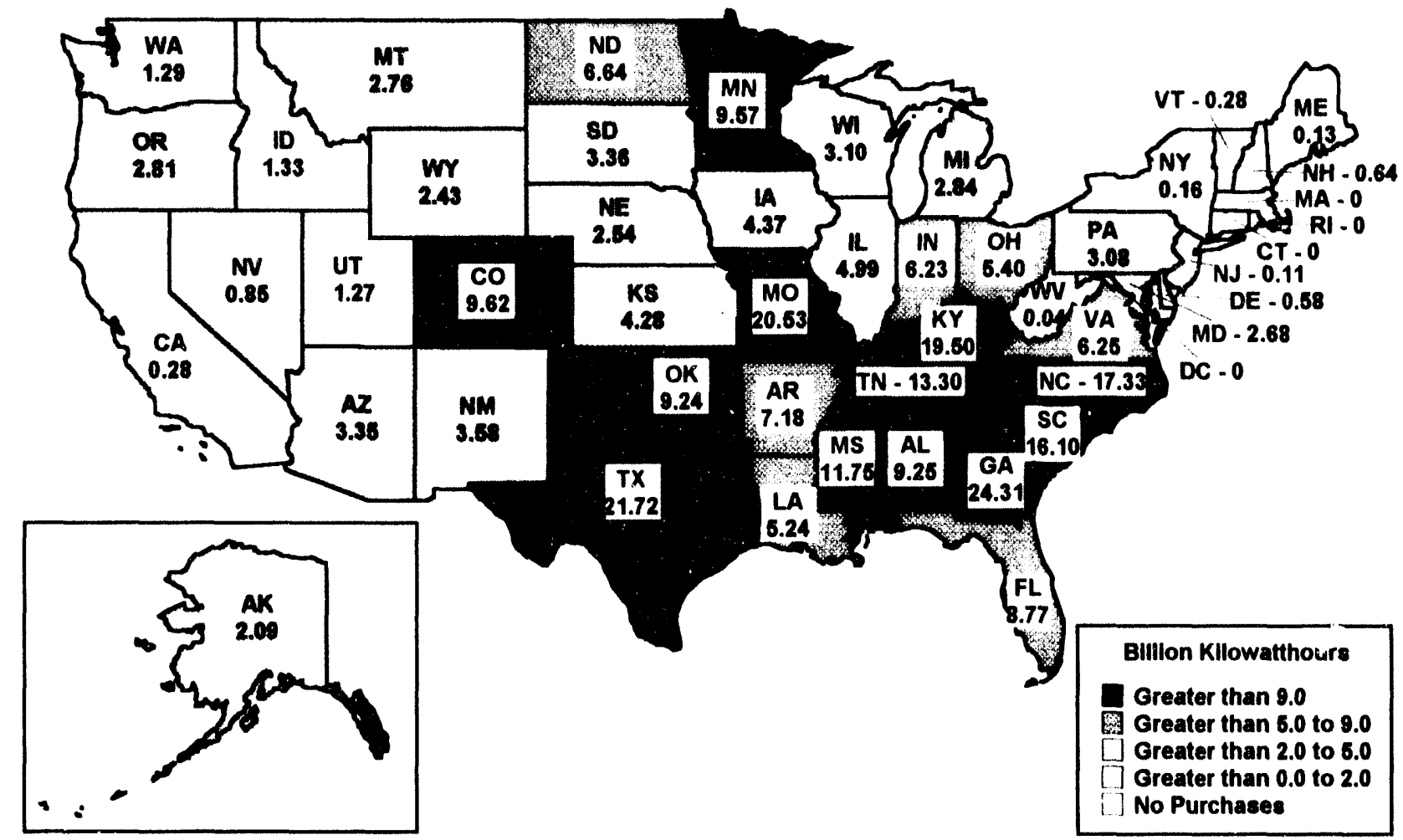

Source: Rural Electrification Administration, REA Form 7, "Financial and Statistical Report," REA Form 12a through 12i, "Electric Power Supply Borrowers," REA Form 12c through 12g. "Electric Distribution Borrowers with Generating Facilities."

${ }^{8}$ There have been fewer than 50 REA borrowers that have paid off their loans. Those utilities are not required to file the REA forms. 
- Dividends on member capital are limited.

- Education is necessary for understanding and support.9

Cooperative entities are recognized by State statutes, which differ by State. Cooperatives can use a variety of generic labels depending on State statutes: mutuals and cooperatives (Washington, Idaho, and Nevada); cooperatives, rural electric membership corporations (North Carolina); rural electric systems, electric power associations (Mississippi), etc. In addition, many cooperatives are incorporated. Federal legislation further defines cooperative activities. Cooperatives are organized for a variety of reasons including insurance, agricultural, telephone, water, and electrical services.

There were about 35 electric cooperatives which predated the establishment of the REA in 1935. A number of these cooperatives were formed as mutual associations. Many of these early efforts, however, were not successful. It was the establishment of the REA and other activities starting in 1935 that initiated the major development phase of cooperative electrification.
This chapter focuses on purchases by cooperative borrowers because, at this time, REA computerizes only individual utility transactions with other utilities for purchased power (Figure 14). Therefore, data on sales for resale are not presented in this chapter. Because REA does not collect information on exchanges and wheeling, data from the Form EIA.861, "Annual Electric Utility Report," were used to provide aggregate data totals for the cooperative borrowers. Form EIA-861 is a mandatory form filed annually by all electric utilities in the United States on a calendar-year basis.

\section{Tables}

This chapter presents data on each of the REA electric borrowers. These cooperatives purchased electricity from other cooperatives, investor-owned, Federally owned, municipal utilities, and State authorities. Electricity purchases by power supply cooperatives are presented in Table 33 and by distribution cooperatives in Table 34. Cooperatives not listed elsewhere are pre-

Figure 15. Electricity Purchase Costs by Cooperative Borrowers by State, 1992

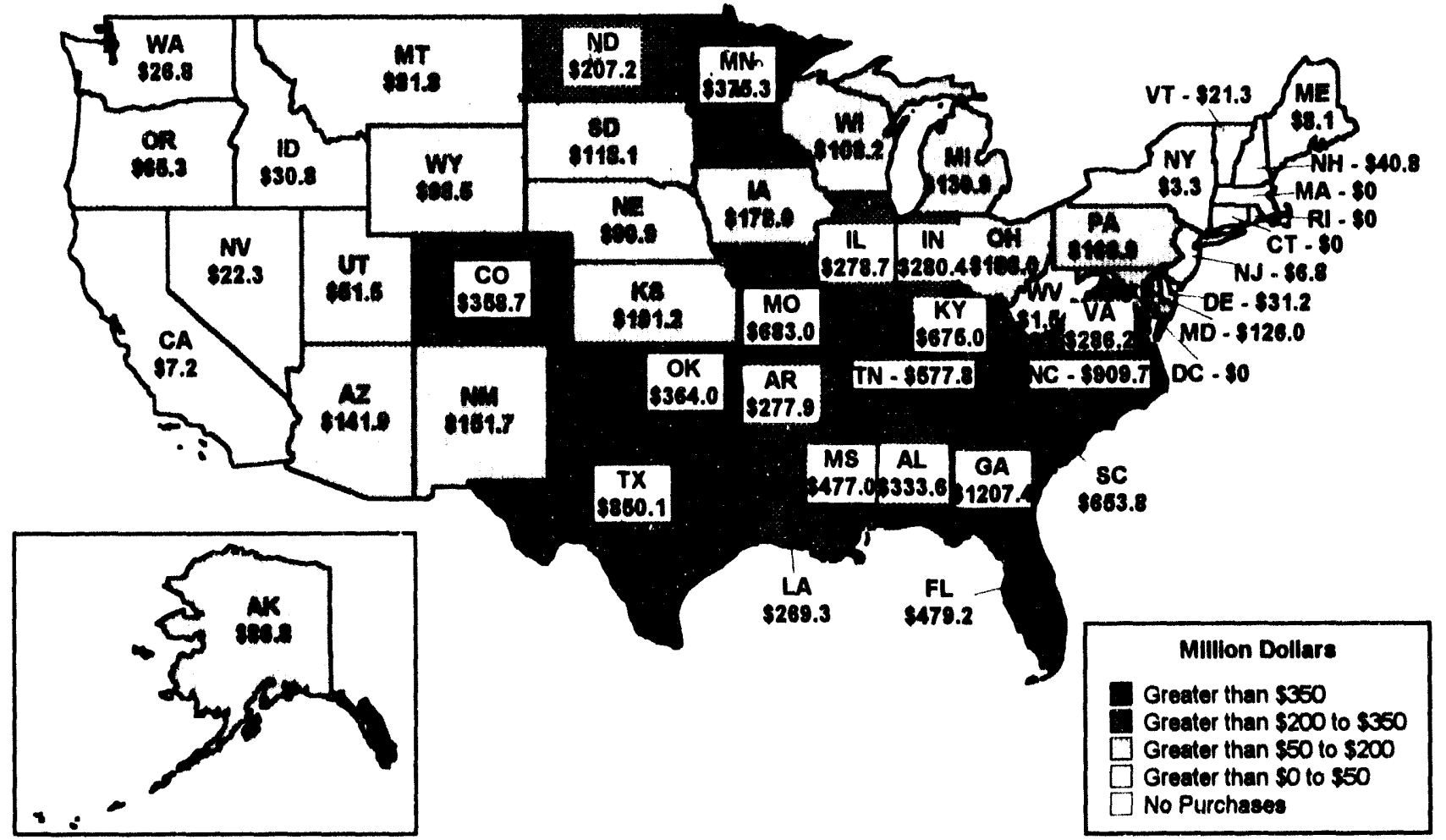

Source: Rural Electrification Administration, REA Form 7, "Financial and Statistical Report," REA Form 12a throgh 121, "Electric Power Supply Borrowers," REA Form 12c through 12g, "Electric Distribution Borrowers with Generating Faclities."

'Information paraphrased and quoted from Agricultural Cooperative Service, U. S. Department of Agriculture, "Cooperative Principles and Legal Foundations," Cooperative Information Report 1, Section I (March 1982), p. 3. 


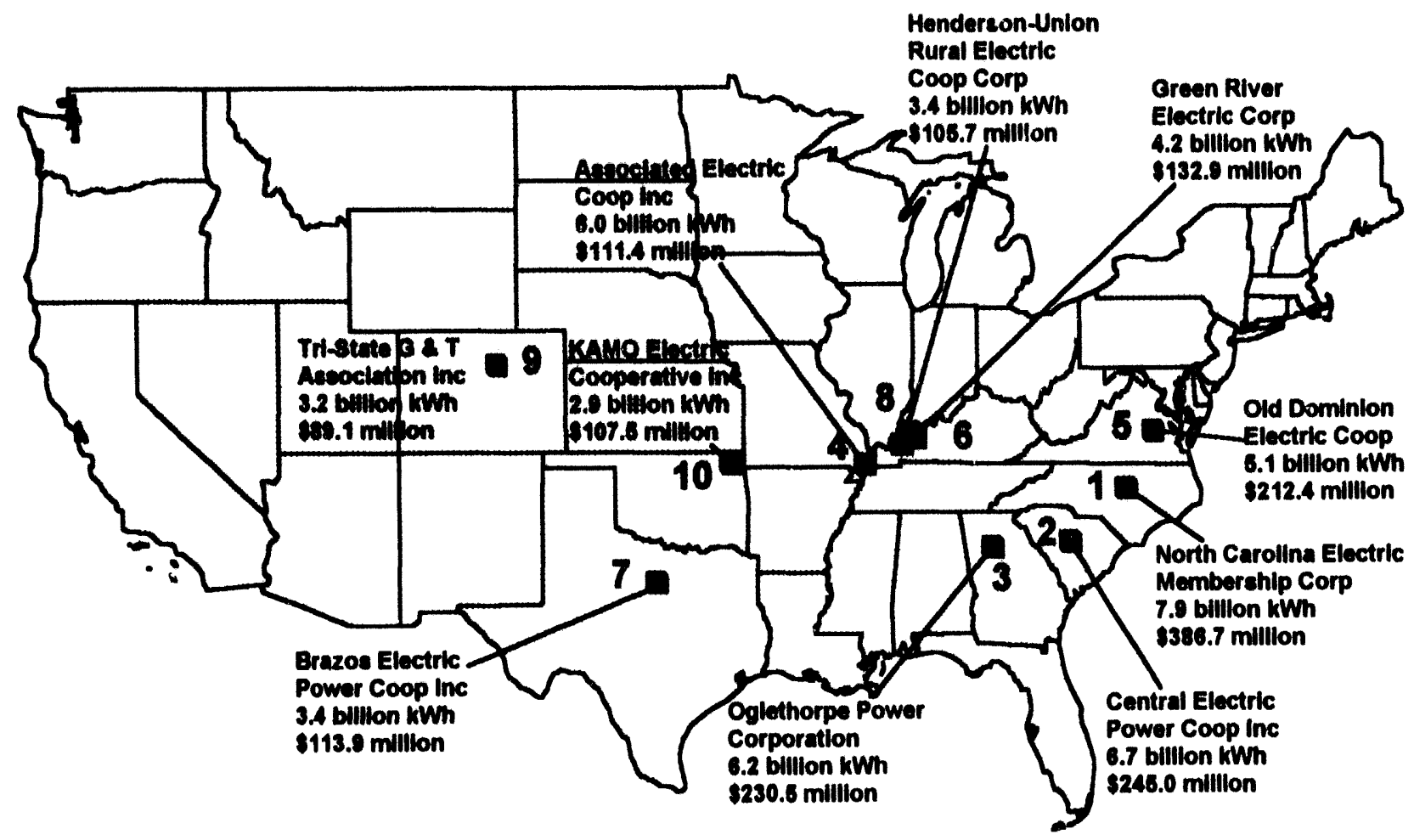

Notes: Number appearing next to utility location indicates rank by kilowatthours (kWh) purchased. Old Dominion Electric Coop is no longer an REA borrower and does not flle a Form 12; source of data is FERC Form 1.

Source: Rural Electrification Administration, REA Form 7, "Financial and Statistical Report," REA Form 12a through 121, "Electric Power Supply Borrowers," REA Form 12c through 12g, "Electric Distribution Borrowers with Generating Facilities."

sented in Table 35. Table 36 provides data on exchanges and transmission service transactions of the cooperative borrowers.

The tables present aggregate data on total quantity and value of each cooperative's electric utility trade (Figure 15). The reported quantities cannot be broken into firm and interruptible trade classifications. Nor is information available on demand, energy, and other revenues and costs. For ease of identification, the cooperative borrowers are listed by State according to the mailing address of the administrative office. This listing does not necessarily reflect the electrical geographics of a cooperative's electrical system.

\section{Highlights}

As a group, power supply cooperatives purchased more electricity than the average distribution cooperative. However, in ranking purchases of electricity by cooperatives, the top 20 in both classes of cooperatives each purchased more than 1 billion kilowatthours.
(The power supply cooperatives acquired nearly 60 billion kilowatthours while the top 20 distribution bought slightly less than 36 billion kilowatthours.) In addition, 13 of the top 20 distribution cooperatives are located in just 5 States in the Southeast (Georgia, Florida, Kentucky, Tennessee and Virginia). Of all power purchased by the top 20 distribution cooperatives, 73.9 percent was purchased by these 13 cooperative utilities (Tables 33, 34, and 35), which reflects a strong regional strength for cooperative utilities (Figure 16).

The most purchases made by a distribution cooperative in 1992 totaled 4.2 billion kilowatthours by the Green River Electric Corporation of Kentucky. That electricity cost $\$ 132.9$ million, which was also the largest total payments made by a distribution cooperative in 1992. The State of Kentucky also had another cooperative in the top five. Henderson-Union Rural Electric Cooperative Corporation was second in amount of purchases but fourth in the cost of those purchases. It paid $\$ 105.7$ million for 3.4 billion kilowatthours. In third place for the amount of electricity purchased, as in 1990, was the Middle Tennessee Electric Membership Corporation. This cooperative ranked fifth in total cost, buying 2.5 billion kilowatthours for $\$ 102.9$ mil- 


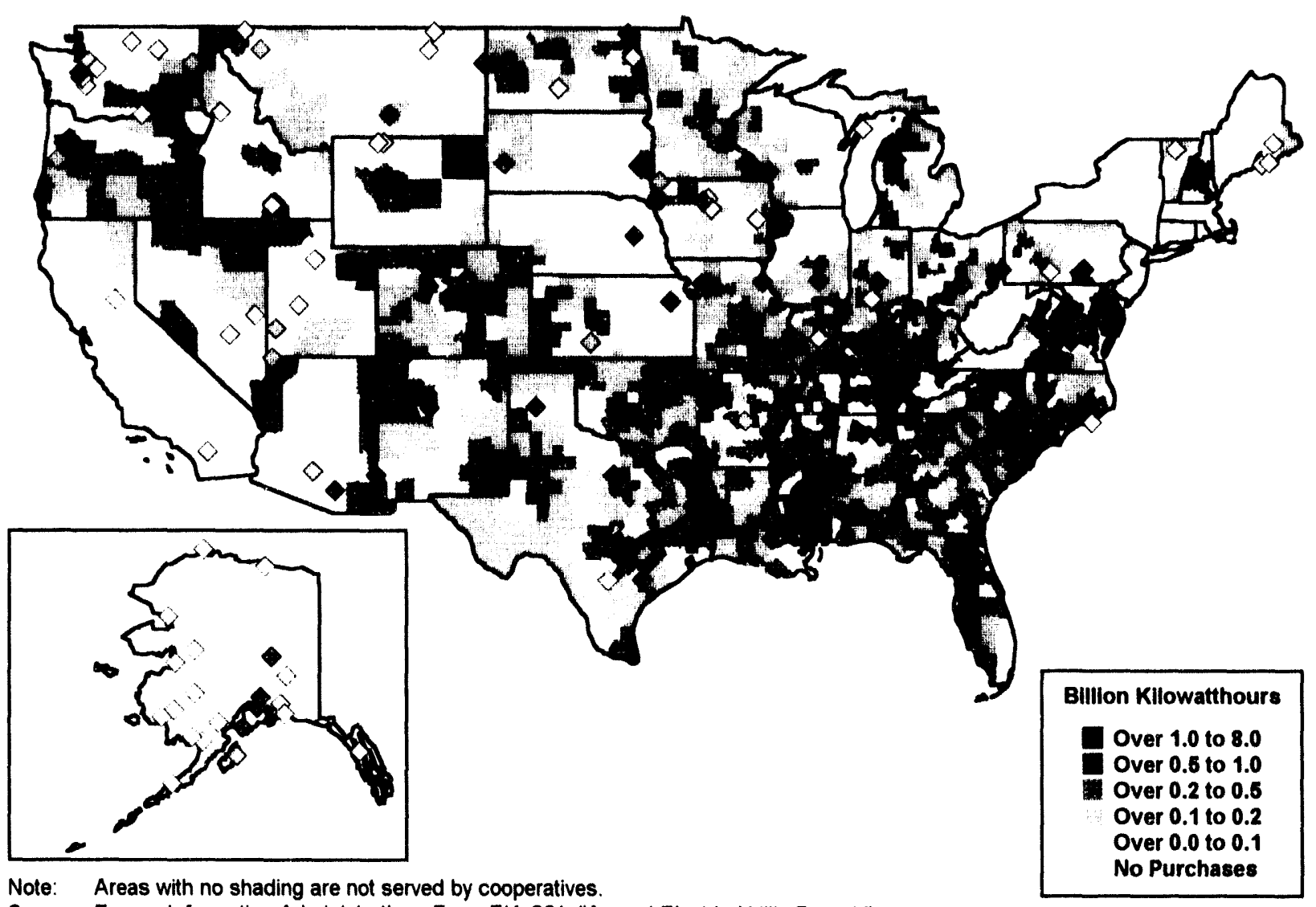

Source: Energy Information Administration, Form EIA-861, "Annual Electric Utility Report."

lion. Of the other top five power purchasers, the Southern Maryland Electric Cooperative bought 2.1 billion kilowatthours for $\$ 96.6$ million and was followed by the Withlacoochee River Electric Cooperative of Florida which purchased just under 2.0 billion kilowatthours for \$114.9 million.

Purchases by power supply cooperatives showed a similar pattern of regional concentrations. Of the top 20 power supply cooperatives, 9 are centered in the States of Missouri, Texas, South Carolina and Kentucky. While most of the top 20 power supply cooperatives are located east of the Mississippi River, the first 3 are in the Southeastern coastal States. The largest power supply cooperative purchaser by quantity was the North Carolina Electric Membership Corporation which spent $\$ 386.7$ million for 7.9 billion kilowatthours. It was followed by the Central Electric Power Coop, Inc. of South Carolina which bought 6.7 billion kilowatthours for $\$ 245$ million. The third largest purchaser was the Oglethorpe Power Corporation of Georgia. That cooperative spent $\$ 230.5$ million for 6.2 billion kilowatthours. The fourth largest purchaser, the Associated Electric Cooperative of Missouri, bought 6 billion kilowatthours but only paid $\$ 111.4$ million which illustrates that there can be large differ- ences in costs among regions. The Brazos Electric Power Cooperative, Inc. of Texas bought 3.4 billion kilowatthours for $\$ 113.9$ million and holds the fifth place.

Most cooperative utilities are not very active in implementing exchange or wheeling transactions with other utilities outside their membership. Nevertheless, the Brazos Electric Power Cooperative, Inc. of Texas exchanged over 17 billion kilowatthours and Tri-State Generation \& Transmission Assn., Inc. of Colorado exchanged about 1.7 billion kilowatthours. The East Kentucky Power Cooperative provided transmission services (wheeling) for about 1.5 billion kilowatthours (Table 36).

While power supply cooperatives operate generation facilities to meet the needs of both bulk power requirements of their membership and supply the end-using customer, no cooperatives were among the top 50 of all utilities for generation and count of end-use customers. In part, this reflects the historical mission of cooperative utilities to serve low-population areas. Yet, these cooperative utilities do provide service to large areas of the United States (Figure 17). 


\section{Summary}

Power supply cooperatives, although small in total numbers, play an important role in the wholesale power resale market. Their large purchases and wideranging redistribution capability have put them in a highly visible position in the cooperative sector of the electric power industry. The amount of electricity a large power supply cooperative purchases is far greater than that of a typical distribution cooperative.

Many of these power suppliers are wholly owned by distribution cooperatives and supply only those cooperatives as wholesale customers. Others are distribution cooperatives with excess generating capability. But as a group, power supply cooperatives continue to purchase more electric power from a wider range of sources.
Regional differences in establishment of cooperatives primarily resulted from the historical development of the electric utility industry. Differences in the volume and type of trade can be related to geographical concentration and the proximity of other utilities and transmission facilities. For instance, some large interconnected transmission systems cross cooperative service territories affording the cooperative multiple sources of power. In contrast, cooperatives that are geographically isolated may only be able to buy from a single source.

Most distribution cooperatives sell power to the ultimate consumers who reside in their service territories. Cooperative service territories generally have a lower density of customers and many miles of distribution lines. Although distribution cooperatives do buy power from nearby investor-owned utilities and government authorities, the large majority of distribution cooperatives purchase most of their supply from power supply cooperatives. 
Table 33. Electriclty Purchases by the Power Supply segment of Cooperative Borrowers, by State, 1992

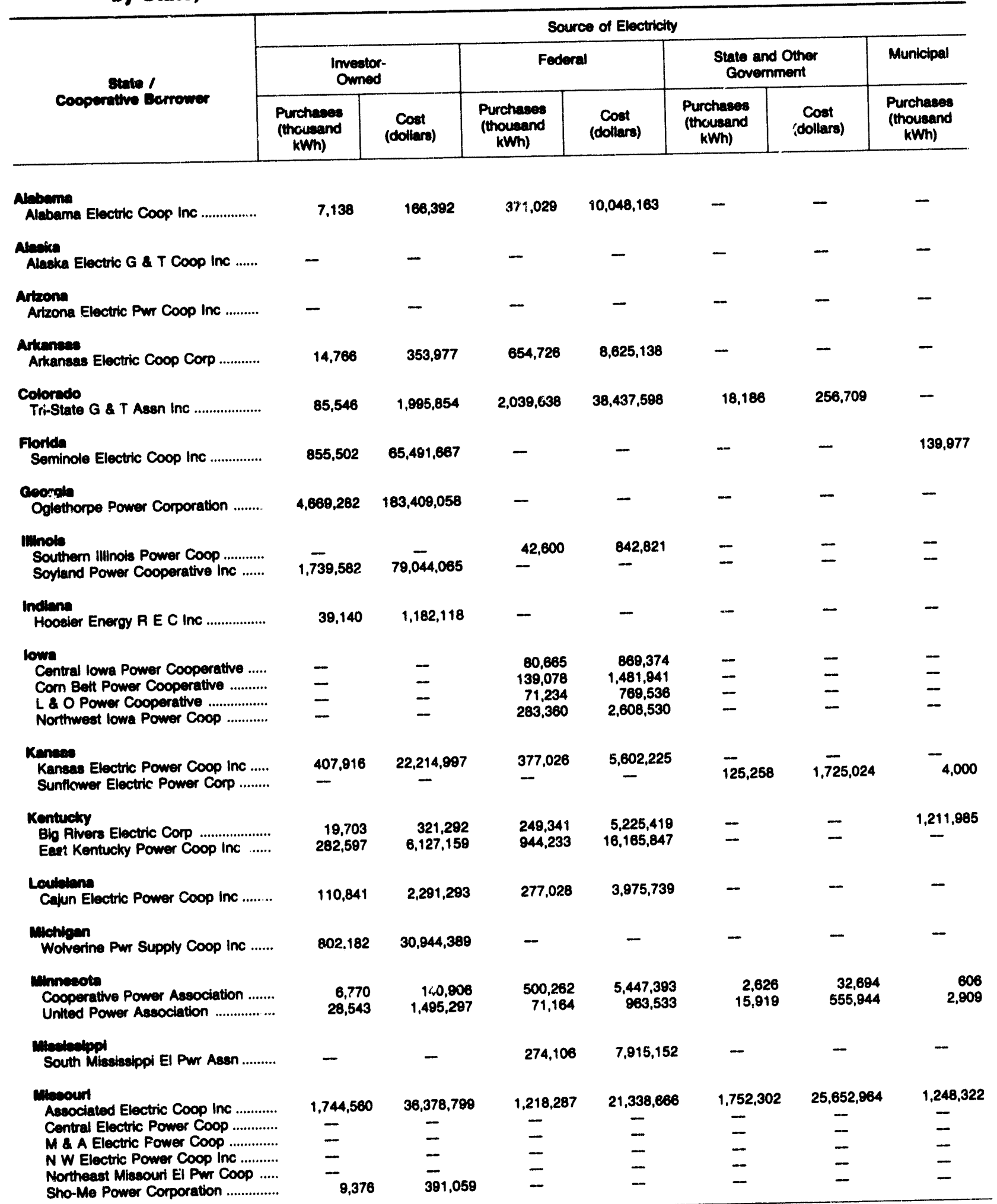

See notes and footnotes at end of table. 
Table 33. Electricity Purchases by the Power Supply Segment of Cooperative Borrowers, by State, 1992 (Continued)

\begin{tabular}{|c|c|c|c|c|c|c|c|}
\hline \multirow{3}{*}{ Cocperitive Eornower } & \multicolumn{7}{|c|}{ Source of Electricity } \\
\hline & \multirow{2}{*}{$\frac{\text { Municipal }}{\text { Cost }}$} & \multicolumn{2}{|c|}{ Cooperative } & \multicolumn{2}{|c|}{ Other' } & \multicolumn{2}{|c|}{ Total } \\
\hline & & $\begin{array}{l}\text { Purchaess } \\
\text { (thoueand } \\
\text { kWh) }\end{array}$ & $\begin{array}{c}\text { Cost } \\
\text { (dollars) }\end{array}$ & $\begin{array}{l}\text { Purchases } \\
\text { (thousend } \\
\text { kWh) }\end{array}$ & $\begin{array}{c}\text { Cost } \\
\text { (dollars) }\end{array}$ & $\begin{array}{l}\text { Purchases } \\
\text { (thousand } \\
\text { kWh) }\end{array}$ & $\begin{array}{c}\text { Cost } \\
\text { (dollars) }\end{array}$ \\
\hline Alrowama Electric Coop Inc ................ & - & $1,603,505$ & $31,314,454$ & 409,245 & $11,541,689$ & $2,380,917$ & $53,070,698$ \\
\hline Alaska Electric G \& T Coop Inc ...... & - & 804,109 & $30,549,978$ & - & - & 804,109 & $30,549,978$ \\
\hline $\begin{array}{l}\text { Ateoria } \\
\text { Artzona Electric Pwr Coop Inc ......... }\end{array}$ & - & - & - & 690,072 & $17,268,105$ & 690,072 & $17,266,105$ \\
\hline $\begin{array}{l}\text { Arteneses } \\
\text { Arkaneas Electric Coop Corp ............ }\end{array}$ & - & 133,365 & $2,201,682$ & 231 & 14,174 & 803,088 & $11,194,971$ \\
\hline $\begin{array}{l}\text { Coloredo } \\
\text { Tri-State G a T Asan Inc ................... }\end{array}$ & - & $1,088,440$ & $48,287,176$ & 746 & 24,498 & $3,232,556$ & $89,011,835$ \\
\hline $\begin{array}{l}\text { Fordela } \\
\text { Seminole Electric Coop Inc ................ }\end{array}$ & $10,034,759$ & 17,804 & 411,826 & 342 & 17,729 & $1,013,725$ & $75,955,981$ \\
\hline $\begin{array}{l}\text { Cocreta } \\
\text { Oolethorpe Power Corporation ......... }\end{array}$ & - & $1,495,031$ & $44,599,170$ & 68,849 & $2,502,087$ & $6,233,262$ & $230,510,324$ \\
\hline $\begin{array}{l}\text { Iminols } \\
\text { Southem Illinois Power Coop ........... } \\
\text { Soyland Power Cooperative Inc ...... }\end{array}$ & - & $\begin{array}{r}782 \\
-\quad\end{array}$ & 38,129 & - & - & $\begin{array}{r}43,392 \\
1,738,582\end{array}$ & $\begin{array}{r}880,850 \\
79,044,065\end{array}$ \\
\hline $\begin{array}{l}\text { Indiene } \\
\text { Hoosier Energy R E C Inc .................. }\end{array}$ & - & 9,543 & 750,140 & - & - & 48,683 & $1,932,258$ \\
\hline $\begin{array}{l}\text { lowe } \\
\text { Central lowa Power Cooperative ..... } \\
\text { Corn Beit Power Cooperative ........... } \\
\text { L \& O Power Cooperative ................ } \\
\text { Northwest lowa Power Coop ........... }\end{array}$ & $\overline{-}$ & $\begin{array}{r}343 \\
110,299 \\
69,978 \\
271,162\end{array}$ & $\begin{array}{r}20,032 \\
1,625,005 \\
3,176,880 \\
12,639,459\end{array}$ & $\begin{array}{l}- \\
- \\
9,346\end{array}$ & $\overline{-}^{5,504}$ & $\begin{array}{r}81,136 \\
249,377 \\
141,212 \\
583,858\end{array}$ & $\begin{array}{r}894,910 \\
3,106,946 \\
3,946,426 \\
15,549,837\end{array}$ \\
\hline $\begin{array}{l}\text { Kenane } \\
\text { Kansas Electric Power Coop Inc ..... } \\
\text { Sunflower Electric Power Corp ........ }\end{array}$ & $\overline{83,289}$ & 13,813 & 227,915 & - & - & $\begin{array}{l}798,755 \\
1.9,258\end{array}$ & $\begin{array}{r}28,045,137 \\
1,808,313\end{array}$ \\
\hline $\begin{array}{l}\text { Kentuchy } \\
\text { Big Rlvers Electric Corp ................... } \\
\text { East Kentucky Power Coop Inc ....... }\end{array}$ & $\begin{array}{l}27,250,486 \\
-\end{array}$ & $\begin{array}{l}36,863 \\
-\end{array}$ & $\begin{array}{l}630,083 \\
-\end{array}$ & $\overline{-}$ & $\overline{-}$ & $\begin{array}{l}1,517,892 \\
1,228,830\end{array}$ & $\begin{array}{l}33,427,280 \\
22,293,006\end{array}$ \\
\hline $\begin{array}{l}\text { Loulatena } \\
\text { Cafun Electric Power Coop inc ........ }\end{array}$ & - & - & - & 12,855 & 386,678 & 400,724 & $6,653,710$ \\
\hline $\begin{array}{l}\text { Ellehiean } \\
\text { Wotverine Pur Supply Coop Inc ...... }\end{array}$ & - & - & - & - & - & 802,182 & $30,944,389$ \\
\hline $\begin{array}{l}\text { Minnesote } \\
\text { Cooperatve Power Association ....... } \\
\text { United Pows Association ................. }\end{array}$ & $\begin{array}{r}9,297 \\
65,691\end{array}$ & $\begin{array}{r}826,979 \\
16,235\end{array}$ & $\begin{array}{r}18,178,088 \\
408,437\end{array}$ & $-4,015$ & $\overline{68,665}$ & $\begin{array}{r}1,137,243 \\
138,785\end{array}$ & $\begin{array}{r}23,808,388 \\
3,553,567\end{array}$ \\
\hline $\begin{array}{l}\text { Mlocicelppi } \\
\text { South Misaissippl EI Pwr Assn ......... }\end{array}$ & - & $1,643,575$ & $35,465,322$ & 40,547 & 653,261 & $1,958,228$ & $44,033,735$ \\
\hline $\begin{array}{l}\text { Wheurt } \\
\text { Aseociated Electric Coop Inc ............ } \\
\text { Central Electric Power Coop ............. } \\
\text { M \& A Electric Power Coop ............ } \\
\text { N W Electric Power Coop Inc ........... } \\
\text { Northeast Mlesourt El Pw Coop ...... } \\
\text { Sho-Me Power Corporation ............... }\end{array}$ & $\begin{array}{l}28,055,247 \\
\overline{-} \\
\overline{-}\end{array}$ & $\begin{array}{r}\overline{1,692,378} \\
968,841 \\
1,020,548 \\
958,528 \\
2,354,264\end{array}$ & $\begin{array}{l}\mathbf{6 6 , 5 7 2 , 5 6 2} \\
37,160,633 \\
36,734,548 \\
31,973,485 \\
83,004,373\end{array}$ & 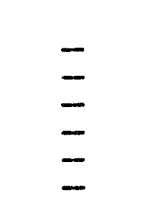 & $\bar{z}$ & $\begin{array}{r}5,963,471 \\
1,682,378 \\
988,841 \\
1,020,548 \\
958,526 \\
2,363,640\end{array}$ & $\begin{array}{r}111,425,676 \\
66,572,562 \\
37,160,633 \\
36,734,548 \\
31,973,485 \\
83,385,432\end{array}$ \\
\hline
\end{tabular}


Table 33. Electricity Purchases by the Power Supply Segment of Cooperative Borrowers, by State, 1992 (Continued)

\begin{tabular}{|c|c|c|c|c|c|c|c|}
\hline \multirow{3}{*}{$\begin{array}{l}\text { State I } \\
\text { Cooperattve Borrower }\end{array}$} & \multicolumn{7}{|c|}{ Source of Electricity } \\
\hline & \multicolumn{2}{|c|}{$\begin{array}{l}\text { Investor. } \\
\text { Owned }\end{array}$} & \multicolumn{2}{|c|}{ Federal } & \multicolumn{2}{|c|}{$\begin{array}{l}\text { State and Other } \\
\text { Government }\end{array}$} & \multirow{2}{*}{$\begin{array}{l}\text { Municipal } \\
\begin{array}{l}\text { Purchases } \\
\text { (thousand } \\
\text { kWh) }\end{array}\end{array}$} \\
\hline & $\begin{array}{l}\text { Purchases } \\
\text { (thousand } \\
\text { kWh) }\end{array}$ & $\begin{array}{c}\text { Cost } \\
\text { (dollars) }\end{array}$ & $\begin{array}{l}\text { Purchases } \\
\text { (thousand } \\
\text { kWh) }\end{array}$ & $\begin{array}{c}\text { Cost } \\
\text { (dollars) }\end{array}$ & $\begin{array}{l}\text { Purchases } \\
\text { (thousand } \\
\text { kWh) }\end{array}$ & $\underset{\text { (dollars) }}{\text { Cost }}$ & \\
\hline $\begin{array}{l}\text { Montana } \\
\text { Upper Missouri G\&T El Coop Inc.... }\end{array}$ & - & - & 323.459 & $3,822,765$ & - & - & - \\
\hline $\begin{array}{l}\text { Nebracka } \\
\text { Nebraska Electric G\&T Coop Inc .... }\end{array}$ & - & - & - & - & $2,339,004$ & $81,040,211$ & - \\
\hline $\begin{array}{l}\text { Now Moxlco } \\
\text { Plains Elec Gen\&Trans Coop inc .... }\end{array}$ & 112,591 & $5,567,326$ & 536,385 & $9,523,997$ & 10,889 & 242,095 & - \\
\hline $\begin{array}{l}\text { North Carolina } \\
\text { North Carolina El Member Corp ....... }\end{array}$ & $7,886,848$ & $386,657,145$ & - & - & - & - & - \\
\hline $\begin{array}{l}\text { North Dakota } \\
\text { Basin Electric Power Coop............... } \\
\text { C6ntral Power Elec Coop Inc .......... } \\
\text { Minnkota Power Coop Inc ............... } \\
\text { Square Butte Elec Coop Inc ................... }\end{array}$ & $\overline{419,473}$ & $\overline{-} \overline{-}$ & $\begin{array}{l}5,879 \\
328,273 \\
571,620 \\
--\end{array}$ & $\begin{array}{l}4,834,525 \\
3,582,007 \\
6,170,356 \\
--\end{array}$ & $\begin{array}{l}2,351 \\
- \\
-\end{array}$ & $\begin{array}{l}67,012 \\
\overline{171}, 719\end{array}$ & $\begin{array}{l}\overline{-} \\
\overline{27,626}\end{array}$ \\
\hline 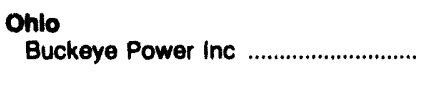 & 419,065 & $7,499,046$ & - & - & - & - & $-\cdots$ \\
\hline $\begin{array}{l}\text { Oklahoma } \\
\text { Kamo Electric Cooperative Inc ........ } \\
\text { Western Farmers Elec Coop Inc ..... }\end{array}$ & $\begin{array}{r}34,874 \\
3,202\end{array}$ & $\begin{array}{r}1,312,265 \\
129,000\end{array}$ & $\overline{876,135}$ & $11, \overline{597,542}$ & $\begin{array}{l}1,393,789 \\
--\end{array}$ & $\begin{array}{l}48,007,969 \\
--\end{array}$ & - \\
\hline $\begin{array}{l}\text { Ponnaylvania } \\
\text { Allegheny Electric Coop Inc .............. }\end{array}$ & 752,729 & $33,271,246$ & - & - & 392,890 & $2,226,724$ & -- \\
\hline $\begin{array}{l}\text { South Carollna } \\
\text { Central Electric Pwr Coop Inc ......... } \\
\text { Saluda River Electric Coop Inc ........ }\end{array}$ & $\begin{array}{r}87,334 \\
1,185,702\end{array}$ & $\begin{array}{r}3,682,146 \\
57,095,442\end{array}$ & 274,437 & $\begin{array}{l}7,529,665 \\
--\end{array}$ & $\stackrel{6,307,822}{-}$ & $233,770,100$ & - \\
\hline $\begin{array}{l}\text { South Dakota } \\
\text { East River Elec Pwr Coop Inc .......... }\end{array}$ & - & - & 728,990 & $7,748,894$ & - & - & - \\
\hline $\begin{array}{l}\text { Toxas } \\
\text { Brazos Electric Power Coop Inc ...... } \\
\text { Northeast Texas Elec Coop Inc ...... } \\
\text { Sam Rayburn G \& T Inc ................ } \\
\text { South Texas Electric Coop Inc ........ } \\
\text { Tex-La Electric Coop-Texas Inc ....... }\end{array}$ & $\begin{array}{r}1,008,916 \\
740,305 \\
29,542 \\
39,970 \\
917,832\end{array}$ & $\begin{array}{r}39,086,700 \\
28,422,727 \\
5,692,330 \\
957,010 \\
36,836,380\end{array}$ & $\begin{array}{l}134,926 \\
380,347 \\
-- \\
259,993\end{array}$ & $\begin{array}{c}1,699,216 \\
5,401,225 \\
\overline{1,894,484} \\
--\end{array}$ & $\begin{array}{l}287,790 \\
\overline{-} \\
\overline{102,363}\end{array}$ & $\begin{array}{l}8,679,170 \\
\overline{-} \\
\overline{1,460,836} \\
-\end{array}$ & $\begin{array}{l}56,212 \\
\overline{-} \\
\overline{15,371}\end{array}$ \\
\hline $\begin{array}{l}\text { Utah } \\
\text { Deseret Generation \& Tran Coop ... }\end{array}$ & 8,516 & 191,976 & 82 & 1,554 & 1,792 & 36,222 & - \\
\hline $\begin{array}{l}\text { Vermont } \\
\text { Vermont G \& T Cooperative Inc ....... }\end{array}$ & 64,147 & $2,761,432$ & - & -- & 18,183 & 583,097 & - \\
\hline $\begin{array}{l}\text { Wisconsin } \\
\text { Dairyland Power Cooperative ............ }\end{array}$ & 130,617 & $1,832,667$ & 25,507 & 377,453 & 140,530 & $1,783,094$ & 9,524 \\
\hline
\end{tabular}

See notes and footnotes at end of table. 
Table 33. Electricity Purchases by the Power Supply Segment of Cooperative Borrowers, by State, 1992 (Continued)

\begin{tabular}{|c|c|c|c|c|c|c|c|}
\hline \multirow{3}{*}{$\begin{array}{l}\text { State / } \\
\text { Cooperattve Borrower }\end{array}$} & \multicolumn{7}{|c|}{ Source of Electrictiy } \\
\hline & \multirow{2}{*}{$\begin{array}{c}\text { Municipal } \\
\text { Cost } \\
\text { (dollars) }\end{array}$} & \multicolumn{2}{|c|}{ Cooperathe } & \multicolumn{2}{|c|}{ Other' } & \multicolumn{2}{|c|}{ Total } \\
\hline & & $\begin{array}{l}\text { Purchases } \\
\text { (thousand } \\
\text { kWh) }\end{array}$ & $\begin{array}{c}\text { Cost } \\
\text { (dollars) }\end{array}$ & $\begin{array}{c}\text { Purchases } \\
\text { (thousand } \\
\text { kWh) }\end{array}$ & $\begin{array}{c}\text { Cost } \\
\text { (dollars) }\end{array}$ & $\begin{array}{l}\text { Purchases } \\
\text { (thousand } \\
\text { kWh) }\end{array}$ & $\begin{array}{c}\text { Cost } \\
\text { (dollars) }\end{array}$ \\
\hline $\begin{array}{l}\text { Montena } \\
\text { Upper Missouri G\&T El Coop Inc .... }\end{array}$ & - & 645,663 & $32,530,384$ & - & - & 969,122 & $36,353,149$ \\
\hline $\begin{array}{l}\text { Nebraska } \\
\text { Nebraska Electric G\&T Coop Inc .... }\end{array}$ & - & - & - & - & - & $2,339,004$ & $81,040,211$ \\
\hline $\begin{array}{l}\text { New Moxico } \\
\text { Plains Elec Gen\&Trans Coop Inc .... }\end{array}$ & - & - & - & 5,201 & 68,205 & 665,166 & $15,401,623$ \\
\hline $\begin{array}{l}\text { North Carollina } \\
\text { North Carolina El Member Corp ...... }\end{array}$ & - & - & - & - & - & $7,886,848$ & $386,657,145$ \\
\hline $\begin{array}{l}\text { North Dakota } \\
\text { Basin Electric Power Coop ............... } \\
\text { Central Power Elec Coop Inc ........... } \\
\text { Minnkota Power Coop Inc ................ } \\
\text { Square Butte Elec Coop Inc ............. }\end{array}$ & 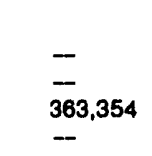 & $\begin{array}{r}1,356 \\
576,329 \\
974,212 \\
9,117\end{array}$ & $\begin{array}{r}10,796 \\
24,998,804 \\
22,586,783 \\
56,065\end{array}$ & $\begin{array}{l}80,449 \\
- \\
-\end{array}$ & $\begin{array}{l}1,371,682 \\
\overline{-} \\
8,869,853 \\
--\end{array}$ & $\begin{array}{r}90,035 \\
904,602 \\
2,652,591 \\
9,117\end{array}$ & $\begin{array}{r}6,284,015 \\
28,580,811 \\
61,032,948 \\
56,065\end{array}$ \\
\hline 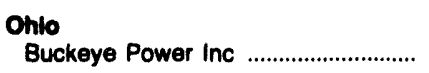 & - & -- & - & - & -- & 419,065 & $7,499,046$ \\
\hline $\begin{array}{l}\text { Oklahoma } \\
\text { Kamo Electric Cooperative Inc ........ } \\
\text { Western Farmers Elec Coop inc ..... }\end{array}$ & - & $1,503,996$ & $\begin{array}{c}58,136,258 \\
-\end{array}$ & - & -- & $\begin{array}{r}2,932,659 \\
879,337\end{array}$ & $\begin{array}{r}107,456,492 \\
11,726,542\end{array}$ \\
\hline $\begin{array}{l}\text { Penneylvania } \\
\text { Allegheny Electric Coop Inc .............. }\end{array}$ & - & -- & - & 2,723 & 72,527 & $1,148,342$ & $35,570,497$ \\
\hline $\begin{array}{l}\text { South Carollna } \\
\text { Central Electric Pwr Coop Inc ......... } \\
\text { Saluda River Electric Coop Inc ........ }\end{array}$ & - & - & - & $--5,882$ & $\overline{929,017}$ & $\begin{array}{l}6,669,593 \\
1,191,584\end{array}$ & $\begin{array}{r}244,981,911 \\
58,024,459\end{array}$ \\
\hline $\begin{array}{l}\text { South Dakota } \\
\text { East River Elec Pwr Coop Inc ......... }\end{array}$ & -- & 667,723 & $33,430,368$ & - & - & $1,396,713$ & $41,179,262$ \\
\hline $\begin{array}{l}\text { Texas } \\
\text { Brazos Electric Power Coop Inc ...... } \\
\text { Northeast Texas Elec Coop Inc ....... } \\
\text { Sam Rayburn G \& T Inc .................. } \\
\text { South Texas Electric Coop Inc ........ } \\
\text { Tex-La Electric Coop-Texas Inc ...... }\end{array}$ & $\begin{array}{l}213,244 \\
-- \\
-- \\
-- \\
250,934\end{array}$ & $\begin{array}{c}1,418,948 \\
-- \\
304,027 \\
1,269,982 \\
-\end{array}$ & $\begin{array}{c}50,545,884 \\
- \\
11,787,141 \\
48,973,322 \\
-\end{array}$ & $\begin{array}{l}485,621 \\
-- \\
298,075 \\
4,643 \\
--\end{array}$ & $\begin{array}{c}13,615,267 \\
- \\
10,430,432 \\
92,724 \\
-\end{array}$ & $\begin{array}{r}3,392,413 \\
1,120,652 \\
631,644 \\
1,676,951 \\
933,203\end{array}$ & $\begin{array}{r}113,639,481 \\
33,823,952 \\
27,909,903 \\
53,378,376 \\
37,087,314\end{array}$ \\
\hline $\begin{array}{l}\text { Utah } \\
\text { Deseret Generation \& Tran Coop ... }\end{array}$ & - & - & -- & 7,425 & 138,635 & 17,815 & 367,487 \\
\hline $\begin{array}{l}\text { Vermont } \\
\text { Vermont G \& T Cooperative Inc ...... }\end{array}$ & - & - & - & 228 & 140,725 & 82,558 & $3,485,254$ \\
\hline $\begin{array}{l}\text { Wieconsin } \\
\text { Dairyland Power Cooperative ........... }\end{array}$ & 141,923 & 213,401 & $2,825,500$ & -- & - & 519,579 & $6,960,637$ \\
\hline
\end{tabular}

\footnotetext{
1 Includes transactions with power pools, utilities in Canada and Mexico, and nonutilities.

- Not Applicable

Notes: -The Department of Agriculture under the Rural Electrification Act is authorized to provide Federal cominitments for long-term financing and to guarantee non-Federal long-term loans to utilities supplying power to rural America. The utilities that received guarantees for their loans are referred to as borrowers by the REA. Nonborrowing cooperatives and other utilities that do not borrow from the REA are not required to file the REA-7 and REA-12 forms. •Power supply borrowers generate electricity and sell power to other utilities. • For identification purposes, the Cooperative Borrowers are listed in the State in which the administrative office is located. - Totals may not equal sum of components because of independent rounding.

Source: Rural Electrification Administration, REA Form 7, "Financial And Statistical Report," REA Form 12a through 12i, "Electric Power Supply Borrowers," Form 12c through 12g, "Electric Distribution Borrowers with Generating Facilities."
} 
Table 34. Electrlclty Purchases by the Distribution Segment of Cooperative Borrowers, by State, 1992

\begin{tabular}{|c|c|c|c|c|c|c|c|}
\hline \multirow{3}{*}{$\begin{array}{c}\text { Btate / } \\
\text { Coopernthe Borrower }\end{array}$} & \multicolumn{7}{|c|}{ Source of Electrictiy } \\
\hline & \multicolumn{2}{|c|}{$\begin{array}{l}\text { Imvestor- } \\
\text { Owned }\end{array}$} & \multicolumn{2}{|c|}{ Federal } & \multicolumn{2}{|c|}{$\begin{array}{l}\text { State and Other } \\
\text { Government }\end{array}$} & \multirow{2}{*}{$\begin{array}{l}\text { Municipal } \\
\begin{array}{c}\text { Purchases } \\
\text { (thousend } \\
\text { kWh) }\end{array}\end{array}$} \\
\hline & $\begin{array}{l}\text { Purchases } \\
\text { (thousand } \\
\text { kWh) }\end{array}$ & $\begin{array}{c}\text { Cost } \\
\text { (dollars) }\end{array}$ & $\begin{array}{l}\text { Purchases } \\
\text { (thousand } \\
\text { kWh) }\end{array}$ & $\begin{array}{c}\text { Cost } \\
\text { (dollars) }\end{array}$ & $\begin{array}{l}\text { Purchases } \\
\text { (thoueand } \\
\text { kWh) }\end{array}$ & $\begin{array}{c}\text { Cost } \\
\text { (dollara) }\end{array}$ & \\
\hline 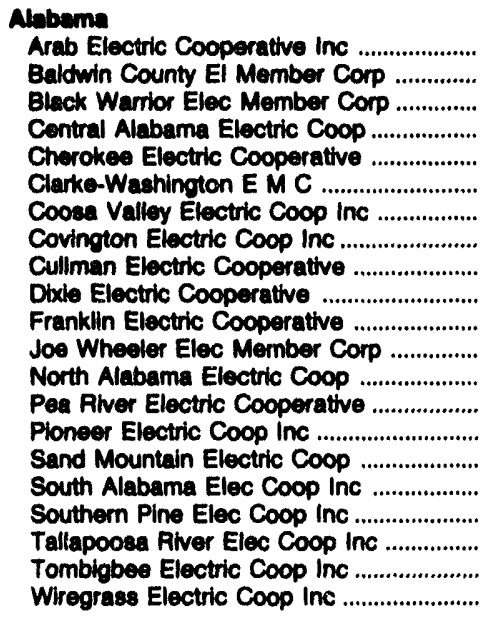 & 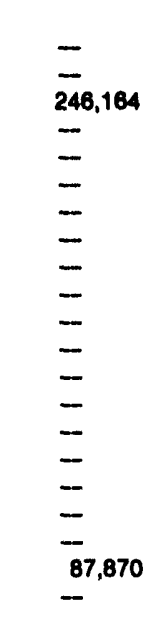 & $\begin{array}{c}\overline{-} \\
\bar{z}, 071,094 \\
\overline{-} \\
\overline{-} \\
\overline{-} \\
\overline{-} \\
\overline{-} \\
\overline{-} \\
\overline{-} \\
\overline{-} \\
3,348,118 \\
-\end{array}$ & $\begin{array}{l}247,081 \\
\overline{30,277} \\
\overline{358,277} \\
- \\
- \\
\overline{608,130} \\
\overline{160,138} \\
715,038 \\
427,045 \\
\overline{-} \\
\overline{427,217} \\
\overline{-} \\
\overline{1} \\
\overline{10,596}\end{array}$ & $\begin{array}{c}11,082,317 \\
\overline{1,136,215} \\
\overline{14,543,773} \\
\overline{-} \\
\overline{2} \\
26,874,698 \\
\overline{194,618} \\
32,333,828 \\
16,354,488 \\
- \\
\overline{-} \\
19,168,788 \\
- \\
- \\
\overline{386,224} \\
-\end{array}$ & $\begin{array}{l}\bar{z} \\
\bar{z} \\
\bar{z} \\
\overline{-} \\
= \\
\overline{-} \\
\overline{-} \\
\overline{-} \\
=\end{array}$ & $\begin{array}{l}\bar{z} \\
\bar{z} \\
\bar{z} \\
\overline{-} \\
\overline{-} \\
\overline{-} \\
\overline{-} \\
\overline{-} \\
\overline{-} \\
-\end{array}$ & $\begin{array}{l}\bar{z} \\
\overline{-} \\
\overline{-} \\
\overline{-} \\
\overline{-} \\
\overline{-} \\
\overline{-} \\
\overline{-} \\
\overline{-} \\
\overline{-}\end{array}$ \\
\hline 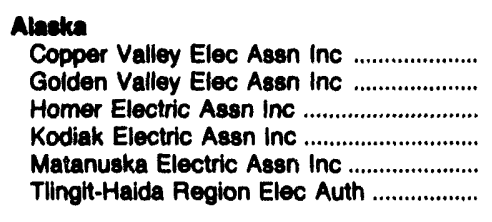 & $\begin{array}{l}\bar{z} \\
\bar{z} \\
\bar{z} \\
2,900\end{array}$ & $\begin{array}{l}\overline{-} \\
\overline{-} \\
\overline{293,452}\end{array}$ & $\begin{array}{l}\overline{-} \\
\bar{z} \\
\overline{25.147}\end{array}$ & $\begin{array}{l}\overline{-} \\
\bar{z} \\
\overline{427,483}\end{array}$ & $\begin{array}{l}37,599 \\
65,828 \\
- \\
100,202 \\
-\end{array}$ & $\begin{array}{c}2,406,351 \\
3,101,194 \\
- \\
6,412,946 \\
- \\
-\end{array}$ & $\begin{array}{l}- \\
-2,532 \\
- \\
- \\
-\end{array}$ \\
\hline 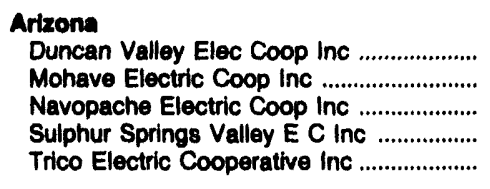 & $\bar{m}=$ & 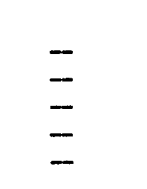 & $\begin{array}{l}- \\
- \\
- \\
-\end{array}$ & $\begin{array}{l}\overline{144,497} \\
- \\
-\end{array}$ & 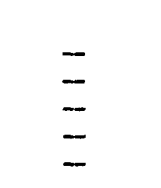 & $\begin{array}{l}- \\
- \\
-\end{array}$ & $\begin{array}{l}- \\
- \\
-\end{array}$ \\
\hline 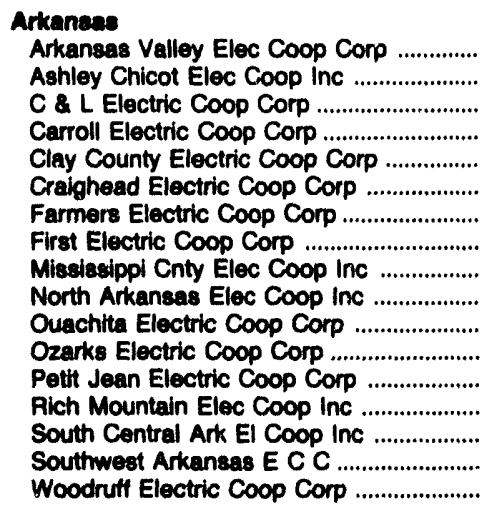 & $\begin{array}{l}504,718 \\
\overline{-} \\
\overline{-} \\
\overline{64,908} \\
\overline{-} \\
5,524 \\
\overline{-} \\
\overline{-} \\
\overline{-}\end{array}$ & $\begin{array}{l}17,844,259 \\
\overline{-} \\
- \\
\overline{-} \\
3,180,473 \\
\overline{-} \\
\overline{277,322} \\
\overline{-} \\
\overline{-} \\
\overline{-} \\
-\end{array}$ & $\begin{array}{l}- \\
- \\
- \\
- \\
- \\
- \\
- \\
-\end{array}$ & $\begin{array}{l}- \\
- \\
- \\
- \\
- \\
- \\
- \\
- \\
- \\
- \\
- \\
-\end{array}$ & $\begin{array}{l}\overline{-} \\
\overline{-} \\
\overline{-} \\
\overline{-} \\
\overline{-} \\
\overline{-} \\
\overline{-} \\
\overline{-} \\
\overline{-}\end{array}$ & $\begin{array}{l}- \\
- \\
- \\
\overline{-} \\
\overline{-} \\
- \\
- \\
- \\
- \\
- \\
- \\
-\end{array}$ & $\begin{array}{l}- \\
- \\
- \\
- \\
- \\
- \\
- \\
- \\
- \\
- \\
- \\
-\end{array}$ \\
\hline $\begin{array}{l}\text { Colliomia } \\
\text { Anza Electric Cooperative Inc .................. } \\
\text { Plumas-Sierra Rural Elec Coop ............... } \\
\text { Surprise Valiey Electric Corp ................... }\end{array}$ & $\overline{-}$ & - & $\begin{array}{r}- \\
95,592 \\
153,287\end{array}$ & $\begin{array}{l}-\overline{2,004,903} \\
3,006,309\end{array}$ & - & $\overline{-}$ & - \\
\hline 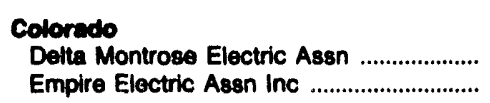 & $\overline{-}$ & $\overline{-}$ & $\overline{-}$ & $\overline{-}$ & $\overline{-}$ & $\overline{-}$ & $\overline{-}$ \\
\hline
\end{tabular}

See notes and footnotes at end of table. 
Table 34. Electriclty Purchases by the Distribution Segment of Cooperatlve Borrowers, by State, 1992 (Continued)

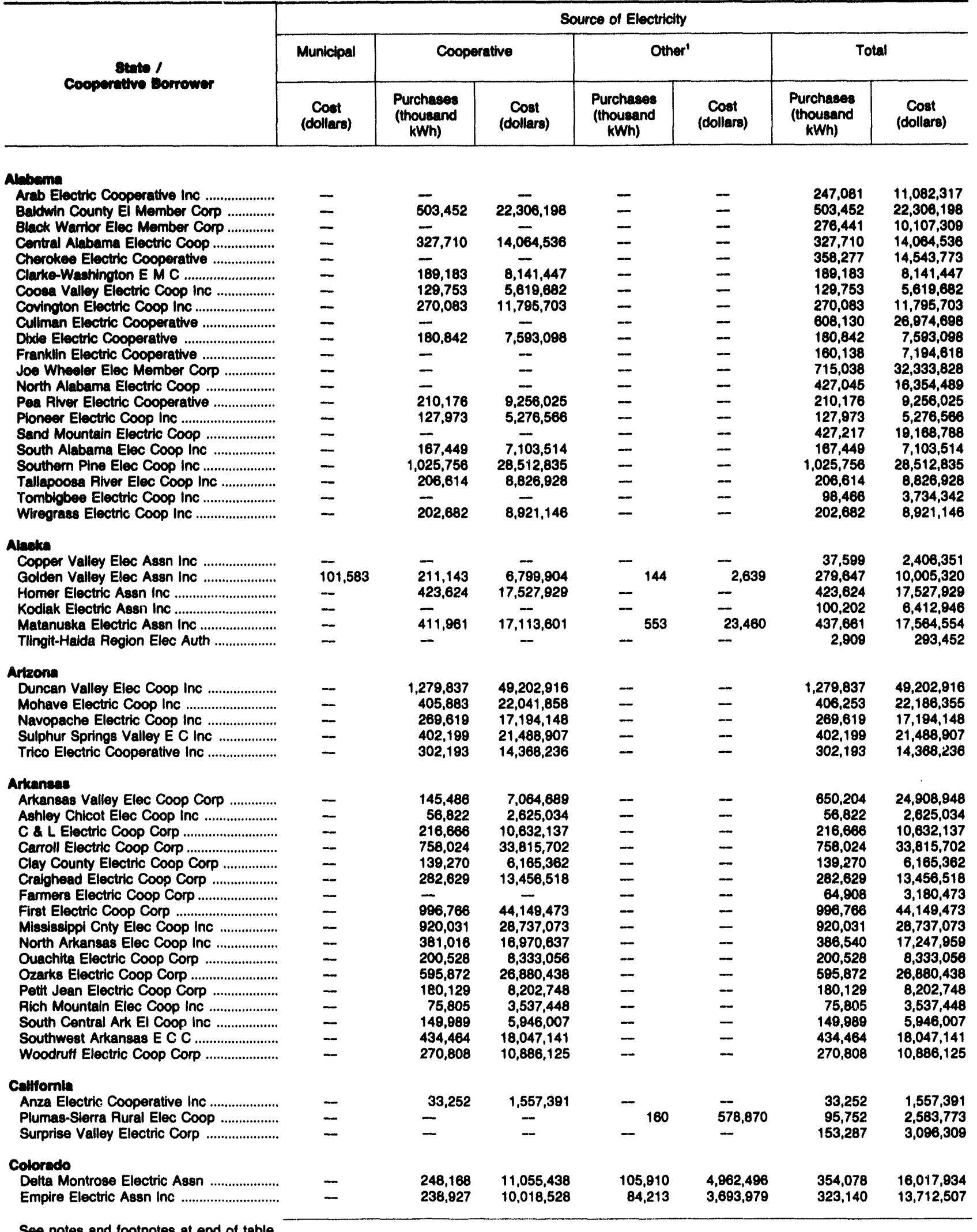


Table 34. Electriclty Purchases by the Distribution Segment of Cooperative Borrowers, by State, 1992 (Continued)

\begin{tabular}{|c|c|c|c|c|c|c|c|}
\hline \multirow{3}{*}{$\begin{array}{c}\text { State / } \\
\text { Cooperative Borrower }\end{array}$} & \multicolumn{7}{|c|}{ Source of Electricity } \\
\hline & \multicolumn{2}{|c|}{$\begin{array}{l}\text { Investor- } \\
\text { Owned }\end{array}$} & \multicolumn{2}{|c|}{ Federal } & \multicolumn{2}{|c|}{$\begin{array}{l}\text { State and Other } \\
\text { Government }\end{array}$} & \multirow{2}{*}{$\begin{array}{l}\text { Municipal } \\
\begin{array}{c}\text { Purchases } \\
\text { (thousand } \\
\text { kWh) }\end{array}\end{array}$} \\
\hline & $\begin{array}{c}\text { Purchases } \\
\text { (thousand } \\
\text { kWh) }\end{array}$ & $\begin{array}{c}\text { Cost } \\
\text { (dollars) }\end{array}$ & $\begin{array}{c}\text { Purchases } \\
\text { (thousand } \\
\text { kWh) }\end{array}$ & $\begin{array}{c}\text { Cost } \\
\text { (dollars) }\end{array}$ & $\begin{array}{c}\text { Purchases } \\
\text { (thousand } \\
\text { kWh) }\end{array}$ & $\begin{array}{c}\text { Cost } \\
\text { (dollars) }\end{array}$ & \\
\hline \multicolumn{8}{|l|}{ Coloredo } \\
\hline Grand Valley Ril Pwr Line Inc ...................... & 70,617 & $3,306,096$ & 5,593 & 108,480 & - & - & - \\
\hline Gunnison County Elec Assn Inc ................ & - & - & - & - & - & - & - \\
\hline 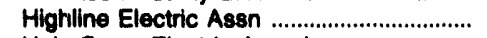 & - & - & - & - & - & - & - \\
\hline 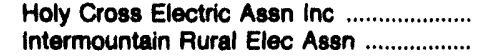 & $\begin{array}{l}422,082 \\
448,578\end{array}$ & $\begin{array}{l}18,463,183 \\
18,799,736\end{array}$ & $\begin{array}{l}30,183 \\
94,983\end{array}$ & $\begin{array}{r}600,071 \\
1,802,956\end{array}$ & - & - & - \\
\hline 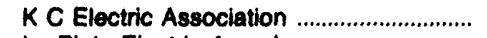 & - & - & - & - & - & - & - \\
\hline La Plata Electric Assn Inc ............................. & - & - & - & - & - & - & -- \\
\hline Morgan County Rural Elec Assn ................ & - & - & - & - & - & - & - \\
\hline 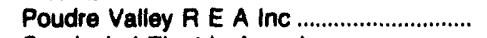 & - & - & - & - & - & - & - \\
\hline San leabel Electric Assn Inc ...................... & - & - & - & - & - & - & - \\
\hline 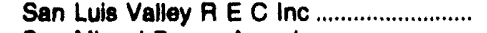 & - & - & - & - & - & - & - \\
\hline San Miguel Power Assn Inc ........................ & - & - & - & - & - & - & - \\
\hline Sangre De Cristo Elec Assn Inc ................ & -- & - & -- & - & - & - & - \\
\hline Southeast Colorado Power Assn ................ & - & - & - & - & - & - & - \\
\hline 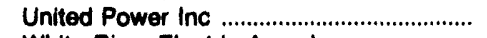 & 78 & 5,722 & - & - & - & - & - \\
\hline White Aiver Electric Assn Inc ..................... & -- & $-\infty$ & - & - & -- & - & - \\
\hline 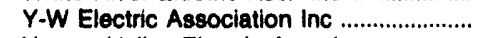 & - & - & - & - & - & - & 18 \\
\hline Yampa Valley Electric Assn Inc .................. & 224,731 & $9,437,755$ & 18,267 & 294,550 & - & - & - \\
\hline \multicolumn{8}{|l|}{ Deloware } \\
\hline Delaware Electric Coop Inc ............................. & - & -- & - & - & - & - & - \\
\hline \multicolumn{8}{|l|}{ Florlda } \\
\hline 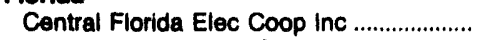 & - & - & 11,555 & 275,324 & - & - & - \\
\hline Choctawhatche Elec Coop Inc ..................... & - & - & - & - & -- & - & - \\
\hline Clay Electric Cooperative Inc ......................... & - & - & - & - & - & - & - \\
\hline Escambia River Elec Coop Inc .................... & - & - & -- & - & - & - & - \\
\hline Gulf Coast Electric Coop Inc ........................ & - & - & - & - & - & - & - \\
\hline Peace River Electric Coop Inc ...................... & - & - & - & - & -- & - & - \\
\hline 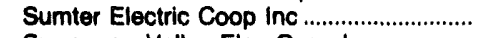 & -- & - & - & - & -- & -- & - \\
\hline Suwannee Valley Elec Coop Inc ............... & - & - & 23,298 & 563,827 & - & - & - \\
\hline 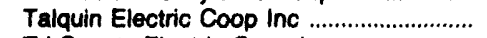 & - & - & 65,885 & $1,591,225$ & -- & -- & 7,735 \\
\hline Tri-County Electric Coop Inc .......................... & - & - & 25,768 & 567,872 & -- & -- & - \\
\hline West Florida El Coop Assn Inc .................... & - & - & - & - & - & -- & - \\
\hline Withlacoochee River Elec Coop ..................... & -- & - & - & - & - & - & - \\
\hline \multicolumn{8}{|l|}{ Ceorgla } \\
\hline Altamaha Electric Member Corp .................. & - & $-\infty$ & 18,093 & 459,313 & -- & -- & - \\
\hline Amicalola Electric Member Corp .................. & - & -- & 20,643 & 487,220 & - & - & -- \\
\hline Blue Ridge Mountain E M C ......................... & - & -- & 329,700 & $13,861,379$ & -- & - & - \\
\hline Canoochee Electric Member Corp ............ & - & - & 15,575 & 391,054 & - & - & - \\
\hline Carroll Electric Member Corp ........................ & - & - & 30,416 & 718,379 & - & -- & - \\
\hline Central Georgia EI Member Corp ............... & - & - & 23,551 & 555,813 & - & - & - \\
\hline Coastal Electric Member Corp ...................... & - & -- & 5,492 & 129,699 & -- & -- & - \\
\hline Cobb Electric Membership Corp ................. & -- & - & 67,910 & $1,602,709$ & -- & -- & - \\
\hline Colquitt Electric Members Corp ................... & -- & - & 69,106 & $1,630,947$ & - & - & - \\
\hline Coweta Fayette EI Member Corp ............... & -- & - & 23,240 & 548,897 & - & - & - \\
\hline Excelsior Electric Member Corp ................... & - & - & 15,217 & 374,787 & -- & -- & - \\
\hline Flint Electric Membership Corp ................... & - & -- & 100,166 & $2,363,970$ & - & - & - \\
\hline Grady County Elec Member Corp ............ & -- & - & 18,767 & 443,238 & - & -- & - \\
\hline 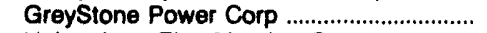 & - & - & 55,097 & $1,300,332$ & - & - & - \\
\hline Habersham Elec Member Corp .................. & -- & - & 18,259 & 413,163 & -- & -- & - \\
\hline Hart County Elec Member Corp .................. & - & - & 33,784 & 797,324 & - & -- & - \\
\hline Irwin County Elec Member Corp ................. & - & - & 14,930 & 352,368 & - & -- & -- \\
\hline Jackson Electric Member Corp ................... & - & - & 85,621 & $2,022,097$ & -- & -- & - \\
\hline Jefferson Electric Member Corp ................ & -- & - & 24,782 & 584,862 & - & -- & - \\
\hline Lamar Electric Membership Corp ............... & -- & - & 12,079 & 285,061 & - & -- & - \\
\hline Little Ocmulgee El Member Corp ............... & - & - & 14,116 & 333,144 & - & - & - \\
\hline Middle Georgia El Member Corp ............... & -- & $-\infty$ & 10,846 & 256,154 & -- & -- & - \\
\hline Mitchell Electric Member Corp .................... & - & - & 32,324 & 764,745 & - & - & - \\
\hline
\end{tabular}

See notes and footnotes at end of table. 
Table 34. Electricity Purchases by the Distribution Segment of Cooperative Borrowers, by State, 1992 (Continued)

\begin{tabular}{|c|c|c|c|c|c|c|c|}
\hline \multirow{3}{*}{$\begin{array}{c}\text { State / } \\
\text { Cooporative Borrower }\end{array}$} & \multicolumn{7}{|c|}{ Source of Electricity } \\
\hline & \multirow{2}{*}{$\frac{\text { Municipal }}{\text { Cost }}$} & \multicolumn{2}{|c|}{ Cooperative } & \multicolumn{2}{|c|}{ Other' } & \multicolumn{2}{|c|}{ Total } \\
\hline & & $\begin{array}{c}\text { Purchases } \\
\text { (thousand } \\
\text { kWh) }\end{array}$ & $\begin{array}{c}\text { Cost } \\
\text { (dollars) }\end{array}$ & $\begin{array}{c}\text { Purchases } \\
\text { (thousand } \\
\text { kWh) }\end{array}$ & $\begin{array}{c}\text { Cost } \\
\text { (dollars) }\end{array}$ & $\begin{array}{c}\text { Purchases } \\
\text { (thousand } \\
\text { kWh) }\end{array}$ & $\begin{array}{c}\text { Cost } \\
\text { (dollara) }\end{array}$ \\
\hline 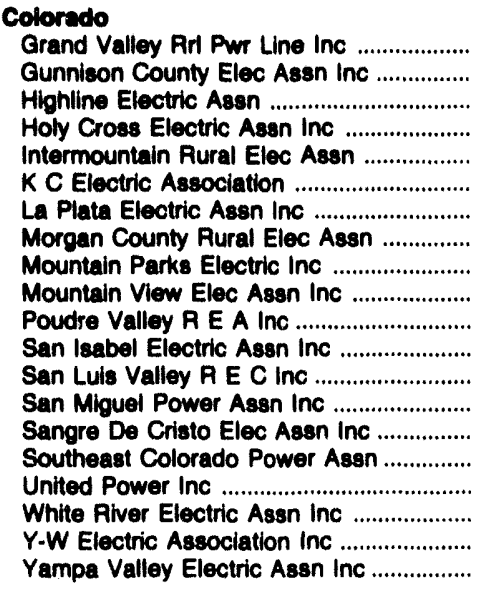 & $\begin{array}{l}- \\
- \\
- \\
- \\
- \\
- \\
- \\
- \\
- \\
- \\
- \\
- \\
- \\
- \\
-\end{array}$ & $\begin{array}{r}-- \\
68,241 \\
260,599 \\
- \\
- \\
156,461 \\
340,799 \\
175,959 \\
206,391 \\
327,879 \\
540,222 \\
\overline{129,647} \\
73,854 \\
61,975 \\
112,381 \\
468,504 \\
57,731 \\
266,591 \\
97\end{array}$ & $\begin{array}{r}\overline{3} \\
3,161,940 \\
12,067,566 \\
- \\
\overline{-} \\
6,386,356 \\
14,478,462 \\
7,407,698 \\
8,176,012 \\
12,995,691 \\
20,466,824 \\
- \\
6,334,521 \\
3,299,636 \\
2,822,610 \\
4,997,248 \\
16,408,054 \\
2,498,325 \\
12,258,835 \\
4,273\end{array}$ & $\begin{array}{r}32,948 \\
41,019 \\
2 \\
255,809 \\
233,382 \\
- \\
118,802 \\
- \\
- \\
-- \\
\overline{209,368} \\
27,797 \\
34,811 \\
- \\
38,287 \\
\overline{2} 3,317 \\
\overline{129,186}\end{array}$ & $\begin{array}{r}1,570,740 \\
1,911,455 \\
24 \\
12,021,246 \\
11,522,407 \\
- \\
5,416,830 \\
- \\
- \\
- \\
\overline{8}, 998,667 \\
1,297,100 \\
1,676,928 \\
- \\
1,652,565 \\
- \\
1, \overline{0} 6,873 \\
\overline{6} \overline{105,947}\end{array}$ & $\begin{array}{r}109,158 \\
109,260 \\
260,601 \\
708,074 \\
776,943 \\
156,461 \\
459,601 \\
175,959 \\
206,391 \\
327,879 \\
540,222 \\
209,368 \\
157,444 \\
108,665 \\
61,975 \\
150,668 \\
468,582 \\
81,048 \\
266,609 \\
372,281\end{array}$ & $\begin{array}{r}4,985,316 \\
5,073,395 \\
12,087,590 \\
31,084,500 \\
32,125,099 \\
6,386,356 \\
19,895,292 \\
7,407,698 \\
8,176,012 \\
12,995,691 \\
20,466,824 \\
8,998,667 \\
7,631,621 \\
4,976,564 \\
2,822,610 \\
6,649,813 \\
16,413,776 \\
3,545,198 \\
12,259,109 \\
15,842,525\end{array}$ \\
\hline $\begin{array}{l}\text { Deloware } \\
\text { Delaware Electric Coop Inc ......................... }\end{array}$ & - & 583,193 & $31,210,041$ & -- & - & 583,193 & $31,210,041$ \\
\hline 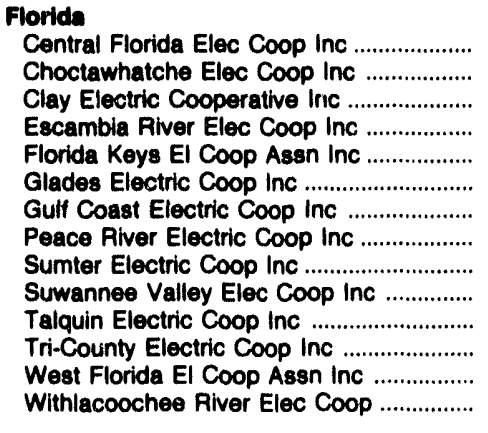 & $\begin{array}{l}- \\
- \\
- \\
- \\
- \\
- \\
- \\
- \\
- \\
557,986 \\
- \\
-\end{array}$ & $\begin{array}{r}246,049 \\
350,750 \\
1,717,653 \\
118,829 \\
- \\
195,078 \\
162,675 \\
246,826 \\
972,659 \\
195,157 \\
553,306 \\
126,521 \\
268,001 \\
1,973,300\end{array}$ & $\begin{array}{r}12,747,483 \\
15,369,139 \\
87,186,379 \\
5,102,139 \\
-- \\
9,643,088 \\
6,945,931 \\
13,553,036 \\
52,591,087 \\
9,946,659 \\
30,324,406 \\
6,481,709 \\
11,713,133 \\
114,864,315\end{array}$ & $\begin{array}{l}-- \\
- \\
- \\
1,598 \\
-- \\
- \\
- \\
- \\
- \\
- \\
- \\
-\end{array}$ & $\begin{array}{l}- \\
-- \\
-- \\
\overline{137,377} \\
-- \\
- \\
- \\
- \\
- \\
- \\
- \\
- \\
-\end{array}$ & $\begin{array}{r}257,604 \\
350,750 \\
1,717,653 \\
118,829 \\
493,175 \\
195,078 \\
162,675 \\
246,826 \\
972,659 \\
218,455 \\
626,926 \\
152,289 \\
268,001 \\
1,973,300\end{array}$ & $\begin{array}{r}13,022,807 \\
15,369,139 \\
87,186,379 \\
5,102,139 \\
23,267,496 \\
9,643,088 \\
6,945,931 \\
13,553,036 \\
52,591,087 \\
10,510,486 \\
32,473,617 \\
7,049,581 \\
11,713,133 \\
114,864,315\end{array}$ \\
\hline 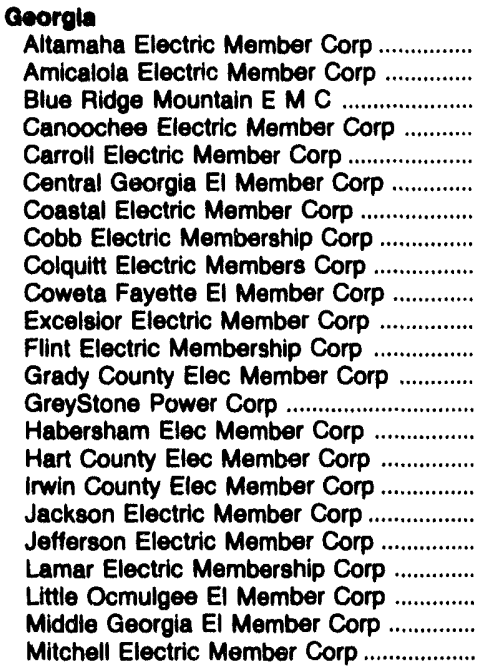 & $\begin{array}{l}- \\
- \\
- \\
- \\
- \\
- \\
- \\
- \\
- \\
- \\
- \\
- \\
- \\
- \\
- \\
-\end{array}$ & $\begin{array}{r}216,171 \\
247,214 \\
202,416 \\
434,430 \\
333,274 \\
127,558 \\
1,648,050 \\
593,279 \\
531,739 \\
176,746 \\
805,797 \\
159,496 \\
845,506 \\
233,909 \\
287,552 \\
109,684 \\
1,734,114 \\
318,030 \\
154,470 \\
88,588 \\
61,883 \\
256,833\end{array}$ & $\begin{array}{r}12,041,857 \\
13,453,454 \\
- \\
11,248,167 \\
23,622,568 \\
19,170,265 \\
6,805,750 \\
107,762,226 \\
32,080,485 \\
33,089,699 \\
9,654,193 \\
44,977,005 \\
9,307,248 \\
50,416,673 \\
12,355,333 \\
16,428,683 \\
5,961,604 \\
97,948,927 \\
19,102,088 \\
8,998,355 \\
4,849,018 \\
3,169,427 \\
14,409,572\end{array}$ & $\begin{array}{l}- \\
-- \\
-- \\
-- \\
-- \\
-- \\
- \\
- \\
- \\
- \\
- \\
- \\
- \\
- \\
- \\
- \\
- \\
-\end{array}$ & $\begin{array}{l}-- \\
-- \\
- \\
- \\
- \\
- \\
- \\
- \\
-- \\
-- \\
- \\
- \\
- \\
-- \\
- \\
- \\
- \\
- \\
-\end{array}$ & $\begin{array}{r}234,264 \\
267,857 \\
329,700 \\
217,991 \\
464,846 \\
356,825 \\
133,050 \\
1,715,960 \\
662,385 \\
554,979 \\
191,963 \\
905,963 \\
178,263 \\
900,603 \\
252,168 \\
321,336 \\
124,614 \\
1,819,735 \\
342,812 \\
166,549 \\
102,704 \\
72,729 \\
289,157\end{array}$ & $\begin{array}{r}12,501,170 \\
13,940,674 \\
13,861,379 \\
11,639,221 \\
24,340,947 \\
19,726,078 \\
6,935,449 \\
109,364,935 \\
33,711,432 \\
33,638,596 \\
10,028,980 \\
47,340,975 \\
9,750,486 \\
51,717,005 \\
12,768,496 \\
17,226,007 \\
6,313,972 \\
99,971,024 \\
19,686,950 \\
9,283,416 \\
5,182,162 \\
3,425,581 \\
15,174,317\end{array}$ \\
\hline
\end{tabular}

See notes and footnotes at end of table. 
Table 34. Electricity Purchases by the Distribution Segment of Cooperative Borrowers, by State, 1982 (Continued)

\begin{tabular}{|c|c|c|c|c|c|c|c|}
\hline \multirow{3}{*}{$\begin{array}{l}\text { State I } \\
\text { cooperative Eorrower }\end{array}$} & \multicolumn{7}{|c|}{ Source of Electricity } \\
\hline & \multicolumn{2}{|c|}{$\begin{array}{l}\text { Imvestor- } \\
\text { Owned }\end{array}$} & \multicolumn{2}{|c|}{ Federal } & \multicolumn{2}{|c|}{$\begin{array}{c}\text { State and Other } \\
\text { Covernment }\end{array}$} & \multirow{2}{*}{$\begin{array}{c}\text { Municipal } \\
\text { Purchasea } \\
\text { (thousand } \\
\text { kWh) }\end{array}$} \\
\hline & $\begin{array}{c}\text { Purchases } \\
\text { (thoumand } \\
\text { kWh) }\end{array}$ & $\begin{array}{c}\text { Cost } \\
\text { (dollare) }\end{array}$ & $\begin{array}{c}\text { Purcheses } \\
\text { (thousend } \\
\text { KWh) }\end{array}$ & $\begin{array}{c}\text { Coat } \\
\text { (dollare) }\end{array}$ & $\begin{array}{c}\text { Purchaces } \\
\text { (thousend } \\
\text { KWh) }\end{array}$ & $\begin{array}{c}\text { Cost } \\
\text { (dollars) }\end{array}$ & \\
\hline 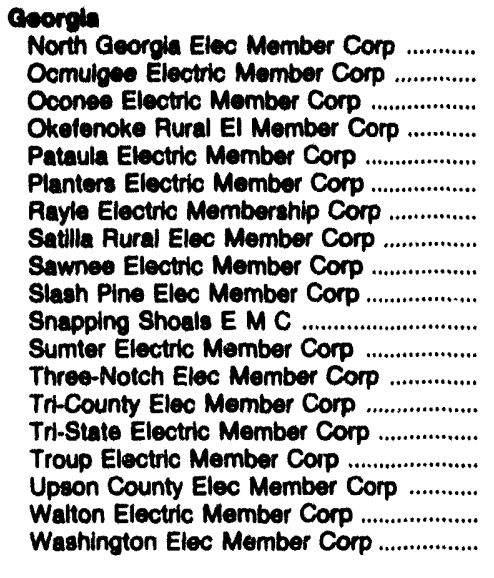 & $\begin{array}{l}- \\
- \\
= \\
= \\
= \\
= \\
= \\
- \\
- \\
- \\
- \\
- \\
-\end{array}$ & $\begin{array}{l}- \\
= \\
= \\
= \\
= \\
- \\
= \\
- \\
24,465 \\
- \\
- \\
-\end{array}$ & $\begin{array}{r}1,848,807 \\
14,794 \\
14,247 \\
16,313 \\
5,819 \\
18,440 \\
18,682 \\
54,550 \\
34,353 \\
8,608 \\
34,850 \\
20,255 \\
19,737 \\
11,179 \\
180,785 \\
21,059 \\
8,198 \\
53,662 \\
25,471\end{array}$ & $\begin{array}{r}79,385,596 \\
349,410 \\
336,244 \\
400,451 \\
137,426 \\
435,510 \\
440,857 \\
1,289,503 \\
811,348 \\
203,159 \\
822,867 \\
478,394 \\
493,565 \\
263,835 \\
7,800,772 \\
497,379 \\
193,428 \\
1,266,465 \\
601,129\end{array}$ & 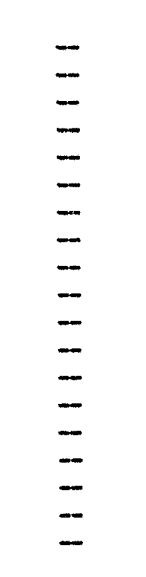 & $\begin{array}{l}\overline{-} \\
\overline{-} \\
\overline{-} \\
\overline{-} \\
\overline{-} \\
\overline{-} \\
\overline{-} \\
\overline{-} \\
- \\
-\end{array}$ & $\begin{array}{l}- \\
- \\
\overline{-} \\
= \\
- \\
- \\
\overline{-} \\
- \\
- \\
\overline{-} \\
-\end{array}$ \\
\hline 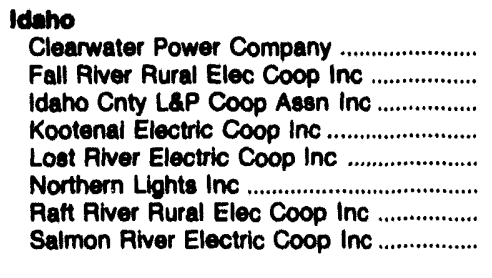 & $\begin{array}{l}- \\
- \\
- \\
-\end{array}$ & $\begin{array}{l}- \\
- \\
- \\
-\end{array}$ & $\begin{array}{r}145,171 \\
163,087 \\
36,114 \\
212,185 \\
71,615 \\
250,738 \\
234,146 \\
214,320\end{array}$ & $\begin{array}{r}3,435,820 \\
3,595,878 \\
788,963 \\
4,950,125 \\
1,594,087 \\
6,448,210 \\
4,829,087 \\
4,877,790\end{array}$ & $\begin{array}{l}- \\
- \\
- \\
- \\
-\end{array}$ & $\begin{array}{l}- \\
- \\
- \\
- \\
-\end{array}$ & $\begin{array}{l}- \\
- \\
- \\
- \\
-\end{array}$ \\
\hline 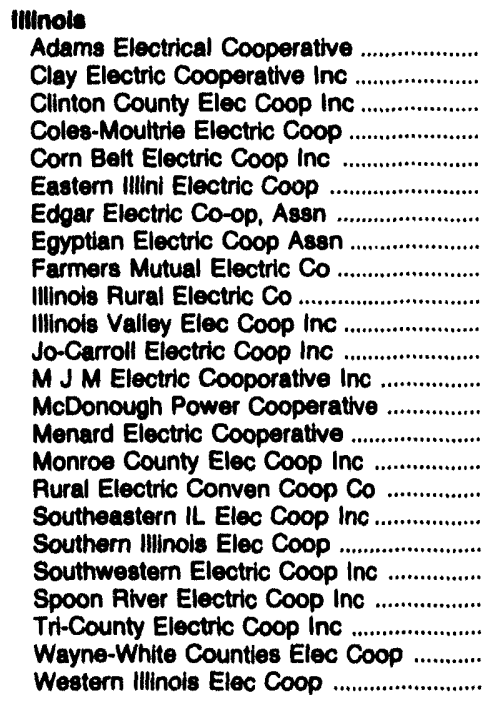 & $\begin{array}{l}- \\
- \\
- \\
- \\
- \\
- \\
- \\
- \\
- \\
- \\
- \\
- \\
- \\
- \\
-\end{array}$ & $\begin{array}{l}- \\
- \\
- \\
- \\
- \\
- \\
- \\
- \\
- \\
- \\
- \\
- \\
- \\
- \\
- \\
- \\
- \\
- \\
-\end{array}$ & $\begin{array}{l}- \\
\bar{z} \\
\bar{z} \\
\bar{z} \\
\overline{-} \\
\bar{z} \\
\bar{z} \\
\overline{-} \\
\bar{z} \\
\bar{z} \\
\bar{z} \\
\overline{-}\end{array}$ & $\begin{array}{l}- \\
-- \\
-- \\
-- \\
- \\
- \\
- \\
- \\
- \\
- \\
- \\
- \\
- \\
- \\
- \\
- \\
-\end{array}$ & $\begin{array}{l}- \\
\overline{-} \\
\overline{-} \\
\overline{-} \\
\overline{-} \\
\overline{-} \\
\overline{-} \\
\overline{-} \\
- \\
\overline{-} \\
- \\
- \\
- \\
-\end{array}$ & $\begin{array}{l}- \\
\overline{-} \\
- \\
- \\
- \\
\overline{-} \\
\overline{-} \\
- \\
- \\
- \\
- \\
- \\
- \\
- \\
\overline{-} \\
-\end{array}$ & 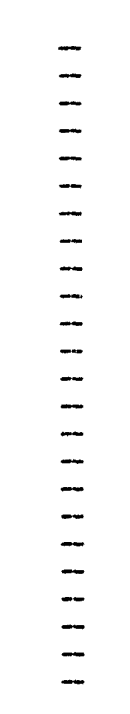 \\
\hline 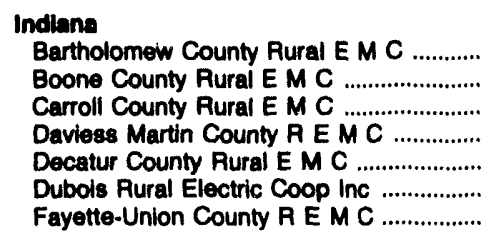 & $\begin{array}{l}- \\
\overline{-} \\
\overline{-}\end{array}$ & $\begin{array}{l}- \\
- \\
- \\
- \\
-\end{array}$ & $\begin{array}{l}- \\
- \\
- \\
-\end{array}$ & $\begin{array}{l}- \\
\overline{-} \\
\overline{-} \\
-\end{array}$ & $\begin{array}{l}- \\
- \\
- \\
- \\
-\end{array}$ & $\begin{array}{l}- \\
\overline{-} \\
\overline{-} \\
\overline{-}\end{array}$ & $\begin{array}{l}- \\
- \\
- \\
- \\
-\end{array}$ \\
\hline
\end{tabular}

See notes and footnotes at end of table. 
Table 34. Electrletty Purchases by the Diotribution 8egment of Cooperative Borrowers, by state, 1902 (Continued)

\begin{tabular}{|c|c|c|c|c|c|c|c|}
\hline \multirow{3}{*}{ Coopernitur É Eorromer } & \multicolumn{7}{|c|}{ Source of Electritity } \\
\hline & \multirow{2}{*}{$\frac{\text { Municipal }}{\text { Cont }}$} & \multicolumn{2}{|c|}{ Cooperative } & \multicolumn{2}{|c|}{ Other' } & \multicolumn{2}{|c|}{ Total } \\
\hline & & $\begin{array}{l}\text { Purchaees } \\
\text { (thouaand } \\
\text { KWl: }\end{array}$ & $\begin{array}{c}\text { Cost } \\
\text { (dollans) }\end{array}$ & $\begin{array}{l}\text { Purchases } \\
\text { (thousand } \\
\text { kWh) }\end{array}$ & $\begin{array}{c}\text { Coot } \\
\text { (dollens) }\end{array}$ & $\begin{array}{l}\text { Purchasese } \\
\text { (thousand } \\
\text { kWh) }\end{array}$ & $\begin{array}{c}\text { Coot } \\
\text { (dollaro) }\end{array}$ \\
\hline 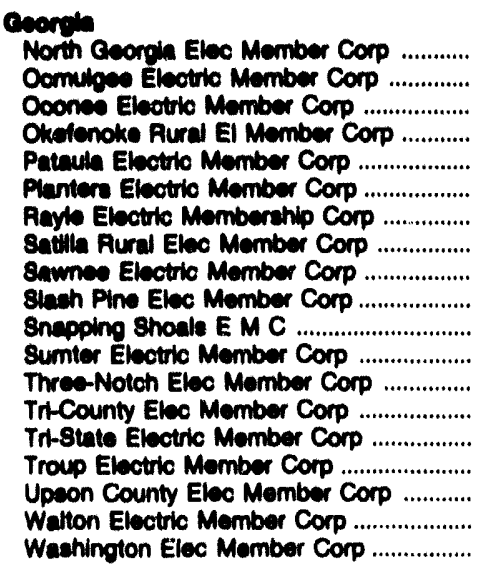 & $\begin{array}{l}\bar{z} \\
\bar{z} \\
= \\
= \\
= \\
= \\
= \\
= \\
= \\
=\end{array}$ & $\begin{array}{r}- \\
92,, 080 \\
198,406 \\
311,768 \\
36,043 \\
141,000 \\
141,160 \\
445,817 \\
818,208 \\
77,643 \\
602,086 \\
194,188 \\
138,758 \\
168,095 \\
\overline{277,419} \\
73,704 \\
1,086,585 \\
270,230\end{array}$ & 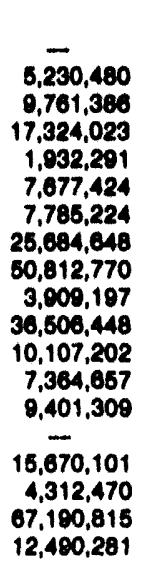 & $\begin{array}{l}\bar{z} \\
\bar{z} \\
\bar{z} \\
\bar{z} \\
\bar{z} \\
\bar{z} \\
\bar{z} \\
\bar{z}\end{array}$ & $\begin{array}{l}\bar{z} \\
\bar{z} \\
\bar{z} \\
\bar{z} \\
\bar{z} \\
\bar{z} \\
\bar{z} \\
\bar{z} \\
\overline{-}\end{array}$ & 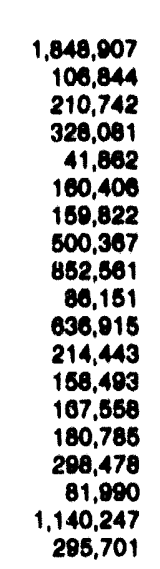 & 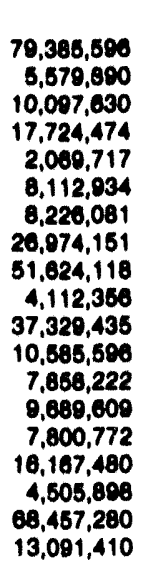 \\
\hline 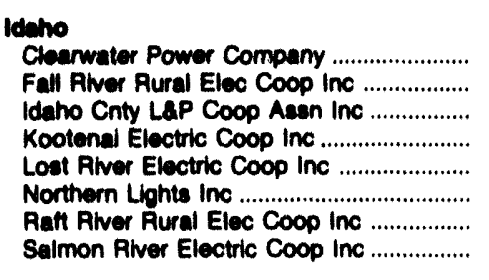 & $\begin{array}{l}\overline{-} \\
\bar{z} \\
\bar{z} \\
\overline{-}\end{array}$ & $\begin{array}{l}\bar{z} \\
\bar{z} \\
\bar{z} \\
\overline{-}\end{array}$ & $\begin{array}{l}\bar{z} \\
\bar{z} \\
\bar{z}\end{array}$ & $\begin{array}{l}\overline{-} \\
\bar{z} \\
\overline{-} \\
\overline{-}\end{array}$ & $\begin{array}{l}\overline{-} \\
\bar{z} \\
\bar{z} \\
\overline{-}\end{array}$ & $\begin{array}{r}145,171 \\
163,087 \\
36,114 \\
212,185 \\
71,615 \\
250,738 \\
234,146 \\
214,320\end{array}$ & $\begin{array}{r}3,435,020 \\
3,595,878 \\
788,863 \\
4,950,125 \\
1,694,087 \\
5,448,210 \\
4,829,087 \\
4,877,790\end{array}$ \\
\hline 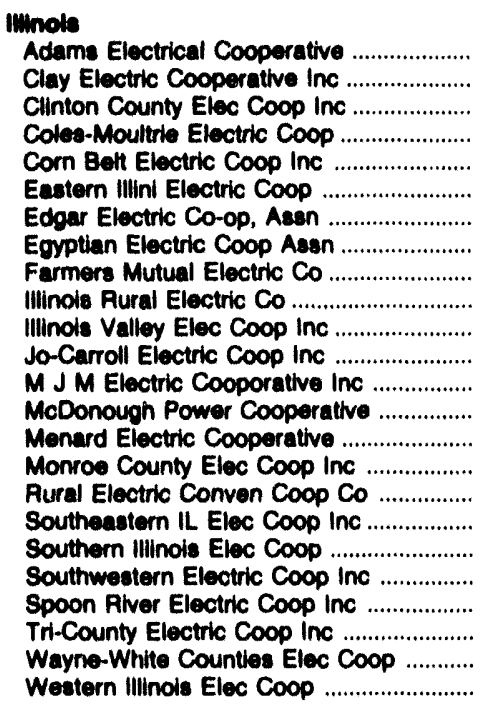 & 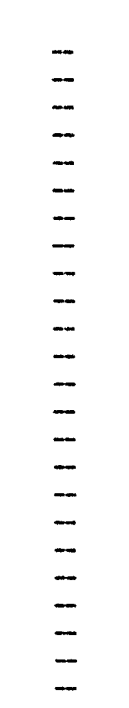 & $\begin{array}{r}86,731 \\
40,761 \\
95,685 \\
139,866 \\
202,289 \\
194,655 \\
62,119 \\
196,217 \\
15,188 \\
108,491 \\
73,974 \\
67,601 \\
92,308 \\
66,812 \\
133,313 \\
65,719 \\
74,088 \\
572,620 \\
143,071 \\
227,903 \\
44,229 \\
240,282 \\
233,135 \\
36,365\end{array}$ & 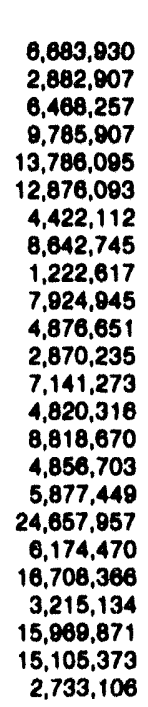 & 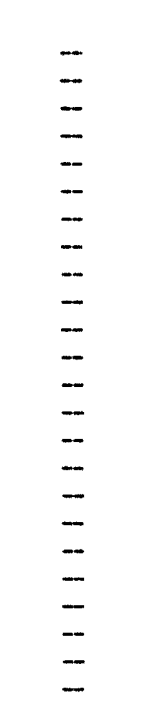 & 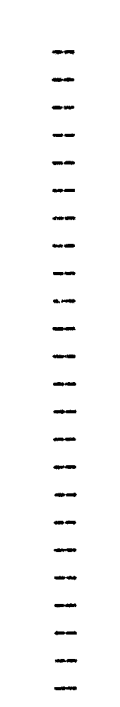 & $\begin{array}{r}86,731 \\
40,761 \\
95,685 \\
139,856 \\
202,296 \\
194,855 \\
62,119 \\
196,217 \\
15,186 \\
106,491 \\
73,874 \\
67,601 \\
92,306 \\
66,812 \\
133,313 \\
65,719 \\
74,088 \\
572,620 \\
143,071 \\
227,803 \\
44,229 \\
240,262 \\
233,135 \\
36,355\end{array}$ & $\begin{array}{r}6,683,930 \\
2,882,907 \\
6,468,257 \\
9,785,907 \\
13,787,056 \\
12,876,093 \\
4,422,112 \\
8,642,745 \\
1,222,617 \\
7,924,946 \\
4,878,651 \\
2,870,236 \\
7,141,273 \\
4,820,316 \\
8,816,670 \\
4,866,703 \\
5,877,449 \\
24,657,957 \\
6,174,470 \\
16,708,368 \\
3,215,134 \\
15,969,871 \\
15,105,373 \\
2,733,106\end{array}$ \\
\hline 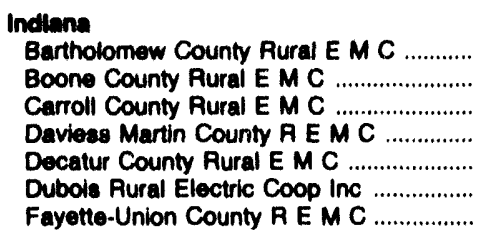 & $\begin{array}{l}\overline{-} \\
\bar{z} \\
\overline{-} \\
\bar{m}\end{array}$ & $\begin{array}{r}153,790 \\
153,189 \\
121,493 \\
113,478 \\
187,334 \\
194,790 \\
59,959\end{array}$ & $\begin{array}{l}7,221,723 \\
6,717,851 \\
5,290,885 \\
5,134,516 \\
7,878,842 \\
8,687,676 \\
2,712,878\end{array}$ & $\begin{array}{l}\overline{-} \\
\overline{-} \\
\overline{-} \\
\overline{-}\end{array}$ & $\begin{array}{l}\overline{-} \\
\overline{-} \\
\overline{-} \\
\overline{-}\end{array}$ & $\begin{array}{r}153,780 \\
153,189 \\
121,493 \\
113,478 \\
187,334 \\
194,780 \\
59,959\end{array}$ & $\begin{array}{l}7,221,723 \\
6,717,851 \\
5,290,885 \\
5,134,516 \\
7,878,842 \\
8,687,676 \\
2,712,878\end{array}$ \\
\hline
\end{tabular}

See notes and footnotes at end of table. 
Table 34. Electriclty Purchaces by the Distribution Sogrient of Cooperative Borrowers, by Stato, 1992 (Continued)

\begin{tabular}{|c|c|c|c|c|c|c|c|}
\hline \multirow{3}{*}{$\begin{array}{l}\text { State I } \\
\text { Coopernttve Eorrower }\end{array}$} & \multicolumn{7}{|c|}{ Source of Electricity } \\
\hline & \multicolumn{2}{|c|}{$\begin{array}{l}\text { Inveator. } \\
\text { Owned }\end{array}$} & \multicolumn{2}{|c|}{ Federal } & \multicolumn{2}{|c|}{$\begin{array}{l}\text { State and Other } \\
\text { Government }\end{array}$} & \multirow{2}{*}{$\begin{array}{l}\text { Municipal } \\
\text { Purchases } \\
\text { (thousand } \\
\text { kWh) }\end{array}$} \\
\hline & $\begin{array}{l}\text { Purchases } \\
\text { (thousand } \\
\text { kWh) }\end{array}$ & $\begin{array}{c}\text { Cost } \\
\text { (dolliars) }\end{array}$ & $\begin{array}{c}\text { Purchases } \\
\text { (thouasend } \\
\text { :Wh) }\end{array}$ & $\begin{array}{c}\text { Coot } \\
\text { (dollara) }\end{array}$ & $\begin{array}{l}\text { Purchases } \\
\text { (thousand } \\
\text { kWh) }\end{array}$ & $\begin{array}{c}\text { Cont } \\
\text { (dollara) }\end{array}$ & \\
\hline \multicolumn{8}{|l|}{ Incitena } \\
\hline $\begin{array}{l}\text { Fulton County Rural E M C } \\
\text { Hancock County REMC }\end{array}$ & - & - & - & - & - & - & - \\
\hline 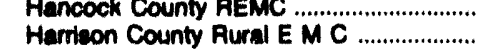 & $\overline{-}$ & - & - & - & - & - & - \\
\hline 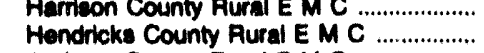 & $\overline{-}$ & $\overline{-}$ & $\overline{-}$ & - & - & - & - \\
\hline 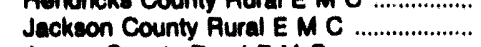 & $\overline{296,395}$ & $11,226,466$ & $\overline{-}$ & $\overline{-}$ & $\overline{-}$ & $\overline{-}$ & $\overline{-}$ \\
\hline Jasper County Rural E M C ........................... & - & - & - & - & - & - & - \\
\hline Jay County Rural E M C & - & - & - & - & - & - & - \\
\hline Johneon County Rural E M C ........................ & - & -- & - & - & - & - & - \\
\hline Kankakee Valley Rural E M C ...................... & - & - & - & - & - & - & - \\
\hline & $=$ & - & - & - & - & - & - \\
\hline & $\overline{-}$ & - & - & - & - & - & - \\
\hline & $\overline{-}$ & - & - & - & - & - & - \\
\hline 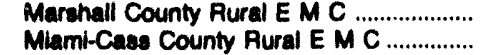 & & $\overline{-}$ & $\bar{z}$ & $\overline{-}$ & $=$ & - & - \\
\hline & $\overline{-}$ & & $\overline{-}$ & $\overline{-}$ & $\bar{z}$ & $\bar{z}$ & $\overline{-}$ \\
\hline & $\overline{-}$ & $\overline{-}$ & $\overline{-}$ & $\overline{-}$ & $\bar{z}$ & $\overline{-}$ & $\bar{z}$ \\
\hline & - & - & - & - & - & - & $\overline{-}$ \\
\hline 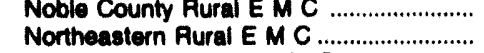 & - & -- & - & - & - & - & $\overline{-}$ \\
\hline 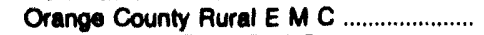 & - & - & - & -. & - & -- & - \\
\hline Parke County Rural E M C ........................... & - & - & - & - & - & - & -- \\
\hline Rush County Rural E M C ............................ & - & - & -- & $\cdots$ & - & - & - \\
\hline Shelby County Rural E M C .......................... & - & - & - & - & - & - & - \\
\hline Southeastern Indiana A E M C ..................... & - & - & -- & -- & -- & - & $-\infty$ \\
\hline 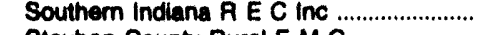 & - & - & - & - & - & - & - \\
\hline Steuben County Rural E M C ....................... & - & - & - & - & - & - & - \\
\hline Sullvan County Rural E M C ....................... & - & - & - & $\cdots$ & - & - & - \\
\hline Tipmont Arl Elec Member Corp .................... & - & - & - & - & -- & - & - \\
\hline United Rural Elec Member Corp ............... & - & - & - & - & - & - & - \\
\hline Utiltites Dist-Western in REMC ...................... & - & - & -- & - & - & - & - \\
\hline Wabash County Rural E M C .......................... & - & -- & - & - & - & - & -- \\
\hline Warren County Rural E M C ......................... & - & - & - & -- & - & - & - \\
\hline Wayne County Rural E M C & $\cdots$ & -- & - & - & - & - & -- \\
\hline White County Rural E M C ............................ & - & - & - & - & - & -- & - \\
\hline \multicolumn{8}{|l|}{ lowa } \\
\hline Adams County Coop Electric Co ............... & - & - & -- & - & -- & - & - \\
\hline Allamakeo-Clayton EI Coop Inc .................. & - & -- & -- & -- & - & - & - \\
\hline Benton County Elec Coop Assn ................ & - & - & -- & - & -- & - & -- \\
\hline Buchanan County Arl Elec Coop .................. & - & - & - & - & - & - & - \\
\hline Butler County Rural Elec Coop ...................... & - & - & - & -- & - & - & - \\
\hline Calhoun County Elec Coop Assn .............. & - & - & - & - & -- & - & - \\
\hline Cedar Valley Electric Coop .......................... & - & - & - & - & - & - & - \\
\hline Chariton Valley Elec Coop Inc ....................... & - & - & -- & -- & - & -- & - \\
\hline Clarke Electric Coop Inc ............................. & - & -- & - & - & -- & - & -- \\
\hline Eastern lowa Lights Power Coop ............... & - & - & - & -- & - & - & - \\
\hline Farmers Electric Coop Inc ............................... & - & - & - & - & - & - & - \\
\hline Franklin Rural Electric Coop .......................... & - & - & - & -- & -- & - & - \\
\hline 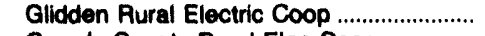 & - & - & -- & - & -- & - & -- \\
\hline Grundy County Rural Elec Coop .................. & - & - & - & $\sim$ & - & - & - \\
\hline Guthrie County Rural E C A .......................... & - & - & - & - & -- & - & - \\
\hline Hancock County Rural Elec Coop ............. & - & - & -- & - & -- & - & - \\
\hline Harrison County Rrt Elec Coop ..................... & - & - & -- & - & - & - & -- \\
\hline Hawkeye Tri-County Elec Coop .................... & - & - & - & $\cdots$ & -- & - & - \\
\hline Humboldt County \& E C & - & - & - & - & - & -- & - \\
\hline Ida County Rural Electric Coop ..................... & - & - & - & - & - & -- & - \\
\hline lowa Lakes Electric Coop ............................... & - & - & - & - & - & - & -- \\
\hline Linn County Rural El Coop Assn ................ & - & - & - & - & - & - & - \\
\hline 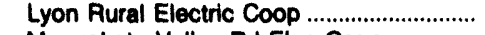 & - & - & - & - & - & - & - \\
\hline Maquoketa Valley Rrl Elec Coop .............. & - & - & - & - & - & - & - \\
\hline Marshall County Ril Elec Coop .................... & - & - & -- & - & - & - & - \\
\hline Midiand Power Cooperative ........................... & - & - & - & - & - & - & - \\
\hline Monona County Rural Elec Coop .............. & -- & -- & - & - & - & - & -- \\
\hline 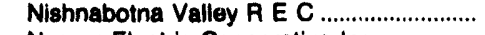 & - & - & -- & - & - & - & - \\
\hline Nyman Electric Cooperative Inc ................ & - & - & - & - & - & -- & - \\
\hline
\end{tabular}

See notes and footnotes at end of table. 
Table 34. Electriclty Purchases by the Diatributlon Segment of Cooperative Borrowers, by 8 tate, 1992 (Continued)

\begin{tabular}{|c|c|c|c|c|c|c|c|}
\hline \multirow{3}{*}{ Cocte / } & \multicolumn{7}{|c|}{ Source of Electricity } \\
\hline & Municipal & Coo & ive & & & & \\
\hline & $\begin{array}{c}\text { Coat } \\
\text { (dollare) }\end{array}$ & $\begin{array}{l}\text { Purchases } \\
\text { (thousand } \\
\text { kWh) }\end{array}$ & $\begin{array}{c}\text { Coot } \\
\text { (dollare) }\end{array}$ & $\begin{array}{c}\text { Purchaces } \\
\text { (thouaand } \\
\text { (WWh) }\end{array}$ & $\begin{array}{c}\text { Cost } \\
\text { (dollara) }\end{array}$ & $\begin{array}{c}\text { Purcheses } \\
\text { (thousand } \\
\text { KWh) }\end{array}$ & $\begin{array}{c}\text { Conl } \\
\text { (dollara) }\end{array}$ \\
\hline
\end{tabular}

\section{Indiene}

Fulton County Rural E M C

Hancook County REMC

Harrison County Rural E M C

Hendricks County Rural E M C

Jackeon County Rurd E M C

Jesoer County Rural E M C

Jay County Rural E M C

Johneon County Rural E M C

Kankakee Valley Rural E M C

Knox County Rural E M C

Koecluako County Rural E M C

Leorange County Rural E M C

Marthall County Rural E M C.

Mumi-Case County Rural E M C.

Morgan County Rural E M C

Newton County Rurai E M C

Noble County Rural E M C

Nonthenatern Rural E M C

Orange County Rural E M C

Parke County Rural E M C

Rush County Rural E M C

Sholby County Rural E M C

Southeastern Indiana A E M C

Southern Indiana $A E C$ inc

Steuben County Rural E M C

Sullvan County Rural E M C

Tipmont Art Elec Member Corp

United Rural Elec Member Corp

Utilties Dist-Western in REMC

Wabash County Rural E M C

Warren County Rural E M C

Wayne County Rural E M C

White County Rural E M C

lowt

Adams County Coop Electric Co

Allamakes-Clayton El Coop Inc

Benton County Elec Coop Aaen

Buchanan County Rit Elec Coop

Butler County Rural Elec Coop...

Cedar Valley Electric Coop

Clarke Electric Coop Inc

Clartern lowa Lights Power Coop ..............

Farmers Electric Coop inc .......

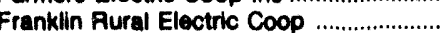

Glidden Rural Electric Coop

Grundy County Rural Elec Coop

Gunthrio ce

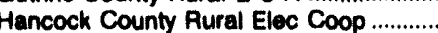

Harrison County Ari Elec Coop

Hawkeye Tr.County Elec Coop

Humboldt County R E C

Rural Electric CoOp

Linn County Rural El C sop Asen ...............

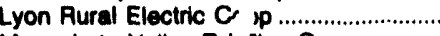

Maquoketa Valley Rrl Elec Coop .............

Mershall County Arl Elec Coop

Midiand Power Cooperattve

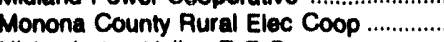

Nishnabotna Valley R E C

Nyman Electric Cooperative Inc

See notes and footnotes at end of table.
$3,159,003$

$\mathbf{5 , 5 2 2 , 0 2 1}$

$13,081,547$

$11,842,580$

283,232

248,089

101,590

101,588
80,718

183,205

156,675

180,123

212,098

73,997

68,105

83,315

329,898

23,204

133,205

339,691

71,802

148,387

70,720

121,346

287,813

113,318

85,100

102.814

260,037

349,067

225,275

116,110

64,134

93,275

92,314

4,892,460

$3,880,280$

$\mathbf{8 , 4 4 1 , 7 8 7}$

$7,816,133$

0,415,030

$9,877,758$

$3,353,819$

$3,213,389$

$3,563,061$

$15,542,224$

$1,135,141$

$5,788,000$

$14,622,582$

$3,322,533$

$6,608,430$

$3,156,459$

$5,604,770$

$3,272,803$

$5,101,684$

$4,015,643$

$4,684,054$

$11,464,449$

$14,679,848$

$10,338,387$

$5,060,244$

$2,897,223$

$4,171,605$

$4,180,811$

26,141

121,846

59,107

79,870

90,376

28,685

61,320

67,687

52,151

317,368

102,272

37,868

36,066

47,458

57,688

43,240

44,124

115,878

40,602

34,585

240,588

162,371

51,787

221,362

68,306

168,243

33,392

70,817

21,213

$1,287,812$

$5,219,396$

$2,977,557$

$\mathbf{3 , 9 6 7 , 4 5 8}$

$4,633,172$

$1,381,174$

$2,420,551$

$2,709,311$

$2,677,758$

$16,107,885$

$4,818,070$

$1,830,182$

$1,796,232$

$2,259,290$

$2,891,482$

$2,035,845$

$1,888,890$

$5,037,977$

$1,918,879$

$1,415,632$

$10,887,466$

$8,810,991$

$1,788,855$

$11,848,595$

$3,514,787$

$8,198,684$

$1,518,715$

$2,945,858$
72,154

120,049

283,232

248,089

296,395

101,590

90,719

183,206

156,675

180,123

212,098

73,097

68,105

83,315

329,889

23,204

133,205

339,681

71,602

149,387

70,720

121,346

287,813

113,318

85,100

102,814

250,037

349,067

225,275

118,110

64,134

93,275

92,314

$1,052,689$
26,141

121,846

59,107

79,878

99,385

28,685

61,320

87,687

52,151

317,368

102,272

37,868

36,066

47,458

57,688

43,240

44,124

115,978

40,602

34,585

240,588

162,373

51,797

221,362

68,306

168,243

33,392

70,817

21,213

$3,159,093$

5,522,021

$13,081,547$

$11,842,580$

$11,226,466$

$4,802,466$

$3,880,280$

$8,441,787$

$7,616,133$

$8,415,030$

$0,877,758$

$3,353,818$

$3,213,389$

$3,563,061$

$15,542,224$

$1,135,141$

$5,788,000$

$14,622,582$

$3,322,533$

$6,608,436$

$3,156,459$

$5,604,770$

$13,272,903$

$5,101,694$

$4,015,643$

$4,684,054$

$11,464,440$

$14,879,949$

$10,338,387$

$5,060,244$

$2,897,223$

$4,171,605$

$4,180,911$ 
Table 34. Electricity Purchaces by the Distribution Segment of Cooperatlve Borrowers, by State, 1992 (Continued)

\begin{tabular}{|c|c|c|c|c|c|c|c|}
\hline \multirow{3}{*}{$\begin{array}{l}\text { stats I } \\
\text { Cocperattve Eorrower }\end{array}$} & \multicolumn{7}{|c|}{ Source of Electricity } \\
\hline & \multicolumn{2}{|c|}{$\begin{array}{l}\text { Inveator. } \\
\text { Owned }\end{array}$} & \multicolumn{2}{|c|}{ Federal } & \multicolumn{2}{|c|}{$\begin{array}{l}\text { State and Other } \\
\text { Government }\end{array}$} & \multirow{2}{*}{$\begin{array}{l}\text { Municipal } \\
\text { Purcheses } \\
\text { (thousand } \\
\text { kWh) }\end{array}$} \\
\hline & $\begin{array}{l}\text { Purchasese } \\
\text { (thousend } \\
\text { kWh) }\end{array}$ & $\begin{array}{c}\text { Coot } \\
\text { (dollare) }\end{array}$ & $\begin{array}{l}\text { Purcheses } \\
\text { (thousend } \\
\text { kWh) }\end{array}$ & $\begin{array}{c}\text { Coat } \\
\text { (dollara) }\end{array}$ & $\begin{array}{c}\text { Purchases } \\
\text { (thousand } \\
\text { kWh) }\end{array}$ & $\begin{array}{c}\text { Coat } \\
\text { (dollare) }\end{array}$ & \\
\hline \multicolumn{8}{|l|}{ lowe } \\
\hline 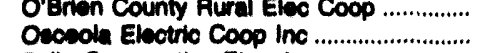 & $\overline{-}$ & $\bar{z}$ & $\overline{-}$ & $\overline{-}$ & $\overline{-}$ & $\overline{-}$ & $\overline{-}$ \\
\hline Polla Cooperative Elec Asen ....................... & - & - & - & -- & - & - & - \\
\hline Pymouth Electric Coop Asen ...................... & - & - & - & - & - & - & - \\
\hline 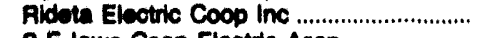 & - & - & - & - & -- & - & - \\
\hline SE lowa Coop Eleotric Acen ......................... & $\overline{-}$ & - & - & - & - & - & - \\
\hline $\begin{array}{l}\text { Sec County Rural Electric Coop } \\
\text { Slan............. Electric Coop Asen }\end{array}$ & $\overline{-}$ & $\overline{-}$ & & $\overline{-}$ & - & - & - \\
\hline 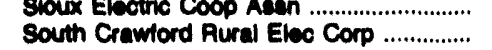 & - & $\overline{-}$ & - & $\overline{-}$ & $\overline{-}$ & $\overline{-}$ & $\overline{-}$ \\
\hline Southern lown Eloc Coop Inc .................... & - & - & - & - & - & - & - \\
\hline T I P Rurel Electric Coop ................................ & - & - & - & - & - & - & - \\
\hline Winnebego Rural Eloc Coop Asen ............ & - & - & - & - & - & - & - \\
\hline Woodoury County Rural E C A ..................... & $\overline{-}$ & - & - & $\overline{-}$ & - & - & - \\
\hline Wripht County Rural Elec Coop .................. & - & - & - & - & - & - & - \\
\hline \multicolumn{8}{|l|}{ Keneas } \\
\hline Ark Valloy Elec Coop Aean inc .................. & - & - & - & - & - & - & - \\
\hline Brown-Alchicon E C A Inc ............................. & - & - & - & - & - & - & - \\
\hline Butter Rural El Coop Aesen Inc ...................... & - & - & - & - & - & - & - \\
\hline C \& W Rural Elec Coop Asen Inc ................. & $\overline{-}$ & - & $\overline{-}$ & $\overline{-}$ & $\overline{-}$ & $\bar{z}$ & $\overline{-}$ \\
\hline $\begin{array}{l}\text { Canoy Valloy El Coop Asen Inc ................... } \\
\text { CMS Electric Cooperative Inc .................... }\end{array}$ & $\overline{-}$ & $\overline{-}$ & $\overline{-}$ & $\overline{-}$ & $\overline{-}$ & $\overline{-}$ & $\overline{-}$ \\
\hline 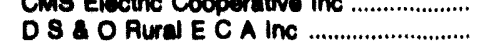 & - & - & - & - & - & - & $=$ \\
\hline Doniphan Elec Coop Aleen Inc ........................ & 15,134 & 571,882 & - & - & - & - & - \\
\hline 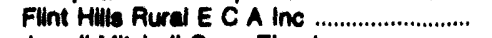 & - & - & - & - & - & - & - \\
\hline Jewell-Mitchell Coop Elec Inc ....................... & - & - & - & - & - & -- & - \\
\hline Kaw Valloy Eloc Coop Inc ............................... & 89,087 & $3,312,329$ & - & - & - & - & - \\
\hline Lene-8coth Electric coop inc ....................... & - & - & - & - & - & - & - \\
\hline Leavenworth-Jeffersoon E C Inc ................. & - & - & - & - & - & -- & - \\
\hline Lyon-Coffey Electric Coop Inc .................... & & $10 \overline{0} m m$ & 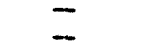 & $\overline{-}$ & $\overline{-}$ & - & - \\
\hline & 432,394 & $18,492,300$ & $\overline{-}$ & $\overline{-}$ & - & $\overline{-}$ & $\overline{-}$ \\
\hline 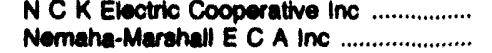 & $\overline{43,133}$ & $1, \overline{571}, 893$ & - & - & - & $\overline{-}$ & $\overline{-}$ \\
\hline Ninneacan Rural E C A Inc ............................... & - & - & - & - & - & - & -- \\
\hline Northwest Kansas E C A Inc ....................... & - & - & - & - & - & - & -- \\
\hline Norton-Docatur Coop El Co inc .................. & - & - & - & - & - & - & - \\
\hline 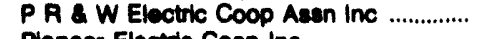 & $\overline{-}$ & $\overline{-}$ & 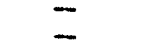 & - & $\bar{z}$ & $\bar{z}$ & - \\
\hline 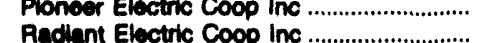 & $\overline{-}$ & $\overline{-}$ & $\overline{-}$ & $\overline{-}$ & $\overline{-}$ & $\bar{z}$ & $\overline{-}$ \\
\hline 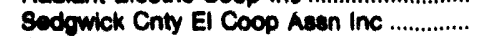 & - & - & - & - & - & - & - \\
\hline Sekan Electric Coop Asen Inc ...................... & - & - & - & - & - & - & - \\
\hline Smoky Hill Elec Coop Aeen Inc .................. & - & - & - & - & - & - & - \\
\hline Sumner-Cowley Elec Coop Inc ................... & 59,927 & $3,222,488$ & - & - & - & - & - \\
\hline Twin Valley Electric Coop Inc ..................... & - & - & - & - & - & - & - \\
\hline 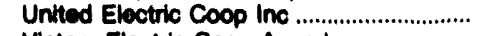 & - & - & - & - & - & - & - \\
\hline Victory Electric Coop Asen Inc ..................... & - & - & - & - & - & -- & - \\
\hline Weatern Coop Elec Asen Inc .......................... & - & - & -- & - & - & - & - \\
\hline Wheatland Electric Coop Inc ......................... & - & - & - & - & - & - & - \\
\hline \multicolumn{8}{|l|}{ Kentuoky } \\
\hline Bip Sandy Rural Elec Coop Corp .............. & - & - & - & - & - & - & - \\
\hline Blive Grase Rural El Coop Corp .................... & - & - & - & - & - & - & - \\
\hline Clark Aural Electric Coop Corp ..................... & - & - & $\overline{-}$ & - & - & - & - \\
\hline Cumberland Valley Rural E C C ................. & - & - & - & - & - & - & - \\
\hline & - & $\overline{-}$ & - & $\bar{z}$ & $\overline{-}$ & $\overline{-}$ & $\overline{-}$ \\
\hline $\begin{array}{l}\text { Fox Creok Rural Elec Coop Corp .............. } \\
\text { Grayeon Rural Elec Coop Corp .................. }\end{array}$ & - & - & - & - & - & - & $\overline{-}$ \\
\hline $\begin{array}{l}\text { Grayeon Aural Elec Coop Corp .................... } \\
\text { Green Rlver Eloctric Corp ......................... }\end{array}$ & - & - & - & - & - & - & - \\
\hline Harricon County Rural E C C .......................... & - & - & - & - & - & -- & - \\
\hline Henderson-Union Rural E C C ....................... & - & - & - & - & - & - & - \\
\hline Hickman-Fulton Counties RECC ................. & - & - & 103,529 & $4,654,240$ & - & - & - \\
\hline Inter-County Rural E C C ............................... & - & - & - & - & - & - & - \\
\hline Jeckeon County Rur & - & - & - & - & - & - & - \\
\hline
\end{tabular}

See notes and tootnotes at end of table. 
Table 34. Electriclty Purchases by the Distribution Segment of Cooperative Borrowers, by State, 1992 (Continued)

\begin{tabular}{|c|c|c|c|c|c|c|c|}
\hline \multirow{3}{*}{$\begin{array}{c}\text { Btite / } \\
\text { Cocpernttve Borrower }\end{array}$} & \multicolumn{7}{|c|}{ Source of Electricity } \\
\hline & \multirow{2}{*}{$\begin{array}{c}\text { Municlpal } \\
\begin{array}{c}\text { Cost } \\
\text { (dollars) }\end{array}\end{array}$} & \multicolumn{2}{|c|}{ Cooperative } & \multicolumn{2}{|c|}{ Other' } & \multicolumn{2}{|c|}{ Total } \\
\hline & & $\begin{array}{c}\text { Purchases } \\
\text { (thousend } \\
\text { kW/h) }\end{array}$ & $\begin{array}{c}\text { Cost } \\
\text { (dollars) }\end{array}$ & $\begin{array}{c}\text { Purchases } \\
\text { (thouseand } \\
\text { kWh) }\end{array}$ & $\begin{array}{c}\text { Cost } \\
\text { (dollars) }\end{array}$ & $\begin{array}{c}\text { Purchases } \\
\text { (thousand } \\
\text { kWh) }\end{array}$ & $\begin{array}{c}\text { Cost } \\
\text { (dollars) }\end{array}$ \\
\hline 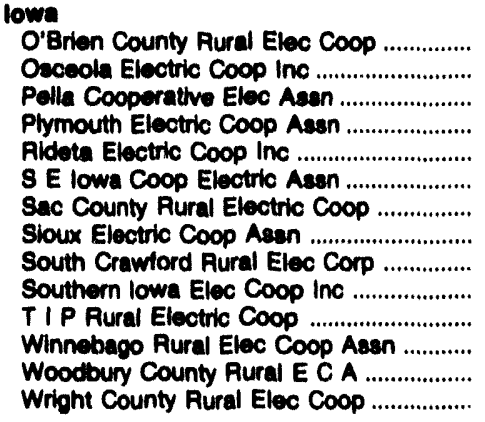 & $\begin{array}{l}= \\
= \\
= \\
= \\
= \\
= \\
=\end{array}$ & $\begin{array}{r}35,955 \\
23,411 \\
35,845 \\
63,017 \\
33,348 \\
154,853 \\
20,501 \\
73,582 \\
66,560 \\
62,425 \\
92,717 \\
38,131 \\
45,108 \\
73,019\end{array}$ & $\begin{array}{r}1,356,482 \\
801,898 \\
1,846,850 \\
2,705,979 \\
1,621,107 \\
5,858,439 \\
1,038,183 \\
3,032,102 \\
2,618,208 \\
2,542,282 \\
4,707,828 \\
1,542,335 \\
1,918,328 \\
2,762,763\end{array}$ & $\begin{array}{ll} & 1 \\
- & \\
- & 9 \\
- & \\
- & \\
- & \\
- & \\
- & \end{array}$ & 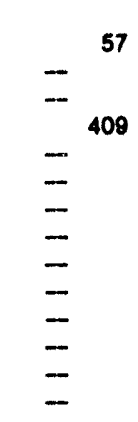 & $\begin{array}{r}35,966 \\
23,411 \\
35,846 \\
63,026 \\
33,348 \\
154,953 \\
20,501 \\
73,562 \\
66,560 \\
62,425 \\
92,717 \\
38,131 \\
45,108 \\
73,019\end{array}$ & $\begin{array}{r}1,366,539 \\
801,898 \\
1,848,850 \\
2,706,388 \\
1,621,107 \\
5,058,439 \\
1,036,183 \\
3,032,102 \\
2,619,209 \\
2,542,282 \\
4,707,828 \\
1,542,335 \\
1,918,328 \\
2,762,763\end{array}$ \\
\hline 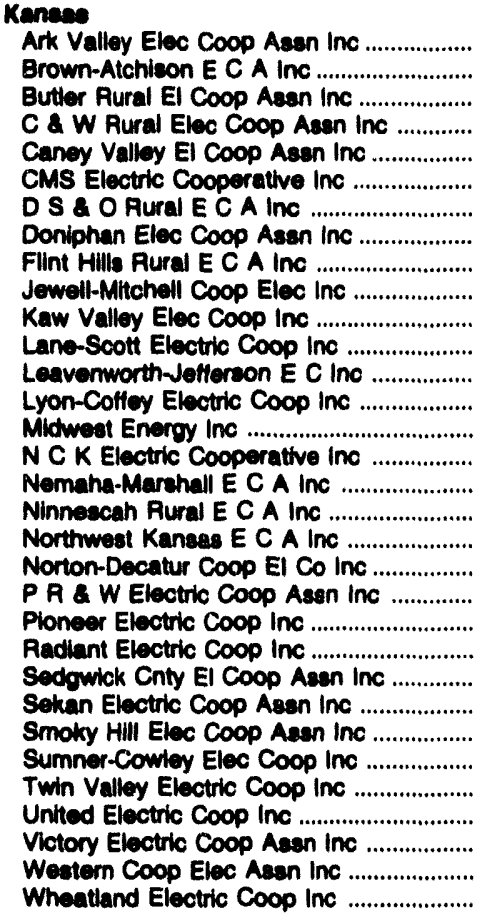 & 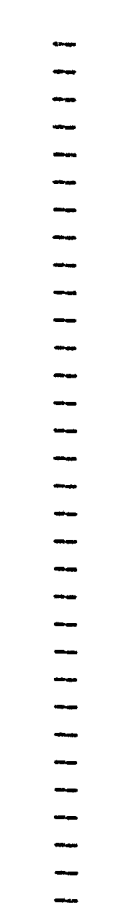 & $\begin{array}{r}70,253 \\
33,395 \\
78,057 \\
36,550 \\
53,218 \\
83,532 \\
85,260 \\
- \\
66,709 \\
45,490 \\
65,371 \\
62,703 \\
78,828 \\
444,539 \\
27,864 \\
51,759 \\
33,258 \\
102,710 \\
30,608 \\
209,732 \\
43,122 \\
62,269 \\
51,469 \\
40,197 \\
23,027 \\
57,464 \\
80,071 \\
124,443 \\
563,097\end{array}$ & $\begin{array}{r}3,615,087 \\
1,763,432 \\
4,334,157 \\
1,868,868 \\
2,677,895 \\
3,835,173 \\
4,769,449 \\
- \\
3,552,940 \\
2,437,160 \\
-\overline{0} \\
4,029,436 \\
3,707,719 \\
4,014,731 \\
10,061,270 \\
1,474,649 \\
- \\
2,672,122 \\
1,987,553 \\
5,196,609 \\
1,742,376 \\
12,390,346 \\
2,145,929 \\
3,565,178 \\
2,687,591 \\
2,021,577 \\
\overline{1}-295,361 \\
2,944,617 \\
4,534,084 \\
7,415,531 \\
31,170,223\end{array}$ & 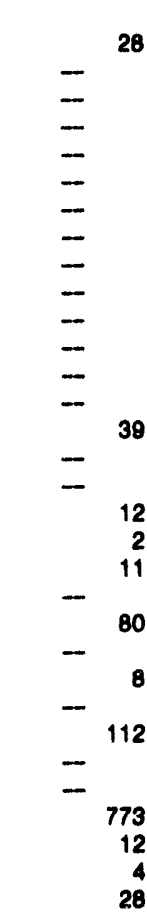 & 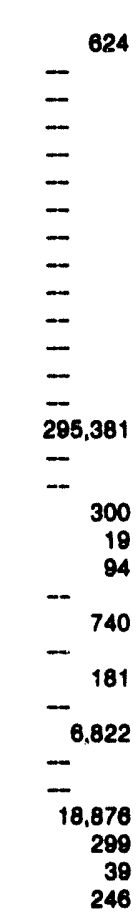 & $\begin{array}{r}70,281 \\
33,395 \\
78,057 \\
36,550 \\
53,219 \\
83,532 \\
85,250 \\
15,134 \\
66,709 \\
45,480 \\
89,087 \\
65,371 \\
62,703 \\
78,028 \\
876,872 \\
27,864 \\
43,133 \\
51,771 \\
33,260 \\
102,721 \\
30,606 \\
209,812 \\
43,122 \\
62,277 \\
51,469 \\
40,309 \\
58,827 \\
23,027 \\
58,237 \\
80,083 \\
124,447 \\
563,125\end{array}$ & $\begin{array}{r}3,615,711 \\
1,763,432 \\
4,334,157 \\
1,688,868 \\
2,677,895 \\
3,835,173 \\
4,769,449 \\
571,882 \\
3,562,940 \\
2,437,160 \\
3,312,329 \\
4,029,435 \\
3,707,719 \\
4,014,731 \\
28,848,951 \\
1,474,649 \\
1,571,893 \\
2,672,422 \\
1,987,572 \\
5,186,703 \\
1,742,376 \\
12,391,086 \\
2,145,029 \\
3,565,359 \\
2,687,581 \\
2,028,399 \\
3,222,488 \\
1,295,361 \\
2,863,493 \\
4,534,383 \\
7,415,570 \\
31,170,469\end{array}$ \\
\hline 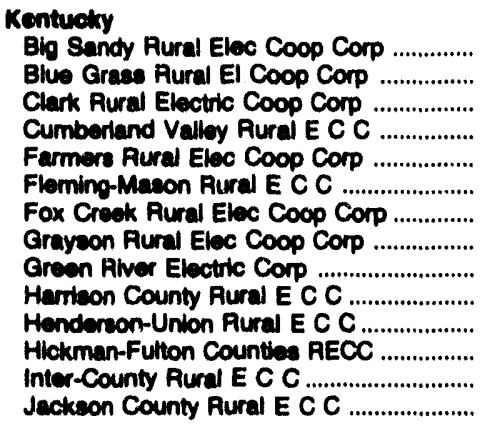 & $\begin{array}{l}\overline{-} \\
\overline{-} \\
\overline{-} \\
\overline{-} \\
\overline{-} \\
\overline{-}\end{array}$ & $\begin{array}{r}202,680 \\
379,799 \\
252,997 \\
365,524 \\
267,341 \\
391,046 \\
124,633 \\
169,000 \\
4,226,071 \\
170,468 \\
3,371,574 \\
\overline{335,324} \\
600,612\end{array}$ & $\begin{array}{r}8,279,302 \\
15,489,743 \\
10,405,761 \\
14,888,855 \\
10,893,167 \\
14,889,677 \\
5,108,769 \\
6,774,671 \\
132,944,112 \\
6,641,033 \\
105,708,234 \\
-\overline{6} \\
9,692,771 \\
24,215,837\end{array}$ & $\begin{array}{l}- \\
\bar{z} \\
\bar{z} \\
\overline{-} \\
\overline{-} \\
\overline{-}\end{array}$ & $\begin{array}{l}- \\
\overline{-} \\
\overline{-} \\
\overline{-} \\
\overline{-} \\
\overline{-} \\
-\end{array}$ & $\begin{array}{r}202,680 \\
379,799 \\
252,897 \\
385,524 \\
287,341 \\
391,846 \\
124,633 \\
169,000 \\
4,226,071 \\
170,468 \\
3,371,574 \\
103,529 \\
235,324 \\
600,612\end{array}$ & $\begin{array}{r}8,279,302 \\
15,489,743 \\
10,405,761 \\
14,888,856 \\
10,893,167 \\
14,889,677 \\
5,108,769 \\
6,774,871 \\
132,844,112 \\
6,641,033 \\
105,708,234 \\
4,654,240 \\
9,602,771 \\
24,215,937\end{array}$ \\
\hline
\end{tabular}

See notes and footnotes at end of table. 
Table 34. Electricity Purchaces by the Distribution Segment of Cooperative Borrowers, by State, 1992 (Continued)

\begin{tabular}{|c|c|c|c|c|c|c|c|}
\hline \multirow{3}{*}{$\begin{array}{c}\text { State / } \\
\text { Cooperathe Borrower }\end{array}$} & \multicolumn{7}{|c|}{ Source of Electrictty } \\
\hline & \multicolumn{2}{|c|}{$\begin{array}{l}\text { Investor- } \\
\text { Owned }\end{array}$} & \multicolumn{2}{|c|}{ Federal } & \multicolumn{2}{|c|}{$\begin{array}{l}\text { State and Other } \\
\text { Government }\end{array}$} & \multirow{2}{*}{$\begin{array}{l}\text { Municlpal } \\
\begin{array}{c}\text { Purchases } \\
\text { (thousend } \\
\text { KWh) }\end{array}\end{array}$} \\
\hline & $\begin{array}{c}\text { Purchases } \\
\text { (thousand } \\
\text { kWh) }\end{array}$ & $\begin{array}{c}\text { Cost } \\
\text { (dollars) }\end{array}$ & $\begin{array}{c}\text { Purcheses } \\
\text { (thousand } \\
\text { kWh) }\end{array}$ & $\begin{array}{c}\text { Cost } \\
\text { (dollars) }\end{array}$ & $\begin{array}{c}\text { Purchases } \\
\text { (thousand } \\
\text { kWh) }\end{array}$ & $\begin{array}{c}\text { Cost } \\
\text { (dollars) }\end{array}$ & \\
\hline \multicolumn{8}{|l|}{ Kentuciny } \\
\hline Jackson Purchase El Coop Corp .................. & - & - & - & - & - & - & - \\
\hline 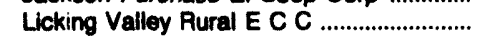 & - & - & - & - & - & - & - \\
\hline 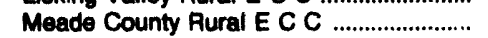 & - & - & - & - & - & - & - \\
\hline Nolin Rural Eloctric Coop Corp ................. & - & - & - & - & - & - & - \\
\hline Owen Electric Cooperative Inc ................... & - & - & - & - & - & - & - \\
\hline Pennyrile Rural Elec Coop Corp ................ & - & - & 748,180 & $33,877,833$ & - & - & - \\
\hline 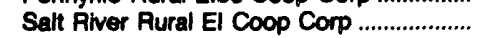 & - & - & - & - & - & - & - \\
\hline 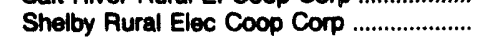 & - & - & - & - & - & - & - \\
\hline South Kentucky Rural E C C ....................... & - & - & - & - & - & - & - \\
\hline 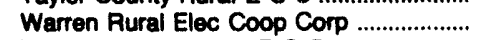 & - & - & 951,778 & $40,557,433$ & - & - & - \\
\hline West Kentucky Rural E C C ........................... & - & - & 721,986 & $29,599,744$ & - & - & - \\
\hline \multicolumn{8}{|l|}{ Loulelane } \\
\hline Bossier Rural Elec Member Corp ................. & - & - & - & - & - & - & - \\
\hline 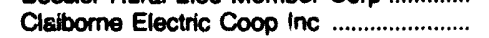 & - & - & - & - & - & - & - \\
\hline Concordia Electric Coop Inc ....................... & - & - & - & - & - & - & - \\
\hline Dixie Electric Membership Corp ................. & - & - & - & - & - & - & - \\
\hline 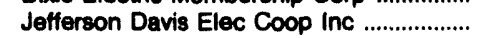 & - & - & - & - & - & - & - \\
\hline Northeast Louisiana Power Coop .............. & - & - & - & - & - & - & - \\
\hline Pointe Coupee Elec Memi si Corp ........... & - & - & - & - & - & - & - \\
\hline South Louisiana Elec Coop Assn .............. & - & - & - & - & - & - & - \\
\hline Southwest Louisiana E M C .......................... & - & - & - & - & - & - & - \\
\hline 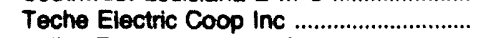 & - & - & - & - & - & - & - \\
\hline Valley Eloctric Member Corp ....................... & - & - & - & - & - & - & - \\
\hline Washington St Tammany E C Inc ................. & - & - & - & - & - & - & - \\
\hline \multicolumn{8}{|l|}{ Maine } \\
\hline Eastern Maine Electric Coop ........................ & 28,030 & $1,542,998$ & - & -- & - & - & - \\
\hline 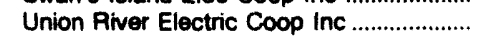 & 8,037 & 665,098 & - & -- & - & - & - \\
\hline \multicolumn{8}{|l|}{ Maryland } \\
\hline 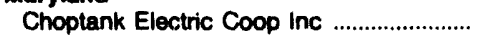 & - & - & - & - & - & - & - \\
\hline Southern Maryland El Coop Inc ................ & $2,122,122$ & $96,601,125$ & - & - & - & $\sim$ & - \\
\hline \multicolumn{8}{|l|}{ Mlehlogn } \\
\hline Alger-Delta Coop Electric Assn .................... & 40,162 & $1,780,087$ & - & - & - & - & 10,603 \\
\hline Cherryland Electric Coop Inc ....................... & - & - & - & - & - & - & - \\
\hline 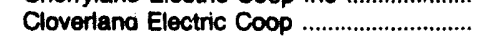 & 175,187 & $3,722,384$ & - & - & - & - & 33 \\
\hline Fruit Belt Electric Coop ................................... & - & - & - & - & - & - & - \\
\hline O \& A Electric Cooperative ............................. & - & - & - & - & - & - & - \\
\hline Oceana Electric Cooperative ....................... & - & - & - & - & - & - & - \\
\hline 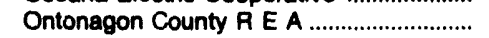 & 21,236 & $1,020,314$ & - & - & - & - & - \\
\hline 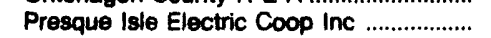 & - & - & - & - & - & - & - \\
\hline 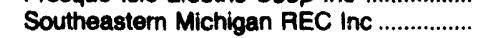 & 29,013 & 887,393 & - & - & - & - & 5,321 \\
\hline Thumb Electric Coop-Michigan .................... & 112,878 & $5,133,596$ & - & - & - & - & - \\
\hline 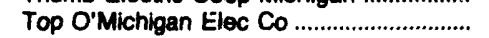 & - & - & - & - & - & - & - \\
\hline 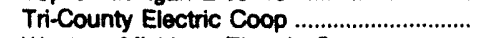 & - & - & - & - & - & - & - \\
\hline Westem Michigan Electric Coop ............... & - & - & - & - & - & - & - \\
\hline \multicolumn{8}{|l|}{ Minnesota } \\
\hline 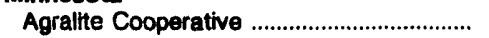 & - & - & - & - & - & - & - \\
\hline 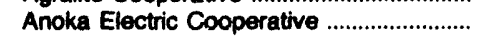 & - & -- & - & - & - & - & - \\
\hline 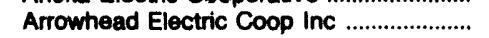 & - & - & - & - & - & - & - \\
\hline 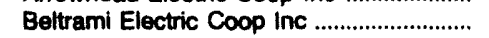 & - & - & - & - & - & - & - \\
\hline 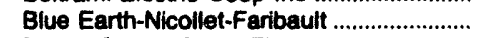 & - & - & - & - & - & - & - \\
\hline 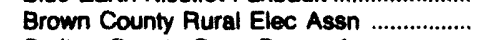 & - & - & - & - & - & - & - \\
\hline Carton County Coop Power Assn ............. & - & - & - & - & - & - & - \\
\hline 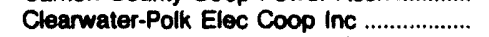 & - & - & - & - & - & - & - \\
\hline Cooperative L8P Assn Lake Cnty ............. & - & - & - & - & - & - & - \\
\hline Crow Wing Coop Power\&light Co ............ & - & - & - & - & - & - & - \\
\hline Dairyland Electric Coop Inc .......................... & - & - & - & - & - & - & - \\
\hline
\end{tabular}

See notes and footnotes at end of table. 
Table 34. Electricity Purchases by the Distribution Segment of Cooperative Borrowers, by State, 1992 (Continued)

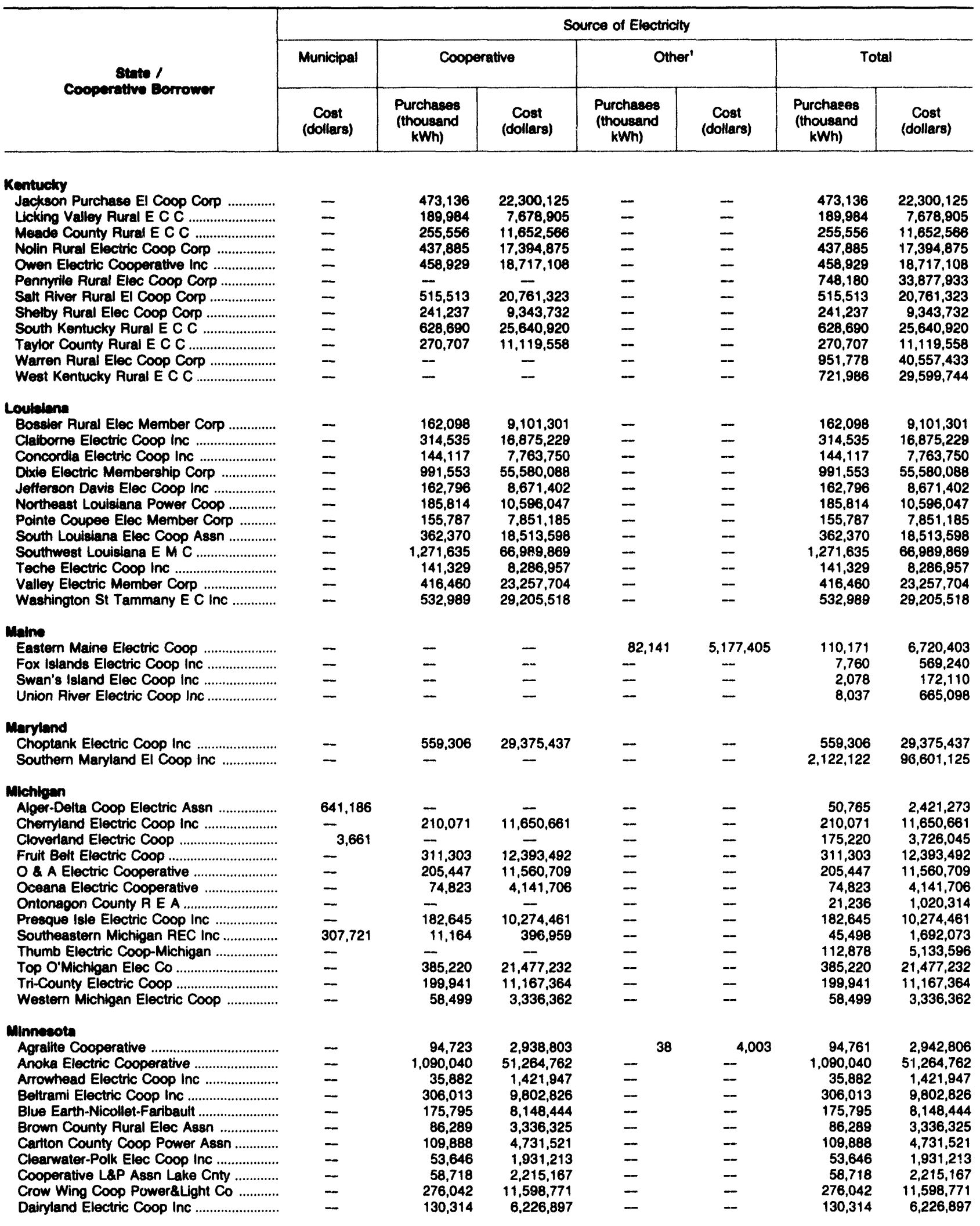

See notes and footnotes at end of table. 
Table 34. Electriclty Purchases by the Distribution Segment of Cooperative Borrowers, by State, 1992 (Continued)

\begin{tabular}{|c|c|c|c|c|c|c|c|}
\hline \multirow{3}{*}{$\begin{array}{c}\text { Stive / } \\
\text { Cooperattre Borrower }\end{array}$} & \multicolumn{7}{|c|}{ Source of Electricity } \\
\hline & \multicolumn{2}{|c|}{$\begin{array}{l}\text { Investor- } \\
\text { Owned }\end{array}$} & \multicolumn{2}{|c|}{ Federal } & \multicolumn{2}{|c|}{$\begin{array}{l}\text { State and Other } \\
\text { Government }\end{array}$} & \multirow{2}{*}{$\begin{array}{c}\text { Municipal } \\
\begin{array}{c}\text { Purchases } \\
\text { (thouseand } \\
\text { KWh) }\end{array}\end{array}$} \\
\hline & $\begin{array}{c}\text { Purchases } \\
\text { (thousand } \\
\text { KWh) }\end{array}$ & $\begin{array}{c}\text { Cost } \\
\text { (dollars) }\end{array}$ & $\begin{array}{l}\text { Purchases } \\
\text { (thousand } \\
\text { kWh) }\end{array}$ & $\begin{array}{l}\text { Cost } \\
\text { (dollars) }\end{array}$ & $\begin{array}{l}\text { Purchases } \\
\text { (thousand } \\
\text { kWh) }\end{array}$ & $\begin{array}{c}\text { Cost } \\
\text { (dollars) }\end{array}$ & \\
\hline
\end{tabular}

Minnesota

Dakota Electric Association

East Contral Electric Asen

Federated Rural Electric Assn

Goodhue County Coop Elec Assn ...........

Itasca-Mantrap Coop Elec Assn ................

Kandfyohi Coop Elec Power Assn .............

Lake Region Coop Elec Assn .

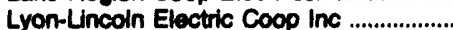

McLeod Cooperattve Power Assn .............

Meeker Coop Light \& Power Asen ............

Mille Lace Electric Coop

Minnesota Valley Coop LEP Assn .........

Minnesota Valley Electric Coop

Nobles Cooperative Electric...

North Itasce Electric Coop Inc

North Pine Electric Coop Inc

North Star Electric CoOp Inc

Northern Electric CoOp Asen

P K M Electric Coop Inc

Red Lake Electric Coop Inc

Red River Valley Coop Pwr Asen ..............

Redwood Electric Cooperative

Renville-sibley Coop Pum Aeen ........

Roseau Electric Coop inc ...........................

Runestone Electric Association ....................

South Central Electric Assn .

Southwestern Minnesote Coop EI

Stearns Cooperative Elec Assn

Steele Waseca Coop Electric

Todd-Wadena Electric CoOp

Traverse Electric Coop Inc

Tri-County Electric Coop

Wild Rice Electric Coop Inc .........................

Wright-Hennepin Coop Elec Assn .............

Misclocipol

Central Electric Power Assn

Coahorna Electric Pow

Cosst Electric Power Assn

Delta Electric Power Assn

Dixie Electric Power Assn ..........................

East Mississippi Elec Pwr Assn ..................

Magnolia Electric Power Assn .

Monroe County Elec Power Assn

Natchez Trace Elec Power Assn ..............

North East Miseiseippi E P A...

Northcentral Mississippl E P A ...................

Peart River Valley El Pur Asen

Singing River Elec Power Assn

Southern Pine Elec Power Assn ................

Southwest Misalseippi E P A

Tallahatchio Valley E P A

Tishomingo County EI Pwr Assn ...............

Tomblgbee Electric Power Asen ................

Twin County Electric Pwr Assn

Yazoo Valley Elec Power Assn

4-County Electric Power Assn

Mlesount

Atchison-Holt Electric Coop

Barry Electric Cooperative...

Barton County Elec Coop inc

See notes and footnotes at end of table.

\begin{tabular}{|c|c|c|c|c|}
\hline- & - & - & - & - \\
\hline- & - & - & - & - \\
\hline- & - & - & - & - \\
\hline $\bar{z}$ & $\bar{z}$ & - & - & - \\
\hline 22,824 & 381,079 & - & $\overline{-}$ & $\overline{-}$ \\
\hline- & - & - & - & - \\
\hline- & - & - & - & - \\
\hline- & - & - & - & - \\
\hline - & - & - & - & - \\
\hline - & $\bar{I}$ & - & - & - \\
\hline 53,710 & 633,667 & - & - & - \\
\hline- & - & - & - & - \\
\hline- & - & - & - & - \\
\hline - & - & - & - & - \\
\hline - & - & - & - & - \\
\hline$\overline{-}$ & - & - & - & - \\
\hline - & - & $\overline{-}$ & $\overline{-}$ & - \\
\hline- & - & - & - & $\overline{-}$ \\
\hline- & - & - & - & - \\
\hline- & - & - & - & - \\
\hline- & -- & - & - & - \\
\hline- & $=$ & $=$ & $\overline{-}$ & - \\
\hline - & - & - & - & - \\
\hline- & - & - & - & \\
\hline- & - & - & - & - \\
\hline - & $=$ & $=$ & $\overline{-}$ & $\overline{-}$ \\
\hline & - & - & - & - \\
\hline- & -- & - & - & \\
\hline- & - & - & - & 一 \\
\hline- & $=$ & $\bar{z}$ & $\overline{-}$ & - \\
\hline
\end{tabular}

$429,421 \quad 18,639,784$

$-$

$-$

$\overline{108,410}$

142,573

279,025

312,921

408,806

$-$

$-$

$-$

514,69

206,861

736,332

$-$

710,027

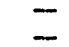

$-$

-

$7,947,205$

6, 219,017

$12,441,97 \theta$

$13,272,715$

$22,081,135$

-

$-$

$-$

$9,224,228$

$32,770,803$

$-$

-

$\overline{023,634}$

-
$\bar{z}$
$\bar{z}$
$\bar{z}$
$\bar{z}$
$\bar{z}$
$\bar{z}$
$\bar{z}$

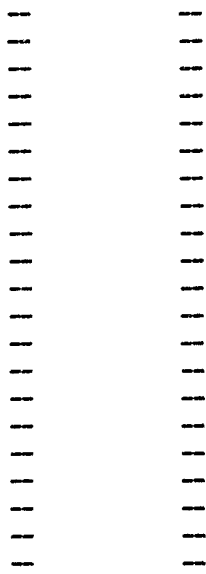

-

$-$

$=$


Table 34. Electricity Purchases by the Dlatribution Segment of Cooperative Borrowers, by State, 1992 (Continued)

\begin{tabular}{|c|c|c|c|c|c|c|c|}
\hline \multirow{3}{*}{ 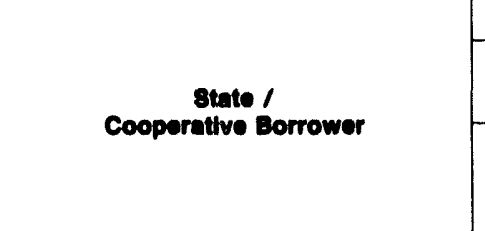 } & \multicolumn{7}{|c|}{ Source of Electricity } \\
\hline & \multirow{2}{*}{$\begin{array}{c}\text { Municipal } \\
\begin{array}{c}\text { Cost } \\
\text { (dollars) }\end{array}\end{array}$} & \multicolumn{2}{|c|}{ Cooperative } & \multicolumn{2}{|c|}{ Other' } & \multicolumn{2}{|c|}{ Total } \\
\hline & & $\begin{array}{l}\text { Purchases } \\
\text { (thousand } \\
\text { KWh) }\end{array}$ & $\begin{array}{c}\text { Cost } \\
\text { (dollars) }\end{array}$ & $\begin{array}{c}\text { Purchases } \\
\text { (thousend } \\
\text { kWh) }\end{array}$ & $\begin{array}{c}\text { Cost } \\
\text { (dollars) }\end{array}$ & $\begin{array}{l}\text { Purchases } \\
\text { (thousand } \\
\text { kWh) }\end{array}$ & $\begin{array}{c}\text { Cost } \\
\text { (dollars) }\end{array}$ \\
\hline 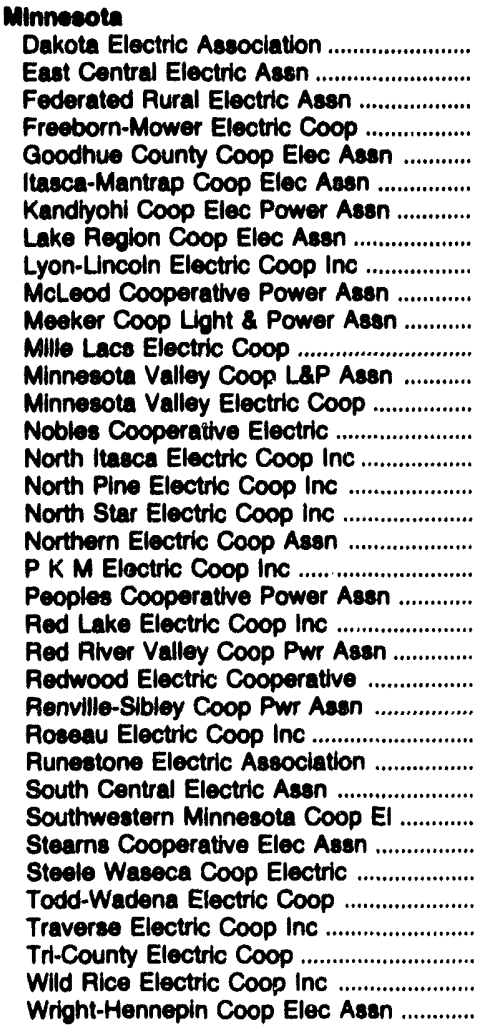 & 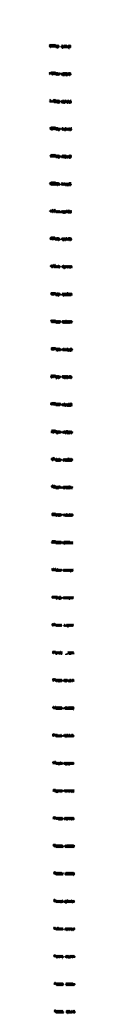 & $\begin{array}{r}998,670 \\
450,641 \\
118,418 \\
122,058 \\
69,364 \\
70,618 \\
109,258 \\
262,180 \\
65,777 \\
109,224 \\
111,512 \\
100,294 \\
76,438 \\
237,850 \\
95,859 \\
31,779 \\
76,805 \\
81,325 \\
188,829 \\
88,381 \\
204,085 \\
98,440 \\
109,150 \\
53,509 \\
66,123 \\
121,712 \\
148,316 \\
87,728 \\
66,444 \\
274,831 \\
121,372 \\
115,002 \\
58,781 \\
238,152 \\
169,069 \\
406,352\end{array}$ & $\begin{array}{r}50,286,554 \\
19,781,737 \\
4,012,064 \\
4,700,746 \\
3,662,632 \\
2,900,348 \\
3,950,875 \\
10,298,727 \\
2,726,513 \\
4,951,296 \\
4,508,065 \\
4,385,206 \\
3,991,704 \\
11,413,604 \\
3,168,306 \\
1,388,968 \\
3,421,730 \\
2,500,819 \\
7,700,241 \\
3,014,915 \\
8,244,912 \\
3,419,336 \\
3,743,529 \\
1,819,334 \\
2,332,386 \\
3,562,214 \\
5,469,474 \\
2,970,480 \\
2,255,327 \\
11,780,737 \\
5,391,137 \\
4,323,186 \\
2,184,651 \\
9,625,811 \\
5,739,436 \\
17,282,125\end{array}$ & 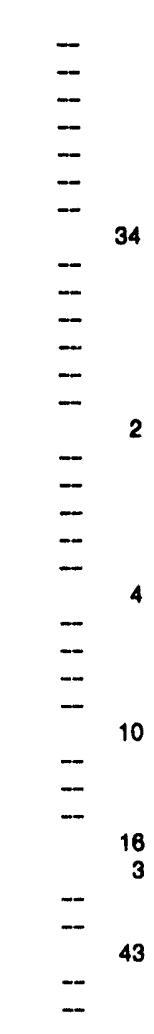 & $\begin{array}{l}- \\
- \\
- \\
- \\
- \\
- \\
2,004 \\
- \\
- \\
- \\
- \\
- \\
- \\
- \\
- \\
- \\
- \\
- \\
- \\
- \\
- \\
- \\
- \\
- \\
- \\
- \\
- \\
-\end{array}$ & $\begin{array}{r}898,670 \\
460,641 \\
118,418 \\
122,058 \\
69,364 \\
83,642 \\
109,258 \\
262,214 \\
65,777 \\
109,224 \\
111,512 \\
100,294 \\
130,148 \\
237,850 \\
96,861 \\
31,779 \\
78,805 \\
81,325 \\
188,829 \\
88,381 \\
204,089 \\
98,440 \\
109,159 \\
53,508 \\
66,123 \\
121,722 \\
148,316 \\
87,728 \\
68,444 \\
274,847 \\
121,375 \\
115,002 \\
58,781 \\
238,195 \\
169,069 \\
400,352\end{array}$ & $\begin{array}{r}50,286,554 \\
19,781,737 \\
4,012,064 \\
4,700,746 \\
3,662,632 \\
3,281,427 \\
3,950,875 \\
10,300,731 \\
2,725,513 \\
4,951,296 \\
4,508,065 \\
4,385,206 \\
4,625,371 \\
11,413,604 \\
3,168,440 \\
1,388,968 \\
3,421,730 \\
2,500,819 \\
7,700,241 \\
3,014,915 \\
8,245,167 \\
3,419,336 \\
3,743,529 \\
1,919,334 \\
2,332,386 \\
3,562,764 \\
5,468,474 \\
2,970,480 \\
2,255,327 \\
11,781,857 \\
5,391,386 \\
4,323,186 \\
2,194,551 \\
9,628,611 \\
5,739,436 \\
17,282,125\end{array}$ \\
\hline 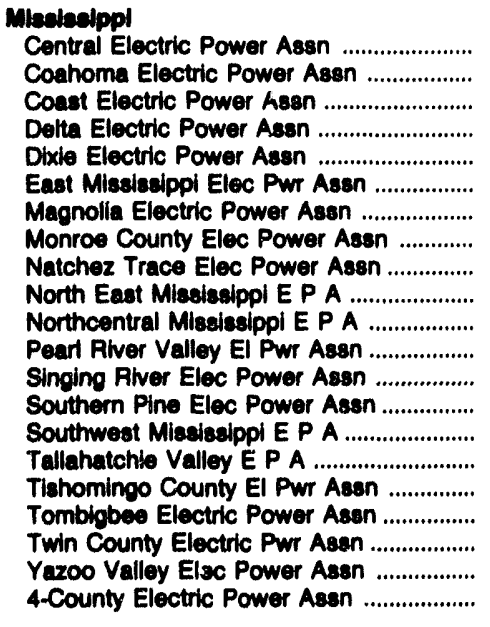 & $\begin{array}{l}- \\
- \\
- \\
- \\
- \\
- \\
- \\
- \\
- \\
- \\
- \\
-\end{array}$ & $\begin{array}{l}\overline{-} \\
89,129 \\
823,751 \\
367,245 \\
491,085 \\
\overline{380,879} \\
- \\
- \\
- \\
\overline{438,535} \\
847,964 \\
1,267,928 \\
348,732 \\
- \\
- \\
\overline{204,508} \\
219,442 \\
-\end{array}$ & $\begin{array}{c}\overline{4,247,779} \\
36,599,257 \\
17,521,188 \\
23,529,563 \\
- \\
17,574,833 \\
- \\
- \\
- \\
20,608,991 \\
39,875,188 \\
55,053,219 \\
16,024,283 \\
- \\
- \\
- \\
9,767,012 \\
7,655,685 \\
-\end{array}$ & $\begin{array}{l}- \\
- \\
- \\
- \\
- \\
- \\
- \\
- \\
- \\
- \\
- \\
- \\
- \\
- \\
-\end{array}$ & $\begin{array}{l}- \\
- \\
- \\
- \\
- \\
- \\
- \\
- \\
- \\
- \\
- \\
- \\
- \\
- \\
-\end{array}$ & $\begin{array}{r}429,421 \\
89,129 \\
823,751 \\
367,245 \\
491,085 \\
495,086 \\
380,879 \\
142,573 \\
279,025 \\
312,821 \\
488,806 \\
438,535 \\
847,964 \\
1,267,826 \\
348,732 \\
514,691 \\
208,961 \\
736,332 \\
204,508 \\
219,442 \\
710,027\end{array}$ & $\begin{array}{r}18,639,784 \\
4,247,779 \\
36,599,257 \\
17,521,188 \\
23,529,563 \\
17,728,009 \\
17,574,833 \\
6,218,017 \\
12,441,979 \\
13,272,715 \\
22,081,135 \\
20,608,891 \\
39,875,188 \\
55,053,219 \\
16,024,283 \\
21,868,444 \\
9,224,228 \\
32,770,803 \\
9,767,012 \\
7,855,885 \\
30,023,684\end{array}$ \\
\hline 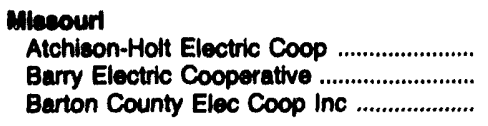 & - & $\begin{array}{r}61,044 \\
118,187 \\
68,356\end{array}$ & $\begin{array}{l}2,389,075 \\
5,246,079 \\
3,267,293\end{array}$ & - & - & $\begin{array}{r}61,044 \\
118,187 \\
68,356\end{array}$ & $\begin{array}{l}2,389,075 \\
5,246,079 \\
3,267,293\end{array}$ \\
\hline
\end{tabular}

Sreg notes and footnotes at end of table. 
Table 34. Electricity Purchases by the Distribution Segment of Cooperative Borrowers, by State, 1992 (Continued)

\begin{tabular}{|c|c|c|c|c|c|c|c|}
\hline \multirow{3}{*}{$\begin{array}{c}\text { State / } \\
\text { Cooperative Borrower }\end{array}$} & \multicolumn{7}{|c|}{ Source of Electricity } \\
\hline & \multicolumn{2}{|c|}{$\begin{array}{l}\text { Investor- } \\
\text { Owned }\end{array}$} & \multicolumn{2}{|c|}{ Federal } & \multicolumn{2}{|c|}{$\begin{array}{l}\text { State and Other } \\
\text { Government }\end{array}$} & \multirow{2}{*}{$\begin{array}{c}\text { Municipal } \\
\begin{array}{c}\text { Purchases } \\
\text { (thousand } \\
\text { kWh) }\end{array}\end{array}$} \\
\hline & $\begin{array}{c}\text { Purchases } \\
\text { (thousand } \\
\text { KWh) }\end{array}$ & $\begin{array}{c}\text { Cost } \\
\text { (dollars) }\end{array}$ & $\begin{array}{c}\text { Purchases } \\
\text { (thousand } \\
\text { kWh) }\end{array}$ & $\begin{array}{c}\text { Cost } \\
\text { (dollars) }\end{array}$ & $\begin{array}{c}\text { Purchases } \\
\text { (thousand } \\
\text { kWh) }\end{array}$ & $\begin{array}{c}\text { Cost } \\
\text { (dollars) }\end{array}$ & \\
\hline
\end{tabular}

\section{Miscourt}

Black River Electric Coop

Boone Electric Cooperative

Callaway Electric Cooperative

Central Missouri Elec Coop Inc

Citizens Electric Corporation

Consolidated Electric Coop

Crawford Electric Coop inc

CO-MO Electric Coop inc

Farmers Electric Coop Inc

ticitive

Grundy Electric Coop Inc

Howard Electric Cooperative

Iniercounty

Laclede Electric Coop inc

Lewis County Rural E C A.

Macon Electric Cooperative

Missouri Rural Electric Coop

New-Mac Electric Coop Inc

Nodaway Worth Elec Coop Inc

North Central MO Elec Coop Inc

Northwest Missouri El Coop Inc

Osage Valley Elec Coop Assn

Ozark Border Electric Coop

Ozark Electric Cooperative Inc

Pemiscot-Dunklin Elec Coop Inc

Platte-Clay Electric Coop inc

Ralls County Electric Coop

inc ......................

Scott-New Madrid-MS Elec Coop ................

Southwest Electric Cooperative

SE-MA-NO Electric Cooperative

Three Rivers Electric Coop .

Tri-County Electric Coop Assn

Webster Electric Cooperative

West Central Electric Coop Inc

White River Valley El Coop Inc

Montana

Beartooth Electric Coop Inc

Big Flat Electric Coop Inc

Elec Coop Inc

Fergus Electric Coop Inc

Flathead Electric Coop Inc

Glacier Electric Coop Inc

Hill County Electric Coop Inc

Lincoln Electric Coop Inc ...

Lower Yellowstone R E A Inc

Marias River Electric Coop Inc

McCone Electric Co-op Inc

Mid-Yellowgtone Elec Coop Inc

Missoula Electric Coop Inc

Park Electric Cooperative Inc

Ravalli County Elec Coop Inc

Sheridan Electric Coop inc

Southeast Electric Coop Inc

Sun River Electric Coop Inc

Valley Electric Coop Inc

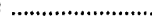

Yellowstone Vlly Elec Coop Inc

See notes and footnotes at end of table.

\begin{tabular}{|c|c|}
\hline- & - \\
\hline- & - \\
\hline- & - \\
\hline$\overline{627,121}$ & $22, \overline{771}, 922$ \\
\hline-- & ${ }^{22, \pi}$ \\
\hline 151 & 11,518 \\
\hline-- & -- \\
\hline- & $\cdots$ \\
\hline- & $\overline{-}$ \\
\hline- & -- \\
\hline- & -- \\
\hline-- & - \\
\hline-- & - \\
\hline- & - \\
\hline$\overline{-}$ & - \\
\hline- & - \\
\hline- & - \\
\hline- & - \\
\hline- & $\bar{z}$ \\
\hline- & - \\
\hline- & - \\
\hline- & -- \\
\hline-- & - \\
\hline- & - \\
\hline 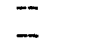 & - \\
\hline -- & $\overline{-}$ \\
\hline- & - \\
\hline- & -- \\
\hline- & - \\
\hline$\ldots$ & - \\
\hline - & -- \\
\hline$\cdots$ & 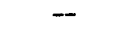 \\
\hline
\end{tabular}

$-$

$\overline{-}$

$-$

$-$

$-$

$-$

$\overline{-}$

$=$

$\overline{-}$

$-$

$=$

$\overline{-}$

$-$

$\bar{z}$

-

$-$

$-$

$\overline{-}$

$\overline{-}$

$\overline{-}$

$-$

$-$

$-$

$-$

$-$

164,829

148,825

$-$

94,960

$-$

$-$

$-$

139,424

-

90,639

$-$

$-$

$-$

116,393

24,647
$-$

$-$

$-$

$-$

$-$

$-$

$-$

$-$

$-$

$-$

$-$

$-$

$-$

$-$

$-$

$3,145,217$

-

$2, \overline{038}, 303$

-

$-$

$-$

$2,608,961$

628,303

$=$

$\overline{-}$

-

$\overline{-}$

$=$

$-$

$=$

$-$

$-$

$=$

$=$

$-$
$=$

$-$

$\overline{-}$

$-$

$\overline{-}$

$\overline{-}$

$-$

$\bar{z}$

$\overline{-}$

$-$

--

$\overline{-}$

-

$-$

$\overline{-}$

$\overline{-}$

\section{$\overline{-}$}

$-$

$124,961 \quad 3,217,393$

-

628,303


Table 34. Electricity Purchases by the Distribution Segment of Cooperative Borrowers, by State, 1992 (Continued)

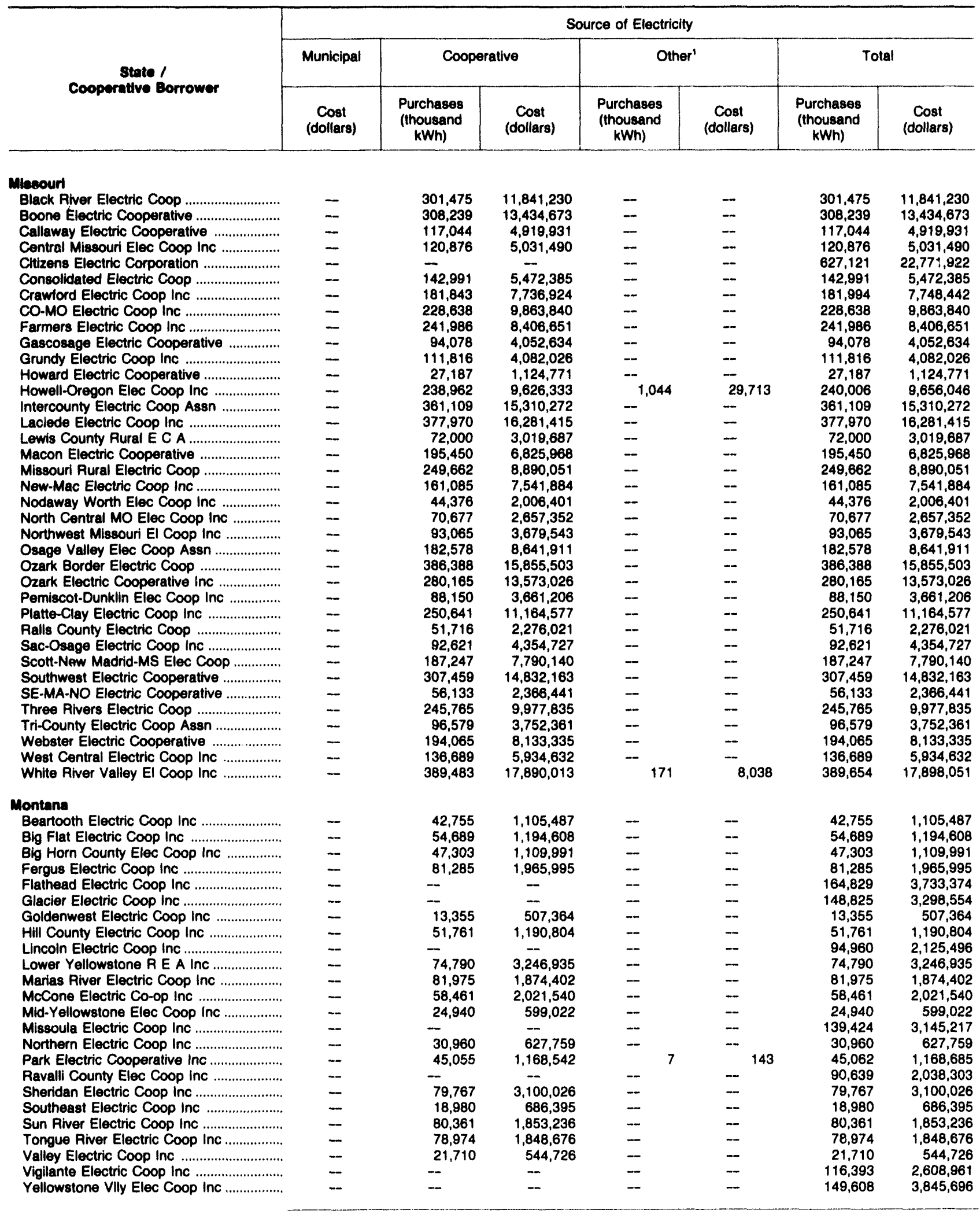

See notes and footnotes at end of table. 
Table 34. Electricity Purchases by the Distribution Segment of Cooperative Borrowers, by State, 1992 (Continued)

\begin{tabular}{|c|c|c|c|c|c|c|c|}
\hline \multirow{3}{*}{$\begin{array}{c}\text { Etate / } \\
\text { Cooperative Borrower }\end{array}$} & \multicolumn{7}{|c|}{ Source of Electricity } \\
\hline & \multicolumn{2}{|c|}{$\begin{array}{l}\text { Investor. } \\
\text { Owned }\end{array}$} & \multicolumn{2}{|c|}{ Federal } & \multicolumn{2}{|c|}{$\begin{array}{c}\text { State and Other } \\
\text { Government }\end{array}$} & \multirow{2}{*}{$\begin{array}{l}\text { Municipal } \\
\begin{array}{c}\text { Purchases } \\
\text { (thousand } \\
\text { kWh) }\end{array}\end{array}$} \\
\hline & $\begin{array}{c}\text { Purchases } \\
\text { (thousand } \\
\text { KWh) }\end{array}$ & $\begin{array}{c}\text { Cost } \\
\text { (dollars) }\end{array}$ & $\begin{array}{l}\text { Purchases } \\
\text { (thousand } \\
\text { KWh) }\end{array}$ & $\begin{array}{c}\text { Cost } \\
\text { (dollars) }\end{array}$ & $\begin{array}{c}\text { Purchases } \\
\text { (thousand } \\
\text { kWh) }\end{array}$ & $\begin{array}{c}\text { Cost } \\
\text { (dollars) }\end{array}$ & \\
\hline \multicolumn{8}{|l|}{ Nebraska } \\
\hline Midwest Electric Member Corp ..................... & - & - & - & - & - & - & - \\
\hline Panhandle Rural El Member Assn ............ & - & - & - & - & - & - & 420 \\
\hline \multicolumn{8}{|l|}{ Neveda } \\
\hline 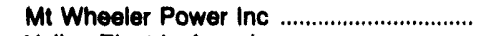 & - & - & - & - & - & $\overline{-}$ & - \\
\hline 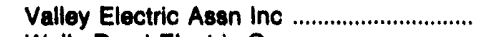 & 159,400 & $5,436,379$ & - & - & 118,616 & $1,885,240$ & - \\
\hline Wells Rural Electric Company ...................... & - & - & 383,716 & $8,088,058$ & $\cdots$ & - & - \\
\hline \multicolumn{8}{|l|}{ Now Hampahire } \\
\hline New Hampshire Elec Coop Inc .................. & 643,736 & $40,845,823$ & - & - & - & - & - \\
\hline \multicolumn{8}{|l|}{ Now Joreey } \\
\hline Sussex Rural Eloctric Coop inc .................... & - & - & $\cdots$ & - & - & - & - \\
\hline \multicolumn{8}{|l|}{ Now Mexico } \\
\hline Central New Mexico El Coop Inc .................. & - & $\sim$ & - & - & - & -- & - \\
\hline Central Valley Elec Coop Inc ....................... & 337,392 & $10,843,409$ & - & - & - & -- & - \\
\hline Columbus Electric Coop Inc ........................ & - & - & - & - & - & -- & - \\
\hline Continental Divide El Coop Inc ................... & $-\infty$ & - & - & - & -- & -- & - \\
\hline Farmers' Elec Coop Inc N Mex ................ & 132,008 & $4,655,341$ & -- & - & - & -- & -- \\
\hline Jemez Mountains Elec Coop Inc ............... & - & - & - & - & - & - & -- \\
\hline Kit Carson Electric Coop Inc ....................... & -- & -ineson & -- & - & - & - & - \\
\hline Lea County Electric Coop Inc .................... & 683,050 & $21,025,163$ & - & - & - & - & -- \\
\hline Mora-San Miguel Elec Coop Inc ................. & - & - & - & $-\infty$ & - & - & - \\
\hline Northern Rio Arriba E Coop Inc .................. & -- & $-\infty$ & -- & -- & -- & - & -- \\
\hline Otero County Electric Coop Inc .................. & - & $\overline{70 n}$ & - & - & - & - & - \\
\hline Roosevelt County Elec Coop Inc .............. & 107,856 & $3,702,211$ & - & - & - & - & - \\
\hline Sierra Electric Coop Inc ............................... & - & - & - & - & -- & -- & - \\
\hline Socorro Electric Coop Inc ............................. & - & - & -- & - & - & $-\infty$ & - \\
\hline Southwestern Electric Coop Inc .................. & - & - & - & -- & - & $-\infty$ & - \\
\hline Springer Electric Coop Inc ........................... & - & - & - & - & - & -- & - \\
\hline \multicolumn{8}{|l|}{ Now York } \\
\hline Delaware County Elec Coop Inc ................. & -- & - & - & -- & 45,043 & 916,733 & - \\
\hline Oneida-Madison Elec Coop Inc .................... & -- & - & - & - & 17,637 & 382,867 & - \\
\hline Otsego Electric Coop Inc ............................ & - & - & - & - & 41,588 & 940,704 & - \\
\hline Steuben Rural Elec Coop Inc ........................ & - & -- & - & - & 59,491 & $1,022,773$ & - \\
\hline \multicolumn{8}{|l|}{ North Carollna } \\
\hline Albemarle Electric Member Corp ................. & - & - & 6,211 & 168,815 & -- & - & -- \\
\hline Blue Aidge Elec Member Corp .................... & - & - & 13,920 & 378,388 & -- & - & - \\
\hline Brunswick Electric Member Corp ............... & - & - & 8,928 & 206,646 & - & - & - \\
\hline Cape Hatteras Elec Member Corp ............. & - & -- & -- & - & -- & -- & - \\
\hline Carteret-Craven El Member Corp ............... & - & - & 6,804 & 157,498 & - & - & - \\
\hline Central Electric Member Corp ..................... & - & - & 3,147 & 72,840 & - & -- & - \\
\hline Crescent Electric Member Corp .................. & - & - & 31,855 & 865,932 & - & - & - \\
\hline Davidson Electric Member Corp .................. & - & - & - & - & -- & - & - \\
\hline Edgecombe-Martin County E M C .............. & - & - & 9,953 & 267,303 & $\cdots$ & - & $-\infty$ \\
\hline Four County Elec Member Corp ................. & -- & - & 10,663 & 246,799 & - & - & - \\
\hline French Broad Elec Member Corp .............. & 346,733 & $16,856,573$ & 13,379 & 431,982 & - & - & - \\
\hline Halifax Electric Member Corp ...................... & -- & - & 6,327 & 164,409 & -- & -- & - \\
\hline Harkers Island El Member Corp ................. & - & - & 42 & 2,486 & - & -- & - \\
\hline Haywood Electric Member Corp ................ & - & - & 5,551 & 170,886 & - & - & - \\
\hline Jones-Onslow Elec Member Corp .............. & - & -- & 13,167 & 304,766 & - & - & - \\
\hline Lumbee River Elec Member Corp ............. & - & -- & 8,471 & 219,227 & - & - & - \\
\hline Pee Dee Electric Member Corp ................... & - & - & 8,304 & 195,293 & - & - & - \\
\hline $\begin{array}{l}\text { Pledmont Electric Member Corp .................. } \\
\text { Pitt \& Greene Elec Member Corn }\end{array}$ & - & - & 2,758 & 63,845 & - & - & - \\
\hline Pitt \& Greene Elec Member Corp ............. & - & - & 4,013 & 92,888 & - & - & - \\
\hline Randolph Electric Member Corp ................. & - & - & 9,164 & 212,113 & - & - & - \\
\hline Roanoke Electric Member Corp ................. & - & - & 13,242 & 355,632 & - & - & - \\
\hline 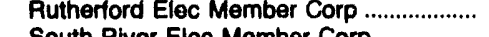 & - & - & 44,583 & $1,212,002$ & - & $-m$ & - \\
\hline South River Elec Member Corp ........................... & - & - & 15,542 & 359,734 & - & - & - \\
\hline Surry-Yadkin Elec Member Corp ............... & - & - & -7510 & - & - & - & - \\
\hline Tideland Electric Member Corp .................. & - & - & 7,519 & 195,534 & - & - & $\cdots$ \\
\hline
\end{tabular}

See notes and footnotes at end of table. 
Table 34. Electricity Purchases by the Distribution Segment of Cooperative Borrowers, by State, 1992 (Continued)

\begin{tabular}{|c|c|c|c|c|c|c|c|}
\hline \multirow{3}{*}{$\begin{array}{l}\text { State I } \\
\text { Cooperattvo Borrower }\end{array}$} & \multicolumn{7}{|c|}{ Source of Electricity } \\
\hline & \multirow{2}{*}{$\begin{array}{c}\text { Municipal } \\
\text { Cost } \\
\text { (dollars) }\end{array}$} & \multicolumn{2}{|c|}{ Cooperative } & \multicolumn{2}{|c|}{ Other' } & \multicolumn{2}{|c|}{ Total } \\
\hline & & $\begin{array}{c}\text { Purchases } \\
\text { (thousand } \\
\text { kWh) }\end{array}$ & $\begin{array}{c}\text { Cost } \\
\text { (dollars) }\end{array}$ & $\begin{array}{l}\text { Purchases } \\
\text { (thousand } \\
\text { kWh) }\end{array}$ & $\begin{array}{c}\text { Cost } \\
\text { (dollars) }\end{array}$ & $\begin{array}{c}\text { Purchases } \\
\text { (thousand } \\
\text { kWh) }\end{array}$ & $\begin{array}{c}\text { Cost } \\
\text { (dollars) }\end{array}$ \\
\hline $\begin{array}{l}\text { Mobraaka } \\
\text { Midwest Electric Member Corp .................. } \\
\text { Panhandle Rural El Member Assn ........... }\end{array}$ & $-5,898$ & $\begin{array}{r}111,519 \\
\theta 2,280\end{array}$ & $\begin{array}{l}5,530,734 \\
4,365,566\end{array}$ & $\overline{-}$ & $\overline{-}$ & $\begin{array}{r}111,619 \\
92,700\end{array}$ & $\begin{array}{l}5,530,734 \\
4,371,462\end{array}$ \\
\hline 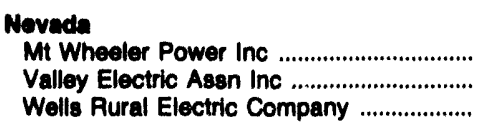 & $\bar{z}$ & $\begin{array}{l}94,379 \\
-\end{array}$ & $\stackrel{5,114,843}{-}$ & $\begin{array}{l}84,434 \\
-\end{array}$ & $\begin{array}{l}1,586,864 \\
- \\
-\end{array}$ & $\begin{array}{l}178,813 \\
279,016 \\
393,716\end{array}$ & $\begin{array}{l}6,701,707 \\
7,321,619 \\
8,088,058\end{array}$ \\
\hline $\begin{array}{l}\text { Now Hampehire } \\
\text { New Hampshire Elec Coop Inc .................. }\end{array}$ & - & - & - & 56 & 1,158 & 643,792 & $40,846,881$ \\
\hline $\begin{array}{l}\text { New Jereey } \\
\text { Sussex Rural Electric Coop Inc .................. }\end{array}$ & - & 108,774 & $6,829,294$ & - & -- & 108,774 & $6,829,294$ \\
\hline 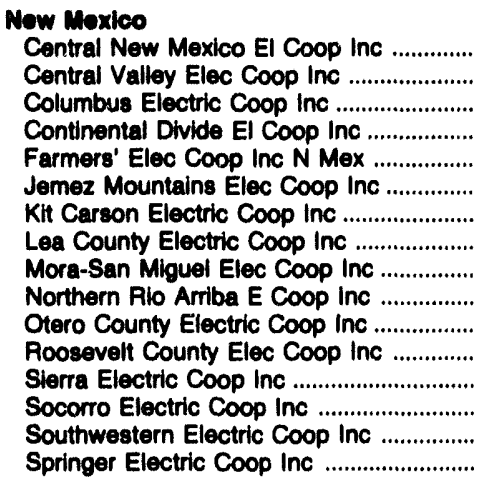 & $\begin{array}{l}\bar{z} \\
\bar{z} \\
\bar{z} \\
\bar{z} \\
\bar{z} \\
\bar{z} \\
\bar{z}\end{array}$ & $\begin{array}{r}95,296 \\
\overline{251,396} \\
221,068 \\
\overline{227,849} \\
188,516 \\
\overline{44,876} \\
22,259 \\
99,310 \\
\overline{34,928} \\
127,710 \\
302,916 \\
55,325\end{array}$ & $\begin{array}{r}6,131,572 \\
\overline{12,378,404} \\
14,150,781 \\
\overline{14,313,982} \\
12,108,336 \\
-\overline{2} \\
2,897,752 \\
1,391,521 \\
6,266,899 \\
-\overline{2,234,122} \\
7,777,014 \\
13,387,925 \\
3,078,722\end{array}$ & $\begin{array}{l}\bar{z} \\
\bar{z} \\
\bar{z} \\
\bar{z} \\
\bar{z} \\
\bar{z} \\
\bar{z}\end{array}$ & $\begin{array}{l}\bar{z} \\
\bar{z} \\
\bar{z} \\
\bar{z} \\
\bar{z} \\
\bar{z} \\
\bar{z} \\
\overline{-}\end{array}$ & $\begin{array}{r}95,296 \\
337,392 \\
251,396 \\
221,068 \\
132,008 \\
227,849 \\
188,516 \\
663,050 \\
44,876 \\
22,259 \\
99,310 \\
107,856 \\
34,928 \\
127,710 \\
302,916 \\
55,325\end{array}$ & $\begin{array}{r}6,131,572 \\
10,843,409 \\
12,378,404 \\
14,150,781 \\
4,655,341 \\
14,313,992 \\
12,108,336 \\
21,025,163 \\
2,897,752 \\
1,391,621 \\
6,268,899 \\
3,702,211 \\
2,234,122 \\
7,777,014 \\
13,387,925 \\
3,078,722\end{array}$ \\
\hline 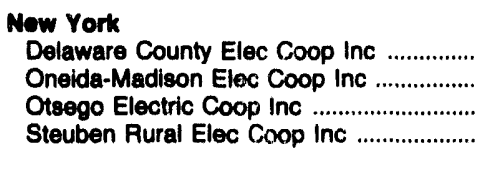 & $\bar{z}$ & $\overline{-}$ & $\bar{z}$ & $\bar{m}=$ & $\bar{z}$ & $\begin{array}{l}45,043 \\
17,637 \\
41,588 \\
59,491\end{array}$ & $\begin{array}{r}916,733 \\
382,867 \\
940,704 \\
1,022,773\end{array}$ \\
\hline 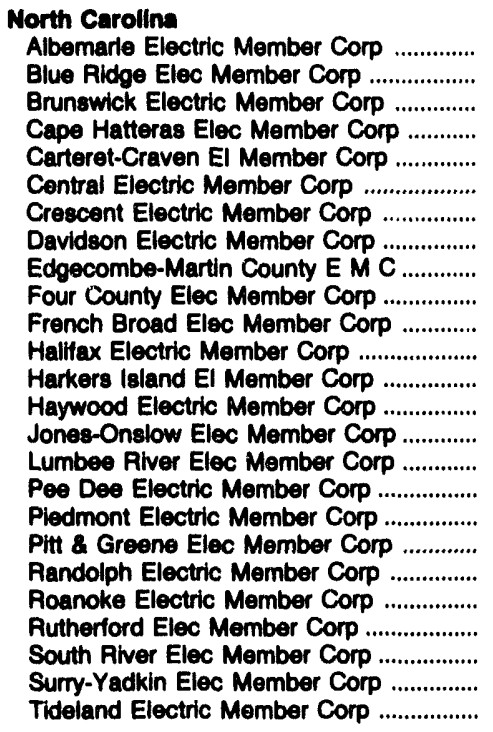 & $\begin{array}{l}\bar{z} \\
\bar{z} \\
\bar{z} \\
\bar{z} \\
\bar{z} \\
\bar{z} \\
\bar{z} \\
\bar{z} \\
\bar{z} \\
= \\
=\end{array}$ & $\begin{array}{r}99,408 \\
838,789 \\
579,839 \\
73,072 \\
355,995 \\
141,592 \\
670,096 \\
463,555 \\
151,625 \\
374,227 \\
96,050 \\
13,684 \\
168,350 \\
570,897 \\
488,727 \\
231,623 \\
273,906 \\
119,390 \\
328,462 \\
159,650 \\
737,642 \\
416,601 \\
231,282 \\
214,144\end{array}$ & $\begin{array}{r}5,613,825 \\
38,063,696 \\
38,282,834 \\
4,151,548 \\
22,270,212 \\
9,029,589 \\
34,073,704 \\
23,227,777 \\
7,984,682 \\
22,659,862 \\
-\overline{4} \\
4,964,869 \\
826,795 \\
8,315,391 \\
36,556,003 \\
31,166,571 \\
13,845,593 \\
14,670,558 \\
7,315,188 \\
20,271,496 \\
8,434,310 \\
36,528,173 \\
27,381,282 \\
12,096,745 \\
12,870,075\end{array}$ & $\begin{array}{l}-675 \\
= \\
= \\
= \\
= \\
= \\
= \\
= \\
= \\
= \\
= \\
= \\
= \\
=\end{array}$ & $\begin{array}{l}23,659 \\
= \\
= \\
- \\
- \\
= \\
= \\
= \\
= \\
= \\
= \\
= \\
= \\
= \\
= \\
-\end{array}$ & $\begin{array}{r}105,619 \\
853,384 \\
588,767 \\
73,072 \\
362,799 \\
144,739 \\
702,852 \\
463,555 \\
161,578 \\
384,890 \\
360,112 \\
102,377 \\
13,726 \\
173,801 \\
584,064 \\
498,198 \\
239,927 \\
276,664 \\
123,403 \\
337,626 \\
172,892 \\
782,225 \\
432,143 \\
231,282 \\
221,663\end{array}$ & $\begin{array}{r}5,780,640 \\
38,465,743 \\
38,489,480 \\
4,151,548 \\
22,427,710 \\
9,102,429 \\
34,939,724 \\
23,227,777 \\
8,251,985 \\
22,906,681 \\
17,288,665 \\
5,129,378 \\
829,281 \\
8,486,277 \\
36,860,769 \\
31,385,798 \\
14,040,886 \\
14,734,403 \\
7,408,076 \\
20,483,609 \\
8,789,942 \\
37,740,175 \\
27,741,016 \\
12,086,746 \\
13,065,609\end{array}$ \\
\hline
\end{tabular}

See notes and footnotes at end of table. 
Table 34. Electriclty Purchases by the Diatribution Segment of Cooperative Borrowers, by State, 1992 (Continued)

\begin{tabular}{|c|c|c|c|c|c|c|c|}
\hline \multirow{3}{*}{$\begin{array}{c}\text { State / } \\
\text { Cooperative Borrower }\end{array}$} & \multicolumn{7}{|c|}{ Source of Electrictiy } \\
\hline & \multicolumn{2}{|c|}{$\begin{array}{l}\text { Investor. } \\
\text { Owned }\end{array}$} & \multicolumn{2}{|c|}{ Federal } & \multicolumn{2}{|c|}{$\begin{array}{l}\text { State and Other } \\
\text { Government }\end{array}$} & \multirow{2}{*}{$\begin{array}{c}\text { Municipal } \\
\begin{array}{c}\text { Purchases } \\
\text { (thousand } \\
\text { kWh) }\end{array}\end{array}$} \\
\hline & $\begin{array}{c}\text { Purchases } \\
\text { (thousand } \\
\text { kWh) }\end{array}$ & $\begin{array}{c}\text { Cost } \\
\text { (dollars) }\end{array}$ & $\begin{array}{c}\text { Purchases } \\
\text { (thousand } \\
\text { kWh) }\end{array}$ & $\begin{array}{c}\text { Cost } \\
\text { (dollars) }\end{array}$ & $\begin{array}{l}\text { Purchases } \\
\text { (thousand } \\
\text { kWh) }\end{array}$ & $\begin{array}{c}\text { Cost } \\
\text { (dollars) }\end{array}$ & \\
\hline \multicolumn{8}{|l|}{ North Carollnm } \\
\hline 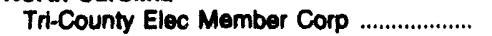 & - & - & 7,864 & 182,013 & - & - & - \\
\hline Union Electric Membership Corp ............... & - & - & 21,284 & 578,561 & - & - & - \\
\hline Wake Electric Membership Corp ................ & - & $\cdots$ & 5,488 & 127,221 & - & - & - \\
\hline \multicolumn{8}{|l|}{ North Dakota } \\
\hline 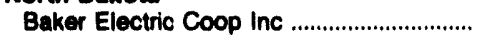 & - & - & - & - & - & - & - \\
\hline Burke-Divide Electric Coop inc ...................... & - & - & - & - & - & - & - \\
\hline 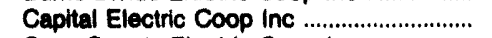 & - & - & 12,748 & 157,176 & - & - & - \\
\hline Cass County Electric Coop Inc ...................... & - & - & - & - & - & -- & - \\
\hline Cavalier Rural Elec Coop Inc .......................... & - & - & - & - & - & - & - \\
\hline James Valley Electric Coop Inc ..................... & 60 & 2,105 & - & - & - & - & - \\
\hline KEM Electric Cooperative Inc ..................... & - & $-\infty$ & 34,104 & 417,447 & - & - & - \\
\hline McKenzie Electric Coop Inc ......................... & - & - & - & - & - & - & - \\
\hline 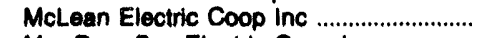 & - & - & - & $-\overline{a n}$ & - & - & -- \\
\hline Mor-Gran-Sou Electric Coop Inc ................ & - & - & 27,810 & 329,675 & - & - & - \\
\hline Mountrail-Williams Elec Coop ....................... & - & - & - & - & $-m$ & - & - \\
\hline Nodak Rural Electric Coop Inc ....................... & - & - & - & - & - & - & -- \\
\hline North Central Elec Coop Inc ......................... & - & - & - & - & - & -- & - \\
\hline 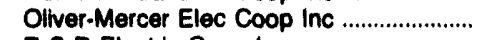 & - & - & 18,525 & 215,889 & - & - & - \\
\hline R S R Electric Coop Inc .............................. & - & - & - & - & -- & -- & - \\
\hline Sheyenne Valley Elec Coop Inc .................... & - & - & - & -- & - & - & - \\
\hline Slope Electric Coop Inc ............................. & - & - & - & - & - & - & -- \\
\hline Tri-County Electric Coop Inc ........................ & - & - & - & - & - & -- & - \\
\hline Verendrye Electric Coop Inc ........................ & - & - & - & - & - & - & - \\
\hline West Plains Electric Coop Inc ..................... & - & 一 & - & - & - & -- & - \\
\hline \multicolumn{8}{|l|}{ Onio } \\
\hline 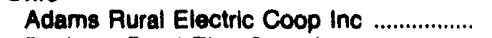 & - & - & - & - & - & - & - \\
\hline Buckeye Rural Elec Coop Inc ....................... & - & - & - & - & - & - & - \\
\hline Butler Rural Electric Coop Inc ...................... & - & - & - & - & - & - & - \\
\hline Carroll Electric Coop Inc ............................. & - & - & - & - & - & - & - \\
\hline Darke Rural Electric Coop Inc ..................... & - & - & - & - & - & - & - \\
\hline Delaware Rural Elec Coop Inc ...................... & - & - & -- & -- & - & - & -- \\
\hline Firelands Electric Coop inc ......................... & - & -- & - & - & - & - & - \\
\hline Frontier Power Company .............................. & - & -- & - & -- & $m$ & - & - \\
\hline Guernsey-Muskingum El Coop Inc ........... & + & - & - & -- & - & -- & - \\
\hline Hancock-Wood Electric Coop Inc .............. & - & -- & - & -- & - & - & - \\
\hline Holmes-Wayne Electric Coop Inc .............. & -- & -- & - & - & -- & $-m$ & - \\
\hline Licking Rural Electric Inc ................................ & - & - & - & - & - & -- & - \\
\hline Logan Cnty Coop P\&L Assn inc .................. & -- & - & -- & - & - & $-\infty$ & - \\
\hline Lorain-Medina R E C Inc ................................ & - & -- & -- & - & -- & -- & - \\
\hline Marion Rural Electric Coop Inc ..................... & -- & -- & -- & - & -- & - & - \\
\hline 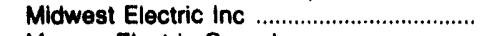 & - & -- & - & - & - & - & - \\
\hline Morrow Electric Coop Inc ............................ & - & -- & - & -- & - & -- & - \\
\hline 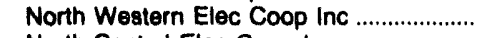 & - & -- & $-\infty$ & - & - & -- & -- \\
\hline North-Central Elec Coop Inc ....................... & - & - & -- & - & - & - & - \\
\hline Paulding-Putman Elec Coop Inc ................... & - & - & -- & - & - & -- & - \\
\hline Pioneer Rural Elec Coop Inc ......................... & - & - & - & -- & - & -- & - \\
\hline South Central Power Company ..................... & - & - & -- & - & - & -- & - \\
\hline TriCounty Rural Elec Coop Inc ...................... & - & - & - & -- & - & - & - \\
\hline Union Rural Electric Coop Inc ..................... & - & - & - & - & - & - & - \\
\hline United Rural Electric Coop Inc ..................... & - & - & - & -- & - & - & -- \\
\hline Washington Electric Coop Inc ....................... & - & - & - & - & - & -- & - \\
\hline \multicolumn{8}{|l|}{ Oklahoma } \\
\hline 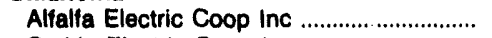 & - & - & - & - & - & -- & -- \\
\hline 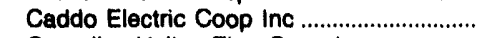 & - & -- & - & - & -- & - & - \\
\hline Canadian Valley Elec Coop Inc .................... & - & -- & $-\infty$ & - & - & - & - \\
\hline 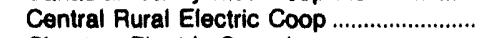 & - & - & -- & - & - & - & -- \\
\hline Choctaw Electric Coop Inc ........................... & - & - & - & - & - & - & - \\
\hline Cimarron Electric Cooperative ....................... & - & - & - & - & - & - & - \\
\hline Cookson Hills Elec Coop Inc ........................ & -- & - & - & - & - & - & - \\
\hline Cotton Electric Coop Inc .............................. & - & - & -- & - & - & - & - \\
\hline East Central Okla EI Coop Inc ..................... & - & - & - & - & - & - & - \\
\hline
\end{tabular}

See notes and footnotes at end of table. 
Tabie 34. Electricity Purchases by the Dletribution Segment of Cooperative Borrowers, by State, 1992 (Continued)

\begin{tabular}{|c|c|c|c|c|c|c|c|}
\hline \multirow{3}{*}{$\begin{array}{c}\text { state / } \\
\text { Cooperattve Borrower }\end{array}$} & \multicolumn{7}{|c|}{ Source of Electricity } \\
\hline & \multirow{2}{*}{$\begin{array}{c}\text { Municipal } \\
\begin{array}{c}\text { Cost } \\
\text { (dollars) }\end{array}\end{array}$} & \multicolumn{2}{|c|}{ Cooperative } & \multicolumn{2}{|c|}{ Other' } & \multicolumn{2}{|c|}{ Total } \\
\hline & & $\begin{array}{l}\text { Purchases } \\
\text { (thousand } \\
\text { kWh) }\end{array}$ & $\begin{array}{c}\text { Cost } \\
\text { (dollars) }\end{array}$ & $\begin{array}{l}\text { Purchuses } \\
\text { (thousand } \\
\text { KWh) }\end{array}$ & $\begin{array}{c}\text { Cost } \\
\text { (dollars) }\end{array}$ & $\begin{array}{c}\text { Purchases } \\
\text { (thousand } \\
\text { KWh) }\end{array}$ & $\begin{array}{c}\text { Cost } \\
\text { (dollars) }\end{array}$ \\
\hline $\begin{array}{l}\text { North Cercolln } \\
\text { Tri-County Elec Member Corp .................. } \\
\text { Union Electric Membership Corp ............. } \\
\text { Wake Electric Membership Corp .............. }\end{array}$ & - & $\begin{array}{l}305,212 \\
460,835 \\
254,398\end{array}$ & $\begin{array}{l}18,638,213 \\
25,225,370 \\
14,441,625\end{array}$ & $m$ & - & $\begin{array}{l}313,076 \\
482,119 \\
259,894\end{array}$ & $\begin{array}{l}18,820,226 \\
25,803,931 \\
14,509,046\end{array}$ \\
\hline 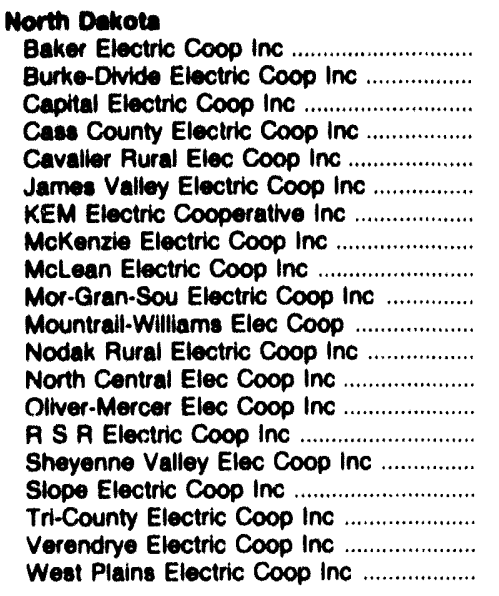 & $\begin{array}{l}- \\
- \\
- \\
- \\
- \\
- \\
- \\
- \\
- \\
- \\
- \\
- \\
-- \\
- \\
- \\
-\end{array}$ & $\begin{array}{r}131,363 \\
51,213 \\
91,681 \\
391,512 \\
40,335 \\
67,642 \\
18,347 \\
273,667 \\
47,438 \\
63,262 \\
141,272 \\
487,305 \\
141,526 \\
160,515 \\
110,802 \\
71,081 \\
51,409 \\
98,927 \\
244,105 \\
210,986\end{array}$ & $\begin{array}{r}5,061,935 \\
1,735,931 \\
4,372,818 \\
11,519,481 \\
1,463,910 \\
2,818,168 \\
807,889 \\
10,701,225 \\
1,948,042 \\
3,278,486 \\
4,744,221 \\
16,109,442 \\
5,608,149 \\
8,285,918 \\
4,483,384 \\
2,464,996 \\
2,095,592 \\
4,060,425 \\
8,478,041 \\
9,862,676\end{array}$ & $\begin{array}{ll}- & \\
- & \\
- & \\
-- & \\
-- & \\
- & 93 \\
& 13 \\
- & \\
- & \\
- & \\
- & 7 \\
- & \\
- & \\
- & \\
- & \end{array}$ & 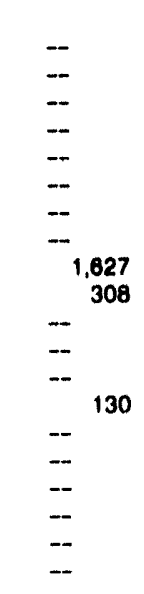 & $\begin{array}{r}131,383 \\
51,213 \\
104,429 \\
391,512 \\
40,335 \\
67,702 \\
52,451 \\
273,667 \\
47,531 \\
91,085 \\
141,272 \\
487,305 \\
141,526 \\
179,047 \\
110,802 \\
71,081 \\
51,409 \\
98,927 \\
244,105 \\
210,986\end{array}$ & $\begin{array}{r}5,061,835 \\
1,735,931 \\
4,529,894 \\
11,518,481 \\
1,463,910 \\
2,920,273 \\
1,325,436 \\
10,701,225 \\
1,949,669 \\
3,608,479 \\
4,744,221 \\
16,109,442 \\
5,608,149 \\
8,501,937 \\
4,483,384 \\
2,464,996 \\
2,095,592 \\
4,060,425 \\
8,478,041 \\
9,862,676\end{array}$ \\
\hline 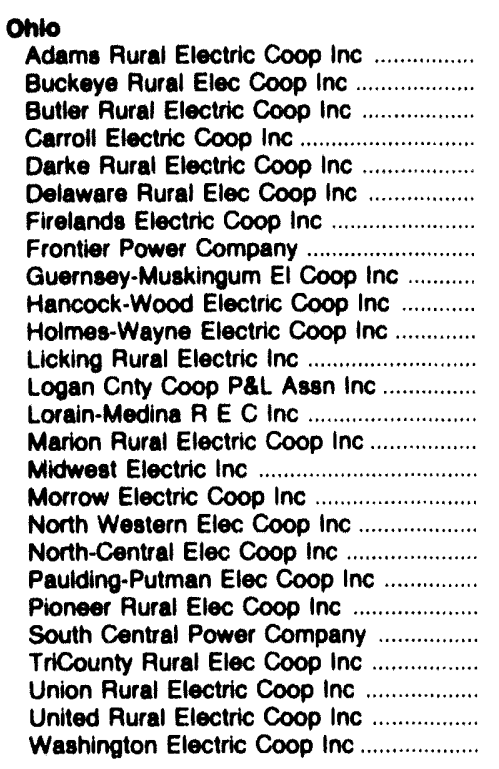 & $\begin{array}{l}- \\
-- \\
-- \\
-- \\
-- \\
-- \\
-- \\
-- \\
-- \\
-- \\
-- \\
-- \\
-- \\
-- \\
-- \\
- \\
-\end{array}$ & $\begin{array}{r}60,318 \\
183,136 \\
150,591 \\
116,597 \\
88,184 \\
74,385 \\
99,200 \\
101,437 \\
134,957 \\
318,541 \\
209,731 \\
237,037 \\
82,607 \\
169,392 \\
51,799 \\
160,123 \\
99,523 \\
72,941 \\
240,058 \\
191,522 \\
387,090 \\
1,228,228 \\
48,298 \\
356,229 \\
47,078 \\
75,612\end{array}$ & $\begin{array}{r}2,278,196 \\
7,129,011 \\
5,937,028 \\
4,183,668 \\
3,366,310 \\
2,757,459 \\
3,418,231 \\
3,566,124 \\
4,930,049 \\
9,980,036 \\
7,481,111 \\
8,619,901 \\
2,945,562 \\
6,048,057 \\
1,921,727 \\
5,846,144 \\
3,654,835 \\
2,548,314 \\
7,632,086 \\
7,625,559 \\
13,219,487 \\
45,308,941 \\
1,757,459 \\
11,866,235 \\
1,745,047 \\
2,750,424\end{array}$ & $\begin{array}{l}-- \\
-- \\
-- \\
-- \\
-- \\
-- \\
-- \\
-- \\
-- \\
-- \\
-- \\
-- \\
- \\
-- \\
- \\
-- \\
-- \\
-- \\
-- \\
-\end{array}$ & $\begin{array}{l}-- \\
-- \\
-- \\
-- \\
-- \\
-- \\
-- \\
-- \\
-- \\
-- \\
- \\
- \\
- \\
- \\
- \\
- \\
-- \\
- \\
-- \\
-\end{array}$ & $\begin{array}{r}60,318 \\
183,136 \\
150,591 \\
116,597 \\
88,184 \\
74,385 \\
99,200 \\
101,437 \\
134,957 \\
318,541 \\
209,731 \\
237,037 \\
82,807 \\
169,392 \\
51,799 \\
160,123 \\
98,523 \\
72,941 \\
240,058 \\
191,522 \\
387,090 \\
1,228,228 \\
48,298 \\
356,229 \\
47,078 \\
75,612\end{array}$ & $\begin{array}{r}2,278,196 \\
7,129,011 \\
5,937,028 \\
4,183,668 \\
3,366,310 \\
2,757,459 \\
3,418,231 \\
3,566,124 \\
4,930,049 \\
9,980,036 \\
7,481,111 \\
8,619,901 \\
2,945,562 \\
6,048,057 \\
1,921,727 \\
5,846,144 \\
3,654,835 \\
2,548,314 \\
7,632,086 \\
7,625,559 \\
13,219,487 \\
45,308,941 \\
1,757,459 \\
11,866,235 \\
1,745,047 \\
2,750,424\end{array}$ \\
\hline 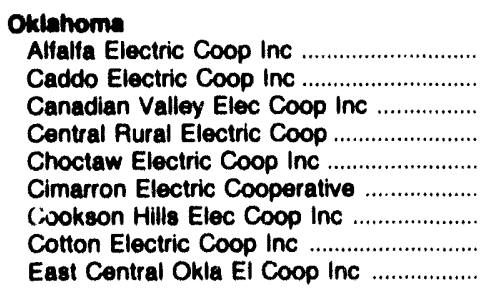 & $\begin{array}{l}- \\
-- \\
- \\
-- \\
-- \\
-- \\
- \\
-\end{array}$ & $\begin{array}{l}127,393 \\
213,998 \\
299,641 \\
228,868 \\
214,721 \\
162,680 \\
177,595 \\
426,553 \\
344,745\end{array}$ & $\begin{array}{r}5,984,157 \\
10,914,832 \\
13,745,370 \\
10,109,234 \\
8,865,641 \\
8,022,185 \\
8,235,647 \\
19,345,149 \\
15,610,441\end{array}$ & $\begin{array}{l}-- \\
-- \\
-- \\
- \\
- \\
- \\
-\end{array}$ & $\begin{array}{l}-- \\
-- \\
- \\
- \\
- \\
-- \\
- \\
-\end{array}$ & $\begin{array}{l}127,393 \\
213,098 \\
299,641 \\
228,868 \\
214,721 \\
182,680 \\
177,595 \\
426,553 \\
344,745\end{array}$ & $\begin{array}{r}5,884,157 \\
10, \theta 14,832 \\
13,745,370 \\
10,109,234 \\
8,865,841 \\
8,022,185 \\
8,235,647 \\
19,345,149 \\
15,610,441\end{array}$ \\
\hline
\end{tabular}

See notes and footnotes at end of table. 
Table 34. Electriclty Purchases by the Distribution segment of Cooporative Borrowers, by 8tate, 1902 (Continued)

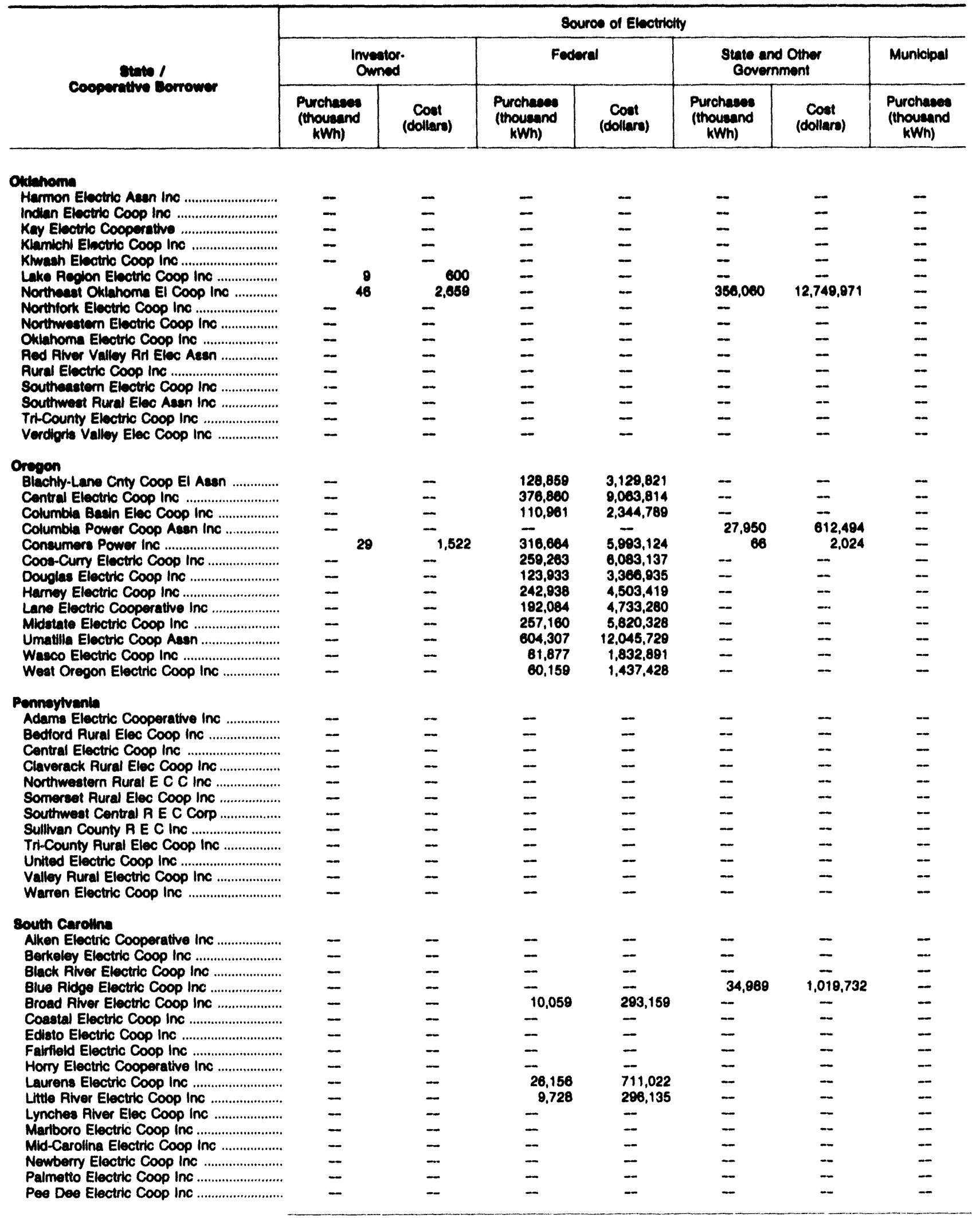

See noter $s$ and footnotes at end of table. 
Table 34. Electriclty Purchases by the Diatribution segment of Cooperative Borrowere, by State, 1992 (Continued)

\begin{tabular}{|c|c|c|c|c|c|c|c|}
\hline \multirow{3}{*}{$\begin{array}{l}\text { Etate I } \\
\text { cooperattve Borrower }\end{array}$} & \multicolumn{7}{|c|}{ Source of Electricty } \\
\hline & \multirow{2}{*}{ 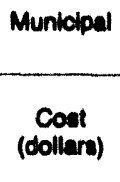 } & \multicolumn{2}{|c|}{ Cooperative } & \multicolumn{2}{|c|}{ Other' } & \multicolumn{2}{|c|}{ Total } \\
\hline & & $\begin{array}{l}\text { Purchasese } \\
\text { (thousand } \\
\text { kWh) }\end{array}$ & $\begin{array}{c}\text { Cont } \\
\text { (dollars) }\end{array}$ & $\begin{array}{l}\text { Purchasese } \\
\text { (thousand } \\
\text { kWh) }\end{array}$ & $\begin{array}{c}\text { Coof } \\
\text { (dollare) }\end{array}$ & $\begin{array}{l}\text { Purchases } \\
\text { (thoueand } \\
\text { kWh) }\end{array}$ & $\begin{array}{c}\text { Cont } \\
\text { (dollarn) }\end{array}$ \\
\hline 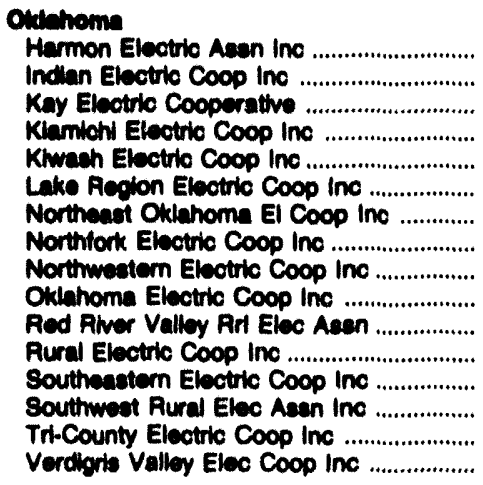 & $\begin{array}{l}\bar{z} \\
\bar{z} \\
\bar{z} \\
\bar{z} \\
\bar{z} \\
=\end{array}$ & $\begin{array}{r}44,893 \\
290,860 \\
111,349 \\
194,402 \\
73,140 \\
210,016 \\
12,052 \\
78,187 \\
200,312 \\
514,200 \\
176,582 \\
217,680 \\
116,041 \\
86,300 \\
230,466 \\
321,827\end{array}$ & 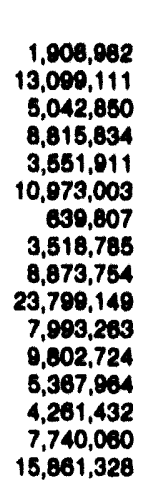 & $\begin{array}{l}\bar{z} \\
= \\
= \\
= \\
= \\
= \\
=\end{array}$ & $\begin{array}{l}\overline{-} \\
= \\
= \\
=187 \\
= \\
= \\
= \\
= \\
=\end{array}$ & $\begin{array}{r}44,603 \\
290,050 \\
111,349 \\
194,602 \\
73,146 \\
210,025 \\
308,904 \\
78,187 \\
200,312 \\
514,205 \\
176,682 \\
217,688 \\
118,041 \\
68,308 \\
230,468 \\
321,627\end{array}$ & 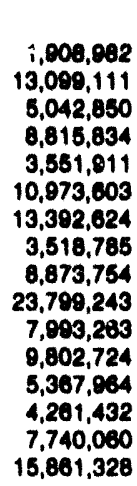 \\
\hline 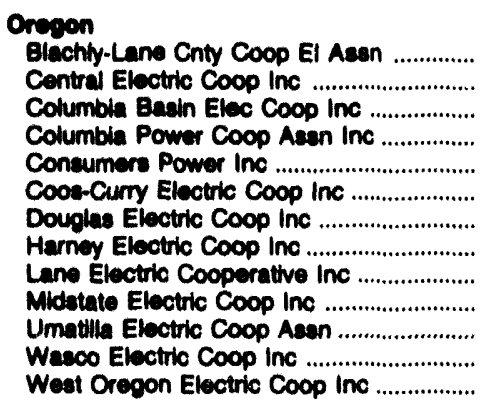 & $\begin{array}{l}\bar{z} \\
\bar{z} \\
\bar{z} \\
\bar{z} \\
\bar{z}\end{array}$ & $\begin{array}{l}= \\
= \\
= \\
= \\
= \\
=\end{array}$ & $\begin{array}{l}\bar{z} \\
\bar{z} \\
\bar{z} \\
\bar{z} \\
\bar{z} \\
\bar{m}\end{array}$ & $\begin{array}{l}\overline{-} \\
= \\
\overline{1} \\
\overline{13,590} \\
\overline{10,426} \\
= \\
= \\
=\end{array}$ & $\begin{array}{l}\overline{-} \\
\overline{-} \\
\overline{58,460} \\
\overline{3} \\
\overline{38,488} \\
\overline{-} \\
\overline{-}\end{array}$ & $\begin{array}{r}128,869 \\
376,860 \\
110,961 \\
27,960 \\
316,759 \\
272,859 \\
123,833 \\
242,938 \\
202,510 \\
257,160 \\
604,307 \\
81,877 \\
60,159\end{array}$ & $\begin{array}{r}3,129,821 \\
9,083,814 \\
2,344,789 \\
612,494 \\
5,998,670 \\
6,141,608 \\
3,366,035 \\
4,503,419 \\
4,771,788 \\
5,820,328 \\
12,046,729 \\
1,832,891 \\
1,437,428\end{array}$ \\
\hline 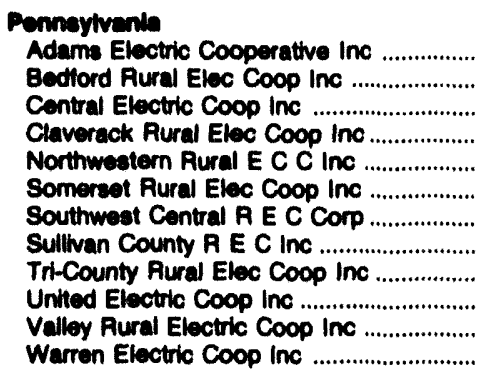 & $\begin{array}{l}\overline{ } \\
\bar{z} \\
\bar{z} \\
\bar{z} \\
\bar{z}\end{array}$ & $\begin{array}{r}317,550 \\
98,258 \\
200,452 \\
158,492 \\
192,071 \\
159,569 \\
271,446 \\
43,445 \\
125,238 \\
130,378 \\
191,561 \\
49,316\end{array}$ & $\begin{array}{r}19,736,565 \\
5,780,265 \\
12,049,401 \\
9,789,806 \\
11,338,075 \\
10,191,682 \\
17,325,682 \\
2,671,055 \\
8,127,036 \\
8,323,051 \\
11,815,626 \\
3,124,082\end{array}$ & $\begin{array}{l}\bar{z} \\
\bar{z} \\
\bar{z} \\
\bar{z} \\
\bar{z}\end{array}$ & $\begin{array}{l}\overline{-} \\
\bar{z} \\
\bar{z} \\
\bar{z} \\
\bar{z} \\
=\end{array}$ & $\begin{array}{r}317,550 \\
96,258 \\
200,452 \\
158,492 \\
192,071 \\
159,569 \\
271,446 \\
43,446 \\
125,238 \\
130,378 \\
191,561 \\
49,316\end{array}$ & $\begin{array}{r}19,736,555 \\
5,760,286 \\
12,049,401 \\
9,789,606 \\
11,338,875 \\
10,191,682 \\
17,325,582 \\
2,671,055 \\
8,127,036 \\
8,323,651 \\
11,615,626 \\
3,124,082\end{array}$ \\
\hline 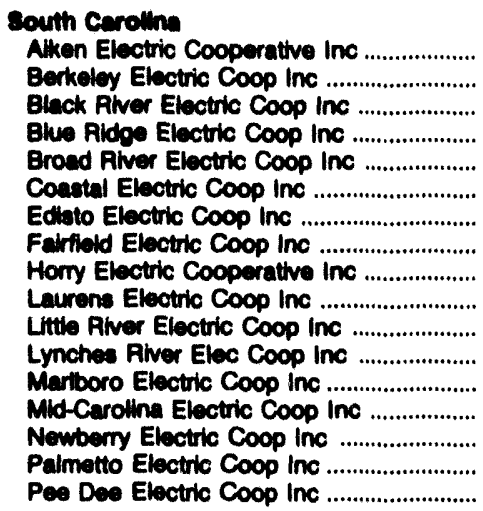 & $\begin{array}{l}\bar{z} \\
\bar{z} \\
\bar{z} \\
\bar{z} \\
\bar{z}\end{array}$ & $\begin{array}{l}484,833 \\
929,944 \\
397,317 \\
537,695 \\
178,780 \\
111,420 \\
208,906 \\
434,464 \\
455,890 \\
429,601 \\
114,680 \\
219,967 \\
432,612 \\
587,330 \\
170,380 \\
765,857 \\
574,007\end{array}$ & $\begin{array}{r}18,677,057 \\
38,791,661 \\
15,823,077 \\
29,727,704 \\
10,169,919 \\
4,571,429 \\
8,515,636 \\
16,081,255 \\
19,572,426 \\
24,082,503 \\
6,472,369 \\
8,734,411 \\
11,495,672 \\
24,591,802 \\
6,392,805 \\
30,054,482 \\
21,025,357\end{array}$ & $\begin{array}{l}\bar{z} \\
\bar{z} \\
\bar{z} \\
\bar{z} \\
\bar{z} \\
\bar{z}\end{array}$ & $\begin{array}{l}\bar{z} \\
\bar{z} \\
\bar{z} \\
\bar{z} \\
\bar{z} \\
= \\
= \\
=\end{array}$ & $\begin{array}{l}484,833 \\
929,844 \\
387,9417 \\
57,31,684 \\
188,839 \\
111,420 \\
208,906 \\
434,464 \\
455,896 \\
455,757 \\
124,408 \\
219,957 \\
432,612 \\
587,330 \\
170,380 \\
765,857 \\
574,007\end{array}$ & $\begin{array}{r}19,677,057 \\
38,791,601 \\
15,823,077 \\
30,747,436 \\
10,457,078 \\
4,571,429 \\
8,515,636 \\
16,081,255 \\
19,572,426 \\
24,793,525 \\
6,788,494 \\
8,734,411 \\
11,495,672 \\
24,591,802 \\
6,392,905 \\
30,054,482 \\
21,825,357\end{array}$ \\
\hline
\end{tabular}

See notes and tootnotes at end of table. 
Table 34. Electricity Purchases by the Dietribution Segment of Cooperative Borrowers, by State, 1982 (Continued)

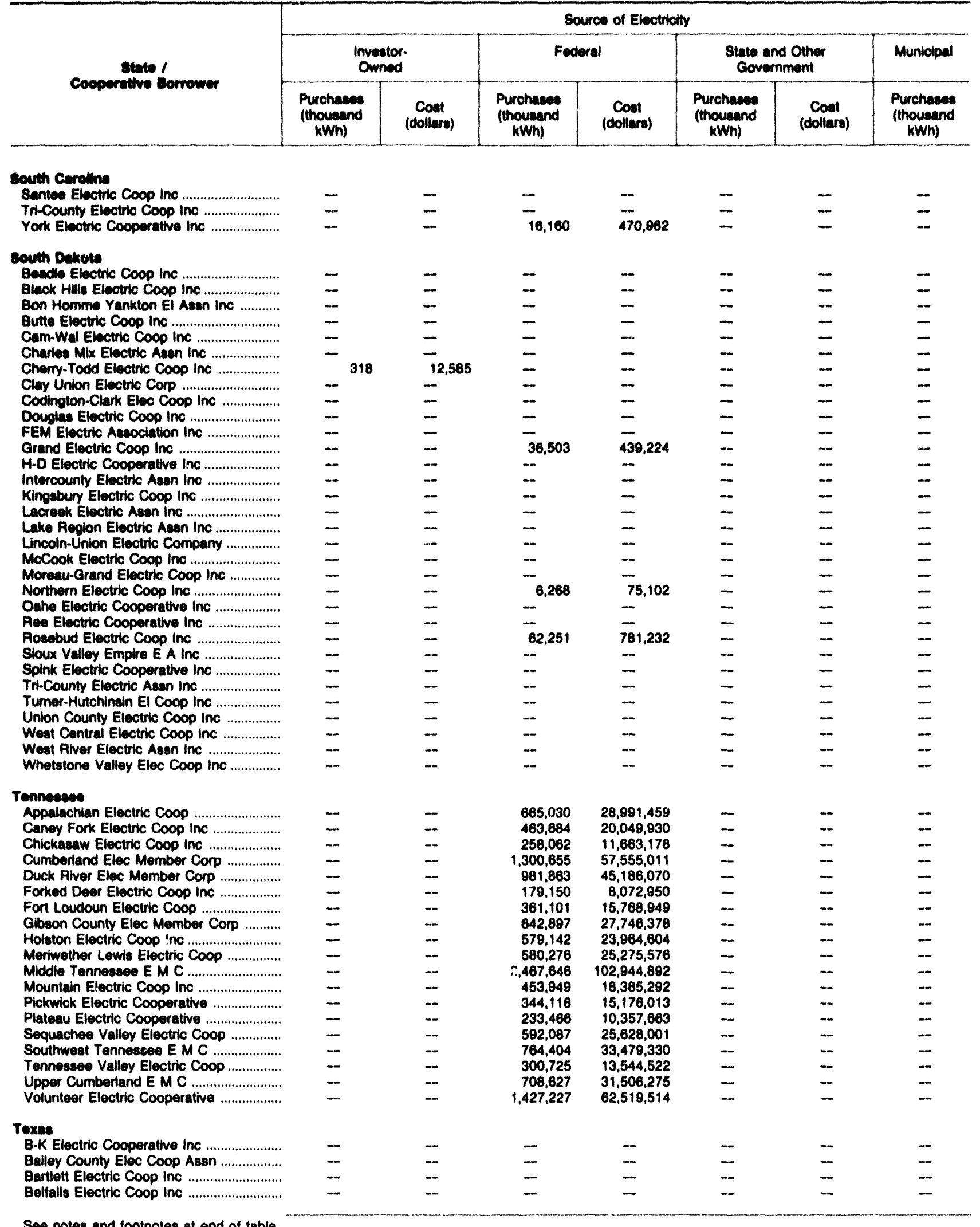

See notes and footnotes at end of table. 
Table 34. Electrleity Purchases by the Distribution Segment of Cooperative Borrowers, by State, 1992 (Continued)

\begin{tabular}{|c|c|c|c|c|c|c|c|}
\hline \multirow{3}{*}{$\begin{array}{l}\text { State I } \\
\text { Cooperattve Eorrower }\end{array}$} & \multicolumn{7}{|c|}{ Source of Electricity } \\
\hline & \multirow{2}{*}{$\begin{array}{c}\text { Municipal } \\
\text { Coat } \\
\text { (dollara) }\end{array}$} & \multicolumn{2}{|c|}{ Cooperative } & \multicolumn{2}{|c|}{ Other' } & \multicolumn{2}{|c|}{ Total } \\
\hline & & $\begin{array}{l}\text { Purchasese } \\
\text { (thousand } \\
\text { kWh) }\end{array}$ & $\begin{array}{c}\text { Cost } \\
\text { (dollars) }\end{array}$ & $\begin{array}{l}\text { Purchases } \\
\text { (thousand } \\
\text { kWh) }\end{array}$ & $\underset{\text { (dollars) }}{\text { Cost }}$ & $\begin{array}{l}\text { Purchases } \\
\text { (thousand } \\
\text { kWh) }\end{array}$ & $\begin{array}{c}\text { Cost } \\
\text { (dollarg) }\end{array}$ \\
\hline $\begin{array}{l}\text { Bowth Caroline } \\
\text { Santee Electric Coop inc......................... } \\
\text { Tri-County Electric Coop line .................... } \\
\text { York Electric Cooperative inc ................... }\end{array}$ & $\bar{z}$ & $\begin{array}{l}561,403 \\
197,258 \\
361,592\end{array}$ & $\begin{array}{r}23,050,657 \\
8,118,334 \\
20,284,635\end{array}$ & $=$ & $\overline{-}$ & $\begin{array}{l}561,403 \\
197,258 \\
377,752\end{array}$ & $\begin{array}{r}23,050,657 \\
8,118,334 \\
20,755,597\end{array}$ \\
\hline 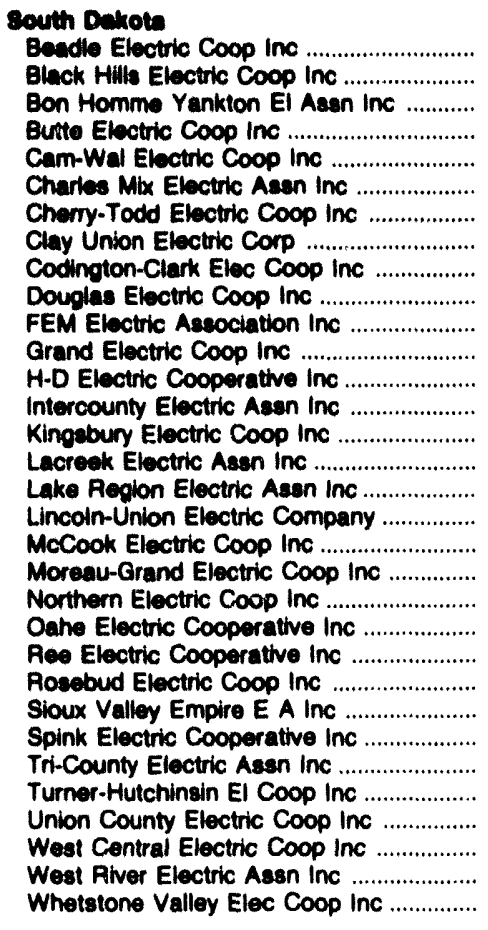 & 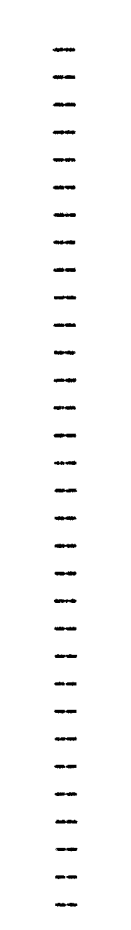 & $\begin{array}{r}34,814 \\
64,811 \\
58,253 \\
59,044 \\
38,697 \\
34,010 \\
71,402 \\
44,481 \\
61,825 \\
17,150 \\
40,463 \\
87,243 \\
52,171 \\
57,379 \\
19,733 \\
62,929 \\
55,147 \\
68,127 \\
25,562 \\
65,647 \\
84,994 \\
42,895 \\
23,552 \\
12,103 \\
227,177 \\
25,572 \\
54,177 \\
75,265 \\
17,737 \\
91,770 \\
129,484 \\
57,056\end{array}$ & $\begin{array}{r}1,488,617 \\
2,485,277 \\
2,405,168 \\
2,277,156 \\
1,661,108 \\
1,405,552 \\
2,890,179 \\
1,834,116 \\
2,492,632 \\
717,199 \\
1,633,671 \\
2,883,312 \\
2,162,140 \\
2,430,671 \\
790,830 \\
2,563,914 \\
2,221,606 \\
2,686,341 \\
1,085,525 \\
2,689,680 \\
3,338,421 \\
1,713,850 \\
1,001,988 \\
752,411 \\
9,454,107 \\
1,096,320 \\
2,206,785 \\
3,148,319 \\
730,863 \\
3,762,146 \\
5,174,926 \\
2,306,579\end{array}$ & $\begin{array}{l}- \\
-288 \\
= \\
= \\
= \\
= \\
= \\
= \\
- \\
= \\
= \\
= \\
- \\
= \\
= \\
= \\
= \\
= \\
= \\
= \\
= \\
= \\
-\end{array}$ & 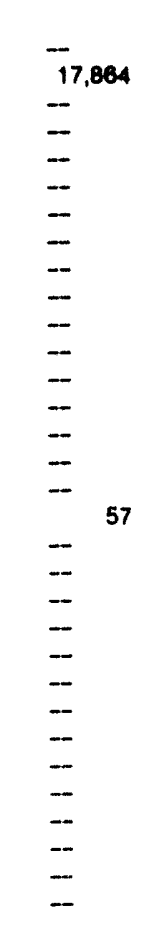 & $\begin{array}{r}34,814 \\
65,099 \\
58,253 \\
59,044 \\
38,697 \\
34,010 \\
71,720 \\
44,481 \\
61,825 \\
17,150 \\
40,483 \\
123,746 \\
52,171 \\
57,379 \\
19,733 \\
62,829 \\
55,147 \\
68,129 \\
25,562 \\
65,647 \\
91,262 \\
42,895 \\
23,552 \\
74,354 \\
227,177 \\
25,572 \\
54,177 \\
75,265 \\
17,737 \\
91,770 \\
129,484 \\
57,056\end{array}$ & $\begin{array}{r}1,489,617 \\
2,503,141 \\
2,405,160 \\
2,277,156 \\
1,661,108 \\
1,405,552 \\
2,902,764 \\
1,834,116 \\
2,492,632 \\
717,199 \\
1,633,671 \\
3,332,536 \\
2,162,140 \\
2,430,671 \\
790,830 \\
2,563,914 \\
2,221,606 \\
2,686,398 \\
1,085,525 \\
2,669,680 \\
3,41,523 \\
1,713,850 \\
1,001,988 \\
1,533,643 \\
9,454,107 \\
1,096,320 \\
2,208,785 \\
3,148,319 \\
730,663 \\
3,762,146 \\
5,174,926 \\
2,306,579\end{array}$ \\
\hline 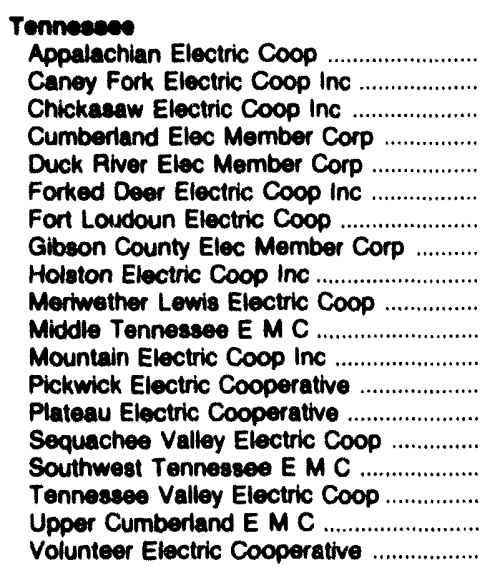 & $\begin{array}{l}\bar{z} \\
= \\
= \\
= \\
\bar{z} \\
= \\
= \\
= \\
= \\
\bar{z} \\
= \\
=\end{array}$ & $\begin{array}{l}\overline{-} \\
\overline{-} \\
\bar{z} \\
\overline{-} \\
\overline{-} \\
\overline{-} \\
\overline{-} \\
\overline{-} \\
\overline{-} \\
\overline{-} \\
\overline{-} \\
-\end{array}$ & $\begin{array}{l}= \\
= \\
= \\
= \\
= \\
= \\
= \\
= \\
= \\
= \\
= \\
= \\
- \\
- \\
-\end{array}$ & $\begin{array}{l}\overline{-} \\
\overline{-} \\
- \\
\overline{-} \\
\overline{-} \\
\overline{-} \\
\overline{-} \\
- \\
\overline{-} \\
\overline{-} \\
-\end{array}$ & 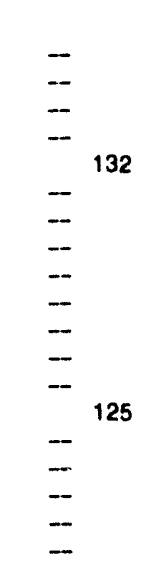 & $\begin{array}{r}665,030 \\
463,684 \\
258,062 \\
1,300,655 \\
981,867 \\
179,150 \\
361,101 \\
642,897 \\
579,142 \\
580,276 \\
2,467,646 \\
453,949 \\
344,118 \\
233,473 \\
592,087 \\
764,404 \\
300,725 \\
708,627 \\
1,427,227\end{array}$ & $\begin{array}{r}28,991,459 \\
20,049,930 \\
11,663,178 \\
57,555,011 \\
45,18,01,202 \\
8,072,950 \\
15,768,949 \\
27,746,378 \\
23,964,604 \\
25,275,576 \\
102,944,892 \\
18,385,292 \\
15,176,013 \\
10,357,788 \\
25,628,001 \\
33,478,330 \\
13,544,522 \\
31,508,275 \\
62,519,514\end{array}$ \\
\hline 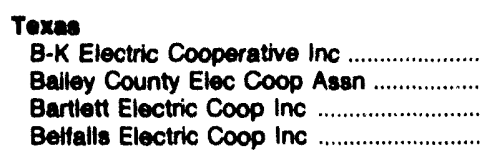 & $\bar{z}$ & $\begin{array}{r}89,857 \\
124,517 \\
73,234 \\
61,546\end{array}$ & $\begin{array}{l}3,303,488 \\
4,942,226 \\
3,070,420 \\
2,410,586\end{array}$ & $\bar{\Xi}$ & $\bar{z}$ & $\begin{array}{r}89,857 \\
124,517 \\
73,234 \\
61,546\end{array}$ & $\begin{array}{l}3,303,488 \\
4,942,226 \\
3,070,420 \\
2,410,586\end{array}$ \\
\hline
\end{tabular}

See notes and footnotes at end of table. 
Table 34. Electricity Purchases by the Diatribution Segment of Cooperative Borrowers, by State, 1992 (Continued)

\begin{tabular}{|c|c|c|c|c|c|c|c|}
\hline \multirow{3}{*}{$\begin{array}{c}\text { Sthte / } \\
\text { Coopertive Borromer }\end{array}$} & \multicolumn{7}{|c|}{ Source of Electricity } \\
\hline & \multicolumn{2}{|c|}{$\begin{array}{l}\text { Investor. } \\
\text { Owned }\end{array}$} & \multicolumn{2}{|c|}{ Federal } & \multicolumn{2}{|c|}{$\begin{array}{l}\text { State and Other } \\
\text { Government }\end{array}$} & \multirow{2}{*}{$\begin{array}{c}\text { Municipal } \\
\begin{array}{c}\text { Purchases } \\
\text { (thousand } \\
\text { kWh) }\end{array}\end{array}$} \\
\hline & $\begin{array}{l}\text { Purchases } \\
\text { (thousand } \\
\text { kWh) }\end{array}$ & $\begin{array}{c}\text { Cost } \\
\text { (dollars) }\end{array}$ & $\begin{array}{l}\text { Purchases } \\
\text { (thouseand } \\
\text { kWh) }\end{array}$ & $\begin{array}{c}\text { Cost } \\
\text { (dollars) }\end{array}$ & $\begin{array}{c}\text { Purchases } \\
\text { (thousand } \\
\text { kWh) }\end{array}$ & $\begin{array}{c}\text { Cost } \\
\text { (dollars) }\end{array}$ & \\
\hline
\end{tabular}

\section{Toxas}

Bowio-Caes Electric Coop Inc

Central Texas Elec Coop Inc ......................

Cherokee County Elec Coop Asan ...........

Coleman County Elec Coop inc.

Comenche County Elec Coop Asen

Concho Valley Elec Coop inc

Cooke County Elec Coop Asan

Deep East Texas Elec Coop Inc

Denton County Elec Coop Inc

Denvitt County Elec Coop inc

Dickens Electric Coop Inc

Erath County EI Coop Assn Inc

Fannin County Electric Coop

Fayette Electric Coop inc

FEC Electric Cooperative

Gate City Electric Coop Inc

Grayson-Collin Elec Coop Inc

.

Hamilton County Elec Coop Assn ............

Hill County Electric Coop.

Houston County Elec Coop Inc ..................

Hunt-Collin Electric Coop inc

J-A-C Electulo Cooperathe Inc ...

Jackson Electric Coop Inc...

Jasper-Newton Elec CoOp inc

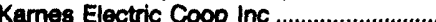

Keufman County Elec Coop inc

Kimble Electric Coop Inc

Elec Coop Assn

Lighthouse Electric Coop inc

Lyntegar Electric Coop Inc

Electric Coop inc

McLennan County Elec Coop Inc ..............

Medina Electric Coop inc

Mid-South Electric Coop Assn

Midwest Electric Coop inc

Nevarro County Eloc Coop ind

Navasota Valley Elec Coop Inc

New Era Electric Coop Inc

North Plains Electric Coop Inc

Nueces Electric Coop Inc

Panola-Harrieon Elec CoOp In

Rio Grande Electric Coop Inc

Rita Blanca Electric Coop Inc

Rusk County Electric Coop inc.

Sam Houston Electric Coop Inc

San Patricio Electric Coop inc

South Plains Electric Coop Inc

Southwest Texas Elec Coop Inc

Stamford Electric Coop Inc

Swisher Electric CoOp inc

Taylor Eloctric Coop inc ...

Tri-County Electric Coop inc

Upahur Rural Elec Coop Corp

Victoria County Elec Coop Co

Wharton County Elec Coop inc

Wise Electric Cooperative Inc

Wood County Electric Coop inc

See notes and footnotes at end of table.

\begin{tabular}{|c|c|c|}
\hline - & - & - \\
\hline - & $\overline{-}$ & - \\
\hline 92,520 & $3,346,878$ & $\overline{-}$ \\
\hline- & - & - \\
\hline 134,843 & $4,906,182$ & - \\
\hline $\bar{z}$ & - & - \\
\hline- & $=$ & $\bar{z}$ \\
\hline 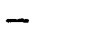 & - & $\underline{-}$ \\
\hline- & - & - \\
\hline - & - & - \\
\hline - & - & - \\
\hline$\overline{-}$ & $\overline{-}$ & - \\
\hline - & - & $\overline{-}$ \\
\hline - & - & - \\
\hline 24,442 & 964,351 & - \\
\hline- & - & - \\
\hline$=$ & $\bar{z}$ & $\bar{z}$ \\
\hline 33,623 & $1,84 \theta, 858$ & - \\
\hline - & - & - \\
\hline 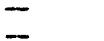 & - & $\overline{-}$ \\
\hline- & $\overline{-}$ & $\overline{-}$ \\
\hline - & -- & - \\
\hline- & $\bar{c}$ & - \\
\hline 13,288 & 655,122 & - \\
\hline $\bar{z}$ & $\overline{-}$ & $\bar{z}$ \\
\hline $\begin{array}{r}12,346 \\
368,305\end{array}$ & 496,816 & $\overline{-}$ \\
\hline $\begin{array}{l}368,305 \\
488,454\end{array}$ & $\begin{array}{l}13,773,451 \\
27,563,041\end{array}$ & $\overline{-}$ \\
\hline 25,328 & $1,038,799$ & - \\
\hline$\overline{57,948}$ & $\overline{0}$ & - \\
\hline- & $2,601,620$ & $\bar{z}$ \\
\hline 115,286 & $4,681,345$ & - \\
\hline - & 一 & - \\
\hline- & - & - \\
\hline - & - & - \\
\hline - & - & - \\
\hline$\overline{111.982}$ & $\overline{007}$ & - \\
\hline- & ${ }^{4,008,004}$ & $\bar{I}$ \\
\hline & - & - \\
\hline- & - & - \\
\hline$\overline{-}$ & - & $\overline{-}$ \\
\hline $\begin{array}{r}180,420 \\
66,992\end{array}$ & $\begin{array}{l}6,355,011 \\
2,489,950\end{array}$ & $\bar{z}$ \\
\hline- & - & - \\
\hline 179,305 & $6,855,784$ & $\overline{-}$ \\
\hline - & $\overline{-}$ & $\overline{-}$ \\
\hline - & - & - \\
\hline- & - & - \\
\hline 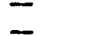 & - & \\
\hline
\end{tabular}

\begin{tabular}{|c|c|c|}
\hline$\overline{237,512}$ & $9, \overline{993}, 818$ & $\overline{-}$ \\
\hline - & - & - \\
\hline - & - & - \\
\hline- & - & - \\
\hline - & - & - \\
\hline- & - & - \\
\hline- & - & - \\
\hline- & - & - \\
\hline 68,045 & $2,609,387$ & - \\
\hline- & - & - \\
\hline- & - & - \\
\hline- & - & - \\
\hline 141,333 & $5,598,385$ & - \\
\hline- & - & - \\
\hline- & - & - \\
\hline- & - & - \\
\hline- & - & - \\
\hline- & - & - \\
\hline- & - & - \\
\hline- & - & - \\
\hline - & - & - \\
\hline- & - & - \\
\hline- & - & - \\
\hline- & - & - \\
\hline- & - & - \\
\hline- & - & - \\
\hline- & - & - \\
\hline 26,339 & $1,063,616$ & - \\
\hline- & - & - \\
\hline- & - & - \\
\hline- & - & - \\
\hline- & - & - \\
\hline$\overline{38}, 005$ & $\overline{1377552}$ & $\overline{-}$ \\
\hline - & - & - \\
\hline- & - & - \\
\hline- & - & - \\
\hline- & - & - \\
\hline- & - & - \\
\hline- & - & - \\
\hline- & - & - \\
\hline- & - & - \\
\hline- & - & - \\
\hline- & - & - \\
\hline- & - & - \\
\hline- & - & - \\
\hline- & - & - \\
\hline- & - & - \\
\hline- & - & - \\
\hline- & - & - \\
\hline- & - & - \\
\hline- & - & - \\
\hline- & - & - \\
\hline- & - & - \\
\hline- & - & - \\
\hline- & - & - \\
\hline- & - & - \\
\hline- & - & - \\
\hline- & $\overline{-}$ & - \\
\hline- & - & \\
\hline
\end{tabular}


Table 34. Electricity Purcisses by the Distribution Segment of Cooperative Borrowers, by State, 1992 (Cointinued)

\begin{tabular}{|c|c|c|c|c|c|c|c|}
\hline \multirow{3}{*}{$\begin{array}{c}\text { State I } \\
\text { Cooperattve Borrower }\end{array}$} & \multicolumn{7}{|c|}{ Source of Electricity } \\
\hline & \multirow{2}{*}{$\frac{\text { Municipal }}{\begin{array}{c}\text { Cost } \\
\text { (dollars) }\end{array}}$} & \multicolumn{2}{|c|}{ Cooperative } & \multicolumn{2}{|c|}{ Other' } & \multicolumn{2}{|c|}{ Total } \\
\hline & & $\begin{array}{c}\text { Purchases } \\
\text { (thousand } \\
\text { kWh) }\end{array}$ & $\begin{array}{c}\text { Cost } \\
\text { (dollars) }\end{array}$ & $\begin{array}{c}\text { Purchases } \\
\text { (thousand } \\
\text { kWh) }\end{array}$ & $\begin{array}{c}\text { Cost } \\
\text { (dollars) }\end{array}$ & $\begin{array}{l}\text { Purchases } \\
\text { (thousand } \\
\text { kWh) }\end{array}$ & $\begin{array}{c}\text { Cost } \\
\text { (dollars) }\end{array}$ \\
\hline \multicolumn{8}{|l|}{ Toxes: } \\
\hline $\begin{array}{l}\text { Bowie-Cass Electric Coop Inc .................... } \\
\text { Central Texas Elec Coop Inc }\end{array}$ & - & 454,537 & $14,879,575$ & - & - & 454,537 & $14,879,575$ \\
\hline Central Texas Elec Coop Inc ........................ & - & - & - & - & - & 237,512 & $9,893,818$ \\
\hline Cherokee County Elec Coop Assn ............ & - & 196,661 & $\mathbf{9 , 7 0 1 , 2 9 7}$ & - & - & 196,661 & $8,701,287$ \\
\hline & - & - & - & - & - & 92,520 & $3,346,878$ \\
\hline Comanche County Elec Coop Assn .......... & - & 152,237 & $5,875,833$ & - & - & 152,237 & $5,875,833$ \\
\hline Concho Valley Elec Coop Inc ......................... & - & - & - & - & - & 134,843 & $4,906,182$ \\
\hline Cooke County Elec Coop Assn ..................... & - & 243,454 & $9,200,646$ & tan & 181 & 243,462 & $9,200,827$ \\
\hline Deep East Texas Elec Coop Inc ................. & - & 548,547 & $24,526,727$ & - & - & 549,547 & $24,526,727$ \\
\hline Denton County Elec Coop ine ....................... & - & 530,227 & $22,937,462$ & - & -- & 530,227 & $22,937,462$ \\
\hline 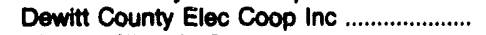 & - & - & - & - & - & 68,045 & $2,609,387$ \\
\hline 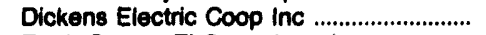 & - & 411,100 & $13,474,309$ & - & - & 411,100 & $13,474,309$ \\
\hline Erath County El Coop Assn Inc .................. & - & 224,529 & $9,077,646$ & - & - & 224,529 & $9,077,646$ \\
\hline & - & 63,434 & $2,989,272$ & - & - & 63,434 & $2,989,272$ \\
\hline 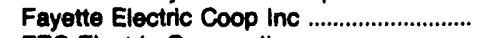 & - & - & - & - & - & 141,333 & $5,588,385$ \\
\hline FEC Electric Cooperative ............................ & - & 366,404 & $17,006,419$ & - & -- & 366.404 & $17,006,419$ \\
\hline Gate City Eloctric Coop Inc ............................. & - & 34,335 & $1,245,712$ & - & - & 34,335 & $1,245,712$ \\
\hline Grayson-Collin Elec Coop Inc ........................ & - & 242,873 & $11,431,846$ & - & - & 242,873 & $11,431,846$ \\
\hline 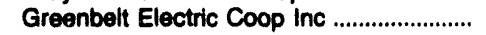 & - & 21,442 & 765,109 & - & - & 45,884 & $1,729,460$ \\
\hline Hamilton County Elec Coop Assn ............. & - & 105,443 & $4,288,176$ & - & - & 105,443 & $4,288,176$ \\
\hline 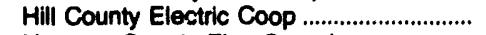 & - & 195,768 & $8,115,829$ & - & - & 195,768 & $8,115,829$ \\
\hline Houston County Elec Coop Inc ...................... & - & 196,162 & $9,400,796$ & - & - & 196,162 & $9,400,796$ \\
\hline Hunt-Collin Electric Coop Inc ....................... & - & - & - & - & - & 33,623 & $1,849,858$ \\
\hline J-A-C Electric Cooperative Inc ....................... & - & 88,070 & $3,311,740$ & - & - & 88,070 & $3,311,740$ \\
\hline Jackson Electric Coop Inc ................................. & - & 144,212 & $7,625,750$ & - & - & 144,212 & $7,625,750$ \\
\hline Jasper-Newton Elec Coop Inc .................... & - & 279,870 & $12,587,412$ & - & - & 279,870 & $12,587,412$ \\
\hline Johnson County Elec Coop Assn ............... & -- & 501,190 & $21,312,546$ & - & - & 501,190 & $21,312,546$ \\
\hline 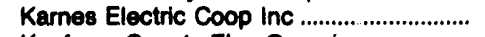 & - & 155,892 & $7,867,843$ & - & - & 155,802 & $7,867,843$ \\
\hline Kaufman County Elec Coop inc .................... & - & 316,573 & $14,489,391$ & - & - & 316,573 & $14,488,391$ \\
\hline 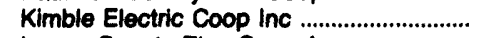 & - & - & - & - & - & 39,627 & $1,718,738$ \\
\hline Lamar County Elec Coop Assn ...................... & - & 96,418 & $4,622,923$ & - & - & 96,418 & $4,622,923$ \\
\hline Lamb County Electric Coop Inc ....................... & - & 175,980 & $6,870,706$ & - & - & 175,880 & $6,870,706$ \\
\hline 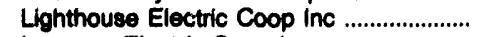 & - & 130,752 & $5,442,327$ & - & -- & 143,098 & $5,939,143$ \\
\hline 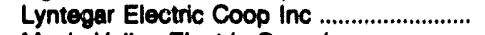 & - & - & - & - & - & 368,305 & $13,773,451$ \\
\hline Magic Valley Electric Coop Inc .................... & - & - & - & - & - & 498,454 & $27,563,041$ \\
\hline 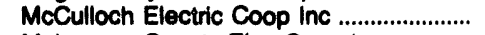 & - & - & - & - & - & 63,333 & $2,416,351$ \\
\hline MeLennan County Elec Coop Inc ................ & - & 139,541 & $5,758,765$ & - & - & 138,541 & $5,758,765$ \\
\hline 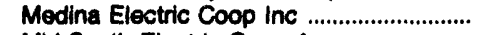 & - & - & - & - & - & 57,948 & $2,661,625$ \\
\hline Mid-South Electric Coop Assn ..................... & - & 248,129 & $9,894,294$ & - & - & 248,129 & $9,894,294$ \\
\hline 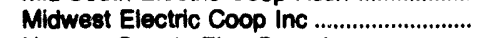 & - & 36,834 & $1,201,343$ & - & - & 152,120 & $5,882,688$ \\
\hline Navarro County Eloc Coop Inc ........................ & - & 198,689 & $7,802,573$ & - & - & 108,689 & $7,802,573$ \\
\hline Navasota Valley Elec Coop Inc ................... & - & 249,638 & $9,631,799$ & - & - & 249,638 & $9,631,799$ \\
\hline New Era Electric Coop Inc ............................ & - & 252,380 & $11,660,973$ & - & 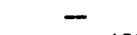 & 252,380 & $11,680,873$ \\
\hline North Plains Electric Coop Inc ........................ & - & 135,367 & $4,655,295$ & & 18 & 135,374 & $4,655,475$ \\
\hline 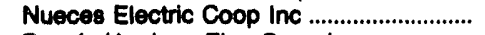 & - & 129,099 & $6,581,607$ & - & - & 129,099 & $6,581,607$ \\
\hline Panola-Harrison Elec Coop Inc ................. & - & 225,116 & $7,487,343$ & - & - & 225,116 & $7,487,343$ \\
\hline Rio Grande Electric Coop Inc ....................... & - & 18,408 & 897,087 & - & - & 131,390 & $5,764,671$ \\
\hline Pita Blanca Electric Coop Inc ......................... & - & 120,467 & $4,240,617$ & - & - & 120,467 & $4,240,617$ \\
\hline 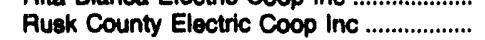 & - & 251,838 & $8,510,783$ & - & - & 251,838 & $8,510,763$ \\
\hline Sam Houston Electric Coop Inc ................... & - & 690,340 & $34,756,059$ & -- & - & 690,340 & $34,756,059$ \\
\hline San Patricio Electric Coop Inc .................... & - & 123,233 & $6,168,624$ & - & -- & 123,233 & $6,168,624$ \\
\hline South Plains Electric Coop Inc ...................... & - & 348,640 & $13,317,066$ & - & - & 349,640 & $13,317,066$ \\
\hline Southwest Texas Elec Coop Inc ............... & - & - & - & - & - & 180,420 & $6,355,011$ \\
\hline 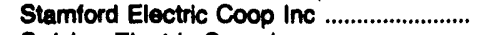 & - & - & - & - & - & 86,892 & $2,489,850$ \\
\hline Swiaher Electric Coop Inc ............................ & - & 141,234 & $5,843,380$ & - & - & 141,234 & $5,843,390$ \\
\hline 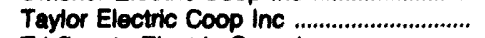 & - & - & - & - & - & 179,305 & $6,855,784$ \\
\hline Tri-County Electric Coop Inc .......................... & - & 635,033 & $25,943,298$ & - & - & 635.033 & $25,943,298$ \\
\hline Upshur Rural Elec Coop Corp ....................... & - & 508,847 & $16,187,301$ & - & - & 508,847 & $16,187,301$ \\
\hline Victoria County Elec Coop Co ......................... & - & 221,643 & $11,735,223$ & - & - & 221,643 & $11,735,223$ \\
\hline Wharton County Elec Coop Inc ..................... & - & 111,088 & $5,837,686$ & - & - & 111,098 & $5,637,686$ \\
\hline Wise Electric Cooperative Inc ....................... & - & 168,023 & $6,887,773$ & & 20 & 168,032 & $6,887,975$ \\
\hline Wood County Electric Coop Inc ..................... & & 351,986 & $12,786,142$ & - & - & 351,966 & $12,786,142$ \\
\hline
\end{tabular}

See notes and footnotes at end of table. 
Table 34. Electricity Purchases by the Distribution Segment of Cooperative Borrowers, by State, 1992 (Continued)

\begin{tabular}{|c|c|c|c|c|c|c|c|}
\hline \multirow{3}{*}{$\begin{array}{l}\text { State / } \\
\text { Cooporntive Borrower }\end{array}$} & \multicolumn{7}{|c|}{ Source of Electricity } \\
\hline & \multicolumn{2}{|c|}{$\begin{array}{l}\text { Investor- } \\
\text { Owned }\end{array}$} & \multicolumn{2}{|c|}{ Federal } & \multicolumn{2}{|c|}{$\begin{array}{l}\text { State and Other } \\
\text { Government }\end{array}$} & \multirow{2}{*}{$\begin{array}{c}\text { Municipal } \\
\begin{array}{c}\text { Purchases } \\
\text { (thousand } \\
\text { kWh) }\end{array}\end{array}$} \\
\hline & $\begin{array}{c}\text { Purchases } \\
\text { (thousand } \\
\text { kWh) }\end{array}$ & $\begin{array}{c}\text { Cost } \\
\text { (dollars) }\end{array}$ & $\begin{array}{c}\text { Purchases } \\
\text { (thousand } \\
\text { kWh) }\end{array}$ & $\begin{array}{c}\text { Cost } \\
\text { (dollars) }\end{array}$ & $\begin{array}{c}\text { Purchases } \\
\text { (thousand } \\
\text { kWh) }\end{array}$ & $\begin{array}{c}\text { Cost } \\
\text { (dollars) }\end{array}$ & \\
\hline \multicolumn{8}{|l|}{ Vtah } \\
\hline 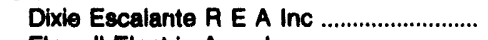 & - & - & - & - & 17,278 & 769,520 & - \\
\hline Flowell Electric Assn Inc ............................. & - & - & 333 & 6,997 & - & - & - \\
\hline Garkane Power Association Inc ................. & - & - & $-\overline{0}$ & Fin & - & - & -- \\
\hline Moon Lake Electric Assn Inc ....................... & - & - & 237,097 & $4,479,530$ & - & - & -- \\
\hline \multicolumn{8}{|l|}{ Vermont } \\
\hline $\begin{array}{l}\text { Vermont Electric Coop Inc ............................ } \\
\text { Washington Electric Coop Inc ................... }\end{array}$ & $\begin{array}{l}12,229 \\
21,819\end{array}$ & $\begin{array}{r}702,974 \\
1,052,380\end{array}$ & - & - & $\begin{array}{l}20,533 \\
16,022\end{array}$ & $\begin{array}{l}263,659 \\
339,050\end{array}$ & 3,373 \\
\hline \multicolumn{8}{|l|}{ Virginia } \\
\hline A \& N Electric Cooperative ......................... & - & - & - & - & - & -- & - \\
\hline BARC Electric Cooperative Inc ..................... & - & - & 8,959 & 179,961 & - & - & - \\
\hline Central Virginia Electric Coop ..................... & 362,979 & $17,302,633$ & 19,058 & 511,832 & - & - & -- \\
\hline Communinty Electric Cooperative .................. & $-\overline{c o n a n}$ & - & 10,133 & 200,716 & - & - & -- \\
\hline Craig-Botetourt Electric Coop ....................... & 54,608 & $2,113,340$ & 4,053 & 78,922 & $-\infty$ & - & - \\
\hline Mecklenburg Electric Coop ......................... & - & - & 27,174 & 729,792 & -- & - & $-\infty$ \\
\hline Northern Neck Elec Coop Inc ..................... & - & - & 9,448 & 253,729 & -- & - & - \\
\hline Northern Virginia Elec Coop ....................... & - & - & 7,828 & 168,295 & - & - & -- \\
\hline Powell Valley Electric Coop ......................... & - & - & 400,377 & $16,558,876$ & -- & - & - \\
\hline Prince George Electric Coop ....................... & - & - & 6,060 & 133,863 & -- & - & - \\
\hline Rappahannock Electric Coop ...................... & - & - & 53,722 & $1,276,271$ & -- & -- & - \\
\hline Shenandoah Valley Elec Coop ................... & - & - & 23,806 & 639,340 & -- & - & - \\
\hline Southside Electric Coop Inc ......................... & - & - & 34,913 & 937,652 & - & - & - \\
\hline \multicolumn{8}{|l|}{ Washington } \\
\hline Benton Rural Electric Assn .......................... & - & -- & 318,932 & $5,992,134$ & - & - & - \\
\hline Big Bend Electric Coop Inc ........................ & - & -- & 401,871 & $7,651,708$ & -- & -- & -- \\
\hline Columbia Rural Elec Assn Inc .................... & -- & - & 209,876 & $3,897,216$ & - & - & - \\
\hline Lincoln Electric Coop Inc ............................. & - & -- & 105,914 & $2,316,899$ & -- & - & - \\
\hline Nespelem Valley Elec Coop Inc .................. & - & -- & 37,815 & 902,131 & -- & - & - \\
\hline Okanogan County Elec Coop Inc ............... & - & - & 35,026 & 822,985 & -- & -- & - \\
\hline Orcas Power \& Light Company .................. & -- & - & 137,771 & $3,250,569$ & - & -- & - \\
\hline Tanner Electric Cooperative ........................ & - & - & 40,277 & 951,619 & - & - & - \\
\hline \multicolumn{8}{|l|}{ West Virginia } \\
\hline Harrison Rural Elec Assn Inc ....................... & 42,684 & $1,534,887$ & - & - & -- & - & - \\
\hline \multicolumn{8}{|l|}{ Wisconsin } \\
\hline Adams-Columbia Electric Coop ................... & 298,824 & $9,640,986$ & - & -- & - & - & -- \\
\hline Barron Electric Cooperative ...................... & - & - & - & - & - & - & - \\
\hline 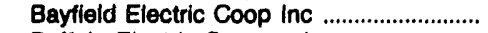 & - & - & -- & - & - & - & - \\
\hline Buffalo Electric Cooperative ....................... & - & $\bar{n}-$ & -- & - & - & - & - \\
\hline Central Wisconsin Elec Coop ..................... & 69,257 & $2,221,712$ & -- & - & - & - & - \\
\hline Chippewa Valley Electric Coop ................... & - & -- & - & -- & - & -- & - \\
\hline Clark Electric Cooperative ........................... & - & - & -- & - & - & - & - \\
\hline Crawford Electric Cooperative .................... & - & - & -- & - & - & -- & -- \\
\hline Dunn County Electric Coop ........................ & - & - & -- & - & $\cdots$ & - & - \\
\hline Eau Claire Electric Coop ............................. & - & -- & -- & - & - & - & - \\
\hline Grant-Lafayette Electric Coop ................... & - & - & - & - & - & - & - \\
\hline Head of The Lakes Elec Coop .................. & - & - & -- & - & - & - & - \\
\hline Jackson Electric Coop Inc ......................... & $\rightarrow$ & - & -- & -- & - & - & - \\
\hline Jump River Electric Coop Inc ..................... & - & - & - & - & - & -- & - \\
\hline Oakdale Electric Cooperative ....................... & $\overline{70}$ & $\overline{-\infty}$ & - & - & - & - & - \\
\hline Oconto Electric Cooperative ...................... & 76,418 & $2,697,600$ & - & - & - & -- & - \\
\hline Pierce-Pepin Electric Coop ........................ & - & - & - & - & - & - & - \\
\hline Polk-Burnett Electric Coop ......................... & - & - & - & - & - & - & - \\
\hline 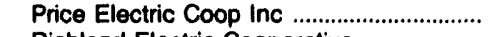 & - & - & -- & - & - & - & - \\
\hline Richland Electric Cooperative ................... & - & $-\overline{-}$ & -- & - & - & - & - \\
\hline Rock County Electric Coop Assn ............... & 61,868 & $2,055,317$ & - & -- & - & ... & -- \\
\hline St Croix County Electric Coop .................. & - & - & - & - & - & -- & - \\
\hline Taylor Electric Cooperative .......................... & $\sim$ & - & - & - & $-\infty$ & - & - \\
\hline Trempealeau Electric Coop ........................ & - & - & - & - & -- & - & - \\
\hline Vernon Electric Cooperative ..................... & - & $-\overline{-}$ & - & - & -- & - & - \\
\hline Washington Island EI Coop Inc ................. & 6,962 & 282,654 & -- & -- & - & - & - \\
\hline
\end{tabular}

See notes and footnotes at end of table. 
Table 34. Electricity Purchases by the Distribution Segment of Cooperative Borrowers, by State, 1992 (Continued)

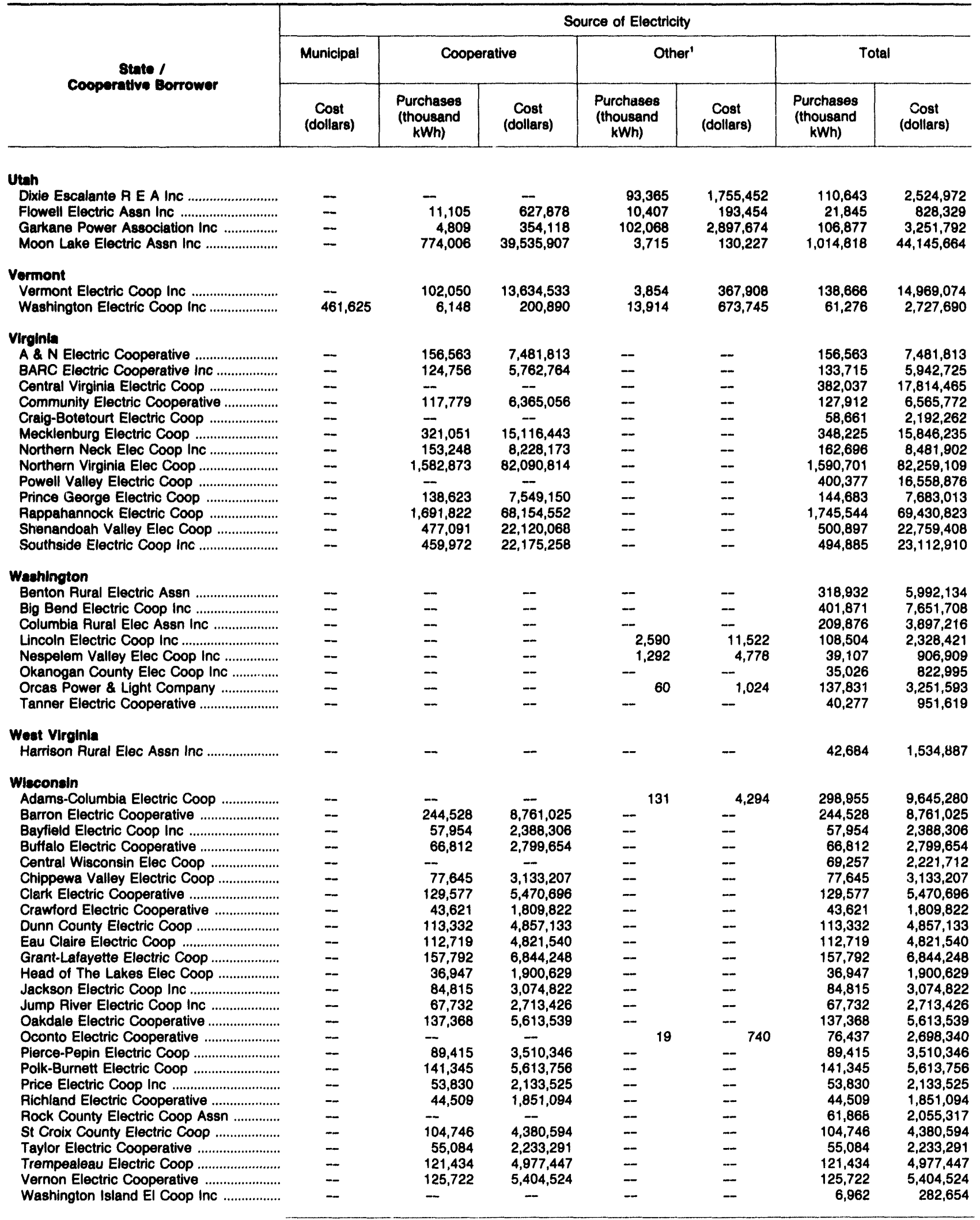

See notes and footnotes at end of table. 
Table 34. Electricity Purchases by the Distribution Segment of Cooperatlve Borrowers, by State, 1992 (Continued)

\begin{tabular}{|c|c|c|c|c|c|c|c|}
\hline \multirow{3}{*}{$\begin{array}{c}\text { State / } \\
\text { Cooporatlve Borrower }\end{array}$} & \multicolumn{7}{|c|}{ Source of Electricity } \\
\hline & \multicolumn{2}{|c|}{$\begin{array}{l}\text { Investor- } \\
\text { Owned }\end{array}$} & \multicolumn{2}{|c|}{ Federal } & \multicolumn{2}{|c|}{$\begin{array}{l}\text { State and Other } \\
\text { Government }\end{array}$} & \multirow{2}{*}{$\begin{array}{c}\text { Municipal } \\
\begin{array}{c}\text { Purchases } \\
\text { (thousand } \\
\text { kWh) }\end{array}\end{array}$} \\
\hline & $\begin{array}{c}\text { Purchases } \\
\text { (thousand } \\
\text { kWh) }\end{array}$ & $\begin{array}{c}\text { Cost } \\
\text { (dollars) }\end{array}$ & $\begin{array}{c}\text { Purchases } \\
\text { (thousand } \\
\text { kWh) }\end{array}$ & $\begin{array}{c}\text { Cost } \\
\text { (dollars) }\end{array}$ & $\begin{array}{c}\text { Purchases } \\
\text { (thousand } \\
\text { kWh) }\end{array}$ & $\begin{array}{c}\text { Cost } \\
\text { (dollars) }\end{array}$ & \\
\hline \multicolumn{8}{|l|}{ Wyoming } \\
\hline 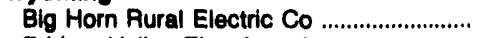 & - & -- & - & - & -- & - & - \\
\hline Bridger Valley Elec Assn inc .......................... & - & - & 39,411 & 694,289 & - & - & - \\
\hline Carbon Power \& Light Inc .......................... & - & - & - & $-\infty$ & - & - & - \\
\hline Garland Light \& Power Company .............. & -- & - & - & - & -- & - & - \\
\hline Hot Springs Rural EI Assn Inc ................... & -- & - & - & - & - & - & - \\
\hline 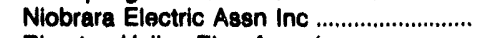 & -- & - & - & - & - & - & - \\
\hline Riverton Valley Elec Assn inc ....................... & -- & - & - & - & - & - & - \\
\hline Rural Electric Company ................................. & - & - & - & - & - & - & - \\
\hline Sheridan-Johnson Arl Elec Assn .............. & - & - & - & - & - & - & - \\
\hline Tri-County Electric Assn Inc ....................... & $\rightarrow$ & - & - & - & - & - & - \\
\hline Wheatland Rural Elec Assn Inc ................ & - & - & - & - & - & - & - \\
\hline Wyrulec Company & - & - & - & - & - & - & - \\
\hline
\end{tabular}

\begin{tabular}{|c|c|c|c|c|c|c|}
\hline \multicolumn{2}{|c|}{ Source of Electricity } \\
\hline Municipal & \multicolumn{2}{|c|}{ Cooperative } & \multicolumn{2}{|c|}{ Other' } & Total \\
\hline $\begin{array}{c}\text { Cost } \\
\text { (dollars) }\end{array}$ & $\begin{array}{c}\text { Purchases } \\
\text { (thousand } \\
\mathrm{kWh} \text { ) }\end{array}$ & $\begin{array}{c}\text { Cost } \\
\text { (dollars) }\end{array}$ & $\begin{array}{c}\text { Purchases } \\
\text { (thousand } \\
\mathrm{kWh}\end{array}$ & $\begin{array}{c}\text { Cost } \\
\text { (dollars) }\end{array}$ & $\begin{array}{c}\text { Purchases } \\
\text { (thousand } \\
\mathrm{kWh})\end{array}$ & $\begin{array}{c}\text { Cost } \\
\text { (dollars) }\end{array}$ \\
\hline
\end{tabular}

Wyoming

Big Horn Rural Electric Co

Bridger Valley Elec Assn Inc

Carbon Power \& Light Inc....

Garland Light \& Power Company

Hot Springs Rural El Assn Inc

Niobrara Electric Assn Inc...

Piverton Valley Elec Assn Inc.

Rural Electric Company

Tri-County Electric Asen Inc

Wheattand Rural Elec Asen Inc

Wyrulec Company

$\begin{array}{lr}-- & 66,546 \\ -- & 40,946 \\ -- & 82,213 \\ -- & 15,728 \\ - & 357,991 \\ -- & 54,207 \\ -- & 255,647 \\ -- & 162,963 \\ -- & 75,686 \\ - & 1,058,433 \\ - & 86,751 \\ - & 131,254\end{array}$

$2,424,602$
$2,299,314$
$3,275,107$
673,467
$12,715,651$
$2,054,820$
$9,300,485$
$6,771,434$
$2,799,601$
$47,130,655$
$3,493,281$
$4,855,268$

$\begin{array}{ll} & 62 \\ - & \\ - & \\ - & \\ - & \\ - & \\ - & 7 \\ - & \\ - & \end{array}$

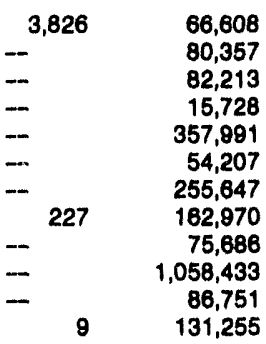

I Includes transactions with power pools, utilities in Canada and Mexico, and nonutilities.

- Not Applicable

Notes: - The Department of Agriculture under the Rural Electrification Act is authorized to provide Federal commitments for long-term financing and to guarantee non-Federal long-term loans to utilities supplying power to rural America. The utilities that recelved guarantees for their loans are referred to as borrowers by the REA. Nonborrowing cooperattves and other utilities that do not borrow from the REA are not required to file the REA-7 and REA-12 forms. - Distribution borrowers do not generate electricity; they purchase electricity from other utilities. $\bullet$ For identification purposes, the Cooperative Borrowers are listed in the State in which the administrative office is located. -Totals may not equal sum of components becaues of independent rounding.

Source: Rural Electrification Administration, REA Form 7, "Financial and Statistical Peport," REA Form 12a through 121, "Electric Power Supply Borrowers," Form 12c through 12g, "Electric Distribution Borrowers with Generating Facilities." 
Table 35 Electricity Purchases by Electrical Cooperatives Not Reported to the Rural Electriflcation Administration, by State, 1992

\begin{tabular}{|c|c|c|c|c|c|c|c|}
\hline \multirow{2}{*}{$\begin{array}{l}\text { State / } \\
\text { Utility }\end{array}$} & \multicolumn{7}{|c|}{$\begin{array}{l}\text { Source of Electricity } \\
\text { (Purchases in thousand } \mathrm{kWh} \text { ) }\end{array}$} \\
\hline & $\begin{array}{l}\text { Investor- } \\
\text { Owned }\end{array}$ & Federal & $\begin{array}{l}\text { State and } \\
\text { Other } \\
\text { Government }\end{array}$ & Municipal & Cooperative & Other' & Total \\
\hline Alacka & - & - & - & - & - & 1,314 & 1,314 \\
\hline $\begin{array}{l}\text { Artzona } \\
\text { Graham County Elec Coop Inc .............................. }\end{array}$ & - & - & - & - & 81,780 & - & 81,760 \\
\hline 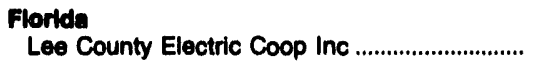 & - & - & - & - & $1,958,931$ & - & $1,958,831$ \\
\hline 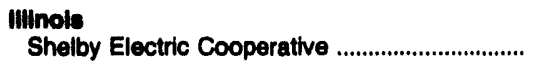 & - & - & - & - & 179,337 & - & 179,337 \\
\hline 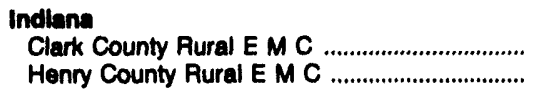 & $\overline{-}$ & $\overline{-}$ & $\overline{-}$ & - & $\begin{array}{l}191,241 \\
135,145\end{array}$ & $\overline{-}$ & $\begin{array}{l}191,241 \\
135,145\end{array}$ \\
\hline 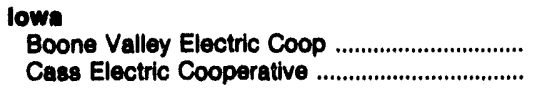 & $\overline{-}$ & $\overline{-}$ & $\overline{-}$ & $\overline{-}$ & $\begin{array}{l}5,800 \\
1,205\end{array}$ & $\overline{-}$ & $\begin{array}{l}5,800 \\
1,205\end{array}$ \\
\hline 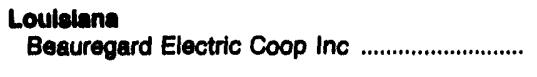 & - & - & - & - & 437,758 & - & 437,758 \\
\hline 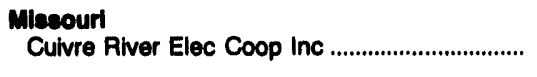 & - & - & - & - & 492,820 & - & 482,820 \\
\hline $\begin{array}{l}\text { Novada } \\
\text { Farmers Mutual Power Assn ................................. }\end{array}$ & - & - & - & - & 1,111 & - & 1,111 \\
\hline $\begin{array}{l}\text { Ponnaylvania } \\
\text { New Enterprise Rural Elec Coop ........................ }\end{array}$ & - & - & - & - & 38,910 & - & 38,910 \\
\hline $\begin{array}{l}\text { Texas } \\
\text { Fort Belknap Electric Coop Inc ............................ }\end{array}$ & - & -- & - & - & 94,413 & - & 94,413 \\
\hline $\begin{array}{l}\text { Virglnis } \\
\text { Old Dominion Electric Coop ................................ }\end{array}$ & - & - & - & - & - & $5,148,808$ & $5,148,808$ \\
\hline
\end{tabular}

1 Includes transactions with power pools, utilities in Canada and Mexico, and nonutilities.

- Not Applicable

Notes: -The Department of Agriculture under the Rural Electrification Act is authorized to provide Federal commitments for long-term financing and to guarantee non-Federal long-term loans to utilities supplying power to rural America. The utilities that recelved guarantees for their loans are referred to as borrowers by the REA. Nonborrowing cooperatives and other utilities that do not borrow from the REA are not required to file the REA-7 and REA-12 forms. Distribution borrowers do not generate electricity; they purchase electricity from other utilities. $\bullet$ For identification purposes, the Cooperative Borrowers are listed in the State in which the administrative oflice is located. -Totals may not equal sum of components because of independent rounding. Source: •Energy Information Administration Form EIA-861, "Annual Electric Utility Report." 
Table 36. Electriclty Exchanges and Wheeling by Cooperative Borrowers, by State, 1992 (Thousand Kilowatthours)

\begin{tabular}{|c|c|c|c|c|}
\hline \multirow{2}{*}{$\begin{array}{l}\text { State I } \\
\text { Cooperattve Borrower }\end{array}$} & \multicolumn{2}{|c|}{ Exchanges } & \multicolumn{2}{|c|}{ Wheoling } \\
\hline & Received & Dellvered & Received & Dellvered \\
\hline 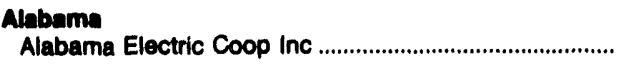 & - & - & 94,037 & 94,037 \\
\hline 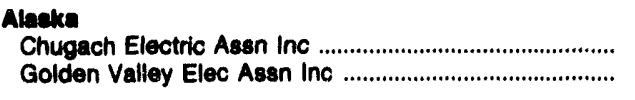 & $\overline{10,347}$ & $\overline{10,675}$ & 174,638 & $\begin{array}{c}174,638 \\
-\end{array}$ \\
\hline 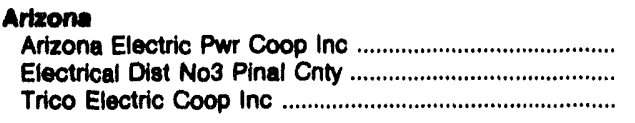 & $\begin{array}{l}18,109 \\
14,738 \\
-\end{array}$ & $\begin{array}{l}31,183 \\
3,290 \\
-\end{array}$ & $\frac{7,200}{7,003}$ & $\frac{6,712}{7,003}$ \\
\hline 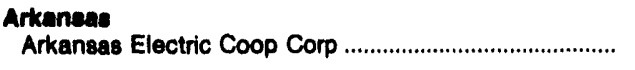 & 5,668 & 11,036 & - & -- \\
\hline 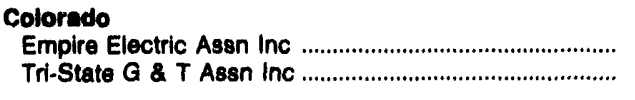 & $\overline{1,734,690}$ & $\overline{1,784,616}$ & $\begin{array}{r}6,804 \\
131,808\end{array}$ & $\begin{array}{r}6,704 \\
126,210\end{array}$ \\
\hline 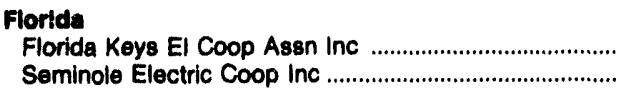 & $\overline{26,446}$ & $\overline{13,430}$ & $\begin{array}{l}439,784 \\
--\end{array}$ & $\begin{array}{c}420,555 \\
-\end{array}$ \\
\hline $\begin{array}{l}\text { Idaho } \\
\text { Idaho Cnty L\&P Coop Assn Inc } \\
\text { Rural Electric Co }\end{array}$ & - & -- & $\begin{array}{l}1,070 \\
1,456\end{array}$ & $\begin{array}{l}1,070 \\
1,456\end{array}$ \\
\hline 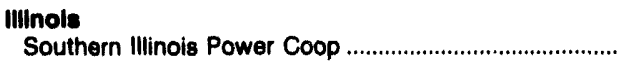 & 161,284 & 48,202 & -- & -- \\
\hline 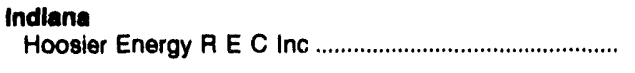 & 104,513 & 129,857 & 185,047 & 176,198 \\
\hline 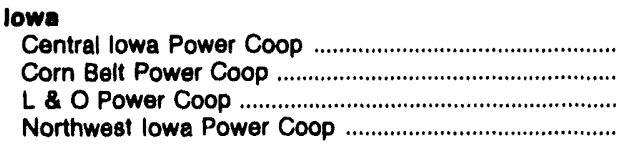 & $\begin{array}{c}1,609,891 \\
80,855 \\
-- \\
130,884\end{array}$ & $\begin{array}{c}1,522,665 \\
283,717 \\
- \\
126,746\end{array}$ & $\begin{array}{r}- \\
\overline{92,526} \\
300,982\end{array}$ & $\begin{array}{r}\overline{-} \\
\overline{86,473} \\
287,958\end{array}$ \\
\hline 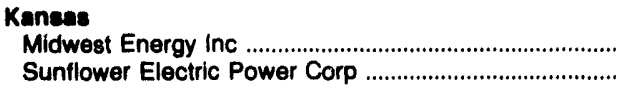 & -252 & -367 & $\overline{86,552}$ & $\overline{86,552}$ \\
\hline 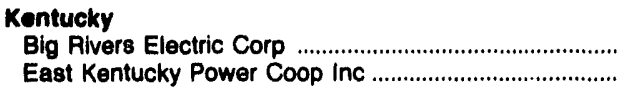 & $\begin{array}{r}52,280 \\
162,900\end{array}$ & $\begin{array}{r}52,717 \\
108,248\end{array}$ & $\begin{array}{r}140,371 \\
1,549,114\end{array}$ & $\begin{array}{r}140,371 \\
1,549,114\end{array}$ \\
\hline 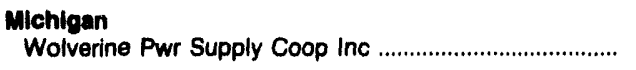 & 143,864 & 5,313 & - & - \\
\hline 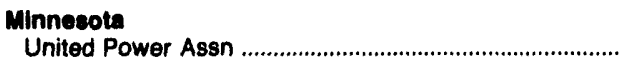 & 7,094 & 64,996 & 55,057 & 24,685 \\
\hline 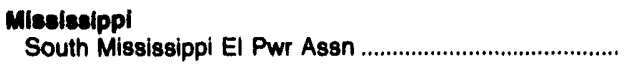 & 25,905 & 25,478 & - & - \\
\hline 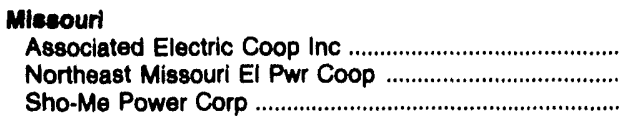 & $\begin{array}{l}360,069 \\
-\end{array}$ & $\begin{array}{l}161,811 \\
- \\
-\end{array}$ & $\begin{array}{r}617,379 \\
163,678 \\
90,111\end{array}$ & $\begin{array}{r}574,338 \\
183,678 \\
90,111\end{array}$ \\
\hline 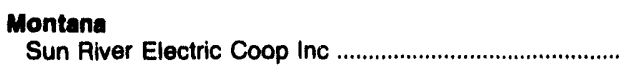 & - & - & 3,035 & 3,035 \\
\hline 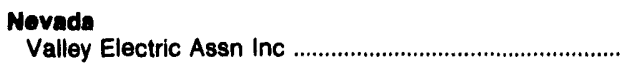 & 24,235 & 19,812 & - & - \\
\hline 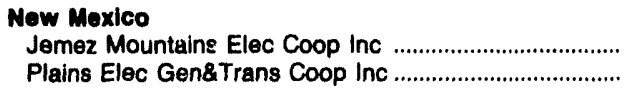 & $\overline{68,731}$ & $\overline{53,632}$ & $\begin{array}{r}76,528 \\
102,193\end{array}$ & $\begin{array}{r}76,528 \\
102,193\end{array}$ \\
\hline
\end{tabular}

See notes and footnotes at end of table. 
Table 36. Electrlclty Exchanges and Wheeling by Cooperative Borrowers, by State, 1992 (Thousand Kilowatthours) (Continued)

\begin{tabular}{|c|c|c|c|c|}
\hline \multirow{2}{*}{$\begin{array}{l}\text { State I' } \\
\text { Cooperative Borrower }\end{array}$} & \multicolumn{2}{|c|}{ Exchanges } & \multicolumn{2}{|c|}{ Wheeling } \\
\hline & Received & Delivered & Received & Delivered \\
\hline 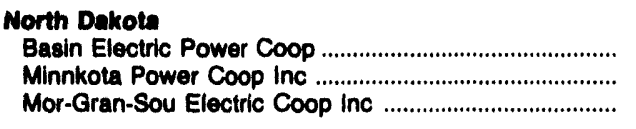 & $\begin{array}{l}33,632 \\
88,131 \\
-\end{array}$ & $\begin{array}{c}100,374 \\
131,140 \\
-\end{array}$ & $\begin{array}{r}-- \\
271,293 \\
129\end{array}$ & $\begin{array}{r}\overline{254,035} \\
129\end{array}$ \\
\hline 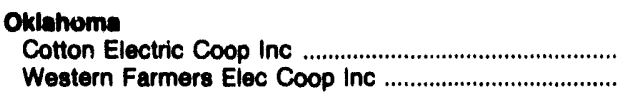 & $\overline{55,777}$ & $\overline{70,779}$ & $\begin{array}{l}6,294 \\
--\end{array}$ & $\begin{array}{l}6,294 \\
--\end{array}$ \\
\hline 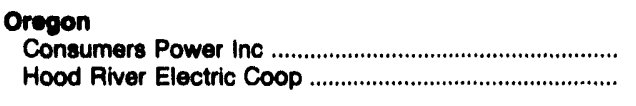 & $-{ }^{66}$ & $\overline{-}$ & $\overline{18,739}$ & $\overline{19,738}$ \\
\hline 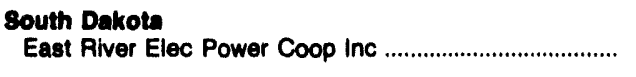 & - & - & 324,967 & 304,361 \\
\hline 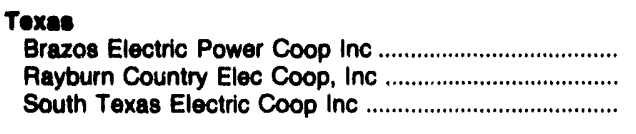 & $\begin{array}{c}17,279,118 \\
254,536 \\
--\end{array}$ & $\begin{array}{r}17,230,447 \\
286,262 \\
318,187\end{array}$ & $\begin{array}{c}169,453 \\
10 \\
161,000\end{array}$ & $\begin{array}{r}163,954 \\
-- \\
165,829\end{array}$ \\
\hline 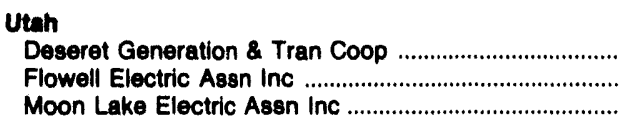 & $\begin{array}{l}6,351 \\
4,280 \\
--\end{array}$ & $\begin{array}{c}17,226 \\
4,280 \\
--\end{array}$ & $\overline{24,850}$ & $\overline{24,850}$ \\
\hline 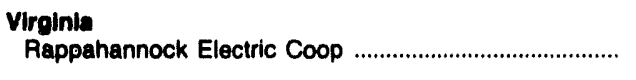 & - & - & 3,714 & 3,645 \\
\hline 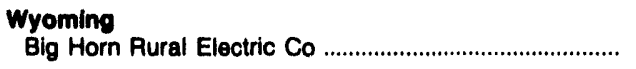 & 9,096 & 8,749 & -- & -- \\
\hline
\end{tabular}

- Not Applicable

Notes: - The Department of Agriculture under the Rural Electrification Act is authorized to provide Federal commitments for long-term financing and to guarantee non-Federal long-term loans to utilities supplying power to rural America. The utilities that received guarantees for their loans are referred to as borrowers by the REA. Nonborrowing cooperatives and other utilities that do not borrow from the REA are not required to file the Rural Electrification Administration, AEA Form 7, "Financial and Statistical Report," the REA Form 12a through 12i, "Electric Power Supply Borrowers," and the Form 12c through 12g, "Electric Distribution Borrowers with Generating Facilities." $\bullet$ For identification purposes, the Cooperative Borrowers are listed in the State in which the administrative office is located. Totals may not equal sum of components because of independent rounding. Source: Energy Information Administration, Form EIA-861, "Annual Eloctric Utility Report." 


\section{Selected Information on the Physical Transmission System}

\section{Overvlew}

This chapter presents data on selected portions of the physical electrical system that investor- and publicly owned electric utilities report on FERC Form 1, "Annual Report of Major Electric Utilities, Licensees and Others," and Form EIA-412, "Annual Report of Public Electric Utilities." Data on cooperative utilities cannot be provided because the filings submitted to the Rural Electrification Administration are not inclusive of the same information requested from the other ownership classes. Summary totals (in NERC regions) of these collected data are presenter' for the first time. Figure 18 highlights the high voltage transmission system in the United States.

This is the first time transmission system information has been presented in this publication. The data reported by utilities were reviewed by EIA staff to ensure its accuracy and consistency. A significant number of utilities were contacted to verify the quality of the data reported. In some cases, utilities revised previously submitted information because of misunderstandings related to the number of circuits and circuit mileage. Utilities are reporting transmission lines that have multiple ownership, and some duplication in reporting statistics is present. Costs reported on transmission line additions sometimes reflect improvement/upgrades of existing lines, not new transmission lines. Costs reported are those used to maintain FERC Account 101, Asset Value of Electric Plant in Service. EIA is working with FERC to improve the quality of transmission system data reported by investor-owned utilities on FERC Form 1. This includes more consistent reporting of line transformer and watthour meter statistics in the categories of "customers use" and "company use."

\section{Tables}

This chapter presents summary (by NERC Region) and individual statistics on voltage of transmission lines
138 kilovolts and above for both alternating and direct current lines. ${ }^{10}$ The data are shown in the categories of overhead and underground/submarine lines for both structural and circuit miles for the following types of utilities.

- Investor-owned electric utilities (Tables 37 and 38)

- Federal power authorities (Tables 39 and 40)

- State and other government utilities (Tables 39 and 40)

- Municipalities (Tables 39 and 40).

Transmission lines added and watthour/line transformer information are not collected from public utilities. Fiscal-year ending dates for each public utility differ and are not included in the tables. Transmission line data are, therefore, not necessarily for the same time period.

Tables 41 and 42 provide summary and detailed information on the transmission lines added by investorowned electric utilities in 1992. Information is presented for lines 138 kilovolts and above for alternating current. No direct current lines were added in 1992. In addition, line cost information is presented and broken into the categories of land, structural, conductors and supporting equipment, and the total. Table $41 \mathrm{ag}-$ gregates the line type by mileage ranges associated with the construction and/or removal of the transmission lines. Public utilities are not required to file this information on the Form EIA-412.

Negative numbers in the tables represent the removal of transmission mileage from the physical electrical system. Some negative numbers in the cost information represent removal of the cost of those lines and equipment from the ratebase. Upgrades to conductors and other equipment for transmission lines currently in operation in the transmission system may be shown without an associated line length.

\footnotetext{
${ }^{10}$ No data are required to be filed on voltages less than 138 kilovolts.
} 


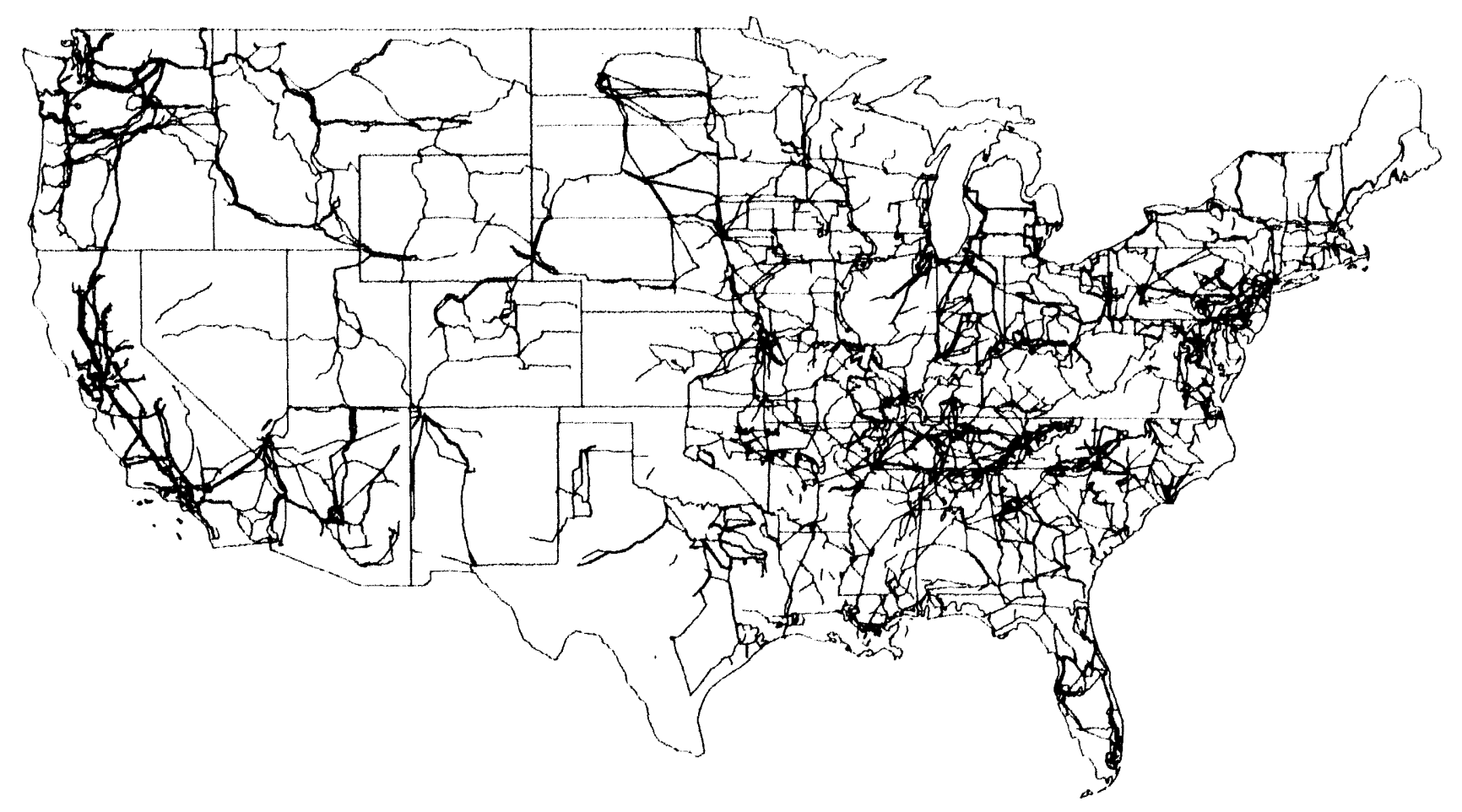

Note: Transmission lines shown are $161,230,345$, and 500 kilovolts, atternating current.

Source: Federal Emergency Management Agency.

Summary and detailed information on line transformer and watthour meter totals are provided by investorowned electric utilities (Tables 43 and 44). This information is broken into the following categories: number of line transformers present at the beginning of the year; additions during the year; reductions during the year; total in use by customers; total in use by the electric company; and summary information on what is kept in stock, locked at the customer's site, and in an inactive status. The line transformer capacity in megavoltamperes is also presented by the same categories.

\section{Highlights}

The 138-kilovolt alternating current (AC) transmission line was reported to be the most frequently used type of high voltage line by investor-owned utilities. Over 53,000 structural miles were in-place with over 65,000 miles of circuit attached to various types of structures. The AC voltages levels of 230 kilovolts and 345 kilovolts followed in a close second and third. The ECAR region had the most 138-kilovolt AC lines with nearly 20,000 structural miles and over 25,000 miles of overhead circuit miles. SERC had the most 230-kilovolt AC lines with nearly 15,000 structural miles (and nearly 18,000 circuit miles), while the 345-kilovolt AC line leader was the ECAR region with nearly 8,000 structural miles and 13,000 circuit miles. Consolidated Edison Company of New York had the most underground miles (over 430 circuit miles of 138-kilovolt and 165 circuit miles of 345-kilovolt lines).

Public electric utilities reported a very different mix of high voltage $\mathrm{AC}$ and direct current (DC) lines. The 230-kilovolt line was the most frequently reported with over 16,000 structural miles in-place. The voltages of 345 kilovolts and 500 kilovolts were second and third with over 6,000 and 5,400 structural miles, respectively. The 138-kilovolt AC line came in fifth for public utilities and 161 kilovolts was fourth. Public utilities also operated the highest direct current voltage. The 1000-kilovolt DC line was in the WSCC region with over 500 structural miles in use. The WSCC region had the largest and widest variety of voltage types in use. The Bonneville Power Administration and the Western Power Administration ranked first and second for total mileage.

Investor-owned electric utilities reported adding over 2,560 miles of new transmission lines of a size of 138 kilovolts or greater to the electrical systems in 1992. The cost for land right-of-way, new structures, conductors and line materials for these additions and up- 
grades was nearly $\mathbf{3 7 5}$ million. Florida Power and Light, whose electrical system was severely damaged by Hurricane Andrew in 1992, led the other reporting utilities in adding new (or in this case replacing) higher transmission voltage support for its electrical system. Public Service Company of Colorado had the largest increase, but those existing lines were acquired through the purchase of assets of a bankrupt cooperative.

Counts of distribution line transformers and watthour meters reported by investor-owned electric utilities were at the levels of over 22 and 92 million, respectively, for the contiguous United States at the end of 1992. Investor-owned utilities removed from service 2.0 and 2.5 percent, respectively, of the line transformers and watthour meters in 1992. Utilities then added 3.2 percent to the beginning year balance for line transformers from purchase, acquisitions, and refurbishment. The ranking of the top five investor-owned utilities for year-end watthour meters follows: Pacific Gas and Electric of California (4.6 million); Southern California Edison (4.3 million); Commonwealth Edison of Illinois (3.7 million); Florida Power and Light (3.6 million); and Consolidated Edison of New York (3.2 million). The top five changed for line transformers at year end, but Pacific Gas and Electric was again first with almost 918,000. Texas Utilities Electric was second with 676,000, and Florida Power and Light had 672,000 for third. Southern California Edison was fourth with 652,000, and Duke Power of North Carolina was fifth with 590,000 line transformers. In addition to third (Florida Power and Light) and fifth (Duke Power), SERC also contained utilities placing sixth (Alabama Power and Light with 543,000), seventh (Georgia Power with 538,000 ) and ninth (Virginia Electric and Power with 523,000) in the year end count of line transformers. Yet the SERC region contained less than 20 percent of the total line transformers in the contiguous United States in 1992.

\section{Summary}

The first time reporting of transmission line statistics by voltage size for both investor-owned and public electric utilities presents the opportunity to see what mix of transmission voltages are in use and where they are in the United States. Some of the voltage sizes represent tests of a particular operating level of $A C$ voltage which did not find widespread usage (154 kilovolts and 170 kilovolts). Other voltage sizes indicate a test of voltages that could become a future mainstay ( 525 kilovolts AC, 550 kilovolts AC, 800 kilovolts AC, and 1000 kilovolts $D C)$.

The inclusion of transmission statistics of public utilities for the first time provides a point of reference to understand the complexity of the transmission system ownership and how the electrical system is interwoven across the United States. Many public utilities acquire electricity through very short transmission lines and substation connections taps attached to the electrical power grid. The ownership of these connections is not captured by these data.

Many other types of electrical equipment used in support for operating the electrical grid are not singled out for collection nor are the relationships of megavoltampere, reactive power, and megawatts highlighted. The information shown in this chapter does not describe the operation of the physical electrical system nor the costing of its services. Both cost allocation and how engineering operations are linked to the contract trade of electricity are issues that are in the forefront for the changing electric power industry. 
Table 37. Tranamiedion Line statibtlos of Investor-Owned Utiltibe, by NERC Reglon, 1902

\begin{tabular}{|c|c|c|c|c|}
\hline \multirow{2}{*}{ 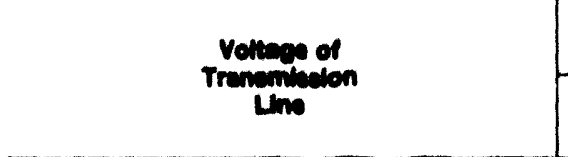 } & \multicolumn{2}{|c|}{ Oremend } & \multicolumn{2}{|c|}{$\begin{array}{l}\text { Underground } \\
\text { Submerim }\end{array}$} \\
\hline & $\begin{array}{c}\text { Structural } \\
\text { Niles }\end{array}$ & $\begin{array}{l}\text { Ciroult } \\
\text { Miles }\end{array}$ & $\begin{array}{l}\text { Structural } \\
\text { Miles }\end{array}$ & $\begin{array}{l}\text { Chout } \\
\text { Miles }\end{array}$ \\
\hline \multicolumn{5}{|c|}{ Invertonowned Utumes in reAn } \\
\hline 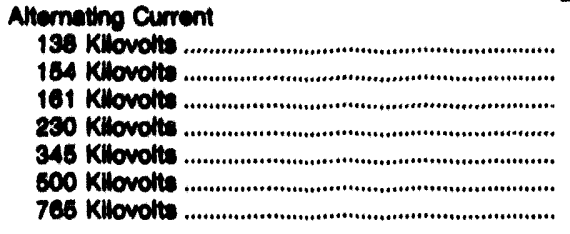 & $\begin{array}{r}10,400 \\
60 \\
865 \\
860 \\
7,916 \\
920 \\
2,023\end{array}$ & $\begin{array}{r}28,788 \\
204 \\
1,404 \\
1,176 \\
12,808 \\
1,038 \\
2,028\end{array}$ & $\begin{array}{l}-61 \\
- \\
- \\
-\end{array}$ & $\begin{array}{l}{ }^{61} \\
= \\
= \\
-\end{array}$ \\
\hline & \multicolumn{4}{|c|}{ Invedtor-owned Uutites in ERcot } \\
\hline \multirow[t]{2}{*}{ 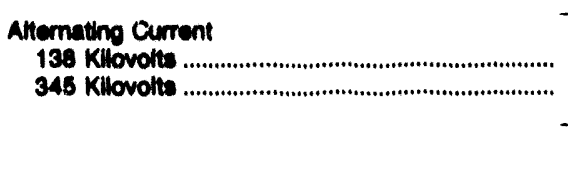 } & $\begin{array}{r}10,900 \\
4,241\end{array}$ & $\begin{array}{r}12,442 \\
7,100\end{array}$ & 33 & -58 \\
\hline & \multicolumn{4}{|c|}{ Investor-owned Utumtes in MAMC } \\
\hline \multirow[t]{2}{*}{ 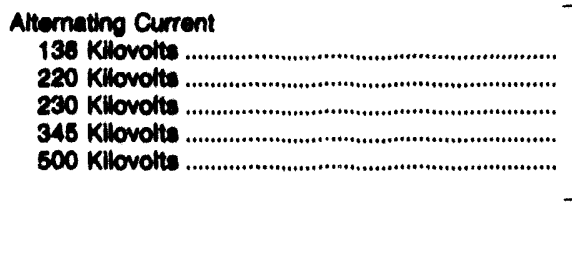 } & $\begin{array}{r}931 \\
447 \\
3,434 \\
170 \\
1,902\end{array}$ & $\begin{array}{r}1,330 \\
837 \\
4,651 \\
176 \\
2.012\end{array}$ & $\begin{array}{r}67 \\
6 \\
-\quad 69 \\
-\quad\end{array}$ & $\begin{array}{r}104 \\
6 \\
-\quad 138 \\
-\end{array}$ \\
\hline & \multicolumn{4}{|c|}{ Invector-owned Uttlibes in MAIN } \\
\hline \multirow[t]{2}{*}{ 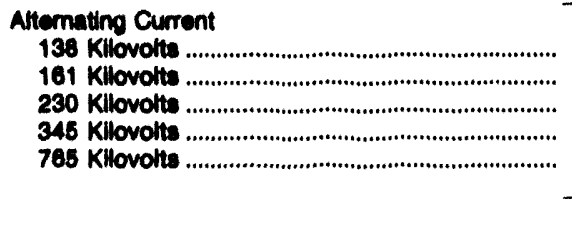 } & $\begin{array}{r}8,082 \\
795 \\
191 \\
3,924 \\
80\end{array}$ & $\begin{array}{r}10,483 \\
801 \\
282 \\
5,311 \\
80\end{array}$ & $\begin{array}{l}239 \\
- \\
- \\
-\end{array}$ & $\begin{array}{l}238 \\
- \\
-\end{array}$ \\
\hline & \multicolumn{4}{|c|}{ Inveotor-Owned Utilites in MAPP } \\
\hline \multirow[t]{2}{*}{ 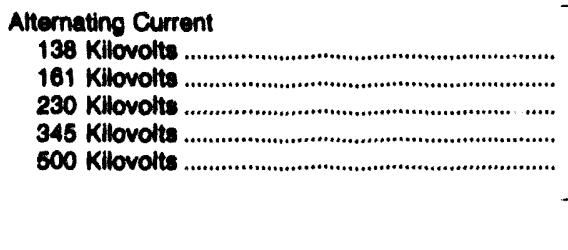 } & $\begin{array}{r}6 \\
2,746 \\
1,543 \\
1,977 \\
273\end{array}$ & $\begin{array}{r}6 \\
3,154 \\
1,548 \\
2,467 \\
273\end{array}$ & $\begin{array}{l}- \\
- \\
- \\
-\end{array}$ & $\begin{array}{l}- \\
- \\
-\end{array}$ \\
\hline & \multicolumn{4}{|c|}{ Investor-Owned Utilties in NPCC } \\
\hline \multirow[t]{2}{*}{ 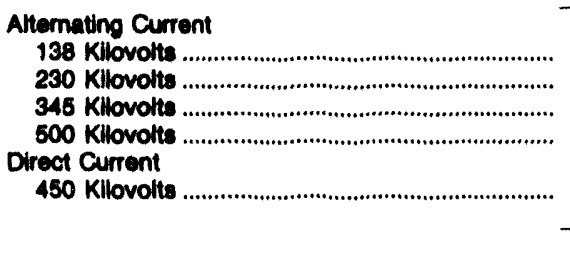 } & $\begin{array}{r}410 \\
1,085 \\
3,313 \\
10 \\
\\
434\end{array}$ & $\begin{array}{r}456 \\
1,207 \\
4,103 \\
10 \\
\\
434\end{array}$ & $\begin{array}{l}-295 \\
-\quad 119 \\
-\end{array}$ & $\begin{array}{r}571 \\
-\quad 108 \\
-\end{array}$ \\
\hline & \multicolumn{4}{|c|}{ Invector-Owned Utiltive in seAC } \\
\hline \multirow[t]{2}{*}{ 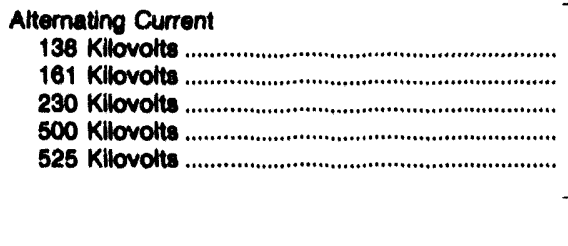 } & $\begin{array}{r}1,510 \\
440 \\
14,686 \\
3,847 \\
575\end{array}$ & $\begin{array}{r}1,533 \\
469 \\
17,622 \\
3,947 \\
575\end{array}$ & $\begin{array}{r} \\
-\quad 35 \\
-\quad 39 \\
-\quad\end{array}$ & $\begin{array}{r} \\
-\quad 45 \\
-\end{array}$ \\
\hline & \multicolumn{4}{|c|}{ Investor-Owned Utiltien In spp } \\
\hline $\begin{array}{l}\text { Alternating Current } \\
138 \text { Kllovolts } \\
181 \text { Kllovolts } \\
230 \text { Kilovolts } \\
245 \text { Kilovolts }\end{array}$ & $\begin{array}{l}7,464 \\
3,694 \\
4,370 \\
3,837\end{array}$ & $\begin{array}{l}8,103 \\
3,868 \\
4,864 \\
4,085\end{array}$ & $\begin{array}{l}- \\
- \\
-\end{array}$ & -10 \\
\hline
\end{tabular}

See notes and footnotes at end of table. 
Table 37. Tranomicalon Line statiotios of Investor-Owned Utilitiee, by NERC Reglon, 1992 (Continued)

\begin{tabular}{|c|c|c|c|c|}
\hline \multirow{2}{*}{$\begin{array}{l}\text { Ventupe of } \\
\text { Tromingenten }\end{array}$} & \multicolumn{2}{|c|}{ Overinend } & \multicolumn{2}{|c|}{$\begin{array}{l}\text { Undenground } \\
\text { Submarin }\end{array}$} \\
\hline & $\begin{array}{c}\text { Struotural } \\
\text { Milnes }\end{array}$ & $\begin{array}{l}\text { Crrout } \\
\text { Mles }\end{array}$ & $\begin{array}{l}\text { Structural } \\
\text { Milies }\end{array}$ & $\begin{array}{l}\text { Crrout } \\
\text { Milbs }\end{array}$ \\
\hline \multicolumn{5}{|c|}{ Imvetor-owned utinites in ape } \\
\hline $\begin{array}{l}\text { Alomating Current } \\
\text { So0 Kllovolts }\end{array}$ & 2,018 & 2,148 & - & - \\
\hline & \multicolumn{4}{|c|}{ Invester-Ommed unnteo in weoc } \\
\hline 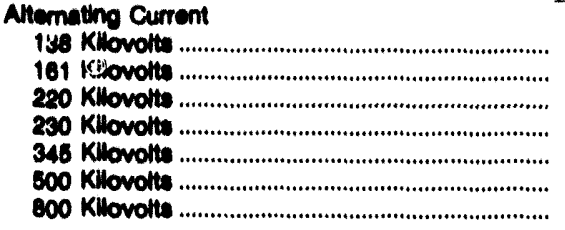 & $\begin{array}{r}4,370 \\
1,281 \\
2,069 \\
12,169 \\
6,363 \\
7,005 \\
313\end{array}$ & $\begin{array}{r}5,328 \\
1,372 \\
2,600 \\
18,440 \\
7,260 \\
9,503 \\
313\end{array}$ & $\begin{array}{l}- \\
- \\
- \\
-\end{array}$ & $\begin{array}{l}- \\
- \\
- \\
-\end{array}$ \\
\hline & \multicolumn{4}{|c|}{ Contiguous U.8. Total } \\
\hline 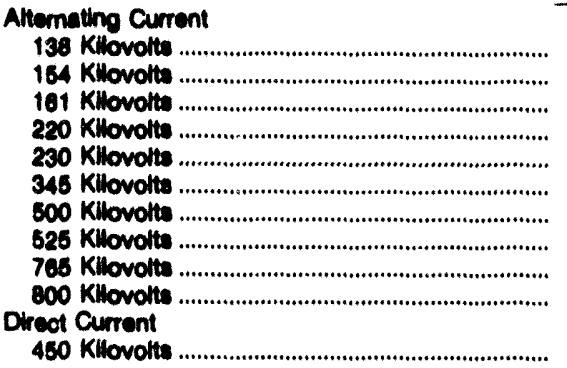 & $\begin{array}{r}53,229 \\
68 \\
9,621 \\
3,113 \\
38,288 \\
31,730 \\
17,158 \\
575 \\
2,113 \\
313 \\
434\end{array}$ & $\begin{array}{r}65,424 \\
204 \\
11,008 \\
3,208 \\
46,778 \\
43,316 \\
10,021 \\
576 \\
2,113 \\
313 \\
434\end{array}$ & $\begin{array}{r}740 \\
-\quad 11 \\
6 \\
162 \\
-\quad 137 \\
- \\
- \\
-\end{array}$ & $\begin{array}{r}1,073 \\
-\quad 11 \\
6 \\
220 \\
-\quad 210 \\
- \\
- \\
-\end{array}$ \\
\hline
\end{tabular}

Notea: The States of Alaka and Hawall are not included. NERC is the North American Electric Reliability Councll. The Alacka Syatem Coordinatino Council is not included. See gloseary for a list of regions and Figure 2 for a map of the reglons. Totals may not equal sum of components because of independent rounding. Voluntary flinge of datribution line and tranamiasion line data below 138 kllovolte are not included in this tabie.

Sources: •Federal Energy Regulatory Commisalon, FERC Form 1, "Annual Report of Major Electric Utilties, Lloensees and Others." Form 1.F. "Annual Report for Nonmajor Pubtlc Utilties and Licensees." 
Table 38. Tranemisalon Line Statistice of Investor-Owned Utilitios, 1992

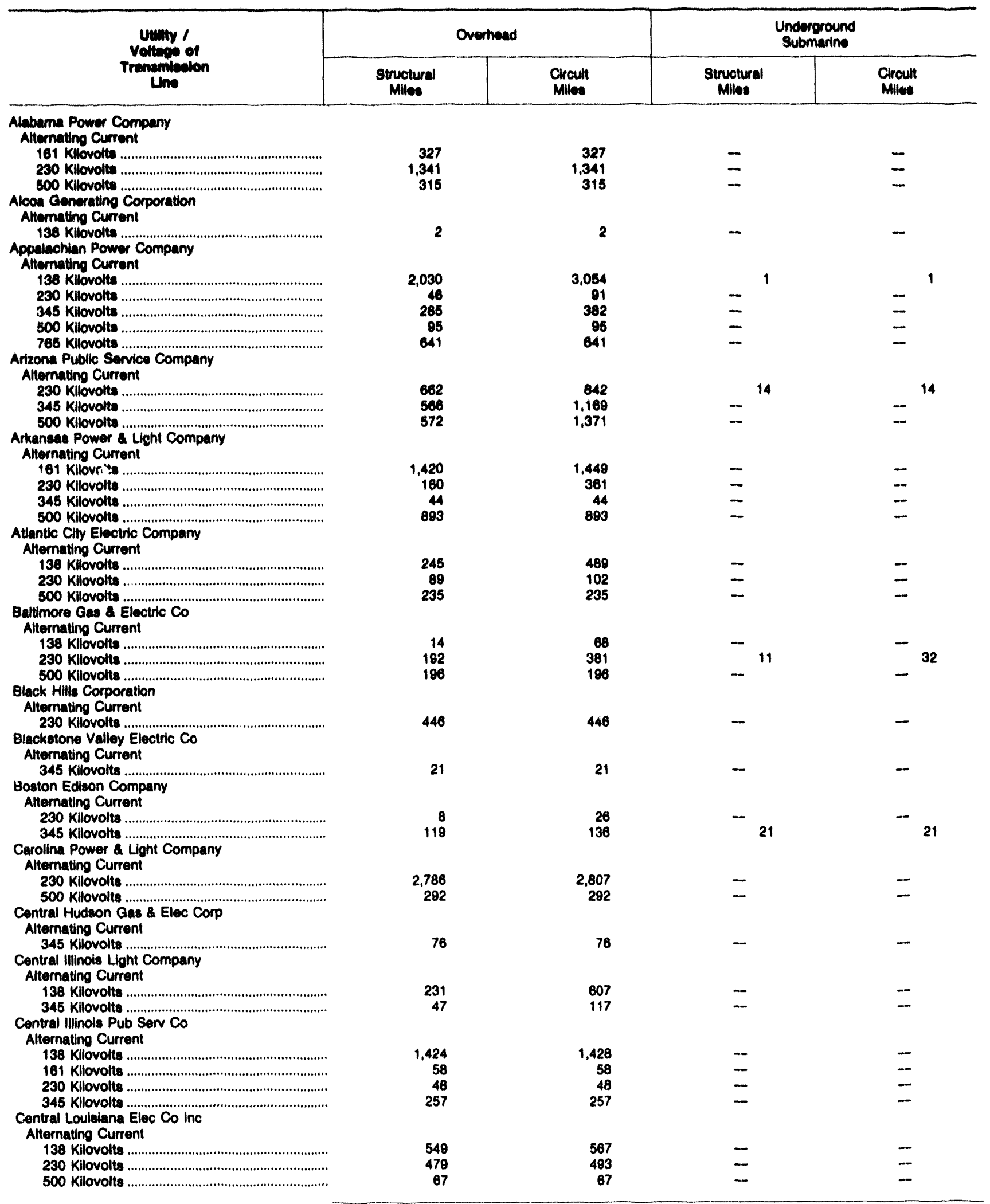

See notes and footnotes at end of table. 
Table 38. Transmiselon Line Statistics of Investor-Owned Utilitles, 1992 (Continued)

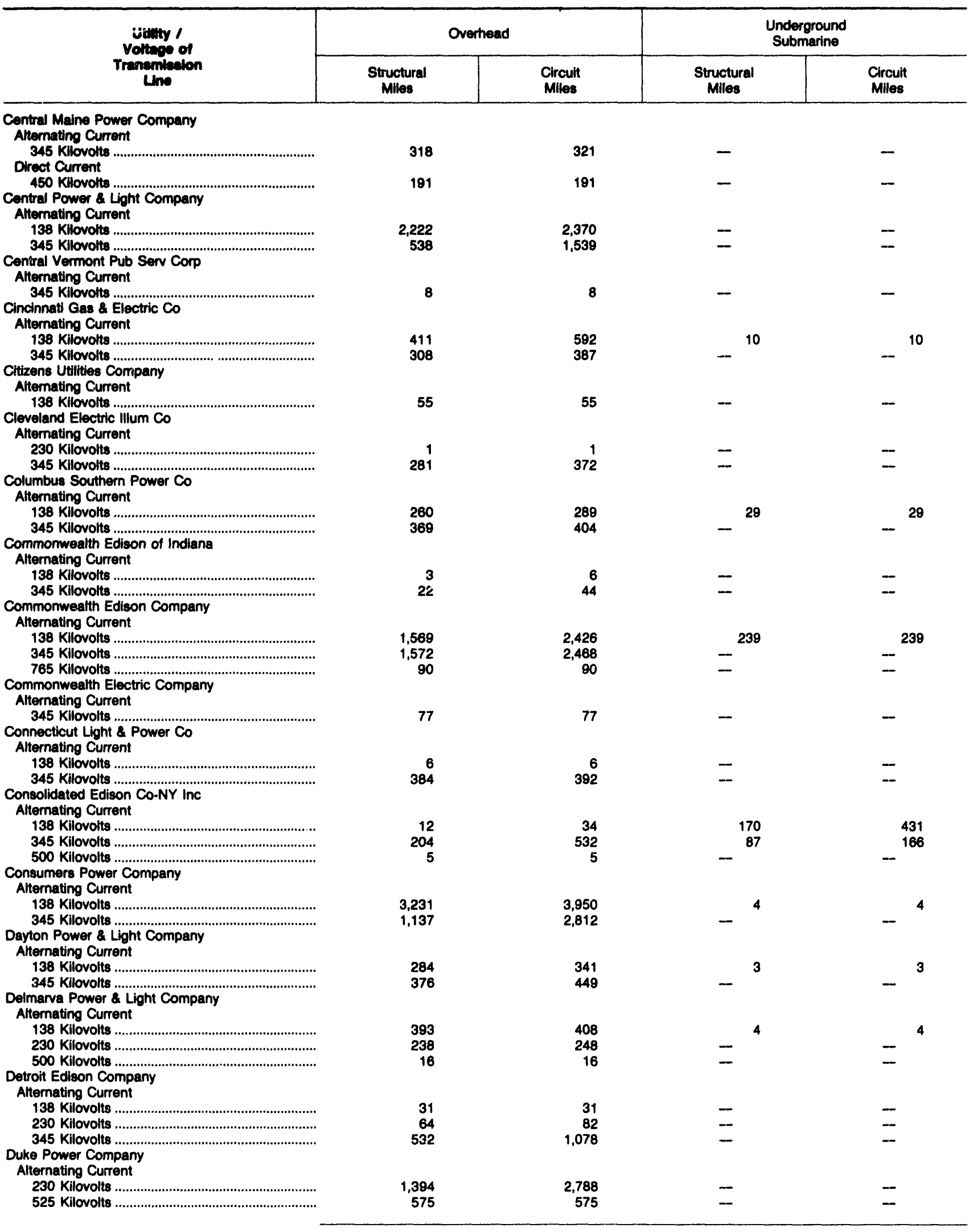

See notes and footnotes at end of table. 
Table 38. Transmission Line Statistics of Investor-Owned Utilities, 1992 (Continued)

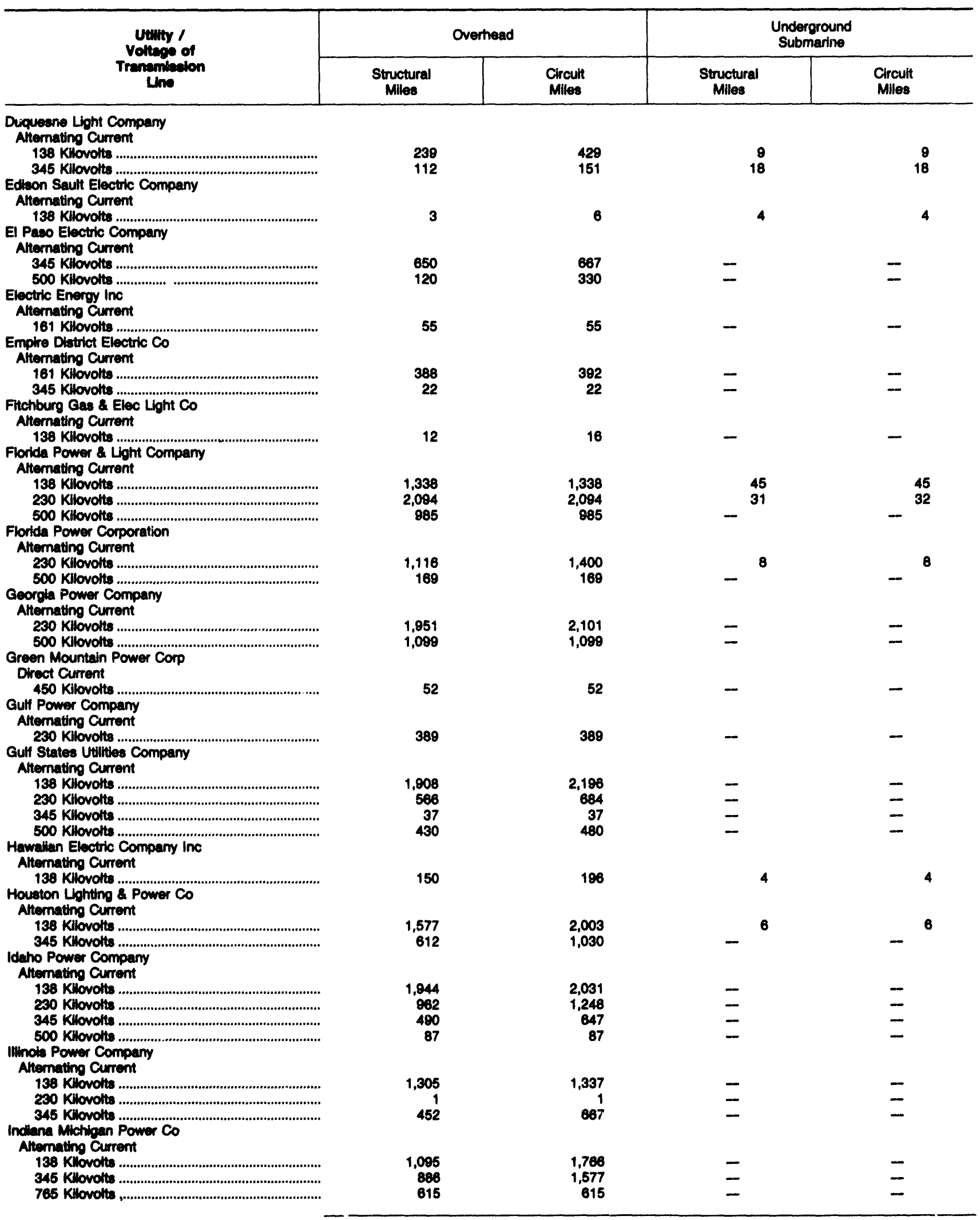

See notes and footnotes at end of table. 
Table 38. Tranamiselon Line Statistics of Investor-Owned Utilities, 1992 (Continued)

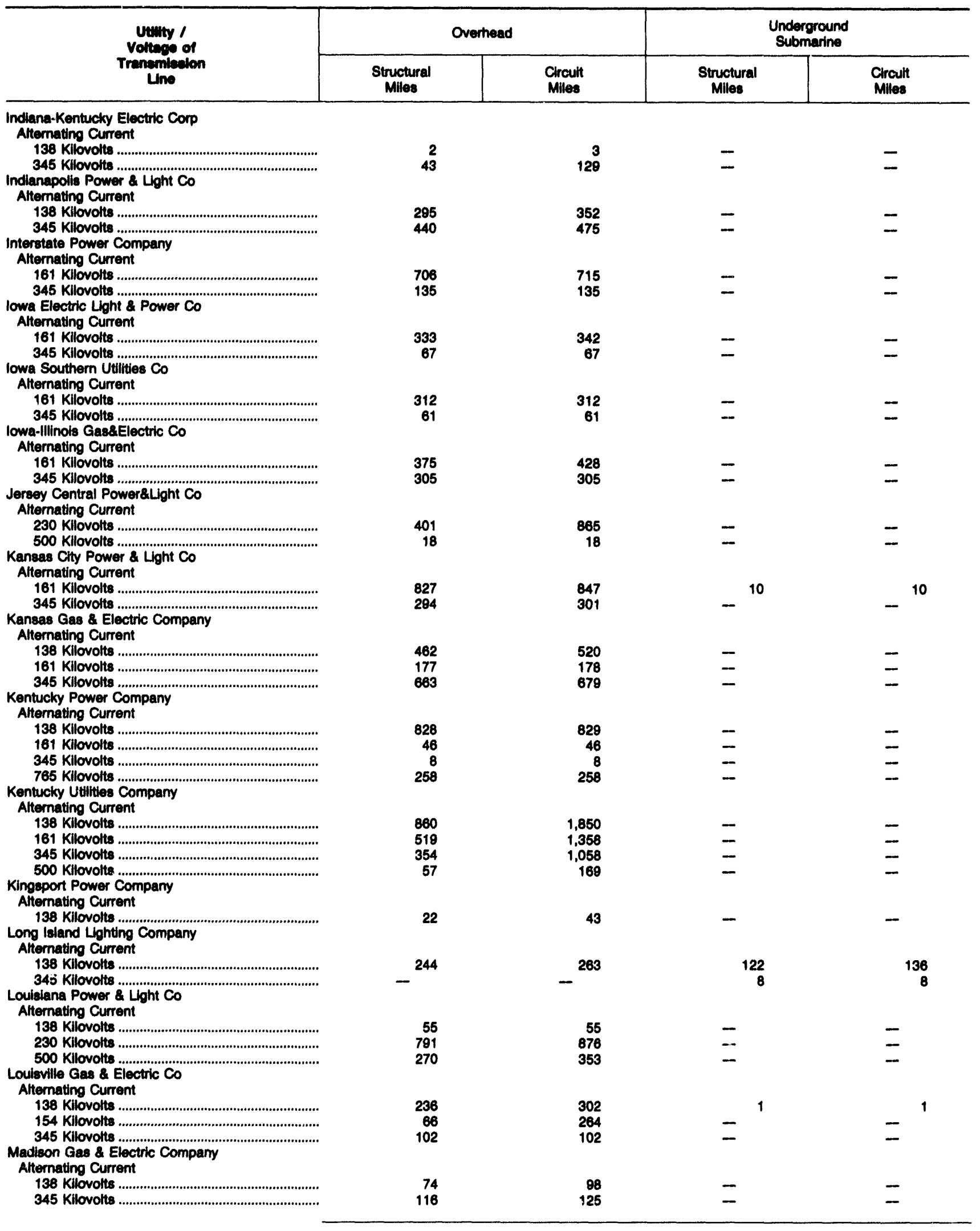

See notes and footnotes at end of table. 
Table 38. Transmission Line Statistics of Investor-Owned UtIIIties, 1992 (Continued)

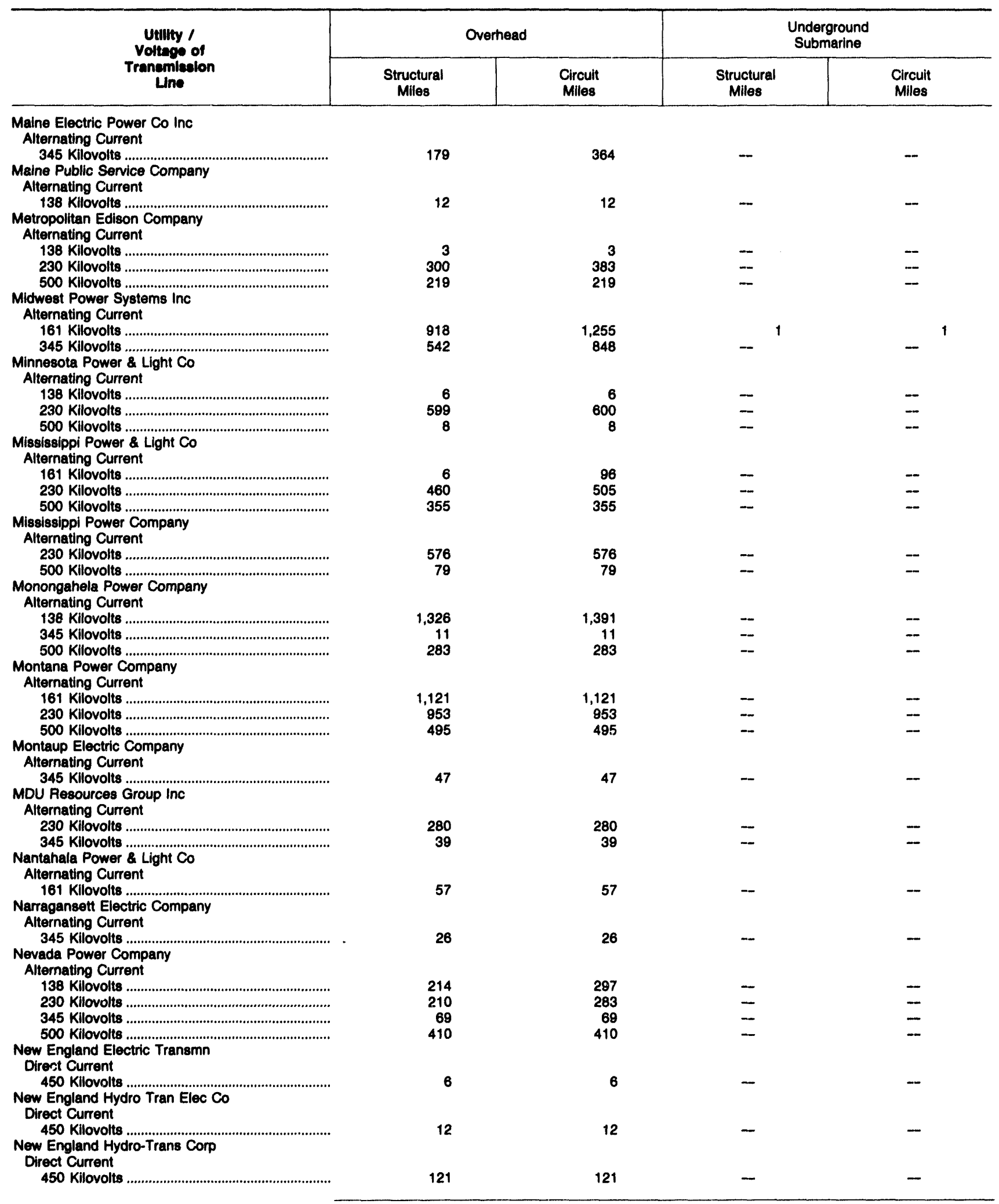

Seo notes and footnotes at end of table. 
Table 38. Transmission Line Statistics of Investor-Owned Utilitles, 1992 (Continued)

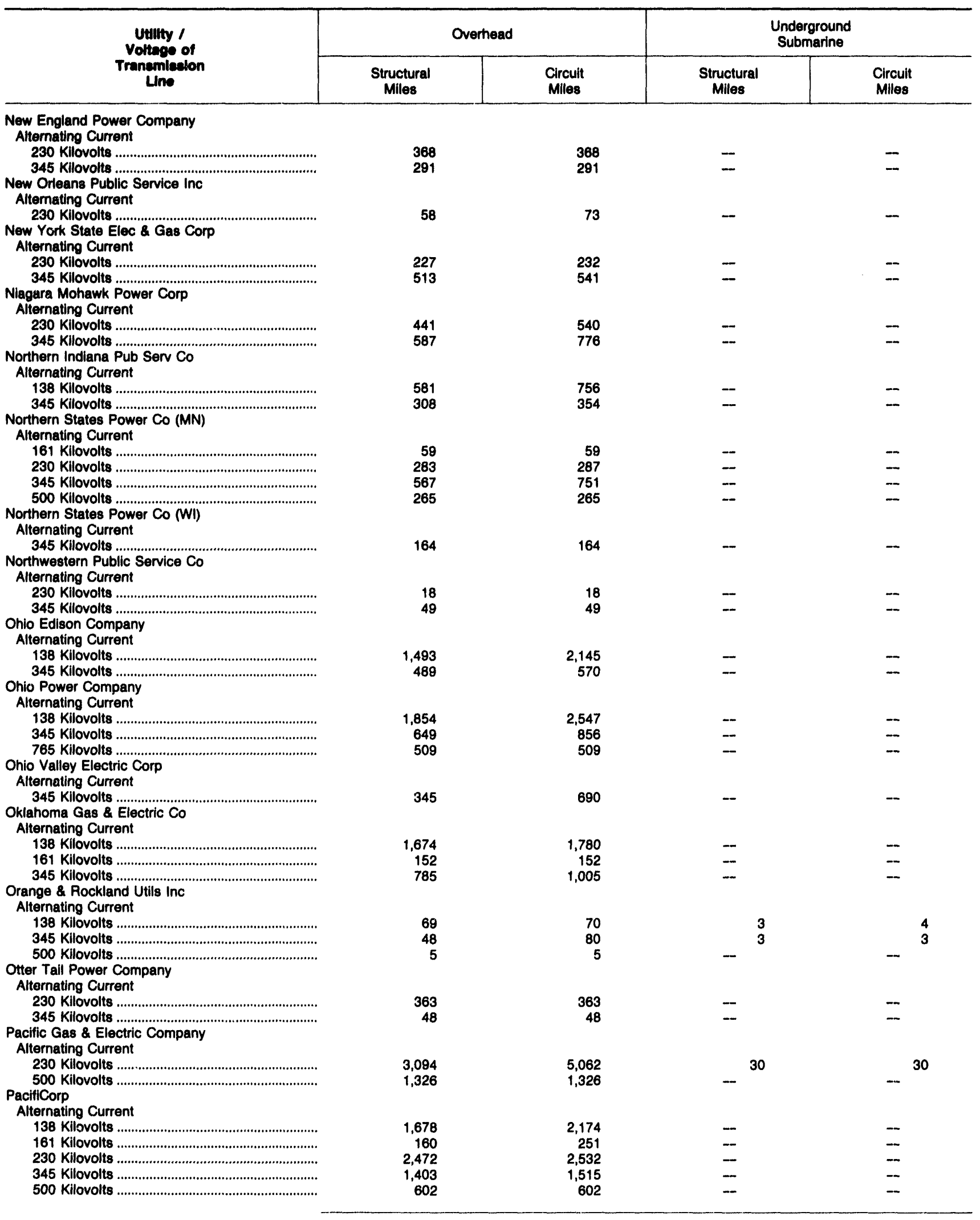

See notes and footnotes at end of table. 
Table 38. Transmisalon Line Statistics of Investor-Owned Utilities, 1992 (Continued)

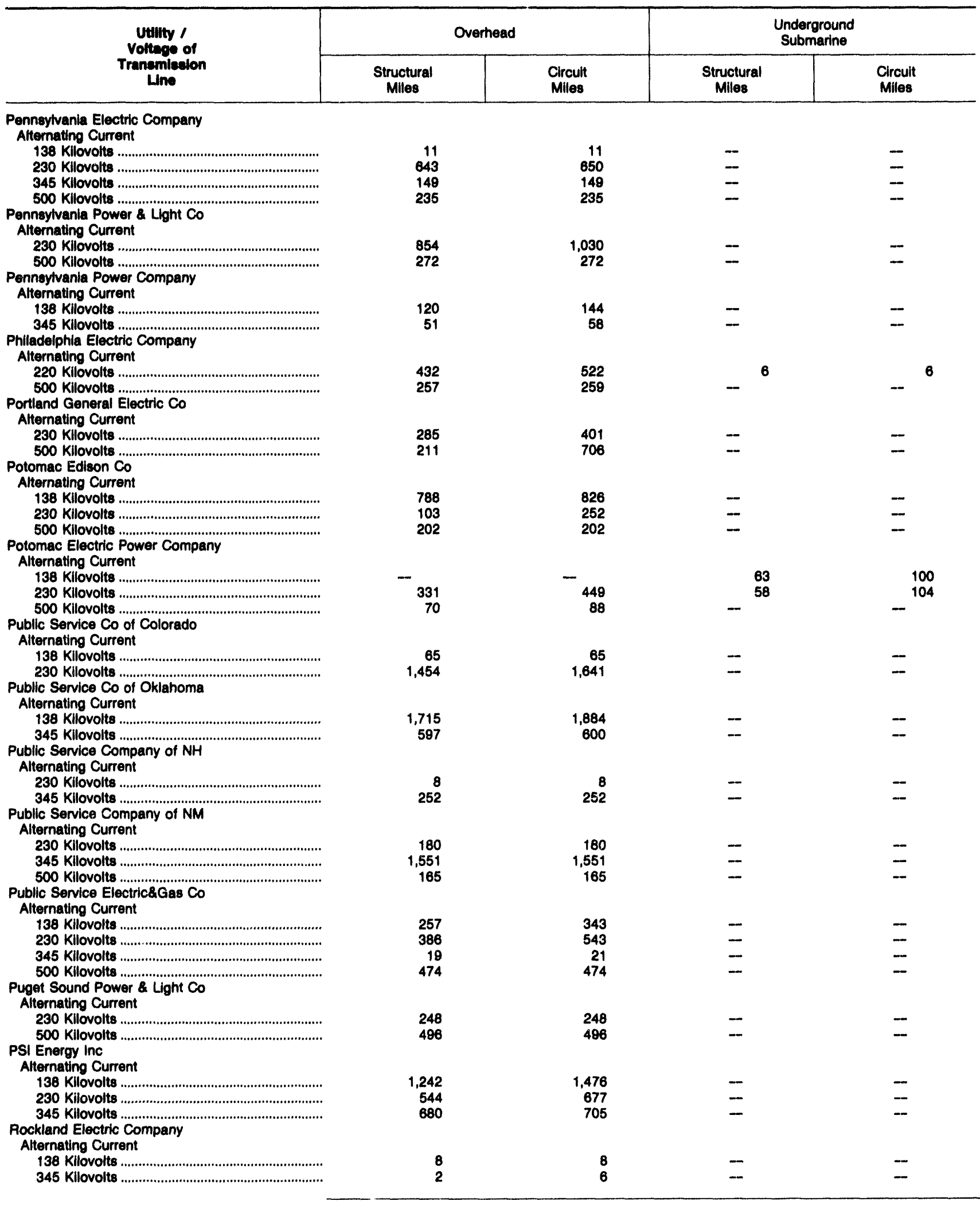

See notes and footnotes at end of table. 
Table 38. Transmission Line Statistics of Investor-Owned Utilities, 1992 (Continued)

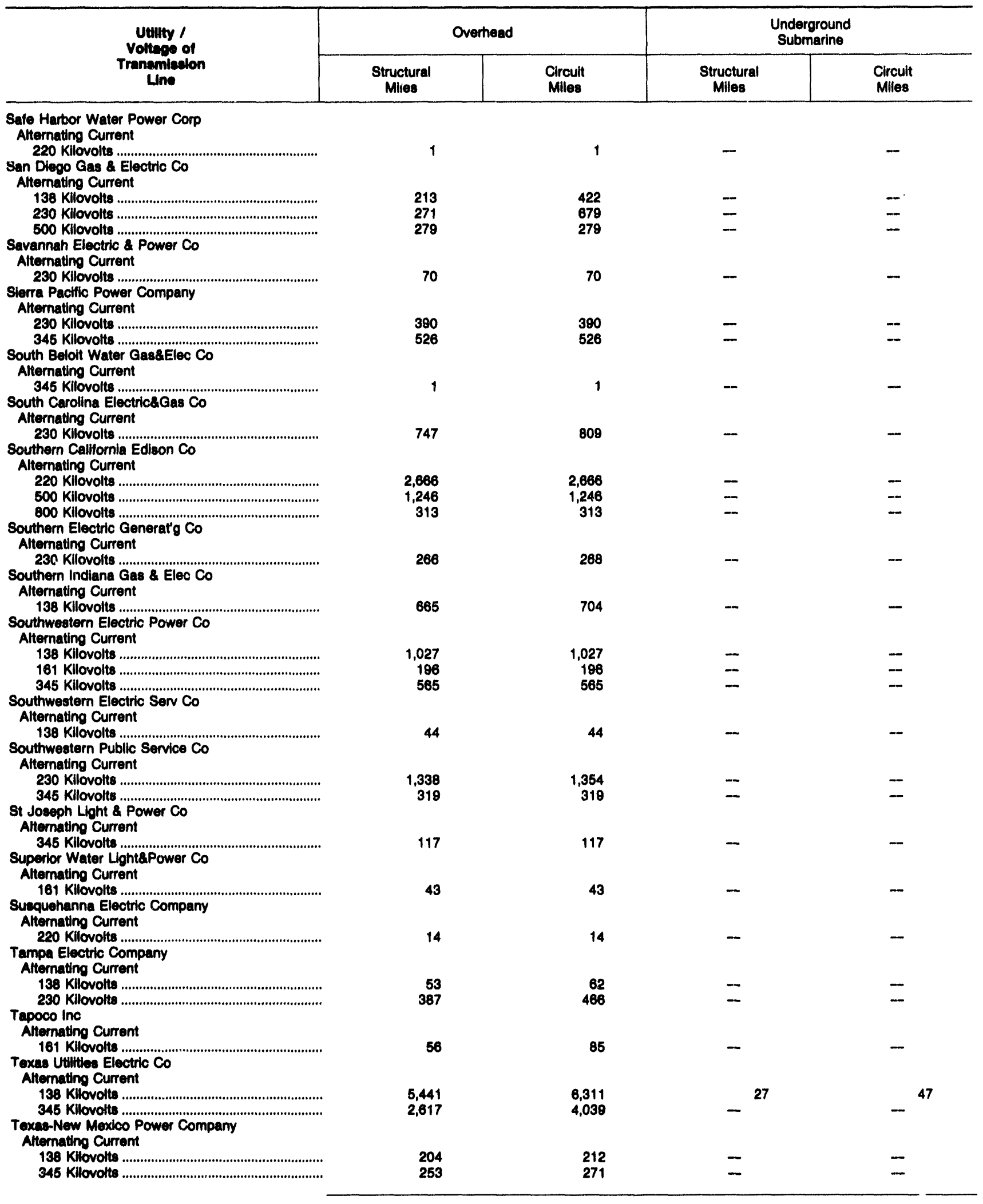

See notes and footnotes at end of table. 
Table 38. Tranamisaion Line Statistics of Investor-Owned Utilities, 1992 (Continued)

\begin{tabular}{|c|c|c|c|c|}
\hline \multirow{2}{*}{$\begin{array}{l}\text { Utally I } \\
\text { Voltage of } \\
\text { Tranamleclon } \\
\text { Uine }\end{array}$} & \multicolumn{2}{|c|}{ Overhead } & \multicolumn{2}{|c|}{$\begin{array}{c}\text { Underground } \\
\text { Submarine }\end{array}$} \\
\hline & $\begin{array}{l}\text { Structural } \\
\text { Milles }\end{array}$ & $\begin{array}{l}\text { Circuit } \\
\text { Miles }\end{array}$ & $\begin{array}{l}\text { Structural } \\
\text { Miles }\end{array}$ & $\begin{array}{l}\text { Circuit } \\
\text { Miles }\end{array}$ \\
\hline \multicolumn{5}{|l|}{$\begin{array}{l}\text { Toledo Edison Company } \\
\text { Alternating Current }\end{array}$} \\
\hline 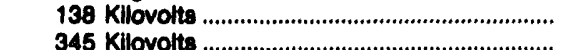 & 389 & 506 & - & - \\
\hline 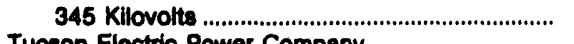 & 134 & 158 & - & - \\
\hline \multicolumn{5}{|l|}{ Tucson Electric Power Company } \\
\hline 138 Kilovolts & 262 & 334 & - & - \\
\hline 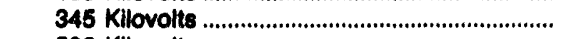 & 1,098 & 1,122 & - & - \\
\hline 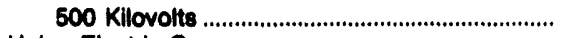 & 1,873 & 1,967 & - & - \\
\hline \multirow{2}{*}{\multicolumn{5}{|c|}{$\begin{array}{l}\text { Union Electric Company } \\
\text { Alternating Current }\end{array}$}} \\
\hline & & & & \\
\hline 161 Kilovolts & 681 & 686 & $=$ & $\overline{-}$ \\
\hline 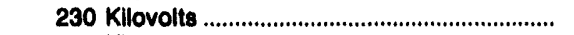 & 90 & 90 & -- & - \\
\hline 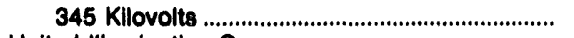 & 658 & 782 & - & -- \\
\hline \multirow{2}{*}{\multicolumn{5}{|c|}{$\begin{array}{l}\text { United Illuminating Company } \\
\text { Alternating Current }\end{array}$}} \\
\hline & & & & \\
\hline $\begin{array}{l}345 \text { Kllovolts } \\
\text { Upper Peninsula Power Company }\end{array}$ & 6 & 6 & - & - \\
\hline \multicolumn{5}{|l|}{$\begin{array}{l}\text { Upper Peninsula Power Company } \\
\text { Alternating Current }\end{array}$} \\
\hline 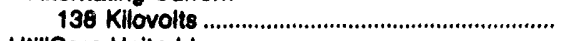 & 348 & 353 & - & - \\
\hline UtilliCorp United Inc & & 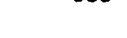 & & \\
\hline \multicolumn{5}{|l|}{ Alternating Current } \\
\hline 138 Kilovolts & 74 & 74 & - & -- \\
\hline 161 Kilovolts & 376 & 406 & - & - \\
\hline 230 Kilovolts & 211 & 211 & - & -- \\
\hline 345 Kilovolts & 58 & 58 & - & - \\
\hline \multirow{2}{*}{\multicolumn{5}{|c|}{$\begin{array}{l}\text { Vermont Electric Power Co Inc } \\
\text { Alternating Current }\end{array}$}} \\
\hline & & & & \\
\hline 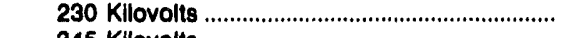 & 33 & 33 & - & -- \\
\hline 345 Kilovolts & 52 & 52 & - & -- \\
\hline \multirow{2}{*}{\multicolumn{5}{|c|}{$\begin{array}{l}\text { Vermont Electric Trans Co Inc } \\
\text { Direct Current } \\
\quad 450 \text { Kilovolts }\end{array}$}} \\
\hline & & & & \\
\hline \\
\hline & & & & \\
\hline 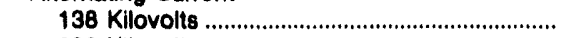 & 119 & 133 & -- & -- \\
\hline 230 Kilovolts & 1,549 & 2,513 & -- & - \\
\hline 500 Kilovolts & 1,008 & 1,008 & -- & - \\
\hline \multirow{2}{*}{\multicolumn{5}{|c|}{$\begin{array}{l}\text { Washington Water Power Company } \\
\text { Alternating Current }\end{array}$}} \\
\hline Alternating Current & & & & \\
\hline 230 Kilovolts & 542 & 544 & -- & - \\
\hline 500 Kilovolts & 113 & 113 & - & - \\
\hline West Penn Power Company & & & & \\
\hline Alternating Current & & & & \\
\hline 138 Kilovolts & 1,098 & 1,335 & -- & - \\
\hline 230 Kilovolts & 72 & 72 & - & -- \\
\hline 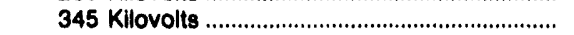 & 6 & 6 & -- & -- \\
\hline 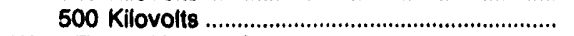 & 273 & 273 & - & -- \\
\hline West Texas Utilities Company & & & 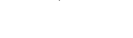 & 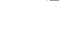 \\
\hline Alternating Current & & & & \\
\hline 138 Kilovolts & 1,502 & 1,502 & - & - \\
\hline 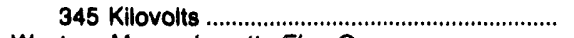 & 221 & 221 & -- & -- \\
\hline Western Massachusetts Elec Co & & & & \\
\hline Alternating Current & & & & \\
\hline 345 Kilovolts & 105 & 105 & - & - \\
\hline Western Resources inc & & & & \\
\hline Alternating Current & & & & \\
\hline 161 Kilovolts & 152 & 152 & - & - \\
\hline 230 Kilovolts & 307 & 307 & - & -- \\
\hline 345 Kilovolts & 336 & 338 & -- & - \\
\hline Wheeling Power Company & & & & \\
\hline Alternating Current & & & & \\
\hline 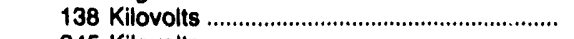 & 95 & 99 & - & - \\
\hline 345 Kilovolts & 9 & 16 & -- & -- \\
\hline 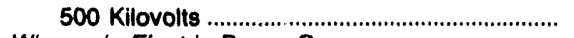 & 16 & 16 & - & - \\
\hline Wisconsin Electric Power Co & & & & \\
\hline Alternating Current & & & & \\
\hline 138 Kilovolts & 1,147 & 1,603 & - & - \\
\hline 230 Killovolts & 52 & 123 & - & - \\
\hline 345 Kilovolts & 575 & 639 & - & - \\
\hline
\end{tabular}

See notes and footnotes at end of table. 
Table 38. Transmiesion Line Statistics of Investor-Owned Utilities, 1992 (Continued)

\begin{tabular}{|c|c|c|c|c|}
\hline \multirow{2}{*}{$\begin{array}{l}\text { Utility I } \\
\text { Voltage of } \\
\text { Tranemiacion } \\
\text { Line }\end{array}$} & \multicolumn{2}{|c|}{ Overhead } & \multicolumn{2}{|c|}{$\begin{array}{l}\text { Underground } \\
\text { Submarine }\end{array}$} \\
\hline & $\begin{array}{l}\text { Struotural } \\
\text { Miles }\end{array}$ & $\begin{array}{l}\text { Circult } \\
\text { Miles }\end{array}$ & $\begin{array}{l}\text { Structural } \\
\text { Miles }\end{array}$ & $\begin{array}{l}\text { Circult } \\
\text { Miles }\end{array}$ \\
\hline \multicolumn{5}{|l|}{$\begin{array}{l}\text { Wisconsin Power \& Light Co } \\
\text { Atternating Current }\end{array}$} \\
\hline 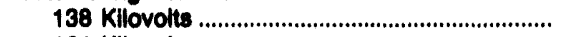 & 651 & 917 & - & - \\
\hline 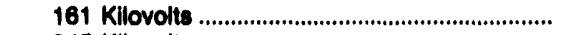 & 1 & 2 & - & - \\
\hline 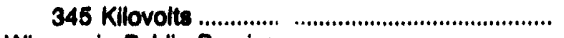 & 100 & 107 & $-\cdots$ & - \\
\hline \multirow{3}{*}{$\begin{array}{l}\text { Wisconsin Public Service orp } \\
\text { Alternating Current } \\
138 \text { Killovolts } \\
345 \text { Kilovolts }\end{array}$} & & & & \\
\hline & 288 & 285 & - & - \\
\hline & 124 & 124 & - & - \\
\hline
\end{tabular}

- Not Applicable

Notes: -Totals may not equal sum of components because of independent rounding. •Voluntary filings of distribution line and transmission line data below 138 kilovolts are not included in this table.

Sources: -Federal Energy Regulatory Commission, FERC Form 1, "Annual Report of Major Electric Utilities, Licensees and Others." •FERC Form 1.F. "Annual Report for Nonmajor Public Utilities and Licensees." 
Table 39. Tranemiealon Line Statiatice of Public Utilities, by NERC Reglon, Fiscal Year 1992

\begin{tabular}{|c|c|c|c|c|}
\hline \multirow{2}{*}{$\begin{array}{l}\text { Voltage of } \\
\text { Trantemiselon } \\
\text { Uns }\end{array}$} & \multicolumn{2}{|c|}{ Overhead } & \multicolumn{2}{|c|}{$\begin{array}{l}\text { Underground } \\
\text { Submarine }\end{array}$} \\
\hline & $\begin{array}{l}\text { Structural } \\
\text { Miles }\end{array}$ & $\begin{array}{l}\text { Circuit } \\
\text { Miles }\end{array}$ & $\begin{array}{c}\text { Structural } \\
\text { Miles }\end{array}$ & $\begin{array}{l}\text { Circ it } \\
\text { Milie s }\end{array}$ \\
\hline & \multicolumn{4}{|c|}{ Publlo villtiee in ECAR } \\
\hline \multirow[t]{2}{*}{ 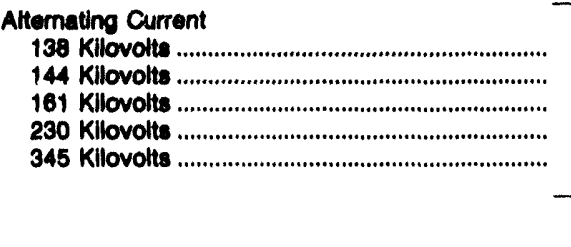 } & $\begin{array}{r}137.87 \\
16.40 \\
13.00 \\
7.00 \\
40.00\end{array}$ & $\begin{array}{r}149.86 \\
16.40 \\
13.00 \\
7.00 \\
40.00\end{array}$ & $\begin{array}{l}6.16 \\
- \\
-\end{array}$ & $\begin{array}{l}6.16 \\
-\end{array}$ \\
\hline & \multicolumn{4}{|c|}{ Publle Utillties in ERCOT } \\
\hline \multirow[t]{2}{*}{$\begin{array}{l}\text { Alternating Current } \\
138 \text { Kllovolts } \\
346 \text { Kilovolts }\end{array}$} & $\begin{array}{r}1,657.09 \\
389.82\end{array}$ & $\begin{array}{r}1,859.25 \\
485.66\end{array}$ & $\overline{-}$ & $\overline{-}$ \\
\hline & \multicolumn{4}{|c|}{ Publlo Utilitios in MAIN } \\
\hline \multirow[t]{2}{*}{ 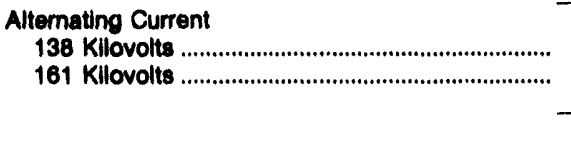 } & $\begin{array}{l}95.86 \\
24.49\end{array}$ & $\begin{array}{r}121.16 \\
24.49\end{array}$ & $\overline{-}$ & - \\
\hline & \multicolumn{4}{|c|}{ Publle Utilities in MAPP } \\
\hline \multirow[t]{2}{*}{ 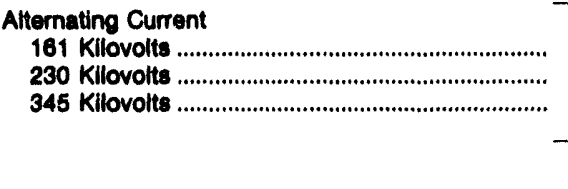 } & $\begin{array}{r}642.03 \\
977.48 \\
2,019.30\end{array}$ & $\begin{array}{r}642.03 \\
977.48 \\
2,033.05\end{array}$ & - & - \\
\hline & \multicolumn{4}{|c|}{ Publle Utilities in NPCC } \\
\hline $\begin{array}{l}\text { Alternating Current } \\
230 \text { Kilovolts } \\
345 \text { Kilovolts } \\
765 \text { Kilovolts }\end{array}$ & $\begin{array}{l}324.29 \\
637.20 \\
154.89\end{array}$ & $\begin{array}{l}324.20 \\
882.20 \\
154.89\end{array}$ & $\overline{26.54}$ & $\begin{array}{l}-\overline{34.38} \\
--\end{array}$ \\
\hline \multirow[t]{2}{*}{ 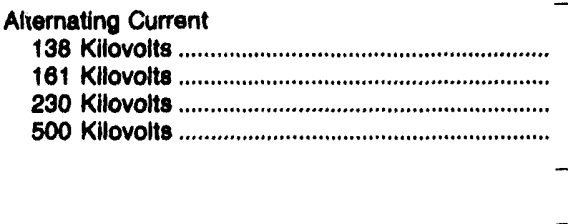 } & $\begin{array}{r}405.18 \\
2.20 \\
1,269.81 \\
74.83\end{array}$ & $\begin{array}{r}495.21 \\
2.20 \\
1,318.98 \\
74.83\end{array}$ & $\begin{array}{l}2.50 \\
- \\
--\end{array}$ & $\begin{array}{c}2.50 \\
- \\
- \\
-\end{array}$ \\
\hline & \multicolumn{4}{|c|}{ Public Utilities in SPP } \\
\hline \multirow[t]{2}{*}{ 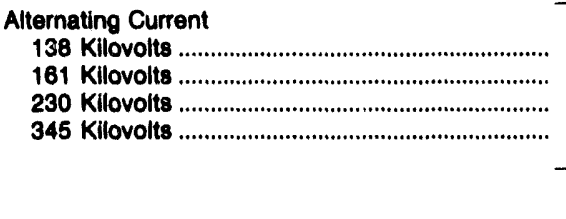 } & $\begin{array}{r}251.94 \\
1,860.33 \\
18.27 \\
158.60\end{array}$ & $\begin{array}{r}251.94 \\
1,968.76 \\
18.27 \\
203.60\end{array}$ & $\bar{z}-$ & $\overline{-}$ \\
\hline & \multicolumn{4}{|c|}{ Publlc Utiblies in Wscc } \\
\hline $\begin{array}{l}\text { Alternating Current } \\
138 \text { Kilovolts } \\
161 \text { Kilovolts } \\
230 \text { Kilovolts } \\
287 \text { Kilovolts } \\
345 \text { Kilovolts } \\
500 \text { Kllovolts }\end{array}$ & $\begin{array}{r}284.47 \\
1,542.09 \\
13,461.34 \\
615.13 \\
2,833.80 \\
5,374.52\end{array}$ & $\begin{array}{r}361.01 \\
1,599.09 \\
14,402.56 \\
615.13 \\
3,038.70 \\
5,755.47\end{array}$ & $\begin{array}{l}35.41 \\
\overline{24.14} \\
\overline{-}\end{array}$ & $\begin{array}{l}65.43 \\
\overline{37.99} \\
- \\
-\end{array}$ \\
\hline $\begin{array}{r}500 \text { Kllovolts } \\
1000 \text { Kllovolts }\end{array}$ & $\begin{array}{l}487.52 \\
529.15\end{array}$ & $\begin{array}{l}487.52 \\
529.15\end{array}$ & - & - \\
\hline
\end{tabular}

See notes and footnotes at end of table. 
Table 39. Tranamleslon Line Statistice of Public Utilities, by NERC Reglon, Flecal Year 1992 (Continued)

\begin{tabular}{|c|c|c|c|c|}
\hline \multirow{2}{*}{$\begin{array}{l}\text { Voltage of } \\
\text { Tranemiesion } \\
\text { Line }\end{array}$} & \multicolumn{2}{|c|}{ Overhead } & \multicolumn{2}{|c|}{$\begin{array}{l}\text { Underground } \\
\text { Submarine }\end{array}$} \\
\hline & $\begin{array}{l}\text { Siructural } \\
\text { Mlles }\end{array}$ & $\begin{array}{l}\text { Circuit } \\
\text { Miles }\end{array}$ & $\begin{array}{c}\text { Structural } \\
\text { Miles }\end{array}$ & $\begin{array}{l}\text { Circult } \\
\text { Miles }\end{array}$ \\
\hline & \multicolumn{4}{|c|}{ Contlouous U.8. Total } \\
\hline $\begin{array}{l}\text { Alternating Current } \\
138 \text { Kllovolts } \\
144 \text { Kllovolts } \\
181 \text { Kllovolts } \\
230 \text { Kllovolts } \\
287 \text { Kllovolts } \\
345 \text { Kllovolts } \\
500 \text { Kllovolts } \\
785 \text { Kllovolts } \\
\text { Direct Current } \\
500 \text { Kllovolts } \\
1000 \text { Kllovolts }\end{array}$ & $\begin{array}{r}2,832.41 \\
16.40 \\
4,084.14 \\
16,058.19 \\
615.13 \\
6,078.82 \\
5,449.35 \\
154.89 \\
\\
487.52 \\
529.15\end{array}$ & $\begin{array}{r}3,238.43 \\
16.40 \\
4,249.57 \\
17,048.58 \\
615.13 \\
6,683.21 \\
5,830.30 \\
154.89 \\
\\
487.52 \\
529.15\end{array}$ & $\begin{array}{l}44.06 \\
- \\
\overline{27.79} \\
\overline{26.54} \\
- \\
- \\
- \\
-\end{array}$ & $\begin{array}{l}74.08 \\
- \\
\overline{41.64} \\
\overline{34.38} \\
-- \\
- \\
- \\
--\end{array}$ \\
\hline
\end{tabular}

Notes: -The States of Alaska and Hawali are not included. -NERC is the North American Electric Reliabllity Council. The Alaska System Coordinating Council is not included. See glossary for a list of regions and Figure 2 for a map of the regions. -Totals may not equal sum of components because of independent rounding. Voluntary filings of distribution line and transmission line data below 138 kilovolts are not included in this table. Sources: •Energy Information Administration, Form ElA-412, "Annual Peport of Public Electric Utilities." 
Table 40. Tranemieslon Line Statiatics of Publlc Utilities, Flacal Year 1992

\begin{tabular}{|c|c|c|c|c|}
\hline \multirow{2}{*}{$\begin{array}{c}\text { Publlo Authortly I } \\
\text { Utility I } \\
\text { Voltases of } \\
\text { Tranamlaelon } \\
\text { Uns }\end{array}$} & \multicolumn{2}{|c|}{ Overhead } & \multicolumn{2}{|c|}{$\begin{array}{l}\text { Underground } \\
\text { Submarine }\end{array}$} \\
\hline & $\begin{array}{l}\text { Structural } \\
\text { Milles }\end{array}$ & $\begin{array}{l}\text { Ciroult } \\
\text { Milles }\end{array}$ & $\begin{array}{c}\text { Structural } \\
\text { Milles }\end{array}$ & $\begin{array}{l}\text { Crrcult } \\
\text { Milles }\end{array}$ \\
\hline \multicolumn{5}{|l|}{ Federal } \\
\hline \multicolumn{5}{|l|}{$\begin{array}{l}\text { Alaaka Power Administration } \\
\text { Alternating Current }\end{array}$} \\
\hline \multirow{2}{*}{\multicolumn{5}{|c|}{$\begin{array}{l}\text { Bonneville Power Admin } \\
\text { Atternating Current }\end{array}$}} \\
\hline & & & & \\
\hline 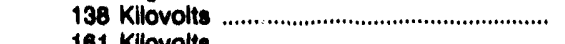 & 92.30 & 92.30 & - & -- \\
\hline $\begin{array}{l}161 \text { Killovolts } \\
230 \text { Kilovolts }\end{array}$ & 46.50 & 46.50 & - & -- \\
\hline 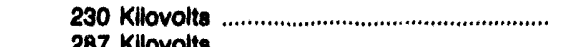 & $4,725.64$ & $4,826.14$ & - & - \\
\hline 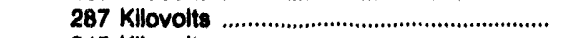 & 227.40 & 227.40 & -- & - \\
\hline 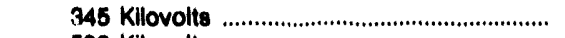 & 343.60 & 423.60 & -- & - \\
\hline 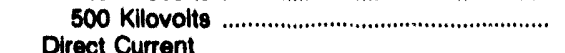 & $4,333.10$ & $4,391.10$ & - & - \\
\hline $\begin{array}{l}\text { Direct Current } \\
1000 \text { Kilovolis ....... }\end{array}$ & 264.40 & 264.40 & $\ldots$ & -- \\
\hline \multicolumn{5}{|l|}{$\begin{array}{l}\text { Southwestern Power Admin } \\
\text { Alternating Current }\end{array}$} \\
\hline 138 Kilovolts & 164.12 & 164.12 & - & -- \\
\hline 161 Kilovolts & $1,120.31$ & $1,179.71$ & - & -- \\
\hline \multicolumn{5}{|l|}{$\begin{array}{l}\text { Western Area Power Admin } \\
\text { Alternating Current }\end{array}$} \\
\hline 138 Kllovolts & 39.10 & 78.20 & - & - \\
\hline 181 Kllovolts ...................................................... & $1,178.79$ & $1,178.79$ & - & -- \\
\hline 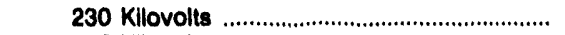 & $6,385.41$ & $6,876.61$ & -- & -- \\
\hline 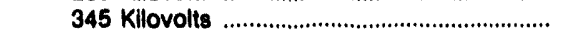 & $1,558.34$ & $1,683.24$ & - & -- \\
\hline 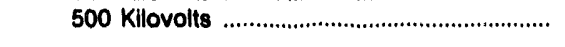 & 288.34 & 288.34 & -- & -- \\
\hline \multicolumn{5}{|l|}{ State and Other Government } \\
\hline \multicolumn{5}{|l|}{ Alaska Energy Authority } \\
\hline 138 Kilovolts & 296.00 & 296.00 & - & -- \\
\hline \multicolumn{5}{|l|}{$\begin{array}{l}\text { Grand River Dam Authority } \\
\text { Alternating Current }\end{array}$} \\
\hline 138 Kilovolts & 61.71 & 61.71 & -. & -- \\
\hline 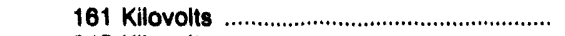 & 287.81 & 332.84 & - & -- \\
\hline 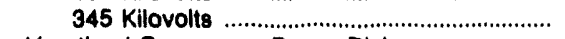 & 113.60 & 113.60 & -- & -- \\
\hline \multicolumn{5}{|l|}{ Heartland Consumers Power Dist } \\
\hline 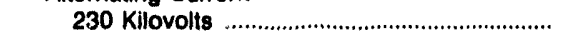 & 210.00 & 210.00 & -- & -- \\
\hline 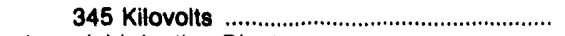 & 536.00 & 536.00 & -- & -- \\
\hline \multirow{2}{*}{\multicolumn{5}{|c|}{ Imperial Irrigation District }} \\
\hline Alternating Current & & & & \\
\hline 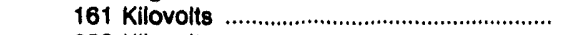 & 302.00 & 359.00 & - & - \\
\hline 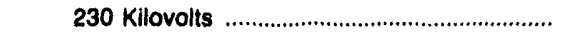 & 144.70 & 144.70 & -- & - \\
\hline 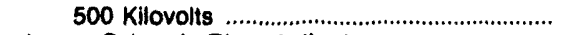 & 161.20 & 322.40 & - & - \\
\hline \multirow{2}{*}{\multicolumn{5}{|c|}{$\begin{array}{l}\text { Lower Colorado River Authority } \\
\text { Alternating Current }\end{array}$}} \\
\hline & & & & \\
\hline 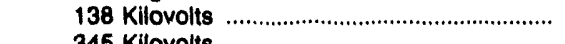 & $1,326.63$ & $1,525.39$ & - & - \\
\hline 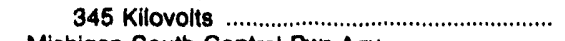 & 210.07 & 305.81 & - & - \\
\hline \multicolumn{5}{|l|}{ Michigan South Central Pwr Agy } \\
\hline \multicolumn{5}{|l|}{ Alternating Current } \\
\hline 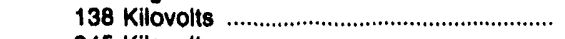 & 2.74 & 3.98 & - & - \\
\hline 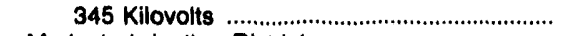 & 40.00 & 40.00 & - & - \\
\hline \multirow{2}{*}{\multicolumn{5}{|c|}{$\begin{array}{l}\text { Modesto Irrigation District } \\
\text { Alternating Current }\end{array}$}} \\
\hline Alternating Current & & & & \\
\hline 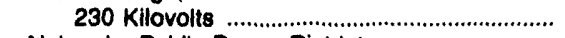 & 65.64 & 65.64 & -- & - \\
\hline \multirow{2}{*}{\multicolumn{5}{|c|}{$\begin{array}{l}\text { Nebraska Public Power District } \\
\text { Alternating Current }\end{array}$}} \\
\hline Alternating Current & & & & \\
\hline 161 Kilovolts & .26 & .26 & - & - \\
\hline 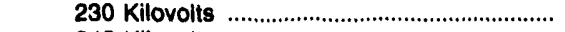 & 676.89 & 676.99 & - & -- \\
\hline 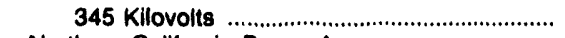 & 886.36 & 886.36 & - & -- \\
\hline Northern California Power Agny & & & & \\
\hline $\begin{array}{l}\text { Alternating Current } \\
230 \text { Kilovolts }\end{array}$ & 41.20 & 44.40 & - & _- \\
\hline
\end{tabular}

See notes and footnotes at end of table. 
Table 40. Tranamieslon Line Statistles of Public Utilities, Flecal Year 1992 (Continued)

\begin{tabular}{|c|c|c|c|c|}
\hline \multirow{2}{*}{$\begin{array}{l}\text { Publlo Authority I } \\
\text { Utility I } \\
\text { Voltegs of } \\
\text { Trunemiedon } \\
\text { Lins }\end{array}$} & \multicolumn{2}{|c|}{ Overhead } & \multicolumn{2}{|c|}{$\begin{array}{l}\text { Underground } \\
\text { Submarine }\end{array}$} \\
\hline & $\begin{array}{c}\text { Structural } \\
\text { Milles }\end{array}$ & $\begin{array}{l}\text { Circuit } \\
\text { Miles }\end{array}$ & $\begin{array}{l}\text { Structural } \\
\text { Miles }\end{array}$ & $\begin{array}{l}\text { Circult } \\
\text { Miles }\end{array}$ \\
\hline
\end{tabular}

\section{State and Other Covernment}

Oklahoma Municipal Power Auth Alternating Current

138 Kilovolts

Omana Public Power District

Alternating Current

161 Kilovolts

345 Kilovolts

Papago Tribal Utility Auth
Alternating Current

230 Kilovolts

Platte River Power Authority

Alternating Current

230 Kilovolts

345 Kilovolts

Power Authority of State of NY
Alternating Current

230 Kilovolts

345 Kilovolts

785 Kilovolts

PUD No 1 of Chelan County

Alternating Current

230 Killovolts

PUD No 1 of Cowlitz County

Alternating Current

230 Kilovolts

PUD No 1 of Douglas County

Alternating Current

230 Kilovolts

PUD No 2 of Grant County

Alternating Current

230 Kllovolts

Sacramento Municipal Util Dist

Alternating Current

230 Kilovolts

South Carolina Pub Serv Auth

Alternating Current

230 Kllovolts

Southern Minnesota Mun P Agny

Alternating Current

161 Kilovolts

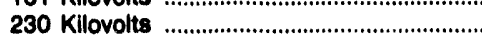

Utah Associated Mun Power Sys

Alternating Current

138 Kilovolts

Utah Municipal Power Agency

Alternating Current

138 Killovolts

345 Kilovolts

Washington Pub Pwr..................

Alternating Current

500 Kllovolts

9.41

9.41

392.58

316.78

10.00

272.20

368.20

324.29

637.20

154.89

23.23

3.83

82.00

139.31

33.00

852.58

122.69

17.30

21.00

292.00

78.40

171.10

23.85

392.58

316.79

10.00

272.20

368.20

324.29

882.20

154.89

23.23

3.83

82.00

139.31

33.00

852.58

122.69

17.30

21.00

292.00

78.40

171.10

23.85

16.20

16.20

180.30

179.85

179.85

Alternating Current

Austin City of

Alternating Current

345 Killovolts

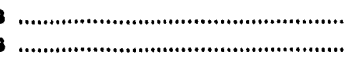

See notes and footnotes at end of table. 
Table 40. Tranamiselon Line 8tatiatice of Publle Utilities, Flecal Yoar 1992 (Continued)

\begin{tabular}{|c|c|c|c|c|}
\hline \multirow{2}{*}{ 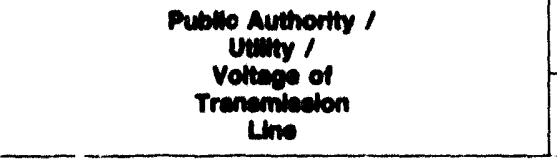 } & \multicolumn{2}{|c|}{ Overhend } & \multicolumn{2}{|c|}{$\begin{array}{l}\text { Underground } \\
\text { Submarine }\end{array}$} \\
\hline & $\begin{array}{l}\text { Structural } \\
\text { Milles }\end{array}$ & $\begin{array}{l}\text { Crroult } \\
\text { Millos }\end{array}$ & $\begin{array}{l}\text { Structural } \\
\text { Milles }\end{array}$ & $\begin{array}{l}\text { Crrcult } \\
\text { Mlles }\end{array}$ \\
\hline \multicolumn{5}{|l|}{ Muniolpeal } \\
\hline 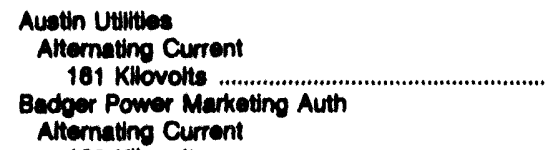 & 36.40 & 30.40 & - & - \\
\hline $\begin{array}{l}138 \text { Kllovolts ................................................. } \\
\text { Bnyan City of } \\
\text { Atternating Current }\end{array}$ & 2.50 & 2.50 & - & - \\
\hline $\begin{array}{l}138 \text { Kllovolts } \\
\text { Codar Falle Mun Electric Uitl } \\
\text { Atternating Current }\end{array}$ & 34.67 & 34.67 & - & - \\
\hline 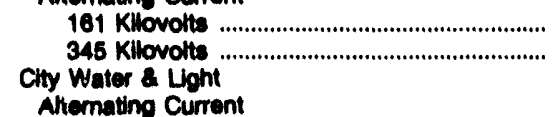 & $\begin{array}{r}4.20 \\
142.45\end{array}$ & $\begin{array}{r}4.20 \\
156.20\end{array}$ & $=$ & $\overline{-}$ \\
\hline 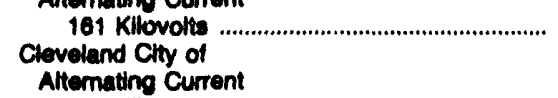 & 339.00 & 339.00 & - & - \\
\hline $\begin{array}{l}138 \text { Kllovolts } \\
\text { Colowwater Board of Public Util } \\
\text { Altennating Current }\end{array}$ & 35.15 & 35.15 & 6.15 & 6.15 \\
\hline $\begin{array}{l}\text { Alimaung Current } \\
130 \text { Kllovolts ........................................... } \\
\text { Colloge Station City of } \\
\text { Alternating Current }\end{array}$ & 2.93 & 2.93 & - & - \\
\hline 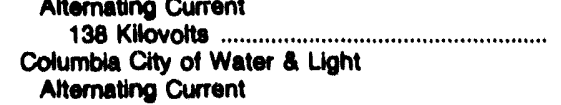 & 11.60 & 11.60 & - & - \\
\hline $\begin{array}{l}161 \text { Kilovolts } \\
\text { Crawtordaville Elec LotsPwi Co } \\
\text { Alternating Current }\end{array}$ & 24.48 & 24.49 & - & - \\
\hline $\begin{array}{l}138 \text { Killovolts } \\
\text { Dation City of } \\
\text { Alternating Current }\end{array}$ & 12.50 & 12.50 & - & - \\
\hline $\begin{array}{l}230 \text { Kilovolts } \\
\text { Danville City of Electric Oiv } \\
\text { Alternating Current }\end{array}$ & 31.55 & 31.55 & - & - \\
\hline $\begin{array}{l}138 \text { Kilovolts } \\
\text { Floresville Elec Lgt \& Pwr Sys } \\
\text { Allernating Current }\end{array}$ & 1.36 & 2.71 & - & - \\
\hline $\begin{array}{l}138 \text { Kilovolts } \\
\text { Gainesville City of } \\
\text { Alternating Current }\end{array}$ & 12.00 & 12.00 & - & - \\
\hline 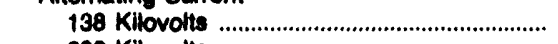 & 127.24 & 206.04 & - & - \\
\hline $\begin{array}{l}230 \text { Kilovolts } \\
\text { Gigndale City of } \\
\text { Direct Current }\end{array}$ & 2.51 & 2.51 & -- & - \\
\hline $\begin{array}{l}1000 \text { Killovolts } \\
\text { Greenville Eloctric Uti Sys } \\
\text { Alternating Current }\end{array}$ & 32.54 & 32.54 & - & - \\
\hline $\begin{array}{l}138 \text { Kilovolts } \\
\text { Hamilton Dept of Public Utilis } \\
\text { Alternating Current }\end{array}$ & 56.00 & 56.00 & - & - \\
\hline $\begin{array}{l}138 \text { Kllovolts } \\
\text { Henderson City Utillty Commiss } \\
\text { Alternating Current }\end{array}$ & 23.25 & 23.25 & - & - \\
\hline $\begin{array}{l}161 \text { Kilovolts } \\
\text { Homestead Clty of } \\
\text { Alternating Current }\end{array}$ & 13.00 & 13.00 & - & - \\
\hline $\begin{array}{l}138 \text { Kilovolts } \\
\text { Hutchinson Utilitios Comm } \\
\text { Alternating Current }\end{array}$ & 2.00 & 2.00 & - & - \\
\hline 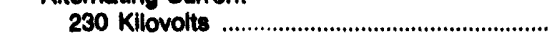 & 38.30 & 38.30 & - & - \\
\hline
\end{tabular}

See notes and footnotes at end of table. 

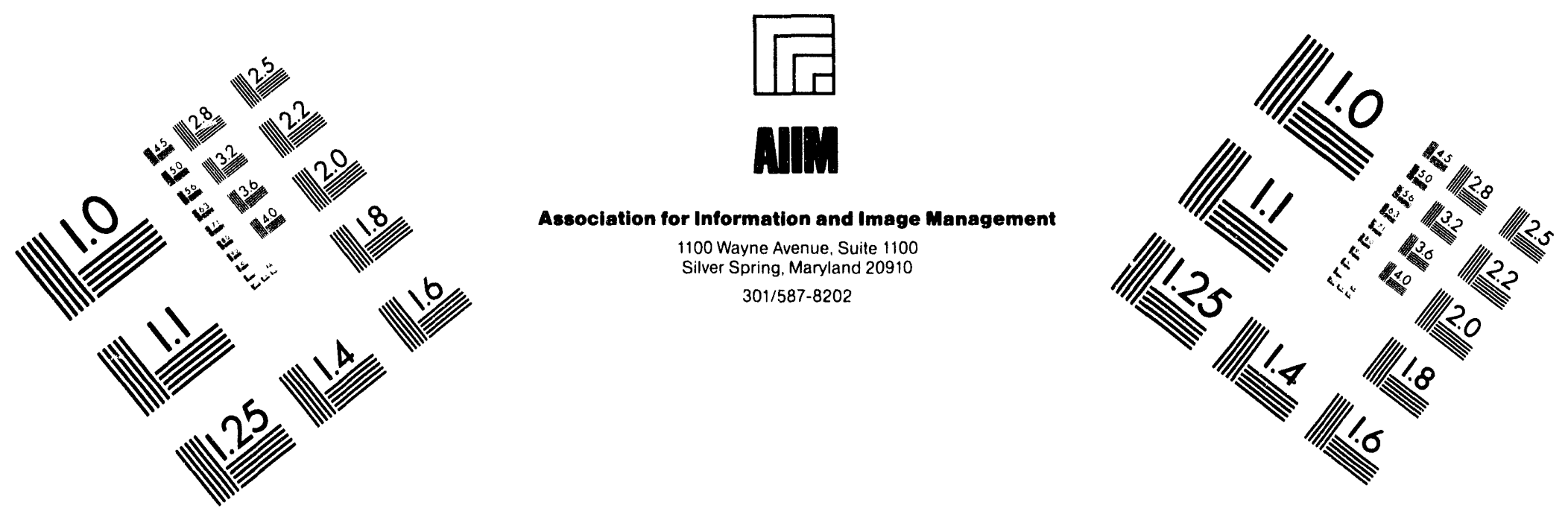

\section{Centimeter}

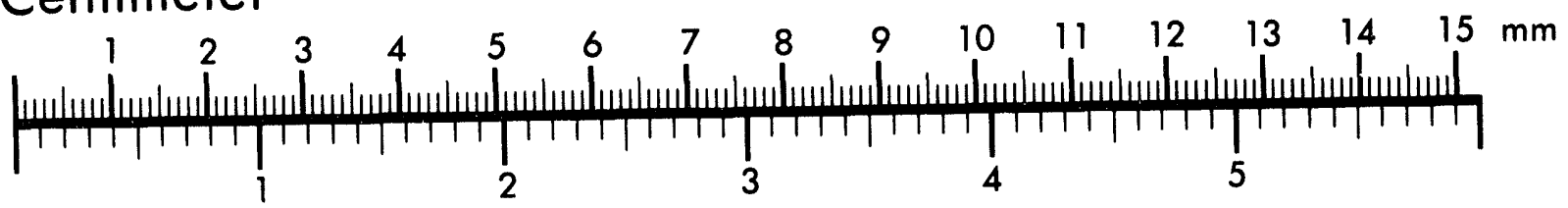
Inches
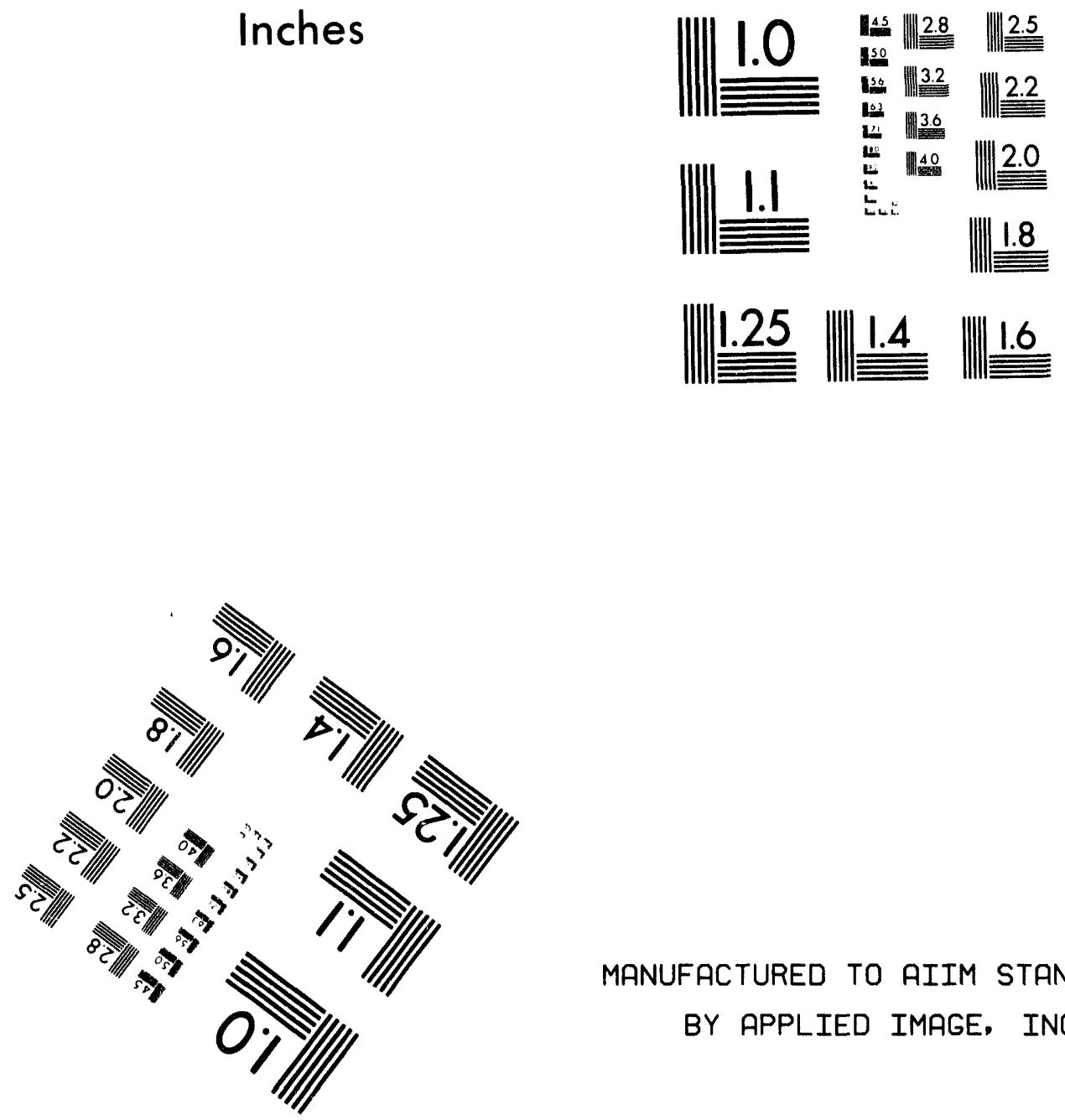

MANUFACTURED TO AIIM STANDARDS

BY APPLIED IMAGE, INC.

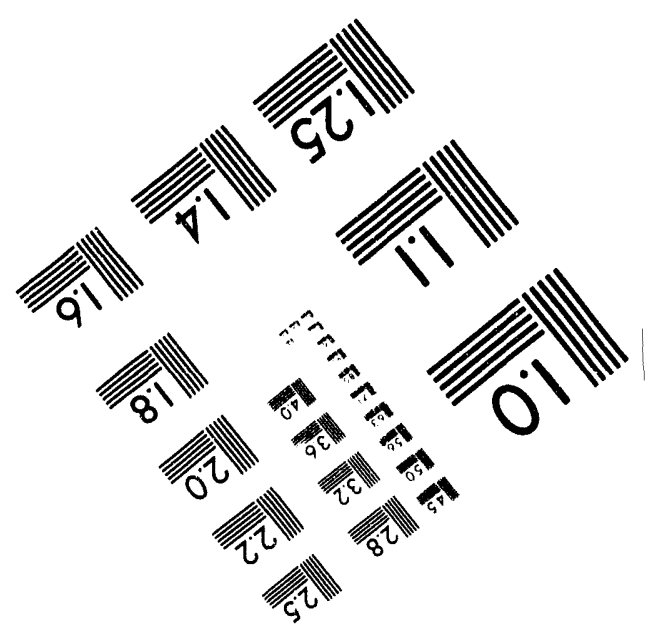



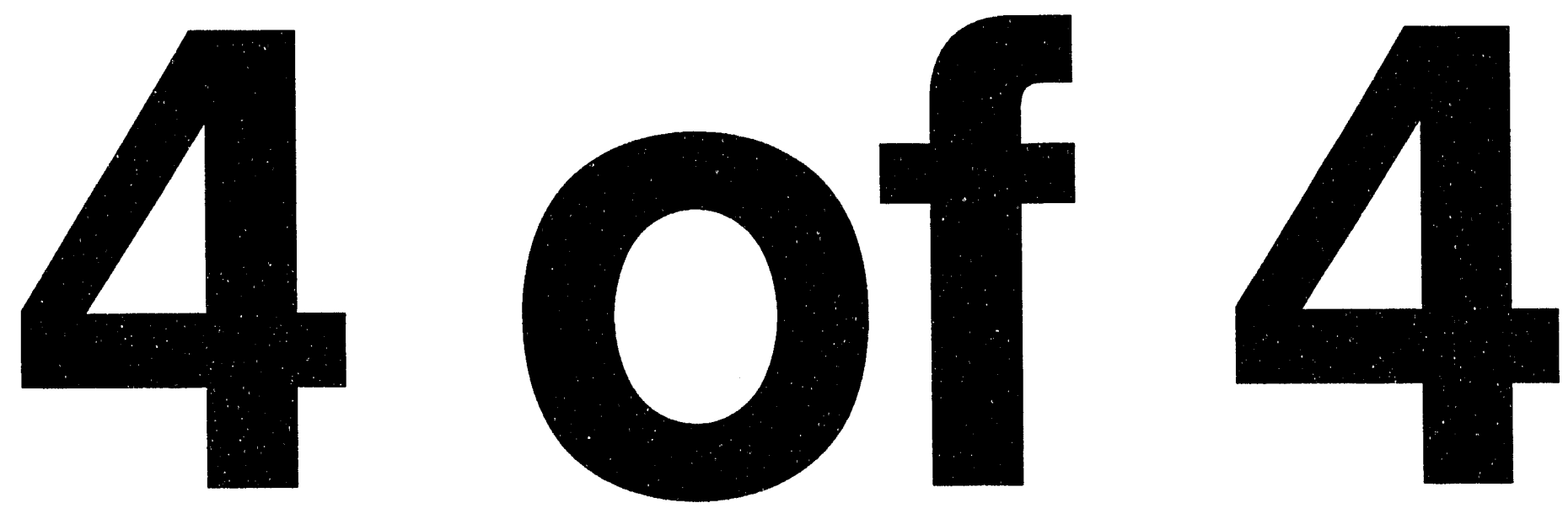
Table 40. Transmission Line Statistics of Public Utilities, Flscal Year 1992 (Continued)

\begin{tabular}{|c|c|c|c|c|}
\hline \multirow{2}{*}{$\begin{array}{c}\text { Public Authority / } \\
\text { Uutlty / } \\
\text { Volises of } \\
\text { Tranamiaclon } \\
\text { Line }\end{array}$} & \multicolumn{2}{|c|}{ Overhead } & \multicolumn{2}{|c|}{$\begin{array}{l}\text { Underground } \\
\text { Submarine }\end{array}$} \\
\hline & $\begin{array}{c}\text { Structural } \\
\text { Miles }\end{array}$ & $\begin{array}{l}\text { Circuit } \\
\text { Miles }\end{array}$ & $\begin{array}{l}\text { Structural } \\
\text { Milles }\end{array}$ & $\begin{array}{l}\text { Circult } \\
\text { Miles }\end{array}$ \\
\hline
\end{tabular}

\section{Municlpal}

Idaho Falls City of

Alternating Current

161 Kilovolts

Independence City of

Alternating Current

161 Kilovolts .

14.80

14.80

Antermountain Power Agency

Altomating Current

230 Kilovolts

Direct Current

500 Kilovolts

Jacksonville Beach City of

Alternating Current

138 Kllovolts

Jacksonville Electric Auth

Alternating Current

138 Kilovolts

230 Killovolts

500 Killovolts

Key West Utility Board of

Alternating Current

138 Kilovolts

Kissimmee Utility Authority

Alternating Current

230 Kilovolts

Lafayette City of

Alternating Current

230 Killovolts

Lake Worth City of

Alternating Current

138 Kilovolts

Lakeland City of

Alternating Current

230 Kilovolts

Lansing Board of Water \& Light

Alternating Current

138 Kilovolts ...

Lincoln Electric System

Alternating Current

161 Kllovolts

345 Kilovolts

Logansport Municipal Utilities

Alternating Current

230 Killovolts

Los Angeles Dept of Wtr \& PW

Alternating Current

138 Kilovolts

230 Kilovolts

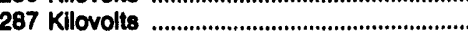

345 Kilovolts

500 Kilovolts

Direct Current

1000 Kilovolts

Louleville Electric Syatem

Alternating Current

161 Kilovolts

Lubbock City of

Alternating Current

230 Kilovolts

22.60

22.60

141.41

141.41

487.52

487.52

41.00

41.00

183.53

182.95

194.76

74.83

222.99
74.83

2.50

3.65

2.50

46.11

46.11

2.96

2.96

14.00

14.00

5.30

5.30

16.81

16.81

40.64

50.04

11.80

11.80

49.70

49.70

7.00

7.00

51.48

87.15

706.96

387.73

100.56

387.73

729.78

568.03

232.21

232.21

2.20

2.20

4.27

Marshall City of

Alternating Current

161 Kilovolts

4.27

17.00

35.41

$\begin{array}{ll}35.41 & 65.43\end{array}$

$\begin{array}{ll}14.47 & 28.32\end{array}$

$-$

$-$

-

$-$

$-$

17.00

See notes and footnotes at end of table. 
Table 40. Tranemiselon Line Statistles of Publlc Utilities, Fiscal Year 1992 (Continued)

\begin{tabular}{|c|c|c|c|c|}
\hline \multirow{2}{*}{$\begin{array}{l}\text { Public Authority I } \\
\text { Utilly I } \\
\text { Voltage of } \\
\text { Tranemieston } \\
\text { Uine }\end{array}$} & \multicolumn{2}{|c|}{ Overhead } & \multicolumn{2}{|c|}{$\begin{array}{l}\text { Underground } \\
\text { Submarine }\end{array}$} \\
\hline & $\begin{array}{l}\text { Structural } \\
\text { Miles }\end{array}$ & $\begin{array}{l}\text { Circult } \\
\text { Miles }\end{array}$ & $\begin{array}{l}\text { Structural } \\
\text { Mlles }\end{array}$ & $\begin{array}{l}\text { Circuit } \\
\text { Miles }\end{array}$ \\
\hline \multicolumn{5}{|l|}{ Munleipal } \\
\hline $\begin{array}{l}\text { Menasha Electric \& Water Util } \\
\text { Alternating Current } \\
138 \text { Kllovolts ... } \\
\text { Metropolitan Water District.................................... } \\
\text { Alternating Current }\end{array}$ & 15.07 & 15.07 & - & - \\
\hline $\begin{array}{l}230 \text { Kilovolts } \\
\text { Murray City Power Department } \\
\text { Alternating Current }\end{array}$ & 305.60 & 305.60 & - & - \\
\hline 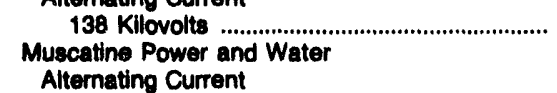 & 1.78 & 3.58 & - & - \\
\hline $\begin{array}{l}161 \text { Kilovolts } \\
\text { Naperville City of } \\
\text { Alternating Current }\end{array}$ & 46.30 & 46.30 & - & - \\
\hline $\begin{array}{l}138 \text { Kilovolts } \\
\text { Natchitoches City of } \\
\text { Alternating Current }\end{array}$ & 18.58 & 18.58 & - & - \\
\hline $\begin{array}{l}138 \text { Kilovolts } \\
\text { New Braunfels Utilities } \\
\text { Alternating Current }\end{array}$ & .50 & .50 & - & -- \\
\hline $\begin{array}{l}138 \text { Kilovolts ... } \\
\text { Orlando Utilities Commission } \\
\text { Alternating Current }\end{array}$ & 34.42 & 37.82 & - & - \\
\hline $\begin{array}{l}230 \text { Kilovolts } \\
\text { Orville City of } \\
\text { Alternating Current }\end{array}$ & 110.24 & 119.37 & - & - \\
\hline $\begin{array}{l}138 \text { Kllovolts } \\
\text { Owensboro Municipal Utilities } \\
\text { Aiternating Current }\end{array}$ & 8.80 & 8.80 & - & - \\
\hline $\begin{array}{l}\text { Provo City Corporation } \\
\text { Alternating Current }\end{array}$ & 16.40 & 16.40 & - & - \\
\hline $\begin{array}{l}138 \text { Kilovolts } \\
\text { Rochester Public Utilitios } \\
\text { Alternating Current }\end{array}$ & .40 & .40 & - & - \\
\hline $\begin{array}{l}161 \text { Kilovolts } \\
\text { San Francisco City \& County of } \\
\text { Alternating Current }\end{array}$ & 27.80 & 27.80 & - & - \\
\hline $\begin{array}{l}230 \text { Kilovolts } \\
\text { Seattle City Light } \\
\text { Alternating Current }\end{array}$ & 50.70 & 101.40 & - & - \\
\hline $\begin{array}{l}230 \text { Kilovolts } \\
\text { Seguin City of } \\
\text { Alternating Current }\end{array}$ & 305.23 & 482.13 & 9.67 & 9.67 \\
\hline $\begin{array}{l}138 \text { Kilovolts } \\
\text { Sikeston Board of Mun Utils } \\
\text { Alternating Current }\end{array}$ & 1.97 & 1.97 & - & - \\
\hline $\begin{array}{l}161 \text { Kilovolts } \\
\text { Springfield Clty of } \\
\text { Alternating Current }\end{array}$ & 28.00 & 28.00 & - & - \\
\hline $\begin{array}{l}161 \text { Kllovolts } \\
345 \text { Kllovolts } \\
\text { Springfield Water Light\&Power } \\
\text { Alternating Current }\end{array}$ & $\begin{array}{l}45.61 \\
45.00\end{array}$ & $\begin{array}{l}49.61 \\
90.00\end{array}$ & - & $\overline{-}$ \\
\hline $\begin{array}{l}138 \text { Kilovolts } \\
\text { Tacoma Dept of Public Utils } \\
\text { Alternating Current }\end{array}$ & 59.71 & 85.01 & - & - \\
\hline $\begin{array}{l}230 \text { Kllovolts } \\
\text { Tallahasseo City of } \\
\text { Alternating Current }\end{array}$ & 31.00 & 83.00 & - & - \\
\hline 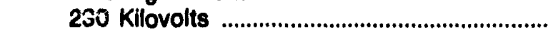 & 39.10 & 39.10 & - & - \\
\hline
\end{tabular}

See notes and footnotes at end of table. 
Table 40. Tranemiselon Line Statistics of Public Utilities, Fiscal Year 1992 (Continued)

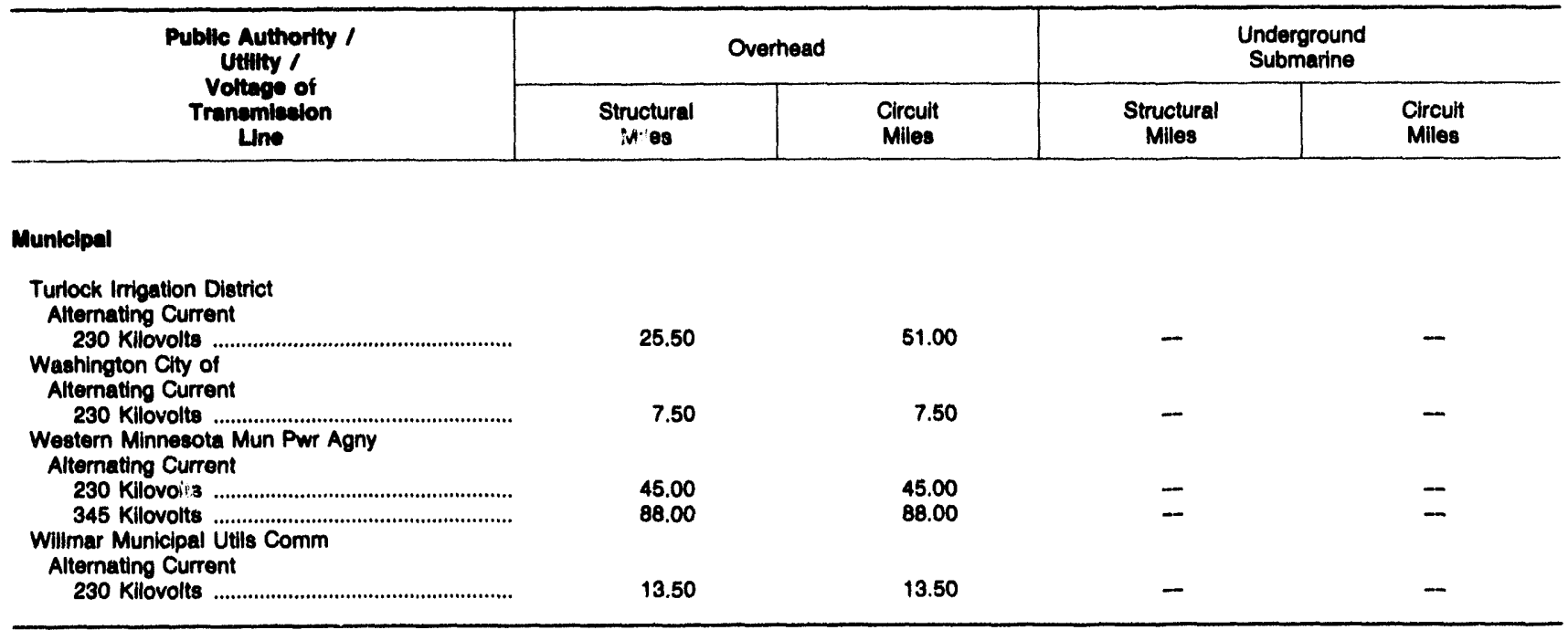

Notes: - Totals may not equal sum of components because of independent rounding. •Voluntary filings of distribution line and transmission line data below 138 kilovolts are not included in this table.

Sources: •Energy Information Administration, Form ElA-412, "Annual Report of Public Electric Utilities." 
Table 41. Transmisalon Lines Added by Investor-Owned Utillties, by NERC Reglon, 1992

\begin{tabular}{|c|c|c|c|c|c|}
\hline \multirow{2}{*}{$\begin{array}{l}\text { Votiage of } \\
\text { Tranembelon Line }\end{array}$} & \multirow{2}{*}{$\begin{array}{l}\text { Lne } \\
\text { Length } \\
\text { (miles) }\end{array}$} & \multicolumn{4}{|c|}{ Line Cost (dollars) } \\
\hline & & Land & Structure & Conductors & Total \\
\hline & \multicolumn{5}{|c|}{ Investor-Owned Utiltibe In ECAR } \\
\hline \multirow[t]{2}{*}{ 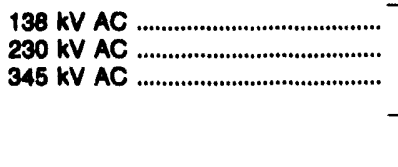 } & $\begin{array}{r}117.66 \\
94.42 \\
20.00\end{array}$ & $\begin{array}{r}4,385,633 \\
3,933,820 \\
246,768\end{array}$ & $\begin{array}{r}13,741,559 \\
11,636,106 \\
5,934,330\end{array}$ & $\begin{array}{r}12,621,654 \\
8,854,043 \\
2,839,381\end{array}$ & $\begin{array}{r}30,748,846 \\
24,423,969 \\
9,020,479\end{array}$ \\
\hline & \multicolumn{5}{|c|}{ Investor-Owned Utilities In ERCOT } \\
\hline \multirow[t]{2}{*}{ 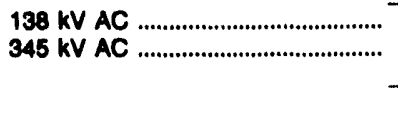 } & $\begin{array}{r}431.05 \\
37.68\end{array}$ & $\begin{array}{l}309,983 \\
-\end{array}$ & $1,125,353$ & 834,949 & $2,270,295$ \\
\hline & \multicolumn{5}{|c|}{ Investor-Owned Utiltiles in MAAC } \\
\hline \multirow[t]{2}{*}{$\begin{array}{l}138 \mathrm{kV} \text { AC } \\
220 \mathrm{kV} \text { AC } \\
280 \mathrm{kV} \text { AC } \\
500 \mathrm{kV} \text { AC }\end{array}$} & $\begin{array}{r}4.63 \\
-\quad 37.71 \\
-\end{array}$ & $\begin{array}{r}75,068 \\
4,951,579 \\
9,285,339 \\
22,331\end{array}$ & $\begin{array}{r}17,228,759 \\
8,510,483 \\
14,519,133 \\
-\end{array}$ & $\begin{array}{r}6,640,694 \\
4,691,544 \\
12,033,269 \\
-\end{array}$ & $\begin{array}{r}26,488,660 \\
18,153,606 \\
35,837,741 \\
22,331\end{array}$ \\
\hline & \multicolumn{5}{|c|}{ Investor-Owned Utillities in MAIN } \\
\hline \multirow[t]{2}{*}{ 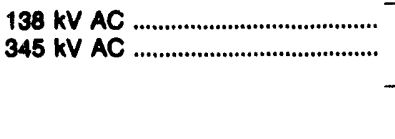 } & $\begin{array}{l}87.88 \\
76.30\end{array}$ & $\begin{array}{l}1,814,806 \\
1,629,103\end{array}$ & $\begin{array}{r}5,395,351 \\
14,085,041\end{array}$ & $\begin{array}{r}4,743,639 \\
15,381,572\end{array}$ & $\begin{array}{l}11,953,786 \\
31,095,716\end{array}$ \\
\hline & \multicolumn{5}{|c|}{ Inveator-Owned Utiltibe in MAPP } \\
\hline \multirow[t]{2}{*}{ 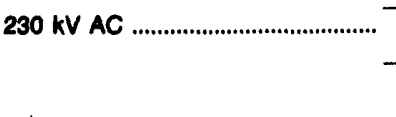 } & 0.05 & - & 65,364 & 63,548 & 128,912 \\
\hline & \multicolumn{5}{|c|}{ Investor-Owned Utiltiles in NPCC } \\
\hline \multirow[t]{2}{*}{$\begin{array}{l}138 \text { kV AC } \\
345 \text { kV AC }\end{array}$} & $\begin{array}{r}4.31 \\
.05\end{array}$ & $\overline{35,650}$ & $\begin{array}{r}8,165,918 \\
238,932\end{array}$ & $\frac{6,477,977}{-}$ & $\begin{array}{r}14,643,895 \\
274,582\end{array}$ \\
\hline & \multicolumn{5}{|c|}{ Invector-Owned Utilities In sERC } \\
\hline \multirow[t]{2}{*}{$\begin{array}{l}138 \text { kV AC } \\
230 \text { kV AC }\end{array}$} & $\begin{array}{l}255.38 \\
158.13\end{array}$ & $\begin{array}{r}303,787 \\
3,181,668\end{array}$ & $\begin{array}{r}5,622,703 \\
14,018,358\end{array}$ & $\begin{array}{r}4,901,778 \\
14,036,102\end{array}$ & $\begin{array}{l}10,806,948 \\
31,236,129\end{array}$ \\
\hline & \multicolumn{5}{|c|}{ Inveetor-Owned Uttlities in SPP } \\
\hline \multirow[t]{2}{*}{ 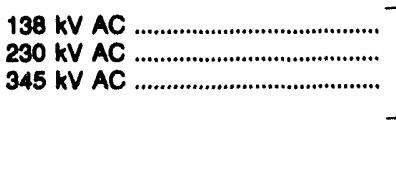 } & $\begin{array}{r}27.95 \\
34.13 \\
102.99\end{array}$ & $\begin{array}{r}, 398,730 \\
642,600 \\
9,540\end{array}$ & $\begin{array}{r}5,170,748 \\
5,489,748 \\
116,535\end{array}$ & $\begin{array}{l}2,932,791 \\
3,637,837 \\
2,595,662\end{array}$ & $\begin{array}{r}11,041,894 \\
9,770,185 \\
2,702,657\end{array}$ \\
\hline & \multicolumn{5}{|c|}{ Investor-Owned Utilties in Wscc } \\
\hline \multirow[t]{2}{*}{ 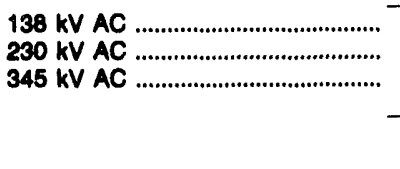 } & $\begin{array}{r}18.70 \\
708.44 \\
346.70\end{array}$ & $\begin{array}{r}330,899 \\
5,861,031 \\
1,498,843\end{array}$ & $\begin{array}{r}1,977,898 \\
37,994,994 \\
21,887,974\end{array}$ & $\begin{array}{r}545,736 \\
25,421,855 \\
8,291,158\end{array}$ & $\begin{array}{r}2,854,531 \\
69,377,880 \\
31,677,975\end{array}$ \\
\hline & \multicolumn{5}{|c|}{ Contiguous U.S. Total } \\
\hline $\begin{array}{l}138 \mathrm{kV} \text { AC } \\
220 \mathrm{kV} \text { AC } \\
230 \mathrm{kV} \text { AC } \\
345 \mathrm{kV} \text { AC } \\
500 \mathrm{kV} \text { AC }\end{array}$ & $\begin{array}{r}947.54 \\
- \\
1,030.88 \\
583.72 \\
-\end{array}$ & $\begin{array}{r}8,618,916 \\
4,851,579 \\
23,004,458 \\
3,418,804 \\
22,331\end{array}$ & $\begin{array}{r}58,428,287 \\
8,510,483 \\
83,723,703 \\
42,262,812 \\
\end{array}$ & $\begin{array}{r}39,699,218 \\
4,691,544 \\
64,046,654 \\
29,107,773 \\
-\end{array}$ & $\begin{array}{r}110,808,865 \\
18,153,606 \\
170,774,816 \\
74,771,409 \\
22,331\end{array}$ \\
\hline
\end{tabular}

- Not Applicable

Notes: The States of Alaska and Hawall are not included. -NERC is the North American Electric Rellability Council. The Alaska System Coordinating Council is not included. See gloseary for a list of reglons and Figure 2 for a map of the reglons. Totals may not equal sum of components because of independent rounding. -Voluntary filings of diatribution line and transmisaion line data below 138 kilovolts are not included in this table.

Sources: -Federal Energy Regulatory Commiseion, FERC Form 1, "Annual Report of Major Electric Utilties, Llcensees and Others." -FERC Form 1-F. "Annual Report for Nonmajor Public Utilities and Licensees." 
Table 42. Transmlesion Lines Added by Investor-Owned Utilities, 1992

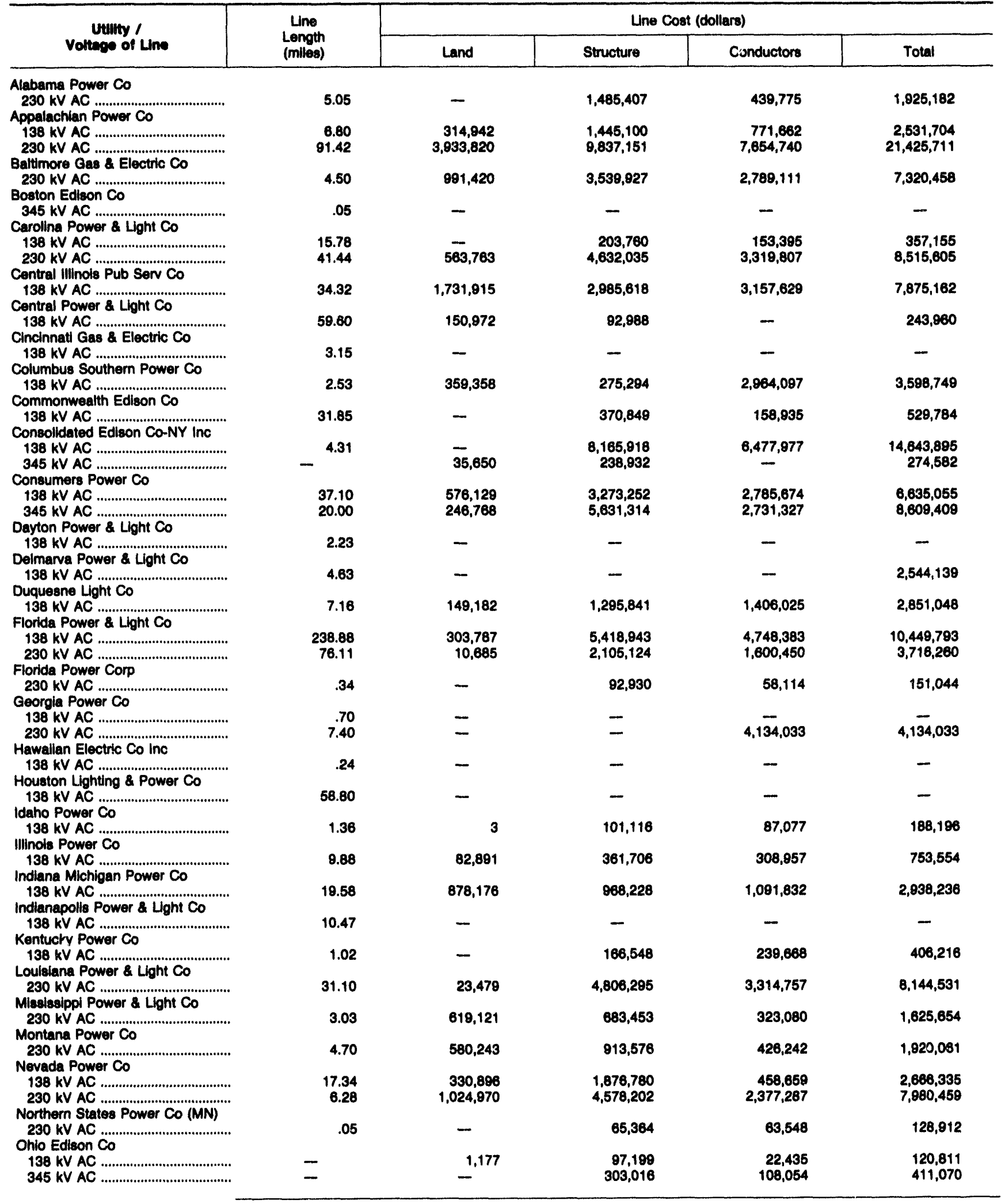

See notes and footnotes at end of table. 
Table 42. Transmission Lines Added by Investor-Owned Utilitles, 1992 (Continued)

\begin{tabular}{|c|c|c|c|c|c|}
\hline \multirow{2}{*}{$\begin{array}{l}\text { Utillty I } \\
\text { Voltage of Line }\end{array}$} & \multirow{2}{*}{$\begin{array}{c}\text { Line } \\
\text { Length } \\
\text { (miles) } \\
\end{array}$} & \multicolumn{4}{|c|}{ Line Cost (dollars) } \\
\hline & & Land & Structure & Conductors & Total \\
\hline \multicolumn{6}{|l|}{$\begin{array}{l}\text { Onio Power Co } \\
138 \mathrm{kV} \mathrm{AC} . . .\end{array}$} \\
\hline \multicolumn{6}{|l|}{ Oklahoma Gas \& Electric Co } \\
\hline 138 kV AC & $-\quad 4.48$ & $\begin{array}{r}1,031,263 \\
9,540\end{array}$ & $\begin{array}{r}4,319,566 \\
116,535\end{array}$ & $\begin{array}{r}1,859,802 \\
2,075\end{array}$ & $\begin{array}{r}7,310,631 \\
109,070\end{array}$ \\
\hline \multicolumn{6}{|l|}{ Pacific Gas \& Electric Co } \\
\hline $\begin{array}{l}230 \mathrm{kV} \text { AC } \\
\text { Pennsylvania Power \& Light Co }\end{array}$ & 17.59 & - & - & - & - \\
\hline $230 \mathrm{kV}$ AC & 22.23 & - & 292,935 & $3,971,452$ & $4,264,387$ \\
\hline \multicolumn{6}{|l|}{ 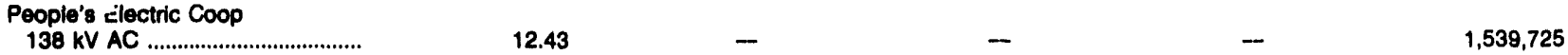 } \\
\hline \multicolumn{6}{|l|}{$\begin{array}{l}\text { Philadelphia Eloctric Co } \\
220 \mathrm{kV} \mathrm{AC}\end{array}$} \\
\hline & - & $4,951,579$ & $8,510,483$ & $4,691,544$ & $18,153,606$ \\
\hline \multicolumn{6}{|l|}{ Potomac Electric Power Co } \\
\hline \multicolumn{4}{|l|}{ Public Service Co of Colorado' } & 145,930 & 187,707 \\
\hline $\begin{array}{l}230 \mathrm{kV} \text { AC } \\
345 \mathrm{kV} \text { AC }\end{array}$ & $\begin{array}{l}655.06 \\
346.70\end{array}$ & $\begin{array}{l}3,567,132 \\
1,498,843\end{array}$ & $\begin{array}{l}31,130,647 \\
21,887,974\end{array}$ & $\begin{array}{r}20,258,072 \\
8,291,158\end{array}$ & $\begin{array}{l}54,955,851 \\
31,677,975\end{array}$ \\
\hline \multicolumn{6}{|l|}{ Public Service Co of Oklahoma } \\
\hline \multicolumn{5}{|l|}{ Public Service Electric\&Gas Co } & $1,864,900$ \\
\hline $\begin{array}{l}138 \mathrm{kV} \text { AC } \\
230 \mathrm{kV} \text { AC }\end{array}$ & $-{ }_{10.41}$ & $\begin{array}{r}75,068 \\
8,293,919\end{array}$ & $\begin{array}{l}17,228,759 \\
10,644,494\end{array}$ & $\begin{array}{l}6,640,684 \\
5,126,776\end{array}$ & $\begin{array}{l}23,944,521 \\
24,065,189\end{array}$ \\
\hline $\begin{array}{l}500 \text { kV AC } \\
\text { Puggt Sound Power \& Light Co }\end{array}$ & \multicolumn{3}{|c|}{$\begin{array}{l}\text { Puget Sound Power \& Light Co } \\
230 \text { kV AC }\end{array}$} & -- & 22,331 \\
\hline & $1,372,569$ & $2,360,254$ & $4,521,509$ \\
\hline $138 \mathrm{kV}$ AC & $\begin{array}{l}4.00 \\
3.00\end{array}$ & 65,923 & $\begin{array}{r}691,702 \\
1,798,955\end{array}$ & $\begin{array}{r}372,455 \\
1,199,303\end{array}$ & $\begin{array}{l}1,130,080 \\
2,998,258\end{array}$ \\
\hline \multicolumn{6}{|l|}{ South Carolina ElectricaGas Co } \\
\hline \multirow{2}{*}{\multicolumn{6}{|c|}{$\begin{array}{l}230 \mathrm{kV} \text { AC } \\
\text { Southern Indiana Gas \& Elec Co } \\
138 \mathrm{kV} \text { AC }\end{array}$}} \\
\hline & 7.08 & - & 590,731 & 355,501 & 946,232 \\
\hline \multicolumn{6}{|l|}{$\begin{array}{l}\text { St Joseph Light \& Power Co } \\
345 \mathrm{kV} \text { AC }\end{array}$} \\
\hline \multicolumn{5}{|l|}{ Texas Utilties Electric Co } & $2,593,587$ \\
\hline $138 \mathrm{kV}$ AC & 277.06 & - & - & - & - \\
\hline 345 kV AC & 37.68 & - & - & - & - \\
\hline \multicolumn{6}{|l|}{$\begin{array}{l}\text { Utilicorp United Inc } \\
138 \text { kV AC }\end{array}$} \\
\hline \multicolumn{6}{|l|}{$\begin{array}{l}138 \mathrm{kV} \text { AC } \\
\text { Virginia Electric \& Power Co }\end{array}$} \\
\hline $\begin{array}{r}230 \mathrm{kV} \mathrm{AC} \\
\text {.... }\end{array}$ & 19.79 & $2,193,922$ & $4,918,673$ & $3,382,987$ & $10,495,582$ \\
\hline \multicolumn{4}{|l|}{$\begin{array}{l}\text { West Penn Power Co } \\
138 \mathrm{kV} \text { AC }\end{array}$} & & \\
\hline West Texas Utilties Co & & & & 100,400 & $3,883,849$ \\
\hline $\begin{array}{l}138 \mathrm{kV} \text { AC } \\
\text { Wisconsin Electric Power Co }\end{array}$ & 35.59 & 159,021 & $1,032,365$ & 834,949 & $2,026,335$ \\
\hline 138 kV AC & 11.83 & - & $1,677,178$ & $1,118,118$ & $2,795,296$ \\
\hline 345 kV AC & 78.30 & $1,629,103$ & $14,085,041$ & $15,381,572$ & $31,095,716$ \\
\hline
\end{tabular}

- Public Service Company of Colorado purchased the transmission assets of a bankrupt cooperative. These numbers reflect the addition of new assets to the ratebase of Public Service Company cf Colorado.

- Not Appllcable

Notes: Totals may not equal sum of components because of independent rounding. • Voluntary filings of distribution line and transmission line date below 138 kilovolts are not included in this table.

Sources: •Federal Energy Regulatory Commission, FERC Form 1, "Annual Report of Major Electric Utilities, Licensees and Others." •FERC Form 1.F, "Annual Report for Nonmajor Public Utilities and Licensees." 
Table 43. Watthour Meter and Line Transformer Statistics of Investor-Owned Utilitles, by NERC Region, 1992 (Count and Megavoltampere)

\begin{tabular}{|c|c|c|c|c|c|c|c|}
\hline $\begin{array}{l}\text { Diotribution } \\
\text { Equlpmont }\end{array}$ & $\begin{array}{l}\text { Number at } \\
\text { Beginning } \\
\text { of Year }\end{array}$ & $\begin{array}{l}\text { Additions } \\
\text { During } \\
\text { Year }\end{array}$ & $\begin{array}{l}\text { Reductions } \\
\text { During } \\
\text { Year }\end{array}$ & $\begin{array}{l}\text { Number at } \\
\text { End of } \\
\text { Year }\end{array}$ & $\begin{array}{l}\text { In Stock, } \\
\text { Locked, } \\
\text { and Inactive }\end{array}$ & $\begin{array}{l}\ln \\
\text { Customers } \\
\text { Use }\end{array}$ & $\begin{array}{l}\text { In } \\
\text { Company } \\
\text { Use }\end{array}$ \\
\hline & \multicolumn{7}{|c|}{ Investor-Owned Utilities In ECAR } \\
\hline \multirow[t]{2}{*}{$\begin{array}{l}\text { Watthour Meter Count .................... } \\
\text { Line Transformer Count ............... } \\
\text { Line Transformer Capacity (MVa) }\end{array}$} & $\begin{array}{r}14,803,337 \\
3,987,528 \\
140,279\end{array}$ & $\begin{array}{r}533,413 \\
136,124 \\
5,088\end{array}$ & $\begin{array}{r}383,896 \\
71,940 \\
2,053\end{array}$ & $\begin{array}{r}14,952,854 \\
4,051,712 \\
143,314\end{array}$ & $\begin{array}{r}718,504 \\
154,358 \\
10,022\end{array}$ & $\begin{array}{r}12,252,192 \\
3,349,143 \\
112,614\end{array}$ & $\begin{array}{r}1,982,158 \\
548,211 \\
20,678\end{array}$ \\
\hline & \multicolumn{7}{|c|}{ Investor-Owned Utillties in ERCOT } \\
\hline \multirow[t]{2}{*}{$\begin{array}{l}\text { Watthour Meter Count .................... } \\
\text { Line Transformer Count ................. } \\
\text { Line Transformer Capacity (MVa) }\end{array}$} & $\begin{array}{r}5,076,196 \\
1,464,513 \\
75,646\end{array}$ & $\begin{array}{r}100,625 \\
46,804 \\
2,816\end{array}$ & $\begin{array}{r}62,370 \\
31,191 \\
1,562\end{array}$ & $\begin{array}{r}5,114,451 \\
1,480,126 \\
76,900\end{array}$ & $\begin{array}{r}198,307 \\
51,307 \\
15,807\end{array}$ & $\begin{array}{r}1,034,082 \\
391,410 \\
13,051\end{array}$ & $\begin{array}{r}3,882,062 \\
1,037,409 \\
48,042\end{array}$ \\
\hline & \multicolumn{7}{|c|}{ Investor-Owned Utilities in MAAC } \\
\hline \multirow[t]{2}{*}{$\begin{array}{l}\text { Watthour Meter Count ................... } \\
\text { Line Transformer Count ................. } \\
\text { Line Transformer Capacity (MVa) }\end{array}$} & $\begin{array}{r}9,748,199 \\
1,910,472 \\
98,869\end{array}$ & $\begin{array}{r}355,165 \\
50,265 \\
3,159\end{array}$ & $\begin{array}{r}224,949 \\
28,694 \\
1,475\end{array}$ & $\begin{array}{r}9,879,715 \\
1,932,043 \\
100,553\end{array}$ & $\begin{array}{r}646,038 \\
65,048 \\
6,218\end{array}$ & $\begin{array}{r}9,227,677 \\
1,690,612 \\
83,270\end{array}$ & $\begin{array}{r}6,000 \\
176,383 \\
11,065\end{array}$ \\
\hline & \multicolumn{7}{|c|}{ Investor-Owned Utillties in MAIN } \\
\hline \multirow[t]{2}{*}{$\begin{array}{l}\text { Watthour Meter Count ................... } \\
\text { Line Transformer Count .................. } \\
\text { Line Transformer Capacity (MVa) }\end{array}$} & $\begin{array}{r}7,957,035 \\
1,677,423 \\
93,878\end{array}$ & $\begin{array}{r}288,591 \\
60,827 \\
3,499\end{array}$ & $\begin{array}{r}166,049 \\
35,648 \\
1,600\end{array}$ & $\begin{array}{r}8,079,577 \\
1,702,602 \\
95,777\end{array}$ & $\begin{array}{r}319,026 \\
70,193 \\
9,147\end{array}$ & $\begin{array}{r}7,758,396 \\
1,630,325 \\
81,093\end{array}$ & $\begin{array}{l}2,155 \\
2,084 \\
5,537\end{array}$ \\
\hline & \multicolumn{7}{|c|}{ Investor-Owned Utilities in MAPP } \\
\hline \multirow[t]{2}{*}{$\begin{array}{l}\text { Watthour Meter Count ................... } \\
\text { Line Transformer Count ................. } \\
\text { Line Transformer Capacity (MVa) }\end{array}$} & $\begin{array}{r}3,113,115 \\
714,196 \\
32,396\end{array}$ & $\begin{array}{r}127,440 \\
18,942 \\
995\end{array}$ & $\begin{array}{r}74,807 \\
11,764 \\
434\end{array}$ & $\begin{array}{r}3,165,748 \\
721,374 \\
32,957\end{array}$ & $\begin{array}{r}156,705 \\
31,544 \\
2,348\end{array}$ & $\begin{array}{r}3,006,583 \\
689,606 \\
30,526\end{array}$ & $\begin{array}{r}2,460 \\
224 \\
83\end{array}$ \\
\hline & \multicolumn{7}{|c|}{ Investor-Owned Utilities in NPCC } \\
\hline \multirow[t]{2}{*}{$\begin{array}{l}\text { Watthour Meter Count ................... } \\
\text { Line Transformer Count ................. } \\
\text { Line Transformer Cioacity (MVa) }\end{array}$} & $\begin{array}{r}13,786,623 \\
2,375,133 \\
116,113\end{array}$ & $\begin{array}{r}408,724 \\
64,432 \\
3,040\end{array}$ & $\begin{array}{r}348,743 \\
41,952 \\
2,931\end{array}$ & $\begin{array}{r}13,846,604 \\
2,397,613 \\
116,222\end{array}$ & $\begin{array}{r}782,098 \\
177,888 \\
13,670\end{array}$ & $\begin{array}{r}\mathbf{9 , 8 7 5 , 7 7 7} \\
1,022,620 \\
\mathbf{5 9 , 5 2 3}\end{array}$ & $\begin{array}{r}3,191,145 \\
1,197,470 \\
43,029\end{array}$ \\
\hline & \multicolumn{7}{|c|}{ Investor-Owned Utilities in SERC } \\
\hline \multirow[t]{2}{*}{$\begin{array}{l}\text { Watthour Meter Count .................... } \\
\text { Line Transformer Count ................. } \\
\text { Line Transformer Capacity (MVa) }\end{array}$} & $\begin{array}{r}14,262,696 \\
4,056,641 \\
186,905\end{array}$ & $\begin{array}{r}531,604 \\
149,666 \\
6,986\end{array}$ & $\begin{array}{r}525,695 \\
99,885 \\
3,712\end{array}$ & $\begin{array}{r}14,268,605 \\
4,106,422 \\
190,179\end{array}$ & $\begin{array}{r}893,647 \\
148,040 \\
10,222\end{array}$ & $\begin{array}{r}11,758,689 \\
3,154,491 \\
143,961\end{array}$ & $\begin{array}{r}1,616,269 \\
803,891 \\
35,996\end{array}$ \\
\hline & \multicolumn{7}{|c|}{ Investor-Owned Uttilites in SPP } \\
\hline \multirow[t]{2}{*}{$\begin{array}{l}\text { Watthour Meter Count .................... } \\
\text { Line Transformer Count ................. } \\
\text { Line Transformer Capacity (MVa) }\end{array}$} & $\begin{array}{r}5,643,843 \\
2,012,059 \\
81,969\end{array}$ & $\begin{array}{r}204,882 \\
58,807 \\
3,248\end{array}$ & $\begin{array}{r}176,285 \\
47,321 \\
1,740\end{array}$ & $\begin{array}{r}5,672,440 \\
2,023,545 \\
83,477\end{array}$ & $\begin{array}{r}352,440 \\
68,485 \\
5,548\end{array}$ & $\begin{array}{r}4,991,770 \\
1,945,041 \\
77,080\end{array}$ & $\begin{array}{r}328,230 \\
10,019 \\
849\end{array}$ \\
\hline & \multicolumn{7}{|c|}{ Investor-Owned Utillities in WSCC } \\
\hline \multirow[t]{2}{*}{$\begin{array}{l}\text { Watthour Meter Count .................... } \\
\text { Line Transformer Count ................ } \\
\text { Line Transformer Capacity (MVa) }\end{array}$} & $\begin{array}{r}17,053,460 \\
3,591,916 \\
132,281\end{array}$ & $\begin{array}{r}635,637 \\
112,181 \\
5,261\end{array}$ & $\begin{array}{r}314,166 \\
57,300 \\
1,651\end{array}$ & $\begin{array}{r}17,374,931 \\
3,646,797 \\
135,891\end{array}$ & $\begin{array}{r}820,961 \\
174,847 \\
10,291\end{array}$ & $\begin{array}{r}15,147,308 \\
2,835,483 \\
97,846\end{array}$ & $\begin{array}{r}1,406,662 \\
636,467 \\
27,754\end{array}$ \\
\hline & \multicolumn{7}{|c|}{ ContIguous U.S. Total } \\
\hline $\begin{array}{l}\text { Watthour Meter Count ................... } \\
\text { Line Transformer Count ............... } \\
\text { Line Transformer Capacity (MVa) }\end{array}$ & $\begin{array}{r}91,445,804 \\
21,789,881 \\
958,336\end{array}$ & $\begin{array}{r}, 186,081 \\
698,048 \\
34,092\end{array}$ & $\begin{array}{r}2,276,960 \\
425,695 \\
17,158\end{array}$ & $\begin{array}{r}92,354,925 \\
22,062,234 \\
975,270\end{array}$ & $\begin{array}{r}4,887,726 \\
941,710 \\
83,273\end{array}$ & $\begin{array}{r}75,052,474 \\
16,708,731 \\
698,964\end{array}$ & $\begin{array}{r}12,417,141 \\
4,412,158 \\
193,033\end{array}$ \\
\hline
\end{tabular}

' In stock, locked meters on customer's prenitises and inactive transformers on system.

Notes: -NERC is the North American Electric Reliability Council. The Alaska System Coordinating Council is not included. See glossary for a list of regions and Figure 2 for a map of the regions. - Totals maly not equal sum of components because of independent rounding. $\bullet M V a$ means megavoltampere.

Sources: •Federal Energy Regulatory Commission, FERC Form 1, "Annual Report of Major Electric Utilities, Licensees and Others." 
Table 44. Watthour Meter and Line Transformer Statistics of Investor-Owned Utilities, 1992 (Count and Megavoltampere)

\begin{tabular}{|c|c|c|c|c|c|c|c|}
\hline $\begin{array}{l}\text { Dietribution } \\
\text { Equlpmont }\end{array}$ & $\begin{array}{l}\text { Number at } \\
\text { Beginning } \\
\text { of Year }\end{array}$ & $\begin{array}{l}\text { Additions } \\
\text { During } \\
\text { Year }\end{array}$ & $\begin{array}{l}\text { Reductions } \\
\text { During } \\
\text { Year }\end{array}$ & $\begin{array}{l}\text { Number at } \\
\text { End of } \\
\text { Yoar }\end{array}$ & $\begin{array}{l}\text { In Stock, } \\
\text { Locked, } \\
\text { and Inactive ' }\end{array}$ & $\begin{array}{l}\text { In } \\
\text { Customers } \\
\text { Use }\end{array}$ & $\begin{array}{l}\ln \\
\text { Company } \\
\text { Use }\end{array}$ \\
\hline \multicolumn{8}{|l|}{ Alabama Power Co } \\
\hline $\begin{array}{l}\text { Watthour Meter Count .................. } \\
\text { Line Transformer Count .............. } \\
\text { Line Transformer Capacity }\end{array}$ & $\begin{array}{r}1,194,695 \\
532,151\end{array}$ & $\begin{array}{l}54,721 \\
24,242\end{array}$ & $\begin{array}{l}34,881 \\
13,852\end{array}$ & $\begin{array}{r}1,214,535 \\
542,541\end{array}$ & $\begin{array}{l}58,189 \\
13,226\end{array}$ & $\begin{array}{r}1,155,888 \\
529,315\end{array}$ & $--^{458}$ \\
\hline $\begin{array}{l}\text { (MVa) } \\
\text { Alaska Electric Light\&Power } \mathrm{Co}\end{array}$ & 16,926 & 945 & 405 & 17,466 & 916 & 16,550 & -- \\
\hline $\begin{array}{l}\text { Line Transformer Count ............... } \\
\text { Line Transformer Capacity }\end{array}$ & 2,738 & 32 & 16 & 2,754 & 220 & 2,507 & 27 \\
\hline $\begin{array}{l}\text { (MVa) } \\
\text { Appalachian Power Co }\end{array}$ & 194 & 3 & - & 197 & 19 & 177 & 1 \\
\hline $\begin{array}{l}\text { Watthour Meter Count ................. } \\
\text { Line Transformer Count .............. } \\
\text { Line Transformer Capacity }\end{array}$ & $\begin{array}{l}868,128 \\
330,445\end{array}$ & $\begin{array}{l}25,524 \\
13,023\end{array}$ & $\begin{array}{r}11,149 \\
6,686\end{array}$ & $\begin{array}{l}882,503 \\
336,782\end{array}$ & $\begin{array}{l}38,291 \\
10,220\end{array}$ & $\begin{array}{l}843,511 \\
326,442\end{array}$ & $\begin{array}{l}701 \\
120\end{array}$ \\
\hline $\begin{array}{l}\text { (MVa) } \\
\text { Arizona Public Service Co }\end{array}$ & 10,510 & 465 & 191 & 10,784 & 836 & 9,930 & 18 \\
\hline $\begin{array}{l}\text { Watthour Meter Count ................. } \\
\text { Line Transformer Count .............. } \\
\text { Line Transformer Capacity }\end{array}$ & $\begin{array}{l}722,908 \\
185,501\end{array}$ & $\begin{array}{r}66,891 \\
5,260\end{array}$ & $\begin{array}{r}41,445 \\
1,161\end{array}$ & $\begin{array}{l}748,354 \\
189,600\end{array}$ & $\begin{array}{l}75,437 \\
15,184\end{array}$ & $\begin{array}{l}672,700 \\
174,416\end{array}$ & 217 \\
\hline $\begin{array}{l}\text { (MVa) ................................... } \\
\text { Arkansas Power \& Light Co }\end{array}$ & 11,448 & 333 & 60 & 11,721 & 1,164 & 10,557 & - \\
\hline $\begin{array}{l}\text { Watthour Meter Count ................. } \\
\text { Line Transformer Count .............. } \\
\text { Line Transformer Capacity }\end{array}$ & $\begin{array}{l}692,873 \\
291,492\end{array}$ & $\begin{array}{r}43,391 \\
6,824\end{array}$ & $\begin{array}{l}64,196 \\
13,371\end{array}$ & $\begin{array}{l}672,068 \\
284,945\end{array}$ & $\begin{array}{l}82,882 \\
10,682\end{array}$ & $\begin{array}{l}585,699 \\
273,156\end{array}$ & $\begin{array}{l}3,487 \\
1,107\end{array}$ \\
\hline $\begin{array}{l}\text { (MVa) ......................................... } \\
\text { Atlantic Clty Electric Co }\end{array}$ & 10,118 & 305 & 334 & 10,089 & 836 & 9,148 & 105 \\
\hline $\begin{array}{l}\text { Watthour Meter Count ................. } \\
\text { Line Transformer Count ............. } \\
\text { Line Transformer Capacity }\end{array}$ & $\begin{array}{l}470,572 \\
126,454\end{array}$ & $\begin{array}{r}29,631 \\
3,693\end{array}$ & $\begin{array}{r}24,999 \\
2,441\end{array}$ & $\begin{array}{l}475,204 \\
127,706\end{array}$ & $\begin{array}{r}10,689 \\
4,186\end{array}$ & $\begin{array}{l}464,350 \\
123,499\end{array}$ & $\begin{array}{r}165 \\
21\end{array}$ \\
\hline $\begin{array}{l}\text { (MVa) } \\
\text { Baltimore Gas \& Electric Co }\end{array}$ & 4,755 & 152 & 126 & 4,781 & 175 & 4,604 & 2 \\
\hline $\begin{array}{l}\text { Watthour Meter Count ................. } \\
\text { Line Transformer Count ............. } \\
\text { Line Transformer Capacity }\end{array}$ & $\begin{array}{r}1,102,550 \\
177,180\end{array}$ & $\begin{array}{r}32,595 \\
5,224\end{array}$ & $\begin{array}{r}11,748 \\
1,899\end{array}$ & $\begin{array}{r}1,123,397 \\
180,505\end{array}$ & $\begin{array}{r}34,230 \\
4,849\end{array}$ & $\begin{array}{c}1,087,033 \\
-\end{array}$ & $\begin{array}{r}2,134 \\
175,656\end{array}$ \\
\hline $\begin{array}{l}\text { (MVa) } \\
\text { Bangor Hydro-Electric Co }\end{array}$ & 11,302 & 390 & 101 & 11,591 & 576 & - & 11,015 \\
\hline $\begin{array}{l}\text { Watthour Meter Count ................. } \\
\text { Line Transformer Count ............. } \\
\text { Line Transformer Capacity }\end{array}$ & $\begin{array}{r}106,481 \\
36,666\end{array}$ & $\begin{array}{l}2,731 \\
1,518\end{array}$ & $\begin{array}{r}2,139 \\
863\end{array}$ & $\begin{array}{r}107,073 \\
37,321\end{array}$ & - & $\begin{array}{r}106,895 \\
37,321\end{array}$ & $\begin{array}{r}78 \\
-\quad\end{array}$ \\
\hline $\begin{array}{l}\text { (MVa) } \\
\text { Black Hills Corp }\end{array}$ & 827 & 43 & 9 & 861 & - & 861 & - \\
\hline $\begin{array}{l}\text { Watthour Meter Count ................ } \\
\text { Line Transformer Count ............ } \\
\text { Line Transformer Capacity }\end{array}$ & $\begin{array}{l}57,193 \\
17,873\end{array}$ & $\begin{array}{r}1,295 \\
727\end{array}$ & $\begin{array}{l}753 \\
453\end{array}$ & $\begin{array}{l}57,735 \\
18,147\end{array}$ & $\begin{array}{l}1,250 \\
1,462\end{array}$ & $\begin{array}{l}56,175 \\
16,685\end{array}$ & $-{ }^{310}$ \\
\hline $\begin{array}{l}\text { (MVa) ............................ } \\
\text { Blackstone Valley Electic }\end{array}$ & 914 & 75 & 14 & 975 & 32 & 943 & - \\
\hline $\begin{array}{l}\text { Watthour Meter Count ................. } \\
\text { Line Transformer Count ............ } \\
\text { Line Transformer Capacity }\end{array}$ & $\begin{array}{l}90,267 \\
12,851\end{array}$ & $\begin{array}{r}3,201 \\
271\end{array}$ & $\begin{array}{r}2,323 \\
531\end{array}$ & $\begin{array}{l}91,145 \\
12,591\end{array}$ & $\begin{array}{l}4,383 \\
1,355\end{array}$ & $\begin{array}{l}86,746 \\
11,217\end{array}$ & $\begin{array}{l}16 \\
19\end{array}$ \\
\hline $\begin{array}{l}(\mathrm{MVa}) \\
\text { Boston Edison Co }\end{array}$ & 750 & 18 & 23 & 745 & 175 & 569 & 1 \\
\hline $\begin{array}{l}\text { Watthour Meter Count ................. } \\
\text { Line Transformer Count ............ } \\
\text { Line Transformer Capacity }\end{array}$ & $\begin{array}{r}764,107 \\
60,152\end{array}$ & $\begin{array}{r}14,555 \\
1,792\end{array}$ & $\begin{array}{r}34,378 \\
1,192\end{array}$ & $\begin{array}{r}744,284 \\
60,752\end{array}$ & $\begin{array}{r}57,864 \\
7,310\end{array}$ & $\begin{array}{c}684,267 \\
-\end{array}$ & $\begin{array}{r}2,153 \\
53,442\end{array}$ \\
\hline $\begin{array}{l}\text { (MVa) ....................................... } \\
\text { Cambridge Electric Light Co }\end{array}$ & 4,851 & 145 & 91 & 4,905 & 656 & - & 4,240 \\
\hline $\begin{array}{l}\text { Watthour Meter Count ................ } \\
\text { Line Transformer Count ............ } \\
\text { Line Transformer Capacity }\end{array}$ & $\begin{array}{r}45,864 \\
2,366\end{array}$ & $\begin{array}{r}1,154 \\
59\end{array}$ & $\begin{array}{r}1,028 \\
91\end{array}$ & $\begin{array}{r}45,890 \\
2,334\end{array}$ & $\begin{array}{r}1,159 \\
453\end{array}$ & $\begin{array}{r}44,804 \\
1,876\end{array}$ & $\begin{array}{r}27 \\
5\end{array}$ \\
\hline $\begin{array}{l}\text { (MVa) ................ } \\
\text { Sarolina Power \& Light Co }\end{array}$ & 162 & 7 & 4 & 165 & 34 & 130 & 1 \\
\hline $\begin{array}{l}\text { Watthour Meter Count ................ } \\
\text { Line Transformer Count ............ } \\
\text { Line Transformer Capacity }\end{array}$ & $\begin{array}{r}1,018,849 \\
373,818\end{array}$ & $\begin{array}{r}57,241 \\
9,303\end{array}$ & $\begin{array}{r}41,689 \\
4,952\end{array}$ & $\begin{array}{r}1,034,401 \\
378,169\end{array}$ & $\begin{array}{l}48,841 \\
11,257\end{array}$ & $\begin{array}{l}985,179 \\
366,912\end{array}$ & $-{ }^{381}$ \\
\hline $\begin{array}{l}\text { (MVa) } \\
\text { Central Hudson Gas \& Elec Corp }\end{array}$ & 14,566 & 521 & 154 & 14,933 & 877 & 14,056 & - \\
\hline $\begin{array}{l}\text { Watthour Meter Count ................. } \\
\text { Line Transformer Count ............ } \\
\text { Line Transformer Capacity }\end{array}$ & $\begin{array}{r}270,674 \\
83,015\end{array}$ & $\begin{array}{l}9,540 \\
1,696\end{array}$ & $\begin{array}{l}4,894 \\
1,075\end{array}$ & $\begin{array}{r}275,320 \\
83,636\end{array}$ & $\begin{array}{r}23,003 \\
3,461\end{array}$ & 252,317 & $\overline{80,175}$ \\
\hline$(\mathrm{MVa})$ & 2,317 & 57 & 34 & 2,340 & 151 & - & 2,189 \\
\hline
\end{tabular}

See notes and footnotes at end of table. 
Table 44. Watthour Meter and Line Transformer Statistics of Investor-Owned Utilities, 1992 (Count and Megavoltampere) (Continued)

\begin{tabular}{|c|c|c|c|c|c|c|c|}
\hline $\begin{array}{l}\text { Diatribution } \\
\text { Equlpment }\end{array}$ & $\begin{array}{l}\text { Number at } \\
\text { Beginning } \\
\text { of Year }\end{array}$ & $\begin{array}{l}\text { Additions } \\
\text { During } \\
\text { Year }\end{array}$ & $\begin{array}{l}\text { Reductions } \\
\text { During } \\
\text { Year }\end{array}$ & $\begin{array}{l}\text { Number at } \\
\text { End of } \\
\text { Year }\end{array}$ & $\begin{array}{l}\text { In Stock, } \\
\text { Locked, } \\
\text { and Inactive }\end{array}$ & $\begin{array}{l}\text { In } \\
\text { Customers } \\
\text { Use }\end{array}$ & $\begin{array}{l}\text { In } \\
\text { Company } \\
\text { Use }\end{array}$ \\
\hline \multicolumn{8}{|l|}{ Central llinols Light Co } \\
\hline $\begin{array}{l}\text { Watthour Mater Count ................. } \\
\text { Line Transformer Count............ } \\
\text { Line Transformer Capacity }\end{array}$ & $\begin{array}{r}201,836 \\
61,629\end{array}$ & $\begin{array}{l}3,784 \\
1,749\end{array}$ & $\begin{array}{l}3,188 \\
1,522\end{array}$ & $\begin{array}{r}202,432 \\
61,856\end{array}$ & $\begin{array}{l}8,485 \\
1,892\end{array}$ & $\begin{array}{r}183,947 \\
59,864\end{array}$ & - \\
\hline $\begin{array}{l}\text { (MVa) } \\
\text { Central Illinols Pub Son Co }\end{array}$ & 2,759 & 103 & 47 & 2,815 & 167 & 2,648 & - \\
\hline $\begin{array}{l}\text { Watthour Meter Count ................. } \\
\text { Line Tranaformer Count ............ } \\
\text { Line Transformer Capacity }\end{array}$ & $\begin{array}{l}333,093 \\
105,879\end{array}$ & $\begin{array}{r}14,569 \\
3,468\end{array}$ & $\begin{array}{l}5,350 \\
2,546\end{array}$ & $\begin{array}{l}342,312 \\
106,801\end{array}$ & $\begin{array}{r}16,947 \\
5,872\end{array}$ & $\begin{array}{l}325,017 \\
100,827\end{array}$ & $\begin{array}{l}348 \\
102\end{array}$ \\
\hline $\begin{array}{l}\text { (MVa) } \\
\text { Central Louisiana Elec Co Inc }\end{array}$ & 4,150 & 164 & 80 & 4,234 & 495 & 3,734 & 5 \\
\hline $\begin{array}{l}\text { Watthour Meter Count ................. } \\
\text { Line Transformer Count ............. } \\
\text { Line Transformer Capacity }\end{array}$ & $\begin{array}{l}221,706 \\
110,529\end{array}$ & $\begin{array}{l}8,902 \\
3,340\end{array}$ & $\begin{array}{l}2,067 \\
1,512\end{array}$ & $\begin{array}{l}228,541 \\
112,357\end{array}$ & $\begin{array}{r}12,687 \\
135\end{array}$ & $\begin{array}{l}215,854 \\
112,222\end{array}$ & -- \\
\hline 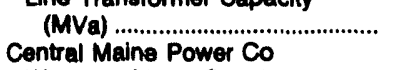 & 3,223 & 128 & 60 & 3,289 & 265 & 3,024 & - \\
\hline $\begin{array}{l}\text { Watthour Meter Count ................ } \\
\text { Line Transformer Count ............. } \\
\text { Line Transformer Capacity }\end{array}$ & $\begin{array}{l}532,586 \\
195,520\end{array}$ & $\begin{array}{r}11,568 \\
6,898\end{array}$ & $\begin{array}{r}13,055 \\
2,907\end{array}$ & $\begin{array}{l}531,099 \\
189,511\end{array}$ & $\begin{array}{r}19,557 \\
5,303\end{array}$ & - & $\begin{array}{l}511,542 \\
194,208\end{array}$ \\
\hline $\begin{array}{l}\text { (MVa) ................................... } \\
\text { Central Power \& Light Co }\end{array}$ & 4,570 & 135 & 70 & 4,635 & 453 & - & 4,182 \\
\hline $\begin{array}{l}\text { Watthour Meter Count ................ } \\
\text { Line Transformer Count ............ } \\
\text { Line Transformer Capacity }\end{array}$ & $\begin{array}{l}621,541 \\
203,226\end{array}$ & $\begin{array}{r}21,046 \\
7,204\end{array}$ & $\begin{array}{r}13,784 \\
2,856\end{array}$ & $\begin{array}{l}628,803 \\
207,474\end{array}$ & $\begin{array}{r}29,353 \\
7,892\end{array}$ & $\begin{array}{l}599,162 \\
199,010\end{array}$ & $\begin{array}{l}288 \\
572\end{array}$ \\
\hline 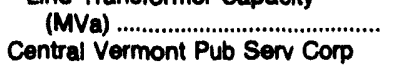 & 7,783 & 357 & 85 & 8,055 & 588 & 7,430 & 37 \\
\hline $\begin{array}{l}\text { Watthour Meter Count ................. } \\
\text { Line Transformer Count............ } \\
\text { Line Transformer Capacity }\end{array}$ & $\begin{array}{r}179,102 \\
63,409\end{array}$ & $\begin{array}{l}9,232 \\
2,888\end{array}$ & $\begin{array}{r}1,546 \\
607\end{array}$ & $\begin{array}{r}186,788 \\
65,690\end{array}$ & $\begin{array}{r}22,754 \\
1,792\end{array}$ & $\begin{array}{r}163,874 \\
63,734\end{array}$ & $\begin{array}{r}160 \\
164\end{array}$ \\
\hline Cincinnat Gas \& Electric Co & 1.160 & 60 & 8 & 1,212 & 64 & 1,142 & 6 \\
\hline $\begin{array}{l}\text { Watthour Meter Count .................. } \\
\text { Line Transformer Count............ } \\
\text { Line Transforme, Capacity }\end{array}$ & $\begin{array}{l}643,781 \\
150,553\end{array}$ & $\begin{array}{r}32,327 \\
5,677\end{array}$ & $\begin{array}{r}19,174 \\
3,456\end{array}$ & $\begin{array}{l}656,934 \\
152,774\end{array}$ & $\begin{array}{l}52,233 \\
19,483\end{array}$ & -- & $\begin{array}{l}604,701 \\
133,291\end{array}$ \\
\hline $\begin{array}{l}\text { (MVa) .......... } \\
\text { Citizens Electric Co of Lewisb }\end{array}$ & 9,374 & 468 & 212 & 9,630 & 1,775 & - & 7,855 \\
\hline $\begin{array}{l}\text { Watthour Meter Count ................. } \\
\text { Line Transformer Count .............. } \\
\text { Line Transformer Capacity }\end{array}$ & $\begin{array}{l}5,834 \\
2,073\end{array}$ & $\begin{array}{r}284 \\
56\end{array}$ & $\begin{array}{r}177 \\
19\end{array}$ & $\begin{array}{l}5,941 \\
2,110\end{array}$ & $\begin{array}{r}146 \\
40\end{array}$ & $\begin{array}{l}5,794 \\
2,060\end{array}$ & $\begin{array}{r}1 \\
10\end{array}$ \\
\hline 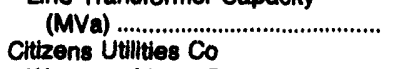 & 68 & 2 & - & 70 & 3 & 67 & - \\
\hline $\begin{array}{l}\text { Watthour Meter Count ................. } \\
\text { Line Transformer Count ............. } \\
\text { Line Transformer Capacity }\end{array}$ & $\begin{array}{r}103,945 \\
35,227\end{array}$ & $\begin{array}{l}4,764 \\
4,445\end{array}$ & $\begin{array}{r}2,038 \\
79\end{array}$ & $\begin{array}{r}108,671 \\
39,593\end{array}$ & $\begin{array}{l}5,987 \\
3,503\end{array}$ & $\begin{array}{l}89,856 \\
31,041\end{array}$ & $\begin{array}{r}10,828 \\
5,049\end{array}$ \\
\hline $\begin{array}{l}\text { (MVa) ....................................... } \\
\text { Cleveland Electric Ilum Co }\end{array}$ & 1,051 & 147 & 8 & 1,190 & 197 & 854 & 139 \\
\hline $\begin{array}{l}\text { Watthour Meter Count ................. } \\
\text { Line Tranaformer Count ............. } \\
\text { Line Tranaformer Capacity }\end{array}$ & $\begin{array}{l}818,644 \\
137,325\end{array}$ & $\begin{array}{r}11,406 \\
2,307\end{array}$ & $\begin{array}{r}10,053 \\
878\end{array}$ & $\begin{array}{l}819,997 \\
138,754\end{array}$ & $\begin{array}{r}29,433 \\
8,043\end{array}$ & $\begin{array}{l}790,388 \\
130,711\end{array}$ & -176 \\
\hline $\begin{array}{l}\text { (MVa) ....................................... } \\
\text { Columbus Southern Power Co }\end{array}$ & 7,557 & 113 & 48 & 7,622 & 1,085 & 6,557 & - \\
\hline $\begin{array}{l}\text { Watthour Meter Count ................. } \\
\text { Line Transtormer Count .............. } \\
\text { Une Transformer Capacity }\end{array}$ & $\begin{array}{l}597,553 \\
142,155\end{array}$ & $\begin{array}{r}30,039 \\
4,719\end{array}$ & $\begin{array}{r}16,121 \\
2,613\end{array}$ & $\begin{array}{l}609,471 \\
144,261\end{array}$ & $\begin{array}{r}22,500 \\
5,482\end{array}$ & $\begin{array}{l}586,841 \\
138,404\end{array}$ & $\begin{array}{l}130 \\
375\end{array}$ \\
\hline 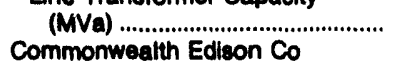 & 6,321 & 189 & 85 & 6,425 & 427 & 5,980 & 18 \\
\hline $\begin{array}{l}\text { Watthour Meter Count ................ } \\
\text { Line Transformer Count ............. } \\
\text { Line Transformer Capecity }\end{array}$ & $\begin{array}{r}3,653,806 \\
524,337\end{array}$ & $\begin{array}{r}138,334 \\
18,314\end{array}$ & $\begin{array}{l}72,313 \\
13,288\end{array}$ & $\begin{array}{r}3,719,827 \\
529,363\end{array}$ & $\begin{array}{r}122,726 \\
11,906\end{array}$ & $\begin{array}{r}3,597,101 \\
517,457\end{array}$ & - \\
\hline $\begin{array}{l}\text { (MVa) ..................................... } \\
\text { Commonweath Electric Co }\end{array}$ & 34,427 & 1.180 & 579 & 35,028 & 1,325 & 33,703 & - \\
\hline $\begin{array}{l}\text { Wattho.. Meter Count ................. } \\
\text { Line Transformer Count ............. } \\
\text { Line Transformer Capacity }\end{array}$ & $\begin{array}{r}328,740 \\
58,841\end{array}$ & $\begin{array}{l}7,609 \\
1,032\end{array}$ & $\begin{array}{l}4,859 \\
1,298\end{array}$ & $\begin{array}{r}332,490 \\
58,575\end{array}$ & $\begin{array}{l}7,682 \\
6,235\end{array}$ & $\begin{array}{r}324,604 \\
52,291\end{array}$ & $\begin{array}{r}204 \\
49\end{array}$ \\
\hline 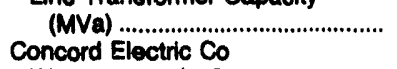 & 2,250 & 43 & 39 & 2,254 & 343 & 1,807 & 4 \\
\hline $\begin{array}{l}\text { Watthour Meter Count ................. } \\
\text { Line Transformer Count ............ } \\
\text { Line Transformer Capacity }\end{array}$ & $\begin{array}{r}25,684 \\
6,839\end{array}$ & $\begin{array}{r}360 \\
83\end{array}$ & $\begin{array}{r}176 \\
37\end{array}$ & $\begin{array}{r}25,848 \\
6,885\end{array}$ & $\begin{array}{r}1,264 \\
282\end{array}$ & $\begin{array}{r}24,571 \\
6,567\end{array}$ & $\begin{array}{l}13 \\
36\end{array}$ \\
\hline 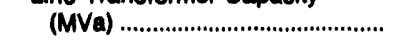 & 178 & 4 & 2 & 181 & 14 & 168 & 1 \\
\hline
\end{tabular}

See notes and footnotes at end of table. 
Table 44. Watthour Meter and Line Transformer Statistics of Investor-Owned Utillties, 1992 (Count and Megavoltampere) (Continued)

\begin{tabular}{|c|c|c|c|c|c|c|c|}
\hline $\begin{array}{l}\text { Distribution } \\
\text { Equipment }\end{array}$ & $\begin{array}{c}\text { Number at } \\
\text { Beginning } \\
\text { of Year }\end{array}$ & $\begin{array}{l}\text { Additions } \\
\text { During } \\
\text { Year }\end{array}$ & $\begin{array}{l}\text { Reductions } \\
\text { During } \\
\text { Year }\end{array}$ & $\begin{array}{l}\text { Number at } \\
\text { End of } \\
\text { Year }\end{array}$ & $\begin{array}{l}\text { In Stock, } \\
\text { Locked, } \\
\text { and Inactive }\end{array}$ & $\begin{array}{l}\text { In } \\
\text { Customers } \\
\text { Use }\end{array}$ & $\begin{array}{l}\text { In } \\
\text { Company } \\
\text { Use }\end{array}$ \\
\hline \multicolumn{8}{|l|}{ Connecticut Light \& Power Co } \\
\hline $\begin{array}{l}\text { Watthour Meter Count ................. } \\
\text { Line Transformer Count .............. } \\
\text { Line Transformer Capacity }\end{array}$ & $\begin{array}{r}1,105,505 \\
220,407\end{array}$ & $\begin{array}{r}40,150 \\
5,379\end{array}$ & $\begin{array}{r}38,329 \\
4,353\end{array}$ & $\begin{array}{r}1,107,326 \\
221,433\end{array}$ & $\begin{array}{r}30,419 \\
6,074\end{array}$ & $\begin{array}{r}1,076,787 \\
214,949\end{array}$ & $\begin{array}{l}120 \\
410\end{array}$ \\
\hline $\begin{array}{l}\text { (MVa) . } \\
\text { Connecticut Valley Elec Co Inc }\end{array}$ & 10,345 & 269 & 607 & 10,007 & 273 & 9,710 & 24 \\
\hline Watthour Meter Count .................. & 12,404 & 204 & 241 & 12,367 & 732 & 11,628 & 7 \\
\hline $\begin{array}{l}\text { Line Transformer Count ............... } \\
\text { Line Transformer Capacity }\end{array}$ & 3,585 & 111 & 53 & 3,643 & 43 & 3,595 & 5 \\
\hline $\begin{array}{l}\text { (MVa) ... Power Co } \\
\text { Conowingo Pow }\end{array}$ & 60 & 2 & 1 & 61 & 1 & 60 & -- \\
\hline $\begin{array}{l}\text { Watthour Meter Count ................ } \\
\text { Line Transformer Count ................ } \\
\text { Line Transformer Capacity }\end{array}$ & $\begin{array}{r}36,816 \\
9,812\end{array}$ & $\begin{array}{r}1,094 \\
294\end{array}$ & $\begin{array}{l}872 \\
195\end{array}$ & $\begin{array}{r}37,038 \\
9,911\end{array}$ & $\begin{array}{r}3,103 \\
222\end{array}$ & $\begin{array}{r}33,925 \\
9,676\end{array}$ & $\begin{array}{l}10 \\
13\end{array}$ \\
\hline $\begin{array}{l}\text { (MVa) .... Edison Co-NY Inc } \\
\text { Consolidated }\end{array}$ & 364 & 31 & 4 & 391 & 24 & 366 & 1 \\
\hline Watthour Meter Count .................... & $3,170,958$ & 88,695 & 69,452 & $3,190,201$ & 221,922 & $2,967,776$ & 503 \\
\hline $\begin{array}{l}\text { Line Transformer Count ............... } \\
\text { Line Transformer Capacity }\end{array}$ & 77,754 & 2,592 & 1,644 & 78,702 & 4,184 & 74,518 & $-\infty$ \\
\hline $\begin{array}{l}\text { (MVa) } \\
\text { Consolidated Water Power Co }\end{array}$ & 22,942 & 722 & 359 & 23,305 & 1,284 & 22,021 & - \\
\hline Watthour Meter Count .................. & 1,252 & - & 5 & 1,247 & 91 & - & 1,156 \\
\hline $\begin{array}{l}\text { Line Transformer Count ............... } \\
\text { Line Transformer Capacity }\end{array}$ & 495 & 13 & 3 & 505 & 140 & -- & 365 \\
\hline $\begin{array}{l}\text { (MVa) ................ } \\
\text { Consumers Power Co }\end{array}$ & 8,346 & 388 & 48 & 8,686 & 3,251 & - & 5,435 \\
\hline Watthour Meter Count .................. & $\begin{array}{r}1,551,215 \\
460,139\end{array}$ & $\begin{array}{l}55,381 \\
19,993\end{array}$ & $\begin{array}{r}31,022 \\
9,164\end{array}$ & $\begin{array}{r}1,575,574 \\
470,968\end{array}$ & $\begin{array}{l}44,975 \\
27,369\end{array}$ & $\begin{array}{r}1,529,938 \\
443,434\end{array}$ & $\begin{array}{l}661 \\
165\end{array}$ \\
\hline Dayton Power \& Light Co & & & & & & & \\
\hline Watthour Meter Count .................. & 488,525 & 13,007 & 22,768 & 478,764 & 18,628 & 460,045 & 91 \\
\hline $\begin{array}{l}\text { Line Transformer Count ............... } \\
\text { Line Transformer Capacity }\end{array}$ & 137,622 & 2,091 & 2,882 & 136,831 & 11,724 & 125,107 & - \\
\hline (MVa) ................................... & 6,472 & 132 & 161 & 6.443 & 675 & 5,768 & - \\
\hline Watthour Meter Count .................. & 412,035 & 14,267 & 11,478 & 414,824 & 27,230 & 387,588 & 6 \\
\hline $\begin{array}{l}\text { Line Transformer Count ............... } \\
\text { Line Transformer Capacity }\end{array}$ & 101,628 & 3,088 & 1,329 & 103,387 & 5,113 & 98,033 & 241 \\
\hline $\begin{array}{l}\text { (MVa) } \\
\text { Detroit Edison Co }\end{array}$ & 4,464 & 148 & 48 & 4,564 & 313 & 4,248 & 3 \\
\hline Watthour Meter Count .................. & $2,277,209$ & 77,313 & 26,195 & $2,328,327$ & 130,566 & $2,196,757$ & 1,004 \\
\hline $\begin{array}{l}\text { Line Transformer Count ................ } \\
\text { Line Transformer Capacity }\end{array}$ & 349,602 & 12,494 & 4,273 & 357,823 & 6,385 & 349,531 & 1,907 \\
\hline $\begin{array}{l}\text { (MVa) } \\
\text { Duke Power Co }\end{array}$ & 19,820 & 671 & 150 & 20,341 & 570 & 19,749 & 22 \\
\hline $\begin{array}{l}\text { Watthour Meter Count ................. } \\
\text { Line Transformer Count .............. } \\
\text { Line Transformer Capacity }\end{array}$ & $\begin{array}{r}1,901,674 \\
585,619\end{array}$ & $\begin{array}{l}68,964 \\
21,467\end{array}$ & $\begin{array}{r}181,929 \\
16,677\end{array}$ & $\begin{array}{r}1,788,709 \\
590,409\end{array}$ & $\begin{array}{l}70,985 \\
14,808\end{array}$ & $\begin{array}{r}1,715,158 \\
575,225\end{array}$ & $\begin{array}{r}2,566 \\
376\end{array}$ \\
\hline $\begin{array}{l}\text { (MVa) } \\
\text { nunuesne Light Co }\end{array}$ & 33,006 & 910 & 522 & 33,394 & 1,365 & 32,010 & 19 \\
\hline $\begin{array}{l}\text { Watthour Meter Count ................. } \\
\text { Line Transformer Count .............. } \\
\text { Line Transformer Capacity }\end{array}$ & $\begin{array}{r}608,663 \\
96,325\end{array}$ & $\begin{array}{l}5,026 \\
3,520\end{array}$ & $\begin{array}{r}9,800 \\
602\end{array}$ & $\begin{array}{r}603,889 \\
99,243\end{array}$ & $\begin{array}{r}17,888 \\
1,622\end{array}$ & - & $\begin{array}{r}586,001 \\
97,621\end{array}$ \\
\hline $\begin{array}{l}\text { (MVa) } \\
\text { Eastern Edison } \mathrm{Co}\end{array}$ & 4,978 & 125 & 14 & 5,089 & 75 & - & 5,014 \\
\hline $\begin{array}{l}\text { Watthour Meter Count ................. } \\
\text { Line Transformer Count .............. } \\
\text { Line Transformer Capacity }\end{array}$ & $\begin{array}{r}203,855 \\
31,119\end{array}$ & $\begin{array}{l}3,819 \\
1,280\end{array}$ & $\begin{array}{l}3,702 \\
1,439\end{array}$ & $\begin{array}{r}203,972 \\
30,960\end{array}$ & $\begin{array}{l}7,243 \\
2,327\end{array}$ & $\begin{array}{r}199,123 \\
28,937\end{array}$ & $\begin{array}{l}22 \\
61\end{array}$ \\
\hline $\begin{array}{l}\text { (MVa) } \\
\text { Edison Sault Electric } \mathrm{Co}_{0}\end{array}$ & 1,621 & 40 & 305 & 1,356 & 199 & 1,156 & 1 \\
\hline $\begin{array}{l}\text { Watthour Meter Count ................ } \\
\text { Line Transformer Count ............ } \\
\text { Line Transformer Capacity }\end{array}$ & $\begin{array}{r}21,542 \\
7,755\end{array}$ & $\begin{array}{l}321 \\
242\end{array}$ & $\begin{array}{r}390 \\
3\end{array}$ & $\begin{array}{r}21,473 \\
7,994\end{array}$ & $\begin{array}{l}649 \\
725\end{array}$ & $\begin{array}{r}20,790 \\
7,238\end{array}$ & $\begin{array}{l}34 \\
31\end{array}$ \\
\hline (MVa) Paso Electric Co & 244 & 7 & - & 251 & 37 & 213 & 1 \\
\hline $\begin{array}{l}\text { Watthour Meter Count ................. } \\
\text { Line Transformer Count ............ } \\
\text { Line Transformer Capacity }\end{array}$ & $\begin{array}{r}290,323 \\
61,036\end{array}$ & $\begin{array}{l}7,187 \\
1,495\end{array}$ & $\begin{array}{r}3,136 \\
37\end{array}$ & $\begin{array}{r}294,374 \\
62,494\end{array}$ & $\begin{array}{l}3,989 \\
1,636\end{array}$ & $\begin{array}{r}290,208 \\
60,729\end{array}$ & $\begin{array}{l}177 \\
129\end{array}$ \\
\hline $\begin{array}{l}\text { (MVa) } \\
\text { Empire District Electric Co }\end{array}$ & 2,505 & 72 & 1 & 2,576 & 170 & 2,405 & 1 \\
\hline $\begin{array}{l}\text { Watthour Meter Count ................ } \\
\text { Line Transformer Count .............. } \\
\text { Line Transformer Capacity }\end{array}$ & $\begin{array}{r}129,535 \\
61,616\end{array}$ & $\begin{array}{l}5,848 \\
2,665\end{array}$ & $\begin{array}{r}2,243 \\
726\end{array}$ & $\begin{array}{r}133,140 \\
63,555\end{array}$ & $\begin{array}{r}15,064 \\
3,222\end{array}$ & $\begin{array}{r}117,940 \\
60,333\end{array}$ & -136 \\
\hline$(\mathrm{MVa})$ & 1,894 & 133 & 16 & 2,011 & 103 & 1,908 & - \\
\hline
\end{tabular}

See notes and footnotes at end of table. 
Table 44. Watthour Meter and Line Transformer Statistics of Investor-Owned UtIlitles, 1992 (Count and Megavoltampere) (Continued)

\begin{tabular}{|c|c|c|c|c|c|c|c|}
\hline $\begin{array}{l}\text { Diatribution } \\
\text { Equlpment }\end{array}$ & $\begin{array}{l}\text { Number at } \\
\text { Beginning } \\
\text { of Year }\end{array}$ & $\begin{array}{l}\text { Additions } \\
\text { During } \\
\text { Year }\end{array}$ & $\begin{array}{l}\text { Reductions } \\
\text { During } \\
\text { Year }\end{array}$ & $\begin{array}{l}\text { Number at } \\
\text { End of } \\
\text { Year }\end{array}$ & $\begin{array}{l}\text { In Stock, } \\
\text { Locked, } \\
\text { and Inactive }\end{array}$ & $\begin{array}{l}\text { In } \\
\text { Customers } \\
\text { Use }\end{array}$ & $\begin{array}{l}\text { In } \\
\text { Company } \\
\text { Use }\end{array}$ \\
\hline \multicolumn{8}{|l|}{ Exeter \& Hampton Electric Co } \\
\hline $\begin{array}{l}\text { Watthour Meter Count ................ } \\
\text { Line Transformer Count ............. } \\
\text { Line Transformer Capacity }\end{array}$ & $\begin{array}{r}36,331 \\
6,850\end{array}$ & $\begin{array}{r}1,563 \\
120\end{array}$ & $\begin{array}{r}651 \\
85\end{array}$ & $\begin{array}{r}37,243 \\
6,885\end{array}$ & $\begin{array}{r}2,954 \\
473\end{array}$ & $\begin{array}{r}34,281 \\
6,408\end{array}$ & $\begin{array}{l}8 \\
4\end{array}$ \\
\hline $\begin{array}{l}\text { (MVa) ... } \\
\text { Fitchburg Gas \& Elec Light Co }\end{array}$ & 255 & 5 & 2 & 258 & 24 & 234 & - \\
\hline $\begin{array}{l}\text { Watthour Meter Count ................. } \\
\text { Line Transformer Count ............. } \\
\text { Line Transformer Capacity }\end{array}$ & $\begin{array}{r}26,491 \\
5,463\end{array}$ & $\begin{array}{l}633 \\
382\end{array}$ & $\begin{array}{l}557 \\
133\end{array}$ & $\begin{array}{r}26,567 \\
5,692\end{array}$ & $\begin{array}{r}1,825 \\
494\end{array}$ & $\begin{array}{l}24,735 \\
--\end{array}$ & $\begin{array}{r}7 \\
5,198\end{array}$ \\
\hline $\begin{array}{l}\text { (MVa) } \\
\text { Florida Power \& Light Co }\end{array}$ & 167 & 2 & 8 & 161 & 19 & - & 142 \\
\hline $\begin{array}{l}\text { Watthour Meter Count ................. } \\
\text { Line Transformer Count ............ } \\
\text { Line Transformer Capacity }\end{array}$ & $\begin{array}{r}3,511,835 \\
655,510\end{array}$ & $\begin{array}{r}147,073 \\
39,735\end{array}$ & $\begin{array}{l}89,284 \\
23,089\end{array}$ & $\begin{array}{r}3,569,624 \\
672,156\end{array}$ & $\begin{array}{r}261,082 \\
17,078\end{array}$ & $\begin{array}{r}3,308,145 \\
653,585\end{array}$ & $\begin{array}{r}397 \\
1,493\end{array}$ \\
\hline $\begin{array}{l}\text { (MVa) } \\
\text { Florida Power Corp }\end{array}$ & 35,390 & 1,842 & 892 & 36,340 & 1,350 & 34,874 & 116 \\
\hline $\begin{array}{l}\text { Watthour Meter Count ................. } \\
\text { Line Transformer Count ............ } \\
\text { Line Transformer Capacity }\end{array}$ & $\begin{array}{r}1,341,948 \\
289,009\end{array}$ & $\begin{array}{l}50,746 \\
12,421\end{array}$ & $\begin{array}{l}24,699 \\
11,237\end{array}$ & $\begin{array}{r}1,367,995 \\
290,193\end{array}$ & $\begin{array}{r}144,023 \\
4,518\end{array}$ & $1,223,536$ & $\begin{array}{r}436 \\
285,675\end{array}$ \\
\hline $\begin{array}{l}\text { (MVa) .... } \\
\text { Georgia Power Co }\end{array}$ & 12,796 & 599 & 542 & 12,853 & 337 & - & 12,516 \\
\hline $\begin{array}{l}\text { Watthour Meter Count ................. } \\
\text { Line Transformer Count ............. } \\
\text { Line Transformer Capacity }\end{array}$ & $\begin{array}{r}1,729,834 \\
534,396\end{array}$ & $\begin{array}{l}32,751 \\
11,272\end{array}$ & $\begin{array}{r}74,857 \\
7,531\end{array}$ & $\begin{array}{r}1,687,728 \\
538,137\end{array}$ & $\begin{array}{l}76,965 \\
25,176\end{array}$ & - & $\begin{array}{r}1,610,763 \\
512,961\end{array}$ \\
\hline $\begin{array}{l}\text { (MVa) } \\
\text { Granite State Electric Co }\end{array}$ & 25,334 & 593 & 434 & 25,493 & 2,496 & - & 22,997 \\
\hline $\begin{array}{l}\text { Watthour Meter Count ................. } \\
\text { Line Transformer Count ............. } \\
\text { Line Transformer Capacity }\end{array}$ & $\begin{array}{r}37,305 \\
8,269\end{array}$ & $\begin{array}{r}1,146 \\
102\end{array}$ & $\begin{array}{r}1,040 \\
199\end{array}$ & $\begin{array}{r}37,411 \\
8,172\end{array}$ & $\begin{array}{l}2,257 \\
1,357\end{array}$ & $\begin{array}{r}34,794 \\
6,769\end{array}$ & $\begin{array}{r}360 \\
46\end{array}$ \\
\hline $\begin{array}{l}\text { (MVa) } \\
\text { Green Mountain Power Corp }\end{array}$ & 330 & 8 & 6 & 332 & 30 & 301 & 1 \\
\hline $\begin{array}{l}\text { Watthour Meter Count ................. } \\
\text { Line Transformer Count ............ } \\
\text { Line Transformer Capacity }\end{array}$ & $\begin{array}{l}82,905 \\
31,229\end{array}$ & $\begin{array}{r}1,133 \\
297\end{array}$ & $\begin{array}{r}740 \\
2,232\end{array}$ & $\begin{array}{l}83,298 \\
29,294\end{array}$ & $\begin{array}{l}3,572 \\
1,321\end{array}$ & $\begin{array}{l}79,574 \\
27,865\end{array}$ & $\begin{array}{l}152 \\
108\end{array}$ \\
\hline $\begin{array}{l}(\mathrm{MVa}) \\
\text { Gulf Power Co }\end{array}$ & 1,027 & 8 & 142 & 893 & 67 & 823 & 3 \\
\hline $\begin{array}{l}\text { Watthour Meter Count .................. } \\
\text { Line Transformer Count .............. } \\
\text { Line Transformer Capacity }\end{array}$ & $\begin{array}{l}322,165 \\
110,186\end{array}$ & $\begin{array}{l}6,657 \\
5,392\end{array}$ & $\begin{array}{l}3,281 \\
2,570\end{array}$ & $\begin{array}{l}325,541 \\
113,008\end{array}$ & $\begin{array}{r}23,540 \\
4,332\end{array}$ & $\begin{array}{l}301,934 \\
108,585\end{array}$ & $\begin{array}{l}67 \\
91\end{array}$ \\
\hline Gulf States Utilities Co & 3,894 & 220 & 60 & 4,054 & 224 & 3,819 & 11 \\
\hline $\begin{array}{l}\text { Watthour Meter Count .................. } \\
\text { Line Transformer Count ............. } \\
\text { Line Transformer Capacity }\end{array}$ & $\begin{array}{l}606,189 \\
284,624\end{array}$ & $\begin{array}{r}19,545 \\
5,147\end{array}$ & $\begin{array}{l}9,656 \\
6,139\end{array}$ & $\begin{array}{l}616,078 \\
283,632\end{array}$ & $\begin{array}{r}29,919 \\
2,511\end{array}$ & $\begin{array}{l}584,359 \\
279,428\end{array}$ & $\begin{array}{l}1,800 \\
1,693\end{array}$ \\
\hline $\begin{array}{l}(\mathrm{MVa}) \\
\text { Hawailan Electric Co Inc }\end{array}$ & 12,586 & 353 & 288 & 12,651 & 342 & 12,264 & 45 \\
\hline $\begin{array}{l}\text { Watthour Meter Count .................. } \\
\text { Line Transformer Count ............. } \\
\text { Line Transformer Capacity }\end{array}$ & $\begin{array}{r}262,663 \\
29,258\end{array}$ & $\begin{array}{l}7,419 \\
1,696\end{array}$ & $\begin{array}{l}3,727 \\
1,190\end{array}$ & $\begin{array}{r}266,355 \\
28,764\end{array}$ & ${ }_{-}^{5,226}$ & $\begin{array}{r}260,381 \\
29,764\end{array}$ & $-{ }^{748}$ \\
\hline (MVa) .................................. & 2,602 & 160 & 73 & 2,689 & - & 2,689 & - \\
\hline Watthour Meter Count ...................... & 1 & 2 & - & 3 & - & -- & 3 \\
\hline $\begin{array}{l}\text { Line Transformer Count ............... } \\
\text { Holyoke Water Power Co }\end{array}$ & 1 & - & - & 1 & - & - & 1 \\
\hline $\begin{array}{l}\text { Watthour Meter Count .................. } \\
\text { Line Transformer Count .............. } \\
\text { Line Transformer Capacity }\end{array}$ & $\begin{array}{l}455 \\
183\end{array}$ & $\begin{array}{l}20 \\
20\end{array}$ & $\begin{array}{r}23 \\
2\end{array}$ & $\begin{array}{l}452 \\
201\end{array}$ & $\begin{array}{r}182 \\
32\end{array}$ & $\begin{array}{l}218 \\
151\end{array}$ & $\begin{array}{l}52 \\
18\end{array}$ \\
\hline $\begin{array}{l}\text { (MVa) .................................. } \\
\text { Houston Lighting \& Power Co }\end{array}$ & 100 & 4 & 2 & 102 & 17 & 81 & 4 \\
\hline $\begin{array}{l}\text { Watthour Meter Count .................. } \\
\text { Line Transformer Count .............. } \\
\text { Line Transformer Capacity }\end{array}$ & $\begin{array}{r}1,714,885 \\
391,999\end{array}$ & $\begin{array}{l}18,782 \\
16,429\end{array}$ & $\begin{array}{r}8,275 \\
15,162\end{array}$ & $\begin{array}{r}1,725,392 \\
393,266\end{array}$ & $\begin{array}{l}50,162 \\
10,095\end{array}$ & - & $\begin{array}{r}1,675,230 \\
383,171\end{array}$ \\
\hline $\begin{array}{l}\text { (MVa) } \\
\text { Idaho Power Co }\end{array}$ & 26,881 & 1,104 & 912 & 27,073 & 12,336 & - & 14,737 \\
\hline $\begin{array}{l}\text { Watthour Meter Count .................. } \\
\text { Line Transformer Count .............. } \\
\text { Line Transformer Capacity }\end{array}$ & $\begin{array}{l}307,976 \\
158,215\end{array}$ & $\begin{array}{r}14,578 \\
5,868\end{array}$ & $\begin{array}{l}7,185 \\
2,816\end{array}$ & $\begin{array}{l}315,369 \\
161,267\end{array}$ & $\begin{array}{l}6,266 \\
4,818\end{array}$ & $\begin{array}{l}307,503 \\
154,847\end{array}$ & $\begin{array}{l}1,600 \\
1,602\end{array}$ \\
\hline $\begin{array}{l}(\mathrm{MVa}) \\
\text { Illinois Power } \mathrm{Co}\end{array}$ & 7,014 & 307 & 101 & 7,220 & 556 & 6,652 & 12 \\
\hline $\begin{array}{l}\text { Watthour Meter Count ................. } \\
\text { Line Transformer Count ............. } \\
\text { Line Transformer Capacity }\end{array}$ & $\begin{array}{l}594,685 \\
190,316\end{array}$ & $\begin{array}{r}14,807 \\
2,777\end{array}$ & $\begin{array}{r}13,373 \\
1,770\end{array}$ & $\begin{array}{l}596,119 \\
191,323\end{array}$ & $\begin{array}{r}33,928 \\
6,628\end{array}$ & $\begin{array}{l}562,191 \\
183,816\end{array}$ & 879 \\
\hline$(\mathrm{MVa})$ & 7,868 & 148 & 69 & 7,947 & 557 & 7,369 & 21 \\
\hline
\end{tabular}

See notes and footnotes at end of table. 
Table 44. Watthour Moter and Line Transformer Statistics of investor-Owned Utilities, 1992 (Count and Megavoltampere) (Continued)

\begin{tabular}{|c|c|c|c|c|c|c|c|}
\hline $\begin{array}{l}\text { Dietribution } \\
\text { Equipment }\end{array}$ & $\begin{array}{l}\text { Number at } \\
\text { Beghning } \\
\text { of Year }\end{array}$ & $\begin{array}{l}\text { Additions } \\
\text { During } \\
\text { Year }\end{array}$ & $\begin{array}{l}\text { Reductions } \\
\text { During } \\
\text { Year }\end{array}$ & $\begin{array}{l}\text { Number at } \\
\text { End of } \\
\text { Yoar }\end{array}$ & $\begin{array}{l}\text { In Stock, } \\
\text { Locked, } \\
\text { and Inactive ' }\end{array}$ & $\begin{array}{l}\text { In } \\
\text { Customers } \\
\text { Uee }\end{array}$ & $\begin{array}{l}\text { In } \\
\text { Company } \\
\text { Use }\end{array}$ \\
\hline \multicolumn{8}{|l|}{ Indiana Michigan Power Co } \\
\hline $\begin{array}{l}\text { Watthour Meter Count ................. } \\
\text { Line Transformer Count .............. } \\
\text { Line Transformer Lapacity }\end{array}$ & $\begin{array}{l}556,409 \\
159,994\end{array}$ & $\begin{array}{r}22,280 \\
4,832\end{array}$ & $\begin{array}{r}23,524 \\
3,718\end{array}$ & $\begin{array}{l}555,165 \\
161,208\end{array}$ & $\begin{array}{r}26,576 \\
2,860\end{array}$ & $\begin{array}{l}527,812 \\
157,131\end{array}$ & $\begin{array}{r}777 \\
1,217\end{array}$ \\
\hline 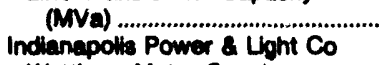 & 6,067 & 199 & 123 & 6,143 & 282 & 5,784 & 77 \\
\hline $\begin{array}{l}\text { Wotthour Moter Count ................ } \\
\text { Line Transformer Count ............. } \\
\text { Line Transformer Capacity }\end{array}$ & $\begin{array}{r}428,489 \\
79,551\end{array}$ & $\begin{array}{r}14,837 \\
2,808\end{array}$ & $\begin{array}{l}8,630 \\
1,131\end{array}$ & $\begin{array}{r}432,786 \\
81,228\end{array}$ & $\begin{array}{r}23,802 \\
4,877\end{array}$ & $\begin{array}{r}408,924 \\
76,107\end{array}$ & $\begin{array}{r}70 \\
244\end{array}$ \\
\hline Interatate Power Co & 5,276 & 237 & 42 & 5,471 & 510 & 4,948 & 13 \\
\hline $\begin{array}{l}\text { Watthour Meter Count .................. } \\
\text { Line Transformer Count .............. } \\
\text { Line Transformer Capacty }\end{array}$ & $\begin{array}{r}185,299 \\
47,519\end{array}$ & $\begin{array}{r}5,512 \\
784\end{array}$ & $\begin{array}{r}5,345 \\
569\end{array}$ & $\begin{array}{r}185,468 \\
47,734\end{array}$ & $\begin{array}{l}6,399 \\
1,947\end{array}$ & $\begin{array}{r}178,718 \\
45,787\end{array}$ & -351 \\
\hline Iown Electric Light \& Power Co & 1,748 & 39 & 13 & 1,775 & 110 & 1,685 & - \\
\hline $\begin{array}{l}\text { Watthour Meter Count ................. } \\
\text { Uns Transformer Count .............. } \\
\text { Une Transformer Cepacty }\end{array}$ & $\begin{array}{r}228,035 \\
68,749\end{array}$ & $\begin{array}{l}5,085 \\
1,945\end{array}$ & $\begin{array}{l}4,208 \\
1,076\end{array}$ & $\begin{array}{r}228,692 \\
69,618\end{array}$ & $\begin{array}{l}8,754 \\
2,861\end{array}$ & $\begin{array}{r}219,521 \\
66,757\end{array}$ & $-{ }_{-}^{617}$ \\
\hline (MVa) ................................ & 2,389 & 89 & 27 & 2,451 & 183 & 2,268 & - \\
\hline $\begin{array}{l}\text { Watthour Meter Count ................... } \\
\text { Line Transformer Count .............. } \\
\text { Line Transformer Capacty }\end{array}$ & $\begin{array}{r}201,777 \\
56,755\end{array}$ & $\begin{array}{l}6,541 \\
2,778\end{array}$ & $\begin{array}{l}3,802 \\
1,921\end{array}$ & $\begin{array}{r}204,516 \\
57,612\end{array}$ & $\begin{array}{r}11,508 \\
3,869\end{array}$ & $\begin{array}{r}193,010 \\
53,643\end{array}$ & $\overline{-}$ \\
\hline (MVa) ............................... & 2,781 & 61 & 69 & 2,783 & 317 & 2,466 & - \\
\hline $\begin{array}{l}\text { Watthour Meter Count .................. } \\
\text { Line Transformer Count .............. } \\
\text { Line Transformer Capacity }\end{array}$ & $\begin{array}{l}888,223 \\
188,292\end{array}$ & $\begin{array}{r}69,351 \\
4,649\end{array}$ & $\begin{array}{r}19,653 \\
4,503\end{array}$ & $\begin{array}{r}1,037,921 \\
198,438\end{array}$ & $\begin{array}{r}115,860 \\
7,927\end{array}$ & $\begin{array}{l}921,811 \\
190,511\end{array}$ & -250 \\
\hline Kansas City Power \& Light Co & 10,253 & 246 & 242 & 10,257 & 722 & 9,535 & - \\
\hline $\begin{array}{l}\text { Watthour Meter Count ................. } \\
\text { Line Transformer Count .............. } \\
\text { Live Transformer Cepacity }\end{array}$ & $\begin{array}{r}473,547 \\
96,668\end{array}$ & $\begin{array}{r}11,185 \\
3,512\end{array}$ & $\begin{array}{r}22,911 \\
2,280\end{array}$ & $\begin{array}{r}481,821 \\
97,901\end{array}$ & $\begin{array}{r}16,742 \\
1,529\end{array}$ & $\begin{array}{r}445,012 \\
96,372\end{array}$ & $\begin{array}{r}67 \\
-\quad\end{array}$ \\
\hline (MVa) & 5,469 & 227 & 95 & 5,601 & 269 & 5,5312 & - \\
\hline $\begin{array}{l}\text { Watthour Meter Count ................. } \\
\text { Line Transformer Count ............... } \\
\text { Line Transformer Capacity }\end{array}$ & $\begin{array}{r}272,971 \\
95,310\end{array}$ & $\begin{array}{l}4,511 \\
2,498\end{array}$ & $\begin{array}{r}3,071 \\
716\end{array}$ & $\begin{array}{r}274,411 \\
97,092\end{array}$ & $\begin{array}{l}4,867 \\
3,250\end{array}$ & $\begin{array}{r}268,210 \\
93,673\end{array}$ & $\begin{array}{l}334 \\
169\end{array}$ \\
\hline $\begin{array}{l}\text { (MVa) } \\
\text { Kentucky Power Co }\end{array}$ & 4,242 & 229 & 24 & 4,447 & 287 & 4,138 & 22 \\
\hline $\begin{array}{l}\text { Watthour Meter Count .................. } \\
\text { Line Transformer Count .............. } \\
\text { Line Transformer Capacity }\end{array}$ & $\begin{array}{r}168,053 \\
78,073\end{array}$ & $\begin{array}{l}5,893 \\
5,174\end{array}$ & $\begin{array}{l}3,920 \\
4,224\end{array}$ & $\begin{array}{r}170,026 \\
78,023\end{array}$ & $\begin{array}{l}7,769 \\
1,792\end{array}$ & $\begin{array}{r}162,204 \\
77,168\end{array}$ & $\begin{array}{l}53 \\
63\end{array}$ \\
\hline $\begin{array}{l}\text { (MVa) } \\
\text { Kentucky Utilities Co }\end{array}$ & 2,404 & 166 & 138 & 2,432 & 156 & 2,274 & 2 \\
\hline $\begin{array}{l}\text { Watthour Meter Count .................. } \\
\text { Line Transformer Count .............. } \\
\text { Line Transformer Capacity }\end{array}$ & $\begin{array}{l}488,112 \\
172,592\end{array}$ & $\begin{array}{r}17,488 \\
4,929\end{array}$ & $\begin{array}{r}4,124 \\
790\end{array}$ & $\begin{array}{l}501,476 \\
176,731\end{array}$ & $\begin{array}{l}54,297 \\
-\end{array}$ & $\begin{array}{l}446,430 \\
176,731\end{array}$ & $-{ }^{749}$ \\
\hline Kingeport Power Co & 5,941 & 180 & 20 & 6,111 & - & 6,111 & - \\
\hline $\begin{array}{l}\text { Watthour Meter Count ................ } \\
\text { Lne Transformer Count ............... } \\
\text { Line Transformer Capacity }\end{array}$ & $\begin{array}{l}40,972 \\
12,880\end{array}$ & $\begin{array}{l}959 \\
431\end{array}$ & $\begin{array}{l}577 \\
759\end{array}$ & $\begin{array}{l}41,354 \\
12,652\end{array}$ & $\begin{array}{r}1,220 \\
472\end{array}$ & $\begin{array}{l}40,111 \\
12,171\end{array}$ & $\begin{array}{r}23 \\
9\end{array}$ \\
\hline $\begin{array}{l}\text { (MVa) ................................. } \\
\text { Lockhart Power Co }\end{array}$ & 543 & 20 & 5 & 558 & 38 & 519 & 1 \\
\hline $\begin{array}{l}\text { Watthour Meter Count ................. } \\
\text { Line Transformer Crunt .............. } \\
\text { Line Transformer Capacity }\end{array}$ & $\begin{array}{l}5,564 \\
3,031\end{array}$ & $\begin{array}{l}21 \% \\
113\end{array}$ & $\begin{array}{l}309 \\
317\end{array}$ & $\begin{array}{l}5,467 \\
2,827\end{array}$ & $\begin{array}{r}52 \\
148\end{array}$ & $\begin{array}{l}5,387 \\
2,679\end{array}$ & -28 \\
\hline $\begin{array}{l}\text { (MVa) .......................................... } \\
\text { Long lsland Lighting Co }\end{array}$ & 83 & 3 & 4 & 82 & 6 & 76 & - \\
\hline $\begin{array}{l}\text { Watthour Meter Count ................... } \\
\text { Line Transformer Count .............. } \\
\text { Line Transformer Capacity }\end{array}$ & $\begin{array}{r}1,148,031 \\
180,642\end{array}$ & $\begin{array}{r}26,054 \\
3,080\end{array}$ & $\begin{array}{r}38,380 \\
3,834\end{array}$ & $\begin{array}{r}1,135,805 \\
179,888\end{array}$ & $\begin{array}{r}7,000 \\
25,697\end{array}$ & $\overline{-}$ & $\begin{array}{r}1,128,805 \\
154,191\end{array}$ \\
\hline (MVa) .............................. & 9,836 & 229 & 231 & 9,834 & 2,092 & - & 7,742 \\
\hline $\begin{array}{l}\text { Watthour Meter Count ................... } \\
\text { Line Transformer Count .............. } \\
\text { Line Transformer Capacity }\end{array}$ & $\begin{array}{l}654,816 \\
181,551\end{array}$ & $\begin{array}{r}28,158 \\
7,214\end{array}$ & $\begin{array}{r}15,326 \\
6,399\end{array}$ & $\begin{array}{l}665,648 \\
182,366\end{array}$ & $\begin{array}{r}58,578 \\
9,780\end{array}$ & $\begin{array}{l}606,981 \\
170,852\end{array}$ & $\begin{array}{r}89 \\
1,634\end{array}$ \\
\hline$(\mathrm{MVa})$ & 8,901 & 444 & 308 & 9,127 & 932 & 8,047 & 148 \\
\hline
\end{tabular}

See notes and footnotes at end of table. 
Table 44. Watthour Meter and Line Transformer Statistics of Investor-Owned Utilities, 1992 (Count and Megavoltampere) (Continued)

\begin{tabular}{|c|c|c|c|c|c|c|c|}
\hline $\begin{array}{l}\text { Dictutbution } \\
\text { Equipment }\end{array}$ & $\begin{array}{l}\text { Number at } \\
\text { Beginning } \\
\text { of Year }\end{array}$ & $\begin{array}{l}\text { Additions } \\
\text { During } \\
\text { Year }\end{array}$ & $\begin{array}{l}\text { Reductions } \\
\text { During } \\
\text { Year }\end{array}$ & $\begin{array}{l}\text { Number at } \\
\text { End of } \\
\text { Year }\end{array}$ & $\begin{array}{l}\text { In Stock, } \\
\text { Locked, } \\
\text { and Inactive }\end{array}$ & $\begin{array}{l}\text { In } \\
\text { Customers } \\
\text { Use }\end{array}$ & $\underset{\text { Use }}{\ln }$ \\
\hline \multicolumn{8}{|l|}{ Louisville Gas \& Electric Co } \\
\hline $\begin{array}{l}\text { Watthour Meter Count ................... } \\
\text { Line Transformer Count ............... } \\
\text { Line Transformer Capacity }\end{array}$ & $\begin{array}{r}358,681 \\
77,556\end{array}$ & $\begin{array}{r}13,620 \\
1,746\end{array}$ & $\begin{array}{r}13,710 \\
1,198\end{array}$ & $\begin{array}{r}358,571 \\
78,104\end{array}$ & $\begin{array}{r}22,399 \\
2,387\end{array}$ & $\begin{array}{r}336,051 \\
75,717\end{array}$ & -121 \\
\hline Madison Gas \& Electric Co & 4,189 & 115 & 39 & 4,265 & 130 & 4,135 & - \\
\hline $\begin{array}{l}\text { Watthour Meter Count ................. } \\
\text { Line Transformer Count ............ } \\
\text { Line Transformer Capacity }\end{array}$ & $\begin{array}{r}116,724 \\
18,661\end{array}$ & $\begin{array}{r}1,890 \\
360\end{array}$ & $\begin{array}{l}855 \\
151\end{array}$ & $\begin{array}{r}117,759 \\
18,870\end{array}$ & $\begin{array}{l}3,107 \\
1,373\end{array}$ & $\begin{array}{r}114,571 \\
17,430\end{array}$ & $\begin{array}{l}81 \\
67\end{array}$ \\
\hline $\begin{array}{l}\text { (MVa) .................................... } \\
\text { Maine Public Service Co }\end{array}$ & 1,148 & 30 & 5 & 1,173 & 120 & 1,050 & 3 \\
\hline $\begin{array}{l}\text { Watthour Meter Count ................. } \\
\text { Line Transformer Count ............. } \\
\text { Line Transformer Capacity }\end{array}$ & $\begin{array}{l}35,235 \\
11,430\end{array}$ & $\begin{array}{r}1,408 \\
187\end{array}$ & $\begin{array}{r}1,283 \\
49\end{array}$ & $\begin{array}{l}35,360 \\
11,568\end{array}$ & $\begin{array}{r}1,602 \\
564\end{array}$ & $\begin{array}{l}33,615 \\
11,004\end{array}$ & $\begin{array}{l}143 \\
-\end{array}$ \\
\hline $\begin{array}{l}\text { (MVa) ................................... } \\
\text { Massachusetts Electric }\end{array}$ & 218 & 5 & 1 & 222 & 27 & 195 & - \\
\hline $\begin{array}{l}\text { Watthour Meter Count ................ } \\
\text { Line Transformer Count ............ } \\
\text { Line Transformer Capacity }\end{array}$ & $\begin{array}{l}896,552 \\
148,470\end{array}$ & $\begin{array}{r}42,878 \\
2,603\end{array}$ & $\begin{array}{r}31,085 \\
3,566\end{array}$ & $\begin{array}{r}1,008,345 \\
147,507\end{array}$ & $\begin{array}{r}35,051 \\
9,990\end{array}$ & $\begin{array}{l}973,083 \\
135,218\end{array}$ & $\begin{array}{r}211 \\
2,299\end{array}$ \\
\hline Maui Electric Co Lid & 7,454 & 142 & 123 & 7,473 & 917 & 6,399 & 157 \\
\hline $\begin{array}{l}\text { Watthour Meter Count ................. } \\
\text { Line Transformer Count ............ } \\
\text { Line Transformer Capacity }\end{array}$ & $\begin{array}{r}51,650 \\
8,880\end{array}$ & $\begin{array}{r}2,655 \\
465\end{array}$ & $\begin{array}{l}255 \\
221\end{array}$ & $\begin{array}{r}54,050 \\
9,124\end{array}$ & - & $\begin{array}{r}54,050 \\
9,124\end{array}$ & - \\
\hline $\begin{array}{l}\text { (MVa) } \\
\text { Metropolitan Edison Co }\end{array}$ & 444 & 31 & 10 & 465 & - & 465 & - \\
\hline $\begin{array}{l}\text { Watthour Meter Count ................. } \\
\text { Line Transformer Count ............. } \\
\text { Line Transformer Capacity }\end{array}$ & $\begin{array}{l}482,005 \\
146,373\end{array}$ & $\begin{array}{r}14,195 \\
3,736\end{array}$ & $\begin{array}{l}8,436 \\
1,605\end{array}$ & $\begin{array}{l}487,764 \\
148,504\end{array}$ & $\begin{array}{r}35,549 \\
3,263\end{array}$ & $\begin{array}{l}449,177 \\
145,241\end{array}$ & 3,038 \\
\hline (MVa) ....................................... & 5,813 & 162 & 71 & 5,904 & 395 & 5,509 & - \\
\hline $\begin{array}{l}\text { Watthour Meter Count ................ } \\
\text { Line Transformer Count ............. } \\
\text { Line Transformer Capacity }\end{array}$ & $\begin{array}{l}446,121 \\
129,342\end{array}$ & $\begin{array}{r}12,466 \\
3,233\end{array}$ & $\begin{array}{r}10,065 \\
1,198\end{array}$ & $\begin{array}{l}448,522 \\
131,377\end{array}$ & $\begin{array}{r}7,331 \\
971\end{array}$ & $\begin{array}{l}441,191 \\
130,406\end{array}$ & - \\
\hline Minnesota Power \& Light Co & 5,710 & 196 & 61 & 5,845 & 73 & 5,772 & - \\
\hline $\begin{array}{l}\text { Watthour Meter Count ................. } \\
\text { Line Transformer Count ............. } \\
\text { Line Transformer Capacity }\end{array}$ & $\begin{array}{r}123,085 \\
37,117\end{array}$ & $\begin{array}{l}4,123 \\
1,353\end{array}$ & $\begin{array}{r}1,770 \\
573\end{array}$ & $\begin{array}{r}125,438 \\
37,897\end{array}$ & $\begin{array}{l}8,887 \\
2,613\end{array}$ & $\begin{array}{r}116,551 \\
35,284\end{array}$ & - \\
\hline Mississippi Power \& Light Co & 1,201 & 58 & 12 & 1,247 & 172 & 1,075 & - \\
\hline $\begin{array}{l}\text { Watthour Meter Count ................ } \\
\text { Line Transformer Count ............... } \\
\text { Line Transformer Capacity }\end{array}$ & $\begin{array}{l}373,806 \\
150,797\end{array}$ & $\begin{array}{r}11,476 \\
4,275\end{array}$ & $\begin{array}{l}5,606 \\
3,073\end{array}$ & $\begin{array}{l}379,676 \\
151,999\end{array}$ & $\begin{array}{r}18,612 \\
3,509\end{array}$ & $\begin{array}{l}360,635 \\
148,490\end{array}$ & -429 \\
\hline Mississippi Power Co & 5,415 & 175 & 101 & 5,489 & 238 & 5,251 & - \\
\hline $\begin{array}{l}\text { Watthour Meter Count ................. } \\
\text { Line Transformer Count ............... } \\
\text { Line Transformer Capacity }\end{array}$ & $\begin{array}{r}186,254 \\
73,388\end{array}$ & $\begin{array}{l}5,161 \\
2,408\end{array}$ & $\begin{array}{l}2,998 \\
2,457\end{array}$ & $\begin{array}{r}188,417 \\
73,339\end{array}$ & $\begin{array}{l}9,831 \\
3,571\end{array}$ & $\begin{array}{r}178,443 \\
69,768\end{array}$ & -143 \\
\hline Monongahela Power $\mathrm{Co}$ & 2,734 & 141 & 81 & 2,794 & - & 2,794 & - \\
\hline $\begin{array}{l}\text { Watthour Meter Count ................. } \\
\text { Line Transformer Count .............. } \\
\text { Line Transformer Capacity }\end{array}$ & $\begin{array}{l}358,626 \\
170,634\end{array}$ & $\begin{array}{r}14,866 \\
6,090\end{array}$ & $\begin{array}{r}12,295 \\
4,225\end{array}$ & $\begin{array}{l}362,197 \\
172,489\end{array}$ & $\begin{array}{r}27,267 \\
3,116\end{array}$ & $\begin{array}{l}334,924 \\
169,383\end{array}$ & - \\
\hline Montana Power Co & 2,902 & 109 & 48 & 2,963 & 126 & 2,837 & - \\
\hline $\begin{array}{l}\text { Watthour Meter Count ................. } \\
\text { Line Transformer Count ............. } \\
\text { Line Transformer Capacity }\end{array}$ & $\begin{array}{l}278,449 \\
105,527\end{array}$ & $\begin{array}{l}7,882 \\
3,854\end{array}$ & $\begin{array}{l}4,726 \\
2,094\end{array}$ & $\begin{array}{l}281,605 \\
107,387\end{array}$ & $\begin{array}{r}17,233 \\
6,476\end{array}$ & $\begin{array}{l}263,957 \\
100,578\end{array}$ & $\begin{array}{l}415 \\
333\end{array}$ \\
\hline Mt Carmel Public Utility Co & 3,408 & 174 & 64 & 3,518 & 452 & 3,042 & 24 \\
\hline $\begin{array}{l}\text { Watthour Meter Count................. } \\
\text { Line Transformer Count ............. } \\
\text { Line Transformer Capacity }\end{array}$ & $\begin{array}{l}6,674 \\
2,433\end{array}$ & $\begin{array}{l}77 \\
30\end{array}$ & $\begin{array}{r}126 \\
14\end{array}$ & $\begin{array}{l}6,625 \\
2,449\end{array}$ & $\begin{array}{r}277 \\
2,434\end{array}$ & 6,328 & $\begin{array}{l}20 \\
15\end{array}$ \\
\hline MDU Resources Group Inc & 68 & 1 & 4 & 65 & 64 & - & 1 \\
\hline $\begin{array}{l}\text { Watthour Meter Count ................. } \\
\text { Line Transformer Count .............. } \\
\text { Line Transformer Capacity }\end{array}$ & $\begin{array}{r}118,341 \\
32,515\end{array}$ & $\begin{array}{l}213 \\
320\end{array}$ & $\overline{-}$ & $\begin{array}{r}118,554 \\
32,835\end{array}$ & $\begin{array}{l}4,672 \\
3,439\end{array}$ & $\begin{array}{r}113,768 \\
29,396\end{array}$ & -114 \\
\hline 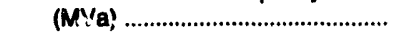 & 1,080 & 64 & - & 1,144 & 136 & 1,008 & - \\
\hline
\end{tabular}

See notes and footnotes at end of table. 
Table 44. Watthour Meter and LIne Transformer Statistics of Investor-Owned Utilities, 1992 (Count and Megavoltampere) (Continued)

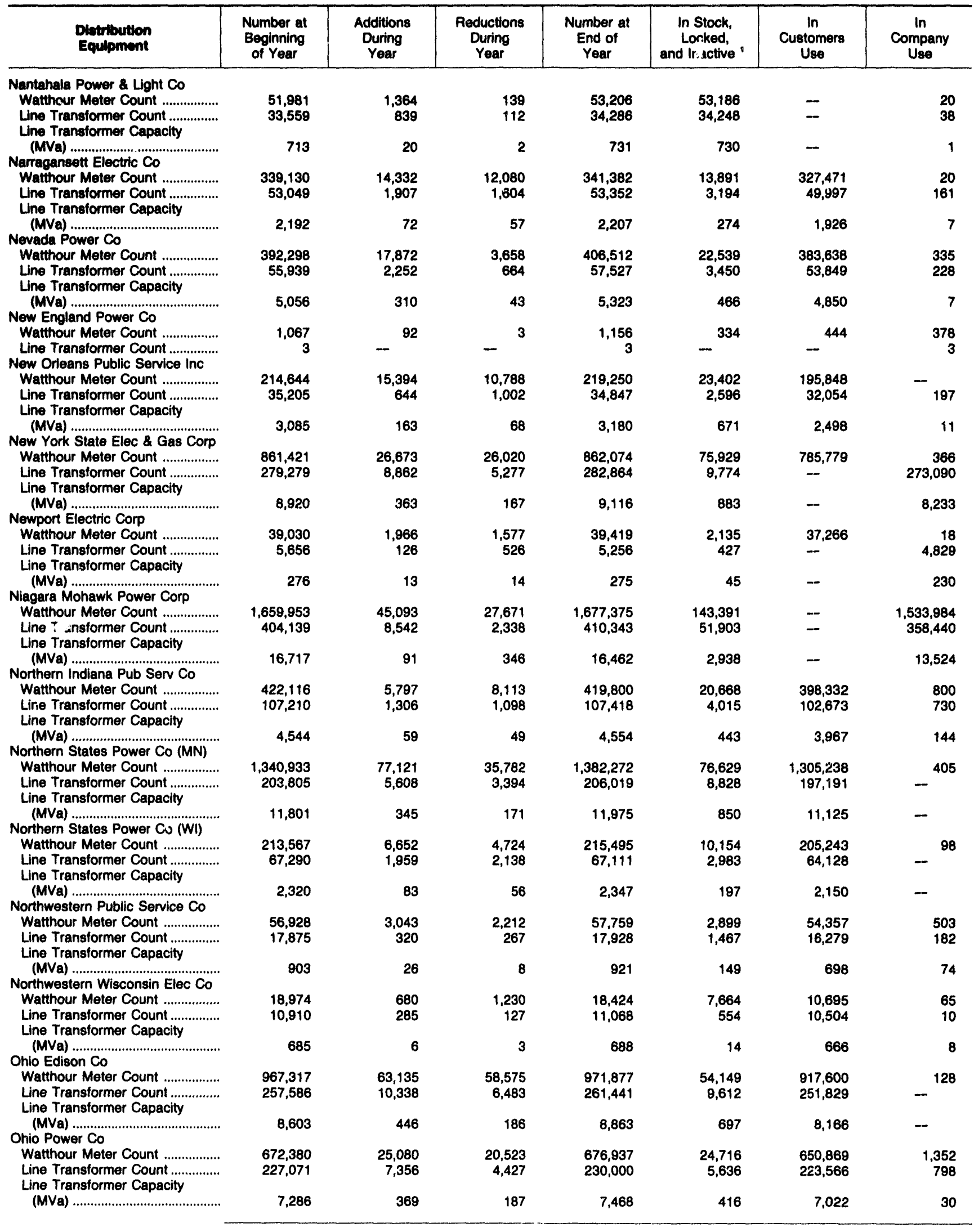

See notes and footnotes at end of table. 
Table 44. Watthour Meter and Line Transformer Statistics of Investor-Owned Utilities, 1992 (Count and Megavoltampere) (Continued)

\begin{tabular}{|c|c|c|c|c|c|c|c|}
\hline $\begin{array}{l}\text { Distribution } \\
\text { Equipment }\end{array}$ & $\begin{array}{l}\text { Number at } \\
\text { Beginning } \\
\text { of Year }\end{array}$ & $\begin{array}{l}\text { Additions } \\
\text { During } \\
\text { Year }\end{array}$ & $\begin{array}{l}\text { Reductions } \\
\text { During } \\
\text { Year }\end{array}$ & $\begin{array}{l}\text { Number at } \\
\text { End of } \\
\text { Year }\end{array}$ & $\begin{array}{l}\text { In Stock, } \\
\text { Locked, } \\
\text { and Inactive }\end{array}$ & $\begin{array}{l}\text { In } \\
\text { Customers } \\
\text { Use }\end{array}$ & $\begin{array}{l}\text { In } \\
\text { Company } \\
\text { Use }\end{array}$ \\
\hline \multicolumn{8}{|l|}{ Orange \& Rockland Utils Inc } \\
\hline $\begin{array}{l}\text { Line Transformer Count ..................... } \\
\text { Line Transformer Capacity }\end{array}$ & $\begin{array}{r}202,505 \\
61,445\end{array}$ & $\begin{array}{l}2,103 \\
1,794\end{array}$ & $\begin{array}{l}2,558 \\
1,317\end{array}$ & $\begin{array}{r}202,650 \\
61,922\end{array}$ & $\begin{array}{l}15,514 \\
13,818\end{array}$ & $\begin{array}{r}18,114 \\
48,104\end{array}$ & $--^{22}$ \\
\hline $\begin{array}{l}\text { (MVa) } \\
\text { Otter Tall Power Co }\end{array}$ & 3,189 & 108 & 83 & 3,214 & 963 & 2,251 & -- \\
\hline 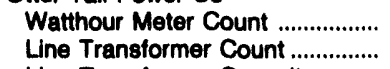 & $\begin{array}{r}165,922 \\
40,338\end{array}$ & $\begin{array}{r}5,516 \\
297\end{array}$ & $\begin{array}{r}5,279 \\
443\end{array}$ & $\begin{array}{r}166,159 \\
40,192\end{array}$ & $\begin{array}{r}10,803 \\
1,758\end{array}$ & $\begin{array}{r}155,074 \\
38,434\end{array}$ & $--^{282}$ \\
\hline $\begin{array}{l}\text { Line Transformer Capacity } \\
\text { (MVa) }\end{array}$ & 1,686 & 24 & 12 & 1,698 & 138 & 1,560 & - \\
\hline Pacific Gas \& Electric Co & & & & & & & \\
\hline $\begin{array}{l}\text { Watthour Meter Count .................... } \\
\text { Line Transformer Count ................. }\end{array}$ & $\begin{array}{r}4,569,258 \\
909,985\end{array}$ & $\begin{array}{r}119,833 \\
19,313\end{array}$ & $\begin{array}{l}68,442 \\
11,365\end{array}$ & $\begin{array}{r}4,620,649 \\
917,933\end{array}$ & $\begin{array}{r}316,541 \\
44,569\end{array}$ & $\begin{array}{r}4,304,108 \\
868,970\end{array}$ & $\overline{4,394}$ \\
\hline $\begin{array}{l}\text { Line Transformer Capacity } \\
\text { (MVa) }\end{array}$ & 35,465 & 785 & 335 & 35,915 & 1,843 & 33,984 & 88 \\
\hline Watthour Meter Count .................. & $1,412,764$ & 144,780 & 57.269 & $1,500,275$ & 98,963 & - & $1,401,312$ \\
\hline $\begin{array}{l}\text { Line Transformer Count ............... } \\
\text { Line Transformer Capacity }\end{array}$ & 443,507 & 29,106 & 10,872 & 461,741 & 48,833 & - & 412,908 \\
\hline $\begin{array}{l}\text { (MVa) } \\
\text { Pennsylvania Electric Co }\end{array}$ & 17,254 & 1,643 & 304 & 18,593 & 2,607 & - & 15,986 \\
\hline $\begin{array}{l}\text { Pennsylvania Electric Co } \\
\text { Watthour Meter Count ................. }\end{array}$ & & & & & & & \\
\hline 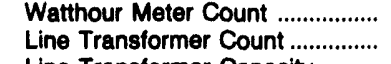 & $\begin{array}{l}585,216 \\
173,632\end{array}$ & $\begin{array}{r}20,432 \\
5,534\end{array}$ & $\begin{array}{r}13,161 \\
3,439\end{array}$ & $\begin{array}{l}592,487 \\
175,727\end{array}$ & $\begin{array}{l}24,614 \\
12,436\end{array}$ & $\begin{array}{l}567,873 \\
163,291\end{array}$ & $\overline{-}$ \\
\hline 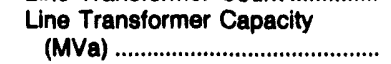 & 6.824 & & & & & & \\
\hline Pennsylvania Power \& Light Co & $0,0<4$ & 210 & 154 & 6,946 & 987 & 5,959 & - \\
\hline 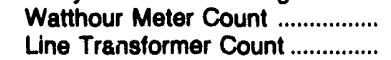 & $1,238,925$ & 44,084 & 33,338 & $1,249,671$ & 41,755 & $1,207,916$ & - \\
\hline $\begin{array}{l}\text { Line Transformer Count .............. } \\
\text { Line Transformer Capacity }\end{array}$ & 387,318 & 10,408 & 4,444 & 393,282 & 4,803 & 388,479 & -- \\
\hline 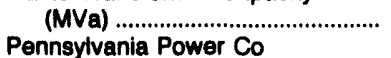 & 11,656 & 419 & 132 & 11,943 & 313 & 11,630 & - \\
\hline Watthour Meter Count ..................... & 163,129 & 7.271 & 9,077 & 161,323 & 6,206 & 155,090 & 27 \\
\hline $\begin{array}{l}\text { Line Transformer Count ................ } \\
\text { Line Transformer Capacity }\end{array}$ & 51,796 & 2,072 & 864 & 53,004 & 1,940 & 51,064 & - \\
\hline$(\mathrm{MVa})$ & 1,355 & 76 & 24 & 1,407 & 110 & 1,297 & - \\
\hline $\begin{array}{l}\text { Philadelphia Electric Co } \\
\text { Watthour Meter Count .................. }\end{array}$ & & & & & & & \\
\hline 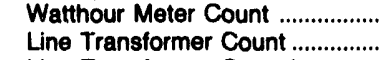 & $\begin{array}{r}1,730,723 \\
162,474\end{array}$ & $\begin{array}{r}35,223 \\
4,004\end{array}$ & $\begin{array}{r}26,569 \\
1,922\end{array}$ & $\begin{array}{r}1,739,377 \\
164,556\end{array}$ & $\begin{array}{r}177,126 \\
5,338\end{array}$ & $\begin{array}{r}1,562,083 \\
158,776\end{array}$ & $\begin{array}{l}168 \\
442\end{array}$ \\
\hline 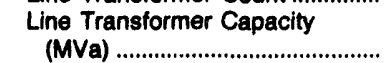 & & & & & & & \\
\hline $\begin{array}{l}\text { (MVa) .............. } \\
\text { Pioneer Power \& Light Co }\end{array}$ & 10,988 & 357 & 123 & 11,222 & 685 & 10,493 & 44 \\
\hline $\begin{array}{l}\text { Pioneer Power \& Light Co } \\
\text { Watthour Meter Count ................. }\end{array}$ & & & & & & & \\
\hline 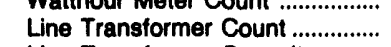 & $\begin{array}{r}1,581 \\
885\end{array}$ & $\begin{array}{l}50 \\
36\end{array}$ & 2 & $\begin{array}{r}1,629 \\
019\end{array}$ & 67 & 1,559 & 3 \\
\hline $\begin{array}{l}\text { Line Transformer Capacity } \\
(\mathrm{MVa})\end{array}$ & & & & & 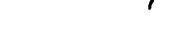 & 911 & -- \\
\hline $\begin{array}{l}\text { (MVa) ........................... } \\
\text { Portland General Electric Co }\end{array}$ & 16 & 1 & - & 17 & - & 17 & - \\
\hline Watthour Meter Count .................. & 638,208 & 46,208 & 33,388 & 651,028 & 28,480 & 622,423 & 125 \\
\hline $\begin{array}{l}\text { Line Transformer Count ............... } \\
\text { Line Transformer Capacity }\end{array}$ & 160,565 & 3,096 & 1,475 & 162,186 & 8,530 & 153,656 & - \\
\hline$(\mathrm{MVa})$ & 6,998 & 170 & 45 & 7,123 & 468 & 6,655 & - \\
\hline $\begin{array}{l}\text { Potomac Edison Co } \\
\text { Wathour Meter Count }\end{array}$ & & & & & & & \\
\hline $\begin{array}{l}\text { Watthour Meter Count ...................... } \\
\text { Line Transformer Count ............... }\end{array}$ & $\begin{array}{l}365,009 \\
154,698\end{array}$ & $\begin{array}{r}12,089 \\
3,790\end{array}$ & $\begin{array}{l}7,897 \\
2,624\end{array}$ & $\begin{array}{l}368,201 \\
155,864\end{array}$ & $\begin{array}{r}15,405 \\
5,949\end{array}$ & $\begin{array}{l}353,758 \\
149,887\end{array}$ & $\begin{array}{l}38 \\
28\end{array}$ \\
\hline $\begin{array}{l}\text { Line Transformer Capacity } \\
\text { (MVa) }\end{array}$ & & & & & & & \\
\hline Potomas: Electric Power Co & 4,605 & 176 & 43 & 4,738 & 353 & 4,379 & 6 \\
\hline 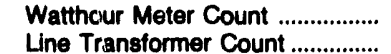 & 696,154 & 25,988 & 16,232 & 705,910 & 94,284 & 611,626 & - \\
\hline $\begin{array}{l}\text { Line Transformer Count ............... } \\
\text { Line Transformer Capacity }\end{array}$ & 104,593 & 1,905 & 1,736 & 104,762 & 9,101 & 95,661 & - \\
\hline (MVa) .... & 11,974 & 309 & 165 & 12,118 & 1,272 & 10,846 & - \\
\hline Public Service Co of Colorado & & & & & & & \\
\hline $\begin{array}{l}\text { Watthour Meter Count ................... } \\
\text { Line Transformer Count ............... }\end{array}$ & 991,914 & 27,882 & 21,617 & 998,179 & 19,683 & 978,496 & $\overline{100} 011$ \\
\hline $\begin{array}{l}\text { Line Transformer Count .............. } \\
\text { Line Transformer Capacity }\end{array}$ & 167,801 & 3,571 & 2,430 & 168,942 & 6,931 & - & 162,011 \\
\hline $\begin{array}{l}\text { (MVa) } \\
\text { Public Service Co of NH }\end{array}$ & 9,132 & 345 & 122 & 9,355 & 696 & - & 8,659 \\
\hline Watthour Meter Count .................. & 504,466 & 9,876 & 8,263 & 506,079 & 21,787 & 484,036 & 256 \\
\hline $\begin{array}{l}\text { Line Transformer Count ............... } \\
\text { Line Transformer Capacity }\end{array}$ & 123,274 & 2,308 & 1,195 & 124,387 & 3,517 & 120,870 & - \\
\hline (MVa) & 4,285 & 82 & 35 & 4,332 & 301 & 4,031 & - \\
\hline
\end{tabular}

See notes and footnotes at end of table. 
Table 44. Watthour Meter and Line Transformer Statistics of Investor-Owned Utilities, 1992 (Count and Megavoltampere) (Continued)

\begin{tabular}{|c|c|c|c|c|c|c|c|}
\hline $\begin{array}{l}\text { Diatribution } \\
\text { Equipment }\end{array}$ & $\begin{array}{l}\text { Number at } \\
\text { Beginning } \\
\text { of Year }\end{array}$ & $\begin{array}{l}\text { Additions } \\
\text { During } \\
\text { Year }\end{array}$ & $\begin{array}{l}\text { Reductions } \\
\text { During } \\
\text { Year }\end{array}$ & $\begin{array}{l}\text { Number at } \\
\text { End of } \\
\text { Year }\end{array}$ & $\begin{array}{l}\text { In Stock, } \\
\text { Locked, } \\
\text { and Inactive }\end{array}$ & $\begin{array}{l}\text { In } \\
\text { Customers } \\
\text { Use }\end{array}$ & $\begin{array}{l}\text { In } \\
\text { Company } \\
\text { Use }\end{array}$ \\
\hline \multicolumn{8}{|l|}{ Public Service Co of NM } \\
\hline $\begin{array}{l}\text { Watthour Meter Count ................. } \\
\text { Line Transformer Count ............ } \\
\text { Line Transformer Capacity }\end{array}$ & $\begin{array}{r}318,762 \\
66,272\end{array}$ & $\begin{array}{r}10,642 \\
1,360\end{array}$ & $\begin{array}{r}1,557 \\
875\end{array}$ & $\begin{array}{r}327,847 \\
66,757\end{array}$ & $\begin{array}{r}4,725 \\
916\end{array}$ & $\begin{array}{r}323,056 \\
65,685\end{array}$ & $\begin{array}{r}66 \\
156\end{array}$ \\
\hline Public Service Co of Oklahoma & 2,704 & 46 & 62 & 2,688 & 85 & 2,580 & 23 \\
\hline $\begin{array}{l}\text { Watthour Meter Count ................. } \\
\text { Line Transformer Count .............. } \\
\text { Line Transformer Cepacity }\end{array}$ & $\begin{array}{l}489,930 \\
140,036\end{array}$ & $\begin{array}{r}11,454 \\
4,796\end{array}$ & $\begin{array}{l}9,099 \\
1,817\end{array}$ & $\begin{array}{l}492,285 \\
143,015\end{array}$ & - & $\begin{array}{l}492,285 \\
143,015\end{array}$ & - \\
\hline $\begin{array}{l}\text { (MVa) } \\
\text { Public Service Electric\&Gas Co }\end{array}$ & 6,274 & 210 & 107 & 6,377 & - & 6,377 & - \\
\hline Watthour Meter Count ................. & $\begin{array}{r}1,935,485 \\
299,963\end{array}$ & $\begin{array}{r}66,732 \\
6,961\end{array}$ & $\begin{array}{r}57,585 \\
4,860\end{array}$ & $\begin{array}{r}1,944,632 \\
302,064\end{array}$ & $\begin{array}{r}79,108 \\
4,396\end{array}$ & $\begin{array}{r}1,865,296 \\
297,668\end{array}$ & -228 \\
\hline $\begin{array}{l}\text { Line Transformer Capacity } \\
\text { (MVa) ........................................ } \\
\text { Puget Sound Power \& Light Co }\end{array}$ & 19,375 & 623 & 294 & 19,704 & 550 & 19,154 & - \\
\hline $\begin{array}{l}\text { Watthour Meter Count ................. } \\
\text { Line Transformer Count ............. } \\
\text { Line Transformer Capactiy }\end{array}$ & $\begin{array}{l}840,601 \\
243,553\end{array}$ & $\begin{array}{r}36,470 \\
8,249\end{array}$ & $\begin{array}{l}8,882 \\
3,846\end{array}$ & $\begin{array}{l}868,189 \\
247,956\end{array}$ & $\begin{array}{r}18,851 \\
6,020\end{array}$ & $\begin{array}{l}849,178 \\
241,471\end{array}$ & $\begin{array}{l}160 \\
465\end{array}$ \\
\hline PSI Energy Inc & 10,285 & 332 & 125 & 10,492 & 405 & 10,071 & 16 \\
\hline $\begin{array}{l}\text { Watthour Meter Count ................. } \\
\text { Line Transformer Count .............. } \\
\text { Line Transformer Capacity }\end{array}$ & $\begin{array}{l}645,543 \\
184,172\end{array}$ & $\begin{array}{r}32,892 \\
4,429\end{array}$ & $\begin{array}{r}21,201 \\
3,744\end{array}$ & $\begin{array}{l}657,234 \\
184,857\end{array}$ & $\begin{array}{r}36,786 \\
5,758\end{array}$ & $\begin{array}{l}619,890 \\
178,518\end{array}$ & $\begin{array}{l}558 \\
581\end{array}$ \\
\hline $\begin{array}{l}\text { (MVa) } \\
\text { Rochester Gas \& Eloctric Corp }\end{array}$ & 7,860 & 221 & 156 & 7,925 & 507 & 7,395 & 23 \\
\hline Watthour Meter Count .................. & 352,767 & 1,948 & - & 354,715 & 23,044 & 331,358 & 313 \\
\hline $\begin{array}{l}\text { Line Transformer Count ............... } \\
\text { Line Transformer Capacity }\end{array}$ & 73,407 & 2,141 & 1,815 & 73,733 & 8,550 & - & 65,183 \\
\hline $\begin{array}{l}\text { (MVa) } \\
\text { Rockland Electric Co }\end{array}$ & 2,897 & 66 & 75 & 2,888 & 713 & - & 2,175 \\
\hline Watthour Meter Count ................. & 64,961 & 1,289 & 701 & 65,549 & 2,344 & 63,205 & - \\
\hline $\begin{array}{l}\text { Line Transformer Count ............... } \\
\text { Line Transformer Capacity }\end{array}$ & 20,680 & 713 & 302 & 21,091 & 3,374 & 17,717 & - \\
\hline San Diego Gas \& Electric Co & 1,033 & 44 & 15 & 1,062 & 203 & 859 & - \\
\hline v'atthour Meter Count ..................... & $1,169,502$ & 20,408 & 11,927 & $1,177,983$ & 60,941 & $1,115,493$ & 1,549 \\
\hline $\begin{array}{l}\text { Line Transformer Count ................ } \\
\text { Line Transformer Capacity }\end{array}$ & 160,527 & 4,380 & 3,340 & 161,567 & 4,114 & 157,453 & - \\
\hline $\begin{array}{l}\text { (MVa) } \\
\text { Savannah Electric \& Power Co }\end{array}$ & 9,702 & 304 & 177 & 9,829 & 420 & 9,409 & - \\
\hline $\begin{array}{l}\text { Line Transformer Count ......................... } \\
\text { Slerra Pacific Power Co }\end{array}$ & $\begin{array}{r}123,101 \\
34,376\end{array}$ & $\begin{array}{l}3,500 \\
1,216\end{array}$ & 1,884 & 33,708 & $\begin{array}{r}9,009 \\
932\end{array}$ & $\begin{array}{r}114,512 \\
32,776\end{array}$ & -31 \\
\hline $\begin{array}{l}\text { Watthour Meter Count ................. } \\
\text { Line Transformer Count ............. } \\
\text { Line Transformer Capacity }\end{array}$ & $\begin{array}{r}267,952 \\
58,944\end{array}$ & $\begin{array}{l}8,624 \\
1,978\end{array}$ & $\begin{array}{l}4,021 \\
1,253\end{array}$ & $\begin{array}{r}272,555 \\
59,669\end{array}$ & $\begin{array}{l}7,866 \\
5,428\end{array}$ & 264,509 & $\begin{array}{r}180 \\
54,241\end{array}$ \\
\hline $\begin{array}{l}\text { (MVa) } \\
\text { South Beloit Water Gas\&Elec Co }\end{array}$ & 3,412 & 155 & 140 & 3,427 & 489 & - & 2,938 \\
\hline Watthour Meter Count ..................... & 6,314 & 221 & - & 6,535 & -- & 6,535 & -- \\
\hline $\begin{array}{l}\text { Line Transformer Count ............... } \\
\text { Line Transformer Capacity }\end{array}$ & 1.977 & 98 & 13 & 2,062 & - & 2,062 & - \\
\hline $\begin{array}{l}\text { (MVa) } \\
\text { South Carolina Electric\&Gas Co }\end{array}$ & 100 & 5 & 2 & 103 & - & 103 & -- \\
\hline $\begin{array}{l}\text { Watthour Meter Count ................ } \\
\text { Line Transformer Count ............. } \\
\text { Line Transformer Capacity }\end{array}$ & $\begin{array}{l}488,000 \\
192,595\end{array}$ & $\begin{array}{r}11,298 \\
5,155\end{array}$ & $\begin{array}{l}5,560 \\
4,684\end{array}$ & $\begin{array}{l}493,738 \\
193,066\end{array}$ & $\begin{array}{r}35,022 \\
4,498\end{array}$ & $\begin{array}{l}458,215 \\
187,162\end{array}$ & $\begin{array}{r}501 \\
1,406\end{array}$ \\
\hline $\begin{array}{l}\text { (MVa) } \\
\text { Southern California Edison Co }\end{array}$ & 7,925 & 259 & 193 & 7,991 & 522 & 7,413 & 56 \\
\hline $\begin{array}{l}\text { Watthour Meter Count ................. } \\
\text { Line Transformer Count .............. }\end{array}$ & $\begin{array}{r}4,224,824 \\
647,706\end{array}$ & $\begin{array}{l}89,811 \\
17,718\end{array}$ & $\begin{array}{l}43,936 \\
13,226\end{array}$ & $\begin{array}{r}4,270,699 \\
652,198\end{array}$ & $\begin{array}{r}130,192 \\
10,948\end{array}$ & $\begin{array}{r}4,140,291 \\
641,250\end{array}$ & $-{ }^{216}$ \\
\hline Southern Indiana Gas \& Elec Co & & & & & & & \\
\hline Watthour Meter Count .................. & 131,088 & 3,557 & 2,844 & 131,801 & 5,449 & 126,296 & 56 \\
\hline $\begin{array}{l}\text { Line Transformer Count ............... } \\
\text { Line Transformer Capacity }\end{array}$ & 45,967 & 1,091 & 622 & 46,436 & 2,022 & 44,414 & - \\
\hline $\begin{array}{l}\text { (MVa) ..... } \\
\text { Southwestern Electric Power Co }\end{array}$ & 1,851 & 37 & 18 & 1,870 & 138 & 1,732 & - \\
\hline $\begin{array}{l}\text { Watthour Meter Count ................. } \\
\text { Line Transformer Count ............ } \\
\text { Line Transformer Capacity }\end{array}$ & $\begin{array}{l}391,681 \\
155,299\end{array}$ & $\begin{array}{r}12,687 \\
4,052\end{array}$ & $\begin{array}{l}9,530 \\
1,512\end{array}$ & $\begin{array}{l}394,838 \\
157,839\end{array}$ & $\begin{array}{l}9,596 \\
1,728\end{array}$ & $\begin{array}{l}385,012 \\
156,111\end{array}$ & $-^{230}$ \\
\hline$(\mathrm{MVa})$ & 6,362 & 212 & 52 & 6,522 & 168 & 6,354 & - \\
\hline
\end{tabular}

See notes and footnotes at end of table. 
Table 44. Watthour Meter and Line Transformer Statistics of Investor-Owned Utilities, 1992 (Count and Megavoltampere) (Continued)

\begin{tabular}{|c|c|c|c|c|c|c|c|}
\hline $\begin{array}{l}\text { Dietribution } \\
\text { Equipment }\end{array}$ & $\begin{array}{l}\text { Number at } \\
\text { Beginning } \\
\text { of Year }\end{array}$ & $\begin{array}{l}\text { Additions } \\
\text { During } \\
\text { Year }\end{array}$ & $\begin{array}{l}\text { Reductions } \\
\text { During } \\
\text { Year }\end{array}$ & $\begin{array}{l}\text { Number at } \\
\text { End of } \\
\text { Year }\end{array}$ & $\begin{array}{l}\text { In Stock, } \\
\text { Locked, } \\
\text { and Inactive }\end{array}$ & $\begin{array}{l}\text { In } \\
\text { Customers } \\
\text { Use }\end{array}$ & $\begin{array}{l}\ln \\
\text { Company } \\
\text { Use }\end{array}$ \\
\hline \multicolumn{8}{|l|}{ Southwestern Electric Serv Co } \\
\hline $\begin{array}{l}\text { Watthour Meter Count ................. } \\
\text { Line Transformer Count ............ } \\
\text { Line Transformer Capacity }\end{array}$ & $\begin{array}{l}40,872 \\
24,694\end{array}$ & $\begin{array}{r}1,011 \\
317\end{array}$ & $\begin{array}{r}1,012 \\
326\end{array}$ & $\begin{array}{l}40,991 \\
24,685\end{array}$ & $\begin{array}{l}1,291 \\
1,324\end{array}$ & $\begin{array}{l}39,585 \\
23,305\end{array}$ & $\begin{array}{r}115 \\
56\end{array}$ \\
\hline $\begin{array}{l}\text { (MVa) ......................... } \\
\text { Southwestern Public Service Co }\end{array}$ & 602 & 19 & 10 & 611 & 58 & 552 & 1 \\
\hline $\begin{array}{l}\text { Watthour Meter Count .................. } \\
\text { Line Transformer Count ............. } \\
\text { Line Transformer Capacity }\end{array}$ & $\begin{array}{l}360,206 \\
122,948\end{array}$ & $\begin{array}{l}6,525 \\
2,872\end{array}$ & $\begin{array}{l}6,757 \\
2,392\end{array}$ & $\begin{array}{l}359,974 \\
123,428\end{array}$ & 14,730 & $\begin{array}{l}345,000 \\
122,318\end{array}$ & $\begin{array}{r}244 \\
1,110\end{array}$ \\
\hline St Joseph Light \& Power Co & 4,335 & 128 & 71 & 4,392 & - & 4,360 & 32 \\
\hline $\begin{array}{l}\text { Watthour Meter Count ................. } \\
\text { Line Transformer Count ............ } \\
\text { Line Transformer Capacity }\end{array}$ & $\begin{array}{l}72,443 \\
21,025\end{array}$ & $\begin{array}{l}196 \\
710\end{array}$ & -740 & $\begin{array}{l}72,639 \\
20,995\end{array}$ & $\begin{array}{r}13,211 \\
1,505\end{array}$ & $\begin{array}{l}59,207 \\
19,490\end{array}$ & -221 \\
\hline $\begin{array}{l}\text { (MVa) ......... } \\
\text { Superior Water Light\&Power Co }\end{array}$ & 877 & 34 & 17 & 894 & 133 & 761 & - \\
\hline $\begin{array}{l}\text { Watthour Meter Count ................. } \\
\text { Line Transformer Count ............ } \\
\text { Line Transformer Capacity }\end{array}$ & $\begin{array}{r}14,133 \\
1,981\end{array}$ & $\begin{array}{r}508 \\
60\end{array}$ & $\begin{array}{r}390 \\
58\end{array}$ & $\begin{array}{r}14,251 \\
1,983\end{array}$ & $\begin{array}{r}1,007 \\
154\end{array}$ & $\begin{array}{r}13,219 \\
1,797\end{array}$ & $\begin{array}{l}25 \\
32\end{array}$ \\
\hline $\begin{array}{l}\text { (MVa) } \\
\text { Tampa Electric Co }\end{array}$ & 81 & 4 & 2 & 83 & 9 & 73 & 1 \\
\hline $\begin{array}{l}\text { Watthour Meter Count ................. } \\
\text { Line Transformer Count ............. } \\
\text { Line Transformer Capacity }\end{array}$ & $\begin{array}{l}513,803 \\
119,014\end{array}$ & $\begin{array}{l}9,565 \\
5,903\end{array}$ & $\begin{array}{l}6,589 \\
3,469\end{array}$ & $\begin{array}{l}516,779 \\
121,448\end{array}$ & $\begin{array}{r}41,915 \\
1,420\end{array}$ & $\begin{array}{l}474,741 \\
119,561\end{array}$ & $\begin{array}{l}123 \\
467\end{array}$ \\
\hline $\begin{array}{l}\text { (MVa) ................................... } \\
\text { Texas Utilities Electric Co }\end{array}$ & 6,355 & 288 & 147 & 6,496 & 140 & 6,319 & 37 \\
\hline $\begin{array}{l}\text { Watthour Meter Count .................. } \\
\text { Line Transformer Count ............. } \\
\text { Line Transformer Capacity }\end{array}$ & $\begin{array}{r}2,283,429 \\
666,542\end{array}$ & $\begin{array}{l}47,159 \\
19,335\end{array}$ & $\begin{array}{r}25,753 \\
9,434\end{array}$ & $\begin{array}{r}2,304,835 \\
676,443\end{array}$ & $\begin{array}{l}98,916 \\
22,833\end{array}$ & - & $\begin{array}{r}2,205,919 \\
653,610\end{array}$ \\
\hline $\begin{array}{l}\text { (MVa) ..................................... } \\
\text { Texas-New Mexico Power Co }\end{array}$ & 34,827 & 1,201 & 467 & 35,561 & 2,294 & - & 33,267 \\
\hline $\begin{array}{l}\text { Watthour Meter Count .................. } \\
\text { Line Transformer Count .............. } \\
\text { Line Transformer Capacity }\end{array}$ & $\begin{array}{r}225,908 \\
98,457\end{array}$ & $\begin{array}{l}7,696 \\
1,623\end{array}$ & $\begin{array}{l}6,799 \\
2,293\end{array}$ & $\begin{array}{r}226,805 \\
97,787\end{array}$ & $\begin{array}{r}10,343 \\
6,787\end{array}$ & $\begin{array}{r}216,462 \\
91,000\end{array}$ & - \\
\hline Toledo Edison Co & 3,115 & 72 & 56 & 3,131 & 354 & 2,777 & - \\
\hline $\begin{array}{l}\text { Watthour Meter Count ................. } \\
\text { Line Transformer Count ............ } \\
\text { Line Transformer Capacity }\end{array}$ & $\begin{array}{r}320,823 \\
71,797\end{array}$ & $\begin{array}{l}6,434 \\
1,466\end{array}$ & 11,126 & $\begin{array}{r}316,131 \\
73,263\end{array}$ & $\begin{array}{r}11,654 \\
2,487\end{array}$ & $\begin{array}{r}304,477 \\
70,776\end{array}$ & - \\
\hline $\begin{array}{l}\text { (MVa) } \\
\text { Tucson Electric Power Co }\end{array}$ & 3,526 & 105 & - & 3,631 & 202 & 3,429 & - \\
\hline $\begin{array}{l}\text { Watthour Meter Count ................ } \\
\text { Line Transformer Count ............. } \\
\text { Line Transformer Capacity }\end{array}$ & $\begin{array}{r}295,352 \\
60,175\end{array}$ & $\begin{array}{r}6,938 \\
982\end{array}$ & $\begin{array}{r}2,220 \\
153\end{array}$ & $\begin{array}{r}300,070 \\
61,004\end{array}$ & $\begin{array}{l}2,019 \\
3,115\end{array}$ & $\begin{array}{r}298,051 \\
57,889\end{array}$ & - \\
\hline 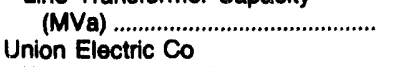 & 3,613 & 88 & 9 & 3,692 & 267 & 3,425 & -- \\
\hline $\begin{array}{l}\text { Watthour Meter Count ................. } \\
\text { Line Transformer Count ............ } \\
\text { Line Transformer Capacity }\end{array}$ & $\begin{array}{r}1,259,102 \\
274,439\end{array}$ & $\begin{array}{l}61,733 \\
16,976\end{array}$ & $\begin{array}{r}43,356 \\
9,810\end{array}$ & $\begin{array}{r}1,277,479 \\
281,605\end{array}$ & $\begin{array}{r}25,442 \\
7,260\end{array}$ & $\begin{array}{r}1,251,970 \\
274,025\end{array}$ & $\begin{array}{r}67 \\
320\end{array}$ \\
\hline $\begin{array}{l}\text { (MVa) ........................... } \\
\text { Union Light Heat \& Power Co }\end{array}$ & 15,608 & 704 & 538 & 15,774 & 731 & 15,007 & 36 \\
\hline $\begin{array}{l}\text { Watthour Meter Count ................. } \\
\text { Line Transformer Count ............. } \\
\text { Line Transformer Capacity }\end{array}$ & $\begin{array}{r}119,288 \\
26,354\end{array}$ & $\begin{array}{l}6,237 \\
1,284\end{array}$ & $\begin{array}{r}4,227 \\
925\end{array}$ & $\begin{array}{r}121,298 \\
26,713\end{array}$ & $\begin{array}{l}7,429 \\
1,473\end{array}$ & - & $\begin{array}{r}113,869 \\
25,240\end{array}$ \\
\hline 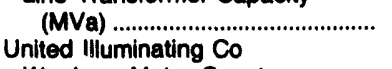 & 1,398 & 84 & 41 & 1,441 & 114 & - & 1,327 \\
\hline $\begin{array}{l}\text { Warthour Meter Count ................. } \\
\text { Line Transformer Count ............. } \\
\text { Line Transformer Capacity }\end{array}$ & $\begin{array}{r}310,173 \\
56,348\end{array}$ & $\begin{array}{r}26,537 \\
1,370\end{array}$ & $\begin{array}{r}11,673 \\
1,144\end{array}$ & $\begin{array}{r}325,037 \\
56,574\end{array}$ & $\begin{array}{r}22,727 \\
3,218\end{array}$ & $\begin{array}{r}301,970 \\
53,187\end{array}$ & $\begin{array}{l}340 \\
169\end{array}$ \\
\hline $\begin{array}{l}\text { (MVa) } \\
\text { Unitll Power Corp }\end{array}$ & 3,430 & 127 & 64 & 3,493 & 441 & 3,041 & 11 \\
\hline $\begin{array}{l}\text { Watthour Moter Count ................. } \\
\text { Upper Peninsula Power Co }\end{array}$ & 2 & - & - & 2 & - & - & 2 \\
\hline $\begin{array}{l}\text { Watthour Meter Count ................. } \\
\text { Line Transformer Count ............. } \\
\text { Line Transformer Capacity }\end{array}$ & $\begin{array}{l}61,882 \\
18,680\end{array}$ & $\begin{array}{r}1,593 \\
468\end{array}$ & $\begin{array}{r}1,007 \\
272\end{array}$ & $\begin{array}{l}62,468 \\
18,876\end{array}$ & $\begin{array}{r}2,977 \\
860\end{array}$ & $\begin{array}{l}59,380 \\
17,984\end{array}$ & $\begin{array}{r}111 \\
32\end{array}$ \\
\hline (MVa) & 429 & 15 & 5 & 439 & 39 & 399 & 1 \\
\hline $\begin{array}{l}\text { Watthour Meter Count ................. } \\
\text { Line Transformer Count ............. } \\
\text { Line Transformer Capacity }\end{array}$ & $\begin{array}{l}367,916 \\
126,472\end{array}$ & $\begin{array}{l}9,586 \\
7,294\end{array}$ & $\begin{array}{l}7,9,92 \\
2,2,1\end{array}$ & $\begin{array}{l}369,540 \\
131,495\end{array}$ & $\begin{array}{l}40,006 \\
20,452\end{array}$ & $\begin{array}{l}328,728 \\
108,701\end{array}$ & $\begin{array}{r}806 \\
2,342\end{array}$ \\
\hline$(\mathrm{MVa})$ & 4,653 & 386 & 117 & 4,922 & 907 & 3,588 & 427 \\
\hline
\end{tabular}

See notes and footnotes at end of table. 
Table 44. Watthour Meter and Line Transformer Statistics of Investor-Owned Utilities, 1992 (Count and Megavoltampere) (Continued)

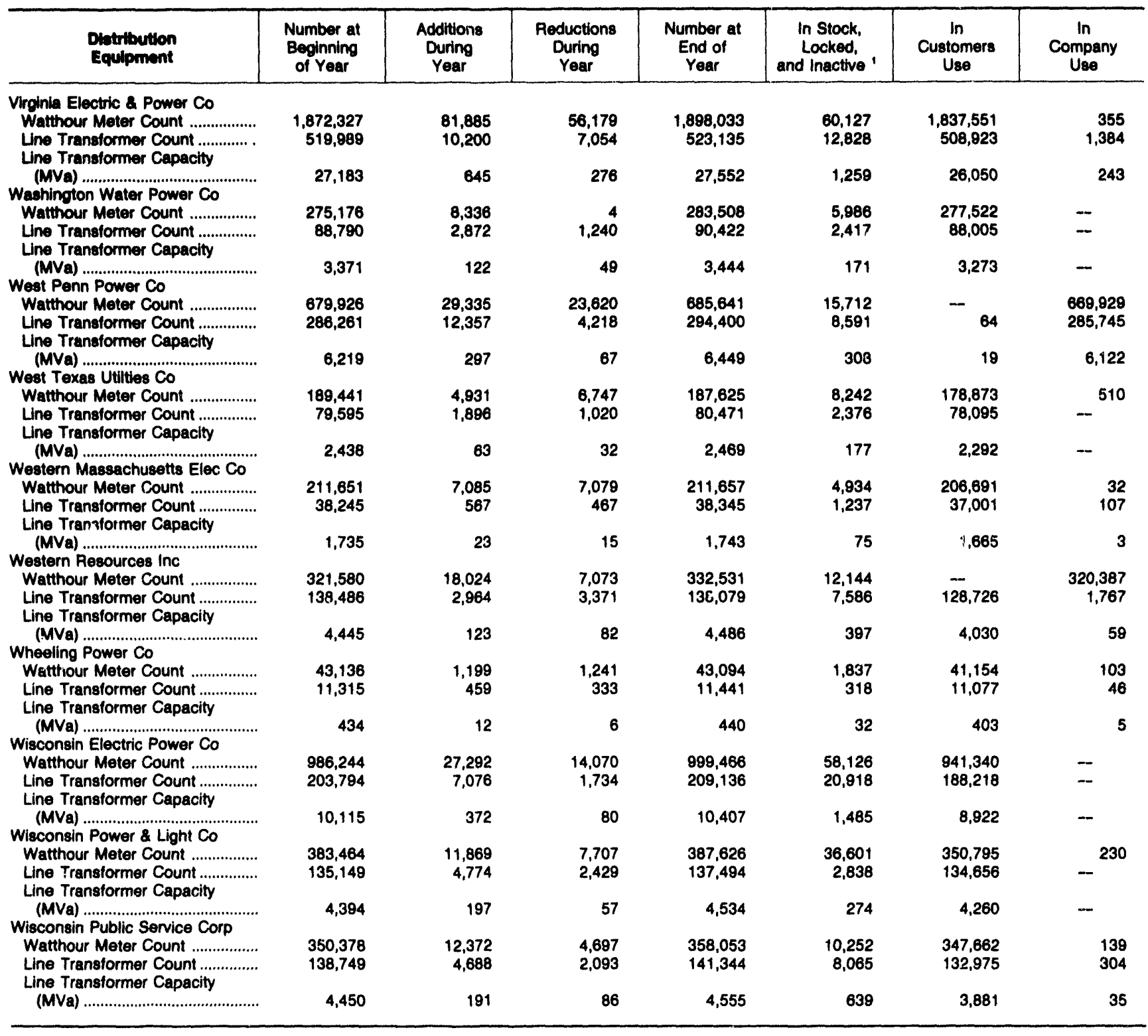

In stock, locked meters on customer's premises and inactive transformers on system.

- Not Applicable

Notes: -Totals may not equal sum of components because of independent rounding. $\bullet$ MVa means megavoltampere.

Sources: •Federal Energy Regulatory Commission, FERC Form 1, "Annual Report of Major Electric Utilities, Licensees and Others." 


\section{Appendix A}

Federal Energy

Regulatory

Commission

Regulation of the

Electric Utility

Industry 


\section{Federal Energy Regulatory Commission Regulation of the Electric Utility Industry}

The responsibility for regulating most interstate wholesale electric trade rests with the Federal Government, and not with State or local governments. Therefore, data on wholesale trade are collected within a framework of Federal laws and regulations. In wholesale trade between electrically connected utilities, if even one utility is electrically connected to a utility that operates in a different State, then trade among any of these utilities is considered interstate and jurisdictional for the Federal Energy Regulatory Commission (FERC) regulatory purposes. Hence virtually all wholesale trade except that in Alaska, Hawaii, and part of Texas is considered interstate.

FERC is responsible for governing interstate trade of wholesale electricity for investor-owned utilities. In order to participate in wholesale electricity trade, utilities must file interconnection agreements (contracts) with the FERC. FERC exercises its regulatory authority directly by approving interconnections between utilities, coordinating agreements to exchange power, and approving wholesale power rates. Authority for bulk power transactions is typically prearranged under interconnection contracts. These agreements define an individual electric utility's overall relationship to other utilities. In addition, they define how each will maintain its part of the electrical system operations. Other contracts are often negotiated to handle specific needs. In fact, one of the most common is for support of full or partial requirements power. Requirements contracts cover electric utilities that have either insufficient or no generating capability to satisfy their customer load. They represent long-term, firm power contractual obligations in which the terms and conditions obligate the selling electric utility to provide the buying electric utility a level of service equivalent to the seller's requirement to support its own retail customers. These contracts may be with more than one electric utility.

The FERC established a Uniform System of Accounts for the financial reporting of electric trade transactions. The transactions reported are complex and involve specifics of contracts, simultaneous energy transactions, the unintended receipt and delivery of energy, and the handling of energy losses. These accounts can be found in the FERC's "Accounting and Reporting Requirements For Public Utilities and Licensees."
FERC requires only investor-owned utilities to file financial information on electric trade. The Rural Electrification Administration (REA) requires its borrowers (usually cooperatives) to file similar information. while EIA requires zimilar information from publir: utilities. Both the REA and the EIA request the reporting of information based on the Uniform System of Accounts.

Data on wholesale electricity trade are collected on Federal survey data forms that are accounting-system based. They were designed for regulatory purposes to meet individual electric utility administrative and rate case needs as opposed to overall aggregate industry statistical and analytical purposes.

The FERC Forms 1 and 1-F are the primary financial reporting forms. Wholesale data are collected on 4 of the approximately 75 survey schedules comprising the FERC Form 1. The wholesale electricity trade schedules are categorized as sales for resale, purchased power, summary of interchange, and transmission of electricity for or by others. These schedules correspond to one or more of the following accounts from the Uniform System of Accounts as follows:

- Sales for Resale (447)

- A. This account shall include the net billing for electricity supplied to other electric utilities or to public authorities for resale purposes.

- B. Records shall be maintained so as to show the quantity of electricity sold and the revenue received from each customer.

- NOTE: Revenues from electricity supplied to other public utilities for use by them and not for distribution shall be included in account 443, Commercial and Industrial Sales, unless supplied under the same contract as and not readily separable from revenues includable in this account.

- Purchased Power (555)

- A. This account shall include the cost at point of receipt by the utility of electricity purchased for resale. It shall include, also, net settlements for exchange of electricity or power, such as economy energy, off-peak energy for on-peak 
energy, spinning reserve capacity, etc. In addition, the account shall include the net settlements for transactions under pooling or interconnection agreements wherein there is a balancing of debits and credits for energy, capacity, etc. Distinct purchases and sales shall not be recorded as exchanges and net amounts are only recorded merely because debit and credit amounts are combined in the voucher settlement.

- B. The records supporting this account shall show, by months, the demands and demand charges, kilowatthours and prices thereof under each purchase contract and the charges and credits under each exchange or power pooling contract.

\section{- Other Electric Revenues (456)}

- 5. Revenues from transmission of electricity of others over transmission facilities of the utility.

\section{- Transmission of Electricity By Others (565)}

- This account shall include amounts payable to others for the transmission of the utility's electricity over transmission facilities owned by others.

\section{Addition of Statistical Classification Codes to the FERC Form 1}

The Federal Energy Regulatory Commission modified the FERC Form 1, "Annual Report of Major Electric Utilities, Licensees and Others" for the 1990 collection year. The Statistical Classification Codes are based on the original contractual terms and conditions of the service. The following codes were added to FERC Form 1:

- RQ - for requirements service. Requirements service is service which the supplier plans to provide on an ongoing basis (i.e., the supplier includes projected load for this service in its system resource planning). In addition, the reliability of requirements service must be the same as, or second only to, the supplier's service to its own ultimate consumers.

- LF - for long-term service. "Long-term" means 5 years or longer and "firm" means that service cannot be interrupted for economic reasons and is intended to remain reliable even under adverse conditions (e.g., the supplier must attempt to buy emergency energy from third parties to maintain deliveries of LF service). This category should not be used for long-term firm service which meets the definition of RQ service. For all transactions identified as LF, provide in a footnote the termination date of the contract defined as the earliest date that either buyer or seller can unilaterally get out of the contract.
- IF - for intermediate-term firm service. The same as LF service except that "intermediate-term" means longer than 1 year but less than 5 years.

- SF - for short-term firm service. Use this category for all firm services where the duration of each period of commitment for service is 1 year or less.

- LU - for long-term service from a designated generating unit. "Long-term" means 5 years or longer. The availability and reliability of service, aside from transmission constraints, must match the availability and reliability of the designated unit.

- IU - for intermediate-term service from a designated generating unit. The same as LU service except that "intermediate-term" means longer than $i$ year but less than 5 years.

- EX - for exchange of electricity. Use this category for transactions involving a balancing of debits and credits for energy, capacity, etc. and any settlements for imbalanced exchanges.

- OS - for other service. Use this category only for those services which cannot be placed in the above-defined categories, such as all nonfirm service regardless of the length of the contract and service from designated units of less than 1 year. Describe the nature of the service in a footnote.

- AD - for out-of-period adjustments. Use this code for any accounting adjustments or "tune-ups" for service provided in prior reporting years. Provide an explanation in a footnote for each adjustment.

\section{FERC Accounting Release Number AR-14}

The Office of the Chief Accountant released an interpretation of Account 555 in Accounting Release Number AR-14 effective January 1, 1991. The format was question and answer. AR-14 is as follows:

Question: The text of Account 555, Purchased Power, states in part: "This account shall include the cost at point of receipt by the utility of electricity purchased for resale. It shall include, also, net settlements for exchange of electricity or power, such as economy energy, off-peak energy for on-peak energy, spinning reserve capacity, etc. In addition, the account shall include the net settlements for transactions under pooling or interconnection agreements wherein there is a balancing of debits and credits for energy, capacity, etc." Does this mean that Account 555 is to include all settlement amounts arising from power pool transactions regardless of how compensation for energy delivered to the power pool or capacity made available to the power pool is determined and all settlement amounts for energy provided under nonpool contractual agreements in which the agreements contain provisions pertaining to both the receipt and delivery of energy? 
Answer: No. The above requirement must be read in conjunction with the sentence in the accounting text that immediately follows the above quote which states: "Distinct purchases and sales shall not be recorded as exchanges and net amounts only recorded merely because debit and credit amounts are combined in the voucher settlement." It is the intent of the text of Account 555 to limit the use of the account to the cost of energy and capacity purchased and net settlements arising from barter transactions. Gross amounts for compensation for energy delivered or capacity made available to a power pool or delivered pursuant to other agreements where settlements are determined through application of specific rate schedules, "splitthe-saving" calculations, simulations, or other nonbarter means must be recorded in the appropriate operating revenue account and not netted with purchased power expenses properly recorded in Account 555. 
Appendix B

State, NERC

Region, and Data

Sources for

Electric Utilities 


\section{State, NERC Region, and Data Sources for Electric Utilities}

Table B1. Investor-Owned Utilities, 1992

\begin{tabular}{|c|c|c|c|}
\hline Utility & State' & NERC Reglon ${ }^{2}$ & Survey Form' \\
\hline 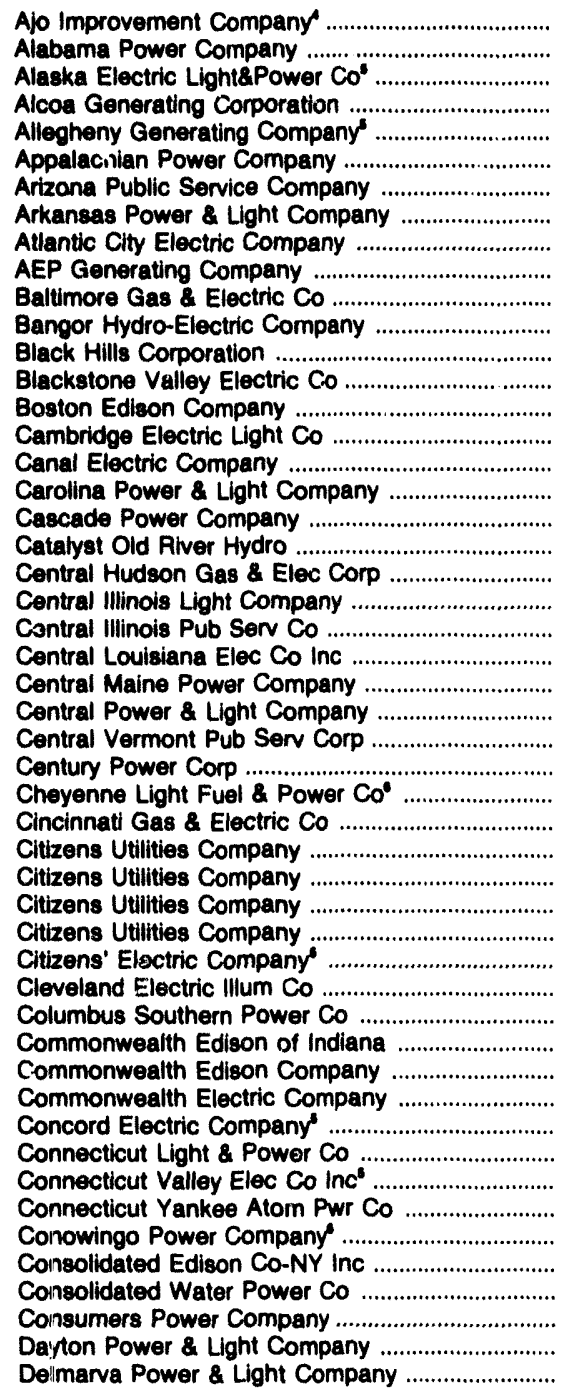 & $\begin{array}{l}\text { Arizona } \\
\text { Alabama } \\
\text { Alaska } \\
\text { Indiana } \\
\text { Maryland } \\
\text { Virginia } \\
\text { Arizona } \\
\text { Arkansas } \\
\text { New Jersey } \\
\text { Ohio } \\
\text { Maryland } \\
\text { Maine } \\
\text { South Dakota } \\
\text { Rhode lsland } \\
\text { Massachusetts } \\
\text { Massachusetts } \\
\text { Massachusetts } \\
\text { North Carolina } \\
\text { North Carolina } \\
\text { Louisiana } \\
\text { New York } \\
\text { Illinois } \\
\text { Illinois } \\
\text { Louisiana } \\
\text { Maine } \\
\text { Texas } \\
\text { Vermont } \\
\text { Arizona } \\
\text { Wyoming } \\
\text { Ohio } \\
\text { Hawaii } \\
\text { Vermont } \\
\text { Idaho } \\
\text { Arizona } \\
\text { Pennsylvania } \\
\text { Ohio } \\
\text { Ohio } \\
\text { Indiana } \\
\text { Illinois } \\
\text { Massachusetts } \\
\text { New Hampshire } \\
\text { Connecticut } \\
\text { New Hampshire } \\
\text { Connecticut } \\
\text { Maryland } \\
\text { New York } \\
\text { Wisconsin } \\
\text { Michigan } \\
\text { Onio } \\
\text { Delaware }\end{array}$ & $\begin{array}{l}\text { WSCC } \\
\text { SERC } \\
\text { ASCC } \\
\text { ECAR } \\
\text { ECAR } \\
\text { ECAR } \\
\text { WSCC } \\
\text { SPP } \\
\text { MAAC } \\
\text { ECAR } \\
\text { MAAC } \\
\text { NPCC } \\
\text { WSCC } \\
\text { NPCC } \\
\text { NPCC } \\
\text { NPCC } \\
\text { NPCC } \\
\text { SERC } \\
\text { SERC } \\
\text { SPP } \\
\text { NPCC } \\
\text { MAIN } \\
\text { MAIN } \\
\text { SPP } \\
\text { NPCC } \\
\text { ERCOT } \\
\text { NPCC } \\
\text { WSCC } \\
\text { WSCC } \\
\text { ECAR } \\
\text { HI } \\
\text { NPCC } \\
\text { WSCC } \\
\text { WSCC } \\
\text { MAAC } \\
\text { ECAR } \\
\text { ECAR } \\
\text { MAIN } \\
\text { MAIN } \\
\text { NPCC } \\
\text { NPCC } \\
\text { NPCC } \\
\text { NPCC } \\
\text { NPCC } \\
\text { MAAC } \\
\text { NPCC } \\
\text { MAIN } \\
\text { ECAR } \\
\text { ECAR } \\
\text { MAAC }\end{array}$ & 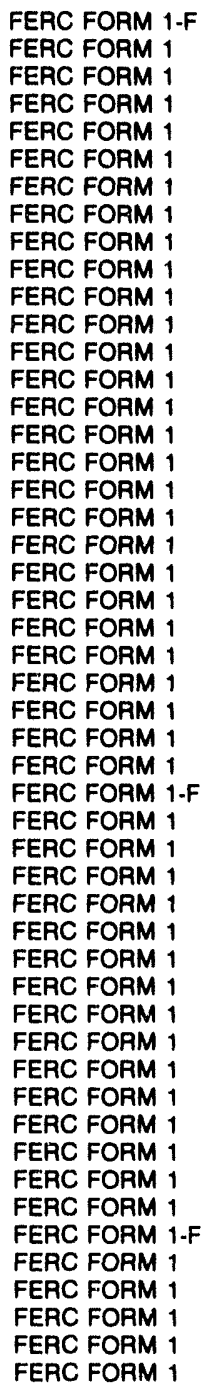 \\
\hline
\end{tabular}

See footnotes at end of table. 
Table B1. Investor-Owned Utillities, 1992 (Continued)

\begin{tabular}{|c|c|c|c|}
\hline Utility & State' & NERC Reglon' & Burvey Form' \\
\hline 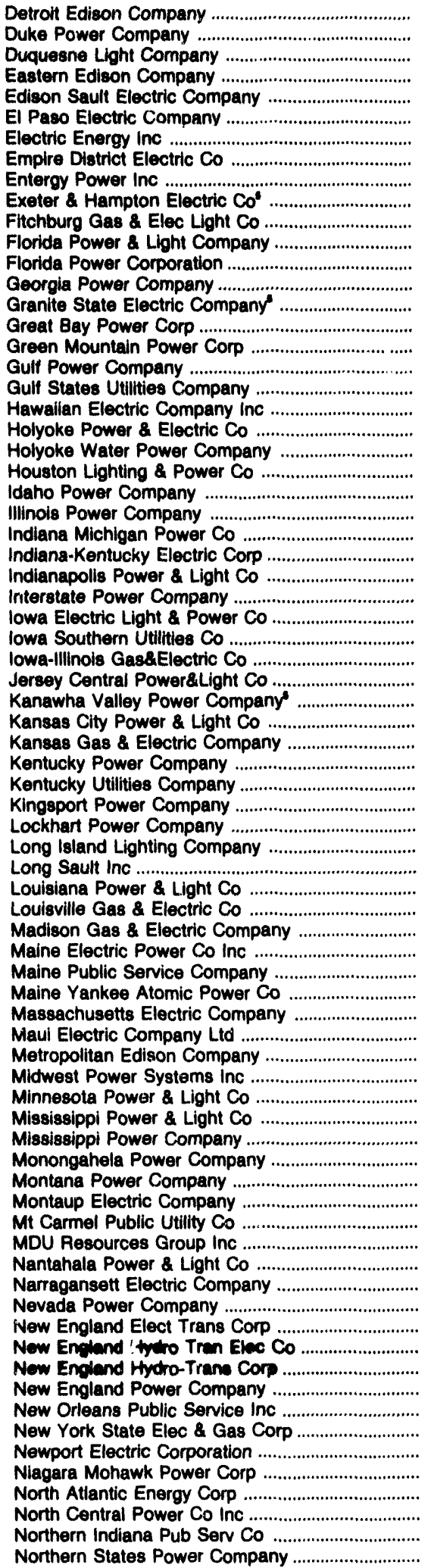 & 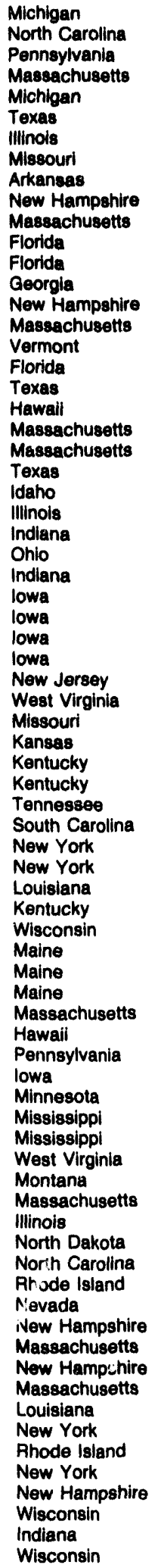 & 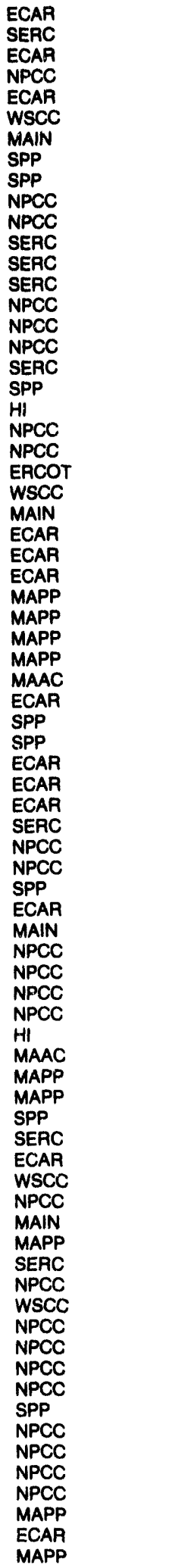 & 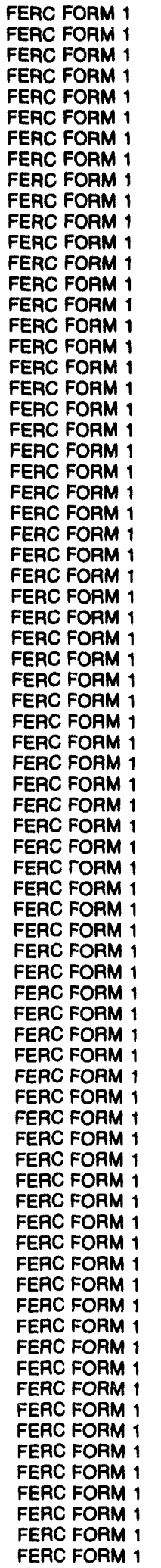 \\
\hline
\end{tabular}

See footnotes at end of table. 
Table B1. Investor-Owned Utilities, 1992 (Continued)

\begin{tabular}{|c|c|c|c|}
\hline Utility & State' & NEAC Roglon' & Survey Form \\
\hline 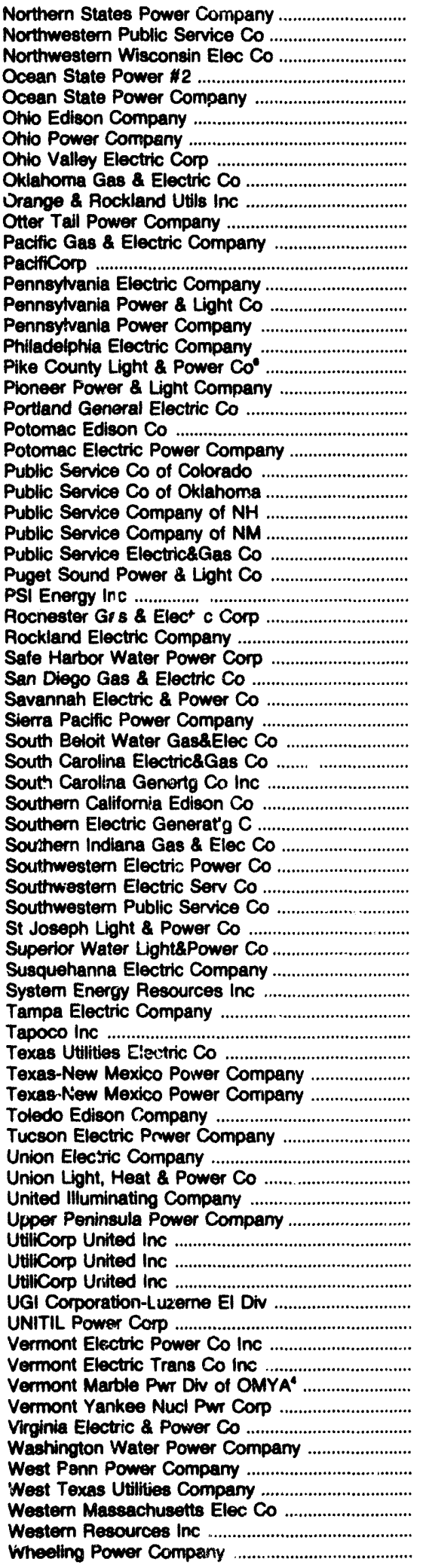 & 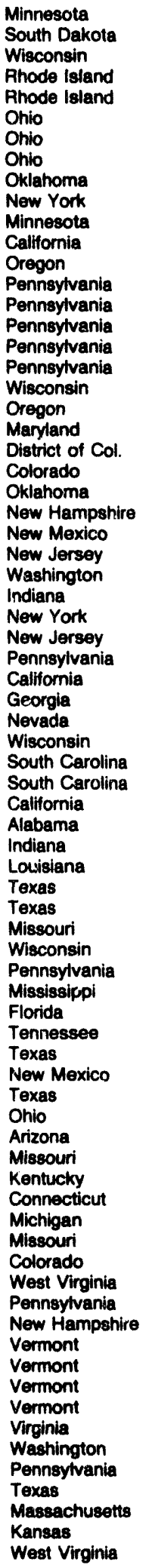 & 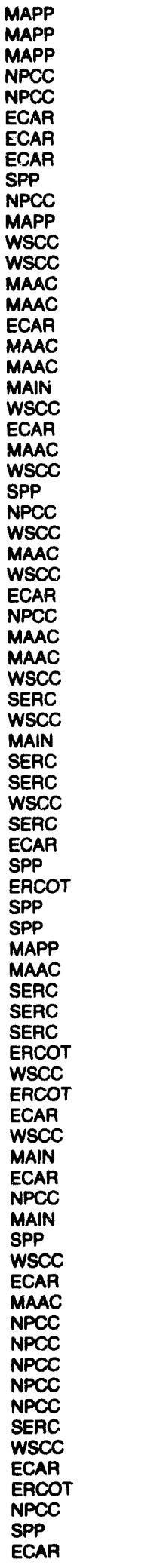 & 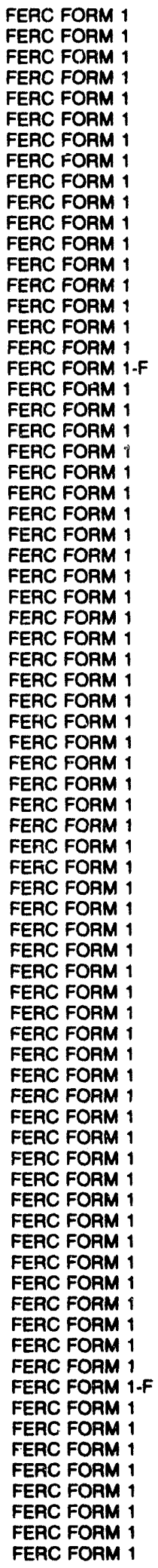 \\
\hline
\end{tabular}

See footnotes at end of table. 
Table B1. Investor-Owned Utilities, 1992 (Continued)

\begin{tabular}{|c|c|c|c|}
\hline Utifly & Stinte' & NERC Reglon ${ }^{2}$ & Survoy Form" \\
\hline 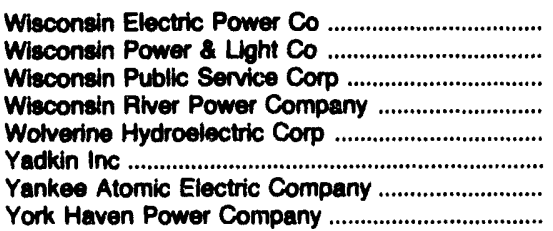 & $\begin{array}{l}\text { Wisconsin } \\
\text { Wisconsin } \\
\text { Wisconsin } \\
\text { Wisconsin } \\
\text { Michigan } \\
\text { North Carolina } \\
\text { Massachusetts } \\
\text { Pennsylvania }\end{array}$ & $\begin{array}{l}\text { MAIN } \\
\text { MAIN } \\
\text { MAIN } \\
\text { MAIN } \\
\text { ECAR } \\
\text { SERC } \\
\text { NPCC } \\
\text { MAAC }\end{array}$ & $\begin{array}{l}\text { FERC FORM } 1 \\
\text { FERC FORM } 1 \\
\text { FERC FORM } 1 \\
\text { FERC FORM } 1 \\
\text { FERC FORM } 1 \\
\text { FERC FORM } 1 \\
\text { FERC FORM } 1 \\
\text { FERC FORM } 1\end{array}$ \\
\hline
\end{tabular}

1 The State in which the corporate office of the utility is located.

2 The principal North American Electric Reliability Council region in which the utility operates. See Glossary for a list of all regions.

- Source of data for this publication

- No bulk povier transactions in 1992.

- A voluntary filing of the FERC Form 1 is done by this electric utility.

- This utility completed pages 310/311 or 326/327 from FERC Form 1.

Note: Some of the investor-owned electric utilities that are required to file as "minor" utilities did not use the FERC Form 1-F, instead they filed the FERC Form 1. Table B1 identifies only the actual form filed with the FERC and not the list of "minor" investor-owned electric utilities.

Sources: -Federal Energy Regulatory Commission, FEAC Form 1, "Annual Report of Major Electric Utilities, Licensees and Others." •FERC Form 1-F, "Annual Report for Nonmajor Public Utilities and Licensees." 
Table B2. Foderal Utilities, 1992

\begin{tabular}{|c|c|c|c|}
\hline Utility & State' & NERC Reglon" & Survoy Form" \\
\hline 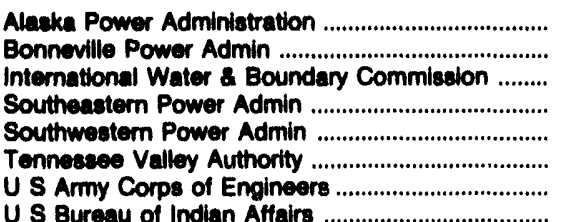 & $\begin{array}{l}\text { Alaska } \\
\text { Oregon } \\
\text { Texas } \\
\text { Georgla } \\
\text { Oklahoma } \\
\text { Tennessee } \\
\text { Michigan }\end{array}$ & $\begin{array}{l}\text { ASCC } \\
\text { WSCC } \\
\text { ERCOT } \\
\text { SERC } \\
\text { SPP } \\
\text { SERC } \\
\text { ECAR }\end{array}$ & $\begin{array}{l}\text { EIA-412 } \\
\text { EIA-412 } \\
\text { None4 } \\
\text { EIA-412 } \\
\text { EIA-412 } \\
\text { EIA-412 } \\
\text { EIA-412 }\end{array}$ \\
\hline 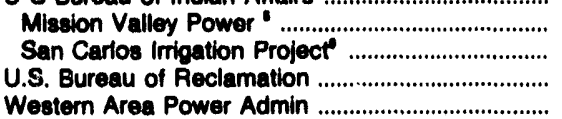 & $\begin{array}{l}\text { Montana } \\
\text { Arizona } \\
\text { District of Columbia } \\
\text { Colorado }\end{array}$ & $\begin{array}{l}\text { WSCC } \\
\text { WSCC } \\
\text { WSCC } \\
\text { WSCC }\end{array}$ & $\begin{array}{l}\text { EIA-412 } \\
\text { EIA-412 } \\
\text { Nonet } \\
\text { EIA-412 }\end{array}$ \\
\hline
\end{tabular}

The State in which the administrative office of the utilly is located.

- The principal North American Electric Reliability Council region in which the utllity operates. See glossary for a list of all regions.

- Source of data for this publication

Data reported by Western Area Power Administration.

- Under P.L. 93-638, the operation and maintenance of the electric utility portion of the irrigation project has been contracted with Mission Valley Power, an entity of the Coniederated Salish and Kootenal Tribes.

- During 1982, portions of the utility were divested by the Federal Government. The off-reservation distribution system is to be transferred to San Cerlos Imigation and Drainage District (a state entity) which will sell it to Arizona Public Service. The on-reservation distributlon system is to be transferred to Gila River Utility Authority (of the Gila River Indian Community) and the San Carlos Apache Tribe. The Coolldge Dam will remain U.S. property and be run by the Bureau of Indlan Affairs.

Note: The Federal Government both produces electricity and sells it at wholesale. Federal producers include the U.S. Army Corps of Engineers, Bureau of Indian Aftairs, Bureau of Reclamation, and the International Boundary and Water Commission. The power marketing administrations of the U.S. Department of Energy are also federally owned utilities; they include the Alaska, Bonneville, Southeastern, Southwestern, and Western Area Power Administrations. The Tennessee Valley Authority also both generates and markets electricity.

Sources: •Energy Information Administration, Form EIA-412, "Annual Report of Public Electric Utilties." •Energy Information Administration, Form

ElA-861, "Annual Electric Utility Report." 
Table B3. State and Other Government Utilities, 1992

\begin{tabular}{|c|c|c|c|}
\hline vatury & State' & NERC Reglon" & survey Form \\
\hline 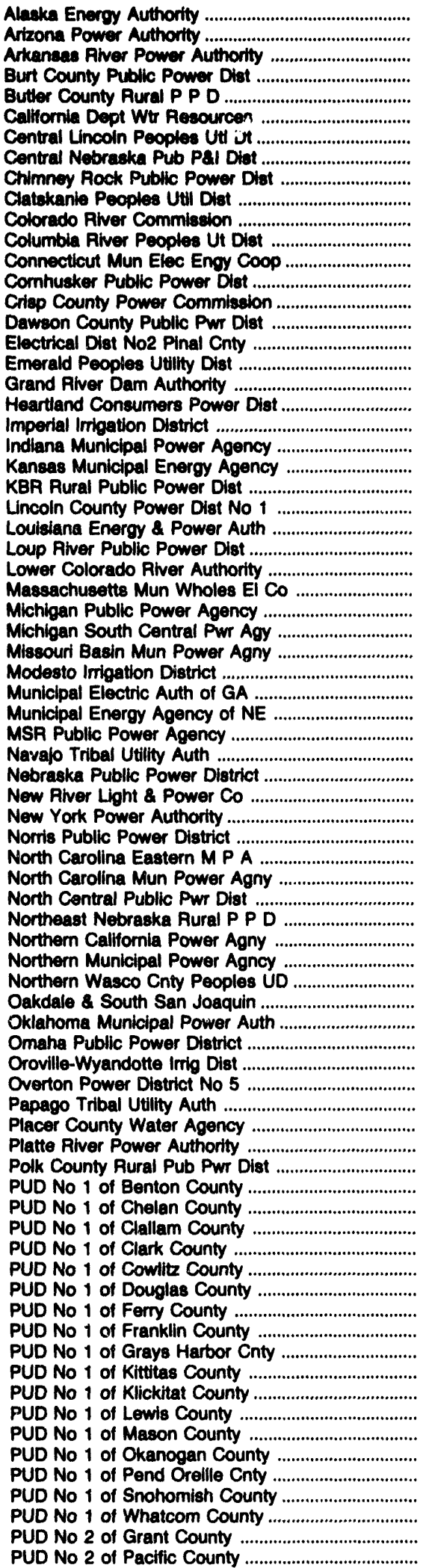 & 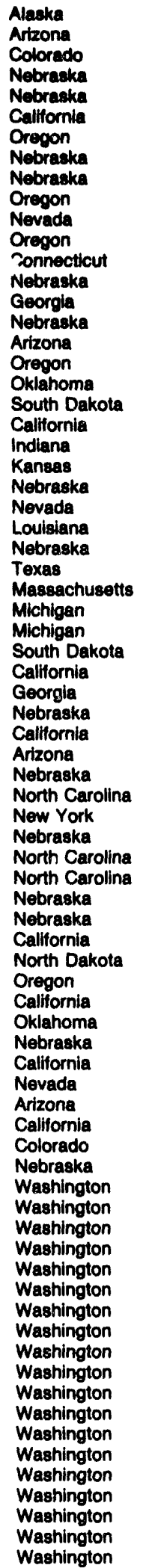 & 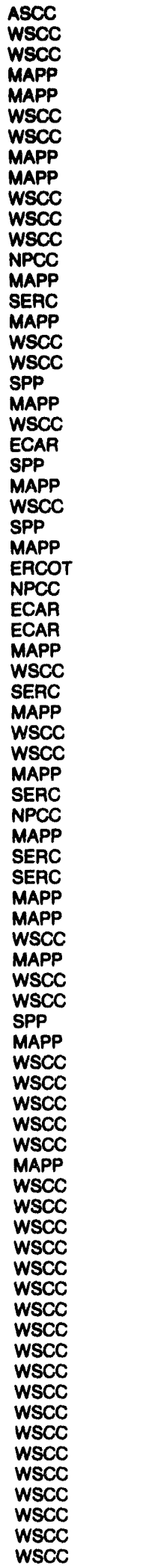 & 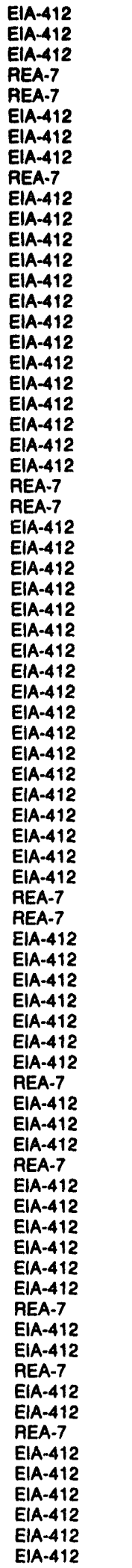 \\
\hline
\end{tabular}

See footnotes at end of table. 
Table B3. State and Other Government Utilities, 1992 (Continued)

\begin{tabular}{|c|c|c|c|}
\hline Uatinty & State' & NERC Reglon" & Burvey Form" \\
\hline 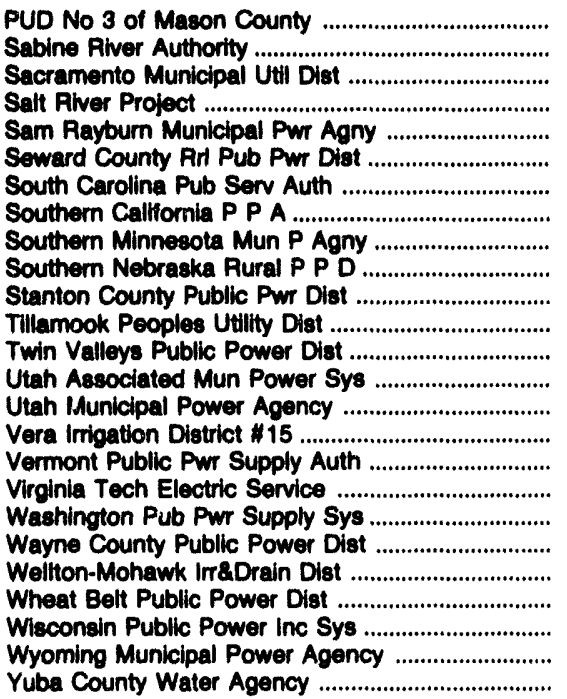 & $\begin{array}{l}\text { Washington } \\
\text { Texas } \\
\text { California } \\
\text { Arizona } \\
\text { Texas } \\
\text { Nebraska } \\
\text { South Carolina } \\
\text { Callornia } \\
\text { Minnesota } \\
\text { Nebraska } \\
\text { Nebraska } \\
\text { Oregon } \\
\text { Nebraska } \\
\text { Utah } \\
\text { Utah } \\
\text { Washington } \\
\text { Vermont } \\
\text { Virginia } \\
\text { Washington } \\
\text { Nebraska } \\
\text { Arizona } \\
\text { Nebraska } \\
\text { Wisconsin } \\
\text { Wyoming } \\
\text { California }\end{array}$ & $\begin{array}{l}\text { WSCC } \\
\text { SPP } \\
\text { WSCC } \\
\text { WSCC } \\
\text { SPP } \\
\text { MAPP } \\
\text { SERC } \\
\text { WSCC } \\
\text { MAPP } \\
\text { MAPP } \\
\text { MAPP } \\
\text { WSCC } \\
\text { MAPP } \\
\text { WSCC } \\
\text { WSCC } \\
\text { WSCC } \\
\text { NPCC } \\
\text { ECAR } \\
\text { WSCC } \\
\text { MAPP } \\
\text { WSCC } \\
\text { WSCC } \\
\text { MAIN } \\
\text { WSCC } \\
\text { WSCC }\end{array}$ & $\begin{array}{l}\text { EIA-412 } \\
\text { EIA-412 } \\
\text { EIA-412 } \\
\text { EIA-412 } \\
\text { EIA-412 } \\
\text { REA-7 } \\
\text { EIA-412 } \\
\text { EIA-412 } \\
\text { EIA-412 } \\
\text { EIA-412 } \\
\text { REA-7 } \\
\text { EIA-412 } \\
\text { REA-7 } \\
\text { EIA-412 } \\
\text { EIA-412 } \\
\text { EIA-412 } \\
\text { EIA-412 } \\
\text { EIA-412 } \\
\text { EIA-412 } \\
\text { REA-7 } \\
\text { REA-7 } \\
\text { REA-7 } \\
\text { EIA-412 } \\
\text { EIA-412 } \\
\text { EIA-412 }\end{array}$ \\
\hline
\end{tabular}

- The State in which the authority is located.

2. The principal North American Electric Reliability Council region in which the utility operates. See glossary for a list of all regions.

- Source of data for this publication

Sources: •Energy Information Administration, Form ElA-412, "Annual Report of Public Electric Utilities." •Rural Electrification Administration, REA Form 7, "Financial and Statistical Report," REA Form 12a through 121, "Electric Power Supply Borrowers," Form 12c through 12g, "Electric Distribution Borrowers with Generating Facilities." •Energy Information Administration, Form EIA-861, "Annual Electric Utility Report." 
Table B4. Municlpal Utilitles, 1992

\begin{tabular}{|c|c|c|c|}
\hline Utillty & State' & NERC Roglon' & survey Form' \\
\hline 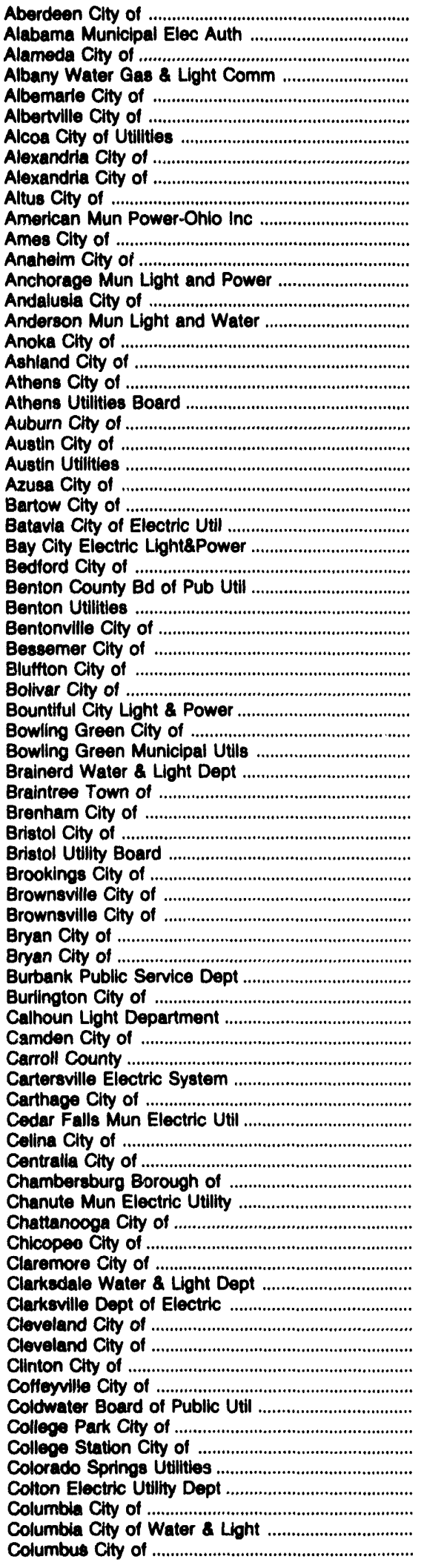 & 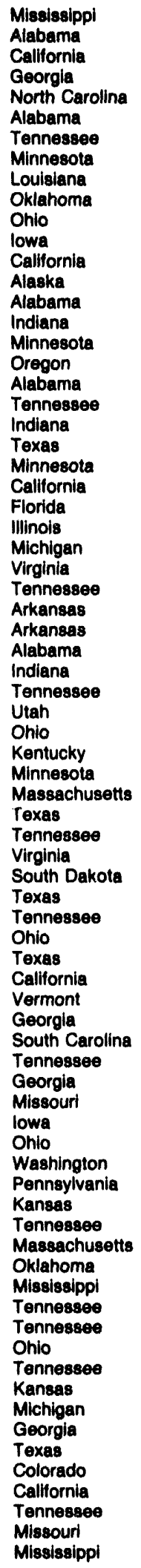 & 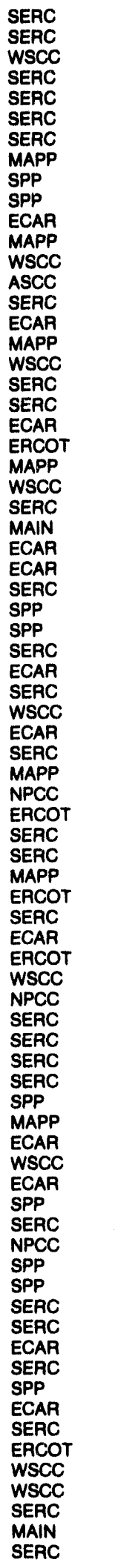 & 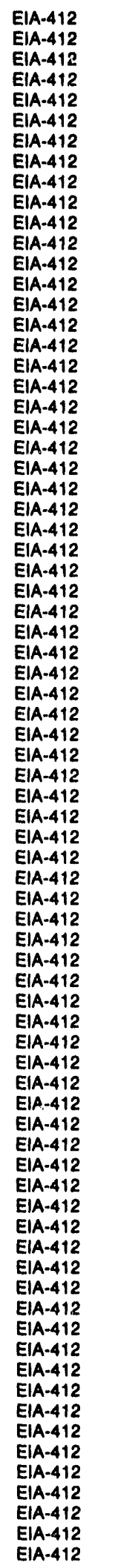 \\
\hline
\end{tabular}

See footnotes at end of table. 
Table B4. Municipal Utilities, 1992 (Continued)

\begin{tabular}{|c|c|c|c|}
\hline Utimy & Stinte' & NERC Roglon" & survey form ${ }^{2}$ \\
\hline 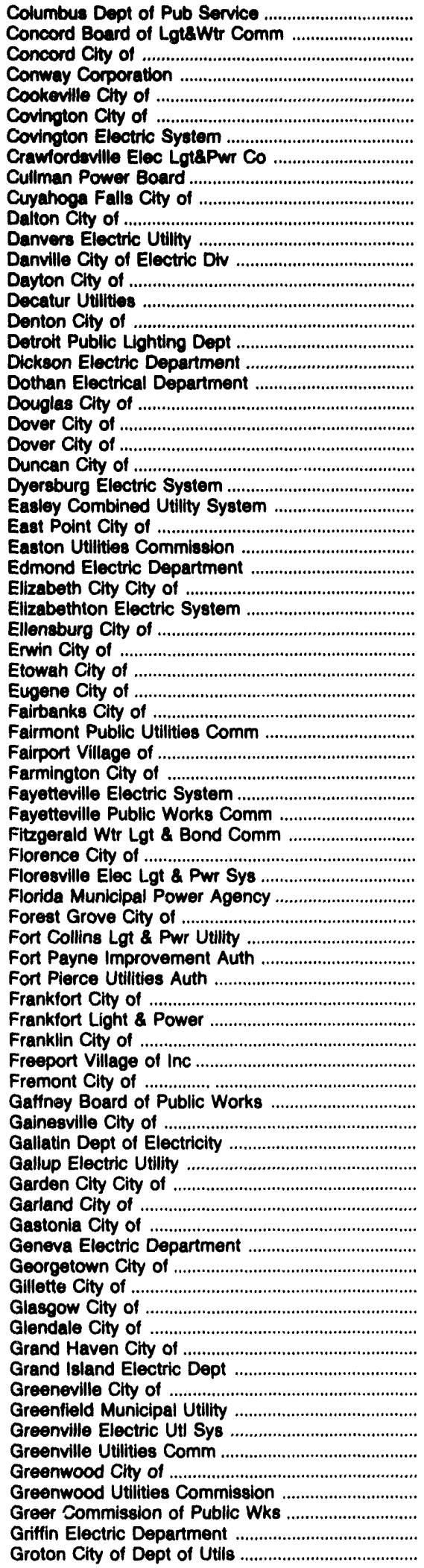 & 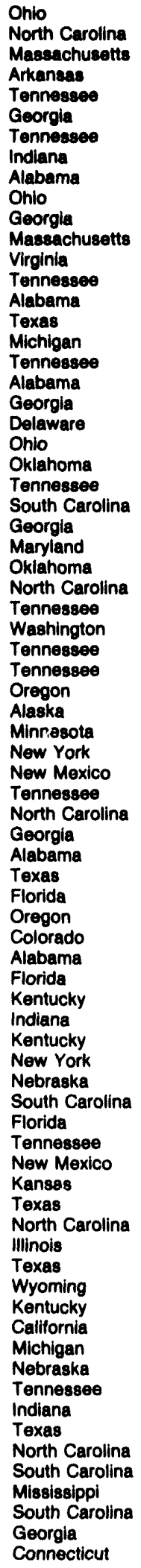 & 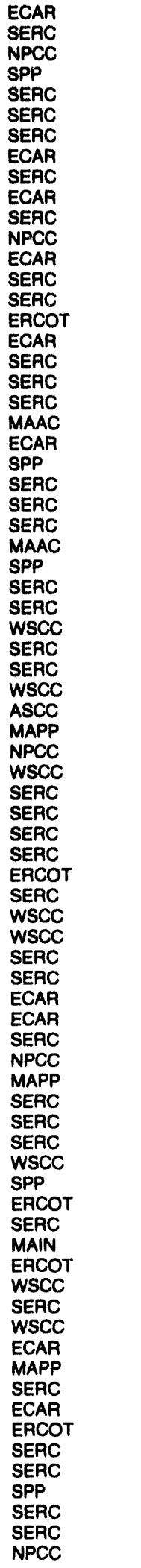 & 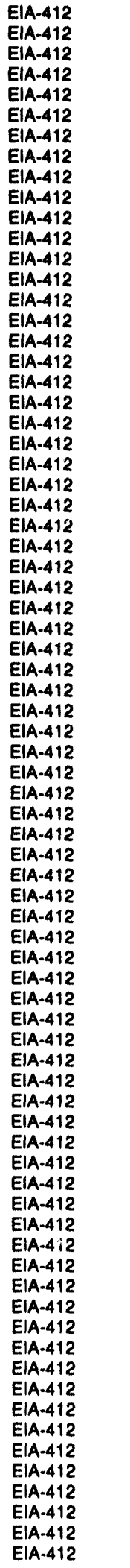 \\
\hline
\end{tabular}

See footnotes at end of table. 
Table B4. Municipal Utilities, 1992 (Continued)

\begin{tabular}{|c|c|c|c|}
\hline Utultsy & Etente' & NERC Reglon" & Survoy Form" \\
\hline 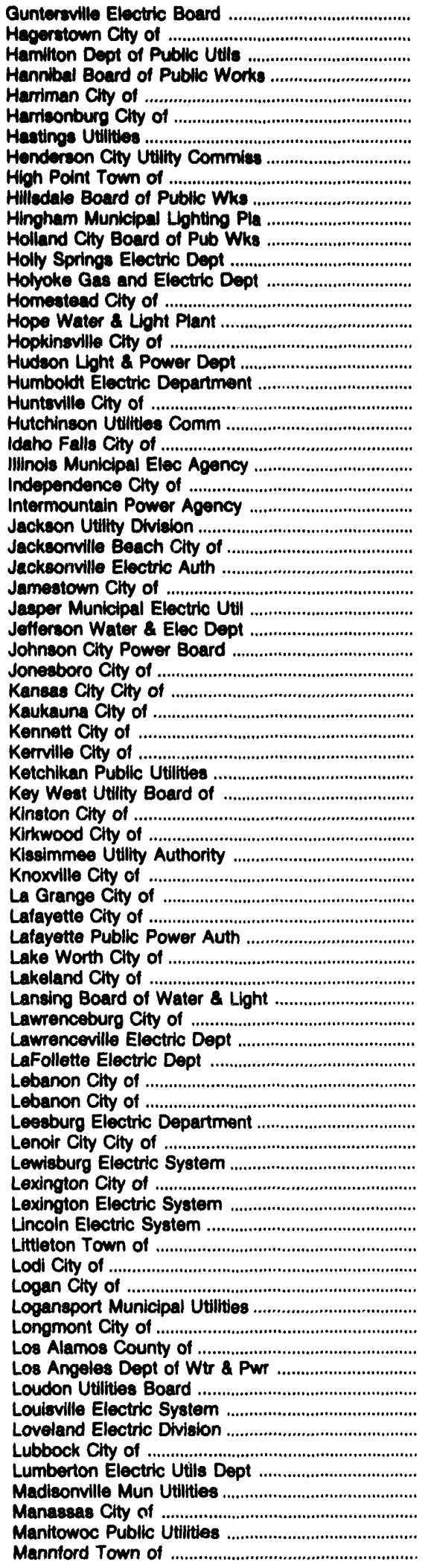 & 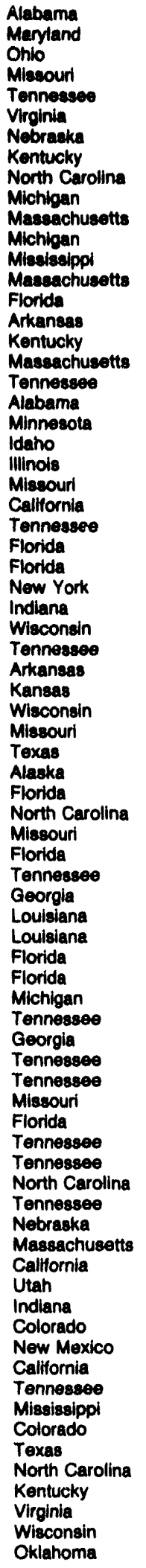 & 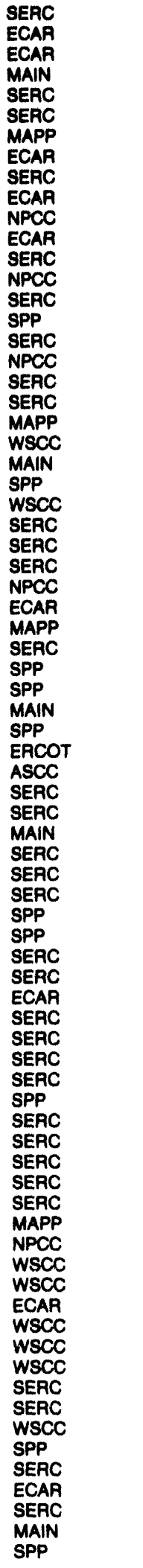 & 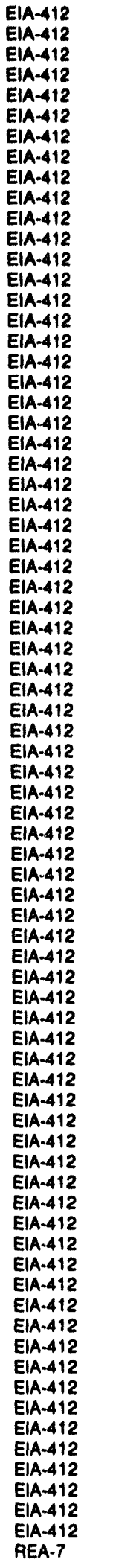 \\
\hline
\end{tabular}

See footnotes at end of table. 
Table B4. Municipal Utilities, 1992 (Continued)

\begin{tabular}{|c|c|c|c|}
\hline Utinty & state' & NERC Reolon" & survoy Form" \\
\hline 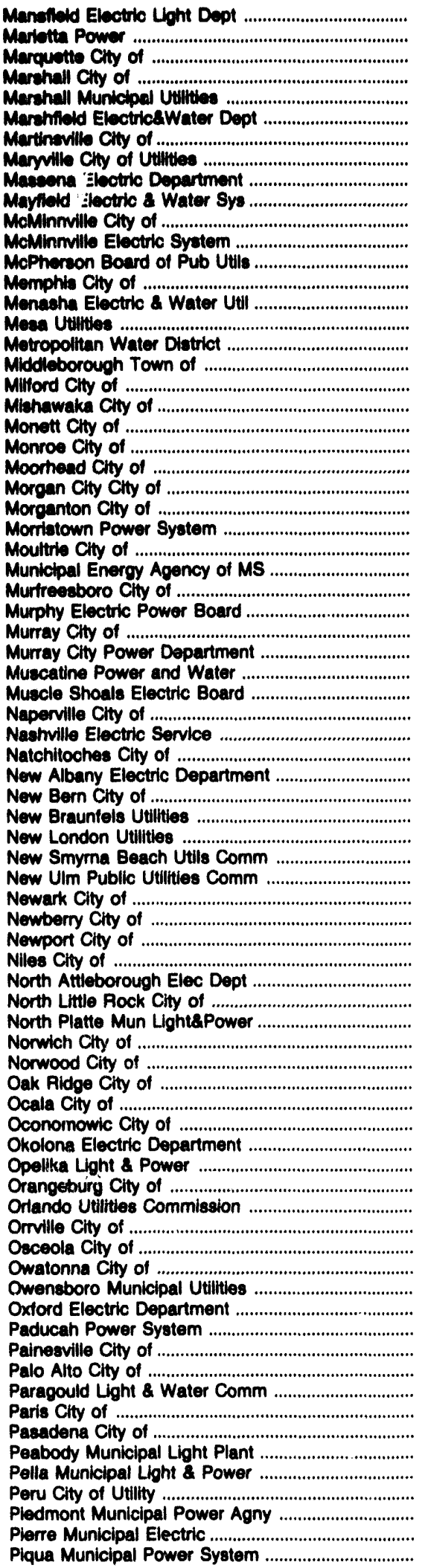 & 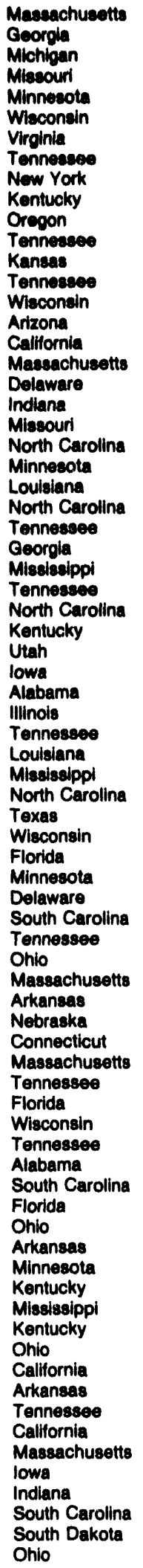 & 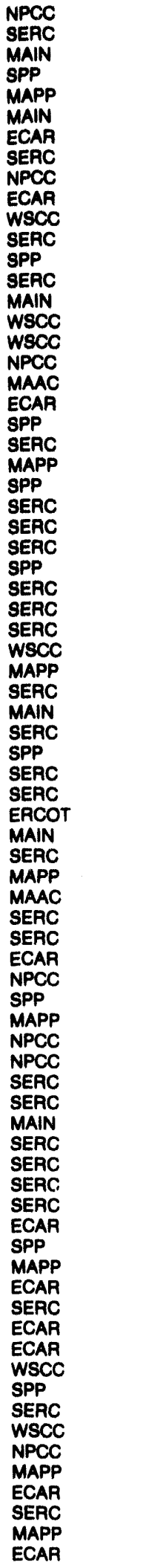 & 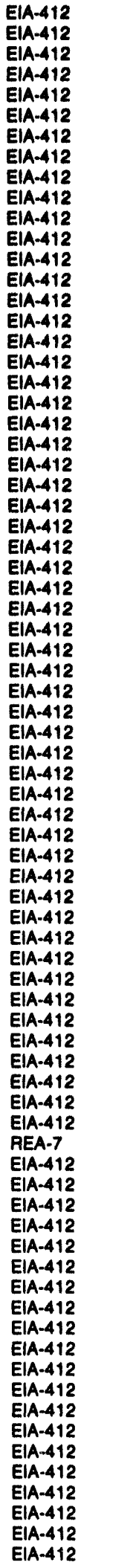 \\
\hline
\end{tabular}

See footnotes at end of table. 
Table B4. Municipal Utilities, 1992 (Continued)

\begin{tabular}{|c|c|c|c|}
\hline Utulty & Etate' & NERC Roclon' & survey Form' \\
\hline 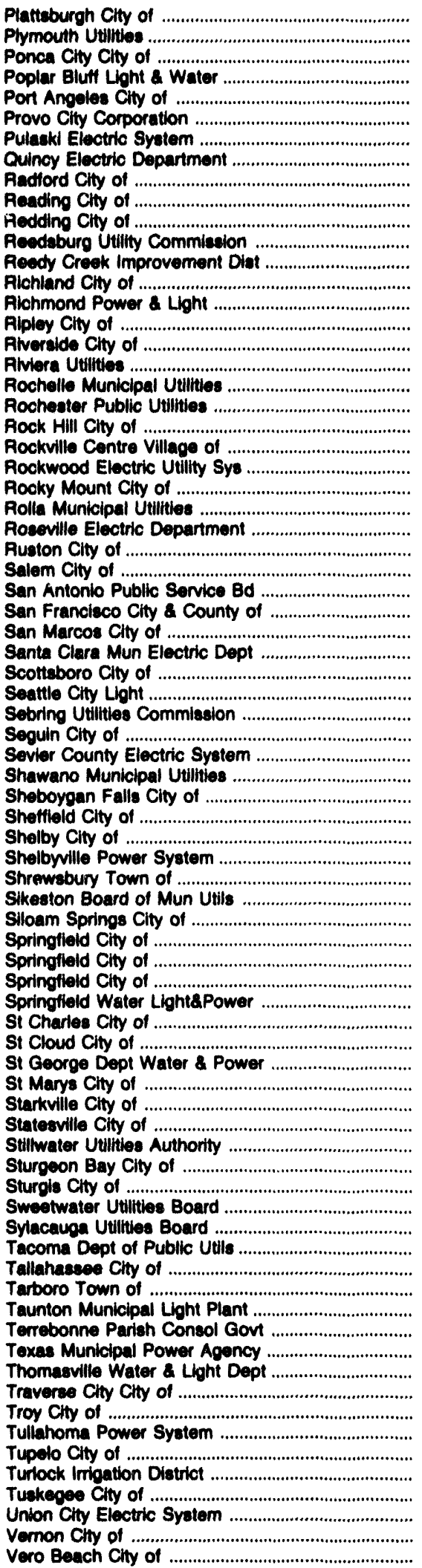 & 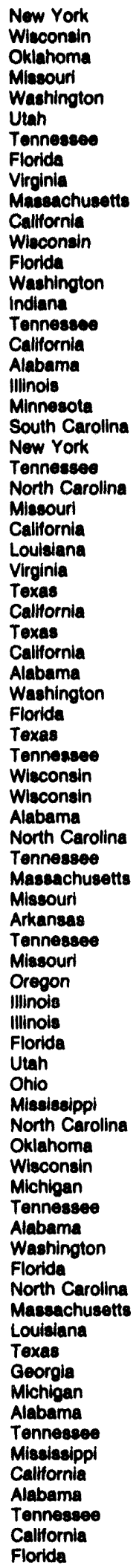 & 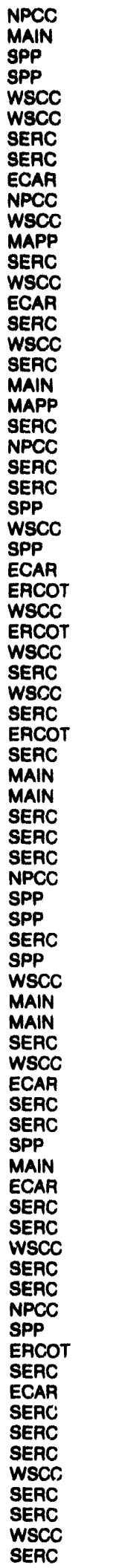 & 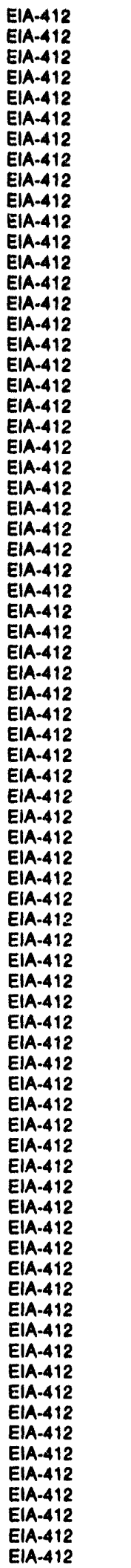 \\
\hline
\end{tabular}

See footnotes at end of table. 
Tabie B4. Municipal Utilitios, 1992 (Continued)

\begin{tabular}{|c|c|c|c|}
\hline Utamily & state' & NERC Roglon" & Survoy Form' \\
\hline 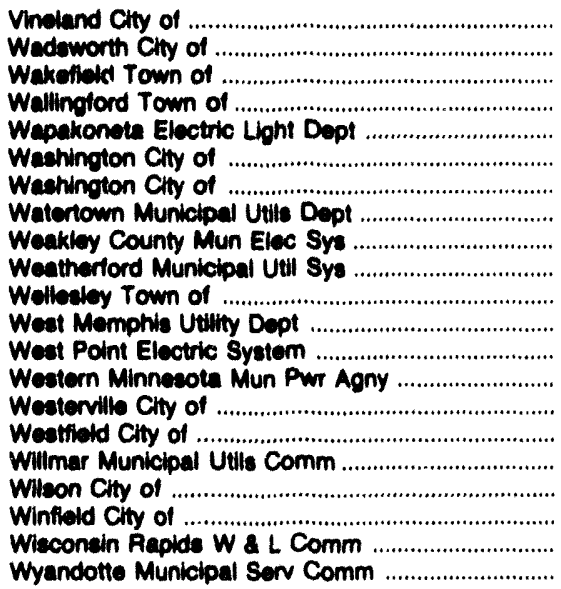 & $\begin{array}{l}\text { Now Jersey } \\
\text { Ohio } \\
\text { Massachusotts } \\
\text { Connecticut } \\
\text { Ohio } \\
\text { Indlana } \\
\text { North Carolina } \\
\text { South Dakota } \\
\text { Tennessee } \\
\text { Texas } \\
\text { Massachusetts } \\
\text { Arkansas } \\
\text { Miecisaippi } \\
\text { Minnesota } \\
\text { Ohio } \\
\text { Masaachusetts } \\
\text { Minnesota } \\
\text { North Carolina } \\
\text { Kansas } \\
\text { Wisconsin } \\
\text { Michigan }\end{array}$ & $\begin{array}{l}\text { MAC } \\
\text { ECAR } \\
\text { NPCC } \\
\text { NPCC } \\
\text { ECAR } \\
\text { ECAR } \\
\text { SERC } \\
\text { MAPP } \\
\text { SERC } \\
\text { ERCOT } \\
\text { NPCC } \\
\text { SPP } \\
\text { SEAC } \\
\text { MAPP } \\
\text { ECAR } \\
\text { NPCC } \\
\text { MAPP } \\
\text { SERC } \\
\text { SPP } \\
\text { MAPP } \\
\text { ECAR }\end{array}$ & $\begin{array}{l}E \mid A-412 \\
E \mid A-412 \\
E \mid A-412 \\
E \mid A-412 \\
E \mid A-412 \\
E \mid A-412 \\
E \mid A-412 \\
E \mid A-412 \\
E \mid A-412 \\
E \mid A-412 \\
E \mid A-412 \\
E \mid A-412 \\
E \mid A-412 \\
E \mid A-412 \\
E \mid A-412 \\
\text { E|A-412 } \\
E \mid A-412 \\
E \mid A-412 \\
E \mid A-412 \\
E \mid A-412 \\
\text { EIA-412 }\end{array}$ \\
\hline
\end{tabular}

The State in which the municipality is located.

- The principal North American Electric Reliability Council region in which the utility operates. See glossary for a liat of all regions.

- Source of data for this publication

Sources: •Energy Information Adminiatration, Form ElA.412, "Annual Report of Public Electric Utilities." •Energy Information Administration, Form ElA-861, "Annual Electric Utility Report." 
Table B6. Cooperative Borrowers, 1992

\begin{tabular}{|c|c|c|c|}
\hline Uatinly & State' & Nanc neqion" & survey form" \\
\hline 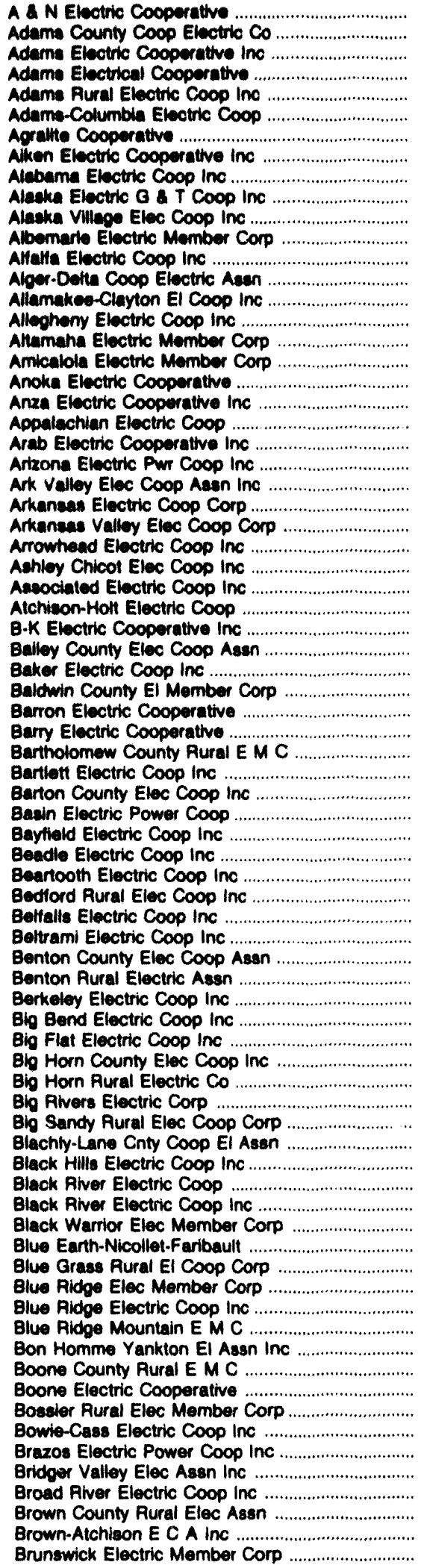 & 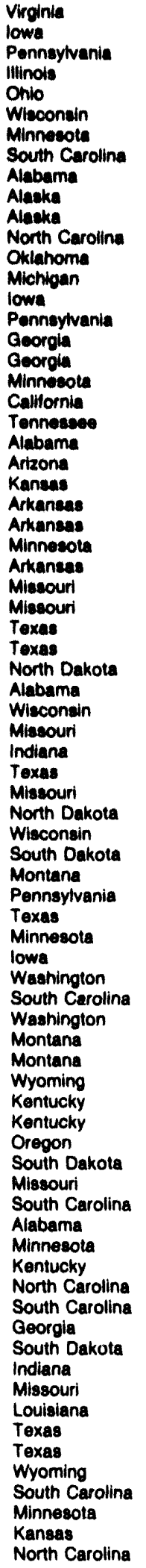 & 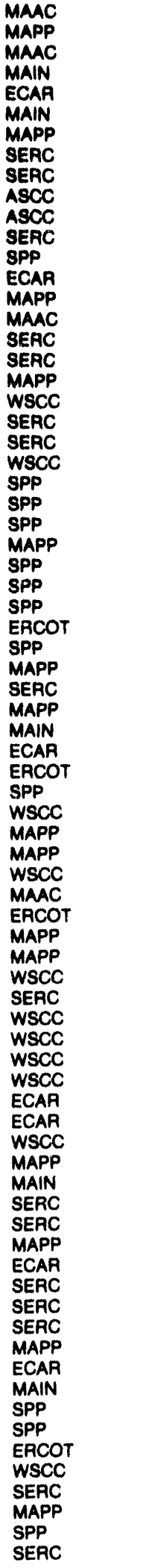 & 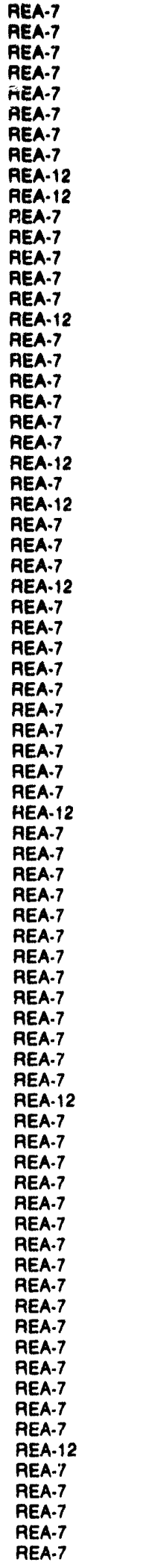 \\
\hline
\end{tabular}

See footnotes at end of table. 
Table B5. Cooporative Borrowers, 1992 (Continued)

\begin{tabular}{|c|c|c|c|}
\hline vinumy & State' & NERC Reglon" & Survoy Form' \\
\hline 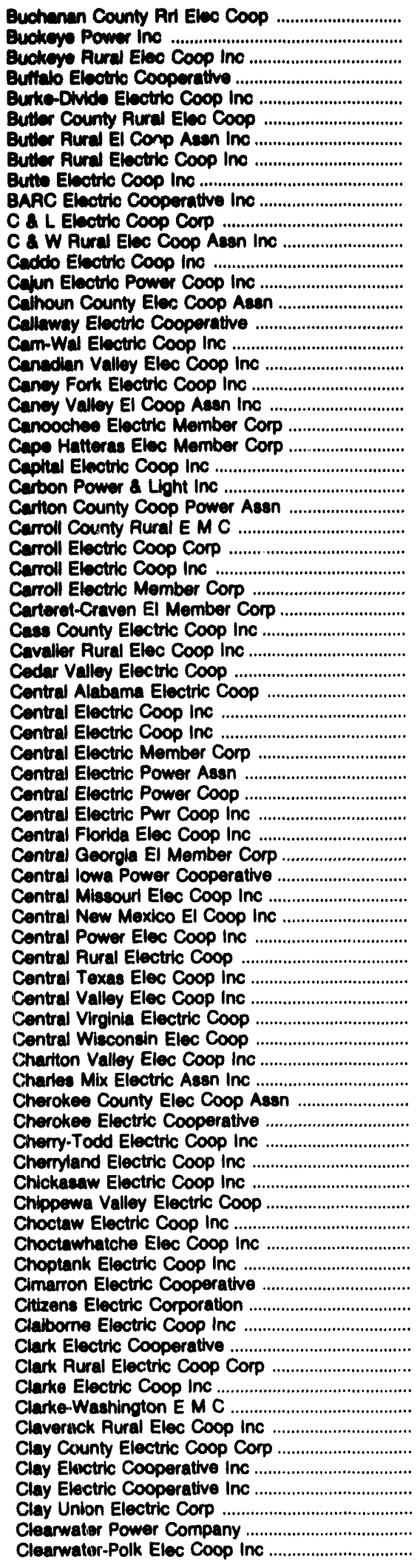 & 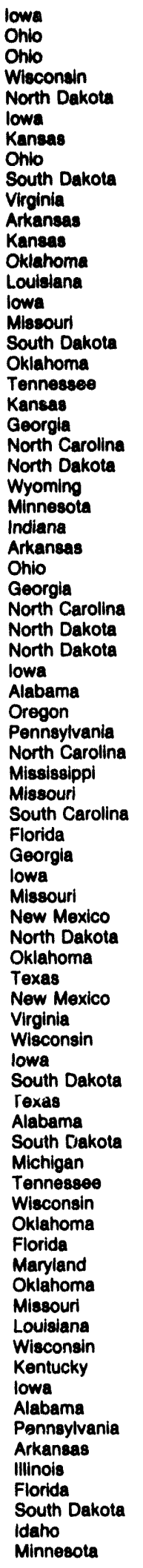 & 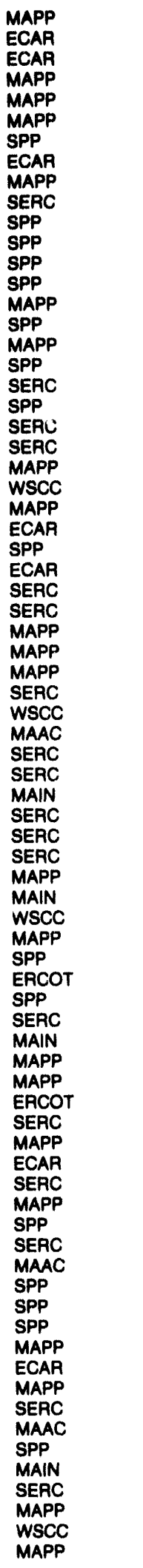 & 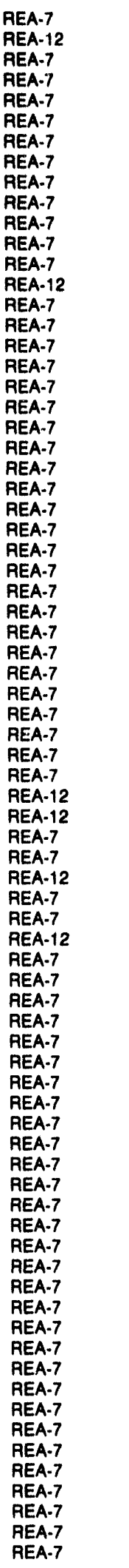 \\
\hline
\end{tabular}

See footnotes at end of table. 
Table B5. Cooperative Borrowers, 1992 (Continued)

\begin{tabular}{|c|c|c|c|}
\hline venity & State' & NERC Reglon" & Survey Form' \\
\hline 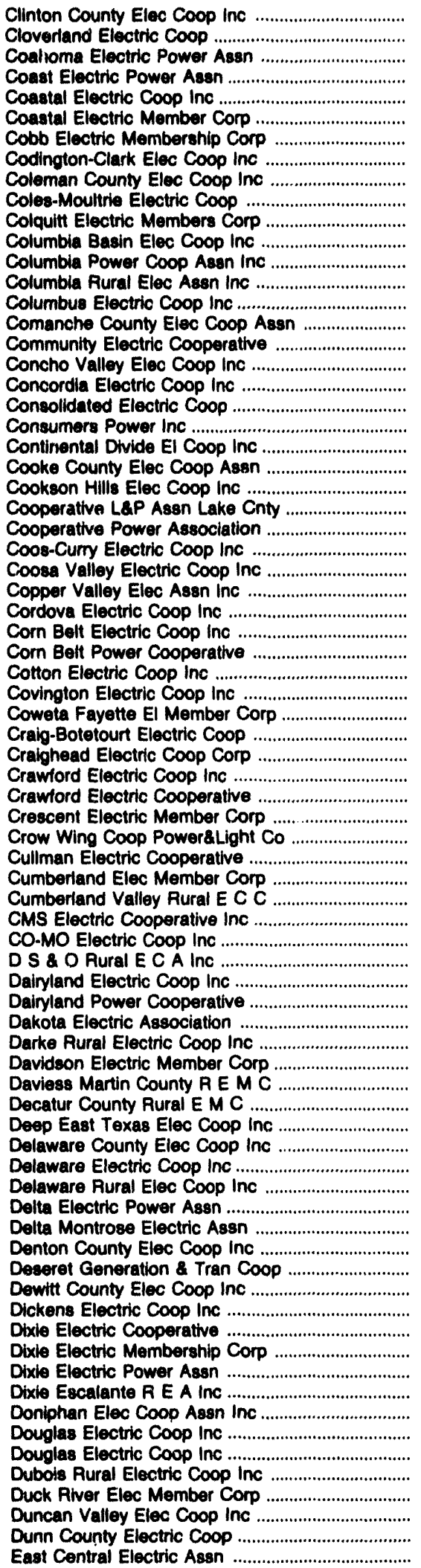 & 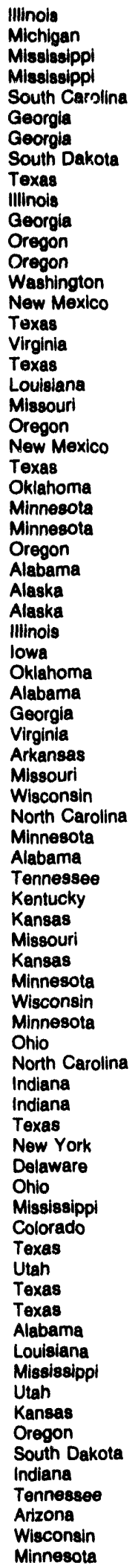 & 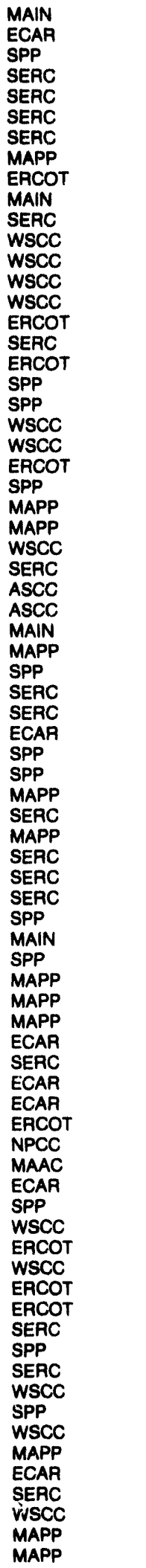 & 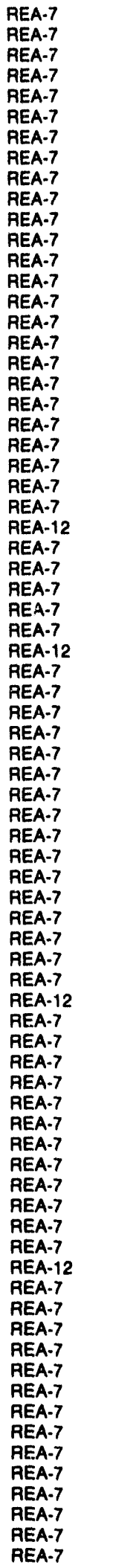 \\
\hline
\end{tabular}

See footnotes at end of table. 
Table B5. Cooperattve Borrowers, 1992 (Continued)

\begin{tabular}{|c|c|c|c|}
\hline Utimty & State' & MEAC Reglon ${ }^{2}$ & Survoy Form' \\
\hline 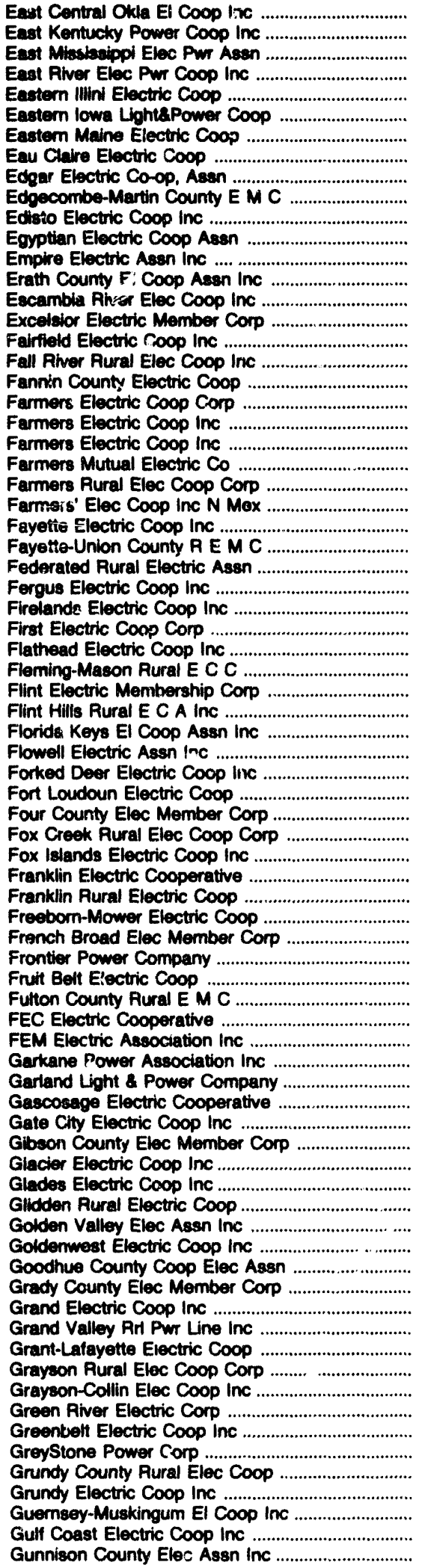 & 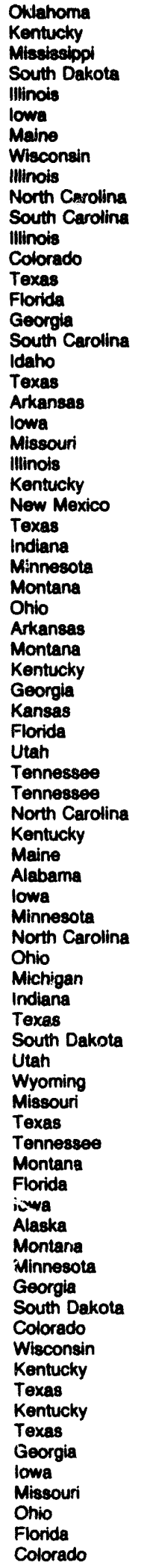 & 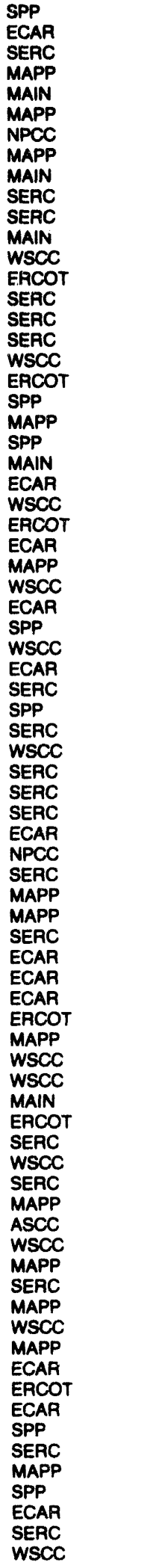 & 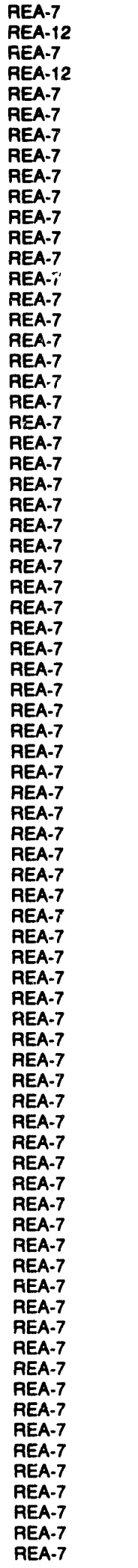 \\
\hline
\end{tabular}

See footnotes at end of table. 
Table B5. Cooperative Borrowers, 1992 (Continued)

\begin{tabular}{|c|c|c|c|}
\hline Utinty & State' & NERC Rogion" & Survey Form" \\
\hline 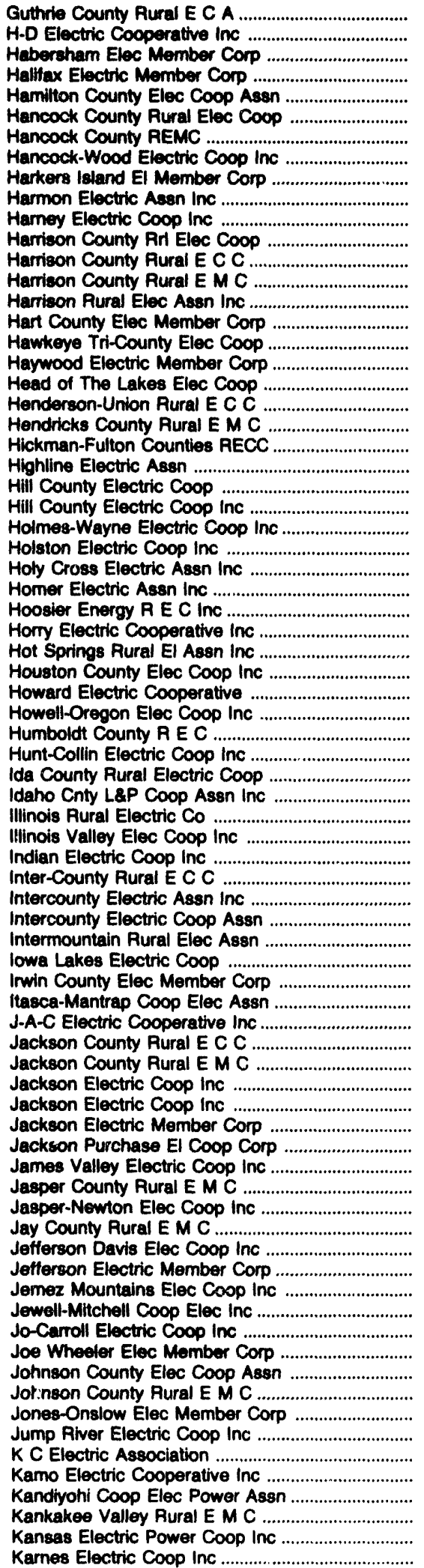 & 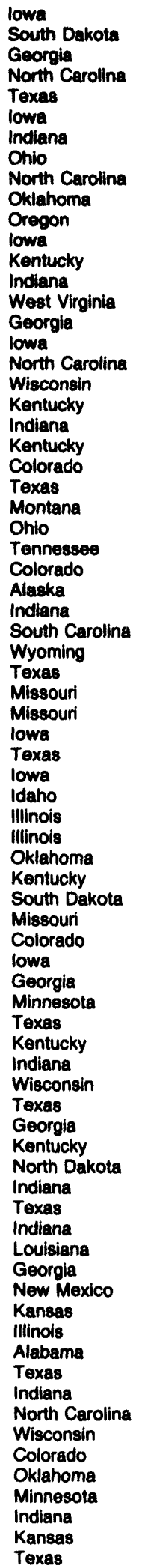 & 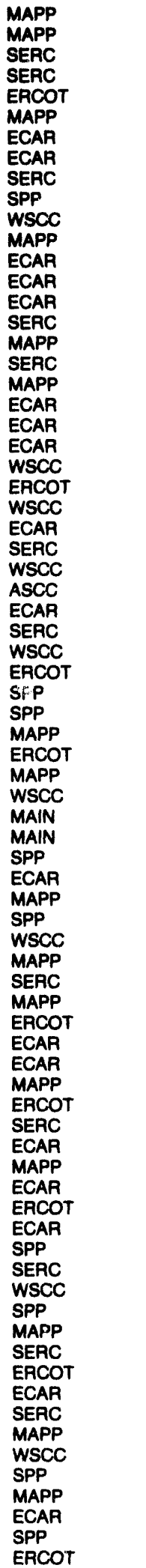 & 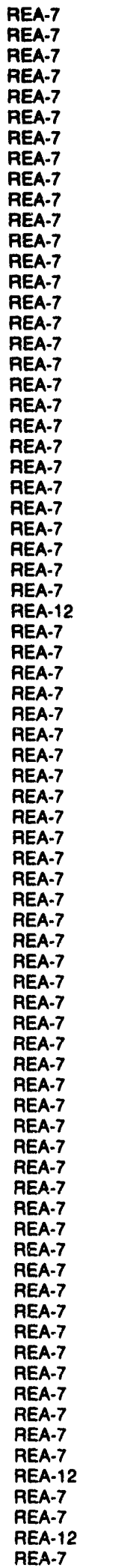 \\
\hline
\end{tabular}

See footnotes at end of table. 


\begin{tabular}{|c|c|c|c|}
\hline Uatumy & State' & MERC Roglon' & Survey Form" \\
\hline 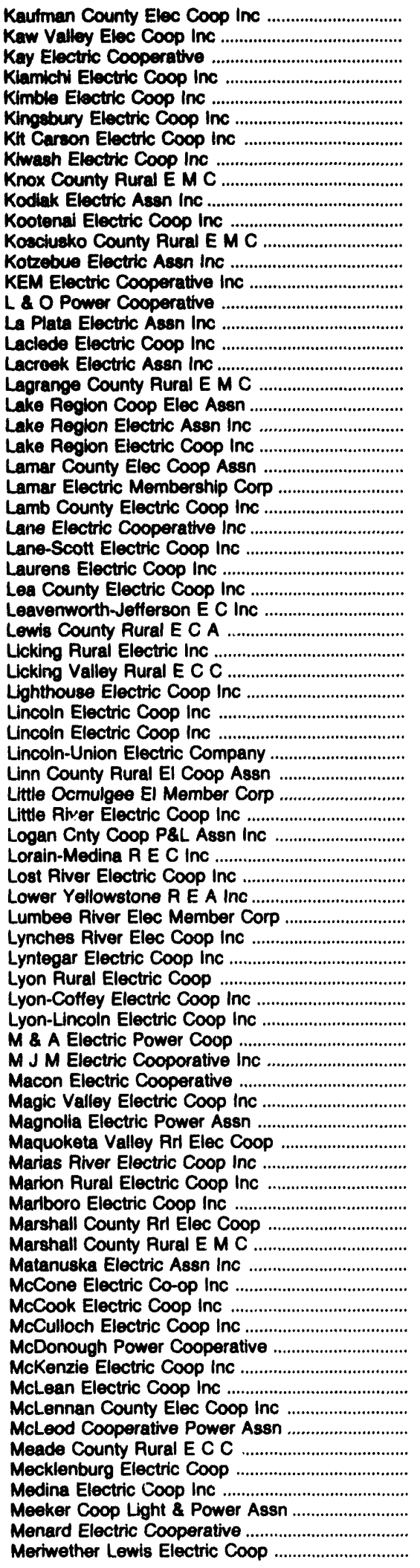 & 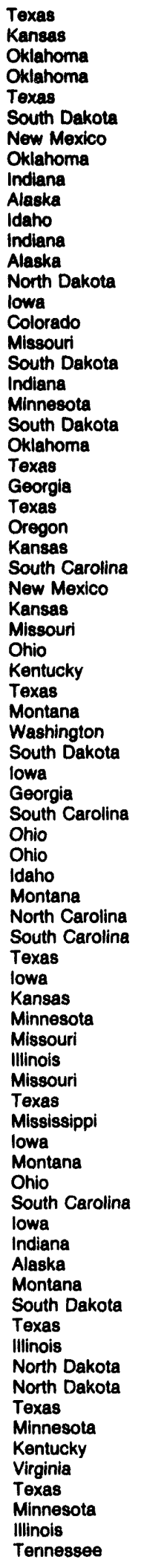 & 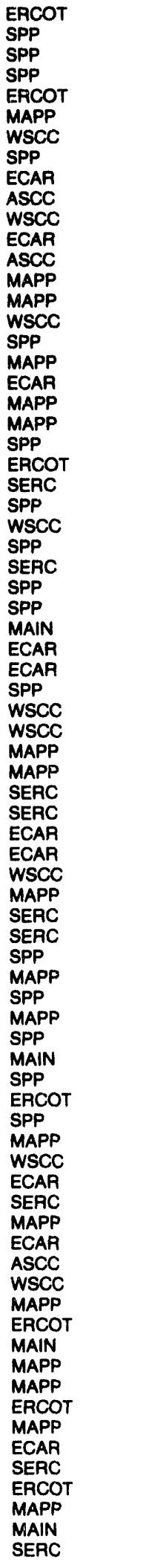 & 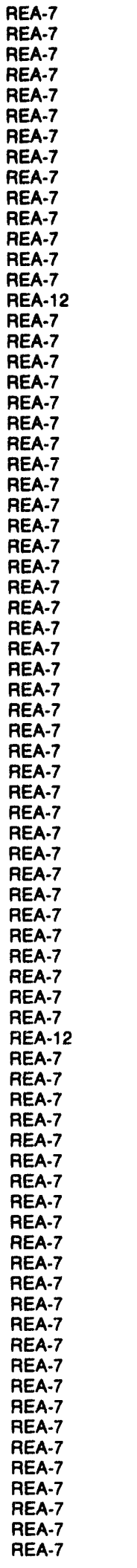 \\
\hline
\end{tabular}

See footnotes at end of table. 
Table B5. Cooperative Borrowers, 1992 (Continued)

\begin{tabular}{|c|c|c|c|}
\hline Uatilty & State' & NERC Roglon" & Survey Form" \\
\hline 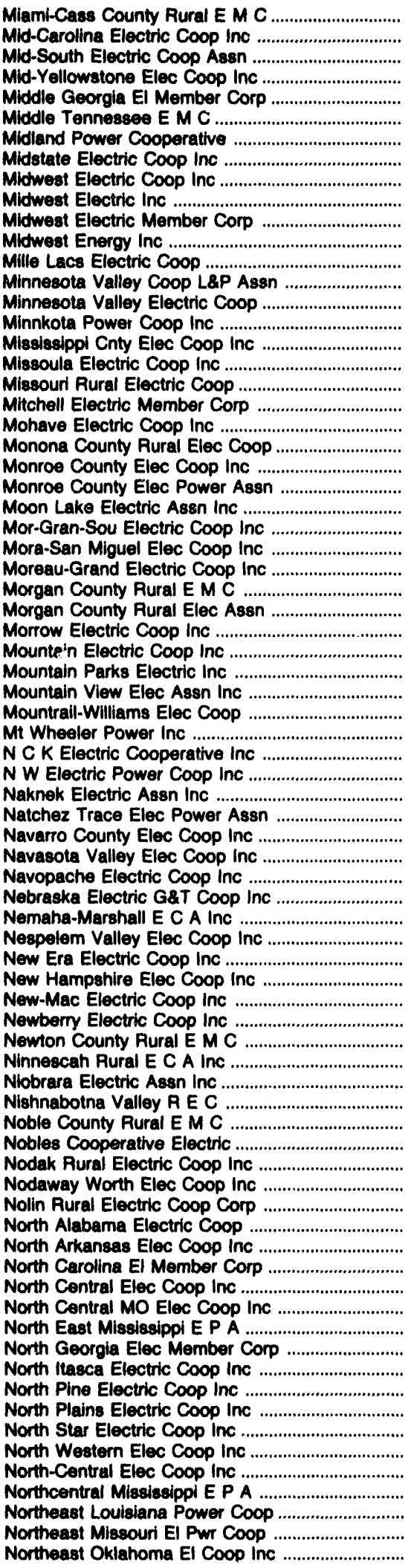 & 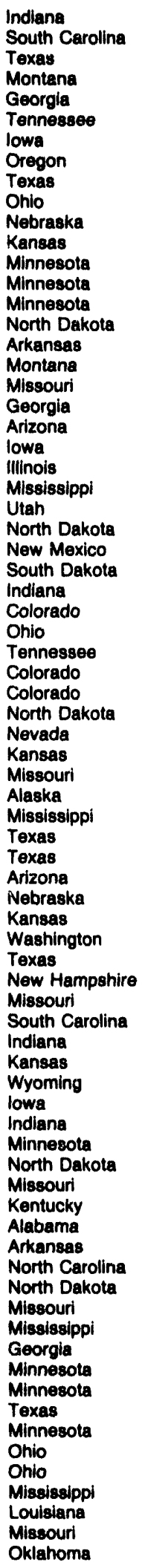 & 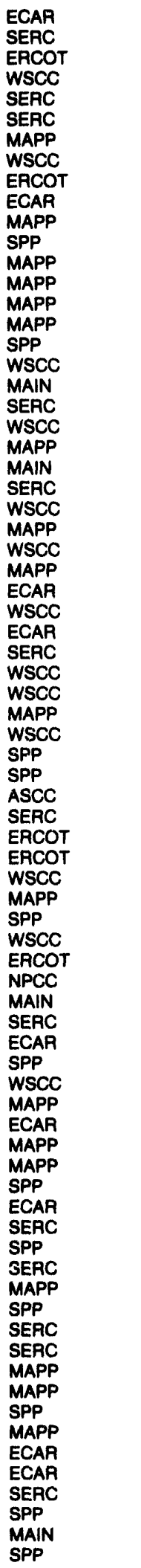 & 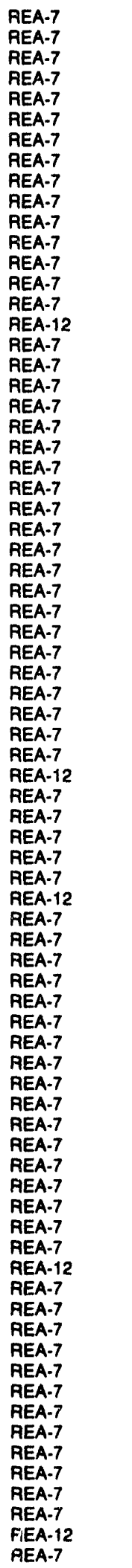 \\
\hline
\end{tabular}

See footnotes at end of table. 
Table B5. Cooperative Borrowers, 1992 (Continued)

\begin{tabular}{|c|c|c|c|}
\hline Utulty & State' & NERC Roglon' & Survey Form" \\
\hline 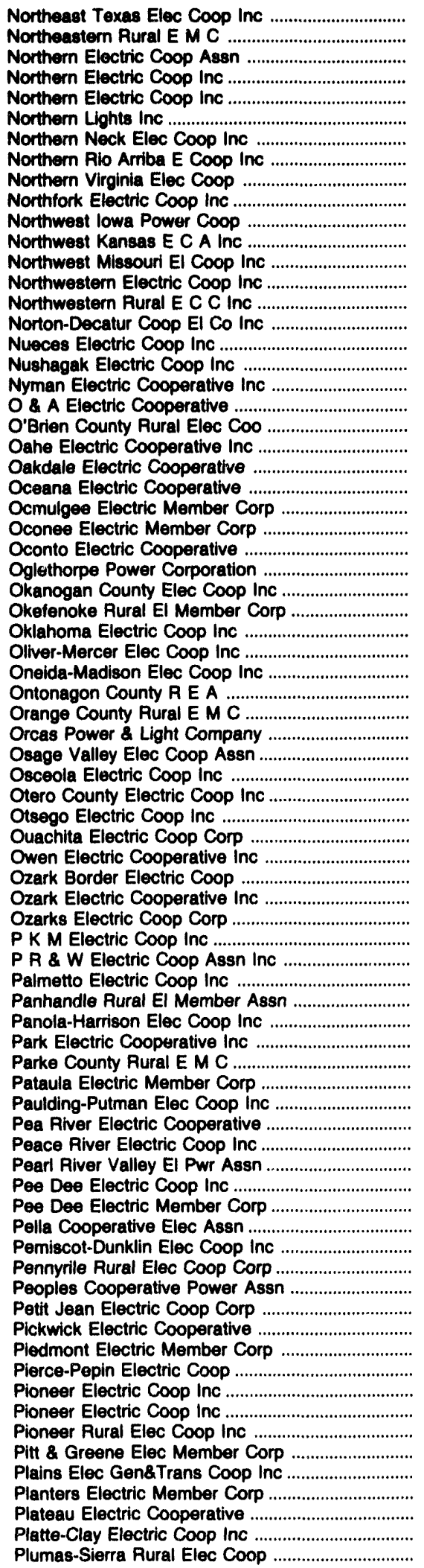 & 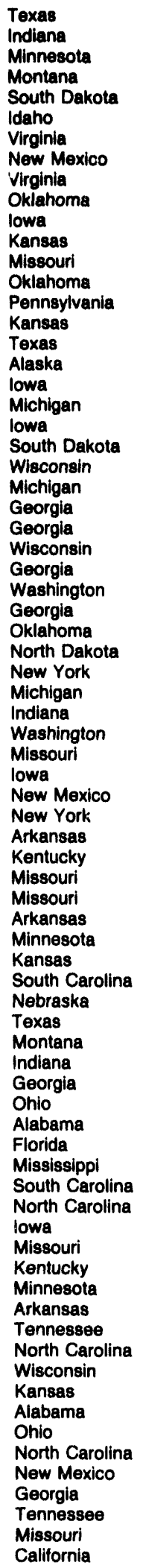 & 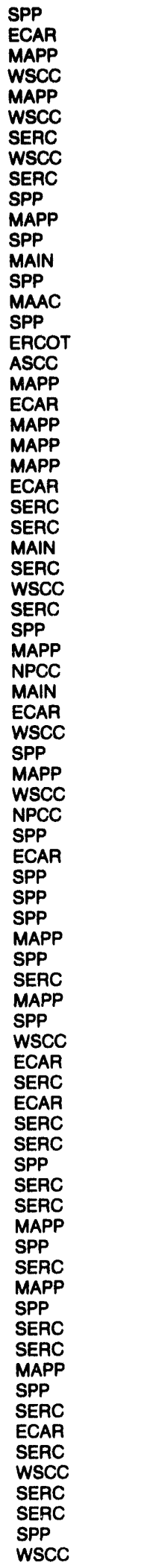 & 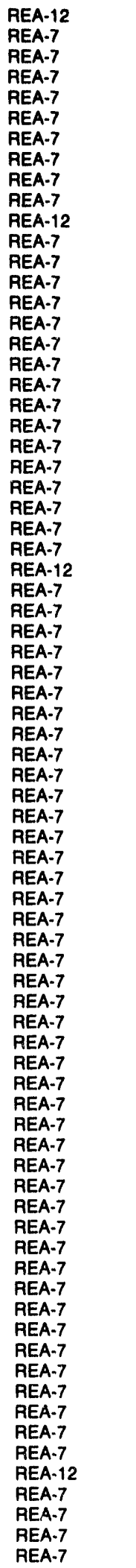 \\
\hline
\end{tabular}

Seo footnotes at end of table. 
Table B5. Cooperative Borrowers, 1992 (Continued)

\begin{tabular}{|c|c|c|c|}
\hline Utility & Stato' & NEAC Reglon" & Survey Form" \\
\hline 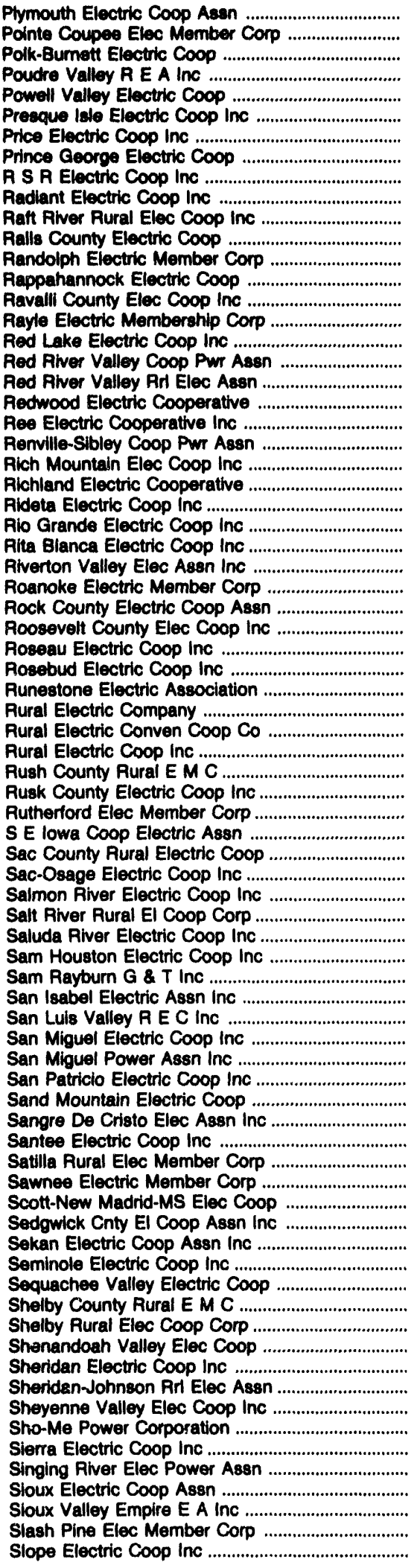 & 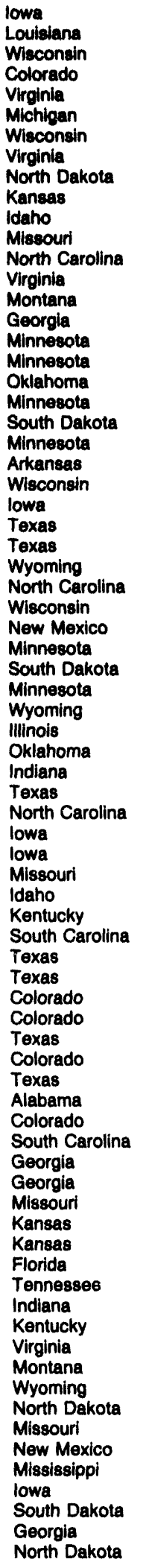 & 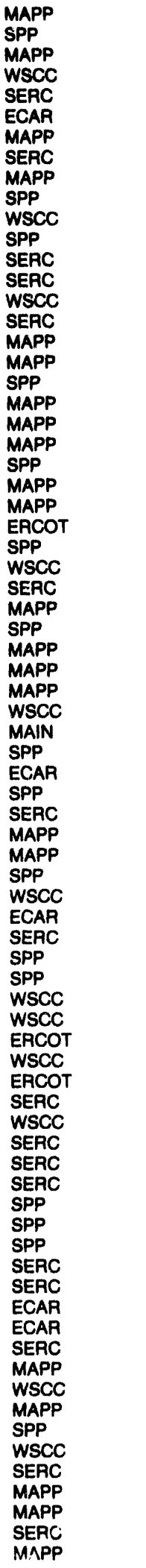 & 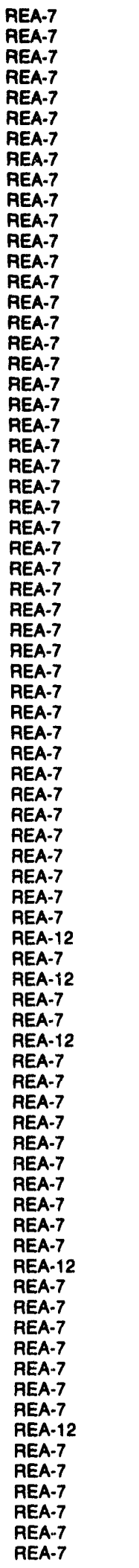 \\
\hline
\end{tabular}

See footnotes at end of table. 
Table B5. Cooperatlve Borrowers, 1992 (Continued)

\begin{tabular}{|c|c|c|c|}
\hline Utulity & State' & NEAC Reglon" & Survoy Form ${ }^{3}$ \\
\hline 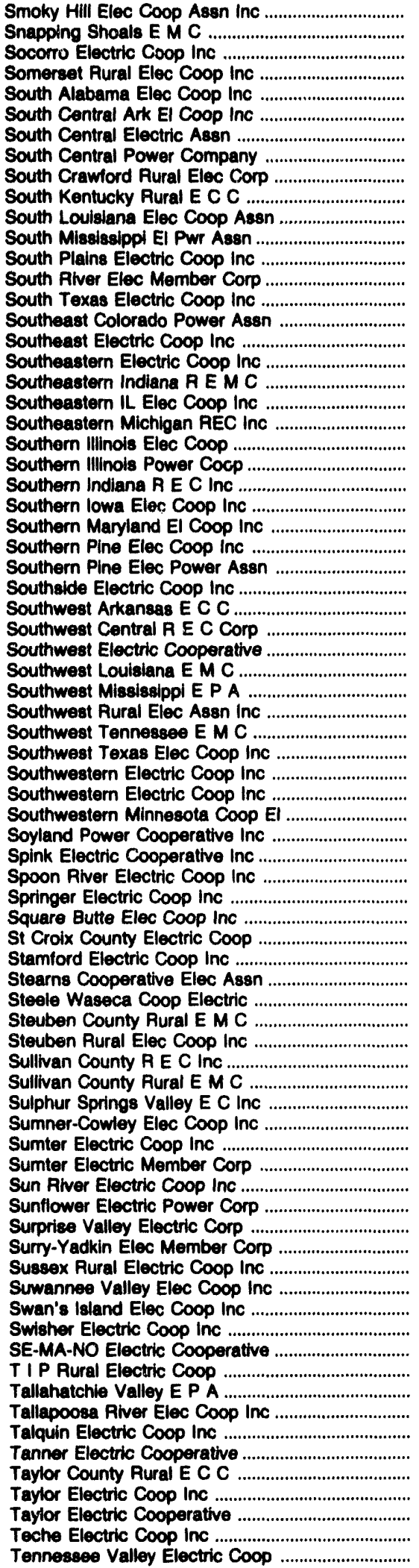 & 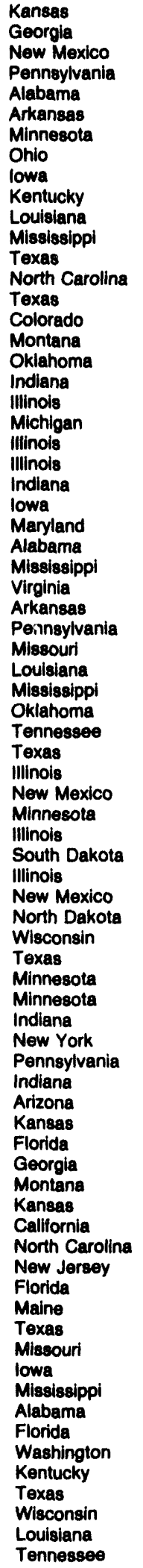 & 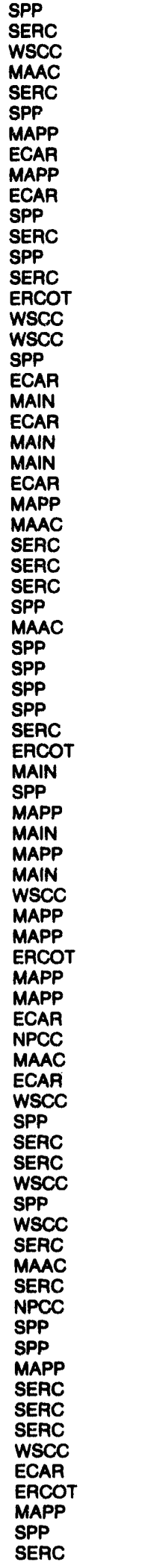 & 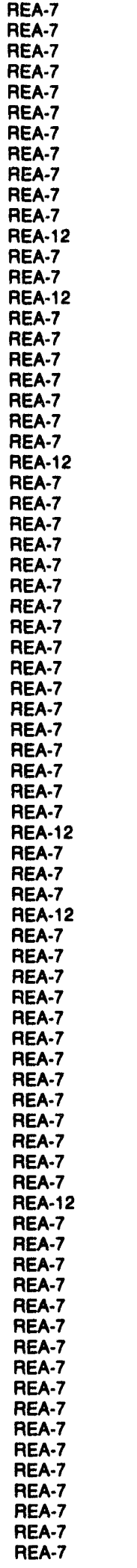 \\
\hline
\end{tabular}

See footnotes at end of table. 
Table B5. Cooperative Borrowers, 1992 (Continued)

\begin{tabular}{|c|c|c|c|}
\hline Uatity & state' & NERC Reglon" & survoy Form' \\
\hline 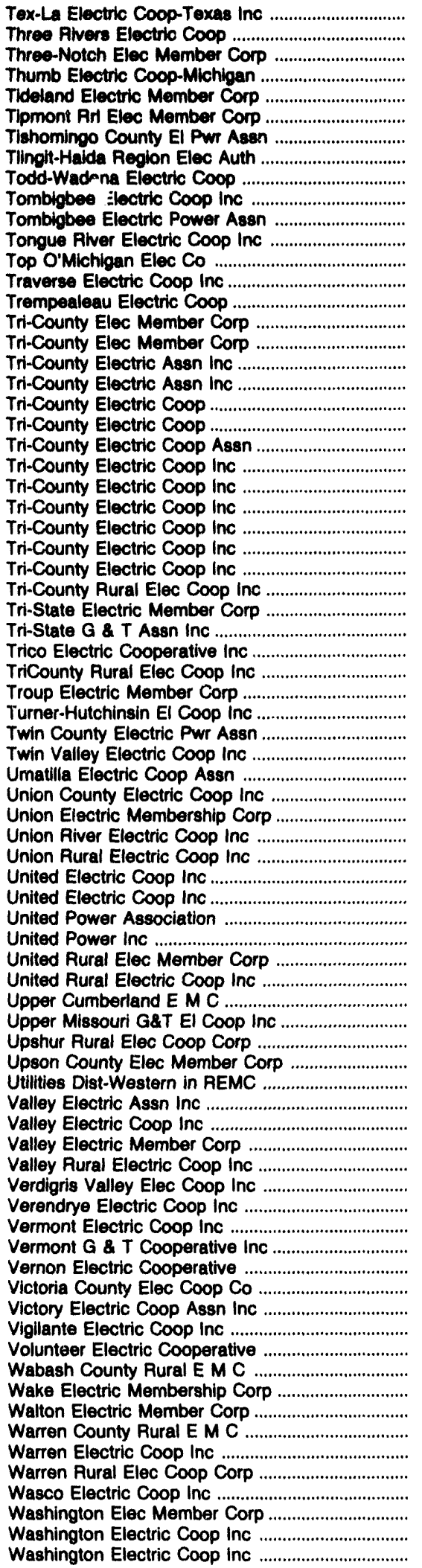 & 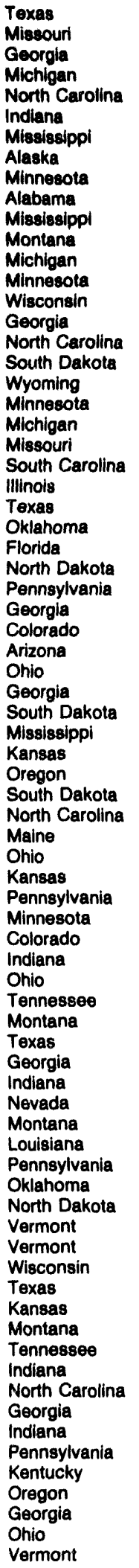 & 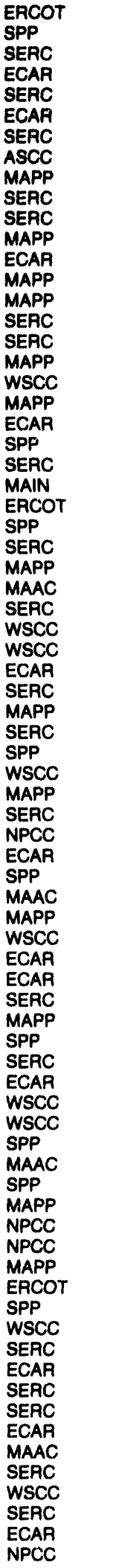 & 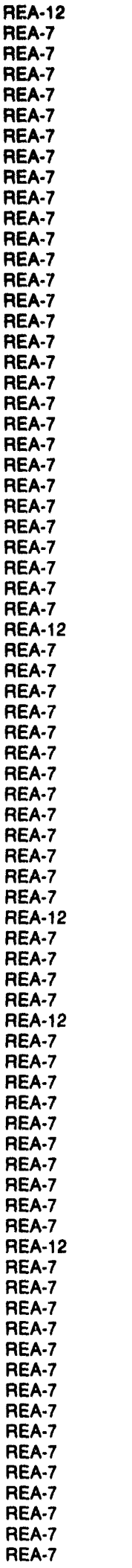 \\
\hline
\end{tabular}

See footnotes at end of table. 
Table B5. Cooperative Borrowers, 1992 (Continued)

\begin{tabular}{|c|c|c|c|}
\hline Uullty & state' & NERC Reglon' & survoy Form' \\
\hline 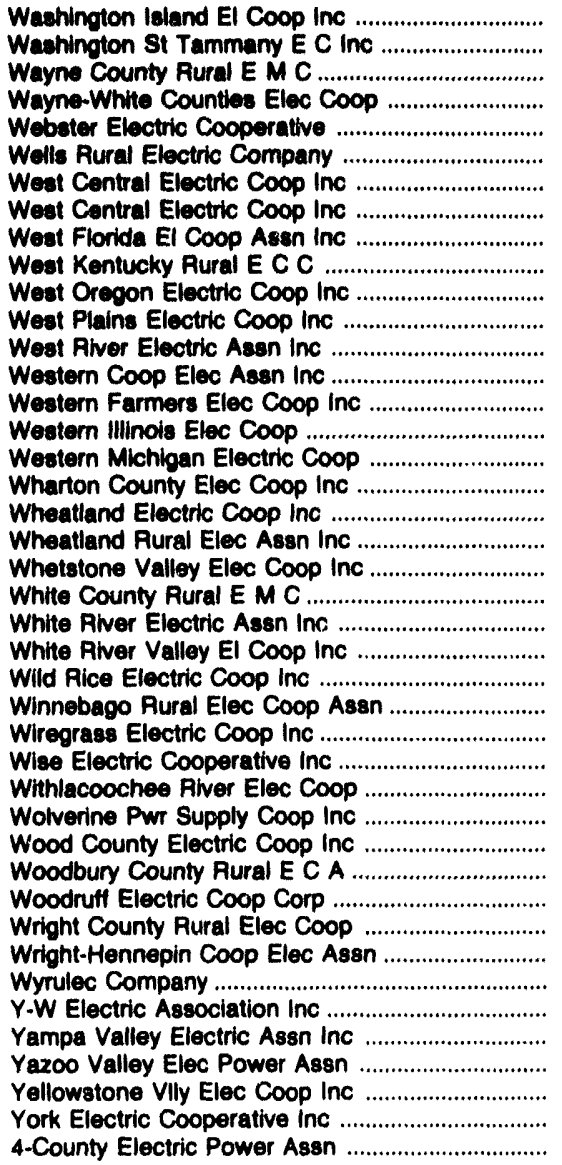 & $\begin{array}{l}\text { Wisconsin } \\
\text { Louisiana } \\
\text { Indlana } \\
\text { Illinole } \\
\text { Missouri } \\
\text { Nevada } \\
\text { Missouri } \\
\text { South Dakota } \\
\text { Florlda } \\
\text { Kentucky } \\
\text { Oregon } \\
\text { North Dakota } \\
\text { South Dakota } \\
\text { Kansas } \\
\text { Oklahoma } \\
\text { Illinois } \\
\text { Michigan } \\
\text { Texas } \\
\text { Kansas } \\
\text { Wyoming } \\
\text { South Dakota } \\
\text { Indiana } \\
\text { Colorado } \\
\text { Missouri } \\
\text { Minnesota } \\
\text { lowa } \\
\text { Alabama } \\
\text { Texas } \\
\text { Florida } \\
\text { Michigan } \\
\text { Texas } \\
\text { lowa } \\
\text { Arkansas } \\
\text { lowa } \\
\text { Minnesota } \\
\text { Wyoming } \\
\text { Colorado } \\
\text { Colorado } \\
\text { Missiasippi } \\
\text { Montana } \\
\text { South Carolina } \\
\text { Mississippi }\end{array}$ & $\begin{array}{l}\text { MAIN } \\
\text { SPP } \\
\text { ECAR } \\
\text { MAIN } \\
\text { SPP } \\
\text { WSCC } \\
\text { SPP } \\
\text { MAPP } \\
\text { SERC } \\
\text { ECAR } \\
\text { WSCC } \\
\text { MAPP } \\
\text { MAPP } \\
\text { SPP } \\
\text { SPP } \\
\text { MAIN } \\
\text { MAIN } \\
\text { ERCOT } \\
\text { SPP } \\
\text { WSCC } \\
\text { MAPP } \\
\text { ECAR } \\
\text { WSCC } \\
\text { SPP } \\
\text { MAPP } \\
\text { MAPP } \\
\text { SERC } \\
\text { ERCOT } \\
\text { SERC } \\
\text { ECAR } \\
\text { SPP } \\
\text { MAPP } \\
\text { SPP } \\
\text { MAPP } \\
\text { MAPP } \\
\text { WSCC } \\
\text { WSCC } \\
\text { WSCC } \\
\text { SERC } \\
\text { WSCC } \\
\text { SERC } \\
\text { SERC }\end{array}$ & $\begin{array}{l}\text { REA-7 } \\
\text { AEA-7 } \\
\text { REA-7 } \\
\text { REA-7 } \\
\text { REA-7 } \\
\text { REA-7 } \\
\text { REA-7 } \\
\text { REA-7 } \\
\text { REA-7 } \\
\text { REA-7 } \\
\text { REA-7 } \\
\text { REA-7 } \\
\text { REA-7 } \\
\text { REA-7 } \\
\text { REA-12 } \\
\text { REA-7 } \\
\text { REA-7 } \\
\text { REA-7 } \\
\text { REA-7 } \\
\text { REA-7 } \\
\text { REA-7 } \\
\text { REA-7 } \\
\text { AEA-7 } \\
\text { REA-7 } \\
\text { REA-7 } \\
\text { REA-7 } \\
\text { REA-7 } \\
\text { REA-7 } \\
\text { REA-7 } \\
\text { REA-12 } \\
\text { REA-7 } \\
\text { REA-7 } \\
\text { REA-7 } \\
\text { REA-7 } \\
\text { REA-7 } \\
\text { REA-7 } \\
\text { REA-7 } \\
\text { REA-7 } \\
\text { REA-7 } \\
\text { REA-7 } \\
\text { REA-7 } \\
\text { REA-7 }\end{array}$ \\
\hline
\end{tabular}

1 The State in which the administrative office of the cooperative borrower is located.

- The principal North American Electric Reliability Council region in which the utlity operates. See glossary for a list of all regions.

Source of data for this publication 1992.

Note: Penoyer Valley Electric Co filed a FERC Form 1-F, "Annual Report for Nonmajor Public Utilities and Licensees" but had no transactions in

Sources: •Rural Electrification Administration, REA Form 7, "Financial and Statistical Report," REA Form 12a through 12i, "Electric Power Supply Borrowers," Form 12c through 12g. "Electric Distribution Borrowers with Generating Facilities." •Energy Information Administration, Form ElA-661, "Annual Electric Utility Report." 
Table B6. Electric Utilities Not Reported Eleowhere In Survoys Roporting Dotalled Traneactions, 1992

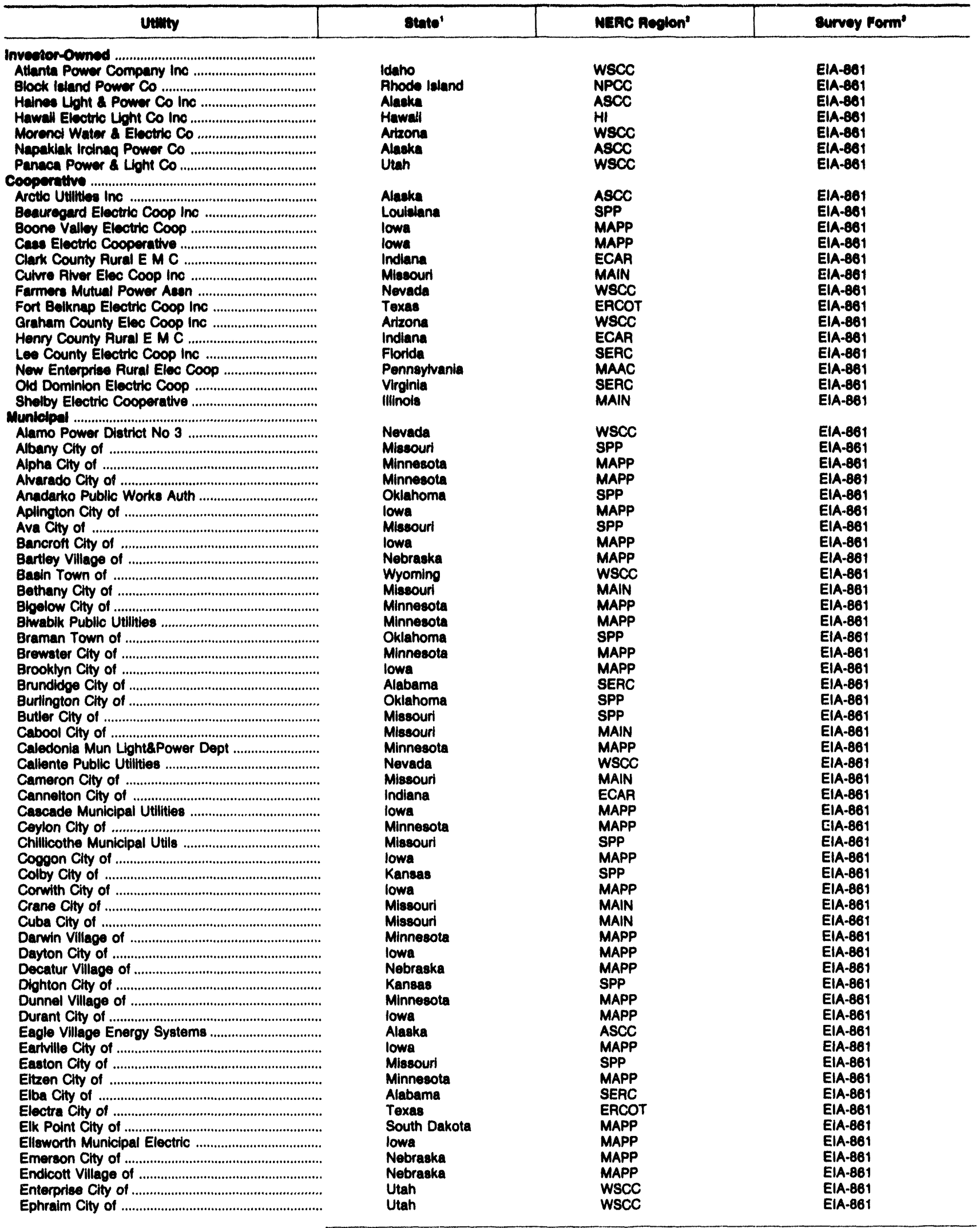

See footnotes at end of table. 
Table B6. Electric Utilities Not Roported Eleowhere in surveye Reporting Detalled Traneactions, 1902 (Continued)

\begin{tabular}{|c|c|c|c|}
\hline unimy & Etate' & ware nesion" & eurvor Form" \\
\hline 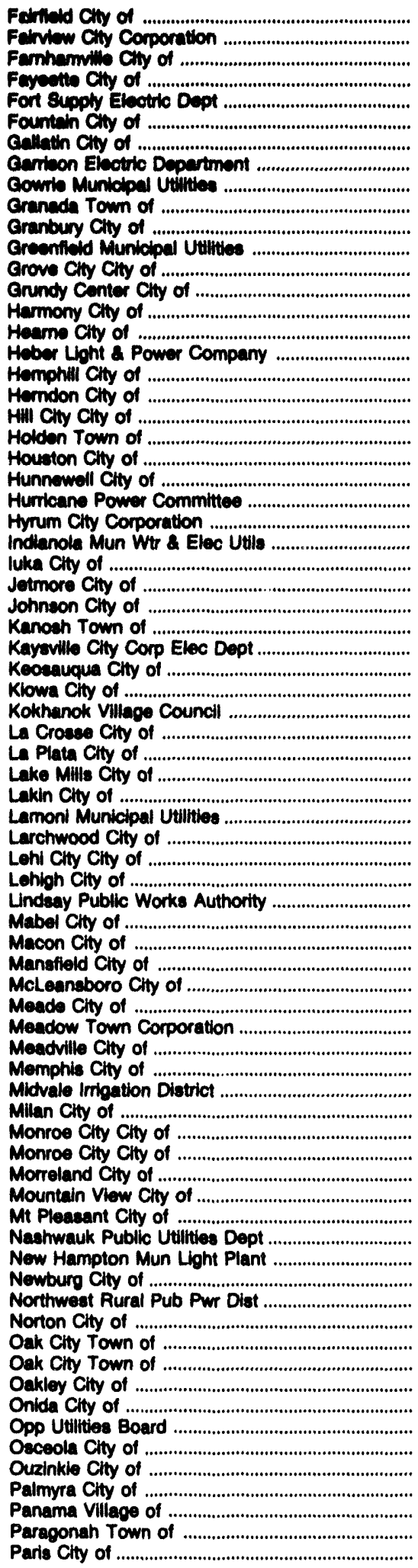 & 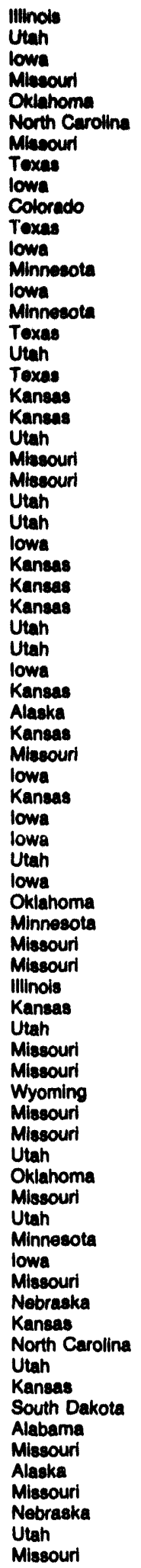 & 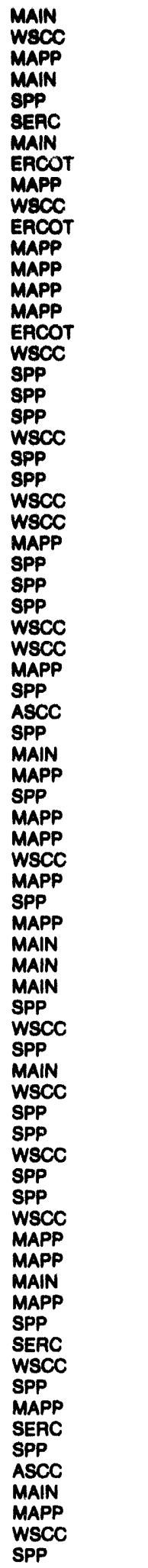 & 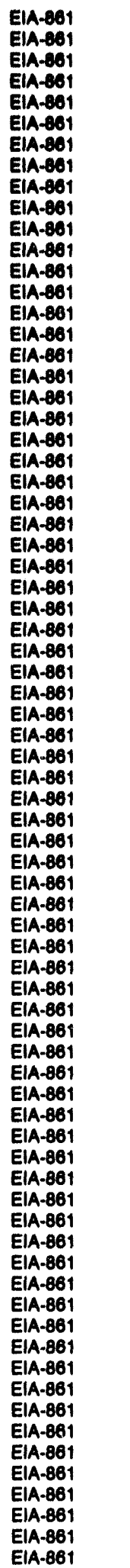 \\
\hline
\end{tabular}

See footnotes at end of table. 
Table B6. Electrle Utilities Not Roported Eleowhere In Survoys Reporting Dotallod Traneactlone, 1992 (Continued)

\begin{tabular}{|c|c|c|c|}
\hline vaniny & Etate' & Nanc Recion' & survey Form' \\
\hline 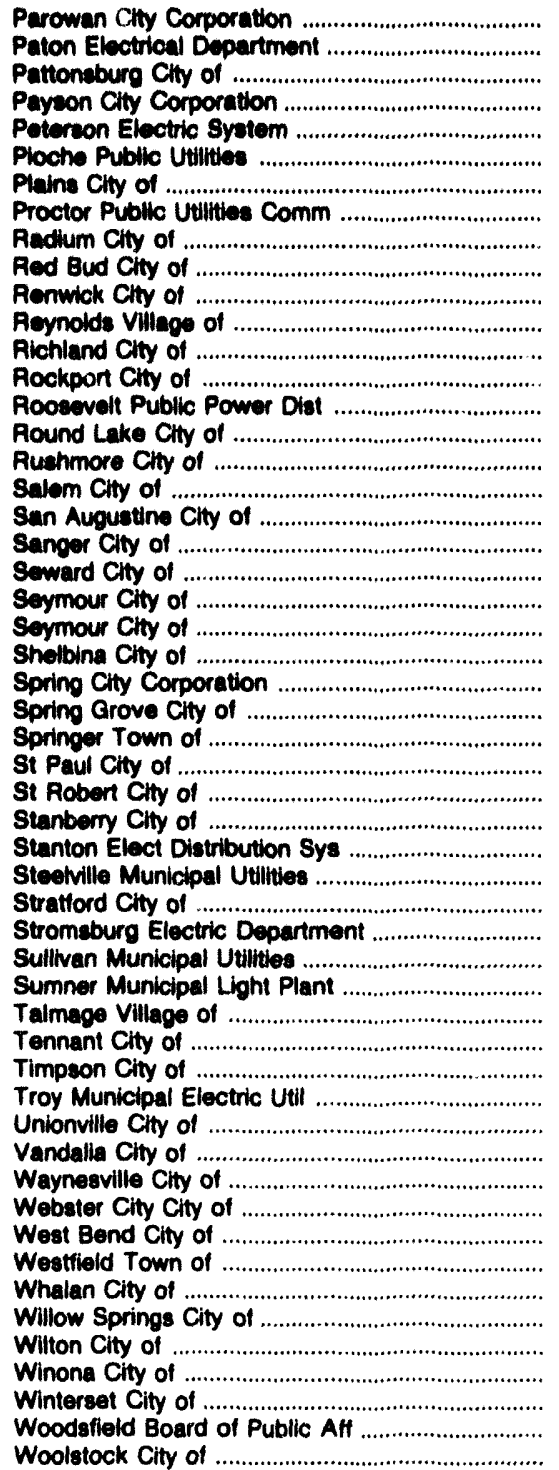 & $\begin{array}{l}\text { Utah } \\
\text { lowa } \\
\text { Mlesouri } \\
\text { Utah } \\
\text { Minnocota } \\
\text { Nevada } \\
\text { Texas } \\
\text { Minnesota } \\
\text { Kansas } \\
\text { Ilinols } \\
\text { lowa } \\
\text { Nebraska } \\
\text { Miseouri } \\
\text { Missouri } \\
\text { Nebraska } \\
\text { Minnesola } \\
\text { Minnesota } \\
\text { Missouri } \\
\text { Texas } \\
\text { Texas } \\
\text { Kansas } \\
\text { Miseouri } \\
\text { Texas } \\
\text { Missouri } \\
\text { Utah } \\
\text { Minnesota } \\
\text { New Mexico } \\
\text { Nebraska } \\
\text { Missouri } \\
\text { Miseouri } \\
\text { lowa } \\
\text { Missouri } \\
\text { lowa } \\
\text { Nebraska } \\
\text { Missouri } \\
\text { lowa } \\
\text { Nebraska } \\
\text { lowa } \\
\text { Texas } \\
\text { Indlana } \\
\text { Missouri } \\
\text { Missouri } \\
\text { Missouri } \\
\text { lowa } \\
\text { lowa } \\
\text { lowa } \\
\text { Minnesota } \\
\text { Missouri } \\
\text { lowa } \\
\text { Missouri } \\
\text { lowa } \\
\text { Ohio } \\
\text { lowa }\end{array}$ & $\begin{array}{l}\text { WSCC } \\
\text { MAPP } \\
\text { MAIN } \\
\text { WSCC } \\
\text { MAPP } \\
\text { WSCC } \\
\text { SPP } \\
\text { MAPP } \\
\text { SPP } \\
\text { MAIN } \\
\text { MAPP } \\
\text { MAPP } \\
\text { MAIN } \\
\text { SPP } \\
\text { MAPP } \\
\text { MAPP } \\
\text { MAPP } \\
\text { SPP } \\
\text { SPP } \\
\text { ERCOT } \\
\text { SPP } \\
\text { SPP } \\
\text { ERCOT } \\
\text { SPP } \\
\text { WSCC } \\
\text { MAPP } \\
\text { SPP } \\
\text { MAPP } \\
\text { MAIN } \\
\text { SPP } \\
\text { MAPP } \\
\text { MAIN } \\
\text { MAPP } \\
\text { MAPP } \\
\text { MAIN } \\
\text { MAPP } \\
\text { MAPP } \\
\text { MAPP } \\
\text { ERCOT } \\
\text { ECAP } \\
\text { SPP } \\
\text { MAIN } \\
\text { SPP } \\
\text { MAPP } \\
\text { MAPP } \\
\text { MAPP } \\
\text { MAPP } \\
\text { SPP } \\
\text { MAPP } \\
\text { SPP } \\
\text { MAPP } \\
\text { ECAR } \\
\text { MAPP }\end{array}$ & 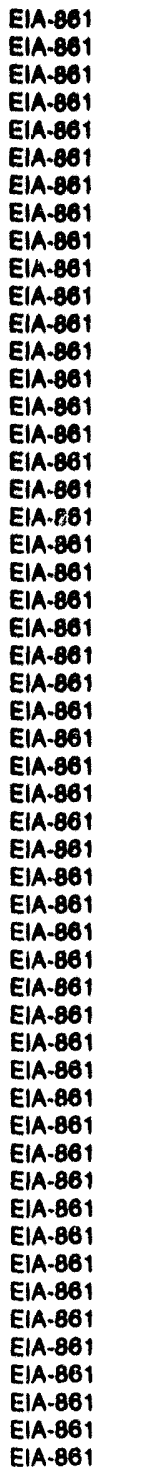 \\
\hline
\end{tabular}

- The State in which the administrative or corporate office of the utility is located.

2 The principal North American Electric Reliability Council region in which the utility operates. See glossary for a list of all regions.

- Source of data for this publication

Note: These electric utilities are not reported elsewhere because they fall below the class of ownership survey threshold and/or information on exchanges and wheeling for selected utilities are only reported on the Form, ElA-861.

Source: -Energy Information Administration, Form ElA-861, "Annual Electric Utility Report." 
Table B7. Eloctric Power Marketers Authorized by FERC, as of December 31, 1993

\begin{tabular}{|c|c|c|c|c|}
\hline Neme & State & $\begin{array}{c}\text { PEAC } \\
\text { Roteronoe }\end{array}$ & $\begin{array}{c}\text { Authorization } \\
\text { Reforence }\end{array}$ & $\begin{array}{c}\text { Dote } \\
\text { Authorlzed }\end{array}$ \\
\hline 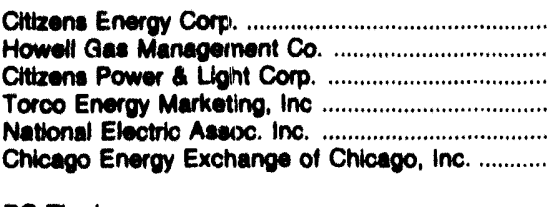 & $\begin{array}{l}\text { Maseachusetts } \\
\text { Texas } \\
\text { Maseschusetts } \\
\text { District of Columbia } \\
\text { Texas } \\
\text { Illinols }\end{array}$ & $\begin{array}{l}\text { EL86-2-000 } \\
\text { EL87-50-000 } \\
\text { ER89-401-000 } \\
\text { EL89-32-000 } \\
\text { ER90-188-000 } \\
\text { ER90-225-000 } \\
\text { ER90-17-000 }\end{array}$ & $\begin{array}{ll}35 & \# 61,198 \\
40 & \# 61,336 \\
48 & \# 61,210 \\
48 & \# 61,294 \\
50 & \# 61,378 \\
51 & \# 61,054\end{array}$ & $\begin{array}{l}5 / 18 / 86 \\
9 / 28 / 87 \\
8 / 08 / 89 \\
9 / 07 / 89 \\
3 / 20 / 90 \\
4 / 19 / 90\end{array}$ \\
\hline 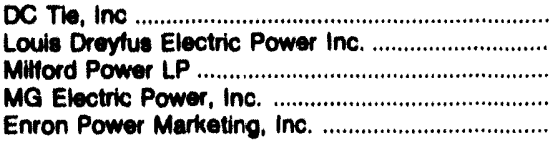 & $\begin{array}{l}\text { District of Columbia } \\
\text { District of Columbia } \\
\text { Massachusetts } \\
\text { New York } \\
\text { Massachusetts }\end{array}$ & $\begin{array}{l}\text { ER91-435-000 } \\
\text { ER92-850-000 } \\
\text { ER93-493-000 } \\
\text { ER93-839-000 } \\
\text { ER94-24-000 }\end{array}$ & $\begin{array}{l}\text { FERC Letter } \\
61 \quad \% 61,303 \\
\text { FERC Letter } \\
\text { FERC Letter } \\
65 \$ 61,305\end{array}$ & $\begin{array}{c}7 / 11 / 91 \\
12 / 02 / 92 \\
9 / 17 / 93 \\
10 / 19 / 93 \\
12 / 02 / 93\end{array}$ \\
\hline
\end{tabular}

Note: The Federal Energy Regulatory Commission has jurisdictional responsibility when an energy marketer takes ownership of electricity. If ownership is nol paseas to the party brokering or coordinating the transaction, then the FERC does not consider the broker involved in interstate tracie. Source: Federal Energy Regulatory Commission. 


\section{Appendix C}

Fundamentals of

Electric Power

Transmission and

Integrating

Nonutility

Generators 


\section{Fundamentals of Electric Power Transmission and Integrating Nonutility Generators}

\section{Fundamentals of the Electric Power Transmission System}

\begin{abstract}
The electric power system in the United States contains three interrelated elements: the generating facilities that produce the power; the transmission network that conducts the flow of power from the points of generation to the points of distribution; and the distribution system that delivers the electric power to the consumers. The transmission net rork is the integrating medium of the power supply system providing the electrical connections between the many geographically separated parts of the electric power generating and distribution systems.
\end{abstract}

The electric transmission network is unlike any other mode of transportation. The flow of electricity is virtually instantaneous, changing magnitude and direction as conditions on the power system dictate. "Electricity distributes itself along paths of least resistance that are determined by a complex electrical relationship involving the relative location and distribution of generation resources, transmission line facilities, and centers of demand. All transmission paths share the power transfer, and the degree of sharing is determined by the relationship of the network components. The system consists of transmission lines, substations with voltage transformers, circuit breakers, and other equipment required to transmit power safely from generation sources to ultimate customers. Transmission voltage levels have increased with improvements in technology and in keeping with the growing demand for electricity.

The transmission system performs several essential functions simultaneously: (1) it supplies the physical means for delivering electricity from the generating sources to the load centers; (2) it integrates generating sources and load centers into a flexible and resilient whole; and (3) it interconnects the physical facilities with those of neighboring systems. Although transmis- sion lines are often added to the network initially to meet a single specific requirement, once added they become an integrated part of the transmission network and their operation becomes interdependent with all the other elements of the network. Operating the system effectively requires significant planning and operational coordination of the generators and transmission facilities to: (1) achieve efficient use of the system, (2) prevent overloading and failure, and (3) maintain adequate reserve transmission and generation capacity to ensure system reliability.

\section{The Need for Coordination of System Operation}

The current electrical system has developed in response to the regulations and economics of the electrical utility and nonutility sectors of the electric power industry, as well as to the changing technical factors that influence the generation, transmission, and distribution of electricity. Since the different electrical systems operate as a unified power grid (there are three in the contiguous United States) and the effects of power flows are not confined to contractual paths or apparent direct paths, handling the ever changing flow of electricity is a critical activity for system operators of the power grids. Large power transfers, for example, can change transmission line loadings hundreds of miles from the direct electrical path connecting the source and destination. Actions by individual utilities or NUGs can affect the operation of all the others on the system.

\section{Control and Operation of Electric Systems}

As electrical energy itself cannot be stored, power must be instantaneously available to end users at any time, in any amount at the proper voltage. As a result, severe demands are imposed on electrical equipment and the transmission network when meeting changing

\footnotetext{
"The frequency of electric power supply in the United States is almost entirely 60 hertz (formerly cycles per second). The frequency of a system depends entirely upon the speed at which the supply generator is rotated by its prime mover. James Robert Eaton, Electric Power Transmission Systems (Englewood Cliffs, New Jersey: Prentice-Hall, Inc., 1972), pp. 2-3.
} 
loads. Monitoring the flow of scheduled electricity, handling customer requirements, and coordinating trade among utilities are the responsibility of the dispatch center. A dispatch center can be operated independently of other electrical systems by a single utility; it can link two or more interconnected utilities, or even unify several power systems with combined load requirements and maintenance programs.

The operators of dispatch centers must continually monitor load patterns to ensure that adequate electricity is available at all times. For most dispatch centers, it is the daily responsibility to (1) record the flow of electricity at the customer load centers and the entering and exiting amounts on its transmission lines, (2) watch the transmission connecting points for each interconnected electrical system, and (3) monitor the power flow from each generation plant. The dispatch center determines the power available from its system, balances the unit-generation marginal costs with buyor-sell opportunities with other utilities, coordinates the bulk power transactions, examines what plants must be dispatched to avoid technical system problems or undue economic costs, and accounts for system power losses. It also projects demand requirements in order to determine how much generating capacity will be needed and when. These projections may be done hourly, daily, weekly, or at longer intervals.

One electrical operating entity (power pool, electric utility, State authority, and/or Federal utility) within a group of interconnected electrical systems takes responsibility for maintaining system frequency for that electrical geographic area, monitors the load, and ensures generation availability to meet load requirements. Some control centers within these conirol areas are highly computerized, automatically loading the generating facilities as needed and maintaining the system at the correct operating frequency. This is important because deviations in the scheduled power flows or from the standard system frequency can automatically cause compensating changes in the output at the generating plants. These deviations can mean there has been a loss or gain of a customer load, a plant or line has suffered a forced outage, or some plant or line has been returned to the system. Any of these changes can require some review or action by these control centers.

Stabilizing system frequency is made easier by coordination with other electric systems and by drawing from a larger base of on-line capability. Load changes are absorbed by all the electrical systems, and many of the increasing and decreasing load changes cancel out or offset each other, so that the effect on the entire interconnected electrical system is less than it would be on an isolated electric utility. Also, this integrated system frees each generating unit from the necessity to make continual large changes in production levels.

\section{Integrating Nonutility Generators with the Bulk Electric System}

Nonutility generation sources continue to be a growing portion of the U.S. electrical generation capacity. This role of NUGs reflects the changing structure of the electric supply system.

\section{Integrating Nonutllity Generators}

NUGs are presenting a new challenge to the operators of the power grids because of the increasing numbers and their growing contribution to wholesale generation. Matching customer load and generation for both daily operations and future planning activities is becoming increasingly complex with the growing NUG role and increasing use of the transmission system. Electrical reliability concerns and the proper integration of NUGs into the supply system have become important issues.

The proper integration of NUGs into the electrical operations of interconnection and dispatching generation can be regarded as engineering problems for which technical solutions are available. However, there are institutional issues associated with the responsibility for serving customers and control of the electrical system. The increasing role of NUGs has altered the traditional view of participants in the electrical supply. It also has raised issues for electrical system operations and transmission line usage.

Utilities have three basic concerns involving the integration of NUGs with the bulk power system, relating primarily to the relationships of the NUGs, utilities, and customers:

- Utilities, with the principal responsibility to operate the system, do not always have full operating control over the NUGs.

- The forces that drive NUG development and operation do not necessarily coincide with the obligations of the utilities to serve customer demands.

- The fulfillment of NUG development plans to support the future generation requirements of the system are not controlled by the utilities, which are obligated to provide sufficient capacity.

The investor-owned utility obligation to serve is part of what was once called the "regulatory compact," which tied the utility exclusivity to a service territory franchise and requires that the rate of return and prices be set by a regulatory body. However, the obligation of NUGs to provide power tends to be contractual. This contractual obligation (power sales contract) must be satisfied to provide an adequate return on investment and to service debt. 
The perspectives of some utility industry organizations and NUG participants on these obligations may differ. Some of these differences can be attributed to positions of the organizations in the market, with respect to their cost structures and existing capacity. Some utilities welcome the opportunity for potential cost savings and diversity of supply options offered by NUGs, others are more concerned about operations and overall system reliability.

In response to such concerns, the North American Electric Reliability Council (NERC), which the utility industry charged to oversee the reliability of the bulk electric supply, has established guidelines on the minimum operating considerations that all utility and nonutility generators must follow to ensure the continued reliability of the system. ${ }^{12}$

\section{Impact of Nonutillty Generation on the Supply System}

The electric utility industry and nonutility industry have worked together to safely and reliably interconnect NUGs. Many utilities are increasingly relying on NUG power as an important source of power. Unanswered questions remain, however, regarding the degree to which increasing NUG capacity can be accommodated in transmission system operations and if the reliability of the electrical supply system will be compromised.

Several factors which utilities may not control can influence the operation of the overall system. The overall level of increase in NUG capacity is just one element. The size of individual facilities has a direct bearing on the potential system impacts; small facilities are less likely to have the same impacts as large ones. At the same time, the locations of individual projects, even small ones, can be critical. Where a facility is sited can affect transmission line loadings and substation equipment operation. Similarly, the timing of power production from a NUG facility can affect the balance of power flows on the system. Moreover, the availability and reliability of NUG power can influence the operation of the system and the requirements for reserve capacity.

The electrical supply system is operated within closely watched tolerances and can require complex and realtime balancing of generation and transmission facilities with fluctuating demand. The substantial interrelationships of all the system components--utility and nonutility--suggest that generation capacity that falls outside the direct control of system operators increases their operational and planning challenges, and may affect system reliability. The extent to which operation, size, location, timing, availability and reliability of NUG power production can be coordinated with system operators will determine the impacts of NUG integration on the bulk electricity supply system.

Technically, all of these factors exert both positive and negative influences on the electrical system, depending on site-specific conditions and timing of actions. For example, a NUG facility could be located specifically to help a utility avoid a transmission or distribution bottleneck. Proper integration of NUGs into the daily operational control and management of the electrical power grid is critical for capturing the benefits and minimizing the disadvantages for all entities connected to the grid.

\footnotetext{
${ }^{12}$ North American Electric Reliability Council, Integrating Nonutility Generators (Princeton, New Jersey, January 1992). The specific guidelines now address both planning a ' operating considerations, and apply to all utility and nonutility sources. The guidelines address a range of needs, from specific design issues, to information needs, and data exchange requirements. The guidelines also cover how the generation sources would be brought on- and off-line during routine and emergency conditions.
} 
Appendix D

Technical Notes 


\section{Technical Notes}

\section{Sources of Data}

The data sources used to produce this publication include selected items from the following Federal Energy Regulatory Commission (FERC), Energy Information Administration (EIA), and Rural Electrification Administration (REA) annual data collection forms:

- Form EIA-412, “Annual Report of Public Electric Utilities"

- Form EIA-861, "Annual Electric Utility Report"

- Form FE-781R, "Annual Report of International Electrical Export/Import Data"

- FERC Form 1, "Annual Report of Major Electric Utilities, Licensees and Others"

- FERC Form 1-F, “Annual Report of Nonmajor Public Utilities and Licensees"

- REA Form 7, "Financial and Statistical Reports - Electric Distribution Borrowers"

- REA Form 12a through 12i, "Electric Power Supply Borrowers," and REA Form 12c through 12g, "Electric Distribution Borrowers with Generating Facilities"

Completion of these forms by electric utilities is mandatory. Individual forms are completed by all U.S. utilities meeting specific ownership and size criteria. Sampling methods are not used as part of these surveys except for minimum threshold reporting; all qualifying utilities (that is, all utilities satisfying reporting criteria) are required to report annually.

Other 1992 data collected on these forms are also published by EIA and REA in the following reports: $\mathrm{Fi}$ nancial Statistics of Selected Investor-Owned Electric Utilities 1992 (DOE/EIA-0437)/1, Financial Statistics of Selected Publicly Owned Electric Utilities 1992 (DOE/EIA-0437)/2, and Annual Report of Energy Purchased by REA Borrowers (REA).

\section{Form EIA-412, "Annual Report of Publlc Electric Utilitles"}

\author{
Summary of Primary Purpose.
}

Form EIA-412 is used to collect detailed accounting, financial, and operating data annually from publicly owned electric utilities in the United States. For the fiscal or calendar year that ended December 31, 1992, new criteria were used to select the 492 reporting respondents for this form. Publicly owned electric utilities engaged in the generation, transmission, or distribution of electricity that had 120 million kilowatthours of sales to ultimate consumers or 120 million kilowatthours of sales for resale for the 2 previous years as reported on Form EIA-861, "Annual Electric Utility Report," must submit Form EIA-412.

\section{Instrument and Design History.}

Federal Power Commission (FPC) Form 1M was implemented as a mandatory survey by the FPC in 1961 . It became the responsibility of the EIA in October 1977 when the FPC was merged into the DOE. Effective January 1980, the FPC Form $1 \mathrm{M}$ was superseded by Form EIA-412, also a mandatory survey, used to collect the same data as those previously reported on FPC Form 1M.

\section{Data Processing}

The processing of data reported on FERC Form 1, Form EIA-412, and Form EIA-861 is the responsibility of the Data Systems Branch, Survey Management Division of the Office of Coal, Nuclear, Electric and Alternate Fuels. The normal processing of FERC Form 1-F is the responsibility of the FERC. Automated systems are used to edit data from the surveys, using deterministic and statistical checks. When these data have passed the editing process, they are aggregated into a master file, which is used as input to this publication's data base. 


\section{Form ElA-861, "Annual Electric UtIIty Report"}

Summary of Primary Purpose.

Form EIA-861 is a census of electric utilities in the United States. The mandatory survey is used to collect information on power production and sales data from approximately 3,232 electric utilities. The data collected are used to maintain and update the EIA electric utility frame data base. This data base supports queries from the Executive Branch, Congress, other public agencies, and the general public. Summary data from Form EIA-861 are also contained in the Electric Power Monthly, the Electric Sales and Revenues 1992, the Financial Statistics of Selected Investor-Owned Electric Utilities 1992, and the Financial Statistics of Selected Publicly Owned Electric Utilities 1992. These reports present totals for electric utilities on a national level, by State, and by ownership type.

\section{Instrument and Design History.}

Form EIA-861 was implemented in January 1985 to collect data as of the end of 1984. The Federal Energy Administration Act of 1974 (Public Law 93-275) defines the legislative authority to collect these data. Detailed comparisons and descriptions of conceptual problems affecting the quality of the data are discussed in the report, An Assessment of the Quality of Selected EIA Data Series: Electric Power Data .

\section{Form FE-781R, "Annual Report of International Electrical Export/Import Data"}

Summary of Primary Purpose.

Form FE-781R is used to collect on an annual basis, monthly international electricity trade information on the gross amounts of electrical energy imports and exports and the costs and revenues associated with these transactions. The use of the format contained in Form FE-781R is optional for reporting purposes; however, submission of the data is mandatory

\section{Instrument and Design History.}

The authority to issue presidential permits pursuant to Executive Order Number 10485 was transferred to the Secretary of Energy by Executive Order Number 12038 (43 FR 4957 February 7, 1987). This responsibility was delegated by the Secretary to the Economic Regulatory Administration (DOE Delegation Order Number 0204-04, October 1, 1977). The authority was redelegated (DOE Delegation Order Number 127) to the Office of Fuels Programs, the Office of Fossil Energy (54 FR 11436 March 20, 1989). The survey uni- verse is defined under Title 10 of the Code of Federal Regulations, Sections 205.308 and 205.325 to include all public utilities or other entities subject to the Department of Energy jurisdiction under Part II of the Federal Power Act, engaged in the export of electrical energy across the international borders of the United States with Canada and Mexico, or, who own the physical facilities which are used to transmit power across the international border.

\section{Data Processing.}

Form FE-781R is mailed to the respondents to collect annually the monthly data for the preceding calendar year. The completed forms are to be returned to the DOE by February 15 . The receipts are manually edited and the data used for the Presidential Permit Program are entered into a machine readable format.

\section{FERC Form 1, "Annual Report of Major Electric Utilities, Licensees and Others"}

\author{
Summary of Primary Purpose.
}

The Federal Energy Regulatory Commission (FERC) Form 1 is used to collect detailed accounting, financial, and operating data annually from "major" investorowned electric utilities having, in each of the last 3 consecutive years, sales or transmission service that exceeds any one of the following: 1 billion kilowatthours of total sales, 100 thousand kilowatthours of sales for resale, 500 thousand kilowatthours of gross interchange out, or 500 thousand kilowatthours of wheeling for others (deliveries plus losses). The FERC Form 1-F is used to collect detailed accounting, financial, and operating data annually from "minor" investor-owned electric utilities that fall below the major category. For the year 1992, jurisdictional filings of the FERC Form 1 and 1-F totaled 224; though not all can be considered traditional electric utilities. See Table B1 (Appendix B) for those that qualified in 1992.

\section{Instrument and Design History.}

FERC Form 1 was implemented in 1935 by the Federal Power Commission (FPC). In October 1977, it became the responsibility of the EIA to publish energy data when the FPC was merged into the Department of Energy (DOE). This mandatory report is prepared in accordance with the FERC's Uniform System of Accounts for private utilities and licensees. In 1990, the wholesale trade schedules were modified. The Summary of Interchange schedule was absorbed by the Purchased Power Schedule and the Transmission For and By Others schedule was changed from a narrative format into two separate tabular collection schedules. In addition to these schedule changes, the statistical 
classifications covering the data filed on these schedules were adjusted.

\section{Uniformity of Treatment.}

The uniformity of treatment accorded the utilities' statements in this report will generally permit ready comparison of items among the utilities included in the compilations. These statements were filed by all "major" investor-owned electric utilities subject to the FERC accounting jurisdiction.

The Uniform System of Accounts prescribed for use by electric utilities under the FERC's jurisdiction was prepared in cooperation with the National Association of Regulatory Utility Commissioners. ${ }^{13}$ In all material respects, the Uniform System of Accounts conforms with the Association's approved system adopted by certain State commissions. The "major" investorowned electric utilities are required to follow the Uniform System of Accounts. In the presentation of individual utility data, the utilities are grouped according to the survey form and the class of ownership to which the utility belongs. The 492 publicly owned electric utilities that filed the EIA-412 in 1992 are requested to follow the Uniform System of Accounts when making their filings.

No attempt has been made to consolidate (in the technical accounting sense) the accounts of the utilities included in the summary statement of this report. The combined totals represent the arithmetic sum of accounts as reported by the individual utilities. Consequently, duplications exist to a limited extent in the composite totals shown in several statements. Therefore, as a result of mergers, consolidations, and sales of properties to public authorities, as well as the introduction of new utilities, the dollar and quantity figures presented in the report will not be representative of exactly the same properties from year to year.

\section{FERC Form 1-F, "Annual Report of Nonmajor Public Utilities and Licensees"}

\section{Summary of Primary Purpose.}

FERC Form 1-F is used to collect limited accounting, financial, and operating data annually from investorowned electric utilities that are not classified as "major," and had total sales in each of the last 3 consecutive years of 10 million kilowatthours or more.

Instrument and Design History.
FERC Form 1-F was implemented by the FPC regulations under the authority of the Federal Power Act as FPC Form 1-F for the year 1961 and thereafter. In October 1977, the FPC was merged into the DOE. This mandatory report is prepared in accordance with the FERC's Uniform System of Accounts for Private Utilities and Licensees.

\section{Publication--FERC Form 1 and Form EIA-412}

Data on plant construction cost, production expenses, generating equipment characteristics, and other relevant operating data are are no longer published.

Financial data can be found in the Financial Statistics of Selected Investor-Owned Electric Utilities 1992 and Financial Statistics of Selected Publicly Owned Electric Utilities 1992. These data include the following: income and earnings, taxes, depreciation and amortization, distribution of salaries and wages, electric utility operating revenues, electric utility operation and maintenance expenses, and generating plant statistics. Planned construction data, year-end balance sheets, and general corporate information are also included.

\section{Information Collected on FERC Form 1, Form EIA-412, and Form EIA-861}

Reported data selected from FERC Form 1 include sales for resale, purchased power, exchanges, and wheeling, while the reported data selected from Form EIA-412 include only sales for resale and purchased power.

"Electric Energy Account Summary data" for the municipals, cooperatives, Federal power marketing administrations, and the Tennessee Valley Authority were summarized from Form EIA-861. Form EIA-861 is used to collect information for EIA on the statistics of electric utilities and their generation, transmission and distribution of electric energy in the United States, its territories, or Puerto Rico. Form EIA-861 is mandatory under Public Law 93-275, the Federal Energy Administration Act of 1974.

\section{Rural Electrification Administration Cooperative Borrowers}

In an effort to broaden the coverage in this report of the electric utility industry, information has been included from the Cooperative Borrowers of the Rural Electrification Administration (REA). These data are

\footnotetext{
${ }^{13}$ The National Association of Regulatory Utility Commissioners includes members of the railroad, public utilities, and public service commissions of the 50 States, the District of Columbia, the Commonwealth of Puerto Rico, and the territory of the Virgin Islands. The following Federal regulatory agencies also were included: the Interstate Commerce Commission, the Federal Energy Regulatory Commission, the Federal Communication Commission, and the Securities and Exchange Commission.
} 
based on financial and statistical information submitted to the REA in 1992.

The operating data collected by the REA furnish information needed for checking the security of government loans, the preparation of estimates used in forecasting expenses for future loans, and allow the REA to fulfill its reporting obligations. The reports prepared by the cooperative borrowers must accurately reflect the financial data as shown by their books of account.

\section{Confidentiality of the Data.}

The data collected on the forms used for input to this report are not confidential.

\section{Quality of Data}

The Office of Coal, Nuclear, Electric and Alternate Fuels is responsible for routine data improvements and quality-assurance activities in accordance with standards established by EIA. These standards are the measuring rod necessary for quality statistics. Dataimprovement efforts include follow-up on nonrespondents, verification of data-keyed input by automatic computerized methods, and editing by subject-matter specialists.

Completed forms received by the Office of Coal, $\mathrm{Nu}$ clear, Electric and Alternate Fuels are sorted, screened for completion of reported information, and keyed onto computer tapes for storage and transfer to data bases on random access storage devices for computer processing. The information coded on the computer tapes is spot-checked against the data reported on the forms to certify accuracy of the tapes. Computerized respondent data files are checked to identify those who fail to respond to the survey. By law, nonrespondents may be fined or otherwise penalized for not filing an EIA data form as prescribed in the instructions. Before invoking the law, EIA tries to obtain the required information by encouraging cooperation of nonrespondents.

The individual utility identification codes for new and existing entities are edited prior to being sent for data entry. Additional edit checks of these data are performed through computer programs. The program edits include both deterministic checks, in which records are checked for the presence of data in required fields, and statistical checks, in which the data are checked against a range of values based on historical data values and for logical or mathematical consistency with data elements reported in the form. Discrepancies arising as a result of these checks are resolved either by the processing office or by a telephone call to the company concerned.

\section{Data Valldation Procedures}

The methodology used to develop this report is based on procedures and techniques routinely employed in data requirement reviews and data validation projects conducted by and for EIA. The four-step process used consists of: (1) identifying patterns of respondent misreporting and inconsistencies in reporting between respondents; (2) analyzing each problem identified to develop probable causes; (3) correcting the identified problems; and (4) modifying the forms and/or instructions to eliminate or minimize future occurrences. The objective of the process is to make improvements that will:

- Improve the readability, accuracy, and meaningfulness of the data collected on wholesale electricity trade

- Reduce respondent burden by means of clarifying instructions, simplifying form layout, and eliminating unnecessary calculations

- Effect data standardization among the various forms used to collect wholesale electricity trade data

- Facilitate the computerization of wholesale electricity trade data to be collected in the future.

A variety of methods were used to identify reporting problems including:

- Manual pre-edit of FERC Form 1 and Form EIA -412 to identify nonstandard responses that could not be computerized as submitted by the respondent

- Manual review of selected forms to characterize patterns of reporting and misreporting and the type of respondents responsible

- Automated statistical reports (such as frequency counts) summarizing respondent reporting regarding individual data items

- Automated interform data validation to identify inconsistencies between aggregate level data reported by a respondent on Form EIA-861 and FERC Form 1

- Automated intraform data validation to identify reporting inconsistencies (such as, total does not equal the sum of the components)

- Automated interform data validation to identify inconsistencies between respondents reporting the same transaction (such as, the buyer and the seller of power reporting different quantities of energy delivered and received)

- Follow-up telephone calls are made to respondents (excluding those who filed FERC Forms) regarding confusing and/or inaccurate entries. Questions on the accuracy of filings made to the FERC are handled at the discretion of FERC staff. 
The causes of misreporting and inconsistent reporting were identified principally by studying in detail the form layouts and instructions, and identifying patterns and trends in misreporting among all respondents. Considerable judgment was necessary in developing probable causes since all respondents could not be systematically contacted regarding errors. As an alternative, explanatory footnotes found in the completed forms, supplementary pages, and manual checks were used to confirm and validate the data. Once probable causes were identified, modification of the forms was straightforward.

\section{Data Edits}

The data used for this publication are limited to those reported by respondent utilities. The following edit checks were used to identify missing or inaccurate data from the information that was initially reported by the respondent utilities.

- Energy--Sales for Resale and Purchased Power

- No single transaction could exceed 4,380 million kilowatthours.

- If sales or purchase transactions were reported, an energy receipt or delivery was required.

- Reported totals or subtotals had to equal the sum of energy transactions.

- Energy--Interchanges (Exchanges)

- No single transaction could exceed 4,380 million kilowatthours.

- If interchange transactions were reported, an energy receipt or delivery was required.

- Net energy received for a transaction had to equal total energy received minus energy delivered.

- Reported totals or subtotals had to equal the sum of energy transactions.

- Revenue--Sales for Resale

- Total revenue for a transaction had to equal the sum of reported revenue-demand, revenueenergy, and revenue-other.

- Total revenue for a transaction divided by the kilowatthours sold for resale had to be in the range of 0.5 to 20 cents per kilowatthour.

- Reported revenue totals or subtotals had to equal the sum of revenue transactions.

- Cost--Purchased Power

- Total cost for a transaction had to equal the sum of demand, energy, and other costs.

- Total cost of a transaction divided by kilowatthours purchased had to be in the range of 0.5 to 20 cents per kilowatthour.
- Reported cost totals or subtotals had to equal the sum of cost transactions.

- Settlement--Interchanged Energy (Exchanges)

- For transactions involving monetary settlement, the settlement amount divided by net kilowatthours received or delivered was required to be in the range of 0.5 to 20 cents per kilowatthour.

- For settlement transactions involving monetary settlement, settlement amount was required to be positive if more energy was received than delivered; negative if more energy was delivered than received.

- Reported totals and subtotals had to equal the sum of settlement transactions.

\section{Adjustments}

Since the Federal forms used to collect electricity trade data are essentially accounting forms, adjustment data are routinely provided so that submitted data will be consistent with the accounting systems of the utilities. Many respondents include adjustments, particularly those related to transactions occurring in the reporting year, as a component of the related transaction. However, adjustments to transactions occurring in previous years are aggregated with current year transactions or are listed separately (that is, rebates, overcharges, reclassifications, fuel cost adjustments, etc.).

\section{Sales for Resale and Purchased Power}

Sales for resale data are collected on FERC Form 1, Form EIA-412, REA Form 7, and REA Form 12. The primary sources of this information are FERC Form 1 and a few of the Form EIA-412's completed by Federal and State agencies. These respondents account for most of the wholesale electricity trade in the United States. The detailed data on sales for resale are not keyed into a machine-readable format by the REA; therefore computer matching was not done.

Since sales for resale on Form EIA-412 is on a fiscalyear basis as opposed to calendar year, the Form EIA-412 data are not consistent with data collected on the other wholesale electricity trade schedules. Also wholesale electricity trade sales cannot be aggregated for all Form EIA-412 respondents because the respondents use different fiscal years. This is also true for the purchased power data. In 1989, the statistical classifications of the Form EIA-412 were modified to gather information on the types of electrical power and energy purchased and sold. The 1990 data reflect the collection of data using the enlarged statistical classification groupings. 
The REA Form 12, unlike FERC Form 1 and Form EIA-412, is not used to collect sales according to statistical classification (that is, firm, durnp, and other power). The REA does not process the sales for resale data reported on the REA Forms 7 and 12. As such, crosschecking between buyers and sellers is less comprehensive. It also should be noted that Form EIA-412 was used to collect statistical classification data for both sales and purchased energy in 1992, while the REA did not collect statistical classification data for either.

The purchased power schedules on Form EIA-412 and REA Forms 7 and 12 does not include interchange (exchange) transactions. Form EIA-412 respondents are asked to report exchanges on a gross basis on the Energy Account Balance schedule. The REA Forms 7 and 12 do not specifically address interchange (exchanges) transactions, but respondents are to report transactions on a net basis, if there is a cash payment.

\section{Interchange (Exchange)}

Both purchase and interchange costs fall under the Uniform System of Accounts No. 555, and were reported on two different schedules until 1990. In 1990, the Summary of Interchange Schedule was deleted from the FERC Form 1 and the information collected on that schedule were shifted to the Purchased Power Schedule. The FERC has determined, for their regulatory accounting purposes, that the term, "interchange," means energy that is only exchanged in kind. Historically, however, "interchange" has been used by the industry to mean all energy transfers between utilities (sales, purchases, exchanges, and wheeling) and it has resulted in inconsistent reporting.

\section{Transmission For Others and By Others (Wheelling)}

Detailed data on the Transmission For or By Others are collected only on FERC Form 1. Before 1990, respondents provided in a narrative format six basic elements of information for each wheeling transaction:

- Name of company and description of service

- Points of origin and termination of service

- Megawatthours received and delivered

- Monetary settlement

- Nonmonetary settlement

- Other explanations needed to describe the transactions.

The FERC Form 1 was modified for the 1990 collection years. The narrative schedule was changed to two separate tabular formats. The Transmission For Others schedule now collects additional information on: payments made by, energy received from; energy deliv- ered to; statistical classification; FERC rate schedule; disaggregation of monetary settlement into demand, energy, other, and total; and points of origin, termination of service, and nonmonetary settlement were all deleted. Transmission By Others now collects information on name of company; megawatthours received and delivered; and expenses for transmission of electricity by others (e.g., demand, energy, other, and total).

\section{Difference between Publlcation Mothodologles}

The Federal Energy Regulatory Commission modified the FERC Form 1 in 1990 in order to handle better their responsibilities under the Federal Power Act. New information was requested that included statistical classification categories which collected data on power and energy transactions based on the original contractual terms and conditions. Because of this new information, the firm, nonfirm, and miscellaneous publication categories used in prior years were adjusted to handle these new classifications.

In prior publications, firm trade was defined as any transaction in which the charges had to include a demand charge. Firm trade data were then separated into two groups. The first group included transactions with demand charges where no quantities of energy were delivered or received (possible under "take or pay contracts"), and the second included quantities of energy that were traded with an "Energy," and sometimes, "Other" charge. (The "Other" charge in the firm category covers associated payments made on utility transactions for expenses associated with operating and/or renting physical facilities such as transmission lines and substations).

The differences between nonfirm electricity trade and the firm categories is that no demand charges are involved in the nonfirm transactions. The energy charge plus any "Other" charges associated with those transactions represented the value of these nonfirm transactions. The last grouping, "Other," includes transactions which did not include a demand or energy charge. (The filing electric utilities usually did not provide descriptive explanations.) However, from the footnotes provided and the account descriptions, data in this category do include: purchasing or maintaining operating reserve expenses; stability and reliability standards expenses; transmission line and other facility rentals; and other system operating charges. ${ }^{14}$

For this publication, the new FERC classifications were used to define differently the firm, economy, and other electricity trade categories. Form redesigns implemented in reporting year 1990 for both FERC Form 1 and Form EIA-412 permitted respondent reported data to be used to differentiate between firm and nonfirm power trades. 
The following list shows the type-of-trade categories reported by the FERC Form 1 and the Form EIA-412 respondents.

\section{- FERC Form 1}

- Requirements (RQ)

- Long-Term (LF)

- Intermediate Firm (IF)

- Short-Term Firm (SF)

- Long-Term

- Intermediate Firm (IF)

- Short-Term Firm (SF)

- Long-Term Unit (LU)

- Intermediate Unit (IU)

- Exchange Power (EX)

- Other Service (OS)

- Adjustments (AD)

- Form EIA-412

- Firm Power (FP)

- Unit Power (UP)

- Economy Power (EP)

- Dump Power (DP)

- Maintenance/Emergency Power (ME)

- Operating Reserve (OR)

- Other Capacity/Energy (OT).

Appendix A provides the FERC Form 1 definitions found on pages 310 and 326. Form EIA-412 definitions are found on Schedules V and VII of the form.

Like previous years, 1992 transactions without energy receipts or deliveries but with demand charges are classified as firm, "demand charge only" trade. FERC Form 1 and Form EIA-412 respondents do not always report type of trade data, which results in missing data. Likewise, data is missing for cooperatives, since they are not required to report the type-of-trade on the $\mathrm{Ru}$ ral Electrification Administration (REA) Forms REA-7 and REA-12. Imputations for missing typeof-trade data are performed according to the following procedures:

- Sales for Resale. If the purchasing utility reported no net generation on the Form EIA-861, "Annual Electric Utility Report," for the reporting year 1990 , then the trade is classified as firm. Otherwise the trade is classified as economy.
- Purchased Power. If the respondent utility (the purchasing utility) reported no net generation on the 1990 Form EIA-861, then the trade is classified as firm. Otherwise the trade is classified as economy.

\section{Data Rovisions}

The foregoing edits identified data errors. Data inaccuracies resulting from transcription and typographical errors, reporting in the wrong units (for example, kilowatthours instead of megawatthours) and arithmetic errors were corrected after discussions with respondents. Many suspected data errors were found to be correct as reported. Explanations provided by respondents included:

- The transaction was an energy or monetary adjustment to information previously reported. Adjustments reflected settlement of contract disputes, reimbursement of overcharges, or accounting errors and often were one-time bookkeeping corrections.

- The transaction involved large demand charges, but a small amount of energy was delivered.

- The transaction was a nonstandard operating condition such as loop and inadvertent energy flow, emergency power deliveries, or sale of energy under a purchase contract.

- The transaction was for access rights or the lease of equipment used in the sale or purchase of energy.

- The transaction was between two associated utilities or a utility and a power pool reporting internal energy, revenue, or cost accounting practices (for example, allocations).

\section{Consistency Checks}

In addition to edit checks, data consistency checks were performed to improve the quality of the data presented in this publication. The consistency checks involved comparing, when possible, Sales for Resale data (revenue and energy) reported by one respondent with the purchased power data (cost and energy) reported by the respective buyers of the energy. Discrepancies of more than 10 percent were discussed with both the selling and the buying utilities to determine if a reporting error had been made. A similar procedure was used to confirm data inconsistencies between utilities that interchanged energy.

\footnotetext{
${ }^{14}$ FERC Forr 1 provides summary totals for electric operation and maintenance expenses. Pages 320-323 of FERC Form 1, under the schedule "Electric Operation and Maintenance Expenses," include information on power production, transmission, and distribution expenses (Uniform System of Accounts 500 to 935). These summary data are reported in the EIA publication, Financial Statistics of Selected Investor-Owned Electric Utilities, DOE/EIA-0437(90). This information cannot be linked to the individual transactions.
} 
Consistency checks identified data errors, but discussions with respondents determined that most of the data were correct as reported. Reasons provided included:

- A sale or purchase by one utility was considered an interchange by the other utility in the transaction.

- Sales, purchases, and interchanges for noninvestor-owned utilities transacted late in the year can be reported in the year in which the transaction occurred or in the following year in which they are booked.

- Data submitted by a utility can cover different reporting periods depending upon the requirements of the forms; Form EIA-412 is a report of the utility's fiscal year while the other forms are for a calendar year.

- Different accounting and reporting procedures are used for recording loop and inadvertent energy flow, equipment and lease charges, service fees, fuel and other adjustments, and corrections to previously reported information.

\section{Corrections}

Corrections were made to the data when missing or inaccurate data were provided by the respondent. Missing and inaccurate information identified during data automation and report preparation were discussed with respondents during the edit process either to correct the data supplied, or to confirm the values. In all cases, the forms were reviewed during the edit process with the respondent, and changes were made at the direction of the respondent.

\section{Explanatory Notes}

Data are reported on FERC Form 1 in accordance with the FERC's Uniform System of Accounts. Data are reported on the REA Forms 7 and 12 in accordance with the REA Uniform System of Accounts (based on the FERC System, with minor modifications). Copies of the Uniform System of Accounts Prescribed for Both Private and Cooperative Borrowing Electric Utilities are available from the U.S. Government Printing Office (GPO).

\section{General Information}

\section{Use of the Glossary}

The terms in the glossary have been defined for general use. Restrictions on the definitions, as used in these data collection systems, are included in each definition when necessary to define the terms as they are used in this report.

\section{Obtaining Coples of the Data}

This publication is available on a subscription basis from the Superintendent of Documents, U.S. Government Printing Office (GPO). Make check or money order payable to the Superintendent of Documents. You may send your order to the GPO or the National Technical Information Center (NTIC). GPO and NTIC prices are subject to change without advance notice.

Superintendent of Documents

U.S. Government Printing Office

Washington, DC 20402

8 a.m. to 4 p.m. eastern time, M-F

(202) 783-3238

FAX (202) 512-2233

National Technical Information Service (NTIS)

Office of Data Base Services

U.S. Department of Commerce

5285 Port Royal Road

Springfield, VA 22161

(703) $487-4650$ 


\section{Glossary}

Accounting System: A system of recording accounting data for a utility or company; or of supplying accounting information for controlling, evaluating, planning, and decision-making.

Administrative and General Expenses: Expenses of an electric utility relating to the overall directions of its corporate offices and administrative affairs, as contrasted with expenses incurred for specialized functions. Examples include office salaries, office supplies, advertising, and other general expenses.

Ampere: The unit of measurement of electrical current produced in a circuit by 1 volt acting through a resistance of $1 \mathrm{ohm}$.

Associated Company (Affliated): A company that is either directly or indirectly controlled by or owned by another firm or holding company.

Automatic Generating Control: The regulation of the power output of electric generators within a prescribed control area in response to changes in system frequency. The line loading or the relation of these to each other, so as to maintain the scheduled system frequency and/or establish interchange with other areas within predetermined limits.

Avallable but not Needed Capability: Net capability of main generating units that are operable but not considered necessary to carry load, and cannot be connected to load within 30 minutes.

Average Water Conditlons: The amount and distribution of precipitation within a drainage basin, and the run-off conditions present, as determined by reviewing the area water supply records over a long period of time.
Backup Power: Electric energy supplied by a utility to replace power and energy lost during an unsched. uled equipment outage.

Base Bill: A charge calculated by taking the rate from the appropriate electric rate schedule and applying it to the level of consumption.

Base Rate: A fixed amount charged each month for any of the classes of utility service provided to a customer.

Baseload: The minimum amount of electric power delivered or required over a given period of time at a steady state.

Baseload Capacity: The generating equipment normally operated to serve loads on a round-the-clock basis.

Baseload Plant: A plant, usually housing highefficiency steam-electric units, which is normally operated to take all or part of the minimum load of a system, and which consequently produces electricity at an essentially constant rate and runs continuously. These units are operated to maximize system mechanical and thermal efficiency and minimize system operating costs.

Black Start Capability: The ability of a generating unit or station to go from a shutdown condition to an operating condition and start delivering power without assistance from the power system.

Block Rate Schedule: An electric rate schedule with a provision for charging a different unit cost for various increasing blocks of demand or energy. A reduced price is charged on succeeding blocks. 
Boller: A device for generating steam for power, processing, or heating purposes; or hot water for heating purposes or hot water supply. Heat from an external combustion source is transmitted to a fluid contained within the tubes found in the boiler shell. This fluid is delivered to an end-use at a desired pressure, temperature and quality.

Boiler Fuel: An energy source to produce heat that is transferred to the boiler vessel in order to generate steam or hot water. Fossil fuel is the primary energy source used to produce heat for boilers.

Borderline Customer: A customer located in the service area of one utility, but supplied by a neighboring utility through an arrangement between the utilities.

Btu (British thermal unit): A standard unit for measuring the quantity of heat energy equal to the quantity of heat required to raise the temperature of 1 pound of water by 1 degree Fahrenheit.

Bulk Power System: The interconnected electrical systems comprising generation and transmission facilities on which faults or disturbances can have a significant effect outside the local area.

Bulk Power Transactions: The wholesale sale, purchase, exchange of electricity, and/or transmission services (wheeling) among electric utilities. Bulk power transactions are used by electric utilities for many different aspects of electric utility operations, from maintaining load to reducing costs.

Bus: An electrical conductor which serves as a common connection for two or more electrical circuits.

Capability: The maximum load that a generating unit, generating station, or other electrical apparatus can carry under specified conditions for a given period of time without exceeding approved limits of temperature and stress.

Capability Margin: The difference between net system capability and system peak load. Nationally, it is the difference between aggregate net system capability and the sum of system peak loads without allowance for time diversity between the loads of the individual systems. Regional computations take into account the diversity between peak loads of the systems that co- ordinate operations. This margin indicates the capability available to provide for scheduled maintenance, emergency outages, system operating requirements, and unforeseen loads.

Capability, Emergency Transfer: The amount of power transfer allowed between areas or within an area when operating to meet NERC emergency criteria contingencies.

Capability, Normal Transfer: The amount of power transfer allowed between areas or within an area when operating to meet NERC normal criteria contingencies.

Capacity: The full-load continuous rating of a generator, prime mover, or other electric equipment under specified conditions as designated by the manufacturer. It is usually indicated on a nameplate attached to the equipment as the full-load rating.

Capacity (Nameplate): The amount of electric power delivered or required for which a generator, turbine, transformer, transmission circuit, station, or system is rated by the manufacturer.

Capacity Charge: An element in a two-part pricing method used in capacity transactions (energy charge is the other element). The capacity (demand) charge is assessed on the amount of capacity being purchased.

Capacity Factor: The ratio of the average load on the generating unit, generating plant, or other electrical apparatus during a specified period of time during its operations.

Capacity Transaction: The acquisition of a specified quantity of generating capacity from another utility for a specified period of time. The utility selling the capacity is obligated to make available to the buyer a specified quantity.

Certificate: A type of permit for public convenience and necessity issued by a utility commission, which authorizes a utility or regulated company to engage in business, construct facilities, provide some services, or abandon service. 
Circuit: A conductor or a system of conductors through which electric current flows.

Circuit-Mile: The total length in miles of separate circuits regardless of the number of conductors used per circuit.

Classes of Service: Customers grouped by similar characteristics in order to be identified for the purpose of setting a common rate for electric service. Usually classified into groups identified as residential, commercial, industrial, governmental, or other similar groupings within each major classification.

Code of Federal Regulations: A compilation of the general and permanent rules of the executive departments and agencies of the Federal Government as published in the Federal Register. The Code is divided into 50 titles that represent broad areas subject to Federal regulation. Title 18 contains the FERC's regulations.

Cogeneration: The sequential or simultaneous process in which useful heat/steam is generated, used in a variety of process applications, and then directed into a turbine to generate electricity and/or mechanical work from the useful thermal energy still available for use.

Cogenerator: A generating facility that produces electricity and another form of useful thermal energy (such as heat or steam), used for industrial, commercial, heating, or cooling purposes.

Combined Cycle: A cogeneration technology in which additional electricity is produced sequentially from the otherwise lost waste heat exiting from one or more gas-fired turbines. The exiting heat flow is routed to an exhaust-fired conventional boiler or to a heat recovery steam generator for utilization by a steam turbine in the production of electricity. This process increases the efficiency of an electric generating system by turning the rejected heat into thermal steam rather than discharging it into the atmosphere.

Commercial and Industrial: Classes of service supplied to a similar grouping of customers. These customer groupings are usually subdivided into smaller segments by classifying such customers as commercial or industrial using the Federal Gov srnment's Office Of Management and Budget's Standard Industrial Classifica- tion Guide and/or a scale of energy usage as yardsticks; other customers are reclassified as commercial or industrial when their demands or annual use exceeds some specified limit. These limits are generally based on a utility's rate schedules, except for those customers who are supplied under special contracts or agreements calling for particular services.

Commingling: The mixing of one utility's generated supply of electric energy with another utility's generated supply within a transmission system.

Commission: An agency of government usually composed of three or more members charged with specific duties, functions, and responsibilities.

Conductor: Metal wires, cables, and bus-bar used for carrying electric current. Conductors may be solid or stranded, that is, built up by an assembly of smaller solid conductors.

Connected Load: The sum of the continuous ratings or the capacities for a system, part of a system, or a customer's electric power consuming apparatus.

Contingency: The unexpected event, usually the loss of one or more segments in the interconnected electrical power system.

Cooperative (electric utility): An electric utility legally established to be owned by and operated for the benefit of those using its services. The utility company will generate, transmit, and/or distribute supplies of electric energy to a specified area not being serviced by another utility. Such ventures are generally exempt from the Federal income tax laws. Most cooperatives have initially been financed by the U.S. Department of Agriculture's Rural Electrification Administration.

Coordination Service: Coordination service generally involves the sale, exchange, or transmission of electricity between two or more electric utilities that typically have sufficient generation and transmission capacity to supply their load requirements under normal conditions.

Coordination Service Pricing: The typical price components of a bulk power coordination sale are an energy charge, a capacity, or reservation charge, and an adder. The price for a particular sale may embody 
some or all of these components. The energy charge is made on a per-kilowatthour basis and is intended to recover the seller's system incremental variable costs of making a sale. Since the nonfuel expenses are usualiy hard to quantify and small relative to fuel expense, energy charges quoted are usually based on fuel cost. A capacity charge is set at a certain level per kilowatt and is normally paid whether or not energy is taken by the buyer. An adder is added to the energy charge to recover the hard-to-quantify nonfuel variable costs. There are three types of adders: percentage, fixed, and split-savings. A percentage adder increases the energy charge by a certain percentage. A fixed adder, adds a fixed amount per kilowatt-hour to the energy charge. Split-savings adders are used only in economy energy transactions. They split production cost savings between the seller and the buyer by adding one-half of the savings to the energy cost.

Cost: The amount paid to acquire resources such as plant and equipment, fuel, or labor services. Fixed costs in the electric utility industry are associated with resources that cannot be changed easily during a short time span (such as plant and equipment) and are independent of the level of generation. Variable costs are associated with resources that can vary during a given time period (such as fuel or labor services) and are directly related to the level of generation.

Cost of Service: A ratemaking concept used for the design and development of rate schedules to ensure that the filed rate schedules recover only the cost of providing the electric service at issue. This concept attempts to correlate the utility's costs and revenue with the service provided to each of the various customer classes.

Current: A flow of electrons in an electrical conductor. The strength or rate of movement of the electricity is measured in amperes.

Deliveries (Electric): Energy generated by one system and delivered to another system through one or more transmission lines.

Demand (Electric): The rate at which electric energy is delivered to or by a system, part of a system, or piece of equipment, at a given instant or averaged over any designated period of time.

Demand Charge: That portion of the consumer's bill for electric service based on the consumer's maximum electric capacity usage and calculated based on the billing demand charges under the applicable rate schedule.

Demand Charge Credit: Compensation received by the buyer when the delivery terms of the contract cannot be met by the seller.

Demand Interval: The time period during which the flow of electricity is measured, usually in 15-, 30-, or 60-minute increments.

Dependable Capacity: The load-carrying ability of a station or system under adverse conditions for a specified period of time.

Diesel-Electric Plant: A generating station that uses diesel engines to drive its electric generators.

Direct Current (DC): An electric current that flows in a constant direction. The magnitude of the current does not vary or has a slight variation.

Dispatching: The operating control of an integrated electric system involving operations such as (1) the assignment of load to specific generating stations and other sources of supply to effect the most economical supply as the total or the significant area loads rise or fall; (2) the control of operations and maintenance of high-voltage lines, substations, and equipment; (3) the operation of principal tie lines and switching; (4) the scheduling of energy transactions with connecting electric utilities.

Distribution system: The portion of the transmission lines and facilities of an electric system off the bulk power system that is dedicated to delivering electric energy to an end-user.

Disturbance: Severe oscillations or sever step changes of current, voltage and/or frequency usually caused by faults.

Disturbance (System): An event resulting in widespread interruptions and characterized by one or more of the following phenomena: the loss of power system stability: cascading outages of circuits; abnormal ranges of frequency or voltage or both. 
Diversity: The electric utility system's load is made up of many individual loads that make demands upon the system usually at different times of the day. The individual loads within the customer classes follow similar usage patterns, but these classes of service place different demands upon the facilities and the system grid. The service requirements of one electrical system can differ greatly from another by time of day usage, facility usage, and/or demands placed upon the system grid.

Diversity Exchange: An exchange of capacity or energy, or both, between systems whose peak loads occur at different times.

Diversity Factor: The ratio of the sum of the noncoincident maximum demands for two or more loads to their coincident maximum demand for the same time period.

Docket: A formal record of a Federal Energy Regulatory Commission proceeding. These records are available for inspection and copying by the public. Each individual case proceeding is identified by an assigned number.

DOE: Department of Energy.

Double-circuit Line: A transmission line having two separate circuits. Since each carries three-phase power, at least six conductors, three per circuit, are required.

Dual-Fired Unit: A generating unit that can produce electricity using two or more input fuels. In some of these units only the primary fuel can be used continuously; the alternate fuel(s) can be used only as a start-up fuel or in emergencies.

Dump Energy: Energy generated in a hydroelectric plant by water that canni: be stored or conserved and which energy is in excess of the needs of the system producing the energy.

Economy Energy: Energy produced and supplied from a more economical source in one system, substituted for that being produced or capable of being produced by a less economical source in another system.
Economy of Scale: A proposition that relatively larger production facilities have lower unit costs than smaller facilities. Economy of scale may exist for any of the phases of operation: generation, transmission, or distribution.

EIA: The Energy Information Administration. An independent agency within the U.S. Department of Energy that develops surveys, collects energy data, and analyzes and models energy issues. The Agency must meet the requests of Congress, other elements within the Department of Energy, the Federal Energy Regulatory Commission, the Executive Branch, its own independent needs, and assist the general public, or other interest groups, without taking a policy position.

Electric Current: The number of electrons per unit of time moving past a point in a conductor.

Electric Energy: The ability of an electric current to produce work, heat, light or other forms of energy. It is measured in kilowatthours.

Electric Expenses: The cost of labor, material, and expenses incurred in operating a facility's prime movers, generators, auxiliary apparatus, switching gear, and other electric equipment for each of the points where electricity enters the transmission or distribution grid.

Electric Plant (Financial): Assets comprising land, building, and equipment permanently employed.

Electric Plant (Physical): A facility containing prime movers, electric generators, and auxiliary equipment for converting mechanical, chemical, and/or fission energy into electric energy.

Electric Power: The rate at which electric energy is generated, transmitted, or consumed. Electric power is measured in watts or kilowatts.

Electric Power Industry: The public, private, and cooperative electric utility systems of the United States taken as a whole. This includes all electric systems serving the public: regulated investor-owned electric utility companies; Federal power projects; State, municipal, and other government-owned systems, including electric public utility districts; electric coopera- 
tives, including generation and transmission entities ( $G \& T^{\prime}$ s); jointly owned electric utility facilities, and electric utility facilities owned by a lessor and leased to an electric utility. Excluded from this list are the special purpose electric facilities or systems that do not offer service to the public.

Electric Power System: An individual electric power entity--a company, an electric cooperative, a public electric supply corporation like the Tennessee Valley Authority, a similar Federal department or agency like the Bonneville Power Administration, the Bureau of Reclamation or the Corps of Engineers, a municipally owned, electric department offering service to the public, or an electric public utility district (a "PUD"); also a jointly owned electric supply project such as the Keystone.

Electric Rate: The price set for a specified amount and type of electricity by class of service in an electric rate schedule or sales contract.

Electric Rate Schedule: A statement of the electric rate and the terms and conditions governing its application, including attendant contract terms and conditions that have been accepted by a regulatory body with appropriate oversight authority.

Electric System Loss: Total electric energy loss from all causes for a electric utility.

Electric Utility: A corporation, person, agency, authority, or other legal entity or instrumentality that owns and/or operates facilities within the United States, its territories, or Puerto Rico for the generation, transmission, distribution, or sale of electric energy, primarily for use by the public and files forms listed in the Code of Federal Regulations, Title 18, Part 141. Facilities that qualify as cogenerators or small power producers under the Public Utility Regulatory Policies Act of 1978 and exempt wholesale generators under the Energy Policy Act of 1992 are not considered electric utilities.

Electrical System Energy Losses: The amount of energy lost during generation, transmission, and distribution of electricity, including plant and unaccounted for use.

Electricity: A form of energy generated by friction, induction, or chemical change that is caused by the presence and motion of elementary charged particles of which matter consists.

Emergency: The failure of an electric power system to generate or deliver electric power as normally intended, resulting in the cutoff or curtailment of service.

Emergency Energy: Electric energy provided for a limited duration, intended only for use during emergency conditions.

Emergency, (Major): A situation usually accompanied by abnormal frequency, abnormal voltage and/or equipment overloads which might seriously affect the reliability of the bulk power system.

Emergency (System): An emergency is considered to exist in an area if firm load may have to be shed because sufficient power or energy is unavailable in that area or in a portion of it after due allowance for purchases.

Energy: The capacity for doing work as measured by the capability of doing work (potential energy) or the conversion of this capability to motion (kinetic energy). Energy has several forms, some of which are easily convertible and can be changed to another form useful for work. Most of the world's convertible energy comes from fossil fuels that are burned to produce heat that is then used as a transfer medium to mechanical or other means in order to accomplish tasks. Electrical energy is usually measured in kilowatthours, while heat energy is usually measured in British thermal units.

Energy Broker System: Introduced into Florida by the Public Service Commission, the energy broker system is a system for exchanging information that allows utilities to efficiently exchange hourly quotations of prices at which each is willing to buy and sell electric energy. For the broker system to operate, utility systems must have in place bilateral agreements between all potential parties and must have transmission arrangements between all potential parties which allow the exchanges to take place.

Energy Charge: That portion of the charge for electric service based upon the electric energy (expressed in kilowatthours) consumed or billed. 
Energy Deliveries: Energy generated by one electric utility system and delivered to another system through one or more transmission lines.

Energy Loss (Power): The difference between energy input and output as a result of transfer of energy between two points. The loss changed to the equivalent power rating is sometimes referred to as capacity loss.

Energy Receipts: Energy generated by one electric utility system and received by another system through one or more transmission lines.

Energy Source: The primary fuel or transfer medium that provides the heat/power that is converted to electricity through chemical, mechanical, or other means. Energy sources include coal, petroleum and petroleum products, gas, water, uranium, wind, sunlight, geothermal, and other sources.

End-User: The final consumer of electricity.

Exchange Energy: Electric energy received by an electric utility system usually in exchange for energy delivered to the other system at another time or place. Exchange energy is to be distinguished from a direct purchase or sale, although accumulated energy balances are sometimes settled in cash.

Facilities Charge: An amount to be paid by the customer in a lump sum, or periodically as reimbursement for facilities furnished. The charge may include operation and maintenance as well as fixed costs.

Facility: A location at which prime movers, electric generators, and/or equipment for converting mechanical, chemical, and/or nuclear energy into electric energy are situated. A facility may contain more than one type of prime mover. For cogenerators, the facility includes the industrial or commercial process.

Failure or Hazard: Any electric power supply equipment or facility failure or other event that, in the judgment of the reporting entity, constitutes a hazard to maintaining the continuity of the bulk electric power supply system such that a load reduction action may become necessary and a reportable outage may occur. The impact of this failure/hazard may require the imposition of a special operating procedure, the extended purchase of emergency power, and/or other bulk power system actions.

Federal Energy Regulatory Commission (FERC): The independent Federal regulatory agency with jurisdiction over interstate electricity sales, wholesale electric rates, hydroelectric licensing, natural gas pricing, oil pipeline rates, and gas pipeline certification.

Federal Power Act: Enacted in 1920, and as amended in 1935, the Act consists of three parts. The first part incorporated the Federal Water Power Act administered by the former Federal Power Commission, whose activities were confined almost entirely to licensing non-Federal hydroelectric projects. Parts II and III were added with the passage of the Public Utility Act. These parts extended the Act's jurisdiction to include regulating the interstate transmission of electrical energy and rates for its sale as wholesale electricity in interstate commerce. The Federal Energy Regulatory Commission is now charged with the administration of this law.

Federal Power Commission: The predecessor agency of the Federal Energy Regulatory Commission. The Federal Power Commission was created by an Act of Congress under the Federal Water Power Act on June 10,1920 . It was charged originally with regulating the electric power and natural gas industries. It was abolished on September 30, 1977, when the Department of Energy was created. Its functions were divided between the Department of Energy and the Federal Energy Regulatory Commission, an independent regulatory agency.

Feeder Line: An electrical line that extends radially from a distribution substation to supply electrical energy within an electric service area or sub-area.

FERC: The Federal Energy Regulatory Commission.

FERC Guidelines: A compilation of the Federal Energy Regulatory Commission's enabling statutes, procedural and program regulations, and orders, opinions and decisions.

File Rate Schedule: The rate for a particular electric service, including attendant contract terms and conditions, accepted for filing by a regulatory body with appropriate oversight authority. 
Filing: Any written application, complaint, declaration, petitic 1 , protest, answer, motion, brief, exception, rate schedule, or other pleading, amendment to a pleading, document, or similar paper that is submitted to a utility commission.

Final Order: A final ruling by FERC that terminates an action, decides some matter litigated by the petitioning parties, operates to some right, or completely disposes of the subject matter.

Firm Power: Electric power intended to meet the load requirement of a utility's customers; there is no planned interruption of services with this type of sale. Utilities may sell capacity for a limited duration and time to other utilities and these types of contractual transactions are also referred to as firm power.

Firm Service: The commitment of generation and/or transmission service to a customer under a filed rate schedule to which the parties to the service anticipate no planned interruption. The allocation of the utility's resources may be system wide, or only for a named unit; the time of availability is usually prescribed as well.

Fiscal Year: A financial year based on a predetermined starting date. The Federal Government's 12-month financial year runs from October 1 through September 30.

Force Majeure Clause: An occurrence generally beyond the control of the buyer or seller in which relief is given by this clause to the parties involved in an agreement. This event, which results in nonperformance of terms in a contract, reliability staudards, or operating agreements, will typically relieve a buyer or seller of liability for damages. Some financial or other compensation relief may be available, if additional terms or conditions are written into the contract concerning unexpected problems beyond the control of the parties.

Forced Outage: The shutdown of a generating unit, transmission line or other facility, for emergency reasons or a condition in which the generating equipment is unavailable for load because of unanticipated breakdown.

Frame: All units (i.e., electric utilities) that comprise the population of interest to a given survey. Subunits may in some instances only be represented in groups, but complete coverage is maintained.

Fuel Emergency: An emergency that exists when supplies of fuels or hydroelectric storage for generation are at a level or estimated to be at a level that would threaten the reliability or adequacy of the bulk electric power supply. The following factors should be taken into account to determine that a fuel emergency exists: (1) Fuel stock or hydroelectric project water storage levels are 50 percent or less of normal for that particular time of the year and a continued downward trend in fuel stock or hydroelectric project water storage level are estimated; or (2) Unscheduled dispatch or emergency generation is causing an abnormal use of a particular fuel type, such that the future supply or stocks of that fuel could reach a level which threatens the reliability or adequacy of bulk electric power supplies.

Full-Forced Outage: The net capability of main generating units that are unavailable for load for emergency reasons.

Gas-Turbine Plant: A plant in which the prime mover is a gas turbine. A gas turbine consists iypically of an axial-flow air compressor, one or more combustion chambers where liquid or gaseous fuel is burned and the hot gases are passed to the turbine; where the hot gases expand to drive the generator and then are used to run the compressor.

Generally Accepted Accounting Principles (GAAP): Defined as the conventions, rules, and procedures necessary to define accepted accounting practice at a particular time; includes both broad guidelines and relatively detailed practices and procedures.

Generating Station: A station which consists of electric generators and auxiliary equipment for converting mechanical, chemical or nuclear energy into electric energy.

Generating Unit: An electric generator together with its prime mover.

Generation: The process of producing electric energy by transforming other forms of energy; also, the amount of electric energy produced, expressed in kilowatthours. 
Generation (Gross): The total amount of electric energy produced by the generating units in a generating station or stations, measured at the generator terminals.

Generation (Net): Gross generation less the electric energy consumed at the generating station for station use.

Generation Rejection: The process of deliberately removing preselected generation from a Power System in response to an abnormal condition in order to maintain the integrity of the system; sometimes also called generator dropping.

Geothermal Energy: Energy from the internal heat of the earth which may be residual heat, friction heat, or a result of radioactive decay. The heat is found in rocks and fluids at various depths and can be extracted by drilling and/or pumping.

Geothermal Plant: A plant in which the prime mover is a steam turbine. The turbine is driven either by steam produced from hot water or by natural steam that derives its energy from heat found in rocks or fluids at various depths beneath the surface of the earth. The energy is extracted by drilling and/or pumping.

Gigawatt (GW): One billion watts.

Gigawatthour (GWh): One billion watthours.

Grid: The layout of an electrical system.

Gross Generation: The total amount of electric energy produced by a generating station or stations, measured at the generator terminals.

Gross Head: A dam's maximum allowed vertical distance between the upstream's surface water (headwater) forebay elevation and the downstream's surface water (tailwater) elevation at the tail-race for reaction wheel dams or the elevation of the jet at impulse wheel dams during specified operation and water conditions.
Head: The product of the water's weight and a usable difference in elevation gives a measurement of the potential energy possessed by water.

Holding Company: A corporation (parent company) that directly or indirectly owns a majority of all of the voting securities of one or more companies located in the same, contiguous States, or operating independent integrated public utility systems in noncontiguous States. A holding company is so structured to bring companies that can best be operated as part of an integrated public utility system under one management. Many States do not permit or authorize a registered utility company to acquire ownership or operation of an electric or gas utility company serving substantially the same territory. The Securities and Exchange Commission, as administrator of the Public Utility Holding Company Act of 1935 , defines a holding company as "a company which...owns, controls...10 percent or more of the outstanding voting securities of a public utility company."

Horsepower: A unit for measuring the rate of work (or power) equivalent to 33,000 foot-pounds per minute or 746 watts.

Hybrid Transmission Line: A double-circuit line which has one alternating current circuit and one direct current circuit. The AC circuit usually serves local loads along the line.

Hydraulic Head: The distance between the respective elevations of the upstream's water surface (headwater) above and the downstream surface water (tailwater) below a hydroelectric power plant.

Hydroelectric Energy: The production of electricity from kinetic energy in flowing water.

Hydroelectric Plant: A plant in which the turbine generators are driven by falling water.

Hydroelectric Plant Capacity: This capacity figure is a function of fluid flow losses, hydraulic turbines, head, and water flow. The minimum net head limits the firm capacity of the plant.

Hydroelectric Power: The harnessing of flowing water to produce mechanical or electrical energy. 
Hydroelectric Project: The complete development of a hydroelectric power site. This includes dams, reservoirs, transmission lines and accessories needed for the maintenance and operation of the powerhouse and any other hydroelectric plant support facilities.

Impedance: The opposition to power flow in an AC circuit. Also, applicable to any device that introduces such opposition, in the form of resistance, reactance, or both. The impedance of a circuit or device is measured as the ratio of voltage to current, where a sinusoidal voltage and current of the same frequency are used for the measurement; measured in ohms.

Inadvertent Power Exchange: An unintended power exchange among utilities that is either not previously agreed upon or in an amount different from the amount agreed upon.

Incremental Energy Costs: The additional cost of producing and/or transmitting electric energy above some previously determined base cost.

Installed Nameplate Capacity: The full-load continuous rating of a generator, prime mover, or other electrical equipment under specified conditions as designated by the manufacturer. Installed nameplate capacity is usually indicated on a nameplate attached physically to the equipment. Installed station capacity does not include auxiliary or house units.

Instantaneous Peak Demand: The maximum demand at the instant of greatest load.

Insulator: A material that is a very poor conductor of electricity. The insulating material is usually a ceramic or fiberglass when used in the transmission line and is designed to support a conductor physically and to separate it electrically from other conductors and supporting material.

Integrated Demand: The summation of the continuously varying instantaneous demand averaged over a specified interval of time. The information is usually determined by examining a demand meter.

Integrated Gasification-Combined Cycle Technology: Coal, water, and oxygen are fed to a gasifier, which produces synthetic natural gas (syngas). This medium Btu gas is cleaned (particulates and sulfur compounds removed) and is fed to a gas turbine. The hot exhaust of the gas turbine and heat recovered from the gasification process are routed through a heat-recovery generator to produce steam, which drives a steam turbine to produce electricity.

Interchange Energy: Kilowatthours delivered to or received by one electric utility or pooling system from another. Settlement may be by payment, returned in kind at a later time or accumulated as energy balances until the end of the stated period.

Interconnected System: A system consisting of two or more individual power systems normally operating with connecting tie lines.

Interconnection: Two or more electric systems having a common transmission line that permits a flow of energy between them. The physical connection of the electric power transmission facilities allows for the sale or exchange of energy.

Interdepartmental Sales: Includes amounts charged by the electric department at tariff or other specified rates for electricity supplied by it to other utility departments.

Interdepartmental Service (Electric): Electricity supplied to departments of the electric utility company other than the electric generating department.

Interlocking Directorates: The holding of a significant position in management or a position on the corporate board of a utility, while simultaneously holding a comparable position with another utility, or with a firm doing business with the utility.

Intermediate Load (Electric System): The range from base load to a point between base load and peak. This point may be the midpoint, a percent of the peak load, or the load over a specified time period.

Internal Combustion Plant: A plant in which the prime mover is an internal combustion engine. An internal combustion engine has one or more cylinders in which the process of combustion take place, converting energy released from the rapid burning of a fuel-air mixture into mechanical energy. Diesel or gasfired engines are the principal types used in electric 
plants. The plant is usually operated during periods of high demand for electricity.

Interruptible Power: Power and usually the associated energy made available by one utility to another which is subject to curtailment or cessation of delivery by the supplier in accordance with a prior agreement with the other party or under specified conditions.

Intervenor: A person, institution, or organization admitted as a participant to a proceeding.

Investor-Owned Electric Utility: A class of utility whose stock is publicly traded and which is organized as a tax-paying business, usually financed by the sale of securities in the capital market. It is regulated and authorized to achieve an allowed rate of return.

Island: A portion of a power system, or several power systems which is (are) electrically separated from the main grid.

Joint-Use Facility: A multiple-purpose hydroelectric plant. An example is a dam that stores water for both flood control and power production.

Jurisdictional Utilities: Utilities regulated by public laws.

Kilowatt (kW): One thousand watts.

Kilowatthour (kWh): One thousand watthours.

Line Loss: Electric energy lost because of the transmission of electricity. Much of the loss is thermal in nature.

Load: The power provided to customers of a system. This may be further identified to more precisely indicate which portion of the system is being considered, (e.g., customer load, station auxiliary load, etc.).

Load, Firm: Power provided to customers that is continuously available on demand and which is subject to interruption only under extreme circumstances.
Load, Interruptible: Those loads that, by contract, can be interrupted in the event of a deficiency on the supplying system.

Load (Electric): The amount of electric power delivered or required at any specific point or points on a system. The requirement originates at the energyconsuming equipment of the customers.

Load Curve: The relationship of power supplied to the time of occurrence which illustrates the varying magnitude of the load during the period covered.

Load Diversity: The difference between the peak of coincident and noncoincident demands of two or more individual loads.

Load Factor: The ratio of the average load to peak load during a specified time interval.

Load Following: Regulation of the power output of electric generators within a prescribed area in response to changes in system frequency, tieline loading, or the relation of these to each other, so as to maintain the scheduled system frequency and/or the established interchange with other areas within predetermined limits.

Load Leveling: Any load control technique that dampens the cyclical daily load flows and increases baseload generation. Peak load pricing and time-of-day charges are two techniques that electric utilities use to reduce peak load and to maximize efficient generation of electricity.

Load Loss (3 hours): Any significant incident on an electric utility system which results in a continuous outage of 3 hours or longer to over 50,000 customers or more than one half of the total customers being served immediately prior to the incident, whichever is less.

Load Management Technique: Utility demand management practices directed at reducing the maximum kilowatt demand on an electric system, and/or modifying the coincident peak demand of one or more classes of service to better meet the utility system capability for a given hour, day, week, season, or year. 
Load Reduction Request: The issuance of any public or private request to any customer or the general public to reduce the use of electricity for reasons of maintaining the continuity of service of the reporting entity's electric power supply system. Requests to a customer(s) served under provisions of an interruptible contract are not a reportable action unless the request is made for reasons of maintaining the continuity of service of the reporting entity's electric power supply.

Load Rellef: Load reduction accomplished by voltage reduction and/or load shedding.

Load Shedding: Deliberate disconnection of customer load in response to an abnormal condition in order to maintain the integrity of the power system. Disconnection may be automatically or manually initiated. The routine use of load control equipment that reduces firm customer load is not considered to be a reportable action.

Loop Flow: The movement of electric power from generator to load by dividing along multiple parallel paths; it especially refers to power flow along an unintended path that loops away from the most direct geographic path, or contract path.

Low Head: Vertical difference of 100 feet or less in the upstream surface water elevation (headwater) and the downstream surface water elevation (tailwater) at a dam.

Maximum Demand: The greatest of all demands of the load that has occurred within a specified period of time.

Maximum Dependable Capacity, Net: The gross electrical output measured at the output terminals of the turbine generator(s) during the most restrictive seasonal conditions, less the station service load.

Median Streamflow: The middle rate of flow of water past a given point for which there have been several greater and lesser rates of flow occurring during a specified period.

Median Water Condition: The middle precipitation and run-off condition for a distribution of water conditions that have happened over a long period time; usually determined by examining water supply records of the periods in question.

Megawatt (MW): One million watts.

Megawatt-Electric (MW): One million watts of electric capacity.

Megawatthour (MWh): One million watthours.

Mill: A monetary cost and billing unit used by utilities; it is equal to $1 / 1000$ of the U.S. dollar (equivalent to $1 / 10$ of 1 cent).

Minimum Streamflow: The minimum rate of flow of water past a given point during a specified period.

Multiple Purpose Project: The development of hydroelectric facilities to serve more than one function. Some of the uses include hydroelectric power, irrigation, water supply, water quality control, and/or fish and wildlife enhancement.

Multiple Purpose Reservoir: Stored water and its usage governed by advanced water resource conservation practices to achieve more than one water control objective. Some of the objectives include flood control, hydro-electric power development, irrigation, recreation usage, and wilderness protection.

Municipality: A city, county, district, or a political subdivision of a State competent under the laws thereof to carry on the business of its charter.

National Association of Regulatory Utility Commissioners: An affiliation of the public service commissioners to promote the uniform treatment of members of the railroad, public utilities, and public service commissions of the 50 States, the District of Columbia, the Commonwealth of Puerto Rico, and the territory of the Virgin Islands.

Natural Streamflow: The rate of flow of water past a given point of an uncontrolled stream, or regulated streamflow adjusted to eliminate the effects of reservoir storage or upstream diversions at a set time interval. 
Net Ewarey for Load: Net generation of main generating units that are system-owned or system-operated plus energy receipts minus energy deliveries.

Net Energy for System: The net generation of a group of plants that are electrically interconnected and centrally controlled, plus system receipts of any electrical energy from outside the system minus any system energy delivered to other electrical systems.

Net Generation: Gross generation less plant use, measured at the high-voltage terminals of a station's stepup transformer. The energy required for pumping at pumped-storage plants is regarded as plant use and must be deducted from the gross generation.

Net Head: The gross head minus all hydraulic losses except those chargeable to the turbine.

Net Summer Capability: The steady hourly output which generating equipment is expected to supply to system load exclusive of auxiliary power as demonstrated by test at the time of summer peak demand.

Net System Capability: The generating station capability of a system at a stated period of time (usually at the time of the system's maximum load) plus capability available at such time from other sources through firm power contacts less firm power obligations at such time to other companies or systems.

Net Winter Capability: The steady hourly output which generating equipment is expected to supply to system load exclusive of auxiliary power as demonstrated by test at the time of winter peak demand.

No-Load Loss: Power and energy lost by an electric system when not operating under demand.

Noncoincident Demand: Sum of two or more demands on individual systems that do not occur in the same demand interval.

Noncoincident Peak Load: The sum of two or more peak loads on individual systems that do not occur in the same time interval. Meaningful only when considering loads within a limited period of time, such as a day, week, month, a heating or cooling season, and usually for not more than 1 year.
Nonfirm Power: Power or power-producing capacity supplied or available under a commitment having limited or no assured availability.

Nonspinning Reserve: The generating capacity not currently running, but capable of being connected to the bus and load within a specified time.

Nonutlilty Generation: Electric generation by endusers, or small power producers under the Public Utility Regulatory Policies Act of 1978, to supply electric power for industrial, commercial, or sales to electric utilities.

Nonutllity Power Producer: An enterprise that has electric generating capacity and is not an electric utility. Nonutility power producers include qualifying cogenerators, qualifying small power producers, and other nonutility generators (including independent power producers) without a designated franchised service area, and which are not otherwise required to file forms listed in the Code of Federal Regulations, Title 18, Part 141.

North American Electric Reliability Council (NERC): A council formed in 1968 by the electric utility industry to promote the reliability and adequacy of the bulk power supply in the electric utility systems of North America. NERC consists of ten regional reliability councils and encompasses essentially all the power systems of the contiguous United States, Canada, and some in Mexico. The data summarized by NERC regions in this publication are limited to that portion applicable to the United States, thereby excluding that portion of NERC data applicable to Canada and Mexico. The NERC Regions are:

ASCC--Alaska System Coordination Cou* cil

ECAR--East Central Area Reliability Coordination Agreement

ERCOT--Electric Reliability Council of Texas

MAIN--Mid-America Interconnected Network

MAAC--Mid-Atlantic Area Council

MAPP--Mid-Continent Area Power Pool

NPCC--Northeast Power Coordinating Council

SERC--Southeastern Electric Reliability Council

SPP--Southwest Power Pool

WSCC--Western Systems Coordinating Council 
Nuclear Power Plant: A facility in which heat produced in a reactor by the fissioning of nuclear fuel is used to drive a steam turbine.

Orf-Peak: Period of relatively low system demand. These periods often occur in daily, weekly, and seasonal patterns; these off-peak periods differ for each individual electric utility.

Ohm: The unit of measurement of electrical resistance. The resistance of a circuit in which a potential difference of 1 volt produces a current of 1 ampere.

On-Peak: Periods of relatively high system demand. These periods often occur in daily, weekly, and seasonal patterns; these on-peak periods differ for each individual electric utility.

Operating Limit: The maximum value of the most critical system operation parameter(s) which meets: precontingency criteria as determined by equipment loading capability and acceptable voltage conditions; transient performance criteria; and post contingency loading and voltage criteria.

Order: A ruling issued by a utility commission granting or denying an application in whole or in part. The order explains the basis for the decision, noting any dispute with the factual assertions of the applicant. Also applied to a final regulation of a utility commission.

Other Generation: Electricity originating from these sources: biomass, fuel cells, geothermal heat, solar power, waste, wind, and wood.

Other Sector: Electricity supplied to public street and highway lighting; other service to public authorities; service to railroads and railways; and interdepartmental service.

Other Service to Public Authorities: Electricity supplied to municipalities or divisions or agencies of State or Federal governments, under special contracts or agreements or service classifications applicable only to public authorities.

Other Unavailable Capability: Net capability of main generating units that are unavailable for load for rea- sons other than full-forced outage or scheduled maintenance. Legal restrictions or other cause make these units unavailable.

Outage: The period during which a generating unit, transmission line, or other facility is out of service.

Output: The amount of power or energy produced by a generating unit, station, or system.

Peak Load: The maximum load during a specifled period of time.

Peak Load Plant: A plant usually housing old, lowefficiency steam units, gas turbines, diesels, or pumpedstorage hydroelectric equipment normally used during the peak-load periods.

Peaking Capacity: Capacity of generating equipment normally operated during the hours of highest daily, weekly, or seasonal loads. Some generating equipment may be operated at certain times as peaking capacity and at other times to serve loads on a 'round-the-clock basis.

Percent Difference: The relative change in a quantity over a specified time period. It is calculated as follows: the current value number has the previous value number subtracted from it, and this new number is divided by the absolute value of the previous value number; then this new number is multiplied by 100 .

Plant: A station at which are located prime movers, electric generators, and auxiliary equipment for converting mechanical, chemical, and/or nuclear energy into electric energy. A station may contain more than one type of prime mover. Electric utility plants exclude stations that satisfy the definition of qualifying facility under the Public Utility Regulatory Policies Act of 1978.

Plant-Use Electricity: The electric energy used in the operation of a plant. This energy total is subtracted from the gross energy production of the plant; for reporting purposes the plant energy production is then reported as a net figure. The energy required for pumping at pumped-storage plants is by definition subtracted, and the energy production for these plants is then reported as a net figure. 
Plant Hours Connected to Load: The number of hours the plant is synchronized to load over a time interval usually of 1 year.

Pole-Mile: $A$ unit of measuring the simple length of a transmission line carrying electric conductors, without regard to the number of conductors carried.

Pondage: The amount of water stored behind a hydroelectric dam of relatively small storage capacity; the dam is usually used for daily or weekly control of the flow of the river.

Power: The rate at which energy is transferred, usually measured in watts. Also used for a measurement of capacity.

Power (Electrical): An electric measurement unit of power called a voltampere is equal to the product of 1 volt and 1 ampere. This is equivalent to 1 Watt for a direct current system and a unit of apparent power is separated into real and reactive power. Real power is the work-producing part of apparent power that measures the rate of supply of energy and is denoted as kilowatts $(\mathrm{kW})$. Reactive power is the portion of apparent power that does no work and is referred to as kilovars; this type of power must be supplied to most types of magnetic equipment, such as motors, and is supplied by generator or by electrostatic equipment. Voltamperes are usually divided by 1,000 and called kilovoltamperes (kVA). Energy is denoted by the product of real power and the length of time utilized; this product is expressed as kilowatthours.

Power Factor: The ratio of real power (kilowatt) to apparent power kilovoltampere for any given load and time.

Power Loss: The difference between power input and output as a result of transfer of energy between two points; sometimes referred to as capacity loss.

Power Pool: An association of two or more interconnected electric systems to provide better system reliability and efficiencies. Depending upon the degree of control authorized by the member utilities, these pools many be run under a single system dispatch to supply power to meet combined load requirements and maintenance programs, or just share the benefits of planned or hourly available wholesale sales of power and energy among the member utilities.
Power Production Plant: All the land and land rights, structures and improvements, boiler or reactor vessel equipment, engines and engine-driven generator, turbogenerator units, accessory electric equipment, and miscellaneous power plant equipment are grouped together for each individual facility.

Power System: A group of one or more generating sources and/or connecting transmission lines operated under common management or supervision to supply load.

Power Transfer Limit: The maximum power that can be transferred from one electric utility system to another without overloading any facility in either system.

Powerhouse: A structure at a hydroelectric plant site that contains the turbine and generator.

Preliminary Permit (Hydroelectric Power): A single site permit granted by the FERC, which gives the recipient priority over anyone else to apply for a hydroelectric license. The preliminary permit enables the recipient to prepare a license application and conduct various studies for economic feasibility and environmental impacts. The period for a preliminary permit may extend to 3 years.

Price: The amount of money or consideration-in-kind for which a service is bought, sold, or offered for sale.

Price Squeeze: Discriminatory rates charged for wholesale electric power that impede competition for retail customers. A price squeeze occurs if an electric utility's price for wholesale service is higher in relation to its wholesale cost than its price for retail service is in relation to its retail costs.

Prime Mover: The engine, turbine, water wheel, or similar machine that drives an electric generator.

Proposed Rates: New electric rate schedule proposed by an applicant to become effective at a future date.

Prudence: Carefulness, precaution, attentiveness, and good judgment, as applied to action or conduct. A legal concept used by regulators to determine the costs to be allowed in a rate base. 
Public Authoritica: Electricity supplied to municipalities or divisions or agencies of State and Federal governments, usually under special contracts or agreements that are applicable only to public authorities.

Public Uttlity: Enterprise providing essential public services, such as electric, gas, telephone, water, and sewer, under legally established monopoly conditions.

Public Utility District: Municipal corporations organized to provide electric service to both incorporated cities and towns and unincorporated rural areas. Public utility districts, sometimes called "People's Utility Districts" or "Public Power Districts," operate in six States.

Publicly Owned Electric Utility: A class of ownership found in the electric power industry. This group includes those utilities operated by municipalities, and State and Federal power agencies.

Pumped-Storage Hydroelectric Plant: A plant that usually generates electric energy during peak-load periods by using water previously pumped into an elevated storage reservoir during off-peak periods when excess generating capacity is available to do so. When additional generating capacity is needed, the water can be released from the reservoir through a conduit to turbine generators located in a power plant at a lower level.

Purchased Power: Power purchased or available for purchase from a source outside the system.

Purchased Power Adjustment: A clause in a rate schedule that provides for adjustments to the bill when energy from other electric system is acquired and its cost varies from a specified unit base amount.

Pure Pumped-Storage Hydroelectric Plant: A plant that produces power only from water that has previously been pumped to an upper reservoir.

Qualifying Facility: A cogeneration facility or small power production facility that meets certain ownership, operating, and efficiency criteria established by the Federal Energy Commission pursuant to the Public Utilities Regulatory Policies Act of 1978 (See the Code of Federal Regulations, title 18, Part 282).
Rate Bawe: The value of property upon which a utility is permitted to earn a specified rate of return as established by a regulatory authority. The rate base generally represents the value of property used by the utility in providing service and may be calculated by any one or a combination of the following accounting methods: fair value, prudent investment, reproduction cost, or original cost. Depending on which method is used, the rate base includes cash working capital, materials and supplies, and deductions for accumulated provisions for depreciation, contributions in aid of construction, customer advances for construction, accumulated deferred income taxes, and accumulated deferred investment tax credits.

Rate Case: A proceeding, usually before a regulatory commission, involving the rates to be charged for a public utility service.

Rate of Return: The ratio of net overating income earned by a utility is calculated as a percentage of its rate base.

Rate of Return on Rate Base: The ratio of net operating income to a specified rate base, expressed as a percentage.

Rate Schedule (Electric): A statement of the financial terms and conditions governing a class or classes of utility services provided to a customer. Approval of the schedule is given by the appropriate ratemaking authority.

Rates: The authorized charges per unit or level of consumption for a specified time period for any of the classes of utility services provided to a customer.

Ratemaking Authority: A utility commission's legal authority to fix, modify, approve, or disapprove rates as determined by the por ers given the commission by a State or Federal legislature.

Rating: A manufacturer's guaranteed performance of a machine, transmission line, or other electrical apparatus, based on design features and test data. The rating will specify such limits as load, voltage, temperature, and frequency. The rating is generally printed on a nameplate attached to equipment and is commonly referred to as the nameplate rating, nameplate capacity, etc. 
Reactance: A phenomenon associated with $\mathrm{AC}$ power characterized by the existence of a time difference between voltage and current variations.

Rectifier: A device for converting alternating current to direct current.

Regulated Streamflow: The rate of flow past a given point during a specified period that is controlled by reservoir water release operations.

Regulation, Procedures, and Practices: A utility commission carries out its regulatory functions through rulemaking and adjudication. Under rulemaking, the utility commission may propose a general rule or regulation change. By law, it must issue a notice of the proposed rule and a request for comments is also made; the Federal Energy Regulatory Commission publishes this in the Federal Register. The final decision must be published. A utility commission may also work on a case-by-case basis from submissions from regulated companies or others. Objections to a proposal may come from the commission or intervenors, in which case the proposal must be presented at a hearing presided over by an administrative law judge. The judge's decision may be adopted, modified, or reversed by the utility commissioners in which case those involved can petition for a rehearing, and may appeal a decision through the courts system to the U.S. Supreme Court.

Reliability (Electric System): A measure of the ability of the system to continue operation while some lines or generators are out of service. Reliability deals with the performance of the system under stress.

Renewable Energy Source: An energy source that is regenerative or virtually inexhaustible. Typical exam. ples are wind, geothermal and water power.

Repowering: Replacement of the combustion technology in an aging generating plant with a new combustion technology, usually resulting in better performance and greater capacity.

Required Rate of Return: The minimum rate of return necessary to induce investors to buy or hold a given security. It equals the risk-free rate of interest, usually represented by the current yield on U.S. Treasury securities, growth potential, plus a risk premium.
Requirements Fower: The firm service needs required by designated load plus losses from the points of supply.

Reserve Generating Capacity: Amount of generating capacity available to meet peak or abnormally high demands for power and to generate power during scheduled or unscheduled outages.

Reserve Margin (Operating): The amount of unused available capability of an electric power system at peak load for a utility system. This figure is calculated by adding running and quick-start capability to the capability available but not needed and then subtracting peak load.

Reservoir: A body of water collected and stored in a natural or artificial lake.

Residential Sector: The residential sector includes private household establishments that consume energy primarily for space heating, water heating, air conditioning, lighting, refrigeration, cooking, and clothes drying. The classification of an individual consumer's account, where the use is both residential and commercial, is based on principal use. Apartment houses are included.

Restoration Time: The time when the major portion of the interrupted load has been restored and the emergency is considered to be ended. However, some of the loads interrupted may not have been restored because of local problems.

Retail Wheeling: An arrangement in which a utility transmits electricity from outside its service territory to a retail customer within its customer service territory.

Retired Hydropower Plant Sites: The site of a plant that formerly produced electrical or mechanical power but is now out of service. Includes plants which have been abandoned, damaged by flood or fire, inundated by new reservoirs, or dismantled.

Reversible Turbine: A hydraulic turbine, normally installed in a pumped-storage plant, which can be used alternatively as a pump or as an engine, turbine, water wheel, or other apparatus that drives an electrical generator. 
Right-Of-Way: The land, and legal right to use and service the land along which a transmission line is located. Transmission line right-of-way is usually acquired in widths that vary with the kilovolt $(\mathrm{kV})$ size of the line.

Rulemaking (Regulations): The authority delegated to administrative agencies by Congress or State legislative bodies to make rules that have the force of law. Frequently, statutory laws that express broad terms of a policy are implemented more specifically by administrative rules, regulations and practices.

Running and Quick-Start Capability: The net capability of generating units that carry load or have quickstart capability. In general, quick-start capability refers to generating units that can be available for load within a 30-minute period.

Run-off: That portion of the precipitation that flows over the land surface and ultimately reaches streams to complete the water cycle. Melting snow is an important source of this water as well as all amounts of surface water that moves to streams or rivers through any given area of a drainage basin.

Rural Electrification Administration (REA): A lending agency in the U.S. Department of Agriculture, REA makes self-liquidation loans to qualified borrowers to finance electric and telephone service to rural areas. REA finances the construction and operation of generating plants, electric transmission and distribution lines, or systems, for the furnishing of initial and continued adequate electric services to persons in rural areas not receiving central station service.

Sales: The amount of kilowatthours sold in a given period of time; usually grouped by classes of service such as residential, commercial, industrial, and other.

Sales for Resale: A type of wholesale sales covering energy supplied to other electric utilities, cooperatives, municipals, and Federal and State electric agencies for resale to ultimate consumers.

Schedule: A statement of the pricing format of electricity and the terms and conditions governing its applications.
Scheduled Outage: The shutdown of a generating unit, transmission line, or other facility, for inspection or maintenance, in accordance with an advance schedule.

Service Area: The territory in which a utility system or distributor is authorized to provide service to consumers.

Single-circuit Line: A transmission line with one electric circuit. For three-phase supply, a single circuit requires at least three conductors, one per phase.

Single-Purpose Project: A hydroelectric project constructed only to generate electricity.

Small Power Producer: Under the Public Utility Regulatory Policies Act, A small power production facility (or small power producer) generates electricity using renewable biomass, conventional hydroelectric, solar, wind, and geothermal energy as a primary energy source. Fossil fuels can be used, but renewable resources must provide at least 75 percent of the total energy input. (See Code of Federal Regulations, Title 18, Part 292.)

Special Contract Rate Schedule: An electric rate schedule for an electric service agreement between a utility and another party in addition to, or independent of any standard rate schedule.

Special Purpose Rate Schedule: An electric rate schedule limited in its application to some particular purpose or process within one, or more than one, type of industry or business.

Stability: The property of a system or element by virtue of which its output will ultimately attain a steady state. The amount of power that can be transferred from one machine to another following a disturbance. The stability of a power system is its ability to develop restoring forces equal to or greater than the disturbing forces so as to maintain a state of equilibrium.

Standby Charge: A charge for the potential use of a utility service, usually done by an agreement with another electric utility system. These services include system backup support, and other running and quickstart capabilities. 
Standby Facility: A facility that is on call to support a utility system and is available to replace or supplement a facility normally in service.

Standby Service: Support service that is available as needed to supplement a customer, a utility system, or to another utility if a schedule or an agreement authorizes the transaction. The service is not regularly used.

State Severance Taxes: Any severance, production, or similar tax, fee, or other levy imposed on the production of crude oil, natural gas, or coal by any State, local government acting under authority of State law, or by an Indian tribe recognized as eligible for services by the Secretary of the Interior.

Station (Electric): A plant containing prime movers, electric generators, and auxiliary equipment for converting mechanical, chemical, and/or nuclear energy into electric energy.

Station Use: Energy that is used in the operation of an electric generating plant. It includes energy consumed for plant lighting, power, and auxiliary facilities, regardless of whether the energy is produced at the plant or comes from another source.

Steam-Electric Plant (Conventional): A plant in which the prime mover is a steam turbine. The steam used to drive the turbine is produced in a boiler where fossil fuels are burned.

Streamflow: The rate at which water passes a given point in a stream, usually expressed in cubic feet per second.

Substation: Facility equipment that switches, changes, or regulates electric voltage.

Subtransmission: A set of transmission lines of voltages between transmission voltages and distribution voltages. Generally, lines in the voltage range of $69 \mathrm{kV}$ to $138 \mathrm{kV}$.

Supporting Structure: The main supporting unit (usually a pole or tower) for transmission line conductors, insulators, and other auxiliary line equipment.
Surplus Energy: Energy generated that is beyond the immediate needs of the producing system. This energy may be supplied by spinning reserve and sold on an interruptible basis.

Suspended Rates: New rates that have been accepted for review by a utility commission. When these rates are suspended, they do not go into effect for a designated period of time, up to a maximum of five months for FERC. Charges under the new rate may be refunded after the resolution of the rate proceeding.

Switching Station: Facility equipment used to tie together two or more electric circuits through switches. The switches are selectively arranged to permit a circuit to be disconnected, or to change the electric connection between the circuits.

System (Electric): Physically connected generation, transmission, and distribution facilities operated as an integrated unit under one central management, or operating supervision.

System Interconnection: A physical connection between two electric systems that permits the transfer of electric energy in either direction.

Tariff: A published volume of rate schedules and general terms and conditions under which a product or service will be supplied.

Terawatthour (TWh): One trillion $\left(10^{12}\right)$ watthours of electric energy.

Thermal Limit: The maximum amount of power a transmission line can carry without suffering heatrelated deterioration of line equipment, particularly conductors.

Three-party Wheeling: An arrangement in which a utility transmits electricity for two other utilities that are not physically connected, where the transmitting utility neither buys nor sells the electricity.

Three-phase Power: Power generated, and transmitted from generator to load, on three conductors. 
Tie Line: A Circuit connecting two or more power systems and/or a generating facility to the grid.

Transfer Capability: The overall capacity of interregional or international power lines, together with the associated electrical system facilities, to transfer power and energy from one electrical system to another.

Transformer: An electrical device for changing the voltage of alternating current.

Transmission: The movement or transfer of electric energy over an interconnected group of lines and associated equipment between points of supply and points at which it is transformed for delivery to consumers, or is delivered to other electric systems. Transmission is considered to end when the energy is transformed for distribution to the consumer.

Transmission Circuit: A conductor used to transport electricity from generating stations to load.

Transmission Line: A set of conductors, insulators, supporting structures, and associated equipment used to move large quantities of power at high voltage, usually over long distances between a generating or receiving point and major substations or delivery points.

Transmission Network: A system of transmission or distribution lines so cross-connected and operated as to permit multiple power supply to any principal point.

Transmission System, Electric: An interconnected group of electric transmission lines and associated equipment for moving or transferring electric energy in bulk between points of supply and points at which it is transformed for delivery over the distribution system lines to consumers, or is delivered to other electric systems.

Turbine: A machine for generating rotary mechanical power from the energy in a stream of fluid (such as water, steam, or hot gas). Turbines convert the kinetic energy of fluids to mechanical energy through the principles of impulse and reaction, or a mixture of the two.
Two-party Wheeling: An arrangement between two utilities in which one utility agrees to transmit electricity owned by the other.

Ultimate Consumer: A consumer that purchases electricity for its own use and not for resale.

Uniform System of Accounts: Prescribed financial rules and regulations established by the Federal Energy Regulatory Commission for utilities subject to its jurisdiction under the authority granted by the Federal Power Act.

Unscheduled Outage Service: Power received by a system from another system to replace power from a generating unit forced out of service.

Used and Useful: A concept used by regulators to determine whether an asset should be included in the utility's rate base. This concept requires that an asset currently provide or be capable of providing a needed service to customers.

Utility (Electric): Privately owned companies and public agencies engaged in the generation, transmission, or distribution of electric power for public use. Public agencies include municipal electric utilities, Federal power projects, rural electrification cooperatives, power districts, and State power authorities and projects.

Utility Generation: Generation by electric systems engaged in selling electric energy to the public.

Volt: The unit of measurement of voltage, electrical force, or pressure. The electrical force that, if steadily applied to a circuit with a resistance of $1 \mathrm{ohm}$, will produce a current of 1 ampere.

Voltage: The difference in electrical potential between any two conductors or between a conductor and ground. It is a measure of the electric energy per electron that electrons can acquire and/or give up as they move between the two conductors.

Voltage Reduction: Any intentional reduction of system voltage by 3 percent or greater for reasons of maintaining the continuity of service of the reporting entity's electric power supply system. 
Waterway: A river, channel, canal, or other navigable body of water used for travel or transport.

Watt: The electrical unit of power. The rate of energy transfer equivalent to 1 ampere flowing under a pressure of 1 volt at unity power factor.

Watthour (Wh): An electrical energy unit of measure equal to 1 watt of power supplied to, or taken from, an electric circuit steadily for 1 hour.

Weir: A dam in a waterway over which water flows and that serves to raise the water level, or direct or regulate flow.

Wheeling: The use of the transmission facilities of one entity to transmit electricity of and for another entity or entities. The most common type of wheeling involves one utility transferring electricity generated by a second utility for sale to a third utility.

Wheeling Charge: An amount charged by one electrical system to transmit the energy of, and for, another system or systems.
Wheeling Service: The movement of electricity from one system to another over transmission facilities of intervening systems. Wheeling service contracts can be established between two or more systems.

Wholesale Sales: Energy supplied to other electric utilities, cooperatives, municipals, and Federal and State electric agencies for resale to ultimate consumers.

Wholesale Wheeling: An arrangement in which electricity is transmitted from a generator to a utility through the transmission facilities of an intervening system.

Wind Energy: Energy produced by harnessing the force of the wind. In a wind energy conversion system such as a windmill, the energy of wind is used to turn the shaft of a generator, which in turn usually produces direct current. This direct current is usually converted to alternating current before being fed into a utility grid system.

Year to Date: The cumulative sum of each month's value starting with January and ending with the current month of the data. 

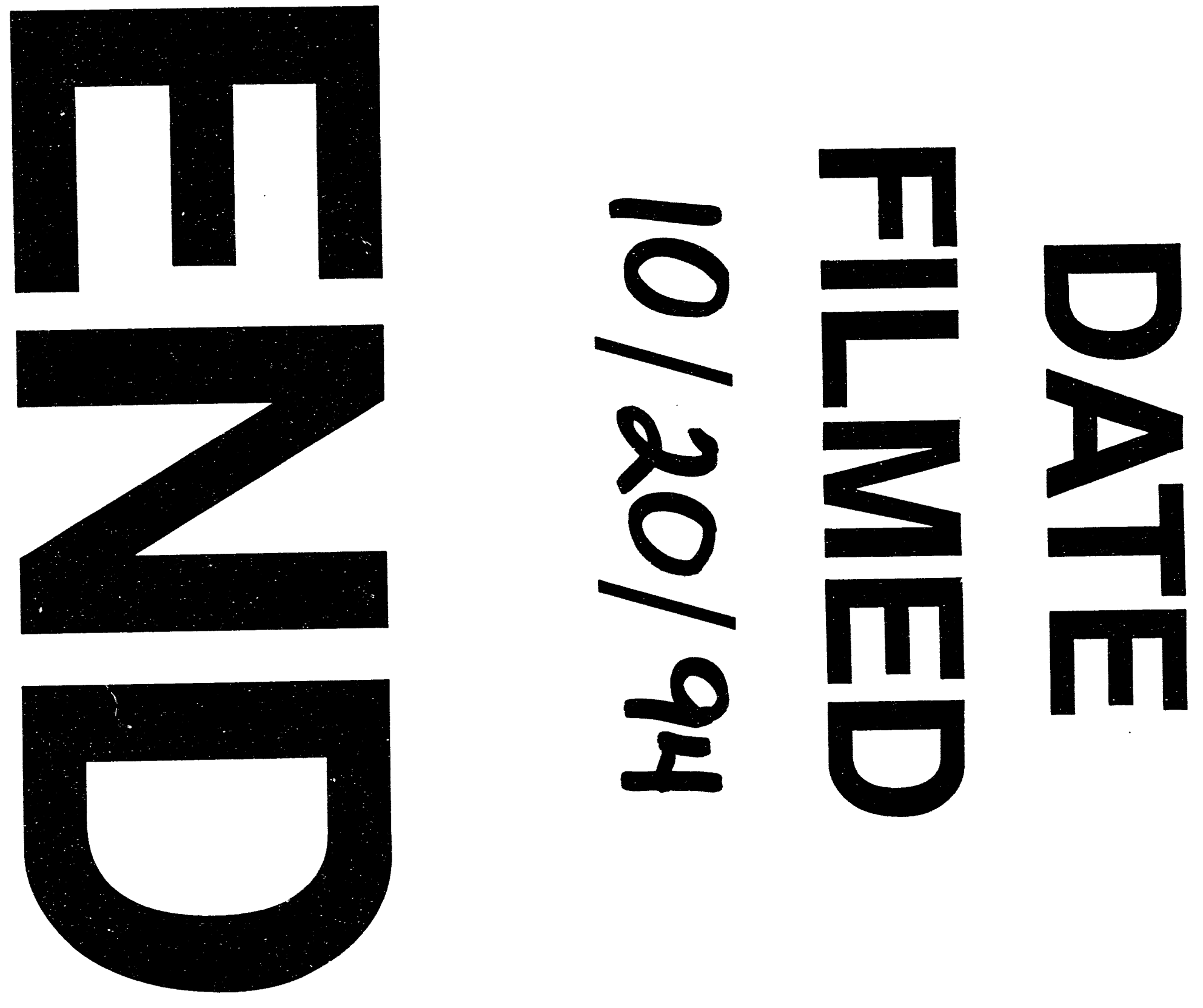


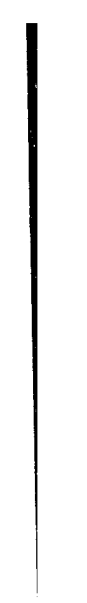

